\title{
State Energy Price and Expenditure Report 1991
}

\section{September 1993}

\author{
Energy Information Administration \\ Office of Energy Markets and End Use \\ - U.S. Department of Energy \\ Washington, DC 20585
}

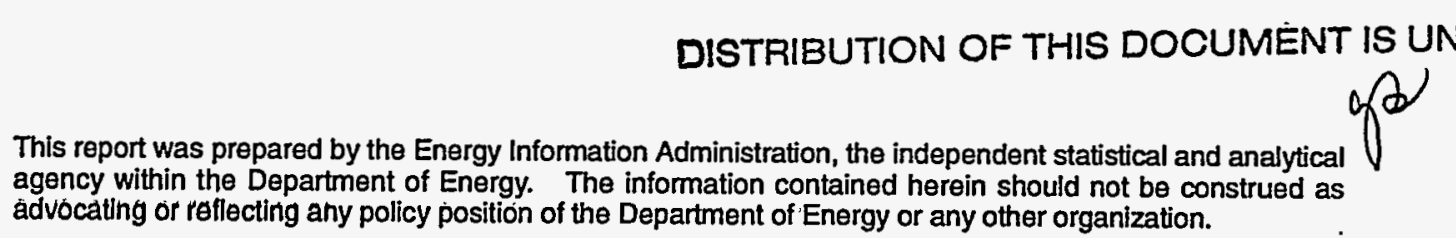





\section{DISCLAIMER}

This report was prepared as an account of work sponsored by an agency of the United States Government. Neither the United States Government nor any agency thereof, nor any of their employees, make any warranty, express or implied, or assumes any legal liability or responsibility for the accuracy, completeness, or usefulness of any information, apparatus, product, or process disclosed, or represents that its use would not infringe privately owned rights. Reference herein to any specific commercial product, process, or service by trade name, trademark, manufacturer, or otherwise does not necessarily constitute or imply its endorsement, recommendation, or favoring by the United States Government or any agency thereof. The views and opinions of authors expressed herein do not necessarily state or reflect those of the United States Government or any agency thereof. 


\section{DISCLAIMER}

Portions of this document may be illegible in electronic image products. Images are produced from the best available original document. 


\section{Contents}

Introduction $\ldots \ldots \ldots \ldots \ldots \ldots \ldots \ldots \ldots \ldots$

U.S. Energy Prices and Expenditures . . . . . . . . . . . . . . . . 5

State Rankings . . . . . . . . . . . . . . . . . . . . . . . . . . 9

Statistical Tables

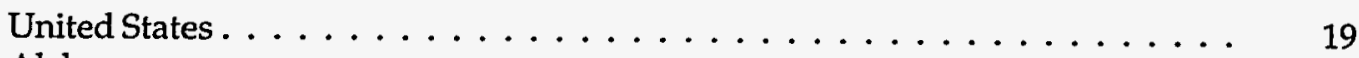

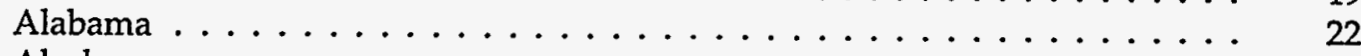

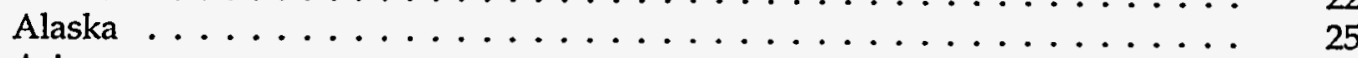

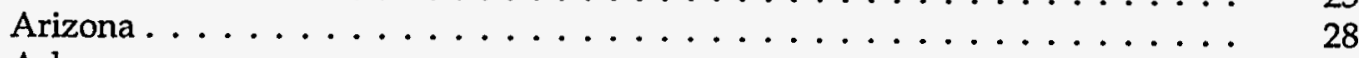

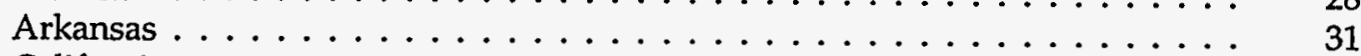

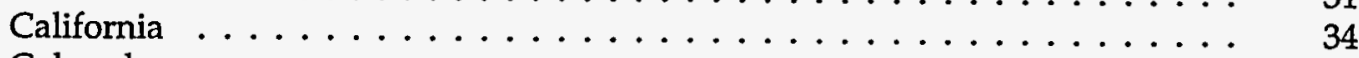

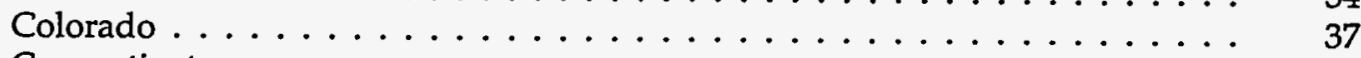

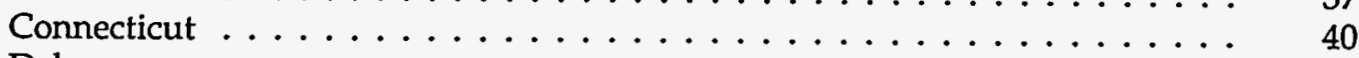

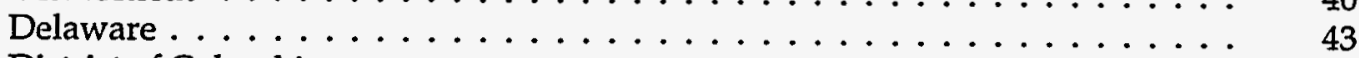

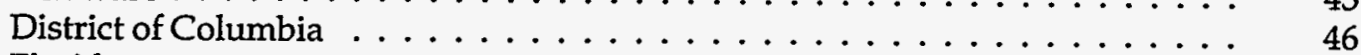

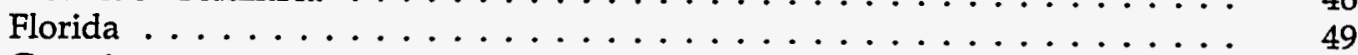

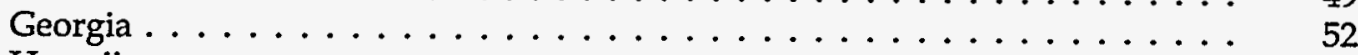

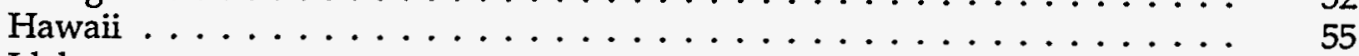

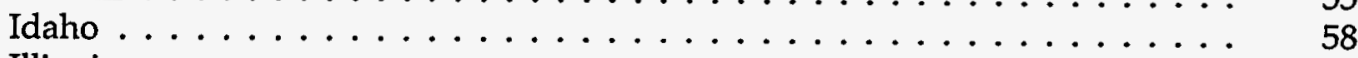

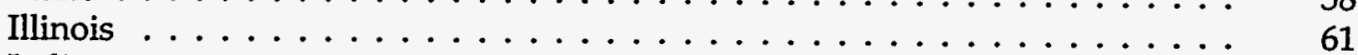

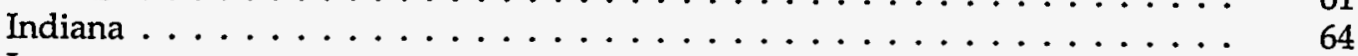

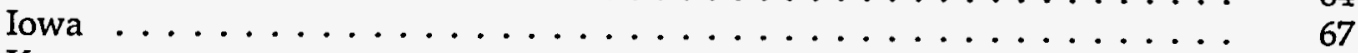

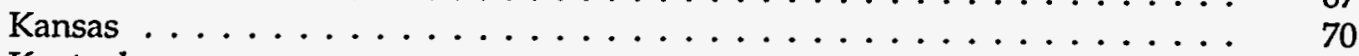

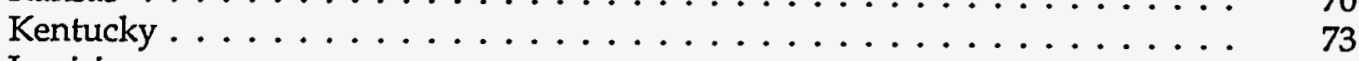

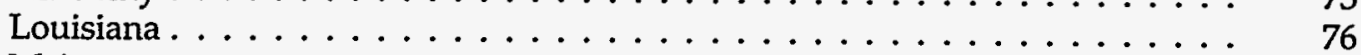

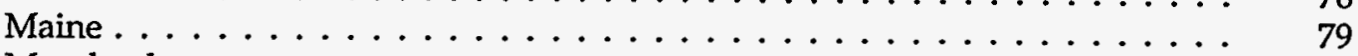

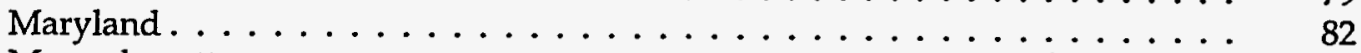

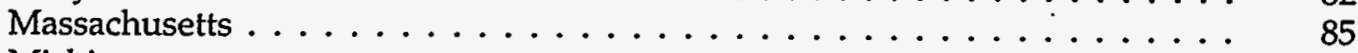

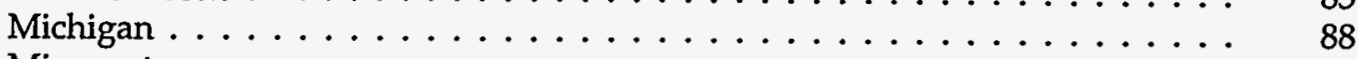

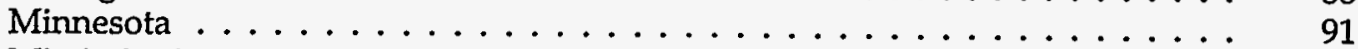

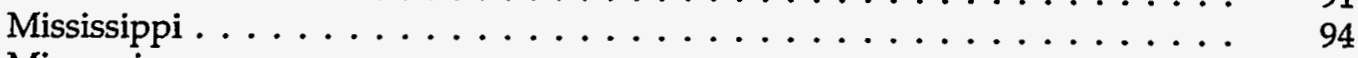

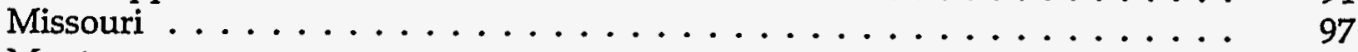

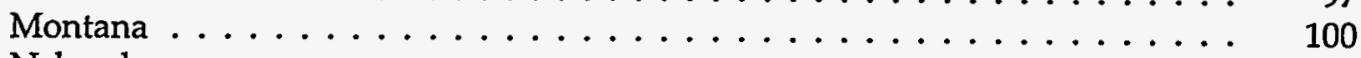

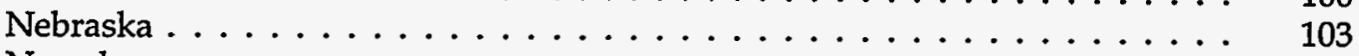

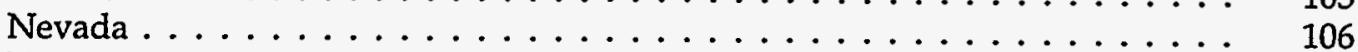

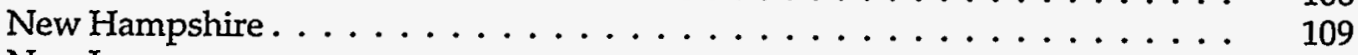

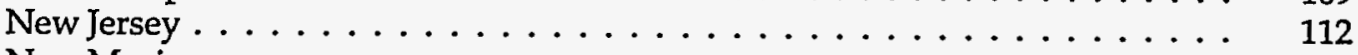

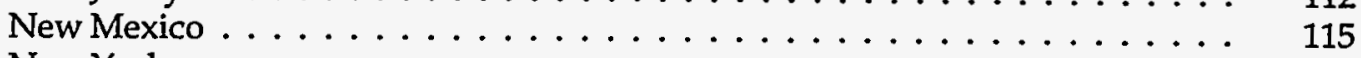

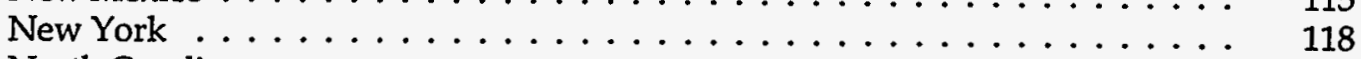

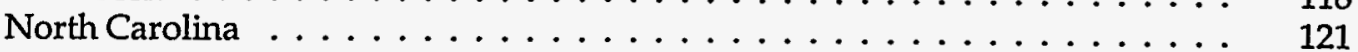

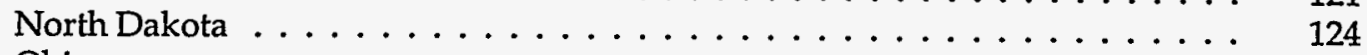

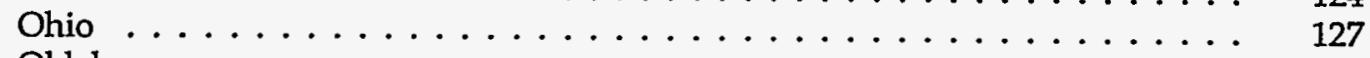

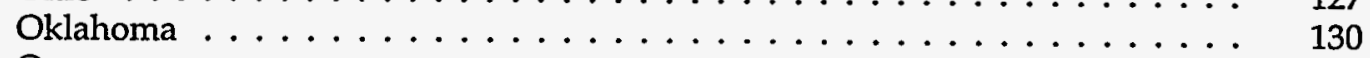

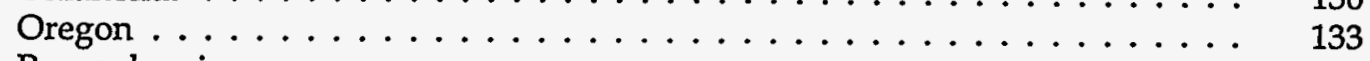

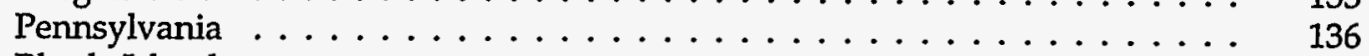

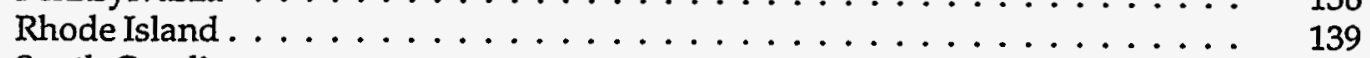

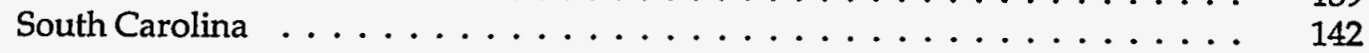




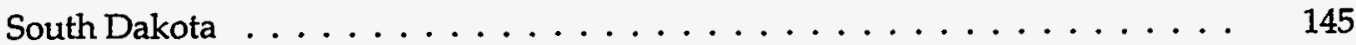

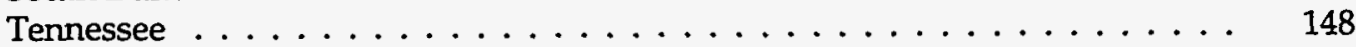

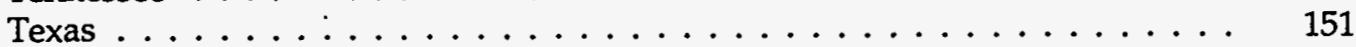

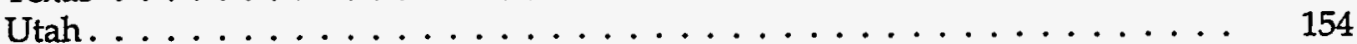

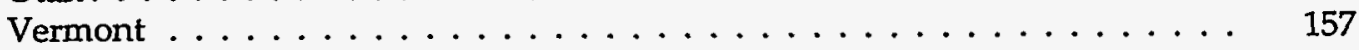

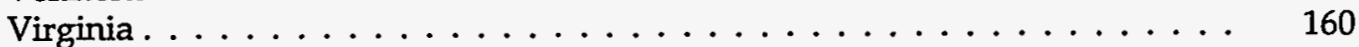

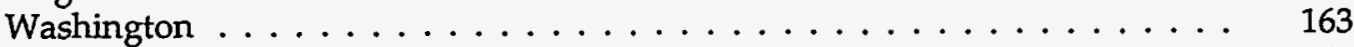

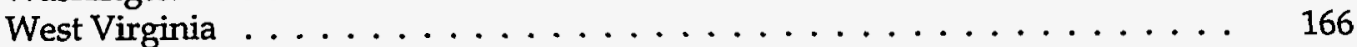

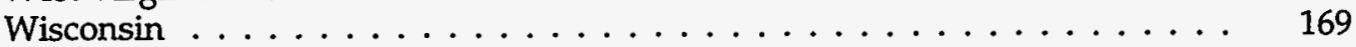

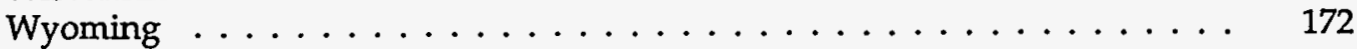

\section{Appendices}

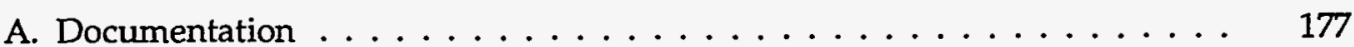

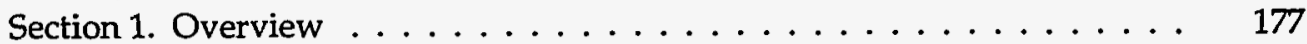

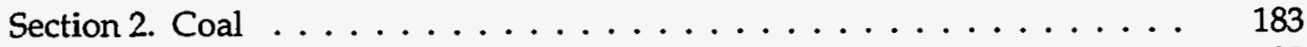

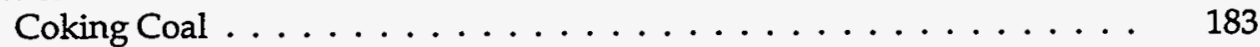

Steam Coal . . . . . . . . . . . . . . . . . . . . . . . . . . . 184

Coal Coke, Imports and Exports . . . . . . . . . . . . . . . . . . . . . . . . . . . . . . . . . . . . . . . . .

Section 3. Natural Gas . . . . . . . . . . . . . . . . . . . . . . . . . . . . 191

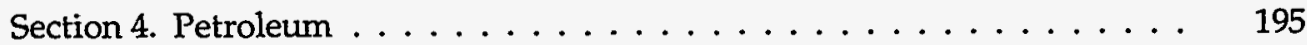

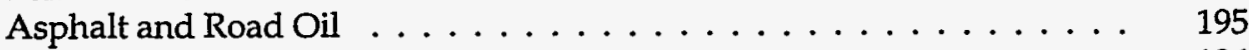

Aviation Gasoline . . . . . . . . . . . . . . . . . . . . . . . . . . . . . . . . . . . . . . . . . . . . . . . . . . . .

Distillate Fuel . . . . . . . . . . . . . . . . . . . . . . . . . . . . 196

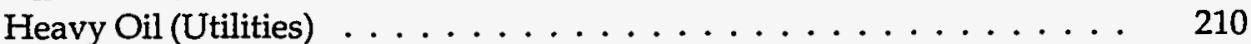

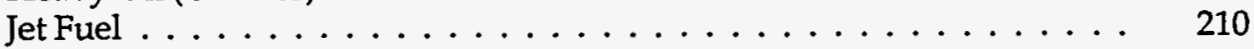

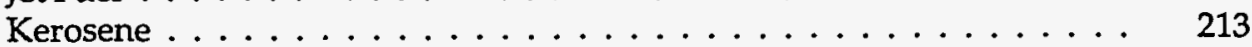

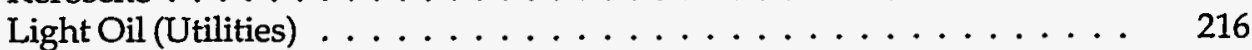

Liquefied Petroleum Gas . . . . . . . . . . . . . . . . . . . . . . . . . . . . . . . . . . . . . . . . . . . . . .

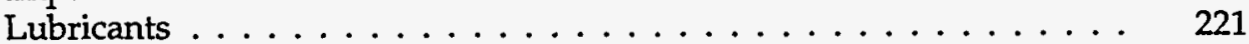

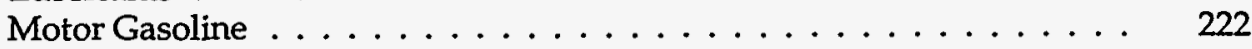

Petroleum Coke (Utilities) . . . . . . . . . . . . . . . . . . . . . . . . . 230

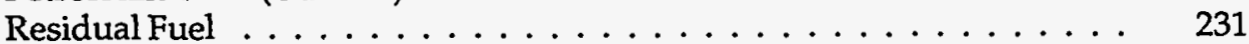

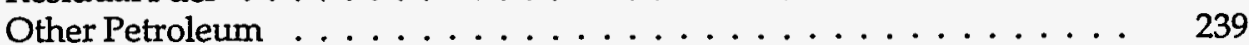

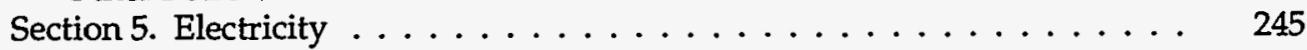

Electricity Consumed by End-Use Sectors . . . . . . . . . . . . . . . . 245

Nuclear Fuel for Generation of Electricity . . . . . . . . . . . . . . . . . . . . . 246

Wood and Waste for Generation of Electricity . . . . . . . . . . . . . 248

Section 6. Consumption Adjustments for Process Fuel and Intermediate

Products .......................... 251

B. Summary of Changes Since the State Energy Price and Expenditure Report $1990 \quad 257$

C. Additional Measures of Energy Consumption, Expenditures, and Prices . . . 263

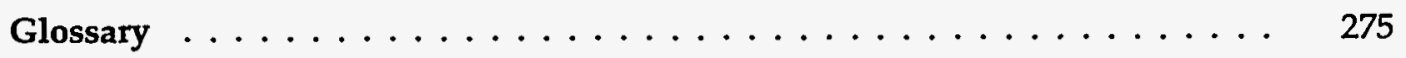




\section{Introduction}

The State Energy Price and Expenditure Report (SEPER) presents energy price and expenditure estimates individually for the 50 States and the District of Columbia and in aggregate for the United States. The price and expenditure estimates are provided by energy source and economic sector and are published for the years 1970, 1975, 1980, and 1985 through 1991. Data for all years, 1970 through 1991, are available on personal computer diskettes. Documentation in Appendix A describes how the price estimates are developed, including sources of data, methods of estimation, and conversion factors applied. This report is an update of the State Energy Price and Expenditure Report 1990, published in September 1992. Changes from the last report are summarized in Appendix B.

Consumption estimates used to calculate expenditures, and the documentation for those estimates, are from the State Energy Data Report 1991, Consumption Estimates (SEDR), published in May 1993. Expenditures are calculated by multiplying the price estimates by the consumption estimates, adjusted to remove process fuel and intermediate product consumption. All expenditures are consumer expenditures, that is, they represent estimates of money directly spent by consumers to purchase energy, generally including taxes (see box on page 2).

Although all prices and expenditures in this report are expressed in nominal dollars, Appendix C, entitled "Additional Measures of Energy Consumption, Expenditures, and Prices," describes the methodology used to develop energy expenditure estimates in constant 1987 dollars and energy price indices in both nominal and real terms. A national-level analysis illustrating the use of these estimates is also presented in Appendix C.

Reliable State data rarely exist, especially as series that are consistent over a long period. Estimates and assumptions are applied to fill data gaps and to maintain consistent definitions in the data series over time. SEPER incorporates the most consistent series and procedures possible. Users of this report (and of the personal computer diskettes) should recognize the limitations imposed on the system due to changing and inadequate data sources. Estimates often are based on a variety of surrogate measures that are selected on the basis of availability, applicability as indicators, continuity over time, and consistency among the various energy commodities. Original source documents for data used in SEPER (cited in the documentation) include descriptions of collection methodologies, universes, imputation or adjustment techniques (if any), and errors associated with the individual processes. Due to the numerous collection forms and procedures associated with these reports, it is not possible to develop a meaningful numerical estimate of the overall errors of the material published in SEPER.

It is also important to note that, even within a State, a single average price may have limited meaning in that it represents a consumption-weighted average over a whole State. For example, urban and rural electricity prices can vary significantly from a State's weighted average, and prices in one region of a State may differ from another because of access to less expensive hydroelectricity. Differences within a State may be greater than differences among adjacent States. Thus, the principal value of the estimates in this report lies in general comparisons among the States, interstate comparisons for a given year, and the analysis of trends over several years.

The five economic sectors used in SEPER correspond to those used in SEDR as follows:

- Residential Sector-The residential sector is considered to consist of all private residences, whether occupied or vacant, owned or rented, including single-family homes, multifamily housing units, and mobile homes. Secondary homes, such as summer homes, are also included. Institutional housing, such as school dormitories, hospitals, and military barracks generally are not included in the residential sector; they are included in the commercial sector.

Note: Throughout this report, the term "State" includes the District of Columbia. 
- Commercial Sector-The commercial sector, as defined economically, consists of business establishments that are not engaged in transportation or in manufacturing or other types of industrial activity (agriculture, mining, or construction). Commercial establishments include hotels, motels, restaurants, wholesale businesses, retail stores, laundries, and other service enterprises; religious and nonprofit organizations; health, social, and educational institutions; and Federal, State, and local governments. Street lights, pumps, bridges, and public services are also included if the establishment operating them is considered commercial.

- Industrial Sector-The industrial sector comprises manufacturing industries, which make up the largest part of the sector, along with mining, construction, agriculture, fisheries, and forestry. Establishments in the sector range from steel mills, to small farms, to companies assembling electronic components.

\section{Taxes in SEPER}

While the objective is to provide price estimates that include all taxes, the data sources used in $S E P E R$ do not treat taxes uniformly. In general, where taxes are included in the source data, they are also included in SEPER. Where taxes are not included but can be separately estimated, they are generally added. In many cases, States and even some localities provide tax exemptions for various kinds of activities or classes of end users, and readers of this report should be careful to consider these exceptions when making detailed comparisons. The Energy Information Administration is continuing to analyze these cases to see if a better representation can be made. The status of tax data in this year's report is summarized below and described more fully in the documentation for each energy source and sector.

\section{End-Use Sectors}

Coal. All steam coal and coking coal prices include taxes in all years. Appropriately, coal imports and exports in the industrial sector do not include end-user taxes.

Natural Gas. Natural gas prices are intended to include all Federal, State, and local taxes, surcharges, and adjustments billed to consumers. However, sales and other taxes itemized directly on customers' bills are frequently not reported as revenues and, therefore, are not included in calculating the prices.

Petroleum. Motor gasoline and diesel fuel prices include excise and other per-gallon taxes but do not include general sales taxes due to wide vari- ation at the local level. Residual fuel oil, distillate fuel oil, and kerosene prices include sales taxes in all years. Residential liquefied petroleum gas prices include taxes in all years, but commercial, industrial, and transportation sector prices do not include taxes from 1985 forward. Jet fuel, aviation gasoline, asphalt and road oil, lubricants and other petroleum products do not include taxes. Other petroleum products are miscellaneous products, petrochemical feedstocks (naphtha, other oils, and still gas), industrial petroleum coke, special naphthas, and waxes:

Electricity. Taxes paid directly by electric utilities (rather than end users) are considered operating costs and are passed on to the end users as part of the price. Depending on jurisdiction, taxes collected from end users and turned over directly to a government authority are not generally included in the reported revenues and, therefore, are not included in calculating the prices.

\section{Electric Utility Sector}

Coal, natural gas, petroleum coke, nuclear, and biomass fuels prices include all taxes, transportation, and handling costs. There are no direct fuel costs (or taxes) for hydroelectric, geothermal, centralized solar, or wind energy. Capital, operation, and maintenance costs and related taxes associated with these energy sources are included indirectly because electricity prices reflect their presence in the rate base. 
- Transportation Sector-The transportation sector consists of private and public vehicles that move people and commodities. Included are automobiles, trucks, buses, motorcycles, railroads and railways (including streetcars), aircraft, ships, barges, and natural gas pipelines.

- Electric Utility Sector-The electric utility sector consists of privately and publicly owned establishments that generate, transmit, distribute, or sell electricity primarily for use by the public and meet the definition of an electric utility. Nonutility power producers are not included in the electric utility sector.

Although end-use allocations of energy consumption and expenditures follow those guidelines as closely as possible, some data are collected using different classifications. For example, electric utilities often classify commercial and industrial users by the quantity of electricity purchases rather than by the business activity of the purchaser. Agricultural use of natural gas is collected and reported in the commercial sector, rather than in the industrial sector. Since agricultural use of natural gas cannot be identified separately, it remains in the commercial sector in this report. Another example is master-metered condominiums, apartments, and establishments with a combination of residential and commercial units. In many cases, residential use is counted in the commercial sector because it is metered and billed as commercial. In those cases, there is no basis for separating residential from commercial use. Readers are advised to consult the SEDR documentation for specific assumptions regarding the consumption estimates.

Where prices for an energy source and sector are not available, comparable prices are substituted. For example, the transportation sector motor gasoline prices are also applied to the commercial and industrial sectors. In some cases, the average of adjacent States' prices is assigned to a missing State price. The documentation elaborates on these price as- sumptions. There are no direct fuel costs for hydroelectric, geothermal, centralized solar, or wind energy. Capital costs associated with these energy sources frequently are extensive and are included indirectly because the prices charged for electricity reflect their presence in the rate base.

Except where specified, it is not generally possible to describe the prices in this report as "wholesale" or "retail." The prices paid in each consuming sector are actually a combination of both sets of prices depending on a number of closely interrelated factors, and the data reflect the combination of prices actually paid by each sector. Almost all residential sector prices are close to retail, reflecting the relatively small quantities of individual purchases and the increased costs of extensive, diffuse, and multilayered distribution systems. Similarly, in the transportation sector almost everyone pays the same retail-like price for motor gasoline, regardless of volume purchased or location of purchase. Conversely, residual fuel oil prices in the transportation sector are certainly more wholesale-like as a result of large deliveries to bulk facilities in major ports. In the same manner, most large industrial and many large commercial expenditures can be thought of as near wholesale, frequently involving direct access to a producer or bulk distribution facility for very large quantities. Many smaller industrial and commercial facilities pay something much closer to retail prices as a result of the small quantities involved and their institutional distance from primary suppliers. Notable exceptions to these relationships include natural gas and electric utilities, which typically establish fixed rates for each of several classes of service depending on representative quantities, service factors, and distribution expenses.

Under contract to the Energy Information Administration, the Battelle Pacific Northwest Laboratories developed many of the price series and estimating procedures incorporated into this report. Descriptions of those data series and procedures are included in the documentation in Appendix A. 



\section{U.S. Energy Prices and Expenditures}

The U.S. energy bill totaled $\$ 467$ billion $^{1}$ in 1991 . The average price paid by consumers for all energy in 1991 was $\$ 8.33$ per million Btu. U.S. energy consumption held at 81 quadrillion Btu per year in 1989, 1990, and 1991. The overall average price of energy jumped by 9 percent in 1990 but rose less than onehalf percent in 1991. Similarly, total expenditures, which had increased by 8 percent in 1990, grew by less than one-half percent in 1991. Americans consumed 322 million Btu per person in 1991, 4 million Btu less than in 1970; however, including inflation,

Table 1. Energy Consumption and Expenditures per Capita, 1970-1991

\begin{tabular}{|c|c|c|c|c|}
\hline & \multicolumn{2}{|c|}{$\begin{array}{l}\text { Consumption } \\
\text { per Capita }\end{array}$} & \multicolumn{2}{|c|}{$\begin{array}{l}\text { Expenditures } \\
\text { per Capita }\end{array}$} \\
\hline & $\begin{array}{c}\text { Million } \\
\text { Btu }\end{array}$ & $\begin{array}{c}\text { Index } \\
1970=1.00\end{array}$ & Dollars & $\begin{array}{c}\text { Index } \\
1970=1.00\end{array}$ \\
\hline 1970 & 326 & 1.00 & 406 & 1.00 \\
\hline 1971 & 328 & 1.00 & 434 & 1.07 \\
\hline 1972 & 341 & 1.04 & 467 & 1.15 \\
\hline 1973 & 352 & 1.08 & 528 & 1.30 \\
\hline 1974 & 340 & 1.04 & 718 & 1.77 \\
\hline 1975 & 328 & 1.00 & 797 & 1.96 \\
\hline 1976 & 342 & 1.05 & 890 & 2.19 \\
\hline 1977 & 347 & 1.06 & 1,002 & 2.47 \\
\hline 1978 & 352 & 1.08 & 1,076 & 2.65 \\
\hline 1979 & 351 & 1.08 & 1,323 & 3.26 \\
\hline 1980 & 335 & 1.03 & 1,650 & 4.06 \\
\hline 1981 & 322 & 0.99 & 1,857 & 4.57 \\
\hline 1982 & 305 & 0.94 & 1,831 & 4.51 \\
\hline 1983 & 301 & 0.92 & 1,775 & 4.37 \\
\hline 1984 & 313 & 0.96 & 1,833 & 4.51 \\
\hline 1985 & 310 & 0.95 & 1,824 & 4.49 \\
\hline 1986 & 308 & 0.94 & 1,581 & 3.89 \\
\hline 1987 & 316 & 0.97 & 1,617 & 3.98 \\
\hline 1988 & 327 & 1.00 & 1,658 & 4.08 \\
\hline 1989 & 328 & 1.00 & 1,750 & 4.31 \\
\hline 1990 & 326 & 1.00 & 1,888 & 4.65 \\
\hline 1991 & 322 & 0.99 & 1,853 & 4.56 \\
\hline
\end{tabular}

Note: Rankings are based on unrounded data.

Source: State Energy Price and Expenditure Data System 1991. they spent over $41 / 2$ times as much money per person for that same amount of energy (Table 1).

Expenditures by Energy Source. Petroleum purchases totaling $\$ 222$ billion accounted for the largest expenditure by energy source in 1991 (Figure 1). Within petroleum, motor gasoline expenditures totaled $\$ 123$ billion or 55 percent of the petroleum total. Distillate fuel expenditures were $\$ 45$ billion (20 percent of petroleum); jet fuel, $\$ 15$ billion (7 percent); and liquefied petroleum gases, $\$ 14$ billion (7 percent). A total of $\$ 185$ billion was spent on electricity, which was generated from many other forms of energy such as coal, nuclear fuel, hydroelectric power, natural gas, and petroleum. Natural gas expenditures totaled $\$ 65$ billion; coal, $\$ 28$ billion; and nuclear fuel, $\$ 4$ billion.

Figure 1. U.S. Energy Expenditures by Source, 1991

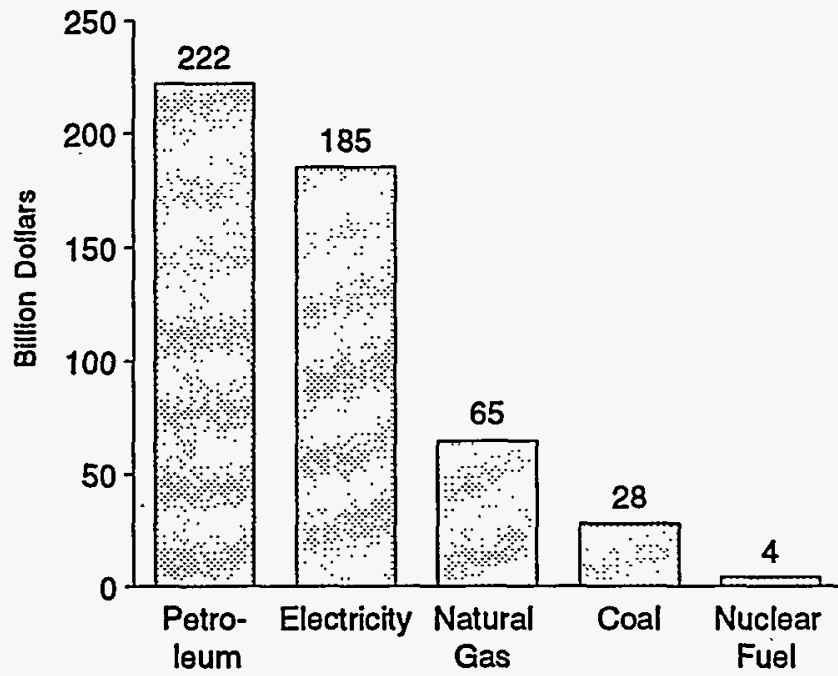

Note: Data are not additive due to expenditure components not shown and the double-counting of primary fuels in electricity. See U.S. expenditures table on page 19. 1991.

\footnotetext{
${ }^{1}$ All prices and expenditures are expressed in current dollars, which are not adjusted to remove the effects of inflation.
} 
Expenditures by End-Use Sector. Transportation sector expenditures continued to exceed all other sectors in 1991, totaling $\$ 171$ billion, followed by $\$ 115$ billion for the residential sector, $\$ 100$ billion for the industrial sector, and $\$ 81$ billion for the commercial sector. In 1991, the transportation and industrial sectors' shares of total expenditures declined while the residential and commercial shares grew (Figure 2). The transportation sector's share dropped by 2 percentage points, reversing the expansion of the previous 2 years. It is the only consuming sector with a smaller share in 1991 than in 1970, down by 6 percentage points. The industrial sector's share consistently decreased after 1984 with an overall decline of 4 percentage points. The residential and commercial sectors' shares of total energy expenditures generally grew during the early 1980's, peaking at 25 percent and 18 percent, respectively, in 1986.

In 1991, electricity purchases accounted for the largest portion of the energy expenses of the residential, commercial, and industrial sectors (Figure 3). Electricity accounted for over half of all energy expenditures in the residential and commercial sectors each year since 1970 with natural gas and petroleum consistently second and third in shares of the two sectors' energy expenses. In the industrial sector more was spent on petroleum than on any
Figure 2. End-Use Sector Shares of U.S. Total Energy Expenditures, 1970-1991

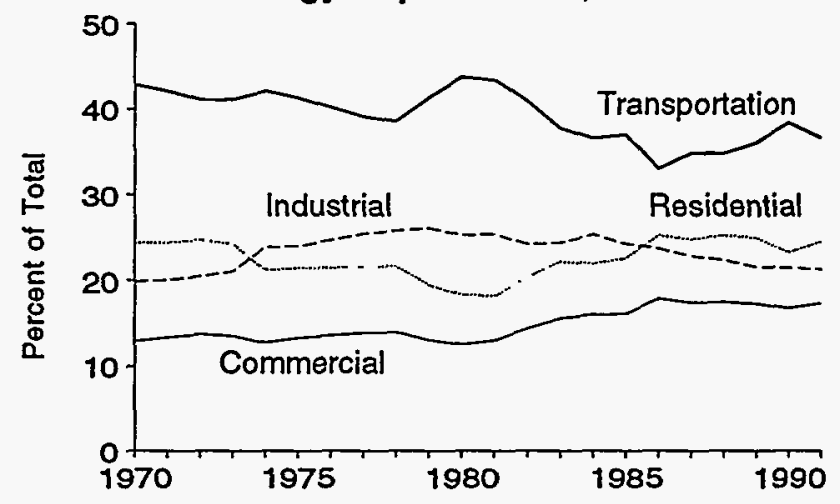
1991.

Source: State Energy Price and Expenditure Data System

other energy source until 1985 when electricity began to take the largest portion of the industrial energy bill, reaching $\$ 44$ billion in 1991 compared to $\$ 32$ billion for petroleum, $\$ 19$ billion for natural gas, and $\$ 4$ billion for coal. In contrast to the industrial sector's diversity of energy sources, the transportation sector was almost exclusively petroleum dependent.

Prices by Energy Source. Of the major energy sources, electricity registered the highest energy

Figure 3. U.S. Energy Expenditures by End-Use Sector and Source, 1991 (Billion Dollars)

Residential

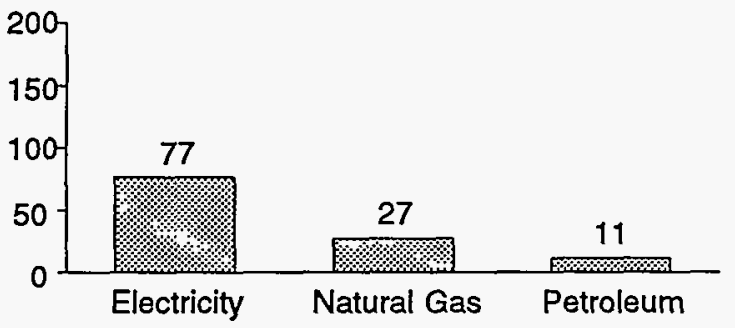

Industrial

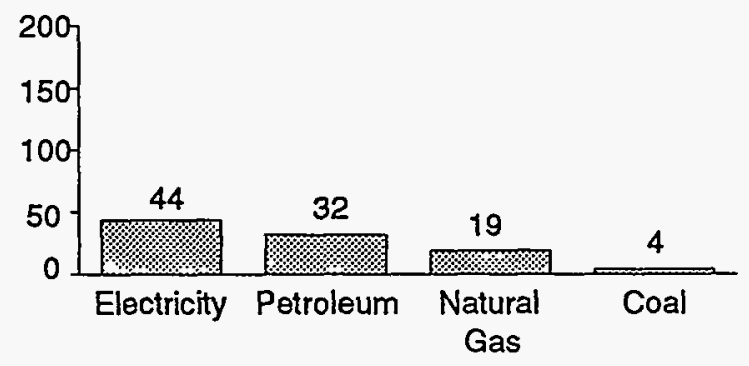

$(s)=$ Less than 0.5 billion dollars.

Source: State Energy Price and Expenditure Data System 1991.

\section{Commercial}

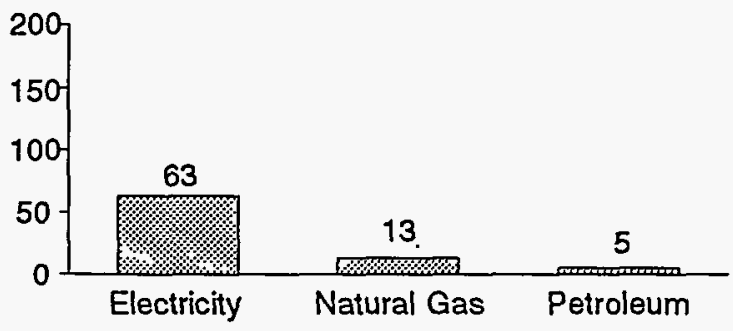

Transportation

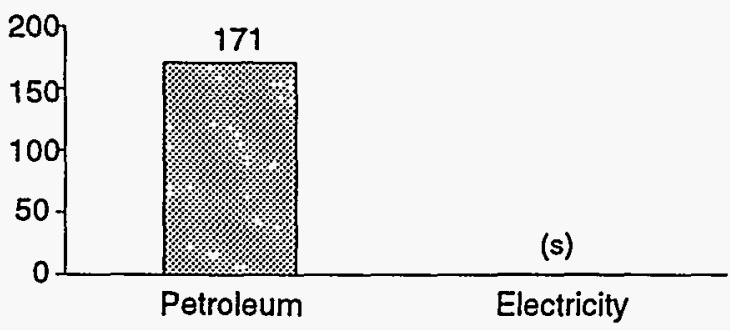


Figure 4. U.S. Energy Prices by Source, 1991

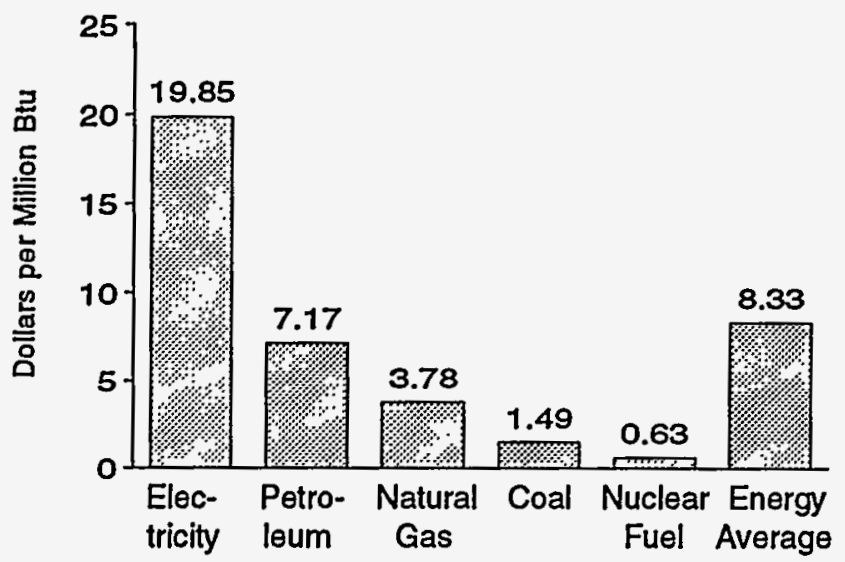

Source: State Energy Price and Expenditure Data System 1991.

price per million Btu. $(\$ 19.85)$, followed by petroleum (\$7.17), natural gas (\$3.78), coal (\$1.49), and nuclear fuel ( $\$ 0.63$ ) (Figure 4). The prices of these major energy sources remained in the same relative order over the 1970 through 1991 period. The price of electricity greatly exceeded other sources' prices because of the significant cost of converting energy from various forms (e.g., fossil fuels, nuclear fuel, hydroelectric energy, and geothermal energy) into electricity as well as somewhat smaller costs for
Figure 5. U.S. Energy Prices by End-Use Sector, 1991

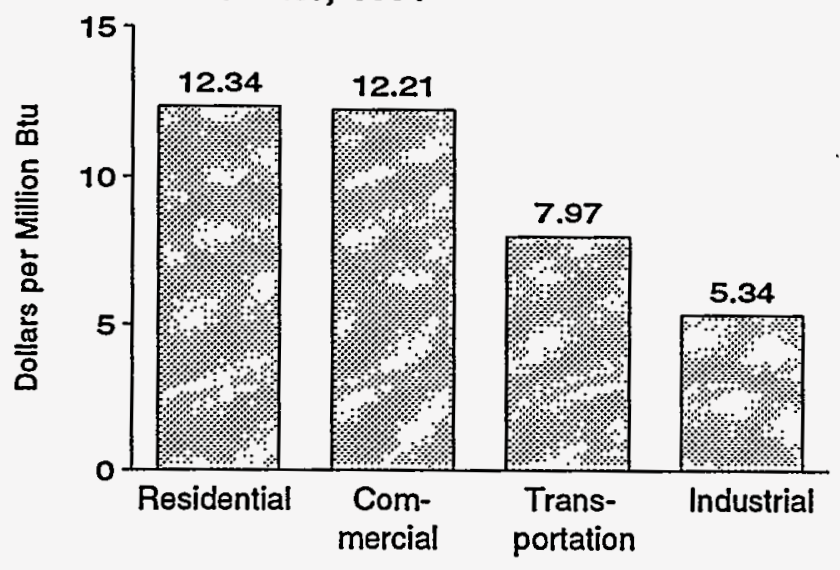

Source: State Energy Price and Expenditure Data System 1991.

transmitting and distributing electricity to end users. In addition, electricity is a premium form of energy because of its flexibility and clean nature at energy consumers' sites.

Prices by End-Use Sector. Because of different mixes of energy sources consumed, the average price paid by major end-use sector in the U.S. economy varied considerably in 1991 (Figure 5). The residential and commercial sectors' average prices were the

Figure 6. U.S. Energy Prices by End-Use Sector and Source, 1991

(Dollars per Million Btu)

\section{Residential}

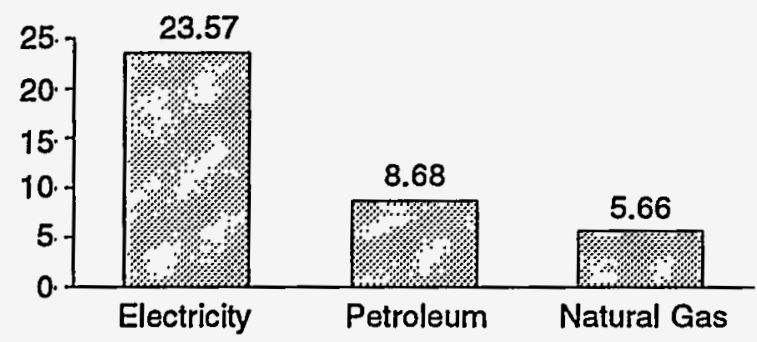

\section{Industrial}

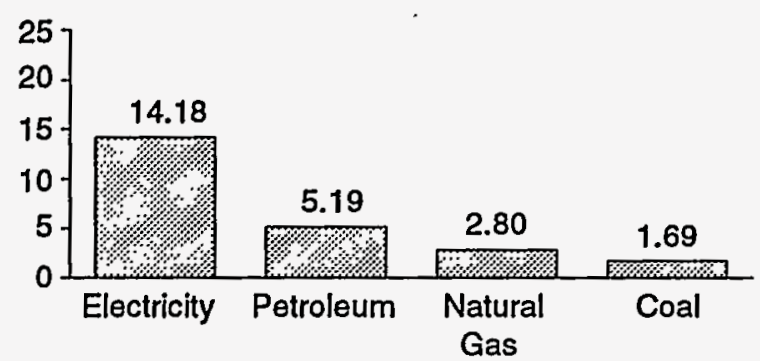

\section{Commercial}

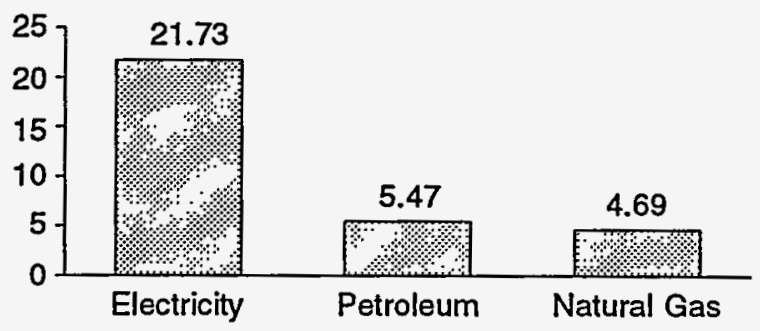

Transportation

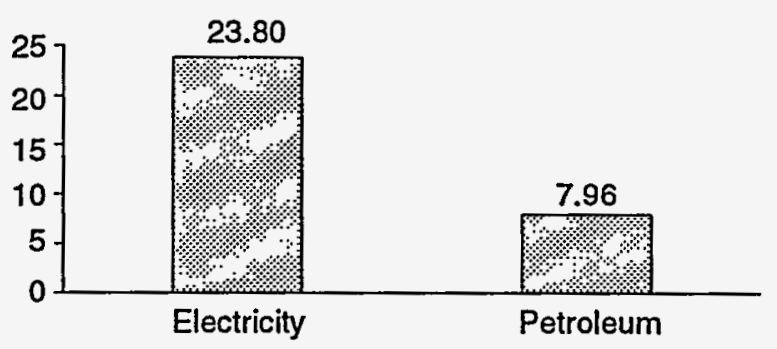

Source: State Energy Price and Expenditure Data System 1991. 
highest, $\$ 12.34$ per million Btu and $\$ 12.21$ per million Btu, respectively. The transportation sector's average price of $\$ 7.97$ per million Btu reflects that sector's reliance on petroleum products. Although the industrial sector consumed more energy than the other sectors, its lower average price of $\$ 5.34$ per million Btu reflects the use of large contracted purchases, which are less costly, and the use of lower-priced fuels, such as coal and residual fuel (Figure 6). The higher average prices paid by residential and commercial consumers are a result, in part, of those sectors' greater reliance on higherpriced electricity. Throughout the 1970's, the residential, commercial, and transportation sectors' average prices for energy were very close, with industrial sector prices about half of the residential prices but following the same year-to-year pattern in price fluctuations. For instance, in 1970, the average price for energy in the residential sector was $\$ 2.12$ per million Btu; the commercial sector, $\$ 1.97$ per million Btu; the transportation sector, $\$ 2.31$ per million $B t u$; and the industrial sector, 83 cents per million Btu. During the 1980's, the transportation sector's average prices dropped significantly below the average residential and commercial prices, following the trend in motor gasoline prices. Industrial prices continued to follow the same fluctuations as the residential and commercial sectors' prices and remained at about half of those prices.

Prices at Electric Utilities. The average price of fuel consumed at electric utilities in 1991 was $\$ 1.37$ per million Btu, compared with the peak price of $\$ 2.01$ per million Btu paid in 1982, when petroleum and natural gas prices were near their highest levels. Petroleum was consistently the highest-priced fuel used by electric utilities during the 1970 through 1991 period. In 1970 through 1975, coal prices were slightly higher than natural gas prices. After 1975, natural gas prices rose rapidly until 1985, while coal prices grew more slowly. The prices of both fuels declined in the second half of the 1980's. Although coal prices have remained at $\$ 1.45$ since 1989 , electric utilities paid decreasing prices for nuclear fuel and natural gas and fluctuating prices for petroleum. In 1991, the price of nuclear fuel was 63 cents per million Btu, $\$ 2.59$ per million Btu for petroleum and $\$ 2.14$ per million Btu for natural gas (Figure 7).

Expenditures at Electric Utilities. Of the $\$ 37$ billion spent by electric utilities to purchase fuel in 1991, 63 percent ( $\$ 23$ billion) was used to purchase coal (Figure 8). Other fuel expenditures were much smaller- $\$ 6$ billion for natural gas, $\$ 4$ billion for nuclear fuel, and $\$ 3$ billion for petroleum products. Electric utility consumption of coal more than
Figure 7. U.S. Prices at Electric Utilities, 1991

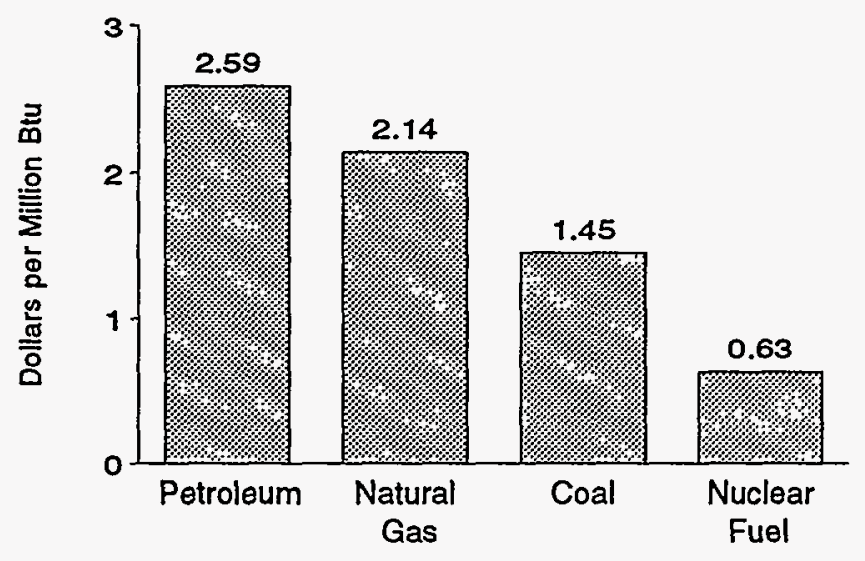

Source: State Energy Price and Expenditure Data System 1991.

doubled during the 1970 through 1991 period. Coal's share of the electric utility annual energy expenditure total ranged from a low of 42 percent in 1977 to a high in 1986 of 64 percent. Petroleum took increasing shares of the electric utility sector fuel budget in the early 1970's and peaked in 1974 at 44 percent. After that, electric utilities generally spent proportionally less each year on petroleum and, by 1991, petroleum purchases were only 8 percent of electric utilities' fuel expenditures. The natural gas share of electric utilities' expenditures ranged from 12 percent in 1974 to 27 percent in 1982. In 1991, natural gas accounted for 17 percent of the electric utility fuel bill. In general, expenditures expanded for coal and nuclear fuel over the entire period covered by this report, while expenditures for natural gas and petroleum contracted after the early 1980's.

Figure 8. U.S. Energy Expenditures at Electric Utilities, 1991

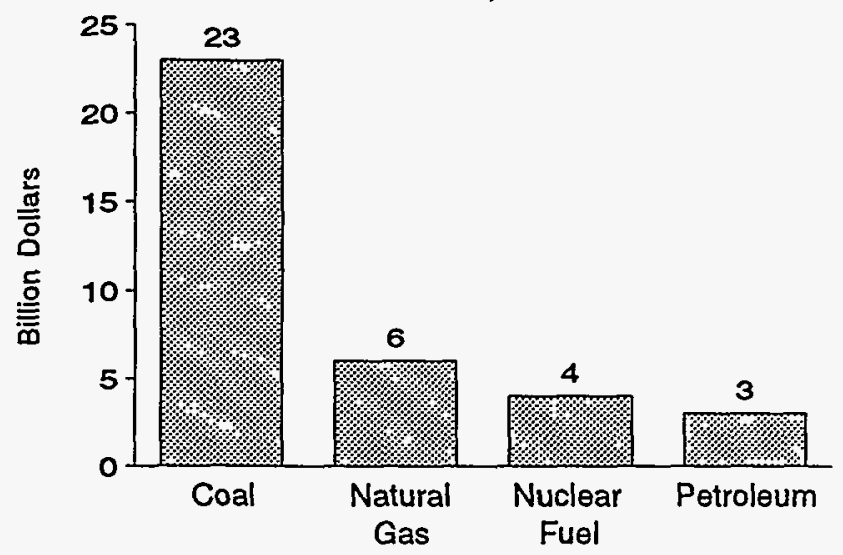

Source: State Energy Price and Expenditure Data System 1991. 


\section{State Rankings}

Energy Prices. The average price paid for energy in 1991 ranged from $\$ 5.69$ per million Btu in Louisiana to $\$ 11.73$ per million Btu in Connecticut (Table 2). In 1970 , the average price for energy ranged from $\$ 0.94$ per million Btu in Louisiana to $\$ 2.40$ per million Btu in Vermont. Some States' ranking positions changed significantly from 1970 to 1991 . For example, the District of Columbia's average price, which ranked 28th in 1970, was 2d highest in 1991, and Pennsylvania's rank rose from $42 \mathrm{~d}$ to 17 th. During the same time period, North Dakota's rank fell from 7th to 48th and South Dakota's fell from 4th to 28th.

Energy Expenditures. State energy expenditures vary widely due to differences in population, climate, and size and type of industry, among other factors. In 1991, energy expenditures ranged from $\$ 1.1$ billion in Vermont to $\$ 47.5$ billion in California (Table 3). The top nine States in the 1970 rankings were still the top nine in 1991, with small variation in their order. The five leading States in energy expenditures in 1991 were California, Texas, New York, Pennsylvania, and Illinois. Their purchases accounted for 35 percent of the Nation's total, about the same as their 34-percent share in 1970.

Energy Expenditures per Capita. Energy expenditures per capita in 1991 ranged from $\$ 1,533$ in Florida to $\$ 3,249$ in Alaska (Table 4). Some States ranked high in their total sum spent on energy but low in the per person rankings, and vice versa; for example, California spent the highest sum for energy in 1991 but ranked 49th in spending per person; New York's total expenditures ranked $3 \mathrm{~d}$, but its per capita expenditures ranked 50th; and Florida was 7 th in expenditures but ranked last in per capita expenditures. On the other hand, Alaska ranked 43d in total expenditures but was 1 st in the per capita ranking. Texas was high on both scales-2d in total expenditures and 5 th in per capita.

Petroleum Prices and Expenditures. In 1991, Statelevel average petroleum prices differed between the highest and lowest cost States by nearly $\$ 3$ per million Btu: the District of Columbia paid the highest,
$\$ 8.82$ per million Btu, while Hawaii paid the lowest, $\$ 5.93$ per million Btu (Table 5). The States that paid the most for petroleum products in 1970 varied from those in 1991. Only Ohio, Iowa, and Nebraska were among the 10 States with the highest petroleum prices in both years. Mississippi paid the $2 \mathrm{~d}$ highest price for petroleum products in 1970 but ranked 42d in petroleum price in 1991. The District of Columbia, which was 50 th in average price for petroleum in 1970, paid the highest price in 1991. Even with its high price, the District of Columbia spent the smallest amount on petroleum products of all the States in 1991 (Table 6). Similarly, while Vermont paid the 2d highest average price for petroleum in 1991, it had the $2 \mathrm{~d}$ smallest petroleum expenditures that year. Nine of the top 10 States that spent the most for all energy in 1991 were also the leading petroleumpurchasing States, in slightly different order. The top five States accounted for 34 percent of all expenditures for petroleum in 1991, unchanged from 1970.

Motor Gasoline Prices and Expenditures. In 1991, motor gasoline prices ranged from $\$ 7.95$ per million Btu in Georgia to $\$ 10.40$ per million Btu in Hawaii, and the U.S. average was $\$ 8.93$ per million Btu (Table 7). In other words, in 1991 motor gasoline purchased in Georgia cost about 31 cents per gallon less than in Hawaii and about 12 cents per gallon less than the U.S. average of $\$ 1.12$ per gallon. The New England States were all in the top 15 rankings in the average price paid for motor gasoline. Expenditures for motor gasoline in 1991 ranged from $\$ 214$ million in the District of Columbia to $\$ 12.8$ billion in California (Table 8). While District of Columbia residents purchased motor gasoline at the Nation's second-highest average price, they spent the smallest amount overall for the commodity. California, with the next to the smallest motor gasoline price average in the Nation, spent the largest amount of money for the product.

Natural Gas Prices and Expenditures. Natural gas price averages ranged from $\$ 1.83$ per million Btu in Alaska to $\$ 6.49$ per million Btu in Connecticut (Table 9). Hawaii paid an unusually high rate (\$14.16 per million Btu) due to its use of small quantities of

Note: Throughout this report, the term "State" includes the District of Columbia. 
liquefied natural gas. All of the Northeastern States with the exception of Vermont fell within the top 10 rankings. Total expenditures for natural gas in 1991 ranged from Maine's $\$ 27$ million to California's $\$ 8.2$ billion (Table 10). California, Texas, and New York spent the most for natural gas, as well as for all petroleum products, motor gasoline, and electricity in 1991. Natural gas expenditures were more concentrated than other energy expenditures in 1991-the top five States accounted for 43 percent of the U.S. total natural gas expenditures versus 36 percent for electricity, 34 percent for petroleum, 34 percent for coal, and 33 percent for motor gasoline.

Coal Prices and Expenditures. In 1991, consumers of coal in Montana were able to purchase the equivalent of a million Btu of coal at a price that was $\$ 3.31$ less than in Alaska: coal prices ranged from 71 cents per million Btu in Montana to $\$ 4.02$ per million Btu in Alaska (Table 11). Coal expenditures ranged from less than one-half million dollars in Rhode Island to $\$ 2.2$ billion in Pennsylvania (Table 12). While the New England States generally ranked high in average price paid for coal, they tended to use little coal, thereby spending small sums for the resource. Pennsylvania, Ohio, Texas, Indiana, and Alabama were the largest purchasers of coal in 1991. Together, they accounted for 34 percent of the Nation's coal expenditures in 1991, much less concentrated than the top five States' share of 49 percent in 1970. In 1970 there were 3 States that consumed no coal, and the 25 States with the smallest coal expenditures accounted for only 3 percent of the total compared with 12 percent in 1991. Texas, which was 30th in coal expenditures in 1970, moved up to 3d place in 1991.

Electricity Prices and Expenditures. Electricity prices recorded the widest range of the major energy sources, varying from $\$ 9.96$ per million Btu in the State of Washington in 1991 to Rhode Island's $\$ 29.75$ per million Btu (Table 13). The large price range, nearly $\$ 20$ per million Btu, reflects the wide price differences of the resources that the States depended on to generate electricity. Washington, for example, relied heavily on hydroelectricity and had the lowest average cost for electricity in both 1970 and 1991. Similarly, almost all of Idaho's electricity came from hydropower; its price ranked 50th in 1991. Montana depended on coal and hydropower for electricity, both low-cost sources of power in 1991. Electricity expenditures in 1991 ranged from $\$ 406$ million in Vermont to $\$ 19.4$ billion in California (Table 14). The five leading State purchasers of electricity accounted for 36 percent of the Nation's total expenditures for electricity, I percent more than in 1970.
Table 2. Energy Prices by State, 1991

(Dollars per Million Btu)

\begin{tabular}{|c|c|c|}
\hline Rank & State & Price \\
\hline 1 & Connecticut & 11.73 \\
\hline 2 & District of Columbia & 11.38 \\
\hline 3 & Vermont & 11.29 \\
\hline 4 & New Hampshire & 11.08 \\
\hline 5 & Arizona & 11.03 \\
\hline 6 & Massachusetts & 10.84 \\
\hline 7 & New York & 10.84 \\
\hline 8 & Florida & 10.55 \\
\hline 9 & Rhode Island & 10.52 \\
\hline 10 & North Carolina & 9.95 \\
\hline 11 & Hawaii & 9.91 \\
\hline 12 & Maryland & 9.67 \\
\hline 13 & Maine & 9.48 \\
\hline 14 & New Jersey & 9.18 \\
\hline 15 & California & 9.14 \\
\hline 16 & Delaware & 8.98 \\
\hline 17 & Pennsylvania & 8.94 \\
\hline 18 & Arkansas & 8.91 \\
\hline 19 & Nevada & 8.79 \\
\hline 20 & South Carolina & 8.78 \\
\hline 21 & Georgia & 8.76 \\
\hline 22 & Virginia & 8.73 \\
\hline 23 & New Mexico & 8.70 \\
\hline 24 & Missouri & 8.67 \\
\hline 25 & Illinois & 8.53 \\
\hline \multirow[t]{2}{*}{26} & Ohio & 8.38 \\
\hline & United States & 8.33 \\
\hline 27 & Tennessee & 8.30 \\
\hline 28 & South Dakota & 8.23 \\
\hline 29 & Michigan & 8.14 \\
\hline 30 & Nebraska & 8.11 \\
\hline 31 & Oregon & 8.07 \\
\hline 32 & Wisconsin & 8.05 \\
\hline 33 & Minnesota & 7.94 \\
\hline 34 & Colorado & 7.93 \\
\hline 35 & Mississippi & 7.93 \\
\hline 36 & Alabama & 7.78 \\
\hline 37 & Montana & 7.74 \\
\hline 38 & Kentucky & 7.68 \\
\hline 39 & Idaho & 7.67 \\
\hline 40 & lowa & 7.43 \\
\hline 41 & Washington & 7.36 \\
\hline 42 & Oklahoma & 7.25 \\
\hline 43 & Kansas & 7.21 \\
\hline 44 & Utah & 6.95 \\
\hline 45 & West Virginia & 6.84 \\
\hline 46 & Indiana & 6.73 \\
\hline 47 & Alaska & 6.49 \\
\hline 48 & North Dakota & 6.48 \\
\hline 49 & Texas & 6.31 \\
\hline 50 & Wyoming & 6.23 \\
\hline 51 & Louisiana & 5.69 \\
\hline
\end{tabular}

Note: Rankings are based on unrounded data. Source: State Energy Price and Expenditure Data System 1991. 
Table 3. Energy Expenditures

by State, 1991

(Million Dollars)

\begin{tabular}{|c|c|c|}
\hline Rank & State & Expenditure \\
\hline 1 & California & 47,460 \\
\hline 2 & Texas & 42,860 \\
\hline 3 & New York & 28,006 \\
\hline 4 & Pennsylvania & 22,279 \\
\hline 5 & Illinois & 21,500 \\
\hline 6 & Ohio & 21,089 \\
\hline 7 & Florida & 20,343 \\
\hline 8 & Michigan & 16,754 \\
\hline 9 & New Jersey & 15,874 \\
\hline 10 & Louisiana & 13,168 \\
\hline 11 & North Carolina & 12,601 \\
\hline 12 & Georgia & 12,479 \\
\hline 13 & Indiana & 11,920 \\
\hline 14 & Virginia & 11,038 \\
\hline 15 & Massachusetts & 10,598 \\
\hline 16 & Missouri & 9,393 \\
\hline 17 & Tennessee & 9,274 \\
\hline 18 & Washington & 8,591 \\
\hline 19 & Alabama & 8,301 \\
\hline 20 & Wisconsin & 8,152 \\
\hline 21 & Maryland & 8,049 \\
\hline 22 & Minnesota & 7,597 \\
\hline 23 & Kentucky & 7,188 \\
\hline 24 & South Carolina & 6,840 \\
\hline 25 & Arizona & 6,432 \\
\hline 26 & Connecticut & 6,200 \\
\hline 27 & Oklahoma & 5,996 \\
\hline 28 & Colorado & 5,415 \\
\hline 29 & lowa & 5,212 \\
\hline 30 & Kansas & 5,143 \\
\hline 31 & Oregon & 5,008 \\
\hline 32 & Mississippi & 4,881 \\
\hline 33 & Arkansas & 4,687 \\
\hline 34 & West Virginia & 3,751 \\
\hline 35 & Nebraska & 3,093 \\
\hline 36 & New Mexico & 2,985 \\
\hline 37 & Utah & 2,868 \\
\hline 38 & Maine & 2,538 \\
\hline 39 & Nevada & 2,405 \\
\hline 40 & Hawaii & 2,039 \\
\hline 41 & Idaho & 1,910 \\
\hline 42 & New Hampshire & 1,906 \\
\hline 43 & Alaska & 1,852 \\
\hline 44 & Rhode Island & 1,756 \\
\hline 45 & Montana & 1,695 \\
\hline 46 & North Dakota & 1,589 \\
\hline 47 & Wyoming & 1,493 \\
\hline 48 & Delaware & 1,387 \\
\hline 49 & South Dakota & 1,270 \\
\hline 50 & District of Columbia & 1,130 \\
\hline \multirow[t]{2}{*}{51} & Vermont & 1,094 \\
\hline & United States & 467,132 \\
\hline
\end{tabular}

Note: Rankings are based on unrounded data. Source: State Energy Price and Expenditure Data System 1991.
Table 4. Energy Expenditures

per Capita by State, 1991

(Dollars per Capita)

\begin{tabular}{|c|c|c|}
\hline Rank & State & Expenditure \\
\hline 1 & Alaska & 3,249 \\
\hline 2 & Wyoming & 3,245 \\
\hline 3 & Louisiana & 3,095 \\
\hline 4 & North Dakota & 2,502 \\
\hline 5 & Texas & 2,471 \\
\hline 6 & Indiana & 2,125 \\
\hline 7 & Montana & 2,096 \\
\hline 8 & West Virginia & 2,081 \\
\hline 9 & Kansas & 2,061 \\
\hline 10 & Maine & 2,057 \\
\hline 11 & New Jersey & 2,047 \\
\hline 12 & Delaware & 2,040 \\
\hline 13 & Alabama & 2,029 \\
\hline 14 & Arkansas & 1,975 \\
\hline 15 & Nebraska & 1,942 \\
\hline 16 & Kentucky & 1,936 \\
\hline 17 & Vermont & 1,930 \\
\hline 18 & Ohio & 1,928 \\
\hline 19 & New Mexico & 1,927 \\
\hline 20 & South Carolina & 1,921 \\
\hline 21 & District of Columbia & 1,899 \\
\hline 22 & Oklahoma & 1,888 \\
\hline 23 & Connecticut & 1,885 \\
\hline 24 & Georgia & 1,884 \\
\hline 25 & Mississippi & 1,882 \\
\hline 26 & Nevada & 1,875 \\
\hline 27 & Tennessee & 1,872 \\
\hline 28 & North Carolina & 1,871 \\
\hline 29 & lowa & 1,865 \\
\hline 30 & Pennsylvania & 1,863 \\
\hline \multirow[t]{2}{*}{31} & Illinois & 1,863 \\
\hline & United States & 1,853 \\
\hline 32 & Idaho & 1,837 \\
\hline 33 & Missouri & 1,821 \\
\hline 34 & South Dakota & 1,804 \\
\hline 35 & Hawaii & 1,793 \\
\hline 36 & Michigan & 1,786 \\
\hline 37 & Massachusetts & 1,767 \\
\hline 38 & Virginia & 1,758 \\
\hline 39 & Rhode Island & 1,747 \\
\hline 40 & New Hampshire & 1,727 \\
\hline 41 & Arizona & 1,716 \\
\hline 42 & Minnesota & 1,714 \\
\hline 43 & Washington & 1,714 \\
\hline 44 & Oregon & 1,714 \\
\hline 45 & Maryland & 1,657 \\
\hline 46 & Wisconsin & 1,645 \\
\hline 47 & Utah & 1,621 \\
\hline 48 & Colorado & 1,603 \\
\hline 49 & California & 1,562 \\
\hline 50 & New York & 1,551 \\
\hline 51 & Florida & 1,533 \\
\hline
\end{tabular}

Note: Rankings are based on unrounded data.

Source: State Energy Price and Expenditure Data System 1991. 
Table 5. Petroleum Prices

by State, 1991

(Dollars per Million Btu)

\begin{tabular}{|c|c|c|}
\hline Rank & State & Price \\
\hline 1 & District of Columbia & 8.82 \\
\hline 2 & Vermont & 8.81 \\
\hline 3 & Rhode Island & 8.50 \\
\hline 4 & Arkansas & 8.39 \\
\hline 5 & Wisconsin & 8.22 \\
\hline 6 & Ohio & 8.17 \\
\hline 7 & North Carolina & 8.12 \\
\hline 8 & lowa & 8.04 \\
\hline 9 & Illinois & 8.04 \\
\hline 10 & Nebraska & 8.03 \\
\hline 11 & Idaho & 8.01 \\
\hline 12 & Colorado & 7.97 \\
\hline 13 & Pennsyivania & 7.96 \\
\hline 14 & Michigan & 7.89 \\
\hline 15 & Minnesota & 7.86 \\
\hline 16 & Tennessee & 7.84 \\
\hline 17 & Arizona & 7.80 \\
\hline 18 & Maryland & 7.72 \\
\hline 19 & North Dakota & 7.68 \\
\hline 20 & West Virginia & 7.67 \\
\hline 21 & Montana & 7.63 \\
\hline 22 & Kentucky & 7.58 \\
\hline 23 & South Carolina & 7.56 \\
\hline 24 & Virginia & 7.53 \\
\hline 25 & Alabama & 7.49 \\
\hline 26 & Connecticut & 7.49 \\
\hline 27 & New Mexico & 7.47 \\
\hline 28 & Nevada & 7.47 \\
\hline 29 & Oregon & 7.43 \\
\hline 30 & Wyoming & 7.42 \\
\hline 31 & Missouri & 7.41 \\
\hline 32 & Oklahoma & 7.41 \\
\hline 33 & South Dakota & 7.38 \\
\hline 34 & Utah & 7.36 \\
\hline 35 & New Hampshire & 7.30 \\
\hline \multirow[t]{2}{*}{36} & Indiana & 7.21 \\
\hline & United States & 7.17 \\
\hline 37 & Washington & 7.16 \\
\hline 38 & Georgia & 7.16 \\
\hline 39 & New York & 7.11 \\
\hline 40 & Massachusetts & 6.98 \\
\hline 41 & California & 6.87 \\
\hline 42 & Mississippi & 6.86 \\
\hline 43 & New Jersey & 6.85 \\
\hline 44 & Alaska & 6.82 \\
\hline 45 & Kansas & 6.80 \\
\hline 46 & Maine & 6.71 \\
\hline 47 & Louisiana & 6.41 \\
\hline 48 & Florida & 6.40 \\
\hline 49 & Delaware & 6.36 \\
\hline 50 & Texas & 5.99 \\
\hline 51 & Hawaii & 5.93 \\
\hline
\end{tabular}

Note: Rankings are based on unrounded data. Source: State Energy Price and Expenditure Data System 1991.
Table 6. Petroleum Expenditures

by State, 1991

(Million Dollars)

\begin{tabular}{|c|c|c|}
\hline Rank & State & Expenditure \\
\hline 1 & Texas & 23,613 \\
\hline 2 & California & 21,023 \\
\hline 3 & New York & 11,822 \\
\hline 4 & Florida & 9,943 \\
\hline 5 & Pennsylvania & 9,244 \\
\hline 6 & Ohio & 8,421 \\
\hline 7 & Illinois & 8,155 \\
\hline 8 & New Jersey & 7,671 \\
\hline 9 & Louisiana & 7,594 \\
\hline 10 & Michigan & 7,034 \\
\hline 11 & North Carolina & 5,849 \\
\hline 12 & Georgia & 5,605 \\
\hline 13 & Virginia & 5,501 \\
\hline 14 & Indiana & 5,387 \\
\hline 15 & Massachusetts & 5,284 \\
\hline 16 & Washington & 4,914 \\
\hline 17 & Missouri & 4,562 \\
\hline 18 & Tennessee & 4,271 \\
\hline 19 & Wisconsin & 3,900 \\
\hline 20 & Minnesota & 3,832 \\
\hline 21 & Alabama & 3,832 \\
\hline 22 & Maryland & 3,809 \\
\hline 23 & Kentucky & 3,592 \\
\hline 24 & Connecticut & 3,126 \\
\hline 25 & South Carolina & 3,057 \\
\hline 26 & Oklahoma & 2,944 \\
\hline 27 & Arizona & 2,739 \\
\hline 28 & Oregon & 2,693 \\
\hline 29 & Colorado & 2,693 \\
\hline 30 & Mississippi & 2,517 \\
\hline 31 & lowa & 2,476 \\
\hline 32 & Kansas & 2,473 \\
\hline 33 & Arkansas & 2,223 \\
\hline 34 & West Virginia & 2,012 \\
\hline 35 & New Mexico & 1,694 \\
\hline 36 & Nebraska & 1,634 \\
\hline 37 & Maine & 1,538 \\
\hline 38 & Hawaii & 1,499 \\
\hline 39 & Utah & 1,420 \\
\hline 40 & Nevada & 1,289 \\
\hline 41 & Alaska & 1,247 \\
\hline 42 & New Hampshire & 1,045 \\
\hline 43 & Idaho & 1,019 \\
\hline 44 & Montana & 972 \\
\hline 45 & North Dakota & 831 \\
\hline 46 & Rhode Island & 780 \\
\hline 47 & Wyoming & 777 \\
\hline 48 & Delaware & 750 \\
\hline 49 & South Dakota & 744 \\
\hline 50 & Vermont & 658 \\
\hline \multirow[t]{2}{*}{51} & District of Columbia & 308 \\
\hline & United States & 222,016 \\
\hline
\end{tabular}

Note: Rankings are based on unrounded data.

Source: State Energy Price and Expenditure Data System 1991. 
Table 7. Motor Gasoline Prices

by State, 1991

(Dollars per Million Btu)

\begin{tabular}{|c|c|c|}
\hline Rank & State & Price \\
\hline 1 & Hawaii & 10.40 \\
\hline 2 & District of Columbia & 10.14 \\
\hline 3 & Rhode Island & 10.09 \\
\hline 4 & Connecticut & 10.04 \\
\hline 5 & Massachusetts & 10.01 \\
\hline 6 & West Virginia & 9.92 \\
\hline 7 & Maine & 9.64 \\
\hline 8 & North Dakota & 9.57 \\
\hline 9 & Maryland & 9.56 \\
\hline 10 & New Hampshire & 9.55 \\
\hline 11 & Louisiana & 9.51 \\
\hline 12 & Delaware & 9.51 \\
\hline 13 & Vermont & 9.48 \\
\hline 14 & Pennsylvania & 9.44 \\
\hline 15 & New York & 9.44 \\
\hline 16 & Nebraska & 9.43 \\
\hline 17 & Minnesota & 9.31 \\
\hline 18 & Colorado & 9.23 \\
\hline 19 & North Carolina & 9.23 \\
\hline 20 & Ohio & 9.23 \\
\hline 21 & Wisconsin & 9.18 \\
\hline 22 & Washington & 9.14 \\
\hline 23 & Oregon & 9.14 \\
\hline 24 & Illinois & 9.13 \\
\hline 25 & Idaho & 9.13 \\
\hline 26 & lowa & 9.11 \\
\hline 27 & New Mexico & 9.10 \\
\hline 28 & Texas & 9.09 \\
\hline 29 & Montana & 9.07 \\
\hline 30 & Virginia & 9.02 \\
\hline 31 & Tennessee & 9.00 \\
\hline 32 & Alaska & 8.99 \\
\hline \multirow[t]{2}{*}{33} & Kentucky & 8.94 \\
\hline & United States & 8.93 \\
\hline 34 & Mississippi & 8.92 \\
\hline 35 & South Dakota & 8.92 \\
\hline 36 & New Jersey & 8.91 \\
\hline 37 & Nevada & 8.83 \\
\hline 38 & Arkansas & 8.81 \\
\hline 39 & Utah & 8.81 \\
\hline 40 & Kansas & 8.72 \\
\hline 41 & Oklahoma & 8.72 \\
\hline 42 & Alabama & 8.69 \\
\hline 43 & Indiana & 8.60 \\
\hline 44 & Arizona & 8.57 \\
\hline 45 & South Carolina & 8.56 \\
\hline 46 & Florida & 8.48 \\
\hline 47 & Wyoming & 8.40 \\
\hline 48 & Michigan & 8.37 \\
\hline 49 & Missouri & 8.30 \\
\hline 50 & California & 8.18 \\
\hline 51 & Georgia & 7.95 \\
\hline
\end{tabular}

Note: Rankings are based on unrounded data.

Source: State Energy Price and Expenditure Data System 1991.
Table 8. Motor Gasoline Expenditures by State, 1991

(Million Dollars)

\begin{tabular}{|c|c|c|}
\hline Rank & State & Expenditure \\
\hline 1 & Califomia & 12,830 \\
\hline 2 & Texas & 9,485 \\
\hline 3 & New York & 6,644 \\
\hline 4 & Florida & 6,297 \\
\hline 5 & Ohio & 5,326 \\
\hline 6 & Pennsylvania & 5,311 \\
\hline 7 & Illinois & 5,005 \\
\hline 8 & Michigan & 4,456 \\
\hline 9 & North Carolina & 3,735 \\
\hline 10 & New Jersey & 3,728 \\
\hline 11 & Georgia & 3,494 \\
\hline 12 & Virginia & 3,340 \\
\hline 13 & Massachusetts & 2,864 \\
\hline 14 & Missouri & 2,786 \\
\hline 15 & Indiana & 2,769 \\
\hline 16 & Tennessee & 2,655 \\
\hline 17 & Washington & 2,604 \\
\hline 18 & Maryland & 2,433 \\
\hline 19 & Wisconsin & 2,406 \\
\hline 20 & Minnesota & 2,398 \\
\hline 21 & Alabama & 2,260 \\
\hline 22 & Louisiana & 2,149 \\
\hline 23 & Kentucky & 2,054 \\
\hline 24 & South Carolina & 1,912 \\
\hline 25 & Arizona & 1,827 \\
\hline 26 & Oklahoma & 1,778 \\
\hline 27 & Colorado & 1,730 \\
\hline 28 & Connecticut & 1,681 \\
\hline 29 & lowa & 1,553 \\
\hline 30 & Oregon & 1,542 \\
\hline 31 & Mississippi & 1,396 \\
\hline 32 & Arkansas & 1,342 \\
\hline 33 & Kansas & 1,284 \\
\hline 34 & West Virginia & 1,007 \\
\hline 35 & New Mexico & 915 \\
\hline 36 & Nebraska & 882 \\
\hline 37 & Utah & 795 \\
\hline 38 & Maine & 715 \\
\hline 39 & Nevada & 712 \\
\hline 40 & New Hampshire & 609 \\
\hline 41 & Idaho & 556 \\
\hline 42 & Montana & 493 \\
\hline 43 & Hawaii & 490 \\
\hline 44 & Rhode Island & 460 \\
\hline 45 & South Dakota & 427 \\
\hline 46 & North Dakota & 415 \\
\hline 47 & Delaware & 389 \\
\hline 48 & Vermont & 337 \\
\hline 49 & Wyoming & 318 \\
\hline 50 & Alaska & 241 \\
\hline \multirow[t]{2}{*}{51} & District of Columbia & 214 \\
\hline & United States & 123,051 \\
\hline
\end{tabular}

Note: Rankings are based on unrounded data.

Source: State Energy Price and Expenditure Data System 1991. 
Table 9. Natural Gas Prices

by State, 1991

(Dollars per Million Btu)

\begin{tabular}{|c|c|c|}
\hline Rank & State & Price \\
\hline 1 & Hawaii & $14.16^{*}$ \\
\hline 2 & Connecticut & 6.49 \\
\hline 3 & New Hampshire & 6.12 \\
\hline 4 & District of Columbia & 6.08 \\
\hline 5 & Rhode Island & 5.94 \\
\hline 6 & Massachusetts & 5.66 \\
\hline 7 & Maine & 5.50 \\
\hline 8 & Pennsylvania & 5.38 \\
\hline 9 & New York & 5.18 \\
\hline 10 & New Jersey & 4.91 \\
\hline 11 & Virginia & 4.76 \\
\hline 12 & Maryland & 4.69 \\
\hline 13 & Georgia & 4.65 \\
\hline 14 & West Virginia & 4.59 \\
\hline 15 & Arizona & 4.58 \\
\hline 16 & Ohio & 4.53 \\
\hline 17 & Missouri & 4.52 \\
\hline 18 & Vermont & 4.51 \\
\hline 19 & California & 4.49 \\
\hline 20 & Michigan & 4.43 \\
\hline 21 & Wisconsin & 4.40 \\
\hline 22 & Illinois & 4.40 \\
\hline 23 & Indiana & 4.25 \\
\hline 24 & South Dakota & 4.21 \\
\hline 25 & Utah & 4.21 \\
\hline 26 & North Dakota & 4.15 \\
\hline 27 & North Carolina & 4.09 \\
\hline 28 & Alabama & 4.08 \\
\hline 29 & Montana & 4.06 \\
\hline 30 & Oregon & 4.01 \\
\hline 31 & Nebraska & 3.93 \\
\hline 32 & Tennessee & 3.91 \\
\hline \multirow[t]{2}{*}{33} & Kentucky & 3.90 \\
\hline & United States & 3.78 \\
\hline 34 & Colorado & 3.74 \\
\hline 35 & Nevada & 3.72 \\
\hline 36 & Minnesota & 3.70 \\
\hline 37 & South Carolina & 3.66 \\
\hline 38 & lowa & 3.65 \\
\hline 39 & New Mexico & 3.62 \\
\hline 40 & Idaho & 3.62 \\
\hline 41 & Washington & 3.55 \\
\hline 42 & Wyoming & 3.48 \\
\hline 43 & Delaware & 3.43 \\
\hline 44 & Arkansas & 3.36 \\
\hline 45 & Kansas & 3.11 \\
\hline 46 & Florida & 2.84 \\
\hline 47 & Oklahoma & 2.78 \\
\hline 48 & Mississippi & 2.59 \\
\hline 49 & Texas & 2.30 \\
\hline 50 & Louisiana & 1.89 \\
\hline 51 & Alaska & 1.83 \\
\hline
\end{tabular}

- Based on small quantities of liquefied natural gas. Note: Rankings are based on unrounded data.

Source: State Energy Price and Expenditure Data System 1991.
Table 10. Natural Gas Expenditures by State, 1991

(Million Dollars)

Rank State Exp

1

2

\section{California}

Texas

New York

8,243

3

4

5

6

7

8

9

10

11

12

13

14

15

16

17

18

19

20

21

22

23

24

24
25

26

27

28

29

30

31

32
33

34

35

36

36

38

39

40

41

42

43

44

45

46

47

48

49

50

51

Illinois

Michigan

6,954

Ohio

4,630

Pennsylvania

4,308

3,538

New Jersey

3,509

2,296

Louisiana 2,209

Indiana $\quad 1,903$

Georgia $\quad 1,471$

Massachusetts $\quad 1,471$

Wisconsin $\quad 1,421$

Oklahoma $\quad 1,263$

Missouri $\quad 1,157$

Minnesota $\quad 1,111$

Florida $\quad 1,004$

Kansas 890

Alabama $\quad 872$

Colorado 854

lowa 833

Tennessee $\quad 830$

Maryland 822

Virginia $\quad 818$

Connecticut $\quad 678$

North Carolina $\quad 676$

Kentucky 659

Arkansas $\quad 635$

Washington 590

Mississippi 524

Utah 513

South Carolina 493

Oregon 472

Arizona 469

West Virginia $\quad 455$

Nebraska 438

New Mexico $\quad 353$

Rhode Island $\quad 331$

Nevada 245

Alaska 212

District of Columbia 188

Wyoming 180

Idaho 174

Montana 169

Delaware $\quad 146$

North Dakota $\quad 110$

South Dakota $\quad 109$

New Hampshire $\quad 87$

Hawaii $\quad 41$

Vermont 32

Maine 27

United States $\quad 64,700$

Note: Rankings are based on unrounded data.

Source: State Energy Price and Expenditure Data System 1991. 
Table 11. Coal Prices

by State, 1991

(Dollars per Million Btu)

\begin{tabular}{|c|c|c|}
\hline Rank & State & Price \\
\hline 1 & Alaska & 4.02 \\
\hline 2 & Rhode Island & 3.51 \\
\hline 3 & Vermont & 3.07 \\
\hline 4 & Maine & 2.67 \\
\hline 5 & Connecticut & 2.19 \\
\hline 6 & California & 1.99 \\
\hline 7 & District of Columbia & 1.96 \\
\hline 8 & Idaho & 1.87 \\
\hline 9 & Florida & 1.86 \\
\hline 10 & New Hampshire & 1.81 \\
\hline 11 & Hawaii & 1.80 \\
\hline 12 & Georgia & 1.80 \\
\hline 13 & Alabama & 1.80 \\
\hline 14 & North Carolina & 1.77 \\
\hline 15 & New Jersey & 1.77 \\
\hline 16 & Massachusetts & 1.75 \\
\hline 17 & Delaware & 1.75 \\
\hline 18 & Illinois & 1.70 \\
\hline 19 & Mississippi & 1.67 \\
\hline 20 & Louisiana & 1.65 \\
\hline 21 & Washington & 1.65 \\
\hline 22 & New York & 1.65 \\
\hline 23 & South Carolina & 1.64 \\
\hline 24 & Michigan & 1.62 \\
\hline 25 & Maryland & 1.62 \\
\hline 26 & Arkansas & 1.61 \\
\hline 27 & Pennsylvania & 1.59 \\
\hline 28 & Virginia & 1.57 \\
\hline 29 & West Virginia & 1.51 \\
\hline 30 & Ohio & 1.49 \\
\hline \multirow[t]{2}{*}{31} & Texas & 1.49 \\
\hline & United States & 1.49 \\
\hline 32 & Indiana & 1.46 \\
\hline 33 & Arizona & 1.43 \\
\hline 34 & Nevada & 1.41 \\
\hline 35 & Wisconsin & 1.41 \\
\hline 36 & New Mexico & 1.38 \\
\hline 37 & Missouri & 1.34 \\
\hline 38 & Oklahoma & 1.32 \\
\hline 39 & Minnesota & 1.30 \\
\hline 40 & Tennessee & 1.28 \\
\hline 41 & Utah & 1.28 \\
\hline 42 & Kentucky & 1.24 \\
\hline 43 & Kansas & 1.23 \\
\hline 44 & South Dakota & 1.22 \\
\hline 45 & Oregon & 1.17 \\
\hline 46 & North Dakota & 1.16 \\
\hline 47 & lowa & 1.15 \\
\hline 48 & Colorado & 1.10 \\
\hline 49 & Wyoming & 0.86 \\
\hline 50 & Nebraska & 0.78 \\
\hline 51 & Montana & 0.71 \\
\hline
\end{tabular}

Note: Rankings are based on unrounded data. Source: State Energy Price and Expenditure Data System 1991.
Table 12. Coal Expenditures

by State, 1991

(Million Dollars)

\begin{tabular}{|c|c|c|}
\hline Rank & State & Expenditure \\
\hline 1 & Pennsylvania & 2,163 \\
\hline 2 & Ohio & 2,110 \\
\hline 3 & Texas & 1,981 \\
\hline 4 & Indiana & 1,958 \\
\hline 5 & Alabama & 1,292 \\
\hline 6 & Illinois & 1,286 \\
\hline 7 & Michigan & 1,232 \\
\hline 8 & West Virginia & 1,209 \\
\hline 9 & Florida & 1,195 \\
\hline 10 & Georgia & 1,161 \\
\hline 11 & Kentucky & 998 \\
\hline 12 & North Carolina & 926 \\
\hline 13 & Tennessee & 725 \\
\hline 14 & Missouri & 718 \\
\hline 15 & Wisconsin & 573 \\
\hline 16 & New York & 567 \\
\hline 17 & Virginia & 558 \\
\hline 18 & Arizona & 498 \\
\hline 19 & South Carolina & 478 \\
\hline 20 & Maryland & 445 \\
\hline 21 & North Dakota & 441 \\
\hline 22 & Utah & 441 \\
\hline 23 & lowa & 398 \\
\hline 24 & Minnesota & 390 \\
\hline 25 & Wyoming & 388 \\
\hline 26 & Oklahoma & 384 \\
\hline 27 & Colorado & 355 \\
\hline 28 & Louisiana & 354 \\
\hline 29 & Arkansas & 347 \\
\hline 30 & Kansas & 330 \\
\hline 31 & New Mexico & 322 \\
\hline 32 & Nevada & 254 \\
\hline 33 & Massachusetts & 205 \\
\hline 34 & Mississippi & 159 \\
\hline 35 & Washington & 147 \\
\hline 36 & California & 127 \\
\hline 37 & Montana & 127 \\
\hline 38 & Nebraska & 118 \\
\hline 39 & New Jersey & 109 \\
\hline 40 & Delaware & 99 \\
\hline 41 & New Hampshire & 63 \\
\hline 42 & Alaska & 51 \\
\hline 43 & Connecticut & 50 \\
\hline 44 & South Dakota & 44 \\
\hline 45 & Oregon & 38 \\
\hline 46 & Maine & 25 \\
\hline 47 & Idaho & 23 \\
\hline 48 & District of Columbia & 3 \\
\hline 49 & Hawaii & 2 \\
\hline 50 & Vermont & 1 \\
\hline \multirow[t]{2}{*}{51} & Rhode Island & * \\
\hline & United States & 27,869 \\
\hline
\end{tabular}

- = Value less than 0.5 million dollars.

Note: Rankings are based on unrounded data.

Source: State Energy Price and Expenditure Data System 1991. 
Table 13. Electricity Prices

by State, 1991

(Dollars per Million Btu)

\begin{tabular}{|c|c|c|}
\hline Rank & State & Price \\
\hline 1 & Rhode Island & 29.75 \\
\hline 2 & Alaska & 28.75 \\
\hline 3 & New York & 28.68 \\
\hline 4 & Connecticut & 28.13 \\
\hline 5 & Massachusetts & 27.94 \\
\hline 6 & New Jersey & 27.76 \\
\hline 7 & California & 27.70 \\
\hline 8 & Hawaii & 27.14 \\
\hline 9 & New Hampshire & 26.76 \\
\hline 10 & Vermont & 25.30 \\
\hline 11 & Maine & 25.21 \\
\hline 12 & Pennsylvania & 23.51 \\
\hline 13 & Arizona & 23.09 \\
\hline 14 & Illinois & 22.41 \\
\hline 15 & Michigan & 21.18 \\
\hline 16 & New Mexico & 21.09 \\
\hline 17 & Florida & 20.99 \\
\hline \multirow[t]{2}{*}{18} & Maryland & 19.97 \\
\hline & United States & 19.85 \\
\hline 19 & Arkansas & 19.76 \\
\hline 20 & Delaware & 19.70 \\
\hline 21 & Kansas & 19.30 \\
\hline 22 & Georgia & 19.28 \\
\hline 23 & North Carolina & 18.96 \\
\hline 24 & Missouri & 18.95 \\
\hline 25 & District of Columbia & 18.34 \\
\hline 26 & Ohio & 17.98 \\
\hline 27 & South Dakota & 17.93 \\
\hline 28 & Texas & 17.91 \\
\hline 29 & Virginia & 17.89 \\
\hline 30 & Mississippi & 17.79 \\
\hline 31 & Louisiana & 17.59 \\
\hline 32 & Colorado & 17.49 \\
\hline 33 & lowa & 17.41 \\
\hline 34 & Oklahoma & 16.99 \\
\hline 35 & North Dakota & 16.90 \\
\hline 36 & Alabama & 16.69 \\
\hline 37 & South Carolina & 16.50 \\
\hline 38 & Nevada & 16.47 \\
\hline 39 & Utah & 16.08 \\
\hline 40 & Minnesota & 16.07 \\
\hline 41 & Nebraska & 16.05 \\
\hline 42 & Wisconsin & 16.01 \\
\hline 43 & Indiana & 15.64 \\
\hline 44 & Tennessee & 15.29 \\
\hline 45 & West Virginia & 14.22 \\
\hline 46 & Kentucky & 12.96 \\
\hline 47 & Wyoming & 12.53 \\
\hline 48 & Oregon & 12.45 \\
\hline 49 & Montana & 12.22 \\
\hline 50 & Idaho & 11.27 \\
\hline 51 & Washington & 9.96 \\
\hline
\end{tabular}

Note: Rankings are based on unrounded data.

Source: State Energy Price and Expenditure Data System 1991.
Table 14. Electricity Expenditures

by State, 1991

(Million Dollars)

\begin{tabular}{|c|c|c|}
\hline Rank & State & Expenditure \\
\hline 1 & California & 19,412 \\
\hline 2 & Texas & 14,271 \\
\hline 3 & New York & 12,665 \\
\hline 4 & Florida & 10,479 \\
\hline 5 & Pennsylvania & 9,235 \\
\hline 6 & Illinois & 8,857 \\
\hline 7 & Ohio & 8,827 \\
\hline 8 & New Jersey & 6,088 \\
\hline 9 & Michigan & 6,042 \\
\hline 10 & North Carolina & 5,973 \\
\hline 11 & Georgia & 5,317 \\
\hline 12 & Virginia & 4,558 \\
\hline 13 & Massachusetts & 4,271 \\
\hline 14 & Indiana & 4,063 \\
\hline 15 & Tennessee & 4,041 \\
\hline 16 & Louisiana & 3,755 \\
\hline 17 & Missouri & 3,654 \\
\hline 18 & Maryland & 3,483 \\
\hline 19 & Alabama & 3,357 \\
\hline 20 & Arizona & 3,249 \\
\hline 21 & South Carolina & 3,212 \\
\hline 22 & Washington & 3,069 \\
\hline 23 & Kentucky & 2,800 \\
\hline 24 & Wisconsin & 2,760 \\
\hline 25 & Minnesota & 2,639 \\
\hline 26 & Connecticut & 2,608 \\
\hline 27 & Oklahoma & 2,267 \\
\hline 28 & Mississippi & 1,940 \\
\hline 29 & Arkansas & 1,860 \\
\hline 30 & Colorado & 1,858 \\
\hline 31 & Oregon & 1,855 \\
\hline 32 & Kansas & 1,839 \\
\hline 33 & lowa & 1,829 \\
\hline 34 & West Virginia & 1,133 \\
\hline 35 & Nebraska & 1,019 \\
\hline 36 & New Mexico & 987 \\
\hline 37 & Maine & 979 \\
\hline 38 & Nevada & 914 \\
\hline 39 & Utah & 857 \\
\hline 40 & New Hampshire & 800 \\
\hline 41 & Hawaii & 769 \\
\hline 42 & Idaho & 694 \\
\hline 43 & Rhode Island & 650 \\
\hline 44 & District of Columbia & 638 \\
\hline 45 & Delaware & 568 \\
\hline 46 & Montana & 546 \\
\hline 47 & Wyoming & 488 \\
\hline 48 & North Dakota & 416 \\
\hline 49 & Alaska & 415 \\
\hline 50 & South Dakota & 409 \\
\hline \multirow[t]{2}{*}{51} & Vermont & 406 \\
\hline & United States & 184,822 \\
\hline
\end{tabular}

Note: Rankings are based on unrounded data.

Source: State Energy Price and Expenditure Data System 1991. 


\section{Statistical Tables}





\begin{tabular}{|c|c|c|c|c|c|c|c|c|c|c|}
\hline Energy Source & 1970 & 1975 & 1980 & 1985 & 1986 & 1987 & 1988 & 1989 & 1990 & 1991 \\
\hline & \multicolumn{10}{|c|}{ Prices in Dollars per Million Btu } \\
\hline 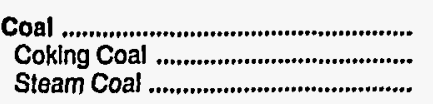 & $\begin{array}{r}0.37 \\
.45 \\
.35\end{array}$ & $\begin{array}{r}1.03 \\
1.65 \\
.90\end{array}$ & $\begin{array}{l}1.47 \\
2.10 \\
1.38\end{array}$ & $\begin{array}{l}1.70 \\
2.03 \\
1.67\end{array}$ & $\begin{array}{l}1.62 \\
1.90 \\
1.60\end{array}$ & $\begin{array}{l}1.54 \\
1.74 \\
1.53\end{array}$ & $\begin{array}{l}1.50 \\
1.79 \\
1.48\end{array}$ & $\begin{array}{l}1.49 \\
1.78 \\
1.47\end{array}$ & $\begin{array}{l}1.49 \\
1.79 \\
1.48\end{array}$ & $\begin{array}{l}1.49 \\
1.83 \\
1.47\end{array}$ \\
\hline 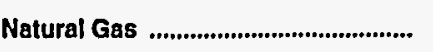 & .59 & 1.18 & 2.86 & 4.61 & 4.07 & 3.77 & 3.78 & 3.85 & 3.85 & 3.78 \\
\hline 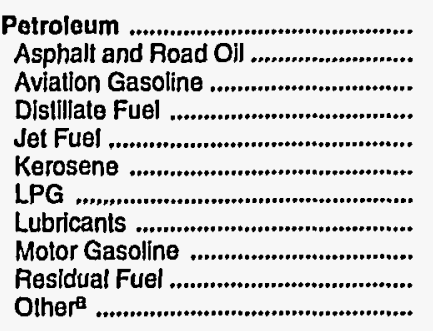 & $\begin{array}{r}1.71 \\
.68 \\
2.17 \\
1.16 \\
.73 \\
1.19 \\
1.46 \\
5.08 \\
2.85 \\
.42 \\
.95\end{array}$ & $\begin{array}{l}3.33 \\
1.89 \\
3.45 \\
2.60 \\
2.05 \\
2.73 \\
2.97 \\
7.48 \\
4.65 \\
1.93 \\
2.43\end{array}$ & $\begin{array}{r}7.35 \\
3.68 \\
9.02 \\
6.70 \\
6.36 \\
6.97 \\
5.64 \\
14.36 \\
9.84 \\
3.88 \\
6.57\end{array}$ & $\begin{array}{r}7.60 \\
4.77 \\
9.99 \\
7.16 \\
5.91 \\
8.17 \\
6.33 \\
17.61 \\
9.01 \\
4.30 \\
7.13\end{array}$ & $\begin{array}{r}5.72 \\
4.34 \\
8.41 \\
5.65 \\
3.92 \\
6.41 \\
6.21 \\
15.59 \\
6.79 \\
2.38 \\
4.59\end{array}$ & $\begin{array}{r}6.00 \\
3.56 \\
7.55 \\
5.89 \\
4.03 \\
6.11 \\
5.85 \\
12.70 \\
7.22 \\
2.86 \\
5.18\end{array}$ & $\begin{array}{r}5.88 \\
3.39 \\
7.41 \\
5.77 \\
3.80 \\
6.15 \\
5.65 \\
14.61 \\
7.32 \\
2.35 \\
4.35\end{array}$ & $\begin{array}{r}6.41 \\
2.95 \\
8.28 \\
6.46 \\
4.39 \\
6.74 \\
5.35 \\
13.30 \\
8.01 \\
2.72 \\
5.11\end{array}$ & $\begin{array}{r}7.44 \\
3.02 \\
9.32 \\
7.67 \\
5.68 \\
8.26 \\
6.53 \\
13.40 \\
9.12 \\
3.16 \\
5.80\end{array}$ & $\begin{array}{r}7.17 \\
3.14 \\
8.71 \\
7.27 \\
4.83 \\
7.83 \\
6.58 \\
15.42 \\
8.93 \\
2.62 \\
5.19\end{array}$ \\
\hline Nuclear Fuel ........................................... & .18 & .24 & .43 & .71 & .70 & .71 & .73 & .70 & .67 & .63 \\
\hline Biomass Fuels at Utilttles .................. & .65 & .92 & 1.74 & .79 & .32 & .95 & .87 & .69 & .52 & .55 \\
\hline 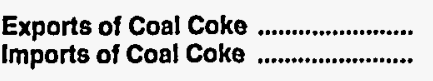 & $\begin{array}{r}1.27 \\
.93\end{array}$ & $\begin{array}{l}2.37 \\
3.47\end{array}$ & $\begin{array}{l}2.54 \\
3.19\end{array}$ & $\begin{array}{l}2.76 \\
2.99\end{array}$ & $\begin{array}{l}2.63 \\
3.11\end{array}$ & $\begin{array}{l}3.37 \\
2.40\end{array}$ & $\begin{array}{l}2.85 \\
2.90\end{array}$ & $\begin{array}{l}2.98 \\
3.79\end{array}$ & $\begin{array}{l}3.53 \\
3.80\end{array}$ & $\begin{array}{l}2.74 \\
3.40\end{array}$ \\
\hline Primary Energy - Flve Sectors ${ }^{b}$.... & 1.08 & 2.19 & 4.58 & 4.92 & 3.96 & 3.97 & 3.88 & 4.12 & 4.52 & 4.34 \\
\hline $\begin{array}{l}\text { Electric Utilly Fuelc } \\
\text { Electricity Purchased by End Users }\end{array}$ & $\begin{array}{r}.32 \\
4.99\end{array}$ & $\begin{array}{r}.96 \\
8.61\end{array}$ & $\begin{array}{r}1.75 \\
13.95\end{array}$ & $\begin{array}{r}1.85 \\
19.05\end{array}$ & $\begin{array}{r}1.55 \\
19.06\end{array}$ & $\begin{array}{r}1.51 \\
18.74\end{array}$ & $\begin{array}{r}1.45 \\
18.68\end{array}$ & $\begin{array}{r}1.48 \\
18.98\end{array}$ & $\begin{array}{r}1.46 \\
19.33\end{array}$ & $\begin{array}{r}1.37 \\
19.85\end{array}$ \\
\hline \multirow[t]{2}{*}{ Total Energy ............................................. } & 1.65 & 3.33 & 6.91 & 8.42 & 7.36 & 7.37 & 7.30 & 7.69 & 8.37 & 8.33 \\
\hline & \multicolumn{10}{|c|}{ Expenditures in Millions of Dollars } \\
\hline $\begin{array}{l}\text { Coal } \\
\text { Coking Coal } \\
\text { Steam Coal }\end{array}$ & $\begin{array}{l}4,594 \\
1,175 \\
3,419\end{array}$ & $\begin{array}{r}13,047 \\
3,692 \\
9,356\end{array}$ & $\begin{array}{r}22,648 \\
3,753 \\
18,895\end{array}$ & $\begin{array}{r}29,719 \\
2,228 \\
27,491\end{array}$ & $\begin{array}{r}27,906 \\
1,825 \\
26,081\end{array}$ & $\begin{array}{r}27,586 \\
1,718 \\
25,868\end{array}$ & $\begin{array}{r}28,371 \\
2,006 \\
26,365\end{array}$ & $\begin{array}{r}28,106 \\
1,934 \\
26,173\end{array}$ & $\begin{array}{r}28,382 \\
1,862 \\
26,520\end{array}$ & $\begin{array}{r}27,869 \\
1,660 \\
26,209\end{array}$ \\
\hline 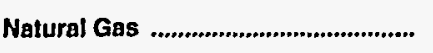 & 10,891 & 20,061 & 51,061 & 72,938 & 59,702 & 58,019 & 61,089 & 65,383 & 64,102 & 64,700 \\
\hline 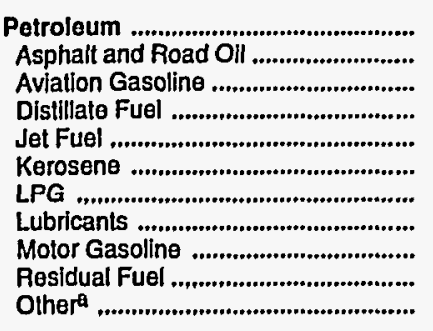 & $\begin{array}{r}48,088 \\
731 \\
218 \\
6,253 \\
1,441 \\
648 \\
2,446 \\
1,532 \\
31,596 \\
2,046 \\
1,177\end{array}$ & $\begin{array}{r}103,858 \\
1,914 \\
245 \\
15,680 \\
4,193 \\
897 \\
5,231 \\
2,277 \\
59,446 \\
10,374 \\
3,602\end{array}$ & $\begin{array}{r}238,408 \\
3,543 \\
580 \\
40,797 \\
13,923 \\
2,291 \\
10,926 \\
5,081 \\
124,408 \\
21,573 \\
15,286\end{array}$ & $\begin{array}{r}223,196 \\
4,916 \\
503 \\
43,611 \\
14,747 \\
1,927 \\
13,108 \\
5,671 \\
118,044 \\
11,495 \\
9,175\end{array}$ & $\begin{array}{r}174,540 \\
4,717 \\
496 \\
34,954 \\
10,505 \\
1,303 \\
12,283 \\
4,909 \\
91,526 \\
7,507 \\
6,339\end{array}$ & $\begin{array}{r}186,402 \\
4,018 \\
344 \\
37,240 \\
11,448 \\
1,196 \\
12,412 \\
4,523 \\
99,809 \\
8,066 \\
7,345\end{array}$ & $\begin{array}{r}189,261 \\
3,848 \\
363 \\
38,362 \\
11,318 \\
1,228 \\
12,301 \\
5,017 \\
103,211 \\
7,258 \\
6,356\end{array}$ & $\begin{array}{r}206,262 \\
3,228 \\
394 \\
43,349 \\
13,434 \\
1,176 \\
11,757 \\
4,682 \\
112,585 \\
8,354 \\
7,304\end{array}$ & $\begin{array}{r}234,509 \\
3,529 \\
419 \\
49,266 \\
17,784 \\
727 \\
13,235 \\
4,857 \\
126,472 \\
8,707 \\
9,513\end{array}$ & $\begin{array}{r}222,016 \\
3,382 \\
363 \\
45,116 \\
14,599 \\
751 \\
14,471 \\
4,999 \\
123,051 \\
6,787 \\
8,498\end{array}$ \\
\hline 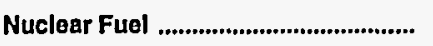 & 44 & 448 & 1,189 & 2,930 & 3,125 & 3,486 & 4,111 & 3,992 & 4,142 & 4,172 \\
\hline Biomass Fuels at Utilities ................. & 2 & 2 & 8 & 11 & 4 & 15 & 15 & 14 & 11 & 12 \\
\hline 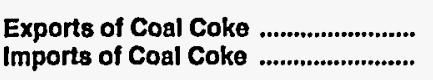 & $\begin{array}{r}-78 \\
4\end{array}$ & $\begin{array}{r}-75 \\
156\end{array}$ & $\begin{array}{r}-130 \\
52\end{array}$ & $\begin{array}{r}-77 \\
43\end{array}$ & $\begin{array}{r}-65 \\
25\end{array}$ & $\begin{array}{r}-48 \\
55\end{array}$ & $\begin{array}{l}-77 \\
194\end{array}$ & $\begin{array}{r}-80 \\
217\end{array}$ & $\begin{array}{r}-50 \\
72\end{array}$ & $\begin{array}{r}-50 \\
93\end{array}$ \\
\hline Primary Energy - Five Sectorg ${ }^{b} . .$. & 63,544 & 137,499 & 313,237 & 328,760 & 265,236 & 275,514 & 282,963 & 303,894 & 331,169 & 318,811 \\
\hline $\begin{array}{l}\text { Electric Utility Fuelc } \\
\text { Electricity Purchased by End Users }\end{array}$ & $\begin{array}{r}-4,316 \\
23,351\end{array}$ & $\begin{array}{r}-16,396 \\
50,680\end{array}$ & $\begin{array}{r}-37,435 \\
98,098\end{array}$ & $\begin{array}{r}-42,558 \\
149,242\end{array}$ & $\begin{array}{r}-35,793 \\
151,806\end{array}$ & $\begin{array}{l}-36,681 \\
154,692\end{array}$ & $\begin{array}{r}-37,435 \\
162,070\end{array}$ & $\begin{array}{r}-38,895 \\
169,340\end{array}$ & $\begin{array}{r}-38,443 \\
176,742\end{array}$ & $\begin{array}{r}-36,501 \\
184,822\end{array}$ \\
\hline 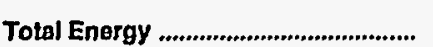 & 82,579 & 171,782 & 373,900 & 435,444 & 381,250 & 393,525 & 407,597 & 434,338 & 469,468 & 467,132 \\
\hline
\end{tabular}

a Includes petroleum coke used at electric utilities.

b Blomass fuels are not included, except those consumed at electric utilities and those added to motor gasoline.

c There are no direct fuel costs for hydroelectric, geothermal, centralized solar, or wind energy.

Nole: Expenditure totals may not equal sum of components due to independent rounding.

Sources: Data sources, estimation procedures, and assumptions are described in the "Documentation" section of this report. 
U Energy Price Estimates by Sector, United States

N 1970, 1975, 1980, and 1985-1991

I (Dollars per Million Btu)

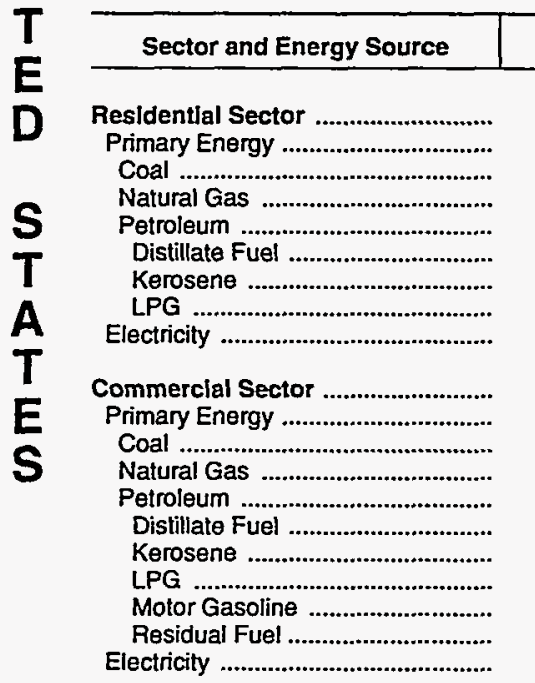

Industrial Sector

Primary Energy

Coal

Coking Coal

Steam Coal ..

Natural Gas

Petroleum

Asphalt and Road Oil ....................

Distillate Fuel ............

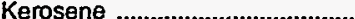

LPG

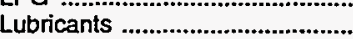

Motor Gasoline

Residual Fuel

Other

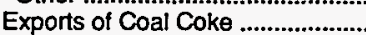

Imports of Coal Coke ......................

Electricity ............................................

Transportation Sector ......................

Primary Energy ...................................

Coal .............................................

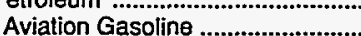

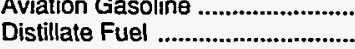

Jet Fuel

LPG

Lubricants

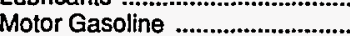

Residual Fuel

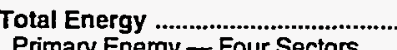

Primary Energy - Four Sectors .....

Electricity

Electric Uttity Sectora ......................

Coal

Natural Gas ...

Petroloum

Heavy Oifb

Light Oib.

um Coke

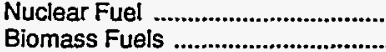

1970

1980

1985

1986

1987

1988

\begin{tabular}{|c|c|}
\hline 1989 & \\
\hline 11.26
\end{tabular}

$2.12 \quad 3.83$

$1.24 \quad 2.13$

$\begin{array}{ll}1.13 & 2.47 \\ 1.06 & 1.67\end{array}$

1.56

$1.39 \quad 2.74$

$1.54 \quad 3.14$

$\begin{array}{lr}2.12 & 4.02 \\ 6.51 & 10.29\end{array}$

7.55

11.14

10.99

10.95
5.70
2.76
5.39
6.71
6.05
6.61
8.64

1988
10.90
5.63
2.64
5.32
6.70
6.11
6.74
8.45

8.64
21.82

21.92

$\begin{array}{rr}91.66 & \mathbf{2 1 . 5 7}\end{array}$

\subsection{6}

$1.97 \quad 4.09$

$\begin{array}{ll}.79 & 1.67 \\ .45 & 1.31\end{array}$

75

$.90 \quad 2.39$

$1.10 \quad 2.42$

$.77 \quad 2.32$

$1.24 \quad 2.60$

$2.86 \quad 4.66$

$\begin{array}{rr}.45 & 1.91 \\ 6.10 & 10.11\end{array}$

10.11

$\begin{array}{ll}.83 & 2.20 \\ .60 & 1.66\end{array}$

$\begin{array}{ll}.60 & 1.66 \\ .45 & 1.50\end{array}$

$\begin{array}{ll}.45 & 1.50 \\ .45 & 1.65\end{array}$

\subsection{8}

4.02
1.55

3.32

5.64

6.45

6.46

5.15

9.77

4.12

16.06

$11.71 \quad 11.35$

5.52

1.80

5.34

6.33

6.35

6.94

8.59

9.01

4.50
21.31

4.72

1.66

4.94

4.55
4.59

$5.90 \quad 5.69$

8.22

2.70

21.10

7.22
3.10

3.10
20.45

10.91

4.41
1.54

4.51

4.41

4.33

4.72

7.80

7.33

2.52
20.34

$\begin{array}{llll}6.09 & 5.40 & 5.19 & 5.03\end{array}$

4.71

3.77
1.87

2.10

1.56

2.52

5.59

3.68

5.54

6.30

5.18

14.36

9.82

3.69

6.58

2.54

3.19

10.81

1.89

2.03

1.81

3.87

6.20

4.77

$6.10 \quad 4.34$

$6.96 \quad 4.44$

$5.66 \quad 5.63$

$17.61 \quad 15.59$

$9.07 \quad 6.80$

$4.24 \quad 2.51$

$\begin{array}{ll}7.16 & 4.61\end{array}$

$2.76 \quad 2.63$

$\begin{array}{rr}2.99 & 3.11\end{array}$

5.19
3.50

1.80

1.90

1.68

1.74

1.64

2.88
4.80

3.56

4.16

4.77

5.16

12.70

7.25
2.87

2.87
5.22

3.37

2.40

13.98

5.03
3.34

1.68

1.79

1.61

2.90

4.48
3.39

3.39

4.61

4.97

14.61

7.32

2.34
4.38

4.38
2.85

2.90

13.78

$12.14 \quad 12.34$

$\begin{array}{lll}2.31 & 4.02 & 8.61\end{array}$

$2.31 \quad 4.02$

8.61

$8.26 \quad 6.22$

6.5

$\begin{array}{lll}8.25 & 6.21 & 6.56\end{array}$

$8 . \overline{25}$

8.25
9.99

9.99
7.50

5.91

8.64

17.61

9.01

4.36

$$
6.21
$$

8.41

6.36

3.92

8.03
15.59

15.59
6.79

2.11

6.56

7.55

6.75

4.03

7.62
12.70

7.22

2.64

$\begin{array}{lll}6.56 & 7.16 & 8.26\end{array}$

$\begin{array}{lll}6.55 & 7.15 & 8.25\end{array}$

6.5 $\quad \overline{15}$

$7.41 \quad 8.28$

$6.59 \quad 7.16$

$3.80 \quad 4.39$

7.77

14.61

7.31
13.30

13.30
8.01

2.32

2.47

22.99

9.32

8.46

5.68

8.46

13.40

2.98

23.49

$\begin{array}{lll}7.30 & 7.69 & 8.37\end{array}$

\subsection{24}

$\begin{array}{rrrr}5.21 & 5.57 & 6.24 & 6.03 \\ 18.68 & 18.98 & 19.33 & 19.85\end{array}$

6.03

$\begin{array}{lll}4.99 & 8.61 & 13.95\end{array}$

$19.05 \quad 19.06$

5.29
18.74

1.45

1.48

$\begin{array}{ll}1.46 & 1.37\end{array}$

$\begin{array}{llll}1.47 & 1.45 & 1.45 & 1.45\end{array}$

$2.26 \quad 2.36 \quad 2.32$

$\begin{array}{lll}2.49 & 2.95 & 3.41\end{array}$

$2.41 \quad 2.85$

3.81

9.89

4.26

3.30

5.62

.83

2.14
2.59

2.46

4.84

.82

.63

Primary Energy - Flve Sectors ${ }^{c}$..

1.08

2.19

4.58

4.92

3.96

3.97

3.88

4.12

4.52

4.34

a There are no direct fuel costs for hydroelectric, geothermal, centralized solar, or wind energy.

beavy oil includes Grade Nos. 4, 5, and 6, and residual fuel oils. Light oil includes Grade No. 2 heating oil, kerosene, and jet fuel.

c Biomass fuels are not included, except those consumed at electric utilities and those added to motor gasoline.

$\rightarrow$ No consumption, including cases where adjustments were made. See "Consumption Adjustments for Process Fuel and Intermediate Products." 


\begin{tabular}{|c|c|c|c|c|c|c|c|c|c|c|}
\hline Sector and Energy Source & 1970 & 1975 & 1980 & 1985 & 1986 & 1987 & 1988 & 1989 & 1990 & 1991 \\
\hline 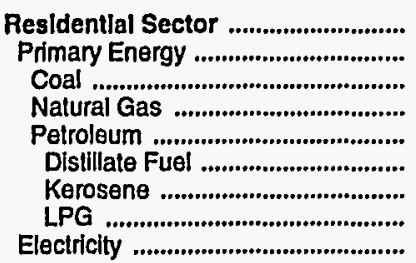 & $\begin{array}{r}20,083 \\
9,731 \\
173 \\
5,272 \\
4,286 \\
2,603 \\
459 \\
1,225 \\
10,352\end{array}$ & $\begin{array}{r}36,844 \\
16,201 \\
209 \\
8,410 \\
7,582 \\
4,954 \\
504 \\
2,124 \\
20,644\end{array}$ & $\begin{array}{r}68,825 \\
30,367 \\
175 \\
17,497 \\
12,695 \\
9,234 \\
887 \\
2,575 \\
38,458\end{array}$ & $\begin{array}{r}98,307 \\
39,636 \\
225 \\
27,136 \\
12,274 \\
7,910 \\
1,391 \\
2,974 \\
58,672\end{array}$ & $\begin{array}{r}96,203 \\
35,427 \\
215 \\
25,147 \\
10,064 \\
6,428 \\
866 \\
2,770 \\
60,776\end{array}$ & $\begin{array}{r}97,552 \\
34,235 \\
181 \\
23,926 \\
10,127 \\
6,236 \\
784 \\
3,107 \\
63,318\end{array}$ & $\begin{array}{r}102,773 \\
35,980 \\
174 \\
25,332 \\
10,474 \\
6,497 \\
967 \\
3,011 \\
66,793\end{array}$ & $\begin{array}{r}108,423 \\
39,180 \\
156 \\
26,953 \\
12,070 \\
7,038 \\
863 \\
4,170 \\
69,243\end{array}$ & $\begin{array}{r}109,265 \\
36,890 \\
186 \\
25,442 \\
11,262 \\
6,703 \\
567 \\
3,992 \\
72,376\end{array}$ & $\begin{array}{r}114,740 \\
37,914 \\
174 \\
26,508 \\
11,233 \\
6,361 \\
611 \\
4,261 \\
76,825\end{array}$ \\
\hline 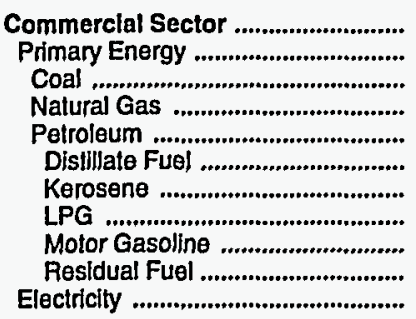 & $\begin{array}{r}10,668 \\
3,333 \\
98 \\
1,844 \\
1,391 \\
646 \\
47 \\
127 \\
247 \\
323 \\
7,335\end{array}$ & $\begin{array}{r}22,835 \\
6,680 \\
162 \\
3,385 \\
3,133 \\
1,423 \\
114 \\
242 \\
415 \\
-939 \\
16,155\end{array}$ & $\begin{array}{r}46,881 \\
16,260 \\
135 \\
8,858 \\
7,267 \\
3,337 \\
262 \\
296 \\
1,046 \\
2,325 \\
30,621\end{array}$ & $\begin{array}{r}70,263 \\
20,139 \\
193 \\
13,368 \\
6,579 \\
3,965 \\
228 \\
496 \\
866 \\
1,025 \\
50,124\end{array}$ & $\begin{array}{r}68,444 \\
16,946 \\
178 \\
11,770 \\
4,998 \\
2,731 \\
295 \\
469 \\
720 \\
784 \\
51,498\end{array}$ & $\begin{array}{r}68,777 \\
16,807 \\
154 \\
11,601 \\
5,053 \\
2,680 \\
277 \\
487 \\
795 \\
813 \\
51,970\end{array}$ & $\begin{array}{r}71,579 \\
17,110 \\
158 \\
12,377 \\
4,576 \\
2,487 \\
123 \\
491 \\
810 \\
665 \\
54,469\end{array}$ & $\begin{array}{r}75,467 \\
17,938 \\
135 \\
12,909 \\
4,894 \\
2,731 \\
153 \\
520 \\
818 \\
672 \\
57,528\end{array}$ & $\begin{array}{r}78,922 \\
18,244 \\
150 \\
12,683 \\
5,412 \\
2,971 \\
80 \\
555 \\
1,011 \\
796 \\
60,678\end{array}$ & $\begin{array}{r}81,488 \\
18,026 \\
132 \\
13,181 \\
4,714 \\
2,697 \\
73 \\
620 \\
767 \\
557 \\
63,462\end{array}$ \\
\hline 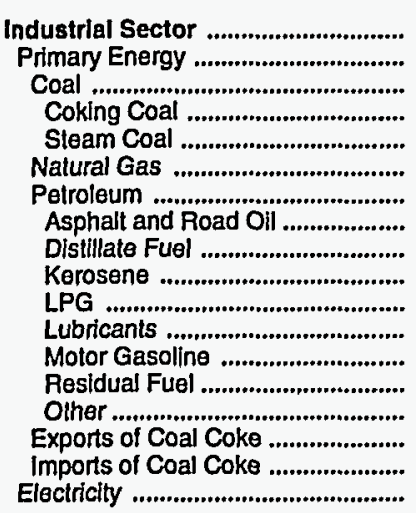 & $\begin{array}{r}16,458 \\
10,834 \\
2,082 \\
1,175 \\
907 \\
2,625 \\
6,202 \\
731 \\
866 \\
142 \\
1,046 \\
786 \\
824 \\
635 \\
1,171 \\
-78 \\
4 \\
5,624\end{array}$ & $\begin{array}{r}41,169 \\
27,409 \\
5,498 \\
3,692 \\
1,806 \\
5,844 \\
15,986 \\
1,914 \\
2,907 \\
278 \\
2,760 \\
1,119 \\
1,039 \\
2,367 \\
3,601 \\
-75 \\
156 \\
13,760\end{array}$ & $\begin{array}{r}94,520 \\
65,657 \\
5,888 \\
3,753 \\
2,135 \\
16,350 \\
43,497 \\
3,543 \\
7,232 \\
1,143 \\
7,967 \\
2,613 \\
1,553 \\
4,175 \\
15,271 \\
-130 \\
52 \\
28,863\end{array}$ & $\begin{array}{r}105,723 \\
6,533 \\
5,244 \\
2,228 \\
3,016 \\
21,615 \\
38,708 \\
4,916 \\
7,208 \\
308 \\
9,399 \\
2,916 \\
1,977 \\
2,817 \\
9,166 \\
-77 \\
43 \\
40,190\end{array}$ & $\begin{array}{r}90,496 \\
51,224 \\
4,759 \\
1,825 \\
2,934 \\
16,479 \\
30,027 \\
4,717 \\
4,478 \\
143 \\
8,836 \\
2,525 \\
1,402 \\
1,598 \\
6,329 \\
-65 \\
25 \\
39,271\end{array}$ & $\begin{array}{r}89,975 \\
50,866 \\
4,466 \\
1,718 \\
2,748 \\
15,909 \\
30,485 \\
4,018 \\
5,082 \\
135 \\
8,662 \\
2,326 \\
1,487 \\
1,441 \\
7,335 \\
-48 \\
55 \\
39,109\end{array}$ & $\begin{array}{r}91,315 \\
50,807 \\
4,749 \\
2,006 \\
2,743 \\
17,257 \\
28,685 \\
3,848 \\
4,652 \\
138 \\
8,627 \\
2,580 \\
1,414 \\
1,082 \\
6,344 \\
-77 \\
194 \\
40,507\end{array}$ & $\begin{array}{r}93,812 \\
51,557 \\
4,651 \\
1,934 \\
2,717 \\
18,770 \\
27,999 \\
3,228 \\
5,473 \\
160 \\
6,907 \\
2,408 \\
1,599 \\
934 \\
7,290 \\
-80 \\
217 \\
42,255\end{array}$ & $\begin{array}{r}100,951 \\
57,593 \\
4,649 \\
1,862 \\
2,787 \\
19,348 \\
33,574 \\
3,529 \\
6,698 \\
81 \\
8,504 \\
2,498 \\
1,685 \\
1,087 \\
9,492 \\
-50 \\
72 \\
43,358\end{array}$ & $\begin{array}{r}99,701 \\
55,497 \\
4,399 \\
1,660 \\
2,739 \\
18,913 \\
32,144 \\
3,382 \\
5,841 \\
67 \\
9,415 \\
2,571 \\
1,729 \\
658 \\
8,480 \\
-50 \\
93 \\
44,204\end{array}$ \\
\hline 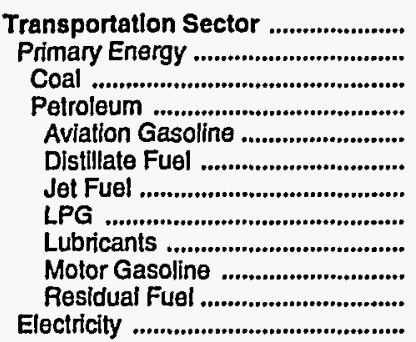 & $\begin{array}{r}35,370 \\
35,330 \\
3 \\
35,327 \\
218 \\
2,058 \\
1,441 \\
49 \\
745 \\
30,525 \\
291 \\
40\end{array}$ & $\begin{array}{r}70,934 \\
70,814 \\
1 \\
70,813 \\
245 \\
5,938 \\
4,150 \\
105 \\
1,158 \\
57,992 \\
1,226 \\
120\end{array}$ & $\begin{array}{r}163,674 \\
163,517 \\
- \\
163,517 \\
580 \\
20,090 \\
13,856 \\
88 \\
2,468 \\
121,809 \\
4,626 \\
156\end{array}$ & $\begin{array}{r}161,150 \\
160,894 \\
- \\
160,894 \\
-503 \\
24,027 \\
14,747 \\
239 \\
2,754 \\
115,201 \\
3,422 \\
256\end{array}$ & $\begin{array}{r}126,107 \\
125,847 \\
125,847 \\
496 \\
41,012 \\
10,505 \\
208 \\
2,384 \\
89,404 \\
1,838 \\
260\end{array}$ & $\begin{array}{r}137,220 \\
136,925 \\
- \\
136,925 \\
344 \\
22,879 \\
11,448 \\
157 \\
2,197 \\
97,527 \\
2,373 \\
295\end{array}$ & $\begin{array}{r}141,930 \\
141,630 \\
- \\
141,630 \\
363 \\
24,310 \\
11,318 \\
172 \\
2,437 \\
100,988 \\
2,042 \\
300\end{array}$ & $\begin{array}{r}156,637 \\
156,324 \\
- \\
156,324 \\
394 \\
27,475 \\
13,434 \\
159 \\
2,274 \\
110,168 \\
2,420 \\
313\end{array}$ & $\begin{array}{r}180,330 \\
179,999 \\
- \\
179,999 \\
419 \\
32,409 \\
17,784 \\
185 \\
2,359 \\
123,775 \\
3,067 \\
331\end{array}$ & $\begin{array}{r}171,203 \\
170,872 \\
- \\
170,872 \\
363 \\
29,829 \\
14,599 \\
175 \\
2,428 \\
120,555 \\
2,922 \\
331\end{array}$ \\
\hline 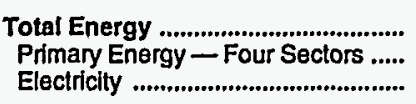 & $\begin{array}{l}62,579 \\
59,228 \\
23,351\end{array}$ & $\begin{array}{r}171,782 \\
121,103 \\
50,680\end{array}$ & $\begin{array}{r}373,900 \\
275,802 \\
98,098\end{array}$ & $\begin{array}{l}435,444 \\
286,202 \\
149,242\end{array}$ & $\begin{array}{l}381,250 \\
229,444 \\
151,806\end{array}$ & $\begin{array}{l}393,525 \\
238,833 \\
154,692\end{array}$ & $\begin{array}{l}407,597 \\
245,528 \\
162,070\end{array}$ & $\begin{array}{l}434,338 \\
264,999 \\
169,340\end{array}$ & $\begin{array}{l}469,468 \\
292,726 \\
176,742\end{array}$ & $\begin{array}{l}467,132 \\
282,310 \\
184,822\end{array}$ \\
\hline 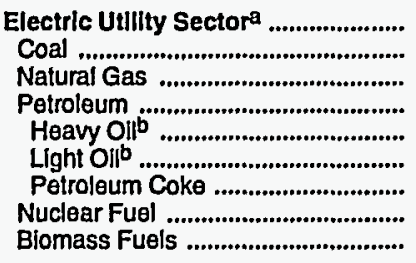 & $\begin{array}{r}4,316 \\
2,237 \\
1,151 \\
882 \\
797 \\
80 \\
6 \\
44 \\
2\end{array}$ & $\begin{array}{r}16,396 \\
7,178 \\
2,422 \\
6,345 \\
5,842 \\
502 \\
1 \\
448 \\
2\end{array}$ & $\begin{array}{r}37,435 \\
16,450 \\
8,357 \\
11,432 \\
10,446 \\
972 \\
14 \\
1,189 \\
8\end{array}$ & $\begin{array}{r}42,558 \\
24,056 \\
10,819 \\
4,742 \\
4,232 \\
502 \\
9 \\
2,930 \\
11\end{array}$ & $\begin{array}{r}35,793 \\
22,755 \\
6,305 \\
3,603 \\
3,288 \\
306 \\
10 \\
3,125 \\
4\end{array}$ & $\begin{array}{r}36,681 \\
22,785 \\
6,583 \\
3,812 \\
3,439 \\
362 \\
11 \\
3,486 \\
15\end{array}$ & $\begin{array}{r}37,435 \\
23,290 \\
6,123 \\
3,896 \\
3,468 \\
416 \\
12 \\
4,111 \\
15\end{array}$ & $\begin{array}{r}38,895 \\
23,165 \\
6,750 \\
4,974 \\
4,328 \\
632 \\
13 \\
3,992 \\
14\end{array}$ & $\begin{array}{r}38,443 \\
23,396 \\
6,631 \\
4,263 \\
3,757 \\
486 \\
20 \\
4,142 \\
11\end{array}$ & $\begin{array}{r}36,501 \\
23,164 \\
6,099 \\
3,054 \\
2,649 \\
387 \\
18 \\
4,172 \\
12\end{array}$ \\
\hline Primary Energy — Five Sectors ${ }^{c}$.. & 63,544 & 137,499 & 313,237 & 328,760 & 265,236 & 275,514 & 282,963 & 303,894 & 331,169 & 318,811 \\
\hline
\end{tabular}

There are no direct fuel costs for hydroelectric, geothermal, centralized solar, or wind energy.

b Heavy oil includes Grade Nos. 4,5, and 6, and residual fuel oils. Wght oil includes Grade No. 2 heating oil, kerosene, and jet fuel.

c Blomass fuels are not included, except those consumed at electric utilities and those added to motor gasoline.

-No consumption, including cases where adjustments were made. See "Consumption Adjustments for Process Fuel and Intermediate Products."

Note: Totals may not equal sum of components due to independent rounding.

Sources: Data sources, estimation procedures, and assumptions are described in the "Documentation" section of this report. 


\section{A Energy Price and Expenditure Estimates by Source, Alabama}

L $1970,1975,1980$, and 1985-1991

\begin{tabular}{|c|c|c|c|c|c|c|c|c|c|c|}
\hline Energy Source & 1970 & 1975 & 1980 & 1985 & 1986 & 1987 & 1988 & 1989 & 1990 & 1991 \\
\hline & \multicolumn{10}{|c|}{ Prices in Dollars per Million Btu } \\
\hline 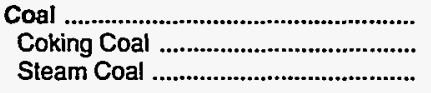 & $\begin{array}{r}0.32 \\
.42 \\
.26\end{array}$ & $\begin{array}{r}1.10 \\
1.50 \\
.94\end{array}$ & $\begin{array}{l}1.70 \\
1.96 \\
1.63\end{array}$ & $\begin{array}{l}2.01 \\
2.02 \\
2.00\end{array}$ & $\begin{array}{l}1.89 \\
1.81 \\
1.89\end{array}$ & $\begin{array}{l}1.87 \\
1.71 \\
1.89\end{array}$ & $\begin{array}{l}1.87 \\
1.70 \\
1.90\end{array}$ & $\begin{array}{l}1.83 \\
1.74 \\
1.84\end{array}$ & $\begin{array}{l}1.83 \\
1.83 \\
1.83\end{array}$ & $\begin{array}{l}1.80 \\
1.80 \\
1.79\end{array}$ \\
\hline Natural Gas & .52 & .96 & 2.90 & 4.73 & 4.50 & 4.27 & 4.03 & 3.92 & 4.07 & 4.08 \\
\hline 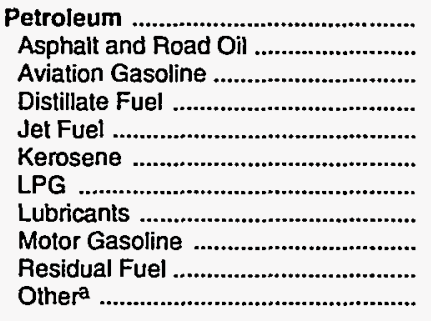 & $\begin{array}{r}2.09 \\
.65 \\
2.17 \\
1.10 \\
.73 \\
.90 \\
2.00 \\
5.08 \\
2.82 \\
.41 \\
.61\end{array}$ & $\begin{array}{l}3.31 \\
1.85 \\
3.45 \\
2.60 \\
2.03 \\
2.45 \\
3.83 \\
7.48 \\
4.26 \\
1.59 \\
1.85\end{array}$ & $\begin{array}{r}7.86 \\
3.02 \\
9.02 \\
6.58 \\
6.39 \\
6.42 \\
6.62 \\
14.36 \\
9.89 \\
2.99 \\
5.80\end{array}$ & $\begin{array}{r}7.96 \\
4.72 \\
9.99 \\
6.66 \\
6.17 \\
8.63 \\
6.98 \\
17.61 \\
9.15 \\
3.80 \\
7.07\end{array}$ & $\begin{array}{r}6.23 \\
4.90 \\
8.41 \\
5.46 \\
4.45 \\
5.06 \\
7.06 \\
15.59 \\
6.99 \\
1.98 \\
4.21\end{array}$ & $\begin{array}{r}6.38 \\
3.37 \\
7.55 \\
5.90 \\
4.39 \\
5.18 \\
7.21 \\
12.70 \\
7.27 \\
2.18 \\
5.03\end{array}$ & $\begin{array}{r}6.22 \\
3.07 \\
7.41 \\
5.54 \\
4.07 \\
5.30 \\
6.97 \\
14.61 \\
7.21 \\
2.49 \\
4.19\end{array}$ & $\begin{array}{r}6.85 \\
2.86 \\
8.28 \\
6.35 \\
4.64 \\
6.05 \\
8.60 \\
13.30 \\
7.96 \\
2.20 \\
5.07\end{array}$ & $\begin{array}{r}7.80 \\
2.94 \\
9.32 \\
7.55 \\
5.99 \\
7.62 \\
10.13 \\
13.40 \\
8.96 \\
2.18 \\
6.17\end{array}$ & $\begin{array}{r}7.49 \\
3.31 \\
8.71 \\
7.09 \\
5.03 \\
7.01 \\
11.25 \\
15.42 \\
8.69 \\
1.74 \\
5.35\end{array}$ \\
\hline 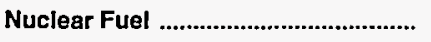 & - & .14 & .33 & .77 & .70 & .69 & .65 & .60 & .56 & .71 \\
\hline Blomass Fuels at Utilities .................. & - & - & - & - & - & - & - & - & - & - \\
\hline Primary Energy - Five Sectors ${ }^{b} \ldots$. & .84 & 1.82 & 3.37 & 3.99 & 3.48 & 3.56 & 3.46 & 3.71 & 4.03 & 3.79 \\
\hline $\begin{array}{l}\text { Electric Utility Fuelc ........................ } \\
\text { Electricity Purchased by End Users }\end{array}$ & $\begin{array}{r}.26 \\
3.51\end{array}$ & $\begin{array}{r}.88 \\
6.87\end{array}$ & $\begin{array}{r}1.17 \\
12.52\end{array}$ & $\begin{array}{r}1.74 \\
16.59\end{array}$ & $\begin{array}{r}1.68 \\
16.66\end{array}$ & $\begin{array}{r}1.69 \\
16.48\end{array}$ & $\begin{array}{r}1.66 \\
16.34\end{array}$ & $\begin{array}{r}1.63 \\
16.43\end{array}$ & $\begin{array}{r}1.60 \\
16.46\end{array}$ & $\begin{array}{r}1.56 \\
16.69\end{array}$ \\
\hline \multirow[t]{2}{*}{ 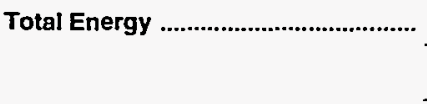 } & 1.37 & 2.84 & 6.41 & 7.90 & 7.15 & 7.11 & 6.89 & 7.28 & 7.87 & 7.78 \\
\hline & \multicolumn{10}{|c|}{ Expenditures in Millions of Dollars } \\
\hline 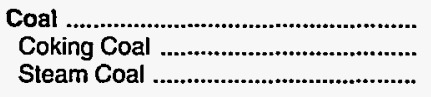 & $\begin{array}{r}215.3 \\
99.4 \\
115.9\end{array}$ & $\begin{array}{l}700.9 \\
269.2 \\
431.7\end{array}$ & $\begin{array}{r}1,121.0 \\
254.7 \\
866.3\end{array}$ & $\begin{array}{r}1,328.5 \\
156.1 \\
1,172.4\end{array}$ & $\begin{array}{r}1,245.2 \\
129.4 \\
1,115.8\end{array}$ & $\begin{array}{r}1,234.1 \\
144.5 \\
1,089.6\end{array}$ & $\begin{array}{r}1,223.2 \\
154.0 \\
1,069.2\end{array}$ & $\begin{array}{r}1,234.0 \\
154.7 \\
1,079.3\end{array}$ & $\begin{array}{r}1,238.1 \\
160.8 \\
1,077.2\end{array}$ & $\begin{array}{r}1,292.2 \\
153.0 \\
1,139.2\end{array}$ \\
\hline 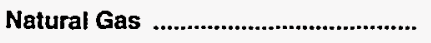 & 143.2 & 227.1 & 676.5 & 923.7 & 796.7 & 763.9 & 826.2 & 839.3 & 841.6 & 872.1 \\
\hline 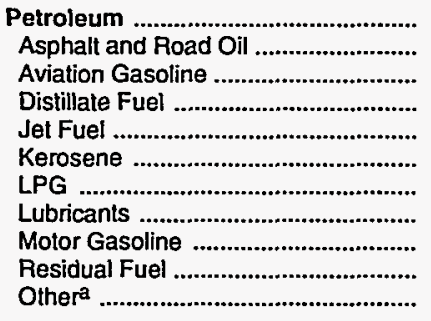 & $\begin{array}{r}731.8 \\
13.7 \\
3.8 \\
54.6 \\
7.2 \\
6.7 \\
57.0 \\
25.0 \\
547.6 \\
8.0 \\
8.2\end{array}$ & \begin{tabular}{r|}
$1,595.1$ \\
33.2 \\
4.3 \\
221.6 \\
19.1 \\
9.4 \\
91.9 \\
47.6 \\
$1,010.7$ \\
127.4 \\
29.9
\end{tabular} & $\begin{array}{r}3,495.8 \\
62.8 \\
11.3 \\
579.2 \\
72.3 \\
45.6 \\
116.3 \\
86.4 \\
2,301.3 \\
135.2 \\
85.3\end{array}$ & $\begin{array}{r}3,353.6 \\
117.7 \\
8.7 \\
631.8 \\
121.6 \\
5.3 \\
91.7 \\
96.4 \\
2,090.3 \\
53.6 \\
136.6\end{array}$ & $\begin{array}{r}2,755.3 \\
113.5 \\
8.7 \\
523.4 \\
93.5 \\
3.7 \\
103.2 \\
83.5 \\
1,705.0 \\
29.3 \\
91.5\end{array}$ & $\begin{array}{r}3,037.6 \\
102.0 \\
5.4 \\
644.1 \\
95.2 \\
4.0 \\
122.7 \\
76.9 \\
1,848.6 \\
31.4 \\
107.1\end{array}$ & $\begin{array}{r}2,962.2 \\
84.1 \\
5.9 \\
658.9 \\
42.2 \\
4.9 \\
112.8 \\
85.3 \\
1,824.2 \\
51.1 \\
92.9\end{array}$ & $\begin{array}{r}3,515.6 \\
85.0 \\
5.5 \\
913.4 \\
52.7 \\
3.9 \\
152.8 \\
79.6 \\
2,067.1 \\
49.1 \\
106.5\end{array}$ & $\begin{array}{r}4,012.8 \\
84.3 \\
5.4 \\
1,118.7 \\
63.1 \\
2.8 \\
152.9 \\
82.6 \\
2,303.4 \\
52.6 \\
146.9\end{array}$ & $\begin{array}{r}3,831.6 \\
116.0 \\
4.8 \\
987.1 \\
50.9 \\
3.8 \\
154.4 \\
85.0 \\
2,260.4 \\
38.5 \\
130.6\end{array}$ \\
\hline 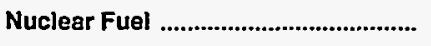 & - & 4.2 & 85.2 & 118.7 & 87.5 & 83.3 & 90.1 & 74.4 & 71.7 & 120.9 \\
\hline Biomass Fuels at Utilities .................. & - & - & - & - & - & - & - & - & - & - \\
\hline Primary Energy - Flve Sectors ${ }^{b}$.... & $1,090.4$ & $2,527.2$ & $5,378.4$ & $5,724.5$ & $4,884.6$ & $5,119.0$ & $5,101.7$ & $5,663.3$ & $6,164.2$ & $6,116.8$ \\
\hline $\begin{array}{l}\text { Electric Utility Fuelc } \\
\text { Electricity Purchased by End Users }\end{array}$ & $\begin{array}{r}-103.4 \\
411.6\end{array}$ & $\begin{array}{r}-385.8 \\
940.2\end{array}$ & $\begin{array}{r}-849.4 \\
2,120.5\end{array}$ & $\begin{array}{r}-1,174.9 \\
2,735.9\end{array}$ & $\begin{array}{r}-1,088.6 \\
2,818.4\end{array}$ & $\begin{array}{r}-1,063.7 \\
2,950.9\end{array}$ & $\begin{array}{r}-1,046.7 \\
3,039.7\end{array}$ & $\begin{array}{r}-1,065.0 \\
3,139.7\end{array}$ & $\begin{array}{r}-1,066.3 \\
3,236.6\end{array}$ & $\begin{array}{r}-1,172.3 \\
3,356.8\end{array}$ \\
\hline 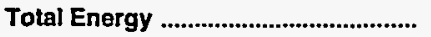 & $1,398.6$ & $3,081.5$ & $6,649.6$ & $7,285.5$ & $6,614.4$ & $7,006.2$ & $7,094.7$ & $7,738.1$ & $8,334.5$ & $8,301.2$ \\
\hline
\end{tabular}

Includes petroleum coke used at electric utilities.

b Biomass fuels are not included, except those consumed at electric utilities and those added to motor gasoline.

c There are no direct fuel costs for hydroelectric, geothermal, centralized solar, or wind energy.

-No consumption, including cases where adjustments were made. See "Consumption Adjustments for Process Fuel and Intermediate Products."

Note: Expenditure totals may not equal sum of components due to independent rounding.

Sources: Data sources, estimation procedures, and assumptions are described in the "Documentation" section of this report. 


\begin{tabular}{|c|c|c|c|c|c|c|c|c|c|c|}
\hline Sector and Energy Source & 1970 & 1975 & 1980 & 1985 & 1986 & 1987 & 1988 & 1989 & 1990 & 1991 \\
\hline 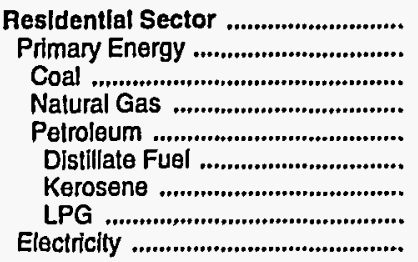 & $\begin{array}{r}2.45 \\
1.37 \\
.81 \\
1.10 \\
2.17 \\
1.24 \\
1.62 \\
2.22 \\
4.62\end{array}$ & $\begin{array}{l}4.48 \\
2.13 \\
1.82 \\
1.52 \\
4.22 \\
2.53 \\
3.31 \\
4.32 \\
8.05\end{array}$ & $\begin{array}{r}9.06 \\
4.53 \\
2.97 \\
3.91 \\
7.89 \\
6.83 \\
9.13 \\
7.75 \\
14.44\end{array}$ & $\begin{array}{r}12.82 \\
6.46 \\
3.19 \\
6.18 \\
8.49 \\
7.68 \\
8.90 \\
8.49 \\
18.74\end{array}$ & $\begin{array}{r}13.02 \\
6.50 \\
2.89 \\
6.30 \\
7.94 \\
5.33 \\
6.18 \\
8.08 \\
18.95\end{array}$ & $\begin{array}{r}12.97 \\
6.73 \\
2.88 \\
6.43 \\
8.54 \\
5.13 \\
5.94 \\
8.68 \\
18.92\end{array}$ & $\begin{array}{r}13.00 \\
6.54 \\
2.62 \\
6.33 \\
7.97 \\
4.93 \\
5.71 \\
8.21 \\
19.06\end{array}$ & $\begin{array}{r}13.24 \\
6.62 \\
2.64 \\
6.09 \\
9.08 \\
5.52 \\
6.40 \\
9.25 \\
19.31\end{array}$ & $\begin{array}{r}13.84 \\
7.12 \\
2.70 \\
6.38 \\
10.91 \\
6.70 \\
7.76 \\
11.04 \\
19.32\end{array}$ & $\begin{array}{r}14.40 \\
7.68 \\
2.81 \\
6.86 \\
12.16 \\
6.16 \\
7.15 \\
12.45 \\
19.61\end{array}$ \\
\hline 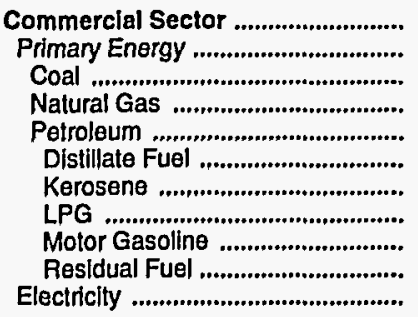 & $\begin{array}{r}1.98 \\
.75 \\
.28 \\
.58 \\
1.53 \\
.97 \\
.75 \\
1.58 \\
2.82 \\
.38 \\
5.39\end{array}$ & $\begin{array}{l}3.97 \\
1.46 \\
1.07 \\
1.04 \\
2.96 \\
2.22 \\
2.24 \\
3.07 \\
4.26 \\
1.69 \\
8.98\end{array}$ & $\begin{array}{r}8.44 \\
3.78 \\
1.73 \\
3.27 \\
6.61 \\
6.22 \\
5.91 \\
5.28 \\
9.89 \\
3.39 \\
16.19\end{array}$ & $\begin{array}{r}11.47 \\
5.40 \\
1.86 \\
5.27 \\
6.17 \\
6.76 \\
8.06 \\
4.97 \\
9.15 \\
4.02 \\
20.01\end{array}$ & $\begin{array}{r}11.41 \\
4.62 \\
1.76 \\
5.21 \\
3.83 \\
3.65 \\
3.97 \\
5.55 \\
6.99 \\
2.16 \\
20.03\end{array}$ & $\begin{array}{r}11.65 \\
4.75 \\
1.63 \\
5.31 \\
4.27 \\
4.04 \\
4.59 \\
4.91 \\
7.27 \\
2.67 \\
19.36\end{array}$ & $\begin{array}{r}11.08 \\
4.39 \\
1.60 \\
5.13 \\
3.50 \\
3.43 \\
3.72 \\
4.89 \\
7.21 \\
1.99 \\
19.29\end{array}$ & $\begin{array}{r}11.84 \\
4.77 \\
1.65 \\
5.01 \\
4.54 \\
4.11 \\
5.15 \\
7.50 \\
7.96 \\
2.38 \\
19.65\end{array}$ & $\begin{array}{r}12.34 \\
5.24 \\
1.65 \\
5.28 \\
5.63 \\
5.96 \\
7.41 \\
8.47 \\
8.96 \\
2.65 \\
19.52\end{array}$ & $\begin{array}{r}13.34 \\
5.64 \\
1.64 \\
5.59 \\
5.87 \\
5.54 \\
6.77 \\
9.38 \\
8.69 \\
2.13 \\
19.82\end{array}$ \\
\hline 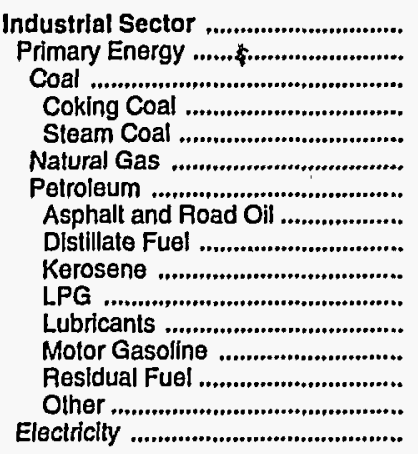 & $\begin{array}{r}.63 \\
.44 \\
.40 \\
.42 \\
.28 \\
.32 \\
.92 \\
.65 \\
.69 \\
.75 \\
1.58 \\
5.08 \\
2.82 \\
.51 \\
.72 \\
2.24\end{array}$ & $\begin{array}{l}1.86 \\
1.36 \\
1.39 \\
1.50 \\
1.07 \\
.73 \\
2.10 \\
1.85 \\
2.04 \\
2.24 \\
3.07 \\
7.48 \\
4.26 \\
1.74 \\
1.85 \\
5.40\end{array}$ & $\begin{array}{r}3.99 \\
2.69 \\
1.89 \\
1.96 \\
1.73 \\
2.46 \\
4.66 \\
3.02 \\
5.28 \\
5.91 \\
5.28 \\
14.36 \\
9.89 \\
3.05 \\
5.80 \\
10.29\end{array}$ & $\begin{array}{r}5.58 \\
3.77 \\
1.95 \\
2.02 \\
1.86 \\
4.09 \\
6.62 \\
4.72 \\
7.02 \\
8.06 \\
4.97 \\
17.61 \\
9.15 \\
4.02 \\
7.07 \\
13.60\end{array}$ & $\begin{array}{r}5.03 \\
3.07 \\
1.78 \\
1.81 \\
1.76 \\
3.54 \\
4.65 \\
4.90 \\
3.46 \\
3.97 \\
5.55 \\
15.59 \\
6.99 \\
2.16 \\
4.21 \\
13.34\end{array}$ & $\begin{array}{r}4.75 \\
2.80 \\
1.67 \\
1.71 \\
1.63 \\
3.01 \\
4.42 \\
3.37 \\
4.00 \\
4.59 \\
4.91 \\
12.70 \\
7.27 \\
2.67 \\
5.03 \\
13.23\end{array}$ & $\begin{array}{r}4.50 \\
2.61 \\
1.65 \\
1.70 \\
1.60 \\
2.92 \\
3.93 \\
3.07 \\
3.24 \\
3.72 \\
4.89 \\
14.61 \\
7.21 \\
1.99 \\
4.19 \\
12.96\end{array}$ & $\begin{array}{r}4.70 \\
2.86 \\
1.71 \\
1.74 \\
1.65 \\
2.93 \\
4.59 \\
2.86 \\
4.49 \\
5.15 \\
7.50 \\
13.30 \\
7.96 \\
2.38 \\
5.07 \\
12.72\end{array}$ & $\begin{array}{r}5.04 \\
3.28 \\
1.76 \\
1.83 \\
1.65 \\
3.07 \\
5.66 \\
2.94 \\
6.45 \\
7.41 \\
8.47 \\
13.40 \\
8.96 \\
2.65 \\
6.17 \\
12.72\end{array}$ & $\begin{array}{r}4.90 \\
3.09 \\
1.74 \\
1.80 \\
1.64 \\
2.92 \\
5.30 \\
3.31 \\
5.90 \\
6.77 \\
9.38 \\
15.42 \\
8.69 \\
2.13 \\
5.35 \\
12.81\end{array}$ \\
\hline 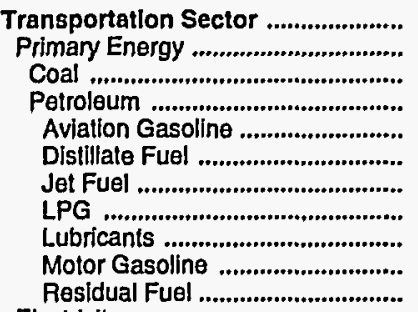 & $\begin{array}{r}2.45 \\
2.45 \\
.28 \\
2.46 \\
2.17 \\
1.33 \\
.73 \\
1.58 \\
5.08 \\
2.82 \\
.34\end{array}$ & $\begin{array}{l}3.67 \\
3.67 \\
1.07 \\
3.67 \\
3.45 \\
2.92 \\
2.03 \\
3.07 \\
7.48 \\
4.26 \\
1.47\end{array}$ & $\begin{array}{r}8.78 \\
8.78 \\
- \\
8.78 \\
9.02 \\
6.99 \\
6.39 \\
5.28 \\
14.36 \\
9.89 \\
2.93\end{array}$ & $\begin{array}{r}8.34 \\
8.34 \\
- \\
8.34 \\
9.99 \\
6.54 \\
6.17 \\
4.97 \\
17.61 \\
9.15 \\
3.72\end{array}$ & $\begin{array}{r}6.65 \\
6.65 \\
-5 \\
6.65 \\
8.41 \\
6.35 \\
4.45 \\
5.55 \\
15.59 \\
6.99 \\
1.85\end{array}$ & $\begin{array}{r}6.89 \\
6.89 \\
- \\
6.89 \\
7.55 \\
6.73 \\
4.39 \\
4.91 \\
12.70 \\
7.27 \\
1.92\end{array}$ & $\begin{array}{r}6.83 \\
6.83 \\
- \\
6.83 \\
7.41 \\
6.41 \\
4.07 \\
4.89 \\
14.61 \\
7.21 \\
2.79\end{array}$ & $\begin{array}{r}7.40 \\
7.40 \\
- \\
7.40 \\
8.28 \\
7.06 \\
4.64 \\
7.50 \\
13.30 \\
7.96 \\
2.15\end{array}$ & $\begin{array}{r}8.36 \\
8.36 \\
- \\
8.36 \\
9.32 \\
8.09 \\
5.99 \\
8.47 \\
13.40 \\
8.96 \\
2.02\end{array}$ & $\begin{array}{r}7.99 \\
7.99 \\
- \\
7.99 \\
8.71 \\
7.57 \\
5.03 \\
9.38 \\
15.42 \\
8.69 \\
1.71\end{array}$ \\
\hline Electricity .............................................. & - & - & - & - & - & - & - & $\rightarrow$ & - & - \\
\hline 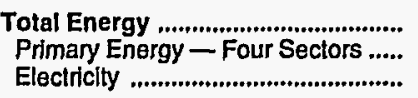 & $\begin{array}{l}1.37 \\
1.09 \\
3.51\end{array}$ & $\begin{array}{l}2.84 \\
2.26 \\
6.87\end{array}$ & $\begin{array}{r}6.41 \\
5.22 \\
12.52\end{array}$ & $\begin{array}{r}7.90 \\
6.01 \\
16.59\end{array}$ & $\begin{array}{r}7.15 \\
5.02 \\
16.66\end{array}$ & $\begin{array}{r}7.11 \\
5.03 \\
16.48\end{array}$ & $\begin{array}{r}6.89 \\
4.81 \\
16.34\end{array}$ & $\begin{array}{r}7.28 \\
5.27 \\
16.43\end{array}$ & $\begin{array}{r}7.87 \\
5.91 \\
16.46\end{array}$ & $\begin{array}{r}7.78 \\
5.71 \\
16.69\end{array}$ \\
\hline 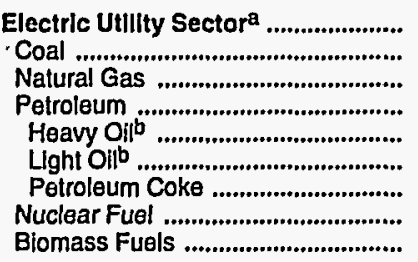 & $\begin{array}{l}.26 \\
.26 \\
.26 \\
.20 \\
.81 \\
.17 \\
- \\
-\end{array}$ & $\begin{array}{r}.88 \\
.92 \\
1.08 \\
2.08 \\
1.69 \\
2.16 \\
-14 \\
-\end{array}$ & $\begin{array}{l}1.17 \\
1.61 \\
2.62 \\
6.35 \\
6 . \overline{5} \\
\overline{33} \\
-\end{array}$ & $\begin{array}{r}1.74 \\
2.02 \\
3.17 \\
6.00 \\
-\overline{0} \\
\overline{0} \\
.77 \\
-\end{array}$ & $\begin{array}{r}1.68 \\
1.91 \\
2.47 \\
3.49 \\
-\overline{49} \\
\overline{-} \\
.70 \\
-\end{array}$ & $\begin{array}{r}1.69 \\
1.92 \\
2.10 \\
4.03 \\
-\overline{4} \\
4.03 \\
- \\
.69 \\
-\end{array}$ & $\begin{array}{r}1.66 \\
1.95 \\
2.07 \\
3.67 \\
- \\
3.67 \\
- \\
.65 \\
-\end{array}$ & $\begin{array}{r}1.63 \\
1.86 \\
2.21 \\
4.14 \\
-\overline{44} \\
\overline{60} \\
-\end{array}$ & $\begin{array}{r}1.60 \\
1.84 \\
2.16 \\
5.57 \\
- \\
5.57 \\
- \\
.56 \\
-\end{array}$ & $\begin{array}{r}1.56 \\
1.81 \\
1.87 \\
5.12 \\
-\overline{2} \\
5.12 \\
.71 \\
-\end{array}$ \\
\hline Prlmary Energy - Flve Sectorsc ... & .84 & 1.82 & 3.37 & 3.99 & 3.48 & 3.56 & 3.46 & 3.71 & 4.03 & 3.79 \\
\hline
\end{tabular}

a There are no direct fuel costs for hydroelectric, geothermal, centralized solar, or wind energy.

b Heavy oil includes Grade Nos. 4, 5, and 6, and residual fuel oils. Light oil includes Grade No. 2 healing oil, kerosene, and jet fuel.

c Blomass fuels are not included, except those consumed at electric utilities and those added to motor gasoline.

- No consumption, including cases where adjustments were made. See "Consumption Adjustments for Process Fuel and Intermediate Products."

Sources: Data sources, estimation procedures, and assumptions are described in the "Documentation" section of this report. 
A Energy Expenditure Estimates by Sector, Alabama

L 1970, 1975, 1980, and 1985-1991

A (Million Dollars)

\begin{tabular}{|c|c|c|c|c|c|c|c|c|c|c|}
\hline Sector and Energy Source & 1970 & 1975 & 1980 & 1985 & 1986 & 1987 & 1988 & 1989 & 1990 & 1991 \\
\hline 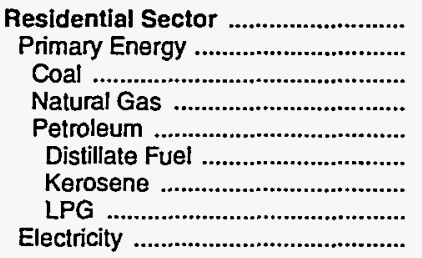 & $\begin{array}{r}289.4 \\
107.7 \\
.9 \\
63.0 \\
43.8 \\
.3 \\
2.2 \\
21.4 \\
41.4 \\
181.7\end{array}$ & $\begin{array}{r}517.3 \\
148.8 \\
.3 \\
82.0 \\
66.4 \\
1.1 \\
2.5 \\
62.8 \\
368.5\end{array}$ & $\begin{array}{r}1,113.2 \\
301.9 \\
5.7 \\
211.7 \\
84.5 \\
.5 \\
10.2 \\
73.7 \\
811.2\end{array}$ & $\begin{array}{r}1,450.9 \\
352.5 \\
3.4 \\
280.1 \\
69.0 \\
1.5 \\
3.7 \\
63.8 \\
1,098.4\end{array}$ & \begin{tabular}{r|}
$1,548.7$ \\
368.3 \\
3.2 \\
291.3 \\
73.7 \\
1.2 \\
2.2 \\
70.3 \\
$1,180.4$
\end{tabular} & $\begin{array}{r}1,667.4 \\
422.2 \\
3.4 \\
325.9 \\
92.9 \\
.9 \\
2.0 \\
90.0 \\
1,245.2\end{array}$ & $\begin{array}{r}1,687.5 \\
410.5 \\
3.5 \\
318.4 \\
88.6 \\
1.2 \\
4.2 \\
83.2 \\
1,277.0\end{array}$ & $\begin{array}{r}1,717.9 \\
410.8 \\
1.6 \\
301.7 \\
107.5 \\
1.1 \\
2.9 \\
103.5 \\
1,307.0\end{array}$ & $\begin{array}{r}1,777.1 \\
411.1 \\
2.4 \\
298.4 \\
110.3 \\
1.0 \\
1.7 \\
107.6 \\
1,366.0\end{array}$ & $\begin{array}{r}1,857.9 \\
432.9 \\
.4 \\
325.4 \\
107.1 \\
.7 \\
2.5 \\
104.0 \\
1,425.0\end{array}$ \\
\hline 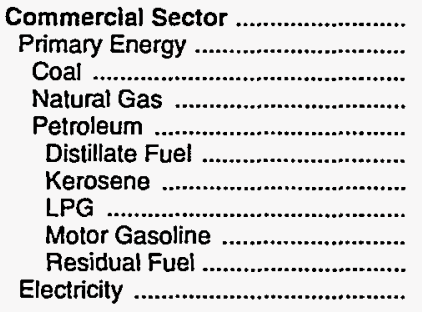 & $\begin{array}{r}131.2 \\
36.6 \\
.5 \\
21.8 \\
14.2 \\
1.5 \\
1.8 \\
5.2 \\
5.8 \\
* \\
94.6\end{array}$ & $\begin{array}{r}263.4 \\
64.4 \\
.3 \\
35.9 \\
28.2 \\
7.1 \\
3.1 \\
7.9 \\
10.1 \\
199.0\end{array}$ & $\begin{array}{r}551.3 \\
154.2 \\
6.2 \\
96.5 \\
51.5 \\
23.2 \\
5.9 \\
8.9 \\
13.4 \\
.1 \\
397.2\end{array}$ & $\begin{array}{r}829.3 \\
228.1 \\
3.6 \\
141.3 \\
83.2 \\
50.8 \\
.7 \\
6.6 \\
12.1 \\
13.0 \\
601.1\end{array}$ & $\begin{array}{r}821.1 \\
186.0 \\
3.6 \\
135.7 \\
46.7 \\
20.6 \\
.6 \\
8.5 \\
9.3 \\
7.6 \\
635.2\end{array}$ & $\begin{array}{r}835.9 \\
179.8 \\
3.5 \\
122.7 \\
53.7 \\
27.1 \\
1.3 \\
9.0 \\
9.9 \\
6.4 \\
656.1\end{array}$ & $\begin{array}{r}862.5 \\
188.5 \\
4.0 \\
135.0 \\
49.5 \\
22.5 \\
.3 \\
8.7 \\
9.2 \\
8.8 \\
674.0\end{array}$ & $\begin{array}{r}944.9 \\
199.9 \\
1.9 \\
136.6 \\
61.5 \\
29.4 \\
.4 \\
14.8 \\
9.3 \\
7.5 \\
745.0\end{array}$ & $\begin{array}{r}981.7 \\
209.7 \\
2.8 \\
131.9 \\
75.1 \\
37.7 \\
.5 \\
14.6 \\
12.1 \\
10.2 \\
772.0\end{array}$ & $\begin{array}{r}1,001.2 \\
193.2 \\
.4 \\
136.1 \\
56.6 \\
31.7 \\
.6 \\
13.8 \\
7.3 \\
3.3 \\
808.0\end{array}$ \\
\hline 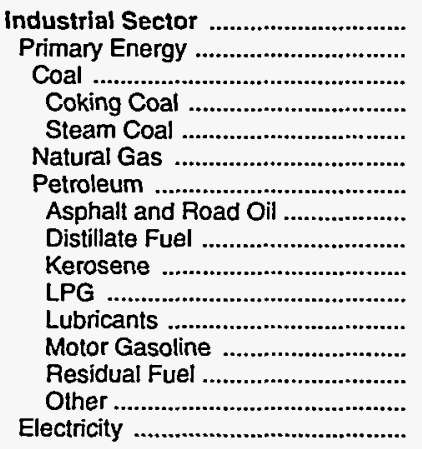 & $\begin{array}{r}369.6 \\
234.3 \\
115.2 \\
99.4 \\
15.8 \\
54.2 \\
64.9 \\
13.7 \\
11.4 \\
2.7 \\
9.9 \\
12.0 \\
3.0 \\
4.4 \\
7.7 \\
135.3\end{array}$ & $\begin{array}{r}1,032.8 \\
660.1 \\
332.8 \\
269.2 \\
63.6 \\
102.4 \\
224.9 \\
33.2 \\
52.4 \\
3.8 \\
20.1 \\
20.0 \\
4.4 \\
61.1 \\
29.9 \\
372.7\end{array}$ & $\begin{array}{r}2,061.3 \\
1,149.2 \\
353.9 \\
254.7 \\
99.2 \\
364.1 \\
431.2 \\
62.8 \\
100.8 \\
29.5 \\
32.8 \\
44.1 \\
5.4 \\
70.5 \\
85.3 \\
912.1\end{array}$ & $\begin{array}{r}2,306.4 \\
1,270.1 \\
272.2 \\
156.1 \\
116.1 \\
498.5 \\
499.4 \\
117.7 \\
150.0 \\
.9 \\
18.4 \\
49.2 \\
24.4 \\
2.2 \\
136.6 \\
1,036.4\end{array}$ & $\begin{array}{r}1,985.0 \\
982.2 \\
241.4 \\
129.4 \\
112.0 \\
366.8 \\
374.0 \\
113.5 \\
82.3 \\
.8 \\
21.5 \\
42.6 \\
15.8 \\
6.0 \\
91.5 \\
1,002.8\end{array}$ & $\begin{array}{r}2,011.9 \\
962.3 \\
251.5 \\
144.5 \\
107.0 \\
312.1 \\
398.6 \\
102.0 \\
103.8 \\
.7 \\
21.9 \\
39.3 \\
16.7 \\
7.0 \\
107.1 \\
1,049.6\end{array}$ & $\begin{array}{r}2,068.4 \\
979.7 \\
268.3 \\
154.0 \\
114.4 \\
367.2 \\
344.1 \\
84.1 \\
82.7 \\
.4 \\
19.3 \\
43.5 \\
14.6 \\
6.6 \\
92.9 \\
1,088.7\end{array}$ & $\begin{array}{r}2,157.9 \\
1,070.1 \\
249.2 \\
154.7 \\
94.5 \\
397.0 \\
424.0 \\
85.0 \\
135.1 \\
.5 \\
32.1 \\
40.6 \\
20.7 \\
3.5 \\
106.5 \\
1,087.7\end{array}$ & $\begin{array}{r}2,333.6 \\
1,235.0 \\
251.7 \\
160.8 \\
90.9 \\
402.3 \\
581.0 \\
84.3 \\
253.1 \\
.6 \\
27.7 \\
42.2 \\
20.8 \\
5.4 \\
146.9 \\
1,098.6\end{array}$ & $\begin{array}{r}2,308.7 \\
1,185.0 \\
252.7 \\
153.0 \\
99.7 \\
402.8 \\
529.6 \\
116.0 \\
186.1 \\
.8 \\
33.5 \\
43.4 \\
18.6 \\
.6 \\
130.6 \\
1,123.7\end{array}$ \\
\hline 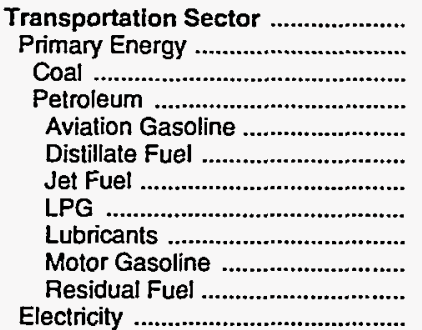 & $\begin{array}{r}608.4 \\
608.4 \\
.1 \\
608.3 \\
3.8 \\
41.3 \\
7.2 \\
.6 \\
13.0 \\
538.8 \\
3.5 \\
\end{array}$ & $\begin{array}{r}1,268.0 \\
1,268.0 \\
1,268.0 \\
4.3 \\
154.6 \\
19.1 \\
1.0 \\
27.6 \\
996.1 \\
65.2 \\
-\end{array}$ & $\begin{array}{r}2,923.8 \\
2,923.8 \\
-\overline{2}, 923.8 \\
11.3 \\
449.8 \\
72.3 \\
.9 \\
42.3 \\
2,282.5 \\
64.6 \\
-\end{array}$ & $\begin{array}{r}2,698.9 \\
2,698.9 \\
-\overline{9} \\
2,698.9 \\
8.7 \\
426.4 \\
121.6 \\
2.9 \\
47.2 \\
2,053.8 \\
38.4 \\
-\end{array}$ & $\begin{array}{r}2,259.6 \\
2,259.6 \\
-\overline{6} \\
2,259.6 \\
8.7 \\
418.0 \\
93.5 \\
3.0 \\
40.9 \\
1,679.9 \\
15.7 \\
-\end{array}$ & $\begin{array}{r}2,491.0 \\
2,491.0 \\
- \\
2,491.0 \\
5.4 \\
510.9 \\
95.2 \\
1.8 \\
37.7 \\
1,822.0 \\
18.0 \\
-\end{array}$ & $\begin{array}{r}2,476.3 \\
2,476.3 \\
- \\
2,476.3 \\
5.9 \\
548.8 \\
42.2 \\
1.6 \\
41.8 \\
1,800.4 \\
35.7 \\
-\end{array}$ & $\begin{array}{r}2,917.4 \\
2,917.4 \\
- \\
2,917.4 \\
5.5 \\
742.6 \\
52.7 \\
2.4 \\
39.0 \\
2,037.0 \\
38.1 \\
-\end{array}$ & $\begin{array}{r}3,242.1 \\
3,242.1 \\
- \\
3,242.1 \\
5.4 \\
822.6 \\
63.1 \\
3.0 \\
40.4 \\
2,270.6 \\
36.9 \\
-\end{array}$ & $\begin{array}{r}3,133.4 \\
3,133.4 \\
-\overline{3}, 133.4 \\
4.8 \\
763.8 \\
50.9 \\
3.2 \\
41.6 \\
2,234.4 \\
34.7 \\
\end{array}$ \\
\hline $\begin{array}{l}\text { Total Energy ..................................... } \\
\text { Primary Energy - Four Sectors ..... } \\
\text { Electricity ............................................. }\end{array}$ & $\begin{array}{r}1,398.6 \\
987.0 \\
411.6\end{array}$ & $\begin{array}{r}3,081.5 \\
2,141.3 \\
940.2\end{array}$ & $\begin{array}{l}6,649.6 \\
4,529.1 \\
2,120.5\end{array}$ & $\begin{array}{l}7,285.5 \\
4,549.6 \\
2,735.9\end{array}$ & $\begin{array}{l}6,614.4 \\
3,796.0 \\
2,818.4\end{array}$ & $\begin{array}{l}7,006.2 \\
4,055.3 \\
2,950.9\end{array}$ & $\begin{array}{l}7,094.7 \\
4,054.9 \\
3,039.7\end{array}$ & $\begin{array}{l}7,738.1 \\
4,598.3 \\
3,139.7\end{array}$ & $\begin{array}{l}8,334.5 \\
5,097.9 \\
3,236.6\end{array}$ & $\begin{array}{l}8,301.2 \\
4,944.5 \\
3,356.8\end{array}$ \\
\hline 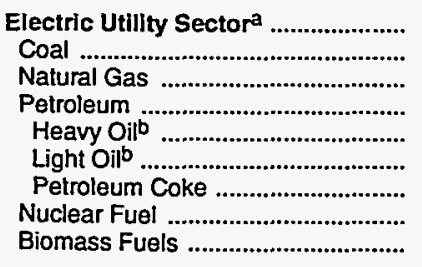 & $\begin{array}{r}103.4 \\
98.6 \\
4.2 \\
.6 \\
- \\
.1 \\
.4 \\
- \\
-\end{array}$ & $\begin{array}{r}385.8 \\
367.5 \\
6.7 \\
7.5 \\
1.0 \\
6.5 \\
- \\
4.2 \\
-\end{array}$ & $\begin{array}{r}849.4 \\
755.2 \\
4.1 \\
4.8 \\
-\overline{8} \\
4 . \overline{-} \\
85 . \overline{2} \\
-\end{array}$ & $\begin{array}{r}1,174.9 \\
1,049.4 \\
3.8 \\
3.1 \\
-\overline{1} \\
3.1 \\
118.7 \\
-\end{array}$ & $\begin{array}{r}1,088.6 \\
996.9 \\
2.8 \\
1.4 \\
-\overline{4} \\
1.4 \\
- \\
87.5 \\
-\end{array}$ & $\begin{array}{r}1,063.7 \\
975.7 \\
3.3 \\
1.4 \\
- \\
1.4 \\
-\overline{3} \\
83.3 \\
-\end{array}$ & $\begin{array}{r}1,046.7 \\
947.4 \\
5.5 \\
3.7 \\
- \\
3.7 \\
-\overline{1} \\
90.1 \\
-\end{array}$ & $\begin{array}{r}1,065.0 \\
981.4 \\
4.0 \\
5.2 \\
- \\
5.2 \\
-\overline{4} \\
74.4 \\
-\end{array}$ & $\begin{array}{r}1,066.3 \\
981.2 \\
9.1 \\
4.3 \\
- \\
4.3 \\
-\overline{7} \\
71.7 \\
-\end{array}$ & $\begin{array}{r}1,172.3 \\
1,038.7 \\
7.8 \\
4.9 \\
-\overline{4} \\
4.9 \\
120.9\end{array}$ \\
\hline Primary Energy - Flve Sectors ${ }^{C}$.. & $1,090.4$ & $2,527.2$ & $5,378.4$ & $5,724.5$ & $4,884.6$ & $5,119.0$ & $5,101.7$ & $5,663.3$ & $6,164.2$ & $6,116.8$ \\
\hline
\end{tabular}

a There are no direct fuel costs for hydroelectric, geothermal, centralized solar, or wind energy.

b Heavy oil includes Grade Nos. 4, 5, and 6, and residual fuel oils. Light oil includes Grade No. 2 heating oil, kerosene, and jet fuel.

c Biomass fuels are not included, except those consumed at electric utilities and those added to motor gasoline.

- No consumption, including cases where adjustments were made. See "Consumption Adjustments for Process Fuel and Intermediate Products."

- Value less than 0.05 million dollars.

Note: Totals may not equal sum of components due to independent rounding.

Sources: Data sources, estimation procedures, and assumptions are described in the "Documentation" section of this report. 


\begin{tabular}{|c|c|c|c|c|c|c|c|c|c|c|}
\hline Energy Source & 1970 & 1975 & 1980 & 1985 & 1986 & 1987 & 1988 & 1989 & 1990 & 1991 \\
\hline & \multicolumn{10}{|c|}{ Prices In Dollars per Million Btu } \\
\hline 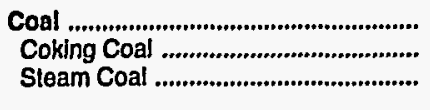 & $\frac{0.92}{-92}$ & $\frac{1.40}{1.40}$ & $\begin{array}{r}1.91 \\
1.91\end{array}$ & $\begin{array}{r}3.65 \\
-\overline{-}\end{array}$ & $\begin{array}{r}3.38 \\
-\overline{-}\end{array}$ & $2 . \overline{0}$ & $\begin{array}{r}2.63 \\
2.63\end{array}$ & $\begin{array}{r}1.95 \\
1.95\end{array}$ & $\begin{array}{r}3.79 \\
3.79\end{array}$ & $\begin{array}{r}4.02 \\
4.02\end{array}$ \\
\hline 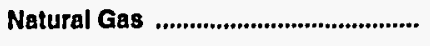 & .67 & .89 & .62 & 1.23 & 1.48 & 1.42 & 1.70 & 1.82 & 1.96 & 1.83 \\
\hline 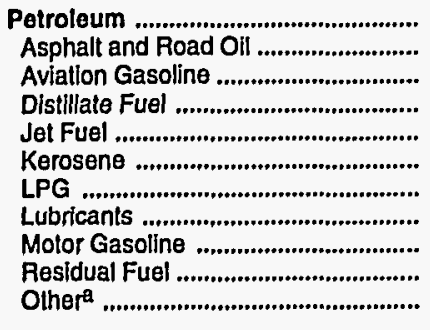 & $\begin{array}{r}1.33 \\
.57 \\
2.17 \\
1.15 \\
.73 \\
1.25 \\
2.09 \\
5.08 \\
3.18 \\
1.37 \\
.43\end{array}$ & $\begin{array}{l}3.00 \\
1.80 \\
3.45 \\
2.88 \\
2.04 \\
3.18 \\
3.80 \\
7.48 \\
5.15 \\
2.34 \\
1.31\end{array}$ & $\begin{array}{r}7.06 \\
3.62 \\
9.02 \\
6.82 \\
6.21 \\
7.29 \\
6.88 \\
14.36 \\
10.20 \\
4.07 \\
4.04\end{array}$ & $\begin{array}{r}7.04 \\
4.47 \\
9.99 \\
7.67 \\
6.07 \\
8.05 \\
13.62 \\
17.61 \\
9.83 \\
4.66 \\
2.79\end{array}$ & $\begin{array}{r}5.29 \\
4.49 \\
8.41 \\
6.71 \\
4.16 \\
6.68 \\
11.59 \\
15.59 \\
7.86 \\
3.04 \\
-\end{array}$ & $\begin{array}{r}5.36 \\
4.25 \\
7.55 \\
6.18 \\
4.13 \\
6.32 \\
11.33 \\
12.70 \\
7.90 \\
3.36 \\
-\end{array}$ & $\begin{array}{r}5.44 \\
3.93 \\
7.41 \\
6.38 \\
4.14 \\
4.57 \\
11.37 \\
14.61 \\
8.14 \\
4.10 \\
-\end{array}$ & $\begin{array}{r}6.23 \\
3.19 \\
8.28 \\
7.62 \\
4.69 \\
7.27 \\
14.88 \\
13.30 \\
8.65 \\
3.90 \\
-\end{array}$ & $\begin{array}{r}7.59 \\
3.14 \\
9.32 \\
8.47 \\
6.17 \\
9.05 \\
15.09 \\
13.40 \\
10.03 \\
5.26 \\
-\end{array}$ & $\begin{array}{r}6.82 \\
3.28 \\
8.71 \\
7.73 \\
5.50 \\
8.68 \\
15.62 \\
15.42 \\
8.99 \\
3.33 \\
-\end{array}$ \\
\hline Nuclear Fuel .......................................... & - & - & - & - & - & - & - & - & - & - \\
\hline Blomass Fuals at Utilities ................. & - & - & - & - & - & - & - & - & - & - \\
\hline Primary Energy - Five Sectors ${ }^{b} \ldots$ & 1.12 & 2.21 & 4.04 & 4.71 & 4.06 & 3.96 & 4.04 & 4.71 & 5.50 & 4.85 \\
\hline $\begin{array}{l}\text { Electric Utility Fuelc ............................ } \\
\text { Electricity Purchased by End Users }\end{array}$ & $\begin{array}{r}.66 \\
9.02\end{array}$ & .95 & $\begin{array}{r}1.25 \\
15.09\end{array}$ & $\begin{array}{r}1.71 \\
24.52\end{array}$ & $\begin{array}{r}1.48 \\
24.89\end{array}$ & $\begin{array}{r}1.59 \\
26.64\end{array}$ & $\begin{array}{r}2.12 \\
26.36\end{array}$ & $\begin{array}{r}1.86 \\
27.06\end{array}$ & $\begin{array}{r}2.38 \\
27.80\end{array}$ & $\begin{array}{r}1.80 \\
28.75\end{array}$ \\
\hline \multirow[t]{2}{*}{ 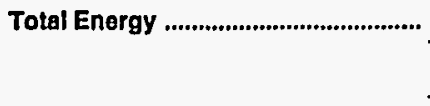 } & 1.39 & 2.71 & 5.06 & 5.99 & 5.26 & 5.28 & 5.42 & 6.24 & 7.01 & 6.49 \\
\hline & \multicolumn{10}{|c|}{ Expenditures in Millions of Dollars } \\
\hline 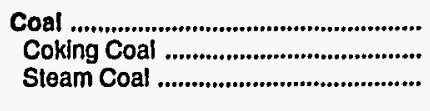 & $\begin{array}{r}12.1 \\
12.1\end{array}$ & $21 . \overline{4}$ & $8 . \overline{2}$ & $\begin{array}{r}42.3 \\
42.3\end{array}$ & $\begin{array}{r}41.1 \\
41.1\end{array}$ & $\begin{array}{r}8.7 \\
\overline{8}\end{array}$ & $\begin{array}{r}11.5 \\
11.5\end{array}$ & $\begin{array}{r}9.2 \\
-\overline{2}\end{array}$ & $\begin{array}{r}47.0 \\
-\overline{0}\end{array}$ & $\begin{array}{r}51.0 \\
51.0\end{array}$ \\
\hline Notural Gas ......................................... & 26.2 & 54.5 & 64.5 & 162.4 & 165.5 & 158.4 & 187.1 & 187.4 & 223.7 & 212.4 \\
\hline 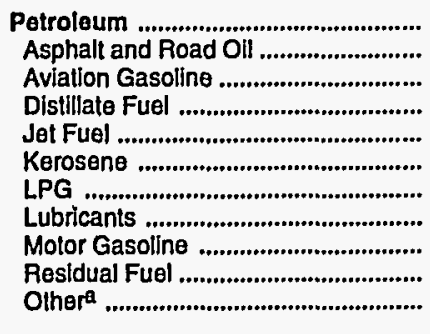 & $\begin{array}{r}122.7 \\
1.0 \\
5.1 \\
33.3 \\
27.5 \\
.2 \\
1.1 \\
1.9 \\
43.8 \\
8.7 \\
.1\end{array}$ & $\begin{array}{r}354.0 \\
3.8 \\
8.1 \\
116.6 \\
85.0 \\
2.2 \\
2.5 \\
6.6 \\
113.0 \\
15.7 \\
.5\end{array}$ & $\begin{array}{r}853.7 \\
7.4 \\
22.7 \\
264.0 \\
335.7 \\
.8 \\
4.2 \\
10.0 \\
196.9 \\
9.4 \\
2.5\end{array}$ & $\begin{array}{r}1,428.2 \\
14.4 \\
24.7 \\
462.1 \\
520.3 \\
.3 \\
15.2 \\
11.2 \\
291.2 \\
84.4 \\
4.5\end{array}$ & $\begin{array}{r}1,266.9 \\
11.1 \\
26.2 \\
292.9 \\
379.8 \\
188.7 \\
10.3 \\
9.7 \\
224.1 \\
124.1 \\
-\end{array}$ & $\begin{array}{r}1,123.8 \\
7.2 \\
7.9 \\
287.5 \\
345.6 \\
171.7 \\
10.4 \\
8.9 \\
215.4 \\
69.2 \\
-\end{array}$ & $\begin{array}{r}1,016.3 \\
18.2 \\
15.2 \\
317.3 \\
394.0 \\
5.0 \\
10.6 \\
9.9 \\
227.7 \\
18.4 \\
-\end{array}$ & $\begin{array}{r}1,270.6 \\
5.8 \\
20.5 \\
490.1 \\
491.0 \\
.1 \\
14.6 \\
9.2 \\
230.7 \\
8.5 \\
-\end{array}$ & $\begin{array}{r}1,554.3 \\
5.6 \\
23.1 \\
571.3 \\
604.3 \\
.1 \\
20.6 \\
9.6 \\
306.6 \\
13.0 \\
-\end{array}$ & $\begin{array}{r}1,247.0 \\
5.6 \\
27.1 \\
441.0 \\
489.0 \\
.4 \\
22.3 \\
9.8 \\
241.1 \\
10.5 \\
-\end{array}$ \\
\hline Nuclear Fuel ........................................ & - & - & - & - & - & - & - & - & - & - \\
\hline Blomass Fuels at UtIlttles ................. & - & - & - & - & - & - & - & - & - & - \\
\hline Primary Energy — Five Sectors ${ }^{b} \ldots$ & 161.0 & 429.9 & 926.4 & $1,632.9$ & $1,473.5$ & $1,290.9$ & $1,215.0$ & $1,467.2$ & $1,824.9$ & $1,510.4$ \\
\hline $\begin{array}{l}\text { Electric Utility Fuelc ........................... } \\
\text { Electriclty Purchased by End Users }\end{array}$ & $\begin{array}{r}-9.9 \\
33.9\end{array}$ & $\begin{array}{r}-27.0 \\
65.9\end{array}$ & $\begin{array}{r}-48.3 \\
129.5\end{array}$ & $\begin{array}{r}-77.0 \\
331.5\end{array}$ & $\begin{array}{r}-66.3 \\
339.3\end{array}$ & $\begin{array}{l}-62.7 \\
353.4\end{array}$ & $\begin{array}{r}-83.9 \\
358.8\end{array}$ & $\begin{array}{r}-78.1 \\
380.1\end{array}$ & $\begin{array}{r}-102.5 \\
400.9\end{array}$ & $\begin{array}{r}-73.2 \\
414.8\end{array}$ \\
\hline Total Energy & 185.0 & 468.9 & $1,007.7$ & $1,887.3$ & $1,746.5$ & $1,581.6$ & $1,489.8$ & $1,769.2$ & $2,123.3$ & $1,852.0$ \\
\hline
\end{tabular}

a Includes petroleum coke used at electric utilities.

b Biomass fuels are not included, except those consumed at electric utilities and those added to motor gasoline.

c There are no direct fuel costs for hydroelectric, geothermal, centralized solar, or wind energy.

- No consumption, including cases where adjustments were made. See "Consumption Adjustments for Process Fuel and Intermediate Products."

Note: Expenditure totals may not equal sum of components due to independent rounding.

Sources: Data sources, estimation procedures, and assumptions are described in the "Documentation" section of this report. 
A Energy Price Estimates by Sector, Alaska

L $1970,1975,1980$, and 1985-1991

A (Dollars per Million Btu)

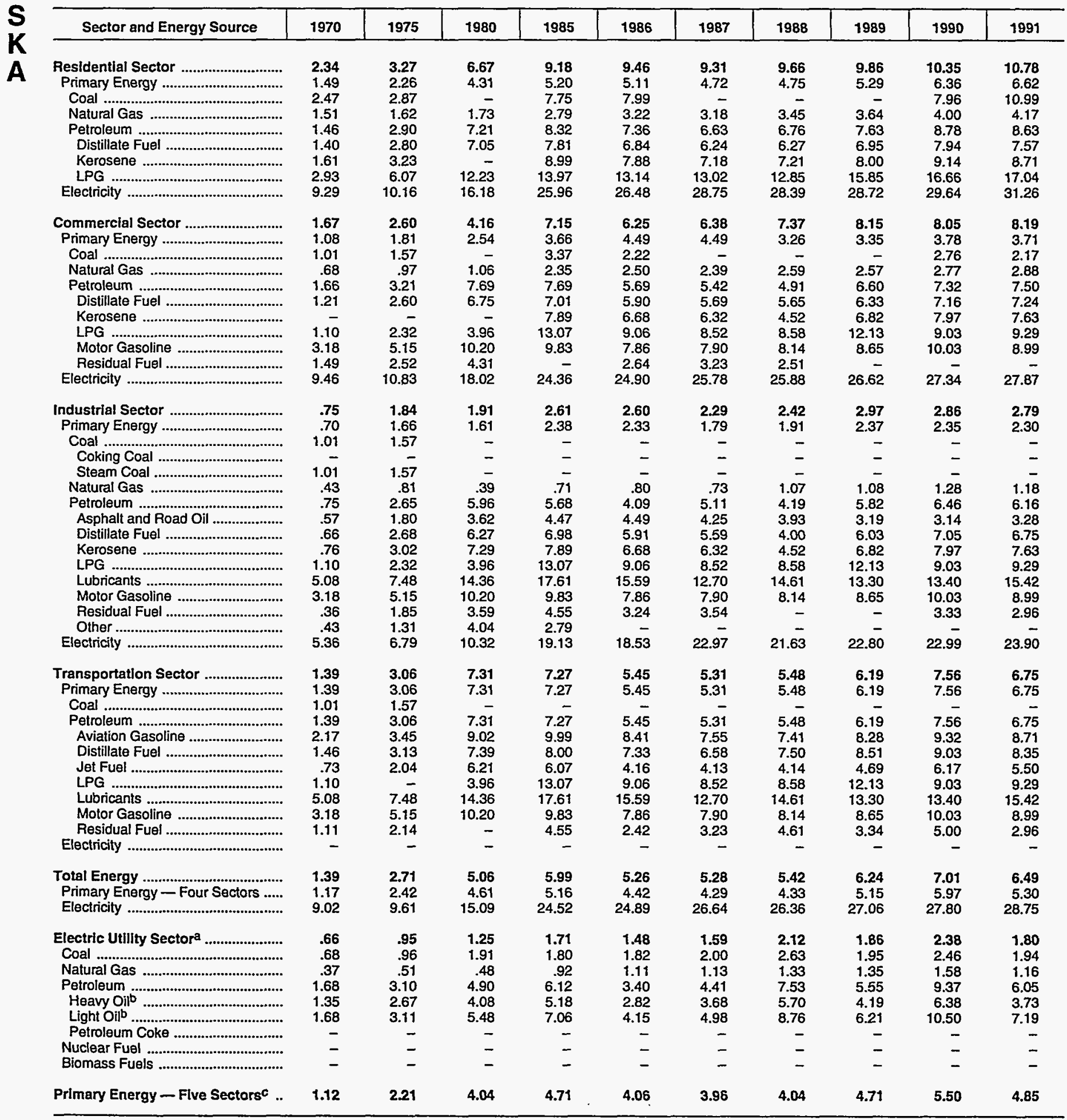

a There are no direct fuel costs for hydroelectric, geothermal, centralized solar, or wind energy.

beavy oil includes Grade Nos. 4, 5, and 6, and residual fuel oils. Light oil includes Grade No. 2 heating oil, kerosene, and jet fuel.

c Biomass fuels are not included, except those consumed at electric utilities and those added to motor gasoline.

-No consumption, including cases where adjustments were made. See "Consumption Adjustments for Process Fuel and Intermediate Products."

Sources: Data sources, estimation procedures, and assumptions are described in the "Documentation" section of this report. 
Energy Expenditure Estimates by Sector, Alaska

$1970,1975,1980$, and 1985-1991

(Million Dollars)

\begin{tabular}{|c|c|c|c|c|c|c|c|c|c|c|}
\hline Sector and Energy Source & 1970 & 1975 & 1980 & 1985 & 1986 & 1987 & 1988 & 1989 & 1990 & 1991 \\
\hline 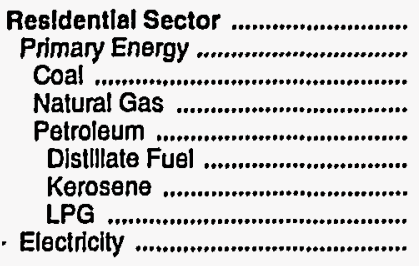 & $\begin{array}{r}38.6 \\
21.9 \\
.4 \\
9.4 \\
12.1 \\
11.1 \\
.2 \\
.9 \\
.9 \\
16.7\end{array}$ & $\begin{array}{r}77.9 \\
46.8 \\
.3 \\
16.9 \\
29.6 \\
26.4 \\
1.7 \\
1.5 \\
1.5\end{array}$ & $\begin{array}{r}124.8 \\
64.5 \\
. \overline{8} \\
13.8 \\
50.8 \\
48.2 \\
. \overline{6} \\
2.6 \\
60.3\end{array}$ & $\begin{array}{r}273.6 \\
125.3 \\
18.7 \\
37.3 \\
69.3 \\
59.6 \\
.1 \\
.1 \\
9.6 \\
148.3\end{array}$ & $\begin{array}{r}257.8 \\
111.0 \\
21.9 \\
39.3 \\
49.7 \\
42.4 \\
7 . \\
7.3 \\
146.8\end{array}$ & $\begin{array}{r}257.4 \\
105.5 \\
39 . \overline{3} \\
66.2 \\
58.6 \\
7.5 \\
151.9\end{array}$ & $\begin{array}{r}252.3 \\
98.3 \\
- \\
43.4 \\
54.9 \\
46.9 \\
.1 \\
7.9 \\
154.0\end{array}$ & $\begin{array}{r}283.4 \\
122.4 \\
- \\
49.3 \\
73.0 \\
61.5 \\
11.6 \\
161.0\end{array}$ & $\begin{array}{r}342.4 \\
174.4 \\
21.7 \\
53.7 \\
98.9 \\
80.7 \\
.1 \\
18.1 \\
168.0\end{array}$ & $\begin{array}{r}349.0 \\
178.0 \\
30.6 \\
56.7 \\
90.7 \\
70.4 \\
.4 \\
19.9 \\
171.0\end{array}$ \\
\hline 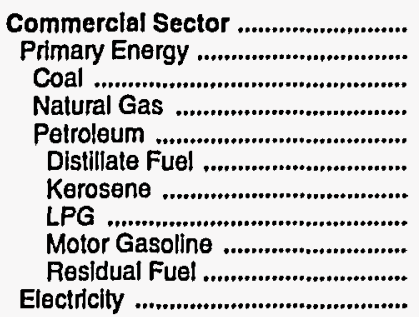 & $\begin{array}{r}39.0 \\
23.5 \\
.3 \\
8.6 \\
14.7 \\
3.0 \\
-\overline{.1} \\
4.1 \\
7.5 \\
15.4\end{array}$ & $\begin{array}{r}66.4 \\
42.1 \\
.3 \\
14.0 \\
27.8 \\
7.6 \\
\overline{.1} \\
11.2 \\
8.9 \\
24.3\end{array}$ & $\begin{array}{r}99.0 \\
54.2 \\
- \\
17 . \overline{5} \\
36.8 \\
22.7 \\
\overline{.1} \\
13.8 \\
.1 \\
44.8\end{array}$ & $\begin{array}{r}274.3 \\
116.6 \\
15.1 \\
48.1 \\
53.4 \\
37.8 \\
.1 \\
1.6 \\
13.8 \\
- \\
157.7\end{array}$ & $\begin{array}{r}484.2 \\
317.9 \\
11.3 \\
52.7 \\
253.9 \\
28.8 \\
188.6 \\
.9 \\
8.3 \\
27.4 \\
166.3\end{array}$ & $\begin{array}{r}464.8 \\
298.2 \\
48 . \overline{7} \\
249.4 \\
34.9 \\
171.6 \\
.9 \\
2.1 \\
39.9 \\
166.6\end{array}$ & $\begin{array}{r}264.7 \\
95.8 \\
54.2 \\
41.6 \\
28.8 \\
4.8 \\
.9 \\
2.2 \\
4.9 \\
168.9\end{array}$ & $\begin{array}{r}276.2 \\
90.2 \\
- \\
55.9 \\
34.4 \\
30.4 \\
1.6 \\
2.4 \\
- \\
186.0\end{array}$ & $\begin{array}{r}323.4 \\
124.3 \\
14.0 \\
56.9 \\
53.5 \\
49.0 \\
1.7 \\
2.7 \\
199.0\end{array}$ & $\begin{array}{r}329.4 \\
121.4 \\
11.2 \\
60.4 \\
49.8 \\
41.1 \\
1.9 \\
6.8 \\
208 . \overline{0}\end{array}$ \\
\hline 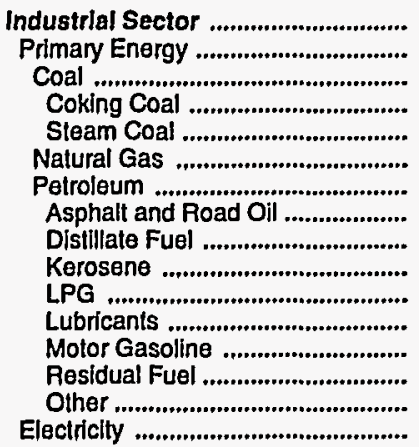 & $\begin{array}{r}25.7 \\
23.9 \\
8.6 \\
-\overline{6} \\
8.6 \\
5.1 \\
10.2 \\
1.0 \\
6.9 \\
.1 \\
.2 \\
. \\
1.8 \\
.1 \\
.1 \\
1.7\end{array}$ & $\begin{array}{r}81.2 \\
70.7 \\
16.5 \\
-\overline{1} \\
16.5 \\
13.5 \\
40.7 \\
3.8 \\
30.8 \\
.5 \\
.8 \\
1.1 \\
2.9 \\
.3 \\
.5\end{array}$ & $\begin{array}{r}128.0 \\
103.5 \\
- \\
- \\
19.5 \\
84.0 \\
7.4 \\
64.0 \\
.8 \\
1.3 \\
1.8 \\
5.9 \\
.3 \\
2.5 \\
24.5\end{array}$ & $\begin{array}{r}255.4 \\
230.0 \\
- \\
- \\
45 . \overline{2} \\
184.8 \\
14.4 \\
71.2 \\
.2 \\
3.3 \\
2.0 \\
21.0 \\
68.3 \\
4.5 \\
25.5\end{array}$ & $\begin{array}{r}215.2 \\
189.0 \\
- \\
- \\
\overline{3} . \overline{9} \\
154.1 \\
11.1 \\
37.7 \\
.1 \\
1.9 \\
1.7 \\
15.9 \\
85.6 \\
-\overline{1} \\
26 . \overline{2}\end{array}$ & $\begin{array}{r}149.5 \\
114.6 \\
- \\
- \\
35.5 \\
79.1 \\
7.2 \\
31.9 \\
1.9 \\
1.9 \\
1.6 \\
19.6 \\
19.8 \\
34 . \overline{9}\end{array}$ & $\begin{array}{r}154.5 \\
118.7 \\
- \\
- \\
\overline{-} \\
48.3 \\
70.4 \\
18.2 \\
46.0 \\
1.6 \\
1.8 \\
2.7 \\
- \\
- \\
35.8\end{array}$ & $\begin{array}{r}146.4 \\
113.4 \\
- \\
- \\
37 . \overline{7} \\
75.7 \\
5.8 \\
64.1 \\
\vdots \\
1.2 \\
1.7 \\
2.9 \\
\overline{-} \\
33.0\end{array}$ & $\begin{array}{r}169.5 \\
135.7 \\
- \\
- \\
58.5 \\
77.2 \\
5.6 \\
64.7 \\
. \\
1.7 \\
2.9 \\
1.7 \\
33.9\end{array}$ & $\begin{array}{r}185.2 \\
149.4 \\
- \\
- \\
- \\
59.1 \\
90.3 \\
5.6 \\
76.3\end{array}$ \\
\hline 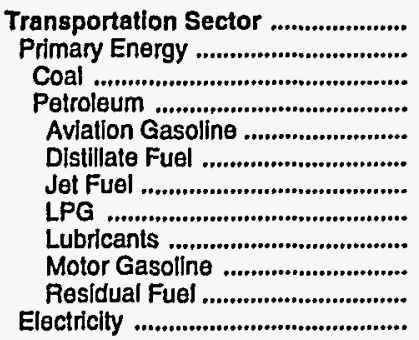 & $\begin{array}{r}81.8 \\
81.8 \\
81.7 \\
5.1 \\
8.5 \\
27.5 \\
. \\
1.8 \\
37.9 \\
.9 \\
-\end{array}$ & $\begin{array}{r}243.3 \\
243.3 \\
243.3 \\
8.1 \\
39.3 \\
85.0 \\
- \\
5.5 \\
98.9 \\
6.5 \\
-\end{array}$ & $\begin{array}{r}655.9 \\
655.9 \\
655.9 \\
22.7 \\
112.1 \\
335.7 \\
.1 \\
8.2 \\
177.1 \\
- \\
-\end{array}$ & $\begin{array}{r}1,084.0 \\
1,084.0 \\
1,084.0 \\
24.7 \\
272.2 \\
520.3 \\
.7 \\
9.1 \\
256.4 \\
.5 \\
-\end{array}$ & $\begin{array}{r}789.3 \\
789.3 \\
789.3 \\
26.2 \\
173.5 \\
379.8 \\
.3 \\
7.9 \\
199.9 \\
1.7 \\
\end{array}$ & $\begin{array}{r}709.8 \\
709.8 \\
709.8 \\
7.9 \\
149.8 \\
345.6 \\
.2 \\
7.3 \\
196.6 \\
2.4 \\
-\end{array}$ & $\begin{array}{r}818.3 \\
818.3 \\
818.3 \\
15.2 \\
173.9 \\
394.0 \\
.3 \\
8.1 \\
222.8 \\
4.1 \\
-\end{array}$ & $\begin{array}{r}1,063.1 \\
1,063.1 \\
-\overline{1} \\
1,063.1 \\
20.5 \\
315.8 \\
491.0 \\
.3 \\
7.6 \\
225.4 \\
2.5 \\
-\end{array}$ & $\begin{array}{r}1,288.0 \\
1,288.0 \\
1,288.0 \\
23.1 \\
347.1 \\
604.3 \\
.2 \\
7.8 \\
301.0 \\
4.4 \\
-\end{array}$ & $\begin{array}{r}988.3 \\
988.3 \\
\overline{-} \\
988.3 \\
27.1 \\
231.0 \\
489.0 \\
.1 \\
8.1 \\
231.6 \\
1.4 \\
-\end{array}$ \\
\hline 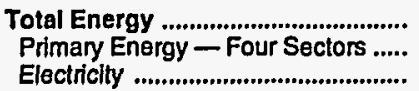 & $\begin{array}{r}185.0 \\
151.2 \\
33.9\end{array}$ & $\begin{array}{r}468.9 \\
403.0 \\
65.9\end{array}$ & $\begin{array}{r}1,007.7 \\
878.2 \\
129.5\end{array}$ & $\begin{array}{r}1,887.3 \\
1,555.9 \\
331.5\end{array}$ & $\begin{array}{r}1,746.5 \\
1,407.2 \\
339.3\end{array}$ & $\begin{array}{r}1,581.6 \\
1,228.1 \\
353.4\end{array}$ & $\begin{array}{r}1,489.8 \\
1,131.1 \\
358.8\end{array}$ & $\begin{array}{r}1,769.2 \\
1,389.1 \\
380.1\end{array}$ & $\begin{array}{r}2,123.3 \\
1,722.4 \\
400.9\end{array}$ & $\begin{array}{r}1,852.0 \\
1,437.2 \\
414.8\end{array}$ \\
\hline 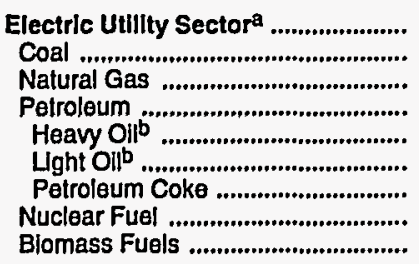 & $\begin{array}{r}9.9 \\
2.9 \\
3.1 \\
3.9 \\
\dot{4} \\
3.9 \\
- \\
-\end{array}$ & $\begin{array}{r}27.0 \\
4.3 \\
10.1 \\
12.6 \\
. \\
12.6 \\
- \\
- \\
-\end{array}$ & $\begin{aligned} 48.3 \\
8.2 \\
13.8 \\
26.3 \\
9.1 \\
17.2 \\
- \\
- \\
-\end{aligned}$ & $\begin{array}{r}77.0 \\
8.4 \\
31.8 \\
36.8 \\
15.5 \\
21.3 \\
- \\
- \\
-\end{array}$ & $\begin{array}{r}66.3 \\
7.8 \\
38.5 \\
19.9 \\
9.4 \\
10.6 \\
- \\
- \\
-\end{array}$ & $\begin{array}{r}62.7 \\
8.7 \\
34.8 \\
19.3 \\
7.1 \\
12.2 \\
- \\
-\end{array}$ & $\begin{array}{r}83.9 \\
11.5 \\
41.3 \\
31.1 \\
9.4 \\
21.6 \\
- \\
- \\
-\end{array}$ & $\begin{array}{r}78.1 \\
9.2 \\
44.5 \\
24.4 \\
6.1 \\
18.3 \\
= \\
=\end{array}$ & $\begin{array}{r}102.5 \\
11.3 \\
54.6 \\
36.6 \\
6.9 \\
29.8 \\
- \\
- \\
-\end{array}$ & $\begin{array}{r}73.2 \\
9.1 \\
36.3 \\
27.8 \\
5.6 \\
22.2 \\
= \\
-\end{array}$ \\
\hline Prlmary Energy - Flve Sectors ${ }^{c}$... & 161.0 & 429.9 & 926.4 & $1,632.9$ & $1,473.5$ & $1,290.9$ & $1,215.0$ & $1,467.2$ & $1,824.9$ & $1,510.4$ \\
\hline
\end{tabular}

There are no direct fuel costs for hydroelectric, geothermal, centralized solar, or wind energy.

b Heavy oll includes Grade Nos. 4,5, and 6, and residual fuel oils. Light oil includes Grade No. 2 heating oil, kerosene, and jel tuel.

c Blomass fuels are not Inciuded, except those consumed at electric utilities and those added to motor gasoline.

- No consumption, including cases where adjustments were made. See "Consumption Adjustments for Process Fuel and Intermediate Products."

"Value less than 0.05 million dollars.

Note: Totals may not equal sum of components due to independent rounding.

Sources: Data sources, estimatlon procedures, and assumptions are described in the "Documentation" section of this report. 


\section{A Energy Price and Expenditure Estimates by Source, Arizona}

R $1970,1975,1980$, and 1985-1991

\begin{tabular}{|c|c|c|c|c|c|c|c|c|c|c|}
\hline Energy Source & 1970 & 1975 & 1980 & 1985 & 1986 & 1987 & 1988 & 1989 & 1990 & 1891 \\
\hline & \multicolumn{10}{|c|}{ Prices In Dollars per Million Btu } \\
\hline 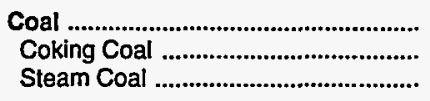 & $\frac{0.21}{-21}$ & $\frac{0.23}{.23}$ & $\begin{array}{r}1.01 \\
1.01\end{array}$ & $\begin{array}{r}1.36 \\
1.36\end{array}$ & $\begin{array}{r}1.49 \\
1.49\end{array}$ & $\frac{1.33}{1.33}$ & $\frac{1.42}{1.42}$ & $\frac{1.38}{1.38}$ & $\frac{1.45}{1.45}$ & $\frac{1.43}{1.43}$ \\
\hline 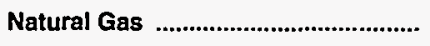 & .54 & 1.01 & 2.86 & 4.92 & 4.12 & 4.10 & 4.45 & 3.92 & 4.52 & 4.58 \\
\hline $\begin{array}{l}\text { Petroleum } \\
\text { Asphalt and Road Oil } \\
\text { Aviation Gasoline } \\
\text { Distillate Fuel } \\
\text { Jet Fuel } \\
\text { Kerosene } \\
\text { LPG } \\
\text { Lubricants } \\
\text { Motor Gasoline } \\
\text { Residual Fuel } \\
\text { Other }\end{array}$ & $\begin{array}{r}1.96 \\
.60 \\
2.17 \\
1.10 \\
.76 \\
1.63 \\
2.08 \\
5.08 \\
2.80 \\
.48 \\
-\end{array}$ & $\begin{array}{l}3.45 \\
1.87 \\
3.45 \\
2.49 \\
2.12 \\
3.19 \\
4.06 \\
7.48 \\
4.62 \\
2.08 \\
1.31\end{array}$ & $\begin{array}{r}8.14 \\
3.63 \\
9.02 \\
6.57 \\
6.59 \\
5.45 \\
6.85 \\
14.36 \\
9.68 \\
3.92 \\
4.04\end{array}$ & $\begin{array}{r}8.08 \\
4.76 \\
9.99 \\
6.45 \\
6.20 \\
5.42 \\
9.98 \\
17.61 \\
9.06 \\
3.79 \\
-\end{array}$ & $\begin{array}{r}6.46 \\
4.33 \\
8.41 \\
5.32 \\
4.25 \\
3.34 \\
9.55 \\
15.59 \\
7.24 \\
2.36 \\
-\end{array}$ & $\begin{array}{r}6.85 \\
3.21 \\
7.55 \\
6.02 \\
4.36 \\
3.28 \\
9.81 \\
12.70 \\
7.77 \\
2.33 \\
-\end{array}$ & $\begin{array}{r}6.54 \\
3.28 \\
7.41 \\
5.97 \\
4.15 \\
4.45 \\
9.05 \\
14.61 \\
7.31 \\
2.37 \\
-\end{array}$ & $\begin{array}{r}7.25 \\
2.84 \\
8.28 \\
6.33 \\
4.80 \\
4.02 \\
11.51 \\
13.30 \\
8.19 \\
2.76 \\
-\end{array}$ & $\begin{array}{r}8.37 \\
2.71 \\
9.32 \\
8.23 \\
6.04 \\
8.14 \\
11.71 \\
13.40 \\
9.22 \\
3.31 \\
-\end{array}$ & $\begin{array}{r}7.80 \\
3.34 \\
8.71 \\
7.78 \\
5.03 \\
7.37 \\
12.08 \\
15.42 \\
8.57 \\
2.65 \\
-\end{array}$ \\
\hline 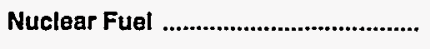 & - & - & - & .65 & .65 & .82 & .77 & .85 & .72 & .70 \\
\hline Blomass Fuels at Utilities .................. & - & - & - & - & - & - & - & - & - & - \\
\hline Primary Energy - Five Sectors ${ }^{b}$.... & 1.29 & 2.25 & 4.49 & 4.58 & 3.68 & 3.74 & 3.35 & 3.93 & 3.98 & 3.64 \\
\hline $\begin{array}{l}\text { Electric Utility Fuelc ......................... } \\
\text { Electricity Purchased by End Users }\end{array}$ & $\begin{array}{r}.33 \\
5.32\end{array}$ & $\begin{array}{r}.84 \\
9.65\end{array}$ & $\begin{array}{r}1.35 \\
15.68\end{array}$ & $\begin{array}{r}1.60 \\
21.15\end{array}$ & $\begin{array}{r}1.26 \\
21.87\end{array}$ & $\begin{array}{r}1.21 \\
21.31\end{array}$ & $\begin{array}{r}1.18 \\
21.73\end{array}$ & $\begin{array}{r}1.38 \\
22.10\end{array}$ & $\begin{array}{r}1.21 \\
22.82\end{array}$ & $\begin{array}{r}1.13 \\
23.09\end{array}$ \\
\hline \multirow[t]{2}{*}{ 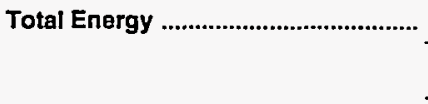 } & 1.97 & 3.87 & 8.42 & 10.03 & 9.17 & 9.72 & 9.75 & 10.35 & 11.30 & 11.03 \\
\hline & \multicolumn{10}{|c|}{ Expenditures in Millions of Dollars } \\
\hline 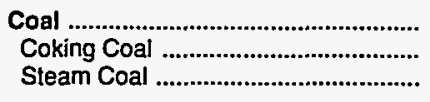 & $\begin{array}{r}1.8 \\
\overline{1}\end{array}$ & $21 . \overline{1}$ & $\begin{array}{r}247.0 \\
247.0\end{array}$ & $\begin{array}{r}466.2 \\
466 . \overline{2}\end{array}$ & $\begin{array}{r}440.9 \\
\overline{-} \\
440.9\end{array}$ & $\begin{array}{r}376.0 \\
-\overline{0}\end{array}$ & $\begin{array}{r}439.6 \\
\overline{-} \\
439.6\end{array}$ & $\begin{array}{r}493.8 \\
- \\
493.8\end{array}$ & $\begin{array}{r}498.5 \\
\overline{498.5}\end{array}$ & $\begin{array}{r}497.6 \\
-\overline{6} \\
497.6\end{array}$ \\
\hline 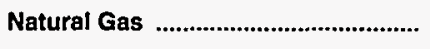 & 96.8 & 148.4 & 434.0 & 580.6 & 379.7 & 426.6 & 487.9 & 510.6 & 464.2 & 468.8 \\
\hline $\begin{array}{l}\text { Petroleum } \\
\text { Asphalt and Road Oil } \\
\text { Aviation Gasoline } \\
\text { Distillate Fuel . } \\
\text { Jet Fuel } \\
\text { Kerosene } \\
\text { LPG } \\
\text { Mubricants } \\
\text { Retor Gasoline } \\
\text { Other }{ }^{2}\end{array}$ & $\begin{array}{r}417.7 \\
14.6 \\
4.7 \\
31.3 \\
27.5 \\
1.5 \\
10.2 \\
10.6 \\
316.9 \\
.3 \\
-\end{array}$ & $\begin{array}{r}1,056.8 \\
28.9 \\
6.2 \\
147.1 \\
82.9 \\
3.8 \\
16.9 \\
21.4 \\
671.9 \\
77.7\end{array}$ & $\begin{array}{r}2,448.1 \\
49.6 \\
12.8 \\
412.0 \\
289.7 \\
2.3 \\
40.0 \\
53.2 \\
1,555.4 \\
33.0 \\
.1\end{array}$ & $\begin{array}{r}2,563.0 \\
81.0 \\
9.3 \\
382.6 \\
244.4 \\
.5 \\
61.9 \\
59.4 \\
1,719.7 \\
4.2 \\
-\end{array}$ & $\begin{array}{r}2,164.6 \\
72.7 \\
9.6 \\
350.5 \\
181.0 \\
1.1 \\
59.2 \\
51.4 \\
1,438.5 \\
.6 \\
-\end{array}$ & $\begin{array}{r}2,356.0 \\
53.1 \\
7.9 \\
373.2 \\
202.5 \\
.9 \\
69.8 \\
47.4 \\
1,599.5 \\
1.8 \\
-\end{array}$ & $\begin{array}{r}2,282.3 \\
58.4 \\
7.0 \\
363.9 \\
195.0 \\
1.4 \\
56.8 \\
52.6 \\
1,546.4 \\
.8 \\
-\end{array}$ & $\begin{array}{r}2,554.2 \\
45.0 \\
8.8 \\
420.6 \\
217.4 \\
1.1 \\
62.2 \\
49.1 \\
1,747.4 \\
2.6 \\
-\end{array}$ & $\begin{array}{r}2,918.1 \\
42.6 \\
9.1 \\
577.1 \\
285.9 \\
.9 \\
58.2 \\
50.9 \\
1,892.9 \\
.5 \\
-\end{array}$ & $\begin{array}{r}2,739.1 \\
48.3 \\
8.3 \\
469.4 \\
263.7 \\
1.5 \\
66.0 \\
52.4 \\
1,827.0 \\
2.4 \\
-\end{array}$ \\
\hline 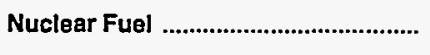 & - & - & - & 8.0 & 69.8 & 118.4 & 190.6 & 71.8 & 158.1 & 188.9 \\
\hline Blomass Fuels at Utilities ................. & - & - & - & - & - & - & - & - & - & - \\
\hline Primary Energy - Five Sectors ${ }^{b}$.... & 516.4 & $1,226.3$ & $3,129.0$ & $3,617.8$ & $3,054.9$ & $3,277.1$ & $3,400.4$ & $3,630.4$ & $4,039.0$ & $3,894.4$ \\
\hline 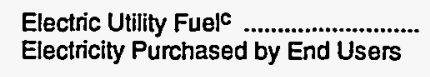 & $\begin{array}{r}-23.5 \\
250.1\end{array}$ & $\begin{array}{r}-129.6 \\
697.1\end{array}$ & $\begin{array}{r}-398.7 \\
1,431.6\end{array}$ & $\begin{array}{r}-580.5 \\
2,381.4\end{array}$ & $\begin{array}{r}-490.6 \\
2,536.2\end{array}$ & $\begin{array}{r}-539.1 \\
2,664.5\end{array}$ & $\begin{array}{r}-671.5 \\
2,886.0\end{array}$ & $\begin{array}{r}-664.3 \\
3,030.3\end{array}$ & $\begin{array}{r}-696.2 \\
3,181.4\end{array}$ & $\begin{array}{r}-711.4 \\
3,249.2\end{array}$ \\
\hline Total Energy & 743.0 & $1,793.8$ & $4,161.9$ & $5,418.7$ & $5,100.5$ & $5,402.4$ & $5,614.9$ & $5,996.4$ & $6,524.2$ & $6,432.3$ \\
\hline
\end{tabular}

Includes petroleum coke used at electric utilities.

biomass fuels are not included, except those consumed at electric utilities and those added to motor gasoline.

c There are no direct fuel costs for hydroelectric, geothermal, centralized solar, or wind energy.

-No consumption, including cases where adjustments were made. See "Consumption Adjustments for Process Fuel and Intermediate Products."

- Value less than 0.05 million dollars.

Note: Expenditure totals may not equal sum of components due to independent rounding.

Sources: Data sources, estimation procedures, and assumptions are described in the "Documentation" section of this report. 


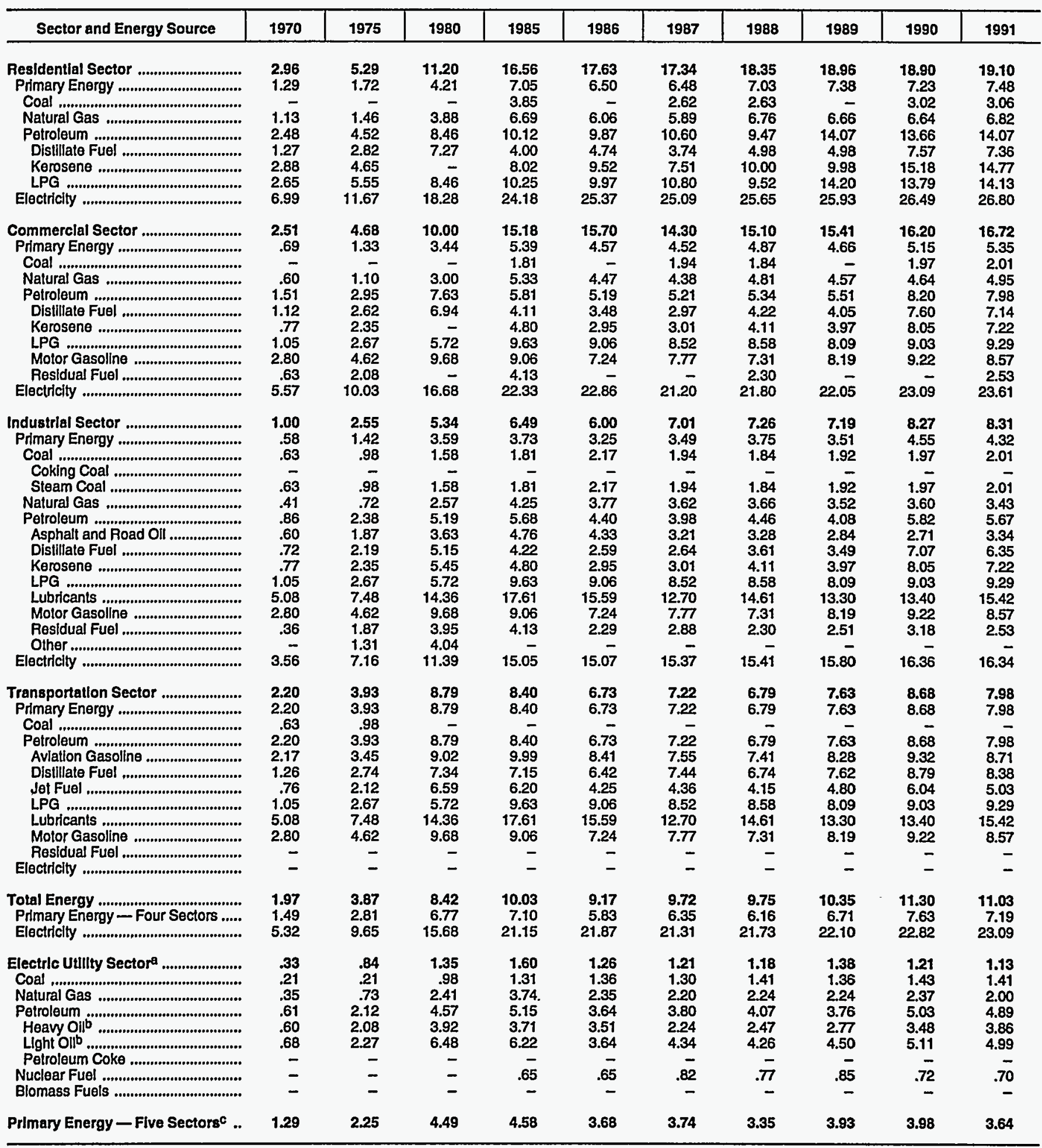

a There are no direct fuel costs for hydroelectric, geothermal, centralized solar, or wind energy.

b Heavy oll Includes Grade Nos. 4, 5, and 6, and residual fuel oils. Light oll includes Grade No. 2 heating oil, kerosene, and jet fuel.

c Blomass fuels are not included, except those consumed at electric utilities and those added to motor gasoline.

-No consumption, including cases where adjustments were made. See "Consumption Adjustments for Process Fuel and Intermediate Products."

Sources: Data sources, estimation procedures, and assumptions are described in the "Documentation" section of this report. 
A Energy Expenditure Estimates by Sector, Arizona

R $1970,1975,1980$, and 1985-1991

(Million Dollars)

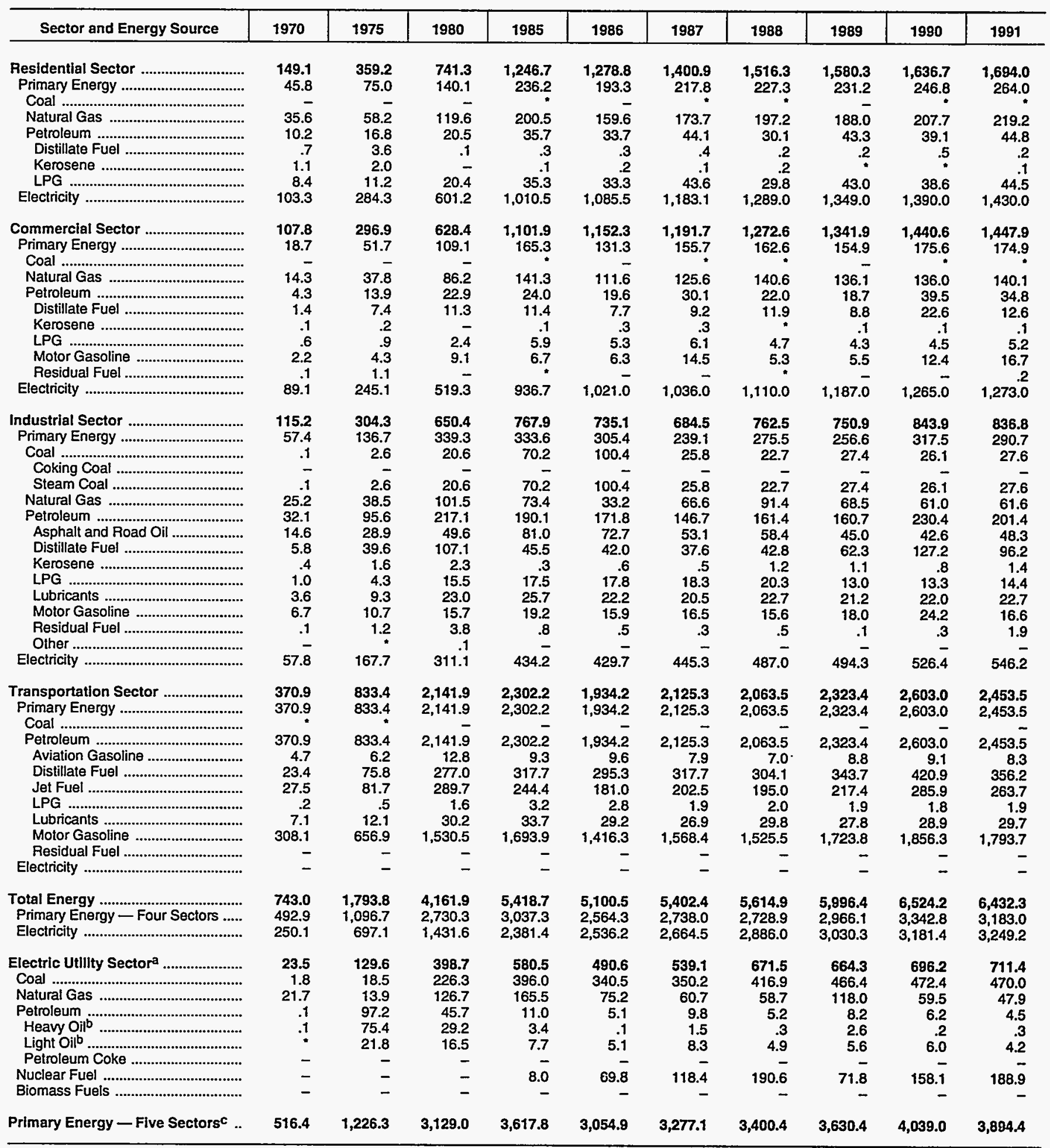

There are no direct fuel costs for hydroelectric, geothermal, centralized solar, or wind energy.

beavy oil includes Grade Nos. 4, 5, and 6, and residual fuel oils. Light oil includes Grade No. 2 heating oil, kerosene, and jet fuel.

c Biomass fuels are not included, except those consumed at electric ulilities and those added to motor gasoline.

$\rightarrow$ No consumption, including cases where adjustments were made. See "Consumption Adjustments for Process Fuel and Intermediate Products."

"Value less than 0.05 million dollars.

Note: Totals may not equal sum of components due to independent rounding.

Sources: Data sources, estimation procedures, and assumptions are described in the "Documentation" section of this report. 


\begin{tabular}{|c|c|c|c|c|c|c|c|c|c|c|}
\hline Energy Source & 1970 & 1975 & 1980 & 1985 & 1986 & 1987 & 1988 & 1989 & 1990 & 1991 \\
\hline & \multicolumn{10}{|c|}{ Prices in Dollars per Million Btu } \\
\hline 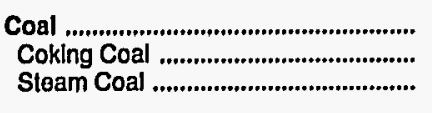 & $\begin{array}{l}- \\
-\end{array}$ & $\frac{1.22}{1.22}$ & $\begin{array}{r}1.44 \\
1 . \overline{4}\end{array}$ & $\begin{array}{r}1.60 \\
1.60\end{array}$ & $\begin{array}{r}1.58 \\
1.58\end{array}$ & $\begin{array}{r}1.57 \\
-57\end{array}$ & $\begin{array}{r}1.60 \\
1.60\end{array}$ & $\begin{array}{r}1.64 \\
1.64\end{array}$ & $\begin{array}{r}1.62 \\
-\overline{2}\end{array}$ & $\begin{array}{r}1.61 \\
1.61\end{array}$ \\
\hline Natural Gas .......................................... & .38 & .79 & 2.27 & 3.83 & 3.53 & $\mathbf{3 . 3 4}$ & 3.43 & 3.39 & 3.27 & 3.36 \\
\hline 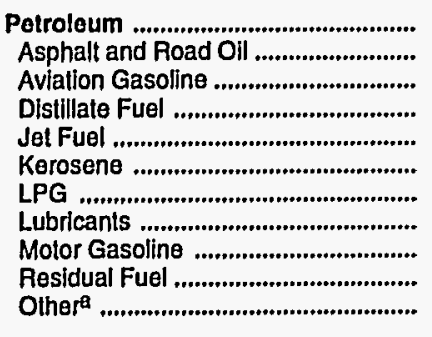 & $\begin{array}{r}1.97 \\
.68 \\
2.17 \\
.98 \\
.72 \\
.88 \\
1.63 \\
5.08 \\
2.74 \\
.43 \\
.86\end{array}$ & $\begin{array}{l}3.31 \\
1.81 \\
3.45 \\
2.39 \\
2.01 \\
2.41 \\
3.12 \\
7.48 \\
4.59 \\
1.72 \\
2.32\end{array}$ & $\begin{array}{r}7.57 \\
3.58 \\
9.02 \\
6.04 \\
6.34 \\
5.51 \\
6.97 \\
14.36 \\
9.93 \\
3.23 \\
6.16\end{array}$ & $\begin{array}{r}8.29 \\
4.21 \\
9.99 \\
7.92 \\
5.96 \\
11.00 \\
8.54 \\
17.61 \\
8.80 \\
4.01 \\
7.81\end{array}$ & $\begin{array}{r}6.18 \\
4.62 \\
8.41 \\
4.76 \\
3.93 \\
3.99 \\
7.54 \\
15.59 \\
6.84 \\
2.37 \\
7.92 \\
\end{array}$ & $\begin{array}{r}6.67 \\
3.33 \\
7.55 \\
5.68 \\
4.07 \\
5.24 \\
7.29 \\
12.70 \\
7.28 \\
2.74 \\
8.40\end{array}$ & $\begin{array}{r}6.39 \\
3.27 \\
7.41 \\
5.13 \\
3.66 \\
4.44 \\
7.70 \\
14.61 \\
7.13 \\
3.08 \\
7.45\end{array}$ & $\begin{array}{r}6.99 \\
2.90 \\
8.28 \\
5.92 \\
4.27 \\
5.07 \\
7.17 \\
13.30 \\
7.69 \\
2.95 \\
8.73\end{array}$ & $\begin{array}{r}8.43 \\
3.01 \\
9.32 \\
7.69 \\
5.90 \\
8.42 \\
10.14 \\
13.40 \\
8.86 \\
2.55 \\
8.40\end{array}$ & $\begin{array}{r}8.39 \\
3.22 \\
8.71 \\
7.43 \\
5.01 \\
7.80 \\
11.20 \\
15.42 \\
8.81 \\
2.30 \\
8.13\end{array}$ \\
\hline Nuclear Fuel ......................................... & - & .24 & .54 & .77 & .71 & .75 & .74 & .75 & .73 & .70 \\
\hline Blomass Fuels at Utilities ................. & - & - & - & - & - & - & - & - & - & - \\
\hline Primary Energy - Five Sectors ${ }^{b} \ldots$ & 1.02 & 2.11 & 4.38 & 4.38 & 3.53 & 3.62 & 3.66 & 3.90 & 4.19 & 4.09 \\
\hline $\begin{array}{l}\text { Electric Utility Fuejc ........................... } \\
\text { Electricity Purchased by End Users }\end{array}$ & $\begin{array}{r}.26 \\
4.78\end{array}$ & $\begin{array}{r}.72 \\
7.80\end{array}$ & $\begin{array}{r}1.46 \\
12.77\end{array}$ & $\begin{array}{r}1.36 \\
18.24\end{array}$ & $\begin{array}{r}1.32 \\
19.61\end{array}$ & $\begin{array}{r}1.28 \\
19.00\end{array}$ & $\begin{array}{r}1.34 \\
18.74\end{array}$ & $\begin{array}{r}1.39 \\
19.04\end{array}$ & $\begin{array}{r}1.32 \\
19.78\end{array}$ & $\begin{array}{r}1.27 \\
19.76\end{array}$ \\
\hline \multirow[t]{2}{*}{ 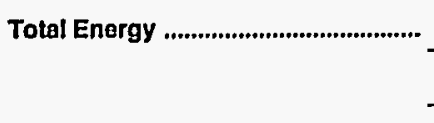 } & 1.52 & 2.98 & 6.68 & 8.41 & 7.52 & 8.03 & 7.48 & 7.72 & 8.74 & 8.91 \\
\hline & \multicolumn{10}{|c|}{ Expenditures in Millions of Dollars } \\
\hline 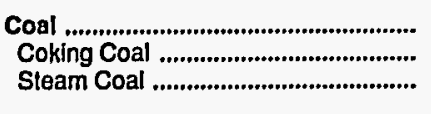 & $\begin{array}{l}- \\
-\end{array}$ & $\begin{array}{r}1.1 \\
1 . \overline{1}\end{array}$ & $\begin{array}{r}52.6 \\
52.6\end{array}$ & $\begin{array}{r}351.1 \\
351 . \overline{1}\end{array}$ & $\begin{array}{r}354.7 \\
\overline{-} \\
354.7\end{array}$ & $\begin{array}{r}330.7 \\
- \\
330.7\end{array}$ & $\begin{array}{r}349.4 \\
- \\
349.4\end{array}$ & $\begin{array}{r}332.8 \\
-\overline{8} \\
332.8\end{array}$ & $\begin{array}{r}344.9 \\
344.9\end{array}$ & $\begin{array}{r}346.9 \\
346.9\end{array}$ \\
\hline Natural Gas & 133.8 & 185.8 & 581.7 & 636.9 & 613.0 & 486.6 & 632.7 & 724.6 & 665.3 & 635.5 \\
\hline 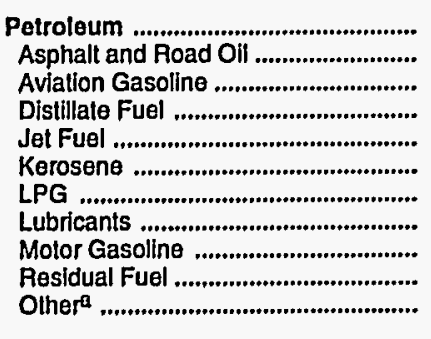 & $\begin{array}{r}469.3 \\
9.4 \\
3.2 \\
31.1 \\
8.5 \\
4.1 \\
62.4 \\
16.4 \\
323.7 \\
2.4 \\
8.0\end{array}$ & $\begin{array}{r}1,130.8 \\
27.4 \\
4.4 \\
133.2 \\
21.7 \\
9.4 \\
109.4 \\
28.0 \\
666.5 \\
97.6 \\
33.2\end{array}$ & $\begin{array}{r}2,318.9 \\
65.9 \\
12.5 \\
376.2 \\
70.0 \\
17.8 \\
123.7 \\
61.0 \\
1,381.9 \\
100.3 \\
109.6\end{array}$ & $\begin{array}{r}2,302.5 \\
35.3 \\
4.4 \\
687.9 \\
65.7 \\
9.7 \\
112.9 \\
68.0 \\
1,235.5 \\
17.0 \\
65.9\end{array}$ & $\begin{array}{r}1,661.8 \\
30.1 \\
4.7 \\
367.7 \\
41.1 \\
1.2 \\
104.3 \\
58.9 \\
1,001.9 \\
11.1 \\
40.9\end{array}$ & $\begin{array}{r}1,803.1 \\
22.5 \\
3.5 \\
444.5 \\
45.8 \\
1.3 \\
93.4 \\
54.3 \\
1,089.6 \\
3.4 \\
44.8\end{array}$ & $\begin{array}{r}1,814.4 \\
29.8 \\
3.7 \\
423.4 \\
44.5 \\
1.3 \\
99.8 \\
60.2 \\
1,108.1 \\
5.8 \\
37.7\end{array}$ & $\begin{array}{r}1,959.1 \\
15.0 \\
4.3 \\
500.9 \\
45.2 \\
1.3 \\
100.3 \\
56.2 \\
1,187.7 \\
5.5 \\
42.7\end{array}$ & $\begin{array}{r}2,285.4 \\
9.9 \\
5.9 \\
638.6 \\
54.5 \\
1.8 \\
128.0 \\
58.3 \\
1,341.8 \\
2.7 \\
43.8\end{array}$ & $\begin{array}{r}2,222.8 \\
11.4 \\
6.3 \\
582.9 \\
41.8 \\
1.6 \\
133.8 \\
60.0 \\
1,342.2 \\
1.1 \\
41.7\end{array}$ \\
\hline Nuclear Fual ..................................... & - & 12.7 & 46.0 & 82.8 & 68.0 & 91.6 & 70.4 & 71.4 & 88.3 & 95.4 \\
\hline Blomass Fuels at UtIlitles ................ & - & - & - & - & - & - & - & - & - & - \\
\hline Primary Energy — Flve Sectors ${ }^{b} \ldots$ & 603.0 & $1,330.3$ & $2,999.2$ & $3,373.2$ & $2,697.5$ & $2,711.9$ & $2,866.9$ & $3,087.9$ & $3,383.9$ & $3,300.5$ \\
\hline $\begin{array}{l}\text { Electric Utllity Fuelc ............................ } \\
\text { Electricity Purchased by End Users }\end{array}$ & $\begin{array}{l}-29.3 \\
217.4\end{array}$ & $\begin{array}{r}-82.2 \\
480.4\end{array}$ & $\begin{array}{r}-286.3 \\
1,149.8\end{array}$ & $\begin{array}{r}-451.4 \\
1,440.1\end{array}$ & $\begin{array}{r}-454.4 \\
1,490.5\end{array}$ & $\begin{array}{r}-459.1 \\
1,521.8\end{array}$ & $\begin{array}{r}-445.9 \\
1,570.6\end{array}$ & $\begin{array}{r}-448.2 \\
1,644.7\end{array}$ & $\begin{array}{r}-476.2 \\
1,789.8\end{array}$ & $\begin{array}{r}-473.8 \\
1,859.8\end{array}$ \\
\hline Total Energy ........................................ & 791.2 & $1,728.5$ & $3,862.7$ & $4,361.9$ & $3,733.6$ & $3,774.6$ & $3,991.7$ & $4,284.4$ & $4,697.5$ & $4,686.5$ \\
\hline
\end{tabular}

a Includes petroleum coke used at electric utilities.

b Biomass fuels are not included, except those consumed at electric utilities and those added to motor gasoline.

c There are no direct fuel costs for hydroelectric, geothermal, centralized solar, or wind energy.

-No consumption, Including cases where adjustments were made. See "Consumption Adjustments for Process Fuel and Intermediate Products."

Note: Expenditure totals may not equal sum of components due to independent rounding.

Sources: Data sources, estimation procedures, and assumptions are described in the "Documentation" section of this report. 


\section{A Energy Price Estimates by Sector, Arkansas \\ R $1970,1975,1980$, and 1985-1991 \\ K (Dollars per Million Btu)}

\begin{tabular}{|c|c|c|c|c|c|c|c|c|c|c|}
\hline Sector and Energy Source & 1970 & 1975 & 1980 & 1985 & 1986 & 1987 & 1988 & 1989 & 1990 & 1991 \\
\hline 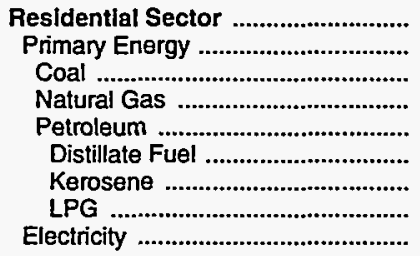 & $\begin{array}{r}1.91 \\
1.07 \\
- \\
.75 \\
1.81 \\
.93 \\
1.40 \\
1.84 \\
6.82\end{array}$ & $\begin{array}{r}3.90 \\
1.82 \\
- \\
1.12 \\
3.44 \\
2.40 \\
2.80 \\
3.51 \\
9.35\end{array}$ & $\begin{array}{r}8.14 \\
3.45 \\
2.97 \\
2.49 \\
8.54 \\
6.54 \\
- \\
8.77 \\
15.58\end{array}$ & $\begin{array}{r}11.53 \\
5.01 \\
3.19 \\
4.35 \\
8.52 \\
10.33 \\
11.07 \\
8.46 \\
21.91\end{array}$ & $\begin{array}{r}12.19 \\
5.22 \\
-\overline{4} \\
4.77 \\
7.31 \\
4.38 \\
4.70 \\
7.36 \\
22.68\end{array}$ & $\begin{array}{r}12.15 \\
4.99 \\
2.88 \\
4.57 \\
7.39 \\
5.30 \\
5.68 \\
7.42 \\
22.46\end{array}$ & $\begin{array}{r}12.11 \\
5.21 \\
2.62 \\
4.78 \\
7.86 \\
4.71 \\
5.05 \\
7.92 \\
22.31\end{array}$ & $\begin{array}{r}12.27 \\
5.15 \\
2.64 \\
4.83 \\
6.93 \\
4.95 \\
5.30 \\
6.97 \\
22.78\end{array}$ & $\begin{array}{r}13.67 \\
5.90 \\
2.70 \\
5.06 \\
10.74 \\
7.69 \\
8.24 \\
10.78 \\
23.65\end{array}$ & $\begin{array}{r}13.75 \\
5.83 \\
2.81 \\
4.90 \\
12.09 \\
7.11 \\
7.62 \\
12.15 \\
23.76\end{array}$ \\
\hline 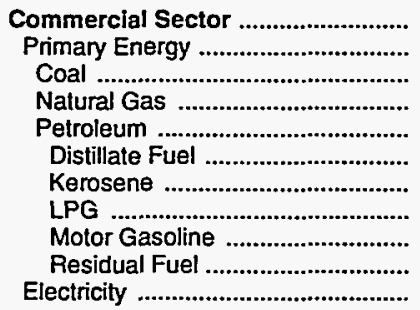 & $\begin{array}{r}1.58 \\
.64 \\
- \\
.52 \\
1.37 \\
.86 \\
.77 \\
1.23 \\
2.74 \\
.42 \\
6.07\end{array}$ & $\begin{array}{r}3.08 \\
1.25 \\
- \\
.90 \\
2.23 \\
2.29 \\
2.32 \\
2.64 \\
4.59 \\
1.75 \\
8.60\end{array}$ & $\begin{array}{r}6.73 \\
2.80 \\
1.89 \\
2.29 \\
5.24 \\
6.25 \\
5.51 \\
5.54 \\
9.93 \\
3.33 \\
14.73\end{array}$ & $\begin{array}{r}10.20 \\
5.34 \\
2.12 \\
4.06 \\
9.10 \\
9.09 \\
10.98 \\
8.65 \\
8.80 \\
-\overline{0} \\
19.06\end{array}$ & $\begin{array}{r}10.81 \\
4.51 \\
- \\
4.33 \\
5.94 \\
3.00 \\
3.31 \\
7.83 \\
6.84 \\
2.42 \\
19.70\end{array}$ & $\begin{array}{r}10.55 \\
4.40 \\
2.02 \\
4.21 \\
5.61 \\
4.18 \\
4.81 \\
7.13 \\
7.28 \\
19.06\end{array}$ & $\begin{array}{r}10.50 \\
4.44 \\
2.04 \\
4.31 \\
5.53 \\
3.31 \\
3.84 \\
7.45 \\
7.13 \\
19.11\end{array}$ & $\begin{array}{r}10.74 \\
4.49 \\
1.98 \\
4.36 \\
5.30 \\
3.69 \\
4.68 \\
7.40 \\
7.69 \\
- \\
19.64\end{array}$ & $\begin{array}{r}11.64 \\
4.94 \\
1.99 \\
4.43 \\
7.86 \\
6.84 \\
8.62 \\
9.42 \\
8.86 \\
20.40\end{array}$ & $\begin{array}{r}11.59 \\
4.70 \\
1.86 \\
4.28 \\
7.86 \\
6.39 \\
7.92 \\
10.22 \\
8.81 \\
20.32\end{array}$ \\
\hline 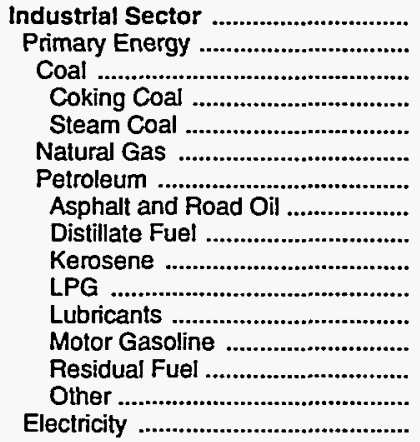 & $\begin{array}{r}.69 \\
.46 \\
- \\
- \\
- \\
.28 \\
.98 \\
.68 \\
.67 \\
.77 \\
1.23 \\
5.08 \\
2.74 \\
.45 \\
.86 \\
2.78\end{array}$ & $\begin{array}{r}1.63 \\
1.29 \\
1.22 \\
-\overline{1.22} \\
.68 \\
2.18 \\
1.81 \\
2.09 \\
2.32 \\
2.64 \\
7.48 \\
4.59 \\
1.63 \\
2.32 \\
5.18\end{array}$ & $\begin{array}{r}4.17 \\
3.26 \\
1.89 \\
-\overline{1.89} \\
2.24 \\
4.95 \\
3.58 \\
4.87 \\
5.51 \\
5.54 \\
14.36 \\
9.93 \\
2.95 \\
6.16 \\
9.15\end{array}$ & $\begin{array}{r}6.73 \\
5.48 \\
2.12 \\
- \\
2.12 \\
3.65 \\
8.32 \\
4.21 \\
9.44 \\
10.98 \\
8.65 \\
17.61 \\
8.80 \\
4.01 \\
7.81 \\
13.74\end{array}$ & $\begin{array}{r}5.43 \\
3.70 \\
2.04 \\
- \\
2.04 \\
3.40 \\
4.36 \\
4.62 \\
2.84 \\
3.31 \\
7.83 \\
15.59 \\
6.84 \\
2.42 \\
7.92 \\
15.53\end{array}$ & $\begin{array}{r}6.14 \\
4.01 \\
2.02 \\
- \\
2.02 \\
3.10 \\
5.16 \\
3.33 \\
4.14 \\
4.81 \\
7.13 \\
12.70 \\
7.28 \\
2.74 \\
8.40 \\
14.56\end{array}$ & $\begin{array}{r}5.21 \\
3.53 \\
2.04 \\
- \\
2.04 \\
3.04 \\
4.55 \\
3.27 \\
3.30 \\
3.84 \\
7.45 \\
14.61 \\
7.13 \\
2.49 \\
7.45 \\
14.14\end{array}$ & $\begin{array}{r}5.31 \\
3.61 \\
1.98 \\
-\overline{1} \\
1.98 \\
3.08 \\
5.36 \\
2.90 \\
4.02 \\
4.68 \\
7.40 \\
13.30 \\
7.69 \\
2.68 \\
8.73 \\
14.34\end{array}$ & $\begin{array}{r}5.94 \\
4.05 \\
1.99 \\
- \\
1.99 \\
2.86 \\
7.62 \\
3.01 \\
7.41 \\
8.62 \\
9.42 \\
13.40 \\
8.86 \\
2.54 \\
8.40 \\
14.93\end{array}$ & $\begin{array}{r}6.21 \\
4.09 \\
1.86 \\
- \\
1.86 \\
3.01 \\
7.56 \\
3.22 \\
6.80 \\
7.92 \\
10.22 \\
15.42 \\
8.81 \\
2.30 \\
8.13 \\
14.80\end{array}$ \\
\hline 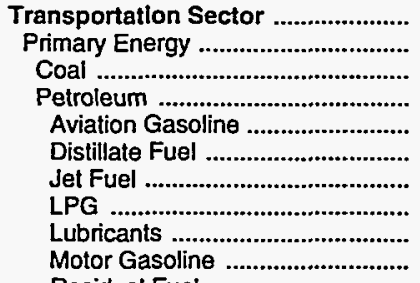 & $\begin{array}{r}2.38 \\
2.38 \\
- \\
2.38 \\
2.17 \\
1.16 \\
.72 \\
1.23 \\
5.08 \\
2.74\end{array}$ & $\begin{array}{l}4.06 \\
4.06 \\
1.22 \\
4.06 \\
3.45 \\
2.53 \\
2.01 \\
2.64 \\
7.48 \\
4.59\end{array}$ & $\begin{array}{r}9.11 \\
9.11 \\
- \\
9.11 \\
9.02 \\
6.70 \\
6.34 \\
5.54 \\
14.36 \\
9.93\end{array}$ & $\begin{array}{r}8.24 \\
8.24 \\
- \\
8.24 \\
9.99 \\
6.56 \\
5.96 \\
8.65 \\
17.61 \\
8.80\end{array}$ & $\begin{array}{r}6.63 \\
6.63 \\
- \\
6.63 \\
8.41 \\
6.09 \\
3.93 \\
7.83 \\
15.59 \\
6.84\end{array}$ & $\begin{array}{r}7.01 \\
7.01 \\
- \\
7.01 \\
7.55 \\
6.59 \\
4.07 \\
7.13 \\
12.70 \\
7.28\end{array}$ & $\begin{array}{r}6.81 \\
6.81 \\
- \\
6.81 \\
7.41 \\
6.21 \\
3.66 \\
7.45 \\
14.61 \\
7.13\end{array}$ & $\begin{array}{r}7.34 \\
7.34 \\
- \\
7.34 \\
8.28 \\
6.71 \\
4.27 \\
7.40 \\
13.30 \\
7.69\end{array}$ & $\begin{array}{r}8.53 \\
8.53 \\
- \\
8.53 \\
9.32 \\
7.87 \\
5.90 \\
9.42 \\
13.40 \\
8.86\end{array}$ & $\begin{array}{r}8.43 \\
8.43 \\
- \\
8.43 \\
8.71 \\
7.64 \\
5.01 \\
10.22 \\
15.42 \\
8.81\end{array}$ \\
\hline 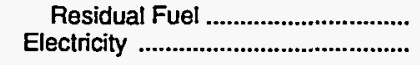 & $\begin{array}{r}.40 \\
-\end{array}$ & $\begin{array}{r}1.57 \\
-\end{array}$ & - & - & - & - & - & - & - & - \\
\hline $\begin{array}{l}\text { Total Energy ................................... } \\
\text { Primary Energy - Four Sectors ...... } \\
\text { Electricity .......................................... }\end{array}$ & $\begin{array}{l}1.52 \\
1.20 \\
4.78\end{array}$ & $\begin{array}{l}2.98 \\
2.41 \\
7.80\end{array}$ & $\begin{array}{r}6.68 \\
5.56 \\
12.77\end{array}$ & $\begin{array}{r}8.41 \\
6.64 \\
18.24\end{array}$ & $\begin{array}{r}7.52 \\
5.34 \\
19.61\end{array}$ & $\begin{array}{r}8.03 \\
5.78 \\
19.00\end{array}$ & $\begin{array}{r}7.48 \\
5.38 \\
18.74\end{array}$ & $\begin{array}{r}7.72 \\
5.63 \\
19.04\end{array}$ & $\begin{array}{r}8.74 \\
6.51 \\
19.78\end{array}$ & $\begin{array}{r}8.91 \\
6.55 \\
19.76\end{array}$ \\
\hline 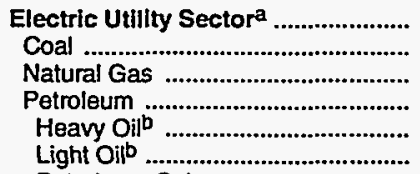 & $\begin{array}{l}.26 \\
.25 \\
.42 \\
.42 \\
.46\end{array}$ & $\begin{array}{r}.72 \\
. \overline{1} \\
1.79 \\
1.78 \\
2.22\end{array}$ & $\begin{array}{l}1.46 \\
1.34 \\
2.15 \\
3.39 \\
3.34 \\
4.34\end{array}$ & $\begin{array}{l}1.36 \\
1.58 \\
2.82 \\
4.99 \\
3.84 \\
5.86\end{array}$ & $\begin{array}{l}1.32 \\
1.56 \\
1.56 \\
2.20 \\
1.65 \\
4.42\end{array}$ & $\begin{array}{l}1.28 \\
1.55 \\
1.52 \\
3.86 \\
2.76 \\
3.86\end{array}$ & $\begin{array}{l}1.34 \\
1.58 \\
1.35 \\
4.43 \\
3.81 \\
5.12\end{array}$ & $\begin{array}{l}1.39 \\
1.63 \\
1.65 \\
3.81 \\
3.26 \\
4.36\end{array}$ & $\begin{array}{l}1.32 \\
1.61 \\
1.54 \\
4.72 \\
2.75 \\
4.94\end{array}$ & $\begin{array}{l}1.27 \\
1.60 \\
1.41 \\
5.61 \\
2.72 \\
5.64\end{array}$ \\
\hline $\begin{array}{l}\text { Petroleum Coke } \\
\text { Nuclear Fuel } \\
\text { Biomass Fuels }\end{array}$ & $\overline{-}$ & $\overline{24}$ & $\overline{.54}$ & $\begin{array}{l}- \\
.77 \\
-\end{array}$ &.$\overline{71}$ & $\overline{75}$ & $\begin{array}{r}. \\
.74 \\
-\end{array}$ & $\overline{75}$ & $\overline{73}$ & $\overline{70}$ \\
\hline Primary Energy - Flve Sectorsc .. & 1.02 & 2.11 & 4.38 & 4.38 & $\mathbf{3 . 5 3}$ & 3.62 & 3.66 & 3.90 & 4.19 & 4.09 \\
\hline
\end{tabular}

a There are no direct fuel costs for hydroelectric, geothermal, centralized solar, or wind energy.

b Heavy oil includes Grade Nos. 4, 5, and 6, and residual fuel oils. Light oil includes Grade No. 2 heating oil, kerosene, and jet fuel.

c Biomass fuels are not included, except those consumed at electric utilities and those added to motor gasoline.

-No consumption, including cases where adjustments were made. See "Consumption Adjustments for Process Fuel and Intermediate Products."

Sources: Data sources, estimation procedures, and assumptions are described in the "Documentation" section of this report. 
Energy Expenditure Estimates by Sector, Arkansas 1970, 1975, 1980, and 1985-1991

(Million Dollars)

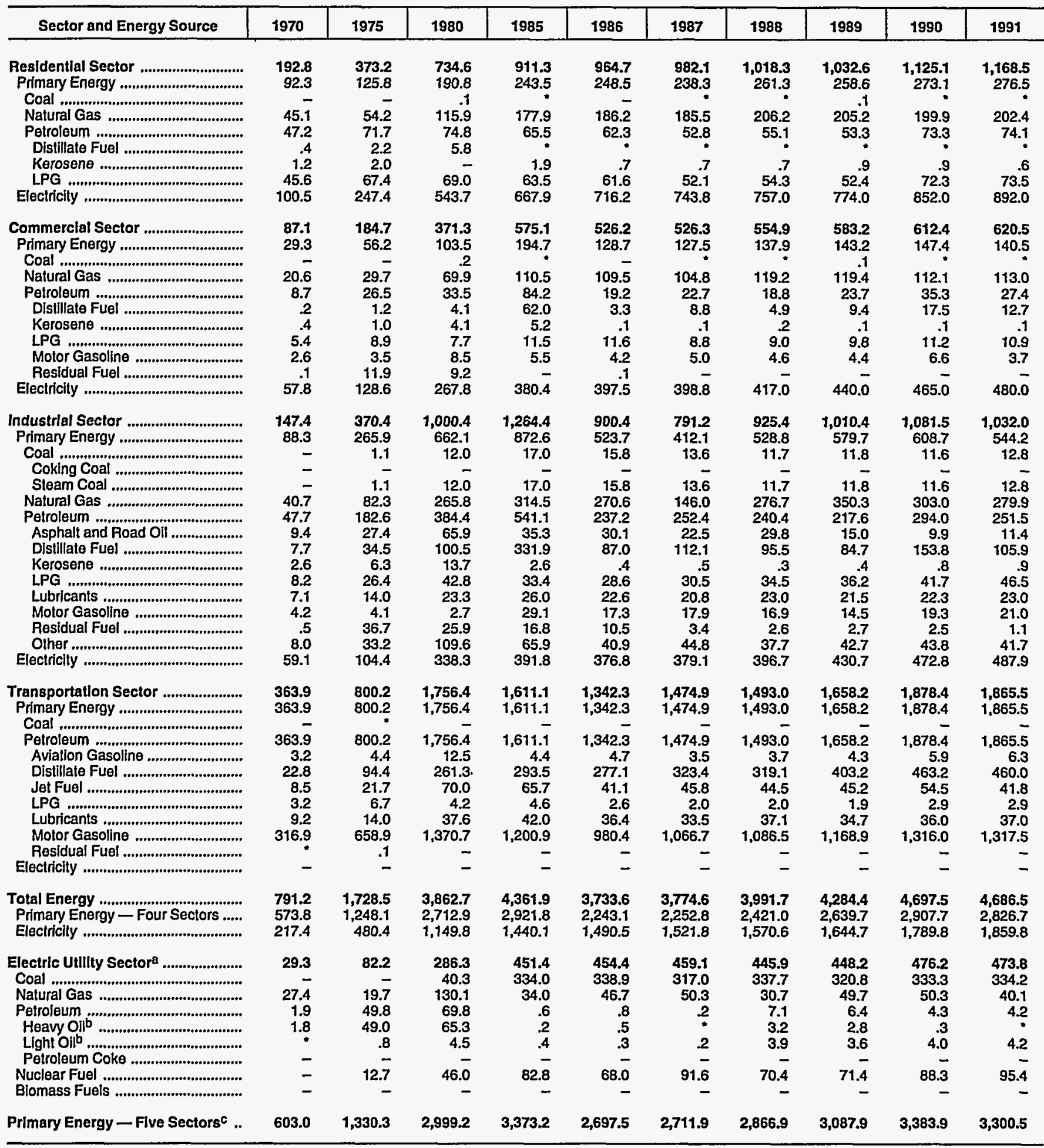

a There are no direct fuel costs for hydroelectric, geothermal, centralized solar, or wind energy.

b Heavy oil includes Grade Nos, 4, 5, and 6, and residual fuel oils. Light oil includes Grade No. 2 heating oil, kerosene, and jet fuel.

c Blomass fuels are not included, except those consumed at electric utilities and those added to motor gasoline.

-No consumption, including cases where adjusiments were made. See "Consumption Adjustments for Process Fuel and intermediate Products."

-Value less than 0.05 million dollars.

Note: Totals may not equal sum of components due to independent rounding

Sources: Data sources, estimation procedures, and assumplions are described in the "Documentation" section of this report. 
C Energy Price and Expenditure Estimates by Source, California

A $1970,1975,1980$, and $1985-1991$

\begin{tabular}{|c|c|c|c|c|c|c|c|c|c|c|}
\hline Energy Source & 1970 & 1975 & 1980 & 1985 & 1986 & 1987 & 1988 & 1989 & 1990 & 1991 \\
\hline & \multicolumn{10}{|c|}{ Prices In Dollars per Million Btu } \\
\hline 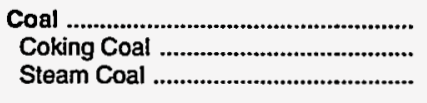 & $\begin{array}{r}0.45 \\
.43 \\
.83\end{array}$ & $\begin{array}{r}1.32 \\
1.38 \\
.92\end{array}$ & $\begin{array}{l}1.91 \\
1.97 \\
1.82\end{array}$ & $\frac{2.30}{2.30}$ & $\begin{array}{r}2.26 \\
2.26\end{array}$ & $\begin{array}{r}2.09 \\
2.09\end{array}$ & $\begin{array}{r}1.96 \\
1.96\end{array}$ & $\begin{array}{c}1.93 \\
- \\
1.93\end{array}$ & $\begin{array}{r}2.01 \\
2.01\end{array}$ & $\begin{array}{c}1.99 \\
1.99\end{array}$ \\
\hline 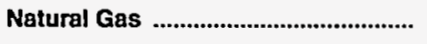 & .56 & 1.25 & 3.54 & 5.01 & 4.02 & 3.77 & 4.05 & 4.10 & 4.31 & 4.49 \\
\hline 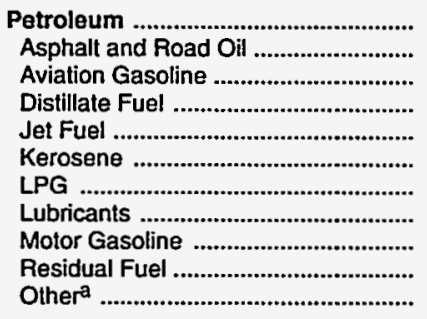 & $\begin{array}{r}1.78 \\
.49 \\
2.17 \\
1.26 \\
.73 \\
1.08 \\
1.84 \\
5.08 \\
2.80 \\
.38 \\
1.04\end{array}$ & $\begin{array}{l}3.53 \\
1.62 \\
3.45 \\
2.97 \\
2.04 \\
2.76 \\
3.08 \\
7.48 \\
4.84 \\
2.38 \\
2.27\end{array}$ & $\begin{array}{r}7.37 \\
3.78 \\
9.02 \\
6.62 \\
6.21 \\
6.44 \\
6.09 \\
14.36 \\
10.19 \\
4.49 \\
6.28\end{array}$ & $\begin{array}{r}7.39 \\
4.78 \\
9.99 \\
6.48 \\
6.01 \\
6.50 \\
9.35 \\
17.61 \\
8.68 \\
4.75 \\
7.43\end{array}$ & $\begin{array}{r}5.72 \\
5.08 \\
8.41 \\
5.55 \\
3.99 \\
7.89 \\
9.52 \\
15.59 \\
6.68 \\
2.71 \\
6.15\end{array}$ & $\begin{array}{r}5.85 \\
5.36 \\
7.55 \\
5.92 \\
4.04 \\
5.51 \\
9.57 \\
12.70 \\
6.95 \\
2.61 \\
6.10\end{array}$ & $\begin{array}{r}5.82 \\
4.53 \\
7.41 \\
5.93 \\
3.84 \\
7.22 \\
9.43 \\
14.61 \\
7.08 \\
2.31 \\
5.18\end{array}$ & $\begin{array}{r}6.31 \\
3.10 \\
8.28 \\
6.72 \\
4.50 \\
10.15 \\
8.90 \\
13.30 \\
7.66 \\
2.68 \\
5.67\end{array}$ & $\begin{array}{r}7.19 \\
3.13 \\
9.32 \\
7.39 \\
5.76 \\
8.42 \\
10.15 \\
13.40 \\
8.57 \\
3.66 \\
5.80\end{array}$ & $\begin{array}{r}6.87 \\
3.18 \\
8.71 \\
7.25 \\
4.80 \\
7.93 \\
10.70 \\
15.42 \\
8.18 \\
2.57 \\
5.73\end{array}$ \\
\hline Nuclear Fuel ........................................... & .19 & .21 & .49 & .96 & .97 & .99 & .90 & .85 & .72 & .67 \\
\hline Biomass Fuels at UtIIItles ................... & .65 & .92 & 1.74 & .79 & .32 & .95 & .87 & $\left({ }^{d}\right)$ & $(d)$ & $\left({ }^{d}\right)$ \\
\hline Primary Energy — Flve Sectors ${ }^{b} \ldots$ & 1.20 & 2.61 & 5.95 & 6.19 & 4.87 & 4.79 & 4.90 & 5.20 & 5.77 & 5.59 \\
\hline $\begin{array}{l}\text { Electric Utility Fuelc .......................... } \\
\text { Electricity Purchased by End Users }\end{array}$ & $\begin{array}{r}.34 \\
4.76\end{array}$ & $\begin{array}{l}1.82 \\
8.71\end{array}$ & $\begin{array}{r}3.99 \\
17.16\end{array}$ & $\begin{array}{r}3.70 \\
22.90\end{array}$ & $\begin{array}{r}2.16 \\
23.21\end{array}$ & $\begin{array}{r}2.02 \\
22.70\end{array}$ & $\begin{array}{r}2.17 \\
23.42\end{array}$ & $\begin{array}{r}2.20 \\
24.91\end{array}$ & $\begin{array}{r}2.17 \\
25.98\end{array}$ & $\begin{array}{r}1.95 \\
27.70\end{array}$ \\
\hline \multirow[t]{2}{*}{ 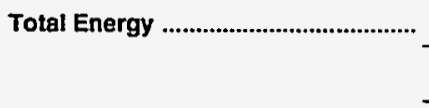 } & 1.73 & 3.47 & 7.71 & 8.91 & 7.79 & 7.63 & 7.86 & B.34 & 9.03 & 9.14 \\
\hline & \multicolumn{10}{|c|}{ Expenditures in Millions of Dollars } \\
\hline 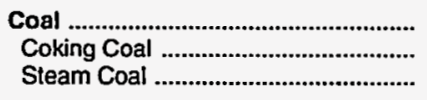 & $\begin{array}{r}27.9 \\
25.6 \\
2.3\end{array}$ & $\begin{array}{r}74.6 \\
67.7 \\
6.9\end{array}$ & $\begin{array}{r}126.7 \\
79.8 \\
46.8\end{array}$ & $\begin{array}{r}104.1 \\
104.1\end{array}$ & $\begin{array}{r}96.0 \\
-\overline{0}\end{array}$ & $\begin{array}{r}94.0 \\
94.0\end{array}$ & $\begin{array}{r}99.5 \\
\overline{-} \\
99.5\end{array}$ & $\begin{array}{r}111.6 \\
- \\
111.6\end{array}$ & $\begin{array}{r}131.1 \\
-\overline{-} \\
131.1\end{array}$ & $\begin{array}{r}127.4 \\
127.4\end{array}$ \\
\hline 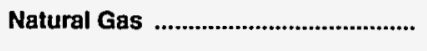 & $1,126.7$ & $2,148.2$ & $6,063.2$ & $9,251.8$ & $6,011.6$ & $7,109.8$ & $7,145.3$ & $7,426.4$ & $7,830.2$ & $8,242.8$ \\
\hline 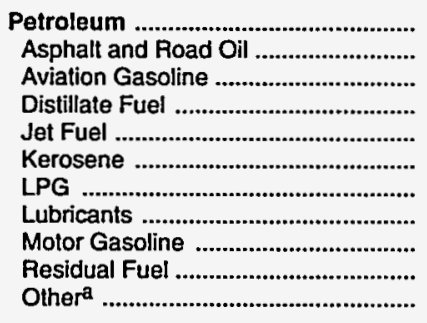 & $\begin{array}{r}4,210.9 \\
39.0 \\
23.9 \\
283.0 \\
242.7 \\
6.1 \\
99.5 \\
122.3 \\
3,149.1 \\
161.1 \\
84.0\end{array}$ & $\begin{array}{r}9,939.3 \\
141.0 \\
28.5 \\
719.4 \\
716.0 \\
31.8 \\
169.8 \\
164.9 \\
6,137.9 \\
1,628.1 \\
202.0\end{array}$ & $\begin{array}{r}24,321.3 \\
462.2 \\
13.0 \\
2,390.8 \\
2,199.3 \\
77.3 \\
365.8 \\
427.3 \\
13,579.1 \\
4,131.7 \\
674.9\end{array}$ & $\begin{array}{r}21,232.6 \\
439.0 \\
68.3 \\
2,729.9 \\
2,257.8 \\
33.8 \\
585.7 \\
476.9 \\
12,192.3 \\
1,953.0 \\
496.0\end{array}$ & $\begin{array}{r}16,756.0 \\
517.9 \\
56.8 \\
2,409.2 \\
1,685.3 \\
22.0 \\
550.7 \\
412.9 \\
9,816.4 \\
957.7 \\
327.1\end{array}$ & $\begin{array}{r}18,140.0 \\
585.1 \\
41.3 \\
2,539.5 \\
1,811.7 \\
21.4 \\
654.6 \\
380.3 \\
10,665.9 \\
1,064.4 \\
375.7\end{array}$ & $\begin{array}{r}18,877.4 \\
461.6 \\
49.1 \\
2,908.9 \\
1,784.5 \\
9.2 \\
637.4 \\
421.9 \\
11,300.4 \\
984.3 \\
320.0\end{array}$ & $\begin{array}{r}20,876.3 \\
308.2 \\
54.4 \\
3,199.9 \\
2,286.8 \\
11.1 \\
646.8 \\
393.8 \\
12.504 .1 \\
1,123.1 \\
348.2\end{array}$ & $\begin{array}{r}23,628.7 \\
308.8 \\
52.0 \\
3,548.4 \\
3,081.3 \\
6.9 \\
646.4 \\
408.5 \\
13,699.5 \\
1,479.4 \\
397.5\end{array}$ & $\begin{array}{r}21,022.9 \\
301.0 \\
47.9 \\
3,181.4 \\
2,503.0 \\
6.3 \\
660.8 \\
420.4 \\
12,829.6 \\
727.3 \\
345.2\end{array}$ \\
\hline Nuclear Fuel & 6.7 & 14.4 & 26.1 & 204.0 & 273.7 & 322.6 & 297.0 & 295.9 & 251.9 & 226.9 \\
\hline Blomass Fuels at Utllitles .................. & .3 & .2 & .4 & $\bullet$ & .1 & .2 & .1 & $\left({ }^{d}\right)$ & (d) & (d) \\
\hline PrImary Energy - Flve Sectors ${ }^{b}$ & $5,372.4$ & $12,176.6$ & $30,537.6$ & $30,792.6$ & $23,137.4$ & $25,666.8$ & $26,419.3$ & $28,710.2$ & $31,841.9$ & $29,619.9$ \\
\hline $\begin{array}{l}\text { Electric Utility Fuelc .......................... } \\
\text { Electricity Purchased by End Users }\end{array}$ & $\begin{array}{r}-282.1 \\
1,886.7\end{array}$ & $\begin{array}{r}-1,553.7 \\
4,328.6\end{array}$ & $\begin{array}{r}-4,018.4 \\
9,560.0\end{array}$ & $\begin{array}{r}-3,498.6 \\
14,143.4\end{array}$ & $\begin{array}{l}-1,694.0 \\
14,220.3\end{array}$ & $\begin{array}{r}-2,060.3 \\
14,334.2\end{array}$ & $\begin{array}{r}-2,131.0 \\
15,574.0\end{array}$ & $\begin{array}{l}-2,168.0 \\
17,085.2\end{array}$ & $\begin{array}{l}-1,882.4 \\
18,417.8\end{array}$ & $\begin{array}{l}-1,572.2 \\
19,412.5\end{array}$ \\
\hline Total Energy & $6,977.0$ & $14,951.5$ & $36,079.3$ & $41,437.4$ & $35,663.8$ & $37,940.7$ & $39,862.2$ & $43,627.4$ & $48,377.4$ & $47,460.2$ \\
\hline
\end{tabular}

a Includes petroleum coke used at electric utilities.

b Biomass fuels are not included, except those consumed at electric utilities and those added to motor gasoline.

c There are no direct fuel costs for hydroelectric, geothermal, centralized solar, or wind energy.

d Utilities used biomass fuels at no charge or received a fee for accepting them.

-No consumption, including cases where adjustments were made. See "Consumption Adjustments for Process Fuel and intermediate Products."

Value less than 0.05 million dollars.

Note: Expenditure totals may not equal sum of components due to independent rounding.

Sources: Data sources, estimation procedures, and assumptions are described in the "Documentation" section of this report. 


\begin{tabular}{|c|c|c|c|c|c|c|c|c|c|c|}
\hline Sector and Energy Source & 1970 & 1975 & 1980 & 1985 & 1986 & 1987 & 1988 & 1989 & 1990 & 1991 \\
\hline 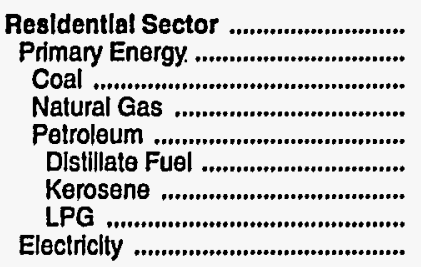 & $\begin{array}{r}1.92 \\
.99 \\
1.31 \\
.93 \\
2.49 \\
1.27 \\
2.57 \\
2.67 \\
6.53\end{array}$ & $\begin{array}{r}3.20 \\
1.55 \\
1.49 \\
4.17 \\
2.80 \\
5.08 \\
4.45 \\
10.68\end{array}$ & $\begin{array}{r}6.76 \\
3.53 \\
5.13 \\
3.37 \\
8.14 \\
6.92 \\
13.04 \\
8.15 \\
17.18\end{array}$ & $\begin{array}{r}10.02 \\
5.61 \\
4.54 \\
5.51 \\
8.53 \\
5.25 \\
9.18 \\
8.66 \\
22.80\end{array}$ & $\begin{array}{r}10.27 \\
5.16 \\
-\overline{-} \\
4.95 \\
10.82 \\
7.58 \\
13.26 \\
10.95 \\
23.26\end{array}$ & $\begin{array}{r}10.42 \\
5.39 \\
3.76 \\
5.13 \\
11.84 \\
6.05 \\
10.57 \\
12.37 \\
23.55\end{array}$ & $\begin{array}{r}11.36 \\
5.72 \\
3.37 \\
5.48 \\
11.04 \\
5.84 \\
10.22 \\
11.39 \\
25.02\end{array}$ & $\begin{array}{r}11.89 \\
5.67 \\
3.66 \\
5.40 \\
11.46 \\
7.63 \\
13.34 \\
11.64 \\
27.69\end{array}$ & $\begin{array}{r}12.67 \\
5.86 \\
3.77 \\
5.60 \\
12.00 \\
5.70 \\
9.96 \\
12.45 \\
29.26\end{array}$ & $\begin{array}{r}13.74 \\
6.41 \\
5.21 \\
6.11 \\
12.39 \\
5.60 \\
9.78 \\
12.75 \\
31.61\end{array}$ \\
\hline 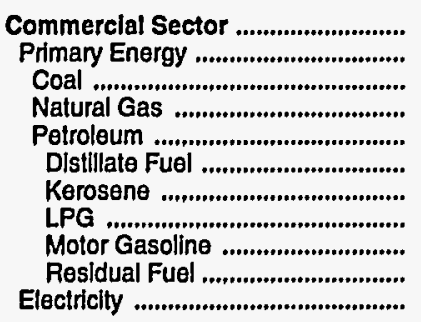 & $\begin{array}{r}2.09 \\
.71 \\
.63 \\
.69 \\
.76 \\
1.12 \\
.78 \\
1.37 \\
2.80 \\
.40 \\
5.02\end{array}$ & $\begin{array}{r}4.36 \\
1.48 \\
- \\
1.22 \\
2.93 \\
2.60 \\
2.50 \\
2.77 \\
4.84 \\
2.45 \\
8.73\end{array}$ & $\begin{array}{r}9.58 \\
4.30 \\
1.82 \\
3.82 \\
6.02 \\
6.60 \\
6.38 \\
5.21 \\
10.19 \\
4.90 \\
17.99\end{array}$ & $\begin{array}{r}15.05 \\
6.42 \\
2.28 \\
6.39 \\
6.71 \\
5.40 \\
6.27 \\
9.65 \\
8.68 \\
3.93 \\
23.61\end{array}$ & $\begin{array}{r}15.17 \\
5.62 \\
- \\
5.65 \\
5.53 \\
5.56 \\
4.69 \\
9.02 \\
6.68 \\
2.16 \\
24.19\end{array}$ & $\begin{array}{r}14.10 \\
5.27 \\
2.09 \\
5.28 \\
5.24 \\
4.81 \\
4.83 \\
8.47 \\
6.95 \\
2.60 \\
23.47\end{array}$ & $\begin{array}{r}13.83 \\
4.68 \\
1.96 \\
4.55 \\
5.28 \\
4.94 \\
4.79 \\
8.53 \\
7.08 \\
2.09 \\
24.15\end{array}$ & $\begin{array}{r}14.66 \\
4.93 \\
1.92 \\
4.71 \\
6.23 \\
6.22 \\
6.05 \\
7.63 \\
7.66 \\
2.30 \\
25.33\end{array}$ & $\begin{array}{r}15.08 \\
5.14 \\
2.00 \\
4.96 \\
6.27 \\
5.72 \\
6.02 \\
9.03 \\
8.57 \\
3.00 \\
26.31\end{array}$ & $\begin{array}{r}15.96 \\
5.43 \\
1.97 \\
5.36 \\
6.01 \\
5.42 \\
5.46 \\
9.29 \\
8.18 \\
2.24 \\
28.12\end{array}$ \\
\hline 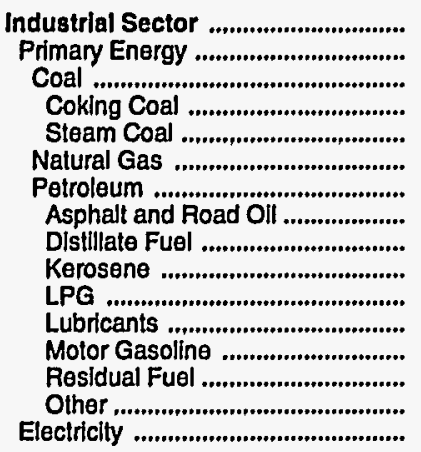 & $\begin{array}{r}.87 \\
.57 \\
.43 \\
.43 \\
.63 \\
.38 \\
.92 \\
.49 \\
.68 \\
.78 \\
1.37 \\
5.08 \\
2.80 \\
.35 \\
1.04 \\
2.90\end{array}$ & $\begin{array}{r}2.24 \\
1.52 \\
1.32 \\
1.38 \\
.92 \\
1.05 \\
2.26 \\
1.62 \\
2.21 \\
2.50 \\
2.77 \\
7.48 \\
4.84 \\
1.66 \\
2.27 \\
6.70\end{array}$ & $\begin{array}{r}6.20 \\
4.34 \\
1.91 \\
1.97 \\
1.82 \\
3.64 \\
5.22 \\
3.78 \\
5.49 \\
6.38 \\
5.21 \\
14.36 \\
10.19 \\
3.16 \\
6.28 \\
16.04\end{array}$ & $\begin{array}{r}7.93 \\
5.19 \\
2.28 \\
- \\
2.28 \\
4.54 \\
6.04 \\
4.78 \\
5.54 \\
6.27 \\
9.65 \\
17.61 \\
8.68 \\
3.93 \\
7.43 \\
22.00\end{array}$ & $\begin{array}{r}7.15 \\
4.22 \\
2.26 \\
- \\
2.26 \\
3.46 \\
5.15 \\
5.08 \\
4.14 \\
4.69 \\
9.02 \\
15.59 \\
6.68 \\
2.16 \\
6.15 \\
21.63\end{array}$ & $\begin{array}{r}6.48 \\
4.18 \\
2.09 \\
- \\
2.09 \\
3.39 \\
5.28 \\
5.36 \\
4.27 \\
4.83 \\
8.47 \\
12.70 \\
6.95 \\
2.60 \\
6.10 \\
20.38\end{array}$ & $\begin{array}{r}6.82 \\
4.20 \\
1.96 \\
- \\
1.96 \\
3.65 \\
5.11 \\
4.53 \\
4.24 \\
4.79 \\
8.53 \\
14.61 \\
7.08 \\
2.09 \\
5.18 \\
20.15\end{array}$ & $\begin{array}{r}7.03 \\
4.18 \\
1.92 \\
- \\
1.92 \\
3.62 \\
5.34 \\
3.10 \\
5.35 \\
6.05 \\
7.63 \\
13.30 \\
7.66 \\
2.30 \\
5.67 \\
20.89\end{array}$ & $\begin{array}{r}7.07 \\
4.34 \\
2.00 \\
- \\
2.00 \\
3.79 \\
5.58 \\
3.13 \\
5.33 \\
6.02 \\
9.03 \\
13.40 \\
8.57 \\
3.00 \\
5.80 \\
21.35\end{array}$ & $\begin{array}{r}7.19 \\
4.26 \\
1.97 \\
- \\
1.97 \\
3.86 \\
5.46 \\
3.18 \\
4.82 \\
5.46 \\
9.29 \\
15.42 \\
8.18 \\
2.24 \\
5.73 \\
22.22\end{array}$ \\
\hline 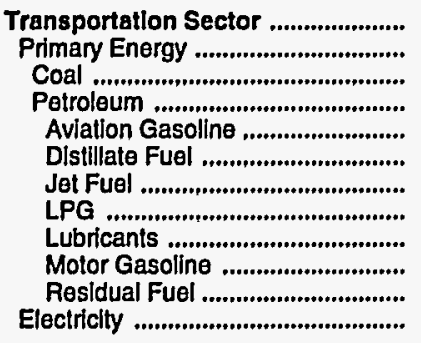 & $\begin{array}{r}2.07 \\
2.07 \\
.63 \\
2.07 \\
2.17 \\
1.42 \\
.73 \\
1.37 \\
5.08 \\
2.80 \\
.36 \\
2.88\end{array}$ & $\begin{array}{r}4.02 \\
4.02 \\
.92 \\
4.02 \\
3.45 \\
3.22 \\
2.04 \\
2.77 \\
7.48 \\
4.84 \\
2.12 \\
4.34\end{array}$ & $\begin{array}{r}8.22 \\
8.22 \\
- \\
8.22 \\
9.02 \\
7.07 \\
6.21 \\
5.21 \\
14.36 \\
10.19 \\
4.14 \\
11.39\end{array}$ & $\begin{array}{r}7.68 \\
7.67 \\
- \\
7.67 \\
9.99 \\
6.90 \\
6.01 \\
9.65 \\
17.61 \\
8.68 \\
5.02 \\
18.29\end{array}$ & $\begin{array}{r}5.82 \\
5.82 \\
- \\
5.82 \\
8.41 \\
5.93 \\
3.99 \\
9.02 \\
15.59 \\
6.68 \\
2.86 \\
18.21\end{array}$ & $\begin{array}{r}5.94 \\
5.94 \\
- \\
5.94 \\
7.55 \\
6.70 \\
4.04 \\
8.47 \\
12.70 \\
6.95 \\
2.60 \\
13.47\end{array}$ & $\begin{array}{r}5.98 \\
5.97 \\
-\overline{7} \\
5.97 \\
7.41 \\
6.51 \\
3.84 \\
8.53 \\
14.61 \\
7.08 \\
2.26 \\
14.99\end{array}$ & $\begin{array}{r}6.50 \\
6.50 \\
- \\
6.50 \\
8.28 \\
7.14 \\
4.50 \\
7.63 \\
13.30 \\
7.66 \\
2.57 \\
13.15\end{array}$ & $\begin{array}{r}7.42 \\
7.42 \\
7 \overrightarrow{7} \\
7.42 \\
8.32 \\
5.21 \\
9.03 \\
13.40 \\
8.57 \\
3.59 \\
13.25\end{array}$ & $\begin{array}{r}7.00 \\
6.99 \\
- \\
6.99 \\
8.71 \\
8.02 \\
4.80 \\
9.29 \\
15.42 \\
8.18 \\
2.58 \\
14.80\end{array}$ \\
\hline $\begin{array}{l}\text { Total Energy ..................................... } \\
\text { Primary Energy - Four Sectors ...... } \\
\text { Electricity ...................................... }\end{array}$ & $\begin{array}{l}1.73 \\
1.40 \\
4.76\end{array}$ & $\begin{array}{l}3.47 \\
2.79 \\
8.71\end{array}$ & $\begin{array}{r}7.71 \\
6.43 \\
17.16\end{array}$ & $\begin{array}{r}8.91 \\
6.77 \\
22.90\end{array}$ & $\begin{array}{r}7.79 \\
5.41 \\
23.21\end{array}$ & $\begin{array}{r}7.63 \\
5.44 \\
22.70\end{array}$ & $\begin{array}{r}7.86 \\
5.51 \\
23.42\end{array}$ & $\begin{array}{r}8.34 \\
5.84 \\
24.91\end{array}$ & $\begin{array}{r}9.03 \\
6.45 \\
25.98\end{array}$ & $\begin{array}{r}9.14 \\
6.24 \\
27.70\end{array}$ \\
\hline 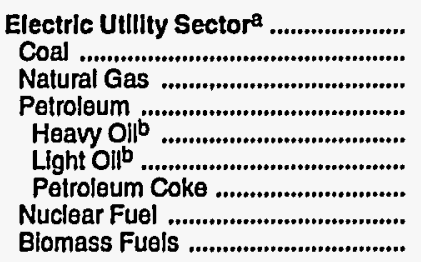 & $\begin{array}{l}.34 \\
-33 \\
.40 \\
.40 \\
.36 \\
\overline{19} \\
.65\end{array}$ & $\begin{array}{r}1.82 \\
1.05 \\
2.50 \\
2.50 \\
2.43 \\
. \overline{21} \\
.92\end{array}$ & $\begin{array}{r}3.99 \\
-\overline{3} \\
5.06 \\
5.03 \\
5.84 \\
\overline{-49} \\
1.74\end{array}$ & $\begin{array}{r}3.70 \\
-\overline{4} \\
5.37 \\
5.31 \\
5.69 \\
-\overline{86} \\
.79\end{array}$ & $\begin{array}{r}2.16 \\
-\overline{2} \\
2.81 \\
3.26 \\
3.16 \\
4.79 \\
- \\
.97 \\
.32\end{array}$ & $\begin{array}{r}2.02 \\
-\overline{2} \\
2.50 \\
2.93 \\
2.82 \\
4.10 \\
- \\
.99 \\
.95\end{array}$ & $\begin{array}{r}2.17 \\
-\overrightarrow{83} \\
2.67 \\
2.66 \\
4.03 \\
\overrightarrow{0} \\
.90 \\
.87\end{array}$ & $\begin{array}{r}2.20 \\
- \\
2.92 \\
3.09 \\
3.08 \\
3.64 \\
-\overline{85} \\
\left..{ }^{d}\right)\end{array}$ & $\begin{array}{r}2.17 \\
- \\
3.03 \\
4.36 \\
4.36 \\
4.57 \\
- \\
.72 \\
\left({ }^{d}\right)\end{array}$ & $\begin{array}{r}1.95 \\
- \\
2.87 \\
3.23 \\
3.06 \\
4.90 \\
- \\
.67 \\
\text { (d) }\end{array}$ \\
\hline Primary Energy - Flve Sectorsc .. & 1.20 & 2.61 & 5.95 & 6.19 & 4.87 & 4.79 & 4.90 & 5.20 & $\mathbf{5 . 7 7}$ & 5.59 \\
\hline
\end{tabular}

a There are no direct fuel costs for hydroelectric, geothermal, centralized solar, or wind energy.

b Heavy oll includes Grade Nos. 4,5, and 6, and residual fuel oils. Light oil includes Grade No. 2 heating oil, kerosene, and jet fuel.

c Biomass fuels are not included, except those consumed at electric utilities and those added to motor gasoline.

d Utilities used biomass fuels at no charge or received a fee for accepting them.

- No consumption, including cases where adjustments were made. See "Consumption Adjustments for Process Fuel and Intermediate Products."

Sources: Data sources, estimation procedures, and assumptions are described in the "Documentation" section of this report. 


\section{Energy Expenditure Estimates by Sector, California}

A $1970,1975,1980$, and 1985-1991

(Million Dollars)

\begin{tabular}{|c|c|c|c|c|c|c|c|c|c|c|}
\hline Sector and Energy Source & 1970 & 1975 & 1980 & 1985 & 1986 & 1987 & 1988 & 1989 & 1990 & 1991 \\
\hline $\begin{array}{l}\text { Resldentlal Sector } \\
\text { Primary Energy } \\
\text { Coal } \\
\text { Natural Gas } \\
\text { Petroleum foum } \\
\text { Distillate Fuel } \\
\text { Kerosene }\end{array}$ & $\begin{array}{r}1,401.1 \\
603.5 \\
1.1 \\
544.3 \\
58.2 \\
3.7 \\
2.4 \\
52.1 \\
797.6\end{array}$ & $\begin{array}{r}2,665.4 \\
1,052.7 \\
993.8 \\
58.9 \\
8.0 \\
6.1 \\
44.7 \\
1,612.8\end{array}$ & $\begin{aligned} 5,063.6 \\
2,014.1 \\
.2 \\
1,861.6 \\
152.4 \\
3.8 \\
1.3 \\
147.2 \\
3,049.5\end{aligned}$ & $\begin{array}{r}7,666.2 \\
3,193.4 \\
2.0 \\
3,016.1 \\
175.3 \\
4.5 \\
3.8 \\
167.0 \\
4,472.8\end{array}$ & $\begin{array}{r}7,138.8 \\
2,573.1 \\
- \\
2,384.7 \\
188.4 \\
10.6 \\
13.8 \\
164.0 \\
4,565.8\end{array}$ & $\begin{array}{r}7,751.8 \\
2,901.0 \\
2,648.3 \\
252.7 \\
10.0 \\
4.9 \\
237.7 \\
4,850.8\end{array}$ & $\begin{array}{r}8,576.9 \\
3,058.9 \\
.2 \\
2,803.9 \\
254.8 \\
7.8 \\
5.9 \\
241.2 \\
5,518.0\end{array}$ & $\begin{array}{r}9,242.7 \\
3,162.7 \\
.3 \\
2,874.8 \\
287.6 \\
10.7 \\
8.2 \\
268.7 \\
6,080.0\end{array}$ & $\begin{array}{r}9,892.5 \\
3,246.6 \\
.8 \\
2,973.8 \\
272.0 \\
7.5 \\
5.0 \\
259.5 \\
6,646.0\end{array}$ & $\begin{array}{r}10,642.8 \\
3,522.8 \\
1.9 \\
3,189.5 \\
331.4 \\
6.5 \\
4.4 \\
320.5 \\
7,120.0\end{array}$ \\
\hline $\begin{array}{l}\text { Commercial Sector } \\
\text { Primary Energy } \\
\text { Coal ... } \\
\text { Natural Gas . } \\
\text { Petroleum foum } \\
\text { Distillate Fuel } \\
\text { Kerosene } \\
\text { LPG } \\
\text { Motor Gasoline } \\
\text { Residual Fuel } \\
\text { Electricity }\end{array}$ & $\begin{array}{r}905.1 \\
208.7 \\
1.0 \\
152.9 \\
54.8 \\
4.3 \\
2.3 \\
4.7 \\
21.8 \\
21.8 \\
696.3\end{array}$ & $\begin{array}{r}2,165.1 \\
442.1 \\
- \\
309.6 \\
132.6 \\
9.8 \\
9.2 \\
4.9 \\
41.2 \\
67.4 \\
1,722.9\end{array}$ & $\begin{array}{r}5,377.9 \\
1,482.7 \\
.1 \\
1,027.9 \\
454.6 \\
124.0 \\
8.0 \\
16.6 \\
96.1 \\
209.9 \\
3,895.2\end{array}$ & $\begin{array}{r}7,528.4 \\
1,598.4 \\
1.8 \\
1,359.7 \\
236.9 \\
110.5 \\
12.5 \\
32.8 \\
80.2 \\
.9 \\
5,930.0\end{array}$ & $\begin{array}{r}7,525.2 \\
1,354.8 \\
1,070 . \overline{4} \\
284.5 \\
182.9 \\
3.0 \\
23.9 \\
61.6 \\
13.0 \\
6,170.4\end{array}$ & $\begin{array}{r}7,719.1 \\
1,487.3 \\
1,153.9 \\
333.4 \\
192.7 \\
4.6 \\
28.7 \\
91.8 \\
15.5 \\
6,231.8\end{array}$ & $\begin{array}{r}8,112.7 \\
1,455.6 \\
.2 \\
1,162.5 \\
293.0 \\
181.9 \\
2.4 \\
31.9 \\
66.0 \\
10.8 \\
6,657.1\end{array}$ & $\begin{array}{r}8,803.6 \\
1,546.9 \\
.3 \\
1,264.5 \\
282.1 \\
167.1 \\
1.4 \\
31.1 \\
71.7 \\
10.8 \\
7,256.7\end{array}$ & $\begin{array}{r}9,683.0 \\
1,750.3 \\
.8 \\
1,459.7 \\
289.9 \\
152.8 \\
.6 \\
33.2 \\
86.3 \\
16.9 \\
7,932.7\end{array}$ & $\begin{array}{r}10,114.4 \\
1,847.1 \\
1.3 \\
1,581.9 \\
263.9 \\
140.5 \\
.7 \\
41.2 \\
70.7 \\
10.7 \\
8,267.3\end{array}$ \\
\hline 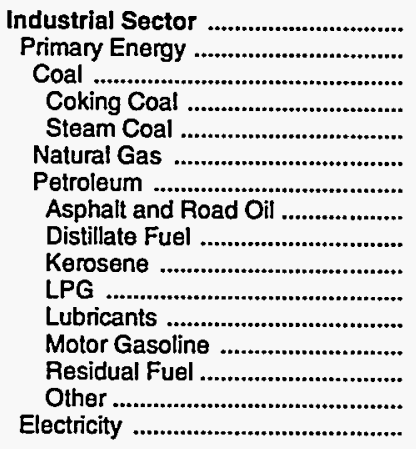 & $\begin{array}{r}920.6 \\
528.3 \\
25.8 \\
25.6 \\
.2 \\
.2 \\
209.3 \\
293.2 \\
39.0 \\
31.2 \\
1.5 \\
41.1 \\
46.6 \\
28.6 \\
21.3 \\
84.0 \\
392.2\end{array}$ & $\begin{array}{r}2,357.8 \\
1,368.8 \\
74.6 \\
67.7 \\
6.9 \\
539.6 \\
754.7 \\
141.0 \\
126.2 \\
16.5 \\
116.1 \\
56.6 \\
34.0 \\
62.4 \\
202.0 \\
988.9\end{array}$ & $\begin{array}{r}6,347.4 \\
3,739.6 \\
126.4 \\
79.8 \\
46.5 \\
1,248.7 \\
2,364.5 \\
462.2 \\
489.1 \\
67.9 \\
191.9 \\
183.1 \\
90.9 \\
204.4 \\
674.9 \\
2,607.7\end{array}$ & $\begin{array}{r}8,227.1 \\
4,501.7 \\
100.3 \\
-\overline{100} \\
100.3 \\
1,745.8 \\
2,655.6 \\
439.0 \\
586.9 \\
17.4 \\
343.2 \\
204.4 \\
139.8 \\
428.9 \\
496.0 \\
3,725.4\end{array}$ & $\begin{array}{r}6,814.4 \\
3,344.8 \\
96.0 \\
-\overline{9} \\
96.0 \\
1,252.1 \\
1,996.7 \\
517.9 \\
346.6 \\
5.2 \\
332.3 \\
176.9 \\
111.5 \\
179.2 \\
327.1 \\
3,469.7\end{array}$ & $\begin{array}{r}7,242.0 \\
4,000.3 \\
94.0 \\
94 . \overline{0} \\
94.0 \\
1,637.5 \\
2,268.8 \\
585.1 \\
443.8 \\
11.9 \\
362.2 \\
163.0 \\
118.6 \\
208.4 \\
375.7 \\
3,241.7\end{array}$ & $\begin{array}{r}6,957.6 \\
3,580.6 \\
99.1 \\
-\overline{1} \\
99.1 \\
1,557.2 \\
1,924.3 \\
461.6 \\
414.5 \\
1.0 \\
336.2 \\
180.8 \\
110.8 \\
99.2 \\
320.0 \\
3,387.0\end{array}$ & $\begin{array}{r}7,370.8 \\
3,632.7 \\
111.0 \\
111 . \overline{0} \\
1,712.8 \\
1,808.9 \\
308.2 \\
504.7 \\
1.5 \\
322.2 \\
168.8 \\
130.0 \\
25.5 \\
348.2 \\
3,738.1\end{array}$ & $\begin{array}{r}7,886.2 \\
4,059.4 \\
129.6 \\
129 . \overline{6} \\
1,967.7 \\
1,962.1 \\
308.8 \\
590.6 \\
1.3 \\
323.2 \\
175.1 \\
141.6 \\
24.1 \\
397.5 \\
3,826.8\end{array}$ & $\begin{array}{r}7,941.4 \\
3,929.8 \\
124.2 \\
124 . \overline{2} \\
2,147.0 \\
1,658.6 \\
301.0 \\
400.2 \\
1.1 \\
273.6 \\
180.2 \\
140.5 \\
16.8 \\
345.2 \\
4,011.5\end{array}$ \\
\hline 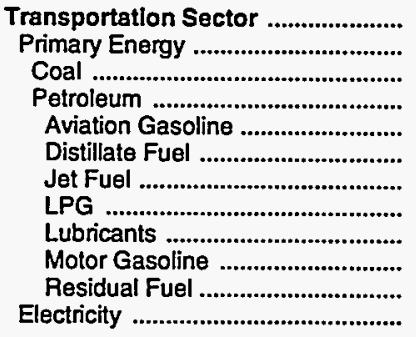 & $\begin{array}{r}3,750.3 \\
3,749.8 \\
.1 \\
3,749.7 \\
23.9 \\
243.7 \\
242.7 \\
1.6 \\
75.7 \\
3,098.8 \\
63.3 \\
.5\end{array}$ & $\begin{array}{r}7,763.2 \\
7,759.2 \\
7,759.2 \\
28.5 \\
573.4 \\
714.6 \\
4.0 \\
108.3 \\
6,062.6 \\
267.8 \\
4.0\end{array}$ & $\begin{array}{r}19,290.4 \\
19,282.8 \\
19,282.8 \\
13.0 \\
1,720.6 \\
2,166.4 \\
10.0 \\
244.2 \\
13,392.0 \\
1,736.6 \\
7.6\end{array}$ & $\begin{array}{r}18,015.7 \\
18,000.4 \\
18,000.4 \\
68.3 \\
2,017.9 \\
2,257.8 \\
42.6 \\
272.5 \\
11,972.3 \\
1,369.0 \\
15.2\end{array}$ & $\begin{array}{r}14,185.3 \\
14,170.8 \\
\overline{1} \\
14,170.8 \\
56.8 \\
1,859.4 \\
1,685.3 \\
30.6 \\
235.9 \\
9,643.3 \\
659.5 \\
14.5\end{array}$ & $\begin{array}{r}15,227.8 \\
15,217.9 \\
- \\
15,217.9 \\
41.3 \\
1,884.6 \\
1,811.7 \\
25.9 \\
217.3 \\
10,455.5 \\
781.5 \\
9.9\end{array}$ & $\begin{array}{r}16,205.0 \\
16,193.1 \\
\overline{1} \\
16,193.1 \\
49.1 \\
2,300.8 \\
1,784.5 \\
28.1 \\
241.1 \\
11,123.6 \\
665.9 \\
11.9\end{array}$ & $\begin{array}{r}18,210.2 \\
18,199.9 \\
18,199.9 \\
54.4 \\
2,511.1 \\
2,286.8 \\
24.9 \\
225.0 \\
12,302.4 \\
795.3 \\
10.3\end{array}$ & $\begin{array}{r}20,915.6 \\
20,903.2 \\
20,903.2 \\
52.0 \\
2,792.5 \\
3,081.3 \\
30.5 \\
233.4 \\
13,471.6 \\
1,241.9 \\
12.4\end{array}$ & $\begin{array}{r}18,761.7 \\
18,748.0 \\
18,748.0 \\
47.9 \\
2,631.2 \\
2,503.0 \\
25.6 \\
240.2 \\
12,618.3 \\
681.8 \\
13.7\end{array}$ \\
\hline 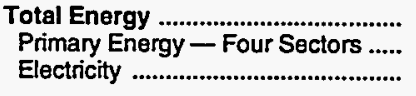 & $\begin{array}{l}6,977.0 \\
5,090.3 \\
1,886.7\end{array}$ & $\begin{array}{r}14,951.5 \\
10,622.9 \\
4,328.6\end{array}$ & $\begin{array}{r}36,079.3 \\
26,519.2 \\
9,560.0\end{array}$ & & $\begin{array}{l}35,663.8 \\
21,443.4 \\
14,220.3\end{array}$ & & & & $\begin{array}{l}48,377.4 \\
29,959.6 \\
18,417.8\end{array}$ & $\begin{array}{l}47,460.2 \\
28,047.8 \\
19,412.5\end{array}$ \\
\hline 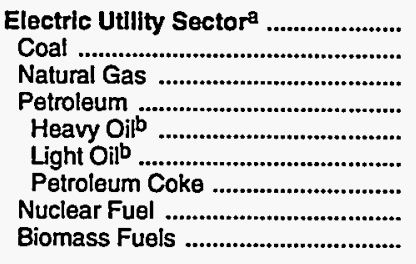 & $\begin{array}{r}282.1 \\
-\overline{1} \\
220.1 \\
54.9 \\
54.7 \\
.2 \\
\overline{6} \\
6.7 \\
.3\end{array}$ & \begin{tabular}{r|}
$1,553.7$ \\
- \\
305.2 \\
$1,234.0$ \\
$1,230.5$ \\
3.4
\end{tabular} & $\begin{array}{r}4,018.4 \\
- \\
1,925.0 \\
2,067.0 \\
1,980.8 \\
86.2\end{array}$ & $\begin{array}{r}3,130 . \overline{1} \\
164.4 \\
154.2 \\
10.2\end{array}$ & $\begin{array}{r}1.304 .4 \\
115.8 \\
106.1 \\
9.7\end{array}$ & $\begin{array}{r}1,670.1 \\
67.3 \\
58.9 \\
8.4\end{array}$ & $\begin{array}{r}2,131.0 \\
1,621 . \overline{7} \\
212.2 \\
208.3 \\
3.9 \\
-\end{array}$ & $\begin{array}{r}2,168.0 \\
-\overline{3} \\
1,574.3 \\
297.8 \\
291.5 \\
6.3 \\
-\overline{-} \\
295.9 \\
(0)\end{array}$ & $\begin{array}{r}1,882.4 \\
1,429.0 \\
201.4 \\
196.4 \\
5.0\end{array}$ & $\begin{array}{r}1,572.2 \\
\overline{1} \\
1,324 . \overline{3} \\
20.9 \\
18.0 \\
3.0 \\
- \\
226.9 \\
\text { (d) }\end{array}$ \\
\hline Primary Energy - Five Sectorsc .. & $5,372.4$ & $12,176.6$ & $30,537.6$ & $30,792.6$ & $23,137.4$ & $25,666.8$ & $26,419.3$ & $28,710.2$ & $31,841.9$ & $29,619.9$ \\
\hline
\end{tabular}

There are no direct fuel costs for hydroelectric, geothermal, centralized solar, or wind energy.

b Heavy oil includes Grade Nos. 4, 5, and 6, and residual fuel oils. Light oil includes Grade No. 2 heating oil, kerosene, and jet fuel.

c Biomass fuels are not included, except those consumed at electric utilities and those added to motor gasoline.

d Utilities used biomass fuels at no charge or received a fee for accepting them.

-No consumption, including cases where adjustments were made. See "Consumption Adjustments for Process Fuel and Intermediate Products."

Value less than 0.05 million dollars.

Note: Totals may not equal sum of components due to independent rounding.

Sources: Data sources, estimation procedures, and assumptions are described in the "Documentation" section of this report. 


\begin{tabular}{|c|c|c|c|c|c|c|c|c|c|c|}
\hline Energy Source & 1970 & 1975 & 1980 & 1985 & 1986 & 1987 & 1988 & 1989 & 1990 & 1991 \\
\hline & \multicolumn{10}{|c|}{ Prices in Dollars per Mtllion Btu } \\
\hline 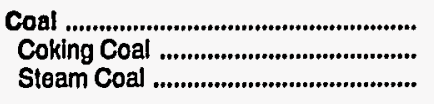 & $\begin{array}{r}0.33 \\
.43 \\
.30\end{array}$ & $\begin{array}{r}0.68 \\
1.38 \\
.53\end{array}$ & $\begin{array}{r}1.00 \\
1.97 \\
.89\end{array}$ & $\begin{array}{r}1.17 \\
1.17\end{array}$ & $\begin{array}{r}1.18 \\
1.18\end{array}$ & $\begin{array}{r}1.14 \\
1.14\end{array}$ & $\begin{array}{r}1.08 \\
1.08\end{array}$ & $\begin{array}{r}1.08 \\
1.08\end{array}$ & $\begin{array}{r}1.07 \\
1.07\end{array}$ & $\begin{array}{r}1.10 \\
1.10\end{array}$ \\
\hline Natural Gas ............................................ & .48 & .98 & 2.98 & 4.71 & 4.54 & 4.26 & 3.95 & 3.85 & 3.92 & 3.74 \\
\hline 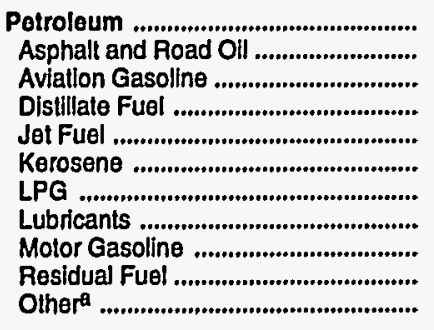 & $\begin{array}{r}1.88 \\
.58 \\
2.17 \\
1.04 \\
.76 \\
.98 \\
1.60 \\
5.08 \\
2.72 \\
.44 \\
1.09\end{array}$ & $\begin{array}{l}3.54 \\
2.01 \\
3.45 \\
2.30 \\
2.12 \\
2.22 \\
3.02 \\
7.48 \\
4.67 \\
1.59 \\
1.98\end{array}$ & $\begin{array}{r}7.93 \\
3.40 \\
9.02 \\
6.45 \\
6.59 \\
5.78 \\
5.88 \\
14.36 \\
9.36 \\
3.88 \\
7.39\end{array}$ & $\begin{array}{r}8.00 \\
4.73 \\
9.99 \\
6.26 \\
5.94 \\
7.69 \\
6.39 \\
17.61 \\
9.28 \\
3.80 \\
6.78\end{array}$ & $\begin{array}{r}6.07 \\
4.30 \\
8.41 \\
5.52 \\
3.92 \\
4.66 \\
6.21 \\
15.59 \\
6.76 \\
2.34 \\
5.24\end{array}$ & $\begin{array}{r}6.47 \\
3.03 \\
7.55 \\
5.86 \\
4.05 \\
4.23 \\
4.80 \\
12.70 \\
7.59 \\
2.63 \\
5.01\end{array}$ & $\begin{array}{r}6.24 \\
3.19 \\
7.41 \\
5.46 \\
3.78 \\
4.10 \\
3.27 \\
14.61 \\
7.34 \\
2.80 \\
4.35\end{array}$ & $\begin{array}{r}7.10 \\
2.73 \\
8.28 \\
6.66 \\
4.30 \\
5.39 \\
5.97 \\
13.30 \\
8.13 \\
1.96 \\
4.04\end{array}$ & $\begin{array}{r}8.07 \\
2.59 \\
9.32 \\
7.70 \\
5.59 \\
6.99 \\
6.54 \\
13.40 \\
9.29 \\
2.64 \\
3.98\end{array}$ & $\begin{array}{r}7.97 \\
3.24 \\
8.71 \\
7.33 \\
4.87 \\
7.06 \\
8.14 \\
15.42 \\
9.23 \\
3.86 \\
3.63\end{array}$ \\
\hline Nuclear Fubl ....................................... & - & - & .21 & - & .27 & .28 & .29 & .28 & - & - \\
\hline Blomass Fuels at Utilitles ................. & - & - & - & .79 & .32 & .95 & .87 & .80 & .80 & .80 \\
\hline Primary Energy - Flve Sector $8^{b} \ldots$ & 1.03 & 2.02 & 4.33 & 4.72 & 3.93 & 4.01 & 3.77 & 3.98 & 4.37 & 4.40 \\
\hline $\begin{array}{l}\text { Electric Utility Fuelc ............................ } \\
\text { Electricity Purchased by End Users }\end{array}$ & $\begin{array}{r}.25 \\
6.09\end{array}$ & $\begin{array}{r}.60 \\
7.95\end{array}$ & $\begin{array}{r}1.12 \\
12.94\end{array}$ & $\begin{array}{r}1.21 \\
17.88\end{array}$ & $\begin{array}{r}1.21 \\
17.69\end{array}$ & $\begin{array}{r}1.15 \\
17.12\end{array}$ & $\begin{array}{r}1.09 \\
17.32\end{array}$ & $\begin{array}{r}1.08 \\
17.48\end{array}$ & $\begin{array}{r}1.08 \\
17.31\end{array}$ & $\begin{array}{r}1.11 \\
17.49\end{array}$ \\
\hline \multirow[t]{2}{*}{ Total Energy .......................................... } & 1.52 & 2.92 & 6.49 & 8.20 & 7.24 & 7.35 & 7.13 & 7.57 & 8.09 & 7.93 \\
\hline & \multicolumn{10}{|c|}{ Expenditures in Millions of Dollars } \\
\hline 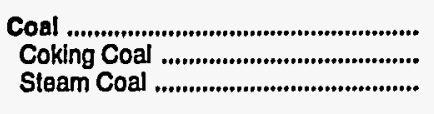 & $\begin{array}{l}38.3 \\
12.0 \\
26.3\end{array}$ & $\begin{array}{r}108.4 \\
39.5 \\
69.0\end{array}$ & $\begin{array}{r}248.2 \\
50.2 \\
197.9\end{array}$ & $\begin{array}{r}349.9 \\
349 . \overline{9}\end{array}$ & $\begin{array}{r}349.5 \\
349.5\end{array}$ & $\begin{array}{r}336.8 \\
\overline{-} \\
336.8\end{array}$ & $\begin{array}{r}337.3 \\
\overline{3} \\
337.3\end{array}$ & $\begin{array}{r}349.0 \\
- \\
349.0\end{array}$ & $\begin{array}{r}353.3 \\
-\overline{3} \\
353.3\end{array}$ & $\begin{array}{r}355.4 \\
- \\
355.4\end{array}$ \\
\hline 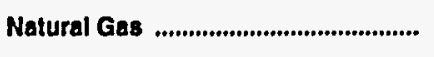 & 128.2 & 262.9 & 706.8 & 931.2 & 810.9 & 793.7 & 817.3 & 837.0 & 820.8 & 854.2 \\
\hline 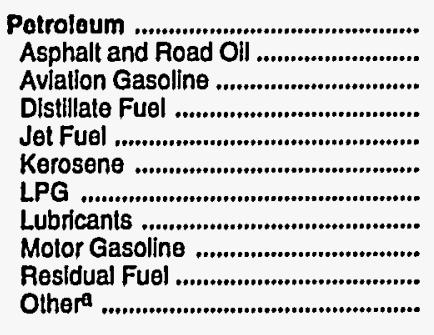 & $\begin{array}{r}503.5 \\
12.3 \\
3.7 \\
30.9 \\
32.0 \\
4.5 \\
27.5 \\
13.0 \\
372.5 \\
3.9 \\
3.1\end{array}$ & $\begin{array}{r}1,139.9 \\
29.8 \\
4.6 \\
118.1 \\
85.7 \\
3.5 \\
55.7 \\
20.8 \\
782.3 \\
32.7 \\
6.7\end{array}$ & $\begin{array}{r}2,577.0 \\
51.6 \\
12.1 \\
422.1 \\
175.9 \\
13.5 \\
83.3 \\
55.8 \\
1,685.6 \\
43.6 \\
33.5\end{array}$ & $\begin{array}{r}2,598.7 \\
97.5 \\
7.1 \\
348.2 \\
264.1 \\
4.0 \\
51.3 \\
62.3 \\
1,742.4 \\
3.7 \\
18.0\end{array}$ & $\begin{array}{r}2,007.3 \\
88.2 \\
7.5 \\
325.5 \\
178.8 \\
1.6 \\
47.3 \\
53.9 \\
1,297.1 \\
1.1 \\
6.3\end{array}$ & $\begin{array}{r}2,134.8 \\
62.6 \\
5.8 \\
336.7 \\
192.0 \\
2.0 \\
39.6 \\
49.7 \\
1,439.3 \\
7.1\end{array}$ & $\begin{array}{r}2,073.9 \\
75.2 \\
6.2 \\
355.7 \\
138.0 \\
2.0 \\
30.4 \\
55.1 \\
1,404.9 \\
.5 \\
6.0\end{array}$ & $\begin{array}{r}2,242.2 \\
53.1 \\
7.6 \\
395.9 \\
129.8 \\
6.9 \\
79.0 \\
51.4 \\
1,512.7 \\
.1 \\
5.8\end{array}$ & $\begin{array}{r}2,580.9 \\
55.9 \\
7.8 \\
465.0 \\
193.0 \\
2.0 \\
71.8 \\
53.4 \\
1,725.8 \\
6.2\end{array}$ & $\begin{array}{r}2,693.0 \\
66.8 \\
6.8 \\
504.2 \\
183.3 \\
2.0 \\
138.8 \\
54.9 \\
1,730.0 \\
1.1 \\
5.0\end{array}$ \\
\hline Nuclear Fuel ........................................ & - & - & 1.5 & - & .2 & .5 & 2.1 & 1.6 & - & - \\
\hline Blomass Fuels at Utillties ................. & - & - & - & - & - & $\cdot$ & $\cdot$ & - & * & - \\
\hline Primary Energy - Flve Sectorsb .... & 670.0 & $1,511.2$ & $3,533.4$ & $3,879.8$ & $3,167.8$ & $3,265.9$ & $3,230.6$ & $3,429.8$ & $3,755.0$ & $3,902.5$ \\
\hline $\begin{array}{l}\text { Electric Utility Fuelc ............................ } \\
\text { Electricity Purchased by End Users }\end{array}$ & $\begin{array}{r}-30.6 \\
222.3\end{array}$ & $\begin{array}{r}-105.4 \\
426.0\end{array}$ & $\begin{array}{r}-272.5 \\
918.2\end{array}$ & $\begin{array}{r}-342.6 \\
1,608.3\end{array}$ & $\begin{array}{r}-340.8 \\
1,621.9\end{array}$ & $\begin{array}{r}-332.3 \\
1,622.8\end{array}$ & $\begin{array}{r}-337.3 \\
1,716.9\end{array}$ & $\begin{array}{r}-350.7 \\
1,775.2\end{array}$ & $\begin{array}{r}-344.7 \\
1,800.4\end{array}$ & $\begin{array}{r}-345.8 \\
1,858.4\end{array}$ \\
\hline Total Energy ...................................... & 861.7 & $1,831.8$ & $4,179.0$ & $5,145.4$ & $4,448.9$ & $4,556.4$ & $4,610.2$ & $4,854.3$ & $5,210.6$ & $5,415.2$ \\
\hline
\end{tabular}

Includes petroleum coke used at electric utilities.

b Biomass fuels are not included, except those consumed at electric utilities and those added to motor gasoline.

c There are no direct fuel costs for hydroelectric, geothermal, centralized solar, or wind energy.

-No consumplion, Including cases where adjustments were made. See "Consumption Adjustments for Process Fuel and Intermediate Products."

- Value less than 0.05 million dollars.

Note: Expenditure totals may not equal sum of components due to independent rounding.

Sources: Data sources, estimation procedures, and assumptions are described in the "Documentation" section of this report. 


\section{Energy Price Estimates by Sector, Colorado}

O $1970,1975,1980$, and 1985-1991

L (Dollars per Million Btu)

\begin{tabular}{|c|c|c|c|c|c|c|c|c|c|c|}
\hline Sector and Energy Source & 1970 & 1975 & 1980 & 1985 & 1986 & 1987 & 1988 & 1989 & 1990 & 1991 \\
\hline 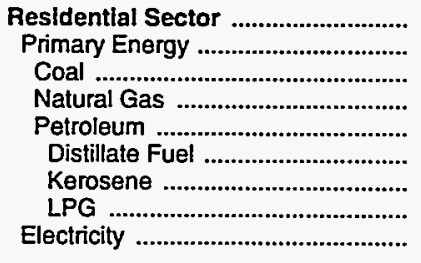 & $\begin{array}{r}1.71 \\
.88 \\
.90 \\
.74 \\
1.74 \\
1.28 \\
1.51 \\
1.79 \\
7.73\end{array}$ & $\begin{array}{l}2.76 \\
1.53 \\
1.58 \\
1.29 \\
3.26 \\
2.84 \\
2.96 \\
3.33 \\
9.94\end{array}$ & $\begin{array}{r}5.72 \\
3.53 \\
2.54 \\
3.26 \\
7.31 \\
6.96 \\
7.98 \\
7.32 \\
15.00\end{array}$ & $\begin{array}{r}8.76 \\
5.18 \\
2.83 \\
5.11 \\
6.71 \\
6.91 \\
9.20 \\
6.55 \\
20.28\end{array}$ & $\begin{array}{r}9.06 \\
5.08 \\
2.78 \\
4.99 \\
6.61 \\
4.32 \\
5.75 \\
6.81 \\
20.60\end{array}$ & $\begin{array}{r}8.61 \\
4.74 \\
2.40 \\
4.74 \\
5.07 \\
3.49 \\
4.65 \\
5.18 \\
20.01\end{array}$ & $\begin{array}{r}8.22 \\
4.27 \\
2.12 \\
4.39 \\
2.63 \\
3.47 \\
4.62 \\
2.51 \\
20.31\end{array}$ & $\begin{array}{r}8.60 \\
4.66 \\
2.43 \\
4.57 \\
6.10 \\
5.09 \\
6.78 \\
6.11 \\
20.56\end{array}$ & $\begin{array}{r}8.69 \\
4.70 \\
2.41 \\
4.55 \\
7.02 \\
6.19 \\
8.24 \\
7.02 \\
20.57\end{array}$ & $\begin{array}{r}8.53 \\
4.64 \\
2.36 \\
4.46 \\
7.30 \\
5.90 \\
7.85 \\
7.32 \\
20.72\end{array}$ \\
\hline 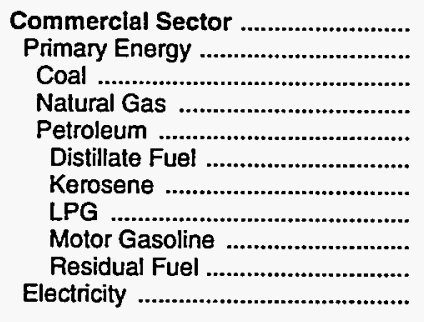 & $\begin{array}{r}1.66 \\
.62 \\
.39 \\
.59 \\
1.28 \\
1.06 \\
.89 \\
1.18 \\
2.72 \\
.38 \\
5.97\end{array}$ & $\begin{array}{r}2.73 \\
1.20 \\
.81 \\
1.10 \\
2.73 \\
2.49 \\
2.11 \\
2.59 \\
4.67 \\
1.93 \\
7.95\end{array}$ & $\begin{array}{r}6.09 \\
3.26 \\
1.20 \\
3.03 \\
7.08 \\
6.48 \\
5.65 \\
4.79 \\
9.36 \\
4.35 \\
14.37\end{array}$ & $\begin{array}{r}9.48 \\
4.64 \\
1.31 \\
4.61 \\
6.16 \\
5.45 \\
5.98 \\
6.12 \\
9.28 \\
4.07 \\
18.34\end{array}$ & $\begin{array}{r}9.51 \\
4.39 \\
1.24 \\
4.41 \\
5.03 \\
4.83 \\
3.67 \\
5.10 \\
6.76 \\
2.34 \\
17.73\end{array}$ & $\begin{array}{r}8.91 \\
4.11 \\
1.39 \\
4.15 \\
4.20 \\
3.61 \\
4.02 \\
4.09 \\
7.59 \\
- \\
17.05\end{array}$ & $\begin{array}{r}8.69 \\
3.77 \\
1.36 \\
3.83 \\
3.62 \\
2.95 \\
3.78 \\
4.21 \\
7.34 \\
- \\
17.03\end{array}$ & $\begin{array}{r}9.17 \\
4.05 \\
1.34 \\
4.00 \\
4.98 \\
3.83 \\
5.08 \\
5.86 \\
8.13 \\
1.80 \\
17.17\end{array}$ & $\begin{array}{r}9.27 \\
4.11 \\
1.29 \\
3.97 \\
6.36 \\
4.95 \\
5.98 \\
5.93 \\
9.29 \\
- \\
16.89\end{array}$ & $\begin{array}{r}9.15 \\
4.11 \\
1.39 \\
3.92 \\
6.48 \\
4.30 \\
6.35 \\
8.68 \\
9.23 \\
- \\
17.07\end{array}$ \\
\hline 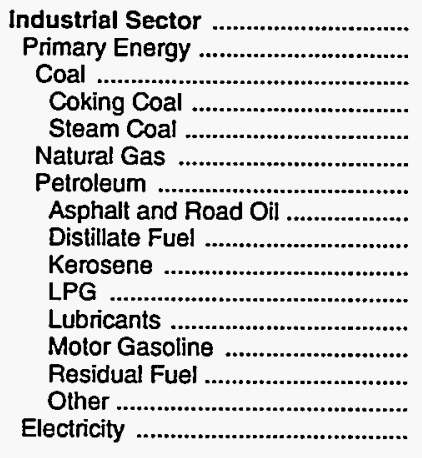 & $\begin{array}{r}.65 \\
.53 \\
.42 \\
.43 \\
.39 \\
.29 \\
.97 \\
.58 \\
.83 \\
.89 \\
1.18 \\
5.08 \\
2.72 \\
.47 \\
1.09 \\
3.50\end{array}$ & $\begin{array}{r}1.74 \\
1.40 \\
1.17 \\
1.38 \\
.81 \\
.72 \\
2.19 \\
2.01 \\
1.96 \\
2.11 \\
2.59 \\
7.48 \\
4.67 \\
1.43 \\
1.98 \\
5.55\end{array}$ & $\begin{array}{r}4.20 \\
3.44 \\
1.66 \\
1.97 \\
1.20 \\
2.65 \\
5.17 \\
3.40 \\
5.33 \\
5.65 \\
4.79 \\
14.36 \\
9.36 \\
3.82 \\
7.39 \\
9.40\end{array}$ & $\begin{array}{r}5.65 \\
4.34 \\
1.31 \\
- \\
1.31 \\
4.01 \\
5.80 \\
4.73 \\
5.25 \\
5.98 \\
6.12 \\
17.61 \\
9.28 \\
4.07 \\
6.78 \\
12.67\end{array}$ & $\begin{array}{r}5.19 \\
3.64 \\
1.24 \\
- \\
1.24 \\
3.82 \\
4.35 \\
4.30 \\
3.23 \\
3.67 \\
5.10 \\
15.59 \\
6.76 \\
2.34 \\
5.24 \\
12.95\end{array}$ & $\begin{array}{r}5.14 \\
3.37 \\
1.39 \\
-\overline{1} \\
3.39 \\
3.60 \\
3.03 \\
3.53 \\
4.02 \\
4.09 \\
12.70 \\
7.59 \\
2.68 \\
5.01 \\
12.78\end{array}$ & $\begin{array}{r}4.97 \\
3.35 \\
1.36 \\
- \\
1.36 \\
3.43 \\
3.83 \\
3.19 \\
3.32 \\
3.78 \\
4.21 \\
14.61 \\
7.34 \\
1.82 \\
4.35 \\
13.22\end{array}$ & $\begin{array}{r}4.79 \\
3.18 \\
1.34 \\
- \\
1.34 \\
2.50 \\
4.40 \\
2.73 \\
4.47 \\
5.08 \\
5.86 \\
13.30 \\
8.13 \\
1.80 \\
4.04 \\
13.32\end{array}$ & $\begin{array}{r}4.94 \\
3.28 \\
1.29 \\
- \\
1.29 \\
2.77 \\
4.46 \\
2.59 \\
5.26 \\
5.98 \\
5.93 \\
13.40 \\
9.29 \\
2.46 \\
3.98 \\
13.17\end{array}$ & $\begin{array}{r}5.13 \\
3.65 \\
1.39 \\
- \\
1.39 \\
2.27 \\
5.56 \\
3.24 \\
5.58 \\
6.35 \\
8.68 \\
15.42 \\
9.23 \\
2.26 \\
3.63 \\
13.38\end{array}$ \\
\hline $\begin{array}{c}\text { Transportation Sector } \\
\text { Primary Energy } \\
\text { Coal }\end{array}$ & $\begin{array}{r}2.17 \\
2.17 \\
.39\end{array}$ & $\begin{array}{r}3.99 \\
3.99 \\
.81\end{array}$ & $\begin{array}{r}8.75 \\
8.75 \\
-\end{array}$ & $\begin{array}{r}8.43 \\
8.43\end{array}$ & $\begin{array}{r}6.38 \\
6.38 \\
-\end{array}$ & $\begin{array}{l}6.98 \\
6.98\end{array}$ & $\begin{array}{l}6.88 \\
6.88\end{array}$ & $\begin{array}{l}7.68 \\
7.68\end{array}$ & $\begin{array}{l}8.76 \\
8.76\end{array}$ & $\begin{array}{l}8.53 \\
8.53\end{array}$ \\
\hline 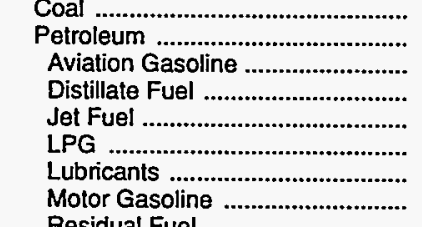 & $\begin{array}{r}.39 \\
2.17 \\
2.17 \\
1.20 \\
.76 \\
1.18 \\
5.08 \\
2.72\end{array}$ & $\begin{array}{r}.81 \\
3.99 \\
3.45 \\
2.49 \\
2.12 \\
2.59 \\
7.48 \\
4.67\end{array}$ & $\begin{array}{r}-\overline{8} \\
9.75 \\
7.02 \\
6.59 \\
4.79 \\
14.36 \\
9.36\end{array}$ & $\begin{array}{r}8 . \overline{-} \\
9.99 \\
6.70 \\
5.94 \\
6.12 \\
17.61 \\
9.28\end{array}$ & $\begin{array}{r}-\overline{6} \\
8.41 \\
6.91 \\
3.92 \\
5.10 \\
15.59 \\
6.76\end{array}$ & $\begin{array}{r}6.98 \\
7.55 \\
7.28 \\
4.05 \\
4.09 \\
12.70 \\
7.59\end{array}$ & $\begin{array}{r}- \\
6.88 \\
7.41 \\
7.13 \\
3.78 \\
4.21 \\
14.61 \\
7.34\end{array}$ & $\begin{array}{r}-\overline{7} \\
7.68 \\
8.28 \\
7.85 \\
4.30 \\
5.86 \\
13.30 \\
8.13\end{array}$ & $\begin{array}{r}- \\
8.76 \\
9.32 \\
8.80 \\
5.59 \\
5.93 \\
13.40 \\
9.29\end{array}$ & $\begin{array}{r}- \\
8.53 \\
8.71 \\
8.39 \\
4.87 \\
8.68 \\
15.42 \\
9.23\end{array}$ \\
\hline 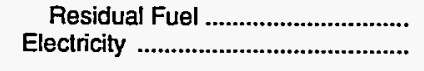 & $\begin{array}{c}.38 \\
-\end{array}$ & $\begin{array}{r}1.86 \\
-\end{array}$ & - & $\begin{array}{r}3.79 \\
-\end{array}$ & 2.01 & - & - & - & - & - \\
\hline $\begin{array}{l}\text { Total Energy .................................. } \\
\text { Primary Energy - Four Sectors ..... } \\
\text { Electricity ......................................... }\end{array}$ & $\begin{array}{l}1.52 \\
1.20 \\
6.09\end{array}$ & $\begin{array}{l}2.92 \\
2.45 \\
7.95\end{array}$ & $\begin{array}{r}6.49 \\
5.69 \\
12.94\end{array}$ & $\begin{array}{r}8.20 \\
6.58 \\
17.88\end{array}$ & $\begin{array}{r}7.24 \\
5.40 \\
17.69\end{array}$ & $\begin{array}{r}7.35 \\
5.58 \\
17.12\end{array}$ & $\begin{array}{r}7.13 \\
5.29 \\
17.32\end{array}$ & $\begin{array}{r}7.57 \\
5.71 \\
17.48\end{array}$ & $\begin{array}{r}8.09 \\
6.31 \\
17.31\end{array}$ & $\begin{array}{r}7.93 \\
6.17 \\
17.49\end{array}$ \\
\hline 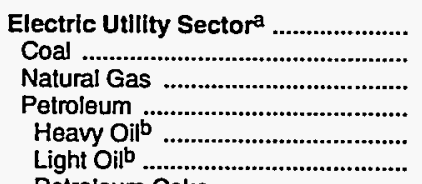 & $\begin{array}{l}.25 \\
.26 \\
.24 \\
.37 \\
.36 \\
.45\end{array}$ & $\begin{array}{r}.60 \\
.48 \\
.59 \\
2.18 \\
1.94 \\
2.56\end{array}$ & $\begin{array}{r}1.12 \\
.86 \\
2.64 \\
5.65 \\
4.38 \\
6.50\end{array}$ & $\begin{array}{l}1.21 \\
1.15 \\
3.53 \\
5.79 \\
4.00 \\
5.92\end{array}$ & $\begin{array}{r}1.21 \\
1.18 \\
3.00 \\
3.37 \\
- \\
3.37\end{array}$ & $\begin{array}{l}1.15 \\
1.12 \\
2.29 \\
3.86 \\
2.35 \\
3.86\end{array}$ & $\begin{array}{l}1.09 \\
1.07 \\
2.29 \\
3.52 \\
2.80 \\
3.88\end{array}$ & $\begin{array}{l}1.08 \\
1.06 \\
2.28 \\
4.07 \\
2.74 \\
4.10\end{array}$ & $\begin{array}{l}1.08 \\
1.06 \\
2.17 \\
5.34 \\
3.09 \\
5.34\end{array}$ & $\begin{array}{l}1.11 \\
1.09 \\
2.15 \\
4.39 \\
3.86 \\
5.13\end{array}$ \\
\hline 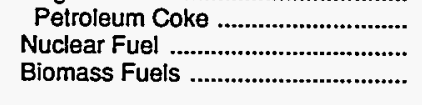 & $\overline{-}$ & - &.$\overline{1}$ & $\frac{-}{79}$ & $\begin{array}{r}-27 \\
.32\end{array}$ & $\begin{array}{r}-28 \\
.95\end{array}$ & $\begin{array}{r}-29 \\
.87\end{array}$ & $\begin{array}{r}-28 \\
.80\end{array}$ & $\begin{array}{r}- \\
.80\end{array}$ & $\overline{-}$ \\
\hline Primary Energy - Flve Sectors ${ }^{c}$.. & 1.03 & 2.02 & 4.33 & 4.72 & 3.93 & 4.01 & 3.77 & 3.98 & 4.37 & 4.40 \\
\hline
\end{tabular}

a There are no direct fuel costs for hydroelectric, geothermal, centralized solar, or wind energy.

Heavy oil includes Grade Nos. 4, 5, and 6, and residual fuel oils. Light oil includes Grade No. 2 heating oil, kerosene, and jet fuel.

c Biomass fuels are not included, except those consumed at electric utilities and those added to motor gasoline.

$\rightarrow$ No consumption, including cases where adjustments were made. See "Consumption Adjustments for Process Fuel and Intermediate Products."

Sources: Data sources, estimation procedures, and assumptions are described in the "Documentation" section of this report. 


\begin{tabular}{|c|c|c|c|c|c|c|c|c|c|c|}
\hline Sector and Energy Source & 1970 & 1975 & 1980 & 1985 & 1986 & 1987 & 1988 & 1989 & 1990 & 1991 \\
\hline 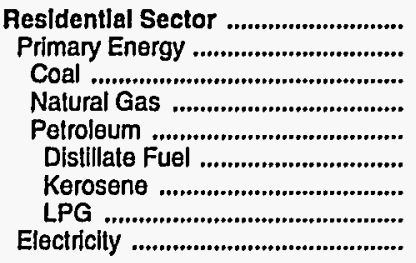 & $\begin{array}{r}185.9 \\
84.1 \\
1.6 \\
59.4 \\
23.1 \\
1.3 \\
1.0 \\
20.9 \\
101.8\end{array}$ & $\begin{array}{r}330.9 \\
156.5 \\
.2 \\
115.6 \\
40.7 \\
4.7 \\
.6 \\
35.4 \\
174.4\end{array}$ & $\begin{array}{r}684.1 \\
341.6 \\
1.9 \\
290.6 \\
49.1 \\
3.2 \\
1.0 \\
44.9 \\
342.5\end{array}$ & $\begin{array}{r}1,116.1 \\
502.9 \\
3.3 \\
459.9 \\
39.6 \\
4.3 \\
2.6 \\
32.8 \\
613.3\end{array}$ & $\begin{array}{r}1,068.0 \\
444.9 \\
2.2 \\
406.6 \\
36.1 \\
1.6 \\
1.0 \\
33.6 \\
623.0\end{array}$ & $\begin{array}{r}t, 069.7 \\
440.2 \\
1.5 \\
408.9 \\
29.8 \\
1.2 \\
.7 \\
27.9 \\
629.5\end{array}$ & $\begin{array}{r}1,088.8 \\
426.9 \\
1.5 \\
410.6 \\
14.8 \\
1.1 \\
.8 \\
12.9 \\
662.0\end{array}$ & $\begin{array}{r}1,136.9 \\
463.9 \\
1.2 \\
424.0 \\
38.8 \\
1.2 \\
1.6 \\
35.9 \\
673.0\end{array}$ & $\begin{array}{r}1,153.3 \\
466.3 \\
1.0 \\
420.1 \\
45.2 \\
1.0 \\
1.0 \\
43.2 \\
687.0\end{array}$ & $\begin{array}{r}1,214.7 \\
500.7 \\
1.2 \\
447.2 \\
52.3 \\
.9 \\
1.1 \\
50.3 \\
714.0\end{array}$ \\
\hline 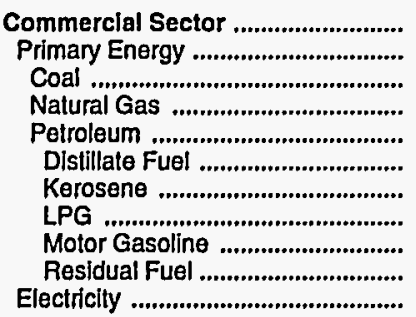 & $\begin{array}{r}134.4 \\
40.9 \\
1.3 \\
33.7 \\
5.9 \\
.9 \\
.7 \\
2.4 \\
1.8 \\
.1 \\
93.5\end{array}$ & $\begin{array}{r}258.5 \\
88.2 \\
.2 \\
75.5 \\
12.4 \\
3.4 \\
.6 \\
4.9 \\
2.7 \\
.9 \\
170.3\end{array}$ & $\begin{array}{r}594.0 \\
237.1 \\
1.7 \\
201.9 \\
33.6 \\
12.8 \\
.2 \\
5.2 \\
15.4 \\
.1 \\
356.8\end{array}$ & $\begin{array}{r}1,129.0 \\
356.8 \\
2.8 \\
317.8 \\
36.2 \\
21.6 \\
.5 \\
5.4 \\
8.6 \\
772.2\end{array}$ & $\begin{array}{r}1,051.7 \\
298.6 \\
1.8 \\
272.8 \\
24.0 \\
11.4 \\
.3 \\
4.4 \\
6.8 \\
1.1 \\
753.0\end{array}$ & $\begin{array}{r}1,036.0 \\
300.9 \\
1.6 \\
267.1 \\
32.3 \\
20.2 \\
.6 \\
3.9 \\
7.6 \\
- \\
735.1\end{array}$ & $\begin{array}{r}1,078.7 \\
294.7 \\
1.8 \\
264.5 \\
28.4 \\
17.5 \\
.3 \\
3.8 \\
6.8 \\
- \\
784.0\end{array}$ & $\begin{array}{r}1,131.2 \\
304.2 \\
1.2 \\
273.3 \\
29.7 \\
12.0 \\
4.5 \\
6.1 \\
7.0 \\
.1 \\
827.0\end{array}$ & $\begin{array}{r}1,128.8 \\
297.8 \\
1.0 \\
264.5 \\
32.2 \\
12.6 \\
.3 \\
6.4 \\
12.9 \\
831.0\end{array}$ & $\begin{array}{r}1,172.8 \\
321.8 \\
1.3 \\
278.6 \\
42.0 \\
14.8 \\
.4 \\
10.5 \\
16.3 \\
-\overline{9}\end{array}$ \\
\hline 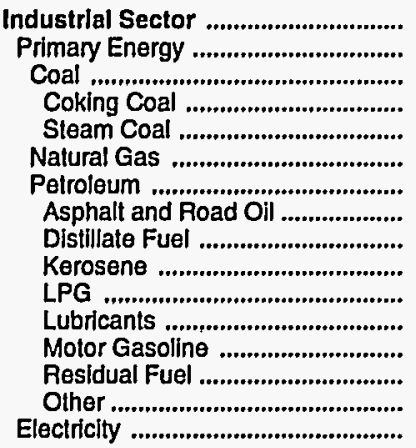 & $\begin{array}{r}121.5 \\
94.6 \\
17.4 \\
12.0 \\
5.4 \\
23.1 \\
54.1 \\
12.3 \\
10.1 \\
2.8 \\
3.6 \\
4.2 \\
14.8 \\
3.0 \\
3.1 \\
26.9\end{array}$ & $\begin{array}{r}314.6 \\
233.2 \\
53.4 \\
39.5 \\
14.0 \\
40.9 \\
138.9 \\
29.8 \\
38.6 \\
2.3 \\
13.6 \\
7.1 \\
21.1 \\
19.8 \\
6.7 \\
81.3\end{array}$ & $\begin{array}{r}769.0 \\
550.2 \\
71.3 \\
50.2 \\
21.1 \\
131.6 \\
347.2 \\
51.6 \\
123.7 \\
12.3 \\
32.4 \\
20.8 \\
34.2 \\
38.8 \\
33.5 \\
218.8\end{array}$ & $\begin{array}{r}631.1 \\
408.4 \\
22.4 \\
-\overline{4} \\
22.4 \\
136.3 \\
249.6 \\
97.5 \\
70.2 \\
.9 \\
11.6 \\
23.2 \\
28.3 \\
18.0 \\
222.7\end{array}$ & $\begin{array}{r}591.7 \\
345.9 \\
20.5 \\
- \\
20.5 \\
117.6 \\
207.7 \\
88.2 \\
64.9 \\
.4 \\
8.2 \\
20.1 \\
19.7 \\
* \\
6.3 \\
245.8\end{array}$ & $\begin{array}{r}552.1 \\
293.8 \\
21.8 \\
-\overline{8} \\
21.8 \\
100.0 \\
172.0 \\
62.6 \\
54.7 \\
.7 \\
7.2 \\
18.5 \\
21.1 \\
7 \\
7.1 \\
258.3\end{array}$ & $\begin{array}{r}618.9 \\
348.0 \\
19.6 \\
\overline{-} \\
19.6 \\
123.2 \\
205.2 \\
75.2 \\
71.4 \\
.8 \\
12.9 \\
20.5 \\
18.4 \\
6.0 \\
670.9\end{array}$ & $\begin{array}{r}623.6 \\
348.5 \\
17.9 \\
\overline{-} \\
17.9 \\
121.1 \\
209.5 \\
53.1 \\
73.5 \\
.8 \\
35.7 \\
19.1 \\
21.6 \\
5.8 \\
575.1\end{array}$ & $\begin{array}{r}631.7 \\
349.3 \\
19.8 \\
\overline{-} \\
19.8 \\
124.4 \\
205.1 \\
55.9 \\
82.2 \\
.6 \\
20.5 \\
19.8 \\
19.8 \\
6 \\
6.2 \\
282.3\end{array}$ & $\begin{array}{r}738.6 \\
445.2 \\
21.6 \\
-\overline{6} \\
21.6 \\
116.1 \\
307.4 \\
66.8 \\
114.8 \\
.6 \\
75.4 \\
20.4 \\
24.4 \\
. \\
5.0 \\
293.4\end{array}$ \\
\hline 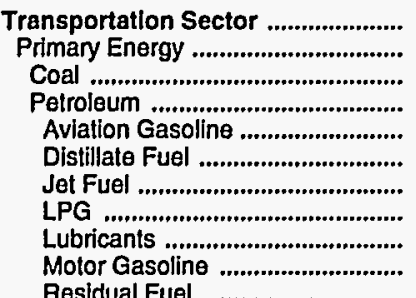 & $\begin{array}{r}419.9 \\
419.9 \\
419.8 \\
3.7 \\
18.6 \\
32.0 \\
.6 \\
8.8 \\
356.0 \\
?\end{array}$ & $\begin{array}{r}927.9 \\
927.9 \\
927.9 \\
4.6 \\
62.3 \\
85.7 \\
1.8 \\
13.7 \\
758.5 \\
13\end{array}$ & $\begin{array}{r}2,131.9 \\
2,131.9 \\
- \\
2,131.9 \\
12.1 \\
272.1 \\
175.9 \\
.8 \\
35.1 \\
1,636.1\end{array}$ & $\begin{array}{r}2,269.2 \\
2,269.2 \\
- \\
2,269.2 \\
7.1 \\
248.2 \\
264.1 \\
1.5 \\
39.1 \\
1,705.6\end{array}$ & $\begin{array}{r}1,737.6 \\
1,737.6 \\
- \\
1,737.6 \\
7.5 \\
245.7 \\
178.8 \\
1.1 \\
33.9 \\
1,270.6\end{array}$ & $\begin{array}{r}1,898.7 \\
1,898.7 \\
- \\
1,898.7 \\
5.8 \\
258.6 \\
192.0 \\
.6 \\
31.2 \\
1,410.6\end{array}$ & $\begin{array}{r}1,823.7 \\
1,823.7 \\
- \\
1,823.7 \\
6.2 \\
264.4 \\
138.0 \\
.8 \\
34.6 \\
1,379.7\end{array}$ & $\begin{array}{r}1,962.6 \\
1,962.6 \\
- \\
1,962.6 \\
7.6 \\
307.5 \\
129.8 \\
1.3 \\
32.3 \\
1,484.1\end{array}$ & $\begin{array}{r}2,296.8 \\
2,296.8 \\
- \\
2,296.8 \\
7.8 \\
367.7 \\
193.0 \\
1.6 \\
33.5 \\
1,693.2\end{array}$ & $\begin{array}{r}2,289.1 \\
2,289.1 \\
- \\
2,289.1 \\
6.8 \\
372.6 \\
183.3 \\
2.6 \\
34.5 \\
1,689.4\end{array}$ \\
\hline 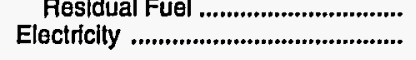 & .2 & $\begin{array}{r}1.2 \\
-\end{array}$ & - & $\begin{array}{r}3.5 \\
-\end{array}$ & - & - & - & - & - & - \\
\hline $\begin{array}{l}\text { Total Energy .................................... } \\
\text { Primary Energy - Four Sectors ..... } \\
\text { Electricity ......................................... }\end{array}$ & $\begin{array}{l}861.7 \\
639.4 \\
222.3\end{array}$ & $\begin{array}{r}1,831.8 \\
1,405.8 \\
426.0\end{array}$ & $\begin{array}{r}4,179.0 \\
3,260.9 \\
918.2\end{array}$ & $\begin{array}{l}5,145.4 \\
3,537.1 \\
1,608.3\end{array}$ & $\begin{array}{l}4,448.9 \\
2,827.0 \\
1,621.9\end{array}$ & $\begin{array}{l}4,556.4 \\
2,933.6 \\
1,622.8\end{array}$ & $\begin{array}{l}4,610.2 \\
2,893.3 \\
1,716.9\end{array}$ & $\begin{array}{l}4,854.3 \\
3,079.1 \\
1,775.2\end{array}$ & $\begin{array}{l}5,210.6 \\
3,410.2 \\
1,800.4\end{array}$ & $\begin{array}{l}5,415.2 \\
3,556.8 \\
1,858.4\end{array}$ \\
\hline 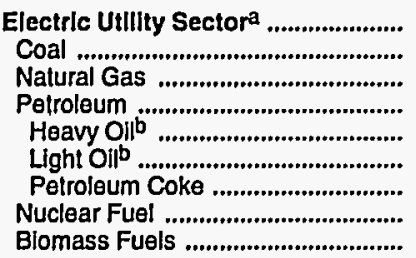 & $\begin{array}{r}30.6 \\
18.0 \\
12.0 \\
.6 \\
.6 \\
.1 \\
- \\
- \\
-\end{array}$ & $\begin{array}{r}105.4 \\
54.5 \\
30.9 \\
20.0 \\
10.8 \\
9.2 \\
- \\
- \\
-\end{array}$ & $\begin{array}{r}272.5 \\
173.3 \\
82.7 \\
15.1 \\
4.7 \\
10.3 \\
-\overline{5} \\
-\end{array}$ & $\begin{array}{r}342.6 \\
321.3 \\
17.2 \\
4.1 \\
.2 \\
3.9 \\
- \\
-\end{array}$ & $\begin{array}{r}340.8 \\
324.9 \\
13.9 \\
1.9 \\
\overline{1.9} \\
\overline{2}\end{array}$ & $\begin{array}{r}332.3 \\
312.0 \\
17.7 \\
2.0 \\
2.0 \\
\overline{.5}\end{array}$ & $\begin{array}{r}337.3 \\
314.3 \\
19.1 \\
1.8 \\
.5 \\
1.3 \\
-\overline{1} \\
2.1\end{array}$ & $\begin{array}{r}350.7 \\
328.7 \\
18.7 \\
1.7 \\
1.7 \\
1 . \overline{6}\end{array}$ & $\begin{array}{r}344.7 \\
331.4 \\
11.8 \\
1.6 \\
1.6 \\
= \\
=\end{array}$ & $\begin{array}{r}345.8 \\
331.4 \\
12.2 \\
2.2 \\
1.1 \\
1.1 \\
- \\
- \\
-\end{array}$ \\
\hline Prlmary Energy - Five Sectors ${ }^{c}$.. & 670.0 & $1,511.2$ & $3,533.4$ & $3,879.8$ & $3,167.8$ & $3,265.9$ & $3,230.6$ & $3,429.8$ & $3,755.0$ & $3,902.5$ \\
\hline
\end{tabular}

a There are no direct fuel costs for hydroelectric, geothermal, centralized solar, or wind energy.

b Heavy oll includes Grade Nos. 4, 5, and 6, and residual fuel oils. Light oil includes Grade No. 2 heating oil, kerosene, and jet fuel.

c Biomass fuels are not included, except those consumed at electric utilities and those added to motor gasoline.

-No consumption, including cases where adjustments were made. See "Consumption Adjustments for Process Fuel and Intermediate Products."

Value less than 0.05 million dollars.

Note: Tolals may not equal sum of components due to independent rounding.

Sources: Data sources, estimation procedures, and assumptions are described in the "Documentation" section of this report. 


\begin{tabular}{|c|c|c|c|c|c|c|c|c|c|c|}
\hline Energy Source & 1970 & 1975 & 1980 & 1985 & 1986 & 1987 & 1988 & 1989 & 1990 & 1991 \\
\hline & \multicolumn{10}{|c|}{ Prices In Dollars per Million Btu } \\
\hline 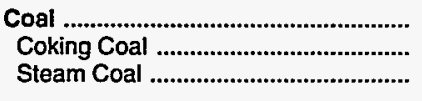 & $\frac{0.48}{-48}$ & $\begin{array}{r}2.09 \\
-\overline{-} \\
2.09\end{array}$ & $\begin{array}{c}3.35 \\
- \\
3.35\end{array}$ & $\begin{array}{r}2.40 \\
2.40\end{array}$ & $\frac{2.32}{-}$ & $\frac{2.45}{2.45}$ & $\begin{array}{r}2.31 \\
2.31\end{array}$ & $\frac{2.16}{2.16}$ & $\begin{array}{r}2.15 \\
2.15\end{array}$ & $\begin{array}{r}2.19 \\
2.19\end{array}$ \\
\hline 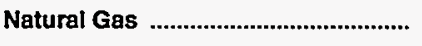 & 1.57 & 2.86 & 4.97 & 7.20 & 6.86 & 5.94 & 6.04 & 6.35 & 6.40 & 6.49 \\
\hline 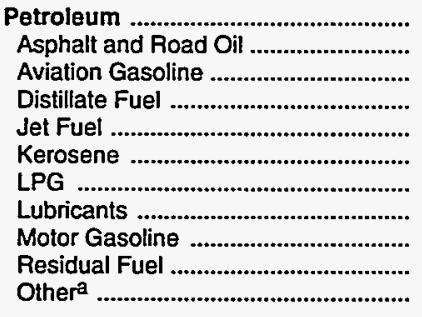 & $\begin{array}{r}1.37 \\
.68 \\
2.17 \\
1.29 \\
.75 \\
1.40 \\
1.98 \\
5.08 \\
2.96 \\
.40 \\
.91\end{array}$ & $\begin{array}{l}3.06 \\
1.91 \\
3.45 \\
2.73 \\
2.11 \\
2.91 \\
3.63 \\
7.48 \\
4.61 \\
2.04 \\
2.97\end{array}$ & $\begin{array}{r}7.09 \\
3.67 \\
9.02 \\
6.82 \\
6.50 \\
7.17 \\
6.85 \\
14.36 \\
10.10 \\
4.66 \\
7.68\end{array}$ & $\begin{array}{r}7.45 \\
5.20 \\
9.99 \\
8.25 \\
6.29 \\
8.79 \\
10.95 \\
17.61 \\
9.37 \\
4.32 \\
7.94\end{array}$ & $\begin{array}{r}5.67 \\
4.86 \\
8.41 \\
6.61 \\
4.50 \\
6.87 \\
10.66 \\
15.59 \\
7.45 \\
2.56 \\
5.19\end{array}$ & $\begin{array}{r}5.99 \\
3.58 \\
7.55 \\
6.28 \\
4.34 \\
6.55 \\
10.02 \\
12.70 \\
7.93 \\
2.97 \\
6.05\end{array}$ & $\begin{array}{r}6.13 \\
3.39 \\
7.41 \\
6.49 \\
4.00 \\
6.33 \\
10.79 \\
14.61 \\
8.91 \\
2.42 \\
5.08\end{array}$ & $\begin{array}{r}6.61 \\
3.20 \\
8.28 \\
7.19 \\
4.67 \\
6.98 \\
11.12 \\
13.30 \\
9.30 \\
2.88 \\
5.91\end{array}$ & $\begin{array}{r}7.61 \\
3.38 \\
9.32 \\
8.50 \\
5.91 \\
8.61 \\
12.31 \\
13.40 \\
10.06 \\
3.04 \\
6.68\end{array}$ & $\begin{array}{r}7.49 \\
3.05 \\
8.71 \\
8.12 \\
5.10 \\
7.87 \\
13.87 \\
15.42 \\
10.04 \\
2.49 \\
5.96\end{array}$ \\
\hline 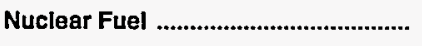 & .13 & .29 & .38 & .91 & .91 & .92 & .86 & .89 & .84 & .80 \\
\hline Blomass Fuels at Utilities .................. & - & - & - & - & - & - & .87 & $(d)$ & $\left({ }^{d}\right)$ & $(d)$ \\
\hline Primary Energy - Five Sectors ${ }^{b}$.... & 1.26 & 2.68 & 5.65 & 5.95 & 4.46 & 4.47 & 4.51 & 4.99 & 5.37 & 5.82 \\
\hline $\begin{array}{l}\text { Electric Utility Fuelc .......................... } \\
\text { Electricity Purchased by End Users }\end{array}$ & $\begin{array}{r}.35 \\
6.27\end{array}$ & $\begin{array}{r}1.35 \\
13.15\end{array}$ & $\begin{array}{r}2.60 \\
19.10\end{array}$ & $\begin{array}{r}2.38 \\
26.62\end{array}$ & $\begin{array}{r}1.54 \\
24.65\end{array}$ & $\begin{array}{r}1.61 \\
24.27\end{array}$ & $\begin{array}{r}1.42 \\
24.55\end{array}$ & $\begin{array}{r}1.64 \\
25.68\end{array}$ & $\begin{array}{r}1.53 \\
26.83\end{array}$ & $\begin{array}{r}1.50 \\
28.13\end{array}$ \\
\hline \multirow[t]{2}{*}{ 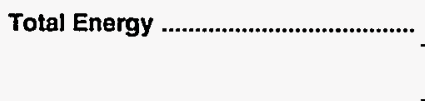 } & 2.09 & 4.53 & 9.07 & 11.20 & 9.66 & 9.61 & 9.97 & 10.49 & 11.57 & 11.73 \\
\hline & \multicolumn{10}{|c|}{ Expenditures In Millions of Dollars } \\
\hline 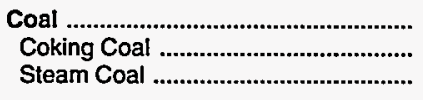 & $\begin{array}{r}23.5 \\
23 . \overline{5}\end{array}$ & $\begin{array}{r}2.7 \\
-\overline{7}\end{array}$ & $\begin{array}{r}1.2 \\
1.2\end{array}$ & $\begin{array}{r}51.1 \\
51 . \overline{1}\end{array}$ & $\begin{array}{r}49.1 \\
-\overline{1}\end{array}$ & $\begin{array}{r}52.4 \\
52.4\end{array}$ & $\begin{array}{r}53.3 \\
- \\
53.3\end{array}$ & $\begin{array}{r}51.1 \\
-\overline{1}\end{array}$ & $\begin{array}{r}55.1 \\
-\overline{1}\end{array}$ & $\begin{array}{r}49.5 \\
\overline{-} \\
49.5\end{array}$ \\
\hline 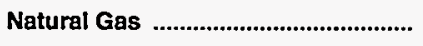 & 96.4 & 183.6 & 368.3 & 577.0 & 553.0 & 556.8 & 546.3 & 620.3 & 642.2 & 678.4 \\
\hline 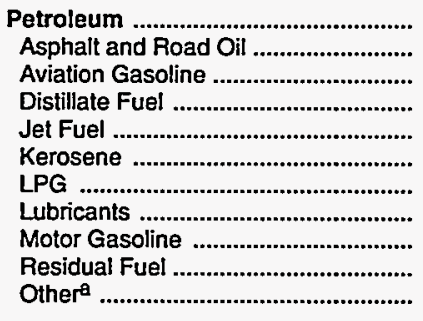 & $\begin{array}{r}802.3 \\
4.6 \\
1.4 \\
181.0 \\
12.3 \\
6.2 \\
13.9 \\
17.5 \\
445.2 \\
89.3 \\
31.0\end{array}$ & $\begin{array}{r}1,641.3 \\
16.0 \\
1.6 \\
343.5 \\
25.4 \\
9.7 \\
29.8 \\
18.0 \\
770.2 \\
417.5 \\
9.7\end{array}$ & $\begin{array}{r}3,612.9 \\
15.3 \\
4.1 \\
885.8 \\
72.5 \\
20.0 \\
37.8 \\
39.7 \\
1,602.8 \\
859.2 \\
75.8\end{array}$ & $\begin{array}{r}3,317.1 \\
72.2 \\
3.6 \\
909.1 \\
38.5 \\
35.5 \\
50.6 \\
44.3 \\
1,525.5 \\
571.4 \\
66.5\end{array}$ & $\begin{array}{r}2,641.8 \\
68.6 \\
3.1 \\
793.6 \\
31.9 \\
21.8 \\
44.0 \\
38.3 \\
1,247.2 \\
358.2 \\
35.2\end{array}$ & $\begin{array}{r}2,727.2 \\
50.9 \\
2.1 \\
775.0 \\
43.7 \\
21.5 \\
57.1 \\
35.3 \\
1,348.0 \\
353.4 \\
40.2\end{array}$ & $\begin{array}{r}2,992.9 \\
41.7 \\
1.8 \\
868.6 \\
48.8 \\
26.0 \\
59.8 \\
39.2 \\
1,539.8 \\
332.8 \\
34.4\end{array}$ & $\begin{array}{r}3,316.7 \\
38.2 \\
1.7 \\
1,073.0 \\
59.2 \\
26.6 \\
64.9 \\
36.5 \\
1,575.7 \\
401.7 \\
39.3\end{array}$ & $\begin{array}{r}3,255.3 \\
35.6 \\
4.4 \\
1,009.5 \\
78.4 \\
15.4 \\
71.1 \\
37.9 \\
1,636.1 \\
317.3 \\
49.6\end{array}$ & $\begin{array}{r}3,126.3 \\
40.0 \\
1.3 \\
938.1 \\
62.9 \\
16.9 \\
75.2 \\
39.0 \\
1,680.7 \\
227.4 \\
44.8\end{array}$ \\
\hline 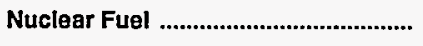 & 5.3 & 26.4 & 49.1 & 125.5 & 182.7 & 203.0 & 205.2 & 186.1 & 177.5 & 105.5 \\
\hline Blomess Fuels at Utilitles .................. & - & - & - & - & - & - & 2.2 & $(d)$ & (d) & (d) \\
\hline Primary Energy - Five Sectors ${ }^{b}$.... & 927.5 & $1,853.8$ & $4,031.6$ & $4,070.8$ & $3,426.7$ & $3,539.5$ & $3,799.9$ & $4,174.1$ & $4,130.1$ & $3,959.7$ \\
\hline $\begin{array}{l}\text { Electric Utility Fuelc } \\
\text { Electricity Purchased by End Users }\end{array}$ & $\begin{array}{l}-76.1 \\
345.0\end{array}$ & $\begin{array}{r}-311.5 \\
829.8\end{array}$ & $\begin{array}{r}-688.1 \\
1,381.4\end{array}$ & $\begin{array}{r}-634.9 \\
2,132.6\end{array}$ & $\begin{array}{r}-521.1 \\
2,062.9\end{array}$ & $\begin{array}{r}-561.8 \\
2,130.0\end{array}$ & $\begin{array}{r}-541.4 \\
2,255.1\end{array}$ & $\begin{array}{r}-588.1 \\
2,398.0\end{array}$ & $\begin{array}{r}-512.4 \\
2,489.0\end{array}$ & $\begin{array}{r}-367.2 \\
2,608.0\end{array}$ \\
\hline 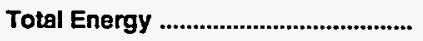 & $1,196.4$ & $2,372.1$ & $4,724.8$ & $5,568.5$ & $4,968.5$ & $5,107.7$ & $5,513.6$ & $5,984.1$ & $6,106.7$ & $6,200.5$ \\
\hline
\end{tabular}

a Includes petroleum coke used at electric utilities.

b Biomass fuels are not included, except those consumed at electric utilities and those added to motor gasoline.

c There are no direct fuel costs for hydroelectric, geothermal, centralized solar, or wind energy.

d Utilities used biomass fuels at no charge or received a fee for accepting them.

-No consumption, including cases where adjustments were made. See "Consumption Adjustments for Process Fuel and Intermediate Products."

Note: Expenditure totals may not equal sum of components due to independent rounding.

Sources: Data sources, estimation procedures, and assumptions are described in the "Documentation" section of this report. 


\begin{tabular}{|c|c|c|c|c|c|c|c|c|c|c|}
\hline Sector and Energy Source & 1970 & 1975 & 1980 & 1985 & 1986 & 1987 & 1988 & 1989 & 1990 & 1991 \\
\hline 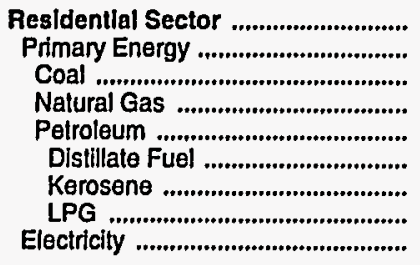 & $\begin{array}{l}2.47 \\
1.62 \\
1.30 \\
1.88 \\
1.53 \\
1.48 \\
1.70 \\
2.70 \\
7.21\end{array}$ & $\begin{array}{r}5.14 \\
3.03 \\
2.62 \\
3.28 \\
2.93 \\
2.84 \\
3.16 \\
5.01 \\
14.49\end{array}$ & $\begin{array}{r}9.39 \\
6.73 \\
4.47 \\
5.72 \\
7.14 \\
7.07 \\
8.15 \\
9.21 \\
20.27\end{array}$ & $\begin{array}{r}13.42 \\
8.60 \\
4.39 \\
8.88 \\
8.48 \\
8.37 \\
8.98 \\
10.41 \\
29.24\end{array}$ & $\begin{array}{r}11.89 \\
7.52 \\
4.19 \\
8.57 \\
7.02 \\
6.90 \\
7.40 \\
10.52 \\
27.20\end{array}$ & $\begin{array}{r}11.59 \\
7.04 \\
3.85 \\
7.96 \\
6.59 \\
6.46 \\
6.94 \\
9.13 \\
26.82\end{array}$ & $\begin{array}{r}11.51 \\
7.05 \\
4.24 \\
7.63 \\
6.77 \\
6.61 \\
7.10 \\
10.96 \\
27.03\end{array}$ & $\begin{array}{r}12.03 \\
7.61 \\
4.21 \\
7.98 \\
7.44 \\
7.23 \\
7.76 \\
12.74 \\
28.09\end{array}$ & $\begin{array}{r}13.66 \\
8.61 \\
4.37 \\
8.31 \\
8.78 \\
8.55 \\
9.18 \\
13.60 \\
29.32\end{array}$ & $\begin{array}{r}14.06 \\
8.55 \\
4.08 \\
8.48 \\
8.61 \\
8.27 \\
8.88 \\
14.99 \\
30.79\end{array}$ \\
\hline 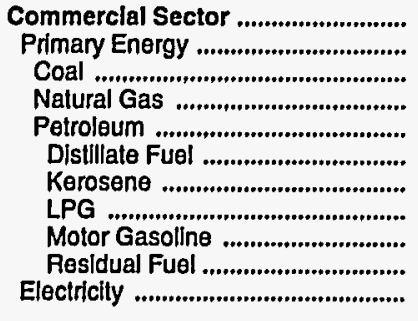 & $\begin{array}{r}2.60 \\
1.13 \\
.79 \\
1.45 \\
1.00 \\
1.09 \\
.79 \\
1.42 \\
2.96 \\
.42 \\
7.15\end{array}$ & $\begin{array}{r}5.93 \\
2.53 \\
2.00 \\
2.64 \\
2.47 \\
2.44 \\
2.67 \\
2.89 \\
4.61 \\
1.97 \\
13.70\end{array}$ & $\begin{array}{r}10.31 \\
5.43 \\
1.67 \\
4.67 \\
6.06 \\
6.37 \\
6.29 \\
5.31 \\
10.10 \\
4.59 \\
19.84\end{array}$ & $\begin{array}{r}13.55 \\
6.54 \\
2.38 \\
6.59 \\
6.54 \\
7.27 \\
7.70 \\
11.48 \\
9.37 \\
4.68 \\
27.30\end{array}$ & $\begin{array}{r}12.33 \\
5.27 \\
2.13 \\
6.24 \\
4.53 \\
5.15 \\
5.24 \\
10.80 \\
7.45 \\
2.79 \\
25.26\end{array}$ & $\begin{array}{r}12.24 \\
5.06 \\
2.73 \\
5.59 \\
4.57 \\
4.83 \\
5.64 \\
10.95 \\
7.93 \\
3.12 \\
24.53\end{array}$ & $\begin{array}{r}12.52 \\
4.87 \\
2.56 \\
5.45 \\
4.30 \\
4.72 \\
5.38 \\
10.63 \\
8.91 \\
2.57 \\
24.74\end{array}$ & $\begin{array}{r}12.91 \\
5.49 \\
2.61 \\
5.88 \\
5.11 \\
5.74 \\
6.40 \\
9.28 \\
9.30 \\
3.04 \\
25.99\end{array}$ & $\begin{array}{r}14.39 \\
6.12 \\
2.63 \\
6.10 \\
6.16 \\
6.87 \\
7.68 \\
10.80 \\
10.06 \\
3.25 \\
27.09\end{array}$ & $\begin{array}{r}15.49 \\
6.64 \\
2.66 \\
6.69 \\
6.60 \\
6.46 \\
7.02 \\
11.94 \\
10.04 \\
2.69 \\
28.21\end{array}$ \\
\hline 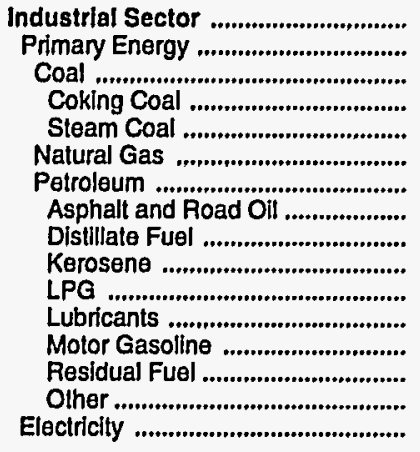 & $\begin{array}{r}1.06 \\
.72 \\
.79 \\
- \\
.79 \\
1.03 \\
.69 \\
.68 \\
.73 \\
.79 \\
1.42 \\
5.08 \\
2.96 \\
.43 \\
.91 \\
4.27\end{array}$ & $\begin{array}{r}3.45 \\
2.29 \\
2.00 \\
- \\
2.00 \\
2.24 \\
2.30 \\
1.91 \\
2.41 \\
2.67 \\
2.89 \\
7.48 \\
4.61 \\
2.12 \\
2.97 \\
10.51\end{array}$ & $\begin{array}{r}7.04 \\
5.13 \\
- \\
- \\
- \\
4.08 \\
5.40 \\
3.67 \\
5.75 \\
6.29 \\
5.31 \\
14.36 \\
10.10 \\
4.55 \\
7.68 \\
16.60\end{array}$ & $\begin{array}{r}9.88 \\
6.10 \\
2.38 \\
- \\
2.38 \\
5.38 \\
6.41 \\
5.20 \\
6.72 \\
7.70 \\
11.48 \\
17.61 \\
9.37 \\
4.68 \\
7.94 \\
21.93\end{array}$ & $\begin{array}{r}8.46 \\
4.69 \\
2.13 \\
- \\
2.13 \\
4.53 \\
4.76 \\
4.86 \\
4.57 \\
5.24 \\
10.80 \\
15.59 \\
7.45 \\
2.79 \\
5.19 \\
20.00\end{array}$ & $\begin{array}{r}8.21 \\
4.57 \\
2.73 \\
- \\
2.73 \\
4.08 \\
4.78 \\
3.58 \\
4.92 \\
5.64 \\
10.95 \\
12.70 \\
7.93 \\
3.12 \\
6.05 \\
19.93\end{array}$ & $\begin{array}{r}8.18 \\
4.31 \\
2.56 \\
-\overline{2} \\
2.56 \\
3.92 \\
4.50 \\
3.39 \\
4.70 \\
5.38 \\
10.63 \\
14.61 \\
8.91 \\
2.57 \\
5.08 \\
20.17\end{array}$ & $\begin{array}{r}8.81 \\
4.74 \\
2.61 \\
- \\
2.61 \\
4.36 \\
4.91 \\
3.20 \\
5.59 \\
6.40 \\
9.28 \\
13.30 \\
9.30 \\
3.04 \\
5.91 \\
21.11\end{array}$ & $\begin{array}{r}9.32 \\
5.17 \\
2.63 \\
- \\
2.63 \\
4.65 \\
5.53 \\
3.38 \\
6.70 \\
7.68 \\
10.80 \\
13.40 \\
10.06 \\
3.25 \\
6.68 \\
22.15\end{array}$ & $\begin{array}{r}8.89 \\
4.88 \\
2.66 \\
- \\
2.66 \\
4.69 \\
5.04 \\
3.05 \\
6.13 \\
7.02 \\
11.94 \\
15.42 \\
10.04 \\
2.69 \\
5.96 \\
23.21\end{array}$ \\
\hline 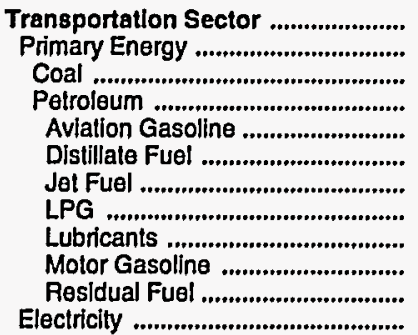 & $\begin{array}{r}2.63 \\
2.63 \\
.79 \\
2.63 \\
2.17 \\
1.39 \\
.75 \\
1.42 \\
5.08 \\
2.96 \\
.38 \\
-\end{array}$ & $\begin{array}{r}4.30 \\
4.30 \\
2.00 \\
4.30 \\
3.45 \\
2.90 \\
2.09 \\
2.89 \\
7.48 \\
4.61 \\
1.72 \\
-\end{array}$ & $\begin{array}{r}9.69 \\
9.69 \\
- \\
9.69 \\
9.02 \\
7.40 \\
6.51 \\
5.31 \\
14.36 \\
10.10 \\
3.88 \\
-\end{array}$ & $\begin{array}{r}9.28 \\
9.28 \\
- \\
9.28 \\
9.99 \\
9.19 \\
6.29 \\
11.48 \\
17.61 \\
9.37 \\
4.06 \\
-\end{array}$ & $\begin{array}{r}7.42 \\
7.42 \\
- \\
7.42 \\
8.41 \\
7.60 \\
4.50 \\
10.80 \\
15.59 \\
7.45 \\
2.32 \\
-\end{array}$ & $\begin{array}{r}7.70 \\
7.70 \\
- \\
7.70 \\
7.55 \\
7.35 \\
4.34 \\
10.95 \\
12.70 \\
7.93 \\
2.73 \\
-\end{array}$ & $\begin{array}{r}8.49 \\
8.49 \\
-\overline{8} \\
8.49 \\
7.41 \\
7.78 \\
4.00 \\
10.63 \\
14.61 \\
8.91 \\
2.20 \\
-\end{array}$ & $\begin{array}{r}8.88 \\
8.88 \\
-\overrightarrow{ } \\
8.88 \\
8.28 \\
8.33 \\
4.67 \\
9.28 \\
13.30 \\
9.30 \\
2.63 \\
-\end{array}$ & $\begin{array}{r}9.75 \\
9.75 \\
- \\
9.75 \\
9.32 \\
9.74 \\
5.91 \\
10.80 \\
13.40 \\
10.06 \\
2.74 \\
-\end{array}$ & $\begin{array}{r}9.68 \\
9.68 \\
- \\
9.68 \\
8.71 \\
9.42 \\
5.10 \\
11.94 \\
15.42 \\
10.04 \\
2.29 \\
-\end{array}$ \\
\hline $\begin{array}{l}\text { Total Energy ....................................... } \\
\text { Primary Energy - Four Sectors ...... } \\
\text { Electricity ........................................... }\end{array}$ & $\begin{array}{l}2.09 \\
1.64 \\
6.27\end{array}$ & $\begin{array}{r}4.53 \\
3.35 \\
13.15\end{array}$ & $\begin{array}{r}9.07 \\
7.45 \\
19.10\end{array}$ & $\begin{array}{r}11.20 \\
8.23 \\
26.62\end{array}$ & $\begin{array}{r}9.66 \\
6.75 \\
24.65\end{array}$ & $\begin{array}{r}9.61 \\
6.71 \\
24.27\end{array}$ & $\begin{array}{r}9.97 \\
7.06 \\
24.55\end{array}$ & $\begin{array}{r}10.49 \\
7.52 \\
25.68\end{array}$ & $\begin{array}{r}11.57 \\
8.32 \\
26.83\end{array}$ & $\begin{array}{r}11.73 \\
8.25 \\
28.13\end{array}$ \\
\hline 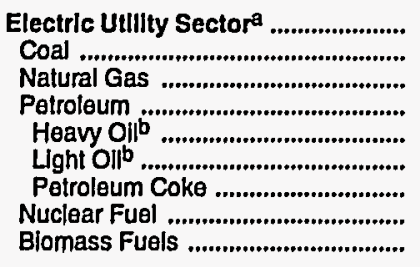 & $\begin{array}{l}.35 \\
.45 \\
.34 \\
.38 \\
.38 \\
.37 \\
-13 \\
-\end{array}$ & $\begin{array}{r}1.35 \\
1.24 \\
1.36 \\
2.02 \\
2.02 \\
2.36 \\
-29 \\
-\end{array}$ & $\begin{array}{r}2.60 \\
\overline{-} \\
4.71 \\
4.70 \\
6.13 \\
\overline{-38} \\
-\end{array}$ & $\begin{array}{r}2.38 \\
2.35 \\
3.39 \\
4.25 \\
4.24 \\
5.88 \\
- \\
.91 \\
-\end{array}$ & $\begin{array}{r}1.54 \\
2.27 \\
2.09 \\
2.51 \\
2.51 \\
3.59 \\
-\overline{.} \\
-\end{array}$ & $\begin{array}{r}1.61 \\
2.42 \\
2.37 \\
2.94 \\
2.93 \\
4.01 \\
- \\
.92 \\
-\end{array}$ & $\begin{array}{r}1.42 \\
2.29 \\
2.17 \\
2.41 \\
2.40 \\
3.63 \\
- \\
.86 \\
.87\end{array}$ & $\begin{array}{r}1.64 \\
2.14 \\
2.51 \\
2.87 \\
2.85 \\
4.26 \\
-\overline{89} \\
.80\end{array}$ & $\begin{array}{r}1.53 \\
2.13 \\
2.70 \\
3.02 \\
3.01 \\
5.67 \\
- \\
.84 \\
\left({ }^{d}\right)\end{array}$ & $\begin{array}{r}1.50 \\
2.17 \\
2.09 \\
2.49 \\
2.47 \\
4.92 \\
- \\
.80 \\
\left({ }^{d}\right)\end{array}$ \\
\hline Primary Energy - Five Sectors ${ }^{c}$.. & 1.26 & 2.68 & 5.65 & 5.95 & 4.46 & 4.47 & 4.51 & 4.99 & 5.37 & 5.82 \\
\hline
\end{tabular}

There are no direct fuel costs for hydroelectric, geothermal, centralized solar, or wind energy.

beavy oil includes Grade Nos. 4, 5, and 6, and residual fuel oils. Light oil includes Grade No. 2 heating oil, kerosene, and jet fuel.

c Blomass fuels are not included, except those consumed at electric utilities and those added to motor gasoline.

d Utilities used biomass fuels at no charge or received a fee for accepting them.

-No consumption, including cases where adjustments were made. See "Consumption Adjustments for Process Fuel and Intermediate Products."

Sources: Data sources, estimation procedures, and assumptions are described in the "Documentation" section of this report. 


\section{Energy Expenditure Estimates by Sector, Connecticut \\ O 1970, 1975, 1980, and 1985-1991}

N (Million Dollars)

\begin{tabular}{|c|c|c|c|c|c|c|c|c|c|c|}
\hline Sector and Energy Source & 1970 & 1975 & 1980 & 1985 & 1986 & 1987 & 1988 & 1989 & 1990 & 1991 \\
\hline $\begin{array}{l}\text { Pesidential Sector } \\
\text { Primary Energy } \\
\text { Coal } \\
\text { Natural Gas } \\
\text { Petroleum } \\
\text { Distillate Fuel } \\
\text { Kerosene } \\
\text { LPG } \\
\text { Electricity }\end{array}$ & $\begin{array}{r}353.6 \\
196.4 \\
.8 \\
59.6 \\
136.0 \\
122.7 \\
5.1 \\
8.2 \\
157.3\end{array}$ & \begin{tabular}{r|}
708.8 \\
340.6 \\
.8 \\
105.8 \\
234.0 \\
214.5 \\
5.2 \\
14.3 \\
368.2
\end{tabular} & $\begin{array}{r}1,342.0 \\
773.7 \\
1.0 \\
187.4 \\
585.3 \\
554.3 \\
10.8 \\
20.1 \\
568.4\end{array}$ & $\begin{array}{r}1,694.5 \\
832.6 \\
2.2 \\
299.8 \\
530.6 \\
475.8 \\
30.8 \\
24.0 \\
861.9\end{array}$ & $\begin{array}{r}1,659.2 \\
816.6 \\
2.2 \\
309.8 \\
504.5 \\
465.2 \\
17.7 \\
21.5 \\
842.6\end{array}$ & $\begin{array}{r}1,662.9 \\
778.0 \\
1.5 \\
296.7 \\
479.8 \\
437.3 \\
16.0 \\
26.5 \\
884.9\end{array}$ & $\begin{array}{r}1,813.3 \\
863.3 \\
.7 \\
310.7 \\
551.8 \\
505.9 \\
16.2 \\
29.7 \\
950.0\end{array}$ & $\begin{array}{r}1,993.1 \\
988.0 \\
.8 \\
335.7 \\
651.6 \\
599.6 \\
12.6 \\
39.4 \\
1,005.0\end{array}$ & $\begin{array}{r}1,981.6 \\
943.6 \\
.8 \\
321.3 \\
621.5 \\
569.1 \\
10.2 \\
42.2 \\
1,038.0\end{array}$ & $\begin{array}{r}2,024.2 \\
927.3 \\
.8 \\
325.0 \\
601.5 \\
541.2 \\
8.8 \\
51.5 \\
1,096.9\end{array}$ \\
\hline $\begin{array}{l}\text { Commercial Sector } \\
\text { Primary Energy } \\
\text { Coal } \\
\text { Natural Gas } \\
\text { Petroleum } \\
\text { Distillate Fuel } \\
\text { Kerosene } \\
\text { LPG } \\
\text { Molor Gasoline } \\
\text { Residual Fuel } \\
\text { Electricity }\end{array}$ & $\begin{array}{r}169.6 \\
56.1 \\
.3 \\
21.3 \\
34.4 \\
29.5 \\
.1 \\
.8 \\
1.5 \\
2.6 \\
113.5\end{array}$ & $\begin{array}{r}398.3 \\
117.9 \\
.4 \\
42.3 \\
75.2 \\
59.7 \\
.2 \\
1.5 \\
5.8 \\
8.1 \\
280.4\end{array}$ & $\begin{array}{r}731.2 \\
254.8 \\
.2 \\
96.1 \\
158.4 \\
107.8 \\
.2 \\
.2 \\
2.0 \\
14.6 \\
33.8 \\
476.4\end{array}$ & $\begin{array}{r}1,195.1 \\
381.8 \\
.8 \\
166.9 \\
214.1 \\
150.2 \\
2.8 \\
4.7 \\
7.0 \\
49.4 \\
813.3\end{array}$ & $\begin{array}{r}1,104.1 \\
305.3 \\
.8 \\
158.9 \\
145.6 \\
105.8 \\
2.0 \\
3.9 \\
5.7 \\
28.2 \\
798.8\end{array}$ & $\begin{array}{r}1,109.9 \\
289.8 \\
.7 \\
158.9 \\
130.2 \\
88.2 \\
3.6 \\
5.6 \\
7.2 \\
25.6 \\
820.1\end{array}$ & $\begin{array}{r}1,145.4 \\
274.4 \\
.3 \\
154.0 \\
120.0 \\
83.1 \\
2.0 \\
5.1 \\
7.7 \\
22.0 \\
871.0\end{array}$ & $\begin{array}{r}1,295.2 \\
351.2 \\
.3 \\
187.1 \\
163.8 \\
114.6 \\
5.3 \\
5.1 \\
9.3 \\
29.6 \\
944.0\end{array}$ & $\begin{array}{r}1,333.1 \\
343.1 \\
.3 \\
185.3 \\
157.5 \\
117.2 \\
2.2 \\
5.9 \\
10.7 \\
21.4 \\
990.0\end{array}$ & $\begin{array}{r}1,405.2 \\
355.2 \\
.3 \\
185.2 \\
169.6 \\
112.2 \\
6.7 \\
7.2 \\
34.6 \\
9.0 \\
1,050.0\end{array}$ \\
\hline 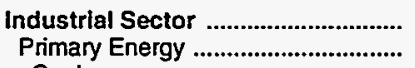 & $\begin{array}{l}193.4 \\
119.2\end{array}$ & $\begin{array}{l}420.1 \\
239.0\end{array}$ & $\begin{array}{l}857.8 \\
521.2\end{array}$ & $\begin{array}{l}861.9 \\
404.6\end{array}$ & $\begin{array}{l}723.9 \\
302.4\end{array}$ & $\begin{array}{l}739.1 \\
314.2\end{array}$ & $\begin{array}{l}722.2 \\
288.2\end{array}$ & $\begin{array}{l}753.4 \\
304.4\end{array}$ & $\begin{array}{l}793.0 \\
332.0\end{array}$ & $\begin{array}{l}806.1 \\
345.1\end{array}$ \\
\hline 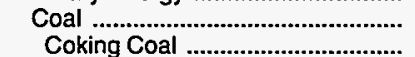 & 2.7 & 1.4 & - & .2 & .4 & .2 & 1.0 & 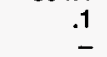 & .1 & .2 \\
\hline 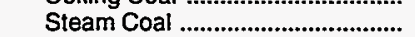 & 2.7 & 1.4 & - &.$\overline{2}$ &.$\overline{4}$ & $\overline{2}$ & $1 . \overline{0}$ &.$\overline{1}$ & $\overline{1}$ &.$\overline{2}$ \\
\hline 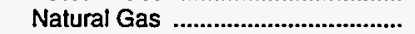 & 15.3 & 34.9 & 84.7 & 105.0 & 82.6 & 83.4 & 78.7 & 89.0 & 122.2 & 158.1 \\
\hline Petroleum …………………………... & 101.1 & 202.7 & 436.4 & 299.4 & 219.3 & 230.6 & 208.5 & 215.4 & 209.8 & 186.8 \\
\hline 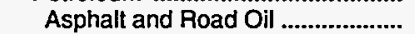 & 4.6 & 16.0 & 15.3 & 72.2 & 68.6 & 50.9 & 41.7 & 38.2 & 35.6 & 40.0 \\
\hline Distillate Fuel .................................... & 8.3 & 27.2 & 108.4 & 42.0 & 28.1 & 48.7 & 36.5 & 47.4 & 39.8 & 38.5 \\
\hline 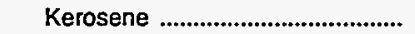 & 1.0 & 4.3 & 9.0 & 1.9 & 2.1 & 2.0 & 7.8 & 8.7 & 2.9 & 1.5 \\
\hline LPG & 4.8 & 13.8 & 15.3 & 20.7 & 17.7 & 24.1 & 23.8 & 19.3 & 21.5 & 14.8 \\
\hline Lubricants .................................. & 10.2 & 9.1 & 18.1 & 20.2 & 17.5 & 16.1 & 17.9 & 16.7 & 17.3 & 17.8 \\
\hline Motor Gasoline ............................. & 4.2 & .9 & 3.5 & 11.1 & 9.3 & 9.8 & 12.5 & 13.5 & 13.8 & 12.6 \\
\hline Residual Fuel ............................... & 37.0 & 121.7 & 191.1 & 64.8 & 40.8 & 38.9 & 33.9 & 32.4 & 29.3 & 16.8 \\
\hline Other & 31.0 & 9.7 & 75.8 & 66.5 & 35.2 & 40.2 & 34.4 & 39.3 & 49.6 & 44.8 \\
\hline Electricity ……………………...................... & 74.3 & 181.2 & 336.6 & 457.4 & 421.5 & 424.9 & 434.0 & 449.0 & 461.0 & 461.0 \\
\hline 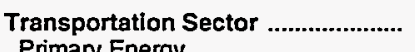 & 479.8 & 844.8 & $1,793.8$ & $1,817.0$ & $1,481.4$ & $1,595.7$ & $1,832.7$ & $1,942.3$ & $1,999.0$ & $1,964.9$ \\
\hline 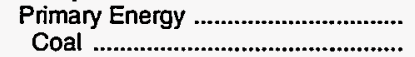 & 479.8 & 844.8 & $1,793.8$ & $\begin{array}{r}1,817.0 \\
-\end{array}$ & $1,481.4$ & $\begin{array}{r}1,595.7 \\
-\end{array}$ & $\begin{array}{r}1,832.7 \\
-\end{array}$ & $\begin{array}{r}1,942.3 \\
-\end{array}$ & $\begin{array}{r}1,999.0 \\
-\end{array}$ & $1,964.9$ \\
\hline Petroleum & 479.8 & 844.8 & $1,793.8$ & $1,817.0$ & $1,481.4$ & $1,595.7$ & $1,832.7$ & $1,942.3$ & $1,999.0$ & $1,964.9$ \\
\hline 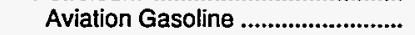 & 1.4 & 1.6 & 4.1 & 3.6 & 3.1 & 2.1 & 1.8 & 1.7 & 4.4 & 1.3 \\
\hline Distillate Fuel .............................. & 18.3 & 40.5 & 111.2 & 238.2 & 192.0 & 197.6 & 238.3 & 306.5 & 281.2 & 243.0 \\
\hline Jet Fuel ........................................ & 12.3 & 23.8 & 70.7 & 38.5 & 31.9 & 43.7 & 48.8 & 59.2 & 78.4 & 62.9 \\
\hline 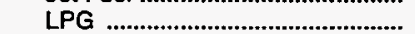 & .1 & .3 & .3 & 1.3 & .9 & .9 & 1.2 & 1.1 & 1.4 & 1.7 \\
\hline Lubricants ................................... & 7.3 & 8.9 & 21.5 & 24.0 & 20.8 & 19.2 & 21.3 & 19.9 & 20.6 & 21.2 \\
\hline 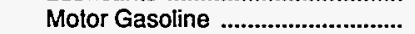 & 439.6 & 763.5 & $1,584.7$ & $1,507.4$ & $1,232.2$ & $1,331.0$ & $1,519.6$ & $1,552.8$ & $1,611.6$ & $1,633.5$ \\
\hline Residual Fuel ....................................... & .9 & 6.3 & 1.3 & 3.9 & .5 & 1.2 & 1.8 & 1.1 & 1.5 & 1.3 \\
\hline 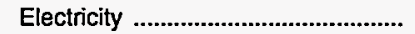 & - & - & - & - & - & - & - & - & - & - \\
\hline 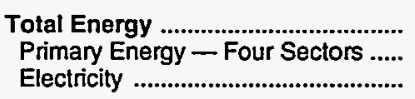 & $\begin{array}{r}1,196.4 \\
851.4 \\
345.0\end{array}$ & $\begin{array}{r}2,372.1 \\
1,542.3 \\
829.8\end{array}$ & $\begin{array}{l}4,724.8 \\
3,343.4 \\
1,381.4\end{array}$ & $\begin{array}{l}5,568.5 \\
3,435.9 \\
2,132.6\end{array}$ & $\begin{array}{l}4,968.5 \\
2,905.6 \\
2,062.9\end{array}$ & $\begin{array}{l}5,107.7 \\
2,977.7 \\
2,130.0\end{array}$ & $\begin{array}{l}5,513.6 \\
3,258.5 \\
2,255.1\end{array}$ & $\begin{array}{l}5,984.1 \\
3,586.0 \\
2,398.0\end{array}$ & $\begin{array}{l}6,106.7 \\
3,617.7 \\
2,489.0\end{array}$ & $\begin{array}{l}6,200.5 \\
3,592.5 \\
2,608.0\end{array}$ \\
\hline 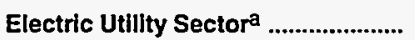 & 76.1 & 311.5 & 688.1 & 634.9 & 521.1 & 561.8 & 541.4 & 588.1 & 512.4 & 367.2 \\
\hline Coal & 19.7 & .1 & - & 47.8 & 45.7 & 50.0 & 51.3 & 49.9 & 54.0 & 48.2 \\
\hline 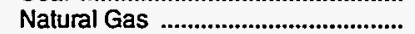 & .1 & .5 & - & 5.4 & 1.6 & 17.9 & 2.8 & 8.5 & 13.5 & 10.2 \\
\hline Petroleum & 51.0 & 284.6 & 639.0 & 456.1 & 291.1 & 290.9 & 280.0 & 343.6 & 267.4 & 203.4 \\
\hline 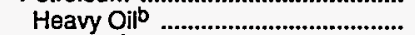 & 48.8 & 281.4 & 633.0 & 453.2 & 288.7 & 287.7 & 275.1 & 338.7 & 265.2 & 200.3 \\
\hline 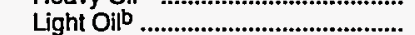 & 2.2 & 3.1 & 6.0 & 2.9 & 2.4 & 3.2 & 4.9 & 4.9 & 2.3 & 3.1 \\
\hline Petroleum Coke ..................................... & - & - & - & - & - & - & - & - & - & - \\
\hline Nuclear Fuel .................................. & 5.3 & 26.4 & 49.1 & 125.5 & 182.7 & 203.0 & 205.2 & 186.1 & 177.5 & 105.5 \\
\hline Primary Energy - Five Sectors $^{c}$... & 927.5 & $1,853.8$ & $4,031.6$ & $4,070.8$ & $3,426.7$ & $3,539.5$ & $3,799.9$ & $4,174.1$ & $4,130.1$ & $3,959.7$ \\
\hline
\end{tabular}

a There are no direct fuel costs for hydroelectric, geothermal, centralized solar, or wind energy.

b Heavy oil includes Grade Nos. 4, 5, and 6, and residual fuel oils. Light oil includes Grade No. 2 heating oil, kerosene, and jet fuel.

c Biomass fuels are not included, except those consumed at electric utilities and those added to motor gasoline.

d Utilities used biomass fuels at no charge or received a fee for accepting them.

-No consumption, including cases where adjustments were made. See "Consumption Adjustments for Process Fuel and Intermediate Products."

Value less than 0.05 million dollars.

Note: Totals may not equal sum of components due to independent rounding.

Sources: Data sources, estimation procedures, and assumptions are described in the "Documentation" section of this report. 
Energy Price and Expenditure Estimates by Source, Delaware $1970,1975,1980$, and 1985-1991

\begin{tabular}{|c|c|c|c|c|c|c|c|c|c|c|}
\hline Energy Source & 1970 & 1975 & 1980 & 1985 & 1986 & 1987 & 1988 & 1989 & 1990 & 1991 \\
\hline & \multicolumn{10}{|c|}{ Prices in Dollars per Million Btu } \\
\hline 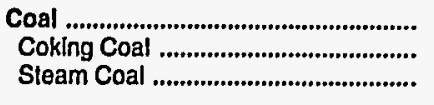 & $\begin{array}{r}0.39 \\
-39\end{array}$ & $\begin{array}{r}1.16 \\
1.16\end{array}$ & $\begin{array}{r}1.57 \\
- \\
1.57\end{array}$ & $\begin{array}{r}1.87 \\
-\overline{1} \\
1.87\end{array}$ & $\begin{array}{l}1.87 \\
1.87\end{array}$ & $\begin{array}{r}1.78 \\
1.78\end{array}$ & $\begin{array}{r}1.77 \\
1.77\end{array}$ & $\begin{array}{r}1.75 \\
- \\
1.75\end{array}$ & $\begin{array}{r}1.76 \\
-\overline{6}\end{array}$ & $\begin{array}{r}1.75 \\
- \\
1.75\end{array}$ \\
\hline 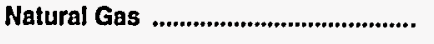 & .91 & 1.60 & 3.37 & 4.87 & 4.43 & 3.93 & 4.04 & 4.06 & 3.82 & 3.43 \\
\hline 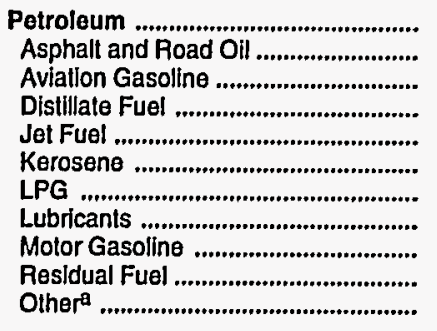 & $\begin{array}{r}1.27 \\
.66 \\
2.17 \\
1.16 \\
.73 \\
1.26 \\
1.25 \\
5.08 \\
2.86 \\
.45 \\
.35\end{array}$ & $\begin{array}{l}2.75 \\
1.76 \\
3.45 \\
2.53 \\
2.03 \\
3.14 \\
3.60 \\
7.48 \\
4.54 \\
1.92 \\
1.68\end{array}$ & $\begin{array}{r}6.00 \\
3.58 \\
9.02 \\
6.77 \\
6.46 \\
8.36 \\
5.20 \\
14.36 \\
9.60 \\
4.23 \\
5.84\end{array}$ & $\begin{array}{r}7.39 \\
4.97 \\
9.99 \\
7.58 \\
6.63 \\
8.65 \\
10.65 \\
17.61 \\
9.39 \\
4.16 \\
4.94\end{array}$ & $\begin{array}{r}5.22 \\
4.16 \\
8.41 \\
5.94 \\
4.87 \\
7.03 \\
10.76 \\
15.59 \\
6.97 \\
2.32 \\
2.88\end{array}$ & $\begin{array}{r}5.52 \\
3.17 \\
7.55 \\
5.70 \\
4.03 \\
6.50 \\
10.36 \\
12.70 \\
7.61 \\
2.73 \\
3.30\end{array}$ & $\begin{array}{r}5.34 \\
3.13 \\
7.41 \\
5.68 \\
4.16 \\
6.47 \\
10.14 \\
14.61 \\
8.07 \\
2.24 \\
2.92\end{array}$ & $\begin{array}{r}6.04 \\
2.90 \\
8.28 \\
6.33 \\
4.80 \\
7.30 \\
11.46 \\
13.30 \\
8.88 \\
2.68 \\
3.45\end{array}$ & $\begin{array}{r}6.91 \\
2.90 \\
9.32 \\
7.58 \\
6.33 \\
8.68 \\
12.13 \\
13.40 \\
10.26 \\
2.71 \\
2.73\end{array}$ & $\begin{array}{r}6.36 \\
3.06 \\
8.71 \\
7.08 \\
5.51 \\
8.11 \\
12.81 \\
15.42 \\
9.51 \\
2.25 \\
2.58\end{array}$ \\
\hline Nuclear Fuel ........................................... & - & - & - & - & - & - & - & - & - & - \\
\hline Blomass Fuols at Utilitles .................. & - & - & - & - & - & - & - & - & - & - \\
\hline Primary Energy - Five Sectors ${ }^{b} \ldots$ & 1.06 & 2.48 & 5.13 & 5.16 & 4.06 & 4.08 & 4.08 & 4.55 & 4.92 & 4.58 \\
\hline $\begin{array}{l}\text { Electric Utility Fuelc ........................... } \\
\text { Electricity Purchased by End Users }\end{array}$ & .39 & $\begin{array}{r}1.63 \\
11.69\end{array}$ & $\begin{array}{r}3.35 \\
18.84\end{array}$ & $\begin{array}{r}2.48 \\
21.42\end{array}$ & $\begin{array}{r}2.01 \\
19.09\end{array}$ & $\begin{array}{r}2.05 \\
18.79\end{array}$ & $\begin{array}{r}1.96 \\
19.54\end{array}$ & $\begin{array}{r}2.12 \\
18.47\end{array}$ & $\begin{array}{r}1.97 \\
19.00\end{array}$ & $\begin{array}{r}1.92 \\
19.70\end{array}$ \\
\hline \multirow[t]{2}{*}{ 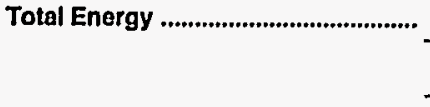 } & 1.69 & 3.89 & 7.28 & 9.16 & 7.64 & 7.47 & 7.88 & 8.32 & 9.09 & 8.98 \\
\hline & \multicolumn{10}{|c|}{ Expenditures in Millions of Dollars } \\
\hline 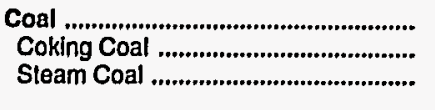 & $\begin{array}{r}14.5 \\
14 . \overline{5}\end{array}$ & $\begin{array}{r}26.6 \\
26.6\end{array}$ & $\begin{array}{r}44.1 \\
44.1\end{array}$ & $\begin{array}{r}133.3 \\
133 . \overline{3}\end{array}$ & $\begin{array}{r}124.1 \\
- \\
124.1\end{array}$ & $\begin{array}{r}125.1 \\
-\overline{1} \\
125.1\end{array}$ & $\begin{array}{r}121.8 \\
-\overline{8} \\
121.8\end{array}$ & $\begin{array}{r}106.4 \\
-\overline{4} \\
106.4\end{array}$ & $\begin{array}{r}104.9 \\
104.9\end{array}$ & $\begin{array}{r}99.1 \\
99.1\end{array}$ \\
\hline 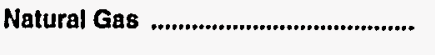 & 24.4 & 34.0 & 102.9 & 188.6 & 145.4 & 144.1 & 118.9 & 143.4 & 150.9 & 145.9 \\
\hline 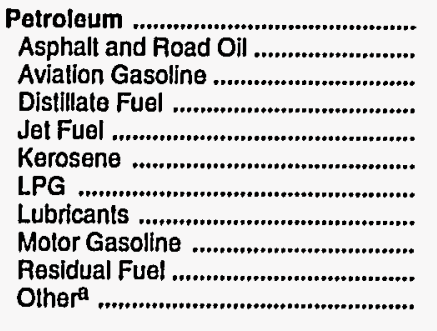 & $\begin{array}{r}173.0 \\
2.3 \\
.2 \\
29.1 \\
8.1 \\
3.1 \\
10.6 \\
3.3 \\
93.8 \\
18.6 \\
3.8\end{array}$ & $\begin{array}{r}435.9 \\
7.6 \\
.3 \\
62.2 \\
18.0 \\
4.9 \\
34.9 \\
3.7 \\
168.4 \\
123.3 \\
12.5\end{array}$ & $\begin{array}{r}1,059.8 \\
8.3 \\
.5 \\
146.5 \\
54.6 \\
14.3 \\
56.7 \\
12.1 \\
333.5 \\
335.5 \\
97.8\end{array}$ & $\begin{array}{r}818.0 \\
27.3 \\
.8 \\
150.8 \\
56.0 \\
34.6 \\
37.9 \\
13.5 \\
372.5 \\
92.7 \\
32.1\end{array}$ & $\begin{array}{r}602.0 \\
16.8 \\
.8 \\
114.3 \\
35.2 \\
13.5 \\
34.2 \\
11.7 \\
282.7 \\
72.6 \\
20.2\end{array}$ & $\begin{array}{r}647.7 \\
12.1 \\
.6 \\
126.9 \\
27.9 \\
13.6 \\
37.9 \\
10.8 \\
314.6 \\
79.3 \\
24.0\end{array}$ & $\begin{array}{r}683.8 \\
8.5 \\
.7 \\
127.3 \\
30.5 \\
12.5 \\
37.0 \\
11.9 \\
347.3 \\
87.1 \\
20.9\end{array}$ & $\begin{array}{r}760.5 \\
10.0 \\
.7 \\
155.3 \\
32.4 \\
11.8 \\
39.7 \\
11.1 \\
380.3 \\
94.4 \\
24.6\end{array}$ & $\begin{array}{r}798.3 \\
10.3 \\
3.6 \\
142.0 \\
44.4 \\
7.8 \\
44.9 \\
11.6 \\
429.3 \\
63.4 \\
41.0\end{array}$ & $\begin{array}{r}750.4 \\
2.9 \\
.8 \\
141.1 \\
37.6 \\
8.6 \\
57.5 \\
11.9 \\
389.2 \\
67.1 \\
33.7\end{array}$ \\
\hline 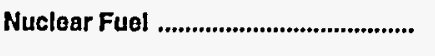 & - & - & - & - & - & - & - & - & - & - \\
\hline Blomass Fuels at Utilities ................. & - & - & - & - & - & - & - & - & - & - \\
\hline Primary Energy - Five Sectors ${ }^{b}$.... & 211.8 & 496.5 & $1,206.8$ & $1,139.9$ & 871.6 & 916.8 & 924.6 & $1,010.3$ & $1,054.2$ & 995.4 \\
\hline $\begin{array}{l}\text { Electric Utility Fuelc ........................... } \\
\text { Electricily Purchased by End Users }\end{array}$ & $\begin{array}{r}-23.1 \\
75.7\end{array}$ & $\begin{array}{r}-106.3 \\
202.1\end{array}$ & $\begin{array}{r}-239.3 \\
368.7\end{array}$ & $\begin{array}{r}-229.9 \\
457.9\end{array}$ & $\begin{array}{r}-179.4 \\
440.0\end{array}$ & $\begin{array}{r}-186.2 \\
446.3\end{array}$ & $\begin{array}{r}-185.6 \\
499.4\end{array}$ & $\begin{array}{r}-191.6 \\
504.3\end{array}$ & $\begin{array}{r}-171.0 \\
533.6\end{array}$ & $\begin{array}{r}-175.9 \\
567.9\end{array}$ \\
\hline Total Energy ........................................... & 264.4 & 592.2 & $1,336.2$ & $1,367.9$ & $1,132.2$ & $1,176.9$ & $1,238.4$ & $1,323.0$ & $1,416.7$ & $1,387.5$ \\
\hline
\end{tabular}

Includes petroleum coke used at electric utilities.

b Blomass fuels are not included, except those consumed at electric utilities and those added to motor gasoline.

c There are no direct fuel costs for hydroelectric, geothermal, centralized solar, or wind energy.

- No consumption, including cases where adjustments were made. See "Consumption Adjustments for Process Fuel and Intermediate Products."

Note: Expenditure totals may not equal sum of components due to independent rounding.

Sources; Data sources, estimation procedures, and assumptions are described in the "Documentation" section of this report. 


\section{Energy Price Estimates by Sector, Delaware \\ E $1970,1975,1980$, and 1985-1991}

L (Dollars per Million Btu)

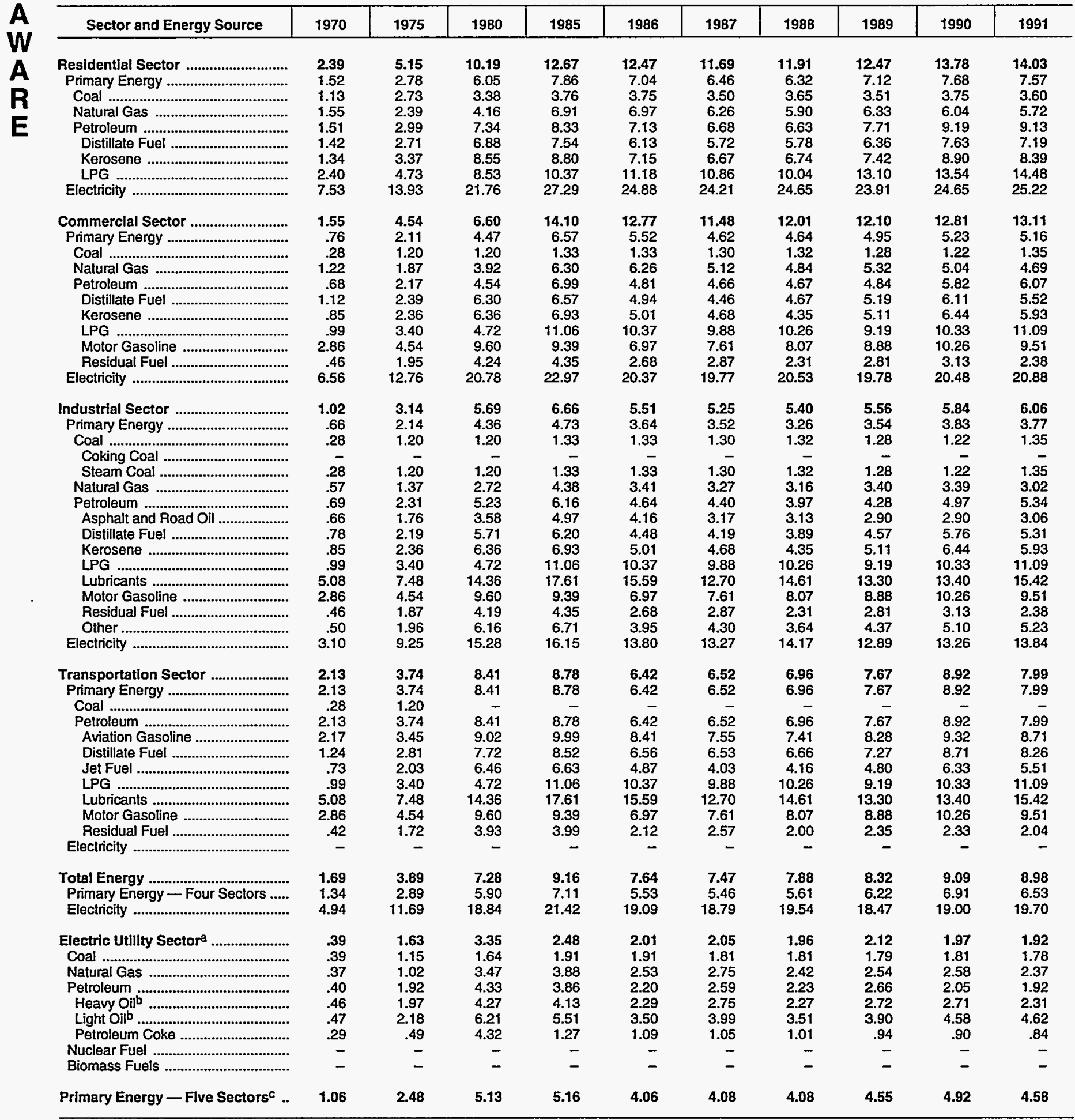

a There are no direct fuel costs for hydroelectric, geothermal, centralized solar, or wind energy.

Heavy oil includes Grade Nos. 4, 5, and 6, and residual fuel oils. Light oil includes Grade No. 2 heating oil, kerosene, and jet fuel.

c Biomass fuels are not included, except those consumed at electric utilities and those added to motor gasoline.

$\rightarrow$ No consumption, including cases where adjustments were made. See "Consumption Adjustments for Process Fuel and Intermediate Products."

Sources: Data sources, estimation procedures, and assumptions are described in the "Documentation" section of this report. 
Energy Expenditure Estimates by Sector, Delaware 1970, 1975, 1980, and 1985-1991

(Million Dollars)

\begin{tabular}{|c|c|c|c|c|c|c|c|c|c|c|}
\hline Sector and Energy Source & 1970 & 1975 & 1980 & 1985 & 1986 & 1987 & 1988 & 1989 & 1990 & 1991 \\
\hline 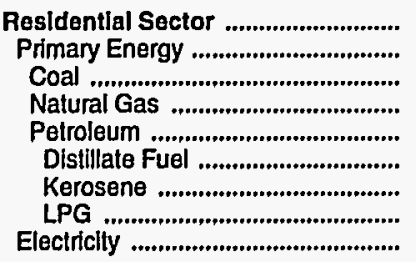 & $\begin{array}{r}65.9 \\
35.9 \\
.1 \\
12.4 \\
23.4 \\
16.8 \\
2.8 \\
3.8 \\
30.0\end{array}$ & $\begin{array}{r}135.5 \\
57.5 \\
.2 \\
16.9 \\
40.4 \\
29.4 \\
4.1 \\
6.9 \\
77.9\end{array}$ & $\begin{array}{r}246.2 \\
107.7 \\
.2 \\
29.7 \\
77.8 \\
52.7 \\
13.3 \\
11.7 \\
138.6\end{array}$ & $\begin{array}{r}336.2 \\
157.1 \\
.2 \\
43.9 \\
113.0 \\
58.5 \\
32.4 \\
22.1 \\
179.1\end{array}$ & $\begin{array}{r}296.5 \\
116.4 \\
.4 \\
48.6 \\
67.5 \\
37.7 \\
12.9 \\
16.8 \\
180.1\end{array}$ & $\begin{array}{r}315.3 \\
122.9 \\
1.3 \\
44.7 \\
76.9 \\
44.7 \\
12.8 \\
19.5 \\
192.4\end{array}$ & $\begin{array}{r}337.6 \\
124.6 \\
.6 \\
45.5 \\
78.4 \\
46.9 \\
11.6 \\
20.0 \\
213.0\end{array}$ & $\begin{array}{r}350.0 \\
136.0 \\
.6 \\
48.8 \\
86.6 \\
48.9 \\
11.3 \\
26.4 \\
214.0\end{array}$ & $\begin{array}{r}346.7 \\
123.7 \\
.7 \\
44.6 \\
78.4 \\
43.0 \\
7.3 \\
28.1 \\
223.0\end{array}$ & $\begin{array}{r}369.3 \\
126.2 \\
.6 \\
42.1 \\
83.5 \\
42.6 \\
7.9 \\
33.0 \\
243.0\end{array}$ \\
\hline 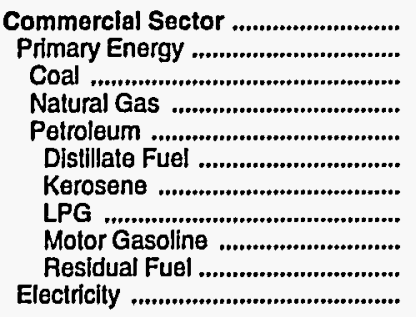 & \begin{tabular}{r|}
34.4 \\
14.5 \\
$\cdot$ \\
3.5 \\
11.0 \\
5.1 \\
.2 \\
.3 \\
.4 \\
5.0 \\
19.9
\end{tabular} & $\begin{array}{r}90.5 \\
32.5 \\
5.6 \\
26.8 \\
10.0 \\
.4 \\
.9 \\
.8 \\
14.7 \\
58.0\end{array}$ & $\begin{array}{r}261.3 \\
154.0 \\
.1 \\
13.1 \\
140.8 \\
23.3 \\
.3 \\
1.1 \\
2.3 \\
113.8 \\
107.3\end{array}$ & $\begin{array}{r}177.9 \\
44.9 \\
.1 \\
22.0 \\
22.8 \\
12.8 \\
2.0 \\
4.2 \\
1.9 \\
1.9 \\
133.0\end{array}$ & $\begin{array}{r}166.5 \\
36.9 \\
.2 \\
22.4 \\
14.3 \\
7.0 \\
.5 \\
2.8 \\
1.4 \\
2.6 \\
129.6\end{array}$ & $\begin{array}{r}171.7 \\
37.8 \\
.8 \\
19.3 \\
17.6 \\
9.4 \\
.4 \\
3.1 \\
1.7 \\
3.0 \\
133.9\end{array}$ & $\begin{array}{r}190.5 \\
39.5 \\
.4 \\
19.9 \\
19.2 \\
10.6 \\
.7 \\
3.6 \\
1.7 \\
2.6 \\
151.0\end{array}$ & $\begin{array}{r}195.4 \\
41.3 \\
.4 \\
22.6 \\
18.4 \\
9.0 \\
.2 \\
3.3 \\
1.8 \\
4.1 \\
154.0\end{array}$ & $\begin{array}{r}207.7 \\
42.7 \\
.4 \\
20.7 \\
21.6 \\
12.0 \\
.4 \\
3.8 \\
1.9 \\
3.6 \\
165.0\end{array}$ & $\begin{array}{r}218.4 \\
42.4 \\
.4 \\
20.5 \\
21.5 \\
14.1 \\
.4 \\
4.5 \\
1.7 \\
.8 \\
176.0\end{array}$ \\
\hline 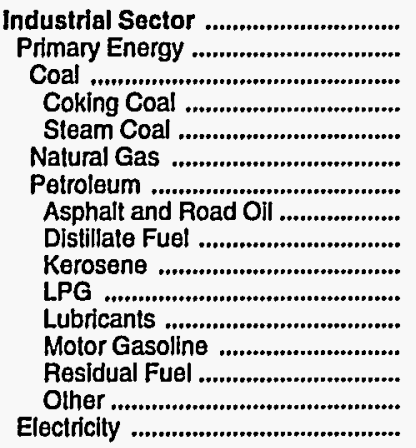 & $\begin{array}{r}57.1 \\
31.3 \\
.2 \\
\overline{.} \\
.2 \\
7.0 \\
24.1 \\
2.3 \\
3.6 \\
.1 \\
6.5 \\
1.3 \\
1.4 \\
7.3 \\
1.6 \\
25.7\end{array}$ & $\begin{array}{r}160.3 \\
94.2 \\
.8 \\
- \\
.8 \\
9.5 \\
83.9 \\
7.6 \\
12.7 \\
.4 \\
26.7 \\
1.4 \\
1.5 \\
21.7 \\
11.8 \\
66.1\end{array}$ & \begin{tabular}{r|}
375.0 \\
252.1 \\
5.4 \\
\\
5.4 \\
34.8 \\
212.0 \\
8.3 \\
20.5 \\
6 \\
43.6 \\
6.6 \\
1.8 \\
45.1 \\
85.6 \\
122.9
\end{tabular} & $\begin{array}{r}355.5 \\
209.8 \\
7.0 \\
-\overline{0} \\
7.0 \\
93.5 \\
109.3 \\
27.3 \\
15.0 \\
.2 \\
11.4 \\
7.3 \\
2.7 \\
16.1 \\
29.4 \\
145.7\end{array}$ & $\begin{array}{r}282.8 \\
152.5 \\
6.7 \\
-\overline{5} \\
6.7 \\
69.7 \\
76.1 \\
16.8 \\
10.8 \\
.1 \\
13.0 \\
6.3 \\
2.2 \\
9.6 \\
17.4 \\
130.3\end{array}$ & $\begin{array}{r}266.8 \\
146.8 \\
7.0 \\
-\overline{0} \\
7.0 \\
57.7 \\
82.0 \\
12.1 \\
10.2 \\
.4 \\
15.2 \\
5.8 \\
2.3 \\
14.3 \\
21.6 \\
119.9\end{array}$ & $\begin{array}{r}263.0 \\
127.6 \\
8.1 \\
-\overline{8} \\
8.1 \\
46.2 \\
73.4 \\
8.5 \\
10.1 \\
.3 \\
13.1 \\
6.5 \\
2.4 \\
13.6 \\
18.9 \\
135.4\end{array}$ & $\begin{array}{r}271.4 \\
135.0 \\
6.5 \\
- \\
6.5 \\
50.2 \\
78.2 \\
10.0 \\
12.0 \\
.3 \\
9.8 \\
6.0 \\
3.0 \\
14.3 \\
22.8 \\
136.3\end{array}$ & $\begin{array}{r}300.9 \\
155.4 \\
6.5 \\
- \\
6.5 \\
56.4 \\
92.5 \\
10.3 \\
14.5 \\
.2 \\
12.8 \\
6.3 \\
2.6 \\
12.6 \\
33.4 \\
145.5\end{array}$ & $\begin{array}{r}286.3 \\
137.4 \\
7.0 \\
- \\
7.0 \\
47.5 \\
82.9 \\
2.9 \\
13.6 \\
.3 \\
19.8 \\
6.5 \\
2.5 \\
10.3 \\
27.0 \\
148.9\end{array}$ \\
\hline 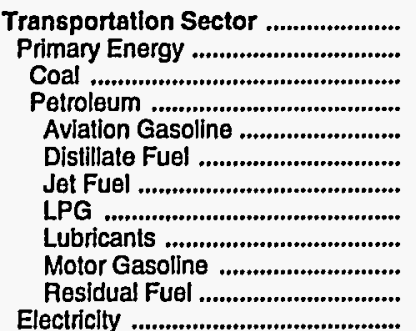 & $\begin{array}{r}107.0 \\
107.0 \\
107.0 \\
.2 \\
2.8 \\
8.1 \\
.1 \\
2.1 \\
92.1 \\
1.8 \\
-\end{array}$ & $\begin{array}{r}206.0 \\
206.0 \\
206.0 \\
.3 \\
8.4 \\
18.0 \\
.5 \\
2.3 \\
166.2 \\
10.4 \\
-\end{array}$ & $\begin{array}{r}453.7 \\
453.7 \\
- \\
453.7 \\
.5 \\
43.3 \\
54.6 \\
.2 \\
5.5 \\
329.4 \\
20.1 \\
\end{array}$ & $\begin{array}{r}498.3 \\
498.3 \\
\overline{3} \\
498.3 \\
.8 \\
61.3 \\
56.0 \\
.2 \\
6.2 \\
367.9 \\
5.8 \\
\end{array}$ & $\begin{array}{r}386.5 \\
386.5 \\
386.5 \\
.8 \\
56.5 \\
35.2 \\
1.7 \\
5.4 \\
279.0 \\
7.8 \\
\end{array}$ & $\begin{array}{r}423.1 \\
423.1 \\
-\overline{1} \\
423.1 \\
.6 \\
59.6 \\
27.9 \\
.1 \\
4.9 \\
310.5 \\
19.4 \\
-\end{array}$ & $\begin{array}{r}447.3 \\
447.3 \\
\overline{-} \\
447.3 \\
.7 \\
56.3 \\
30.5 \\
.2 \\
5.5 \\
343.2 \\
11.0 \\
-\end{array}$ & $\begin{array}{r}506.3 \\
506.3 \\
- \\
506.3 \\
.7 \\
79.1 \\
32.4 \\
.2 \\
5.1 \\
375.5 \\
13.1 \\
-\end{array}$ & $\begin{array}{r}561.4 \\
561.4 \\
- \\
561.4 \\
3.6 \\
69.6 \\
44.4 \\
.2 \\
5.3 \\
424.9 \\
13.4 \\
-\end{array}$ & $\begin{array}{r}513.6 \\
513.6 \\
- \\
513.6 \\
.8 \\
67.6 \\
37.6 \\
.3 \\
5.5 \\
385.0 \\
16.9 \\
-\end{array}$ \\
\hline $\begin{array}{l}\text { Total Energy ...................................... } \\
\text { Primary Energy - Four Sectors ...... } \\
\text { Electricity ......................................... }\end{array}$ & $\begin{array}{r}264.4 \\
188.8 \\
75.7\end{array}$ & $\begin{array}{l}592.2 \\
390.2 \\
202.1\end{array}$ & $\begin{array}{r}1,336.2 \\
967.5 \\
368.7\end{array}$ & $\begin{array}{r}1,367.9 \\
910.0 \\
457.9\end{array}$ & $\begin{array}{r}1,132.2 \\
692.2 \\
440.0\end{array}$ & $\begin{array}{r}1,176.9 \\
730.6 \\
446.3\end{array}$ & $\begin{array}{r}1,238.4 \\
739.0 \\
499.4\end{array}$ & $\begin{array}{r}1,323.0 \\
818.7 \\
504.3\end{array}$ & $\begin{array}{r}1,416.7 \\
883.2 \\
533.6\end{array}$ & $\begin{array}{r}1,387.5 \\
819.6 \\
567.9\end{array}$ \\
\hline 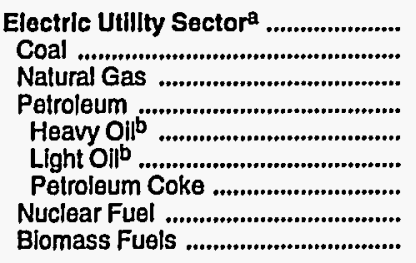 & $\begin{array}{r}23.1 \\
14.2 \\
1.4 \\
7.5 \\
4.5 \\
.8 \\
2.2 \\
- \\
-\end{array}$ & $\begin{array}{r}106.3 \\
25.6 \\
1.9 \\
78.8 \\
76.4 \\
1.7 \\
.7 \\
- \\
-\end{array}$ & $\begin{array}{r}239.3 \\
38.5 \\
25.3 \\
175.6 \\
156.5 \\
6.8 \\
12.2 \\
- \\
-\end{array}$ & $\begin{array}{r}229.9 \\
125.9 \\
29.3 \\
74.7 \\
68.8 \\
3.2 \\
2.7 \\
- \\
-\end{array}$ & $\begin{array}{r}179.4 \\
116.9 \\
4.8 \\
57.7 \\
52.6 \\
2.3 \\
2.8 \\
- \\
-\end{array}$ & $\begin{array}{r}186.2 \\
115.9 \\
22.3 \\
48.0 \\
42.6 \\
3.1 \\
2.4 \\
- \\
-\end{array}$ & $\begin{array}{r}185.6 \\
112.8 \\
7.3 \\
65.5 \\
60.0 \\
3.5 \\
2.0 \\
- \\
-\end{array}$ & $\begin{array}{r}191.6 \\
98.8 \\
21.9 \\
70.9 \\
62.8 \\
6.3 \\
1.8 \\
- \\
-\end{array}$ & $\begin{array}{r}171.0 \\
97.3 \\
29.3 \\
44.4 \\
33.9 \\
2.9 \\
7.6 \\
- \\
-\end{array}$ & $\begin{array}{r}175.9 \\
91.1 \\
35.8 \\
49.0 \\
39.1 \\
3.2 \\
6.7 \\
- \\
-\end{array}$ \\
\hline Primary Energy - Five Sectorsc .. & 211.8 & 496.5 & $1,206.8$ & $1,139.9$ & 871.6 & 916.8 & 924.6 & $1,010.3$ & $1,054.2$ & 995.4 \\
\hline
\end{tabular}

a There are no direct fuel costs for hydroelectric, geothermal, centralized solar, or wind energy.

b Heavy oll includes Grade Nos. 4, 5, and 6, and residual fuel oils. Light oil includes Grade No. 2 heating oil, kerosene, and jet fuel

c Blomass fuels are not included, except those consumed at electric utilities and those added to motor gasoline.

-Nó consumption, including cases where adjustments were made. See "Consumption Adjustments for Process Fuel and Intermediate Products."

Value less than 0.05 million dollars.

Note: Totals may not equal sum of components due to independent rounding.

Sources: Data sources, estimation procedures, and assumptions are described in the "Documentation" section of this report. 


\section{Energy Price and Expenditure Estimates by Source, District of Columbia 1970, 1975, 1980, and 1985-1991}

\begin{tabular}{|c|c|c|c|c|c|c|c|c|c|c|}
\hline Energy Source & 1970 & 1975 & 1980 & 1985 & 1986 & 1987 & 1988 & 1989 & 1990 & 1991 \\
\hline & \multicolumn{10}{|c|}{ Prices in Dollars per Million Btu } \\
\hline Coal & 0.29 & 1.32 & 1.76 & 2.01 & 2.00 & 1.93 & 1.94 & 1.97 & 1.96 & 1.96 \\
\hline 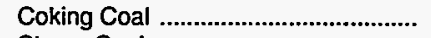 & - & - & - & - & - & - & - & - & - & - \\
\hline 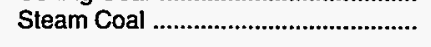 & .29 & 1.32 & 1.76 & 2.01 & 2.00 & 1.93 & 1.94 & 1.97 & 1.96 & 1.96 \\
\hline 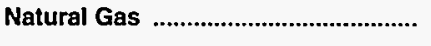 & 1.27 & 2.13 & 4.36 & 7.30 & 6.77 & 6.06 & 6.00 & 6.36 & 6.40 & 6.08 \\
\hline 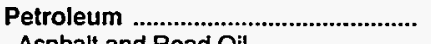 & 1.19 & 3.30 & 7.86 & 8.74 & 6.31 & 6.80 & 6.98 & 7.27 & 8.77 & 8.82 \\
\hline Asphatt and Road Oil ............................ & .67 & 1.80 & 3.58 & 4.95 & 4.18 & 3.22 & 3.16 & 2.88 & 2.94 & 3.10 \\
\hline 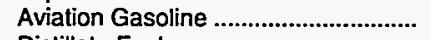 & - & - & - & - & - & - & - & - & - & - \\
\hline 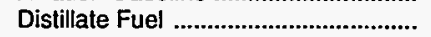 & 1.09 & 2.61 & 7.18 & 7.95 & 6.22 & 6.26 & 6.04 & 6.42 & 8.26 & 8.46 \\
\hline Jet Fuel & .73 & - & 6.46 & 5.80 & 4.01 & 4.01 & 3.75 & - & 5.47 & - \\
\hline 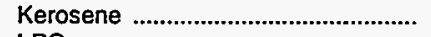 & 1.41 & 2.74 & 8.50 & 7.72 & 6.92 & 6.85 & 6.30 & 5.05 & 6.21 & 7.46 \\
\hline LPG & 1.49 & 3.37 & 6.00 & 11.49 & 11.11 & 10.80 & 11.00 & 10.23 & 10.89 & 11.78 \\
\hline 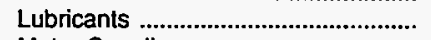 & 5.08 & 7.48 & 14.36 & 17.61 & 15.59 & 12.70 & 14.61 & 13.30 & 13.40 & 15.42 \\
\hline Motor Gasoline & 2.86 & 4.85 & 9.97 & 10.19 & 8.11 & 8.36 & 8.80 & 9.50 & 10.66 & 10.14 \\
\hline 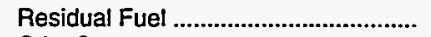 & .50 & 1.97 & 4.46 & 4.36 & 2.95 & 3.27 & 2.41 & 2.79 & 3.22 & 2.61 \\
\hline Othera & - & - & - & - & - & - & - & - & - & - \\
\hline 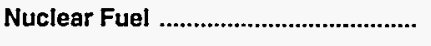 & - & - & - & - & - & - & - & - & - & - \\
\hline Blomass Fuels at Utilities ................. & - & - & - & - & - & - & - & - & - & - \\
\hline Primary Energy - Five Sectors ${ }^{b} \ldots$ & 1.06 & 2.85 & 6.36 & 7.83 & 6.41 & 6.38 & 6.50 & 6.78 & 7.59 & 7.39 \\
\hline $\begin{array}{l}\text { Electric Ulility Fuelc ....................... } \\
\text { Electricity Purchased by End Users }\end{array}$ & $\begin{array}{r}.43 \\
5.39\end{array}$ & $\begin{array}{r}1.92 \\
10.74\end{array}$ & $\begin{array}{r}4.59 \\
14.92\end{array}$ & $\begin{array}{r}4.24 \\
20.89\end{array}$ & $\begin{array}{r}2.28 \\
20.14\end{array}$ & $\begin{array}{r}3.04 \\
18.84\end{array}$ & $\begin{array}{r}2.40 \\
18.00\end{array}$ & $\begin{array}{r}2.89 \\
17.22\end{array}$ & $\begin{array}{r}3.12 \\
17.38\end{array}$ & $\begin{array}{r}2.46 \\
18.34\end{array}$ \\
\hline \multirow[t]{2}{*}{ 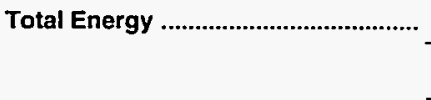 } & 1.72 & 4.33 & 8.75 & 11.67 & 10.38 & 10.24 & 10.44 & 10.52 & 11.27 & 11.38 \\
\hline & \multicolumn{10}{|c|}{ Expenditures in Millions of Dollars } \\
\hline Coal & 8.4 & 13.4 & 5.8 & 7.0 & 2.7 & 3.4 & 1.5 & 2.9 & 3.4 & 3.2 \\
\hline Coking Coal & - & - & - & - & - & - & - & - & - & - \\
\hline 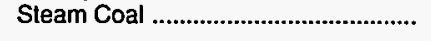 & 8.4 & 13.4 & 5.8 & 7.0 & 2.7 & 3.4 & 1.5 & 2.9 & 3.4 & 3.2 \\
\hline 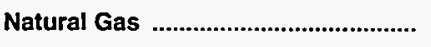 & 33.5 & 55.7 & 121.8 & 211.5 & 200.6 & 188.7 & 197.1 & 213.1 & 184.5 & 188.5 \\
\hline Petroleum & 154.1 & 250.8 & 374.9 & 337.1 & 297.6 & 290.5 & 292.0 & 310.9 & 326.2 & 307.6 \\
\hline 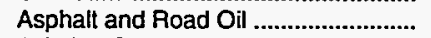 & .1 & .2 & .4 & .9 & .9 & .7 & .7 & .5 & .6 & .5 \\
\hline 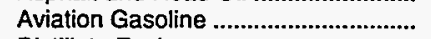 & - & - & - & - & - & - & - & - & - & - \\
\hline 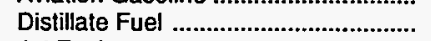 & 31.4 & 48.1 & 95.6 & 103.2 & 86.8 & 70.6 & 65.7 & 68.8 & 74.0 & 76.3 \\
\hline Jet Fuel & $\because$ & - & 12.1 & .2 & 11.4 & 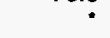 & .1 & - & .2 & - \\
\hline Kerosene & .4 & 1.7 & 12.9 & 3.0 & .5 & .5 & .5 & 1.7 & .4 & .3 \\
\hline LPG & $\because$ & .1 & .1 & .2 & .1 & .2 & .2 & .2 & .2 & .2 \\
\hline 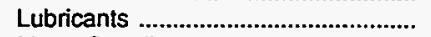 & 1.7 & 2.7 & 5.3 & 5.9 & 5.1 & 4.7 & 5.2 & 4.9 & 5.1 & 5.2 \\
\hline 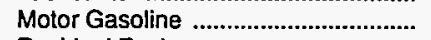 & 85.4 & 146.4 & 203.3 & 203.4 & 165.2 & 185.9 & 201.8 & 209.4 & 225.1 & 214.1 \\
\hline 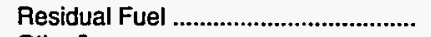 & 35.1 & 51.6 & 45.2 & 20.3 & 27.5 & 27.9 & 17.7 & 25.4 & 20.7 & 10.9 \\
\hline Other ${ }^{a}$ & - & - & - & - & - & - & - & - & - & - \\
\hline 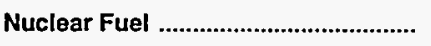 & - & - & - & - & - & - & - & - & - & - \\
\hline Blomass Fuels at Utilities ................... & - & - & - & - & - & - & - & - & - & - \\
\hline Primary Energy - Five Sectors ${ }^{b}$.... & 195.9 & 319.9 & 502.4 & 555.6 & 500.8 & 482.6 & 490.6 & 526.9 & 514.1 & 499.3 \\
\hline $\begin{array}{l}\text { Electric Utility Fuelc .......................... } \\
\text { Electricity Purchased by End Users }\end{array}$ & $\begin{array}{r}-18.0 \\
99.2\end{array}$ & $\begin{array}{r}-31.7 \\
212.3\end{array}$ & $\begin{array}{r}-45.1 \\
356.5\end{array}$ & $\begin{array}{r}-8.3 \\
585.3\end{array}$ & $\begin{array}{r}-6.7 \\
590.1\end{array}$ & $\begin{array}{r}-11.7 \\
578.9\end{array}$ & $\begin{array}{l}-16.4 \\
576.0\end{array}$ & $\begin{array}{r}-26.8 \\
567.0\end{array}$ & $\begin{array}{r}-17.0 \\
584.0\end{array}$ & $\begin{array}{r}-7.6 \\
638.0\end{array}$ \\
\hline Total Energy & 277.2 & 500.5 & 813.8 & $1,132.6$ & $1,084.2$ & $1,049.8$ & $1,050.2$ & $1,067.1$ & $1,081.1$ & $1,129.7$ \\
\hline
\end{tabular}

\footnotetext{
a Includes petroleum coke used at electric utilities.

biomass fuels are not included, except those consumed at electric utilities and those added to motor gasoline.

c There are no direct fuel costs for hydroelectric, geothermal, centralized solar, or wind energy.

-No consumption, including cases where adjustments were made. See "Consumption Adjustments for Process Fuel and Intermediate Products."

- Value less than 0.05 million dollars.

Note: Expenditure totals may not equal sum of components due to independent rounding.

Sources: Data sources, estimation procedures, and assumptions are described in the "Documentation" section of this report.
} 


\begin{tabular}{|c|c|c|c|c|c|c|c|c|c|c|}
\hline Sector and Energy Source & 1970 & 1975 & 1980 & 1985 & 1986 & 1987 & 1988 & 1989 & 1990 & 1991 \\
\hline 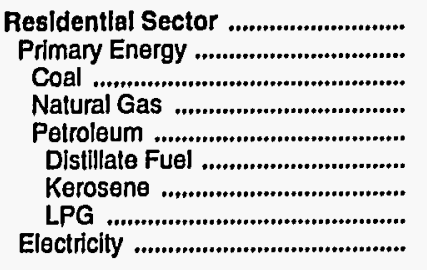 & $\begin{array}{l}2.01 \\
1.42 \\
1.05 \\
1.43 \\
1.42 \\
1.42 \\
1.50 \\
2.57 \\
7.02\end{array}$ & $\begin{array}{r}3.79 \\
2.44 \\
1.75 \\
2.30 \\
2.71 \\
2.71 \\
3.37 \\
4.61 \\
12.65\end{array}$ & $\begin{array}{r}7.12 \\
5.15 \\
3.18 \\
4.56 \\
7.41 \\
7.40 \\
8.55 \\
9.81 \\
17.32\end{array}$ & $\begin{array}{r}9.77 \\
7.67 \\
3.28 \\
7.80 \\
8.74 \\
8.74 \\
8.88 \\
13.53 \\
20.31\end{array}$ & $\begin{array}{r}9.60 \\
7.29 \\
3.22 \\
7.42 \\
7.13 \\
7.12 \\
7.23 \\
13.81 \\
19.97\end{array}$ & $\begin{array}{r}9.26 \\
6.88 \\
3.10 \\
6.99 \\
7.03 \\
7.02 \\
7.13 \\
13.84 \\
19.15\end{array}$ & $\begin{array}{r}9.18 \\
6.84 \\
3.07 \\
6.88 \\
7.02 \\
7.00 \\
7.11 \\
14.13 \\
18.41\end{array}$ & $\begin{array}{r}9.41 \\
7.26 \\
3.25 \\
7.37 \\
7.57 \\
7.54 \\
7.66 \\
14.13 \\
17.59\end{array}$ & $\begin{array}{r}9.54 \\
7.05 \\
3.36 \\
7.12 \\
8.26 \\
8.24 \\
8.37 \\
12.58 \\
17.82\end{array}$ & $\begin{array}{r}9.96 \\
6.98 \\
3.09 \\
7.03 \\
8.60 \\
8.58 \\
8.71 \\
13.46 \\
19.29\end{array}$ \\
\hline 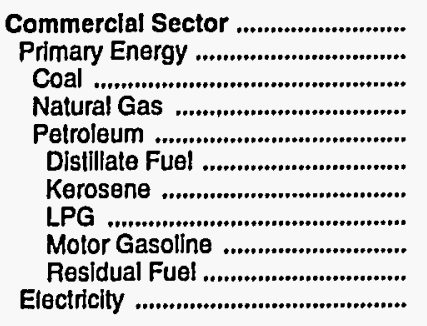 & $\begin{array}{r}1.40 \\
.71 \\
.11 \\
1.09 \\
.61 \\
1.12 \\
1.33 \\
1.03 \\
2.86 \\
.46 \\
6.86\end{array}$ & $\begin{array}{l}4.63 \\
2.11 \\
1.25 \\
1.96 \\
2.28 \\
2.39 \\
2.70 \\
2.75 \\
4.85 \\
2.02 \\
12.49\end{array}$ & $\begin{array}{r}8.62 \\
4.45 \\
1.19 \\
4.21 \\
6.60 \\
6.55 \\
8.50 \\
5.13 \\
9.97 \\
4.43 \\
18.41\end{array}$ & $\begin{array}{r}12.94 \\
5.98 \\
1.33 \\
6.62 \\
6.42 \\
6.73 \\
7.51 \\
10.88 \\
10.19 \\
5.16 \\
22.82\end{array}$ & $\begin{array}{r}11.30 \\
4.88 \\
1.33 \\
5.84 \\
4.19 \\
4.95 \\
5.12 \\
10.37 \\
8.11 \\
3.34 \\
21.78\end{array}$ & $\begin{array}{r}10.77 \\
4.48 \\
1.30 \\
4.94 \\
4.15 \\
4.91 \\
5.70 \\
9.88 \\
8.36 \\
3.50 \\
20.19\end{array}$ & $\begin{array}{r}11.34 \\
4.71 \\
1.33 \\
4.98 \\
4.22 \\
4.49 \\
5.17 \\
10.26 \\
8.80 \\
3.23 \\
19.29\end{array}$ & $\begin{array}{r}11.06 \\
5.01 \\
1.28 \\
5.25 \\
4.98 \\
5.19 \\
4.46 \\
9.19 \\
9.50 \\
3.70 \\
18.37\end{array}$ & $\begin{array}{r}11.78 \\
5.54 \\
1.21 \\
5.59 \\
6.43 \\
7.11 \\
5.25 \\
10.33 \\
10.66 \\
3.91 \\
18.53\end{array}$ & $\begin{array}{r}11.65 \\
5.02 \\
1.34 \\
5.14 \\
5.44 \\
6.02 \\
6.21 \\
11.09 \\
10.14 \\
3.38 \\
19.42\end{array}$ \\
\hline 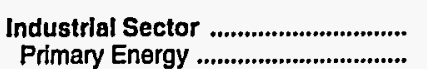 & $\begin{array}{r}1.19 \\
.49\end{array}$ & $\begin{array}{l}4.29 \\
1.63\end{array}$ & $\begin{array}{r}10.20 \\
6.16\end{array}$ & $\begin{array}{r}17.08 \\
8.31\end{array}$ & $\begin{array}{r}16.15 \\
6.08\end{array}$ & $\begin{array}{r}15.65 \\
6.90\end{array}$ & $\begin{array}{r}15.03 \\
7.08\end{array}$ & $\begin{array}{r}14.56 \\
7.77\end{array}$ & $\begin{array}{r}14.72 \\
8.61\end{array}$ & $\begin{array}{r}15.57 \\
8.36\end{array}$ \\
\hline Coal & .11 & 1.25 & 1.20 & - & - & - & - & - & - & - \\
\hline 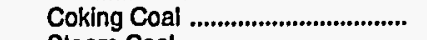 & - & - & - & - & - & - & - & - & - & - \\
\hline Steam Coal ............................................... & .11 & 1.25 & 1.20 & - & - & - & - & - & - & - \\
\hline Natural Gas .............................................. & .67 & 1.36 & 2.45 & - & - & - & - & - & - & - \\
\hline 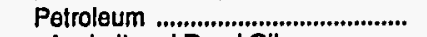 & .66 & 2.08 & 7.60 & 8.31 & 6.08 & 6.90 & 7.08 & 7.77 & 8.61 & 8.36 \\
\hline 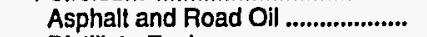 & .67 & 1.80 & 3.58 & 4.95 & 4.18 & 3.22 & 3.16 & 2.88 & 2.94 & 3.10 \\
\hline 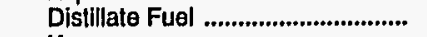 & 1.22 & 2.50 & 7.63 & 6.73 & 4.59 & 5.11 & 4.63 & 5.37 & 6.14 & 5.56 \\
\hline Kerosene .......................................... & 1.33 & 2.70 & 8.50 & 7.51 & 5.12 & 5.70 & 5.17 & - & - & 6.21 \\
\hline 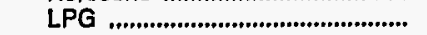 & 1.03 & 2.75 & 5.13 & 10.88 & 10.37 & 9.88 & 10.26 & 9.19 & 10.33 & 11.09 \\
\hline Lubricants ............................................... & 5.08 & 7.48 & 14.36 & 17.61 & 15.59 & 12.70 & 14.61 & 13.30 & 13.40 & 15.42 \\
\hline Motor Gasoline .................................. & - & - & & 10.19 & 8.11 & 8.36 & 8.80 & 9.50 & 10.66 & 10.14 \\
\hline 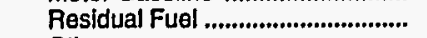 & .59 & 1.82 & 3.97 & 5.16 & - & - & - & 3.70 & 3.91 & 3.38 \\
\hline 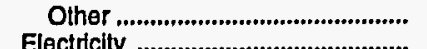 & $\overline{0}$ & $\overline{0}$ & & & $17 \overline{3}$ & $16 \overline{37}$ & $15 \overline{5}$ & $15 \overline{00}$ & $15 \overline{17}$ & $15 \overline{94}$ \\
\hline 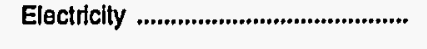 & 3.80 & 8.42 & 11.65 & 17.86 & 17.34 & 16.37 & 15.55 & 15.00 & 15.17 & 15.94 \\
\hline 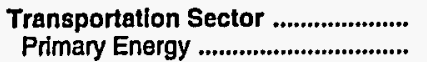 & $\begin{array}{l}2.74 \\
2.74\end{array}$ & $\begin{array}{l}4.43 \\
4.43\end{array}$ & $\begin{array}{l}9.44 \\
9.40\end{array}$ & $\begin{array}{l}9.85 \\
9.68\end{array}$ & $\begin{array}{l}7.77 \\
7.59\end{array}$ & $\begin{array}{l}8.35 \\
8.22\end{array}$ & $\begin{array}{l}8.68 \\
8.56\end{array}$ & $\begin{array}{l}9.19 \\
9.08\end{array}$ & $\begin{array}{l}10.54 \\
10.44\end{array}$ & $\begin{array}{l}10.42 \\
10.29\end{array}$ \\
\hline 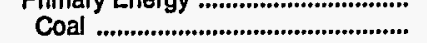 & .11 & 1.25 & - & - & - & - & - & - & - & \\
\hline 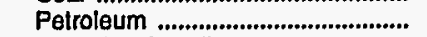 & 2.74 & 4.43 & 9.40 & 9.68 & 7.59 & 8.22 & 8.56 & 9.08 & 10.44 & 10.29 \\
\hline 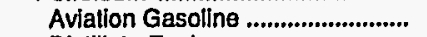 & - & - & - & - & - & - & - & - & - & \\
\hline 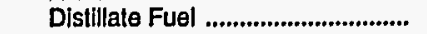 & 1.32 & 2.81 & 7.70 & 8.78 & 7.67 & 7.25 & 7.19 & 7.49 & 9.33 & 10.66 \\
\hline Jet Fuel ......................................... & .73 & - & 6.46 & 5.80 & 4.01 & 4.01 & 3.75 & - & 5.47 & - \\
\hline 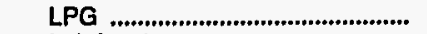 & 1.03 & 2.75 & 5.13 & 10.88 & 10.37 & 9.88 & 10.26 & 9.19 & 10.33 & 11.09 \\
\hline 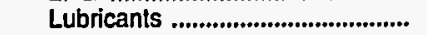 & 5.08 & 7.48 & 14.36 & 17.61 & 15.59 & 12.70 & 14.61 & 13.30 & 13.40 & 15.42 \\
\hline 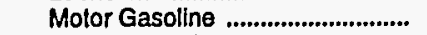 & 2.86 & 4.85 & 9.97 & 10.19 & 8.11 & 8.36 & 8.80 & 9.50 & 10.66 & 10.14 \\
\hline Residual Fuel ....................................... & .45 & 1.81 & 4.20 & 3.75 & 2.05 & & 2.12 & 2.57 & 2.88 & \\
\hline 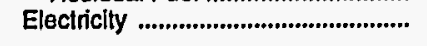 & - & - & 12.62 & 20.73 & 20.40 & 17.14 & 16.91 & 16.91 & 16.54 & 18.09 \\
\hline 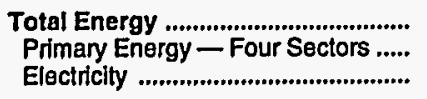 & $\begin{array}{l}1.72 \\
1.25 \\
5.39\end{array}$ & $\begin{array}{r}4.33 \\
3.01 \\
10.74\end{array}$ & $\begin{array}{r}8.75 \\
6.62 \\
14.92\end{array}$ & $\begin{array}{r}11.67 \\
7.93 \\
20.89\end{array}$ & $\begin{array}{r}10.38 \\
6.58 \\
20.14\end{array}$ & $\begin{array}{r}10.24 \\
6.56 \\
18.84\end{array}$ & $\begin{array}{r}10.44 \\
6.91 \\
18.00\end{array}$ & $\begin{array}{r}10.52 \\
7.30 \\
17.22\end{array}$ & $\begin{array}{r}11.27 \\
7.98 \\
17.38\end{array}$ & $\begin{array}{r}11.38 \\
7.63 \\
18.34\end{array}$ \\
\hline 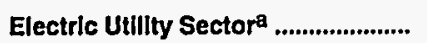 & .43 & 1.92 & 4.59 & 4.24 & 2.28 & 3.04 & 2.40 & 2.89 & 3.12 & 2.46 \\
\hline 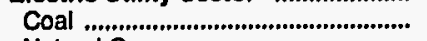 & .39 & 1.50 & - & - & - & - & - & - & - & - \\
\hline 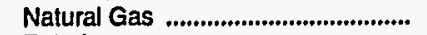 & - & - & - & - & $\overline{-}$ & - & - & $\bar{n}$ & $\overline{0}$ & - \\
\hline Pelroleum & .47 & 2.01 & 4.59 & 4.24 & 2.28 & 3.04 & 2.40 & $\begin{array}{l}2.89 \\
2.71\end{array}$ & $\begin{array}{l}3.12 \\
3.02\end{array}$ & $\begin{array}{l}2.46 \\
2.22\end{array}$ \\
\hline 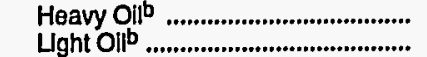 & $\begin{array}{l}.47 \\
.46\end{array}$ & $\begin{array}{l}2.01 \\
2.11\end{array}$ & $\begin{array}{l}4.49 \\
5.95\end{array}$ & $\begin{array}{l}3.94 \\
5.43\end{array}$ & $\begin{array}{l}2.16 \\
2.98\end{array}$ & $\begin{array}{l}2.93 \\
3.83\end{array}$ & $\begin{array}{l}2.22 \\
3.47\end{array}$ & $\begin{array}{l}2.71 \\
4.08\end{array}$ & $\begin{array}{l}3.02 \\
4.29\end{array}$ & $\begin{array}{l}2.22 \\
4.55\end{array}$ \\
\hline Petroleum Coke .................................... & - & - & - & - & - & - & - & - & - & - \\
\hline Nuclear Fuel ....................................... & - & - & - & - & - & - & - & - & - & - \\
\hline 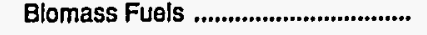 & - & - & - & - & - & - & - & - & - & - \\
\hline Primary Energy - Flve Sectorsc .. & 1.06 & 2.85 & 6.36 & $\mathbf{7 . 8 3}$ & 6.41 & 6.38 & 6.50 & 6.78 & 7.59 & 7.39 \\
\hline
\end{tabular}

a There are no direct fuel costs for hydroelectric, geothermal, centralized solar, or wind energy.

b Heavy oll includes Grade Nos. 4, 5, and 6, and residual fuel oils. Light oil includes Grade No. 2 heating oil, kerosene, and jet fuel.

c Blomass fuels are not included, except those consumed at electric utilities and those added to motor gasoline.

- No consumption, including cases where adjustments were made. See "Consumption Adjustments for Process Fuel and Intermediate Products."

Sources: Data sources, estimation procedures, and assumptions are described in the "Documentation" section of this report. 


\section{Energy Expenditure Estimates by Sector, District of Columbia}

$1970,1975,1980$, and 1985-1991

$\mathbf{S}$ (Million Dollars)

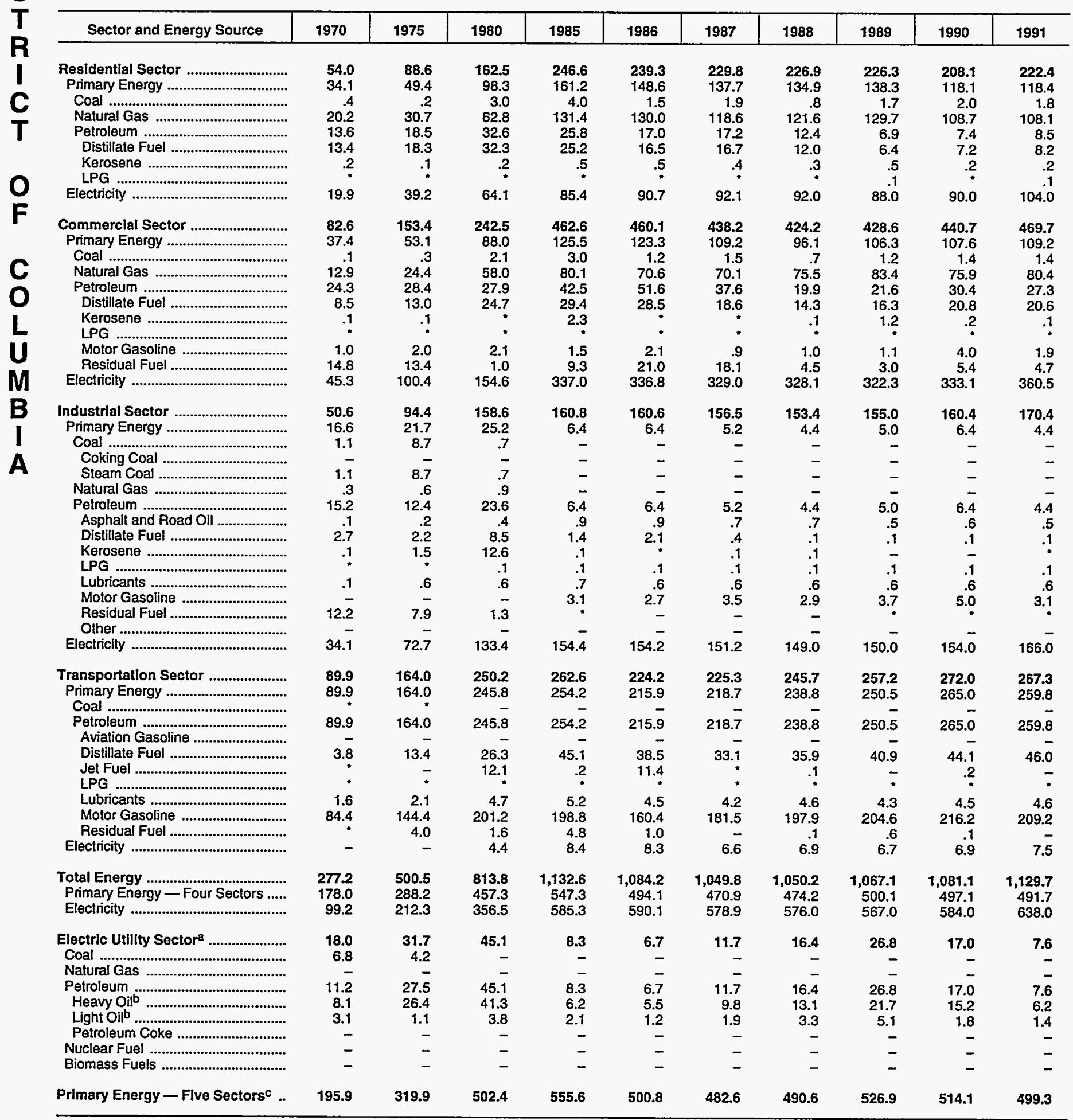

There are no direct fuel costs for hydroelectric, geothermal, centralized solar, or wind energy.

b Heavy oil includes Grade Nos. 4, 5, and 6, and residual fuel oils. Light oil includes Grade No. 2 heating oil, kerosene, and jet fuel.

C Biomass fuels are not included, except those consumed at electric utilities and those added to motor gasoline.

-No consumption, including cases where adjustments were made. See "Consumption Adjustments for Process Fuel and Intermediate Products."

- Value less than 0.05 million dollars.

Note: Totals may not equal sum of components due to independent rounding.

Sources: Data sources, estimation procedures, and assumptions are described in the "Documentation" section of this report. 


\begin{tabular}{|c|c|c|c|c|c|c|c|c|c|c|}
\hline Energy Source & 1970 & 1975 & 1980 & 1985 & 1986 & 1987 & 1988 & 1989 & 1990 & 1991 \\
\hline & \multicolumn{10}{|c|}{ Prices in Dollars per Million Btu } \\
\hline 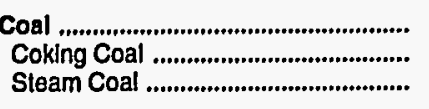 & $\frac{0.31}{-31}$ & $\begin{array}{r}1.01 \\
1.01\end{array}$ & $\begin{array}{r}1.80 \\
1.80\end{array}$ & $\frac{2.12}{2.12}$ & $\begin{array}{r}1.93 \\
1.93\end{array}$ & $\begin{array}{r}1.82 \\
1.82\end{array}$ & $\begin{array}{r}1.78 \\
1.78\end{array}$ & $\begin{array}{r}1.79 \\
1.79\end{array}$ & $\begin{array}{r}1.85 \\
1.85\end{array}$ & $\begin{array}{r}1.86 \\
1.86\end{array}$ \\
\hline Natural Gas ............................................. & .49 & 1.00 & 2.19 & 3.73 & 2.76 & 3.21 & 2.80 & 3.02 & 3.21 & 2.84 \\
\hline 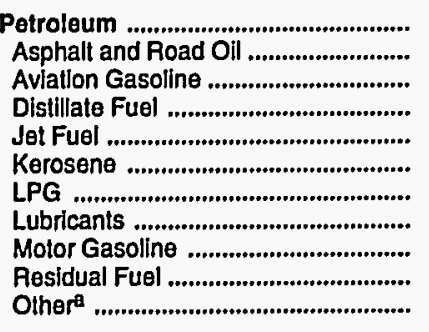 & $\begin{array}{r}1.53 \\
.67 \\
2.17 \\
1.08 \\
.73 \\
1.28 \\
2.61 \\
5.08 \\
2.81 \\
.33 \\
1.35\end{array}$ & $\begin{array}{l}3.02 \\
1.78 \\
3.45 \\
2.53 \\
2.03 \\
3.12 \\
5.14 \\
7.48 \\
4.39 \\
1.84 \\
3.11\end{array}$ & $\begin{array}{r}6.66 \\
3.57 \\
9.02 \\
6.91 \\
6.46 \\
8.45 \\
7.48 \\
14.36 \\
9.80 \\
3.61 \\
7.97\end{array}$ & $\begin{array}{r}7.46 \\
4.99 \\
9.99 \\
6.78 \\
5.90 \\
7.46 \\
10.91 \\
17.61 \\
9.03 \\
3.90 \\
8.30\end{array}$ & $\begin{array}{r}5.32 \\
4.16 \\
8.41 \\
5.90 \\
4.19 \\
7.78 \\
10.27 \\
15.59 \\
6.70 \\
2.15 \\
5.32\end{array}$ & $\begin{array}{r}5.82 \\
3.20 \\
7.55 \\
6.12 \\
4.07 \\
7.70 \\
10.98 \\
12.70 \\
7.11 \\
2.90 \\
6.18\end{array}$ & $\begin{array}{r}5.49 \\
3.13 \\
7.41 \\
5.83 \\
3.82 \\
7.94 \\
11.20 \\
14.61 \\
7.12 \\
2.17 \\
5.21\end{array}$ & $\begin{array}{r}5.95 \\
2.87 \\
8.28 \\
6.16 \\
4.43 \\
7.27 \\
10.15 \\
13.30 \\
7.68 \\
2.64 \\
5.86\end{array}$ & $\begin{array}{r}6.95 \\
2.91 \\
9.32 \\
7.86 \\
5.64 \\
10.50 \\
11.70 \\
13.40 \\
8.85 \\
2.92 \\
6.66\end{array}$ & $\begin{array}{r}6.40 \\
3.06 \\
8.71 \\
7.27 \\
4.93 \\
11.47 \\
12.19 \\
15.42 \\
8.48 \\
2.20 \\
5.89\end{array}$ \\
\hline Nuclear Fuel .......................................... & - & .17 & .35 & .65 & .73 & .69 & .94 & .66 & .64 & .66 \\
\hline Blomass Fuels at Utillties ................. & - & - & - & - & - & - & - & - & - & - \\
\hline Primary Energy - Flve Sectors b .... & 1.20 & 2.44 & 5.12 & 5.17 & 3.96 & 4.19 & 3.92 & 4.23 & 4.81 & 4.43 \\
\hline $\begin{array}{l}\text { Electric Utility Fuelc ........................... } \\
\text { Electricity Purchased by End Users }\end{array}$ & $\begin{array}{r}.33 \\
5.67\end{array}$ & $\begin{array}{r}1.35 \\
10.46\end{array}$ & $\begin{array}{r}2.40 \\
16.24\end{array}$ & $\begin{array}{r}2.21 \\
22.59\end{array}$ & $\begin{array}{r}1.76 \\
21.37\end{array}$ & $\begin{array}{r}1.96 \\
20.55\end{array}$ & $\begin{array}{r}1.72 \\
20.70\end{array}$ & $\begin{array}{r}1.90 \\
20.45\end{array}$ & $\begin{array}{r}1.98 \\
20.62\end{array}$ & $\begin{array}{r}1.81 \\
20.99\end{array}$ \\
\hline \multirow[t]{2}{*}{ Total Energy ........................................ } & 2.35 & 4.62 & 8.84 & 10.84 & 9.31 & 9.35 & 9.22 & 9.70 & 10.54 & 10.55 \\
\hline & \multicolumn{10}{|c|}{ Expenditures in Millions of Dollars } \\
\hline 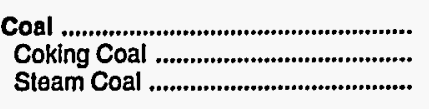 & $35 . \overline{8}$ & $\begin{array}{r}135.0 \\
135.0\end{array}$ & $\begin{array}{r}405.4 \\
405.4\end{array}$ & 999.9 & $\begin{array}{r}887.5 \\
- \\
887.5\end{array}$ & $\begin{array}{r}1,066.6 \\
\overline{-} \\
1,066.6\end{array}$ & $\begin{array}{r}1,088.2 \\
- \\
1,088.2\end{array}$ & $\begin{array}{r}1,128.3 \\
\frac{-}{1,128.3}\end{array}$ & $\begin{array}{r}1,155.6 \\
- \\
1,155.6\end{array}$ & $\begin{array}{r}1,194.6 \\
-\overline{6} \\
1,194.6\end{array}$ \\
\hline Natural Gas ......................................... & 170.1 & 283.6 & 693.8 & $1,081.0$ & 787.7 & 964.7 & 825.1 & 976.6 & $1,081.4$ & $1,004.3$ \\
\hline 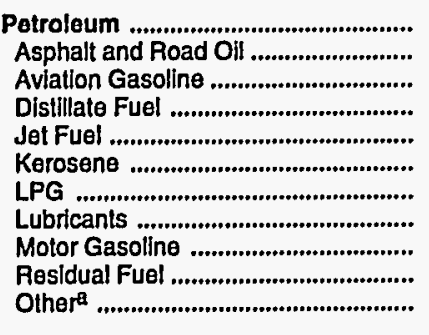 & $\begin{array}{r}1,632.1 \\
18.0 \\
34.4 \\
98.0 \\
96.6 \\
26.6 \\
77.1 \\
33.6 \\
1,125.2 \\
112.8 \\
9.8\end{array}$ & $\begin{array}{r}4,168.9 \\
43.3 \\
33.4 \\
343.6 \\
275.6 \\
15.5 \\
142.5 \\
54.0 \\
2,319.6 \\
915.2 \\
26.1\end{array}$ & $\begin{array}{r}11,051.3 \\
106.4 \\
61.0 \\
1,183.7 \\
1,302.3 \\
45.6 \\
294.6 \\
122.7 \\
5,627.4 \\
2,193.5 \\
114.0\end{array}$ & $\begin{array}{r}9,831.1 \\
220.8 \\
42.4 \\
1,198.8 \\
762.5 \\
107.1 \\
388.1 \\
137.0 \\
5,947.5 \\
911.5 \\
115.5\end{array}$ & $\begin{array}{r}7,989.0 \\
227.3 \\
43.4 \\
1,089.5 \\
587.3 \\
63.5 \\
394.0 \\
118.6 \\
4,614.0 \\
767.6 \\
83.7\end{array}$ & $\begin{array}{r}8,540.0 \\
160.8 \\
29.6 \\
1,172.6 \\
604.7 \\
49.7 \\
353.3 \\
109.2 \\
5,132.4 \\
831.7 \\
95.9\end{array}$ & $\begin{array}{r}8,680.4 \\
164.6 \\
33.0 \\
1,169.0 \\
685.3 \\
51.9 \\
327.9 \\
121.2 \\
5,310.7 \\
735.4 \\
81.4\end{array}$ & $\begin{array}{r}9,426.1 \\
123.5 \\
40.8 \\
1,269.2 \\
835.0 \\
35.2 \\
300.6 \\
113.1 \\
5,731.5 \\
888.0 \\
89.3\end{array}$ & $\begin{array}{r}10,919.2 \\
131.3 \\
38.0 \\
1,574.3 \\
1,013.5 \\
19.6 \\
328.5 \\
117.3 \\
6,581.4 \\
1,001.8 \\
113.4\end{array}$ & $\begin{array}{r}9,943.4 \\
148.5 \\
31.4 \\
1,328.7 \\
724.3 \\
15.4 \\
350.5 \\
120.7 \\
6,297.1 \\
826.2 \\
100.6\end{array}$ \\
\hline Nuclear Fuel ......................................... & - & 15.8 & 63.8 & 165.1 & 172.9 & 138.6 & 265.0 & 148.5 & 149.2 & 146.2 \\
\hline Blomass Fuels at Utilities ................. & - & - & - & - & - & - & - & - & - & - \\
\hline Primary Energy - Flve Sectorsb .... & $1,838.1$ & $4,603.2$ & $12,214.3$ & $12,077.1$ & $9,837.1$ & $10,709.9$ & $10,858.8$ & $11,679.4$ & $13,305.3$ & $12,288.5$ \\
\hline $\begin{array}{l}\text { Electric Utility Fuelc ........................... } \\
\text { Electricity Purchased by End Users }\end{array}$ & $\begin{array}{r}-196.0 \\
971.7\end{array}$ & $\begin{array}{r}-1,114.2 \\
2,532.9\end{array}$ & $\begin{array}{r}-2,439.2 \\
5,029.8\end{array}$ & $\begin{array}{r}-2,244.7 \\
8,548.0\end{array}$ & $\begin{array}{r}-1,980.3 \\
8,480.2\end{array}$ & $\begin{array}{r}-2,256.9 \\
8,586.9\end{array}$ & $\begin{array}{r}-2,200.2 \\
9,197.0\end{array}$ & $\begin{array}{r}-2,433.9 \\
9,661.0\end{array}$ & $\begin{array}{r}-2,516.8 \\
10,098.0\end{array}$ & $\begin{array}{r}-2,424.6 \\
10,479.0\end{array}$ \\
\hline Total Energy ........................................ & $2,613.8$ & $6,021.9$ & $14,804.8$ & $18,380.3$ & $16,336.9$ & $17,039.8$ & $17,855.6$ & $18,906.5$ & $20,886.5$ & $20,342.9$ \\
\hline
\end{tabular}

a Includes petroleum coke used at electric utilities.

b Blomass fuels are not included, except those consumed at electric utilities and those added to motor gasoline.

c There are no direct fuel costs for hydroelectric, geothermal, centralized solar, or wind energy.

-No consumption, Including cases where adjustments were made. See "Consumption Adjustments for Process Fuel and Intermediate Products."

Note: Expenditure totals may not equal sum of components due to independent rounding.

Sources: Data sources, estimation procedures, and assumptions are described in the "Documentation" section of this report. 


\section{F Energy Price Estimates by Sector, Florida}

L $1970,1975,1980$, and 1985-1991

O (Dollars per Million Btu)

\begin{tabular}{|c|c|c|c|c|c|c|c|c|c|c|}
\hline Sector and Energy Source & 1970 & 1975 & 1980 & 1985 & 1986 & 1987 & 1988 & 1989 & 1990 & 1991 \\
\hline 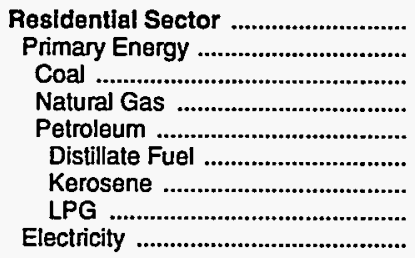 & $\begin{array}{r}4.60 \\
2.37 \\
- \\
2.42 \\
2.35 \\
1.25 \\
1.63 \\
3.11 \\
6.10\end{array}$ & $\begin{array}{r}9.04 \\
4.19 \\
- \\
2.54 \\
5.10 \\
2.62 \\
3.27 \\
6.32 \\
10.92\end{array}$ & $\begin{array}{r}14.66 \\
7.48 \\
3.12 \\
4.49 \\
9.24 \\
6.92 \\
8.92 \\
10.34 \\
16.74\end{array}$ & $\begin{array}{r}21.57 \\
8.81 \\
3.31 \\
6.72 \\
10.04 \\
6.73 \\
9.34 \\
10.70 \\
24.73\end{array}$ & $\begin{array}{r}20.66 \\
8.86 \\
3.00 \\
6.70 \\
10.13 \\
7.67 \\
10.63 \\
10.35 \\
23.36\end{array}$ & $\begin{array}{r}20.36 \\
9.33 \\
2.96 \\
6.83 \\
10.88 \\
6.75 \\
9.36 \\
12.01 \\
22.73\end{array}$ & $\begin{array}{r}20.80 \\
9.44 \\
2.95 \\
6.94 \\
11.10 \\
7.17 \\
9.94 \\
12.01 \\
22.91\end{array}$ & $\begin{array}{r}20.84 \\
9.14 \\
3.05 \\
7.42 \\
10.24 \\
6.56 \\
9.10 \\
10.81 \\
22.67\end{array}$ & $\begin{array}{r}21.25 \\
10.49 \\
3.10 \\
7.79 \\
12.38 \\
9.59 \\
13.30 \\
12.55 \\
22.78\end{array}$ & $\begin{array}{r}21.65 \\
10.91 \\
2.94 \\
8.17 \\
12.75 \\
8.95 \\
12.40 \\
13.05 \\
23.18\end{array}$ \\
\hline 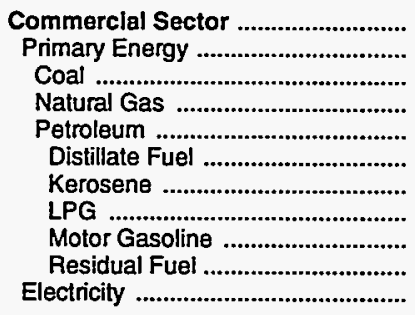 & $\begin{array}{r}3.53 \\
1.07 \\
- \\
.89 \\
1.23 \\
.98 \\
.61 \\
1.27 \\
2.81 \\
.33 \\
6.24\end{array}$ & $\begin{array}{r}7.13 \\
2.03 \\
- \\
1.58 \\
2.52 \\
2.26 \\
2.38 \\
2.51 \\
4.39 \\
1.85 \\
11.44\end{array}$ & $\begin{array}{r}12.27 \\
4.68 \\
1.77 \\
3.21 \\
6.24 \\
6.30 \\
6.41 \\
5.46 \\
9.80 \\
3.71 \\
17.38\end{array}$ & $\begin{array}{r}15.73 \\
5.62 \\
2.04 \\
4.80 \\
6.27 \\
5.79 \\
6.49 \\
11.24 \\
9.03 \\
4.08 \\
22.03\end{array}$ & $\begin{array}{r}14.30 \\
4.49 \\
1.98 \\
4.11 \\
4.83 \\
5.01 \\
5.99 \\
10.15 \\
6.70 \\
2.27 \\
20.45\end{array}$ & $\begin{array}{r}14.17 \\
4.68 \\
1.87 \\
4.28 \\
5.06 \\
4.85 \\
5.64 \\
9.27 \\
7.11 \\
2.98 \\
19.57\end{array}$ & $\begin{array}{r}14.62 \\
4.44 \\
1.84 \\
4.20 \\
4.65 \\
4.46 \\
5.32 \\
9.94 \\
7.12 \\
2.29 \\
19.75\end{array}$ & $\begin{array}{r}14.97 \\
4.71 \\
1.86 \\
4.47 \\
4.93 \\
4.60 \\
5.80 \\
9.15 \\
7.68 \\
2.75 \\
19.40\end{array}$ & $\begin{array}{r}15.25 \\
5.55 \\
1.89 \\
4.64 \\
6.36 \\
7.29 \\
8.04 \\
10.15 \\
8.85 \\
3.09 \\
19.57\end{array}$ & $\begin{array}{r}15.41 \\
4.91 \\
1.89 \\
4.48 \\
5.40 \\
5.88 \\
7.13 \\
10.59 \\
8.48 \\
2.37 \\
19.85\end{array}$ \\
\hline 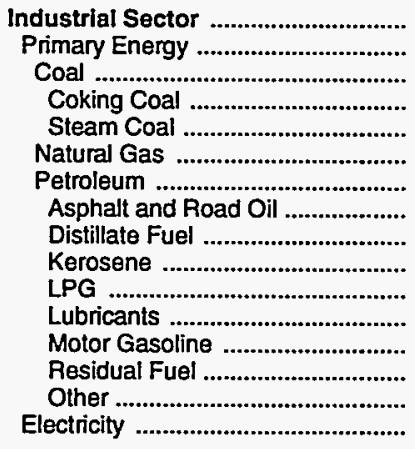 & $\begin{array}{r}.93 \\
.55 \\
- \\
- \\
\overline{.37} \\
.69 \\
.67 \\
.56 \\
.61 \\
1.27 \\
5.08 \\
2.81 \\
.37 \\
1.35 \\
3.56\end{array}$ & $\begin{array}{r}2.71 \\
1.64 \\
.53 \\
- \\
.53 \\
.95 \\
2.18 \\
1.78 \\
2.20 \\
2.38 \\
2.51 \\
7.48 \\
4.39 \\
1.75 \\
3.11 \\
7.57\end{array}$ & $\begin{array}{r}5.48 \\
3.88 \\
1.77 \\
1.77 \\
2.61 \\
4.71 \\
3.57 \\
5.75 \\
6.41 \\
5.46 \\
14.36 \\
9.80 \\
3.44 \\
7.97 \\
13.38\end{array}$ & $\begin{array}{r}7.10 \\
4.99 \\
2.04 \\
2 . \overline{-} \\
3.71 \\
6.11 \\
4.99 \\
5.81 \\
6.49 \\
11.24 \\
17.61 \\
9.03 \\
4.08 \\
8.30 \\
16.63\end{array}$ & $\begin{array}{r}6.42 \\
4.16 \\
1.98 \\
-\overline{0} \\
1.98 \\
2.85 \\
5.06 \\
4.16 \\
5.36 \\
5.99 \\
10.15 \\
15.59 \\
6.70 \\
2.27 \\
5.32 \\
16.29\end{array}$ & $\begin{array}{r}6.11 \\
3.93 \\
1.87 \\
1.87 \\
3.01 \\
4.84 \\
3.20 \\
5.05 \\
5.64 \\
9.27 \\
12.70 \\
7.11 \\
2.98 \\
6.18 \\
14.99\end{array}$ & $\begin{array}{r}5.63 \\
3.50 \\
1.84 \\
1 . \overline{1} \\
1.84 \\
2.63 \\
4.35 \\
3.13 \\
4.77 \\
5.32 \\
9.94 \\
14.61 \\
7.12 \\
2.29 \\
5.21 \\
14.93\end{array}$ & $\begin{array}{r}5.96 \\
3.67 \\
1.86 \\
1.86 \\
2.88 \\
4.68 \\
2.87 \\
5.19 \\
5.80 \\
9.15 \\
13.30 \\
7.68 \\
2.75 \\
5.86 \\
14.84\end{array}$ & $\begin{array}{r}6.12 \\
4.06 \\
1.89 \\
1.89 \\
3.29 \\
5.20 \\
2.91 \\
7.20 \\
8.04 \\
10.15 \\
13.40 \\
8.85 \\
3.09 \\
6.66 \\
14.90\end{array}$ & $\begin{array}{r}5.95 \\
3.73 \\
1.89 \\
\overline{1} \\
1.89 \\
2.83 \\
4.91 \\
3.06 \\
6.38 \\
7.13 \\
10.59 \\
15.42 \\
8.48 \\
2.37 \\
5.89 \\
15.22\end{array}$ \\
\hline 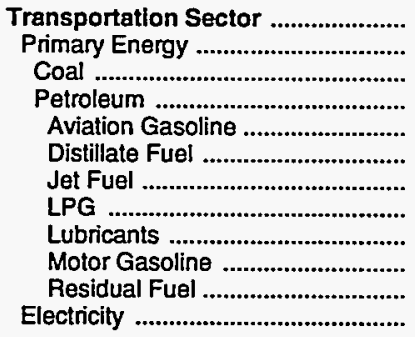 & $\begin{array}{r}2.19 \\
2.19 \\
2.19 \\
2.17 \\
1.44 \\
.73 \\
1.27 \\
5.08 \\
2.81 \\
.29 \\
-\end{array}$ & $\begin{array}{r}3.79 \\
3.79 \\
.53 \\
3.79 \\
3.45 \\
2.89 \\
2.03 \\
2.51 \\
7.48 \\
4.39 \\
1.60 \\
-\end{array}$ & $\begin{array}{r}8.39 \\
8.39 \\
-\overline{1} \\
8.39 \\
9.02 \\
7.72 \\
6.46 \\
5.46 \\
14.36 \\
9.80 \\
3.14 \\
-\end{array}$ & $\begin{array}{r}8.19 \\
8.19 \\
- \\
8.19 \\
9.99 \\
7.24 \\
5.90 \\
11.24 \\
17.61 \\
9.03 \\
3.76 \\
22.04\end{array}$ & $\begin{array}{r}6.12 \\
6.12 \\
-\overline{12} \\
6.12 \\
8.41 \\
6.24 \\
4.19 \\
10.15 \\
15.59 \\
6.70 \\
2.00 \\
19.81\end{array}$ & $\begin{array}{r}6.42 \\
6.42 \\
- \\
6.42 \\
7.55 \\
6.66 \\
4.07 \\
9.27 \\
12.70 \\
7.11 \\
2.82 \\
19.74\end{array}$ & $\begin{array}{r}6.28 \\
6.28 \\
- \\
6.28 \\
7.41 \\
6.25 \\
3.82 \\
9.94 \\
14.61 \\
7.12 \\
1.94 \\
19.83\end{array}$ & $\begin{array}{r}6.79 \\
6.79 \\
- \\
6.79 \\
8.28 \\
6.64 \\
4.43 \\
9.15 \\
13.30 \\
7.68 \\
2.33 \\
19.67\end{array}$ & $\begin{array}{r}7.93 \\
7.93 \\
- \\
7.93 \\
9.32 \\
8.21 \\
5.64 \\
10.15 \\
13.40 \\
8.85 \\
2.56 \\
20.05\end{array}$ & $\begin{array}{r}7.62 \\
7.62 \\
7.62 \\
7.71 \\
7.75 \\
4.93 \\
10.59 \\
15.42 \\
8.48 \\
1.95 \\
20.07\end{array}$ \\
\hline 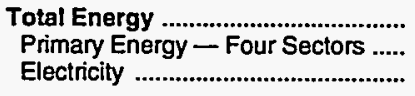 & $\begin{array}{l}2.35 \\
1.74 \\
5.67\end{array}$ & $\begin{array}{r}4.62 \\
3.28 \\
10.46\end{array}$ & $\begin{array}{r}8.84 \\
7.16 \\
16.24\end{array}$ & $\begin{array}{r}10.84 \\
7.46 \\
22.59\end{array}$ & $\begin{array}{r}9.31 \\
5.78 \\
21.37\end{array}$ & $\begin{array}{r}9.35 \\
6.02 \\
20.55\end{array}$ & $\begin{array}{r}9.22 \\
5.81 \\
20.70\end{array}$ & $\begin{array}{r}9.70 \\
6.26 \\
20.45\end{array}$ & $\begin{array}{r}10.54 \\
7.23 \\
20.62\end{array}$ & $\begin{array}{r}10.55 \\
6.90 \\
20.99\end{array}$ \\
\hline $\begin{array}{l}\text { Electric Utility Sectora } \\
\text { Coal } \\
\text { Natural Gas } \\
\text { Petroleum } \\
\text { Heavy Oib } \\
\text { Light Oib } \\
\text { Petroleum Coke } \\
\text { Nuclear Fuel } \\
\text { Biomass Fuels }\end{array}$ & $\begin{array}{r}.33 \\
.31 \\
.35 \\
.33 \\
.33 \\
.36 \\
- \\
- \\
-\end{array}$ & $\begin{array}{r}1.35 \\
1.01 \\
.72 \\
1.88 \\
1.85 \\
2.21 \\
\overline{-17} \\
-\end{array}$ & $\begin{array}{r}2.40 \\
1.80 \\
1.53 \\
3.80 \\
3.72 \\
5.76 \\
-35 \\
.\end{array}$ & $\begin{array}{r}2.21 \\
2.12 \\
3.25 \\
3.96 \\
3.87 \\
5.71 \\
-65 \\
.65\end{array}$ & $\begin{array}{r}1.76 \\
1.93 \\
2.08 \\
2.18 \\
2.15 \\
3.41 \\
- \\
.73 \\
-\end{array}$ & $\begin{array}{r}1.96 \\
1.81 \\
2.71 \\
2.94 \\
2.91 \\
3.93 \\
-69 \\
-\end{array}$ & $\begin{array}{r}1.72 \\
1.78 \\
2.10 \\
2.23 \\
2.20 \\
3.54 \\
-94 \\
-94\end{array}$ & $\begin{array}{r}1.90 \\
1.79 \\
2.46 \\
2.77 \\
2.69 \\
4.27 \\
.66 \\
-\end{array}$ & $\begin{array}{r}1.98 \\
1.85 \\
2.53 \\
3.08 \\
2.99 \\
5.09 \\
- \\
.64 \\
-\end{array}$ & $\begin{array}{r}1.81 \\
1.86 \\
2.13 \\
2.31 \\
2.23 \\
4.63 \\
- \\
.66 \\
-\end{array}$ \\
\hline Primary Energy - Flve Sectors ${ }^{c}$.. & 1.20 & 2.44 & 5.12 & 5.17 & 3.96 & 4.19 & 3.92 & 4.23 & 4.81 & 4.43 \\
\hline
\end{tabular}

a There are no direct fuel costs for hydroelectric, geothermal, centralized solar, or wind energy.

b Heavy oil includes Grade Nos. 4, 5, and 6, and residual fuel oils. Light oil includes Grade No. 2 heating oil, kerosene, and jet fuel.

c Biomass fuels are not included, except those consumed at electric utilities and those added to motor gasoline

-No consumption, including cases where adjustments were made. See "Consumption Adjustments for Process Fuel and Intermediate Products."

Sources: Data sources, estimation procedures, and assumptions are described in the "Documentation" section of this report. 


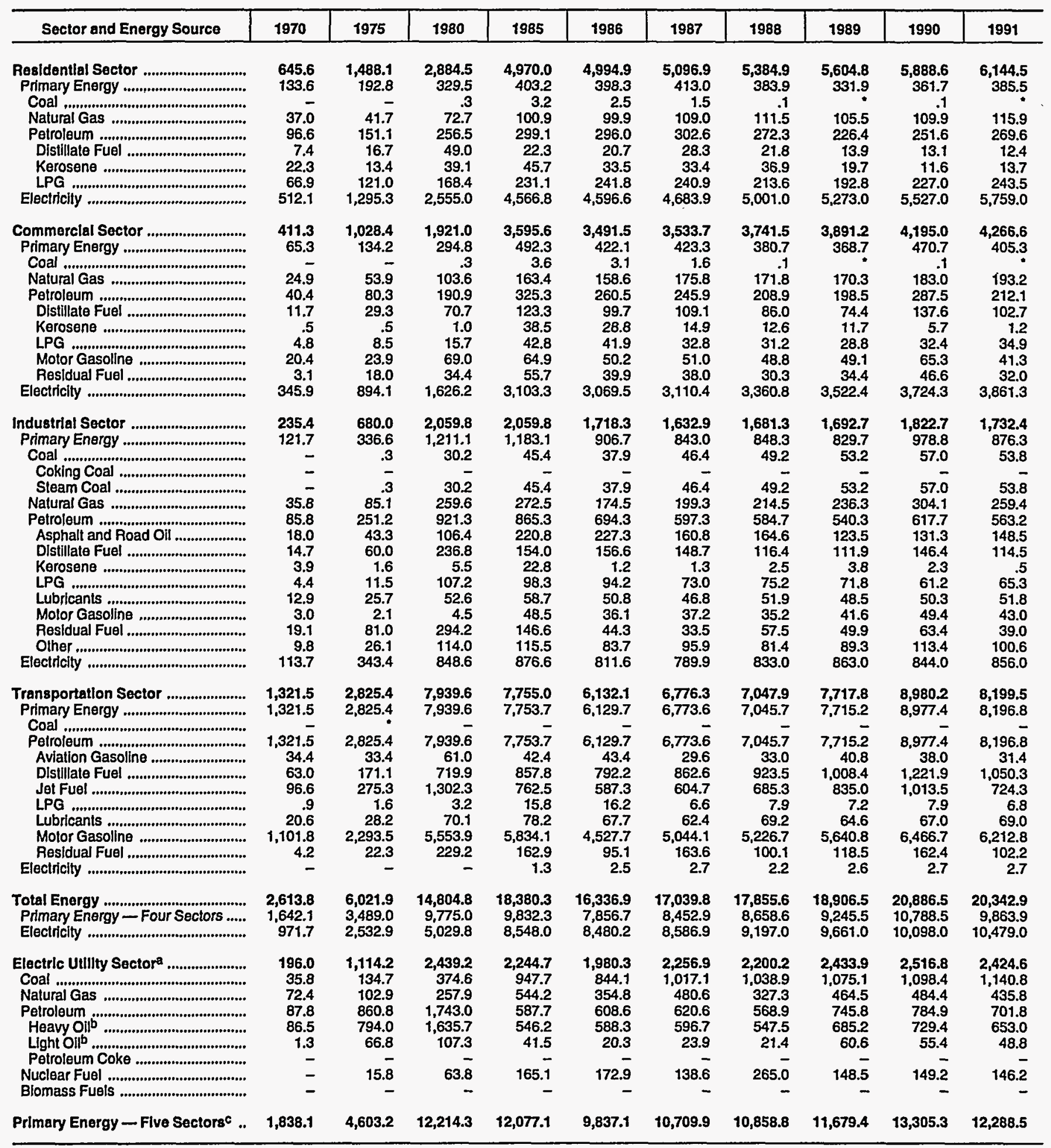

a There are no direct fuel costs for hydroelectric, geothermal, centralized solar, or wind energy.

b Heavy oil includes Grade Nos. 4, 5, and 6, and residual fuel oils. Light oil includes Grade No. 2 heating oil, kerosene, and jet fuel.

$c$ Biomass fuels are not included, except those consumed at electric utilities and those added to motor gasoline.

- No consumption, including cases where adjustments were made. See "Consumption Adjustments for Process Fuel and Intermediate Products."

-Value less than 0.05 million dollars.

Note: Totals may not equal sum of components due to independent rounding.

Sources: Data sources, estimation procedures, and assumptions are described in the "Documentation" section of this report. 


\section{G Energy Price and Expenditure Estimates by Source, Georgia \\ E $1970,1975,1980$, and 1985-1991}

\begin{tabular}{|c|c|c|c|c|c|c|c|c|c|c|}
\hline Energy Source & 1970 & 1975 & 1980 & 1985 & 1986 & 1987 & 1988 & 1989 & 1990 & 1991 \\
\hline & \multicolumn{10}{|c|}{ Prices in Dollars per Million Btu } \\
\hline Coal & 0.39 & 0.95 & 1.50 & 1.87 & 1.81 & 1.79 & 1.75 & 1.75 & 1.79 & 1.80 \\
\hline 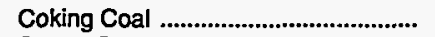 & - & - & - & - & - & - & - & - & - & - \\
\hline Steam Coal & .39 & .95 & 1.50 & 1.87 & 1.81 & 1.79 & 1.75 & 1.75 & 1.79 & 1.80 \\
\hline Natural Gas & .58 & 1.02 & 3.06 & 5.25 & 4.95 & 4.78 & 4.72 & 4.77 & 4.80 & 4.65 \\
\hline Petroleum & 1.94 & 3.65 & 8.02 & 7.56 & 5.71 & 5.95 & 5.74 & 6.35 & 7.33 & 7.16 \\
\hline 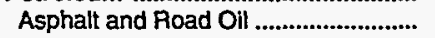 & .76 & 1.74 & 3.72 & 4.97 & 4.19 & 3.21 & 3.15 & 2.85 & 2.86 & 3.18 \\
\hline 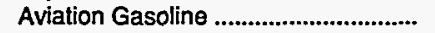 & 2.17 & 3.45 & 9.02 & 9.99 & 8.41 & 7.55 & 7.41 & 8.28 & 9.32 & 8.71 \\
\hline 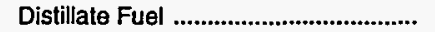 & 1.06 & 2.71 & 7.00 & 6.68 & 5.43 & 5.71 & 5.37 & 5.79 & 7.16 & 6.74 \\
\hline Jet Fuel & .73 & 2.03 & 6.46 & 5.66 & 3.51 & 3.77 & 3.54 & 4.12 & 5.45 & 4.61 \\
\hline Kerosene & .85 & 2.38 & 6.51 & 9.35 & 6.14 & 6.26 & 6.22 & 6.17 & 7.60 & 7.05 \\
\hline LPG & 2.01 & 3.58 & 6.40 & 9.48 & 8.82 & 8.77 & 8.37 & 9.09 & 10.16 & 10.58 \\
\hline 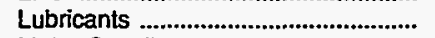 & 5.08 & 7.48 & 14.36 & 17.61 & 15.59 & 12.70 & 14.61 & 13.30 & 13.40 & 15.42 \\
\hline 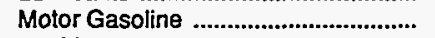 & 2.80 & 4.73 & 9.91 & 8.76 & 6.29 & 6.66 & 6.51 & 7.12 & 8.24 & 7.95 \\
\hline 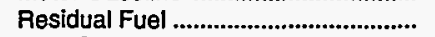 & .38 & 1.70 & 3.27 & 4.13 & 2.23 & 2.90 & 2.29 & 2.61 & 2.52 & 2.11 \\
\hline Other ${ }^{2}$ & 2.53 & 3.33 & 8.56 & 9.22 & 6.46 & 7.19 & 6.14 & 7.35 & 7.56 & 7.08 \\
\hline Nuclear Fuel & - & .13 & .45 & .72 & .84 & .94 & 1.01 & 1.00 & .87 & .73 \\
\hline Blomass Fuels at Utilitles .................. & - & - & - & - & - & - & - & - & - & - \\
\hline Primary Energy - Five Sectors ${ }^{b} \ldots$ & 1.24 & 2.28 & 4.55 & 4.63 & 3.89 & 3.85 & 3.81 & 3.91 & 4.26 & 4.17 \\
\hline $\begin{array}{l}\text { Electric Utility Fuelc } \\
\text { Electricity Purchased by End Users }\end{array}$ & $\begin{array}{r}.35 \\
4.58\end{array}$ & $\begin{array}{r}.91 \\
8.93\end{array}$ & $\begin{array}{r}1.38 \\
12.75\end{array}$ & $\begin{array}{r}1.73 \\
17.09\end{array}$ & $\begin{array}{r}1.71 \\
16.91\end{array}$ & $\begin{array}{r}1.63 \\
17.44\end{array}$ & $\begin{array}{r}1.61 \\
18.29\end{array}$ & $\begin{array}{r}1.53 \\
18.70\end{array}$ & $\begin{array}{r}1.53 \\
19.25\end{array}$ & $\begin{array}{r}1.46 \\
19.28\end{array}$ \\
\hline \multirow[t]{2}{*}{ 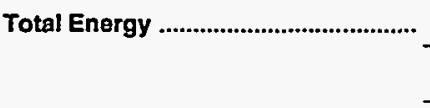 } & 1.86 & 3.68 & 7.34 & 8.48 & 7.43 & 7.57 & 7.56 & 8.10 & 8.83 & 8.76 \\
\hline & \multicolumn{10}{|c|}{ Expenditures In Millions of Dollars } \\
\hline Coal & 75.7 & 295.9 & 784.3 & $1,359.4$ & $1,249.4$ & $1,267.9$ & $1,220.7$ & $1,182.3$ & $1,282.3$ & $1,161.0$ \\
\hline 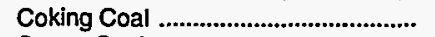 & - & & - & - & - & - & - & - & - & \\
\hline 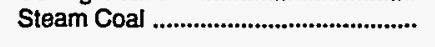 & 75.7 & 295.9 & 784.3 & $1,359.4$ & $1,249.4$ & $1,267.9$ & $1,220.7$ & $1,182.3$ & $1,282.3$ & $1,161.0$ \\
\hline 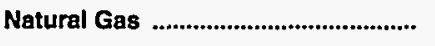 & 195.4 & 336.1 & 970.9 & $1,467.5$ & $1,370.2$ & $1,437.2$ & $1,508.1$ & $1,489.8$ & $1,466.3$ & $1,471.1$ \\
\hline 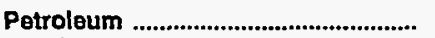 & $1,070.5$ & $2,396.2$ & $5,638.0$ & $5,799.6$ & $4,340.2$ & $4,728.1$ & $4,762.7$ & $5,121.2$ & $6,017.1$ & $5,605.0$ \\
\hline 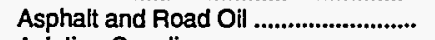 & 19.7 & 48.4 & 118.2 & 151.2 & 156.7 & 127.4 & 126.4 & 93.9 & 121.2 & 109.6 \\
\hline 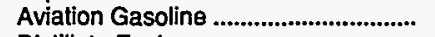 & 6.6 & 6.9 & 17.6 & 10.7 & 10.8 & 8.3 & 8.5 & 8.8 & 9.2 & 8.0 \\
\hline 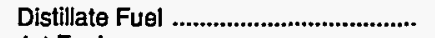 & 79.1 & 254.0 & 792.6 & 924.0 & 776.2 & 865.8 & 872.4 & 948.5 & $1,188.7$ & $1,057.5$ \\
\hline 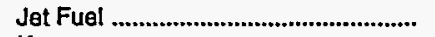 & 42.8 & 147.4 & 598.1 & 518.0 & 351.4 & 419.2 & 405.8 & 405.7 & 567.9 & 373.6 \\
\hline 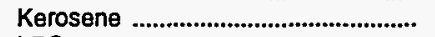 & 2.2 & 3.3 & 20.4 & 19.5 & 11.8 & 7.8 & 11.9 & 11.0 & 8.5 & 7.8 \\
\hline LPG & 56.5 & 108.2 & 175.0 & 231.5 & 202.9 & 202.5 & 202.4 & 242.2 & 216.8 & 256.4 \\
\hline 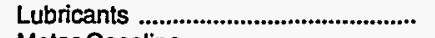 & 31.5 & 51.1 & 108.8 & 121.4 & 105.1 & 96.9 & 107.4 & 100.3 & 104.0 & 107.1 \\
\hline Motor Gasoline & 795.3 & $1,628.9$ & $3,409.4$ & $3,356.1$ & $2,543.8$ & $2,796.6$ & $2,859.1$ & $3,122.4$ & $3,580.3$ & $3,493.7$ \\
\hline $\begin{array}{l}\text { Residual Fuel } \\
\text { Other }\end{array}$ & $\begin{array}{l}24.5 \\
12.4\end{array}$ & $\begin{array}{r}115.5 \\
32.3\end{array}$ & $\begin{array}{l}185.0 \\
212.9\end{array}$ & $\begin{array}{l}285.0 \\
182.0\end{array}$ & $\begin{array}{r}46.8 \\
134.7\end{array}$ & $\begin{array}{r}53.7 \\
149.9\end{array}$ & $\begin{array}{r}40.8 \\
127.9\end{array}$ & $\begin{array}{r}38.9 \\
149.6\end{array}$ & $\begin{array}{r}51.4 \\
169.0\end{array}$ & $\begin{array}{r}32.8 \\
158.7\end{array}$ \\
\hline 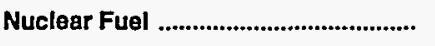 & - & 4.3 & 41.7 & 79.4 & 66.0 & 154.6 & 164.6 & 267.5 & 230.0 & 205.1 \\
\hline Blomass Fuels at Utilitles ................. & - & - & - & - & - & - & - & - & - & - \\
\hline Primary Energy - Flve Sectors ${ }^{b}$.... & $1,341.6$ & $3,032.5$ & $7,434.9$ & $8,705.8$ & $7,025.7$ & $7,587.8$ & $7,656.0$ & $8,060.8$ & $8,995.7$ & $8,442.3$ \\
\hline $\begin{array}{l}\text { Electric Utility Fuelc ......................... } \\
\text { Electricity Purchased by End Users }\end{array}$ & $\begin{array}{r}-88.1 \\
491.7\end{array}$ & $\begin{array}{r}-372.6 \\
1,265.9\end{array}$ & $\begin{array}{r}-837.7 \\
2,227.3\end{array}$ & $\begin{array}{r}-1,379.9 \\
3,690.2\end{array}$ & $\begin{array}{r}-1,256.5 \\
3,916.7\end{array}$ & $\begin{array}{r}-1,347.1 \\
4,236.8\end{array}$ & $\begin{array}{r}-1,305.3 \\
4,608.9\end{array}$ & $\begin{array}{r}-1,372.2 \\
4,885.1\end{array}$ & $\begin{array}{r}-1,425.9 \\
5,254.7\end{array}$ & $\begin{array}{r}-1,280.3 \\
5,317.3\end{array}$ \\
\hline 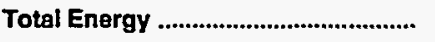 & $1,745.3$ & $3,925.8$ & $8,824.5$ & $11,016.0$ & $9,685.8$ & $10,477.5$ & $10,959.6$ & $11,573.8$ & $12,824.4$ & $12,479.3$ \\
\hline
\end{tabular}

a Includes petroleum coke used at electric utilities.

biomass fuels are not included, except those consumed at electric utilities and those added to motor gasoline.

c There are no direct fuel costs for hydroelectric, geothermal, centralized solar, or wind energy.

-No consumption, including cases where adjustments were made. See "Consumption Adjustments for Process Fuel and Intermediate Products."

Note: Expenditure totals may not equal sum of components due to independent rounding.

Sources: Data sources, estimation procedures, and assumptions are described in the "Documentation" section of this report. 


\begin{tabular}{|c|c|c|c|c|c|c|c|c|c|c|}
\hline Sector and Energy Source & 1970 & 1975 & 1980 & 1985 & 1986 & 1987 & 1988 & 1989 & 1990 & -1991 \\
\hline 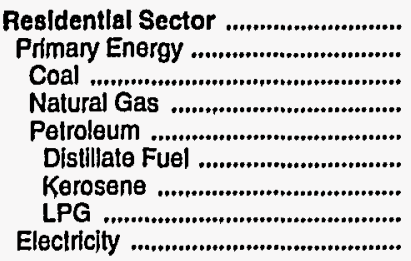 & $\begin{array}{l}2.33 \\
1.21 \\
1.00 \\
1.02 \\
2.22 \\
1.24 \\
1.48 \\
2.35 \\
5.18\end{array}$ & $\begin{array}{l}4.35 \\
1.89 \\
3.23 \\
1.46 \\
4.20 \\
2.61 \\
3.35 \\
4.40 \\
9.01\end{array}$ & $\begin{array}{r}7.88 \\
4.17 \\
3.12 \\
3.57 \\
7.53 \\
6.92 \\
8.77 \\
7.64 \\
13.85\end{array}$ & $\begin{array}{r}12.10 \\
6.87 \\
3.31 \\
6.42 \\
9.11 \\
7.51 \\
10.25 \\
9.22 \\
18.91\end{array}$ & $\begin{array}{r}12.58 \\
6.78 \\
3.00 \\
6.46 \\
8.63 \\
5.43 \\
7.41 \\
9.26 \\
19.67\end{array}$ & $\begin{array}{r}12.28 \\
6.51 \\
2.96 \\
6.18 \\
8.68 \\
5.45 \\
7.44 \\
9.22 \\
19.80\end{array}$ & $\begin{array}{r}12.38 \\
6.31 \\
2.95 \\
6.07 \\
8.04 \\
5.54 \\
7.56 \\
8.36 \\
20.58\end{array}$ & $\begin{array}{r}12.98 \\
6.59 \\
3.05 \\
6.09 \\
9.61 \\
5.34 \\
7.29 \\
10.21 \\
21.18\end{array}$ & $\begin{array}{r}14.28 \\
7.05 \\
3.10 \\
6.64 \\
9.77 \\
6.70 \\
9.15 \\
10.16 \\
21.86\end{array}$ & $\begin{array}{r}14.10 \\
7.00 \\
2.94 \\
6.52 \\
10.18 \\
6.25 \\
8.53 \\
10.57 \\
21.98\end{array}$ \\
\hline 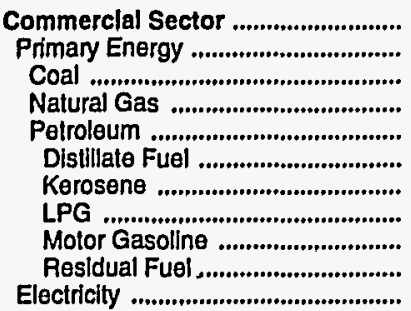 & $\begin{array}{r}2.60 \\
.84 \\
.50 \\
.72 \\
1.44 \\
.97 \\
.63 \\
1.58 \\
2.80 \\
.32 \\
5.85\end{array}$ & $\begin{array}{r}4.98 \\
1.36 \\
1.31 \\
1.07 \\
2.85 \\
2.25 \\
2.22 \\
2.83 \\
4.73 \\
1.73 \\
10.79\end{array}$ & $\begin{array}{r}7.69 \\
3.47 \\
1.60 \\
3.12 \\
7.00 \\
6.31 \\
6.06 \\
5.27 \\
9.91 \\
3.44 \\
14.64\end{array}$ & $\begin{array}{r}12.23 \\
5.83 \\
1.80 \\
5.57 \\
6.81 \\
6.46 \\
7.24 \\
9.84 \\
8.76 \\
4.20 \\
19.94\end{array}$ & $\begin{array}{r}11.32 \\
5.12 \\
1.76 \\
5.46 \\
4.09 \\
3.55 \\
4.24 \\
8.26 \\
6.29 \\
2.46 \\
18.17\end{array}$ & $\begin{array}{r}12.05 \\
5.06 \\
1.73 \\
5.26 \\
4.49 \\
3.92 \\
4.56 \\
8.12 \\
6.66 \\
2.90 \\
19.81\end{array}$ & $\begin{array}{r}12.77 \\
5.06 \\
1.75 \\
5.32 \\
4.29 \\
3.45 \\
4.12 \\
8.40 \\
6.51 \\
2.37 \\
20.90\end{array}$ & $\begin{array}{r}13.79 \\
5.27 \\
1.73 \\
5.33 \\
5.04 \\
3.74 \\
4.72 \\
7.64 \\
7.12 \\
2.72 \\
21.33\end{array}$ & $\begin{array}{r}14.58 \\
5.77 \\
1.78 \\
5.61 \\
6.53 \\
5.09 \\
5.62 \\
10.15 \\
8.24 \\
3.04 \\
21.58\end{array}$ & $\begin{array}{r}14.78 \\
5.65 \\
1.79 \\
5.52 \\
6.39 \\
4.11 \\
4.98 \\
10.59 \\
7.95 \\
2.26 \\
21.68\end{array}$ \\
\hline 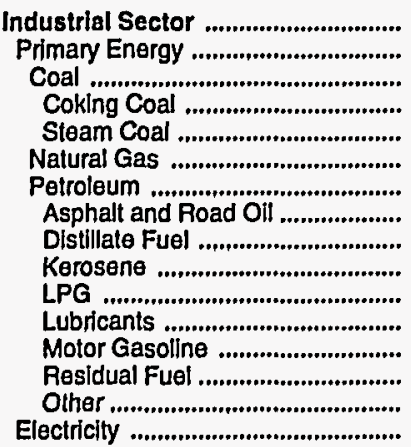 & $\begin{array}{r}.86 \\
.58 \\
.50 \\
- \\
.50 \\
.40 \\
.81 \\
.76 \\
.58 \\
.63 \\
1.58 \\
5.08 \\
2.80 \\
.40 \\
2.53 \\
2.91\end{array}$ & $\begin{array}{r}2.30 \\
1.43 \\
1.31 \\
- \\
1.31 \\
.82 \\
2.23 \\
1.74 \\
2.05 \\
2.22 \\
2.83 \\
7.48 \\
4.73 \\
1.69 \\
3.33 \\
7.33\end{array}$ & $\begin{array}{r}4.97 \\
3.81 \\
1.60 \\
-\overline{1} \\
1.60 \\
2.75 \\
5.36 \\
3.72 \\
5.44 \\
6.06 \\
5.27 \\
14.36 \\
9.91 \\
3.44 \\
8.56 \\
10.43\end{array}$ & $\begin{array}{r}6.47 \\
4.89 \\
1.80 \\
- \\
1.80 \\
4.41 \\
6.14 \\
4.97 \\
6.48 \\
7.24 \\
9.84 \\
17.61 \\
8.76 \\
4.20 \\
9.22 \\
13.09\end{array}$ & $\begin{array}{r}6.03 \\
4.00 \\
1.76 \\
- \\
1.76 \\
3.80 \\
5.18 \\
4.19 \\
3.80 \\
4.24 \\
8.26 \\
15.59 \\
6.29 \\
2.46 \\
6.46 \\
12.95\end{array}$ & $\begin{array}{r}5.83 \\
3.78 \\
1.73 \\
- \\
1.73 \\
3.57 \\
5.00 \\
3.21 \\
4.08 \\
4.56 \\
8.12 \\
12.70 \\
6.66 \\
2.90 \\
7.19 \\
13.01\end{array}$ & $\begin{array}{r}5.84 \\
3.64 \\
1.75 \\
- \\
1.75 \\
3.52 \\
4.72 \\
3.15 \\
3.68 \\
4.12 \\
8.40 \\
14.61 \\
6.51 \\
2.37 \\
6.14 \\
13.68\end{array}$ & $\begin{array}{r}6.03 \\
3.81 \\
1.73 \\
- \\
1.73 \\
3.64 \\
5.07 \\
2.85 \\
4.23 \\
4.72 \\
7.64 \\
13.30 \\
7.12 \\
2.72 \\
7.35 \\
13.72\end{array}$ & $\begin{array}{r}6.03 \\
3.84 \\
1.78 \\
- \\
1.78 \\
3.50 \\
5.29 \\
2.86 \\
5.03 \\
5.62 \\
10.15 \\
13.40 \\
8.24 \\
3.04 \\
7.56 \\
14.16\end{array}$ & $\begin{array}{r}6.01 \\
3.74 \\
1.79 \\
-\overline{1} \\
3.79 \\
3.25 \\
5.51 \\
3.18 \\
4.46 \\
4.98 \\
10.59 \\
15.42 \\
7.95 \\
2.26 \\
7.08 \\
14.01\end{array}$ \\
\hline 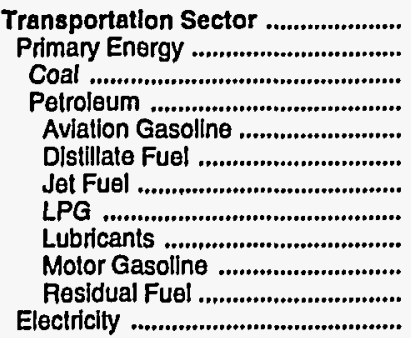 & $\begin{array}{r}2.33 \\
2.33 \\
.50 \\
2.33 \\
2.17 \\
1.32 \\
.73 \\
1.58 \\
5.08 \\
2.80 \\
.28 \\
-\end{array}$ & $\begin{array}{l}4.11 \\
4.11 \\
1.31 \\
4.11 \\
3.45 \\
3.02 \\
2.03 \\
2.83 \\
7.48 \\
4.73 \\
1.52 \\
-\end{array}$ & $\begin{array}{r}8.73 \\
8.73 \\
- \\
8.73 \\
9.02 \\
7.48 \\
6.46 \\
5.27 \\
14.36 \\
9.91 \\
2.91 \\
10.06\end{array}$ & $\begin{array}{r}7.90 \\
7.90 \\
- \\
7.90 \\
9.99 \\
6.74 \\
5.66 \\
9.84 \\
17.61 \\
8.76 \\
3.38 \\
12.92\end{array}$ & $\begin{array}{r}5.79 \\
5.79 \\
- \\
5.79 \\
8.41 \\
5.88 \\
3.51 \\
8.26 \\
15.59 \\
6.29 \\
1.67 \\
13.59\end{array}$ & $\begin{array}{r}6.09 \\
6.08 \\
- \\
6.08 \\
7.55 \\
6.09 \\
3.77 \\
8.12 \\
12.70 \\
6.66 \\
2.81 \\
21.39\end{array}$ & $\begin{array}{r}5.90 \\
5.89 \\
- \\
5.89 \\
7.41 \\
5.74 \\
3.54 \\
8.40 \\
14.61 \\
6.51 \\
1.95 \\
21.42\end{array}$ & $\begin{array}{r}6.50 \\
6.50 \\
- \\
6.50 \\
8.28 \\
6.18 \\
4.12 \\
7.64 \\
13.30 \\
7.12 \\
2.34 \\
22.70\end{array}$ & $\begin{array}{r}7.66 \\
7.65 \\
- \\
7.65 \\
9.32 \\
7.67 \\
5.45 \\
10.15 \\
13.40 \\
8.24 \\
1.85 \\
23.84\end{array}$ & $\begin{array}{r}7.37 \\
7.36 \\
- \\
7.36 \\
8.71 \\
7.21 \\
4.61 \\
10.59 \\
15.42 \\
7.95 \\
1.94 \\
24.88\end{array}$ \\
\hline $\begin{array}{l}\text { Total Energy ................................... } \\
\text { Primary Energy - Four Sectors ..... } \\
\text { Electricity ......................................... }\end{array}$ & $\begin{array}{l}1.86 \\
1.51 \\
4.58\end{array}$ & $\begin{array}{l}3.68 \\
2.88 \\
8.93\end{array}$ & $\begin{array}{r}7.34 \\
6.42 \\
12.75\end{array}$ & $\begin{array}{r}8.48 \\
6.76 \\
17.09\end{array}$ & $\begin{array}{r}7.43 \\
5.38 \\
16.91\end{array}$ & $\begin{array}{r}7.57 \\
5.47 \\
17.44\end{array}$ & $\begin{array}{r}7.56 \\
5.30 \\
18.29\end{array}$ & $\begin{array}{r}8.10 \\
5.73 \\
18.70\end{array}$ & $\begin{array}{r}8.83 \\
6.42 \\
19.25\end{array}$ & $\begin{array}{r}8.76 \\
6.23 \\
19.28\end{array}$ \\
\hline 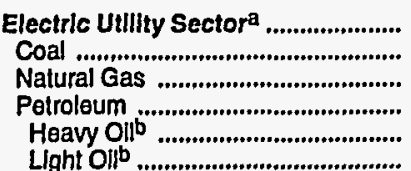 & $\begin{array}{l}.35 \\
.38 \\
.29 \\
.31 \\
.31 \\
.39\end{array}$ & $\begin{array}{r}.91 \\
.93 \\
.71 \\
1.85 \\
1.74 \\
2.30\end{array}$ & $\begin{array}{l}1.38 \\
1.50 \\
2.56 \\
4.48 \\
3.47 \\
6.22\end{array}$ & $\begin{array}{l}1.73 \\
1.88 \\
4.30 \\
5.22 \\
3.59 \\
5.65\end{array}$ & $\begin{array}{l}1.71 \\
1.81 \\
2.00 \\
2.32 \\
1.82 \\
3.46\end{array}$ & $\begin{array}{l}1.63 \\
1.79 \\
3.71 \\
3.42 \\
3.09 \\
3.90\end{array}$ & $\begin{array}{l}1.61 \\
1.75 \\
2.73 \\
2.95 \\
2.24 \\
3.39\end{array}$ & $\begin{array}{l}1.53 \\
1.75 \\
3.16 \\
4.37 \\
2.77 \\
4.52\end{array}$ & $\begin{array}{l}1.53 \\
1.79 \\
2.97 \\
4.26 \\
2.18 \\
5.44\end{array}$ & $\begin{array}{l}1.46 \\
1.80 \\
2.76 \\
4.49 \\
2.22 \\
4.74\end{array}$ \\
\hline Potroleum Coke .............................. & - & - & $\overline{1}$ & - & $\overline{0}$ & $\overline{0}$ & - & - & - & - \\
\hline 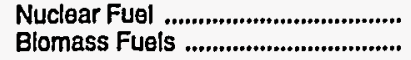 & $\overline{-}$ & .13 & .45 & .72 & .84 & $\begin{array}{r}.94 \\
-\end{array}$ & $\begin{array}{r}1.01 \\
-\end{array}$ & 1.00 & $\begin{array}{r}.87 \\
-\end{array}$ & .73 \\
\hline Prlmary Energy - Flve Sectorsc .. & 1.24 & 2.28 & 4.55 & 4.63 & 3.89 & 3.85 & 3.81 & 3.91 & 4.26 & 4.17 \\
\hline
\end{tabular}

a There are no direct fuel costs for hydroelectric, geothermal, centralized solar, or wind energy.

b Heavy oil includes Grade Nos. 4, 5, and 6, and residual fuel oils. Light oil includes Grade No. 2 heating oil, kerosene, and jet fuel.

c Blomass fuels are not included, except those consumed at electric utilities and those added to motor gasoline.

-No consumption, Including cases where adjustments were made. See "Consumption Adjustments for Process Fuel and Intermediate Products."

Sources: Data sources, estimation procedures, and assumptions are described in the "Documentation" section of this report. 


\section{G Energy Expenditure Estimates by Sector, Georgia \\ E 1970, 1975, 1980, and 1985-1991 \\ O (Million Dollars)}

\section{$\mathbf{R}$

\begin{tabular}{|c|}
\hline Sector and Energy Source \\
\hline $\begin{array}{l}\text { Resldentlal Sector } \\
\text { Primary Energy } \\
\text { Coal } \\
\text { Natural Gas } \\
\text { Petroleum } \\
\text { Distillate Fuel } \\
\text { Kerosene } \\
\text { LPG }\end{array}$ \\
\hline
\end{tabular}

Commercial Sector

Primary Energy

Coal

Natural Gas

Petroleum ...

Distillate Fuel

Kerosene

LPG

Motor Gasoline

Residual Fuel .

Electricity

Industrial Sector

Primary Energy

Coal

Coking Coal

Steam Coal

Natural Gas

Petroleum

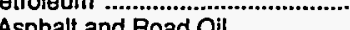

il ...................

Distillate Fuel

Kerosene

LPG ........

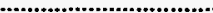

Motor Gasoline

Residual Fue

Other .

Electricity .

Transportation Sector .......................

Primary Energy

Coal

Petroleum

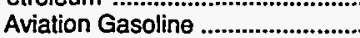

Distillate Fuel .

Jet Fuel

LPG

Lubricants

Motor Gasoline

Residual Fuel

Electricity

Total Energy .

Primary Energy - Four Sectors

Electricity

Electric Utility Sector ${ }^{2}$........................

Coal

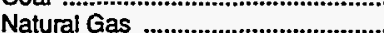

Petroleum

Heavy Oib

Light Oilb

Nuclear Fue!

Biomass Fuels

\begin{tabular}{l|l|l|l|l|l|l|}
\hline 1970 & 1975 & 1980 & 1985 & \\
\hline
\end{tabular}

1970

1975

$$
135
$$

132.3

706.7
1.1

1.1

91.6

39.7

1.8

1.0
36.9

220.7

206.7

43.5

1.0
28.6

28.6
13.9

4.0

.1

5.1
.2

163.1

270.4

162.5

6.0

$6 . \overline{0}$

6.0
58.0

98.6

19.7

13.5

1.1
14.5

14.5

14.6

1.8
21.0

21.0
12.4

107.9

915.2

$1,982.9$

$1,982.8$

915.

6.6
59.6
42.8

16.

16.9
788.3

788.3
.3

6.9
181.7

147.4

1.1
23.4

$1,618.2$

4.1

\begin{tabular}{c|c}
1980 \\
$1,406.8$
\end{tabular}

1985

1986

1987

1988

(2989

\begin{tabular}{|l|l|l|}
\hline 1989 & 1990 & 1991 \\
\hline
\end{tabular}

$2,234.3 \quad 2,463.4$

2,603.5 2,7

2,743.0

$2,866.9$

$2,990.0$

$3,063.3$

$\begin{array}{rr}1.4 & .6 \\ 130.5 & 332.0\end{array}$

717.9

$2,463.4$
731.1

780.2

$\begin{array}{rr}1.1 & .2 \\ 555.0 & 591.3\end{array}$

1.0

638.0

141.3
10.9

.7
672.5
130.8

$\begin{array}{rr}161.8 & 139.7 \\ 15.5 & 11.5\end{array}$

15.0
131.3

11.5
8.5

8.5
119.6
1732.3

5.5
124.9
1.823 .2

99.7
946.6

$1,516.4$

$1,732.3$

$1,823.2$

1,565.1 1,483.8

$\begin{array}{rr}1,565.1 & 1,483.8 \\ 407.6 & 351.9 \\ 1.1 & .2\end{array}$

$1,683.5$

371.4
1.0

294.8

295.1

111.4

58.2

24.7

14.2

12.4
157.5

282.6

69.1
20.5
1.7

18.8

11.9
16.1
131.9

$597 . .5$

$1,157.5$

$1,131.9$

$2,604.7 \quad 2,161.0$

$\begin{array}{rr}1,590.8 & 1,111.2 \\ 69.5 & 77.8\end{array}$

$1,173.0$
26.5

$77 . \overline{8}$

69.5

613.4

151.2

135.3

135.3
2.7

2.7
67.9

61.4

57.5
249.9

249.9
182.0

$1,013.9$

682.6

$1,013.9$

$4,731.6$

$\begin{array}{ll}4,612.0 & 3,577.6 \\ 4,609.6 & 3,574.9\end{array}$

$4,609 . \overline{6}$

$4,731 . \overline{1}$

17.6

616.2

$4,609.6$
10.7

707.4

$3,574 . \overline{9}$

10.8
654.4

654.4

351.4
5.7

7.5

7.5

60.0
$3,284.4$

52.0
$2,493.6$

$2,493.6$
7.1

21.5
2.4

75.6
22.9

22.9
.9

19.4

14.3

18.2

$1,312.1$

2,215.3

$1,117.6$

84.1

$84 . \overline{1}$

501.3

532.3

127.4

84.8

1.5
54.4

49.0

49.0
42.6

42.6
22.7

22.7
149.9

$1,097.7$

$3,975.2$

$3,971.4$

971

$3,971.4$
8.3

8.3
743.7

419.2

3.9
47.9

47.9
$2,739.7$

8.8

3.8
3.7

8.3
8.8
113.6
$1,939.0$

$1,939.0$

$1,853.2$
377.5

37.5
.8

305.0

71.7

24.1

.5

20.1
15.5

11.4

$1,475.7$

2,316.9

$1,127$.

-

91.6

526.1
509.3

126.4

72.8

2.6

64.5

54.3

39.5

21.4

127.9

$4,046.4$

$4,042.1$

$4,042 . \overline{1}$

8.5
761.0

761.0
405.8

4.2

53.1

$2,804.1$

5.5

$1,745.3$

3,925.8

$2,659.9$

$8,824.5 \quad 11,016.0$

$9,685.8 \quad 10,477.5$

$10,959.6$

$0,959.6$

.3
648.0

169.6

8.3

7.3

153.9
$2,049.0$

$1,974.0$

353.8

.4
290.4

290.4
63.1

21.3

2.0
20.3

15.1

4.4
620.2

2,378.8

$2,378.8$
$1,167.8$

88.0

$88 . \overline{0}$

549.2

530.6

93.9

97.2

1.7
64.7

50.7 


\begin{tabular}{|c|c|c|c|c|c|c|c|c|c|c|}
\hline Energy Source & 1970 & 1975 & 1980 & 1985 & 1986 & 1987 & 1988 & 1989 & 1990 & 1991 \\
\hline & \multicolumn{10}{|c|}{ Prices In Dollars per Million Btu } \\
\hline 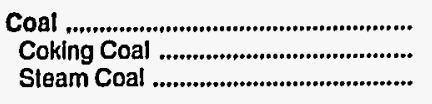 & $\begin{array}{l}- \\
-\end{array}$ & $\begin{array}{l}- \\
-\end{array}$ & $\overline{-}$ & $\frac{2.15}{2.15}$ & $\begin{array}{r}2.09 \\
2.09\end{array}$ & $\begin{array}{r}1.95 \\
1.95\end{array}$ & $\begin{array}{r}1.81 \\
1.81\end{array}$ & $\begin{array}{r}1.76 \\
-\overline{6}\end{array}$ & $\frac{1.82}{1.82}$ & $\begin{array}{r}1.80 \\
1.80\end{array}$ \\
\hline Natural Gas ........................................... & - & - & 13.06 & 14.20 & 11.96 & 11.89 & 11.52 & 11.41 & 12.24 & 14.16 \\
\hline 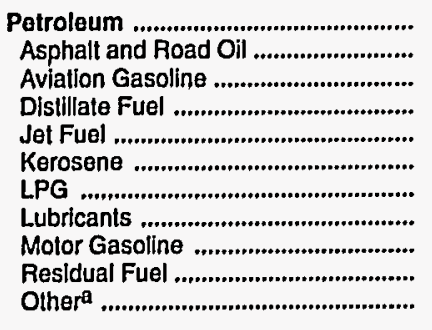 & $\begin{array}{r}1.09 \\
.56 \\
2.17 \\
1.04 \\
.73 \\
.85 \\
2.53 \\
5.08 \\
3.32 \\
.40 \\
.51\end{array}$ & $\begin{array}{l}2.53 \\
1.77 \\
3.45 \\
2.30 \\
2.04 \\
2.50 \\
3.77 \\
7.48 \\
5.44 \\
1.59 \\
1.39\end{array}$ & $\begin{array}{r}6.17 \\
3.61 \\
9.02 \\
6.58 \\
6.21 \\
6.38 \\
6.32 \\
14.36 \\
10.81 \\
3.80 \\
2.49\end{array}$ & $\begin{array}{r}6.79 \\
4.47 \\
9.99 \\
7.88 \\
6.21 \\
6.86 \\
13.79 \\
17.61 \\
11.14 \\
4.81 \\
2.51\end{array}$ & $\begin{array}{r}5.05 \\
4.49 \\
8.41 \\
6.33 \\
4.36 \\
4.18 \\
12.99 \\
15.59 \\
9.63 \\
2.83 \\
1.53\end{array}$ & $\begin{array}{r}5.20 \\
4.24 \\
7.55 \\
6.06 \\
4.30 \\
4.60 \\
12.77 \\
12.70 \\
9.47 \\
3.41 \\
1.50\end{array}$ & $\begin{array}{r}4.84 \\
3.95 \\
7.41 \\
6.26 \\
4.04 \\
4.71 \\
12.61 \\
14.61 \\
9.55 \\
2.80 \\
1.45\end{array}$ & $\begin{array}{r}5.38 \\
3.18 \\
8.28 \\
6.88 \\
4.59 \\
5.64 \\
14.94 \\
13.30 \\
10.42 \\
3.20 \\
1.74\end{array}$ & $\begin{array}{r}6.50 \\
3.15 \\
9.32 \\
8.09 \\
5.99 \\
6.77 \\
15.55 \\
13.40 \\
11.71 \\
4.01 \\
2.02\end{array}$ & $\begin{array}{r}5.93 \\
3.29 \\
8.71 \\
7.97 \\
5.17 \\
6.22 \\
15.30 \\
15.42 \\
10.40 \\
3.21 \\
1.72\end{array}$ \\
\hline Nucloar Fuel ........................................ & - & - & - & - & - & - & - & - & - & - \\
\hline Biomass Fuels at UtIlitles .................. & .65 & .92 & - & .79 & - & - & - & $(0)$ & $(d)$ & - \\
\hline Primary Energy — Five Sectors ${ }^{b}$.... & 1.09 & 2.53 & 6.25 & 6.85 & 5.13 & 5.26 & 4.90 & 5.43 & 6.55 & 6.01 \\
\hline $\begin{array}{l}\text { Electric Utility Fuejc ........................... } \\
\text { Electricity Purchased by End Users }\end{array}$ & $\begin{array}{r}.41 \\
6.98\end{array}$ & $\begin{array}{r}1.58 \\
12.80\end{array}$ & $\begin{array}{r}3.97 \\
22.01\end{array}$ & $\begin{array}{r}4.94 \\
29.81\end{array}$ & $\begin{array}{r}2.98 \\
23.66\end{array}$ & $\begin{array}{r}3.54 \\
24.49\end{array}$ & $\begin{array}{r}2.95 \\
22.53\end{array}$ & $\begin{array}{r}3.32 \\
23.76\end{array}$ & $\begin{array}{r}4.33 \\
26.53\end{array}$ & $\begin{array}{r}3.44 \\
27.14\end{array}$ \\
\hline \multirow[t]{2}{*}{ 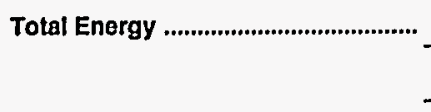 } & 1.73 & 3.95 & 8.71 & 10.30 & 8.49 & 8.63 & 7.84 & 8.60 & 10.03 & 9.91 \\
\hline & \multicolumn{10}{|c|}{ Expenditures in Millions of Dollars } \\
\hline 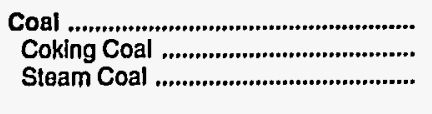 & $\begin{array}{l}- \\
-\end{array}$ & $\overline{-}$ & $\begin{array}{l}- \\
-\end{array}$ & $\frac{2.4}{2.4}$ & $\begin{array}{r}0.8 \\
\overline{8}\end{array}$ & $\begin{array}{r}3.1 \\
-\overline{1}\end{array}$ & $\begin{array}{r}2.2 \\
2 . \overline{2}\end{array}$ & $\begin{array}{r}1.4 \\
\overline{1.4}\end{array}$ & $\begin{array}{r}1.3 \\
\overline{1} \\
1.3\end{array}$ & $\begin{array}{r}1.7 \\
\overline{1.7}\end{array}$ \\
\hline 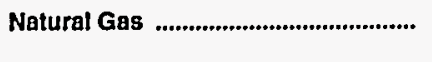 & - & - & 39.4 & 38.1 & 32.0 & 33.1 & 32.4 & 33.2 & 36.5 & 41.2 \\
\hline 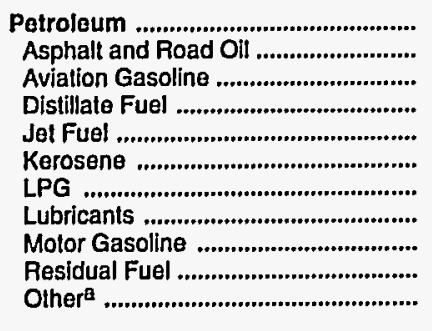 & $\begin{array}{r}207.0 \\
1.4 \\
1.5 \\
9.9 \\
58.4 \\
.7 \\
8.5 \\
2.2 \\
99.2 \\
24.7 \\
.5\end{array}$ & $\begin{array}{r}522.2 \\
4.4 \\
2.0 \\
25.6 \\
170.3 \\
1.1 \\
10.3 \\
4.7 \\
193.5 \\
108.5 \\
1.7\end{array}$ & $\begin{array}{r}1,499.6 \\
6.8 \\
9.1 \\
228.7 \\
492.4 \\
.3 \\
31.1 \\
8.2 \\
410.7 \\
308.6 \\
3.7\end{array}$ & $\begin{array}{r}1,547.4 \\
9.1 \\
7.8 \\
211.5 \\
462.1 \\
.1 \\
6.5 \\
9.1 \\
444.3 \\
395.4 \\
1.5\end{array}$ & $\begin{array}{r}1,100.1 \\
8.1 \\
11.8 \\
168.2 \\
248.5 \\
.1 \\
5.8 \\
7.9 \\
398.5 \\
250.6 \\
.4\end{array}$ & $\begin{array}{r}1,149.7 \\
11.2 \\
9.5 \\
142.6 \\
277.1 \\
.1 \\
7.2 \\
7.3 \\
406.1 \\
287.8 \\
.9\end{array}$ & $\begin{array}{r}1,243.2 \\
9.2 \\
10.5 \\
215.1 \\
271.7 \\
. \\
8.0 \\
8.1 \\
425.8 \\
293.7 \\
1.0\end{array}$ & $\begin{array}{r}1,425.0 \\
6.3 \\
12.0 \\
227.7 \\
341.8 \\
. \\
10.0 \\
7.6 \\
478.8 \\
339.8 \\
1.2\end{array}$ & $\begin{array}{r}1,742.5 \\
8.0 \\
12.8 \\
321.4 \\
425.3 \\
. \\
9.8 \\
7.8 \\
530.3 \\
425.7 \\
1.4\end{array}$ & $\begin{array}{r}1,498.6 \\
8.4 \\
11.5 \\
335.8 \\
333.7 \\
. \\
10.9 \\
8.1 \\
490.0 \\
299.3 \\
.9\end{array}$ \\
\hline 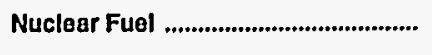 & - & - & - & - & - & - & - & - & - & - \\
\hline Biomess Fuels at Utilities ...................... & .2 & 2 & - & 2 & - & - & - & $(d)$ & (d) & - \\
\hline Primary Energy - Five Sectors ${ }^{b}$.... & 207.2 & 522.4 & $1,538.9$ & $1,588.1$ & $1,132.9$ & $1,185.9$ & $1,277.9$ & $1,459.6$ & $1,780.3$ & $1,541.4$ \\
\hline $\begin{array}{l}\text { Electric Utility Fuel }{ }^{c} \text {............................ } \\
\text { Electricity Purchased by End Users }\end{array}$ & $\begin{array}{r}-17.4 \\
87.4\end{array}$ & $\begin{array}{r}-92.4 \\
225.3\end{array}$ & $\begin{array}{r}-275.8 \\
456.9\end{array}$ & $\begin{array}{r}-342.5 \\
654.7\end{array}$ & $\begin{array}{r}-215.9 \\
538.6\end{array}$ & $\begin{array}{r}-269.6 \\
570.6\end{array}$ & $\begin{array}{r}-240.5 \\
565.5\end{array}$ & $\begin{array}{r}-282.8 \\
629.6\end{array}$ & $\begin{array}{r}-371.8 \\
732.1\end{array}$ & $\begin{array}{r}-271.5 \\
768.9\end{array}$ \\
\hline Total Energy ........................................... & 277.2 & 655.3 & $1,720.0$ & $1,900.4$ & $1,455.6$ & $1,487.0$ & $1,602.9$ & $1,806.3$ & $2,140.5$ & $2,038.8$ \\
\hline
\end{tabular}

a Includes petroleum coke used at electric utilities.

b Biomass fuels are not included, except those consumed at electric utilities and those added to motor gasoline.

c There are no direct fuel costs for hydroelectric, geothermal, centralized solar, or wind energy.

d Utilities used biomass fuels at no charge or received a fee for accepling them.

- No consumption, including cases where adjustments were made. See "Consumption Adjustments for Process Fuel and Intermediate Products."

value less than 0.05 million dollars.

Note: Expenditure totals may not equal sum of components due to independent rounding.

Sources: Data sources, estimation procedures, and assumptions are described in the "Documentation" section of this report. 
W (Dollars per Million Btu)

\begin{tabular}{|c|c|c|c|c|c|c|c|c|c|c|}
\hline Sector and Energy Source & 1970 & 1975 & 1980 & 1985 & 1986 & 1987 & 1988 & 1989 & 1990 & 1991 \\
\hline 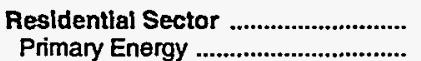 & $\begin{array}{l}7.07 \\
4.11\end{array}$ & $\begin{array}{r}13.13 \\
6.19\end{array}$ & $\begin{array}{l}20.08 \\
12.48\end{array}$ & $\begin{array}{l}30.90 \\
16.13\end{array}$ & $\begin{array}{l}25.56 \\
14.55\end{array}$ & $\begin{array}{l}25.90 \\
14.49\end{array}$ & $\begin{array}{l}24.35 \\
14.16\end{array}$ & $\begin{array}{l}25.71 \\
15.65\end{array}$ & $\begin{array}{l}28.41 \\
16.47\end{array}$ & $\begin{array}{l}29.57 \\
19.93\end{array}$ \\
\hline Coal & - & - & - & - & - & - & - & - & - & - \\
\hline 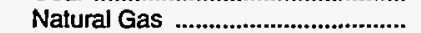 & - & - & 13.50 & 16.74 & 14.82 & 14.89 & 14.55 & 14.50 & 15.37 & 21.23 \\
\hline 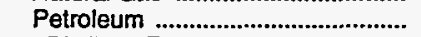 & 4.11 & 6.19 & 11.61 & 15.01 & 14.06 & 13.93 & 13.67 & 17.02 & 17.90 & 18.31 \\
\hline Distillate Fuel ................................... & 1.27 & 2.80 & 6.92 & 7.57 & 5.86 & 5.83 & 5.77 & 6.49 & 7.69 & 7.48 \\
\hline 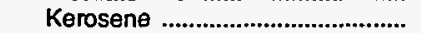 & - & - & - & - & - & - & - & - & - & 14.47 \\
\hline LPG & 4.12 & 6.20 & 11.63 & 15.04 & 14.16 & 14.02 & 13.84 & 17.07 & 17.94 & 18.36 \\
\hline 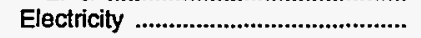 & 8.22 & 14.59 & 23.64 & 33.29 & 27.17 & 27.63 & 25.89 & 27.19 & 30.01 & 30.83 \\
\hline Commercial Sector ........................... & 5.65 & 12.66 & 17.85 & 25.75 & 20.08 & 18.22 & 10.53 & 12.25 & 16.04 & 20.70 \\
\hline 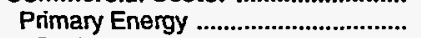 & 1.55 & 3.37 & 8.75 & 11.10 & 8.34 & 7.26 & 4.32 & 4.94 & 6.50 & 8.64 \\
\hline Coal & - & - & - & - & - & - & - & - & - & - \\
\hline 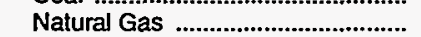 & - & - & 12.70 & 13.34 & 11.08 & 11.01 & 10.69 & 10.59 & 11.45 & 12.37 \\
\hline 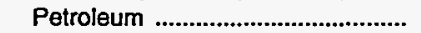 & 1.55 & 3.37 & 6.60 & 7.46 & 5.21 & 4.92 & 3.38 & 3.90 & 5.13 & 6.50 \\
\hline 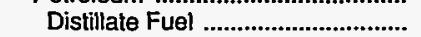 & 1.12 & 2.60 & 6.60 & 6.61 & 4.95 & 4.64 & 4.87 & 5.76 & 6.67 & 6.26 \\
\hline 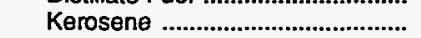 & .85 & 2.50 & - & 6.86 & 4.18 & 4.60 & 4.71 & 5.64 & 6.77 & 6.08 \\
\hline LPG & .91 & 1.91 & 3.81 & 9.63 & 9.06 & 8.52 & 8.58 & 8.09 & 9.03 & 9.29 \\
\hline Motor Gasoline & 3.32 & 5.44 & 10.81 & 11.14 & 9.63 & 9.47 & 9.55 & 10.42 & 11.71 & 10.40 \\
\hline 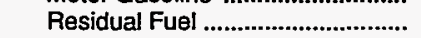 & .42 & 1.59 & 3.86 & 4.60 & 2.81 & 3.33 & 2.71 & 3.09 & 3.83 & 3.11 \\
\hline 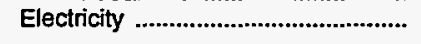 & 9.92 & 16.50 & 26.40 & 34.41 & 27.29 & 27.52 & 25.60 & 26.97 & 29.67 & 30.23 \\
\hline 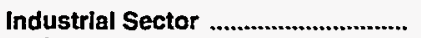 & 1.55 & 4.80 & 8.67 & 13.02 & 9.12 & 9.58 & 8.73 & 11.13 & 11.84 & 11.86 \\
\hline 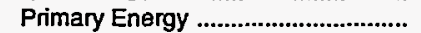 & .60 & 2.05 & 4.44 & 4.93 & 3.41 & 3.74 & 3.39 & 3.87 & 4.57 & 4.03 \\
\hline Coal & - & - & - & 2.15 & 2.09 & 1.95 & 1.81 & 1.76 & 1.82 & 1.80 \\
\hline 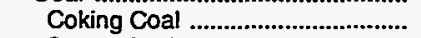 & - & - & - & - & - & - & - & - & - & - \\
\hline 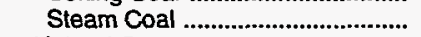 & - & - & - & 2.15 & 2.09 & 1.95 & 1.81 & 1.76 & 1.82 & 1.80 \\
\hline 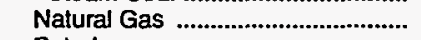 & - & - & - & - & - & - & - & - & - & - \\
\hline Petroleum & .60 & 2.05 & 4.44 & 5.15 & 3.45 & 3.92 & 3.50 & 4.00 & 4.69 & 4.17 \\
\hline Asphalt and Road Oil .................... & .56 & 1.77 & 3.61 & 4.47 & 4.49 & 4.24 & 3.95 & 3.18 & 3.15 & 3.29 \\
\hline 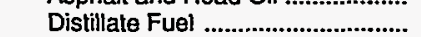 & .74 & 2.22 & 5.49 & 6.07 & 3.70 & 4.07 & 4.16 & 4.99 & 5.99 & 5.38 \\
\hline 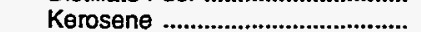 & .85 & 2.50 & 6.38 & 6.86 & 4.18 & 4.60 & 4.71 & 5.64 & 6.77 & 6.08 \\
\hline LPG & .91 & 1.91 & 3.81 & 9.63 & 9.06 & 8.52 & 8.58 & 8.09 & 9.03 & 9.29 \\
\hline 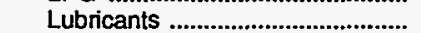 & 5.08 & 7.48 & 14.36 & 17.61 & 15.59 & 12.70 & 14.61 & 13.30 & 13.40 & 15.42 \\
\hline 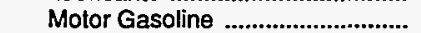 & 3.32 & 5.44 & 10.81 & 11.14 & 9.63 & 9.47 & 9.55 & 10.42 & 11.71 & 10.40 \\
\hline 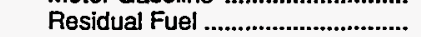 & .42 & 1.92 & 3.82 & 4.60 & 2.81 & 3.33 & 2.71 & 3.09 & 3.83 & 3.11 \\
\hline Other & .51 & 1.39 & 2.49 & 2.51 & 1.53 & 1.50 & 1.45 & 1.74 & 2.02 & 1.72 \\
\hline 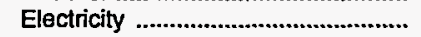 & 4.59 & 9.84 & 18.63 & 25.08 & 18.96 & 20.08 & 18.20 & 19.42 & 22.21 & 22.60 \\
\hline Transportation Sector ..................... & 1.34 & 2.96 & 7.40 & 7.81 & 6.41 & 6.36 & 6.22 & 6.70 & 7.92 & 7.34 \\
\hline 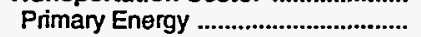 & 1.34 & 2.96 & 7.40 & 7.81 & 6.41 & 6.36 & 6.22 & 6.70 & 7.92 & 7.34 \\
\hline Coal & - & - & - & - & - & - & - & - & - & - \\
\hline Petroleum & 1.34 & 2.96 & 7.40 & 7.81 & 6.41 & 6.36 & 6.22 & 6.70 & 7.92 & 7.34 \\
\hline 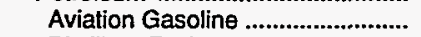 & 2.17 & 3.45 & 9.02 & 9.99 & 8.41 & 7.55 & 7.41 & 8.28 & 9.32 & 8.71 \\
\hline 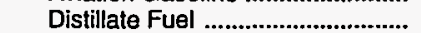 & 1.37 & 2.63 & 7.39 & 8.53 & 7.31 & 8.21 & 7.95 & 8.73 & 9.69 & 10.11 \\
\hline 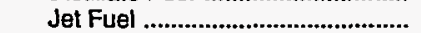 & .73 & 2.04 & 6.21 & 6.21 & 4.36 & 4.30 & 4.04 & 4.59 & 5.99 & 5.17 \\
\hline LPG & .91 & 1.91 & 3.81 & 9.63 & 9.06 & 8.52 & 8.58 & 8.09 & 9.03 & 9.29 \\
\hline Lubricants ...................................... & 5.08 & 7.48 & 14.36 & 17.61 & 15.59 & 12.70 & 14.61 & 13.30 & 13.40 & 15.42 \\
\hline Motor Gasoline .............................. & 3.32 & 5.44 & 10.81 & 11.14 & 9.63 & 9.47 & 9.55 & 10.42 & 11.71 & 10.40 \\
\hline 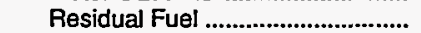 & .37 & 1.37 & 3.27 & 4.65 & 2.62 & 3.18 & 2.49 & 2.81 & 3.51 & 2.85 \\
\hline 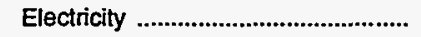 & - & - & - & - & - & - & - & - & - & - \\
\hline 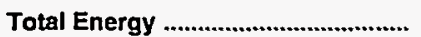 & 1.73 & 3.95 & 8.71 & 10.30 & 8.49 & 8.63 & 7.84 & 8.60 & 10.03 & 9.91 \\
\hline Primary Energy - Four Sectors ..... & 1.29 & 2.90 & 7.15 & 7.66 & 6.17 & 6.15 & 5.79 & 6.41 & 7.58 & 7.16 \\
\hline 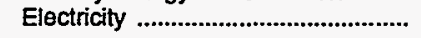 & 6.98 & 12.80 & 22.01 & 29.81 & 23.66 & 24.49 & 22.53 & 23.76 & 26.53 & 27.14 \\
\hline Electric Utility Sectora ...................... & .41 & 1.58 & 3.97 & 4.94 & 2.98 & 3.54 & 2.95 & 3.32 & 4.33 & 3.44 \\
\hline 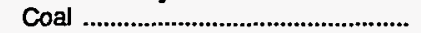 & - & - & - & - & - & - & - & - & - & - \\
\hline Natural Gas & - & - & - & - & - & - & - & - & - & - \\
\hline 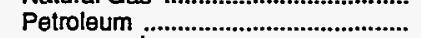 & .40 & 1.58 & 3.97 & 4.95 & 2.98 & 3.54 & 2.95 & 3.32 & 4.33 & 3.44 \\
\hline 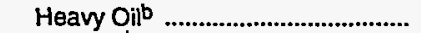 & .40 & 1.57 & 3.87 & 4.86 & 2.86 & 3.44 & 2.87 & 3.29 & 4.15 & 3.31 \\
\hline Light Oilb & .43 & 1.71 & 5.19 & 6.40 & 4.69 & 4.63 & 3.82 & 3.61 & 5.79 & 4.34 \\
\hline 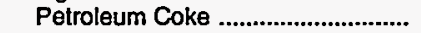 & - & - & - & - & - & - & - & - & - & - \\
\hline 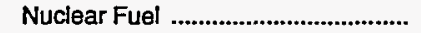 & - & - & - & - & - & - & - & - & - & - \\
\hline Biomass Fuels ....................................... & .65 & .92 & - & .79 & - & - & - & $(d)$ & (d) & - \\
\hline Primary Energy - Five Sectorsc .. & 1.09 & 2.53 & 6.25 & 6.85 & 5.13 & 5.26 & 4.90 & 5.43 & 6.55 & 6.01 \\
\hline
\end{tabular}

a There are no direct fuel costs for hydroelectric, geothermal, centralized solar, or wind energy.

b Heavy oil includes Grade Nos. 4, 5, and 6, and residual fuel oils. Light oil includes Grade No. 2 heating oil, kerosene, and jet fuel.

c Biomass fuels are not included, except those consumed at electric utilities and those added to motor gasoline.

d Utilities used biomass fuels at no charge or received a fee for accepting them.

-No consumption, including cases where adjustments were made. See "Consumption Adjustments for Process Fuel and Intermediate Products."

Sources: Data sources, estimation procedures, and assumptions are described in the "Documentation" section of this report. 
Energy Expenditure Estimates by Sector, Hawaii $1970,1975,1980$, and 1985-1991

(Million Dollars)

\begin{tabular}{|c|c|c|c|c|c|c|c|c|c|c|}
\hline Sector and Energy Source & 1970 & 1975 & 1980 & 1985 & 1986 & 1987 & 1988 & 1989 & 1990 & 1991 \\
\hline 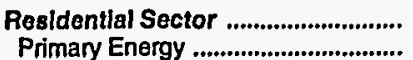 & $\begin{array}{r}43.0 \\
7.0\end{array}$ & $\begin{array}{r}90.1 \\
7.4\end{array}$ & $\begin{array}{r}185.3 \\
36.8\end{array}$ & $\begin{array}{r}230.2 \\
16.8\end{array}$ & $\begin{array}{r}196.1 \\
14.3\end{array}$ & $\begin{array}{r}211.0 \\
15.6\end{array}$ & $\begin{array}{r}205.7 \\
15.7\end{array}$ & $\begin{array}{r}225.6 \\
17.6\end{array}$ & $\begin{array}{r}255.6 \\
17.6\end{array}$ & $\begin{array}{r}273.2 \\
21.2\end{array}$ \\
\hline Coal & - & - & - & - & - & - & - & - & - & - \\
\hline Natural Gas ..................................... & - & - & 18.4 & 11.3 & 9.3 & 9.4 & 8.8 & 8.8 & 9.3 & 12.5 \\
\hline Petroleum & 7.0 & 7.4 & 18.4 & 5.5 & 4.9 & 6.2 & 6.8 & 8.8 & 8.3 & 8.7 \\
\hline 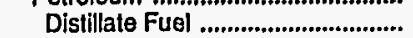 & 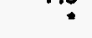 & $\because$ & 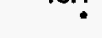 & $\because$ & 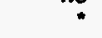 & $\because$ & .1 & 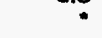 & 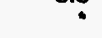 & 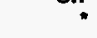 \\
\hline Kerosene ...................................... & - & - & - & - & - & - & - & - & - & • \\
\hline LPG & 7.0 & 7.4 & 18.4 & 5.5 & 4.9 & 6.1 & 6.8 & 8.8 & 8.3 & 8.7 \\
\hline 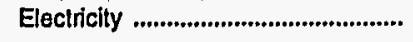 & 36.0 & 82.8 & 148.5 & 213.4 & 181.9 & 195.4 & 190.0 & 208.0 & 238.0 & 252.0 \\
\hline Commerclal Sector .......................... & 30.3 & 67.7 & 172.7 & 225.4 & 202.5 & 223.2 & 255.2 & 271.1 & 299.5 & 297.8 \\
\hline Primary Energy ,................................ & 4.2 & 5.3 & 41.0 & 36.1 & 32.0 & 40.8 & 74.2 & 73.1 & 71.4 & 54.9 \\
\hline 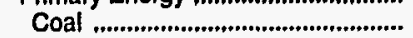 & - & - & - & - & - & - & - & - & - & - \\
\hline Natural Gas ................................... & - & - & 21.0 & 26.8 & 22.7 & 23.7 & 23.6 & 24.4 & 27.2 & 28.7 \\
\hline 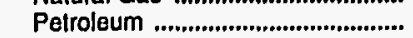 & 4.2 & 5.3 & 20.0 & 9.3 & 9.3 & 17.1 & 50.6 & 48.8 & 44.2 & 26.2 \\
\hline 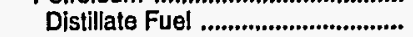 & 1.1 & 1.3 & 15.3 & 5.2 & 5.2 & 13.1 & 17.1 & 16.6 & 19.7 & 22.3 \\
\hline 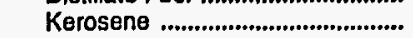 & .4 & .6 & - & .1 & .1 & 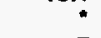 & $\because$ & 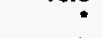 & 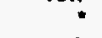 & 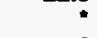 \\
\hline LPG & 3 & .4 & 1.1 & .6 & .6 & .7 & .7 & .7 & .7 & .8 \\
\hline Motor Gasoline ............................. & 2.3 & 2.8 & 3.1 & 2.8 & 2.3 & 2.2 & 2.7 & 2.8 & 3.6 & 2.7 \\
\hline Residual Fuel ................................... & .1 & .2 & .6 & .6 & 1.2 & 1.1 & 30.0 & 28.6 & 20.1 & .4 \\
\hline 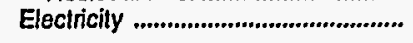 & 26.1 & 62.5 & 131.7 & 189.3 & 170.5 & 182.4 & 181.0 & 198.0 & 228.1 & 242.9 \\
\hline Industrlal Sector ............................. & 36.0 & 110.9 & 275.7 & 325.7 & 244.1 & 257.5 & 258.6 & 274.4 & 344.2 & 341.2 \\
\hline Primary Energy ................................ & 10.7 & 30.8 & 99.1 & 73.8 & 57.9 & 64.6 & 64.1 & 50.8 & 78.1 & 67.2 \\
\hline 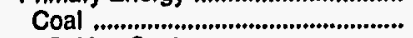 & - & - & - & 2.4 & .8 & 3.1 & 2.2 & 1.4 & 1.3 & 1.7 \\
\hline Coking Coal ................................... & - & - & - & - & - & - & - & - & - & - \\
\hline Steam Coal ................................... & - & - & - & 2.4 & .8 & 3.1 & 2.2 & 1.4 & 1.3 & 1.7 \\
\hline Natural Gas ..................................... & - & - & - & - & - & - & - & - & - & - \\
\hline Petroleum ......................................... & 10.7 & 30.8 & 99.1 & 71.3 & 57.0 & 61.5 & 61.9 & 49.4 & 76.9 & 65.6 \\
\hline Asphalt and Road Oil ..................... & 1.4 & 4.4 & 6.8 & 9.1 & 8.1 & 11.2 & 9.2 & 6.3 & 8.0 & 8.4 \\
\hline Distillate Fuel ................................. & 2.8 & 7.3 & 43.0 & 16.5 & 11.1 & 18.0 & 18.2 & 14.9 & 28.2 & 21.5 \\
\hline Kerosene ....................................... & .3 & .4 & .3 & 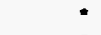 & 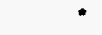 & 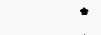 & 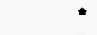 & 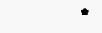 & $\div$ & $\cdot$ \\
\hline 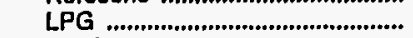 & 1.2 & 2.4 & 11.3 & .2 & .2 & .3 & .3 & .3 & .4 & 1.0 \\
\hline 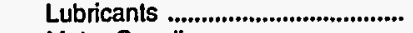 & .1 & 1.3 & 1.7 & 1.9 & 1.7 & 1.5 & 1.7 & 1.6 & 1.6 & 1.7 \\
\hline Motor Gasoline .............................. & .9 & 1.5 & 2.8 & 6.1 & 5.1 & 5.3 & 5.5 & 7.0 & 8.2 & 8.2 \\
\hline Residual Fuel ................................ & 3.5 & 11.7 & 29.4 & 36.0 & 30.3 & 24.4 & 25.9 & 18.2 & 29.1 & 23.9 \\
\hline 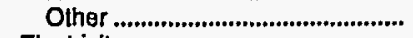 & .5 & 1.7 & 3.7 & 1.5 & .4 & .9 & 1.0 & 1.2 & 1.4 & .9 \\
\hline Electricity .............................................. & 25.3 & 80.1 & 176.7 & 252.0 & 186.3 & 192.9 & 194.5 & 223.6 & 266.0 & 274.0 \\
\hline $\begin{array}{l}\text { Transportatlon Sector ........................ } \\
\text { Primary Energy .................................. }\end{array}$ & $\begin{array}{l}167.9 \\
1679\end{array}$ & $\begin{array}{l}386.6 \\
386.6\end{array}$ & $1,086.3$ & $1,119.0$ & $\begin{array}{l}812.9 \\
8129\end{array}$ & 795.4 & 883.5 & $1,035.2$ & $1,241.3$ & $1,126.6$ \\
\hline & - & 200. & 1,000.0 & $1,10.0$ & 0 & 290.4 & - & $1,000.2$ & $\begin{array}{r}1,241.3 \\
-\end{array}$ & $1,120.0$ \\
\hline 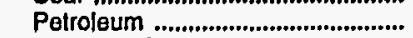 & 167.9 & 386.6 & $1,086.3$ & $1,119.0$ & 812.9 & 795.4 & 883.5 & $1,035.2$ & $1,241.3$ & $1,126.6$ \\
\hline Aviation Gasoline ............................. & 1.5 & 2.0 & 9.1 & 7.8 & 11.8 & 9.5 & 10.5 & 12.0 & 12.8 & 11.5 \\
\hline Distillate Fuel ................................. & 5.7 & 12.7 & 143.5 & 161.7 & 129.4 & 82.7 & 151.3 & 166.8 & 218.5 & 248.7 \\
\hline 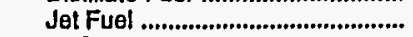 & 58.4 & 170.3 & 492.4 & 462.1 & 248.5 & 277.1 & 271.7 & 341.8 & 425.3 & 333.7 \\
\hline LPG & .1 & .2 & .4 & .2 & .2 & .2 & .3 & .3 & .4 & .5 \\
\hline Lubricants ..................................... & 2.1 & 3.4 & 6.5 & 7.2 & 6.3 & 5.8 & 6.4 & 6.0 & 6.2 & 6.4 \\
\hline Motor Gasoline ............................ & 96.0 & 189.2 & 404.9 & 435.4 & 391.0 & 398.5 & 417.6 & 468.9 & 518.5 & 479.1 \\
\hline Residual Fuel ................................. & 4.1 & 8.7 & 29.7 & 44.6 & 25.7 & 21.6 & 25.6 & 39.5 & 59.5 & 46.7 \\
\hline 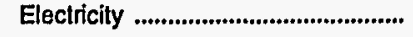 & - & - & - & - & - & - & - & - & - & - \\
\hline 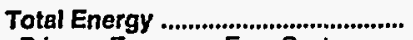 & 277.2 & 655.3 & $1,720.0$ & $1,900.4$ & $1,455.6$ & $1,487.0$ & $1,602.9$ & $1,806.3$ & $2,140.5$ & $2,038.8$ \\
\hline Primary Energy - Four Sectors ...... & 189.8 & 430.0 & $1,263.2$ & $1,245.7$ & 917.0 & 916.3 & $1,037.4$ & $1,176.7$ & $1,408.4$ & $1,269.9$ \\
\hline Electricity ................................................ & 87.4 & 225.3 & 456.9 & 654.7 & 538.6 & 570.6 & 565.5 & 629.6 & 732.1 & 768.9 \\
\hline Electrlc Utllity Sector ${ }^{2}$..................... & 17.4 & 92.4 & 275.8 & 342.5 & 215.9 & 269.6 & 240.5 & 282.8 & 371.8 & 271.5 \\
\hline Coal & - & - & - & - & - & - & - & - & - & - \\
\hline Natural Gas ........................................... & - & - & - & - & - & - & - & - & - & - \\
\hline Petroleum ............................................ & 17.2 & 92.2 & 275.8 & 342.3 & 215.9 & 269.6 & 240.5 & 282.8 & 371.8 & 271.5 \\
\hline 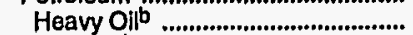 & 17.0 & 87.9 & 248.9 & 314.2 & 193.4 & 240.7 & 212.2 & 253.5 & 316.8 & 228.3 \\
\hline 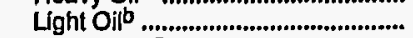 & .2 & 4.3 & 26.8 & 28.0 & 22.5 & 28.9 & 28.3 & 29.3 & 55.0 & 43.2 \\
\hline Petroleum Coke ................................ & - & - & - & - & - & - & - & - & - & - \\
\hline 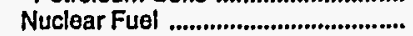 & - & - & - & - & - & - & - & & & - \\
\hline Biomass Fuels ...................................... & .2 & .2 & - & .2 & - & - & - & (d) & (d) $^{d}$ & - \\
\hline Primary Energy - Flve Sectors ${ }^{c}$.. & 207.2 & 522.4 & $1,538.9$ & $1,588.1$ & $1,132.9$ & $1,185.9$ & $1,277.9$ & $1,459.6$ & $1,780.3$ & $1,541.4$ \\
\hline
\end{tabular}

a There are no direct fuel costs for hydroelectric, geothermal, centralized solar, or wind energy.

b Heavy oil includes Grade Nos. 4, 5, and 6, and residual fuel oils. Light oil includes Grade No. 2 heating oil, kerosene, and jet fuel.

c Blomass fuels are not included, except those consumed at electric utilities and those added to motor gasoline.

d Uilities used biomass fuels at no charge or received a fee for accepting them.

- No consumption, including cases where adjustments were made. See "Consumption Adjustments for Process Fuel and intermediate Products."

Value less than 0.05 million dollars.

Note: Totals may not equal sum of components due to independent rounding.

Sources: Data sources, estimation procedures, and assumptions are described in the "Documentation" section of this report. 
I Energy Price and Expenditure Estimates by Source, Idaho

D $1970,1975,1980$, and $1985-1991$

\begin{tabular}{|c|c|c|c|c|c|c|c|c|c|c|}
\hline Energy Source & 1970 & 1975 & 1980 & 1985 & 1986 & 1987 & 1988 & 1989 & 1990 & 1991 \\
\hline & \multicolumn{10}{|c|}{ Prices in Dollars per Million Btu } \\
\hline 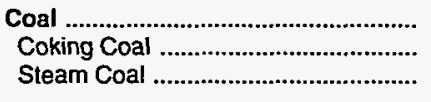 & $\begin{array}{r}0.59 \\
.59\end{array}$ & $\begin{array}{r}0.98 \\
-98\end{array}$ & $\begin{array}{r}1.78 \\
1.78\end{array}$ & $\begin{array}{r}1.70 \\
- \\
1.70\end{array}$ & $\begin{array}{r}2.01 \\
-\overline{1} \\
2.01\end{array}$ & $\begin{array}{r}1.97 \\
- \\
1.97\end{array}$ & $\begin{array}{r}1.78 \\
-\overline{8}\end{array}$ & $\begin{array}{r}1.82 \\
1.82\end{array}$ & $\begin{array}{r}1.76 \\
1.76\end{array}$ & $\begin{array}{r}1.87 \\
1.87\end{array}$ \\
\hline Natural Gas & .66 & 1.43 & 3.87 & 5.07 & 4.22 & 5.86 & 4.49 & 3.57 & 3.43 & 3.62 \\
\hline 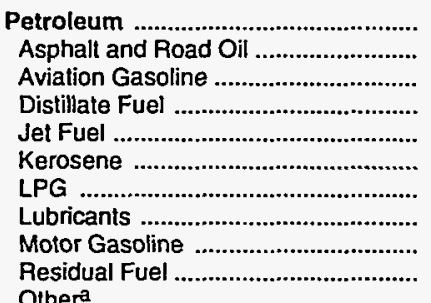 & $\begin{array}{r}1.92 \\
.60 \\
2.17 \\
1.01 \\
.76 \\
.82 \\
2.41 \\
5.08 \\
2.81 \\
.34\end{array}$ & $\begin{array}{l}3.64 \\
1.88 \\
3.45 \\
2.55 \\
2.12 \\
2.59 \\
3.81 \\
7.48 \\
4.81 \\
2.01\end{array}$ & $\begin{array}{r}8.11 \\
3.64 \\
9.02 \\
6.54 \\
6.59 \\
- \\
6.39 \\
14.36 \\
9.79 \\
4.45\end{array}$ & $\begin{array}{r}8.50 \\
4.78 \\
9.99 \\
7.62 \\
6.68 \\
8.03 \\
9.45 \\
17.61 \\
9.31 \\
3.67\end{array}$ & $\begin{array}{r}6.49 \\
4.40 \\
8.41 \\
5.73 \\
4.40 \\
7.54 \\
7.91 \\
15.59 \\
7.07 \\
2.38\end{array}$ & $\begin{array}{r}6.53 \\
3.43 \\
7.55 \\
5.75 \\
4.39 \\
7.63 \\
9.05 \\
12.70 \\
7.26 \\
2.57\end{array}$ & $\begin{array}{r}6.78 \\
3.39 \\
7.41 \\
5.80 \\
4.24 \\
7.74 \\
9.03 \\
14.61 \\
7.61 \\
1.93\end{array}$ & $\begin{array}{r}7.28 \\
2.79 \\
8.28 \\
6.58 \\
4.93 \\
8.35 \\
9.92 \\
13.30 \\
8.21 \\
1.66\end{array}$ & $\begin{array}{r}8.05 \\
2.64 \\
9.32 \\
7.65 \\
6.07 \\
9.73 \\
10.09 \\
13.40 \\
9.15 \\
2.51\end{array}$ & $\begin{array}{r}8.01 \\
3.28 \\
8.71 \\
7.29 \\
5.50 \\
9.11 \\
10.31 \\
15.42 \\
9.13 \\
2.31\end{array}$ \\
\hline Other ${ }^{2}$ & 4.14 & - & - & - & - & - & - & - & - & - \\
\hline 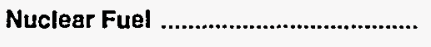 & - & - & - & - & - & - & - & - & - & - \\
\hline Blomass Fuels at Utilities ................. & - & - & - & - & - & - & - & - & - & - \\
\hline Primary Energy - Five Sectors ${ }^{b} \ldots$ & 1.50 & 2.80 & 6.57 & 7.23 & 5.72 & 6.10 & 5.93 & 6.04 & 6.56 & 6.48 \\
\hline $\begin{array}{l}\text { Electric Utility Fuelc } \\
\text { Electricity Purchased by End Users }\end{array}$ & $\begin{array}{r}.35 \\
2.95\end{array}$ & $\begin{array}{l}1.89 \\
4.11\end{array}$ & $\begin{array}{l}3.87 \\
7.39\end{array}$ & $\begin{array}{r}5.60 \\
10.66\end{array}$ & $\begin{array}{r}4.25 \\
10.94\end{array}$ & $\begin{array}{r}2.78 \\
10.81\end{array}$ & $\begin{array}{r}4.07 \\
10.84\end{array}$ & $\begin{array}{r}4.45 \\
11.10\end{array}$ & $\begin{array}{r}5.38 \\
11.15\end{array}$ & $\begin{array}{r}5.04 \\
11.27\end{array}$ \\
\hline \multirow[t]{2}{*}{ 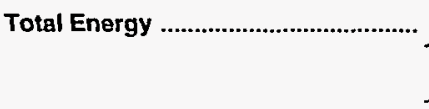 } & 1.77 & 3.03 & 6.75 & 8.16 & 7.12 & 7.36 & 7.26 & 7.39 & 7.77 & 7.67 \\
\hline & \multicolumn{10}{|c|}{ Expenditures in Millions of Dollars } \\
\hline Coal & 4.7 & 13.1 & 17.1 & 15.1 & 17.3 & 17.4 & 17.3 & 17.7 & 17.7 & 22.9 \\
\hline $\begin{array}{l}\text { Coking Coal } \\
\text { Steam Coal }\end{array}$ & $4 . \overline{7}$ & $13 . \overline{1}$ & $17 . \overline{1}$ & $15 . \overline{1}$ & $17 \overline{-}$ & $17 \overline{4}$ & $17 . \overline{3}$ & $17 \overline{\overline{7}}$ & $17 \overline{\overline{7}}$ & $22 . \overline{9}$ \\
\hline 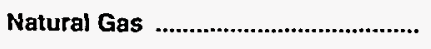 & 29.5 & 84.6 & 182.6 & 192.9 & 138.4 & 200.0 & 167.7 & 149.1 & 142.4 & 173.8 \\
\hline 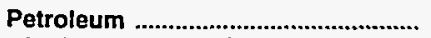 & 202.2 & 456.2 & 913.5 & 880.6 & 685.2 & 700.0 & 748.3 & 847.5 & 967.7 & $1,019.4$ \\
\hline 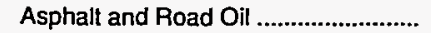 & 4.5 & 11.0 & 19.2 & 20.0 & 15.9 & 11.4 & 9.0 & 15.4 & 22.4 & 21.5 \\
\hline 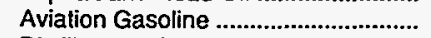 & 1.7 & 2.1 & 7.4 & 4.0 & 3.7 & 2.9 & 1.9 & 2.3 & 1.9 & 1.7 \\
\hline 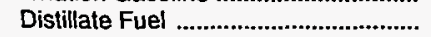 & 32.9 & 112.3 & 215.6 & 247.7 & 197.2 & 213.7 & 219.9 & 263.3 & 319.4 & 361.4 \\
\hline Jet Fuel & 3.9 & 11.0 & 44.9 & 40.7 & 26.8 & 27.7 & 27.3 & 33.4 & 38.1 & 31.5 \\
\hline Kerosene & 1.1 & 2.1 & - & .3 & .3 & .4 & .4 & .2 & .5 & .2 \\
\hline LPG & 9.6 & 16.7 & 23.3 & 26.5 & 21.2 & 20.6 & 24.6 & 30.6 & 22.3 & 30.3 \\
\hline 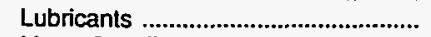 & 4.7 & 7.4 & 15.8 & 17.7 & 15.3 & 14.1 & 15.6 & 14.6 & 15.1 & 15.6 \\
\hline 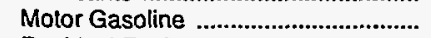 & 142.8 & 285.0 & 570.0 & 521.6 & 404.5 & 408.1 & 448.7 & 487.3 & 547.2 & 556.4 \\
\hline 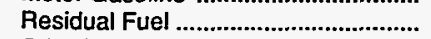 & .6 & 8.6 & 17.1 & 2.0 & .3 & 1.0 & .7 & .5 & .7 & .6 \\
\hline Other ${ }^{2}$ & .4 & - & - & - & - & - & - & - & - & - \\
\hline 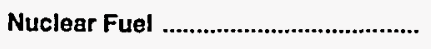 & - & - & - & - & - & - & - & - & - & - \\
\hline Biomass Fuels at Utilities ................. & - & - & - & - & - & - & - & - & - & - \\
\hline Primary Energy - Five Sectors ${ }^{b}$.... & 236.4 & 553.9 & $1,113.1$ & $1,088.6$ & 840.9 & 917.4 & 933.3 & $1,014.4$ & $1,127.9$ & $1,216.1$ \\
\hline 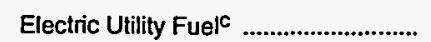 & * & -.1 & -.2 & -.2 & $\cdot$ & $\cdot$ & $\cdot$ & -.1 & • & • \\
\hline Electricity Purchased by End Users & 105.8 & -175.4 & 345.9 & 596.4 & 589.5 & 593.8 & 635.1 & 675.0 & 685.0 & 694.0 \\
\hline 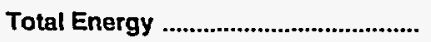 & 342.2 & 729.2 & $1,458.8$ & $1,684.9$ & $1,430.4$ & $1,511.2$ & $1,568.3$ & $1,689.3$ & $1,812.8$ & $1,910.1$ \\
\hline
\end{tabular}

includes petroleum coke used at electric utilities.

b Biomass fuels are not included, except those consumed at electric utilities and those added to motor gasoline.

c There are no direct fuel costs for hydroelectric, geothemal, centralized solar, or wind energy.

-No consumption, including cases where adjustments were made. See "Consumption Adjustments for Process Fuel and Intermediate Products."

"Value less than 0.05 million dollars.

Note: Expenditure totals may not equal sum of components due to independent rounding.

Sources: Data sources, estimation procedures, and assumptions are described in the "Documentation" section of this report. 
Energy Price Estimates by Sector, Idaho

$1970,1975,1980$, and 1985-1991

(Dollars per Million Btu)

\begin{tabular}{|c|c|c|c|c|c|c|c|c|c|c|}
\hline Sector and Energy Source & 1970 & 1975 & 1980 & 1985 & 1986 & 1987 & 1988 & 1989 & 1990 & 1991 \\
\hline 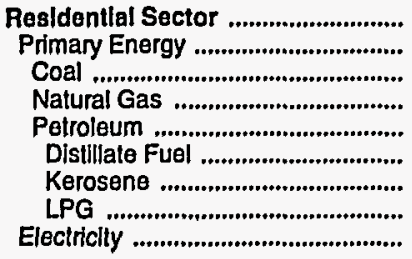 & $\begin{array}{r}2.58 \\
1.54 \\
.99 \\
1.31 \\
1.91 \\
1.40 \\
- \\
2.83 \\
4.81\end{array}$ & $\begin{array}{r}3.43 \\
2.45 \\
1.78 \\
2.07 \\
3.25 \\
2.82 \\
- \\
4.17 \\
5.27\end{array}$ & $\begin{array}{r}7.14 \\
5.28 \\
2.56 \\
4.73 \\
6.97 \\
6.60 \\
- \\
7.84 \\
8.54\end{array}$ & $\begin{array}{r}10.30 \\
6.91 \\
1.97 \\
6.57 \\
7.84 \\
7.29 \\
12.00 \\
9.55 \\
12.60\end{array}$ & $\begin{array}{r}10.28 \\
6.04 \\
1.91 \\
5.89 \\
6.51 \\
5.59 \\
9.20 \\
9.70 \\
13.14\end{array}$ & $\begin{array}{r}10.39 \\
5.71 \\
1.54 \\
5.41 \\
6.40 \\
5.21 \\
8.58 \\
10.64 \\
13.45\end{array}$ & $\begin{array}{r}10.44 \\
5.61 \\
1.26 \\
5.38 \\
6.51 \\
5.21 \\
8.58 \\
10.38 \\
13.88\end{array}$ & $\begin{array}{r}10.57 \\
5.77 \\
1.56 \\
4.92 \\
7.95 \\
5.89 \\
9.70 \\
12.49 \\
14.11\end{array}$ & $\begin{array}{r}10.83 \\
5.95 \\
1.55 \\
4.91 \\
8.58 \\
7.37 \\
12.14 \\
11.73 \\
14.27\end{array}$ & $\begin{array}{r}10.61 \\
6.04 \\
1.49 \\
5.02 \\
8.45 \\
7.20 \\
11.86 \\
12.24 \\
14.33\end{array}$ \\
\hline 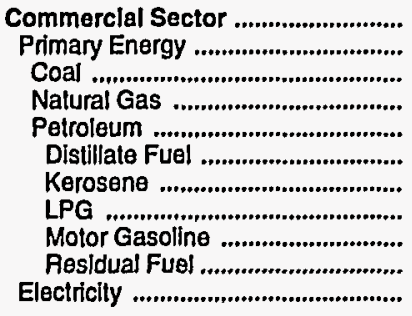 & $\begin{array}{r}2.12 \\
.95 \\
.50 \\
.96 \\
1.35 \\
1.21 \\
.82 \\
1.55 \\
2.81 \\
4.10\end{array}$ & $\begin{array}{r}2.91 \\
1.66 \\
.87 \\
1.47 \\
3.01 \\
2.62 \\
2.59 \\
3.25 \\
4.81 \\
4 . \overline{8}\end{array}$ & $\begin{array}{r}6.49 \\
4.53 \\
1.70 \\
4.36 \\
5.65 \\
6.41 \\
- \\
5.72 \\
9.79 \\
4.63 \\
8.33\end{array}$ & $\begin{array}{r}9.16 \\
5.70 \\
1.69 \\
5.42 \\
7.37 \\
6.82 \\
7.00 \\
9.38 \\
9.31 \\
3.67 \\
12.10\end{array}$ & $\begin{array}{r}9.20 \\
4.88 \\
2.01 \\
4.68 \\
6.15 \\
5.74 \\
4.85 \\
6.76 \\
7.07 \\
2.38 \\
12.58\end{array}$ & $\begin{array}{r}9.04 \\
4.65 \\
1.98 \\
4.44 \\
5.39 \\
4.72 \\
5.02 \\
7.98 \\
7.26 \\
2.57 \\
12.25\end{array}$ & $\begin{array}{r}8.68 \\
4.71 \\
1.82 \\
4.43 \\
5.88 \\
4.41 \\
5.06 \\
7.99 \\
7.61 \\
1.93 \\
12.06\end{array}$ & $\begin{array}{r}8.88 \\
4.64 \\
1.84 \\
4.09 \\
6.54 \\
5.27 \\
5.77 \\
7.58 \\
8.21 \\
1.66 \\
12.58\end{array}$ & $\begin{array}{r}9.23 \\
4.61 \\
1.77 \\
4.07 \\
6.96 \\
6.24 \\
6.53 \\
8.30 \\
9.15 \\
2.51 \\
12.54\end{array}$ & $\begin{array}{r}9.01 \\
4.98 \\
1.88 \\
4.28 \\
7.18 \\
5.66 \\
6.93 \\
8.68 \\
9.13 \\
2.31 \\
12.54\end{array}$ \\
\hline 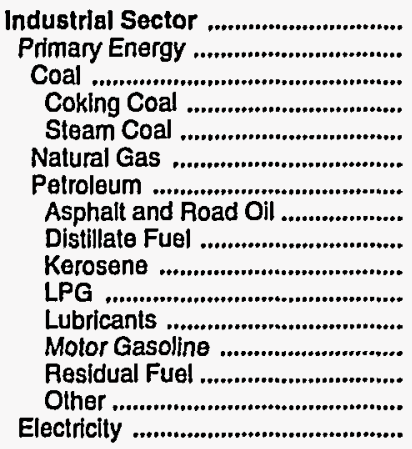 & $\begin{array}{r}.96 \\
.69 \\
.50 \\
.50 \\
.50 \\
.42 \\
.96 \\
.60 \\
.77 \\
.82 \\
1.55 \\
5.08 \\
2.81 \\
.34 \\
4.14 \\
1.84\end{array}$ & $\begin{array}{r}1.97 \\
1.81 \\
.87 \\
- \\
.87 \\
1.11 \\
2.60 \\
1.88 \\
2.40 \\
2.59 \\
3.25 \\
7.48 \\
4.81 \\
2.01 \\
- \\
2.70\end{array}$ & $\begin{array}{r}4.53 \\
4.30 \\
1.70 \\
-\overline{1} \\
1.70 \\
3.58 \\
6.02 \\
3.64 \\
6.02 \\
\overline{5} \\
5.72 \\
14.36 \\
9.79 \\
3.76 \\
5 . \overline{4} \\
5.44\end{array}$ & $\begin{array}{r}5.68 \\
4.80 \\
1.69 \\
- \\
1.69 \\
4.32 \\
6.60 \\
4.78 \\
6.15 \\
7.00 \\
9.38 \\
17.61 \\
9.31 \\
3.67 \\
- \\
7.69\end{array}$ & $\begin{array}{r}5.00 \\
3.78 \\
2.01 \\
-\overline{2} \\
2.01 \\
3.23 \\
4.92 \\
4.40 \\
4.26 \\
4.85 \\
6.76 \\
15.59 \\
7.07 \\
2.38 \\
- \\
7.70\end{array}$ & $\begin{array}{r}5.80 \\
5.03 \\
1.98 \\
- \\
1.98 \\
6.59 \\
4.82 \\
3.43 \\
4.41 \\
5.02 \\
7.98 \\
12.70 \\
7.26 \\
2.57 \\
- \\
7.56\end{array}$ & $\begin{array}{r}5.20 \\
4.11 \\
1.82 \\
- \\
1.82 \\
4.18 \\
4.93 \\
3.39 \\
4.44 \\
5.06 \\
7.99 \\
14.61 \\
7.61 \\
1.93 \\
- \\
7.53\end{array}$ & $\begin{array}{r}4.88 \\
3.67 \\
1.84 \\
- \\
1.84 \\
2.84 \\
5.05 \\
2.79 \\
5.07 \\
5.77 \\
7.58 \\
13.30 \\
8.21 \\
1.66 \\
- \\
7.67\end{array}$ & $\begin{array}{r}4.82 \\
3.65 \\
1.77 \\
-\overline{1} \\
1.77 \\
2.65 \\
5.12 \\
2.64 \\
5.74 \\
6.53 \\
8.30 \\
13.40 \\
9.15 \\
2.51 \\
- \\
7.69\end{array}$ & $\begin{array}{r}4.99 \\
4.09 \\
1.88 \\
-\overline{1} \\
1.88 \\
2.85 \\
5.88 \\
3.28 \\
6.10 \\
6.93 \\
8.68 \\
15.42 \\
9.13 \\
2.31 \\
- \\
7.68\end{array}$ \\
\hline 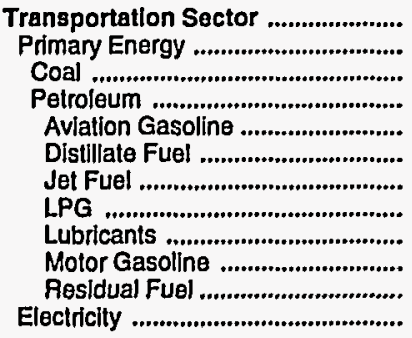 & $\begin{array}{r}2.47 \\
2.47 \\
.50 \\
2.47 \\
2.17 \\
1.81 \\
.76 \\
1.55 \\
5.08 \\
2.81 \\
.39 \\
-\end{array}$ & $\begin{array}{r}4.25 \\
4.25 \\
.87 \\
4.25 \\
3.45 \\
2.68 \\
2.12 \\
3.25 \\
7.48 \\
4.81 \\
- \\
-\end{array}$ & $\begin{array}{r}8.98 \\
8.98 \\
- \\
8.98 \\
9.02 \\
6.95 \\
6.59 \\
5.72 \\
14.36 \\
9.79 \\
- \\
-\end{array}$ & $\begin{array}{r}9.05 \\
9.05 \\
- \\
9.05 \\
9.99 \\
8.70 \\
6.68 \\
9.38 \\
17.61 \\
9.31 \\
- \\
-\end{array}$ & $\begin{array}{r}6.90 \\
6.90 \\
- \\
6.90 \\
8.41 \\
6.86 \\
4.40 \\
6.76 \\
15.59 \\
7.07 \\
- \\
-\end{array}$ & $\begin{array}{r}7.05 \\
7.05 \\
- \\
7.05 \\
7.55 \\
7.06 \\
4.39 \\
7.98 \\
12.70 \\
7.26 \\
- \\
-\end{array}$ & $\begin{array}{r}7.33 \\
7.33 \\
- \\
7.33 \\
7.41 \\
7.23 \\
4.24 \\
7.99 \\
14.61 \\
7.61 \\
- \\
-\end{array}$ & $\begin{array}{r}7.96 \\
7.96 \\
7.96 \\
8.28 \\
8.08 \\
4.93 \\
7.58 \\
13.30 \\
8.21 \\
- \\
-\end{array}$ & $\begin{array}{r}8.99 \\
8.99 \\
-\overline{8} \\
9.99 \\
9.27 \\
6.07 \\
8.30 \\
13.40 \\
9.15 \\
- \\
-\end{array}$ & $\begin{array}{r}8.84 \\
8.84 \\
-\overline{8} \\
8.84 \\
8.71 \\
8.74 \\
5.50 \\
8.68 \\
15.42 \\
9.13 \\
- \\
-.\end{array}$ \\
\hline $\begin{array}{l}\text { Total Energy ...................................... } \\
\text { Primary Energy }- \text { Four Sectors ...... } \\
\text { Electricity ........................................ }\end{array}$ & $\begin{array}{l}1.77 \\
1.50 \\
2.95\end{array}$ & $\begin{array}{l}3.03 \\
2.80 \\
4.11\end{array}$ & $\begin{array}{l}6.75 \\
6.57 \\
7.39\end{array}$ & $\begin{array}{r}8.16 \\
7.23 \\
10.66\end{array}$ & $\begin{array}{r}7.12 \\
5.72 \\
10.94\end{array}$ & $\begin{array}{r}7.36 \\
6.10 \\
10.81\end{array}$ & $\begin{array}{r}7.26 \\
5.93 \\
10.84\end{array}$ & $\begin{array}{r}7.39 \\
6.04 \\
11.10\end{array}$ & $\begin{array}{r}7.77 \\
6.56 \\
11.15\end{array}$ & $\begin{array}{r}7.67 \\
6.48 \\
11.27\end{array}$ \\
\hline 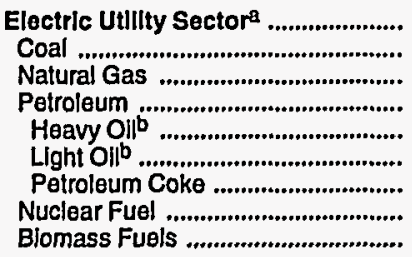 & $\begin{array}{r}.35 \\
- \\
.35 \\
\overline{-} \\
.35 \\
- \\
-\end{array}$ & $\begin{array}{r}1.89 \\
\overline{-} \\
1.38 \\
2.20 \\
2 . \overline{0} \\
- \\
-\end{array}$ & $\begin{array}{r}3.87 \\
-\overline{4} \\
6.39 \\
\overline{-} \\
6.39 \\
- \\
-\end{array}$ & $\begin{array}{r}5.60 \\
-\overline{4} \\
6.07 \\
\overline{-} \\
6.07 \\
- \\
- \\
-\end{array}$ & $\begin{array}{r}4.25 \\
- \\
4.70 \\
3.74 \\
- \\
3.74 \\
- \\
- \\
-\end{array}$ & $\begin{array}{r}2.78 \\
- \\
2.31^{\circ} \\
4.25 \\
- \\
4.25 \\
- \\
- \\
-\end{array}$ & $\begin{array}{r}4.07 \\
- \\
\overline{-} \\
4.07 \\
\overline{-} \\
4.07 \\
- \\
- \\
-\end{array}$ & $\begin{array}{r}4.45 \\
- \\
-\overline{4} \\
4.45 \\
\overline{4} \\
4.45 \\
- \\
- \\
-\end{array}$ & $\begin{array}{r}5.38 \\
- \\
- \\
5.38 \\
- \\
5.38 \\
- \\
- \\
-\end{array}$ & $\begin{array}{r}5.04 \\
\overline{-} \\
5.04 \\
-\overline{4} \\
5.04 \\
\overline{-} \\
\overline{-}\end{array}$ \\
\hline Primary Energy - Five Sectorsc ... & 1.50 & 2.80 & 6.57 & 7.23 & 5.72 & 6.10 & 5.93 & 6.04 & 6.56 & 6.48 \\
\hline
\end{tabular}

a There are no direct fuel costs for hydroelectric, geothermal, centralized solar, or wind energy.

b Heavy oil includes Grade Nos, 4, 5, and 6, and residual fuel oils. Light oil includes Grade No. 2 heating oil, kerosene, and jet fuel.

c Blomass fuels are not included, except those consumed at electric utilities and those added to motor gasoline.

-No consumption, including cases where adjustments were made. See "Consumption Adjustments for Process Fuel and Intermediate Products."

Sources: Data sources, estimation procedures, and assumptions are described in the "Documentation" section of this report. 
Energy Expenditure Estimates by Sector, Idaho

$1970,1975,1980$, and 1985-1991

(Million Dollars)

\begin{tabular}{|c|c|c|c|c|c|c|c|c|c|c|}
\hline Sector and Energy Source & 1970 & 1975 & 1980 & 1985 & 1986 & 1987 & 1988 & 1989 & 1990 & 1991 \\
\hline Residential Sector & 65.3 & 129.9 & 210.6 & 341.1 & 319.0 & 305.3 & 332.3 & 358.0 & 354.7 & 392.0 \\
\hline 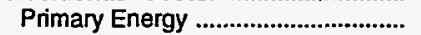 & 26.6 & 60.4 & 66.8 & 92.5 & 75.4 & 66.4 & 74.3 & 83.0 & 80.7 & 99.9 \\
\hline Coal & 1.5 & 2.7 & 2.3 & .7 & .6 & .3 & .8 & 1.0 & .7 & .8 \\
\hline 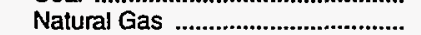 & 10.7 & 30.7 & 36.8 & 53.5 & 43.8 & 38.5 & 42.2 & 44.4 & 43.3 & 53.1 \\
\hline Petroleum ............................................. & 14.4 & 27.0 & 27.8 & 38.3 & 31.1 & 27.5 & 31.4 & 37.6 & 36.6 & 46.1 \\
\hline 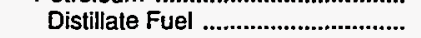 & 6.8 & 16.0 & 18.7 & 27.0 & 20.6 & 17.4 & 18.7 & 19.1 & 22.7 & 29.5 \\
\hline 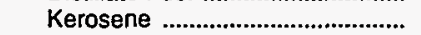 & - & & - & .1 & .3 & .3 & .4 & .1 & .4 & .1 \\
\hline LPG & 7.6 & 11.0 & 9.1 & 11.3 & 10.2 & 9.8 & 12.3 & 18.4 & 13.5 & 16.5 \\
\hline Electricity & 38.6 & 69.5 & 143.8 & 248.5 & 243.5 & 239.0 & 258.0 & 275.0 & 274.0 & 292.0 \\
\hline Commercial Sector ................................... & 40.9 & 90.3 & 170.8 & 265.7 & 248.3 & 246.3 & 269.3 & 281.3 & 281.7 & 298.2 \\
\hline 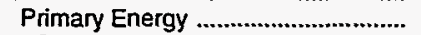 & 11.7 & 31.5 & 57.8 & 76.1 & 57.9 & 53.6 & 67.3 & 68.3 & 58.7 & 77.2 \\
\hline 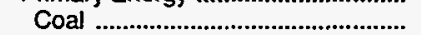 & 1.4 & 2.4 & 2.8 & 1.2 & 1.1 & .7 & 2.0 & 2.1 & 1.6 & 1.9 \\
\hline 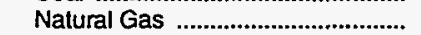 & 5.9 & 18.8 & 26.4 & 51.2 & 40.9 & 34.4 & 37.3 & 37.9 & 35.7 & 42.4 \\
\hline Petroleum …………………………........... & 4.3 & 10.2 & 28.6 & 23.8 & 15.9 & 18.5 & 27.9 & 28.3 & 21.5 & 32.9 \\
\hline Distillate Fuel ...................................... & 2.1 & 5.2 & 8.1 & 14.5 & 9.5 & 11.6 & 11.1 & 10.7 & 12.4 & 14.3 \\
\hline 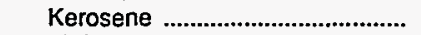 & .5 & 1.2 & - & .1 & • & .1 & & • & * & \\
\hline 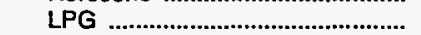 & .7 & 1.5 & 1.2 & 2.0 & 1.3 & 1.3 & 1.7 & 2.0 & 1.7 & 2.1 \\
\hline 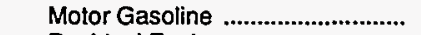 & 1.0 & 2.3 & 5.1 & 6.6 & 5.0 & 5.4 & 15.1 & 15.4 & 7.1 & 16.5 \\
\hline 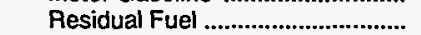 & - & - & 14.2 & .6 & • & .2 & .1 & .3 & .3 & \\
\hline 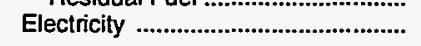 & 29.2 & 58.8 & 113.0 & 189.6 & 190.4 & 192.7 & 202.0 & 213.0 & 223.0 & 221.0 \\
\hline 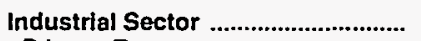 & 84.4 & 191.8 & 369.3 & 384.4 & 324.0 & 407.7 & 380.6 & 394.5 & 407.1 & 470.1 \\
\hline 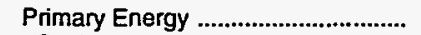 & 46.5 & 144.7 & 280.3 & 226.1 & 168.4 & 245.7 & 205.6 & 207.5 & 219.1 & 289.1 \\
\hline 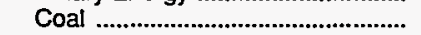 & 1.8 & 8.0 & 12.0 & 13.2 & 15.6 & 16.5 & 14.5 & 14.7 & 15.4 & 20.2 \\
\hline Coking Coal ....................................... & - & - & & & & - & - & - & - & - \\
\hline 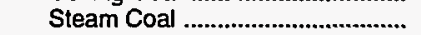 & 1.8 & 8.0 & 12.0 & 13.2 & 15.6 & 16.5 & 14.5 & 14.7 & 15.4 & 20.2 \\
\hline Natural Gas ........................................... & 12.8 & 35.0 & 119.2 & 88.1 & 53.7 & 127.0 & 88.2 & 66.9 & 63.4 & 78.4 \\
\hline 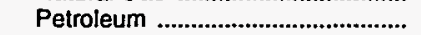 & 31.8 & 101.7 & 149.0 & 124.8 & 99.1 & 102.2 & 102.9 & 125.9 & 140.2 & 190.5 \\
\hline 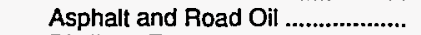 & 4.5 & 11.0 & 19.2 & 20.0 & 15.9 & 11.4 & 9.0 & 15.4 & 22.4 & 21.5 \\
\hline 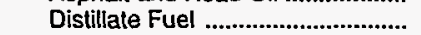 & 14.3 & 55.0 & 77.5 & 62.7 & 53.0 & 61.6 & 63.6 & 79.0 & 91.1 & 132.9 \\
\hline 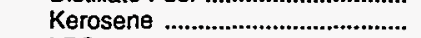 & .5 & .9 & 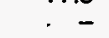 & .1 & & & & & .1 & .1 \\
\hline LPG & 1.2 & 3.9 & 12.6 & 11.3 & 8.1 & 8.5 & 9.4 & 9.2 & 5.6 & 10.5 \\
\hline 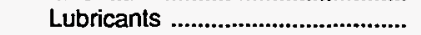 & 1.0 & 2.0 & 3.8 & 4.3 & 3.7 & 3.4 & 3.8 & 3.5 & 3.7 & 3.8 \\
\hline Motor Gasoline .................................. & 9.2 & 20.2 & 32.9 & 25.0 & 18.1 & 16.5 & 16.3 & 18.7 & 16.8 & 21.1 \\
\hline 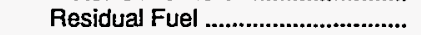 & .6 & 8.6 & 3.0 & 1.4 & .3 & .9 & .6 & .2 & .4 & .6 \\
\hline Other & .4 & - & - & - & - & - & & - & - & - \\
\hline 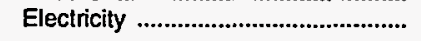 & 37.9 & 47.1 & 89.1 & 158.3 & 155.6 & 162.1 & 175.0 & 187.0 & 188.0 & 181.0 \\
\hline 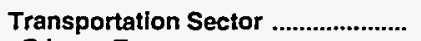 & 151.6 & 317.3 & 708.1 & 693.7 & 539.2 & 551.8 & 586.1 & 655.5 & 769.3 & 749.8 \\
\hline 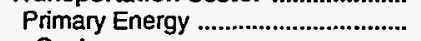 & 151.6 & 317.3 & 708.1 & 693.7 & 539.2 & 551.8 & 586.1 & 655.5 & 769.3 & 749.8 \\
\hline Coal & & & & & & & & & & \\
\hline 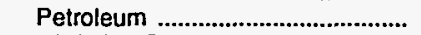 & 151.6 & 317.3 & 708.1 & 693.7 & 539.2 & 551.8 & 586.1 & 655.5 & 769.3 & 749.8 \\
\hline 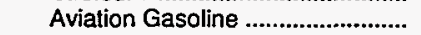 & 1.7 & 2.1 & 7.4 & 4.0 & 3.7 & 2.9 & 1.9 & 2.3 & 1.9 & 1.7 \\
\hline 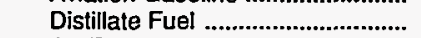 & 9.7 & 36.0 & 111.3 & 143.5 & 114.1 & 123.1 & 126.5 & 154.3 & 193.1 & 184.7 \\
\hline 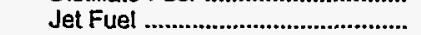 & 3.9 & 11.0 & 44.9 & 40.7 & 26.8 & 27.7 & 27.3 & 33.4 & 38.1 & 31.5 \\
\hline 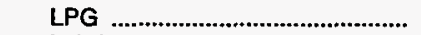 & .1 & .3 & .5 & 2.0 & 1.6 & 1.0 & 1.2 & 1.1 & 1.5 & 1.2 \\
\hline 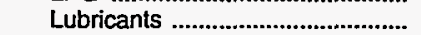 & 3.7 & 5.4 & 12.0 & 13.4 & 11.6 & 10.7 & 11.9 & 11.1 & 11.5 & 11.8 \\
\hline 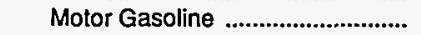 & 132.6 & 262.5 & 532.0 & 490.1 & 381.3 & 386.3 & 417.3 & 453.2 & 523.3 & 518.8 \\
\hline Residual Fuel ...................................... & * & - & - & - & - & - & - & - & - & - \\
\hline Electricity & - & - & - & - & - & - & - & - & - & - \\
\hline 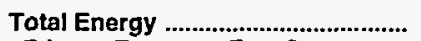 & 342.2 & 729.2 & $1,458.8$ & $1,684.9$ & $1,430.4$ & $1,511.2$ & $1,568.3$ & $1,689.3$ & $1,812.8$ & $1,910.1$ \\
\hline Primary Energy — Four Sectors ..... & 236.4 & 553.8 & $1,113.0$ & $1,088.5$ & 840.9 & 917.4 & 933.3 & $1,014.3$ & $1,127.8$ & $1,216.1$ \\
\hline 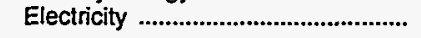 & 105.8 & 175.4 & 345.9 & 596.4 & 589.5 & 593.8 & 635.1 & 675.0 & 685.0 & 694.0 \\
\hline Electric Uttlity Sectora & - & .1 & .2 & .2 & - & • & - & .1 & - & - \\
\hline 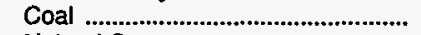 & - & - & - & - & - & - & - & - & - & - \\
\hline 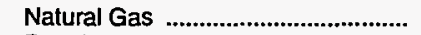 & - & • & .2 & 1 & • & • & - & - & - & - \\
\hline Petroleum & • & .1 & 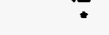 & $\because$ & - & - & * & .1 & - & - \\
\hline 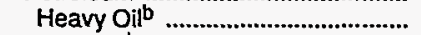 & - & - & - & - & - & - & - & - & - & - \\
\hline Light Oilb ........................................ & - & .1 & - & - & - & - & - & .1 & - & - \\
\hline Petroleum Coke .................................... & - & - & - & - & - & - & - & - & - & - \\
\hline 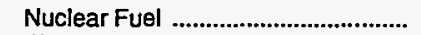 & - & - & - & - & - & - & - & - & - & - \\
\hline 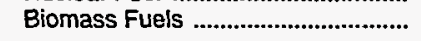 & - & - & - & - & - & - & - & - & - & - \\
\hline Primary Energy - Five Sectors ${ }^{c}$.. & 236.4 & 553.9 & $1,113.1$ & $1,088.6$ & 840.9 & 917.4 & 933.3 & $1,014.4$ & $1,127.9$ & $1,216.1$ \\
\hline
\end{tabular}

There are no direct fuel costs for hydroelectric, geothermal, centralized solar, or wind energy.

beavy oil includes Grade Nos. 4, 5, and 6, and residual fuel oils. Light oil includes Grade No. 2 heating oil, kerosene, and jet fuel.

c Biomass fuels are not included, except those consumed at electric utilities and those added to motor gasoline.

- No consumption, including cases where adjusiments were made. See "Consumption Adjustments for Process Fuel and Intermediate Products."

-Value less than 0.05 million dollars.

Note: Totals may not equal sum of components due to independent rounding.

Sources: Data sources, estimation procedures, and assumptions are described in the "Documentation" section of this report. 
Energy Price and Expenditure Estimates by Source, Illinois $1970,1975,1980$, and 1985-1991

\begin{tabular}{|c|c|c|c|c|c|c|c|c|c|c|}
\hline Energy Source & 1970 & 1975 & 1980 & 1985 & 1986 & 1987 & 1988 & 1989 & 1990 & 1991 \\
\hline & \multicolumn{10}{|c|}{ Prices in Dollars per Million Btu } \\
\hline 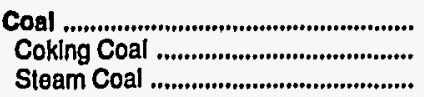 & $\begin{array}{r}0.36 \\
.42 \\
.35\end{array}$ & $\begin{array}{r}0.89 \\
1.49 \\
.82\end{array}$ & $\begin{array}{l}1.64 \\
1.93 \\
1.63\end{array}$ & $\begin{array}{l}2.12 \\
2.08 \\
2.12\end{array}$ & $\begin{array}{l}2.06 \\
2.05 \\
2.06\end{array}$ & $\begin{array}{l}1.93 \\
1.92 \\
1.93\end{array}$ & $\begin{array}{l}1.84 \\
2.02 \\
1.82\end{array}$ & $\begin{array}{l}1.77 \\
1.92 \\
1.75\end{array}$ & $\begin{array}{l}1.72 \\
1.84 \\
1.70\end{array}$ & $\begin{array}{l}1.70 \\
1.99 \\
1.67\end{array}$ \\
\hline Natural Gas ........................................ & .72 & 1.38 & 3.33 & 5.00 & 4.59 & 4.36 & 4.10 & 4.41 & 4.57 & 4.40 \\
\hline 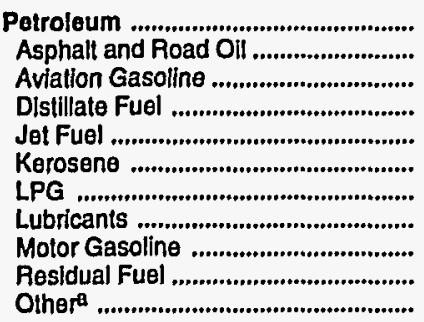 & $\begin{array}{r}1.86 \\
.64 \\
2.17 \\
1.11 \\
.74 \\
1.13 \\
1.39 \\
5.08 \\
3.05 \\
.60 \\
1.29\end{array}$ & $\begin{array}{l}3.39 \\
1.99 \\
3.45 \\
2.58 \\
2.09 \\
2.82 \\
2.68 \\
7.48 \\
4.73 \\
1.68 \\
2.36\end{array}$ & $\begin{array}{r}7.67 \\
3.83 \\
9.02 \\
6.88 \\
6.38 \\
6.67 \\
5.16 \\
14.36 \\
9.81 \\
4.92 \\
6.78\end{array}$ & $\begin{array}{r}8.49 \\
4.91 \\
9.99 \\
7.62 \\
6.00 \\
8.49 \\
8.75 \\
17.61 \\
9.03 \\
5.22 \\
7.79\end{array}$ & $\begin{array}{r}6.51 \\
4.57 \\
8.41 \\
5.75 \\
4.22 \\
5.75 \\
7.27 \\
15.59 \\
6.78 \\
4.10 \\
5.79\end{array}$ & $\begin{array}{r}6.69 \\
3.34 \\
7.55 \\
6.00 \\
4.21 \\
6.17 \\
6.86 \\
12.70 \\
7.23 \\
3.27 \\
6.23\end{array}$ & $\begin{array}{r}6.69 \\
3.51 \\
7.41 \\
5.97 \\
3.84 \\
6.20 \\
6.95 \\
14.61 \\
7.27 \\
2.63 \\
5.24\end{array}$ & $\begin{array}{r}7.25 \\
2.92 \\
8.28 \\
6.67 \\
4.32 \\
6.90 \\
7.38 \\
13.30 \\
8.00 \\
2.87 \\
6.13\end{array}$ & $\begin{array}{r}8.48 \\
3.20 \\
9.32 \\
8.17 \\
5.84 \\
8.36 \\
9.13 \\
13.40 \\
9.35 \\
3.00 \\
6.44\end{array}$ & $\begin{array}{r}8.04 \\
3.38 \\
8.71 \\
7.43 \\
4.75 \\
7.68 \\
8.16 \\
15.42 \\
9.13 \\
2.71 \\
5.95\end{array}$ \\
\hline Nuclear Fuel ......................................... & .15 & .18 & .33 & .64 & .64 & .64 & .66 & .60 & .57 & .49 \\
\hline Blomass Fuels at UtIlitles ................ & .65 & - & - & - & - & - & - & - & - & - \\
\hline Primary Energy - Five Sectors ${ }^{b}$.... & 1.08 & 2.00 & 4.32 & 4.83 & 4.04 & 3.96 & 3.75 & 3.85 & 4.34 & 4.01 \\
\hline $\begin{array}{l}\text { Electric Utility Fuelc ........................... } \\
\text { Electricity Purchased by End Users }\end{array}$ & $\begin{array}{r}.32 \\
5.98\end{array}$ & $\begin{array}{r}.69 \\
9.35\end{array}$ & $\begin{array}{r}1.60 \\
15.33\end{array}$ & $\begin{array}{r}1.67 \\
21.07\end{array}$ & $\begin{array}{r}1.61 \\
22.37\end{array}$ & $\begin{array}{r}1.42 \\
22.86\end{array}$ & $\begin{array}{r}1.23 \\
21.49\end{array}$ & $\begin{array}{r}1.13 \\
22.07\end{array}$ & $\begin{array}{r}1.12 \\
22.02\end{array}$ & $\begin{array}{r}1.06 \\
22.41\end{array}$ \\
\hline \multirow[t]{2}{*}{ Total Energy .......................................... } & 1.69 & 3.15 & 6.69 & 8.45 & 7.73 & 7.89 & $\mathbf{7 . 5 3}$ & 7.96 & 8.67 & 8.53 \\
\hline & \multicolumn{10}{|c|}{ Expenditures in Millions of Dollars } \\
\hline 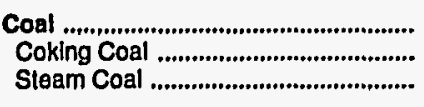 & $\begin{array}{r}329.4 \\
41.6 \\
287.8\end{array}$ & $\begin{array}{l}750.5 \\
120.7 \\
629.8\end{array}$ & $\begin{array}{r}1,388.1 \\
93.7 \\
1,294.5\end{array}$ & $\begin{array}{r}1,720.0 \\
131.6 \\
1,588.3\end{array}$ & $\begin{array}{r}1,654.0 \\
122.9 \\
1,531.1\end{array}$ & $\begin{array}{r}1,508.1 \\
129.3 \\
1,378.8\end{array}$ & $\begin{array}{r}1,370.3 \\
141.7 \\
1,228.7\end{array}$ & $\begin{array}{r}1,261.3 \\
131.2 \\
1,130.1\end{array}$ & $\begin{array}{r}1,283.1 \\
116.4 \\
1,166.8\end{array}$ & $\begin{array}{r}1,285.6 \\
119.4 \\
1,166.2\end{array}$ \\
\hline Natural Gas & 831.7 & $1,512.9$ & $3,601,8$ & $4,873.0$ & $4,234.0$ & $3,771.4$ & $3,933.5$ & $4,376.3$ & $4,271.0$ & $4,307.8$ \\
\hline 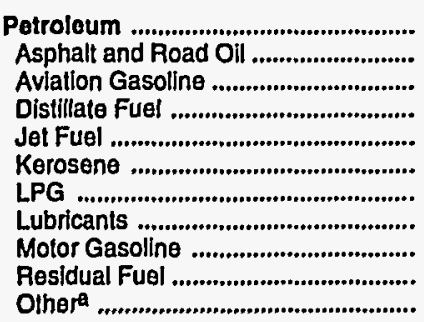 & $\begin{array}{r}2,593.2 \\
53.8 \\
2.9 \\
287.9 \\
95.2 \\
22.9 \\
148.4 \\
100.3 \\
1,715.3 \\
89.2 \\
77.2\end{array}$ & $\begin{array}{r}5,050.2 \\
135.0 \\
1.4 \\
770.9 \\
292.9 \\
41.9 \\
329.8 \\
141.6 \\
2,945.6 \\
223.0 \\
168.1\end{array}$ & $\begin{array}{r}10,370.9 \\
205.7 \\
6.0 \\
1,464.6 \\
710.2 \\
22.9 \\
702.6 \\
302.4 \\
5,622.7 \\
764.2 \\
569.7\end{array}$ & $\begin{array}{r}8,843.8 \\
244.5 \\
10.7 \\
1,427.6 \\
92.2 \\
36.3 \\
837.5 \\
337.5 \\
5,272.2 \\
157.5 \\
427.8\end{array}$ & $\begin{array}{r}6,989.9 \\
187.4 \\
8.9 \\
1,174.5 \\
48.4 \\
13.2 \\
841.8 \\
292.2 \\
3,869.4 \\
181.9 \\
372.4\end{array}$ & $\begin{array}{r}7,426.5 \\
140.1 \\
6.1 \\
1,191.9 \\
46.8 \\
10.6 \\
1,026.4 \\
269.2 \\
4,186.1 \\
126.4 \\
422.9\end{array}$ & $\begin{array}{r}7,708.1 \\
130.4 \\
7.0 \\
1,169.7 \\
85.3 \\
12.3 \\
1,123.6 \\
298.6 \\
4,436.3 \\
82.2 \\
362.6\end{array}$ & $\begin{array}{r}7,574.2 \\
156.0 \\
8.0 \\
1,342.8 \\
109.3 \\
14.4 \\
343.5 \\
278.7 \\
4,852.9 \\
60.6 \\
408.0\end{array}$ & $\begin{array}{r}9,506.1 \\
176.9 \\
7.7 \\
2,024.7 \\
130.1 \\
8.2 \\
416.8 \\
289.1 \\
5,913.3 \\
59.1 \\
480.2\end{array}$ & $\begin{array}{r}8,154.5 \\
177.5 \\
7.7 \\
1,564.0 \\
171.4 \\
8.8 \\
432.6 \\
297.5 \\
5,004.8 \\
53.1 \\
437.0\end{array}$ \\
\hline Nuclear Fuel ,...................................... & 4.1 & 45.2 & 99.4 & 270.5 & 294.6 & 347.5 & 488.7 & 484.5 & 436.4 & 381.5 \\
\hline Blomass Fuels at Utilltles ................. & * & - & - & - & - & - & - & - & - & - \\
\hline Primary Energy - Five Sectors ${ }^{b}$.... & $3,758.5$ & $7,358.8$ & $15,460.2$ & $15,707.3$ & $13,172.5$ & $13,053.6$ & $13,500.6$ & $13,696.2$ & $15,496.5$ & $14,129.4$ \\
\hline $\begin{array}{l}\text { Electric Utility Fuelc } \\
\text { Electricity Purchased by End Use.............. }\end{array}$ & $\begin{array}{r}-254.5 \\
1,417.5\end{array}$ & $\begin{array}{r}-689.6 \\
2,644.9\end{array}$ & $\begin{array}{r}-1,794.2 \\
4,948.6\end{array}$ & $\begin{array}{r}-1,856.1 \\
7,063.0\end{array}$ & $\begin{array}{r}-1,849.5 \\
7,689.4\end{array}$ & $\begin{array}{r}-1,678.7 \\
8,095.9\end{array}$ & $\begin{array}{r}-1,638.8 \\
7,995.8\end{array}$ & $\begin{array}{r}-1,550.4 \\
8,157.9\end{array}$ & $\begin{array}{r}-1,548.3 \\
8,307.1\end{array}$ & $\begin{array}{r}-1,486.4 \\
8,856.5\end{array}$ \\
\hline Total Energy ....................................... & $4,921.4$ & $9,314.1$ & $18,614.6$ & $20,914.2$ & $19,012.3$ & $19,470.9$ & $19,857.6$ & $20,303.6$ & $22,255.3$ & $21,499.5$ \\
\hline
\end{tabular}

a Includes petroleum coke used at electric utilities.

b Biomass fuels are not included, except those consumed at electric utilities and those added to motor gasoline.

c There are no direct fuel costs for hydroelectric, geothermal, centralized solar, or wind energy.

-No consumption, including cases where adjustments were made. See "Consumption Adjustments for Process Fuel and Intermediate Products."

-Value less than 0.05 million dollars.

Note: Expenditure totals may not equal sum of components due to independent rounding.

Sources: Data sources, estimation procedures, and assumptions are described in the "Documentation" section of this report. 
I Energy Price Estimates by Sector, Illinois

L $\quad 1970,1975,1980$, and 1985-1991

L (Dollars per Million Btu)

\begin{tabular}{|c|c|c|c|c|c|c|c|c|c|c|}
\hline Sector and Energy Source & 1970 & 1975 & 1980 & 1985 & 1986 & 1987 & 1988 & 1989 & 1990 & 1991 \\
\hline 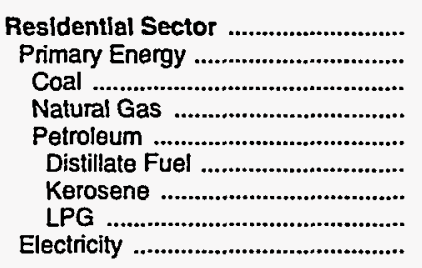 & $\begin{array}{l}1.91 \\
1.11 \\
1.03 \\
1.02 \\
1.48 \\
1.21 \\
1.65 \\
2.02 \\
7.97\end{array}$ & $\begin{array}{r}3.06 \\
1.83 \\
2.11 \\
1.57 \\
2.95 \\
2.57 \\
3.18 \\
3.72 \\
11.41\end{array}$ & $\begin{array}{r}6.05 \\
3.77 \\
2.15 \\
3.53 \\
7.02 \\
6.91 \\
8.71 \\
7.07 \\
17.78\end{array}$ & $\begin{array}{r}9.05 \\
5.47 \\
2.34 \\
5.34 \\
7.76 \\
7.38 \\
9.07 \\
7.82 \\
26.42\end{array}$ & $\begin{array}{r}9.15 \\
5.01 \\
2.05 \\
4.96 \\
6.00 \\
5.47 \\
6.72 \\
6.55 \\
27.74\end{array}$ & $\begin{array}{r}9.79 \\
4.82 \\
2.27 \\
4.74 \\
6.54 \\
6.04 \\
7.43 \\
6.94 \\
29.82\end{array}$ & $\begin{array}{r}9.12 \\
4.60 \\
2.08 \\
4.52 \\
6.35 \\
5.87 \\
7.22 \\
6.77 \\
28.54\end{array}$ & $\begin{array}{r}9.06 \\
4.91 \\
2.20 \\
4.81 \\
7.32 \\
6.12 \\
7.53 \\
8.16 \\
29.21\end{array}$ & $\begin{array}{r}9.65 \\
5.05 \\
2.26 \\
4.95 \\
7.74 \\
7.36 \\
9.05 \\
7.89 \\
29.07\end{array}$ & $\begin{array}{r}9.67 \\
4.94 \\
2.19 \\
4.86 \\
7.10 \\
7.10 \\
8.73 \\
7.02 \\
28.92\end{array}$ \\
\hline 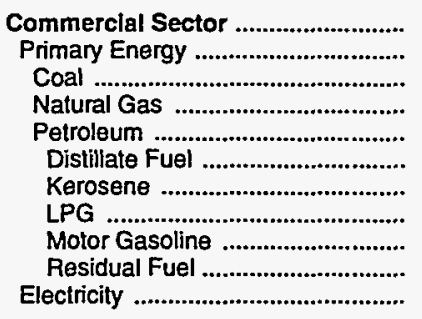 & $\begin{array}{r}1.90 \\
.73 \\
.46 \\
.73 \\
.85 \\
1.04 \\
.82 \\
1.11 \\
3.05 \\
.60 \\
6.61\end{array}$ & $\begin{array}{r}3.62 \\
1.43 \\
1.19 \\
1.28 \\
2.01 \\
2.39 \\
2.51 \\
2.29 \\
4.73 \\
1.36 \\
10.38\end{array}$ & $\begin{array}{r}7.37 \\
3.68 \\
1.71 \\
3.27 \\
6.41 \\
6.49 \\
5.93 \\
4.93 \\
9.81 \\
5.51 \\
16.70\end{array}$ & $\begin{array}{r}10.24 \\
4.98 \\
1.71 \\
4.84 \\
6.46 \\
6.11 \\
6.70 \\
8.89 \\
9.03 \\
4.14 \\
22.36\end{array}$ & $\begin{array}{r}10.73 \\
4.40 \\
1.60 \\
4.47 \\
4.29 \\
4.03 \\
4.77 \\
7.35 \\
6.78 \\
2.38 \\
23.72\end{array}$ & $\begin{array}{r}10.99 \\
4.29 \\
1.44 \\
4.33 \\
4.57 \\
4.22 \\
4.94 \\
6.85 \\
7.23 \\
2.93 \\
22.95\end{array}$ & $\begin{array}{r}10.09 \\
4.10 \\
1.35 \\
4.12 \\
4.47 \\
4.00 \\
4.55 \\
6.96 \\
7.27 \\
2.39 \\
21.38\end{array}$ & $\begin{array}{r}11.06 \\
4.46 \\
1.37 \\
4.45 \\
5.45 \\
4.72 \\
6.08 \\
7.08 \\
8.00 \\
2.43 \\
22.24\end{array}$ & $\begin{array}{r}11.18 \\
4.63 \\
1.39 \\
4.54 \\
6.61 \\
5.67 \\
7.39 \\
9.55 \\
9.35 \\
2.29 \\
22.18\end{array}$ & $\begin{array}{r}11.65 \\
4.53 \\
1.41 \\
4.47 \\
6.09 \\
4.91 \\
6.27 \\
8.57 \\
9.13 \\
2.63 \\
22.68\end{array}$ \\
\hline 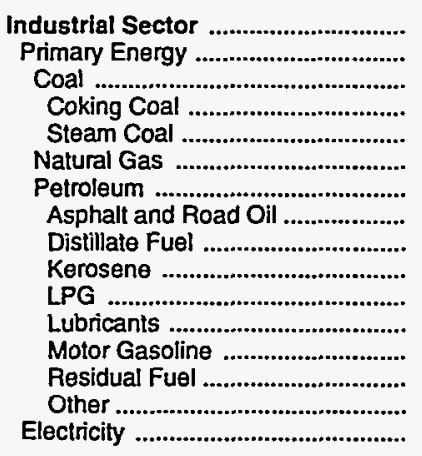 & $\begin{array}{r}.95 \\
.74 \\
.44 \\
.42 \\
.46 \\
.49 \\
1.15 \\
.64 \\
.76 \\
.82 \\
1.11 \\
5.08 \\
3.05 \\
.59 \\
1.29 \\
3.56\end{array}$ & $\begin{array}{l}2.23 \\
1.78 \\
1.33 \\
1.49 \\
1.19 \\
1.19 \\
2.52 \\
1.99 \\
2.33 \\
2.51 \\
2.29 \\
7.48 \\
4.73 \\
2.14 \\
2.36 \\
6.43\end{array}$ & $\begin{array}{r}4.94 \\
4.04 \\
1.79 \\
1.93 \\
1.71 \\
3.10 \\
5.58 \\
3.83 \\
5.37 \\
5.93 \\
4.93 \\
14.36 \\
9.81 \\
3.78 \\
6.78 \\
11.82\end{array}$ & $\begin{array}{r}6.69 \\
5.14 \\
1.88 \\
2.08 \\
1.71 \\
4.57 \\
7.62 \\
4.91 \\
6.05 \\
6.70 \\
8.89 \\
17.61 \\
9.03 \\
4.14 \\
7.79 \\
15.35\end{array}$ & $\begin{array}{r}6.23 \\
4.44 \\
1.78 \\
2.05 \\
1.60 \\
4.06 \\
6.13 \\
4.57 \\
4.31 \\
4.77 \\
7.35 \\
15.59 \\
6.78 \\
2.38 \\
5.79 \\
16.46\end{array}$ & $\begin{array}{r}6.01 \\
4.30 \\
1.64 \\
1.92 \\
1.44 \\
3.77 \\
5.96 \\
3.34 \\
4.46 \\
4.94 \\
6.85 \\
12.70 \\
7.23 \\
2.93 \\
6.23 \\
16.54\end{array}$ & $\begin{array}{r}5.64 \\
4.08 \\
1.63 \\
2.02 \\
1.35 \\
3.36 \\
5.92 \\
3.51 \\
4.10 \\
4.55 \\
6.96 \\
14.61 \\
7.27 \\
2.39 \\
5.24 \\
15.14\end{array}$ & $\begin{array}{r}5.80 \\
3.84 \\
1.61 \\
1.92 \\
1.37 \\
3.65 \\
5.65 \\
2.92 \\
5.49 \\
6.08 \\
7.08 \\
13.30 \\
8.00 \\
2.43 \\
6.13 \\
15.75\end{array}$ & $\begin{array}{r}6.23 \\
4.31 \\
1.58 \\
1.84 \\
1.39 \\
4.01 \\
6.43 \\
3.20 \\
6.67 \\
7.39 \\
9.55 \\
13.40 \\
9.35 \\
2.29 \\
6.44 \\
15.83\end{array}$ & $\begin{array}{r}6.03 \\
4.07 \\
1.63 \\
1.99 \\
1.41 \\
3.70 \\
6.21 \\
3.38 \\
5.65 \\
6.27 \\
8.57 \\
15.42 \\
9.13 \\
2.63 \\
5.95 \\
16.09\end{array}$ \\
\hline 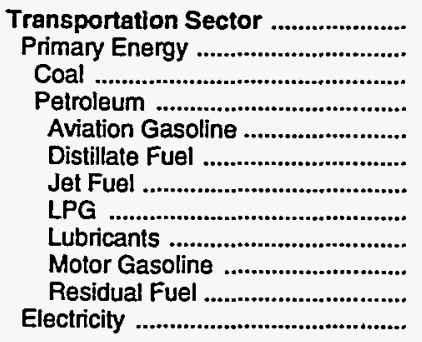 & $\begin{array}{r}2.47 \\
2.47 \\
.46 \\
2.47 \\
2.17 \\
1.39 \\
.74 \\
1.11 \\
5.08 \\
3.05 \\
.57 \\
4.08\end{array}$ & $\begin{array}{l}4.07 \\
4.06 \\
1.19 \\
4.06 \\
3.45 \\
2.84 \\
2.08 \\
2.29 \\
7.48 \\
4.73 \\
1.61 \\
6.11\end{array}$ & $\begin{array}{r}9.00 \\
8.99 \\
-\overline{8.99} \\
9.02 \\
7.45 \\
6.38 \\
4.93 \\
14.36 \\
9.81 \\
5.32 \\
11.82\end{array}$ & $\begin{array}{r}9.00 \\
8.98 \\
- \\
8.98 \\
9.99 \\
8.52 \\
6.00 \\
8.89 \\
17.61 \\
9.03 \\
5.88 \\
19.14\end{array}$ & $\begin{array}{r}6.84 \\
6.81 \\
6.81 \\
8.41 \\
6.60 \\
4.22 \\
7.35 \\
15.59 \\
6.78 \\
4.86 \\
20.87\end{array}$ & $\begin{array}{r}7.21 \\
7.19 \\
7.19 \\
7.55 \\
6.89 \\
4.21 \\
6.85 \\
12.70 \\
7.23 \\
3.32 \\
20.31\end{array}$ & $\begin{array}{r}7.20 \\
7.18 \\
7.18 \\
7.41 \\
6.91 \\
3.84 \\
6.96 \\
14.61 \\
7.27 \\
2.59 \\
18.70\end{array}$ & $\begin{array}{r}7.81 \\
7.79 \\
7.79 \\
8.28 \\
7.20 \\
4.32 \\
7.08 \\
13.30 \\
8.00 \\
2.90 \\
19.82\end{array}$ & $\begin{array}{r}9.18 \\
9.16 \\
9.16 \\
9.32 \\
8.73 \\
5.84 \\
9.55 \\
13.40 \\
9.35 \\
3.11 \\
19.64\end{array}$ & $\begin{array}{r}8.82 \\
8.80 \\
8.80 \\
8.71 \\
8.21 \\
4.75 \\
8.57 \\
15.42 \\
9.13 \\
2.39 \\
20.04\end{array}$ \\
\hline 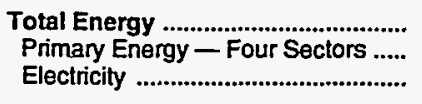 & $\begin{array}{l}1.69 \\
1.31 \\
5.98\end{array}$ & $\begin{array}{l}3.15 \\
2.49 \\
9.35\end{array}$ & $\begin{array}{r}6.69 \\
5.56 \\
15.33\end{array}$ & $\begin{array}{r}8.45 \\
6.48 \\
21.07\end{array}$ & $\begin{array}{r}7.73 \\
5.35 \\
22.37\end{array}$ & $\begin{array}{r}7.89 \\
5.38 \\
22.86\end{array}$ & $\begin{array}{r}7.53 \\
5.23 \\
21.49\end{array}$ & $\begin{array}{r}7.96 \\
5.57 \\
22.07\end{array}$ & $\begin{array}{r}8.67 \\
6.37 \\
22.02\end{array}$ & $\begin{array}{r}8.53 \\
5.95 \\
22.41\end{array}$ \\
\hline 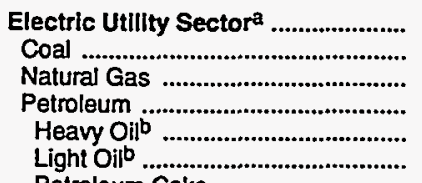 & $\begin{array}{l}.32 \\
.30 \\
.35 \\
.63 \\
.60 \\
.67\end{array}$ & $\begin{array}{r}.69 \\
.75 \\
1.13 \\
1.63 \\
1.35 \\
2.21\end{array}$ & $\begin{array}{l}1.60 \\
1.62 \\
3.19 \\
5.64 \\
5.60 \\
6.38\end{array}$ & $\begin{array}{l}1.67 \\
2.17 \\
5.19 \\
6.03 \\
6.03 \\
6.05\end{array}$ & $\begin{array}{l}1.61 \\
2.12 \\
4.62 \\
5.09 \\
5.24 \\
3.64\end{array}$ & $\begin{array}{l}1.42 \\
2.00 \\
3.66 \\
3.63 \\
3.56 \\
4.22\end{array}$ & $\begin{array}{l}1.23 \\
1.91 \\
3.27 \\
3.12 \\
2.95 \\
3.77\end{array}$ & $\begin{array}{l}1.13 \\
1.81 \\
3.26 \\
3.59 \\
3.36 \\
4.39\end{array}$ & $\begin{array}{l}1.12 \\
1.75 \\
2.67 \\
3.99 \\
3.63 \\
5.26\end{array}$ & $\begin{array}{l}1.06 \\
1.71 \\
2.10 \\
3.03 \\
2.73 \\
4.71\end{array}$ \\
\hline 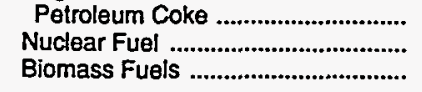 & $\begin{array}{r}. \overline{15} \\
.65\end{array}$ & $\overline{.18}$ & $\overline{.33}$ & $\overline{64}$ &.$\overline{64}$ &.$\overline{64}$ &.$\overline{6}$ &.$\overline{-}$ &.$\overline{-}$ &.$\overline{49}$ \\
\hline Primary Energy - Five Sectors ${ }^{c}$.. & 1.08 & 2.00 & 4.32 & 4.83 & 4.04 & 3.96 & 3.75 & 3.85 & 4.34 & 4.01 \\
\hline
\end{tabular}

There are no direct fuel costs for hydroelectric, geothemal, centralized solar, or wind energy.

beavy oil includes Grade Nos. 4, 5, and 6, and residual fuel oils. Light oil includes Grade No. 2 heating oil, kerosene, and jet fuel.

c Biomass fuels are not included, except those consumed at electric utilities and those added to motor gasoline.

-No consumption, including cases where adjustments were made. See "Consumption Adjustments for Process Fuel and Intermediate Products."

Sources: Data sources, estimation procedures, and assumptions are described in the "Documentation" section of this report. 


\begin{tabular}{|c|c|c|c|c|c|c|c|c|c|c|}
\hline Sector and Energy Source & 1970 & 1975 & 1980 & 1985 & 1986 & 1987 & 1988 & 1989 & 1990 & 1991 \\
\hline 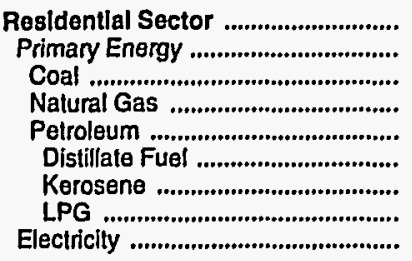 & $\begin{array}{r}1,252.7 \\
639.8 \\
18.2 \\
459.4 \\
162.2 \\
84.1 \\
12.5 \\
65.7 \\
612.9\end{array}$ & $\begin{array}{r}2,144.7 \\
1,118.4 \\
12.7 \\
772.0 \\
333.7 \\
185.3 \\
22.1 \\
126.3 \\
1,026.4\end{array}$ & $\begin{array}{r}3,801.3 \\
1,985.7 \\
3.1 \\
1,728.1 \\
254.4 \\
141.3 \\
7.9 \\
105.2 \\
1,815.6\end{array}$ & $\begin{array}{r}5,412.9 \\
2,710.7 \\
4.9 \\
2,480.4 \\
225.4 \\
97.1 \\
29.2 \\
99.1 \\
2,702.2\end{array}$ & $\begin{array}{r}5,299.9 \\
2,368.9 \\
4.3 \\
2,214.8 \\
149.8 \\
70.0 \\
7.7 \\
72.2 \\
2,930.9\end{array}$ & $\begin{array}{r}5,378.6 \\
2,123.7 \\
5.2 \\
1,961.9 \\
156.7 \\
67.1 \\
6.3 \\
83.3 \\
3,254.9\end{array}$ & $\begin{array}{r}5,597.1 \\
2,288.0 \\
4.4 \\
2,126.8 \\
156.9 \\
72.6 \\
8.9 \\
75.4 \\
3,309.0\end{array}$ & $\begin{array}{r}5,862.9 \\
2,635.9 \\
5.4 \\
2,459.9 \\
170.6 \\
56.4 \\
8.9 \\
105.4 \\
3,227.0\end{array}$ & $\begin{array}{r}5,650.6 \\
2,390.6 \\
4.7 \\
2,237.3 \\
148.5 \\
51.5 \\
5.2 \\
91.8 \\
3,260.0\end{array}$ & $\begin{array}{r}6,017.8 \\
2,468.8 \\
4.5 \\
2,311.5 \\
152.8 \\
50.8 \\
5.8 \\
96.3 \\
3,549.0\end{array}$ \\
\hline 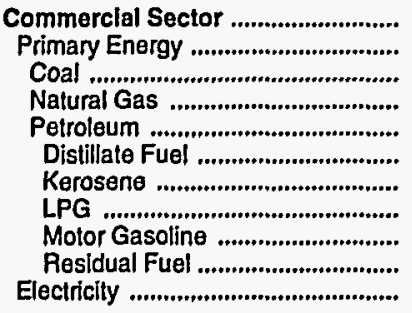 & $\begin{array}{r}733.8 \\
226.9 \\
15.2 \\
144.9 \\
66.8 \\
22.9 \\
.2 \\
6.4 \\
8.5 \\
28.8 \\
506.9\end{array}$ & $\begin{array}{r}1,419.3 \\
424.6 \\
13.4 \\
283.2 \\
128.0 \\
54.4 \\
.7 \\
13.7 \\
16.8 \\
42.4 \\
994.7\end{array}$ & $\begin{array}{r}2,802.2 \\
1,002.3 \\
4.5 \\
761.8 \\
236.0 \\
79.4 \\
.5 \\
12.9 \\
51.9 \\
91.1 \\
1,799.9\end{array}$ & $\begin{array}{r}3,768.8 \\
1,280.5 \\
6.7 \\
1,073.9 \\
200.0 \\
141.5 \\
3.6 \\
19.9 \\
26.1 \\
8.9 \\
2,488.3\end{array}$ & $\begin{array}{r}3,761.9 \\
1,038.3 \\
6.2 \\
934.7 \\
97.4 \\
46.6 \\
2.6 \\
14.3 \\
20.5 \\
13.3 \\
2,723.6\end{array}$ & $\begin{array}{r}3,748.0 \\
938.7 \\
6.1 \\
838.7 \\
94.0 \\
40.5 \\
1.2 \\
14.5 \\
21.0 \\
16.8 \\
2,809.3\end{array}$ & $\begin{array}{r}3,752.5 \\
997.5 \\
5.3 \\
901.9 \\
90.3 \\
45.6 \\
1.5 \\
13.7 \\
20.9 \\
8.7 \\
2,755.0\end{array}$ & $\begin{array}{r}3,869.2 \\
979.0 \\
6.2 \\
892.6 \\
80.3 \\
38.8 \\
2.2 \\
16.1 \\
19.7 \\
3.5 \\
2,890.1\end{array}$ & $\begin{array}{r}3,992.1 \\
1,036.8 \\
5.4 \\
929.3 \\
102.1 \\
51.1 \\
1.1 \\
19.6 \\
27.4 \\
3.0 \\
2,955.3\end{array}$ & $\begin{array}{r}4,139.6 \\
979.5 \\
5.3 \\
884.0 \\
90.3 \\
48.3 \\
1.4 \\
20.7 \\
19.1 \\
.6 \\
3,160.0\end{array}$ \\
\hline 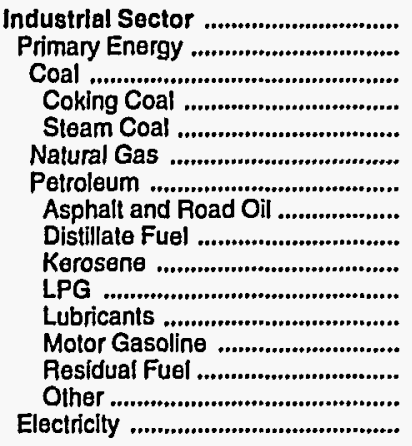 & $\begin{array}{r}1,057.8 \\
763.5 \\
115.5 \\
41.6 \\
73.9 \\
179.9 \\
468.1 \\
53.8 \\
47.4 \\
10.2 \\
74.2 \\
62.1 \\
96.4 \\
46.8 \\
77.2 \\
294.3\end{array}$ & $\begin{array}{r}2,224.5 \\
1,606.3 \\
230.2 \\
120.7 \\
109.5 \\
418.0 \\
958.1 \\
135.0 \\
150.9 \\
19.2 \\
185.6 \\
75.7 \\
106.5 \\
117.0 \\
168.1 \\
618.3\end{array}$ & $\begin{array}{r}4,777.0 \\
3,454.9 \\
228.7 \\
93.7 \\
135.1 \\
1,049.4 \\
2,176.7 \\
205.7 \\
240.0 \\
14.4 \\
581.2 \\
170.6 \\
180.7 \\
214.4 \\
569.7 \\
1,322.1\end{array}$ & $\begin{array}{r}5,325.8 \\
3,476.0 \\
266.9 \\
131.6 \\
135.2 \\
1,287.4 \\
1,921.8 \\
244.5 \\
224.0 \\
3.5 \\
705.0 \\
190.4 \\
82.5 \\
44.3 \\
427.8 \\
1,849.8\end{array}$ & $\begin{array}{r}5,118.5 \\
3,108.6 \\
264.1 \\
122.9 \\
141.2 \\
1,055.7 \\
1,788.8 \\
187.4 \\
231.3 \\
2.8 \\
745.2 \\
164.8 \\
56.0 \\
28.8 \\
372.4 \\
2,009.9\end{array}$ & $\begin{array}{r}5,210.2 \\
3,201.7 \\
260.4 \\
129.3 \\
131.1 \\
959.0 \\
1,982.3 \\
140.1 \\
248.8 \\
3.1 \\
920.9 \\
151.8 \\
59.5 \\
35.2 \\
422.9 \\
2,008.4\end{array}$ & $\begin{array}{r}5,037.5 \\
3,128.7 \\
278.9 \\
141.7 \\
137.2 \\
885.8 \\
1,964.0 \\
130.4 \\
186.8 \\
1.9 \\
1,026.0 \\
168.4 \\
57.2 \\
30.6 \\
362.6 \\
1,908.8\end{array}$ & $\begin{array}{r}4,511.8 \\
2,494.9 \\
251.0 \\
131.2 \\
119.8 \\
1,000.6 \\
1,243.3 \\
156.0 \\
220.6 \\
3.3 \\
215.1 \\
157.2 \\
59.6 \\
23.6 \\
408.0 \\
2,016.8\end{array}$ & $\begin{array}{r}4,877.1 \\
2,809.1 \\
237.9 \\
116.4 \\
121.6 \\
1,079.6 \\
1,491.6 \\
176.9 \\
295.8 \\
2.0 \\
294.0 \\
163.0 \\
61.7 \\
18.0 \\
480.2 \\
2,068.0\end{array}$ & $\begin{array}{r}4,879.5 \\
2,755.9 \\
255.8 \\
119.4 \\
136.4 \\
1,084.8 \\
1,415.4 \\
177.5 \\
252.6 \\
1.7 \\
305.9 \\
167.8 \\
64.4 \\
8.5 \\
437.0 \\
2,123.6\end{array}$ \\
\hline 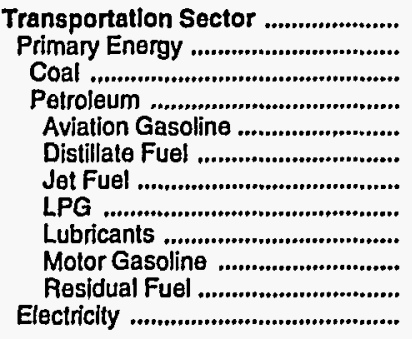 & \begin{tabular}{r|}
$1,877.0$ \\
$1,873.7$ \\
.2 \\
$1,873.5$ \\
2.9 \\
123.2 \\
95.2 \\
2.2 \\
38.2 \\
$1,610.4$ \\
1.5 \\
3.3
\end{tabular} & $\begin{array}{r}3,525.5 \\
3,519.9 \\
3,519.9 \\
1.4 \\
338.4 \\
285.7 \\
4.1 \\
65.9 \\
2,822.2 \\
2.2 \\
5.5\end{array}$ & $\begin{array}{r}7,234.1 \\
7,223.2 \\
7,223.2 \\
6.0 \\
978.7 \\
704.0 \\
3.2 \\
131.8 \\
5,390.1 \\
9.4 \\
10.9\end{array}$ & $\begin{array}{r}6,406.7 \\
6,383.9 \\
- \\
6,383.9 \\
10.7 \\
949.7 \\
92.2 \\
13.5 \\
147.1 \\
5,163.7 \\
6.9 \\
22.7\end{array}$ & $\begin{array}{r}4,832.1 \\
4,807.2 \\
-\overline{-} \\
4,807.2 \\
8.9 \\
816.9 \\
48.4 \\
10.1 \\
127.4 \\
3,792.9 \\
2.6 \\
24.9\end{array}$ & $\begin{array}{r}5,134.1 \\
5,110.8 \\
- \\
5,110.8 \\
6.1 \\
825.1 \\
46.8 \\
7.7 \\
117.4 \\
4,105.6 \\
2.1 \\
23.3\end{array}$ & $\begin{array}{r}5,470.5 \\
5,447.5 \\
- \\
5,447.5 \\
7.0 \\
852.6 \\
85.3 \\
8.5 \\
130.2 \\
4,358.2 \\
5.7 \\
22.9\end{array}$ & $\begin{array}{r}6,059.7 \\
6,035.8 \\
- \\
6,035.8 \\
8.0 \\
1,015.4 \\
109.3 \\
6.9 \\
121.5 \\
4,773.6 \\
1.0 \\
23.9\end{array}$ & $\begin{array}{r}7,735.5 \\
7,711.7 \\
-\overline{7} \\
7,711.7 \\
7.7 \\
1,611.3 \\
130.1 \\
11.4 \\
126.0 \\
5,824.2 \\
1.0 \\
23.7\end{array}$ & $\begin{array}{r}6,462.7 \\
6,438.8 \\
- \\
6,438.8 \\
7.7 \\
1,198.8 \\
171.4 \\
9.6 \\
129.7 \\
4,921.3 \\
.2 \\
23.9\end{array}$ \\
\hline $\begin{array}{l}\text { Total Energy .................................... } \\
\text { Primany Energy - Four Sectors ...... } \\
\text { Electricity ......................................... }\end{array}$ & $\begin{array}{l}4,921.4 \\
3,504.0 \\
1,417.5\end{array}$ & $\begin{array}{l}9,314.1 \\
6,669.2 \\
2,644.9\end{array}$ & $\begin{array}{r}18,614.6 \\
13,666.0 \\
4,948.6\end{array}$ & $\begin{array}{r}20,914.2 \\
13,851.2 \\
7,063.0\end{array}$ & $\begin{array}{r}19,012.3 \\
11,323.0 \\
7,689.4\end{array}$ & $\begin{array}{r}19,470.9 \\
11,375.0 \\
8,095.9\end{array}$ & $\begin{array}{r}19,857.6 \\
11,861.8 \\
7,995.8\end{array}$ & $\begin{array}{r}20,303.6 \\
12,145.7 \\
8,157.9\end{array}$ & $\begin{array}{r}22,255.3 \\
13,948.2 \\
8,307.1\end{array}$ & $\begin{array}{r}21,499.5 \\
12,643.0 \\
8,856.5\end{array}$ \\
\hline 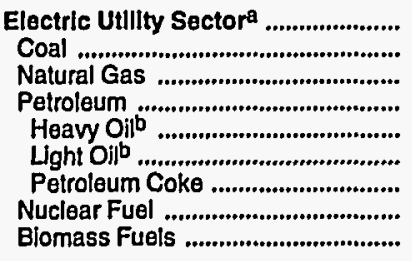 & $\begin{array}{r}254.5 \\
180.2 \\
47.7 \\
22.5 \\
12.2 \\
10.3 \\
4 . \overline{1}\end{array}$ & $\begin{array}{r}689.6 \\
494.2 \\
39.8 \\
110.5 \\
61.4 \\
49.1 \\
45 . \overline{2} \\
-\end{array}$ & $\begin{array}{r}1,794.2 \\
1,151.8 \\
62.5 \\
480.6 \\
449.3 \\
31.3 \\
- \\
99.4 \\
-\end{array}$ & $\begin{array}{r}1,856.1 \\
1,441.6 \\
31.3 \\
112.7 \\
97.4 \\
15.4 \\
- \\
270.5 \\
-\end{array}$ & $\begin{array}{r}1,849.5 \\
1,379.4 \\
28.8 \\
146.8 \\
137.1 \\
9.7 \\
- \\
294.6 \\
-\end{array}$ & $\begin{array}{r}1,678.7 \\
1,236.5 \\
11.9 \\
82.8 \\
72.3 \\
10.4 \\
- \\
347.5 \\
-\end{array}$ & $\begin{array}{r}1,638.8 \\
1,081.7 \\
19.0 \\
49.3 \\
37.2 \\
12.1 \\
- \\
488.7 \\
-\end{array}$ & $\begin{array}{r}1,550.4 \\
998.7 \\
23.1 \\
44.1 \\
32.5 \\
11.6 \\
- \\
484.5 \\
-\end{array}$ & $\begin{array}{r}1,548.3 \\
1,035.1 \\
24.8 \\
52.1 \\
37.0 \\
15.0 \\
- \\
436.4 \\
-\end{array}$ & $\begin{array}{r}1,486.4 \\
1,020.0 \\
27.6 \\
57.3 \\
43.7 \\
13.6 \\
- \\
381.5 \\
-\end{array}$ \\
\hline Prlmary Energy - Flve Sectorsc .. & $3,758.5$ & $7,358.8$ & $15,460.2$ & $15,707.3$ & $13,172.5$ & $13,053.6$ & $13,500.6$ & $13,696.2$ & $15,496.5$ & $14,129.4$ \\
\hline
\end{tabular}

a There are no direct fuel costs for hydroelectric, geothemal, centralized solar, or wind energy.

b Heavy oil includes Grade Nos. 4, 5, and 6, and residual fuel oils. Light oil includes Grade No. 2 heating oil, kerosene, and jet fuel.

c Biomass fuels are not included, except those consumed at electric utilities and those added to motor gasoline.

- No consumption, including cases where adjustments were made. See "Consumption Adjustments for Process Fuel and Intermediate Products."

Value less than 0.05 million dollars.

Note: Totals may not equal sum of components due to independent rounding.

Sources: Data sources, estimation procedures, and assumplions are described in the "Documentation" section of this report. 


\section{Energy Price and Expenditure Estimates by Source, Indiana}

N 1970, 1975, 1980, and 1985-1991

\begin{tabular}{|c|c|c|c|c|c|c|c|c|c|c|}
\hline Energy Source & 1970 & 1975 & 1980 & 1985 & 1986 & 1987 & 1988 & 1989 & 1990 & 1991 \\
\hline & \multicolumn{10}{|c|}{ Prices in Dollars per Million Btu } \\
\hline 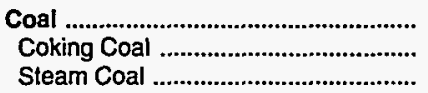 & $\begin{array}{r}0.36 \\
.44 \\
.32\end{array}$ & $\begin{array}{r}1.09 \\
1.76 \\
.73\end{array}$ & $\begin{array}{l}1.53 \\
2.13 \\
1.31\end{array}$ & $\begin{array}{l}1.77 \\
2.24 \\
1.64\end{array}$ & $\begin{array}{l}1.67 \\
2.05 \\
1.58\end{array}$ & $\begin{array}{l}1.55 \\
1.92 \\
1.46\end{array}$ & $\begin{array}{l}1.57 \\
2.02 \\
1.44\end{array}$ & $\begin{array}{l}1.49 \\
1.92 \\
1.37\end{array}$ & $\begin{array}{l}1.46 \\
1.84 \\
1.37\end{array}$ & $\begin{array}{l}1.46 \\
1.99 \\
1.36\end{array}$ \\
\hline 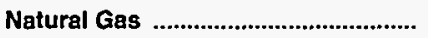 & .68 & 1.16 & 2.88 & 4.71 & 4.59 & 4.08 & 4.31 & 4.52 & 4.26 & 4.25 \\
\hline $\begin{array}{l}\text { Petroleum } \\
\text { Asphalt and Road Oil } \\
\text { Aviation Gasoline } \\
\text { Distillate Fuel } \\
\text { Jet Fuel } \\
\text { Kerosene } \\
\text { LPG } \\
\text { Lubricants } \\
\text { Motor Gasoline } \\
\text { Residual Fuel } \\
\text { Other }\end{array}$ & $\begin{array}{r}2.01 \\
.76 \\
2.17 \\
1.03 \\
.74 \\
1.48 \\
1.86 \\
5.08 \\
2.98 \\
.57 \\
.77\end{array}$ & $\begin{array}{l}3.51 \\
2.05 \\
3.45 \\
2.49 \\
2.08 \\
3.01 \\
3.34 \\
7.48 \\
4.75 \\
1.81 \\
1.94\end{array}$ & $\begin{array}{r}7.78 \\
3.72 \\
9.02 \\
6.85 \\
6.38 \\
7.94 \\
6.08 \\
14.36 \\
10.00 \\
3.63 \\
4.63\end{array}$ & $\begin{array}{r}7.87 \\
4.81 \\
9.99 \\
7.66 \\
5.81 \\
8.03 \\
8.71 \\
17.61 \\
8.85 \\
4.40 \\
6.17\end{array}$ & $\begin{array}{r}5.76 \\
3.94 \\
8.41 \\
5.77 \\
3.80 \\
5.51 \\
7.81 \\
15.59 \\
6.48 \\
2.62 \\
4.04\end{array}$ & $\begin{array}{r}5.95 \\
3.30 \\
7.55 \\
6.11 \\
3.96 \\
6.13 \\
7.44 \\
12.70 \\
6.90 \\
2.64 \\
4.18\end{array}$ & $\begin{array}{r}5.92 \\
3.33 \\
7.41 \\
5.77 \\
3.74 \\
6.11 \\
7.45 \\
14.61 \\
6.94 \\
2.10 \\
3.44\end{array}$ & $\begin{array}{r}6.48 \\
3.03 \\
8.28 \\
6.41 \\
4.33 \\
6.96 \\
8.81 \\
13.30 \\
7.60 \\
2.34 \\
4.01\end{array}$ & $\begin{array}{r}7.40 \\
3.14 \\
9.32 \\
7.59 \\
5.62 \\
8.32 \\
9.63 \\
13.40 \\
8.74 \\
2.66 \\
4.26\end{array}$ & $\begin{array}{r}7.21 \\
3.14 \\
8.71 \\
7.14 \\
4.76 \\
7.65 \\
9.37 \\
15.42 \\
8.60 \\
2.37 \\
4.18\end{array}$ \\
\hline Nuclear Fuel & - & - & - & - & - & - & - & - & - & - \\
\hline Blomass Fuels at Utillties ................... & - & - & - & - & - & - & - & - & - & - \\
\hline Primary Energy - Five Sectors ${ }^{b} \ldots$ & .93 & 1.90 & 3.67 & 4.12 & 3.49 & 3.42 & 3.37 & 3.54 & 3.72 & 3.65 \\
\hline $\begin{array}{l}\text { Electric Utility Fuelc } \\
\text { Electricity Purchased by End Users }\end{array}$ & $\begin{array}{r}.26 \\
5.15\end{array}$ & $\begin{array}{r}.62 \\
7.08\end{array}$ & $\begin{array}{r}1.30 \\
12.32\end{array}$ & $\begin{array}{r}1.66 \\
16.95\end{array}$ & $\begin{array}{r}1.59 \\
17.74\end{array}$ & $\begin{array}{r}1.47 \\
16.91\end{array}$ & $\begin{array}{r}1.44 \\
16.53\end{array}$ & $\begin{array}{r}1.37 \\
15.92\end{array}$ & $\begin{array}{r}1.38 \\
15.75\end{array}$ & $\begin{array}{r}1.36 \\
15.64\end{array}$ \\
\hline \multirow[t]{2}{*}{ Total Energy } & 1.43 & 2.82 & 5.64 & 6.94 & 6.28 & 6.09 & 6.06 & 6.31 & 6.71 & 6.73 \\
\hline & \multicolumn{10}{|c|}{ Expenditures in MIllions of Dollars } \\
\hline 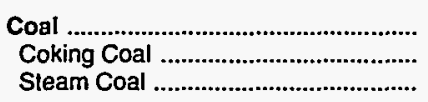 & $\begin{array}{l}364.5 \\
151.8 \\
212.7\end{array}$ & $\begin{array}{r}1,155.2 \\
651.7 \\
503.5\end{array}$ & $\begin{array}{r}1,775.9 \\
684.0 \\
1,091.9\end{array}$ & $\begin{array}{r}2,108.1 \\
560.1 \\
1,548.0\end{array}$ & $\begin{array}{r}1,881.9 \\
429.4 \\
1,452.5\end{array}$ & $\begin{array}{r}1,812.4 \\
454.8 \\
1,357.7\end{array}$ & $\begin{array}{r}1,989.6 \\
561.8 \\
1,427.8\end{array}$ & $\begin{array}{r}1,911.0 \\
502.0 \\
1,409.0\end{array}$ & $\begin{array}{r}1,983.5 \\
437.9 \\
1,545.6\end{array}$ & $\begin{array}{r}1,958.1 \\
438.9 \\
1,519.2\end{array}$ \\
\hline 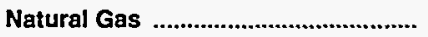 & 359.0 & 532.0 & $1,343.1$ & $1,995.4$ & $1,776.1$ & $1,639.6$ & $1,920.7$ & $2,043.8$ & $1,875.7$ & $1,903.3$ \\
\hline 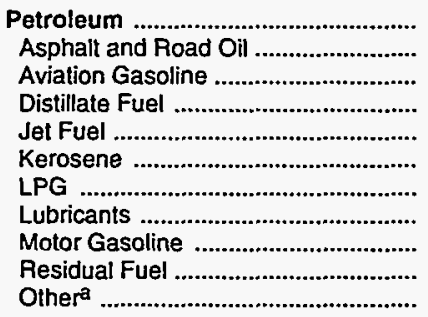 & $\begin{array}{r}1,314.0 \\
30.7 \\
4.0 \\
176.3 \\
10.6 \\
17.9 \\
62.9 \\
48.8 \\
921.2 \\
14.2 \\
27.4\end{array}$ & $\begin{array}{r}2,643.4 \\
82.5 \\
3.8 \\
473.9 \\
30.4 \\
14.3 \\
150.9 \\
72.8 \\
1,614.2 \\
120.0 \\
80.6\end{array}$ & $\begin{array}{r}5,383.4 \\
127.5 \\
11.8 \\
1,227.3 \\
76.5 \\
29.7 \\
174.3 \\
155.7 \\
3,162.9 \\
261.7 \\
155.9\end{array}$ & $\begin{array}{r}5,348.7 \\
170.2 \\
19.8 \\
1,373.4 \\
507.4 \\
33.3 \\
153.5 \\
173.8 \\
2,694.3 \\
57.9 \\
165.1\end{array}$ & $\begin{array}{r}4,174.6 \\
158.5 \\
18.4 \\
1,067.7 \\
400.2 \\
22.9 \\
172.4 \\
150.5 \\
2,043.5 \\
44.8 \\
95.8\end{array}$ & $\begin{array}{r}4,508.9 \\
166.4 \\
14.4 \\
1,126.8 \\
428.4 \\
20.9 \\
164.1 \\
138.6 \\
2,289.7 \\
43.7 \\
116.0\end{array}$ & $\begin{array}{r}4,319.1 \\
153.5 \\
16.2 \\
965.7 \\
350.1 \\
24.7 \\
182.0 \\
153.8 \\
2,340.0 \\
29.9 \\
103.4\end{array}$ & $\begin{array}{r}4,849.7 \\
128.4 \\
12.0 \\
1,235.7 \\
429.8 \\
25.7 \\
261.9 \\
143.5 \\
2,460.7 \\
35.7 \\
116.4\end{array}$ & $\begin{array}{r}5,737.5 \\
178.1 \\
14.2 \\
1,447.3 \\
569.3 \\
17.4 \\
331.5 \\
148.9 \\
2,827.0 \\
47.7 \\
156.0\end{array}$ & $\begin{array}{r}5,387.4 \\
146.8 \\
13.3 \\
1,347.8 \\
459.8 \\
17.6 \\
313.4 \\
153.2 \\
2,769.5 \\
31.0 \\
135.0\end{array}$ \\
\hline 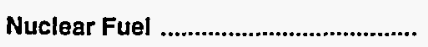 & - & - & - & - & - & - & - & - & - & - \\
\hline Blomass Fuels at Utilities ................. & - & - & - & - & - & - & - & - & - & - \\
\hline Primary Energy - Five Sectors ${ }^{b}$.... & $2,037.6$ & $4,330.6$ & $8,502.3$ & $9,452.2$ & $7,832.5$ & $7,960.9$ & $8,229.4$ & $8,804.5$ & $9,596.6$ & $9,248.8$ \\
\hline $\begin{array}{l}\text { Electric Utility Fuelc .......................... } \\
\text { Electricity Purchased by End Users }\end{array}$ & $\begin{array}{r}-136.5 \\
657.3\end{array}$ & $\begin{array}{r}-372.6 \\
1,252.3\end{array}$ & $\begin{array}{r}-951.4 \\
2,524.5\end{array}$ & $\begin{array}{r}-1,359.6 \\
3,647.8\end{array}$ & $\begin{array}{r}-1,270.9 \\
3,846.6\end{array}$ & $\begin{array}{r}-1,194.4 \\
3,848.9\end{array}$ & $\begin{array}{r}-1,261.8 \\
3,993.9\end{array}$ & $\begin{array}{r}-1,254.3 \\
3,912.8\end{array}$ & $\begin{array}{r}-1,404.9 \\
3,927.0\end{array}$ & $\begin{array}{r}-1,391.5 \\
4,062.7\end{array}$ \\
\hline 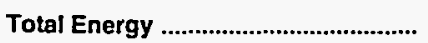 & $2,558.3$ & $5,210.2$ & $10,075.4$ & $11,740.5$ & $10,408.3$ & $10,615.4$ & $10,961.6$ & $11,463.0$ & $12,118.7$ & $11,920.0$ \\
\hline
\end{tabular}

a Includes petroleum coke used at electric utilities.

b Biomass fuels are not included, except those consumed at electric utilities and those added to motor gasoline.

c There are no direct fuel costs for hydroelectric, geothermal, centralized solar, or wind energy.

-No consumption, including cases where adjustments were made. See "Consumption Adjustments for Process Fuel and Intermediate Products."

Note: Expenditure totals may not equal sum of components due to independent rounding.

Sources: Data sources, estimation procedures, and assumptions are described in the "Documentation" section of this report. 
Energy Price Estimates by Sector, Indiana $1970,1975,1980$, and 1985-1991

(Dollars per Million Btu)

\begin{tabular}{|c|c|c|c|c|c|c|c|c|c|c|}
\hline Sector and Energy Source & 1970 & 1975 & 1980 & 1985 & 1986 & 1987 & 1988 & 1989 & 1990 & 1991 \\
\hline 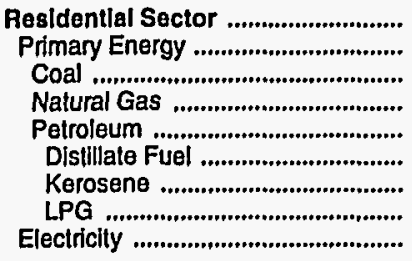 & $\begin{array}{l}2.02 \\
1.18 \\
1.10 \\
1.00 \\
1.52 \\
1.21 \\
1.59 \\
2.10 \\
6.56\end{array}$ & $\begin{array}{l}3.20 \\
2.00 \\
2.52 \\
1.47 \\
3.02 \\
2.57 \\
3.11 \\
3.92 \\
8.55\end{array}$ & $\begin{array}{r}6.42 \\
4.10 \\
2.43 \\
3.19 \\
7.31 \\
7.18 \\
8.55 \\
7.37 \\
13.86\end{array}$ & $\begin{array}{r}9.82 \\
5.81 \\
2.77 \\
5.50 \\
8.04 \\
7.50 \\
8.79 \\
8.76 \\
20.37\end{array}$ & $\begin{array}{r}10.02 \\
5.55 \\
2.83 \\
5.47 \\
6.40 \\
5.66 \\
6.64 \\
7.64 \\
21.14\end{array}$ & $\begin{array}{r}9.88 \\
5.10 \\
2.57 \\
4.91 \\
6.38 \\
5.71 \\
6.69 \\
7.32 \\
21.38\end{array}$ & $\begin{array}{r}9.72 \\
5.25 \\
2.54 \\
5.08 \\
6.49 \\
5.71 \\
6.69 \\
7.46 \\
20.95\end{array}$ & $\begin{array}{r}9.87 \\
5.78 \\
2.46 \\
5.41 \\
8.06 \\
6.30 \\
7.38 \\
9.78 \\
20.31\end{array}$ & $\begin{array}{r}10.13 \\
5.73 \\
2.62 \\
5.28 \\
8.95 \\
7.52 \\
8.81 \\
10.09 \\
20.13\end{array}$ & $\begin{array}{r}10.16 \\
5.71 \\
2.62 \\
5.38 \\
8.02 \\
6.95 \\
8.15 \\
8.97 \\
19.72\end{array}$ \\
\hline 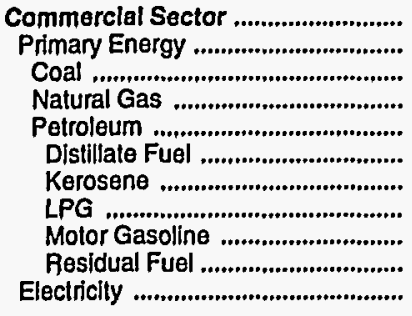 & $\begin{array}{r}1.78 \\
.86 \\
.52 \\
.83 \\
1.09 \\
1.04 \\
.81 \\
1.28 \\
2.98 \\
.70 \\
6.58\end{array}$ & $\begin{array}{l}3.03 \\
1.56 \\
1.36 \\
1.26 \\
2.27 \\
2.39 \\
2.41 \\
2.63 \\
4.75 \\
1.74 \\
8.53\end{array}$ & $\begin{array}{r}6.18 \\
3.69 \\
1.58 \\
2.99 \\
5.51 \\
6.66 \\
6.14 \\
5.10 \\
10.00 \\
4.35 \\
13.36\end{array}$ & $\begin{array}{r}8.72 \\
5.03 \\
1.60 \\
5.00 \\
6.30 \\
6.04 \\
6.69 \\
8.66 \\
8.85 \\
4.40 \\
17.51\end{array}$ & $\begin{array}{r}9.09 \\
4.54 \\
1.53 \\
4.90 \\
4.60 \\
3.94 \\
4.20 \\
7.93 \\
6.48 \\
2.61 \\
18.42\end{array}$ & $\begin{array}{r}9.06 \\
4.22 \\
1.38 \\
4.41 \\
4.90 \\
4.17 \\
4.98 \\
7.55 \\
6.90 \\
2.61 \\
18.34\end{array}$ & $\begin{array}{r}9.43 \\
4.26 \\
1.47 \\
4.53 \\
4.66 \\
3.70 \\
4.55 \\
7.44 \\
6.94 \\
2.08 \\
18.72\end{array}$ & $\begin{array}{r}9.46 \\
4.56 \\
1.46 \\
4.70 \\
5.42 \\
4.79 \\
5.07 \\
7.81 \\
7.60 \\
2.31 \\
18.18\end{array}$ & $\begin{array}{r}9.69 \\
4.55 \\
1.44 \\
4.52 \\
6.85 \\
5.32 \\
6.80 \\
9.36 \\
8.74 \\
2.64 \\
17.97\end{array}$ & $\begin{array}{r}9.73 \\
4.52 \\
1.44 \\
4.55 \\
5.96 \\
4.73 \\
5.94 \\
9.61 \\
8.60 \\
2.38 \\
17.64\end{array}$ \\
\hline 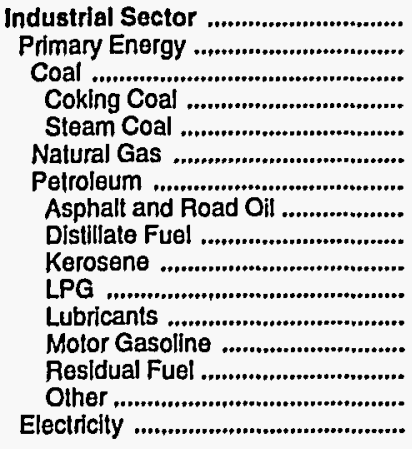 & $\begin{array}{r}.75 \\
.58 \\
.47 \\
.44 \\
.52 \\
.47 \\
1.05 \\
.76 \\
.74 \\
.81 \\
1.28 \\
5.08 \\
2.98 \\
.50 \\
.80 \\
3.52\end{array}$ & $\begin{array}{r}2.01 \\
1.64 \\
1.68 \\
1.76 \\
1.36 \\
.91 \\
2.30 \\
2.05 \\
2.24 \\
2.41 \\
2.63 \\
7.48 \\
4.75 \\
1.86 \\
1.94 \\
5.67\end{array}$ & $\begin{array}{r}3.67 \\
2.76 \\
1.99 \\
2.13 \\
1.58 \\
2.63 \\
4.76 \\
3.72 \\
5.57 \\
6.14 \\
5.10 \\
14.36 \\
10.00 \\
3.43 \\
4.63 \\
11.00\end{array}$ & $\begin{array}{r}4.87 \\
3.39 \\
2.04 \\
2.24 \\
1.60 \\
4.04 \\
6.42 \\
4.81 \\
6.03 \\
6.69 \\
8.66 \\
17.61 \\
8.85 \\
4.40 \\
6.17 \\
14.54\end{array}$ & $\begin{array}{r}4.71 \\
3.01 \\
1.87 \\
2.05 \\
1.53 \\
3.79 \\
4.73 \\
3.94 \\
3.79 \\
4.20 \\
7.93 \\
15.59 \\
6.48 \\
2.61 \\
4.25 \\
15.13\end{array}$ & $\begin{array}{r}4.24 \\
2.79 \\
1.75 \\
1.92 \\
1.38 \\
3.38 \\
4.51 \\
3.30 \\
4.49 \\
4.98 \\
7.55 \\
12.70 \\
6.90 \\
2.61 \\
4.38 \\
13.35\end{array}$ & $\begin{array}{r}4.12 \\
2.86 \\
1.87 \\
2.02 \\
1.47 \\
3.69 \\
4.39 \\
3.33 \\
4.10 \\
4.55 \\
7.44 \\
14.61 \\
6.94 \\
2.08 \\
3.62 \\
12.43\end{array}$ & $\begin{array}{r}4.19 \\
2.93 \\
1.79 \\
1.92 \\
1.46 \\
3.83 \\
4.64 \\
3.03 \\
4.57 \\
5.07 \\
7.81 \\
13.30 \\
7.60 \\
2.31 \\
4.34 \\
11.98\end{array}$ & $\begin{array}{r}4.31 \\
3.06 \\
1.72 \\
1.84 \\
1.44 \\
3.57 \\
5.23 \\
3.14 \\
6.14 \\
6.80 \\
9.36 \\
13.40 \\
8.74 \\
2.64 \\
4.93 \\
11.94\end{array}$ & $\begin{array}{r}4.37 \\
3.08 \\
1.82 \\
1.99 \\
1.44 \\
3.48 \\
5.25 \\
3.14 \\
5.36 \\
5.94 \\
9.61 \\
15.42 \\
8.60 \\
2.38 \\
4.42 \\
11.83\end{array}$ \\
\hline 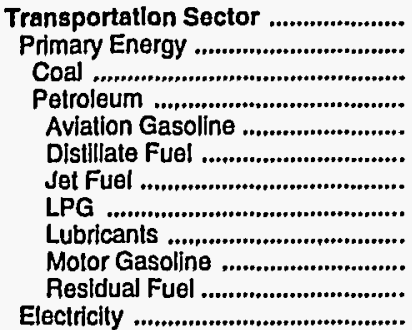 & $\begin{array}{r}2.66 \\
2.66 \\
.52 \\
2.67 \\
2.17 \\
1.23 \\
.74 \\
1.28 \\
5.08 \\
2.98 \\
.65 \\
-\end{array}$ & $\begin{array}{r}4.35 \\
4.35 \\
1.36 \\
4.35 \\
3.45 \\
2.69 \\
2.08 \\
2.63 \\
7.48 \\
4.75 \\
1.53 \\
-\end{array}$ & $\begin{array}{r}9.25 \\
9.25 \\
- \\
9.25 \\
9.02 \\
7.17 \\
6.38 \\
5.10 \\
14.36 \\
10.00 \\
3.87 \\
-\end{array}$ & $\begin{array}{r}8.27 \\
8.27 \\
- \\
8.27 \\
9.99 \\
8.28 \\
5.81 \\
8.66 \\
17.61 \\
8.85 \\
4.85 \\
-\end{array}$ & $\begin{array}{r}6.03 \\
6.03 \\
- \\
6.03 \\
8.41 \\
6.46 \\
3.80 \\
7.93 \\
15.59 \\
6.48 \\
3.25 \\
-\end{array}$ & $\begin{array}{r}6.35 \\
6.35 \\
- \\
6.35 \\
7.55 \\
6.76 \\
3.96 \\
7.55 \\
12.70 \\
6.90 \\
3.22 \\
-\end{array}$ & $\begin{array}{r}6.31 \\
6.31 \\
- \\
6.31 \\
7.41 \\
6.31 \\
3.74 \\
7.44 \\
14.61 \\
6.94 \\
2.37 \\
-\end{array}$ & $\begin{array}{r}6.88 \\
6.88 \\
-\overline{6} \\
6.88 \\
8.28 \\
6.89 \\
4.33 \\
7.81 \\
13.30 \\
7.60 \\
2.66 \\
-\end{array}$ & $\begin{array}{r}8.03 \\
8.03 \\
- \\
8.03 \\
9.32 \\
8.00 \\
5.62 \\
9.36 \\
13.40 \\
8.74 \\
2.80 \\
23.88\end{array}$ & $\begin{array}{r}7.76 \\
7.76 \\
- \\
7.76 \\
8.71 \\
7.71 \\
4.76 \\
9.61 \\
15.42 \\
8.60 \\
2.29 \\
23.17\end{array}$ \\
\hline $\begin{array}{l}\text { Total Energy ................................... } \\
\text { Primary Energy - Four Sectors ...... } \\
\text { Electriclty ......................................... }\end{array}$ & $\begin{array}{l}1.43 \\
1.15 \\
5.15\end{array}$ & $\begin{array}{l}2.82 \\
2.37 \\
7.08\end{array}$ & $\begin{array}{r}5.64 \\
4.78 \\
12.32\end{array}$ & $\begin{array}{r}6.94 \\
5.48 \\
16.95\end{array}$ & $\begin{array}{r}6.28 \\
4.56 \\
17.74\end{array}$ & $\begin{array}{r}6.09 \\
4.46 \\
16.91\end{array}$ & $\begin{array}{r}6.06 \\
4.45 \\
16.53\end{array}$ & $\begin{array}{r}6.31 \\
4.80 \\
15.92\end{array}$ & $\begin{array}{r}6.71 \\
5.26 \\
15.75\end{array}$ & $\begin{array}{r}6.73 \\
5.20 \\
15.64\end{array}$ \\
\hline 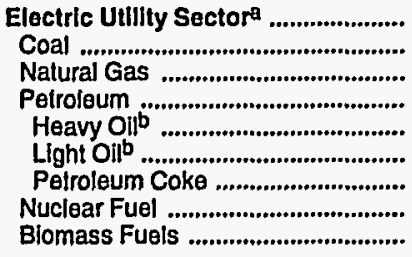 & $\begin{array}{l}.26 \\
.25 \\
.35 \\
.58 \\
.75 \\
.77 \\
.24 \\
- \\
-\end{array}$ & $\begin{array}{r}.62 \\
.59 \\
.82 \\
1.83 \\
1.74 \\
2.12 \\
- \\
- \\
-\end{array}$ & $\begin{array}{r}1.30 \\
1.26 \\
2.51 \\
5.99 \\
-\overrightarrow{5} \\
- \\
- \\
-\end{array}$ & $\begin{array}{r}1.66 \\
1.64 \\
4.15 \\
5.87 \\
-\overline{5} \\
- \\
- \\
-\end{array}$ & $\begin{array}{r}1.59 \\
1.58 \\
4.54 \\
2.55 \\
-\overline{3} \\
3.89 \\
.80 \\
- \\
-\end{array}$ & $\begin{array}{r}1.47 \\
1.47 \\
2.82 \\
2.71 \\
-\overline{12} \\
4.12 \\
.99 \\
- \\
-\end{array}$ & $\begin{array}{r}1.44 \\
1.43 \\
2.59 \\
2.37 \\
- \\
3.63 \\
.93 \\
- \\
-\end{array}$ & $\begin{array}{r}1.37 \\
1.36 \\
2.69 \\
2.30 \\
-\overline{4} \\
4.22 \\
.74 \\
- \\
-\end{array}$ & $\begin{array}{r}1.38 \\
1.36 \\
2.58 \\
2.03 \\
-\overline{12} \\
5.12 \\
- \\
-\end{array}$ & $\begin{array}{r}1.36 \\
1.34 \\
2.38 \\
2.86 \\
- \\
4.94 \\
.81 \\
- \\
-\end{array}$ \\
\hline Primary Energy - Five Sectors $c$.. & .93 & 1.90 & 3.67 & 4.12 & 3.49 & 3.42 & 3.37 & 3.54 & 3.72 & 3.65 \\
\hline
\end{tabular}

There are no direct fuel costs for hydroelectric, geothermal, centralized solar, or wind energy.

b Heavy oil includes Grade Nos. 4, 5, and 6, and residual fuel oils. Light oil includes Grade No. 2 heating oil, kerosene, and jet fuel.

c Biomass fuels are not included, except those consumed at electric utilities and those added to motor gasoline.

- No consumption, including cases where adjustments were made. See "Consumption Adjustments for Process Fuel and Intermediate Products."

Sources: Data sources, estimation procedures, and assumptions are described in the "Documentation" section of this report. 
I Energy Expenditure Estimates by Sector, Indiana

N 1970, 1975, 1980, and 1985-1991

D (Million Dollars)

\begin{tabular}{|c|c|c|c|c|c|c|c|c|c|c|}
\hline Sector and Energy Source & 1970 & 1975 & 1980 & 1985 & 1986 & 1987 & 1988 & 1989 & 1990 & 1991 \\
\hline 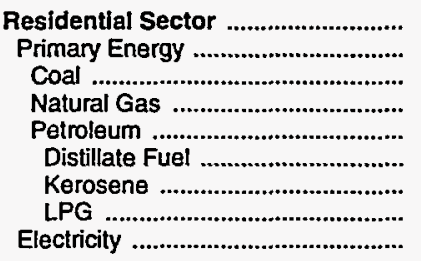 & $\begin{array}{r}591.5 \\
289.6 \\
6.3 \\
160.3 \\
123.1 \\
56.3 \\
16.6 \\
50.2 \\
301.8\end{array}$ & $\begin{array}{r}971.1 \\
493.7 \\
17.6 \\
237.0 \\
239.1 \\
129.4 \\
12.6 \\
97.1 \\
477.5\end{array}$ & $\begin{array}{r}1,771.6 \\
860.9 \\
4.1 \\
516.3 \\
340.4 \\
225.8 \\
23.8 \\
90.8 \\
910.8\end{array}$ & $\begin{array}{r}2,406.9 \\
1,030.6 \\
11.3 \\
810.4 \\
208.9 \\
111.8 \\
23.2 \\
73.9 \\
1,376.4\end{array}$ & $\begin{array}{r}2,446.8 \\
967.4 \\
12.2 \\
772.9 \\
182.3 \\
95.1 \\
14.8 \\
72.4 \\
1,479.4\end{array}$ & $\begin{array}{r}2,430.6 \\
886.1 \\
11.1 \\
688.3 \\
186.8 \\
91.8 \\
15.3 \\
79.6 \\
1,544.4\end{array}$ & $\begin{array}{r}2,617.2 \\
1,010.2 \\
12.7 \\
792.6 \\
204.8 \\
92.9 \\
19.7 \\
92.3 \\
1,607.0\end{array}$ & $\begin{array}{r}2,665.9 \\
1,121.9 \\
10.0 \\
857.6 \\
254.3 \\
84.9 \\
22.3 \\
147.1 \\
1,544.0\end{array}$ & $\begin{array}{r}2,503.3 \\
984.3 \\
11.4 \\
755.8 \\
217.0 \\
75.3 \\
13.9 \\
127.9 \\
1,519.0\end{array}$ & $\begin{array}{r}2,644.7 \\
1,014.7 \\
9.0 \\
799.6 \\
206.2 \\
78.4 \\
14.6 \\
113.1 \\
1,630.0\end{array}$ \\
\hline 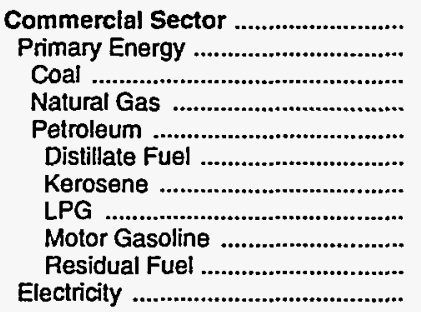 & $\begin{array}{r}247.2 \\
100.8 \\
5.5 \\
64.5 \\
30.7 \\
16.9 \\
.8 \\
5.4 \\
3.9 \\
3.7 \\
146.4\end{array}$ & $\begin{array}{r}444.7 \\
180.7 \\
17.6 \\
87.7 \\
75.3 \\
41.9 \\
1.0 \\
11.5 \\
3.0 \\
18.0 \\
264.0\end{array}$ & $\begin{array}{r}854.4 \\
379.3 \\
5.0 \\
206.9 \\
167.4 \\
77.0 \\
1.1 \\
11.1 \\
11.7 \\
66.5 \\
475.1\end{array}$ & $\begin{array}{r}1,233.2 \\
500.8 \\
12.1 \\
350.9 \\
137.8 \\
92.8 \\
5.1 \\
12.9 \\
16.3 \\
10.7 \\
732.3\end{array}$ & $\begin{array}{r}1,224.7 \\
411.7 \\
12.1 \\
320.3 \\
79.3 \\
42.2 \\
3.3 \\
13.3 \\
16.6 \\
4.0 \\
813.0\end{array}$ & $\begin{array}{r}1,212.8 \\
371.0 \\
11.0 \\
288.8 \\
71.2 \\
33.9 \\
1.4 \\
14.5 \\
16.8 \\
4.6 \\
841.8\end{array}$ & $\begin{array}{r}1,414.3 \\
410.3 \\
13.7 \\
329.9 \\
66.8 \\
28.8 \\
2.0 \\
16.2 \\
16.5 \\
3.1 \\
1,004.0\end{array}$ & $\begin{array}{r}1,423.2 \\
439.2 \\
11.0 \\
351.9 \\
76.4 \\
32.2 \\
1.1 \\
20.7 \\
17.1 \\
5.1 \\
984.0\end{array}$ & $\begin{array}{r}1,391.2 \\
403.1 \\
11.6 \\
309.4 \\
82.1 \\
33.2 \\
1.3 \\
20.9 \\
25.6 \\
1.0 \\
988.1\end{array}$ & $\begin{array}{r}1,423.0 \\
398.8 \\
9.1 \\
315.5 \\
74.3 \\
32.4 \\
1.5 \\
21.4 \\
16.0 \\
3.1 \\
1,024.2\end{array}$ \\
\hline 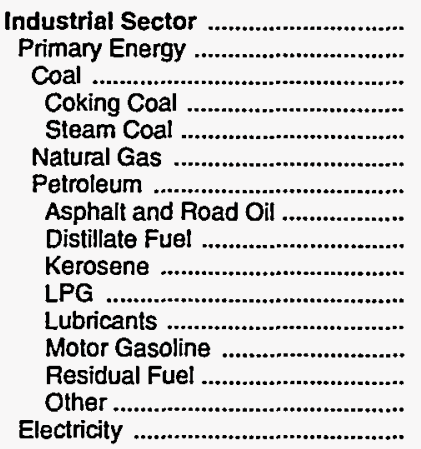 & $\begin{array}{r}743.8 \\
534.7 \\
228.6 \\
151.8 \\
76.9 \\
123.9 \\
182.2 \\
30.7 \\
43.8 \\
.5 \\
6.9 \\
30.0 \\
35.0 \\
8.2 \\
27.1 \\
209.0\end{array}$ & $\begin{array}{r}1,966.2 \\
1,455.4 \\
776.8 \\
651.7 \\
125.1 \\
198.3 \\
480.3 \\
82.5 \\
121.5 \\
.8 \\
41.0 \\
38.2 \\
31.5 \\
84.2 \\
80.6 \\
510.8\end{array}$ & $\begin{array}{r}3,446.0 \\
2,307.4 \\
845.6 \\
684.0 \\
161.6 \\
615.0 \\
846.8 \\
127.5 \\
162.6 \\
4.7 \\
70.8 \\
95.5 \\
39.5 \\
190.3 \\
155.9 \\
1,138.6\end{array}$ & $\begin{array}{r}3,867.5 \\
2,328.4 \\
744.0 \\
560.1 \\
183.9 \\
829.5 \\
754.9 \\
170.2 \\
157.8 \\
5.0 \\
62.1 \\
106.5 \\
41.9 \\
46.2 \\
165.1 \\
1,539.1\end{array}$ & $\begin{array}{r}3,451.2 \\
1,897.0 \\
600.2 \\
429.4 \\
170.7 \\
677.9 \\
618.9 \\
158.5 \\
118.2 \\
4.8 \\
82.5 \\
92.2 \\
28.3 \\
39.9 \\
94.6 \\
1,554.2\end{array}$ & $\begin{array}{r}3,383.0 \\
1,920.3 \\
609.8 \\
454.8 \\
155.0 \\
658.8 \\
651.8 \\
166.4 \\
148.4 \\
4.2 \\
66.4 \\
85.0 \\
30.3 \\
36.9 \\
114.3 \\
1,462.6\end{array}$ & $\begin{array}{r}3,469.4 \\
2,086.5 \\
720.2 \\
561.8 \\
158.4 \\
789.2 \\
577.1 \\
153.5 \\
101.6 \\
3.0 \\
69.4 \\
94.3 \\
29.3 \\
24.5 \\
101.6 \\
1,382.9\end{array}$ & $\begin{array}{r}3,473.9 \\
2,089.1 \\
657.8 \\
502.0 \\
155.7 \\
823.2 \\
608.1 \\
128.4 \\
128.2 \\
2.3 \\
89.7 \\
88.0 \\
30.2 \\
27.0 \\
114.4 \\
1,384.8\end{array}$ & $\begin{array}{r}3,637.3 \\
2,218.3 \\
589.5 \\
437.9 \\
151.6 \\
793.3 \\
835.5 \\
178.1 \\
162.8 \\
2.1 \\
177.5 \\
91.3 \\
28.5 \\
43.2 \\
151.9 \\
1,419.0\end{array}$ & $\begin{array}{r}3,530.2 \\
2,122.6 \\
584.3 \\
438.9 \\
145.4 \\
764.3 \\
774.0 \\
146.8 \\
166.3 \\
1.6 \\
173.4 \\
93.9 \\
32.0 \\
26.6 \\
133.3 \\
1,407.6\end{array}$ \\
\hline 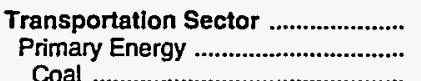 & $\begin{array}{l}975.9 \\
975.9\end{array}$ & $\begin{array}{l}1,828.2 \\
1,828.2\end{array}$ & $\begin{array}{r}4,003.3 \\
4,003.3\end{array}$ & $\begin{array}{l}4,232.9 \\
4,232.9\end{array}$ & $\begin{array}{l}3,285.6 \\
3,285.6\end{array}$ & $\begin{array}{l}3,589.0 \\
3,589.0\end{array}$ & $\begin{array}{l}3,460.6 \\
3,460.6\end{array}$ & $\begin{array}{l}3,899.9 \\
3,899.9\end{array}$ & $\begin{array}{l}4,587.0 \\
4,586.1\end{array}$ & $\begin{array}{l}4,322.0 \\
4,321.2\end{array}$ \\
\hline 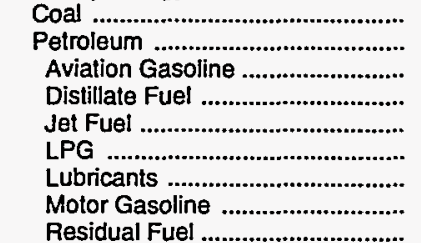 & $\begin{array}{r}.4 \\
975.5 \\
4.0 \\
58.1 \\
10.6 \\
.5 \\
18.8 \\
882.2 \\
1.3\end{array}$ & $\begin{array}{r}.1 \\
1,828.1 \\
3.8 \\
175.3 \\
30.4 \\
1.2 \\
34.6 \\
1,579.7 \\
3.2\end{array}$ & $\begin{array}{r}4,003.3 \\
11.8 \\
736.5 \\
76.5 \\
1.6 \\
60.3 \\
3,111.7 \\
4.9\end{array}$ & $\begin{array}{r}-\overline{-} \\
4,232.9 \\
19.8 \\
996.8 \\
507.4 \\
4.6 \\
67.3 \\
2,636.0 \\
.9\end{array}$ & $\begin{array}{r}3,285.6 \\
18.4 \\
805.0 \\
400.2 \\
4.2 \\
58.2 \\
1,998.6 \\
1.0\end{array}$ & $\begin{array}{r}- \\
3,589.0 \\
14.4 \\
844.2 \\
428.4 \\
3.6 \\
53.6 \\
2,242.6 \\
2.3\end{array}$ & $\begin{array}{r}- \\
3,460.6 \\
16.2 \\
734.3 \\
350.1 \\
4.1 \\
59.5 \\
2,294.2 \\
2.3\end{array}$ & $\begin{array}{r}- \\
3,899.9 \\
12.0 \\
981.3 \\
429.8 \\
4.4 \\
55.5 \\
2,413.4 \\
3.5\end{array}$ & $\begin{array}{r}- \\
4,586.1 \\
14.2 \\
1,163.3 \\
569.3 \\
5.2 \\
57.6 \\
2,772.9 \\
3.5\end{array}$ & $\begin{array}{r}- \\
4,321.2 \\
13.3 \\
1,060.6 \\
459.8 \\
5.5 \\
59.3 \\
2,721.5 \\
1.3\end{array}$ \\
\hline Electricity & - & - & - & - & - & - & - & - & .9 & .8 \\
\hline 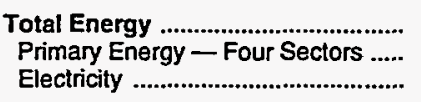 & $\begin{array}{r}2,558.3 \\
1,901.0 \\
657.3\end{array}$ & $\begin{array}{l}5,210.2 \\
3,958.0 \\
1,252.3\end{array}$ & $\begin{array}{r}10,075.4 \\
7,550.9 \\
2,524.5\end{array}$ & $\begin{array}{r}11,740.5 \\
8,092.7 \\
3,647.8\end{array}$ & $\begin{array}{r}10,408.3 \\
6,561.7 \\
3,846.6\end{array}$ & $\begin{array}{r}10,615.4 \\
6,766.5 \\
3,848.9\end{array}$ & $\begin{array}{r}10,961.6 \\
6,967.6 \\
3,993.9\end{array}$ & $\begin{array}{r}11,463.0 \\
7,550.2 \\
3,912.8\end{array}$ & $\begin{array}{r}12,118.7 \\
8,191.7 \\
3,927.0\end{array}$ & $\begin{array}{r}11,920.0 \\
7,857.4 \\
4,062.7\end{array}$ \\
\hline 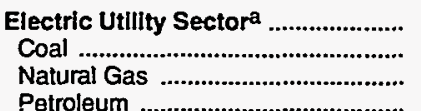 & $\begin{array}{r}136.5 \\
123.7 \\
10.3 \\
2.5\end{array}$ & $\begin{array}{r}372.6 \\
343.1 \\
9.0 \\
20.6\end{array}$ & $\begin{array}{r}951.4 \\
921.2 \\
4.8 \\
25.4\end{array}$ & $\begin{array}{r}1,359.6 \\
1,340.7 \\
4.7 \\
14.2\end{array}$ & $\begin{array}{r}1,270.9 \\
1,257.4 \\
5.0 \\
8.5\end{array}$ & $\begin{array}{r}1,194.4 \\
1,180.5 \\
3.7 \\
10.1\end{array}$ & $\begin{array}{r}1,261.8 \\
1,243.0 \\
9.0 \\
9.8\end{array}$ & $\begin{array}{r}1,254.3 \\
1,232.3 \\
11.0\end{array}$ & $\begin{array}{r}1,404.9 \\
1,371.0 \\
17.1 \\
16.7\end{array}$ & $\begin{array}{r}1,391.5 \\
1,355.8 \\
23.9 \\
11.8\end{array}$ \\
\hline 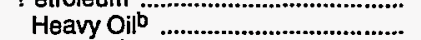 & 1.0 & 14.7 & 20.4 & $\begin{array}{l}14.2 \\
-\end{array}$ & 0.2 & 10.1 & $\begin{array}{l}9.8 \\
-\end{array}$ & 11. & 10.7 & 17.8 \\
\hline $\begin{array}{l}\text { Light Oilb } \\
\text { Petroleum Coke }\end{array}$ & $\begin{array}{r}1.2 \\
.4\end{array}$ & 5.9 & 25.4 & 14.2 & $\begin{array}{l}7.3 \\
1.2\end{array}$ & $\begin{array}{l}8.5 \\
1.7\end{array}$ & $\begin{array}{l}8.0 \\
1.8\end{array}$ & $\begin{array}{l}9.1 \\
2.0\end{array}$ & $\begin{array}{r}12.6 \\
4.1\end{array}$ & $\begin{array}{r}10.1 \\
1.7\end{array}$ \\
\hline 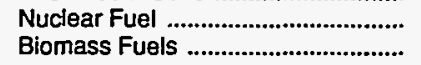 & - & $\begin{array}{l}- \\
-\end{array}$ & - & - & - & - & - & - & - & - \\
\hline Primary Energy - Five Sectorsc .. & $2,037.6$ & $4,330.6$ & $8,502.3$ & $9,452.2$ & $7,832.5$ & $7,960.9$ & $8,229.4$ & $8,804.5$ & $9,596.6$ & $9,248.8$ \\
\hline
\end{tabular}

a There are no direct fuel costs for hydroelectric, geothermal, centralized solar, or wind energy.

b Heavy oil includes Grade Nos. 4, 5, and 6, and residual fuel oils. Light oil includes Grade No. 2 heating oil, kerosene, and jet fuel.

c Biomass fuels are not included, except those consumed at electric utilities and those added to motor gasoline.

No consumption, including cases where adjustments were made. See "Consumption Adjustments for Process Fuel and Intermediate Products."

Note: Totals may not equal sum of components due to independent rounding.

Sources: Data sources, estimation procedures, and assumptions are described in the "Documentation" section of this report. 


\begin{tabular}{|c|c|c|c|c|c|c|c|c|c|c|}
\hline Enargy Source & 1970 & 1975 & 1980 & 1985 & 1986 & 1987 & 1988 & 1989 & 1990 & 1991 \\
\hline & \multicolumn{10}{|c|}{ Prices In Dollars per Million Btu } \\
\hline 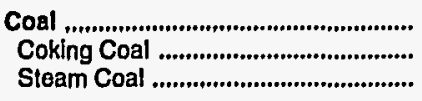 & $\begin{array}{r}0.36 \\
.36\end{array}$ & $\begin{array}{r}0.95 \\
.95\end{array}$ & $\begin{array}{r}1.42 \\
1 . \overline{4}\end{array}$ & $\begin{array}{r}1.52 \\
1.52\end{array}$ & $\begin{array}{r}1.41 \\
1.41\end{array}$ & $\begin{array}{r}1.31 \\
1.31\end{array}$ & $\begin{array}{r}1.28 \\
1.28\end{array}$ & $\frac{1.25}{1.25}$ & $\begin{array}{r}1.16 \\
1.16\end{array}$ & $\begin{array}{r}1.15 \\
1.15\end{array}$ \\
\hline Natural Gas ....,....................................... & .57 & 1.00 & 2.79 & 4.60 & 4.22 & 3.63 & 3.80 & 3.57 & 3.81 & 3.65 \\
\hline 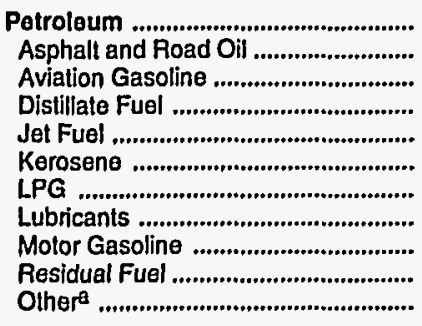 & $\begin{array}{r}2.11 \\
.72 \\
2.17 \\
1.01 \\
.75 \\
1.31 \\
1.59 \\
5.08 \\
2.83 \\
.61 \\
2.15\end{array}$ & $\begin{array}{l}3.74 \\
2.06 \\
3.45 \\
2.45 \\
2.09 \\
2.74 \\
3.00 \\
7.48 \\
4.59 \\
1.88 \\
3.21\end{array}$ & $\begin{array}{r}8.19 \\
3.77 \\
9.02 \\
6.41 \\
6.47 \\
6.23 \\
5.57 \\
14.36 \\
9.97 \\
3.19 \\
7.65\end{array}$ & $\begin{array}{r}8.15 \\
5.03 \\
9.99 \\
6.16 \\
6.28 \\
6.95 \\
7.32 \\
17.61 \\
9.47 \\
4.07 \\
8.17\end{array}$ & $\begin{array}{r}6.54 \\
3.89 \\
8.41 \\
5.47 \\
4.36 \\
5.79 \\
7.23 \\
15.59 \\
7.13 \\
2.86 \\
6.06\end{array}$ & $\begin{array}{r}6.69 \\
3.55 \\
7.55 \\
5.95 \\
4.27 \\
5.83 \\
5.85 \\
12.70 \\
7.36 \\
2.48 \\
6.86\end{array}$ & $\begin{array}{r}6.52 \\
3.46 \\
7.41 \\
5.48 \\
4.12 \\
5.43 \\
5.81 \\
14.61 \\
7.37 \\
2.38 \\
5.86\end{array}$ & $\begin{array}{r}7.43 \\
3.00 \\
8.28 \\
6.27 \\
4.57 \\
5.39 \\
6.83 \\
13.30 \\
8.40 \\
2.25 \\
6.59\end{array}$ & $\begin{array}{r}8.26 \\
3.12 \\
9.32 \\
7.37 \\
6.11 \\
5.91 \\
5.86 \\
13.40 \\
9.38 \\
2.36 \\
7.12\end{array}$ & $\begin{array}{r}8.04 \\
3.15 \\
8.71 \\
6.66 \\
5.21 \\
5.94 \\
7.45 \\
15.42 \\
9.11 \\
2.24 \\
6.45\end{array}$ \\
\hline Nucloar Fuel ,.................................... & - & .25 & .39 & .94 & .75 & .71 & .68 & .67 & .66 & .66 \\
\hline Blomass Fuels at Utllitles ................. & .65 & .92 & 1.74 & .79 & .32 & .95 & .87 & 1.47 & 1.60 & 1.67 \\
\hline Prlmary Energy - Five Sectors ${ }^{b} \ldots$ & 1.19 & 2.16 & 4.60 & 4.87 & 4.09 & 3.87 & 3.79 & 3.98 & 4.24 & 4.03 \\
\hline $\begin{array}{l}\text { Electric Utility Fuelc ............................ } \\
\text { Electricity Purchased by End Users }\end{array}$ & $\begin{array}{r}.30 \\
6.39\end{array}$ & 9.75 & $\begin{array}{r}1.32 \\
13.97\end{array}$ & $\begin{array}{r}1.46 \\
19.02\end{array}$ & $\begin{array}{r}1.31 \\
19.25\end{array}$ & $\begin{array}{r}1.21 \\
18.30\end{array}$ & $\begin{array}{r}1.19 \\
17.59\end{array}$ & $\begin{array}{r}1.18 \\
17.30\end{array}$ & $\begin{array}{r}1.10 \\
17.37\end{array}$ & $\begin{array}{r}1.07 \\
17.41\end{array}$ \\
\hline \multirow[t]{2}{*}{ 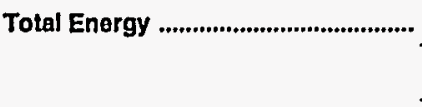 } & 1.80 & 3.12 & 6.72 & 8.03 & 7.28 & 7.06 & 6.87 & 7.12 & 7.65 & 7.43 \\
\hline & \multicolumn{10}{|c|}{ Expenditures in Millions of Dollars } \\
\hline 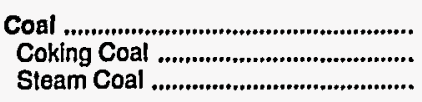 & $\begin{array}{r}47.5 \\
47 . \overline{5}\end{array}$ & $\begin{array}{r}125.4 \\
125 . \overline{4}\end{array}$ & $\begin{array}{r}333.3 \\
333.3\end{array}$ & $\begin{array}{r}407.9 \\
407.9\end{array}$ & $\begin{array}{r}370.6 \\
370.6\end{array}$ & $\begin{array}{r}375.2 \\
375.2\end{array}$ & $\begin{array}{r}390.6 \\
-\overline{6}\end{array}$ & $\begin{array}{r}397.8 \\
-\overline{8}\end{array}$ & $\begin{array}{r}386.2 \\
-\overline{2} \\
386.2\end{array}$ & $\begin{array}{r}397.9 \\
397.9\end{array}$ \\
\hline Natural Gas ...................................... & 190.2 & 332.4 & 719.9 & $1,003.4$ & 851.8 & 713.4 & 874.1 & 777.2 & 803.0 & 832.9 \\
\hline 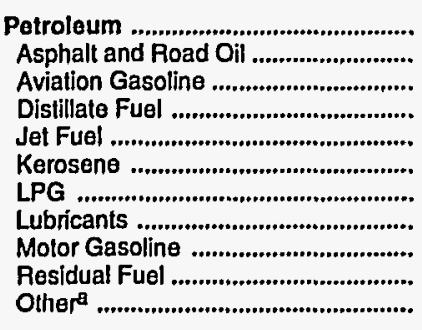 & $\begin{array}{r}730.5 \\
13.9 \\
2.8 \\
80.7 \\
3.0 \\
3.6 \\
66.2 \\
21.6 \\
530.1 \\
1.5 \\
7.1\end{array}$ & $\begin{array}{r}1,403.5 \\
31.4 \\
3.3 \\
207.6 \\
9.8 \\
3.3 \\
152.2 \\
29.8 \\
942.1 \\
7.2 \\
16.8\end{array}$ & $\begin{array}{r}3,029.5 \\
42.5 \\
8.4 \\
594.5 \\
29.6 \\
6.0 \\
228.5 \\
62.1 \\
1,853.2 \\
8.3 \\
196.2\end{array}$ & $\begin{array}{r}2,585.0 \\
67.6 \\
4.2 \\
555.9 \\
20.9 \\
6.1 \\
224.5 \\
69.3 \\
1,565.7 \\
4.7 \\
66.2\end{array}$ & $\begin{array}{r}2,090.9 \\
52.6 \\
6.4 \\
508.6 \\
14.5 \\
3.8 \\
230.9 \\
60.0 \\
1,174.7 \\
9.1 \\
30.2\end{array}$ & $\begin{array}{r}2,059.9 \\
42.1 \\
4.2 \\
546.5 \\
18.7 \\
3.6 \\
130.5 \\
55.3 \\
1,222.9 \\
1.8 \\
34.2\end{array}$ & $\begin{array}{r}2,079.7 \\
50.8 \\
5.4 \\
508.9 \\
16.5 \\
3.3 \\
140.2 \\
61.3 \\
1,260.5 \\
3.9 \\
28.8\end{array}$ & $\begin{array}{r}2,315.0 \\
34.1 \\
4.6 \\
546.1 \\
19.3 \\
2.2 \\
181.4 \\
57.3 \\
1,435.8 \\
2.6 \\
31.6\end{array}$ & $\begin{array}{r}2,510.1 \\
31.8 \\
4.7 \\
653.6 \\
30.7 \\
2.7 \\
135.6 \\
59.4 \\
1,552.2 \\
1.9 \\
37.6\end{array}$ & $\begin{array}{r}2,475.9 \\
32.6 \\
3.6 \\
566.3 \\
24.7 \\
1.7 \\
197.7 \\
61.1 \\
1,552.8 \\
1.4 \\
33.9\end{array}$ \\
\hline Nuclear Fuel ........................................... & - & 6.3 & 10.9 & 19.6 & 24.4 & 19.3 & 23.0 & 22.6 & 21.3 & 29.2 \\
\hline Blomass Fuels at Utilities .................. & .3 & .4 & .5 & .5 & .2 & .7 & .5 & .4 & .3 & .3 \\
\hline Primary Energy — Flve Sectors ${ }^{b} \ldots$ & 968.4 & $1,868.0$ & $4,094.1$ & $4,016.3$ & $3,337.9$ & $3,168.5$ & $3,368.0$ & $3,512.8$ & $3,720.9$ & $3,736.4$ \\
\hline $\begin{array}{l}\text { Electric Utility Fuelc } \\
\text { Electricity Purchased by End Users }\end{array}$ & $\begin{array}{r}-50.4 \\
337.5\end{array}$ & $\begin{array}{r}-132.5 \\
624.4\end{array}$ & $\begin{array}{r}-313.1 \\
1,184.5\end{array}$ & $\begin{array}{r}-366.7 \\
1,666.6\end{array}$ & $\begin{array}{r}-335.2 \\
1,731.1\end{array}$ & $\begin{array}{r}-326.2 \\
1,687.4\end{array}$ & $\begin{array}{r}-354.1 \\
1,731.0\end{array}$ & $\begin{array}{r}-350.2 \\
1,695.0\end{array}$ & $\begin{array}{r}-340.8 \\
1,745.1\end{array}$ & $\begin{array}{r}-353.3 \\
1,829.0\end{array}$ \\
\hline 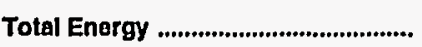 & $1,255.5$ & $2,359.9$ & $4,965.5$ & $5,316.2$ & $4,733.9$ & $4,529.6$ & $4,744.9$ & $4,857.7$ & $5,125.2$ & $5,212.1$ \\
\hline
\end{tabular}

a Includes petroleum coke used at electric utilities.

b Biomass fuels are not included, except those consumed at electric utilities and those added to motor gasoline.

c There are no direct fuel costs for hydroelectric, geothermal, centralized solar, or wind energy.

-No consumption, including cases where adjustments were made. See "Consumption Adjustments for Process Fuel and Intermediate Products."

Note; Expenditure totals may not equal sum of components due to independent rounding.

Sources: Data sources, estimation procedures, and assumptions are described in the "Documentation" section of this report. 
I Energy Price Estimates by Sector, lowa

O 1970, 1975, 1980, and 1985-1991

W (Dollars per Million Btu)

A

\begin{tabular}{|c|c|c|c|c|c|c|c|c|c|c|}
\hline Sector and Energy Source & 1970 & 1975 & 1980 & 1985 & 1986 & 1987 & 1988 & 1989 & 1990 & 1991 \\
\hline 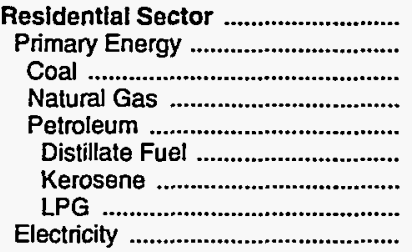 & $\begin{array}{r}2.06 \\
1.16 \\
1.27 \\
.96 \\
1.63 \\
1.22 \\
1.57 \\
1.85 \\
7.75\end{array}$ & $\begin{array}{r}3.44 \\
1.94 \\
3.69 \\
1.42 \\
3.25 \\
2.56 \\
2.99 \\
3.54 \\
10.46\end{array}$ & $\begin{array}{r}6.87 \\
4.09 \\
3.31 \\
3.18 \\
6.84 \\
6.79 \\
8.10 \\
6.86 \\
16.13\end{array}$ & $\begin{array}{r}9.65 \\
5.38 \\
3.41 \\
5.33 \\
5.81 \\
5.94 \\
7.33 \\
5.62 \\
22.53\end{array}$ & $\begin{array}{r}9.65 \\
5.11 \\
3.12 \\
5.07 \\
5.46 \\
5.32 \\
6.57 \\
5.51 \\
22.56\end{array}$ & $\begin{array}{r}9.97 \\
4.75 \\
2.89 \\
4.71 \\
5.17 \\
5.33 \\
6.57 \\
5.00 \\
22.91\end{array}$ & $\begin{array}{r}9.51 \\
4.76 \\
2.51 \\
4.76 \\
5.14 \\
4.80 \\
5.92 \\
5.31 \\
22.23\end{array}$ & $\begin{array}{r}9.65 \\
5.14 \\
2.60 \\
4.65 \\
7.39 \\
4.86 \\
5.99 \\
8.68 \\
22.19\end{array}$ & $\begin{array}{r}10.29 \\
5.19 \\
2.41 \\
4.96 \\
6.73 \\
5.73 \\
7.07 \\
7.19 \\
22.89\end{array}$ & $\begin{array}{r}9.90 \\
4.96 \\
2.32 \\
4.77 \\
6.08 \\
5.32 \\
6.57 \\
6.39 \\
22.74\end{array}$ \\
\hline 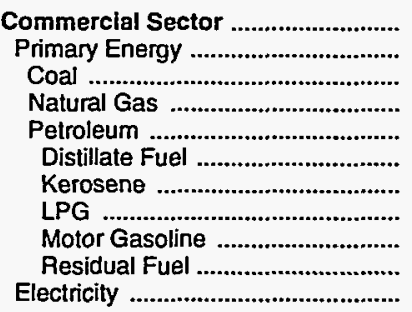 & $\begin{array}{r}1.80 \\
.77 \\
.41 \\
.68 \\
1.30 \\
1.05 \\
.81 \\
1.16 \\
2.83 \\
.66 \\
7.68\end{array}$ & $\begin{array}{r}2.94 \\
1.29 \\
1.24 \\
1.05 \\
2.71 \\
2.40 \\
2.30 \\
2.46 \\
4.59 \\
1.69 \\
10.55\end{array}$ & $\begin{array}{r}6.33 \\
3.38 \\
1.59 \\
2.84 \\
6.57 \\
6.44 \\
5.52 \\
4.88 \\
9.97 \\
3.80 \\
15.93\end{array}$ & $\begin{array}{r}9.24 \\
4.84 \\
1.66 \\
4.80 \\
6.28 \\
5.10 \\
5.86 \\
8.25 \\
9.47 \\
4.07 \\
21.88\end{array}$ & $\begin{array}{r}9.40 \\
4.40 \\
1.57 \\
4.41 \\
5.60 \\
3.81 \\
4.37 \\
8.25 \\
7.13 \\
2.86 \\
21.81\end{array}$ & $\begin{array}{r}8.87 \\
3.84 \\
1.52 \\
3.84 \\
5.23 \\
4.18 \\
5.02 \\
6.45 \\
7.36 \\
2.49 \\
19.95\end{array}$ & $\begin{array}{r}8.38 \\
3.91 \\
1.42 \\
4.00 \\
5.00 \\
3.41 \\
4.08 \\
6.23 \\
7.37 \\
2.38 \\
19.06\end{array}$ & $\begin{array}{r}8.43 \\
3.88 \\
1.35 \\
3.86 \\
5.03 \\
3.65 \\
4.54 \\
5.21 \\
8.40 \\
2.25 \\
18.63\end{array}$ & $\begin{array}{r}8.58 \\
3.93 \\
1.34 \\
4.00 \\
5.10 \\
4.32 \\
5.41 \\
4.85 \\
9.38 \\
2.36 \\
18.33\end{array}$ & $\begin{array}{r}8.63 \\
4.28 \\
1.31 \\
3.96 \\
7.01 \\
3.78 \\
4.70 \\
8.34 \\
9.11 \\
2.24 \\
18.24\end{array}$ \\
\hline 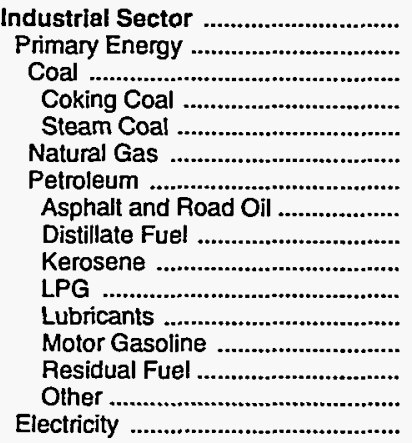 & $\begin{array}{r}1.04 \\
.83 \\
.41 \\
- \\
.41 \\
.36 \\
1.48 \\
.72 \\
.75 \\
.81 \\
1.16 \\
5.08 \\
2.83 \\
.57 \\
2.15 \\
3.87\end{array}$ & $\begin{array}{r}2.01 \\
1.61 \\
1.24 \\
- \\
1.24 \\
.77 \\
2.85 \\
2.06 \\
2.15 \\
2.30 \\
2.46 \\
7.48 \\
4.59 \\
1.92 \\
3.21 \\
6.31\end{array}$ & $\begin{array}{r}4.69 \\
3.96 \\
1.59 \\
- \\
1.59 \\
2.51 \\
6.27 \\
3.77 \\
5.28 \\
5.52 \\
4.88 \\
14.36 \\
9.97 \\
2.88 \\
7.65 \\
10.47\end{array}$ & $\begin{array}{r}5.86 \\
4.64 \\
1.66 \\
- \\
1.66 \\
3.87 \\
6.85 \\
5.03 \\
5.32 \\
5.86 \\
8.25 \\
17.61 \\
9.47 \\
4.07 \\
8.17 \\
13.50\end{array}$ & $\begin{array}{r}5.36 \\
3.89 \\
1.57 \\
- \\
1.57 \\
3.36 \\
5.41 \\
3.89 \\
3.97 \\
4.37 \\
8.25 \\
15.59 \\
7.13 \\
2.86 \\
6.06 \\
14.16\end{array}$ & $\begin{array}{r}4.78 \\
3.38 \\
1.52 \\
- \\
1.52 \\
2.80 \\
5.33 \\
3.55 \\
4.56 \\
5.02 \\
6.45 \\
12.70 \\
7.36 \\
2.49 \\
6.86 \\
12.71\end{array}$ & $\begin{array}{r}4.62 \\
3.30 \\
1.42 \\
- \\
1.42 \\
3.09 \\
4.72 \\
3.46 \\
3.70 \\
4.08 \\
6.23 \\
14.61 \\
7.37 \\
2.38 \\
5.86 \\
12.15\end{array}$ & $\begin{array}{r}4.30 \\
2.93 \\
1.35 \\
- \\
1.35 \\
2.51 \\
4.95 \\
3.00 \\
4.12 \\
4.54 \\
5.21 \\
13.30 \\
8.40 \\
2.25 \\
6.59 \\
11.81\end{array}$ & $\begin{array}{r}4.55 \\
3.18 \\
1.34 \\
- \\
1.34 \\
2.85 \\
5.37 \\
3.12 \\
4.92 \\
5.41 \\
4.85 \\
13.40 \\
9.38 \\
2.36 \\
7.12 \\
11.65\end{array}$ & $\begin{array}{r}4.47 \\
3.15 \\
1.31 \\
- \\
1.31 \\
2.63 \\
5.70 \\
3.15 \\
4.27 \\
4.70 \\
8.34 \\
15.42 \\
9.11 \\
2.24 \\
6.45 \\
11.76\end{array}$ \\
\hline 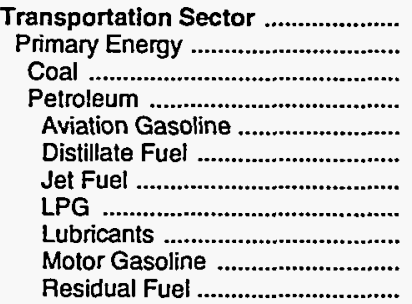 & $\begin{array}{r}2.60 \\
2.60 \\
.41 \\
2.60 \\
2.17 \\
1.27 \\
.75 \\
1.16 \\
5.08 \\
2.83 \\
.66\end{array}$ & $\begin{array}{r}4.24 \\
4.24 \\
1.24 \\
4.24 \\
3.45 \\
2.65 \\
2.09 \\
2.46 \\
7.48 \\
4.59 \\
-\end{array}$ & $\begin{array}{r}9.34 \\
9.34 \\
- \\
9.34 \\
9.02 \\
6.97 \\
6.47 \\
4.88 \\
14.36 \\
9.97 \\
-\end{array}$ & $\begin{array}{r}8.95 \\
8.95 \\
- \\
8.95 \\
9.99 \\
6.85 \\
6.28 \\
8.25 \\
17.61 \\
9.47 \\
-\end{array}$ & $\begin{array}{r}7.13 \\
7.13 \\
- \\
7.13 \\
8.41 \\
6.76 \\
4.36 \\
8.25 \\
15.59 \\
7.13 \\
-\end{array}$ & $\begin{array}{r}7.30 \\
7.30 \\
- \\
7.30 \\
7.55 \\
7.01 \\
4.27 \\
6.45 \\
12.70 \\
7.36 \\
2.33\end{array}$ & $\begin{array}{r}7.27 \\
7.27 \\
- \\
7.27 \\
7.41 \\
6.77 \\
4.12 \\
6.23 \\
14.61 \\
7.37 \\
-\end{array}$ & $\begin{array}{r}8.19 \\
8.19 \\
- \\
8.19 \\
8.28 \\
7.55 \\
4.57 \\
5.21 \\
13.30 \\
8.40 \\
2.08\end{array}$ & $\begin{array}{r}9.20 \\
9.20 \\
- \\
9.20 \\
9.32 \\
8.74 \\
6.11 \\
4.85 \\
13.40 \\
9.38 \\
1.82\end{array}$ & $\begin{array}{r}8.93 \\
8.93 \\
- \\
8.93 \\
8.71 \\
8.32 \\
5.21 \\
8.34 \\
15.42 \\
9.11 \\
-\end{array}$ \\
\hline 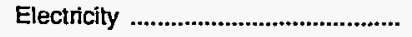 & - & - & - & - & - & - & - & - & - & - \\
\hline $\begin{array}{l}\text { Total Energy .................................. } \\
\text { Primary Energy - Four Sectors ..... } \\
\text { Electricity ........................................... }\end{array}$ & $\begin{array}{l}1.80 \\
1.42 \\
6.39\end{array}$ & $\begin{array}{l}3.12 \\
2.52 \\
9.11\end{array}$ & $\begin{array}{r}6.72 \\
5.78 \\
13.97\end{array}$ & $\begin{array}{r}8.03 \\
6.36 \\
19.02\end{array}$ & $\begin{array}{r}7.28 \\
5.36 \\
19.25\end{array}$ & $\begin{array}{r}7.06 \\
5.17 \\
18.30\end{array}$ & $\begin{array}{r}6.87 \\
5.09 \\
17.59\end{array}$ & $\begin{array}{r}7.12 \\
5.41 \\
17.30\end{array}$ & $\begin{array}{r}7.65 \\
5.94 \\
17.37\end{array}$ & $\begin{array}{r}7.43 \\
5.67 \\
17.41\end{array}$ \\
\hline 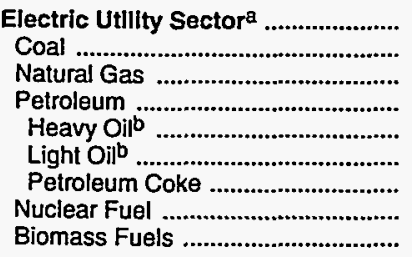 & $\begin{array}{l}.30 \\
.32 \\
.27 \\
.74 \\
.70 \\
.75 \\
- \\
- \\
.65\end{array}$ & $\begin{array}{r}.75 \\
.84 \\
.68 \\
2.05 \\
1.93 \\
2.11 \\
- \\
.25 \\
.92\end{array}$ & $\begin{array}{r}1.32 \\
1.39 \\
2.41 \\
5.41 \\
3.78 \\
6.06 \\
- \\
.39 \\
1.74\end{array}$ & $\begin{array}{r}1.46 \\
1.48 \\
3.61 \\
5.88 \\
3.99 \\
5.93 \\
-\overline{94} \\
.79\end{array}$ & $\begin{array}{r}1.31 \\
1.37 \\
3.42 \\
3.62 \\
- \\
3.62 \\
- \\
.75 \\
.32\end{array}$ & $\begin{array}{r}1.21 \\
1.25 \\
2.15 \\
4.06 \\
- \\
4.06 \\
- \\
.71 \\
.95\end{array}$ & $\begin{array}{r}1.19 \\
1.24 \\
2.03 \\
3.72 \\
- \\
3.72 \\
- \\
.68 \\
.87\end{array}$ & $\begin{array}{r}1.18 \\
1.22 \\
2.67 \\
4.45 \\
- \\
4.45 \\
- \\
.67 \\
1.47\end{array}$ & $\begin{array}{r}1.10 \\
1.12 \\
3.04 \\
5.18 \\
- \\
5.18 \\
- \\
.66 \\
1.60\end{array}$ & $\begin{array}{r}1.07 \\
1.10 \\
2.69 \\
4.38 \\
-\overline{8} \\
4.38 \\
- \\
.66 \\
1.67\end{array}$ \\
\hline Primary Energy - Five Sectorsc .. & 1.19 & 2.16 & 4.60 & 4.87 & 4.09 & 3.87 & 3.79 & 3.98 & 4.24 & 4.03 \\
\hline
\end{tabular}

a There are no direct fuel costs for hydroelectric, geothermal, centralized solar, or wind energy.

b Heavy oil includes Grade Nos. 4, 5, and 6, and residual fuel oils. Light oil includes Grade No. 2 heating oil, kerosene, and jet fuel.

c Biomass fuels are not included, except those consumed at electric utilities and those added to motor gasoline.

-No consumption, including cases where adjustments were made. See "Consumption Adjustments for Process Fuel and Intermediate Products."

Sources: Data sources, estimation procedures, and assumptions are described in the "Documentation" section of this report. 


\begin{tabular}{|c|c|c|c|c|c|c|c|c|c|c|}
\hline Sector and Energy Source & 1970 & 1975 & 1980 & 1985 & 1986 & 1987 & 1988 & 1989 & 1990 & 1991 \\
\hline 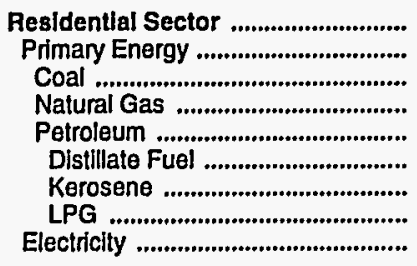 & $\begin{array}{r}332.2 \\
160.9 \\
1.6 \\
92.9 \\
66.4 \\
15.8 \\
2.9 \\
47.6 \\
171.3\end{array}$ & $\begin{array}{r}554.3 \\
256.7 \\
3.3 \\
134.7 \\
118.8 \\
26.9 \\
2.3 \\
89.5 \\
297.5\end{array}$ & $\begin{array}{r}1,020.5 \\
467.9 \\
2.1 \\
271.2 \\
194.6 \\
94.5 \\
2.2 \\
98.0 \\
552.6\end{array}$ & $\begin{array}{r}1,303.6 \\
546.3 \\
7.1 \\
424.1 \\
115.1 \\
49.7 \\
4.8 \\
60.6 \\
757.4\end{array}$ & $\begin{array}{r}1,267.5 \\
497.1 \\
5.9 \\
379.8 \\
111.3 \\
43.0 \\
2.8 \\
65.5 \\
770.4\end{array}$ & $\begin{array}{r}1,188.2 \\
403.1 \\
7.1 \\
309.9 \\
86.1 \\
37.8 \\
2.1 \\
46.1 \\
785.1\end{array}$ & $\begin{array}{r}1,275.3 \\
465.3 \\
7.2 \\
364.6 \\
93.4 \\
31.2 \\
2.6 \\
59.6 \\
810.0\end{array}$ & $\begin{array}{r}1,293.9 \\
506.9 \\
3.7 \\
363.8 \\
139.3 \\
30.2 \\
1.4 \\
107.8 \\
787.0\end{array}$ & $\begin{array}{r}1,281.3 \\
460.3 \\
5.0 \\
356.2 \\
99.1 \\
26.6 \\
1.0 \\
71.5 \\
821.0\end{array}$ & $\begin{array}{r}1,355.7 \\
489.7 \\
4.4 \\
379.0 \\
106.3 \\
27.5 \\
1.3 \\
77.6 \\
866.0\end{array}$ \\
\hline 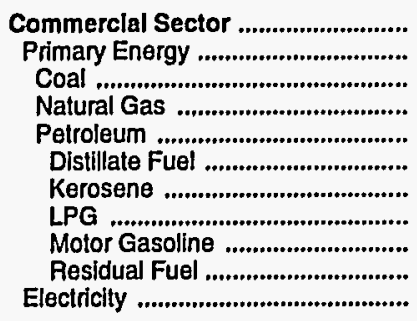 & $\begin{array}{r}151.3 \\
55.5 \\
1.0 \\
39.4 \\
15.1 \\
5.5 \\
.1 \\
5.3 \\
4.0 \\
.3 \\
95.8\end{array}$ & $\begin{array}{r}287.6 \\
103.3 \\
2.0 \\
71.1 \\
30.2 \\
10.1 \\
.1 \\
11.0 \\
7.8 \\
1.2 \\
184.3\end{array}$ & $\begin{array}{r}505.7 \\
206.7 \\
1.9 \\
144.0 \\
60.8 \\
28.2 \\
.2 \\
12.3 \\
18.3 \\
1.9 \\
299.0\end{array}$ & $\begin{array}{r}769.7 \\
298.9 \\
6.4 \\
231.3 \\
61.2 \\
33.4 \\
.2 \\
15.7 \\
11.8 \\
470.8\end{array}$ & $\begin{array}{r}731.0 \\
243.6 \\
5.5 \\
194.7 \\
43.4 \\
15.1 \\
17.3 \\
10.2 \\
.7 \\
487.4\end{array}$ & $\begin{array}{r}651.1 \\
193.9 \\
6.9 \\
147.3 \\
39.7 \\
18.5 \\
.2 \\
10.5 \\
10.3 \\
.3 \\
457.3\end{array}$ & $\begin{array}{r}691.9 \\
227.9 \\
7.2 \\
181.2 \\
39.5 \\
13.6 \\
.1 \\
12.3 \\
13.1 \\
.3 \\
464.0\end{array}$ & $\begin{array}{r}680.2 \\
216.2 \\
3.5 \\
180.0 \\
32.7 \\
10.4 \\
.1 \\
11.4 \\
10.3 \\
.5 \\
464.0\end{array}$ & $\begin{array}{r}682.8 \\
211.8 \\
5.1 \\
177.1 \\
29.5 \\
12.5 \\
1.2 \\
8.5 \\
6.9 \\
.5 \\
471.0\end{array}$ & $\begin{array}{r}749.6 \\
255.6 \\
4.6 \\
185.8 \\
65.2 \\
12.4 \\
.1 \\
17.9 \\
34.8 \\
.1 \\
494.0\end{array}$ \\
\hline 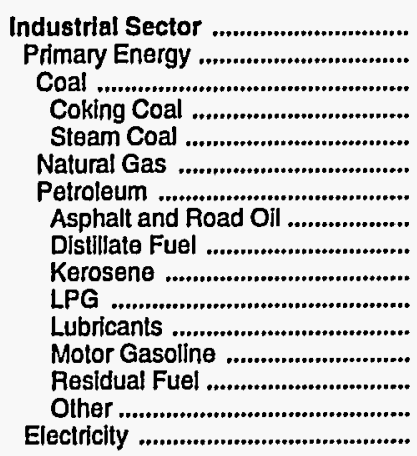 & $\begin{array}{r}272.8 \\
202.4 \\
17.8 \\
17 . \overline{8} \\
36.3 \\
148.2 \\
13.9 \\
25.8 \\
.7 \\
13.0 \\
6.8 \\
80.0 \\
.9 \\
7.1 \\
70.5\end{array}$ & $\begin{array}{r}533.1 \\
390.5 \\
35.1 \\
35.1 \\
35.1 \\
94.6 \\
260.8 \\
31.4 \\
58.6 \\
.9 \\
51.2 \\
7.0 \\
91.5 \\
3.4 \\
16.8 \\
142.6\end{array}$ & $\begin{array}{r}1,335.6 \\
1,002.7 \\
51.6 \\
-\overline{51.6} \\
288.2 \\
662.9 \\
42.5 \\
144.4 \\
3.7 \\
117.6 \\
16.7 \\
136.7 \\
5.0 \\
196.2 \\
332.9\end{array}$ & $\begin{array}{r}1,374.5 \\
936.1 \\
59.1 \\
-\overline{5} \\
59.1 \\
340.2 \\
536.8 \\
67.6 \\
148.4 \\
1.1 \\
145.5 \\
18.6 \\
84.8 \\
4.6 \\
66.2 \\
438.4\end{array}$ & $\begin{array}{r}1,245.5 \\
772.2 \\
55.6 \\
- \\
55.6 \\
272.6 \\
444.0 \\
52.6 \\
135.4 \\
1.0 \\
143.8 \\
16.1 \\
56.5 \\
8.4 \\
30.2 \\
473.2\end{array}$ & $\begin{array}{r}1,114.8 \\
669.8 \\
64.8 \\
-\overline{6} \\
64.8 \\
249.1 \\
355.8 \\
42.1 \\
131.6 \\
1.3 \\
72.8 \\
14.9 \\
57.5 \\
1.4 \\
34.2 \\
445.1\end{array}$ & $\begin{array}{r}1,166.4 \\
709.5 \\
59.4 \\
-\overline{5} \\
59.4 \\
317.3 \\
332.9 \\
50.8 \\
110.8 \\
.5 \\
67.2 \\
16.5 \\
54.6 \\
3.6 \\
28.8 \\
457.0\end{array}$ & $\begin{array}{r}1,044.9 \\
600.9 \\
72.7 \\
-\overline{7} \\
72.7 \\
226.9 \\
301.3 \\
34.1 \\
98.7 \\
.6 \\
61.2 \\
15.4 \\
57.5 \\
2.1 \\
31.6 \\
444.0\end{array}$ & $\begin{array}{r}1,096.6 \\
643.6 \\
71.3 \\
71 . \overline{-} \\
71.3 \\
259.0 \\
313.3 \\
31.8 \\
118.5 \\
.6 \\
54.8 \\
16.0 \\
52.5 \\
1.4 \\
37.6 \\
453.0\end{array}$ & $\begin{array}{r}1,160.6 \\
691.6 \\
77.9 \\
-\overline{7} \\
77.9 \\
258.2 \\
355.4 \\
32.6 \\
114.6 \\
.4 \\
100.8 \\
16.4 \\
55.5 \\
1.2 \\
33.9 \\
469.0\end{array}$ \\
\hline 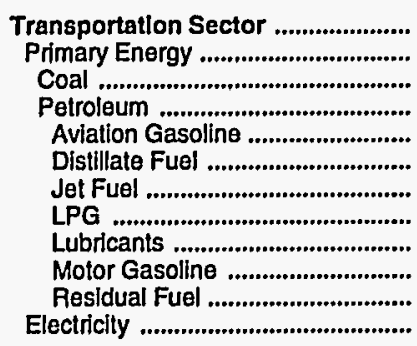 & $\begin{array}{r}499.2 \\
499.2 \\
499.1 \\
2.1 \\
2.8 \\
32.2 \\
3.0 \\
.3 \\
14.8 \\
446.0 \\
.1 \\
-\end{array}$ & $\begin{array}{r}984.9 \\
984.9 \\
984.9 \\
3.3 \\
105.7 \\
9.8 \\
.5 \\
22.7 \\
842.8 \\
- \\
-\end{array}$ & $\begin{array}{r}2,103.8 \\
2,103.8 \\
2,103.8 \\
8.4 \\
321.6 \\
29.6 \\
.6 \\
45.4 \\
1,698.2 \\
- \\
-\end{array}$ & $\begin{array}{r}1,868.4 \\
1,868.4 \\
- \\
1,868.4 \\
4.2 \\
320.9 \\
20.9 \\
2.7 \\
50.7 \\
1,469.1 \\
- \\
-\end{array}$ & $\begin{array}{r}1,489.9 \\
1,489.9 \\
- \\
1,489.9 \\
6.4 \\
312.8 \\
14.5 \\
4.2 \\
43.9 \\
1,108.0 \\
- \\
-\end{array}$ & $\begin{array}{r}1,575.5 \\
1,575.5 \\
\\
1,575.5 \\
4.2 \\
355.8 \\
18.7 \\
1.1 \\
40.4 \\
1,155.2 \\
.1 \\
-\end{array}$ & $\begin{array}{r}1,611.3 \\
1,611.3 \\
- \\
1,611.3 \\
5.4 \\
350.6 \\
16.5 \\
1.0 \\
44.9 \\
1,192.8 \\
= \\
-\end{array}$ & $\begin{array}{r}1,838.7 \\
1,838.7 \\
1,838.7 \\
4.6 \\
403.9 \\
19.3 \\
1.0 \\
41.9 \\
1,368.0 \\
-\end{array}$ & $\begin{array}{r}2,064.5 \\
2,064.5 \\
- \\
2,064.5 \\
4.7 \\
492.3 \\
30.7 \\
.7 \\
43.4 \\
1,492.7 \\
-\end{array}$ & $\begin{array}{r}1,946.2 \\
1,946.2 \\
-\overline{1} \\
1,946.2 \\
3.6 \\
409.1 \\
24.7 \\
1.5 \\
44.7 \\
1,462.6 \\
-\end{array}$ \\
\hline 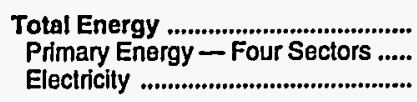 & $\begin{array}{r}1,255.5 \\
918.0 \\
337.5\end{array}$ & $\begin{array}{r}2,359.9 \\
1,735.4 \\
624.4\end{array}$ & $\begin{array}{l}4,965.5 \\
3,781.0 \\
1,184.5\end{array}$ & $\begin{array}{l}5,316.2 \\
3,649.7 \\
1,666.6\end{array}$ & $\begin{array}{l}4,733.9 \\
3,002.8 \\
1,731.1\end{array}$ & $\begin{array}{l}4,529.6 \\
2,842.2 \\
1,687.4\end{array}$ & $\begin{array}{l}4,744.9 \\
3,013.9 \\
1,731.0\end{array}$ & $\begin{array}{l}4,857.7 \\
3,162.6 \\
1,695.0\end{array}$ & $\begin{array}{l}5,125.2 \\
3,380.2 \\
1,745.1\end{array}$ & $\begin{array}{l}5,212.1 \\
3,383.1 \\
1,829.0\end{array}$ \\
\hline 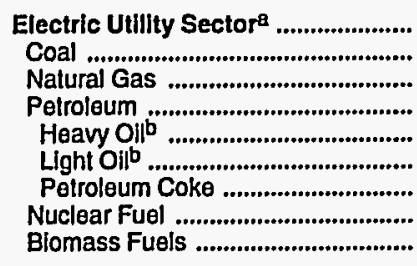 & $\begin{array}{r}50.4 \\
27.0 \\
21.5 \\
1.6 \\
.2 \\
1.4 \\
- \\
\overline{3}\end{array}$ & $\begin{array}{r}132.5 \\
85.0 \\
32.0 \\
8.8 \\
2.6 \\
6.2 \\
- \\
6.3 \\
.4\end{array}$ & $\begin{array}{r}313.1 \\
277.7 \\
16.6 \\
7.4 \\
1.5 \\
5.9 \\
10.9 \\
.5\end{array}$ & $\begin{array}{r}366.7 \\
335.3 \\
7.7 \\
3.6 \\
.1 \\
3.5 \\
19.6 \\
.5\end{array}$ & $\begin{array}{r}335.2 \\
303.6 \\
4.7 \\
2.2 \\
-\overline{2} \\
2.2 \\
24.4 \\
.2\end{array}$ & $\begin{array}{r}326.2 \\
296.4 \\
7.1 \\
2.7 \\
-\overline{7} \\
2.7 \\
19 . \overline{3} \\
.7\end{array}$ & $\begin{array}{r}354.1 \\
316.7 \\
11.1 \\
2.7 \\
-\overline{-} \\
2.7 \\
23.0 \\
.5\end{array}$ & $\begin{array}{r}350.2 \\
317.9 \\
6.5 \\
2.9 \\
2.9 \\
22.6 \\
.4\end{array}$ & $\begin{array}{r}340.8 \\
304.8 \\
10.7 \\
3.7 \\
3 . \overline{7} \\
-\bar{y} \\
21.3 \\
.3\end{array}$ & $\begin{array}{r}353.3 \\
311.1 \\
9.9 \\
2.8 \\
2 . \overline{-} \\
29 . \overline{2} \\
.3\end{array}$ \\
\hline Primary Energy - Flve Sectors ${ }^{c}$.. & 968.4 & $1,868.0$ & $4,094.1$ & $4,016.3$ & $3,337.9$ & $3,168.5$ & $3,368.0$ & $3,512.8$ & $3,720.9$ & $3,736.4$ \\
\hline
\end{tabular}

a There are no direct fuel costs for hydroelectric, geothermal, centralized solar, or wind energy.

b Heavy oll includes Grade Nos. 4, 5, and 6, and residual fuel oils. Light oil includes Grade No. 2 heating oil, kerosene, and jet fuel.

c Biomass fuels are not included, except those consumed at electric utilities and those added to motor gasoline.

- No consumption, including cases where adjustments were made. See "Consumption Adjustments for Process Fuel and Intermediate Products."

- Value less than 0.05 million dollars.

Note: Totals may not equal sum of components due to independent rounding.

Sources: Data sources, estimation procedures, and assumptions are described in the "Documentation" section of this report. 
K Energy Price and Expenditure Estimates by Source, Kansas

A $1970,1975,1980$, and 1985-1991

\begin{tabular}{|c|c|c|c|c|c|c|c|c|c|c|}
\hline Energy Source & 1970 & 1975 & 1980 & 1985 & 1986 & 1987 & 1988 & 1989 & 1990 & 1991 \\
\hline & \multicolumn{10}{|c|}{ Prices In Dollars per Million Btu } \\
\hline 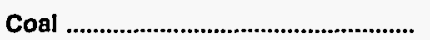 & 0.34 & 0.68 & 1.08 & 1.41 & 1.33 & 1.27 & 1.24 & 1.24 & 1.24 & 1.23 \\
\hline 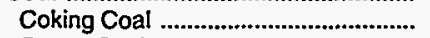 & - & - & - & - & - & - & - & - & - & - \\
\hline 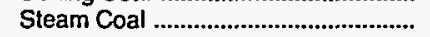 & .34 & .68 & 1.08 & 1.41 & 1.33 & 1.27 & 1.24 & 1.24 & 1.24 & 1.23 \\
\hline Natural Gas & .39 & .67 & 2.14 & 3.58 & 3.30 & 3.15 & 3.03 & 3.36 & 3.30 & 3.11 \\
\hline 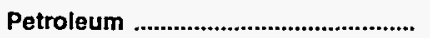 & 1.97 & 3.42 & 7.39 & 7.17 & 6.32 & 6.46 & 6.14 & 6.84 & 7.22 & 6.80 \\
\hline 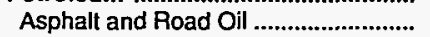 & .74 & 2.10 & 4.02 & 5.22 & 3.88 & 3.70 & 3.49 & 3.00 & 2.94 & 2.98 \\
\hline 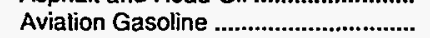 & 2.17 & 3.45 & 9.02 & 9.99 & 8.41 & 7.55 & 7.41 & 8.28 & 9.32 & 8.71 \\
\hline 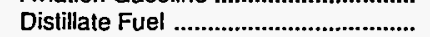 & .99 & 2.43 & 6.52 & 6.42 & 5.54 & 5.66 & 5.16 & 6.30 & 7.50 & 6.85 \\
\hline Jet Fuel & .75 & 2.09 & 6.47 & 5.94 & 3.73 & 4.01 & 3.81 & 4.27 & 5.57 & 4.81 \\
\hline Kerosene & .96 & 2.61 & 5.25 & 6.88 & 4.84 & 3.52 & 3.54 & 5.95 & 6.43 & 5.76 \\
\hline LPG & 1.27 & 2.65 & 4.41 & 4.28 & 8.04 & 7.51 & 7.51 & 7.24 & 4.39 & 3.69 \\
\hline Lubricants & 5.08 & 7.48 & 14.36 & 17.61 & 15.59 & 12.70 & 14.61 & 13.30 & 13.40 & 15.42 \\
\hline 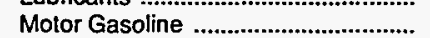 & 2.64 & 4.50 & 9.27 & 9.28 & 6.72 & 7.05 & 6.78 & 7.66 & 8.90 & 8.72 \\
\hline 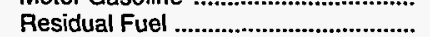 & .47 & 1.60 & 3.24 & 3.91 & 2.28 & 2.36 & 1.89 & 2.06 & 2.10 & 1.47 \\
\hline Other ${ }^{\mathrm{a}}$ & .83 & 1.76 & 3.95 & 6.62 & 6.77 & 6.57 & 5.60 & 6.66 & 6.47 & 6.33 \\
\hline 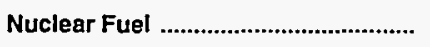 & - & - & - & .84 & .63 & .51 & .45 & .32 & .30 & .31 \\
\hline Biomsss Fuels at Utilities ................. & - & - & - & - & - & - & - & - & - & - \\
\hline Primary Energy — Five Sectors ${ }^{b} \ldots$. & .98 & 1.89 & 3.88 & 4.29 & 3.75 & $\mathbf{3 . 7 1}$ & 3.61 & 3.80 & 3.94 & 3.78 \\
\hline $\begin{array}{l}\text { Electric Utility Fuelc } \\
\text { Electricity Purchased by End Users }\end{array}$ & $\begin{array}{r}.30 \\
5.74\end{array}$ & $\begin{array}{r}.72 \\
7.72\end{array}$ & $\begin{array}{r}1.38 \\
13.75\end{array}$ & $\begin{array}{r}1.44 \\
19.07\end{array}$ & $\begin{array}{r}1.22 \\
20.53\end{array}$ & $\begin{array}{r}1.16 \\
20.06\end{array}$ & $\begin{array}{r}1.13 \\
19.22\end{array}$ & $\begin{array}{r}1.04 \\
18.83\end{array}$ & $\begin{array}{r}1.08 \\
19.31\end{array}$ & $\begin{array}{r}1.12 \\
19.30\end{array}$ \\
\hline \multirow[t]{2}{*}{ 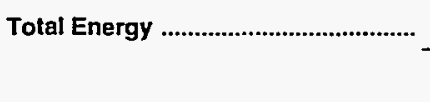 } & 1.54 & 2.86 & 5.93 & 7.21 & 7.03 & 6.91 & 6.70 & 7.22 & 7.51 & 7.21 \\
\hline & \multicolumn{10}{|c|}{ Expenditures in Millions of Dollars } \\
\hline 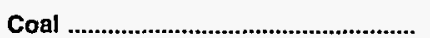 & 3.7 & 42.5 & 207.0 & 365.8 & 334.3 & 340.5 & 334.5 & 330.4 & 338.3 & 329.6 \\
\hline 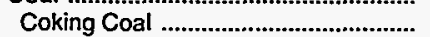 & - & - & - & - & - & - & - & - & - & - \\
\hline 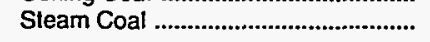 & 3.7 & 42.5 & 207.0 & 365.8 & 334.3 & 340.5 & 334.5 & 330.4 & 338.3 & 329.6 \\
\hline 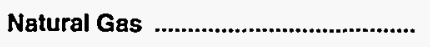 & 175.6 & 248.1 & 808.1 & 960.1 & 768.5 & 809.7 & 772.4 & 828.9 & 871.6 & 889.7 \\
\hline 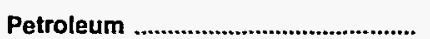 & 535.3 & $1,162.8$ & $2,507.7$ & $2,703.5$ & $2,327.9$ & $2,389.8$ & $2,467.2$ & $2,595.8$ & $2,664.4$ & $2,473.3$ \\
\hline Asphalt and Road Oil ............................. & 10.7 & 30.1 & 80.5 & 58.9 & 68.4 & 64.2 & 101.4 & 61.9 & 75.7 & $\begin{array}{r}2,47.5 \\
73.5\end{array}$ \\
\hline 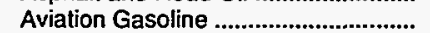 & 3.6 & 3.1 & 10.1 & 6.9 & 6.9 & 4.6 & 5.5 & 6.5 & 6.4 & 5.5 \\
\hline 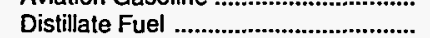 & 43.3 & 159.8 & 560.3 & 561.8 & 461.6 & 550.4 & 498.4 & 578.8 & 723.0 & 627.2 \\
\hline 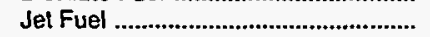 & 6.4 & 15.0 & 89.3 & 147.6 & 148.2 & 96.7 & 89.2 & 91.5 & 115.4 & 78.6 \\
\hline 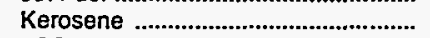 & 1.7 & 1.5 & 14.6 & 2.2 & 2.1 & 1.4 & .8 & 1.9 & 1.0 & .8 \\
\hline LPG & 37.1 & 83.9 & 131.0 & 369.0 & 474.6 & 432.4 & 509.8 & 492.6 & 244.3 & 240.4 \\
\hline 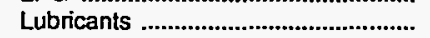 & 20.2 & 35.1 & 88.1 & 98.3 & 85.1 & 78.4 & 86.9 & 81.1 & 84.2 & 86.6 \\
\hline 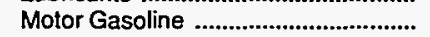 & 399.6 & 756.2 & $1,440.7$ & $1,375.2$ & $1,005.0$ & $1,077.4$ & $1,099.1$ & $1,200.6$ & $1,330.7$ & $1,284.4$ \\
\hline 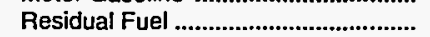 & 1.5 & 49.8 & 17.9 & 1.3 & 4.4 & 3.8 & 7.1 & 3.6 & 2.4 & .8 \\
\hline Other ${ }^{\mathrm{a}}$ & 11.2 & 28.3 & 75.3 & 82.2 & 71.7 & 80.4 & 69.0 & 77.3 & 81.3 & 75.5 \\
\hline Nuclear Fuel .......................................... & - & - & - & 34.9 & 47.7 & 35.7 & 32.4 & 33.3 & 25.3 & 19.4 \\
\hline Blomass Fuels at Utilitles ................. & - & - & - & - & - & - & - & - & - & - \\
\hline Primary Energy - Five Sectors ${ }^{b}$ & 714.6 & $1,453.4$ & $3,522.7$ & $4,064.3$ & $3,478.4$ & $3,575.7$ & $3,606.4$ & $3,788.3$ & $3,899.5$ & $3,712.0$ \\
\hline $\begin{array}{l}\text { Electric Utility Fuelc } \\
\text { Electricity Purchased by End Users }\end{array}$ & $\begin{array}{r}-53.9 \\
259.0\end{array}$ & $\begin{array}{r}-159.5 \\
444.0\end{array}$ & $\begin{array}{r}-394.3 \\
986.7\end{array}$ & $\begin{array}{r}-453.4 \\
1,520.6\end{array}$ & $\begin{array}{r}-412.3 \\
1,656.3\end{array}$ & $\begin{array}{r}-405.4 \\
1,658.4\end{array}$ & $\begin{array}{r}-404.4 \\
1,680.9\end{array}$ & $\begin{array}{r}-400.5 \\
1,646.7\end{array}$ & $\begin{array}{r}-410.7 \\
1,774.8\end{array}$ & $\begin{array}{r}-408.0 \\
1,839.4\end{array}$ \\
\hline 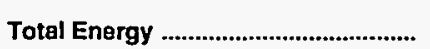 & 919.7 & $1,737.9$ & $4,115.0$ & $5,131.5$ & $4,722.4$ & $4,828.6$ & $4,883.0$ & $5,034.5$ & $5,263.6$ & $5,143.4$ \\
\hline
\end{tabular}

ancludes petroleum coke used at electric utilities.

b Biomass fuels are not included, except those consumed at electric utilities and those added to motor gasoline.

c There are no direct fuel costs for hydroelectric, geothermal, centralized solar, or wind energy.

-No consumption, including cases where adjustments were made. See "Consumption Adjustments for Process Fuel and Intermediate Products."

Note: Expenditure totals may not equal sum of components due to independent rounding.

Sources: Data sources, estimation procedures, and assumptions are described in the "Documentation" section of this report. 
Energy Price Estimates by Sector, Kansas

$1970,1975,1980$, and 1985-1991

(Dollars per Million Btu)

\begin{tabular}{|c|c|c|c|c|c|c|c|c|c|c|}
\hline Sector and Energy Source & 1970 & 1975 & 1980 & 1985 & 1986 & 1987 & 1988 & 1989 & 1990 & 1991 \\
\hline 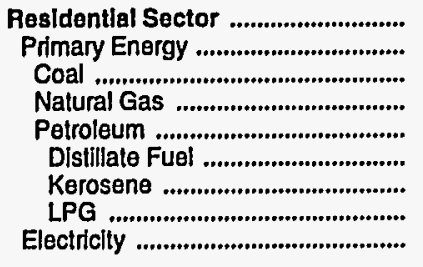 & $\begin{array}{r}1.68 \\
.82 \\
.91 \\
.69 \\
1.52 \\
1.19 \\
1.40 \\
1.53 \\
7.17\end{array}$ & $\begin{array}{l}2.53 \\
1.39 \\
- \\
1.05 \\
3.27 \\
2.62 \\
2.84 \\
3.30 \\
9.23\end{array}$ & $\begin{array}{r}5.48 \\
2.79 \\
2.15 \\
2.38 \\
6.83 \\
6.85 \\
7.68 \\
6.83 \\
15.75\end{array}$ & $\begin{array}{r}8.70 \\
4.29 \\
2.31 \\
4.12 \\
6.54 \\
6.43 \\
7.47 \\
6.52 \\
21.98\end{array}$ & $\begin{array}{r}9.57 \\
4.04 \\
2.33 \\
3.95 \\
5.39 \\
5.45 \\
6.33 \\
5.37 \\
24.01\end{array}$ & $\begin{array}{r}9.34 \\
3.95 \\
1.94 \\
3.90 \\
4.69 \\
3.53 \\
4.09 \\
4.73 \\
23.63\end{array}$ & $\begin{array}{r}9.36 \\
4.13 \\
1.80 \\
4.08 \\
4.79 \\
3.55 \\
4.12 \\
4.84 \\
22.94\end{array}$ & $\begin{array}{r}9.38 \\
4.49 \\
1.85 \\
4.20 \\
8.46 \\
6.16 \\
7.15 \\
8.57 \\
22.46\end{array}$ & $\begin{array}{r}10.16 \\
4.68 \\
1.88 \\
4.48 \\
7.80 \\
6.22 \\
7.23 \\
7.86 \\
22.95\end{array}$ & $\begin{array}{r}9.95 \\
4.49 \\
1.82 \\
4.33 \\
6.95 \\
5.78 \\
6.71 \\
6.98 \\
22.93\end{array}$ \\
\hline 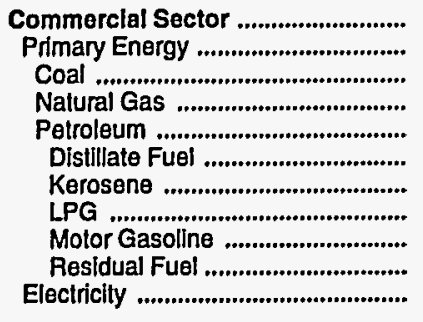 & $\begin{array}{r}1.56 \\
.53 \\
.45 \\
.46 \\
1.22 \\
1.03 \\
.69 \\
.83 \\
2.64 \\
.50 \\
6.02\end{array}$ & $\begin{array}{r}2.75 \\
.89 \\
- \\
.68 \\
2.63 \\
2.45 \\
2.27 \\
1.91 \\
4.50 \\
1.56 \\
8.26\end{array}$ & $\begin{array}{r}5.62 \\
2.27 \\
1.32 \\
1.91 \\
6.51 \\
6.49 \\
5.22 \\
3.58 \\
9.27 \\
14 . \overline{81}\end{array}$ & $\begin{array}{r}8.49 \\
3.41 \\
1.69 \\
3.15 \\
5.90 \\
5.52 \\
6.35 \\
4.14 \\
9.28 \\
19.87\end{array}$ & $\begin{array}{r}8.94 \\
3.07 \\
1.74 \\
2.90 \\
5.43 \\
3.85 \\
4.35 \\
8.25 \\
6.72 \\
2.28 \\
21.03\end{array}$ & $\begin{array}{r}8.71 \\
3.01 \\
1.58 \\
2.88 \\
5.19 \\
2.77 \\
3.32 \\
7.76 \\
7.05 \\
2.36 \\
20.37\end{array}$ & $\begin{array}{r}8.36 \\
3.17 \\
1.42 \\
3.07 \\
4.61 \\
2.52 \\
3.01 \\
7.73 \\
6.78 \\
1.67 \\
19.25\end{array}$ & $\begin{array}{r}8.62 \\
3.32 \\
1.28 \\
3.16 \\
5.81 \\
4.54 \\
5.39 \\
7.13 \\
7.66 \\
2.04 \\
19.17\end{array}$ & $\begin{array}{r}9.20 \\
3.48 \\
1.18 \\
3.36 \\
5.48 \\
4.69 \\
5.88 \\
4.10 \\
8.90 \\
2.13 \\
19.65\end{array}$ & $\begin{array}{r}9.04 \\
3.37 \\
1.26 \\
3.28 \\
4.74 \\
4.10 \\
5.11 \\
3.43 \\
8.72 \\
1.48 \\
19.56\end{array}$ \\
\hline 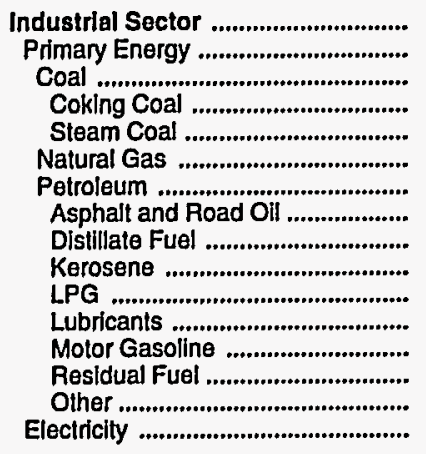 & $\begin{array}{r}.78 \\
.60 \\
.45 \\
- \\
.45 \\
.27 \\
1.24 \\
.74 \\
.64 \\
.69 \\
.83 \\
5.08 \\
2.64 \\
.43 \\
.83 \\
3.50\end{array}$ & $\begin{array}{r}1.84 \\
1.43 \\
.92 \\
- \\
.92 \\
.55 \\
2.48 \\
2.10 \\
2.13 \\
2.27 \\
1.91 \\
7.48 \\
4.50 \\
1.84 \\
1.76 \\
5.62\end{array}$ & $\begin{array}{r}3.94 \\
3.26 \\
1.32 \\
1.32 \\
2.35 \\
4.74 \\
4.02 \\
4.99 \\
5.22 \\
3.58 \\
14.36 \\
9.27 \\
2.53 \\
3.95 \\
10.68\end{array}$ & $\begin{array}{r}5.23 \\
4.34 \\
1.69 \\
-\overline{1} \\
1.69 \\
3.54 \\
5.18 \\
5.22 \\
5.76 \\
6.35 \\
4.14 \\
17.61 \\
9.28 \\
3.86 \\
6.62 \\
14.74\end{array}$ & $\begin{array}{r}5.98 \\
4.92 \\
1.74 \\
1.74 \\
3.17 \\
6.46 \\
3.88 \\
3.95 \\
4.35 \\
8.25 \\
15.59 \\
6.72 \\
2.28 \\
6.77 \\
15.76\end{array}$ & $\begin{array}{r}5.44 \\
4.42 \\
1.58 \\
- \\
1.58 \\
2.93 \\
6.00 \\
3.70 \\
3.02 \\
3.32 \\
7.76 \\
12.70 \\
7.05 \\
2.36 \\
6.57 \\
15.33\end{array}$ & $\begin{array}{r}5.23 \\
4.24 \\
1.42 \\
-\overline{1} \\
1.42 \\
2.39 \\
5.67 \\
3.49 \\
2.74 \\
3.01 \\
7.73 \\
14.61 \\
6.78 \\
1.67 \\
5.60 \\
14.68\end{array}$ & $\begin{array}{r}5.70 \\
4.72 \\
1.28 \\
1.28 \\
3.08 \\
6.07 \\
3.00 \\
4.89 \\
5.39 \\
7.13 \\
13.30 \\
7.66 \\
2.04 \\
6.66 \\
14.17\end{array}$ & $\begin{array}{r}4.89 \\
3.77 \\
1.18 \\
1 . \overline{8} \\
2.88 \\
4.69 \\
2.94 \\
5.34 \\
5.88 \\
4.10 \\
13.40 \\
8.90 \\
2.13 \\
6.47 \\
14.50\end{array}$ & $\begin{array}{r}4.54 \\
3.44 \\
1.26 \\
-\overline{-} \\
1.26 \\
2.64 \\
4.23 \\
2.98 \\
4.64 \\
5.11 \\
3.43 \\
15.42 \\
8.72 \\
1.48 \\
6.33 \\
14.51\end{array}$ \\
\hline 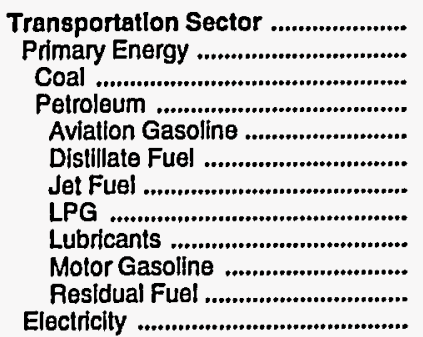 & $\begin{array}{r}2.34 \\
2.34 \\
.45 \\
2.34 \\
2.17 \\
1.18 \\
.75 \\
.83 \\
5.08 \\
2.64 \\
.49 \\
-\end{array}$ & $\begin{array}{r}4.13 \\
4.13 \\
.92 \\
4.13 \\
3.45 \\
2.70 \\
2.09 \\
1.91 \\
7.48 \\
4.50 \\
1.66 \\
-\end{array}$ & $\begin{array}{r}8.58 \\
8.58 \\
- \\
8.58 \\
9.02 \\
7.05 \\
6.47 \\
3.58 \\
14.36 \\
9.27 \\
3.82 \\
-\end{array}$ & $\begin{array}{r}8.38 \\
8.38 \\
-\overline{8} \\
8.38 \\
9.99 \\
6.75 \\
5.94 \\
4.14 \\
17.61 \\
9.28 \\
- \\
-\end{array}$ & $\begin{array}{r}6.30 \\
6.30 \\
- \\
6.30 \\
8.41 \\
6.43 \\
3.73 \\
8.25 \\
15.59 \\
6.72 \\
2.34 \\
\end{array}$ & $\begin{array}{r}6.74 \\
6.74 \\
-\overline{6} \\
7.74 \\
6.65 \\
4.01 \\
7.76 \\
12.70 \\
7.05 \\
- \\
-\end{array}$ & $\begin{array}{r}6.47 \\
6.47 \\
-\overline{6} \\
6.47 \\
7.41 \\
6.21 \\
3.81 \\
7.73 \\
14.61 \\
6.78 \\
-\end{array}$ & $\begin{array}{r}7.23 \\
7.23 \\
7.23 \\
8.28 \\
6.87 \\
4.27 \\
7.13 \\
13.30 \\
7.66 \\
- \\
-\end{array}$ & $\begin{array}{r}8.49 \\
8.49 \\
-\overline{8.49} \\
9.32 \\
8.28 \\
5.57 \\
4.10 \\
13.40 \\
8.90 \\
-\end{array}$ & $\begin{array}{r}8.32 \\
8.32 \\
- \\
8.32 \\
8.71 \\
7.94 \\
4.81 \\
3.43 \\
15.42 \\
8.72 \\
- \\
-\end{array}$ \\
\hline 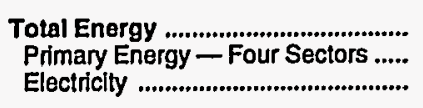 & $\begin{array}{l}1.54 \\
1.20 \\
5.74\end{array}$ & $\begin{array}{l}2.86 \\
2.36 \\
7.72\end{array}$ & $\begin{array}{r}5.93 \\
5.03 \\
13.75\end{array}$ & $\begin{array}{r}7.21 \\
5.71 \\
19.07\end{array}$ & $\begin{array}{r}7.03 \\
5.19 \\
20.53\end{array}$ & $\begin{array}{r}6.91 \\
5.15 \\
20.06\end{array}$ & $\begin{array}{r}6.70 \\
4.99 \\
19.22\end{array}$ & $\begin{array}{r}7.22 \\
5.55 \\
18.83\end{array}$ & $\begin{array}{r}7.51 \\
5.73 \\
19.31\end{array}$ & $\begin{array}{r}7.21 \\
5.35 \\
19.30\end{array}$ \\
\hline 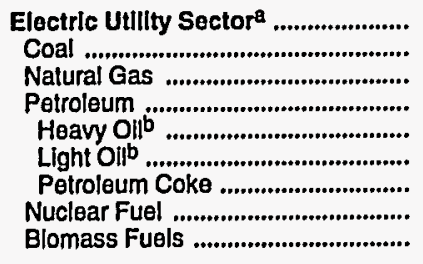 & $\begin{array}{c}.30 \\
.31 \\
.30 \\
.52 \\
.47 \\
.62 \\
- \\
- \\
-\end{array}$ & $\begin{array}{r}.72 \\
.67 \\
.48 \\
1.69 \\
1.55 \\
2.08 \\
.65 \\
= \\
-\end{array}$ & $\begin{array}{r}1.38 \\
1.07 \\
1.78 \\
4.60 \\
3.78 \\
5.74 \\
= \\
=\end{array}$ & $\begin{array}{r}1.44 \\
1.40 \\
2.88 \\
5.39 \\
3.99 \\
5.55 \\
- \\
.84 \\
-\end{array}$ & $\begin{array}{r}1.22 \\
1.32 \\
2.50 \\
3.27 \\
2.33 \\
3.36 \\
- \\
.63 \\
-\end{array}$ & $\begin{array}{r}1.16 \\
1.27 \\
2.17 \\
3.62 \\
2.39 \\
3.87 \\
-\overline{.51} \\
-\end{array}$ & $\begin{array}{r}1.13 \\
1.24 \\
2.06 \\
3.12 \\
2.73 \\
3.43 \\
- \\
.45 \\
-\end{array}$ & $\begin{array}{r}1.04 \\
1.24 \\
1.98 \\
3.81 \\
2.12 \\
4.33 \\
-32 \\
-\end{array}$ & $\begin{array}{r}1.08 \\
1.24 \\
1.76 \\
4.86 \\
1.85 \\
5.40 \\
-30 \\
-\end{array}$ & $\begin{array}{r}1.12 \\
1.23 \\
1.71 \\
4.25 \\
1.41 \\
4.32 \\
- \\
.31 \\
-\end{array}$ \\
\hline Primary Energy — Flve Sectors ${ }^{c}$.. & .98 & 1.89 & 3.88 & 4.29 & 3.75 & 3.71 & 3.61 & 3.80 & 3.94 & 3.78 \\
\hline
\end{tabular}

a There are no direct fuel costs for hydroelectric, geothermal, centralized solar, or wind energy.

b Heavy oil includes Grade Nos. 4, 5, and 6, and residual fuel oils. Light oil includes Grade No. 2 heating oil, kerosene, and jet fuel.

c Blomass fuels are not included, except those consumed at electric utilities and those added to motor gasoline.

- No consumption, Including cases where adjustments were made. See "Consumption Adjustments for Process Fuel and Intermediate Products."

Sources: Data sources, estimation procedures, and assumptions are described in the "Documentation" section of this report. 
K Energy Expenditure Estimates by Sector, Kansas

A 1970, 1975, 1980, and 1985-1991

$\mathrm{N}$ (Million Dollars)

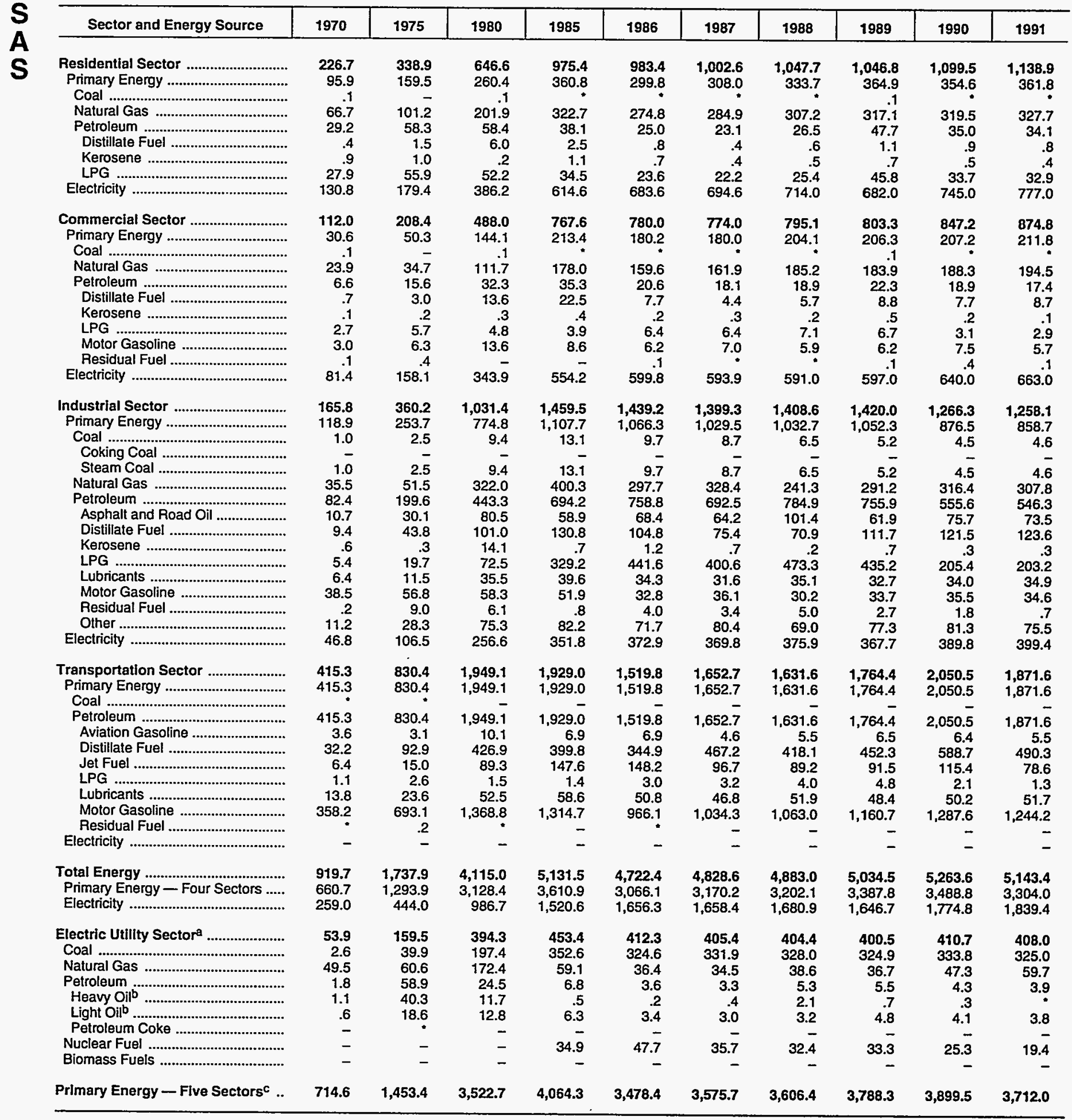

a There are no direct fuel costs for hydroelectric, geothermal, centralized solar, or wind energy.

b Heavy oil includes Grade Nos. 4, 5, and 6, and residual fuel oils. Light oil includes Grade No. 2 heating oil, kerosene, and jet fuel.

c Biomass fuels are not included, except those consumed at electric utilities and those added to motor gasoline.

"Value less than 0.05 million dollars.

Note: Totals may not equal sum of components due to independent rounding.

Sources: Data sources, estimation procedures, and assumptions are described in the "Documentation" section of this report. 
Energy Price and Expenditure Estimates by Source, Kentucky 1970, 1975, 1980, and 1985-1991

\begin{tabular}{|c|c|c|c|c|c|c|c|c|c|c|}
\hline Energy Source & 1970 & 1975 & 1980 & 1985 & 1986 & 1. 1987 & 1988 & 1989 & 1990 & 1991 \\
\hline & \multicolumn{10}{|c|}{ Prices in Dollars per Million Btu } \\
\hline 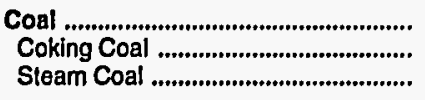 & $\begin{array}{r}0.26 \\
.38 \\
.25\end{array}$ & $\begin{array}{r}0.75 \\
1.60 \\
.70\end{array}$ & $\begin{array}{l}1.37 \\
1.81 \\
1.35\end{array}$ & $\begin{array}{l}1.48 \\
1.93 \\
1.46\end{array}$ & $\begin{array}{l}1.39 \\
1.75 \\
1.38\end{array}$ & $\begin{array}{l}1.33 \\
1.63 \\
1.32\end{array}$ & $\begin{array}{l}1.26 \\
1.73 \\
1.24\end{array}$ & $\begin{array}{l}1.22 \\
1.75 \\
1.20\end{array}$ & $\begin{array}{l}1.27 \\
1.80 \\
1.24\end{array}$ & $\begin{array}{l}1.24 \\
1.72 \\
1.23\end{array}$ \\
\hline 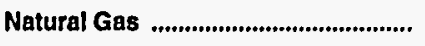 & .65 & 1.02 & 2.85 & 4.77 & 4.38 & 3.91 & 3.89 & 4.07 & 4.12 & 3.90 \\
\hline 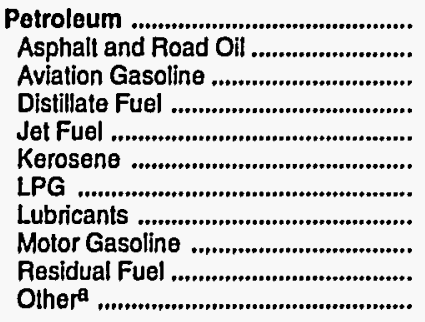 & $\begin{array}{r}2.12 \\
.67 \\
2.17 \\
1.21 \\
.73 \\
1.42 \\
1.90 \\
5.08 \\
2.93 \\
.56 \\
1.07\end{array}$ & $\begin{array}{l}3.81 \\
1.80 \\
3.45 \\
2.58 \\
2.03 \\
2.94 \\
3.60 \\
7.48 \\
4.69 \\
2.06 \\
2.49\end{array}$ & $\begin{array}{r}7.82 \\
3.56 \\
9.02 \\
6.41 \\
6.39 \\
7.55 \\
5.85 \\
14.36 \\
9.65 \\
3.64 \\
6.83\end{array}$ & $\begin{array}{r}7.87 \\
4.74 \\
9.99 \\
6.81 \\
6.17 \\
8.05 \\
6.58 \\
17.61 \\
8.80 \\
4.89 \\
7.08\end{array}$ & $\begin{array}{r}6.23 \\
4.90 \\
8.41 \\
5.39 \\
4.39 \\
5.04 \\
6.97 \\
15.59 \\
6.69 \\
3.08 \\
6.35\end{array}$ & $\begin{array}{r}6.59 \\
3.19 \\
7.55 \\
5.91 \\
4.16 \\
5.47 \\
7.97 \\
12.70 \\
7.36 \\
2.98 \\
5.68\end{array}$ & $\begin{array}{r}6.51 \\
3.05 \\
7.41 \\
5.81 \\
3.93 \\
5.64 \\
7.19 \\
14.61 \\
7.42 \\
2.91 \\
4.86\end{array}$ & $\begin{array}{r}6.97 \\
2.86 \\
8.28 \\
6.49 \\
4.45 \\
6.34 \\
6.00 \\
13.30 \\
8.08 \\
3.05 \\
5.25\end{array}$ & $\begin{array}{r}7.95 \\
2.94 \\
9.32 \\
7.53 \\
5.82 \\
6.97 \\
6.85 \\
13.40 \\
9.25 \\
3.61 \\
5.14\end{array}$ & $\begin{array}{r}7.58 \\
3.30 \\
8.71 \\
6.95 \\
4.92 \\
6.41 \\
6.27 \\
15.42 \\
8.94 \\
3.12 \\
4.85\end{array}$ \\
\hline Nuclear Fual ....................................... & - & - & - & - & - & - & - & - & - & - \\
\hline Blomass Fuels at Utilitles ................. & - & - & - & - & - & - & - & - & - & - \\
\hline Primary Energy - Five Sectors ${ }^{b}$.... & .94 & 1.88 & 4.00 & 3.97 & 3.28 & 3.38 & 3.27 & $\mathbf{3 . 5 5}$ & 3.78 & 3.63 \\
\hline $\begin{array}{l}\text { Electric Utility Fuelc ............................ } \\
\text { Electricity Purchased by End Users }\end{array}$ & $\frac{.22}{3.37}$ & $\begin{array}{r}.64 \\
5.32\end{array}$ & $\begin{array}{r}1.32 \\
10.07\end{array}$ & $\begin{array}{r}1.43 \\
14.84\end{array}$ & $\begin{array}{r}1.34 \\
15.26\end{array}$ & $\begin{array}{r}1.26 \\
15.18\end{array}$ & $\begin{array}{r}1.20 \\
15.08\end{array}$ & $\begin{array}{r}1.14 \\
14.17\end{array}$ & $\begin{array}{r}1.20 \\
13.17\end{array}$ & $\begin{array}{r}1.18 \\
12.96\end{array}$ \\
\hline \multirow[t]{2}{*}{ Total Energy ......................................... } & 1.66 & 3.27 & 6.74 & 8.03 & 7.24 & 7.34 & 7.29 & 7.43 & 7.85 & 7.68 \\
\hline & \multicolumn{10}{|c|}{ Expenditures in Millions of Dollars } \\
\hline 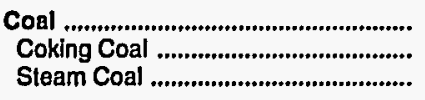 & $\begin{array}{r}138.9 \\
16.4 \\
122.4\end{array}$ & $\begin{array}{r}421.0 \\
52.1 \\
368.8\end{array}$ & $\begin{array}{r}878.8 \\
44.0 \\
834.8\end{array}$ & $\begin{array}{r}1,061.1 \\
60.5 \\
1,000.6\end{array}$ & $\begin{array}{r}1,045.4 \\
52.2 \\
993.2\end{array}$ & $\begin{array}{r}993.3 \\
57.1 \\
936.2\end{array}$ & $\begin{array}{r}1,033.6 \\
60.4 \\
973.2\end{array}$ & $\begin{array}{r}933.9 \\
60.6 \\
873.3\end{array}$ & $\begin{array}{r}1,018.6 \\
56.9 \\
961.8\end{array}$ & $\begin{array}{r}998.3 \\
39.4 \\
958.9\end{array}$ \\
\hline Natural Gas ........................................ & 136.7 & 185.7 & 511.8 & 722.4 & 630.7 & 587.4 & 637.0 & 689.6 & 656.4 & 659.4 \\
\hline 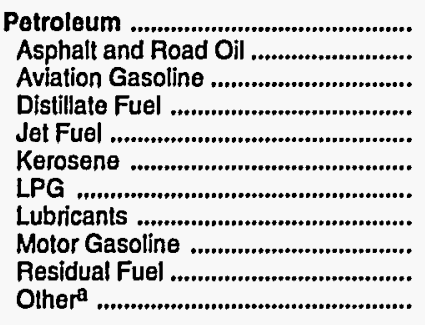 & \begin{tabular}{r|}
760.8 \\
13.8 \\
3.6 \\
58.0 \\
12.6 \\
24.9 \\
67.5 \\
26.0 \\
517.3 \\
3.2 \\
33.8
\end{tabular} & $\begin{array}{r}1,554.6 \\
31.3 \\
2.2 \\
164.1 \\
24.6 \\
26.3 \\
143.7 \\
47.6 \\
1,005.6 \\
11.1 \\
98.2\end{array}$ & $\begin{array}{r}3,870.9 \\
47.8 \\
5.1 \\
855.7 \\
104.4 \\
124.6 \\
216.4 \\
92.0 \\
2,019.1 \\
20.9 \\
385.0\end{array}$ & $\begin{array}{r}3,341.5 \\
58.9 \\
3.3 \\
863.2 \\
119.3 \\
68.8 \\
129.0 \\
102.7 \\
1,845.7 \\
9.5 \\
141.1\end{array}$ & $\begin{array}{r}2,658.4 \\
74.3 \\
3.6 \\
640.8 \\
87.8 \\
31.1 \\
127.8 \\
88.9 \\
1,494.7 \\
8.9 \\
100.5\end{array}$ & $\begin{array}{r}2,963.7 \\
57.2 \\
2.4 \\
705.8 \\
113.5 \\
20.1 \\
193.8 \\
81.9 \\
1,658.9 \\
11.2 \\
118.8\end{array}$ & $\begin{array}{r}3,127.9 \\
53.0 \\
2.3 \\
835.7 \\
110.8 \\
31.2 \\
173.7 \\
90.9 \\
1,722.4 \\
7.4 \\
100.5\end{array}$ & $\begin{array}{r}3,449.2 \\
52.4 \\
2.2 \\
1,063.4 \\
127.7 \\
33.9 \\
136.7 \\
84.8 \\
1,841.4 \\
6.3 \\
100.4\end{array}$ & $\begin{array}{r}3,742.7 \\
59.1 \\
2.4 \\
1,026.1 \\
188.2 \\
22.4 \\
152.0 \\
88.0 \\
2,079.8 \\
8.9 \\
115.9\end{array}$ & $\begin{array}{r}3,591.9 \\
61.3 \\
2.2 \\
917.5 \\
177.9 \\
20.0 \\
164.7 \\
90.5 \\
2,054.4 \\
5.4 \\
98.0\end{array}$ \\
\hline 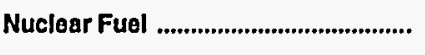 & - & - & - & - & - & - & - & - & - & - \\
\hline Blomass Fuels at Utilitles ................. & - & - & - & - & - & - & - & - & - & - \\
\hline Primary Energy - Five Sectors ${ }^{b}$.... & $1,036.3$ & $2,161.3$ & $5,261.6$ & $5,125.0$ & $4,334.5$ & $4,544.4$ & $4,798.5$ & $5,072.7$ & $5,417.8$ & $5,249.7$ \\
\hline $\begin{array}{l}\text { Electric Utility Fuelc ............................. } \\
\text { Electricity Purchased by End Users }\end{array}$ & $\begin{array}{r}-90.6 \\
354.9\end{array}$ & $\begin{array}{r}-309.8 \\
852.2\end{array}$ & $\begin{array}{r}-743.7 \\
1,698.6\end{array}$ & $\begin{array}{r}-883.4 \\
2,528.3\end{array}$ & $\begin{array}{r}-886.1 \\
2,552.6\end{array}$ & $\begin{array}{r}-832.6 \\
2,596.4\end{array}$ & $\begin{array}{r}-884.5 \\
2,747.0\end{array}$ & $\begin{array}{r}-768.0 \\
2,792.2\end{array}$ & $\begin{array}{r}-859.2 \\
2,707.4\end{array}$ & $\begin{array}{r}-862.0 \\
2,800.1\end{array}$ \\
\hline 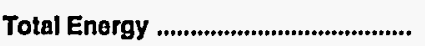 & $1,300.6$ & $2,703.7$ & $6,216.4$ & $6,769.9$ & $6,001.0$ & $6,308.1$ & $6,661.0$ & $7,096.9$ & $7,265.9$ & 7,187.7 \\
\hline
\end{tabular}

Includes petroleum coke used at electric utilities.

b Biomass fuels are not included, except those consumed at electric utilities and those added to motor gasoline.

c There are no direct fuel costs for hydroelectric, geothermal, centralized solar, or wind energy.

-No consumption, including cases where adjustments were made. See "Consumption Adjustments for Process Fuel and Intermediate Products."

Note: Expenditure totals may not equal sum of components due to independent rounding.

Sources: Data sources, estimation procedures, and assumptions are described in the "Documentation" section of this report. 
K Energy Price Estimates by Sector, Kentucky

E 1970,1975, 1980, and 1985-1991

N (Dollars per Million Btu)

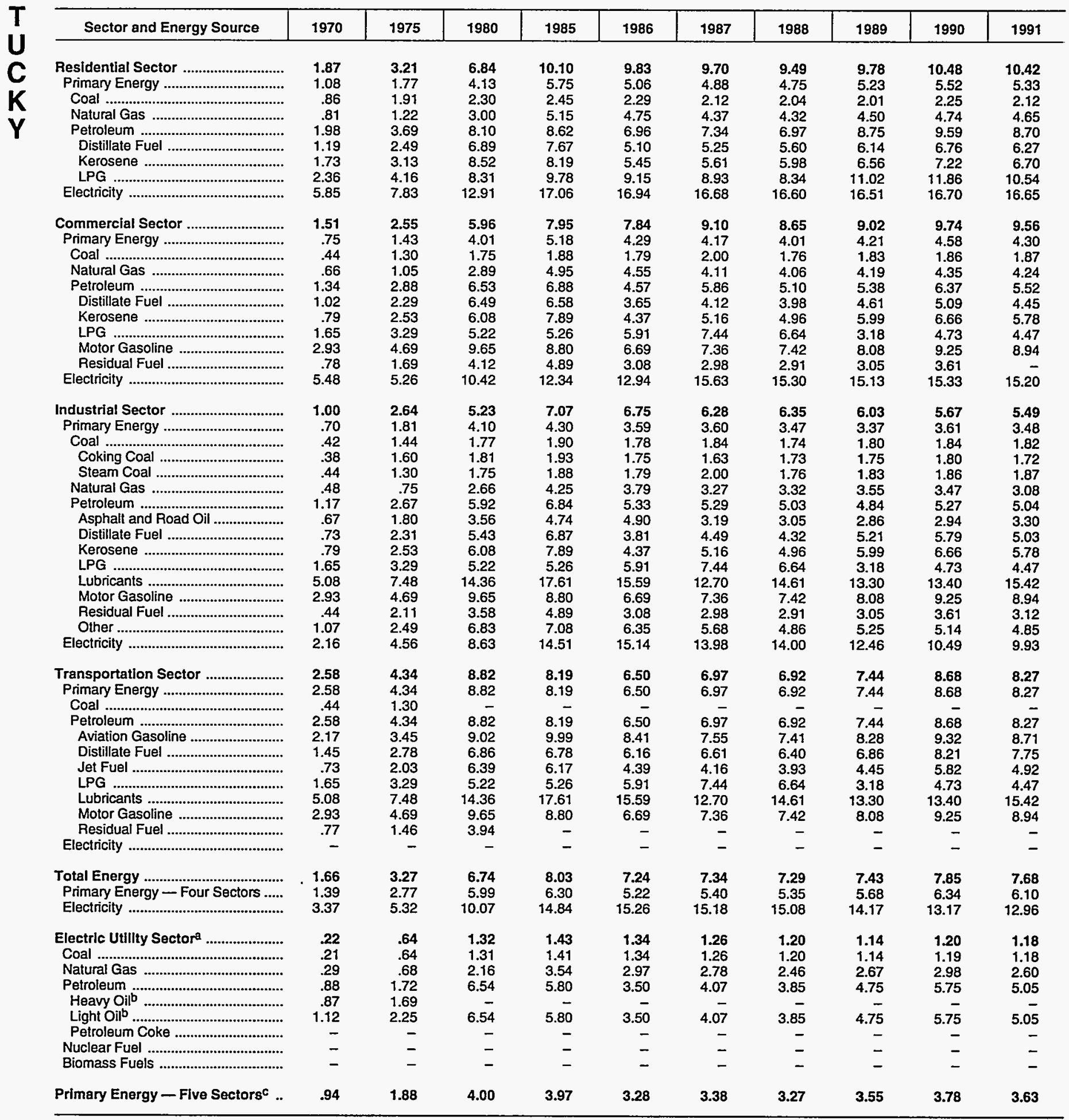

There are no direct fuel costs for hydroelectric, geothermal, centralized solar, or wind energy.

Heavy oil includes Grade Nos. 4, 5, and 6, and residual fuel oils. Light oil includes Grade No. 2 heating oil, kerosene, and jet fuel.

c Biomass fuels are not included, except those consumed at electric utilities and those added to motor gasoline.

-No consumption, including cases where adjustments were made. See "Consumption Adjustments for Process Fuel and Intermediate Products."

Sources: Data sources, estimation procedures, and assumptions are described in the "Documentation" section of this report. 


\begin{tabular}{|c|c|c|c|c|c|c|c|c|c|c|}
\hline Sector and Energy Source & 1970 & 1975 & 1980 & 1985 & 1986 & 1987 & 1988 & 1989 & 1990 & 1991 \\
\hline 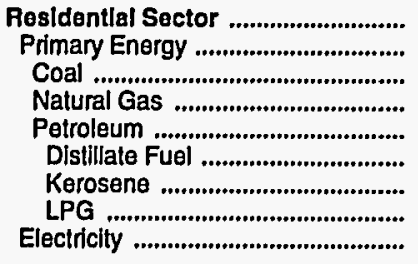 & $\begin{array}{r}268.1 \\
128.6 \\
3.8 \\
71.6 \\
53.1 \\
2.8 \\
20.4 \\
30.0 \\
139.6\end{array}$ & $\begin{array}{r}441.0 \\
185.0 \\
4.6 \\
97.1 \\
83.2 \\
6.4 \\
19.0 \\
57.8 \\
256.0\end{array}$ & $\begin{array}{r}986.8 \\
410.9 \\
5.6 \\
224.9 \\
180.4 \\
32.9 \\
84.6 \\
63.0 \\
575.9\end{array}$ & $\begin{array}{r}1,301.7 \\
455.5 \\
5.2 \\
318.9 \\
131.4 \\
36.8 \\
38.7 \\
55.9 \\
846.2\end{array}$ & $\begin{array}{r}1,279.1 \\
394.4 \\
5.7 \\
292.7 \\
95.9 \\
20.3 \\
20.7 \\
54.9 \\
884.7\end{array}$ & $\begin{array}{r}1,302.6 \\
387.4 \\
5.3 \\
267.7 \\
114.4 \\
23.2 \\
14.2 \\
77.0 \\
915.1\end{array}$ & $\begin{array}{r}1,361.4 \\
409.4 \\
6.3 \\
286.8 \\
116.2 \\
28.9 \\
21.9 \\
65.4 \\
952.0\end{array}$ & $\begin{array}{r}1,400.1 \\
447.1 \\
3.9 \\
304.6 \\
138.6 \\
26.7 \\
21.7 \\
90.3 \\
953.0\end{array}$ & $\begin{array}{r}1,354.2 \\
396.3 \\
2.9 \\
276.4 \\
117.0 \\
25.4 \\
13.1 \\
78.5 \\
958.0\end{array}$ & $\begin{array}{r}1,474.0 \\
415.0 \\
3.4 \\
289.6 \\
122.0 \\
25.7 \\
14.4 \\
81.9 \\
1,059.0\end{array}$ \\
\hline 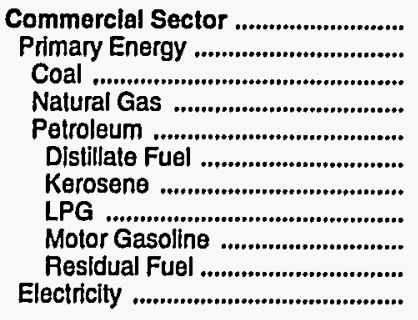 & $\begin{array}{r}111.2 \\
46.5 \\
3.5 \\
28.3 \\
14.6 \\
5.0 \\
1.8 \\
3.7 \\
4.1 \\
.1 \\
64.8\end{array}$ & $\begin{array}{r}193.0 \\
76.6 \\
5.6 \\
40.8 \\
30.2 \\
12.2 \\
3.0 \\
8.1 \\
6.8 \\
.1 \\
116.4\end{array}$ & $\begin{array}{r}563.7 \\
263.8 \\
7.7 \\
114.9 \\
141.1 \\
99.6 \\
21.4 \\
7.0 \\
12.7 \\
.5 \\
299.9\end{array}$ & $\begin{array}{r}663.3 \\
264.6 \\
7.4 \\
172.1 \\
85.1 \\
58.3 \\
4.1 \\
5.3 \\
17.5 \\
398.7\end{array}$ & $\begin{array}{r}647.1 \\
209.3 \\
8.4 \\
154.4 \\
46.6 \\
21.8 \\
3.7 \\
6.3 \\
14.2 \\
.6 \\
437.7\end{array}$ & $\begin{array}{r}739.8 \\
193.3 \\
9.2 \\
141.8 \\
42.3 \\
12.8 \\
2.0 \\
11.3 \\
16.2 \\
546.5\end{array}$ & $\begin{array}{r}777.8 \\
212.8 \\
10.1 \\
150.4 \\
52.3 \\
22.6 \\
4.0 \\
9.2 \\
15.8 \\
.7 \\
565.0\end{array}$ & $\begin{array}{r}796.1 \\
208.1 \\
6.6 \\
157.2 \\
44.2 \\
17.4 \\
5.6 \\
4.6 \\
16.7 \\
588.0\end{array}$ & $\begin{array}{r}812.2 \\
198.2 \\
4.5 \\
143.8 \\
50.0 \\
19.4 \\
3.6 \\
5.5 \\
21.5 \\
614.0\end{array}$ & $\begin{array}{r}852.2 \\
198.3 \\
5.6 \\
149.6 \\
43.0 \\
18.6 \\
3.3 \\
6.1 \\
15.0 \\
654.0\end{array}$ \\
\hline 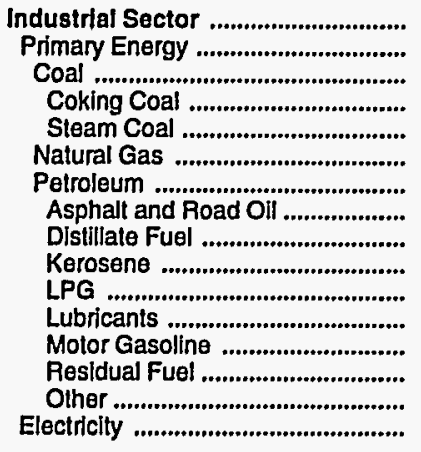 & $\begin{array}{r}341.1 \\
190.6 \\
44.0 \\
16.4 \\
27.5 \\
34.3 \\
112.3 \\
13.8 \\
8.9 \\
2.7 \\
33.5 \\
14.6 \\
3.2 \\
1.8 \\
33.8 \\
150.5\end{array}$ & $\begin{array}{r}923.2 \\
443.4 \\
102.3 \\
52.1 \\
50.1 \\
47.5 \\
293.6 \\
31.3 \\
44.7 \\
4.2 \\
77.0 \\
23.5 \\
4.8 \\
9.9 \\
98.2 \\
479.9\end{array}$ & $\begin{array}{r}1,994.8 \\
1,172.0 \\
134.6 \\
44.0 \\
90.6 \\
167.8 \\
869.6 \\
47.8 \\
203.6 \\
18.6 \\
146.2 \\
46.9 \\
4.5 \\
17.1 \\
385.0 \\
822.8\end{array}$ & $\begin{array}{r}2,306.0 \\
1,022.6 \\
178.3 \\
60.5 \\
117.9 \\
227.4 \\
617.0 \\
58.9 \\
224.3 \\
26.0 \\
65.9 \\
52.4 \\
39.0 \\
9.5 \\
141.1 \\
1,283.4\end{array}$ & $\begin{array}{r}2,002.6 \\
772.6 \\
151.4 \\
52.2 \\
99.2 \\
182.3 \\
439.0 \\
74.3 \\
110.1 \\
6.6 \\
64.9 \\
45.3 \\
28.9 \\
8.3 \\
100.5 \\
1,230.1\end{array}$ & $\begin{array}{r}1,975.6 \\
840.8 \\
152.5 \\
57.1 \\
95.4 \\
176.9 \\
511.4 \\
57.2 \\
142.4 \\
4.0 \\
103.5 \\
41.8 \\
32.6 \\
11.2 \\
118.8 \\
1,134.8\end{array}$ & $\begin{array}{r}2,037.8 \\
807.7 \\
138.4 \\
60.4 \\
77.9 \\
198.6 \\
470.7 \\
53.0 \\
131.0 \\
5.3 \\
97.4 \\
46.3 \\
30.6 \\
6.6 \\
100.5 \\
1,230.0\end{array}$ & $\begin{array}{r}2,071.5 \\
820.3 \\
162.5 \\
60.6 \\
102.0 \\
226.9 \\
430.8 \\
52.4 \\
145.2 \\
6.7 \\
41.0 \\
43.2 \\
35.6 \\
6.3 \\
100.4 \\
1,251.2\end{array}$ & $\begin{array}{r}2,048.9 \\
913.5 \\
160.0 \\
56.9 \\
103.2 \\
235.4 \\
518.1 \\
59.1 \\
175.7 \\
5.7 \\
66.9 \\
44.8 \\
41.0 \\
8.9 \\
115.9 \\
1,135.4\end{array}$ & $\begin{array}{r}1,923.8 \\
836.7 \\
134.6 \\
39.4 \\
95.2 \\
219.6 \\
482.6 \\
61.3 \\
153.1 \\
2.4 \\
75.7 \\
46.2 \\
40.6 \\
5.4 \\
98.0 \\
1,087.1\end{array}$ \\
\hline 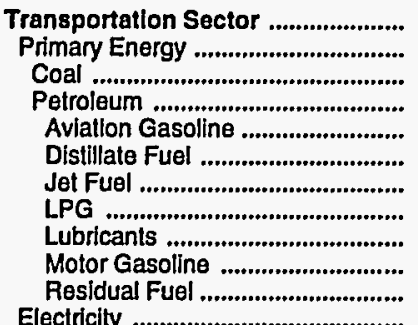 & $\begin{array}{r}580.1 \\
580.1 \\
.1 \\
580.0 \\
3.6 \\
41.4 \\
12.6 \\
.3 \\
11.4 \\
510.0 \\
.7\end{array}$ & $\begin{array}{r}1,146.5 \\
1,146.5 \\
1,146.5 \\
2.2 \\
100.8 \\
24.6 \\
.8 \\
24.0 \\
994.0 \\
. \\
\end{array}$ & $\begin{array}{r}2,671.1 \\
2,671.1 \\
2,-\overline{1} \\
2,671.1 \\
5.1 \\
511.0 \\
104.4 \\
.2 \\
45.1 \\
2,002.0 \\
3.4\end{array}$ & $\begin{array}{r}2,498.9 \\
2,498.9 \\
-\overline{0} \\
2,498.9 \\
3.3 \\
534.7 \\
119.3 \\
1.9 \\
50.4 \\
1,789.3 \\
-\end{array}$ & $\begin{aligned} 2,072.1 \\
2,072.1 \\
- \\
2,072.1 \\
3.6 \\
483.8 \\
87.8 \\
1.7 \\
43.6 \\
1,451.6 \\
-\end{aligned}$ & $\begin{array}{r}2,290.2 \\
2,290.2 \\
- \\
2,290 . \overline{2} \\
2.4 \\
522.1 \\
113.5 \\
1.9 \\
40.2 \\
1,610.1 \\
-\end{array}$ & $\begin{array}{r}2,484.1 \\
2,484.1 \\
2,484 . \overline{1} \\
2.3 \\
648.7 \\
110.8 \\
1.8 \\
44.5 \\
1,676.0 \\
\end{array}$ & $\begin{array}{r}2,829.1 \\
2,829.1 \\
2,829 . \overline{1} \\
2.2 \\
867.7 \\
127.7 \\
.9 \\
41.6 \\
1,789.1 \\
-\end{array}$ & $\begin{array}{r}3,050.5 \\
3,050.5 \\
- \\
3,050.5 \\
2.4 \\
798.4 \\
188.2 \\
1.1 \\
43.1 \\
2,017.3 \\
-\end{array}$ & $\begin{array}{r}2,937.7 \\
2,937.7 \\
- \\
2,937.7 \\
2.2 \\
713.4 \\
177.9 \\
.8 \\
44.4 \\
1,998.8 \\
-\end{array}$ \\
\hline 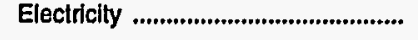 & - & - & - & - & - & - & - & - & - & - \\
\hline 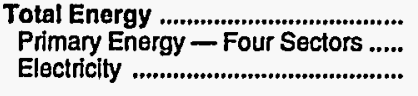 & $\begin{array}{r}1,300.6 \\
945.7 \\
354.9\end{array}$ & $\begin{array}{r}2,703.7 \\
1,851.5 \\
852.2\end{array}$ & $\begin{array}{l}6,216.4 \\
4,517.8 \\
1,698.6\end{array}$ & $\begin{array}{l}6,769.9 \\
4,241.6 \\
2,528.3\end{array}$ & $\begin{array}{l}6,001.0 \\
3,448.4 \\
2,552.6\end{array}$ & $\begin{array}{l}6,308.1 \\
3,711.7 \\
2,596.4\end{array}$ & $\begin{array}{l}6,661.0 \\
3,914.0 \\
2,747.0\end{array}$ & $\begin{array}{l}7,096.9 \\
4,304.7 \\
2,792.2\end{array}$ & $\begin{array}{l}7,265.9 \\
4,558.5 \\
2,707.4\end{array}$ & $\begin{array}{l}7,187.7 \\
4,387.6 \\
2,800.1\end{array}$ \\
\hline 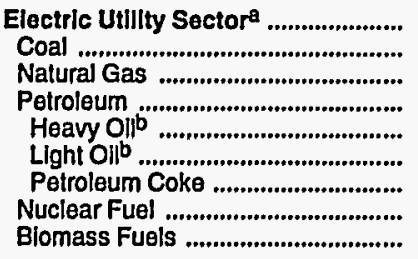 & $\begin{array}{r}90.6 \\
87.4 \\
2.5 \\
.7 \\
.7 \\
- \\
- \\
-\end{array}$ & $\begin{array}{r}309.8 \\
308.4 \\
.2 \\
1.2 \\
1.1 \\
.1 \\
- \\
= \\
-\end{array}$ & $\begin{array}{r}743.7 \\
730.9 \\
4.2 \\
8.6 \\
- \\
8.6 \\
= \\
= \\
-\end{array}$ & $\begin{array}{r}883.4 \\
870.2 \\
4.1 \\
9.1 \\
-\overline{9} \\
- \\
- \\
-\end{array}$ & $\begin{array}{r}886.1 \\
879.9 \\
1.3 \\
4.8 \\
4 . \overline{8} \\
- \\
- \\
-\end{array}$ & $\begin{array}{r}832.6 \\
826.3 \\
1.0 \\
5.3 \\
- \\
5.3 \\
- \\
- \\
-\end{array}$ & $\begin{array}{r}884.5 \\
878.9 \\
1.1 \\
4.5 \\
4 . \overline{5} \\
- \\
-\end{array}$ & $\begin{array}{r}768.0 \\
760.8 \\
.9 \\
6.4 \\
6.4 \\
- \\
-\end{array}$ & $\begin{array}{r}859.2 \\
851.3 \\
.9 \\
7.1 \\
7.1 \\
- \\
- \\
-\end{array}$ & $\begin{array}{r}862.0 \\
854.7 \\
.6 \\
6.7 \\
6.7 \\
= \\
\overline{-}\end{array}$ \\
\hline Primary Energy - Five Sectorsc .. & $1,036.3$ & $2,161.3$ & $5,261.6$ & $5,125.0$ & $4,334.5$ & $4,544.4$ & 4,798.5 & $5,072.7$ & $5,417.8$ & $5,249.7$ \\
\hline
\end{tabular}

a There are no direct fuel costs for hydroelectric, geothermal, centralized solar, or wind energy.

b Heavy oil includes Grade Nos. 4, 5, and 6, and residual fuel oils. Light oil includes Grade No. 2 heating oil, kerosene, and jet fuel.

c Biomass fuels are not included, except those consumed at electric utilities and those added to motor gasoline.

-No consumption, including cases where adjustments were made. See "Consumption Adjustments for Process Fuel and Intermediate Products."

"Value less than 0.05 million dollars.

Note: Totals may not equal sum of components due to independent rounding.

Sources: Data sources, estimation procedures, and assumptions are described in the "Documentation" section of this report. 


\section{Energy Price and Expenditure Estimates by Source, Louisiana}

O $1970,1975,1980$, and $1985-1991$

\begin{tabular}{|c|c|c|c|c|c|c|c|c|c|c|}
\hline Energy Source & 1970 & 1975 & 1980 & 1985 & 1986 & 1987 & 1988 & 1989 & 1990 & 1991 \\
\hline & \multicolumn{10}{|c|}{ Prices in Dollars per Million Btu } \\
\hline 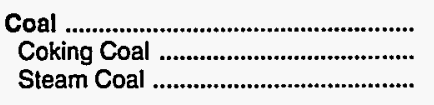 & $\begin{array}{l}- \\
-\end{array}$ & $\begin{array}{l}- \\
-\end{array}$ & $\begin{array}{r}1.26 \\
-\bar{c}\end{array}$ & $\begin{array}{r}2.14 \\
-\overline{4}\end{array}$ & $\frac{1.80}{1.80}$ & $\begin{array}{r}1.66 \\
1.66\end{array}$ & $\begin{array}{r}1.57 \\
1.57\end{array}$ & $\begin{array}{r}1.62 \\
1.62\end{array}$ & $\begin{array}{r}1.68 \\
1.68\end{array}$ & $\begin{array}{r}1.65 \\
1.65\end{array}$ \\
\hline Natural Gas .............................................. & .27 & .75 & 1.61 & 3.09 & 2.15 & 2.00 & 2.14 & 2.12 & 2.11 & 1.89 \\
\hline 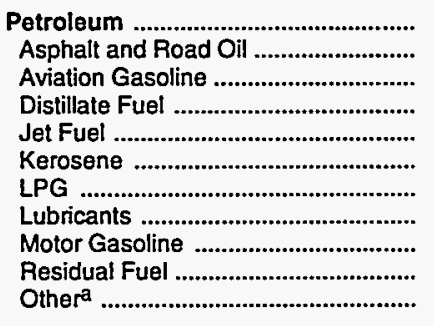 & $\begin{array}{r}1.48 \\
.66 \\
2.17 \\
.86 \\
.72 \\
.60 \\
1.12 \\
5.08 \\
2.86 \\
.45 \\
.70\end{array}$ & $\begin{array}{l}2.80 \\
1.78 \\
3.45 \\
2.34 \\
2.01 \\
2.02 \\
2.52 \\
7.48 \\
4.49 \\
1.62 \\
2.40\end{array}$ & $\begin{array}{r}5.98 \\
3.62 \\
9.02 \\
6.02 \\
6.34 \\
5.53 \\
5.30 \\
14.36 \\
9.89 \\
3.31 \\
6.28\end{array}$ & $\begin{array}{r}6.23 \\
4.17 \\
9.99 \\
5.01 \\
5.70 \\
3.57 \\
5.12 \\
17.61 \\
9.36 \\
3.60 \\
6.57\end{array}$ & $\begin{array}{r}4.84 \\
4.57 \\
8.41 \\
4.84 \\
3.67 \\
3.77 \\
5.68 \\
15.59 \\
7.04 \\
1.39 \\
3.81\end{array}$ & $\begin{array}{r}5.29 \\
3.15 \\
7.55 \\
5.45 \\
3.90 \\
4.92 \\
5.85 \\
12.70 \\
7.14 \\
2.55 \\
4.63\end{array}$ & $\begin{array}{r}5.15 \\
3.14 \\
7.41 \\
5.61 \\
3.61 \\
5.33 \\
5.77 \\
14.61 \\
7.25 \\
2.55 \\
3.86\end{array}$ & $\begin{array}{r}5.48 \\
2.89 \\
8.28 \\
6.12 \\
4.12 \\
5.88 \\
5.47 \\
13.30 \\
7.90 \\
2.52 \\
4.71\end{array}$ & $\begin{array}{r}6.79 \\
2.91 \\
9.32 \\
7.61 \\
5.79 \\
7.60 \\
7.82 \\
13.40 \\
9.47 \\
2.10 \\
5.85\end{array}$ & $\begin{array}{r}6.41 \\
3.08 \\
8.71 \\
7.20 \\
4.67 \\
6.95 \\
7.84 \\
15.42 \\
9.51 \\
2.84 \\
5.03\end{array}$ \\
\hline Nuclear Fuel .......................................... & - & - & - & .86 & 1.04 & .97 & .92 & .90 & .88 & .84 \\
\hline Blomass Fuels at Utilitles ................. & - & - & - & - & - & - & - & - & - & - \\
\hline Primary Energy - Five Sectors ${ }^{b}$ & .65 & 1.60 & 3.70 & 4.48 & 3.32 & 3.41 & 3.40 & 3.46 & 4.02 & 3.78 \\
\hline $\begin{array}{l}\text { Electric Utility Fuel } \\
\text { Electricity Purchased by End Users }\end{array}$ & $\begin{array}{r}.21 \\
4.69\end{array}$ & $\begin{array}{r}.73 \\
6.24\end{array}$ & $\begin{array}{r}2.19 \\
11.49\end{array}$ & $\begin{array}{r}2.46 \\
18.25\end{array}$ & $\begin{array}{r}1.67 \\
17.40\end{array}$ & $\begin{array}{r}1.46 \\
17.33\end{array}$ & $\begin{array}{r}1.44 \\
17.30\end{array}$ & $\begin{array}{r}1.51 \\
17.64\end{array}$ & $\begin{array}{r}1.49 \\
17.77\end{array}$ & $\begin{array}{r}1.40 \\
17.59\end{array}$ \\
\hline \multirow[t]{2}{*}{ 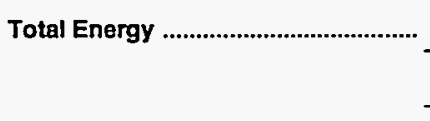 } & .94 & 2.03 & 4.50 & 6.33 & 5.06 & 5.21 & 5.24 & 5.28 & 6.00 & 5.69 \\
\hline & \multicolumn{10}{|c|}{ Expenditures in Millions of Dollars } \\
\hline 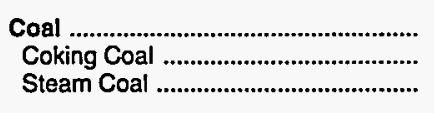 & $\begin{array}{l}- \\
-\end{array}$ & $\begin{array}{l}- \\
-\end{array}$ & $3 . \overline{1}$ & $340 . \overline{0}$ & $\begin{array}{r}308.6 \\
- \\
308.6\end{array}$ & $\begin{array}{r}286.7 \\
- \\
286.7\end{array}$ & $\begin{array}{r}332.9 \\
332.9\end{array}$ & $\begin{array}{r}335.6 \\
- \\
335.6\end{array}$ & $\begin{array}{r}351.2 \\
-\overline{2} \\
351.2\end{array}$ & $\begin{array}{r}353.8 \\
353.8\end{array}$ \\
\hline 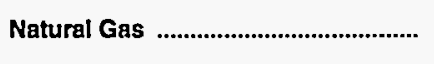 & 376.4 & $1,036.2$ & $2,396.3$ & $3,152.5$ & $2,249.7$ & $2,095.4$ & $2,203.4$ & $2,440.3$ & $2,466.5$ & $2,209.1$ \\
\hline 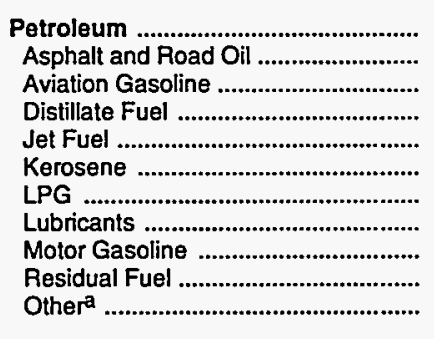 & $\begin{array}{r}964.9 \\
9.7 \\
4.9 \\
59.1 \\
23.4 \\
8.5 \\
199.3 \\
49.0 \\
523.4 \\
31.1 \\
56.5\end{array}$ & $\begin{array}{r}2,705.4 \\
33.2 \\
5.1 \\
268.9 \\
67.9 \\
27.7 \\
481.4 \\
82.9 \\
1,018.8 \\
280.0 \\
439.5\end{array}$ & $\begin{array}{r}8,196.5 \\
46.8 \\
11.6 \\
752.1 \\
306.8 \\
179.2 \\
1,012.5 \\
174.1 \\
2,449.2 \\
1,265.9 \\
1,998.3\end{array}$ & $\begin{array}{r}6,761.1 \\
50.8 \\
8.6 \\
980.8 \\
410.5 \\
3.8 \\
1,293.9 \\
194.3 \\
2,424.2 \\
546.9 \\
847.3\end{array}$ & $\begin{array}{r}5,607.7 \\
54.3 \\
7.0 \\
984.5 \\
369.3 \\
4.8 \\
1,248.6 \\
168.2 \\
1,847.2 \\
228.6 \\
695.2\end{array}$ & $\begin{array}{r}5,921.2 \\
47.5 \\
5.0 \\
1,162.3 \\
415.1 \\
2.0 \\
1,138.3 \\
154.9 \\
1,804.3 \\
377.3 \\
814.4\end{array}$ & $\begin{array}{r}6,036.3 \\
49.9 \\
4.6 \\
1,270.6 \\
435.5 \\
7.8 \\
1,105.1 \\
171.9 \\
1,862.1 \\
416.0 \\
712.8\end{array}$ & $\begin{array}{r}6,130.2 \\
44.4 \\
4.8 \\
1,319.7 \\
518.2 \\
5.6 \\
874.0 \\
160.4 \\
1,944.6 \\
406.6 \\
851.9\end{array}$ & $\begin{array}{r}7,787.4 \\
32.3 \\
5.1 \\
1,739.2 \\
845.1 \\
3.5 \\
1,318.8 \\
166.4 \\
2,174.1 \\
302.9 \\
1,200.0\end{array}$ & $\begin{array}{r}7,594.5 \\
30.6 \\
4.1 \\
1,458.1 \\
852.2 \\
3.4 \\
1,392.3 \\
171.3 \\
2,148.7 \\
457.3 \\
1,076.5\end{array}$ \\
\hline 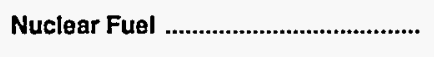 & - & - & - & 22.9 & 119.4 & 128.5 & 135.7 & 119.8 & 133.6 & 125.4 \\
\hline Biomass Fuols at Utilities ..................... & - & - & - & - & - & - & - & - & - & - \\
\hline Primary Energy - Five Sectors ${ }^{b}$.... & $1,341.4$ & $3,741.6$ & $10,595.9$ & $10,276.5$ & $8,285.4$ & $8,431.7$ & $8,708.2$ & $9,025.8$ & $10,738.7$ & $10,282.8$ \\
\hline $\begin{array}{l}\text { Electric Utility Fuelc } \\
\text { Electricity Purchased by End Use............ }\end{array}$ & $\begin{array}{r}-72.9 \\
435.9\end{array}$ & $\begin{array}{r}-303.4 \\
710.5\end{array}$ & $\begin{array}{r}-1,079.1 \\
1,899.6\end{array}$ & $\begin{array}{r}-1,168.2 \\
3,664.5\end{array}$ & $\begin{array}{r}-942.4 \\
3,408.9\end{array}$ & $\begin{array}{r}-811.9 \\
3,379.5\end{array}$ & $\begin{array}{r}-886.9 \\
3,430.5\end{array}$ & $\begin{array}{r}-881.3 \\
3,604.0\end{array}$ & $\begin{array}{r}-931.6 \\
3,739.3\end{array}$ & $\begin{array}{r}-869.3 \\
3,754.6\end{array}$ \\
\hline Total Energy & $1,704.4$ & $4,148.6$ & $11,416.4$ & $12,772.8$ & $10,751.9$ & $10,999.3$ & $11,251.8$ & $11,748.4$ & $13,546.3$ & $13,168.1$ \\
\hline
\end{tabular}

a Includes petroleum coke used at electric utilities.

b Biomass fuels are not included, except those consumed at electric utilities and those added to motor gasoline.

c There are no direct fuel costs for hydroelectric, geothermal, centralized solar, or wind energy.

-No consumption, including cases where adjustments were made. See "Consumption Adjustments for Process Fuel and Intermediate Products."

Note: Expenditure totals may not equal sum of components due to independent rounding.

Sources: Data sources, estimation procedures, and assumptions are described in the "Documentation" section of this report. 
Energy Price Estimates by Sector, Louisiana 1970, 1975, 1980, and 1985-1991

(Dollars per Million Btu)

\begin{tabular}{|c|c|c|c|c|c|c|c|c|c|c|}
\hline Sector and Energy Source & 1970 & 1975 & 1980 & 1985 & 1986 & 1987 & 1988 & 1989 & 1990 & 1991 \\
\hline 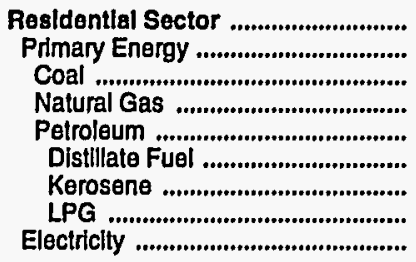 & $\begin{array}{r}2.29 \\
.90 \\
-75 \\
.75 \\
.19 \\
.96 \\
1.60 \\
2.20 \\
6.58\end{array}$ & $\begin{array}{r}3.31 \\
1.55 \\
- \\
1.33 \\
4.36 \\
2.24 \\
3.40 \\
4.39 \\
7.96\end{array}$ & $\begin{array}{r}7.84 \\
3.56 \\
2.97 \\
3.28 \\
8.52 \\
6.65 \\
- \\
8.54 \\
13.81\end{array}$ & $\begin{array}{r}13.04 \\
5.58 \\
-\overline{5} \\
7.47 \\
7.54 \\
3.24 \\
4.77 \\
7.67 \\
20.27\end{array}$ & $\begin{array}{r}13.52 \\
5.76 \\
2.89 \\
5.56 \\
8.90 \\
5.05 \\
7.44 \\
8.99 \\
20.75\end{array}$ & $\begin{array}{r}13.34 \\
5.56 \\
-\overline{5.35} \\
9.10 \\
5.00 \\
7.37 \\
9.13 \\
21.05\end{array}$ & $\begin{array}{r}13.47 \\
5.72 \\
2.62 \\
5.51 \\
9.28 \\
6.69 \\
9.85 \\
9.28 \\
20.89\end{array}$ & $\begin{array}{r}14.10 \\
5.98 \\
- \\
5.72 \\
10.27 \\
5.62 \\
8.28 \\
10.44 \\
21.50\end{array}$ & $\begin{array}{r}14.77 \\
6.12 \\
-\overline{5.85} \\
11.29 \\
6.46 \\
9.50 \\
11.43 \\
21.70\end{array}$ & $\begin{array}{r}14.56 \\
5.87 \\
2.81 \\
5.51 \\
12.75 \\
5.94 \\
8.75 \\
12.88 \\
21.68\end{array}$ \\
\hline 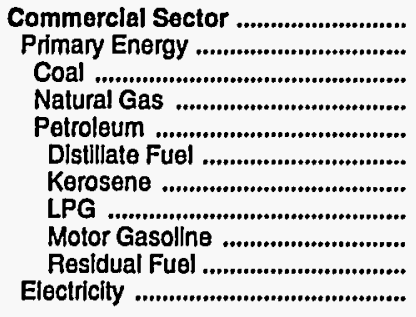 & $\begin{array}{r}1.62 \\
.48 \\
- \\
.37 \\
1.04 \\
.89 \\
.59 \\
1.06 \\
2.86 \\
.49 \\
5.07\end{array}$ & $\begin{array}{r}2.89 \\
1.25 \\
- \\
.77 \\
2.19 \\
2.14 \\
2.01 \\
2.44 \\
4.49 \\
1.76 \\
6.99\end{array}$ & $\begin{array}{r}5.54 \\
3.40 \\
1.24 \\
2.60 \\
3.77 \\
6.36 \\
5.53 \\
5.22 \\
9.89 \\
3.55 \\
12.08\end{array}$ & $\begin{array}{r}12.08 \\
4.28 \\
- \\
5.09 \\
3.37 \\
2.85 \\
3.45 \\
5.08 \\
9.36 \\
4.12 \\
20.24\end{array}$ & $\begin{array}{r}11.78 \\
4.25 \\
1.83 \\
5.05 \\
3.39 \\
3.20 \\
3.49 \\
5.62 \\
7.04 \\
2.14 \\
19.24\end{array}$ & $\begin{array}{r}12.77 \\
4.58 \\
- \\
4.78 \\
4.17 \\
3.95 \\
4.54 \\
5.79 \\
7.14 \\
2.67 \\
19.25\end{array}$ & $\begin{array}{r}13.42 \\
4.87 \\
1.85 \\
4.94 \\
4.70 \\
4.66 \\
5.11 \\
5.70 \\
7.25 \\
1.91 \\
19.56\end{array}$ & $\begin{array}{r}14.02 \\
4.86 \\
-\overline{8} \\
4.98 \\
4.45 \\
4.19 \\
5.32 \\
5.36 \\
7.90 \\
2.29 \\
19.99\end{array}$ & $\begin{array}{r}14.77 \\
5.41 \\
- \\
5.04 \\
6.48 \\
5.74 \\
7.24 \\
7.76 \\
9.47 \\
2.62 \\
20.57\end{array}$ & $\begin{array}{r}14.46 \\
4.96 \\
1.73 \\
4.68 \\
5.91 \\
5.34 \\
6.61 \\
7.75 \\
9.51 \\
2.03 \\
20.30\end{array}$ \\
\hline 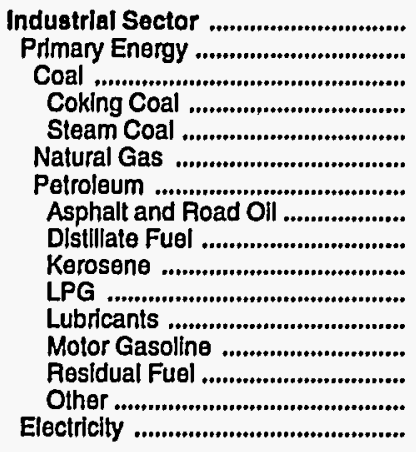 & $\begin{array}{r}.48 \\
.42 \\
- \\
- \\
-23 \\
.97 \\
.97 \\
.66 \\
.51 \\
.59 \\
1.06 \\
5.08 \\
2.86 \\
.49 \\
.70 \\
2.49\end{array}$ & $\begin{array}{r}1.40 \\
1.32 \\
- \\
- \\
.74 \\
2.41 \\
1.78 \\
1.81 \\
2.01 \\
2.44 \\
7.48 \\
4.49 \\
1.72 \\
2.40 \\
3.99\end{array}$ & $\begin{array}{r}3.30 \\
3.07 \\
1.24 \\
-\overline{1.24} \\
1.24 \\
5.68 \\
3.62 \\
4.89 \\
5.53 \\
5.22 \\
14.36 \\
9.89 \\
3.68 \\
6.28 \\
9.02\end{array}$ & $\begin{array}{r}4.64 \\
3.96 \\
1.46 \\
- \\
1.46 \\
2.92 \\
5.33 \\
4.17 \\
2.96 \\
3.45 \\
5.08 \\
17.61 \\
9.36 \\
4.12 \\
6.57 \\
14.93\end{array}$ & $\begin{array}{r}3.56 \\
3.02 \\
1.83 \\
-\overline{1} \\
1.83 \\
1.84 \\
4.65 \\
4.57 \\
3.00 \\
3.49 \\
5.62 \\
15.59 \\
7.04 \\
2.14 \\
3.81 \\
12.63\end{array}$ & $\begin{array}{r}3.59 \\
3.07 \\
2.03 \\
- \\
2.03 \\
1.73 \\
5.02 \\
3.15 \\
3.90 \\
4.54 \\
5.79 \\
12.70 \\
7.14 \\
2.67 \\
4.63 \\
12.30\end{array}$ & $\begin{array}{r}3.64 \\
3.10 \\
1.85 \\
- \\
1.85 \\
1.91 \\
4.75 \\
3.14 \\
4.39 \\
5.11 \\
5.70 \\
14.61 \\
7.25 \\
1.91 \\
3.86 \\
12.25\end{array}$ & $\begin{array}{r}3.52 \\
2.96 \\
1.55 \\
1.55 \\
1.59 \\
4.98 \\
2.89 \\
4.57 \\
5.32 \\
5.36 \\
13.30 \\
7.90 \\
2.29 \\
4.71 \\
12.46\end{array}$ & $\begin{array}{r}4.14 \\
3.64 \\
1.56 \\
- \\
1.56 \\
1.92 \\
6.61 \\
2.91 \\
6.22 \\
7.24 \\
7.76 \\
13.40 \\
9.47 \\
2.62 \\
5.85 \\
12.27\end{array}$ & $\begin{array}{r}3.86 \\
3.34 \\
1.73 \\
- \\
1.73 \\
1.66 \\
6.21 \\
3.08 \\
5.68 \\
6.61 \\
7.75 \\
15.42 \\
9.51 \\
2.03 \\
5.03 \\
12.15\end{array}$ \\
\hline 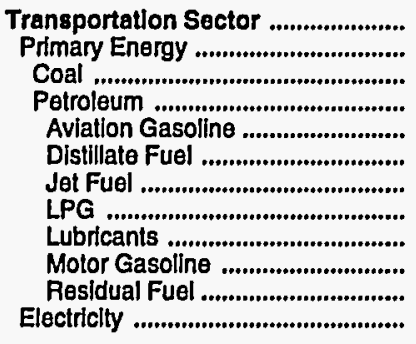 & $\begin{array}{r}1.97 \\
1.97 \\
-\overline{1} \\
1.97 \\
2.17 \\
1.09 \\
.72 \\
1.06 \\
5.08 \\
2.86 \\
.44 \\
5.07\end{array}$ & $\begin{array}{r}3.28 \\
3.28 \\
-\overline{3} \\
3.28 \\
3.45 \\
2.54 \\
2.01 \\
2.44 \\
7.48 \\
4.49 \\
1.54 \\
6.99\end{array}$ & $\begin{array}{r}6.87 \\
6.87 \\
-\overline{6} \\
6.87 \\
9.02 \\
6.84 \\
6.34 \\
5.22 \\
14.36 \\
9.89 \\
3.05 \\
12.08\end{array}$ & $\begin{array}{r}7.16 \\
7.16 \\
- \\
7.16 \\
9.99 \\
6.38 \\
5.70 \\
5.08 \\
17.61 \\
9.36 \\
3.40 \\
20.24\end{array}$ & $\begin{array}{r}5.06 \\
5.06 \\
-\overline{5} \\
5.06 \\
8.41 \\
6.35 \\
3.67 \\
5.62 \\
15.59 \\
7.04 \\
1.34 \\
19.24\end{array}$ & $\begin{array}{r}5.50 \\
5.50 \\
- \\
5.50 \\
7.55 \\
6.56 \\
3.90 \\
5.79 \\
12.70 \\
7.14 \\
2.54 \\
17.55\end{array}$ & $\begin{array}{r}5.43 \\
5.43 \\
- \\
5.43 \\
7.41 \\
6.40 \\
3.61 \\
5.70 \\
14.61 \\
7.25 \\
2.61 \\
18.24\end{array}$ & $\begin{array}{r}5.80 \\
5.80 \\
- \\
5.80 \\
8.28 \\
6.97 \\
4.12 \\
5.36 \\
13.30 \\
7.90 \\
2.53 \\
17.80\end{array}$ & $\begin{array}{r}6.91 \\
6.91 \\
- \\
6.91 \\
9.32 \\
8.48 \\
5.79 \\
7.76 \\
13.40 \\
9.47 \\
2.07 \\
20.18\end{array}$ & $\begin{array}{r}6.52 \\
6.52 \\
- \\
6.52 \\
8.71 \\
8.21 \\
4.67 \\
7.75 \\
15.42 \\
9.51 \\
2.87 \\
18.68\end{array}$ \\
\hline $\begin{array}{l}\text { Total Energy ....................................... } \\
\text { Primary Energy - Four Sectors ..... } \\
\text { Electricity .......................................... }\end{array}$ & $\begin{array}{r}.94 \\
.74 \\
4.69\end{array}$ & $\begin{array}{l}2.03 \\
1.78 \\
6.24\end{array}$ & $\begin{array}{r}4.50 \\
4.02 \\
11.49\end{array}$ & $\begin{array}{r}6.33 \\
5.01 \\
18.25\end{array}$ & $\begin{array}{r}5.06 \\
3.81 \\
17.40\end{array}$ & $\begin{array}{r}5.21 \\
3.98 \\
17.33\end{array}$ & $\begin{array}{r}5.24 \\
4.02 \\
17.30\end{array}$ & $\begin{array}{r}5.28 \\
4.03 \\
17.64\end{array}$ & $\begin{array}{r}6.00 \\
4.79 \\
17.77\end{array}$ & $\begin{array}{r}5.69 \\
4.48 \\
17.59\end{array}$ \\
\hline 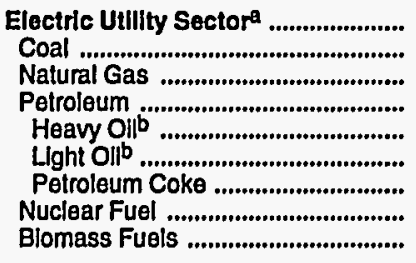 & $\begin{array}{l}.21 \\
. \\
.21 \\
.57 \\
.55 \\
.60 \\
- \\
- \\
-\end{array}$ & $\begin{array}{r}.73 \\
.64 \\
1.76 \\
1.76 \\
1.92 \\
= \\
- \\
-\end{array}$ & $\begin{array}{r}2.19 \\
2.01 \\
3.68 \\
3.58 \\
4.37 \\
- \\
-\end{array}$ & $\begin{array}{r}2.46 \\
2.19 \\
2.73 \\
5.08 \\
3.51 \\
5.84 \\
- \\
.86 \\
-\end{array}$ & $\begin{array}{r}1.67 \\
1.79 \\
1.85 \\
1.63 \\
1.45 \\
3.20 \\
-\overline{0} \\
-\end{array}$ & $\begin{array}{r}1.46 \\
1.64 \\
1.60 \\
3.24 \\
2.72 \\
3.63 \\
- \\
.97 \\
-\end{array}$ & $\begin{array}{r}1.44 \\
1.55 \\
1.63 \\
3.35 \\
2.98 \\
4.42 \\
- \\
.92 \\
-\end{array}$ & $\begin{array}{r}1.51 \\
1.63 \\
1.70 \\
4.04 \\
2.85 \\
5.01 \\
- \\
.90 \\
-\end{array}$ & $\begin{array}{r}1.49 \\
1.70 \\
1.66 \\
4.15 \\
2.46 \\
5.01 \\
-88 \\
-\end{array}$ & $\begin{array}{r}1.40 \\
1.65 \\
1.53 \\
4.32 \\
3.30 \\
4.56 \\
-\overline{8} \\
-\end{array}$ \\
\hline Primary Energy - Flve Sectorsc .. & .65 & 1.60 & 3.70 & 4.48 & 3.32 & 3.41 & 3.40 & 3.46 & 4.02 & 3.78 \\
\hline
\end{tabular}

There are no direct fuel costs for hydroelectric, geothermal, centralized solar, or wind energy.

b Heavy oll Includes Grade Nos. 4, 5, and 6, and residual fuel oils. Light oil includes Grade No. 2 heating oil, kerosene, and jet fuel.

C Blomass fuels are not included, except those consumed at electric utilities and those added to motor gasoline.

-No consumption, Including cases where adjustments were made. See "Consumption Adjustments for Process Fuel and Intermediate Products."

Sources: Data sources, estimation procedures, and assumptions are described in the "Documentation" section of this report. 
L Energy Expenditure Estimates by Sector, Louisiana

O $1970,1975,1980$, and 1985-1991

(Million Dollars)

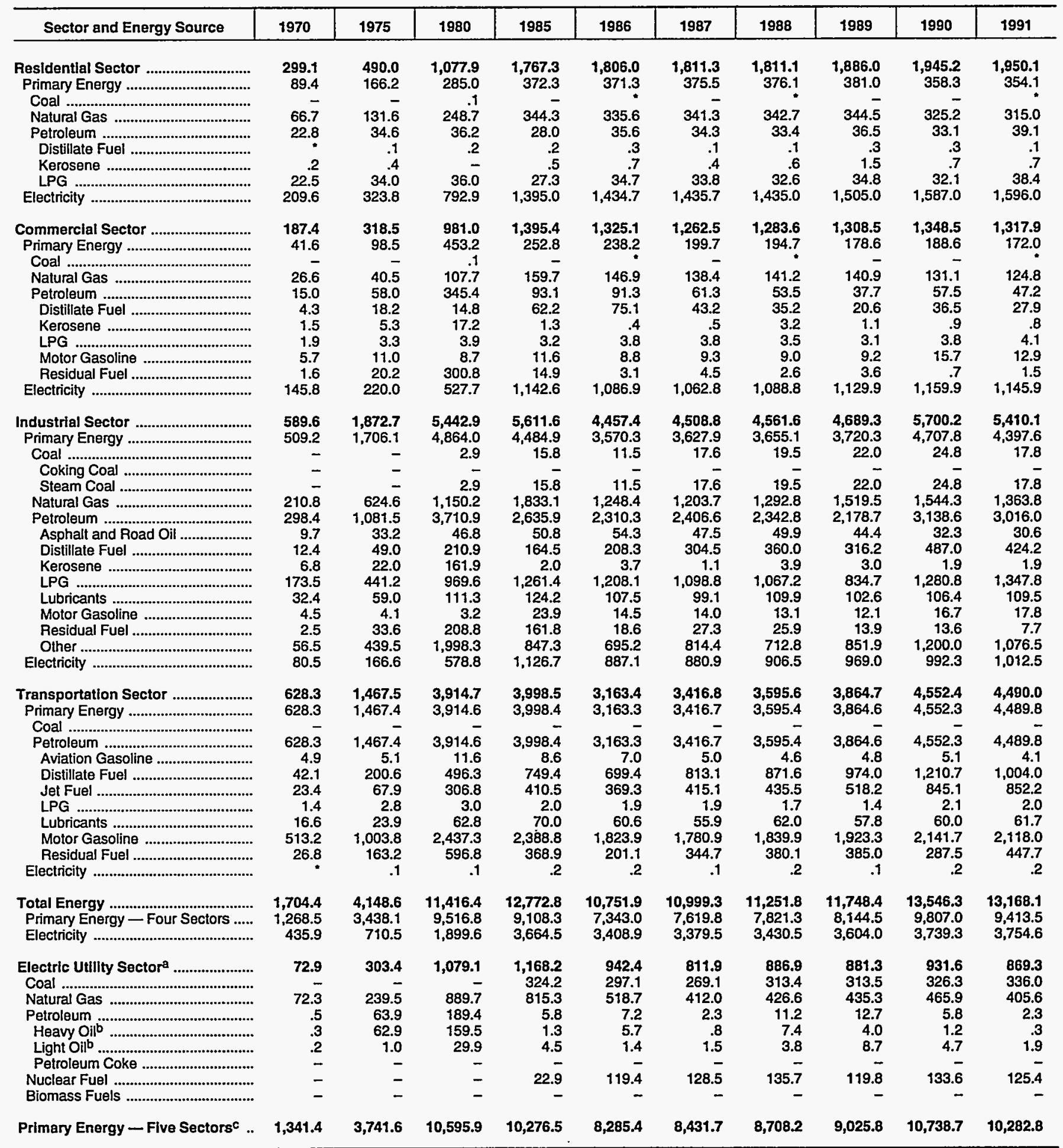

a There are no direct fuel costs for hydroelectric, geothermal, centralized solar, or wind energy.

b Heavy oil includes Grade Nos. 4,5, and 6, and residual fuel oils. Light oil includes Grade No. 2 heating oil, kerosene, and jet fuel.

c Biomass fuels are not included, except those consumed at electric utilities and those added to motor gasoline.

- No consumption, including cases where adjustments were made. See "Consumption Adjustments for Process Fuel and Intermediate Products."

- Value less than 0.05 million dollars.

Note: Totals may not equal sum of components due to independent rounding.

Sources: Data sources, estimation procedures, and assumptions are described in the "Documentation" section of this report. 


\begin{tabular}{|c|c|c|c|c|c|c|c|c|c|c|}
\hline Energy Sourco & 1970 & 1975 & 1980 & 1985 & 1986 & 1987 & 1988 & 1989 & 1990 & 1991 \\
\hline & \multicolumn{10}{|c|}{ Prices in Dollars per Million Btu } \\
\hline 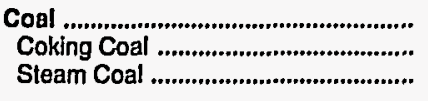 & $\frac{1.06}{1.06}$ & $2 . \overline{60}$ & $\begin{array}{r}1.89 \\
1.89\end{array}$ & $\begin{array}{r}2.58 \\
2.58\end{array}$ & $2 . \overline{-27}$ & $2 . \overline{48}$ & $2 . \overrightarrow{-63}$ & 2.66 & $\begin{array}{r}2.72 \\
2 . \overline{-}\end{array}$ & $\begin{array}{r}2.67 \\
2.67\end{array}$ \\
\hline 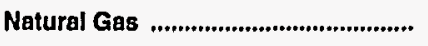 & 1.48 & 2.03 & 5.03 & 7.41 & 7.06 & 6.45 & 5.64 & $\mathbf{5 . 7 8}$ & 6.05 & 5.50 \\
\hline 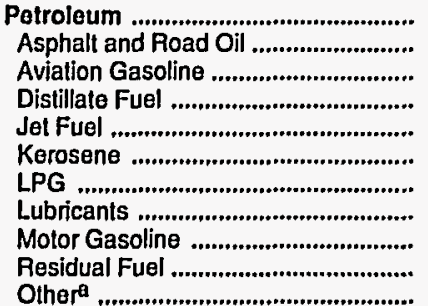 & $\begin{array}{r}1.46 \\
.73 \\
2.17 \\
1.37 \\
.75 \\
1.53 \\
2.37 \\
5.08 \\
3.02 \\
.38 \\
.89\end{array}$ & $\begin{array}{l}3.04 \\
1.99 \\
3.45 \\
2.78 \\
2.09 \\
3.10 \\
4.20 \\
7.48 \\
4.56 \\
1.79 \\
-\end{array}$ & $\begin{array}{r}7.00 \\
3.73 \\
9.02 \\
6.83 \\
6.51 \\
7.82 \\
7.86 \\
14.36 \\
9.69 \\
4.10 \\
-\end{array}$ & $\begin{array}{r}7.38 \\
5.21 \\
9.99 \\
8.00 \\
6.10 \\
8.78 \\
10.89 \\
17.61 \\
9.35 \\
4.37 \\
-\end{array}$ & $\begin{array}{r}5.28 \\
4.88 \\
8.41 \\
6.06 \\
4.50 \\
6.60 \\
10.53 \\
15.59 \\
7.38 \\
2.44 \\
\end{array}$ & $\begin{array}{r}5.85 \\
3.55 \\
7.55 \\
6.21 \\
4.41 \\
6.58 \\
10.66 \\
12.70 \\
7.72 \\
2.93 \\
-\end{array}$ & $\begin{array}{r}5.65 \\
3.35 \\
7.41 \\
6.23 \\
4.13 \\
6.61 \\
10.59 \\
14.61 \\
8.15 \\
2.33 \\
-\end{array}$ & $\begin{array}{r}6.17 \\
3.23 \\
8.28 \\
6.98 \\
4.79 \\
7.77 \\
10.31 \\
13.30 \\
8.69 \\
2.80 \\
\end{array}$ & $\begin{array}{r}6.99 \\
3.34 \\
9.32 \\
7.87 \\
5.92 \\
8.62 \\
13.04 \\
13.40 \\
9.74 \\
2.86 \\
\end{array}$ & $\begin{array}{r}6.71 \\
3.05 \\
8.71 \\
7.56 \\
5.07 \\
8.29 \\
14.45 \\
15.42 \\
9.64 \\
2.33 \\
-\end{array}$ \\
\hline 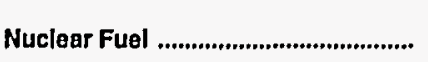 & - & .32 & .58 & .62 & .58 & .60 & .64 & .54 & .46 & .43 \\
\hline Blomass Fuels at Utilltles .................. & - & - & - & - & - & - & - & - & - & - \\
\hline Primary Energy - Five Sectors ${ }^{b}$.... & 1.45 & 2.54 & $\mathbf{5 . 7 0}$ & 5.84 & 4.21 & 4.96 & 4.78 & 4.85 & 5.77 & 5.21 \\
\hline $\begin{array}{l}\text { Electric Utility Fuelc .......................... } \\
\text { Electricity Purchased by End Users }\end{array}$ & $\begin{array}{r}.35 \\
5.92\end{array}$ & $\begin{array}{r}.71 \\
9.70\end{array}$ & $\begin{array}{r}1.82 \\
16.30\end{array}$ & $\begin{array}{r}1.60 \\
20.16\end{array}$ & $\begin{array}{r}1.11 \\
19.88\end{array}$ & $\begin{array}{r}1.50 \\
19.10\end{array}$ & $\begin{array}{r}1.27 \\
19.62\end{array}$ & $\begin{array}{r}1.21 \\
20.53\end{array}$ & $\begin{array}{r}1.17 \\
22.42\end{array}$ & $\begin{array}{r}.73 \\
25.21\end{array}$ \\
\hline \multirow[t]{2}{*}{ Total Energy ........................................... } & 1.95 & 3.77 & 8.44 & 9.49 & 7.45 & 8.05 & 7.77 & 8.55 & 9.49 & 9.48 \\
\hline & \multicolumn{10}{|c|}{ Expenditures in Millions of Dollars } \\
\hline 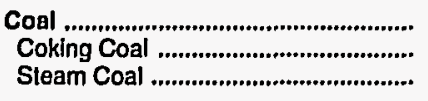 & $2 . \overline{3}$ & $\begin{array}{r}3.4 \\
-\overline{4}\end{array}$ & $5 . \overline{7}$ & $\begin{array}{r}13.2 \\
13.2\end{array}$ & $\begin{array}{r}21.1 \\
-\overline{1}\end{array}$ & $\begin{array}{r}17.0 \\
-\overline{0}\end{array}$ & $\begin{array}{r}18.2 \\
18.2\end{array}$ & $\begin{array}{r}18.0 \\
18.0\end{array}$ & $\begin{array}{r}18.0 \\
18.0\end{array}$ & $\begin{array}{r}25.0 \\
25.0\end{array}$ \\
\hline Natural Gas ........................................... & 1.9 & 4.0 & 11.2 & 19.3 & 17.9 & 17.6 & 18.5 & 21.3 & 26.4 & 26.5 \\
\hline 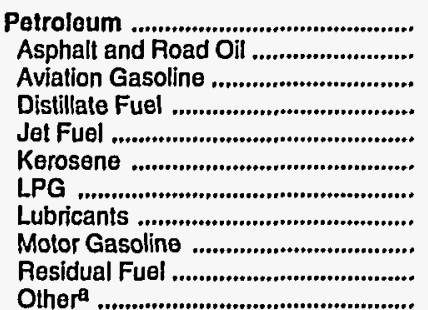 & $\begin{array}{r}337.1 \\
3.4 \\
1.0 \\
94.2 \\
9.4 \\
15.5 \\
5.7 \\
5.2 \\
174.9 \\
27.5 \\
.4\end{array}$ & $\begin{array}{r}675.1 \\
9.2 \\
1.2 \\
186.5 \\
22.7 \\
18.2 \\
15.1 \\
7.6 \\
303.1 \\
111.7 \\
\end{array}$ & $\begin{array}{r}1,388.1 \\
10.8 \\
3.7 \\
422.8 \\
66.7 \\
22.4 \\
25.2 \\
17.1 \\
598.7 \\
220.7 \\
-\end{array}$ & $\begin{array}{r}1,509.0 \\
75.6 \\
2.1 \\
446.4 \\
54.4 \\
51.8 \\
26.5 \\
19.1 \\
615.9 \\
217.2 \\
-\end{array}$ & $\begin{array}{r}1,270.4 \\
23.8 \\
2.4 \\
405.7 \\
39.6 \\
25.0 \\
39.8 \\
16.5 \\
520.9 \\
196.6 \\
-\end{array}$ & $\begin{array}{r}1,331.6 \\
20.1 \\
2.0 \\
432.6 \\
43.7 \\
26.5 \\
50.8 \\
15.2 \\
570.3 \\
170.3 \\
-\end{array}$ & $\begin{array}{r}1,536.0 \\
35.2 \\
2.5 \\
497.8 \\
47.7 \\
37.5 \\
62.2 \\
16.9 \\
658.8 \\
177.4 \\
-\end{array}$ & $\begin{array}{r}1,556.6 \\
21.5 \\
2.9 \\
498.7 \\
59.5 \\
41.7 \\
59.6 \\
15.8 \\
647.4 \\
209.6 \\
-\end{array}$ & $\begin{array}{r}1,675.5 \\
14.3 \\
2.9 \\
549.8 \\
82.9 \\
32.1 \\
65.7 \\
16.3 \\
718.6 \\
192.7 \\
-\end{array}$ & $\begin{array}{r}1,537.9 \\
20.0 \\
1.9 \\
456.3 \\
67.1 \\
34.9 \\
77.0 \\
16.8 \\
714.7 \\
149.1 \\
-\end{array}$ \\
\hline (4) & & & & & & & & & & \\
\hline Nuclear Fuel ............................................ & - & 16.1 & 27.9 & 35.7 & 38.9 & 25.9 & 34.2 & 40.0 & 24.1 & 29.0 \\
\hline Blomass Fuels at UtIlities ................. & - & - & - & - & - & - & - & - & - & - \\
\hline Primary Energy - Five Sectors ${ }^{b}$.... & 341.3 & 698.6 & $1,432.8$ & $1,577.2$ & $1,348.3$ & $1,392.1$ & $1,606.9$ & $1,635.9$ & $1,744.0$ & $1,618.4$ \\
\hline $\begin{array}{l}\text { Electric Utility Fuelc .......................... } \\
\text { Electricity Purchased by End Users }\end{array}$ & $\begin{array}{r}-10.6 \\
102.3\end{array}$ & $\begin{array}{l}-48.1 \\
216.1\end{array}$ & $\begin{array}{r}-129.8 \\
455.3\end{array}$ & $\begin{array}{r}-127.6 \\
675.7\end{array}$ & $\begin{array}{r}-105.0 \\
692.1\end{array}$ & $\begin{array}{r}-106.3 \\
698.4\end{array}$ & $\begin{array}{r}-107.3 \\
754.0\end{array}$ & $\begin{array}{r}-128.9 \\
801.0\end{array}$ & $\begin{array}{r}-86.5 \\
882.0\end{array}$ & $\begin{array}{l}-59.3 \\
979.1\end{array}$ \\
\hline 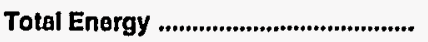 & 433.1 & 866.7 & $1,758.3$ & $2,125.3$ & $1,935.4$ & $1,984.2$ & $2,253.6$ & $2,308.0$ & $2,539.5$ & $2,538.1$ \\
\hline
\end{tabular}

a Includes petroleum coke used at electric utilities.

b Biomass fuels are not included, except those consumed at electric utilities and those added to molor gasoline.

c There are no direct fuel costs for hydroelectric, geothermal, centralized solar, or wind energy.

- No consumplion, including cases where adjustments were made. See "Consumption Adjustments for Process Fuel and Intermediate Products."

Nole: Expenditure totals may not equal sum of components due to independent rounding.

Sources: Data sources, estimation procedures, and assumptions are described in the "Documentation" section of this report. 


\section{Energy Price Estimates by Sector, Maine \\ A 1970, 1975, 1980, and 1985-1991}

I (Dollars per Million Btu)

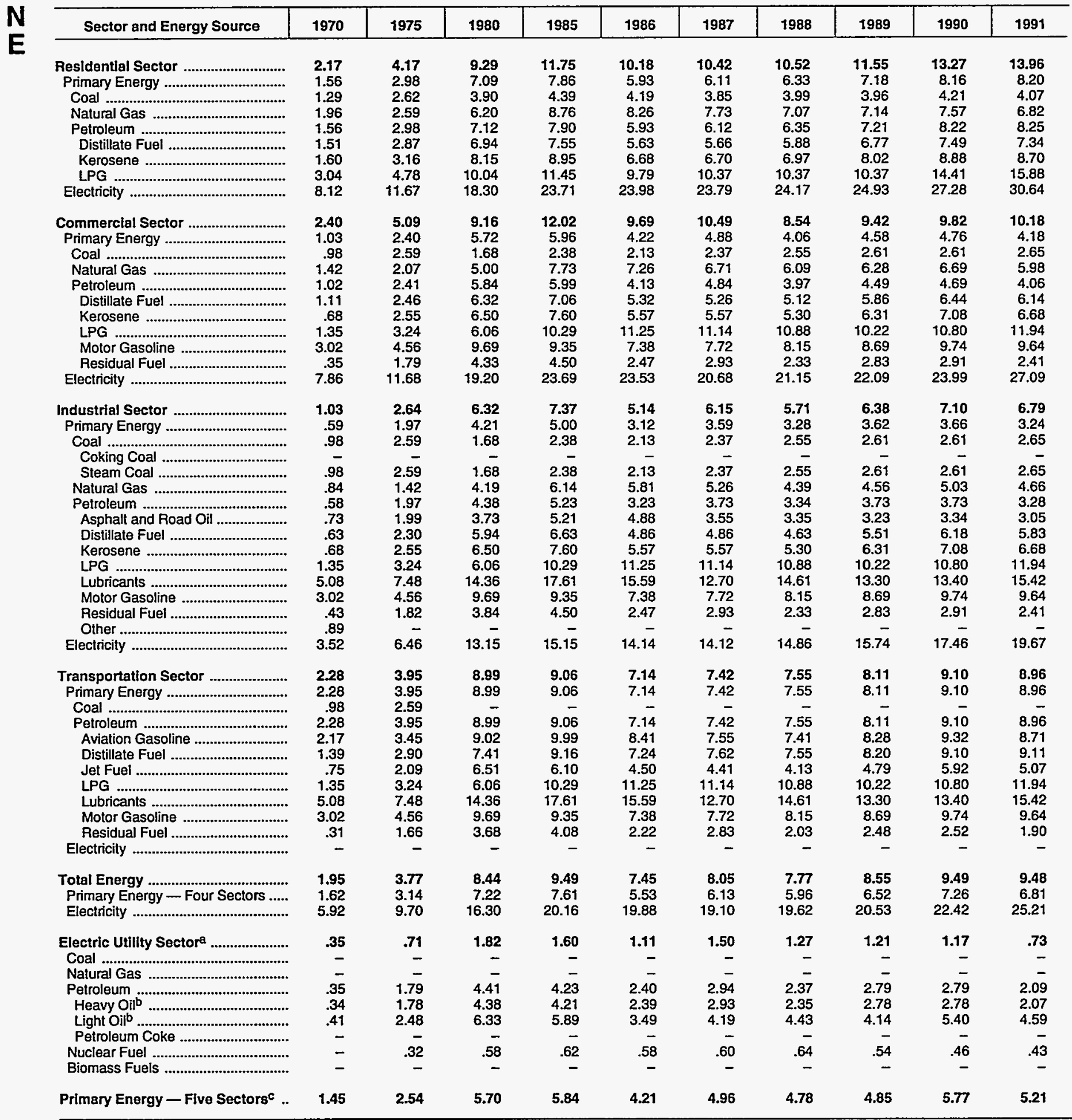

There are no direct fuel costs for hydroelectric, geothermal, centralized solar, or wind energy.

b Heavy oil includes Grade Nos. 4, 5, and 6, and residual fuel oils. Light oil includes Grade No. 2 heating oil, kerosene, and jet fuel.

c Biomass fuels are not included, except those consumed at electric utilities and those added to motor gasoline.

- No consumption, including cases where adjustments were made. See "Consumption Adjustments for Process Fuel and Intermediate Products."

Sources: Data sources, estimation procedures, and assumptions are described in the "Documentation" section of this report. 
Energy Expenditure Estimates by Sector, Maine

1970, 1975, 1980, and 1985-1991

(Million Dollars)

\begin{tabular}{|c|c|c|c|c|c|c|c|c|c|c|}
\hline Sector and Energy Source & 1970 & 1975 & 1980 & 1985 & 1986 & 1987 & 1988 & 1989 & 1990 & 1991 \\
\hline 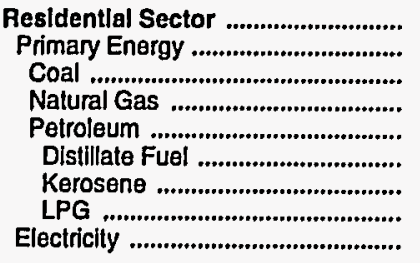 & $\begin{array}{r}137.9 \\
90.2 \\
.7 \\
1.0 \\
88.4 \\
69.1 \\
14.9 \\
4.4 \\
47.7\end{array}$ & $\begin{array}{r}257.0 \\
158.0 \\
.8 \\
1.9 \\
155.3 \\
127.9 \\
16.7 \\
10.7 \\
99.0\end{array}$ & $\begin{array}{r}482.8 \\
295.6 \\
1.1 \\
3.5 \\
291.0 \\
257.7 \\
18.7 \\
14.6 \\
187.2\end{array}$ & $\begin{array}{r}558.7 \\
282.1 \\
2.2 \\
4.8 \\
275.1 \\
214.6 \\
46.2 \\
14.4 \\
276.6\end{array}$ & $\begin{array}{r}528.2 \\
235.5 \\
2.6 \\
4.6 \\
228.3 \\
186.4 \\
23.6 \\
18.2 \\
292.7\end{array}$ & $\begin{array}{r}543.1 \\
240.7 \\
2.0 \\
4.2 \\
234.5 \\
179.9 \\
24.0 \\
30.5 \\
302.4\end{array}$ & $\begin{array}{r}597.5 \\
275.5 \\
1.6 \\
4.1 \\
269.8 \\
204.6 \\
31.0 \\
34.3 \\
322.0\end{array}$ & $\begin{array}{r}642.3 \\
301.3 \\
1.1 \\
4.6 \\
295.6 \\
223.9 \\
36.6 \\
35.2 \\
341.0\end{array}$ & $\begin{array}{r}666.0 \\
300.0 \\
1.9 \\
4.9 \\
293.2 \\
219.8 \\
28.3 \\
45.1 \\
366.0\end{array}$ & $\begin{array}{r}708.3 \\
309.3 \\
.8 \\
5.0 \\
303.5 \\
220.4 \\
29.2 \\
53.9 \\
399.0\end{array}$ \\
\hline 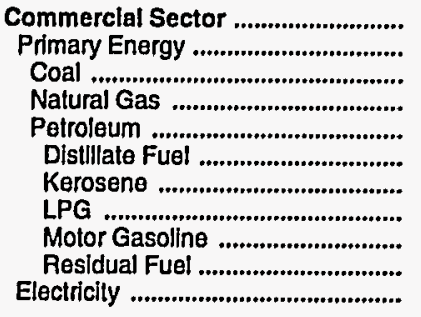 & $\begin{array}{r}39.9 \\
13.7 \\
.4 \\
.6 \\
12.7 \\
10.8 \\
.3 \\
.3 \\
.6 \\
.6 \\
26.1\end{array}$ & $\begin{array}{r}93.9 \\
31.5 \\
.7 \\
1.1 \\
29.7 \\
23.1 \\
.6 \\
1.3 \\
1.0 \\
3.7 \\
62.5\end{array}$ & $\begin{array}{r}210.3 \\
97.8 \\
.5 \\
4.4 \\
92.9 \\
67.7 \\
2.6 \\
1.6 \\
2.5 \\
18.6 \\
112.5\end{array}$ & $\begin{array}{r}280.6 \\
91.6 \\
1.6 \\
9.1 \\
80.9 \\
39.9 \\
4.2 \\
2.3 \\
5.1 \\
29.4 \\
189.0\end{array}$ & $\begin{array}{r}290.7 \\
90.7 \\
2.0 \\
9.1 \\
79.6 \\
48.4 \\
.8 \\
3.7 \\
4.1 \\
22.7 \\
199.9\end{array}$ & $\begin{array}{r}266.1 \\
79.8 \\
1.7 \\
8.7 \\
69.3 \\
45.5 \\
1.3 \\
5.8 \\
3.8 \\
13.0 \\
186.4\end{array}$ & $\begin{array}{r}305.0 \\
107.0 \\
1.4 \\
9.1 \\
96.4 \\
53.3 \\
4.8 \\
6.3 \\
4.5 \\
27.5 \\
198.0\end{array}$ & $\begin{array}{r}328.6 \\
115.6 \\
1.0 \\
10.5 \\
104.2 \\
55.3 \\
3.4 \\
6.1 \\
5.3 \\
34.1 \\
213.0\end{array}$ & $\begin{array}{r}362.7 \\
129.7 \\
1.6 \\
11.3 \\
116.8 \\
63.4 \\
2.7 \\
6.0 \\
5.1 \\
39.6 \\
233.0\end{array}$ & $\begin{array}{r}379.1 \\
115.1 \\
.4 \\
11.2 \\
103.5 \\
51.6 \\
4.7 \\
7.2 \\
2.7 \\
37.3 \\
264.1\end{array}$ \\
\hline 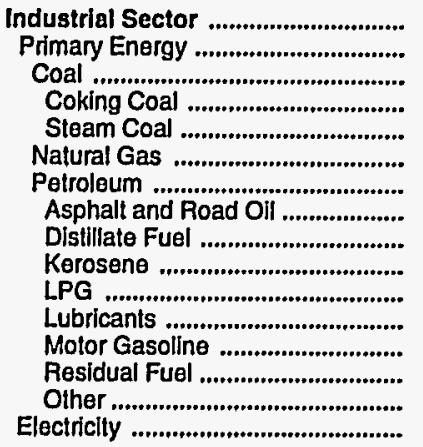 & $\begin{array}{r}55.3 \\
26.9 \\
1.1 \\
- \\
1.1 \\
.3 \\
25.5 \\
3.4 \\
2.9 \\
.2 \\
.9 \\
1.7 \\
2.2 \\
13.8 \\
.4 \\
28.4\end{array}$ & $\begin{array}{r}151.1 \\
96.5 \\
2.0 \\
2 . \overline{2} \\
1.0 \\
93.5 \\
9.2 \\
9.2 \\
.9 \\
3.0 \\
2.7 \\
1.9 \\
66.8 \\
- \\
54.6\end{array}$ & $\begin{array}{r}317.1 \\
161.4 \\
4.1 \\
- \\
4.1 \\
3.2 \\
154.2 \\
10.8 \\
26.4 \\
1.1 \\
8.9 \\
5.6 \\
3.8 \\
97.6 \\
155.7\end{array}$ & $\begin{array}{r}437.5 \\
227.3 \\
9.3 \\
- \\
9.3 \\
5.4 \\
212.6 \\
75.6 \\
17.6 \\
1.4 \\
9.2 \\
6.3 \\
6.1 \\
96.3 \\
- \\
210.2\end{array}$ & $\begin{array}{r}395.2 \\
195.7 \\
16.5 \\
- \\
16.5 \\
4.2 \\
175.0 \\
23.8 \\
15.7 \\
.6 \\
17.0 \\
5.4 \\
5.1 \\
107.4 \\
- \\
199.5\end{array}$ & $\begin{array}{r}376.0 \\
166.4 \\
13.3 \\
- \\
13.3 \\
4.7 \\
148.5 \\
20.1 \\
26.0 \\
1.2 \\
13.9 \\
5.0 \\
5.5 \\
76.8 \\
- \\
209.6\end{array}$ & $\begin{array}{r}429.0 \\
195.0 \\
15.1 \\
15.1 \\
5.2 \\
174.7 \\
35.2 \\
33.3 \\
1.7 \\
20.4 \\
5.5 \\
5.7 \\
72.9 \\
-\overline{0} \\
234.0\end{array}$ & $\begin{array}{r}440.3 \\
193.3 \\
15.9 \\
15 . \overline{-} \\
6.3 \\
171.1 \\
21.5 \\
34.6 \\
1.7 \\
17.2 \\
5.2 \\
6.4 \\
84.6 \\
-\overline{5} \\
247.0\end{array}$ & $\begin{array}{r}461.6 \\
178.6 \\
14.5 \\
- \\
14.5 \\
10.2 \\
153.9 \\
14.3 \\
25.5 \\
1.1 \\
14.0 \\
5.4 \\
4.8 \\
88.9 \\
- \\
283.0\end{array}$ & $\begin{array}{r}504.2 \\
188.2 \\
23.9 \\
-\overline{23.9} \\
10.4 \\
153.9 \\
20.0 \\
26.4 \\
1.0 \\
15.2 \\
5.5 \\
5.1 \\
80.7 \\
316.0\end{array}$ \\
\hline 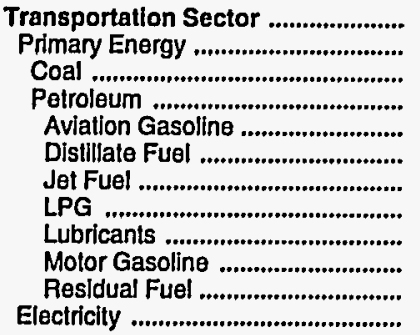 & $\begin{array}{r}199.9 \\
199.9 \\
* \\
199.9 \\
1.0 \\
11.2 \\
9.4 \\
3.5 \\
172.1 \\
2.7 \\
-\end{array}$ & $\begin{array}{r}364.6 \\
364.6 \\
364.6 \\
1.2 \\
25.8 \\
22.7 \\
4.9 \\
300.2 \\
9.8 \\
-\end{array}$ & $\begin{array}{r}748.1 \\
748.1 \\
- \\
748.1 \\
3.7 \\
68.8 \\
66.7 \\
.2 \\
11.5 \\
592.4 \\
4.8 \\
-\end{array}$ & $\begin{array}{r}848.5 \\
848.5 \\
848.5 \\
2.1 \\
273.3 \\
54.4 \\
.6 \\
12.8 \\
604.7 \\
.5 \\
-\end{array}$ & $\begin{array}{r}721.3 \\
721.3 \\
- \\
721.3 \\
2.4 \\
154.4 \\
39.6 \\
.9 \\
11.1 \\
511.8 \\
1.0 \\
-\end{array}$ & $\begin{array}{r}798.9 \\
798.9 \\
- \\
798.9 \\
2.0 \\
180.4 \\
43.7 \\
.6 \\
10.2 \\
561.1 \\
.9 \\
-\end{array}$ & $\begin{array}{r}922.0 \\
922.0 \\
-\overline{9} \\
922.0 \\
2.5 \\
205.3 \\
47.7 \\
1.2 \\
11.3 \\
648.7 \\
5.4 \\
-\end{array}$ & $\begin{array}{r}896.8 \\
896.8 \\
- \\
896.8 \\
2.9 \\
183.8 \\
59.5 \\
1.1 \\
10.6 \\
635.8 \\
3.1 \\
-\end{array}$ & $\begin{array}{r}1,049.2 \\
1,049.2 \\
- \\
1,049.2 \\
2.9 \\
240.6 \\
82.9 \\
.7 \\
11.0 \\
708.7 \\
2.4 \\
-\end{array}$ & $\begin{array}{r}946.6 \\
946.6 \\
-\overrightarrow{6} \\
946.6 \\
1.9 \\
157.3 \\
67.1 \\
.7 \\
11.3 \\
706.9 \\
1.4 \\
-\end{array}$ \\
\hline $\begin{array}{l}\text { Total Energy ...................................... } \\
\text { Primary Energy - Four Sectors ...... } \\
\text { Electricity .......................................... }\end{array}$ & $\begin{array}{l}433.1 \\
330.7 \\
102.3\end{array}$ & $\begin{array}{l}866.7 \\
650.6 \\
216.1\end{array}$ & $\begin{array}{r}1,758.3 \\
1,303.0 \\
455.3\end{array}$ & $\begin{array}{r}2,125.3 \\
1,449.6 \\
675.7\end{array}$ & $\begin{array}{r}1,935.4 \\
1,243.3 \\
692.1\end{array}$ & $\begin{array}{r}1,984.2 \\
1,285.8 \\
698.4\end{array}$ & $\begin{array}{r}2,253.6 \\
1,499.6 \\
754.0\end{array}$ & $\begin{array}{r}2,308.0 \\
1,507.0 \\
801.0\end{array}$ & $\begin{array}{r}2,539.5 \\
1,657.5 \\
882.0\end{array}$ & $\begin{array}{r}2,538.1 \\
1,559.1 \\
979.1\end{array}$ \\
\hline 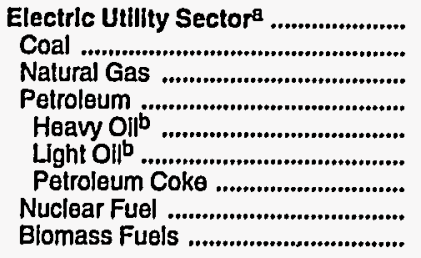 & $\begin{array}{r}10.6 \\
\overline{-} \\
10 . \overline{6} \\
10.3 \\
.2 \\
= \\
-\end{array}$ & $\begin{array}{r}48.1 \\
\overline{-} \\
32.0 \\
31.4 \\
.6 \\
\overline{-} \\
16.1 \\
-\end{array}$ & $\begin{array}{r}129.8 \\
-\overline{-} \\
101 . \overline{9} \\
99 . \overline{7} \\
2 . \overline{2} \\
27 . \overline{9} \\
-\end{array}$ & $\begin{array}{r}127.6 \\
\overline{-} \\
91 . \overline{9} \\
90.9 \\
1.0 \\
35 . \overline{7} \\
-\end{array}$ & $\begin{array}{r}105.0 \\
- \\
- \\
66.1 \\
65.5 \\
.7 \\
\overline{7} \\
38.9 \\
-\end{array}$ & $\begin{array}{r}106.3 \\
- \\
\overline{-} \\
80.4 \\
79.6 \\
.8 \\
\overline{8} \\
25.9 \\
-\end{array}$ & $\begin{array}{r}107.3 \\
- \\
\overline{-} \\
73.0 \\
71.7 \\
1.3 \\
\overline{-} \\
34.2 \\
-\end{array}$ & $\begin{array}{r}128.9 \\
- \\
88.9 \\
87.8 \\
1.1 \\
\overline{-} \\
40.0 \\
-\end{array}$ & $\begin{array}{r}86.5 \\
- \\
-\overline{4} \\
62.4 \\
61.8 \\
.6 \\
- \\
24.1 \\
-\end{array}$ & $\begin{array}{r}59.3 \\
- \\
- \\
30.3 \\
29.8 \\
.6 \\
\overline{-} \\
29.0 \\
-\end{array}$ \\
\hline Primary Energy - Flue Sectors ${ }^{c}$.. & 341.3 & 698.6 & $1,432.8$ & $1,577.2$ & $1,348.3$ & $1,392.1$ & $1,606.9$ & $1,635.9$ & $1,744.0$ & $1,618.4$ \\
\hline
\end{tabular}

a There are no direct fuel costs for hydroelectric, geothermal, centralized solar, or wind energy.

b Heavy oll Includes Grade Nos. 4, 5, and 6, and residual fuel oils. Light oil includes Grade No. 2 heating oil, kerosene, and jet fue!.

c Biomass fuels are not included, except those consumed at electric utilities and those added to motor gasoline.

- No consumption, Including cases where adjustments were made. See "Consumption Adjustments for Process Fuel and Intermediate Products."

- Value less than 0.05 million dollars.

Note: Totals may not equal sum of components due to independent rounding.

Sources: Data sources, estimation procedures, and assumptions are described in the "Documentation" section of this report. 
M Energy Price and Expenditure Estimates by Source, Maryland

A $1970,1975,1980$, and 1985-1991

\begin{tabular}{|c|c|c|c|c|c|c|c|c|c|c|}
\hline Energy Source & 1970 & 1975 & 1980 & 1985 & 1986 & 1987 & 1988 & 1989 & 1990 & 1991 \\
\hline & \multicolumn{10}{|c|}{ Prices in Dollars per Million Btu } \\
\hline 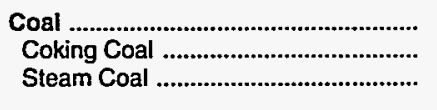 & $\begin{array}{r}0.45 \\
.58 \\
.34\end{array}$ & $\begin{array}{l}1.69 \\
2.14 \\
1.28\end{array}$ & $\begin{array}{l}1.77 \\
2.38 \\
1.50\end{array}$ & $\begin{array}{l}1.75 \\
1.88 \\
1.71\end{array}$ & $\begin{array}{l}1.67 \\
1.76 \\
1.64\end{array}$ & $\begin{array}{l}1.56 \\
1.59 \\
1.56\end{array}$ & $\begin{array}{l}1.57 \\
1.61 \\
1.56\end{array}$ & $\begin{array}{l}1.60 \\
1.67 \\
1.59\end{array}$ & $\begin{array}{l}1.62 \\
1.71 \\
1.61\end{array}$ & $\begin{array}{l}1.62 \\
1.75 \\
1.61\end{array}$ \\
\hline Natural Gas & 1.07 & 1.94 & 3.81 & 6.29 & 5.99 & 5.31 & 4.92 & 5.12 & 5.07 & 4.69 \\
\hline $\begin{array}{l}\text { Petroleum } \\
\text { Asphalt and Road Oil } \\
\text { Aviation Gasoline } \\
\text { Distillate Fuel } \\
\text { Jet Fuel } \\
\text { Kerosene } \\
\text { LPG }\end{array}$ & $\begin{array}{r}1.62 \\
.60 \\
2.17 \\
1.20 \\
.73 \\
1.46 \\
1.87 \\
5.08 \\
2.85 \\
.43 \\
1.53\end{array}$ & $\begin{array}{l}3.30 \\
1.89 \\
3.45 \\
2.61 \\
2.04 \\
3.24 \\
3.71 \\
7.48 \\
4.86 \\
1.87 \\
2.65\end{array}$ & $\begin{array}{r}7.66 \\
3.73 \\
9.02 \\
6.87 \\
6.46 \\
7.88 \\
6.81 \\
14.36 \\
9.93 \\
4.04 \\
8.91\end{array}$ & $\begin{array}{r}8.13 \\
4.95 \\
9.99 \\
7.83 \\
5.80 \\
8.63 \\
11.26 \\
17.61 \\
9.50 \\
4.06 \\
7.31\end{array}$ & $\begin{array}{r}6.41 \\
4.18 \\
8.41 \\
6.13 \\
4.01 \\
7.11 \\
10.99 \\
15.59 \\
7.55 \\
2.30 \\
6.35\end{array}$ & $\begin{array}{r}6.49 \\
3.22 \\
7.55 \\
6.34 \\
4.01 \\
6.89 \\
10.82 \\
12.70 \\
7.80 \\
3.03 \\
5.73\end{array}$ & $\begin{array}{r}6.49 \\
3.16 \\
7.41 \\
6.32 \\
3.75 \\
6.68 \\
9.68 \\
14.61 \\
8.18 \\
2.27 \\
5.05\end{array}$ & $\begin{array}{r}6.89 \\
2.90 \\
8.28 \\
6.81 \\
4.38 \\
7.27 \\
13.83 \\
13.30 \\
8.84 \\
2.68 \\
7.05\end{array}$ & $\begin{array}{r}7.91 \\
2.97 \\
9.32 \\
8.07 \\
5.47 \\
8.65 \\
11.54 \\
13.40 \\
9.88 \\
3.03 \\
6.23\end{array}$ & $\begin{array}{r}7.72 \\
2.89 \\
8.71 \\
7.64 \\
4.78 \\
8.35 \\
12.51 \\
15.42 \\
9.56 \\
2.16 \\
6.37\end{array}$ \\
\hline 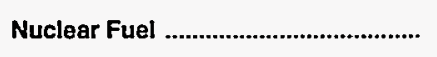 & - & .23 & .44 & .59 & .56 & .49 & .49 & .52 & .61 & .50 \\
\hline Blomass Fuels at Utllities ................... & - & - & - & .79 & .32 & .95 & .87 & 1.27 & - & - \\
\hline Primary Energy - Flve Sectors ${ }^{b}$.... & 1.17 & 2.62 & 4.92 & 5.39 & 4.34 & 4.38 & 4.27 & 4.96 & 5.45 & 4.92 \\
\hline $\begin{array}{l}\text { Electric Utility Fuelc } \\
\text { Electricity Purchased by End Users }\end{array}$ & $\begin{array}{r}.40 \\
5.76\end{array}$ & $\begin{array}{r}1.36 \\
11.19\end{array}$ & $\begin{array}{r}1.66 \\
15.47\end{array}$ & $\begin{array}{r}1.65 \\
18.60\end{array}$ & $\begin{array}{r}1.32 \\
18.66\end{array}$ & $\begin{array}{r}1.43 \\
17.61\end{array}$ & $\begin{array}{r}1.35 \\
17.12\end{array}$ & $\begin{array}{r}1.83 \\
17.53\end{array}$ & $\begin{array}{r}1.88 \\
18.47\end{array}$ & $\begin{array}{r}1.46 \\
19.97\end{array}$ \\
\hline \multirow[t]{2}{*}{ Total Energy } & 1.79 & 4.02 & 7.70 & 9.10 & 8.20 & 7.93 & 7.94 & 8.54 & 9.35 & 9.67 \\
\hline & \multicolumn{10}{|c|}{ Expenditures in Millions of Dollars } \\
\hline Coal & $\begin{array}{r}139.6 \\
79.6 \\
60.0\end{array}$ & $\begin{array}{l}333.2 \\
200.6 \\
132.6\end{array}$ & $\begin{array}{l}416.8 \\
168.9 \\
247.9\end{array}$ & $\begin{array}{l}448.6 \\
107.4 \\
341.2\end{array}$ & $\begin{array}{r}458.3 \\
91.5 \\
366.7\end{array}$ & $\begin{array}{r}451.2 \\
82.3 \\
368.9\end{array}$ & $\begin{array}{r}473.4 \\
82.8 \\
390.6\end{array}$ & $\begin{array}{r}473.0 \\
77.4 \\
395.6\end{array}$ & $\begin{array}{r}463.9 \\
57.6 \\
406.3\end{array}$ & $\begin{array}{r}444.8 \\
46.6 \\
398.3\end{array}$ \\
\hline 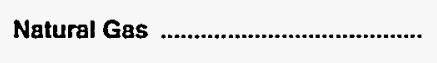 & 168.5 & 270.5 & 607.5 & 966.9_. & 933.1 & 913.8 & 864.2 & 990.5 & 884.5 & 822.0 \\
\hline 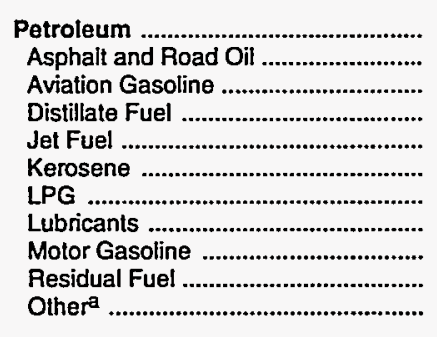 & $\begin{array}{r}860.4 \\
11.1 \\
3.4 \\
138.3 \\
18.1 \\
19.3 \\
13.0 \\
19.2 \\
556.7 \\
58.7 \\
22.5\end{array}$ & $\begin{array}{r}1,942.2 \\
40.8 \\
3.6 \\
317.1 \\
34.6 \\
21.9 \\
33.0 \\
34.6 \\
1,115.0 \\
314.0 \\
27.6\end{array}$ & \begin{tabular}{r|}
$4,059.4$ \\
65.3 \\
7.9 \\
872.6 \\
126.3 \\
52.2 \\
51.5 \\
63.1 \\
$2,296.3$ \\
415.8 \\
108.3
\end{tabular} & \begin{tabular}{r|}
$3,877.0$ \\
148.6 \\
3.8 \\
807.7 \\
125.7 \\
61.0 \\
73.3 \\
70.4 \\
$2,275.7$ \\
201.9 \\
108.9
\end{tabular} & $\begin{array}{r}3,107.1 \\
144.7 \\
4.3 \\
621.0 \\
86.7 \\
37.7 \\
57.2 \\
60.9 \\
1,860.2 \\
105.5 \\
128.8\end{array}$ & $\begin{array}{r}3,318.6 \\
102.9 \\
3.3 \\
668.0 \\
84.0 \\
47.2 \\
68.9 \\
56.1 \\
1,970.1 \\
173.2 \\
144.8\end{array}$ & $\begin{array}{r}3,439.5 \\
91.3 \\
3.5 \\
682.5 \\
93.7 \\
57.8 \\
59.9 \\
62.3 \\
2.114 .9 \\
148.8 \\
124.8\end{array}$ & $\begin{array}{r}3,896.9 \\
86.7 \\
3.5 \\
816.8 \\
107.2 \\
41.5 \\
108.8 \\
58.1 \\
2,278.9 \\
254.9 \\
140.5\end{array}$ & $\begin{array}{r}3,959.5 \\
98.7 \\
3.5 \\
799.5 \\
110.9 \\
22.9 \\
84.4 \\
60.3 \\
2,446.9 \\
188.4 \\
144.1\end{array}$ & $\begin{array}{r}3,808.9 \\
71.0 \\
3.3 \\
770.9 \\
89.9 \\
22.5 \\
91.4 \\
62.1 \\
2,433.3 \\
127.0 \\
137.4\end{array}$ \\
\hline 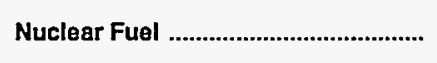 & - & 11.3 & 52.5 & 62.9 & 77.3 & 53.2 & 62.1 & 15.1 & 8.2 & 48.2 \\
\hline 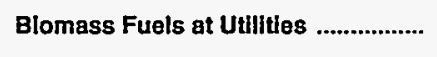 & - & - & - & .1 & .1 & .5 & .5 & .2 & - & - \\
\hline Primary Energy - Five Sectors ${ }^{b} \ldots$. & $1,168.5$ & $2,557.3$ & $5,136.2$ & $5,355.5$ & $4,575.8$ & $4,737.3$ & $4,839.7$ & $5,375.6$ & $5,316.2$ & $5,124.0$ \\
\hline Electric Utility Fuel ${ }^{\mathbb{c}}$ & $\begin{array}{r}-91.0 \\
442.4\end{array}$ & $\begin{array}{r}-352.5 \\
1,042.3\end{array}$ & $\begin{array}{r}-544.9 \\
1,825.5\end{array}$ & $\begin{array}{r}-536.2 \\
2,496.0\end{array}$ & $\begin{array}{r}-495.4 \\
2,671.2\end{array}$ & $\begin{array}{r}-517.2 \\
2,698.1\end{array}$ & $\begin{array}{r}-544.1 \\
2,779.1\end{array}$ & $\begin{array}{r}-646.8 \\
2,946.0\end{array}$ & $\begin{array}{r}-568.0 \\
3,122.0\end{array}$ & $\begin{array}{r}-558.0 \\
3.483 .1\end{array}$ \\
\hline Total Energy & $1,519.9$ & $3,247.1$ & $6,416.7$ & $7,315.3$ & $6,751.7$ & $6,918.2$ & $7,074.7$ & $7,674.8$ & $7,870.1$ & B,049.1 \\
\hline
\end{tabular}

a Includes petroleum coke used at electric utilities.

b Biomass fuels are not included, except those consumed at electric utilities and those added to motor gasoline.

c There are no direct fuel costs for hydroelectric, geothermal, centralized solar, or wind energy.

-No consumption, including cases where adjustments were made. See "Consumption Adjustments for Process Fuel and Intermediate Products."

Note: Expenditure totals may not equal sum of components due to independent rounding.

Sources: Data sources, estimation procedures, and assumptions are described in the "Documentation" section of this report. 
Energy Price Estimates by Sector, Maryland

1970, 1975, 1980, and 1985-1991

(Dollars per Million Btu)

\begin{tabular}{|c|c|c|c|c|c|c|c|c|c|c|}
\hline Sector and Energy Source & 1970 & 1975 & 1980 & 1985 & 1986 & 1987 & 1988 & 1989 & 1990 & 1991 \\
\hline 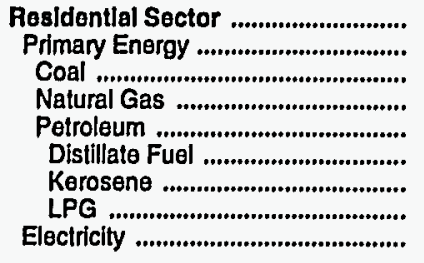 & $\begin{array}{l}2.34 \\
1.46 \\
1.05 \\
1.42 \\
1.50 \\
1.42 \\
1.50 \\
2.57 \\
7.02\end{array}$ & $\begin{array}{r}4.62 \\
2.58 \\
1.75 \\
2.30 \\
2.92 \\
2.71 \\
3.37 \\
4.61 \\
12.65\end{array}$ & $\begin{array}{r}8.54 \\
5.71 \\
3.18 \\
4.38 \\
7.31 \\
7.06 \\
8.55 \\
9.81 \\
17.32\end{array}$ & $\begin{array}{r}11.76 \\
7.54 \\
3.28 \\
7.01 \\
8.62 \\
8.24 \\
8.84 \\
11.42 \\
21.32\end{array}$ & $\begin{array}{r}11.51 \\
6.83 \\
3.22 \\
6.64 \\
7.32 \\
6.92 \\
7.42 \\
11.25 \\
21.14\end{array}$ & $\begin{array}{r}11.04 \\
6.43 \\
3.10 \\
6.16 \\
7.02 \\
6.56 \\
7.03 \\
11.25 \\
20.19\end{array}$ & $\begin{array}{r}10.69 \\
6.11 \\
3.07 \\
5.72 \\
6.85 \\
6.59 \\
7.07 \\
9.17 \\
19.68\end{array}$ & $\begin{array}{r}11.58 \\
6.83 \\
3.25 \\
6.11 \\
8.31 \\
7.10 \\
7.62 \\
17.98 \\
20.10\end{array}$ & $\begin{array}{r}12.68 \\
7.13 \\
3.36 \\
6.28 \\
9.04 \\
8.47 \\
9.09 \\
12.58 \\
21.17\end{array}$ & $\begin{array}{r}13.46 \\
6.90 \\
3.09 \\
6.01 \\
8.99 \\
8.21 \\
8.80 \\
13.46 \\
23.15\end{array}$ \\
\hline 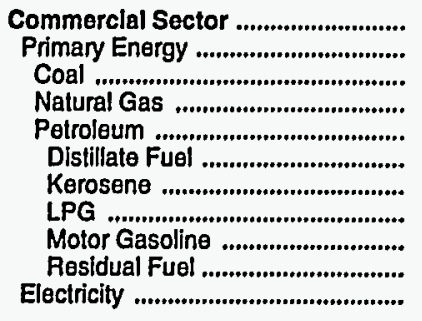 & $\begin{array}{r}2.60 \\
.98 \\
.07 \\
1.09 \\
.93 \\
1.12 \\
.88 \\
1.03 \\
2.85 \\
.43 \\
6.86\end{array}$ & $\begin{array}{r}5.77 \\
2.13 \\
1.06 \\
1.96 \\
2.31 \\
2.39 \\
2.53 \\
2.75 \\
4.86 \\
1.83 \\
12.49\end{array}$ & $\begin{array}{r}9.78 \\
4.74 \\
1.19 \\
3.88 \\
5.81 \\
6.39 \\
6.24 \\
5.13 \\
9.93 \\
4.16 \\
18.41\end{array}$ & $\begin{array}{r}13.15 \\
6.20 \\
1.33 \\
6.17 \\
6.87 \\
6.77 \\
6.95 \\
11.07 \\
9.50 \\
4.41 \\
22.00\end{array}$ & $\begin{array}{r}12.50 \\
4.88 \\
1.33 \\
5.54 \\
4.32 \\
4.70 \\
4.71 \\
10.71 \\
7.55 \\
2.56 \\
21.81\end{array}$ & $\begin{array}{r}10.74 \\
4.40 \\
1.30 \\
4.94 \\
4.17 \\
4.58 \\
4.63 \\
10.30 \\
7.80 \\
3.14 \\
19.92\end{array}$ & $\begin{array}{r}11.33 \\
4.50 \\
1.33 \\
4.93 \\
4.18 \\
4.27 \\
4.26 \\
10.26 \\
8.18 \\
2.39 \\
19.27\end{array}$ & $\begin{array}{r}11.11 \\
4.99 \\
1.28 \\
5.27 \\
4.78 \\
5.45 \\
5.79 \\
9.42 \\
8.84 \\
2.82 \\
19.63\end{array}$ & $\begin{array}{r}12.21 \\
5.50 \\
1.21 \\
5.21 \\
6.07 \\
6.28 \\
6.60 \\
10.33 \\
9.88 \\
3.13 \\
19.94\end{array}$ & $\begin{array}{r}11.61 \\
5.21 \\
1.34 \\
4.92 \\
6.09 \\
5.87 \\
6.08 \\
11.09 \\
9.56 \\
2.36 \\
20.88\end{array}$ \\
\hline 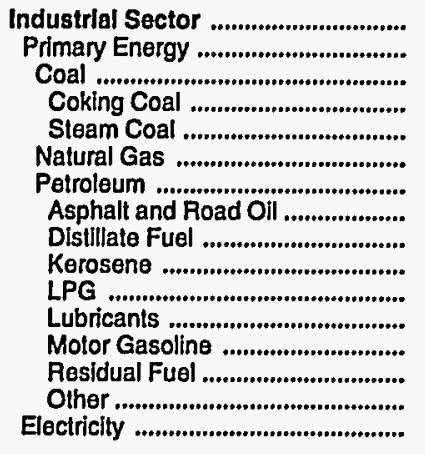 & $\begin{array}{r}.91 \\
.63 \\
.50 \\
.58 \\
.07 \\
.67 \\
.84 \\
.60 \\
.81 \\
.88 \\
1.03 \\
5.08 \\
2.85 \\
.43 \\
1.53 \\
3.80\end{array}$ & $\begin{array}{l}2.79 \\
2.06 \\
2.05 \\
2.14 \\
1.06 \\
1.35 \\
2.40 \\
1.89 \\
2.34 \\
2.53 \\
2.75 \\
7.48 \\
4.86 \\
2.08 \\
2.65 \\
8.42\end{array}$ & $\begin{array}{r}5.00 \\
3.64 \\
2.15 \\
2.38 \\
1.19 \\
3.19 \\
5.77 \\
3.73 \\
5.60 \\
6.24 \\
5.13 \\
14.36 \\
9.93 \\
4.37 \\
8.91 \\
11.65\end{array}$ & $\begin{array}{r}6.36 \\
4.42 \\
1.75 \\
1.88 \\
1.33 \\
5.51 \\
6.33 \\
4.95 \\
6.22 \\
6.95 \\
11.07 \\
17.61 \\
9.50 \\
4.41 \\
7.31 \\
13.92\end{array}$ & $\begin{array}{r}6.14 \\
4.03 \\
1.65 \\
1.76 \\
1.33 \\
5.43 \\
5.17 \\
4.18 \\
4.22 \\
4.71 \\
10.71 \\
15.59 \\
7.55 \\
2.56 \\
6.35 \\
14.12\end{array}$ & $\begin{array}{r}5.71 \\
3.66 \\
1.50 \\
1.59 \\
1.30 \\
4.97 \\
4.74 \\
3.22 \\
4.14 \\
4.63 \\
10.30 \\
12.70 \\
7.80 \\
3.14 \\
5.73 \\
-13.43\end{array}$ & $\begin{array}{r}5.52 \\
3.43 \\
1.54 \\
1.61 \\
1.33 \\
4.17 \\
4.47 \\
3.16 \\
3.82 \\
4.26 \\
10.26 \\
14.61 \\
8.18 \\
2.39 \\
5.05 \\
12.97\end{array}$ & $\begin{array}{r}6.29 \\
3.88 \\
1.57 \\
1.67 \\
1.28 \\
4.67 \\
5.10 \\
2.90 \\
5.18 \\
5.79 \\
9.42 \\
13.30 \\
8.84 \\
2.82 \\
7.05 \\
13.83\end{array}$ & $\begin{array}{r}6.56 \\
3.83 \\
1.50 \\
1.71 \\
1.21 \\
4.45 \\
4.98 \\
2.97 \\
5.91 \\
6.60 \\
10.33 \\
13.40 \\
9.88 \\
3.13 \\
6.23 \\
14.94\end{array}$ & $\begin{array}{r}7.10 \\
3.53 \\
1.55 \\
1.75 \\
1.34 \\
3.42 \\
5.18 \\
2.89 \\
5.44 \\
6.08 \\
11.09 \\
15.42 \\
9.56 \\
2.36 \\
6.37 \\
16.11\end{array}$ \\
\hline 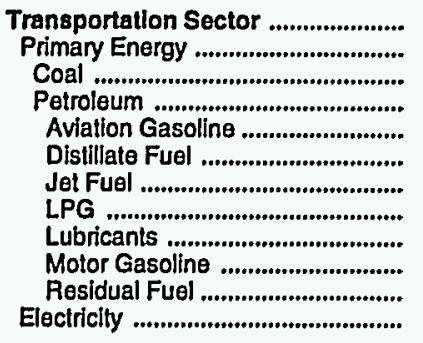 & $\begin{array}{r}2.30 \\
2.30 \\
.07 \\
2.30 \\
2.17 \\
1.32 \\
.73 \\
1.03 \\
5.08 \\
2.85 \\
.39 \\
-\end{array}$ & $\begin{array}{r}4.30 \\
4.30 \\
1.06 \\
4.30 \\
3.45 \\
2.81 \\
2.03 \\
2.75 \\
7.48 \\
4.86 \\
1.61 \\
-\end{array}$ & $\begin{array}{r}8.92 \\
8.92 \\
- \\
8.92 \\
9.02 \\
7.69 \\
6.46 \\
5.13 \\
14.36 \\
9.93 \\
3.53 \\
12.62\end{array}$ & $\begin{array}{r}9.01 \\
9.00 \\
- \\
9.00 \\
9.99 \\
8.64 \\
5.80 \\
11.07 \\
17.61 \\
9.50 \\
3.88 \\
17.74\end{array}$ & $\begin{array}{r}7.11 \\
7.10 \\
7.10 \\
8.41 \\
6.68 \\
4.01 \\
10.71 \\
15.59 \\
7.55 \\
2.09 \\
17.80\end{array}$ & $\begin{array}{r}7.34 \\
7.33 \\
-\overline{7} \\
7.33 \\
7.55 \\
7.32 \\
4.01 \\
10.30 \\
12.70 \\
7.80 \\
2.85 \\
24.06\end{array}$ & $\begin{array}{r}7.51 \\
7.50 \\
- \\
7.50 \\
7.41 \\
7.40 \\
3.75 \\
10.26 \\
14.61 \\
8.18 \\
2.07 \\
23.33\end{array}$ & $\begin{array}{r}8.09 \\
8.07 \\
- \\
8.07 \\
8.28 \\
7.61 \\
4.38 \\
9.42 \\
13.30 \\
8.84 \\
2.39 \\
23.37\end{array}$ & $\begin{array}{r}9.25 \\
9.24 \\
- \\
9.24 \\
9.32 \\
8.97 \\
5.47 \\
10.33 \\
13.40 \\
9.88 \\
2.72 \\
24.42\end{array}$ & $\begin{array}{r}8.97 \\
8.95 \\
- \\
8.95 \\
8.71 \\
8.44 \\
4.78 \\
11.09 \\
15.42 \\
9.56 \\
1.97 \\
25.65\end{array}$ \\
\hline $\begin{array}{l}\text { Total Energy ...................................... } \\
\text { Primary Energy - Four Sectors ...... } \\
\text { Electricity ....................................... }\end{array}$ & $\begin{array}{l}1.79 \\
1.39 \\
5.76\end{array}$ & $\begin{array}{r}4.02 \\
3.09 \\
11.19\end{array}$ & $\begin{array}{r}7.70 \\
6.42 \\
15.47\end{array}$ & $\begin{array}{r}9.10 \\
7.19 \\
18.60\end{array}$ & $\begin{array}{r}8.20 \\
6.00 \\
18.66\end{array}$ & $\begin{array}{r}7.93 \\
5.87 \\
17.61\end{array}$ & $\begin{array}{r}7.94 \\
5.89 \\
17.12\end{array}$ & $\begin{array}{r}8.54 \\
6.47 \\
17.53\end{array}$ & $\begin{array}{r}9.35 \\
7.06 \\
18.47\end{array}$ & $\begin{array}{r}9.67 \\
6.94 \\
19.97\end{array}$ \\
\hline 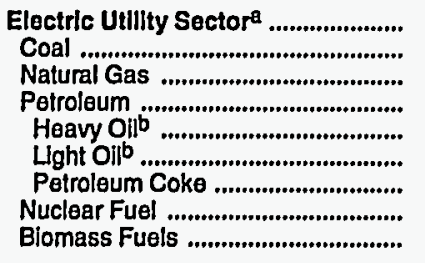 & $\begin{array}{l}.40 \\
.39 \\
.32 \\
.44 \\
.44 \\
.48 \\
- \\
-\end{array}$ & $\begin{array}{r}1.36 \\
1.30 \\
1.10 \\
1.86 \\
1.85 \\
2.18 \\
-23 \\
-\end{array}$ & $\begin{array}{r}1.66 \\
1.54 \\
2.50 \\
4.41 \\
4.21 \\
5.97 \\
. \overline{44}\end{array}$ & $\begin{array}{r}1.65 \\
1.75 \\
3.73 \\
4.22 \\
4.02 \\
5.53 \\
- \\
.59 \\
.79\end{array}$ & $\begin{array}{r}1.32 \\
1.67 \\
2.74 \\
2.41 \\
2.25 \\
3.39 \\
- \\
.56 \\
.32\end{array}$ & $\begin{array}{r}1.43 \\
1.58 \\
2.68 \\
3.17 \\
3.06 \\
3.81 \\
-\overline{49} \\
.95\end{array}$ & $\begin{array}{r}1.35 \\
1.58 \\
2.60 \\
2.46 \\
2.33 \\
3.49 \\
-\overline{.49} \\
.87\end{array}$ & $\begin{array}{r}1.83 \\
1.61 \\
2.60 \\
2.86 \\
2.72 \\
4.12 \\
- \\
.52 \\
1.27\end{array}$ & $\begin{array}{r}1.88 \\
1.65 \\
2.45 \\
3.28 \\
3.10 \\
5.29 \\
- \\
.61 \\
-\end{array}$ & $\begin{array}{r}1.46 \\
1.63 \\
2.26 \\
2.32 \\
2.17 \\
4.44 \\
- \\
.50 \\
-\end{array}$ \\
\hline Prlmary Energy - Flve Sectorsc .. & 1.17 & 2.62 & 4.92 & 5.39 & 4.34 & 4.38 & 4.27 & 4.96 & 5.45 & 4.92 \\
\hline
\end{tabular}

There are no direct fuel costs for hydroelectric, geothermal, centralized solar, or wind energy.

beavy oil includes Grade Nos. 4, 5, and 6, and residual fuel oils. Light oil includes Grade No. 2 heating oil, kerosene, and jet fuel.

c Blomass fuels are not included, except those consumed at electric utilities and those added to motor gasoline.

- No consumption, including cases where adjustments were made. See "Consumption Adjustments for Process Fuel and Intermediate Products."

Sources: Data sources, estimation procedures, and assumptions are described in the "Documentation" section of this report. 
M Energy Expenditure Estimates by Sector, Maryland

A $1970,1975,1980$, and 1985-1991

R (Million Dollars)

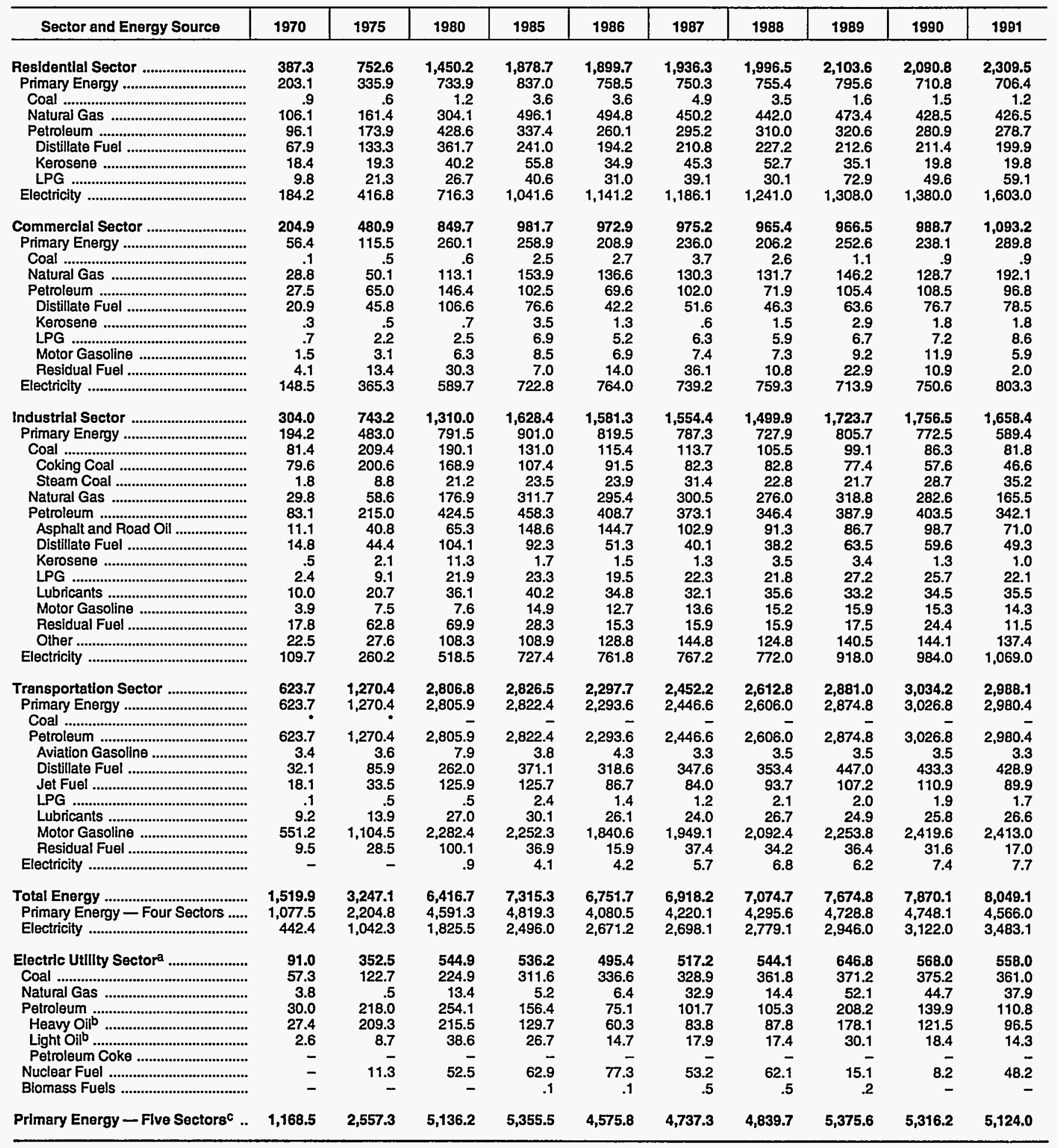

a There are no direct fuel costs for hydroelectric, geothermal, centralized solar, or wind energy.

b Heavy oil includes Grade Nos. 4, 5, and 6, and residual fuel oils. Light oil includes Grade No. 2 heating oil, kerosene, and jet fuel.

c Biomass fuels are not included, except those consumed at electric utilities and those added to motor gasoline.

-No consumption, including cases where adjustments were made. See "Consumption Adjustments for Process Fuel and Intermediate Products."

Value less than 0.05 million dollars.

Note: Totals may not equal sum of components due to independent rounding.

Sources: Data sources, estimation procedures, and assumptions are described in the "Documentation" section of this report. 
Energy Price and Expenditure Estimates by Source, Massachusetts 1970, 1975, 1980, and 1985-1991

\begin{tabular}{|c|c|c|c|c|c|c|c|c|c|c|}
\hline Enargy Source & 1970 & 1975 & 1980 & 1985 & 1986 & 1987 & 1988 & 1989 & 1990 & 1991 \\
\hline & \multicolumn{10}{|c|}{ Prices In Dollars per Million Btu } \\
\hline 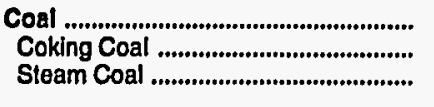 & $\begin{array}{c}0.55 \\
.55\end{array}$ & $\frac{1.57}{1.57}$ & $\begin{array}{r}2.03 \\
2.03\end{array}$ & $\begin{array}{r}2.03 \\
2.03\end{array}$ & $\begin{array}{r}1.81 \\
1.81\end{array}$ & $\begin{array}{r}1.66 \\
1.66\end{array}$ & $\frac{1.66}{1.66}$ & $\frac{1.64}{1.64}$ & $\begin{array}{r}1.77 \\
1.77\end{array}$ & $\begin{array}{r}1.75 \\
1.75\end{array}$ \\
\hline Natural Gas .............................................. & 1.58 & 2.86 & 4.88 & 6.25 & 6.35 & 5.23 & 5.46 & 5.33 & 5.55 & 5.66 \\
\hline 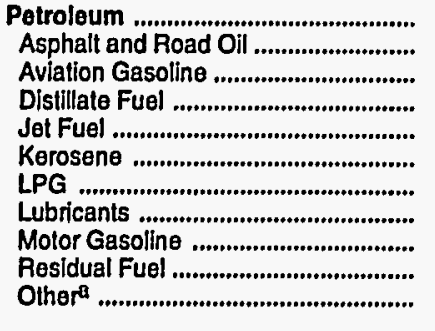 & $\begin{array}{r}1.24 \\
.63 \\
2.17 \\
1.34 \\
.75 \\
1.36 \\
2.31 \\
5.08 \\
2.86 \\
.39 \\
1.86\end{array}$ & $\begin{array}{l}2.95 \\
1.95 \\
3.45 \\
2.74 \\
2.10 \\
2.99 \\
3.74 \\
7.48 \\
4.73 \\
1.95 \\
3.28\end{array}$ & $\begin{array}{r}6.60 \\
3.73 \\
9.02 \\
6.87 \\
6.51 \\
7.06 \\
6.62 \\
14.36 \\
9.69 \\
3.84 \\
8.66\end{array}$ & $\begin{array}{r}7.28 \\
5.21 \\
9.99 \\
8.05 \\
6.04 \\
8.65 \\
11.77 \\
17.61 \\
9.18 \\
4.04 \\
9.25\end{array}$ & $\begin{array}{r}5.13 \\
4.87 \\
8.41 \\
6.00 \\
4.18 \\
6.21 \\
10.13 \\
15.59 \\
7.25 \\
2.31 \\
6.58\end{array}$ & $\begin{array}{r}5.60 \\
3.57 \\
7.55 \\
5.86 \\
4.12 \\
6.60 \\
11.06 \\
12.70 \\
7.56 \\
2.77 \\
7.32\end{array}$ & $\begin{array}{r}5.56 \\
3.38 \\
7.41 \\
5.87 \\
4.02 \\
6.46 \\
11.01 \\
14.61 \\
7.90 \\
2.27 \\
6.31\end{array}$ & $\begin{array}{r}6.11 \\
3.20 \\
8.28 \\
6.55 \\
4.61 \\
7.10 \\
12.00 \\
13.30 \\
8.50 \\
2.64 \\
7.21\end{array}$ & $\begin{array}{r}7.13 \\
3.36 \\
9.32 \\
7.99 \\
5.83 \\
8.41 \\
12.12 \\
13.40 \\
9.53 \\
2.88 \\
7.50\end{array}$ & $\begin{array}{r}6.98 \\
3.05 \\
8.71 \\
7.53 \\
5.00 \\
7.17 \\
13.73 \\
15.42 \\
10.01 \\
2.21 \\
6.95\end{array}$ \\
\hline Nuclear Fuel ........................................ & .20 & .18 & .41 & .60 & .88 & .92 & .96 & .70 & .62 & .61 \\
\hline Blomass Fuels at Utilitles ................. & - & - & - & - & - & - & - & - & - & - \\
\hline Pilmary Energy - Flve Sectors ${ }^{b}$.... & 1.25 & 2.83 & 6.04 & 6.22 & 4.96 & 5.10 & 5.12 & 5.40 & 5.99 & 5.92 \\
\hline $\begin{array}{l}\text { Electric Utilty Fuelc ............................ } \\
\text { Electricity Purchased by End Users }\end{array}$ & $\begin{array}{r}.37 \\
7.29\end{array}$ & $\begin{array}{r}1.66 \\
13.93\end{array}$ & $\begin{array}{r}3.41 \\
21.13\end{array}$ & $\begin{array}{r}2.74 \\
24.34\end{array}$ & $\begin{array}{r}2.03 \\
22.70\end{array}$ & $\begin{array}{r}2.26 \\
23.46\end{array}$ & $\begin{array}{r}2.04 \\
22.86\end{array}$ & $\begin{array}{r}2.18 \\
24.39\end{array}$ & $\begin{array}{r}2.15 \\
25.93\end{array}$ & $\begin{array}{r}1.85 \\
27.94\end{array}$ \\
\hline \multirow[t]{2}{*}{ Total Energy ........................................ } & 1.90 & 4.15 & 8.90 & 10.09 & 8.39 & 8.71 & 8.85 & 9.45 & 10.52 & 10.84 \\
\hline & \multicolumn{10}{|c|}{ Expenditures in Millions of Dollars } \\
\hline 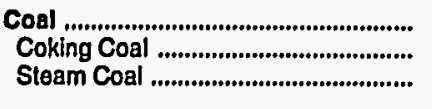 & $\begin{array}{r}11.7 \\
11 . \overline{7}\end{array}$ & $\begin{array}{r}38.5 \\
38.5\end{array}$ & $\begin{array}{r}46.4 \\
46 . \overline{4}\end{array}$ & $\begin{array}{r}223.8 \\
-\overline{8}\end{array}$ & $\begin{array}{r}180.9 \\
- \\
180.9\end{array}$ & $\begin{array}{r}195.2 \\
-\overline{2} \\
195.2\end{array}$ & $\begin{array}{r}194.2 \\
-\overline{2} \\
194.2\end{array}$ & $\begin{array}{r}198.4 \\
198 . \overline{4}\end{array}$ & $\begin{array}{r}200.4 \\
200.4\end{array}$ & $\begin{array}{r}204.7 \\
- \\
204.7\end{array}$ \\
\hline Natural Gas ........................................ & 234.1 & 441.3 & 901.9 & $1,395.1$ & $1,203.3$ & $1,215.6$ & $1,174.4$ & $1,367.2$ & $1,479.7$ & $1,470.6$ \\
\hline 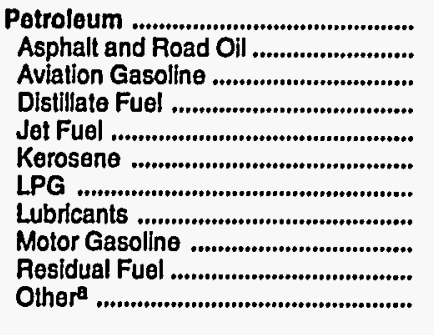 & $\begin{array}{r}1,537.4 \\
12.0 \\
3.0 \\
461.9 \\
33.3 \\
16.3 \\
15.9 \\
29.2 \\
743.8 \\
210.9 \\
11.2\end{array}$ & $\begin{array}{r}3,325.6 \\
23.7 \\
4.0 \\
934.7 \\
95.0 \\
14.7 \\
32.2 \\
35.7 \\
1,357.3 \\
808.9 \\
19.6\end{array}$ & $\begin{array}{r}6,042.8 \\
30.5 \\
12.5 \\
1,504.7 \\
315.8 \\
27.9 \\
51.7 \\
73.2 \\
2,619.1 \\
1,306.9 \\
100.5\end{array}$ & $\begin{array}{r}5,682.3 \\
36.4 \\
6.8 \\
1,550.3 \\
238.4 \\
36.2 \\
72.9 \\
81.7 \\
2,643.9 \\
915.4 \\
100.5\end{array}$ & $\begin{array}{r}4,569.6 \\
36.0 \\
6.2 \\
1,241.9 \\
163.4 \\
29.1 \\
84.0 \\
70.7 \\
2,147.8 \\
721.3 \\
69.3\end{array}$ & $\begin{array}{r}4,732.3 \\
35.0 \\
4.7 \\
1,290.7 \\
182.9 \\
23.3 \\
106.6 \\
65.2 \\
2,284.5 \\
661.9 \\
77.6\end{array}$ & $\begin{array}{r}4,777.1 \\
39.6 \\
4.8 \\
1,258.0 \\
212.1 \\
15.3 \\
95.4 \\
72.3 \\
2,465.8 \\
548.3 \\
65.4\end{array}$ & $\begin{array}{r}5,340.3 \\
30.3 \\
4.9 \\
1,540.7 \\
260.8 \\
15.8 \\
113.3 \\
67.5 \\
2,601.2 \\
633.1 \\
72.8\end{array}$ & $\begin{array}{r}5,579.2 \\
29.9 \\
4.5 \\
1,567.8 \\
323.3 \\
14.7 \\
115.7 \\
70.0 \\
2,789.2 \\
581.2 \\
83.0\end{array}$ & $\begin{array}{r}5,283.6 \\
40.0 \\
2.0 \\
1,456.0 \\
238.4 \\
15.0 \\
95.2 \\
72.0 \\
2,864.2 \\
424.7 \\
76.1\end{array}$ \\
\hline 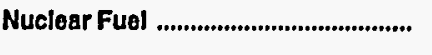 & 2.7 & 7.5 & 14.3 & 39.8 & 23.1 & 11.3 & 11.5 & 22.7 & 33.6 & 29.0 \\
\hline Blomass Fuels at Utillties ................. & - & - & - & - & - & - & - & - & - & - \\
\hline Primary Energy - Five Sectors ${ }^{b} \ldots$ & $1,785.8$ & $3,812.9$ & $7,005.4$ & $7,341.1$ & $5,976.9$ & $6,154.4$ & $6,157.1$ & $6,928.7$ & $7,292.9$ & $6,987.8$ \\
\hline $\begin{array}{l}\text { Electric Utility Fuejc ............................ } \\
\text { Electricity Purchased by End Users }\end{array}$ & $\begin{array}{r}-112.4 \\
612.9\end{array}$ & $\begin{array}{r}-524.9 \\
1.401 .0\end{array}$ & $\begin{array}{r}-1,191.4 \\
2,398.4\end{array}$ & $\begin{array}{r}-1,011.3 \\
3,166.2\end{array}$ & $\begin{array}{r}-698.3 \\
3,101.9\end{array}$ & $\begin{array}{r}-782.8 \\
3,392.0\end{array}$ & $\begin{array}{r}-704.3 \\
3,489.0\end{array}$ & $\begin{array}{r}-862.8 \\
3,801.0\end{array}$ & $\begin{array}{r}-801.1 \\
4,021.1\end{array}$ & $\begin{array}{r}-661.1 \\
4,271.0\end{array}$ \\
\hline Total Enorgy ............................................. & $2,286.3$ & $4,689.0$ & $8,212.4$ & $9,496.0$ & $8,380.5$ & $8,763.6$ & $8,941.8$ & $9,866.9$ & $10,512.8$ & $10,597.7$ \\
\hline
\end{tabular}

a Includes petroleum coke used at electric utilities.

Biomass fuels are not included, except those consumed at electric utilities and those added to motor gasoline.

c There are no direct fuel costs for hydroelectric, geothermal, centralized solar, or wind energy.

-No consumption, including cases where adjustments were made. See "Consumption Adjustments for Process Fuel and Intermediate Products."

Note: Expenditure totals may not equal sum of components due to independent rounding.

Sources: Data sources, estimation procedures, and assumptions are described in the "Documentation" section of this report. 
M Energy Price Estimates by Sector, Massachusetts

A $1970,1975,1980$, and $1985-1991$

S (Dollars per Million Btu)

\begin{tabular}{|c|c|c|c|c|c|c|c|c|c|c|}
\hline Sector and Energy Source & 1970 & 1975 & 1980 & 1985 & 1986 & 1987 & 1988 & 1989 & 1990 & 1991 \\
\hline 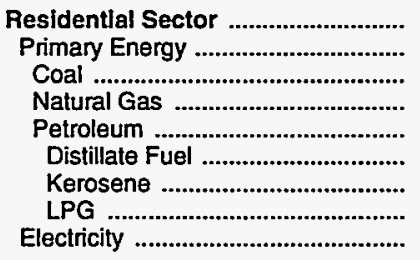 & $\begin{array}{l}2.24 \\
1.61 \\
1.05 \\
1.90 \\
1.52 \\
1.49 \\
1.62 \\
3.04 \\
8.59\end{array}$ & $\begin{array}{r}4.22 \\
2.96 \\
2.62 \\
3.14 \\
2.89 \\
2.85 \\
3.16 \\
4.92 \\
15.30\end{array}$ & $\begin{array}{r}8.65 \\
6.36 \\
4.47 \\
5.33 \\
7.10 \\
7.05 \\
8.15 \\
8.99 \\
22.18\end{array}$ & $\begin{array}{r}11.05 \\
7.93 \\
4.39 \\
7.65 \\
8.24 \\
8.10 \\
8.94 \\
11.43 \\
26.16\end{array}$ & $\begin{array}{r}9.81 \\
6.75 \\
4.19 \\
7.23 \\
6.33 \\
6.22 \\
6.86 \\
9.01 \\
24.38\end{array}$ & $\begin{array}{r}9.82 \\
6.37 \\
3.85 \\
6.45 \\
6.32 \\
6.10 \\
6.73 \\
10.88 \\
25.52\end{array}$ & $\begin{array}{r}9.85 \\
6.35 \\
3.99 \\
6.28 \\
6.43 \\
6.22 \\
6.86 \\
10.95 \\
24.88\end{array}$ & $\begin{array}{r}10.65 \\
7.10 \\
3.96 \\
6.91 \\
7.29 \\
7.01 \\
7.74 \\
12.96 \\
26.70\end{array}$ & $\begin{array}{r}11.97 \\
7.99 \\
4.21 \\
7.56 \\
8.45 \\
8.21 \\
9.06 \\
13.36 \\
28.31\end{array}$ & $\begin{array}{r}12.46 \\
7.95 \\
4.07 \\
7.81 \\
8.11 \\
7.80 \\
8.61 \\
14.73 \\
30.47\end{array}$ \\
\hline 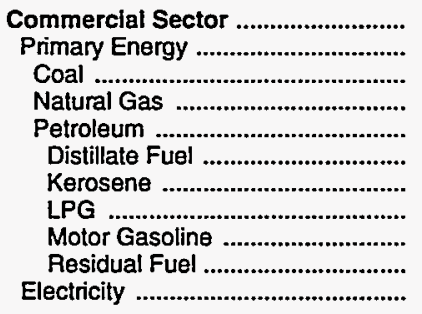 & $\begin{array}{r}1.64 \\
.83 \\
.89 \\
1.40 \\
.72 \\
1.10 \\
.81 \\
1.54 \\
2.86 \\
.37 \\
8.05\end{array}$ & $\begin{array}{r}4.51 \\
2.32 \\
2.62 \\
2.64 \\
2.22 \\
2.44 \\
2.62 \\
2.84 \\
4.73 \\
1.89 \\
14.39\end{array}$ & $\begin{array}{r}9.35 \\
5.04 \\
1.67 \\
4.65 \\
5.37 \\
6.36 \\
6.12 \\
5.52 \\
9.69 \\
3.81 \\
22.08\end{array}$ & $\begin{array}{r}12.93 \\
6.36 \\
2.38 \\
6.88 \\
6.08 \\
6.89 \\
7.61 \\
12.27 \\
9.18 \\
4.31 \\
25.20\end{array}$ & $\begin{array}{r}11.33 \\
5.09 \\
2.11 \\
6.49 \\
4.16 \\
4.85 \\
5.20 \\
11.25 \\
7.25 \\
2.46 \\
23.40\end{array}$ & $\begin{array}{r}11.79 \\
5.00 \\
2.35 \\
5.75 \\
4.38 \\
4.84 \\
5.81 \\
11.25 \\
7.56 \\
2.93 \\
23.49\end{array}$ & $\begin{array}{r}11.33 \\
4.83 \\
2.51 \\
5.93 \\
3.94 \\
4.58 \\
5.45 \\
11.10 \\
7.90 \\
2.36 \\
22.72\end{array}$ & $\begin{array}{r}11.59 \\
5.06 \\
2.57 \\
5.67 \\
4.63 \\
5.45 \\
5.68 \\
10.43 \\
8.50 \\
2.69 \\
24.05\end{array}$ & $\begin{array}{r}12.63 \\
5.50 \\
2.62 \\
6.14 \\
5.04 \\
6.37 \\
7.68 \\
10.80 \\
9.53 \\
3.05 \\
25.44\end{array}$ & $\begin{array}{r}12.63 \\
5.17 \\
2.65 \\
5.94 \\
4.62 \\
5.85 \\
6.18 \\
11.94 \\
10.01 \\
2.28 \\
27.38\end{array}$ \\
\hline $\begin{array}{l}\text { Industrial Sector } \\
\text { Primary Energy }\end{array}$ & $\begin{array}{r}1.05 \\
.64 \\
.89\end{array}$ & $\begin{array}{l}3.42 \\
2.26 \\
2.62\end{array}$ & $\begin{array}{l}8.40 \\
5.20 \\
1.67\end{array}$ & $\begin{array}{l}8.68 \\
5.54 \\
2.38\end{array}$ & $\begin{array}{l}6.66 \\
3.85 \\
2.11\end{array}$ & $\begin{array}{l}7.64 \\
4.43 \\
2.35\end{array}$ & $\begin{array}{l}8.29 \\
4.32 \\
2.51\end{array}$ & $\begin{array}{l}8.83 \\
4.51 \\
2.57\end{array}$ & $\begin{array}{l}9.52 \\
5.03 \\
2.62\end{array}$ & $\begin{array}{l}9.45 \\
4.47 \\
2.65\end{array}$ \\
\hline 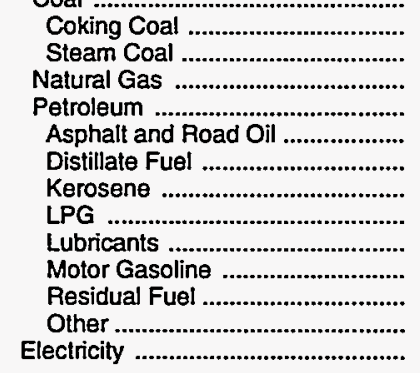 & $\begin{array}{r}.05 \\
.89 \\
1.03 \\
.60 \\
.63 \\
.74 \\
.81 \\
1.54 \\
5.08 \\
2.86 \\
.42 \\
1.86 \\
4.88\end{array}$ & $\begin{array}{r}2.02 \\
2.62 \\
2.28 \\
2.25 \\
1.95 \\
2.36 \\
2.62 \\
2.84 \\
7.48 \\
4.73 \\
2.05 \\
3.28 \\
11.21\end{array}$ & $\begin{array}{r}1.01 \\
1.67 \\
4.09 \\
5.92 \\
3.73 \\
5.59 \\
6.12 \\
5.52 \\
14.36 \\
9.69 \\
4.14 \\
8.66 \\
18.21\end{array}$ & $\begin{array}{r}2.00 \\
- \\
2.38 \\
5.24 \\
5.83 \\
5.21 \\
6.64 \\
7.61 \\
12.27 \\
17.61 \\
9.18 \\
4.31 \\
9.25 \\
20.47\end{array}$ & $\begin{array}{r}\overline{1} \\
2.11 \\
4.88 \\
3.67 \\
4.87 \\
4.54 \\
5.20 \\
11.25 \\
15.59 \\
7.25 \\
2.46 \\
6.58 \\
19.18\end{array}$ & $\begin{array}{r}2.00 \\
- \\
2.35 \\
4.13 \\
4.63 \\
3.57 \\
5.07 \\
5.81 \\
11.25 \\
12.70 \\
7.56 \\
2.93 \\
7.32 \\
20.32\end{array}$ & $\begin{array}{r}2.51 \\
- \\
2.51 \\
3.91 \\
4.62 \\
3.38 \\
4.75 \\
5.45 \\
11.10 \\
14.61 \\
7.90 \\
2.36 \\
6.31 \\
19.94\end{array}$ & $\begin{array}{r}2.0 / \\
- \\
2.57 \\
3.93 \\
4.93 \\
3.20 \\
4.96 \\
5.68 \\
10.43 \\
13.30 \\
8.50 \\
2.69 \\
7.21 \\
21.40\end{array}$ & $\begin{array}{r}2.04 \\
- \\
2.62 \\
4.00 \\
5.92 \\
3.36 \\
6.71 \\
7.68 \\
10.80 \\
13.40 \\
9.53 \\
3.05 \\
7.50 \\
23.14\end{array}$ & $\begin{array}{r}2.05 \\
- \\
2.65 \\
3.84 \\
5.34 \\
3.05 \\
5.40 \\
6.18 \\
11.94 \\
15.42 \\
10.01 \\
2.28 \\
6.95 \\
24.99\end{array}$ \\
\hline $\begin{array}{l}\text { Transportation Sector ....................... } \\
\text { Primary Energy ................................. }\end{array}$ & $\begin{array}{l}2.38 \\
2.37\end{array}$ & $\begin{array}{l}4.25 \\
4.24\end{array}$ & $\begin{array}{l}9.02 \\
9.01\end{array}$ & $\begin{array}{l}8.86 \\
8.84\end{array}$ & $\begin{array}{l}6.95 \\
6.93\end{array}$ & $\begin{array}{l}7.12 \\
7.09\end{array}$ & $\begin{array}{l}7.32 \\
7.28\end{array}$ & $\begin{array}{l}7.81 \\
7.77\end{array}$ & $\begin{array}{l}8.91 \\
8.88\end{array}$ & $\begin{array}{l}9.31 \\
9.27\end{array}$ \\
\hline 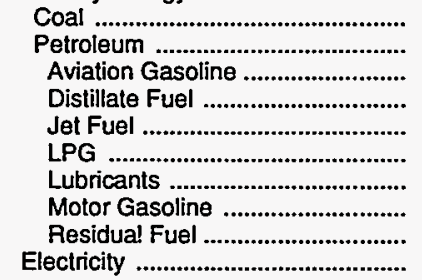 & $\begin{array}{r}.89 \\
2.37 \\
2.17 \\
1.35 \\
.75 \\
1.54 \\
5.08 \\
2.86 \\
.34 \\
5.66\end{array}$ & $\begin{array}{r}2.62 \\
4.24 \\
3.45 \\
2.90 \\
2.09 \\
2.84 \\
7.48 \\
4.73 \\
1.72 \\
14.77\end{array}$ & $\begin{array}{r}-\overline{9} \\
9.01 \\
9.02 \\
7.40 \\
6.51 \\
5.52 \\
14.36 \\
9.69 \\
3.22 \\
21.74\end{array}$ & $\begin{array}{r}-\overline{8.84} \\
9.99 \\
9.24 \\
6.04 \\
12.27 \\
17.61 \\
9.18 \\
3.77 \\
23.83\end{array}$ & $\begin{array}{r}-\overline{3} \\
6.93 \\
8.41 \\
7.07 \\
4.18 \\
11.25 \\
15.59 \\
7.25 \\
2.09 \\
22.13\end{array}$ & $\begin{array}{r}7.09 \\
7.55 \\
6.82 \\
4.12 \\
11.25 \\
12.70 \\
7.56 \\
2.55 \\
30.14\end{array}$ & $\begin{array}{r}- \\
7.28 \\
7.41 \\
6.90 \\
4.02 \\
11.10 \\
14.61 \\
7.90 \\
2.00 \\
28.58\end{array}$ & $\begin{array}{r}- \\
7.77 \\
8.28 \\
7.43 \\
4.61 \\
10.43 \\
13.30 \\
8.50 \\
2.33 \\
30.55\end{array}$ & $\begin{array}{r}-\overline{8} \\
8.88 \\
9.32 \\
9.37 \\
5.83 \\
10.80 \\
13.40 \\
9.53 \\
2.44 \\
31.94\end{array}$ & $\begin{array}{r}-\overline{9} \\
8.27 \\
9.22 \\
5.00 \\
11.94 \\
15.42 \\
10.01 \\
1.94 \\
34.15\end{array}$ \\
\hline $\begin{array}{l}\text { Total Energy ................................... } \\
\text { Primary Energy - Four Sectors ...... } \\
\text { Electricity ............................................ }\end{array}$ & $\begin{array}{l}1.90 \\
1.49 \\
7.29\end{array}$ & $\begin{array}{r}4.15 \\
3.19 \\
13.93\end{array}$ & $\begin{array}{r}8.90 \\
7.18 \\
21.13\end{array}$ & $\begin{array}{r}10.09 \\
7.80 \\
24.34\end{array}$ & $\begin{array}{r}8.39 \\
6.12 \\
22.70\end{array}$ & $\begin{array}{r}8.71 \\
6.23 \\
23.46\end{array}$ & $\begin{array}{r}8.85 \\
6.36 \\
22.86\end{array}$ & $\begin{array}{r}9.45 \\
6.83 \\
24.39\end{array}$ & $\begin{array}{r}10.52 \\
7.69 \\
25.93\end{array}$ & $\begin{array}{r}10.84 \\
7.67 \\
27.94\end{array}$ \\
\hline 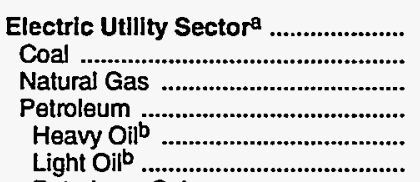 & $\begin{array}{l}.37 \\
.31 \\
.33 \\
.38 \\
.38 \\
.43\end{array}$ & $\begin{array}{l}1.66 \\
1.31 \\
1.32 \\
1.93 \\
1.93 \\
2.17\end{array}$ & $\begin{array}{l}3.41 \\
1.95 \\
3.40 \\
3.86 \\
3.84 \\
6.00\end{array}$ & $\begin{array}{l}2.74 \\
1.97 \\
3.41 \\
3.97 \\
3.91 \\
5.80\end{array}$ & $\begin{array}{l}2.03 \\
1.78 \\
2.27 \\
2.27 \\
2.23 \\
3.54\end{array}$ & $\begin{array}{l}2.26 \\
1.61 \\
2.40 \\
2.73 \\
2.71 \\
3.22\end{array}$ & $\begin{array}{l}2.04 \\
1.61 \\
2.22 \\
2.32 \\
2.26 \\
3.63\end{array}$ & $\begin{array}{l}2.18 \\
1.60 \\
2.37 \\
2.72 \\
2.64 \\
4.22\end{array}$ & $\begin{array}{l}2.15 \\
1.73 \\
2.40 \\
2.91 \\
2.86 \\
5.41\end{array}$ & $\begin{array}{l}1.85 \\
1.73 \\
2.18 \\
2.24 \\
2.20 \\
4.58\end{array}$ \\
\hline 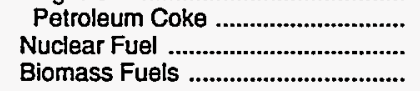 &.$\overline{-}$ &.$\overline{18}$ &.$\overline{-}$ &.$\overline{60}$ & $\begin{array}{r}-88 \\
-\end{array}$ & $\begin{array}{r}- \\
.92 \\
-\end{array}$ & $\begin{array}{c}- \\
-96 \\
-\end{array}$ & $\begin{array}{r}- \\
.70 \\
-\end{array}$ & .62 &. \\
\hline Primary Energy - Flve Sectors ${ }^{c}$.. & 1.25 & 2.83 & 6.04 & 6.22 & 4.96 & 5.10 & 5.12 & $\mathbf{5 . 4 0}$ & 5.99 & 5.92 \\
\hline
\end{tabular}

a There are no direct fuel costs for hydroelectric, geothermal, centralized solar, or wind energy.

b Heavy oil includes Grade Nos. 4, 5, and 6, and residual fuel oils. Light oil includes Grade No. 2 heating oil, kerosene, and jet fuel.

c Biomass fuels are not included, except those consumed at electric utilities and those added to motor gasoline.

-No consumplion, including cases where adjustments were made. See "Consumption Adjustments for Process Fuel and Intermediate Products."

Sources: Data sources, estimation procedures, and assumptions are described in the "Documentation" section of this report. 


\begin{tabular}{|c|c|c|c|c|c|c|c|c|c|c|}
\hline Sector and Energy Source & 1970 & 1975 & 1980 & 1985 & 1986 & 1987 & 1988 & 1989 & 1990 & 1991 \\
\hline 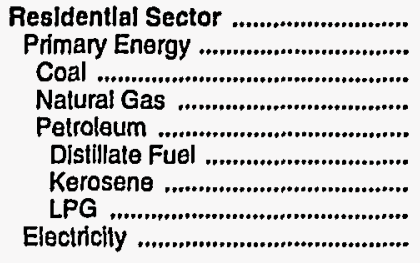 & $\begin{array}{r}793.8 \\
520.1 \\
2.6 \\
158.6 \\
358.9 \\
334.9 \\
13.2 \\
10.7 \\
273.7\end{array}$ & $\begin{array}{r}1,501.2 \\
945.4 \\
3.3 \\
284.4 \\
657.7 \\
628.7 \\
10.6 \\
18.4 \\
555.7\end{array}$ & $\begin{array}{r}2,362.9 \\
1,487.2 \\
5.2 \\
511.9 \\
970.1 \\
932.9 \\
14.9 \\
22.3 \\
875.7\end{array}$ & \begin{tabular}{r|}
$2,844.0$ \\
$1,692.1$ \\
7.2 \\
765.7 \\
919.2 \\
847.8 \\
29.3 \\
42.1 \\
$1,151.9$
\end{tabular} & $\begin{array}{r}2,620.5 \\
1,488.8 \\
4.1 \\
758.4 \\
726.3 \\
669.5 \\
19.5 \\
37.3 \\
1,131.8\end{array}$ & $\begin{array}{r}2,694.3 \\
1,433.9 \\
3.1 \\
696.2 \\
734.6 \\
660.3 \\
20.2 \\
54.1 \\
1,260.4\end{array}$ & $\begin{array}{r}2,759.2 \\
1,442.2 \\
3.2 \\
702.8 \\
736.2 \\
671.1 \\
11.7 \\
53.4 \\
1,317.0\end{array}$ & \begin{tabular}{r|}
$3,165.3$ \\
$1,728.2$ \\
2.7 \\
799.5 \\
926.0 \\
838.4 \\
11.9 \\
75.7 \\
$1,437.0$
\end{tabular} & $\begin{array}{r}3,243.9 \\
1,738.9 \\
3.1 \\
835.2 \\
900.6 \\
826.4 \\
8.4 \\
65.8 \\
1,505.0\end{array}$ & $\begin{array}{r}3,264.1 \\
1,665.1 \\
1.5 \\
835.0 \\
828.6 \\
755.8 \\
7.4 \\
65.4 \\
1,599.0\end{array}$ \\
\hline 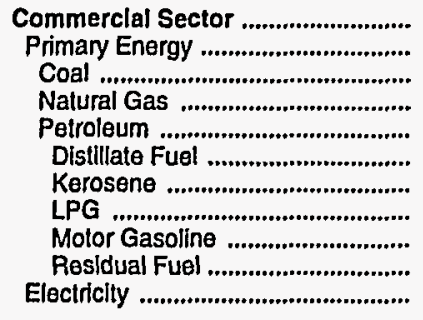 & $\begin{array}{r}390.4 \\
176.2 \\
1.7 \\
50.1 \\
124.4 \\
86.4 \\
.5 \\
1.0 \\
1.5 \\
35.0 \\
214.2\end{array}$ & $\begin{array}{r}964.2 \\
404.6 \\
2.6 \\
100.1 \\
301.8 \\
187.9 \\
.7 \\
1.9 \\
2.7 \\
108.6 \\
559.6\end{array}$ & $\begin{array}{r}1,645.4 \\
662.0 \\
2.0 \\
252.5 \\
407.5 \\
278.0 \\
1.0 \\
2.4 \\
9.7 \\
116.3 \\
983.4\end{array}$ & $\begin{array}{r}1,971.0 \\
631.5 \\
3.9 \\
291.5 \\
336.2 \\
228.9 \\
4.6 \\
8.0 \\
9.1 \\
85.6 \\
1,339.5\end{array}$ & $\begin{array}{r}1,881.7 \\
557.8 \\
1.9 \\
290.4 \\
265.4 \\
188.4 \\
8.6 \\
8.2 \\
7.2 \\
53.0 \\
1,323.9\end{array}$ & $\begin{array}{r}1,938.2 \\
519.6 \\
1.5 \\
275.4 \\
242.7 \\
171.1 \\
1.6 \\
9.9 \\
7.7 \\
52.4 \\
1,418.6\end{array}$ & $\begin{array}{r}1,995.7 \\
540.8 \\
2.1 \\
298.9 \\
239.9 \\
169.8 \\
2.2 \\
9.6 \\
7.6 \\
50.8 \\
1,454.9\end{array}$ & $\begin{array}{r}2,225.4 \\
636.9 \\
1.9 \\
302.4 \\
332.6 \\
246.1 \\
2.1 \\
10.8 \\
8.4 \\
65.4 \\
1,588.6\end{array}$ & $\begin{array}{r}2,357.0 \\
660.3 \\
2.2 \\
321.4 \\
336.6 \\
231.3 \\
5.5 \\
9.4 \\
3.4 \\
87.0 \\
1,696.7\end{array}$ & $\begin{array}{r}2,498.4 \\
679.7 \\
.8 \\
328.2 \\
350.8 \\
259.4 \\
7.0 \\
9.4 \\
9.6 \\
65.4 \\
1,818.6\end{array}$ \\
\hline 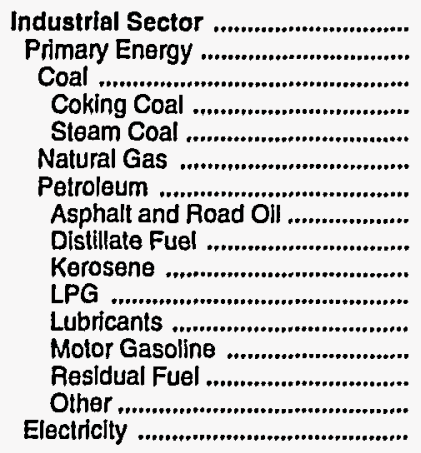 & $\begin{array}{r}277.7 \\
154.3 \\
3.2 \\
- \\
3.2 \\
23.5 \\
127.6 \\
12.0 \\
12.5 \\
2.5 \\
4.0 \\
15.6 \\
1.7 \\
68.1 \\
11.2 \\
123.4\end{array}$ & $\begin{array}{r}660.3 \\
380.0 \\
6.9 \\
- \\
6.9 \\
55.0 \\
318.1 \\
23.7 \\
36.5 \\
3.4 \\
11.6 \\
16.0 \\
2.0 \\
205.3 \\
19.6 \\
280.3\end{array}$ & $\begin{array}{r}989.3 \\
461.9 \\
4.0 \\
- \\
4.0 \\
120.2 \\
337.7 \\
30.5 \\
61.5 \\
12.0 \\
26.5 \\
32.9 \\
4.6 \\
69.3 \\
100.5 \\
527.3\end{array}$ & $\begin{array}{r}1,330.1 \\
669.7 \\
10.4 \\
- \\
10.4 \\
177.8 \\
481.4 \\
36.4 \\
40.4 \\
2.3 \\
19.8 \\
36.7 \\
17.7 \\
227.8 \\
100.5 \\
660.4\end{array}$ & $\begin{array}{r}1,199.0 \\
565.5 \\
5.9 \\
- \\
5.9 \\
119.7 \\
439.9 \\
36.0 \\
41.4 \\
1.1 \\
36.0 \\
31.8 \\
14.4 \\
210.0 \\
69.3 \\
633.5\end{array}$ & $\begin{array}{r}1,295.8 \\
599.7 \\
9.8 \\
- \\
9.8 \\
145.1 \\
444.8 \\
35.0 \\
114.2 \\
1.4 \\
40.5 \\
29.3 \\
15.5 \\
131.4 \\
77.6 \\
696.2\end{array}$ & $\begin{array}{r}1,141.4 \\
444.4 \\
8.9 \\
-\overline{8} \\
8.9 \\
127.3 \\
308.3 \\
39.6 \\
67.9 \\
1.5 \\
30.0 \\
32.4 \\
17.3 \\
54.2 \\
65.4 \\
697.0\end{array}$ & $\begin{array}{r}1,222.3 \\
464.3 \\
7.3 \\
- \\
7.3 \\
144.8 \\
312.1 \\
30.3 \\
73.3 \\
1.8 \\
24.5 \\
30.3 \\
21.2 \\
57.9 \\
72.8 \\
758.0\end{array}$ & $\begin{array}{r}1,329.7 \\
527.7 \\
4.8 \\
-\overline{8} \\
4.8 \\
183.5 \\
339.4 \\
29.9 \\
85.0 \\
.8 \\
38.1 \\
31.4 \\
20.6 \\
50.6 \\
83.0 \\
802.0\end{array}$ & $\begin{array}{r}1,300.7 \\
465.7 \\
5.6 \\
- \\
5.6 \\
218.5 \\
241.7 \\
40.0 \\
37.6 \\
.6 \\
17.4 \\
32.3 \\
17.4 \\
20.2 \\
76.1 \\
835.0\end{array}$ \\
\hline 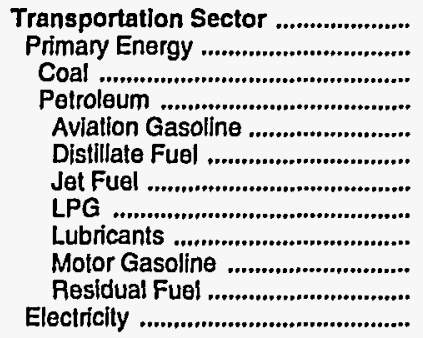 & $\begin{array}{r}824.4 \\
822.8 \\
822.8 \\
3.0 \\
25.2 \\
33.3 \\
.2 \\
13.6 \\
740.6 \\
7.0 \\
1.6\end{array}$ & $\begin{array}{r}1,563.4 \\
1,558.0 \\
1,558.0 \\
4.0 \\
75.8 \\
94.4 \\
.3 \\
19.6 \\
1,352.5 \\
11.3 \\
5.4\end{array}$ & $\begin{array}{r}3,214.8 \\
3,202.9 \\
3,202.9 \\
12.5 \\
211.1 \\
315.5 \\
.5 \\
40.4 \\
2,604.7 \\
18.2 \\
11.9\end{array}$ & $\begin{array}{r}3,350.9 \\
3,336.5 \\
- \\
3,336.5 \\
6.8 \\
405.4 \\
238.4 \\
3.1 \\
45.0 \\
2,617.1 \\
20.7 \\
14.4\end{array}$ & $\begin{array}{r}2,679.3 \\
2,666.6 \\
- \\
2,666.6 \\
6.2 \\
321.4 \\
163.4 \\
2.5 \\
39.0 \\
2,126.1 \\
8.0 \\
12.7\end{array}$ & $\begin{array}{r}2,835.3 \\
2,818.4 \\
- \\
2,818.4 \\
4.7 \\
324.0 \\
182.9 \\
2.1 \\
35.9 \\
2,261.4 \\
7.4 \\
16.8\end{array}$ & $\begin{array}{r}3,045.5 \\
3,025.4 \\
- \\
3,025.4 \\
4.8 \\
316.8 \\
212.1 \\
2.5 \\
39.8 \\
2,440.9 \\
8.5 \\
20.1\end{array}$ & $\begin{array}{r}3,253.9 \\
3,236.5 \\
-\overline{3,236.5} \\
4.9 \\
342.3 \\
260.8 \\
2.4 \\
37.2 \\
2,571.6 \\
17.4 \\
17.4\end{array}$ & $\begin{array}{r}3,582.2 \\
3,564.9 \\
- \\
3,564.9 \\
4.5 \\
409.8 \\
323.3 \\
2.3 \\
38.6 \\
2,765.1 \\
21.2 \\
17.4\end{array}$ & $\begin{array}{r}3,534.5 \\
3,516.2 \\
- \\
3,516.2 \\
2.0 \\
390.5 \\
238.4 \\
3.0 \\
39.7 \\
2,837.2 \\
5.4 \\
18.3\end{array}$ \\
\hline $\begin{array}{l}\text { Total Energy ..................................... } \\
\text { Primary Energy - Four Sectors ...... } \\
\text { Electricity ......................................... }\end{array}$ & $\begin{array}{r}2,286.3 \\
1,673.3 \\
612.9\end{array}$ & $\begin{array}{l}4,689.0 \\
3,288.0 \\
1,401.0\end{array}$ & $\begin{array}{l}8,212.4 \\
5,814.0 \\
2,398.4\end{array}$ & $\begin{array}{l}9,496.0 \\
6,329.8 \\
3,166.2\end{array}$ & $\begin{array}{l}8,380.5 \\
5,278.6 \\
3,101.9\end{array}$ & $\begin{array}{l}8,763.6 \\
5,371.6 \\
3,392.0\end{array}$ & $\begin{array}{l}8,941.8 \\
5,452.8 \\
3,489.0\end{array}$ & $\begin{array}{l}9,866.9 \\
6,065.9 \\
3,801.0\end{array}$ & $\begin{array}{r}10,512.8 \\
6,491.8 \\
4,021.1\end{array}$ & $\begin{array}{r}10,597.7 \\
6,326.7 \\
4,271.0\end{array}$ \\
\hline 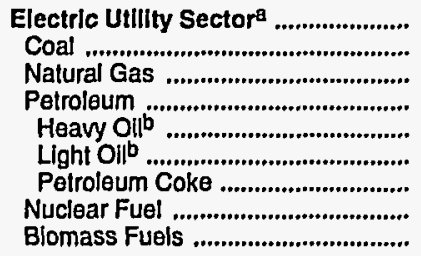 & $\begin{array}{r}112.4 \\
4.2 \\
1.9 \\
103.7 \\
100.8 \\
2.9 \\
-\overline{7} \\
2.7 \\
-\end{array}$ & $\begin{array}{r}524.9 \\
25.6 \\
1.9 \\
490.0 \\
483.6 \\
6.3 \\
\overline{7.5} \\
-\end{array}$ & $\begin{array}{r}1,191.4 \\
35.2 \\
17.3 \\
1,124.6 \\
1,103.1 \\
21.5 \\
-\overline{3} \\
14.3\end{array}$ & $\begin{array}{r}1,011.3 \\
202.4 \\
160.1 \\
609.0 \\
581.3 \\
27.8 \\
-\overline{8} \\
39.8 \\
-\end{array}$ & $\begin{array}{r}698.3 \\
169.0 \\
34.7 \\
471.5 \\
450.3 \\
21.1 \\
- \\
23.1 \\
-\end{array}$ & $\begin{array}{r}782.8 \\
180.8 \\
98.9 \\
491.8 \\
470.7 \\
21.1 \\
- \\
11.3 \\
-\end{array}$ & $\begin{array}{r}704.3 \\
180.0 \\
45.4 \\
467.3 \\
434.9 \\
32.4 \\
- \\
11.5 \\
-\end{array}$ & $\begin{array}{r}862.8 \\
186.5 \\
120.5 \\
533.1 \\
492.5 \\
40.6 \\
\overrightarrow{-} \\
22.7 \\
-\end{array}$ & $\begin{array}{r}801.1 \\
190.3 \\
139.5 \\
437.7 \\
422.3 \\
15.4 \\
- \\
33.6 \\
-\end{array}$ & $\begin{array}{r}661.1 \\
196.7 \\
89.0 \\
346.4 \\
333.8 \\
12.6 \\
-\overline{2} \\
29.0 \\
-\end{array}$ \\
\hline Primary Energy - Flve Sectorsc .. & $1,785.8$ & $3,812.9$ & $7,005.4$ & $7,341.1$ & $5,976.9$ & $6,154.4$ & $6,157.1$ & $6,928.7$ & $7,292.9$ & $6,987.8$ \\
\hline
\end{tabular}

a There are no direct fuel costs for hydroelectric, geothermal, centralized solar, or wind energy.

beavy oil includes Grade Nos. 4, 5, and 6, and residual fuel oils. Light oil includes Grade No. 2 heating oil, kerosene, and jet fuel.

c Biomass fuels are not included, except those consumed at electric utilities and those added to motor gasoline.

- No consumption, including cases where adjustments were made. See "Consumption Adjustments for Process Fuel and Intermediate Products."

- Value less than 0.05 million dollars.

Note: Totals may not equal sum of components due to independent rounding.

Sources: Data sources, estimation procedures, and assumptions are described in the "Documentation" section of this report. 


\section{Energy Price and Expenditure Estimates by Source, Michigan \\ I 1970, 1975, 1980, and 1985-1991}

\begin{tabular}{|c|c|c|c|c|c|c|c|c|c|c|}
\hline Energy Source & 1970 & 1975 & 1980 & 1985 & 1986 & 1987 & 1988 & 1989 & 1990 & 1991 \\
\hline & \multicolumn{10}{|c|}{ Prices in Dollars per Million Btu } \\
\hline Coal & 0.44 & 1.23 & 1.71 & 1.92 & 1.84 & 1.76 & 1.76 & 1.72 & 1.63 & 1.62 \\
\hline Coking Coal ....................................... & .55 & 2.07 & 2.27 & 2.08 & 1.95 & 1.85 & 1.73 & 1.75 & 1.80 & 1.72 \\
\hline 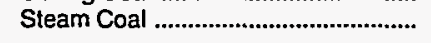 & .42 & 1.04 & 1.62 & 1.90 & 1.83 & 1.75 & 1.76 & 1.72 & 1.63 & 1.62 \\
\hline Natural Gas & .77 & 1.42 & 3.05 & 5.70 & 5.12 & 4.96 & 4.80 & 4.61 & 4.33 & 4.43 \\
\hline $\begin{array}{l}\text { Petroleum } \\
\text { Asphalt and Road Oil }\end{array}$ & $\begin{array}{r}2.00 \\
.84\end{array}$ & $\begin{array}{l}3.72 \\
2.12\end{array}$ & $\begin{array}{l}8.48 \\
4.04\end{array}$ & $\begin{array}{l}8.67 \\
5.00\end{array}$ & $\begin{array}{l}6.54 \\
3.59\end{array}$ & $\begin{array}{l}6.77 \\
3.40\end{array}$ & $\begin{array}{l}6.65 \\
3.21\end{array}$ & $\begin{array}{l}7.28 \\
2.84\end{array}$ & $\begin{array}{l}8.21 \\
3.06\end{array}$ & $\begin{array}{l}7.89 \\
2.87\end{array}$ \\
\hline 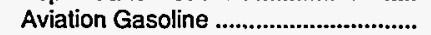 & 2.17 & 3.45 & 9.02 & 9.99 & 8.41 & 7.55 & 7.41 & 8.28 & 9.32 & 8.71 \\
\hline 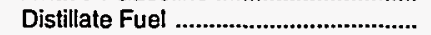 & 1.09 & 2.49 & 6.76 & 7.71 & 5.73 & 6.09 & 5.74 & 6.37 & 7.53 & 6.98 \\
\hline Jet Fuel & .74 & 2.08 & 6.38 & 6.09 & 4.20 & 4.09 & 3.85 & 4.39 & 5.65 & 4.94 \\
\hline 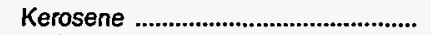 & .89 & 2.60 & 6.29 & 8.52 & 6.56 & 6.37 & 6.41 & 7.06 & 8.28 & 7.60 \\
\hline 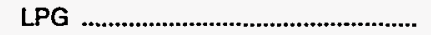 & 1.89 & 3.76 & 6.62 & 8.74 & 8.22 & 7.88 & 7.73 & 9.79 & 10.26 & 10.13 \\
\hline 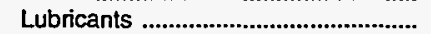 & 5.08 & 7.48 & 14.36 & 17.61 & 15.59 & 12.70 & 14.61 & 13.30 & 13.40 & 15.42 \\
\hline 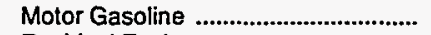 & 2.71 & 4.72 & 10.09 & 9.10 & 6.79 & 7.13 & 7.15 & 7.77 & 8.78 & 8.37 \\
\hline 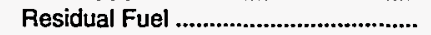 & .59 & 1.96 & 3.90 & 4.45 & 2.84 & 3.00 & 2.86 & 3.01 & 3.00 & 2.54 \\
\hline Other ${ }^{3}$ & 1.25 & 2.92 & 7.80 & 8.21 & 5.71 & 6.46 & 5.50 & 6.00 & 6.69 & 5.95 \\
\hline 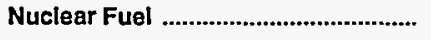 & .36 & .28 & .49 & .80 & .77 & .77 & .88 & .78 & .79 & .65 \\
\hline Blomass Fuels at Utlities ................... & - & - & - & - & - & - & - & - & - & - \\
\hline Primary Energy - Five Sectors ${ }^{b}$..... & 1.12 & 2.23 & 4.39 & 5.18 & 4.30 & 4.26 & 4.21 & 4.33 & 4.49 & 4.38 \\
\hline $\begin{array}{l}\text { Electric Utility Fuelc } \\
\text { Electricity Purchased by End Users }\end{array}$ & $\begin{array}{r}.38 \\
5.55\end{array}$ & $\begin{array}{l}1.02 \\
9.78\end{array}$ & $\begin{array}{r}1.58 \\
15.40\end{array}$ & $\begin{array}{r}1.72 \\
19.88\end{array}$ & $\begin{array}{r}1.66 \\
20.35\end{array}$ & $\begin{array}{r}1.60 \\
19.26\end{array}$ & $\begin{array}{r}1.60 \\
19.39\end{array}$ & $\begin{array}{r}1.52 \\
19.91\end{array}$ & $\begin{array}{r}1.41 \\
20.85\end{array}$ & $\begin{array}{r}1.32 \\
21.18\end{array}$ \\
\hline \multirow[t]{2}{*}{ 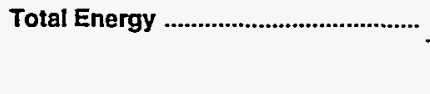 } & 1.70 & 3.33 & 6.61 & 8.48 & 7.50 & 7.60 & 7.44 & 7.72 & 8.07 & 8.14 \\
\hline & \multicolumn{10}{|c|}{ Expenditures in Millians of Dollars } \\
\hline Coal & 363.6 & 925.0 & $1,299.2$ & $1,499.0$ & $1,496.4$ & $1,475.4$ & $1,458.6$ & $1,375.0$ & $1,283.4$ & $1,231.9$ \\
\hline 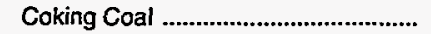 & 73.4 & 290.3 & 250.1 & 149.7 & 140.3 & 53.0 & 38.0 & 44.2 & 51.3 & 26.3 \\
\hline 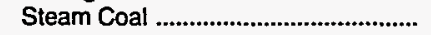 & 290.3 & 634.7 & $1,049.1$ & $1,349.3$ & $1,356.1$ & $1,422.4$ & $1,420.6$ & $1,330.8$ & $1,232.1$ & $1,205.6$ \\
\hline 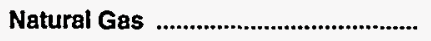 & 620.2 & $1,235.6$ & $2,596.2$ & $3,954.1$ & $3,376.6$ & $3,189.8$ & $3,507.2$ & $3,517.4$ & $3,433.8$ & $3,538.2$ \\
\hline Petroleum & $1,925.7$ & $4,020.3$ & $8,007.8$ & $7,044.2$ & $5,582.6$ & $5,948.9$ & $6,087.6$ & $6,594.1$ & $7,156.4$ & $7,034.3$ \\
\hline 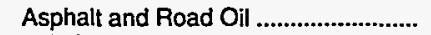 & 21.5 & 54.6 & 94.0 & 92.2 & 80.6 & 79.0 & 61.2 & 72.8 & 80.1 & 66.0 \\
\hline 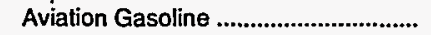 & 7.9 & 6.0 & 22.2 & 10.1 & 10.6 & 9.2 & 9.0 & 11.2 & 10.1 & 9.0 \\
\hline 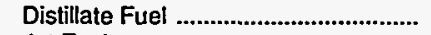 & 240.6 & 610.6 & $1,087.9$ & $1,140.8$ & 883.6 & 897.7 & 923.9 & 922.8 & $1,022.2$ & $1,015.5$ \\
\hline 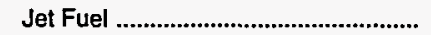 & 30.4 & 66.8 & 236.9 & 223.6 & 167.7 & 191.9 & 185.4 & 227.7 & 319.7 & 272.0 \\
\hline 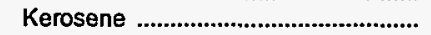 & 15.7 & 19.9 & 44.0 & 24.5 & 15.6 & 15.2 & 17.2 & 20.7 & 12.7 & 15.5 \\
\hline LPG & 43.9 & 103.9 & 163.6 & 440.4 & 462.2 & 501.6 & 482.0 & 656.8 & 545.0 & 660.7 \\
\hline 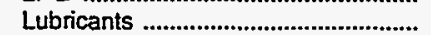 & 97.3 & 124.9 & 285.1 & 318.2 & 275.4 & 253.7 & 281.5 & 262.7 & 272.5 & 280.5 \\
\hline 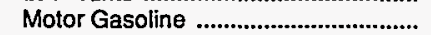 & $1,378.2$ & $2,686.4$ & $5,144.7$ & $4,465.3$ & $3,423.8$ & $3,706.1$ & $3,848.7$ & $4,127.1$ & $4,581.8$ & $4,456.4$ \\
\hline 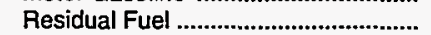 & 33.7 & 217.0 & 315.1 & 56.0 & 50.6 & 51.2 & 74.0 & 75.1 & 44.2 & 23.2 \\
\hline Othera & 56.4 & 130.2 & 614.4 & 273.2 & 212.6 & 243.2 & 204.7 & 217.2 & 268.1 & 235.5 \\
\hline 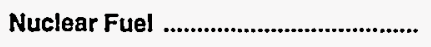 & 1.5 & 22.2 & 85.1 & 117.0 & 102.1 & 119.2 & 169.0 & 178.2 & 181.4 & 189.1 \\
\hline Biomass Fuels at Utilitles ...................... & - & - & - & - & - & - & - & - & - & - \\
\hline Primary Energy - Five Sectors ${ }^{b}$.... & $2,911.0$ & $6,203.2$ & $11,988.3$ & $12,614.4$ & $10,557.8$ & $10,733.3$ & $11,222.5$ & $11,664.7$ & $12,055.0$ & $11,993.5$ \\
\hline $\begin{array}{l}\text { Electric Utility Fuelc } \\
\text { Electricity Purchased by End Users }\end{array}$ & $\begin{array}{r}-223.6 \\
1,041.7\end{array}$ & $\begin{array}{r}-733.0 \\
2,139.6\end{array}$ & $\begin{array}{r}-1,246.3 \\
3,647.5\end{array}$ & $\begin{array}{r}-1,312.2 \\
4,993.3\end{array}$ & $\begin{array}{r}-1,294.4 \\
5,231.3\end{array}$ & $\begin{array}{r}-1,413.6 \\
5,127.9\end{array}$ & $\begin{array}{r}-1,448.5 \\
5,397.5\end{array}$ & $\begin{array}{r}-1,403.8 \\
5,572.4\end{array}$ & $\begin{array}{r}-1,280.4 \\
5,796.9\end{array}$ & $\begin{array}{r}-1,281.2 \\
6,041.6\end{array}$ \\
\hline 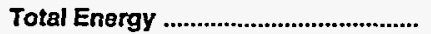 & $3,729.2$ & $7,609.8$ & $14,389.5$ & $16,295.4$ & $14,494.6$ & $14,447.6$ & $15,171.4$ & $15,833,2$ & $16,571.4$ & $16,753.8$ \\
\hline
\end{tabular}

Includes petroleum coke used at electric utilities.

b Biomass fuels are not included, except those consumed at electric utilities and those added to motor gasoline.

c There are no direct fuel costs for hydroelectric, geothermal, centralized solar, or wind energy.

-No consumption, including cases where adjustments were made. See "Consumption Adjustments for Process Fuel and Intermediate Products."

Note: Expenditure totals may not equal sum of components due to independent rounding.

Sources: Data sources, estimation procedures, and assumptions are described in the "Documentation" section of this report. 


\begin{tabular}{|c|c|c|c|c|c|c|c|c|c|c|}
\hline Sector and Energy Source & 1970 & 1975 & 1980 & 1985 & 1986 & 1987 & 1988 & 1989 & 1990 & 1991 \\
\hline 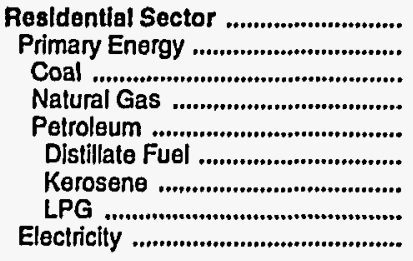 & $\begin{array}{l}1.74 \\
1.10 \\
1.43 \\
1.00 \\
1.35 \\
1.23 \\
1.56 \\
2.07 \\
6.99\end{array}$ & $\begin{array}{r}3.14 \\
1.93 \\
3.06 \\
1.58 \\
2.78 \\
2.51 \\
3.12 \\
4.29 \\
11.32\end{array}$ & $\begin{array}{r}5.56 \\
3.72 \\
3.70 \\
3.13 \\
7.25 \\
7.05 \\
8.43 \\
8.07 \\
16.76\end{array}$ & $\begin{array}{r}8.82 \\
6.41 \\
3.86 \\
6.14 \\
8.30 \\
7.66 \\
8.77 \\
9.63 \\
21.62\end{array}$ & $\begin{array}{r}8.39 \\
5.69 \\
3.45 \\
5.53 \\
6.81 \\
6.07 \\
6.96 \\
8.16 \\
22.13\end{array}$ & $\begin{array}{r}8.33 \\
5.51 \\
3.48 \\
5.29 \\
6.79 \\
5.81 \\
6.65 \\
8.10 \\
21.45\end{array}$ & $\begin{array}{r}8.11 \\
5.34 \\
3.45 \\
5.14 \\
6.61 \\
5.81 \\
6.65 \\
7.74 \\
21.68\end{array}$ & $\begin{array}{r}8.21 \\
5.46 \\
3.46 \\
4.93 \\
9.10 \\
6.40 \\
7.32 \\
12.10 \\
22.17\end{array}$ & $\begin{array}{r}8.53 \\
5.37 \\
3.39 \\
4.80 \\
9.43 \\
7.57 \\
8.66 \\
11.37 \\
22.94\end{array}$ & $\begin{array}{r}8.71 \\
5.36 \\
3.15 \\
4.88 \\
8.56 \\
7.06 \\
8.09 \\
10.11 \\
23.64\end{array}$ \\
\hline 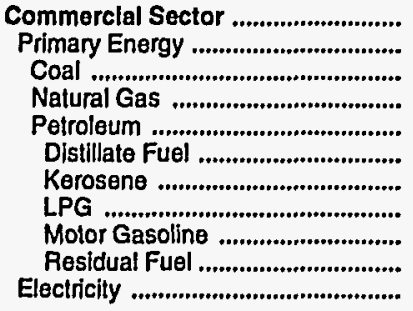 & $\begin{array}{r}2.11 \\
.88 \\
.53 \\
.83 \\
1.23 \\
1.05 \\
.74 \\
1.39 \\
2.71 \\
.64 \\
7.12\end{array}$ & $\begin{array}{r}3.40 \\
1.63 \\
1.49 \\
1.45 \\
2.69 \\
2.33 \\
2.44 \\
2.51 \\
4.72 \\
1.97 \\
11.41\end{array}$ & $\begin{array}{r}6.38 \\
3.53 \\
1.82 \\
3.13 \\
6.86 \\
6.53 \\
6.14 \\
5.16 \\
10.09 \\
3.97 \\
17.60\end{array}$ & $\begin{array}{r}10.12 \\
5.68 \\
1.99 \\
5.61 \\
6.84 \\
6.24 \\
7.21 \\
8.32 \\
9.10 \\
4.39 \\
23.36\end{array}$ & $\begin{array}{r}10.26 \\
4.90 \\
1.93 \\
5.03 \\
4.66 \\
3.67 \\
4.70 \\
8.25 \\
6.79 \\
3.02 \\
24.24\end{array}$ & $\begin{array}{r}9.12 \\
4.91 \\
1.73 \\
4.88 \\
5.58 \\
4.36 \\
5.39 \\
7.76 \\
7.13 \\
2.90 \\
22.31\end{array}$ & $\begin{array}{r}9.43 \\
4.81 \\
1.73 \\
4.81 \\
5.17 \\
4.03 \\
5.11 \\
7.73 \\
7.15 \\
3.20 \\
22.48\end{array}$ & $\begin{array}{r}9.49 \\
4.69 \\
1.68 \\
4.61 \\
5.77 \\
4.32 \\
6.24 \\
8.30 \\
7.77 \\
3.13 \\
23.20\end{array}$ & $\begin{array}{r}10.17 \\
4.62 \\
1.77 \\
4.43 \\
6.93 \\
5.35 \\
6.66 \\
9.36 \\
8.78 \\
3.15 \\
24.21\end{array}$ & $\begin{array}{r}10.22 \\
4.67 \\
1.80 \\
4.53 \\
6.63 \\
4.74 \\
5.90 \\
10.15 \\
8.37 \\
2.79 \\
24.16\end{array}$ \\
\hline 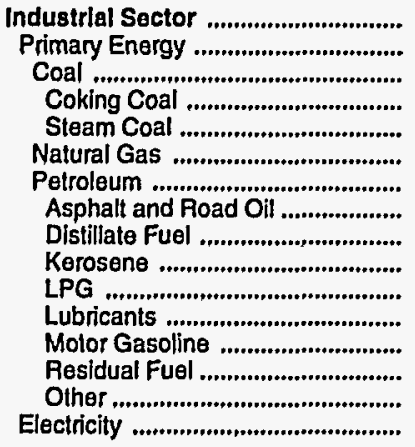 & $\begin{array}{r}1.01 \\
.71 \\
.54 \\
.55 \\
.53 \\
.53 \\
1.27 \\
.84 \\
.68 \\
.74 \\
1.39 \\
5.08 \\
2.71 \\
.54 \\
1.25 \\
3.74\end{array}$ & $\begin{array}{l}2.54 \\
1.81 \\
1.82 \\
2.07 \\
1.49 \\
1.22 \\
2.83 \\
2.12 \\
2.27 \\
2.44 \\
2.51 \\
7.48 \\
4.72 \\
1.98 \\
2.92 \\
7.83\end{array}$ & $\begin{array}{r}5.00 \\
3.68 \\
2.04 \\
2.27 \\
1.82 \\
2.87 \\
6.78 \\
4.04 \\
5.56 \\
6.14 \\
5.16 \\
14.36 \\
10.09 \\
3.23 \\
7.80 \\
13.18\end{array}$ & $\begin{array}{r}7.02 \\
4.75 \\
2.03 \\
2.08 \\
1.99 \\
4.95 \\
8.01 \\
5.00 \\
6.51 \\
7.21 \\
8.32 \\
17.61 \\
9.10 \\
4.39 \\
8.21 \\
16.75\end{array}$ & $\begin{array}{r}6.49 \\
4.08 \\
1.94 \\
1.95 \\
1.93 \\
4.40 \\
6.24 \\
3.59 \\
4.24 \\
4.70 \\
8.25 \\
15.59 \\
6.79 \\
3.02 \\
5.71 \\
16.88\end{array}$ & $\begin{array}{r}6.92 \\
4.25 \\
1.76 \\
1.85 \\
1.73 \\
4.29 \\
6.30 \\
3.40 \\
4.87 \\
5.39 \\
7.76 \\
12.70 \\
7.13 \\
2.90 \\
6.46 \\
15.95\end{array}$ & $\begin{array}{r}6.53 \\
4.10 \\
1.73 \\
1.73 \\
1.73 \\
4.19 \\
6.11 \\
3.21 \\
4.61 \\
5.11 \\
7.73 \\
14.61 \\
7.15 \\
3.20 \\
5.50 \\
15.94\end{array}$ & $\begin{array}{r}6.68 \\
4.16 \\
1.69 \\
1.75 \\
1.68 \\
4.01 \\
6.41 \\
2.84 \\
5.63 \\
6.24 \\
8.30 \\
13.30 \\
7.77 \\
3.13 \\
6.00 \\
16.28\end{array}$ & $\begin{array}{r}6.43 \\
4.07 \\
1.78 \\
1.80 \\
1.77 \\
3.72 \\
6.83 \\
3.06 \\
6.01 \\
6.66 \\
9.36 \\
13.40 \\
8.78 \\
3.15 \\
6.69 \\
17.14\end{array}$ & $\begin{array}{r}6.79 \\
4.37 \\
1.78 \\
1.72 \\
1.80 \\
3.85 \\
7.03 \\
2.87 \\
5.32 \\
5.90 \\
10.15 \\
15.42 \\
8.37 \\
2.79 \\
5.95 \\
17.25\end{array}$ \\
\hline 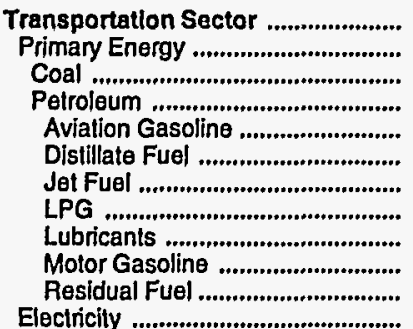 & $\begin{array}{r}2.50 \\
2.50 \\
.53 \\
2.50 \\
2.17 \\
1.27 \\
.74 \\
1.39 \\
5.08 \\
2.71 \\
.57 \\
-\end{array}$ & $\begin{array}{r}4.46 \\
4.46 \\
1.49 \\
4.46 \\
3.45 \\
2.77 \\
2.08 \\
2.51 \\
7.48 \\
4.72 \\
1.76 \\
-\end{array}$ & $\begin{array}{r}9.63 \\
9.63 \\
- \\
9.63 \\
9.02 \\
7.19 \\
6.38 \\
5.16 \\
14.36 \\
10.09 \\
3.49 \\
-\end{array}$ & $\begin{array}{r}8.96 \\
8.96 \\
- \\
8.96 \\
9.99 \\
8.55 \\
6.09 \\
8.32 \\
17.61 \\
9.10 \\
4.38 \\
-\end{array}$ & $\begin{array}{r}6.73 \\
6.73 \\
- \\
6.73 \\
8.41 \\
6.69 \\
4.20 \\
8.25 \\
15.59 \\
6.79 \\
2.45 \\
-\end{array}$ & $\begin{array}{r}6.96 \\
6.96 \\
-\overline{6} \\
6.96 \\
7.55 \\
6.86 \\
4.09 \\
7.76 \\
12.70 \\
7.13 \\
2.90 \\
-\end{array}$ & $\begin{array}{r}6.93 \\
6.93 \\
- \\
6.93 \\
7.41 \\
6.50 \\
3.85 \\
7.73 \\
14.61 \\
7.15 \\
2.26 \\
-\end{array}$ & $\begin{array}{r}7.48 \\
7.48 \\
- \\
7.48 \\
8.28 \\
6.94 \\
4.39 \\
8.30 \\
13.30 \\
7.77 \\
2.49 \\
-\end{array}$ & $\begin{array}{r}8.51 \\
8.51 \\
- \\
8.51 \\
9.32 \\
8.24 \\
5.65 \\
9.36 \\
13.40 \\
8.78 \\
2.42 \\
-\end{array}$ & $\begin{array}{r}8.12 \\
8.12 \\
- \\
8.12 \\
8.71 \\
7.88 \\
4.94 \\
10.15 \\
15.42 \\
8.37 \\
2.09 \\
-\end{array}$ \\
\hline Electricily ............................................ & - & - & - & - & - & - & - & - & - & - \\
\hline $\begin{array}{l}\text { Total Enorgy .................................... } \\
\text { Primary Energy - Four Sectors ..... } \\
\text { Electricity ........................................ }\end{array}$ & $\begin{array}{l}1.70 \\
1.34 \\
5.55\end{array}$ & $\begin{array}{l}3.33 \\
2.65 \\
9.78\end{array}$ & $\begin{array}{r}6.61 \\
5.54 \\
15.40\end{array}$ & $\begin{array}{r}8.48 \\
6.77 \\
19.88\end{array}$ & $\begin{array}{r}7.50 \\
5.52 \\
20.35\end{array}$ & $\begin{array}{r}7.60 \\
5.70 \\
19.26\end{array}$ & $\begin{array}{r}7.44 \\
5.55 \\
19.39\end{array}$ & $\begin{array}{r}7.72 \\
5.79 \\
19.91\end{array}$ & $\begin{array}{r}8.07 \\
6.07 \\
20.85\end{array}$ & $\begin{array}{r}8.14 \\
6.04 \\
21.18\end{array}$ \\
\hline 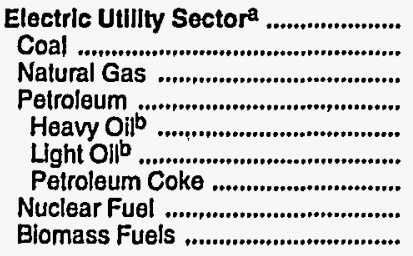 & $\begin{array}{l}.38 \\
.36 \\
.42 \\
.63 \\
.63 \\
.65 \\
- \\
.36\end{array}$ & $\begin{array}{r}1.02 \\
.92 \\
1.28 \\
1.98 \\
1.97 \\
2.05 \\
- \\
.28 \\
-\end{array}$ & $\begin{array}{r}1.58 \\
1.56 \\
2.74 \\
4.24 \\
4.10 \\
6.07 \\
-49 \\
-\end{array}$ & $\begin{array}{r}1.72 \\
1.88 \\
4.43 \\
5.15 \\
4.64 \\
5.60 \\
-\overline{80} \\
-\end{array}$ & $\begin{array}{r}1.66 \\
1.81 \\
3.64 \\
2.83 \\
2.58 \\
3.44 \\
- \\
.77 \\
-\end{array}$ & $\begin{array}{r}1.60 \\
1.75 \\
2.76 \\
3.35 \\
3.14 \\
3.98 \\
- \\
.77 \\
-\end{array}$ & $\begin{array}{r}1.60 \\
1.76 \\
2.56 \\
2.79 \\
2.64 \\
3.59 \\
-\overline{8} \\
-\end{array}$ & $\begin{array}{r}1.52 \\
1.72 \\
1.75 \\
3.15 \\
2.97 \\
4.21 \\
- \\
.78 \\
-\end{array}$ & $\begin{array}{r}1.41 \\
1.60 \\
2.11 \\
3.26 \\
2.89 \\
4.60 \\
- \\
.79 \\
-\end{array}$ & $\begin{array}{r}1.32 \\
1.59 \\
1.96 \\
2.89 \\
2.44 \\
4.51 \\
- \\
.65 \\
-\end{array}$ \\
\hline Primary Energy - Five Sectorsc .. & 1.12 & 2.23 & 4.39 & 5.18 & 4.30 & 4.26 & 4.21 & 4.33 & 4.49 & 4.38 \\
\hline
\end{tabular}

There are no direct fuel costs for hydroelectric, geothermal, centralized solar, or wind energy.

beavy oil includes Grade Nos. 4, 5, and 6, and residual fuel oils. Light oil includes Grade No. 2 heating oil, kerosene, and jet fuel.

c Biomass fuels are not included, except those consumed at electric utilities and those added to motor gasoline.

-No consumption, including cases where adjustments were made. See "Consumption Adjustments for Process Fuel and Intermediate Products."

Sources: Data sources, estimation procedures, and assumptions are described in the "Documentation" section of this report. 


\section{Energy Expenditure Estimates by Sector, Michigan}

\section{$1970,1975,1980$, and 1985-1991}

(Million Dollars)

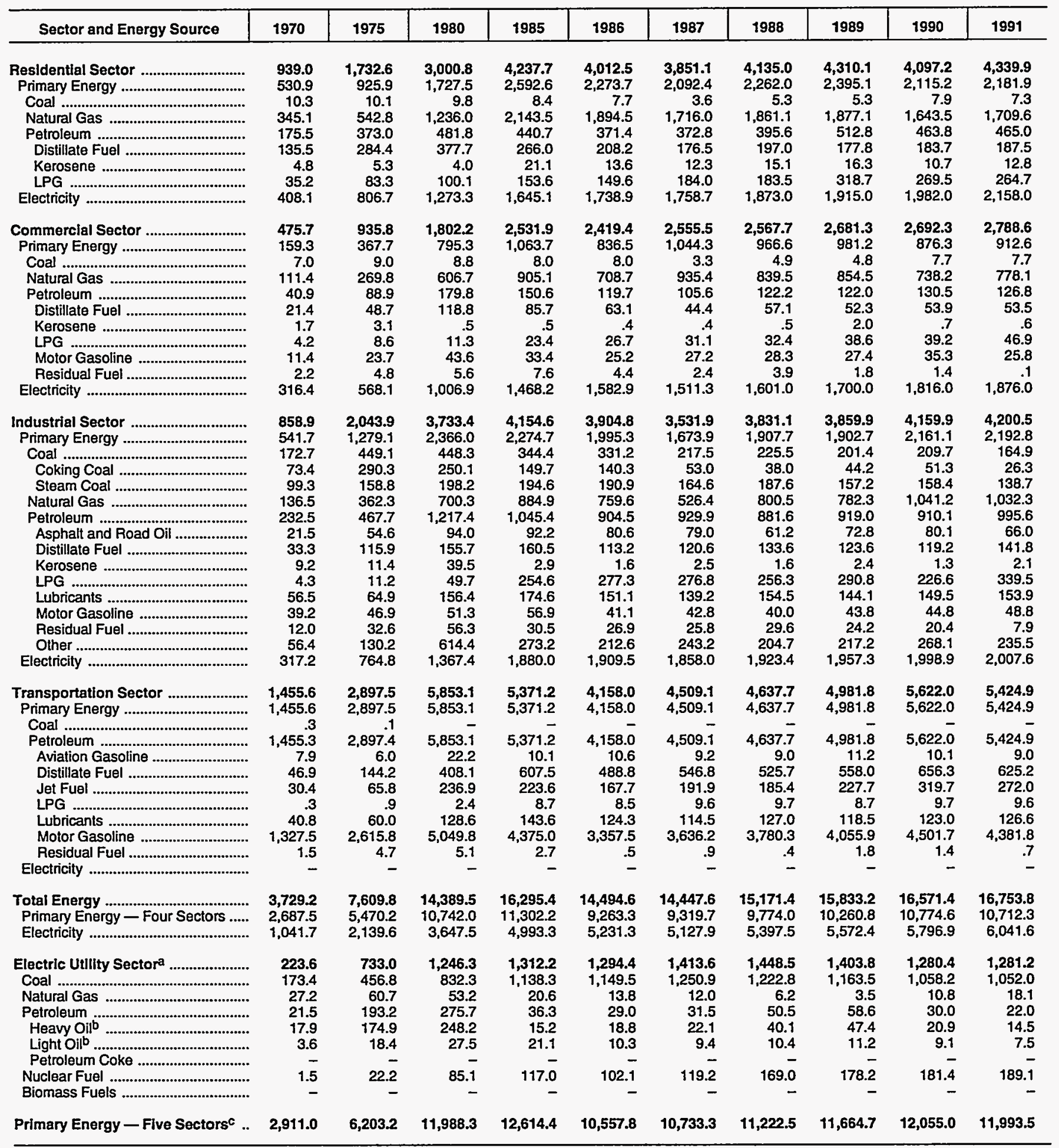

a There are no direct fuel costs for hydroelectric, geothermal, centralized solar, or wind energy.

b Heavy oil includes Grade Nos. 4, 5, and 6, and residual fuel oils. Light oil includes Grade No. 2 heating oil, kerosene, and jet fuel.

c Biomass fuels are not included, except those consumed at electric utilities and those added to motor gasoline.

- No consumption, including cases where adjustments were made. See "Consumption Adjustments for Process Fuel and intermediate Products."

Note: Totals may not equal sum of components due to independent rounding.

Sources: Data sources, estimation procedures, and assumptions are described in the "Documentation" section of this report. 


\begin{tabular}{|c|c|c|c|c|c|c|c|c|c|c|}
\hline Enorgy Source & 1970 & 1975 & 1980 & 1985 & 1986 & 1987 & 1988 & 1989 & 1990 & 1991 \\
\hline & \multicolumn{10}{|c|}{ Prices In Dollars per Million Btu } \\
\hline 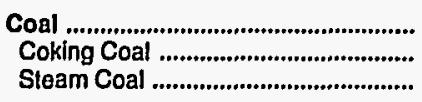 & $\begin{array}{r}0.41 \\
.53 \\
.40\end{array}$ & $\begin{array}{r}0.83 \\
1.80 \\
.69\end{array}$ & $\frac{1.12}{1.12}$ & $\begin{array}{r}1.51 \\
-\overline{1.51}\end{array}$ & $\frac{1.44}{1.44}$ & $\begin{array}{r}1.26 \\
1.26\end{array}$ & $\begin{array}{r}1.25 \\
1.25\end{array}$ & $\frac{1.26}{1.26}$ & $\begin{array}{r}1.32 \\
1.32\end{array}$ & $\begin{array}{r}1.30 \\
1.30\end{array}$ \\
\hline Natural Gas ........,................................ & .66 & 1.17 & 2.85 & 5.13 & 4.61 & 3.72 & 3.87 & 3.87 & 3.87 & 3.70 \\
\hline 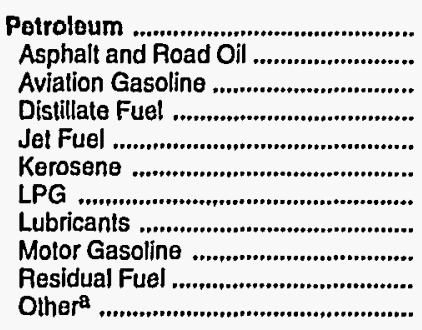 & $\begin{array}{r}2.01 \\
.68 \\
2.17 \\
1.08 \\
.75 \\
1.34 \\
1.80 \\
5.08 \\
2.97 \\
.59 \\
.74\end{array}$ & $\begin{array}{l}3.57 \\
2.19 \\
3.45 \\
2.51 \\
2.09 \\
2.78 \\
3.67 \\
7.48 \\
4.63 \\
1.80 \\
1.72\end{array}$ & $\begin{array}{r}7.88 \\
3.31 \\
9.02 \\
6.72 \\
6.47 \\
7.05 \\
5.82 \\
14.36 \\
9.55 \\
3.52 \\
3.82\end{array}$ & $\begin{array}{r}8.45 \\
4.99 \\
9.99 \\
7.55 \\
5.93 \\
8.30 \\
8.08 \\
17.61 \\
9.73 \\
4.05 \\
4.75\end{array}$ & $\begin{array}{r}6.46 \\
3.86 \\
8.41 \\
5.62 \\
4.04 \\
6.12 \\
7.92 \\
15.59 \\
7.42 \\
2.88 \\
6.09\end{array}$ & $\begin{array}{r}6.66 \\
3.53 \\
7.55 \\
5.98 \\
4.15 \\
6.19 \\
6.78 \\
12.70 \\
7.71 \\
2.43 \\
5.17\end{array}$ & $\begin{array}{r}6.75 \\
3.60 \\
7.41 \\
5.81 \\
3.94 \\
5.87 \\
7.37 \\
14.61 \\
7.81 \\
2.36 \\
3.59\end{array}$ & $\begin{array}{r}7.45 \\
3.01 \\
8.28 \\
6.54 \\
4.47 \\
6.81 \\
8.64 \\
13.30 \\
8.72 \\
2.38 \\
2.37\end{array}$ & $\begin{array}{r}8.23 \\
3.31 \\
9.32 \\
7.89 \\
5.68 \\
8.12 \\
8.85 \\
13.40 \\
9.56 \\
2.50 \\
2.32\end{array}$ & $\begin{array}{r}7.86 \\
3.19 \\
8.71 \\
7.06 \\
4.87 \\
7.34 \\
7.89 \\
15.42 \\
9.31 \\
2.11 \\
1.85\end{array}$ \\
\hline Nuclear Fuel .....,................................. & - & .24 & .44 & .50 & .50 & .51 & .49 & .49 & .48 & .47 \\
\hline Blomass Fuels at Uttlitles ................. & .65 & .92 & 1.74 & - & - & .95 & .87 & 1.02 & .91 & .98 \\
\hline Primary Energy - Flve Sectors ${ }^{b}$.... & 1.27 & 2.13 & 4.42 & 5.27 & 4.36 & 4.02 & 3.97 & 4.23 & 4.51 & 4.42 \\
\hline $\begin{array}{l}\text { Electric Utility Fue|c } \\
\text { Electricity Purchased by End Users............. }\end{array}$ & $\begin{array}{r}.34 \\
6.10\end{array}$ & 8.52 & $\begin{array}{r}.91 \\
13.26\end{array}$ & $\begin{array}{r}1.09 \\
15.81\end{array}$ & $\begin{array}{r}1.03 \\
16.07\end{array}$ & $\begin{array}{r}.99 \\
15.72\end{array}$ & $\begin{array}{r}.99 \\
15.80\end{array}$ & $\begin{array}{r}1.01 \\
15.57\end{array}$ & $\begin{array}{r}1.03 \\
15.68\end{array}$ & $\begin{array}{r}1.02 \\
16.07\end{array}$ \\
\hline \multirow[t]{2}{*}{ Total Energy ....................................... } & 1.86 & 3.18 & 6.91 & 8.42 & 7.30 & 7.29 & 7.36 & 7.62 & 8.10 & 7.94 \\
\hline & \multicolumn{10}{|c|}{ Expenditures in Millions of Dollars } \\
\hline 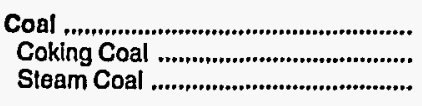 & $\begin{array}{r}74.1 \\
8.6 \\
65.5\end{array}$ & $\begin{array}{r}159.8 \\
45.4 \\
114.4\end{array}$ & $\begin{array}{r}270.7 \\
270 . \overline{7}\end{array}$ & $\begin{array}{r}341.4 \\
341.4\end{array}$ & $\begin{array}{r}290.7 \\
\overline{-} \\
290 . \overline{7}\end{array}$ & $\begin{array}{r}323.6 \\
323.6\end{array}$ & $\begin{array}{r}378.2 \\
378.2\end{array}$ & $\begin{array}{r}405.5 \\
\overline{-} \\
405.5\end{array}$ & $\begin{array}{r}429.1 \\
-\overline{1}\end{array}$ & $\begin{array}{r}390.2 \\
390 . \overline{2}\end{array}$ \\
\hline Natural Gas , .......................................... & 220.6 & 381.4 & 785.0 & $1,283.0$ & $1,084.8$ & 856.2 & $1,051.7$ & $1,108.8$ & $1,066.5$ & $1,110.6$ \\
\hline 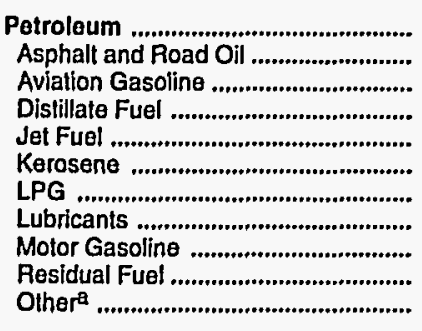 & $\begin{array}{r}988.1 \\
20.0 \\
3.0 \\
140.5 \\
14.7 \\
12.8 \\
60.1 \\
28.5 \\
688.9 \\
14.9 \\
4.6\end{array}$ & $\begin{array}{r}1,902.1 \\
67.2 \\
3.7 \\
355.7 \\
66.5 \\
13.5 \\
124.8 \\
45.5 \\
1,172.9 \\
38.4 \\
13.7\end{array}$ & $\begin{array}{r}3,784.8 \\
78.4 \\
8.8 \\
837.2 \\
188.3 \\
8.5 \\
163.0 \\
97.5 \\
2,319.4 \\
56.3 \\
27.5\end{array}$ & $\begin{array}{r}3,928.6 \\
165.1 \\
7.8 \\
852.5 \\
261.4 \\
8.6 \\
154.0 \\
108.8 \\
2,335.5 \\
15.8 \\
19.1\end{array}$ & $\begin{array}{r}3,044.9 \\
140.3 \\
9.6 \\
617.2 \\
178.6 \\
4.3 \\
179.0 \\
94.2 \\
1,784.2 \\
22.2 \\
15.4\end{array}$ & $\begin{array}{r}3,067.0 \\
137.2 \\
6.8 \\
636.3 \\
132.8 \\
3.2 \\
133.0 \\
86.8 \\
1,899.3 \\
13.6 \\
18.1\end{array}$ & $\begin{array}{r}3,198.3 \\
117.1 \\
6.2 \\
672.8 \\
114.7 \\
5.1 \\
149.8 \\
96.3 \\
2,005.6 \\
14.4 \\
16.2\end{array}$ & $\begin{array}{r}3,501.5 \\
98.5 \\
6.6 \\
730.9 \\
118.0 \\
12.5 \\
190.7 \\
89.8 \\
2,224.8 \\
12.4 \\
17.3\end{array}$ & $\begin{array}{r}3,855.2 \\
132.5 \\
10.0 \\
849.2 \\
164.0 \\
1.9 \\
187.9 \\
93.2 \\
2,385.6 \\
12.2 \\
18.5\end{array}$ & $\begin{array}{r}3,832.3 \\
106.6 \\
8.3 \\
872.2 \\
135.7 \\
2.3 \\
186.3 \\
95.9 \\
2,398.1 \\
10.0 \\
17.0\end{array}$ \\
\hline Nuclear Fuel ....................................... & - & 25.5 & 48.6 & 62.5 & 59.2 & 63.1 & 64.2 & 57.4 & 61.7 & 60.7 \\
\hline Blomass Fuels at Utilities .................. & .1 & $\bullet$ & * & - & - & .4 & 1.4 & 2.6 & 3.7 & 4.1 \\
\hline Prlmary Energy - Fiva Sectors ${ }^{b}$.... & $1,282.9$ & $2,468.9$ & $4,889.2$ & $5,615.6$ & $4,479.6$ & $4,310.4$ & $4,693.7$ & $5,075.8$ & $5,416.3$ & $5,397.9$ \\
\hline $\begin{array}{l}\text { Electric Utility Fuelc .......................... } \\
\text { Electricity Purchased by End Users }\end{array}$ & $\begin{array}{r}-65.3 \\
427.5\end{array}$ & $\begin{array}{r}-144.2 \\
769.9\end{array}$ & $\begin{array}{r}-311.3 \\
1,481.2\end{array}$ & $\begin{array}{r}-355.5 \\
2,062.8\end{array}$ & $\begin{array}{r}-307.0 \\
2,097.2\end{array}$ & $\begin{array}{r}-362.5 \\
2,211.9\end{array}$ & $\begin{array}{r}-419.5 \\
2,433.7\end{array}$ & $\begin{array}{r}-433.2 \\
2,409.9\end{array}$ & $\begin{array}{r}-454.0 \\
2,491.2\end{array}$ & $\begin{array}{r}-439.2 \\
2,638.6\end{array}$ \\
\hline 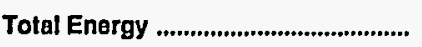 & $1,645.1$ & $3,094.6$ & $6,059.1$ & $7,322.9$ & $6,269.7$ & $6,159.8$ & $6,708.0$ & $7,052.5$ & $7,453.6$ & $7,597.2$ \\
\hline
\end{tabular}

Includes petroleum coke used at electric utilities.

b Blomass fuels are not included, except those consumed at electric utilities and those added to motor gasoline.

c There are no direct fuel costs for hydroelectric, geothermal, centralized solar, or wind energy.

- No consumption, including cases where adjustments were made. See "Consumption Adjustments for Process Fuel and Intermediate Products."

"Value less than 0.05 million dollars.

Note: Expenditure totals may not equal sum of components due to independent rounding.

Sources; Data sources, estimation procedures, and assumptions are described in the "Documentation" section of this report. 
M Energy Price Estimates by Sector, Minnesota $1970,1975,1980$, and 1985-1991

N (Dollars per Million Btu)

\begin{tabular}{|c|c|c|c|c|c|c|c|c|c|c|}
\hline Sector and Energy Source & 1970 & 1975 & 1980 & 1985 & 1986 & 1987 & 1988 & 1989 & 1990 & 1991 \\
\hline 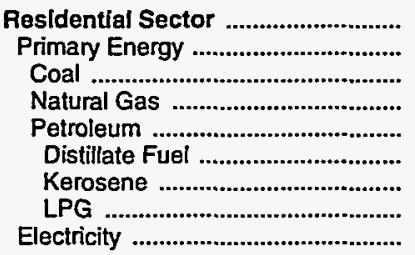 & $\begin{array}{l}2.17 \\
1.29 \\
1.55 \\
1.09 \\
1.54 \\
1.26 \\
1.52 \\
2.03 \\
7.30\end{array}$ & $\begin{array}{l}3.38 \\
2.15 \\
3.04 \\
1.57 \\
3.12 \\
2.55 \\
2.91 \\
4.22 \\
9.90\end{array}$ & $\begin{array}{r}6.92 \\
4.47 \\
4.32 \\
3.24 \\
7.25 \\
7.20 \\
8.02 \\
7.34 \\
16.06\end{array}$ & $\begin{array}{r}9.34 \\
6.22 \\
4.10 \\
5.78 \\
7.81 \\
7.79 \\
8.82 \\
7.79 \\
19.01\end{array}$ & $\begin{array}{r}8.98 \\
5.57 \\
3.89 \\
5.28 \\
6.51 \\
6.05 \\
6.85 \\
7.53 \\
19.41\end{array}$ & $\begin{array}{r}8.98 \\
5.01 \\
3.81 \\
4.58 \\
6.22 \\
5.70 \\
6.45 \\
7.39 \\
19.38\end{array}$ & $\begin{array}{r}8.76 \\
4.95 \\
3.51 \\
4.61 \\
6.03 \\
5.62 \\
6.36 \\
7.03 \\
19.82\end{array}$ & $\begin{array}{r}8.74 \\
5.24 \\
3.49 \\
4.54 \\
7.43 \\
6.30 \\
7.13 \\
10.03 \\
19.71\end{array}$ & $\begin{array}{r}9.25 \\
5.32 \\
3.46 \\
4.61 \\
7.97 \\
7.75 \\
8.77 \\
8.35 \\
19.94\end{array}$ & $\begin{array}{r}8.97 \\
5.07 \\
3.81 \\
4.47 \\
7.12 \\
6.96 \\
7.88 \\
7.42 \\
20.28\end{array}$ \\
\hline 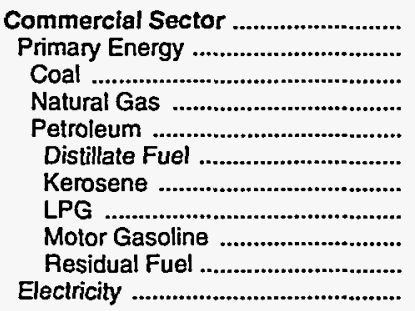 & $\begin{array}{r}1.42 \\
.76 \\
.47 \\
.69 \\
1.13 \\
1.05 \\
.89 \\
1.20 \\
2.97 \\
.58 \\
7.72\end{array}$ & $\begin{array}{r}2.56 \\
1.39 \\
.87 \\
1.16 \\
2.61 \\
2.34 \\
2.54 \\
2.61 \\
4.63 \\
1.97 \\
10.38\end{array}$ & $\begin{array}{r}5.37 \\
3.47 \\
1.77 \\
2.89 \\
6.73 \\
6.60 \\
- \\
4.87 \\
9.55 \\
4.48 \\
12.95\end{array}$ & $\begin{array}{r}7.84 \\
5.39 \\
2.04 \\
5.18 \\
6.62 \\
6.34 \\
6.81 \\
8.32 \\
9.73 \\
4.10 \\
17.53\end{array}$ & $\begin{array}{r}7.61 \\
4.61 \\
1.96 \\
4.62 \\
5.08 \\
4.21 \\
4.32 \\
8.25 \\
7.42 \\
2.86 \\
17.81\end{array}$ & $\begin{array}{r}7.43 \\
3.98 \\
1.85 \\
3.89 \\
4.96 \\
4.37 \\
4.96 \\
6.15 \\
7.71 \\
2.43 \\
17.19\end{array}$ & $\begin{array}{r}7.22 \\
4.03 \\
1.85 \\
4.00 \\
4.74 \\
3.97 \\
4.40 \\
7.73 \\
7.81 \\
2.37 \\
17.52\end{array}$ & $\begin{array}{r}7.09 \\
4.04 \\
1.86 \\
3.98 \\
5.18 \\
4.72 \\
5.20 \\
7.13 \\
8.72 \\
2.38 \\
17.61\end{array}$ & $\begin{array}{r}7.66 \\
4.57 \\
2.05 \\
3.96 \\
7.65 \\
5.75 \\
6.44 \\
9.36 \\
9.56 \\
2.50 \\
17.69\end{array}$ & $\begin{array}{r}7.33 \\
3.93 \\
1.87 \\
3.76 \\
5.52 \\
4.88 \\
5.74 \\
8.34 \\
9.31 \\
2.11 \\
18.01\end{array}$ \\
\hline 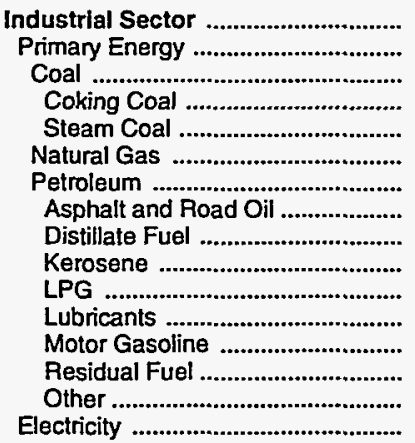 & $\begin{array}{r}1.11 \\
.78 \\
.49 \\
.53 \\
.47 \\
.42 \\
1.16 \\
.68 \\
.83 \\
.89 \\
1.20 \\
5.08 \\
2.97 \\
.55 \\
.82 \\
4.17\end{array}$ & $\begin{array}{r}2.33 \\
1.73 \\
1.33 \\
1.80 \\
.87 \\
.83 \\
2.62 \\
2.19 \\
2.39 \\
2.54 \\
2.61 \\
7.48 \\
4.63 \\
1.74 \\
1.77 \\
6.73\end{array}$ & $\begin{array}{r}5.08 \\
3.60 \\
1.77 \\
-\overline{1} \\
1.77 \\
2.51 \\
5.08 \\
3.31 \\
5.66 \\
5.92 \\
4.87 \\
14.36 \\
9.55 \\
2.97 \\
3.82 \\
11.22\end{array}$ & $\begin{array}{r}6.97 \\
5.00 \\
2.04 \\
- \\
2.04 \\
4.04 \\
6.44 \\
4.99 \\
6.18 \\
6.81 \\
8.32 \\
17.61 \\
9.73 \\
4.10 \\
4.75 \\
12.65\end{array}$ & $\begin{array}{r}6.27 \\
4.05 \\
1.96 \\
-\overline{1} \\
1.96 \\
3.42 \\
4.85 \\
3.86 \\
3.92 \\
4.32 \\
8.25 \\
15.59 \\
7.42 \\
2.86 \\
6.09 \\
12.76\end{array}$ & $\begin{array}{r}5.97 \\
3.55 \\
1.85 \\
- \\
1.85 \\
2.55 \\
4.60 \\
3.53 \\
4.50 \\
4.96 \\
6.15 \\
12.70 \\
7.71 \\
2.43 \\
5.17 \\
12.50\end{array}$ & $\begin{array}{r}6.16 \\
3.62 \\
1.85 \\
- \\
1.85 \\
2.79 \\
4.65 \\
3.60 \\
4.00 \\
4.40 \\
7.73 \\
14.61 \\
7.81 \\
2.37 \\
4.36 \\
12.32\end{array}$ & $\begin{array}{r}6.07 \\
3.60 \\
1.86 \\
- \\
1.86 \\
2.84 \\
4.70 \\
3.01 \\
4.72 \\
5.20 \\
7.13 \\
13.30 \\
8.72 \\
2.38 \\
4.40 \\
12.02\end{array}$ & $\begin{array}{r}6.22 \\
3.91 \\
2.05 \\
- \\
2.05 \\
2.96 \\
5.28 \\
3.31 \\
5.85 \\
6.44 \\
9.36 \\
13.40 \\
9.56 \\
2.50 \\
4.22 \\
12.14\end{array}$ & $\begin{array}{r}6.33 \\
3.83 \\
1.87 \\
- \\
1.87 \\
2.75 \\
5.20 \\
3.19 \\
5.21 \\
5.74 \\
8.34 \\
15.42 \\
9.31 \\
2.11 \\
3.74 \\
12.48\end{array}$ \\
\hline 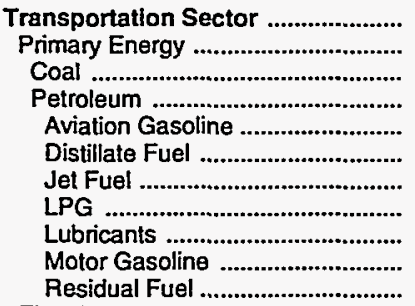 & $\begin{array}{r}2.64 \\
2.64 \\
.47 \\
2.64 \\
2.17 \\
1.24 \\
.75 \\
1.20 \\
5.08 \\
2.97 \\
.57\end{array}$ & $\begin{array}{r}4.13 \\
4.13 \\
.87 \\
4.13 \\
3.45 \\
2.67 \\
2.09 \\
2.61 \\
7.48 \\
4.63 \\
1.70\end{array}$ & $\begin{array}{r}8.88 \\
8.88 \\
- \\
8.88 \\
9.02 \\
7.16 \\
6.47 \\
4.87 \\
14.36 \\
9.55 \\
3.81\end{array}$ & $\begin{array}{r}9.16 \\
9.16 \\
- \\
9.16 \\
9.99 \\
8.68 \\
5.93 \\
8.32 \\
17.61 \\
9.73 \\
3.91\end{array}$ & $\begin{array}{r}6.98 \\
6.98 \\
- \\
6.98 \\
8.41 \\
6.78 \\
4.04 \\
8.25 \\
15.59 \\
7.42 \\
3.65\end{array}$ & $\begin{array}{r}7.34 \\
7.34 \\
- \\
7.34 \\
7.55 \\
7.15 \\
4.15 \\
6.15 \\
12.70 \\
7.71 \\
2.25\end{array}$ & $\begin{array}{r}7.48 \\
7.48 \\
- \\
7.48 \\
7.41 \\
7.21 \\
3.94 \\
7.73 \\
14.61 \\
7.81 \\
1.83\end{array}$ & $\begin{array}{r}8.31 \\
8.31 \\
- \\
8.31 \\
8.28 \\
7.83 \\
4.47 \\
7.13 \\
13.30 \\
8.72 \\
1.84\end{array}$ & $\begin{array}{r}9.21 \\
9.21 \\
- \\
9.21 \\
9.32 \\
9.19 \\
5.68 \\
9.36 \\
13.40 \\
9.56 \\
-\end{array}$ & $\begin{array}{r}8.84 \\
8.84 \\
- \\
8.84 \\
8.71 \\
8.28 \\
4.87 \\
8.34 \\
15.42 \\
9.31 \\
1.98\end{array}$ \\
\hline 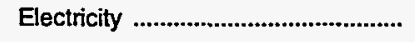 & - & - & - & - & - & - & - & - & - & - \\
\hline 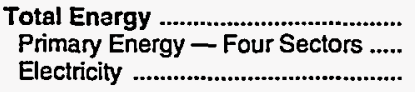 & $\begin{array}{l}1.86 \\
1.50 \\
6.10\end{array}$ & $\begin{array}{l}3.18 \\
2.63 \\
8.64\end{array}$ & $\begin{array}{r}6.91 \\
5.98 \\
13.26\end{array}$ & $\begin{array}{r}8.42 \\
7.12 \\
15.81\end{array}$ & $\begin{array}{r}7.30 \\
5.73 \\
16.07\end{array}$ & $\begin{array}{r}7.29 \\
5.60 \\
15.72\end{array}$ & $\begin{array}{r}7.36 \\
5.64 \\
15.80\end{array}$ & $\begin{array}{r}7.62 \\
6.02 \\
15.57\end{array}$ & $\begin{array}{r}8.10 \\
6.52 \\
15.68\end{array}$ & $\begin{array}{r}7.94 \\
6.26 \\
16.07\end{array}$ \\
\hline 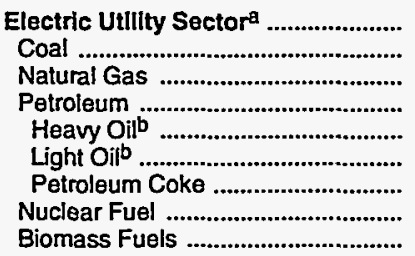 & $\begin{array}{l}.34 \\
.34 \\
.26 \\
.73 \\
.74 \\
.85 \\
.28 \\
- \\
.65\end{array}$ & $\begin{array}{r}.52 \\
.62 \\
.64 \\
2.03 \\
1.95 \\
2.26 \\
.54 \\
.24 \\
.92\end{array}$ & $\begin{array}{r}.91 \\
1.04 \\
1.99 \\
4.86 \\
4.46 \\
5.80 \\
- \\
.44 \\
1.74\end{array}$ & $\begin{array}{r}1.09 \\
1.43 \\
3.69 \\
5.96 \\
3.99 \\
5.97 \\
- \\
.50 \\
-\end{array}$ & $\begin{array}{r}1.03 \\
1.36 \\
2.95 \\
3.91 \\
- \\
3.91 \\
- \\
.50 \\
-\end{array}$ & $\begin{array}{r}.99 \\
1.21 \\
2.08 \\
4.46 \\
2.39 \\
4.47 \\
- \\
.51 \\
.95\end{array}$ & $\begin{array}{r}.99 \\
1.20 \\
1.98 \\
2.07 \\
2.06 \\
3.69 \\
.82 \\
.49 \\
.87\end{array}$ & $\begin{array}{r}1.01 \\
1.20 \\
2.15 \\
1.16 \\
2.12 \\
4.42 \\
.77 \\
.49 \\
1.02\end{array}$ & $\begin{array}{r}1.03 \\
1.25 \\
1.92 \\
1.25 \\
1.85 \\
5.33 \\
.76 \\
.48 \\
.91\end{array}$ & $\begin{array}{r}1.02 \\
1.26 \\
1.70 \\
1.09 \\
2.16 \\
4.63 \\
.77 \\
.47 \\
.98\end{array}$ \\
\hline Primary Energy - Flve Sectors ${ }^{c}$.. & 1.27 & 2.13 & 4.42 & 5.27 & 4.36 & 4.02 & 3.97 & 4.23 & 4.51 & 4.42 \\
\hline
\end{tabular}

There are no direct fuel costs for hydroelectric, geothermal, centralized solar, or wind energy.

b Heavy oil includes Grade Nos. 4, 5, and 6, and residual fuel oils. Light oil includes Grade No. 2 heating oil, kerosene, and jet fuel.

$c$ Biomass fuels are not included, except those consumed at electric utilities and those added to motor gasoline.

-No consumption, including cases where adjustments were made. See "Consumption Adjustments for Process Fuel and intermediate Products."

Sources: Data sources, estimation procedures, and assumptions are described in the "Documentation" section of this report. 


\begin{tabular}{|c|c|c|c|c|c|c|c|c|c|c|}
\hline Sector and Energy Source & 1970 & 1975 & 1980 & 1985 & 1986 & 1987 & 1988 & 1989 & 1990 & 1991 \\
\hline 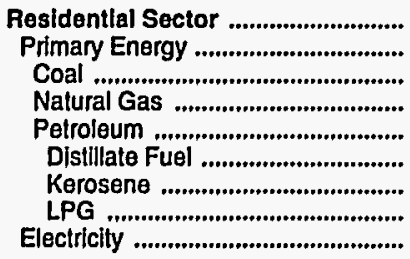 & $\begin{array}{r}455.3 \\
230.2 \\
6.5 \\
111.5 \\
112.2 \\
52.9 \\
10.3 \\
48.9 \\
225.0\end{array}$ & $\begin{array}{r}739.9 \\
395.8 \\
4.8 \\
179.5 \\
211.6 \\
107.6 \\
9.2 \\
94.8 \\
344.1\end{array}$ & $\begin{array}{r}1,315.8 \\
672.0 \\
4.5 \\
333.8 \\
333.7 \\
249.5 \\
5.2 \\
79.0 \\
643.8\end{array}$ & $\begin{array}{r}1,732.8 \\
872.5 \\
6.0 \\
618.7 \\
247.8 \\
173.6 \\
6.8 \\
67.4 \\
860.3\end{array}$ & $\begin{array}{r}1,649.1 \\
771.0 \\
5.0 \\
545.1 \\
221.0 \\
141.0 \\
3.4 \\
76.6 \\
878.1\end{array}$ & $\begin{array}{r}1,535.7 \\
621.0 \\
4.1 \\
411.9 \\
205.0 \\
129.1 \\
2.7 \\
73.2 \\
914.7\end{array}$ & $\begin{array}{r}1,748.4 \\
734.4 \\
5.2 \\
508.9 \\
220.3 \\
143.2 \\
4.1 \\
73.0 \\
1,014.0\end{array}$ & $\begin{array}{r}1,825.3 \\
831.4 \\
5.9 \\
534.3 \\
291.2 \\
164.9 \\
10.9 \\
115.4 \\
994.0\end{array}$ & \begin{tabular}{r|}
$1,745.8$ \\
734.8 \\
3.9 \\
495.3 \\
235.7 \\
145.5 \\
1.5 \\
88.8 \\
$1,011.0$
\end{tabular} & $\begin{array}{r}1,868.5 \\
785.4 \\
2.2 \\
529.5 \\
253.7 \\
166.2 \\
1.8 \\
85.7 \\
1,083.0\end{array}$ \\
\hline 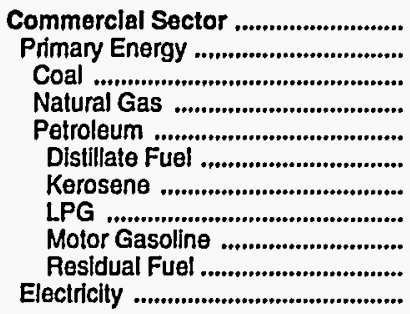 & $\begin{array}{r}163.0 \\
79.2 \\
3.7 \\
53.2 \\
22.3 \\
10.7 \\
1.3 \\
5.1 \\
3.7 \\
1.4 \\
83.7\end{array}$ & $\begin{array}{r}325.9 \\
154.3 \\
2.5 \\
104.2 \\
47.6 \\
24.1 \\
1.7 \\
10.3 \\
8.6 \\
2.8 \\
171.6\end{array}$ & $\begin{array}{r}522.6 \\
269.7 \\
3.4 \\
183.6 \\
82.7 \\
55.5 \\
9.3 \\
9.3 \\
17.1 \\
.9 \\
252.8\end{array}$ & $\begin{array}{r}990.3 \\
543.4 \\
5.6 \\
400.2 \\
137.6 \\
101.1 \\
.9 \\
12.7 \\
17.1 \\
5.8 \\
446.9\end{array}$ & $\begin{array}{r}871.4 \\
408.1 \\
4.7 \\
343.8 \\
59.6 \\
26.4 \\
.1 \\
14.8 \\
12.7 \\
5.5 \\
463.3\end{array}$ & $\begin{array}{r}779.5 \\
308.4 \\
3.7 \\
256.4 \\
48.2 \\
25.7 \\
.2 \\
10.7 \\
9.7 \\
2.0 \\
471.2\end{array}$ & $\begin{array}{r}895.6 \\
381.5 \\
5.1 \\
322.4 \\
54.1 \\
25.5 \\
.1 \\
14.2 \\
9.9 \\
4.4 \\
514.0\end{array}$ & $\begin{array}{r}910.3 \\
402.3 \\
5.9 \\
340.7 \\
55.7 \\
28.4 \\
.1 \\
14.5 \\
8.7 \\
4.0 \\
508.0\end{array}$ & $\begin{array}{r}978.4 \\
446.4 \\
4.3 \\
310.5 \\
131.7 \\
31.5 \\
.2 \\
17.6 \\
78.3 \\
4.1 \\
532.0\end{array}$ & $\begin{array}{r}948.8 \\
385.8 \\
2.0 \\
327.2 \\
56.6 \\
25.9 \\
.1 \\
17.0 \\
9.7 \\
3.9 \\
563.0\end{array}$ \\
\hline 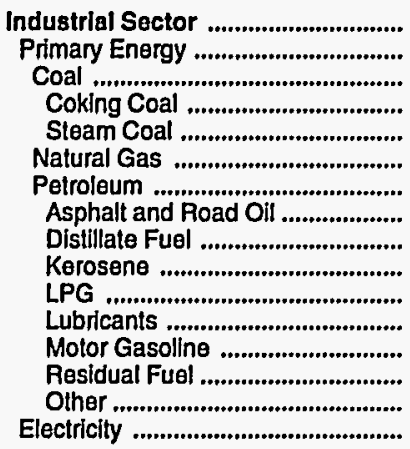 & $\begin{array}{r}323.8 \\
205.0 \\
20.8 \\
8.6 \\
12.2 \\
40.6 \\
143.6 \\
20.0 \\
37.5 \\
1.2 \\
5.6 \\
9.1 \\
56.3 \\
9.4 \\
4.4 \\
118.8\end{array}$ & $\begin{array}{r}725.0 \\
470.8 \\
67.6 \\
45.4 \\
22.2 \\
83.5 \\
319.7 \\
67.2 \\
111.0 \\
2.5 \\
18.8 \\
11.4 \\
76.1 \\
19.0 \\
13.5 \\
254.2\end{array}$ & $\begin{array}{r}1,356.2 \\
771.6 \\
31.9 \\
-\overline{31.9} \\
251.7 \\
488.1 \\
78.4 \\
188.2 \\
3.3 \\
73.5 \\
28.2 \\
67.1 \\
22.0 \\
27.5 \\
584.6\end{array}$ & $\begin{array}{r}1,611.5 \\
855.9 \\
43.3 \\
-\overline{43.3} \\
259.4 \\
553.2 \\
165.1 \\
172.5 \\
.9 \\
70.2 \\
31.4 \\
87.8 \\
6.2 \\
19.1 \\
755.7\end{array}$ & $\begin{array}{r}1,460.3 \\
704.6 \\
39.6 \\
-\overline{39} \\
39.6 \\
190.8 \\
474.1 \\
140.3 \\
128.8 \\
.8 \\
83.8 \\
27.2 \\
62.0 \\
15.8 \\
15.4 \\
755.8\end{array}$ & $\begin{array}{r}1,458.2 \\
632.1 \\
31.3 \\
-\overline{3} \\
31.3 \\
176.0 \\
424.8 \\
137.2 \\
124.2 \\
.3 \\
47.5 \\
25.1 \\
60.9 \\
11.5 \\
18.1 \\
826.1\end{array}$ & $\begin{array}{r}1,550.4 \\
644.7 \\
28.0 \\
28 . \overline{-} \\
28.0 \\
210.1 \\
406.6 \\
117.1 \\
122.7 \\
.8 \\
60.6 \\
27.8 \\
52.3 \\
9.9 \\
15.4 \\
905.7\end{array}$ & $\begin{array}{r}1,559.9 \\
652.0 \\
35.5 \\
-\overline{3} \\
35.5 \\
224.2 \\
392.3 \\
98.5 \\
127.4 \\
1.5 \\
59.2 \\
26.0 \\
57.4 \\
8.2 \\
14.1 \\
907.9\end{array}$ & $\begin{array}{r}1,726.8 \\
778.5 \\
48.8 \\
-\overline{4} \\
48.8 \\
250.7 \\
479.0 \\
132.5 \\
160.6 \\
.3 \\
79.7 \\
26.9 \\
55.8 \\
8.0 \\
15.2 \\
948.3\end{array}$ & $\begin{array}{r}1,740.8 \\
748.2 \\
28.5 \\
-\overline{2} \\
28.5 \\
243.8 \\
475.9 \\
106.6 \\
170.2 \\
.3 \\
82.1 \\
27.7 \\
70.5 \\
6.0 \\
12.5 \\
992.6\end{array}$ \\
\hline 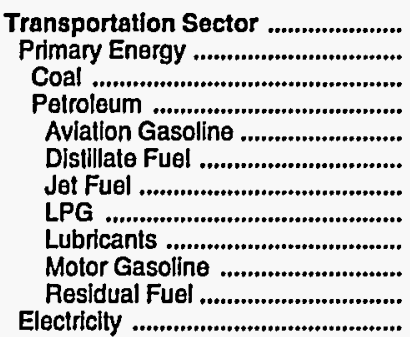 & $\begin{array}{r}703.1 \\
703.1 \\
703.1 \\
3.0 \\
36.6 \\
14.7 \\
.4 \\
19.3 \\
628.9 \\
.1 \\
-\end{array}$ & $\begin{array}{r}1,303.8 \\
1,303.8 \\
1, \\
1,303.7 \\
3.7 \\
104.1 \\
66.5 \\
.9 \\
34.1 \\
1,088.1 \\
6.2 \\
\end{array}$ & $\begin{aligned} 2,864.5 \\
2,864.5 \\
2,864.5 \\
8.8 \\
338.4 \\
188.3 \\
1.2 \\
69.3 \\
2,235.3 \\
23.2 \\
\end{aligned}$ & $\begin{array}{r}2,988.3 \\
2,988.3 \\
2,988.3 \\
7.8 \\
403.6 \\
261.4 \\
3.7 \\
77.4 \\
2,230.6 \\
3.8 \\
\end{array}$ & $\begin{aligned} 2,288.9 \\
2,288.9 \\
-\overline{2} \\
2,288.9 \\
9.6 \\
319.6 \\
178.6 \\
3.8 \\
67.0 \\
1,709.5 \\
.8\end{aligned}$ & $\begin{array}{r}2,386.3 \\
2,386.3 \\
2,386.3 \\
6.8 \\
354.7 \\
132.8 \\
1.6 \\
61.7 \\
1,828.6 \\
.1\end{array}$ & $\begin{array}{r}2,513.5 \\
2,513.5 \\
-\overline{2,513.5} \\
6.2 \\
378.6 \\
114.7 \\
2.1 \\
68.4 \\
1,943.4 \\
.1\end{array}$ & $\begin{array}{r}2,757.0 \\
2,757.0 \\
-\overline{0} \\
2,757.0 \\
6.6 \\
408.2 \\
118.0 \\
1.6 \\
63.9 \\
2,158.7 \\
-\end{array}$ & $\begin{array}{r}3,002.6 \\
3,002.6 \\
3,002.6 \\
10.0 \\
508.9 \\
164.0 \\
1.9 \\
66.3 \\
2,251.5 \\
=\end{array}$ & $\begin{array}{r}3,039.2 \\
3,039.2 \\
- \\
3,039.2 \\
8.3 \\
507.6 \\
135.7 \\
1.6 \\
68.2 \\
2,318.0\end{array}$ \\
\hline 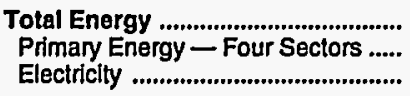 & $\begin{array}{r}1,645.1 \\
1,217.6 \\
427.5\end{array}$ & $\begin{array}{r}3,094.6 \\
2,324.7 \\
769.9\end{array}$ & $\begin{array}{l}6,059.1 \\
4,577.9 \\
1,481.2\end{array}$ & $\begin{array}{l}7,322.9 \\
5,260.1 \\
2,062.8\end{array}$ & $\begin{array}{l}6,269.7 \\
4,172.5 \\
2,097.2\end{array}$ & $\begin{array}{l}6,159.8 \\
3,947.8 \\
2,211.9\end{array}$ & $\begin{array}{l}6,708.0 \\
4,274.2 \\
2,433.7\end{array}$ & $\begin{array}{l}7,052.5 \\
4,642.6 \\
2,409.9\end{array}$ & $\begin{array}{l}7,453.6 \\
4,962.4 \\
2,491.2\end{array}$ & $\begin{array}{l}7,597.2 \\
4,958.6 \\
2,638.6\end{array}$ \\
\hline 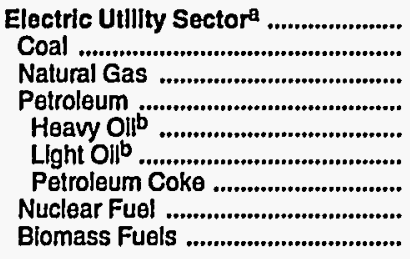 & $\begin{array}{r}65.3 \\
43.1 \\
15.3 \\
6.9 \\
3.9 \\
2.7 \\
.2 \\
. \overline{1}\end{array}$ & $\begin{array}{r}144.2 \\
84.9 \\
14.2 \\
19.5 \\
10.4 \\
8.9 \\
.2 \\
25.5\end{array}$ & $\begin{array}{r}311.3 \\
230.9 \\
16.0 \\
15.8 \\
10.1 \\
5.6 \\
-\overline{-} \\
48.6\end{array}$ & $\begin{array}{r}355.5 \\
286.5 \\
4.7 \\
1.7 \\
4 \\
1.7 \\
62.5 \\
-\end{array}$ & $\begin{array}{r}307.0 \\
241.4 \\
5.1 \\
1.4 \\
1 . \overline{-} \\
59 . \overline{-} \\
-\end{array}$ & $\begin{array}{r}362.5 \\
284.5 \\
11.8 \\
2.6 \\
. \\
2.6 \\
63.1 \\
.4\end{array}$ & $\begin{array}{r}419.5 \\
339.9 \\
10.4 \\
3.7 \\
.1 \\
2.8 \\
.8 \\
64.2 \\
1.4\end{array}$ & $\begin{array}{r}433.2 \\
358.3 \\
9.5 \\
5.4 \\
.1 \\
2.1 \\
3.2 \\
57.4 \\
2.6\end{array}$ & $\begin{array}{r}454.0 \\
372.2 \\
10.1 \\
6.2 \\
6 \\
2.8 \\
3.3 \\
61.7 \\
3.7\end{array}$ & $\begin{array}{r}439.2 \\
357.4 \\
10.1 \\
6.9\end{array}$ \\
\hline Primary Enorgy - Flve Sectors ${ }^{c}$.. & $1,282.9$ & $2,468.9$ & $4,889.2$ & $5,615.6$ & $4,479.6$ & $4,310.4$ & 4,693.7 & $5,075.8$ & $5,416.3$ & $5,397.9$ \\
\hline
\end{tabular}

a There are no direct fuel costs for hydroelectric, geothermal, centralized solar, or wind energy.

b Heavy oil includes Grade Nos. 4, 5, and 6, and residual fuel oils. Light oil includes Grade No. 2 heating oil, kerosene, and jet fuel.

c Biomass fuels are not included, except those consumed at electric utilities and those added to motor gasoline.

- No consumption, including cases where adjustments were made. See "Consumption Adjustments for Process Fuel and Intermediate Products."

Value less than 0.05 million dollars.

Note: Totals may not equal sum of components due to independent rounding.

Sources: Data sources, estimation procedures, and assumptions are described in the "Documentation" section of this report. 


\section{Energy Price and Expenditure Estimates by Source, Mississippi \\ I $1970,1975,1980$, and 1985-1991}

\begin{tabular}{|c|c|c|c|c|c|c|c|c|c|c|}
\hline Energy Source & 1970 & 1975 & 1980 & 1985 & 1986 & 1987 & 1988 & 1989 & 1990 & 1991 \\
\hline & \multicolumn{10}{|c|}{ Prices In Dollars per Million Btu } \\
\hline 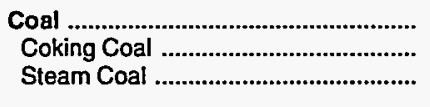 & $\begin{array}{r}0.26 \\
.26\end{array}$ & $\begin{array}{c}0.83 \\
.83\end{array}$ & $\frac{1.83}{1.83}$ & $\begin{array}{r}2.50 \\
2.50\end{array}$ & $\begin{array}{r}2.27 \\
2.27\end{array}$ & $\frac{1.94}{1.94}$ & $\begin{array}{r}1.81 \\
1 . \overline{81}\end{array}$ & $\begin{array}{r}1.67 \\
- \\
1.67\end{array}$ & $\begin{array}{r}1.66 \\
- \\
1.66\end{array}$ & $\begin{array}{r}1.67 \\
1.67\end{array}$ \\
\hline 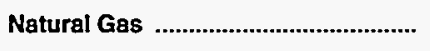 & .38 & .87 & 2.55 & 3.76 & 3.40 & $\mathbf{3 . 3 3}$ & 3.19 & 2.89 & 2.76 & 2.59 \\
\hline 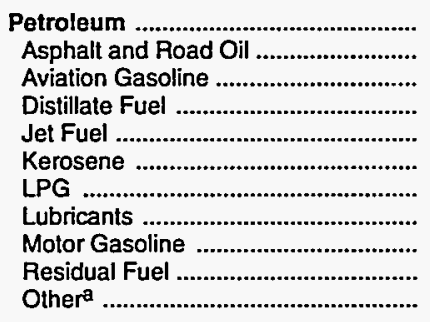 & $\begin{array}{r}2.12 \\
.66 \\
2.17 \\
1.32 \\
.73 \\
.83 \\
1.83 \\
5.08 \\
2.84 \\
.45 \\
.50\end{array}$ & $\begin{array}{l}3.10 \\
1.77 \\
3.45 \\
2.24 \\
2.03 \\
2.03 \\
3.42 \\
7.48 \\
4.34 \\
1.67 \\
1.48\end{array}$ & $\begin{array}{r}7.04 \\
3.56 \\
9.02 \\
6.89 \\
6.39 \\
6.99 \\
6.31 \\
14.36 \\
10.53 \\
2.84 \\
3.39\end{array}$ & $\begin{array}{r}7.59 \\
4.81 \\
9.99 \\
6.68 \\
5.84 \\
8.35 \\
7.40 \\
17.61 \\
8.75 \\
4.06 \\
4.93\end{array}$ & $\begin{array}{r}5.56 \\
4.90 \\
8.41 \\
5.00 \\
3.41 \\
5.49 \\
8.07 \\
15.59 \\
6.54 \\
2.01 \\
3.74\end{array}$ & $\begin{array}{r}5.92 \\
3.27 \\
7.55 \\
5.44 \\
3.97 \\
5.52 \\
8.46 \\
12.70 \\
6.97 \\
1.94 \\
4.33\end{array}$ & $\begin{array}{r}5.86 \\
3.03 \\
7.41 \\
5.29 \\
3.43 \\
4.45 \\
8.78 \\
14.61 \\
7.27 \\
2.79 \\
3.71\end{array}$ & $\begin{array}{r}6.51 \\
2.86 \\
8.28 \\
5.74 \\
3.92 \\
4.44 \\
9.03 \\
13.30 \\
8.07 \\
2.41 \\
4.36\end{array}$ & $\begin{array}{r}7.21 \\
2.93 \\
9.32 \\
6.75 \\
5.16 \\
5.54 \\
6.38 \\
13.40 \\
9.21 \\
2.33 \\
5.19\end{array}$ & $\begin{array}{r}6.86 \\
3.29 \\
8.71 \\
6.41 \\
4.59 \\
5.35 \\
7.29 \\
15.42 \\
8.92 \\
1.75 \\
4.33\end{array}$ \\
\hline Nuclear Fuel ......................................... & - & - & - & 1.13 & 1.15 & .89 & 1.40 & 1.17 & 1.11 & .88 \\
\hline Blomass Fuels at Utillties .................. & - & - & - & - & - & - & - & $\rightarrow$ & - & - \\
\hline Primary Energy - Five Sectors ${ }^{b} \ldots$ & 1.15 & 2.27 & 4.92 & 5.17 & 4.14 & 4.06 & 4.01 & 4.30 & 4.57 & 4.31 \\
\hline $\begin{array}{l}\text { Electric Utility Fuelc .......................... } \\
\text { Electricity Purchased by End Users }\end{array}$ & $\begin{array}{r}.27 \\
4.44\end{array}$ & $\begin{array}{l}1.24 \\
7.58\end{array}$ & $\begin{array}{r}2.16 \\
13.69\end{array}$ & $\begin{array}{r}2.30 \\
17.05\end{array}$ & $\begin{array}{r}2.05 \\
18.01\end{array}$ & $\begin{array}{r}1.57 \\
17.87\end{array}$ & $\begin{array}{r}1.70 \\
18.21\end{array}$ & $\begin{array}{r}1.56 \\
18.25\end{array}$ & $\begin{array}{r}1.54 \\
18.05\end{array}$ & $\begin{array}{r}1.35 \\
17.79\end{array}$ \\
\hline \multirow[t]{2}{*}{ 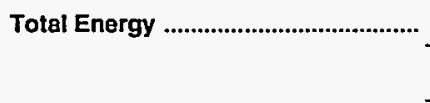 } & 1.71 & 3.20 & 7.32 & 8.20 & 7.25 & 7.37 & 7.29 & 7.75 & 8.25 & 7.93 \\
\hline & \multicolumn{10}{|c|}{ Expenditures in Millions of Dollars } \\
\hline 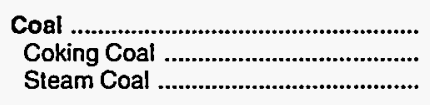 & $\begin{array}{r}3.5 \\
3 . \overline{5}\end{array}$ & $\begin{array}{r}27.5 \\
27 . \overline{5}\end{array}$ & $\begin{array}{r}137.6 \\
137 . \overline{6}\end{array}$ & $\begin{array}{r}273.2 \\
- \\
273.2\end{array}$ & $\begin{array}{r}246.5 \\
-\overline{2} \\
246.5\end{array}$ & $\begin{array}{r}237.6 \\
-\overline{6} \\
237.6\end{array}$ & $\begin{array}{r}234.1 \\
- \\
234.1\end{array}$ & $\begin{array}{r}161.3 \\
- \\
161.3\end{array}$ & $\begin{array}{r}172.3 \\
- \\
172.3\end{array}$ & $\begin{array}{r}159.4 \\
- \\
159.4\end{array}$ \\
\hline 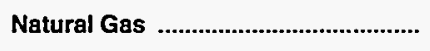 & 111.2 & 154.3 & 553.4 & 710.7 & 581.3 & 530.0 & 515.9 & 520.5 & 557.5 & 524.3 \\
\hline 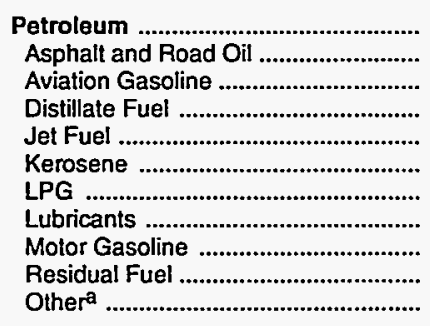 & $\begin{array}{r}\mathbf{5 1 8 . 8} \\
7.7 \\
3.5 \\
46.2 \\
6.3 \\
12.5 \\
59.4 \\
16.2 \\
362.5 \\
1.9 \\
2.7\end{array}$ & $\begin{array}{r}1,099.2 \\
30.4 \\
3.5 \\
127.6 \\
16.3 \\
16.5 \\
102.3 \\
30.9 \\
633.5 \\
126.6 \\
11.6\end{array}$ & $\begin{array}{r}2,475.0 \\
48.1 \\
9.4 \\
383.8 \\
53.3 \\
9.6 \\
125.9 \\
57.1 \\
1,481.0 \\
284.7 \\
22.3\end{array}$ & $\begin{array}{r}2,336.5 \\
65.5 \\
5.4 \\
619.3 \\
134.1 \\
4.1 \\
124.4 \\
63.7 \\
1,267.2 \\
33.5 \\
19.2\end{array}$ & $\begin{array}{r}1,805.1 \\
61.9 \\
5.8 \\
431.5 \\
93.8 \\
2.7 \\
107.4 \\
55.1 \\
980.8 \\
53.0 \\
13.1\end{array}$ & $\begin{array}{r}2,031.1 \\
47.2 \\
4.3 \\
530.5 \\
171.0 \\
2.4 \\
114.3 \\
50.8 \\
1,070.9 \\
24.8 \\
14.9\end{array}$ & $\begin{array}{r}2,181.6 \\
52.8 \\
4.8 \\
586.0 \\
154.5 \\
2.2 \\
125.8 \\
56.3 \\
1,126.9 \\
59.2 \\
12.9\end{array}$ & $\begin{array}{r}2,273.6 \\
37.5 \\
6.4 \\
571.9 \\
144.6 \\
1.6 \\
166.3 \\
52.6 \\
1,229.4 \\
48.8 \\
14.5\end{array}$ & $\begin{array}{r}2,579.3 \\
48.8 \\
6.2 \\
633.8 \\
201.1 \\
1.7 \\
164.4 \\
54.5 \\
1,399.1 \\
50.2 \\
19.3\end{array}$ & $\begin{array}{r}2,516.8 \\
55.2 \\
4.8 \\
576.4 \\
205.4 \\
1.9 \\
152.1 \\
56.1 \\
1,396.0 \\
51.3 \\
17.5\end{array}$ \\
\hline 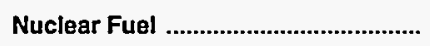 & - & - & - & 53.1 & 50.6 & 74.1 & 143.8 & 97.9 & 87.9 & 86.7 \\
\hline Biomass Fuels at Utilities ................. & - & - & - & - & - & - & - & - & - & - \\
\hline Primary Energy - Five Sectors ${ }^{b}$..... & 633.5 & $1,281.1$ & $3,166.1$ & $3,373.5$ & $2,683.5$ & $2,872.8$ & $3,075.5$ & $3,053.3$ & $3,397.0$ & $3,287.3$ \\
\hline $\begin{array}{l}\text { Electric Utility Fuelc .......................... } \\
\text { Electricity Purchased by End Users }\end{array}$ & $\begin{array}{r}-31.7 \\
225.9\end{array}$ & $\begin{array}{r}-154.7 \\
486.0\end{array}$ & $\begin{array}{r}-438.6 \\
1,075.9\end{array}$ & $\begin{array}{r}-476.1 \\
1,455.8\end{array}$ & $\begin{array}{r}-420.1 \\
1,582.5\end{array}$ & $\begin{array}{r}-381.2 \\
1,606.2\end{array}$ & $\begin{array}{r}-453.7 \\
1,694.2\end{array}$ & $\begin{array}{r}-355.7 \\
1,786.8\end{array}$ & $\begin{array}{r}-387.1 \\
1,914.5\end{array}$ & $\begin{array}{r}-346.5 \\
1,940.5\end{array}$ \\
\hline Total Energy & 827.7 & $1,612.4$ & $3,803.3$ & $4,353.2$ & $3,846.0$ & $4,097.8$ & $4,316.0$ & $4,484.4$ & $4,924.4$ & $4,881.3$ \\
\hline
\end{tabular}

a Includes petroleum coke used at electric utilities.

biomass fuels are not included, except those consumed at electric utilities and those added to motor gasoline.

c There are no direct fuel costs for hydroelectric, geothermal, centralized solar, or wind energy.

-No consumption, including cases where adjustments were made. See "Consumption Adjustments for Process Fuel and Inlermediate Products."

Note: Expenditure totals may not equal sum of components due to independent rounding.

Sources: Data sources, estimation procedures, and assumptions are described in the "Documentation" section of this report. 


\begin{tabular}{|c|c|c|c|c|c|c|c|c|c|c|}
\hline Sector and Energy Source & 1970 & 1975 & 1980 & 1985 & 1986 & 1987 & 1988 & 1989 & 1990 & 1991 \\
\hline 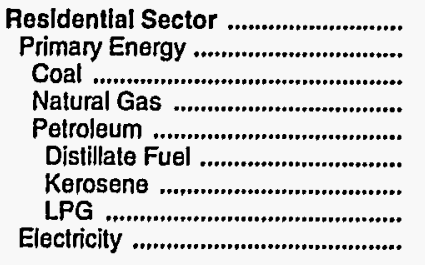 & $\begin{array}{r}2.39 \\
1.31 \\
- \\
.86 \\
2.14 \\
1.24 \\
2.06 \\
2.16 \\
5.06\end{array}$ & $\begin{array}{r}4.43 \\
2.34 \\
- \\
1.38 \\
3.98 \\
2.49 \\
3.79 \\
4.10 \\
8.06\end{array}$ & $\begin{array}{r}9.08 \\
4.45 \\
2.97 \\
3.36 \\
8.41 \\
6.89 \\
10.48 \\
8.35 \\
14.38\end{array}$ & $\begin{array}{r}12.18 \\
5.85 \\
2.74 \\
5.33 \\
7.78 \\
7.07 \\
10.96 \\
7.71 \\
18.12\end{array}$ & $\begin{array}{r}13.06 \\
6.09 \\
2.89 \\
5.47 \\
8.58 \\
5.15 \\
7.99 \\
8.61 \\
19.13\end{array}$ & $\begin{array}{r}13.10 \\
6.62 \\
2.88 \\
6.01 \\
8.82 \\
4.89 \\
7.58 \\
8.89 \\
19.01\end{array}$ & $\begin{array}{r}13.14 \\
6.49 \\
2.62 \\
5.76 \\
9.11 \\
4.42 \\
6.85 \\
9.17 \\
19.15\end{array}$ & $\begin{array}{r}13.38 \\
6.08 \\
2.64 \\
4.95 \\
9.69 \\
3.86 \\
5.99 \\
9.77 \\
20.00\end{array}$ & $\begin{array}{r}13.93 \\
6.17 \\
2.70 \\
5.16 \\
9.47 \\
4.59 \\
7.12 \\
9.49 \\
20.19\end{array}$ & $\begin{array}{r}14.03 \\
6.20 \\
2.81 \\
5.06 \\
10.61 \\
4.23 \\
6.55 \\
10.70 \\
20.16\end{array}$ \\
\hline 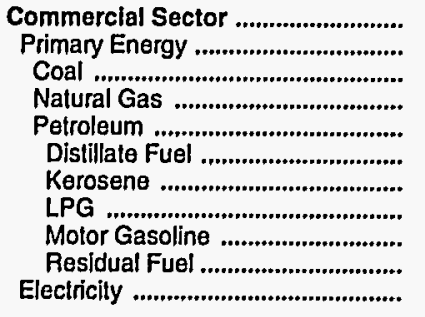 & $\begin{array}{r}1.96 \\
.70 \\
- \\
.57 \\
1.39 \\
.96 \\
- \\
1.33 \\
2.84 \\
.49 \\
5.53\end{array}$ & $\begin{array}{r}3.34 \\
1.29 \\
-\overline{9} \\
.92 \\
2.17 \\
2.18 \\
- \\
2.66 \\
4.34 \\
1.72 \\
8.59\end{array}$ & $\begin{array}{r}6.70 \\
3.17 \\
1.65 \\
2.97 \\
3.36 \\
6.27 \\
- \\
4.92 \\
10.53 \\
3.02 \\
15.87\end{array}$ & $\begin{array}{r}11.75 \\
5.37 \\
1.85 \\
4.95 \\
6.23 \\
5.74 \\
7.17 \\
7.19 \\
8.75 \\
4.33 \\
19.50\end{array}$ & $\begin{array}{r}12.54 \\
4.83 \\
1.78 \\
4.88 \\
4.69 \\
3.26 \\
3.51 \\
7.60 \\
6.54 \\
2.12 \\
20.66\end{array}$ & $\begin{array}{r}12.09 \\
5.15 \\
1.70 \\
5.21 \\
5.04 \\
3.86 \\
4.38 \\
7.94 \\
6.97 \\
2.18 \\
20.22\end{array}$ & $\begin{array}{r}12.75 \\
4.92 \\
1.68 \\
4.94 \\
4.94 \\
3.08 \\
3.34 \\
8.34 \\
7.27 \\
1.94 \\
21.34\end{array}$ & $\begin{array}{r}12.66 \\
4.58 \\
1.70 \\
4.59 \\
4.57 \\
2.88 \\
3.61 \\
8.42 \\
8.07 \\
2.71 \\
21.18\end{array}$ & $\begin{array}{r}13.17 \\
4.52 \\
1.74 \\
4.34 \\
5.09 \\
4.08 \\
5.08 \\
5.02 \\
9.21 \\
- \\
21.33\end{array}$ & $\begin{array}{r}13.11 \\
4.26 \\
1.74 \\
4.15 \\
4.65 \\
3.80 \\
4.64 \\
5.67 \\
8.92 \\
2.03 \\
21.24\end{array}$ \\
\hline 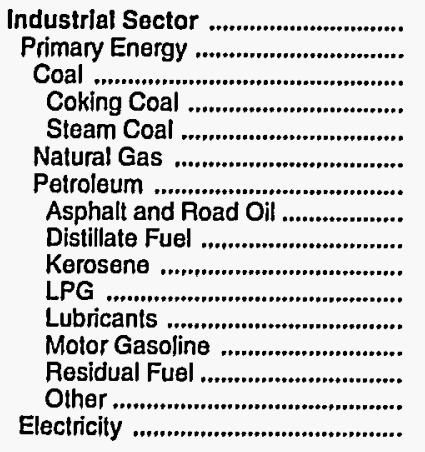 & $\begin{array}{r}.70 \\
.50 \\
.33 \\
.33 \\
.33 \\
.29 \\
.94 \\
.66 \\
.74 \\
.79 \\
1.33 \\
5.08 \\
2.84 \\
.40 \\
.50 \\
2.94\end{array}$ & $\begin{array}{r}1.94 \\
1.32 \\
1.11 \\
1.11 \\
.71 \\
2.05 \\
1.77 \\
1.70 \\
1.86 \\
2.66 \\
7.48 \\
4.34 \\
1.77 \\
1.48 \\
6.39\end{array}$ & $\begin{array}{r}4.90 \\
3.61 \\
1.65 \\
- \\
1.65 \\
2.66 \\
4.61 \\
3.56 \\
5.55 \\
6.21 \\
4.92 \\
14.36 \\
10.53 \\
2.82 \\
3.39 \\
11.42\end{array}$ & $\begin{array}{r}6.13 \\
4.72 \\
1.85 \\
- \\
1.85 \\
3.68 \\
6.45 \\
4.81 \\
6.24 \\
7.17 \\
7.19 \\
17.61 \\
8.75 \\
4.33 \\
4.93 \\
13.94\end{array}$ & $\begin{array}{r}5.44 \\
3.57 \\
1.78 \\
- \\
1.78 \\
3.08 \\
4.39 \\
4.90 \\
3.06 \\
3.51 \\
7.60 \\
15.59 \\
6.54 \\
2.12 \\
3.74 \\
14.63\end{array}$ & $\begin{array}{r}5.45 \\
3.43 \\
1.70 \\
- \\
1.70 \\
2.71 \\
4.48 \\
3.27 \\
3.81 \\
4.38 \\
7.94 \\
12.70 \\
6.97 \\
2.18 \\
4.33 \\
14.74\end{array}$ & $\begin{array}{r}5.08 \\
3.07 \\
1.68 \\
- \\
1.68 \\
2.50 \\
3.90 \\
3.03 \\
2.90 \\
3.34 \\
8.34 \\
14.61 \\
7.27 \\
1.94 \\
3.71 \\
14.84\end{array}$ & $\begin{array}{r}5.15 \\
3.19 \\
1.70 \\
- \\
1.70 \\
2.46 \\
4.36 \\
2.86 \\
3.14 \\
3.61 \\
8.42 \\
13.30 \\
8.07 \\
2.71 \\
4.36 \\
14.18\end{array}$ & $\begin{array}{r}5.26 \\
3.42 \\
1.74 \\
- \\
1.74 \\
2.49 \\
4.61 \\
2.93 \\
4.42 \\
5.08 \\
5.02 \\
13.40 \\
9.21 \\
3.02 \\
5.19 \\
13.63\end{array}$ & $\begin{array}{r}5.20 \\
3.25 \\
1.74 \\
- \\
1.74 \\
2.28 \\
4.72 \\
3.29 \\
4.04 \\
4.64 \\
5.67 \\
15.42 \\
8.92 \\
2.03 \\
4.33 \\
13.16\end{array}$ \\
\hline 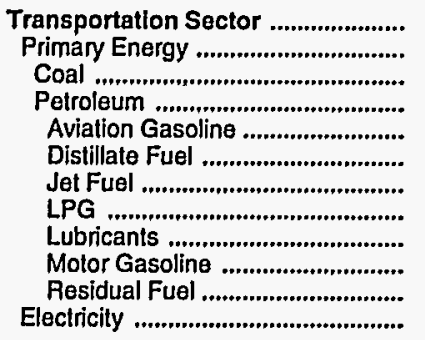 & $\begin{array}{r}2.64 \\
2.64 \\
.33 \\
2.64 \\
2.17 \\
2.02 \\
.73 \\
1.33 \\
5.08 \\
2.84 \\
.43 \\
-\end{array}$ & $\begin{array}{r}3.91 \\
3.91 \\
1.11 \\
3.91 \\
3.45 \\
2.75 \\
2.03 \\
2.66 \\
7.48 \\
4.34 \\
1.49 \\
-\end{array}$ & $\begin{array}{r}8.71 \\
8.71 \\
- \\
8.71 \\
9.02 \\
7.67 \\
6.39 \\
4.92 \\
14.36 \\
10.53 \\
2.55 \\
-\end{array}$ & $\begin{array}{r}7.97 \\
7.97 \\
-\overline{7} \\
7.97 \\
9.99 \\
7.05 \\
5.84 \\
7.19 \\
17.61 \\
8.75 \\
4.03 \\
-\end{array}$ & $\begin{array}{r}5.91 \\
5.91 \\
- \\
5.91 \\
8.41 \\
5.96 \\
3.41 \\
7.60 \\
15.59 \\
6.54 \\
1.90 \\
-\end{array}$ & $\begin{array}{r}6.20 \\
6.20 \\
- \\
6.20 \\
7.55 \\
6.44 \\
3.97 \\
7.94 \\
12.70 \\
6.97 \\
1.92 \\
-\end{array}$ & $\begin{array}{r}6.31 \\
6.31 \\
- \\
6.31 \\
7.41 \\
6.42 \\
3.43 \\
8.34 \\
14.61 \\
7.27 \\
2.79 \\
-\end{array}$ & $\begin{array}{r}7.11 \\
7.11 \\
- \\
7.11 \\
8.28 \\
7.12 \\
3.92 \\
8.42 \\
13.30 \\
8.07 \\
2.15 \\
-\end{array}$ & $\begin{array}{r}8.14 \\
8.14 \\
- \\
8.14 \\
9.32 \\
8.25 \\
5.16 \\
5.02 \\
13.40 \\
9.21 \\
2.01 \\
-\end{array}$ & $\begin{array}{r}7.39 \\
7.39 \\
- \\
7.39 \\
8.71 \\
7.73 \\
4.59 \\
5.67 \\
15.42 \\
8.92 \\
1.71 \\
-\end{array}$ \\
\hline $\begin{array}{l}\text { Total Energy .................................... } \\
\text { Primary Energy - Four Sectors ...... } \\
\text { Electricity ......................................... }\end{array}$ & $\begin{array}{l}1.71 \\
1.39 \\
4.44\end{array}$ & $\begin{array}{l}3.20 \\
2.56 \\
7.58\end{array}$ & $\begin{array}{r}7.32 \\
6.18 \\
13.69\end{array}$ & $\begin{array}{r}8.20 \\
6.50 \\
17.05\end{array}$ & $\begin{array}{r}7.25 \\
5.11 \\
18.01\end{array}$ & $\begin{array}{r}7.37 \\
5.35 \\
17.87\end{array}$ & $\begin{array}{r}7.29 \\
5.25 \\
18.21\end{array}$ & $\begin{array}{r}7.75 \\
5.61 \\
18.25\end{array}$ & $\begin{array}{r}8.25 \\
6.13 \\
18.05\end{array}$ & $\begin{array}{r}7.93 \\
5.80 \\
17.79\end{array}$ \\
\hline 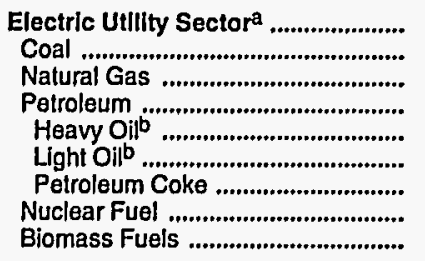 & $\begin{array}{l}.27 \\
.26 \\
.27 \\
.48 \\
.48 \\
.61 \\
- \\
- \\
-\end{array}$ & $\begin{array}{r}1.24 \\
.82 \\
.83 \\
1.70 \\
1.69 \\
2.08 \\
- \\
- \\
-\end{array}$ & $\begin{array}{r}2.16 \\
1.84 \\
2.11 \\
3.06 \\
3.03 \\
5.47 \\
- \\
- \\
-\end{array}$ & $\begin{array}{r}2.30 \\
2.54 \\
-\quad .79 \\
4.78 \\
4.16 \\
5.97 \\
-\overline{13} \\
1.13 \\
-\end{array}$ & $\begin{array}{r}2.05 \\
2.29 \\
2.31 \\
2.13 \\
2.06 \\
4.45 \\
-\overline{15} \\
1.15 \\
-\end{array}$ & $\begin{array}{r}1.57 \\
1.96 \\
1.85 \\
2.38 \\
2.07 \\
3.76 \\
- \\
.89 \\
-\end{array}$ & $\begin{array}{r}1.70 \\
1.81 \\
1.85 \\
3.26 \\
3.18 \\
4.99 \\
- \\
1.40 \\
-\end{array}$ & $\begin{array}{r}1.56 \\
1.67 \\
1.83 \\
2.65 \\
2.48 \\
5.32 \\
-\overline{1.17} \\
-\end{array}$ & $\begin{array}{r}1.54 \\
1.65 \\
1.76 \\
2.44 \\
2.35 \\
4.80 \\
- \\
1.11 \\
-\end{array}$ & $\begin{array}{r}1.35 \\
1.67 \\
1.57 \\
2.25 \\
1.94 \\
4.74 \\
- \\
.88 \\
-\end{array}$ \\
\hline PrImary Energy - Flve Sectors ${ }^{c}$... & 1.15 & 2.27 & 4.92 & 5.17 & 4.14 & 4.06 & 4.01 & 4.30 & 4.57 & 4.31 \\
\hline
\end{tabular}

a There are no direct fuel costs for hydroelectric, geothermal, centralized solar, or wind energy.

b Heavy oil includes Grade Nos. 4, 5, and 6, and residual fuel oils. Light oil includes Grade No. 2 heating oil, kerosene, and jet fuel.

c Biomass fuels are not included, except those consumed at electric utilities and those added to motor gasoline.

-No consumption, including cases where adjustments were made. See "Consumption Adjustments for Process Fuel and Intermediate Products."

Sources; Data sources, estimation procedures, and assumptions are described in the "Documentation" section of this report. 


\section{Energy Expenditure Estimates by Sector, Mississippi 1970, 1975, 1980, and 1985-1991}

$\mathbf{S}$ (Million Dollars)

\begin{tabular}{|c|c|c|c|c|c|c|c|c|c|c|}
\hline Sector and Energy Source & 1970 & 1975 & 1980 & 1985 & 1986 & 1987 & 1988 & 1989 & 1990 & 1991 \\
\hline 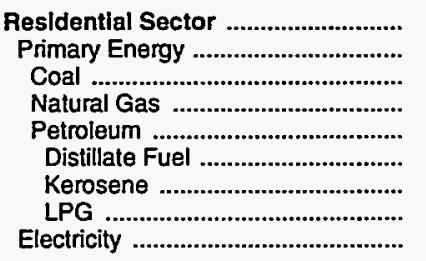 & $\begin{array}{r}194.5 \\
75.9 \\
- \\
32.4 \\
43.5 \\
.6 \\
.9 \\
42.0 \\
118.7\end{array}$ & $\begin{array}{r}334.1 \\
111.5 \\
-\overline{4} \\
71.6 \\
70.0 \\
2.8 \\
2.7 \\
64.4 \\
222.5\end{array}$ & $\begin{array}{r}661.9 \\
173.0 \\
102.6 \\
70.4 \\
.3 \\
2.6 \\
67.5 \\
488.9\end{array}$ & $\begin{array}{r}841.3 \\
195.3 \\
140.4 \\
54.9 \\
.1 \\
1.7 \\
53.2 \\
646.0\end{array}$ & $\begin{array}{r}905.7 \\
196.3 \\
.1 \\
141.4 \\
54.9 \\
.1 \\
1.7 \\
53.1 \\
709.4\end{array}$ & $\begin{array}{r}951.1 \\
229.3 \\
.1 \\
162.3 \\
66.9 \\
.5 \\
1.2 \\
65.3 \\
721.8\end{array}$ & $\begin{array}{r}974.4 \\
228.5 \\
.3 \\
157.3 \\
70.9 \\
.1 \\
1.1 \\
69.7 \\
746.0\end{array}$ & $\begin{array}{r}1,002.9 \\
216.9 \\
134.2 \\
82.6 \\
.1 \\
.8 \\
81.7 \\
786.0\end{array}$ & $\begin{array}{r}1,053.3 \\
208.3 \\
- \\
133.5 \\
74.8 \\
. \\
74.3 \\
845.0\end{array}$ & $\begin{array}{r}1,068.0 \\
207.1 \\
134.2 \\
72.9 \\
: \\
.8 \\
72.0 \\
861.0\end{array}$ \\
\hline 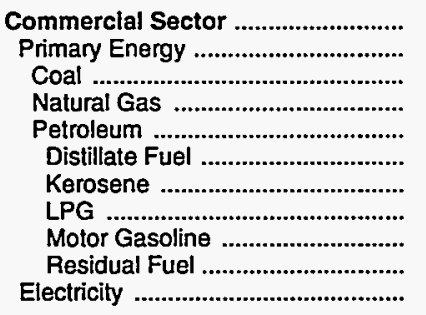 & $\begin{array}{r}77.5 \\
20.5 \\
- \\
13.9 \\
6.7 \\
.6 \\
\overline{-} \\
4.6 \\
1.4 \\
.1 \\
57.0\end{array}$ & $\begin{array}{r}161.8 \\
45.1 \\
-\overline{6} \\
22.6 \\
22.5 \\
3.0 \\
- \\
7.4 \\
2.4 \\
9.7 \\
116.7\end{array}$ & $\begin{array}{r}420.3 \\
143.5 \\
64.1 \\
79.4 \\
.9 \\
7 . \overline{0} \\
6.8 \\
64.7 \\
276.8\end{array}$ & $\begin{array}{r}544.5 \\
136.6 \\
84.1 \\
52.5 \\
35.7 \\
1.6 \\
8.8 \\
6.2 \\
.3 \\
407.9\end{array}$ & $\begin{array}{r}556.5 \\
109.9 \\
.1 \\
84.1 \\
25.7 \\
8.4 \\
.4 \\
8.3 \\
7.5 \\
1.2 \\
446.7\end{array}$ & $\begin{array}{r}571.0 \\
131.2 \\
.1 \\
94.8 \\
36.3 \\
17.9 \\
.2 \\
10.3 \\
7.7 \\
.3 \\
439.8\end{array}$ & $\begin{array}{r}597.6 \\
120.6 \\
.3 \\
90.9 \\
29.3 \\
10.7 \\
.1 \\
11.2 \\
7.1 \\
.2 \\
477.0\end{array}$ & $\begin{array}{r}630.0 \\
117.0 \\
8 \\
83.1 \\
33.8 \\
14.3 \\
.1 \\
12.4 \\
6.8 \\
.2 \\
513.0\end{array}$ & $\begin{array}{r}646.7 \\
107.7 \\
78.6 \\
29.1 \\
14.0 \\
.2 \\
6.9 \\
8.0 \\
- \\
539.0\end{array}$ & $\begin{array}{r}642.1 \\
100.1 \\
75.9 \\
24.1 \\
13.4 \\
.2 \\
6.7 \\
3.8 \\
542.0\end{array}$ \\
\hline 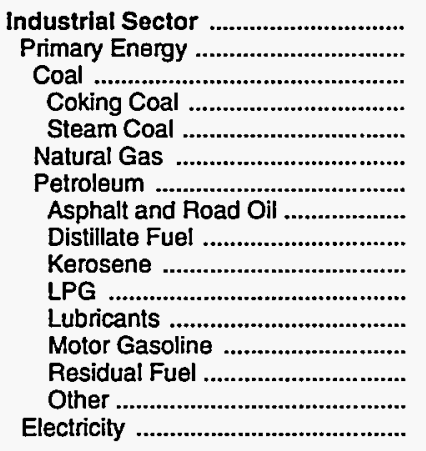 & $\begin{array}{r}146.6 \\
96.4 \\
.4 \\
- \\
.4 \\
37.6 \\
58.4 \\
7.7 \\
13.3 \\
11.6 \\
10.5 \\
7.5 \\
4.6 \\
.5 \\
2.7 \\
50.2\end{array}$ & $\begin{array}{r}365.8 \\
219.1 \\
.6 \\
- \\
.6 \\
63.2 \\
155.3 \\
30.4 \\
43.4 \\
13.8 \\
25.9 \\
17.0 \\
5.0 \\
8.3 \\
11.6 \\
146.7\end{array}$ & $\begin{array}{r}803.2 \\
492.9 \\
2.0 \\
- \\
2.0 \\
182.6 \\
308.3 \\
48.1 \\
111.3 \\
7.0 \\
48.6 \\
29.7 \\
4.1 \\
37.3 \\
22.3 \\
310.2\end{array}$ & $\begin{array}{r}1,151.1 \\
749.2 \\
10.7 \\
- \\
10.7 \\
330.6 \\
407.8 \\
65.5 \\
196.0 \\
.8 \\
56.4 \\
33.1 \\
34.5 \\
2.2 \\
19.2 \\
401.9\end{array}$ & $\begin{array}{r}937.2 \\
510.8 \\
10.3 \\
- \\
10.3 \\
241.5 \\
259.0 \\
61.9 \\
79.5 \\
.6 \\
40.7 \\
28.7 \\
21.6 \\
13.0 \\
13.1 \\
426.5\end{array}$ & $\begin{array}{r}920.6 \\
476.0 \\
11.3 \\
- \\
11.3 \\
194.7 \\
270.0 \\
47.2 \\
122.7 \\
1.1 \\
34.1 \\
26.4 \\
23.0 \\
.6 \\
14.9 \\
444.6\end{array}$ & $\begin{array}{r}946.9 \\
475.6 \\
10.5 \\
-\overline{1} \\
10.5 \\
204.7 \\
260.4 \\
52.8 \\
93.1 \\
1.1 \\
40.8 \\
29.3 \\
24.2 \\
6.2 \\
12.9 \\
471.2\end{array}$ & $\begin{array}{r}992.8 \\
505.1 \\
10.5 \\
- \\
10.5 \\
218.8 \\
275.9 \\
37.5 \\
90.8 \\
.8 \\
68.7 \\
27.3 \\
23.8 \\
12.4 \\
14.5 \\
487.7\end{array}$ & $\begin{array}{r}1,133.0 \\
602.4 \\
10.9 \\
- \\
10.9 \\
226.3 \\
365.1 \\
48.8 \\
145.9 \\
1.0 \\
80.8 \\
28.4 \\
27.8 \\
13.1 \\
19.3 \\
530.5\end{array}$ & $\begin{array}{r}1,081.4 \\
543.9 \\
9.8 \\
- \\
9.8 \\
213.6 \\
320.6 \\
55.2 \\
113.6 \\
.9 \\
71.1 \\
29.2 \\
31.4 \\
1.6 \\
17.5 \\
537.5\end{array}$ \\
\hline 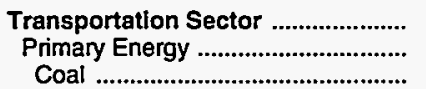 & $\begin{array}{l}409.1 \\
409.1\end{array}$ & $\begin{array}{r}750.7 \\
750.7\end{array}$ & $\begin{array}{r}1,918.0 \\
1,918.0 \\
-\end{array}$ & $\begin{array}{r}1,816.3 \\
1,816.3 \\
-\end{array}$ & $\begin{array}{r}1,446.5 \\
1,446.5 \\
-\end{array}$ & $\begin{array}{r}1,655.1 \\
1,655.1 \\
-\end{array}$ & $\begin{array}{r}1,797.1 \\
1,797.1 \\
\end{array}$ & $\begin{array}{r}1,858.7 \\
1,858.7 \\
-\end{array}$ & $\begin{array}{r}2,091.5 \\
2,091.5\end{array}$ & $\begin{array}{r}2,089.7 \\
2,089.7 \\
\text { - }\end{array}$ \\
\hline 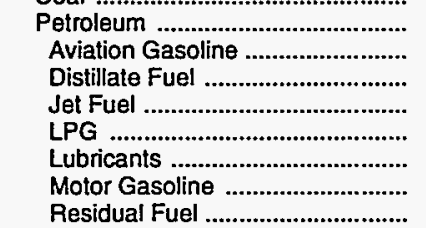 & $\begin{array}{r}409.1 \\
3.5 \\
31.6 \\
6.3 \\
2.4 \\
8.7 \\
356.5\end{array}$ & $\begin{array}{r}750.7 \\
3.5 \\
75.1 \\
16.3 \\
4.6 \\
13.9 \\
626.2 \\
11.1\end{array}$ & $\begin{array}{r}1,918.0 \\
9.4 \\
269.0 \\
53.3 \\
2.7 \\
27.4 \\
1,470.2 \\
86.0\end{array}$ & $\begin{array}{r}1,816.3 \\
5.4 \\
385.5 \\
134.1 \\
6.0 \\
30.6 \\
1,226.6 \\
28.1\end{array}$ & $\begin{array}{r}1,446.5 \\
5.8 \\
342.3 \\
93.8 \\
5.3 \\
26.5 \\
951.8 \\
21.1\end{array}$ & $\begin{array}{r}1,655.1 \\
4.3 \\
388.7 \\
171.0 \\
4.6 \\
24.4 \\
1,040.3 \\
21.9\end{array}$ & $\begin{array}{r}1,797.1 \\
4.8 \\
480.4 \\
154.5 \\
4.1 \\
27.1 \\
1,095.6 \\
30.7\end{array}$ & $\begin{array}{r}1,858.7 \\
6.4 \\
463.9 \\
144.6 \\
3.5 \\
25.2 \\
1,198.8 \\
16.2\end{array}$ & $\begin{array}{r}2,091.5 \\
6.2 \\
472.5 \\
201.1 \\
2.4 \\
26.2 \\
1,363.4 \\
19.7\end{array}$ & $\begin{array}{r}2,089.7 \\
4.8 \\
447.1 \\
205.4 \\
2.2 \\
27.0 \\
1,360.9 \\
42.3\end{array}$ \\
\hline Electricity ........................................... & - & - & - & - & - & - & - & - & - & - \\
\hline 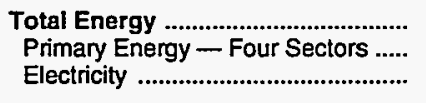 & $\begin{array}{l}827.7 \\
601.9 \\
225.9\end{array}$ & $\begin{array}{r}1,612.4 \\
1,126.4 \\
486.0\end{array}$ & $\begin{array}{l}3,803.3 \\
2,727.5 \\
1,075.9\end{array}$ & $\begin{array}{l}4,353.2 \\
2,897.4 \\
1,455.8\end{array}$ & $\begin{array}{l}3,846.0 \\
2,263.5 \\
1,582.5\end{array}$ & $\begin{array}{l}4,097.8 \\
2,491.6 \\
1,606.2\end{array}$ & $\begin{array}{l}4,316.0 \\
2,621.8 \\
1,694.2\end{array}$ & $\begin{array}{l}4,484.4 \\
2,697.6 \\
1,786.8\end{array}$ & $\begin{array}{l}4,924.4 \\
3,009.9 \\
1,914.5\end{array}$ & $\begin{array}{l}4,881.3 \\
2,940.8 \\
1,940.5\end{array}$ \\
\hline 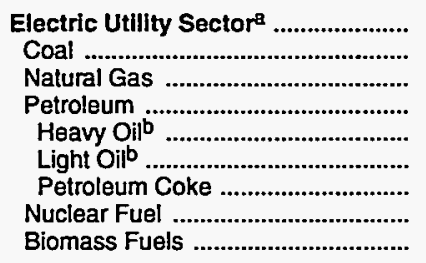 & $\begin{array}{r}31.7 \\
3.1 \\
27.3 \\
1.3 \\
1.2 \\
- \\
- \\
- \\
-\end{array}$ & $\begin{array}{r}154.7 \\
26.9 \\
26.9 \\
100.8 \\
97.6 \\
3.2 \\
- \\
- \\
-\end{array}$ & $\begin{array}{r}438.6 \\
135.5 \\
204.2 \\
98.9 \\
96.7 \\
2.2 \\
- \\
- \\
-\end{array}$ & $\begin{array}{r}476.1 \\
262.4 \\
155.6 \\
4.9 \\
2.8 \\
2.1 \\
- \\
53.1 \\
-\end{array}$ & $\begin{array}{r}420.1 \\
236.1 \\
114.4 \\
18.9 \\
17.8 \\
1.2 \\
-\overline{6} \\
50.6 \\
-\end{array}$ & $\begin{array}{r}381.2 \\
226.0 \\
78.2 \\
2.8 \\
2.0 \\
.8 \\
- \\
74.1 \\
-\end{array}$ & $\begin{array}{r}453.7 \\
223.0 \\
63.0 \\
23.8 \\
22.2 \\
1.7 \\
- \\
143.8 \\
-\end{array}$ & $\begin{array}{r}355.7 \\
150.8 \\
84.5 \\
22.6 \\
19.9 \\
2.7 \\
-\overline{9} \\
97.9 \\
-\end{array}$ & $\begin{array}{r}387.1 \\
161.3 \\
119.1 \\
18.8 \\
17.4 \\
1.4 \\
- \\
87.9 \\
-\end{array}$ & $\begin{array}{r}346.5 \\
149.6 \\
100.6 \\
9.5 \\
7.4 \\
2.2 \\
- \\
86.7 \\
-\end{array}$ \\
\hline Primary Energy - Five Sectors ${ }^{c}$.. & 633.5 & $1,281.1$ & $3,166.1$ & $3,373.5$ & $2,683.5$ & $2,872.8$ & $3,075.5$ & $3,053.3$ & $3,397.0$ & $3,287.3$ \\
\hline
\end{tabular}

a There are no direct fuel costs for hydroelectric, geothermal, centralized solar, or wind energy.

b Heavy oil includes Grade Nos. 4, 5, and 6, and residual fuel oils. Light oil includes Grade No. 2 heating oil, kerosene, and jet fuel.

c Biomass fuels are not included, except those consumed at electric utilities and those added to motor gasoline.

-No consumption, including cases where adjustments were made. See "Consumption Adjustments for Process Fuel and Intermediate Products."

- Value less than 0.05 million dollars.

Note: Totals may not equal sum of components due to independent rounding.

Sources: Data sources, estimation procedures, and assumptions are described in the "Documentation" section of this report. 


\begin{tabular}{|c|c|c|c|c|c|c|c|c|c|c|}
\hline Energy Source & 1970 & 1975 & 1980 & 1985 & 1986 & 1987 & 1988 & 1989 & 1990 & 1991 \\
\hline & \multicolumn{10}{|c|}{ Prices In Dollars per Million Btu } \\
\hline 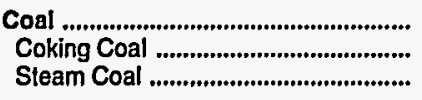 & $\begin{array}{r}0.29 \\
.38 \\
.28\end{array}$ & $\begin{array}{r}0.62 \\
1.60 \\
.60\end{array}$ & $\begin{array}{l}1.22 \\
1.81 \\
1.21\end{array}$ & $\begin{array}{l}1.51 \\
1.93 \\
1.51\end{array}$ & $\begin{array}{l}1.46 \\
1.75 \\
1.46\end{array}$ & $\begin{array}{l}1.40 \\
1.63 \\
1.40\end{array}$ & $\begin{array}{r}1.37 \\
1.37\end{array}$ & $\frac{1.35}{1.35}$ & $\begin{array}{r}1.35 \\
1.35\end{array}$ & $\begin{array}{r}1.34 \\
1.34\end{array}$ \\
\hline Natural Gas ...................................... & .64 & 1.16 & 2.95 & 4.94 & 4.52 & 4.28 & 4.37 & 4.51 & 4.69 & 4.52 \\
\hline 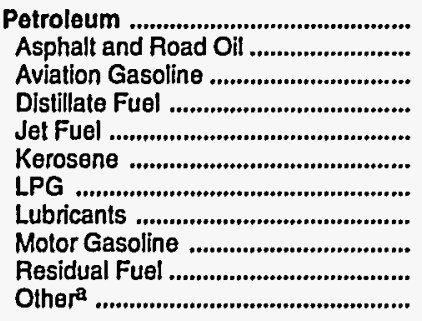 & $\begin{array}{r}1.97 \\
.71 \\
2.17 \\
1.05 \\
.75 \\
.89 \\
1.79 \\
5.08 \\
2.73 \\
.56 \\
1.44\end{array}$ & $\begin{array}{l}3.66 \\
2.05 \\
3.45 \\
2.52 \\
2.09 \\
2.45 \\
3.06 \\
7.48 \\
4.55 \\
1.78 \\
2.76\end{array}$ & $\begin{array}{r}8.04 \\
3.59 \\
9.02 \\
6.61 \\
6.47 \\
6.43 \\
6.32 \\
14.36 \\
9.33 \\
3.33 \\
7.23\end{array}$ & $\begin{array}{r}7.88 \\
4.77 \\
9.99 \\
6.70 \\
5.90 \\
7.78 \\
8.12 \\
17.61 \\
8.56 \\
4.09 \\
7.85\end{array}$ & $\begin{array}{r}5.77 \\
3.81 \\
8.41 \\
5.26 \\
3.95 \\
5.80 \\
6.61 \\
15.59 \\
6.11 \\
2.25 \\
5.14\end{array}$ & $\begin{array}{r}6.21 \\
3.70 \\
7.55 \\
5.61 \\
4.13 \\
5.48 \\
6.71 \\
12.70 \\
6.81 \\
2.60 \\
5.42\end{array}$ & $\begin{array}{r}6.08 \\
3.53 \\
7.41 \\
5.37 \\
3.89 \\
5.14 \\
7.00 \\
14.61 \\
6.82 \\
2.14 \\
4.62\end{array}$ & $\begin{array}{r}6.71 \\
3.12 \\
8.28 \\
6.19 \\
4.36 \\
6.65 \\
7.34 \\
13.30 \\
7.45 \\
2.35 \\
5.62\end{array}$ & $\begin{array}{r}7.78 \\
3.11 \\
9.32 \\
7.49 \\
5.68 \\
8.34 \\
8.91 \\
13.40 \\
8.61 \\
2.54 \\
5.90\end{array}$ & $\begin{array}{r}7.41 \\
3.22 \\
8.71 \\
6.94 \\
4.81 \\
7.42 \\
7.95 \\
15.42 \\
8.30 \\
2.15 \\
5.53\end{array}$ \\
\hline Nuclear Fuel ........................................ & - & - & - & .82 & .82 & .52 & .73 & .71 & .74 & .71 \\
\hline Blomass Fuels at UtIlitles ................ & - & - & - & - & - & - & - & - & - & - \\
\hline Primery Energy - Flve Sectors ${ }^{b} \ldots$ & 1.16 & 2.08 & 4.38 & 4.58 & 3.72 & 3.84 & 3.77 & 4.04 & 4.52 & 4.30 \\
\hline $\begin{array}{l}\text { Electric Utility Fuelc ........................... } \\
\text { Electricity Purchased by End Users }\end{array}$ & $\begin{array}{r}.26 \\
6.17\end{array}$ & $\begin{array}{r}.57 \\
8.64\end{array}$ & $\begin{array}{r}1.25 \\
13.91\end{array}$ & $\begin{array}{r}1.41 \\
17.16\end{array}$ & $\begin{array}{r}1.38 \\
18.45\end{array}$ & $\begin{array}{r}1.31 \\
18.90\end{array}$ & $\begin{array}{r}1.29 \\
18.94\end{array}$ & $\begin{array}{r}1.26 \\
18.92\end{array}$ & $\begin{array}{r}1.27 \\
18.94\end{array}$ & $\begin{array}{r}1.24 \\
18.95\end{array}$ \\
\hline \multirow[t]{2}{*}{ Total Energy ......................................... } & 1.84 & 3.32 & 7.20 & 8.30 & 7.34 & 7.68 & 7.62 & 8.04 & 8.83 & 8.67 \\
\hline & \multicolumn{10}{|c|}{ Expenditures in Millions of Dollars } \\
\hline 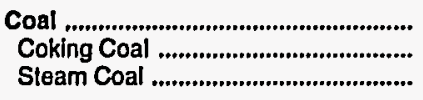 & $\begin{array}{r}80.3 \\
3.1 \\
77.2\end{array}$ & $\begin{array}{r}266.8 \\
11.9 \\
254.9\end{array}$ & $\begin{array}{r}647.4 \\
9.6 \\
637.7\end{array}$ & $\begin{array}{r}800.8 \\
12.0 \\
788.8\end{array}$ & $\begin{array}{r}750.1 \\
10.8 \\
739.3\end{array}$ & $\begin{array}{r}741.4 \\
3.4 \\
738.0\end{array}$ & $\begin{array}{r}751.9 \\
751.9\end{array}$ & $\begin{array}{r}740.3 \\
740.3\end{array}$ & $\begin{array}{r}728.0 \\
\overline{-} \\
728.0\end{array}$ & $\begin{array}{r}717.7 \\
-\overline{7} \\
717.7\end{array}$ \\
\hline Natural Gas & 265.4 & 423.0 & 928.2 & $1,284.0$ & $1,089.0$ & 994.0 & $1,090.0$ & $1,124.5$ & $1,107.8$ & $1,157.2$ \\
\hline 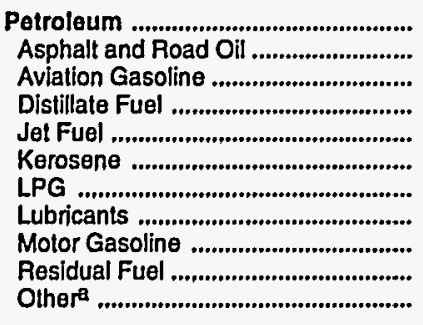 & $\begin{array}{r}1,127.1 \\
26.8 \\
2.0 \\
99.1 \\
34.1 \\
3.2 \\
79.5 \\
35.5 \\
803.2 \\
11.4 \\
32.4\end{array}$ & $\begin{array}{r}2,208.7 \\
73.3 \\
3.2 \\
261.8 \\
98.2 \\
3.9 \\
147.5 \\
58.3 \\
1,490.4 \\
21.7 \\
50.4\end{array}$ & $\begin{array}{r}4,698.9 \\
95.3 \\
7.4 \\
708.2 \\
229.5 \\
11.5 \\
211.7 \\
139.6 \\
2,889.0 \\
23.2 \\
383.7\end{array}$ & $\begin{array}{r}4,438.7 \\
135.9 \\
6.8 \\
765.2 \\
196.6 \\
6.6 \\
163.3 \\
155.8 \\
2,699.9 \\
18.8 \\
289.7\end{array}$ & $\begin{array}{r}3,361.9 \\
116.9 \\
6.9 \\
562.0 \\
149.9 \\
2.5 \\
142.1 \\
134.9 \\
2,034.0 \\
7.8 \\
204.9\end{array}$ & $\begin{array}{r}3,715.3 \\
106.8 \\
5.1 \\
629.7 \\
174.6 \\
2.3 \\
152.9 \\
124.2 \\
2,274.5 \\
11.1 \\
234.2\end{array}$ & $\begin{array}{r}3,811.4 \\
132.6 \\
6.1 \\
663.8 \\
160.8 \\
2.9 \\
167.6 \\
137.8 \\
2,326.8 \\
10.1 \\
203.0\end{array}$ & $\begin{array}{r}4,179.0 \\
94.1 \\
8.4 \\
798.1 \\
179.6 \\
4.3 \\
224.3 \\
128.6 \\
2,492.9 \\
8.3 \\
240.4\end{array}$ & $\begin{array}{r}4,751.8 \\
92.2 \\
5.9 \\
905.3 \\
213.8 \\
2.1 \\
221.8 \\
133.4 \\
2,879.3 \\
10.0 \\
288.0\end{array}$ & $\begin{array}{r}4,561.6 \\
86.7 \\
5.1 \\
820.7 \\
202.3 \\
2.7 \\
249.0 \\
137.3 \\
2,786.0 \\
7.4 \\
264.4\end{array}$ \\
\hline Nuclear Fuel ..................................... & - & - & - & 71.3 & 63.1 & 34.9 & 70.3 & 63.1 & 62.9 & 76.5 \\
\hline Biomass Fuels at Utilities .................. & - & - & - & - & - & - & - & - & - & - \\
\hline Prlmary Energy - Five Sectors ${ }^{b}$.... & $1,472.7$ & $2,898.5$ & $6,274.5$ & $6,594.8$ & $5,264.1$ & $5,485.5$ & $5,723.7$ & $6,107.0$ & $6,650.6$ & $6,513.0$ \\
\hline $\begin{array}{l}\text { Electric Utility Fuejc ............................ } \\
\text { Electricity Purchased by End Users }\end{array}$ & $\begin{array}{r}-76.3 \\
542.4\end{array}$ & $\begin{array}{r}-234.0 \\
974.3\end{array}$ & $\begin{array}{r}-639.6 \\
2,022.4\end{array}$ & $\begin{array}{r}-811.7 \\
2,712.0\end{array}$ & $\begin{array}{r}-761.7 \\
3,038.6\end{array}$ & $\begin{array}{r}-733.5 \\
3,232.6\end{array}$ & $\begin{array}{r}-781.9 \\
3,377.0\end{array}$ & $\begin{array}{r}-762.9 \\
3,396.0\end{array}$ & $\begin{array}{r}-754.7 \\
3,485.0\end{array}$ & $\begin{array}{r}-774.2 \\
3,654.0\end{array}$ \\
\hline Total Energy ....................................... & $1,938.9$ & $3,638.8$ & $7,657.4$ & $8,495.2$ & $7,541.0$ & $7,984.6$ & $8,318.8$ & $8,740.1$ & $9,380.9$ & $9,392.9$ \\
\hline
\end{tabular}

a Includes petroleum coke used at electric utilities.

b Biomass fuels are not included, except those consumed at electric utilities and those added to motor gasoline.

c There are no direct fuel costs for hydroelectric, geothermal, centralized solar, or wind energy.

- No consumption, including cases where adjustments were made. See "Consumption Adjustments for Process Fuel and Intermediate Products."

- Value less than 0.05 million dollars.

Note: Expenditure totals may not equal sum of components due to independent rounding

Sources: Data sources, estimation procedures, and assumptions are described in the "Documentation" section of this report. 
M Energy Price Estimates by Sector, Missouri

I 1970, 1975, 1980, and 1985-1991

S (Dollars per Million Btu)

\begin{tabular}{|c|c|c|c|c|c|c|c|c|c|c|}
\hline Sector and Energy Source & 1970 & 1975 & 1980 & 1985 & 1986 & 1987 & 1988 & 1989 & 1990 & 1991 \\
\hline 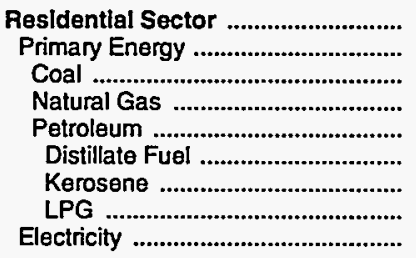 & $\begin{array}{r}2.09 \\
1.13 \\
.86 \\
.96 \\
1.81 \\
1.19 \\
1.43 \\
1.95 \\
7.86\end{array}$ & $\begin{array}{r}3.39 \\
1.85 \\
1.72 \\
1.48 \\
3.14 \\
2.62 \\
2.88 \\
3.26 \\
10.06\end{array}$ & $\begin{array}{r}6.88 \\
3.80 \\
1.70 \\
3.23 \\
7.01 \\
6.85 \\
7.95 \\
7.06 \\
15.21\end{array}$ & $\begin{array}{r}9.66 \\
5.60 \\
1.73 \\
5.40 \\
7.33 \\
6.70 \\
8.45 \\
7.53 \\
19.27\end{array}$ & $\begin{array}{r}10.09 \\
5.04 \\
1.54 \\
4.98 \\
5.60 \\
4.95 \\
6.24 \\
5.81 \\
20.87\end{array}$ & $\begin{array}{r}10.44 \\
4.85 \\
1.37 \\
4.71 \\
6.05 \\
4.66 \\
5.87 \\
6.41 \\
21.59\end{array}$ & $\begin{array}{r}10.34 \\
4.89 \\
1.25 \\
4.73 \\
6.37 \\
4.71 \\
5.94 \\
6.82 \\
21.49\end{array}$ & $\begin{array}{r}10.37 \\
5.17 \\
1.43 \\
4.80 \\
7.74 \\
5.88 \\
7.41 \\
8.02 \\
21.50\end{array}$ & $\begin{array}{r}11.22 \\
5.63 \\
1.56 \\
5.15 \\
9.33 \\
7.27 \\
9.16 \\
9.61 \\
21.56\end{array}$ & $\begin{array}{r}11.24 \\
5.55 \\
1.53 \\
5.10 \\
8.34 \\
6.75 \\
8.51 \\
8.54 \\
21.66\end{array}$ \\
\hline 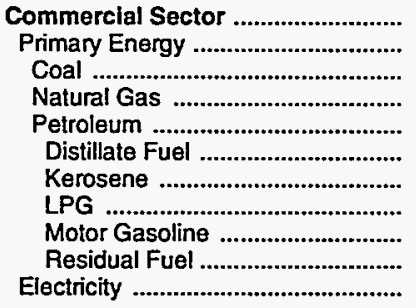 & $\begin{array}{r}1.66 \\
.69 \\
.49 \\
.62 \\
.93 \\
1.03 \\
.82 \\
1.27 \\
2.73 \\
.57 \\
7.00\end{array}$ & $\begin{array}{l}2.87 \\
1.36 \\
1.17 \\
1.14 \\
2.38 \\
2.45 \\
2.40 \\
2.49 \\
4.55 \\
1.77 \\
9.46\end{array}$ & $\begin{array}{r}6.87 \\
3.32 \\
1.58 \\
2.88 \\
5.74 \\
6.49 \\
6.10 \\
5.42 \\
9.33 \\
3.47 \\
14.33\end{array}$ & $\begin{array}{r}10.26 \\
5.06 \\
1.56 \\
4.88 \\
6.54 \\
5.75 \\
6.61 \\
9.12 \\
8.56 \\
4.11 \\
17.94\end{array}$ & $\begin{array}{r}10.43 \\
4.38 \\
1.38 \\
4.35 \\
4.84 \\
3.86 \\
4.36 \\
8.17 \\
6.11 \\
2.25 \\
19.02\end{array}$ & $\begin{array}{r}10.48 \\
4.04 \\
1.27 \\
4.06 \\
4.54 \\
3.66 \\
4.39 \\
7.29 \\
6.81 \\
2.61 \\
19.04\end{array}$ & $\begin{array}{r}10.58 \\
4.08 \\
1.27 \\
4.17 \\
4.31 \\
3.35 \\
4.00 \\
7.27 \\
6.82 \\
2.20 \\
19.06\end{array}$ & $\begin{array}{r}10.98 \\
4.30 \\
1.29 \\
4.31 \\
5.33 \\
4.41 \\
5.49 \\
6.32 \\
7.45 \\
2.41 \\
19.03\end{array}$ & $\begin{array}{r}11.38 \\
4.56 \\
1.31 \\
4.48 \\
6.44 \\
5.47 \\
6.86 \\
7.82 \\
8.61 \\
2.60 \\
18.98\end{array}$ & $\begin{array}{r}11.15 \\
4.48 \\
1.34 \\
4.46 \\
5.66 \\
4.79 \\
5.96 \\
6.94 \\
8.30 \\
2.21 \\
18.79\end{array}$ \\
\hline 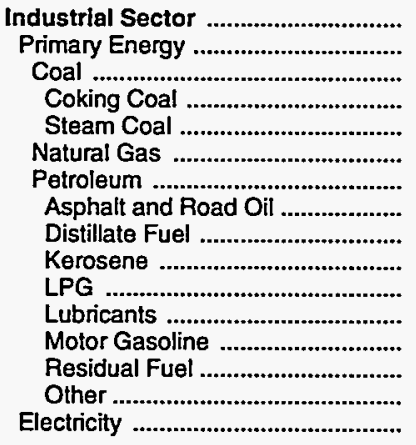 & $\begin{array}{r}1.12 \\
.77 \\
.47 \\
.38 \\
.49 \\
.40 \\
1.19 \\
.71 \\
.77 \\
.82 \\
1.27 \\
5.08 \\
2.73 \\
.53 \\
1.44 \\
4.01\end{array}$ & $\begin{array}{r}2.40 \\
1.75 \\
1.24 \\
1.60 \\
1.17 \\
.80 \\
2.69 \\
2.05 \\
2.25 \\
2.40 \\
2.49 \\
7.48 \\
4.55 \\
1.82 \\
2.77 \\
6.46\end{array}$ & $\begin{array}{r}5.40 \\
4.53 \\
1.61 \\
1.81 \\
1.58 \\
2.61 \\
6.39 \\
3.59 \\
5.83 \\
6.10 \\
5.42 \\
14.36 \\
9.33 \\
3.09 \\
7.30 \\
11.21\end{array}$ & $\begin{array}{r}6.39 \\
5.04 \\
1.62 \\
1.93 \\
1.56 \\
4.14 \\
6.93 \\
4.77 \\
6.00 \\
6.61 \\
9.12 \\
17.61 \\
8.56 \\
4.11 \\
7.85 \\
13.14\end{array}$ & $\begin{array}{r}5.78 \\
3.96 \\
1.44 \\
1.75 \\
1.38 \\
3.75 \\
5.03 \\
3.81 \\
3.96 \\
4.36 \\
8.17 \\
15.59 \\
6.11 \\
2.25 \\
5.14 \\
14.02\end{array}$ & $\begin{array}{r}5.83 \\
3.98 \\
1.29 \\
1.63 \\
1.27 \\
3.61 \\
5.05 \\
3.70 \\
3.99 \\
4.39 \\
7.29 \\
12.70 \\
6.81 \\
2.61 \\
5.42 \\
14.36\end{array}$ & $\begin{array}{r}5.61 \\
3.83 \\
1.27 \\
- \\
1.27 \\
3.79 \\
4.60 \\
3.53 \\
3.64 \\
4.00 \\
7.27 \\
14.61 \\
6.82 \\
2.20 \\
4.62 \\
14.45\end{array}$ & $\begin{array}{r}6.08 \\
4.23 \\
1.29 \\
-\overline{1} \\
1.29 \\
4.10 \\
5.16 \\
3.12 \\
4.99 \\
5.49 \\
6.32 \\
13.30 \\
7.45 \\
2.41 \\
5.63 \\
14.50\end{array}$ & $\begin{array}{r}6.35 \\
4.54 \\
1.31 \\
-\overline{1} \\
1.31 \\
4.14 \\
5.60 \\
3.11 \\
6.23 \\
6.86 \\
7.82 \\
13.40 \\
8.61 \\
2.60 \\
5.90 \\
14.50\end{array}$ & $\begin{array}{r}6.25 \\
4.40 \\
1.34 \\
- \\
1.34 \\
4.04 \\
5.39 \\
3.22 \\
5.42 \\
5.96 \\
6.94 \\
15.42 \\
8.30 \\
2.21 \\
5.53 \\
14.37\end{array}$ \\
\hline $\begin{array}{l}\text { Transportation Sector } \\
\text { Primary Energy }\end{array}$ & $\begin{array}{l}2.32 \\
2.32\end{array}$ & $\begin{array}{l}4.07 \\
4.07\end{array}$ & $\begin{array}{l}8.76 \\
8.76\end{array}$ & $\begin{array}{l}8.19 \\
8.19\end{array}$ & $\begin{array}{l}5.98 \\
5.98\end{array}$ & $\begin{array}{l}6.55 \\
6.55\end{array}$ & $\begin{array}{l}6.52 \\
6.52\end{array}$ & $\begin{array}{l}7.06 \\
7.06\end{array}$ & $\begin{array}{l}8.28 \\
8.28\end{array}$ & $\begin{array}{l}7.90 \\
7.90\end{array}$ \\
\hline 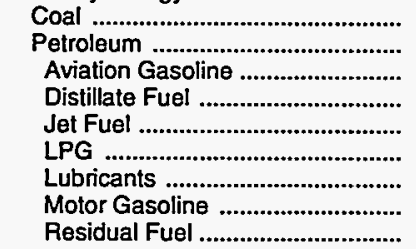 & $\begin{array}{r}.49 \\
2.32 \\
2.17 \\
1.24 \\
.75 \\
1.27 \\
5.08 \\
2.73 \\
.55\end{array}$ & $\begin{array}{l}1.17 \\
4.07 \\
3.45 \\
2.72 \\
2.09 \\
2.49 \\
7.48 \\
4.55 \\
1.73\end{array}$ & $\begin{array}{r}- \\
8.76 \\
9.02 \\
6.97 \\
6.47 \\
5.42 \\
14.36 \\
9.33 \\
3.38\end{array}$ & $\begin{array}{r}8.19 \\
9.99 \\
7.04 \\
5.90 \\
9.12 \\
17.61 \\
8.56 \\
3.88\end{array}$ & $\begin{array}{r}5 . \overline{8} \\
8.41 \\
5.75 \\
3.95 \\
8.17 \\
15.59 \\
6.11 \\
2.24\end{array}$ & $\begin{array}{r}- \\
6.55 \\
7.55 \\
6.33 \\
4.13 \\
7.29 \\
12.70 \\
6.81 \\
-\end{array}$ & $\begin{array}{r}- \\
6.52 \\
7.41 \\
6.10 \\
3.89 \\
7.27 \\
14.61 \\
6.82 \\
1.80\end{array}$ & $\begin{array}{r}7.06 \\
8.28 \\
6.54 \\
4.36 \\
6.32 \\
13.30 \\
7.45 \\
2.08\end{array}$ & $\begin{array}{r}8 . \overline{8} \\
9.32 \\
7.87 \\
5.68 \\
7.82 \\
13.40 \\
8.61 \\
1.65\end{array}$ & $\begin{array}{r}7.90 \\
8.71 \\
7.42 \\
4.81 \\
6.94 \\
15.42 \\
8.30\end{array}$ \\
\hline 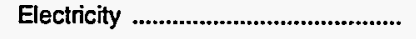 & - & - & - & - & - & - & - & - & - & - \\
\hline $\begin{array}{l}\text { Total Energy ................................... } \\
\text { Primary Energy - Four Sectors ...... } \\
\text { Electricity ............................................ }\end{array}$ & $\begin{array}{l}1.84 \\
1.44 \\
6.17\end{array}$ & $\begin{array}{l}3.32 \\
2.71 \\
8.64\end{array}$ & $\begin{array}{r}7.20 \\
6.13 \\
13.91\end{array}$ & $\begin{array}{r}8.30 \\
6.68 \\
17.16\end{array}$ & $\begin{array}{r}7.34 \\
5.22 \\
18.45\end{array}$ & $\begin{array}{r}7.68 \\
5.47 \\
18.90\end{array}$ & $\begin{array}{r}7.62 \\
5.41 \\
18.94\end{array}$ & $\begin{array}{r}8.04 \\
5.89 \\
18.92\end{array}$ & $\begin{array}{r}8.83 \\
6.71 \\
18.94\end{array}$ & $\begin{array}{r}8.67 \\
6.44 \\
18.95\end{array}$ \\
\hline 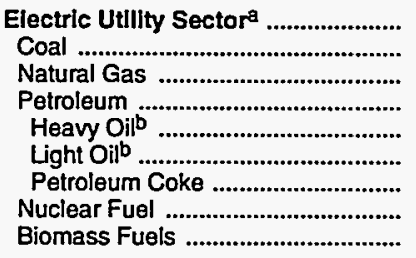 & $\begin{array}{l}.26 \\
.25 \\
.26 \\
.62 \\
.55 \\
.69 \\
- \\
- \\
-\end{array}$ & $\begin{array}{r}.57 \\
.54 \\
.58 \\
2.05 \\
1.74 \\
2.26 \\
.65 \\
- \\
-\end{array}$ & $\begin{array}{r}1.25 \\
1.19 \\
2.22 \\
5.07 \\
3.45 \\
6.01 \\
.67 \\
- \\
-\end{array}$ & $\begin{array}{r}1.41 \\
1.50 \\
3.31 \\
5.60 \\
3.99 \\
5.76 \\
1.38 \\
.82 \\
-\end{array}$ & $\begin{array}{r}1.38 \\
1.47 \\
2.92 \\
3.38 \\
2.32 \\
3.48 \\
- \\
.82 \\
-\end{array}$ & $\begin{array}{r}1.31 \\
1.41 \\
2.96 \\
3.93 \\
2.41 \\
4.14 \\
- \\
.52 \\
-\end{array}$ & $\begin{array}{r}1.29 \\
1.38 \\
2.77 \\
3.35 \\
1.83 \\
3.55 \\
.97 \\
.73 \\
-\end{array}$ & $\begin{array}{r}1.26 \\
1.35 \\
2.46 \\
3.90 \\
2.12 \\
4.45 \\
.80 \\
.71 \\
-\end{array}$ & $\begin{array}{r}1.27 \\
1.35 \\
1.72 \\
4.99 \\
1.80 \\
5.11 \\
- \\
.74 \\
-\end{array}$ & $\begin{array}{r}1.24 \\
1.34 \\
1.49 \\
4.23 \\
1.33 \\
4.78 \\
- \\
.71 \\
-\end{array}$ \\
\hline Primary Energy - Five Sectors ${ }^{c} .$. & 1.16 & 2.08 & 4.38 & 4.58 & 3.72 & 3.84 & $3 . \pi$ & 4.04 & 4.52 & 4.30 \\
\hline
\end{tabular}

a There are no direct fuel costs for hydroelectric, geothermal, centralized solar, or wind energy.

b Heavy oil includes Grade Nos. 4, 5, and 6, and residual fuel oils. Light oil includes Grade No. 2 heating oil, kerosene, and jet fuel.

c Biomass fuels are not included, except those consumed at electric utilities and those added to motor gasoline.

-No consumption, including cases where adjustments were made. See "Consumption Adjustments for Process Fuel and intermediate Products."

Sources: Data sources, estimation procedures, and assumptions are described in the "Documentation" section of this report. 


\begin{tabular}{|c|c|c|c|c|c|c|c|c|c|c|}
\hline Sector and Energy Source & 1970 & 1975 & 1980 & 1985 & 1986 & 1987 & 1988 & 1989 & 1990 & 1991 \\
\hline 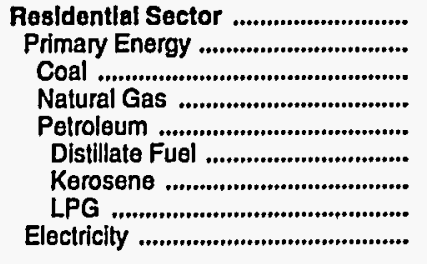 & $\begin{array}{r}486.6 \\
227.1 \\
.6 \\
150.9 \\
75.5 \\
9.1 \\
.6 \\
65.9 \\
259.5\end{array}$ & $\begin{array}{r}840.7 \\
371.9 \\
2.0 \\
232.0 \\
137.9 \\
21.9 \\
.5 \\
115.5 \\
468.8\end{array}$ & $\begin{array}{r}1,622.0 \\
654.0 \\
1.1 \\
471.2 \\
181.7 \\
49.7 \\
2.6 \\
129.4 \\
967.9\end{array}$ & $\begin{array}{r}2,052.0 \\
836.7 \\
2.2 \\
703.3 \\
131.2 \\
31.8 \\
4.5 \\
94.8 \\
1,215.3\end{array}$ & $\begin{array}{r}2,102.6 \\
716.4 \\
1.3 \\
606.9 \\
108.3 \\
23.6 \\
2.0 \\
82.6 \\
1,386.2\end{array}$ & $\begin{array}{r}2,167.1 \\
670.6 \\
2.6 \\
552.4 \\
115.6 \\
17.8 \\
1.8 \\
96.1 \\
1,496.5\end{array}$ & $\begin{array}{r}2,293.5 \\
728.5 \\
2.1 \\
610.8 \\
115.6 \\
17.4 \\
2.0 \\
96.2 \\
1,565.0\end{array}$ & $\begin{array}{r}2,339.4 \\
794.3 \\
2.8 \\
625.1 \\
166.5 \\
16.2 \\
2.9 \\
147.4 \\
1,545.0\end{array}$ & $\begin{array}{r}2,363.1 \\
770.1 \\
3.4 \\
604.1 \\
162.6 \\
15.0 \\
1.5 \\
146.1 \\
1,593.0\end{array}$ & $\begin{array}{r}2,539.4 \\
811.3 \\
3.0 \\
620.3 \\
188.1 \\
16.9 \\
1.8 \\
169.4 \\
1,728.0\end{array}$ \\
\hline 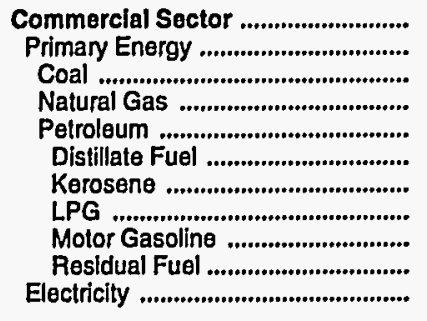 & $\begin{array}{r}227.1 \\
79.8 \\
.6 \\
54.9 \\
24.2 \\
6.5 \\
2.0 \\
7.5 \\
2.2 \\
6.0 \\
147.3\end{array}$ & $\begin{array}{r}400.9 \\
154.4 \\
2.5 \\
104.7 \\
47.2 \\
16.9 \\
2.4 \\
15.5 \\
3.8 \\
8.5 \\
246.5\end{array}$ & $\begin{array}{r}943.7 \\
308.8 \\
1.8 \\
22.7 \\
84.4 \\
37.9 \\
5.9 \\
17.5 \\
10.9 \\
12.1 \\
634.8\end{array}$ & $\begin{array}{r}1,319.4 \\
388.6 \\
3.6 \\
299.5 \\
85.5 \\
49.1 \\
1.2 \\
20.3 \\
11.8 \\
3.1 \\
930.8\end{array}$ & $\begin{array}{r}1,384.3 \\
340.5 \\
2.1 \\
272.1 \\
66.3 \\
33.3 \\
.2 \\
20.5 \\
10.4 \\
1.8 \\
1,043.8\end{array}$ & $\begin{array}{r}1,436.8 \\
315.8 \\
4.4 \\
239.2 \\
72.1 \\
39.6 \\
.2 \\
19.3 \\
11.2 \\
2.0 \\
1,121.0\end{array}$ & $\begin{array}{r}1,526.4 \\
333.4 \\
4.1 \\
268.1 \\
61.2 \\
32.5 \\
.4 \\
18.1 \\
8.9 \\
1.4 \\
1,193.0\end{array}$ & $\begin{array}{r}1,549.7 \\
331.8 \\
4.7 \\
273.6 \\
53.5 \\
23.8 \\
.4 \\
20.5 \\
8.3 \\
.5 \\
1,218.0\end{array}$ & $\begin{array}{r}1,587.5 \\
335.5 \\
5.3 \\
269.0 \\
61.2 \\
28.2 \\
.3 \\
21.0 \\
10.7 \\
1.0 \\
1,252.0\end{array}$ & $\begin{array}{r}1,633.6 \\
350.6 \\
4.8 \\
284.4 \\
61.4 \\
31.0 \\
.1 \\
24.3 \\
5.6 \\
.4 \\
1,283.0\end{array}$ \\
\hline 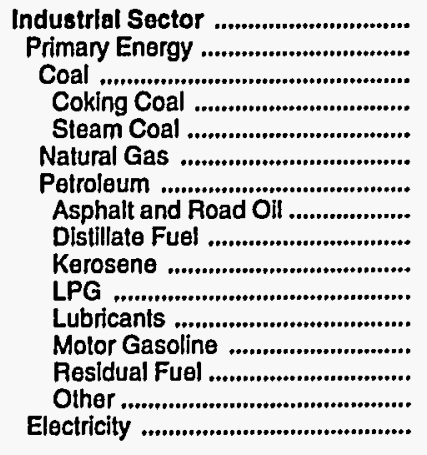 & $\begin{array}{r}346.6 \\
211.0 \\
20.4 \\
3.1 \\
17.3 \\
42.9 \\
147.7 \\
26.8 \\
25.4 \\
.7 \\
5.6 \\
12.8 \\
39.7 \\
4.4 \\
32.4 \\
135.6\end{array}$ & $\begin{array}{r}697.7 \\
438.8 \\
56.8 \\
11.9 \\
44.9 \\
71.3 \\
310.7 \\
73.3 \\
75.7 \\
1.0 \\
15.8 \\
22.3 \\
64.7 \\
7.5 \\
50.4 \\
259.0\end{array}$ & $\begin{array}{r}1,543.2 \\
1,123.6 \\
58.0 \\
9.6 \\
48.4 \\
201.1 \\
864.5 \\
95.3 \\
162.3 \\
3.0 \\
63.4 \\
58.4 \\
91.4 \\
7.5 \\
383.3 \\
419.6\end{array}$ & $\begin{array}{r}1,646.7 \\
1,080.8 \\
66.7 \\
12.0 \\
54.7 \\
276.4 \\
737.7 \\
135.9 \\
139.7 \\
.8 \\
43.8 \\
65.2 \\
48.4 \\
14.4 \\
289.7 \\
565.9\end{array}$ & $\begin{array}{r}1,383.5 \\
774.9 \\
56.3 \\
10.8 \\
45.5 \\
206.6 \\
512.0 \\
116.9 \\
63.1 \\
.2 \\
34.3 \\
56.4 \\
30.8 \\
5.3 \\
204.9 \\
608.6\end{array}$ & $\begin{array}{r}1,401.9 \\
786.8 \\
45.2 \\
3.4 \\
41.8 \\
198.3 \\
543.4 \\
106.8 \\
73.1 \\
.3 \\
34.0 \\
52.0 \\
34.2 \\
8.8 \\
234.2 \\
615.0\end{array}$ & $\begin{array}{r}1,434.2 \\
815.2 \\
45.3 \\
-\overline{-} \\
45.3 \\
206.7 \\
563.3 \\
132.6 \\
79.7 \\
.6 \\
50.5 \\
57.6 \\
31.9 \\
7.3 \\
203.0 \\
619.0\end{array}$ & $\begin{array}{r}1,471.9 \\
838.9 \\
42.5 \\
42.5 \\
222.8 \\
573.6 \\
94.1 \\
93.9 \\
1.0 \\
53.7 \\
53.8 \\
30.4 \\
6.4 \\
240.3 \\
633.0\end{array}$ & $\begin{array}{r}1,543.8 \\
903.8 \\
39.9 \\
39.9 \\
228.5 \\
635.3 \\
92.2 \\
109.2 \\
.3 \\
51.4 \\
55.8 \\
29.8 \\
8.6 \\
288.0 \\
640.0\end{array}$ & $\begin{array}{r}1,508.9 \\
865.9 \\
38.6 \\
-\overline{3} \\
38.6 \\
233.3 \\
594.0 \\
86.7 \\
93.0 \\
.8 \\
52.1 \\
57.4 \\
33.0 \\
6.6 \\
264.4 \\
643.0\end{array}$ \\
\hline 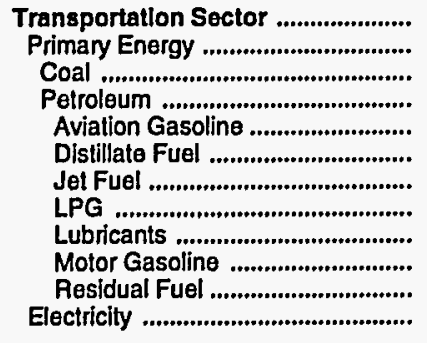 & $\begin{array}{r}878.6 \\
878.6 \\
878.5 \\
2.0 \\
57.5 \\
34.1 \\
.4 \\
22.7 \\
761.4 \\
.6 \\
-\end{array}$ & $\begin{array}{r}1,699.4 \\
1,699.4 \\
\vdots \\
1,699.4 \\
3.2 \\
137.9 \\
98.2 \\
.7 \\
36.0 \\
1,421.9 \\
1.5 \\
-\end{array}$ & $\begin{array}{r}3,548.5 \\
3,548.5 \\
3,548.5 \\
7.4 \\
439.5 \\
229.5 \\
1.3 \\
81.2 \\
2,786.6 \\
3.0 \\
-\end{array}$ & $\begin{array}{r}3,477.1 \\
3,477.1 \\
3,477.1 \\
6.8 \\
537.8 \\
196.6 \\
4.5 \\
90.6 \\
2,639.7 \\
.9 \\
-\end{array}$ & $\begin{array}{r}2,670.6 \\
2,670.6 \\
2, \overline{-} \\
2,670.6 \\
6.9 \\
437.4 \\
149.9 \\
4.7 \\
78.4 \\
1,992.9 \\
.4 \\
-\end{array}$ & $\begin{array}{r}2,978.8 \\
2,978.8 \\
- \\
2,978.8 \\
5.1 \\
494.3 \\
174.6 \\
3.5 \\
72.3 \\
2,229.1 \\
- \\
-\end{array}$ & $\begin{array}{r}3,064.6 \\
3,064.6 \\
- \\
3,064.6 \\
6.1 \\
528.0 \\
160.8 \\
2.7 \\
80.2 \\
2,286.0 \\
1.0 \\
-\end{array}$ & $\begin{array}{r}3,379.1 \\
3,379.1 \\
- \\
3,379.1 \\
8.4 \\
658.5 \\
179.6 \\
2.7 \\
74.8 \\
2,454.2 \\
.9 \\
-\end{array}$ & $\begin{array}{r}3,886.5 \\
3,886.5 \\
- \\
3,886.5 \\
5.9 \\
746.7 \\
213.8 \\
3.4 \\
77.6 \\
2,838.7 \\
.4 \\
-\end{array}$ & $\begin{array}{r}3,711.0 \\
3,711.0 \\
3,711.0 \\
5.1 \\
673.0 \\
202.3 \\
3.3 \\
79.9 \\
2,747.4 \\
-\end{array}$ \\
\hline 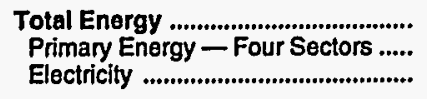 & $\begin{array}{r}1,938.9 \\
1,396.4 \\
542.4\end{array}$ & $\begin{array}{r}3,638.8 \\
2,664.5 \\
974.3\end{array}$ & $\begin{array}{l}7,657.4 \\
5,635.0 \\
2,022.4\end{array}$ & $\begin{array}{l}8,495.2 \\
5,783.1 \\
2,712.0\end{array}$ & $\begin{array}{l}7,541.0 \\
4,502.4 \\
3,038.6\end{array}$ & $\begin{array}{l}7,984.6 \\
4,752.0 \\
3,232.6\end{array}$ & $\begin{array}{l}8,318.8 \\
4,941.8 \\
3,377.0\end{array}$ & $\begin{array}{l}8,740.1 \\
5,344.1 \\
3,396.0\end{array}$ & $\begin{array}{l}9,380.9 \\
5,895.9 \\
3,485.0\end{array}$ & $\begin{array}{l}9,392.9 \\
5,738.9 \\
3,654.0\end{array}$ \\
\hline 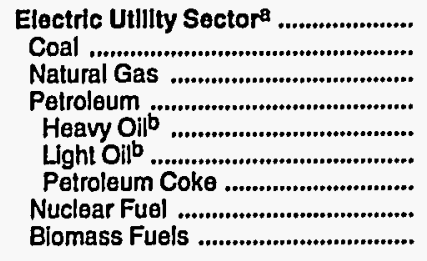 & $\begin{array}{r}76.3 \\
58.6 \\
16.6 \\
1.1 \\
.5 \\
.6 \\
- \\
-\end{array}$ & $\begin{array}{r}234.0 \\
205.4 \\
15.0 \\
13.5 \\
4.1 \\
9.3 \\
.1 \\
- \\
-\end{array}$ & $\begin{array}{r}639.6 \\
586.4 \\
33.3 \\
19.9 \\
.6 \\
18.8 \\
.4 \\
- \\
-\end{array}$ & $\begin{array}{r}811.7 \\
728.4 \\
4.8 \\
7.2 \\
.4 \\
6.8\end{array}$ & $\begin{array}{r}761.7 \\
690.4 \\
3.4 \\
4.8 \\
.3 \\
4.5 \\
-\end{array}$ & $\begin{array}{r}733.5 \\
689.2 \\
4.0 \\
5.4 \\
.4 \\
5.0 \\
- \\
34.9 \\
-\end{array}$ & $\begin{array}{r}781.9 \\
700.4 \\
4.5 \\
6.7 \\
.4 \\
6.3\end{array}$ & $\begin{array}{r}762.9 \\
690.4 \\
3.1 \\
6.3 \\
.5 \\
5.7 \\
.1 \\
63.1 \\
-\end{array}$ & $\begin{array}{r}754.7 \\
679.4 \\
6.2 \\
6.3 \\
.1 \\
6.2 \\
- \\
62.9 \\
-\end{array}$ & $\begin{array}{r}774.2 \\
671.3 \\
19.2 \\
7.2 \\
.4 \\
6.8 \\
76.5 \\
76\end{array}$ \\
\hline Prlmary Energy - Five Sectorsc .. & $1,472.7$ & $2,898.5$ & $6,274.5$ & $6,594.8$ & $5,264.1$ & $5,485.5$ & $5,723.7$ & $6,107.0$ & $6,650.6$ & $6,513.0$ \\
\hline
\end{tabular}

There are no direct fuel costs for hydroelectric, geothermal, centralized solar, or wind energy.

b Heavy oil includes Grade Nos. 4, 5, and 6, and residual fuel oils. Light oil includes Grade No. 2 heating oil, kerosene, and jet fuel.

c Blomass fuels are not included, except those consumed at electric utilities and those added to motor gasoline.

- No consumption, including cases where adjustments were made. See "Consumption Adjustments for Process Fuel and Intermediate Products."

Value less than 0.05 million dollars.

Nole: Totals may not equal sum of components due to independent rounding.

Sources: Data sources, estimation procedures, and assumptions are described in the "Documentation" section of this report. 
M Energy Price and Expenditure Estimates by Source, Montana

O 1970, 1975, 1980, and 1985-1991

$\mathbf{N}$
$\mathbf{T}$
$\mathbf{A}$
$\mathbf{N}$
$\mathbf{A}$

\begin{tabular}{|c|c|c|c|c|c|c|c|c|c|c|}
\hline Energy Source & 1970 & 1975 & 1980 & 1985 & 1986 & 1987 & 1988 & 1989 & 1990 & 1991 \\
\hline & \multicolumn{10}{|c|}{ Prices in Dollars per Million Btu } \\
\hline Coal' & 0.22 & 0.33 & 0.53 & 0.75 & 0.74 & 0.68 & 0.57 & 0.61 & 0.70 & 0.71 \\
\hline 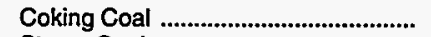 & - & - & - & - & - & - & - & - & - & - \\
\hline 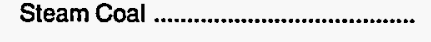 & .22 & .33 & .53 & .75 & .74 & .68 & .57 & .61 & .70 & .71 \\
\hline 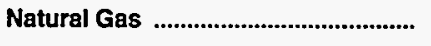 & .57 & 1.07 & 3.14 & 4.84 & 4.32 & 4.05 & 3.92 & 3.95 & 4.16 & 4.06 \\
\hline 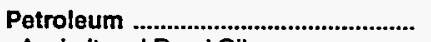 & 1.89 & 3.41 & 7.13 & 7.37 & 6.00 & 6.39 & 6.51 & 6.88 & 7.82 & 7.63 \\
\hline 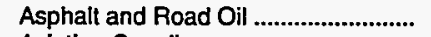 & .59 & 1.84 & 3.67 & 4.83 & 4.35 & 3.07 & 3.29 & 2.75 & 2.64 & 3.29 \\
\hline 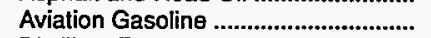 & 2.17 & 3.45 & 9.02 & 9.99 & 8.41 & 7.55 & 7.41 & 8.28 & 9.32 & 8.71 \\
\hline 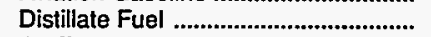 & 1.14 & 2.58 & 6.59 & 6.39 & 5.68 & 6.40 & 6.32 & 6.51 & 7.58 & 7.34 \\
\hline 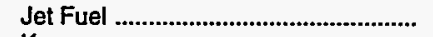 & .76 & 2.12 & 6.59 & 6.64 & 4.81 & 4.38 & 4.33 & 4.79 & 6.26 & 5.47 \\
\hline 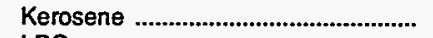 & .94 & 2.63 & - & 11.07 & 5.91 & 6.18 & 5.61 & 5.74 & 6.62 & 7.18 \\
\hline LPG & 2.10 & 3.62 & 6.49 & 7.60 & 6.60 & 6.15 & 6.15 & 7.23 & 9.06 & 9.92 \\
\hline 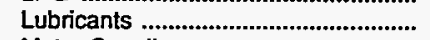 & 5.08 & 7.48 & 14.36 & 17.61 & 15.59 & 12.70 & 14.61 & 13.30 & 13.40 & 15.42 \\
\hline Motor Gasoline & 2.89 & 4.78 & 9.99 & 9.16 & 6.74 & 7.57 & 7.75 & 8.59 & 9.56 & 9.07 \\
\hline 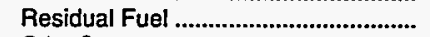 & .39 & 1.99 & 3.28 & 3.03 & 2.63 & 2.34 & 2.12 & 2.16 & 3.03 & 2.36 \\
\hline Other ${ }^{\mathrm{a}}$ & .52 & 1.40 & 2.38 & 2.17 & 1.53 & 1.50 & 1.45 & 1.74 & 2.02 & 1.72 \\
\hline 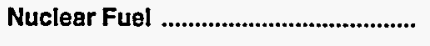 & - & - & - & - & - & - & - & - & - & - \\
\hline Blomass Fuels at Utilities ..................... & .65 & .92 & 1.74 & .79 & .32 & .95 & .87 & $(d)$ & $\left({ }^{d}\right)$ & (d) \\
\hline Primary Energy - Five Sectors ${ }^{b}$.... & 1.26 & 2.41 & 4.79 & 4.69 & 3.39 & 3.47 & 3.03 & 3.32 & 3.82 & 3.63 \\
\hline $\begin{array}{l}\text { Electric Utility Fuelc ............................ } \\
\text { Electricity Purchased by End Users }\end{array}$ & 2.88 & $\begin{array}{r}.34 \\
4.05\end{array}$ & $\begin{array}{r}.72 \\
5.80\end{array}$ & $\begin{array}{r}.72 \\
10.72\end{array}$ & $\begin{array}{r}.68 \\
11.18\end{array}$ & $\begin{array}{r}.66 \\
11.81\end{array}$ & $\begin{array}{r}.55 \\
12.09\end{array}$ & $\begin{array}{r}.58 \\
12.00\end{array}$ & $\begin{array}{r}.68 \\
11.67\end{array}$ & $\begin{array}{r}.68 \\
12.22\end{array}$ \\
\hline \multirow[t]{2}{*}{ 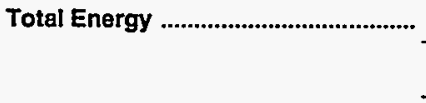 } & 1.56 & 2.78 & 6.02 & 7.48 & 6.74 & 7.05 & 7.12 & 7.24 & 7.81 & 7.74 \\
\hline & \multicolumn{10}{|c|}{ Expenditures in Millions of Dollars } \\
\hline Coal & 2.6 & 6.2 & 31.8 & 74.0 & 98.0 & 90.8 & 103.9 & 108.3 & 116.1 & 127.3 \\
\hline 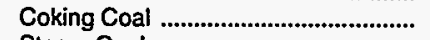 & - & - & - & - & - & - & - & - & - & - \\
\hline 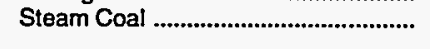 & 2.6 & 6.2 & 31.8 & 74.0 & 98.0 & 90.8 & 103.9 & 108.3 & 116.1 & 127.3 \\
\hline 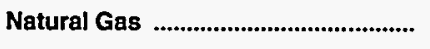 & 45.1 & 78.2 & 166.0 & 204.7 & 157.7 & 140.4 & 149.0 & 164.9 & 162.9 & 169.1 \\
\hline Petroleum ............................................. & 204.2 & 461.4 & $1,050.4$ & $1,062.3$ & 738.5 & 769.7 & 775.7 & 887.4 & 993.8 & 972.3 \\
\hline Asphalt and Road Oil .............................. & 5.3 & 11.3 & 24.9 & 46.9 & 57.4 & 33.5 & 32.2 & 31.9 & 26.0 & 29.5 \\
\hline Aviation Gasoline & .5 & 1.4 & 7.3 & 4.6 & 4.4 & 3.1 & 4.0 & 4.0 & 5.2 & 4.8 \\
\hline 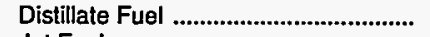 & 31.9 & 114.2 & 288.2 & 421.3 & 231.9 & 244.3 & 232.4 & 291.2 & 327.6 & 355.6 \\
\hline Jet Fuel & 2.7 & 9.7 & 34.1 & 25.2 & 23.3 & 17.7 & 19.6 & 20.0 & 24.8 & 22.0 \\
\hline 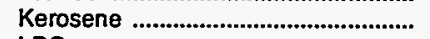 & 2.0 & 1.8 & - & .6 & .7 & .3 & .1 & .1 & .3 & .1 \\
\hline LPG & 9.9 & 17.1 & 41.9 & 39.8 & 33.9 & 35.8 & 31.8 & 44.8 & 58.7 & 35.4 \\
\hline 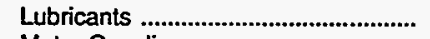 & 6.2 & 9.4 & 21.5 & 24.0 & 20.8 & 19.2 & 21.3 & 19.9 & 20.6 & 21.2 \\
\hline Motor Gasoline & 140.7 & 266.6 & 546.8 & 490.2 & 359.8 & 407.0 & 425.5 & 464.8 & 515.5 & 493.3 \\
\hline 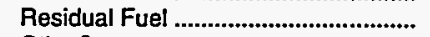 & .7 & 17.6 & 68.3 & 2.4 & .6 & .2 & .1 & .2 & .2 & \\
\hline Othera & 4.4 & 12.3 & 17.4 & 7.1 & 5.5 & 8.6 & 8.7 & 10.5 & 14.8 & 10.3 \\
\hline Nuclear Fuel .......................................... & - & - & - & - & - & - & - & - & - & - \\
\hline Blomass Fuels at Utilities .................. & .5 & .1 & .3 & .5 & .2 & .5 & .5 & $\left({ }^{d}\right)$ & $\left({ }^{d}\right)$ & (d) \\
\hline Primary Energy - Five Sectors ${ }^{b} \ldots$ & 252.4 & 545.9 & $1,248.6$ & $1,341.5$ & 994.4 & $1,001.4$ & $1,029.1$ & $1,160.6$ & $1,272.9$ & $1,268.7$ \\
\hline $\begin{array}{l}\text { Electric Utility Fuelc .......................... } \\
\text { Electricity Purchased by End Users }\end{array}$ & $\begin{array}{l}-3.4 \\
84.1\end{array}$ & $\begin{array}{r}-6.4 \\
119.8\end{array}$ & $\begin{array}{r}-44.3 \\
207.7\end{array}$ & $\begin{array}{r}-69.2 \\
488.6\end{array}$ & $\begin{array}{r}-87.0 \\
515.8\end{array}$ & $\begin{array}{r}-85.9 \\
488.8\end{array}$ & $\begin{array}{r}-99.1 \\
521.6\end{array}$ & $\begin{array}{r}-102.2 \\
519.2\end{array}$ & $\begin{array}{r}-110.6 \\
510.4\end{array}$ & $\begin{array}{r}-119.3 \\
545.8\end{array}$ \\
\hline 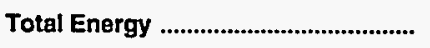 & 333.1 & 659.3 & $1,412.0$ & $1,760.9$ & $1,423.2$ & $1,404.3$ & $1,451.6$ & $1,577.6$ & $1,672.6$ & $1,695.3$ \\
\hline
\end{tabular}

a Includes petroleum coke used at electric utilities.

biomass fuels are not included, except those consumed at electric utilities and those added to motor gasoline.

c There are no direct fuel costs for hydroelectric, geothermal, centralized solar, or wind energy.

d Utilities used biomass fuels at no charge or received a fee for accepting them.

-No consumption, including cases where adjustments were made. See "Consumption Adjustments for Process Fuel and Intermediate Products."

Note: Expenditure totals may not equal sum of components due to independent rounding.

Sources: Data sources, estimation procedures, and assumptions are described in the "Documentation" section of this report. 


\begin{tabular}{|c|c|c|c|c|c|c|c|c|c|c|}
\hline Sector and Energy Source & 1970 & 1975 & 1980 & 1985 & 1986 & 1987 & 1988 & 1989 & 1990 & 1991 \\
\hline 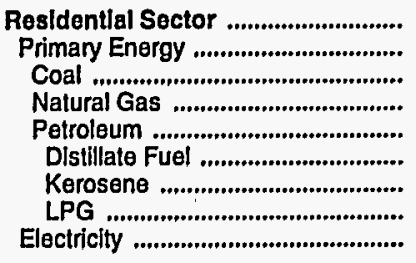 & $\begin{array}{r}1.87 \\
1.06 \\
.80 \\
.88 \\
2.03 \\
1.28 \\
-\overline{2.35} \\
6.57\end{array}$ & $\begin{array}{r}2.73 \\
1.74 \\
1.06 \\
1.27 \\
3.37 \\
2.84 \\
-\overline{8} \\
7.02\end{array}$ & $\begin{array}{r}5.36 \\
3.91 \\
1.35 \\
3.02 \\
7.08 \\
6.92 \\
- \\
7.21 \\
9.04\end{array}$ & $\begin{array}{r}8.27 \\
5.40 \\
.98 \\
4.82 \\
8.10 \\
7.92 \\
11.40 \\
8.18 \\
13.77\end{array}$ & $\begin{array}{r}8.13 \\
4.77 \\
.70 \\
4.46 \\
6.08 \\
4.87 \\
7.00 \\
7.12 \\
14.68\end{array}$ & $\begin{array}{r}8.74 \\
4.69 \\
1.44 \\
4.33 \\
6.10 \\
5.15 \\
7.41 \\
6.62 \\
16.19\end{array}$ & $\begin{array}{r}8.44 \\
4.51 \\
.95 \\
4.20 \\
5.88 \\
4.61 \\
6.62 \\
6.54 \\
15.89\end{array}$ & $\begin{array}{r}8.44 \\
4.85 \\
1.25 \\
4.29 \\
7.10 \\
5.08 \\
7.31 \\
8.51 \\
15.77\end{array}$ & $\begin{array}{r}8.91 \\
5.28 \\
.34 \\
4.47 \\
8.69 \\
6.42 \\
9.23 \\
9.98 \\
15.97\end{array}$ & $\begin{array}{r}9.02 \\
5.14 \\
.28 \\
4.40 \\
8.48 \\
6.11 \\
8.79 \\
10.42 \\
16.86\end{array}$ \\
\hline 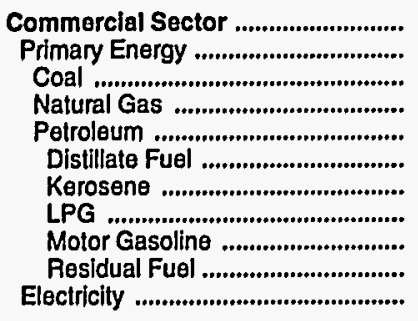 & $\begin{array}{r}1.51 \\
.78 \\
.48 \\
.60 \\
1.64 \\
1.06 \\
.94 \\
1.49 \\
2.89 \\
.34 \\
5.74\end{array}$ & $\begin{array}{r}2.39 \\
1.49 \\
.79 \\
1.07 \\
2.89 \\
2.49 \\
2.63 \\
2.77 \\
4.78 \\
2.03 \\
6.39\end{array}$ & $\begin{array}{r}5.12 \\
3.76 \\
2.04 \\
3.12 \\
6.87 \\
6.45 \\
- \\
5.85 \\
9.99 \\
4.42 \\
8.50\end{array}$ & $\begin{array}{r}8.25 \\
5.39 \\
1.66 \\
5.10 \\
6.09 \\
6.25 \\
6.85 \\
7.19 \\
9.16 \\
3.03 \\
12.49\end{array}$ & $\begin{array}{r}8.92 \\
4.66 \\
2.05 \\
4.48 \\
5.48 \\
5.45 \\
4.14 \\
6.17 \\
6.74 \\
2.63 \\
13.45\end{array}$ & $\begin{array}{r}8.52 \\
4.52 \\
1.96 \\
4.26 \\
5.65 \\
5.32 \\
5.93 \\
5.77 \\
7.57 \\
2.34 \\
14.04\end{array}$ & $\begin{array}{r}8.23 \\
4.30 \\
1.78 \\
4.20 \\
5.02 \\
3.92 \\
5.02 \\
5.75 \\
7.75 \\
2.12 \\
13.46\end{array}$ & $\begin{array}{r}7.88 \\
4.29 \\
1.76 \\
4.28 \\
5.16 \\
3.83 \\
5.08 \\
5.98 \\
8.59 \\
2.16 \\
13.46\end{array}$ & $\begin{array}{r}8.41 \\
4.70 \\
1.72 \\
4.52 \\
6.93 \\
5.13 \\
6.20 \\
8.30 \\
9.56 \\
3.03 \\
13.49\end{array}$ & $\begin{array}{r}8.57 \\
4.39 \\
1.81 \\
4.23 \\
6.16 \\
4.46 \\
6.58 \\
8.68 \\
9.07 \\
2.36 \\
14.36\end{array}$ \\
\hline 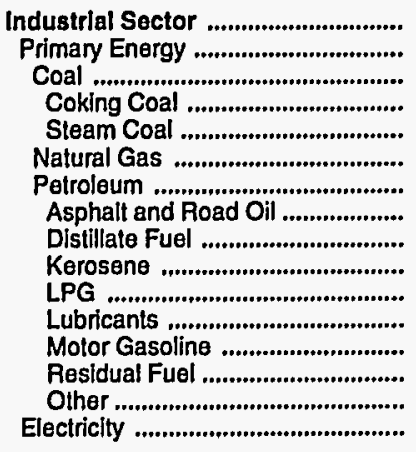 & $\begin{array}{r}.80 \\
.64 \\
.48 \\
\overline{.48} \\
.48 \\
.33 \\
.95 \\
.59 \\
.87 \\
.94 \\
1.49 \\
5.08 \\
2.89 \\
.45 \\
.52 \\
1.33\end{array}$ & $\begin{array}{r}1.79 \\
1.75 \\
.79 \\
- \\
.79 \\
.93 \\
2.32 \\
1.84 \\
2.44 \\
2.63 \\
2.77 \\
7.48 \\
4.78 \\
1.99 \\
1.40 \\
1.96\end{array}$ & $\begin{array}{r}3.73 \\
3.91 \\
2.04 \\
-\overline{2} \\
2.04 \\
3.11 \\
4.23 \\
3.67 \\
5.19 \\
- \\
5.85 \\
14.36 \\
9.99 \\
3.28 \\
2.38 \\
3.05\end{array}$ & $\begin{array}{r}5.91 \\
5.49 \\
1.66 \\
- \\
1.66 \\
4.71 \\
5.89 \\
4.83 \\
6.02 \\
6.85 \\
7.19 \\
17.61 \\
9.16 \\
3.03 \\
2.17 \\
7.35\end{array}$ & $\begin{array}{r}5.00 \\
3.93 \\
2.05 \\
- \\
2.05 \\
3.92 \\
4.24 \\
4.35 \\
3.64 \\
4.14 \\
6.17 \\
15.59 \\
6.74 \\
2.63 \\
1.53 \\
7.52\end{array}$ & $\begin{array}{r}5.33 \\
3.88 \\
1.96 \\
- \\
1.96 \\
3.36 \\
4.19 \\
3.07 \\
5.21 \\
5.93 \\
5.77 \\
12.70 \\
7.57 \\
2.34 \\
1.50 \\
8.42\end{array}$ & $\begin{array}{r}5.49 \\
3.60 \\
1.78 \\
- \\
1.78 \\
3.01 \\
3.98 \\
3.29 \\
4.41 \\
5.02 \\
5.75 \\
14.61 \\
7.75 \\
2.12 \\
1.45 \\
9.33\end{array}$ & $\begin{array}{r}5.24 \\
3.69 \\
1.76 \\
- \\
1.76 \\
2.93 \\
4.05 \\
2.75 \\
4.46 \\
5.08 \\
5.98 \\
13.30 \\
8.59 \\
2.16 \\
1.74 \\
9.15\end{array}$ & $\begin{array}{r}5.42 \\
4.22 \\
1.72 \\
- \\
1.72 \\
3.19 \\
4.71 \\
2.64 \\
5.45 \\
6.20 \\
8.30 \\
13.40 \\
9.56 \\
3.03 \\
2.02 \\
8.39\end{array}$ & $\begin{array}{r}5.55 \\
4.35 \\
1.81 \\
-\overline{1} \\
1.81 \\
3.13 \\
4.97 \\
3.29 \\
5.79 \\
6.58 \\
8.68 \\
15.42 \\
9.07 \\
2.36 \\
1.72 \\
8.54\end{array}$ \\
\hline 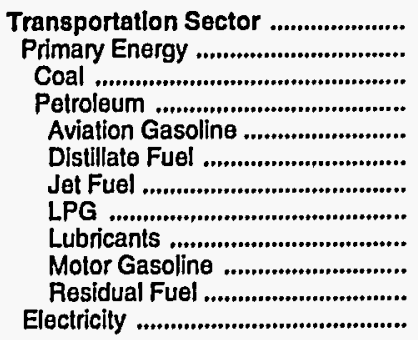 & $\begin{array}{r}2.34 \\
2.34 \\
.48 \\
2.34 \\
2.17 \\
1.24 \\
.76 \\
1.49 \\
5.08 \\
2.89 \\
.34 \\
-\end{array}$ & $\begin{array}{r}4.02 \\
4.02 \\
.79 \\
4.02 \\
3.45 \\
2.65 \\
2.12 \\
2.77 \\
7.48 \\
4.78 \\
2.01 \\
-\end{array}$ & $\begin{array}{r}8.92 \\
8.92 \\
- \\
8.92 \\
9.02 \\
7.15 \\
6.59 \\
5.85 \\
14.36 \\
9.99 \\
- \\
-\end{array}$ & $\begin{array}{r}8.42 \\
8.42 \\
- \\
8.42 \\
9.99 \\
6.80 \\
6.64 \\
7.19 \\
17.61 \\
9.16 \\
4.01 \\
-\end{array}$ & $\begin{array}{r}6.78 \\
6.78 \\
- \\
6.78 \\
8.41 \\
6.84 \\
4.81 \\
6.17 \\
15.59 \\
6.74 \\
- \\
-\end{array}$ & $\begin{array}{r}7.34 \\
7.34 \\
- \\
7.34 \\
7.55 \\
7.09 \\
4.38 \\
5.77 \\
12.70 \\
7.57 \\
- \\
-\end{array}$ & $\begin{array}{r}7.52 \\
7.52 \\
- \\
7.52 \\
7.41 \\
7.31 \\
4.33 \\
5.75 \\
14.61 \\
7.75 \\
- \\
-\end{array}$ & $\begin{array}{r}8.31 \\
8.31 \\
- \\
8.31 \\
8.28 \\
8.13 \\
4.79 \\
5.98 \\
13.30 \\
8.59 \\
- \\
-\end{array}$ & $\begin{array}{r}9.33 \\
9.33 \\
- \\
9.33 \\
9.32 \\
9.18 \\
6.26 \\
8.30 \\
13.40 \\
9.56 \\
- \\
-\end{array}$ & $\begin{array}{r}8.93 \\
8.93 \\
- \\
8.93 \\
8.71 \\
8.94 \\
5.47 \\
8.68 \\
15.42 \\
9.07 \\
- \\
-\end{array}$ \\
\hline $\begin{array}{l}\text { Total Energy ...................................... } \\
\text { Primary Energy - Four Sectors ...... } \\
\text { Electricity ...................................... }\end{array}$ & $\begin{array}{l}1.56 \\
1.35 \\
2.88\end{array}$ & $\begin{array}{l}2.78 \\
2.59 \\
4.05\end{array}$ & $\begin{array}{l}6.02 \\
6.06 \\
5.80\end{array}$ & $\begin{array}{r}7.48 \\
6.70 \\
10.72\end{array}$ & $\begin{array}{r}6.74 \\
5.50 \\
11.18\end{array}$ & $\begin{array}{r}7.05 \\
5.80 \\
11.81\end{array}$ & $\begin{array}{r}7.12 \\
5.79 \\
12.09\end{array}$ & $\begin{array}{r}7.24 \\
6.06 \\
12.00\end{array}$ & $\begin{array}{r}7.81 \\
6.82 \\
11.67\end{array}$ & $\begin{array}{r}7.74 \\
6.59 \\
12.22\end{array}$ \\
\hline 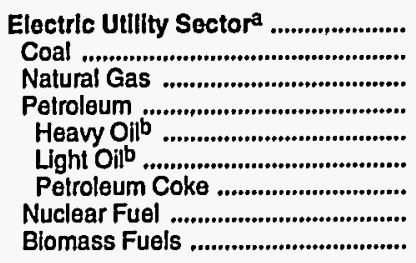 & $\begin{array}{r}.23 \\
.19 \\
.27 \\
.33 \\
.33 \\
.36 \\
- \\
- \\
.65\end{array}$ & $\begin{array}{r}.34 \\
.30 \\
.38 \\
2.00 \\
1.99 \\
2.30 \\
- \\
- \\
.92\end{array}$ & $\begin{array}{r}.72 \\
.44 \\
3.87 \\
5.01 \\
- \\
5.01 \\
- \\
- \\
1.74\end{array}$ & $\begin{array}{r}.72 \\
.71 \\
.59 \\
6.11 \\
- \\
6.11 \\
- \\
- \\
.79\end{array}$ & $\begin{array}{r}.68 \\
.67 \\
.86 \\
4.28 \\
- \\
4.28 \\
- \\
- \\
.32\end{array}$ & $\begin{array}{r}.66 \\
.65 \\
.92 \\
4.11 \\
- \\
4.11 \\
- \\
- \\
.95\end{array}$ & $\begin{array}{r}.55 \\
.55 \\
1.26 \\
4.03 \\
-\overline{3} \\
4.03 \\
- \\
-\overline{87}\end{array}$ & $\begin{array}{r}.58 \\
.58 \\
1.14 \\
4.31 \\
-\overline{1} \\
4.31 \\
- \\
(\bar{d})\end{array}$ & $\begin{array}{r}.68 \\
.67 \\
1.45 \\
5.43 \\
-\overline{5} \\
5.43 \\
- \\
\overline{(d)}\end{array}$ & $\begin{array}{r}.68 \\
.67 \\
3.94 \\
4.72 \\
-\overline{2} \\
4.72 \\
\overline{-} \\
\left({ }^{d}\right)\end{array}$ \\
\hline Primary Energy - Five Sectorsc .. & 1.26 & 2.41 & 4.79 & 4.69 & 3.39 & 3.47 & 3.03 & 3.32 & 3.82 & 3.63 \\
\hline
\end{tabular}

a There are no direct fuel costs for hydroelectric, geothermal, centralized solar, or wind energy.

beavy oil includes Grade Nos. 4, 5, and 6, and residual fuel oils. Light oil includes Grade No. 2 heating oil, kerosene, and jet fuel.

c Biomass fuels are not included, except those consumed at electric utilities and those added to motor gasoline.

d Utilities used biomass fuels at no charge or received a fee for accepting them.

- No consumption, including cases where adjustments were made. See "Consumption Adjustments for Process Fuel and Intermediate Products."

Sources: Data sources, estimation procedures, and assumptions are described in the "Documentation" section of this report. 
M Energy Expenditure Estimates by Sector, Montana

O 1970, 1975, 1980, and 1985-1991

N (Million Dollars)

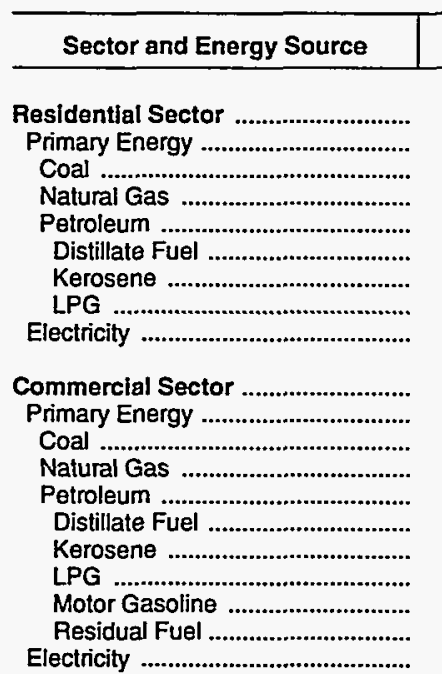

Industrial Sector

Primary Energy

Coal

Coking Coal

.............................

Natural Gas

Petroleum

Asphalt and Road Oil .....................

Distillate Fuel

Kerosene

LPG

Lubricants

Jicane

Residual Fuel .

Other .

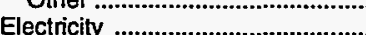

Transportation Sector ......................

Primary Energy .

Coal

etroleum ..........................................

Aviation Gasoline ..........................

Distillate Fuel

Jet Fuel

Lubricants

Motor Gasoline

Residual Fuel

Electricity

Total Energy .....................................

Primary Energy - Four Sectors .....

Electricity

Electric Utillty Sector ${ }^{a}$

Coal

Natural Gas

Petroleum

Heavy Oilb

Light Oib

Petroleum Coke

Nuclear Fuel

Biomass Fuels

Primary Energy - Five Sectorsc ..

\begin{tabular}{|c|c|c|c|c|c|c|c|c|c|}
\hline 1970 & 1975 & 1980 & 1985 & 1986 & 1987 & 1988 & 1989 & 1990 & 1991 \\
\hline $\begin{array}{l}66.7 \\
32.3\end{array}$ & $\begin{array}{r}106.4 \\
55.1\end{array}$ & $\begin{array}{r}187.8 \\
98.0\end{array}$ & $\begin{array}{l}297.4 \\
127.6\end{array}$ & $\begin{array}{l}263.0 \\
102.0\end{array}$ & $\begin{array}{r}265.8 \\
92.4\end{array}$ & $\begin{array}{r}275.2 \\
96.2\end{array}$ & $\begin{array}{l}302.9 \\
116.9\end{array}$ & $\begin{array}{l}300.8 \\
117.7\end{array}$ & $\begin{array}{l}321.5 \\
122.5\end{array}$ \\
\hline .1 & .1 & .1 & 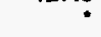 & .1 & .1 & .1 & .5 & .1 & .1 \\
\hline 22.5 & 31.2 & 58.9 & 93.2 & 74.8 & 67.7 & 72.7 & 79.5 & 77.3 & 83.2 \\
\hline 9.7 & 23.8 & 38.9 & 34.3 & 27.1 & 24.6 & 23.4 & 36.9 & 40.3 & 39.2 \\
\hline 1.9 & 9.7 & 17.0 & 15.9 & 10.0 & 7.4 & 6.3 & 10.8 & 10.8 & 12.7 \\
\hline- & - & - & .6 & .5 & .1 & .1 & 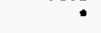 & .1 & 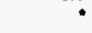 \\
\hline $\begin{array}{r}7.9 \\
34.4\end{array}$ & $\begin{array}{l}14.0 \\
51.3\end{array}$ & 21.9 & $\begin{array}{r}17.8 \\
169.8\end{array}$ & $\begin{array}{r}16.6 \\
161.0\end{array}$ & $\begin{array}{r}17.2 \\
1734\end{array}$ & $\begin{array}{r}17.1 \\
1790\end{array}$ & $\begin{array}{r}26.0 \\
1860\end{array}$ & 29.4 & 26.5 \\
\hline & & & & & & & & & \\
\hline 41.3 & 73.1 & 127.1 & 296.5 & 280.0 & 206.1 & 209.4 & 210.9 & 219.9 & 232.1 \\
\hline 18.0 & 37.2 & 66.4 & 115.7 & 75.4 & 63.3 & 62.4 & 69.9 & 70.9 & 69.1 \\
\hline .1 & .1 & .4 & .2 & .5 & .2 & .2 & 1.2 & 1.2 & 1.0 \\
\hline 11.5 & 20.4 & 44.9 & 75.5 & 56.1 & 47.7 & 51.8 & 57.3 & 56.4 & 55.9 \\
\hline 6.5 & 16.7 & 21.2 & 40.0 & 18.8 & 15.5 & 10.4 & 11.4 & 13.3 & 12.2 \\
\hline 1.7 & 9.7 & 13.0 & 31.4 & 12.8 & 9.5 & 4.5 & 4.5 & 4.6 & 5.3 \\
\hline .5 & .8 & - & 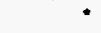 & .2 & 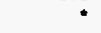 & 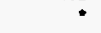 & 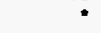 & 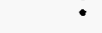 & 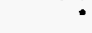 \\
\hline .9 & 1.8 & 3.1 & 2.8 & 2.5 & 2.6 & 2.6 & 3.2 & 4.3 & 3.9 \\
\hline 3.3 & 4.4 & 4.8 & 3.5 & 2.7 & 3.2 & 3.1 & 3.5 & 4.2 & 3.0 \\
\hline 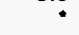 & 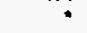 & .2 & 2.4 & .6 & .2 & .1 & .2 & .2 & 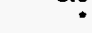 \\
\hline 23.3 & 35.9 & 60.7 & 180.8 & 204.5 & 142.7 & 147.0 & 141.0 & 149.0 & 163.0 \\
\hline 67.2 & 156.8 & 329.7 & 493.1 & 336.7 & 342.8 & 348.1 & 387.9 & 399.9 & 418.9 \\
\hline 40.7 & 124.1 & 272.6 & 355.2 & 186.5 & 170.1 & 152.5 & 195.7 & 221.5 & 235.0 \\
\hline .3 & .8 & 6.0 & 6.8 & 11.6 & 6.7 & 7.0 & 6.4 & 6.9 & 9.4 \\
\hline - & - & - & - & - & - & - & - & - & - \\
\hline .3 & .8 & 6.0 & 6.8 & 11.6 & 6.7 & 7.0 & 6.4 & 6.9 & 9.4 \\
\hline 10.4 & 26.1 & 45.2 & 35.7 & 26.3 & 24.4 & 24.1 & 27.6 & 28.4 & 28.7 \\
\hline 30.0 & 97.2 & 221.4 & 312.7 & 148.5 & 139.0 & 121.4 & 161.7 & 186.2 & 196.9 \\
\hline 5.3 & 11.3 & 24.9 & 46.9 & 57.4 & 33.5 & 32.2 & 31.9 & 26.0 & 29.5 \\
\hline 6.5 & 35.5 & 58.2 & 203.3 & 45.1 & 54.7 & 41.6 & 72.3 & 87.2 & 120.0 \\
\hline 1.5 & 1.0 & - & 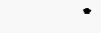 & 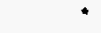 & .2 & .1 & .1 & .2 & .1 \\
\hline .9 & .8 & 15.9 & 17.9 & 13.6 & 15.2 & 11.1 & 14.3 & 22.9 & 3.6 \\
\hline 1.4 & 2.1 & 4.4 & 5.0 & 4.3 & 4.0 & 4.4 & 4.1 & 4.3 & 4.4 \\
\hline 9.6 & 19.4 & 32.5 & 32.6 & 22.6 & 22.8 & 23.4 & 28.4 & 30.7 & 29.1 \\
\hline .4 & 14.9 & 68.1 & 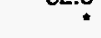 & 2 & ? & 20.7 & 20.1 & ? & ? \\
\hline 4.4 & 12.3 & 17.4 & 7.1 & 5.5 & 8.6 & 8.7 & 10.5 & 14.8 & 10.3 \\
\hline 26.5 & 32.6 & 57.1 & 138.0 & 150.2 & 172.7 & 195.6 & 192.2 & 178.4 & 183.9 \\
\hline 157.9 & 323.1 & 767.3 & 673.8 & 543.5 & 589.6 & 618.9 & 675.9 & 752.1 & 722.8 \\
\hline 157.9 & 323.1 & 767.3 & 673.8 & 543.5 & 589.6 & 618.9 & 675.9 & 752.1 & 722.8 \\
\hline & $\because$ & - & - & - & - & - & - & - & 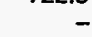 \\
\hline 157.9 & 323.1 & 767.3 & 673.8 & 543.5 & 589.6 & 618.9 & 675.9 & 752.1 & 722.8 \\
\hline .5 & 1.4 & 7.3 & 4.6 & 4.4 & 3.1 & 4.0 & 4.0 & 5.2 & 4.8 \\
\hline 21.9 & 59.2 & 198.3 & 169.4 & 163.5 & 171.7 & 178.4 & 202.0 & 223.1 & 216.6 \\
\hline 2.7 & 9.7 & 34.1 & 25.2 & 23.3 & 17.7 & 19.6 & 20.0 & 24.8 & 22.0 \\
\hline .2 & .5 & 1.0 & 1.3 & 1.2 & .8 & 1.0 & 1.2 & 2.0 & 1.5 \\
\hline 4.7 & 7.3 & 17.1 & 19.1 & 16.5 & 15.2 & 16.9 & 15.8 & 16.3 & 16.8 \\
\hline 127.7 & 242.9 & 509.5 & 454.2 & 334.6 & 381.1 & 398.9 & 432.9 & 480.6 & 461.2 \\
\hline .3 & 2.0 & - & 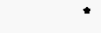 & - & - & - & - & - & - \\
\hline- & - & - & - & - & - & - & - & - & - \\
\hline $\begin{array}{r}333.1 \\
249.0 \\
84.1\end{array}$ & $\begin{array}{l}659.3 \\
539.5 \\
119.8\end{array}$ & $\begin{array}{r}1,412.0 \\
1,204.3 \\
207.7\end{array}$ & $\begin{array}{r}1,760.9 \\
1,272.3 \\
488.6\end{array}$ & $\begin{array}{r}1,423.2 \\
907.4 \\
515.8\end{array}$ & $\begin{array}{r}1,404.3 \\
915.5 \\
488.8\end{array}$ & $\begin{array}{r}1,451.6 \\
930.0 \\
521.6\end{array}$ & $\begin{array}{r}1,577.6 \\
1,058.4 \\
519.2\end{array}$ & $\begin{array}{r}1,672.6 \\
1,162.2 \\
510.4\end{array}$ & $\begin{array}{r}1,695.3 \\
1,149.5 \\
545.8\end{array}$ \\
\hline 3.4 & 6.4 & 44.3 & 69.2 & 87.0 & 85.9 & 99.1 & 102.2 & 110.6 & 119.3 \\
\hline 2.2 & 5.2 & 25.3 & 67.1 & 85.7 & 83.8 & 96.7 & 100.2 & 107.9 & 116.9 \\
\hline .7 & .5 & 17.0 & .3 & .4 & .5 & .4 & .5 & .7 & 1.3 \\
\hline .1 & .7 & 1.7 & 1.4 & .6 & 1.1 & 1.5 & 1.5 & 2.0 & 1.1 \\
\hline .1 & .7 & - & - & - & - & - & - & - & - \\
\hline • & - & 1.7 & 1.4 & .6 & 1.1 & 1.5 & 1.5 & 2.0 & 1.1 \\
\hline- & - & - & - & - & - & - & - & - & - \\
\hline - & - & - & - & - & - & - & - & - & - \\
\hline .5 & .1 & .3 & .5 & .2 & .5 & .5 & (d) & $\left(^{d}\right)$ & $\left({ }^{d}\right)$ \\
\hline 252.4 & 545.9 & $1,248.6$ & $1,341.5$ & 994.4 & $1,001.4$ & $1,029.1$ & $1,160.6$ & $1,272.9$ & $1,268.7$ \\
\hline
\end{tabular}

a There are no direct fuel costs for hydroelectric, geothermal, centralized solar, or wind energy.

beavy oil includes Grade Nos. 4, 5, and 6, and residual fuel oils. Light oil includes Grade No. 2 heating oil, kerosene, and jet fuel.

c Biomass fuels are not included, except those consumed at electric utilities and those added to motor gasoline.

d Utilities used biomass fuels at no charge or received a fee for accepting them.

-No consumption, including cases where adjustments were made. See "Consumption Adjustments for Process Fuel and intermediate Products."

-Value less than 0.05 million dollars.

Note: Totals may not equal sum of components due to independent rounding.

Sources: Data sources, estimation procedures, and assumptions are described in the "Documentation" section of this report. 


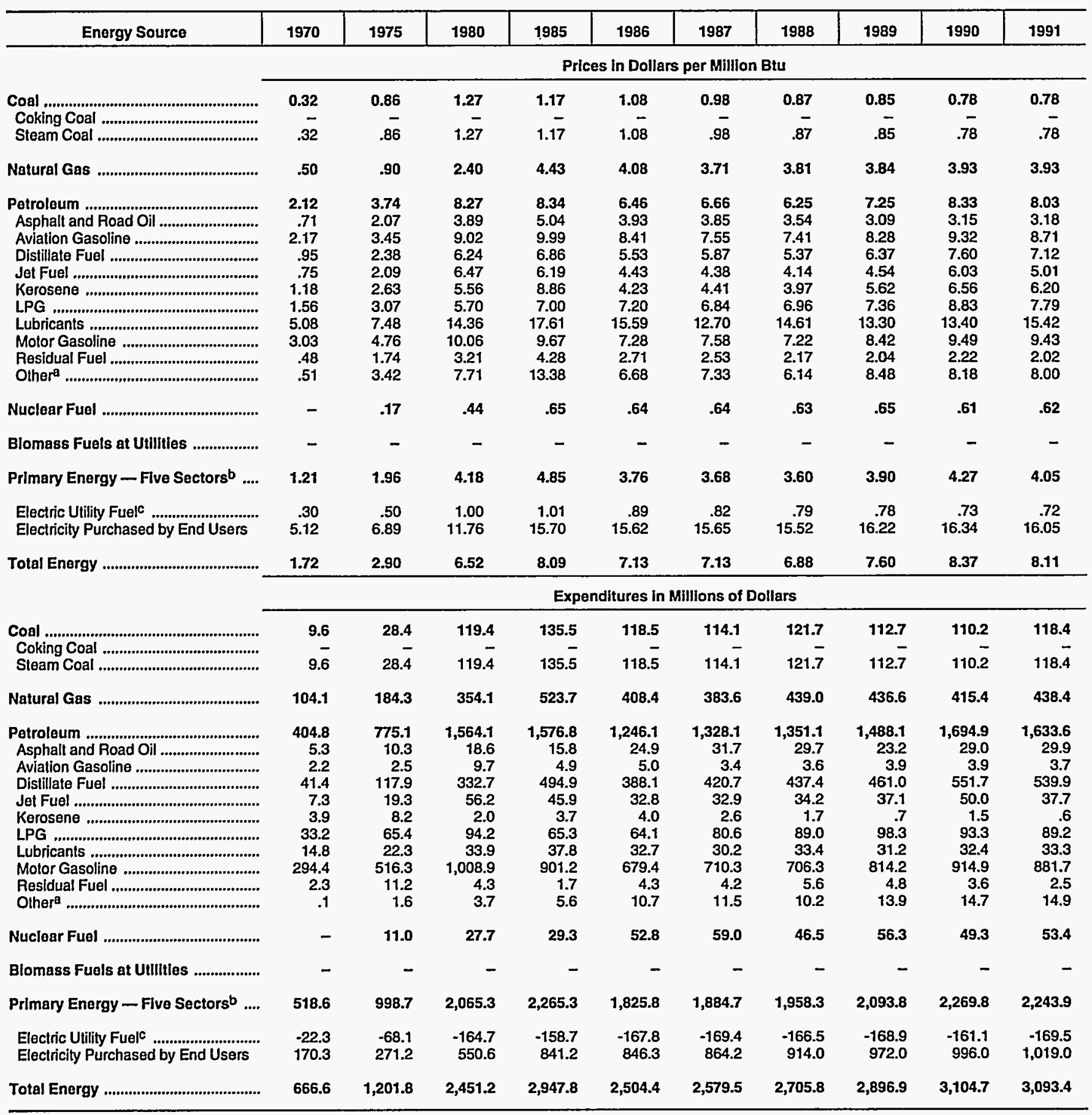

Includes petroleum coke used at electric utilities.

b Biomass fuels are not included, except those consumed at electric utilities and those added to motor gasoline.

c There are no direct fuel costs for hydroelectric, geothermal, centralized solar, or wind energy.

- No consumption, including cases where adjustments were made. See "Consumption Adjustments for Process Fuel and Intermediate Products."

Nole: Expenditure totals may not equal sum of components due to independent rounding.

Sources; Data sources, estimation procedures, and assumptions are described in the "Documentation" section of this report. 
N Energy Price Estimates by Sector, Nebraska

E 1970,1975, 1980, and 1985-1991

B (Dollars per Million Btu)

$\mathbf{R}$

\begin{tabular}{|c|c|c|c|c|c|c|c|c|c|c|}
\hline Sector and Energy Source & 1970 & 1975 & 1980 & 1985 & 1986 & 1987 & 1988 & 1989 & 1990 & 1991 \\
\hline $\begin{array}{l}\text { Resldential Sector } \\
\text { Primary Energy } \\
\text { Coal } \\
\text { Natural Gas . } \\
\text { Petroleum } \\
\text { Distillate Fuel } \\
\text { Kerosene } \\
\text { LPG }\end{array}$ & $\begin{array}{r}1.84 \\
1.04 \\
1.08 \\
.84 \\
1.69 \\
1.19 \\
1.39 \\
1.78 \\
6.21\end{array}$ & $\begin{array}{l}2.95 \\
1.74 \\
2.15 \\
1.29 \\
3.39 \\
2.62 \\
2.74 \\
3.57 \\
8.13\end{array}$ & $\begin{array}{r}5.83 \\
3.32 \\
3.60 \\
2.78 \\
6.84 \\
6.85 \\
7.55 \\
6.82 \\
13.22\end{array}$ & $\begin{array}{r}8.83 \\
5.37 \\
2.76 \\
5.10 \\
7.49 \\
7.92 \\
9.74 \\
7.12 \\
17.30\end{array}$ & $\begin{array}{r}8.64 \\
4.68 \\
2.40 \\
4.62 \\
5.21 \\
4.88 \\
6.00 \\
5.34 \\
17.27\end{array}$ & $\begin{array}{r}8.82 \\
4.52 \\
2.43 \\
4.43 \\
5.18 \\
4.50 \\
5.54 \\
5.35 \\
17.52\end{array}$ & $\begin{array}{r}8.75 \\
4.60 \\
2.49 \\
4.53 \\
5.23 \\
4.27 \\
5.25 \\
5.48 \\
17.42\end{array}$ & $\begin{array}{r}8.99 \\
4.85 \\
2.42 \\
4.54 \\
7.21 \\
5.37 \\
6.61 \\
7.82 \\
18.05\end{array}$ & $\begin{array}{r}9.45 \\
4.96 \\
2.42 \\
4.67 \\
7.57 \\
6.74 \\
8.28 \\
7.79 \\
18.23\end{array}$ & $\begin{array}{r}9.19 \\
4.94 \\
2.36 \\
4.71 \\
6.79 \\
6.25 \\
7.69 \\
6.92 \\
17.86\end{array}$ \\
\hline $\begin{array}{l}\text { Commercial Sector } \\
\text { Primary Energy } \\
\text { Coal } \\
\text { Natural Gas } \\
\text { Petroleum } \\
\text { Distillate Fuel } \\
\text { Kerosene } \\
\text { LPG } \\
\text { Motor Gasoline } \\
\text { Residual Fuel } \\
\text { Electricity }\end{array}$ & $\begin{array}{r}1.36 \\
.59 \\
.16 \\
.52 \\
1.09 \\
1.03 \\
.79 \\
1.09 \\
3.03 \\
.50 \\
4.87\end{array}$ & $\begin{array}{r}2.36 \\
1.17 \\
.81 \\
1.00 \\
2.60 \\
2.45 \\
2.39 \\
2.46 \\
4.76 \\
1.75 \\
6.96\end{array}$ & $\begin{array}{r}5.01 \\
2.62 \\
1.69 \\
2.33 \\
6.81 \\
6.49 \\
5.17 \\
5.19 \\
10.06 \\
3.22 \\
12.86\end{array}$ & $\begin{array}{r}8.34 \\
4.68 \\
2.46 \\
4.29 \\
7.21 \\
6.79 \\
7.81 \\
6.92 \\
9.67 \\
- \\
16.78\end{array}$ & $\begin{array}{r}8.22 \\
4.05 \\
1.70 \\
3.95 \\
5.18 \\
3.49 \\
4.01 \\
8.25 \\
7.28 \\
- \\
16.55\end{array}$ & $\begin{array}{r}8.31 \\
3.90 \\
1.63 \\
3.76 \\
5.28 \\
3.54 \\
4.24 \\
7.76 \\
7.58 \\
2.60 \\
16.43\end{array}$ & $\begin{array}{r}8.04 \\
3.88 \\
1.56 \\
3.81 \\
5.00 \\
3.04 \\
3.63 \\
7.73 \\
7.22 \\
2.13 \\
16.22\end{array}$ & $\begin{array}{r}8.57 \\
3.95 \\
1.47 \\
3.82 \\
5.60 \\
4.03 \\
5.02 \\
7.13 \\
8.42 \\
2.02 \\
16.93\end{array}$ & $\begin{array}{r}8.87 \\
4.17 \\
1.48 \\
3.92 \\
7.01 \\
5.07 \\
6.36 \\
9.36 \\
9.49 \\
2.22 \\
17.22\end{array}$ & $\begin{array}{r}8.57 \\
4.08 \\
1.54 \\
3.93 \\
6.51 \\
4.44 \\
5.53 \\
8.34 \\
9.43 \\
2.03 \\
16.82\end{array}$ \\
\hline 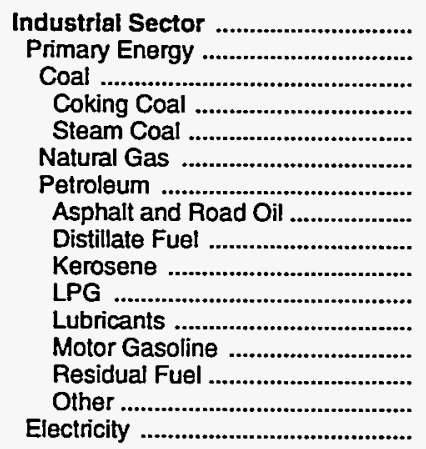 & $\begin{array}{r}.88 \\
.69 \\
.16 \\
- \\
.16 \\
.32 \\
1.26 \\
.71 \\
.73 \\
.79 \\
1.09 \\
5.08 \\
3.03 \\
.40 \\
.51 \\
3.42\end{array}$ & $\begin{array}{r}1.77 \\
1.48 \\
.81 \\
-\overline{1} \\
.81 \\
.69 \\
2.93 \\
2.07 \\
2.25 \\
2.39 \\
2.46 \\
7.48 \\
4.76 \\
1.74 \\
3.42 \\
4.96\end{array}$ & $\begin{array}{r}4.50 \\
3.87 \\
1.69 \\
- \\
1.69 \\
2.21 \\
5.88 \\
3.89 \\
4.94 \\
5.17 \\
5.19 \\
14.36 \\
10.06 \\
3.13 \\
7.71 \\
8.71\end{array}$ & $\begin{array}{r}6.42 \\
5.59 \\
2.46 \\
-\overline{2} \\
2.46 \\
3.67 \\
7.47 \\
5.04 \\
7.09 \\
7.81 \\
6.92 \\
17.61 \\
9.67 \\
4.28 \\
13.38 \\
11.47\end{array}$ & $\begin{array}{r}5.21 \\
4.12 \\
1.70 \\
- \\
1.70 \\
3.28 \\
4.81 \\
3.93 \\
3.64 \\
4.01 \\
8.25 \\
15.59 \\
7.28 \\
2.81 \\
6.68 \\
11.42\end{array}$ & $\begin{array}{r}5.00 \\
3.99 \\
1.63 \\
- \\
1.63 \\
2.81 \\
5.02 \\
3.85 \\
3.85 \\
4.24 \\
7.76 \\
12.70 \\
7.58 \\
2.60 \\
7.33 \\
11.34\end{array}$ & $\begin{array}{r}4.80 \\
3.76 \\
1.56 \\
- \\
1.56 \\
2.90 \\
4.52 \\
3.54 \\
3.30 \\
3.63 \\
7.73 \\
14.61 \\
7.22 \\
2.13 \\
6.14 \\
11.28\end{array}$ & $\begin{array}{r}5.44 \\
4.20 \\
1.47 \\
1.47 \\
2.96 \\
5.29 \\
3.09 \\
4.56 \\
5.02 \\
7.13 \\
13.30 \\
8.42 \\
2.02 \\
8.48 \\
12.34\end{array}$ & $\begin{array}{r}6.11 \\
4.85 \\
1.48 \\
- \\
1.48 \\
3.02 \\
6.14 \\
3.15 \\
5.78 \\
6.36 \\
9.36 \\
13.40 \\
9.49 \\
2.22 \\
8.18 \\
12.31\end{array}$ & $\begin{array}{r}5.72 \\
4.45 \\
1.54 \\
- \\
1.54 \\
2.80 \\
5.60 \\
3.18 \\
5.02 \\
5.53 \\
8.34 \\
15.42 \\
9.43 \\
2.03 \\
8.00 \\
12.19\end{array}$ \\
\hline 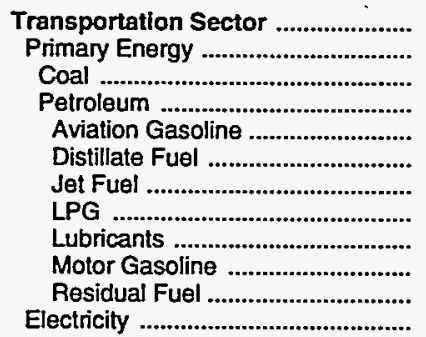 & $\begin{array}{r}2.51 \\
2.51 \\
.16 \\
2.51 \\
2.17 \\
1.14 \\
.75 \\
1.09 \\
5.08 \\
3.03 \\
.50 \\
-\end{array}$ & $\begin{array}{r}4.15 \\
4.15 \\
.81 \\
4.15 \\
3.45 \\
2.50 \\
2.09 \\
2.46 \\
7.48 \\
4.76 \\
1.74 \\
-\end{array}$ & $\begin{array}{r}9.20 \\
9.20 \\
-\overline{-} \\
9.20 \\
9.02 \\
7.06 \\
6.47 \\
5.19 \\
14.36 \\
10.06 \\
- \\
-\end{array}$ & $\begin{array}{r}8.70 \\
8.70 \\
- \\
8.70 \\
9.99 \\
6.68 \\
6.19 \\
6.92 \\
17.61 \\
9.67 \\
= \\
-\end{array}$ & $\begin{array}{r}7.10 \\
7.10 \\
7.10 \\
8.41 \\
6.79 \\
4.43 \\
8.25 \\
15.59 \\
7.28 \\
2.32 \\
-\end{array}$ & $\begin{array}{r}7.31 \\
7.31 \\
- \\
7.31 \\
7.55 \\
7.02 \\
4.38 \\
7.76 \\
12.70 \\
7.58 \\
2.29\end{array}$ & $\begin{array}{r}6.90 \\
6.90 \\
- \\
6.90 \\
7.41 \\
6.47 \\
4.14 \\
7.73 \\
14.61 \\
7.22 \\
-\end{array}$ & $\begin{array}{r}7.95 \\
7.95 \\
- \\
7.95 \\
8.28 \\
7.39 \\
4.54 \\
7.13 \\
13.30 \\
8.42 \\
- \\
-\end{array}$ & $\begin{array}{r}9.10 \\
9.10 \\
- \\
9.10 \\
9.32 \\
8.66 \\
6.03 \\
9.36 \\
13.40 \\
9.49 \\
-\end{array}$ & $\begin{array}{r}8.96 \\
8.96 \\
- \\
8.96 \\
8.71 \\
8.44 \\
5.01 \\
8.34 \\
15.42 \\
9.43 \\
- \\
-\end{array}$ \\
\hline 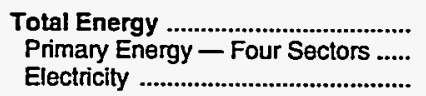 & $\begin{array}{l}1.72 \\
1.40 \\
5.12\end{array}$ & $\begin{array}{l}2.90 \\
2.48 \\
6.89\end{array}$ & $\begin{array}{r}6.52 \\
5.77 \\
11.76\end{array}$ & $\begin{array}{r}8.09 \\
6.78 \\
15.70\end{array}$ & $\begin{array}{r}7.13 \\
5.58 \\
15.62\end{array}$ & $\begin{array}{r}7.13 \\
5.60 \\
15.65\end{array}$ & $\begin{array}{r}6.88 \\
5.36 \\
15.52\end{array}$ & $\begin{array}{r}7.60 \\
5.99 \\
16.22\end{array}$ & $\begin{array}{r}8.37 \\
6.80 \\
16.34\end{array}$ & $\begin{array}{r}8.11 \\
6.53 \\
16.05\end{array}$ \\
\hline 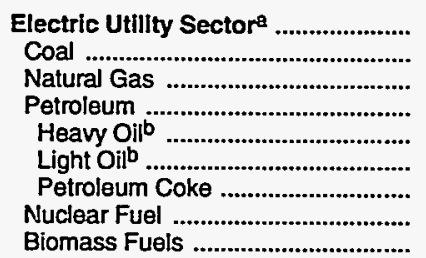 & $\begin{array}{r}.30 \\
.35 \\
.27 \\
.54 \\
.49 \\
.63 \\
- \\
- \\
-\end{array}$ & $\begin{array}{r}.50 \\
.87 \\
.63 \\
1.77 \\
1.73 \\
1.85 \\
.17 \\
-\end{array}$ & $\begin{array}{r}1.00 \\
1.24 \\
1.82 \\
4.14 \\
3.21 \\
6.19 \\
-44 \\
-\end{array}$ & $\begin{array}{r}1.01 \\
1.11 \\
3.58 \\
5.89 \\
-\overline{5} \\
- \\
.65 \\
-\end{array}$ & $\begin{array}{r}.89 \\
1.04 \\
3.21 \\
3.05 \\
2.32 \\
3.87 \\
-64 \\
-\end{array}$ & $\begin{array}{r}.82 \\
.94 \\
2.56 \\
2.87 \\
2.29 \\
3.98 \\
- \\
.64 \\
-\end{array}$ & $\begin{array}{r}.79 \\
.84 \\
2.69 \\
2.83 \\
2.34 \\
3.47 \\
-63 \\
-\end{array}$ & $\begin{array}{r}.78 \\
.83 \\
2.36 \\
3.09 \\
2.15 \\
4.35 \\
-65 \\
-\end{array}$ & $\begin{array}{r}.73 \\
.75 \\
2.01 \\
6.89 \\
1.85 \\
7.03 \\
- \\
.61 \\
-\end{array}$ & $\begin{array}{r}.72 \\
.74 \\
1.97 \\
4.20 \\
1.41 \\
4.57 \\
- \\
.62 \\
-\end{array}$ \\
\hline Primary Energy — Flve Sectors ${ }^{c}$... & 1.21 & 1.96 & 4.18 & 4.85 & 3.76 & 3.68 & 3.60 & 3.90 & 4.27 & 4.05 \\
\hline
\end{tabular}

a There are no direct fuel costs for hydroelectric, geothermal, centralized solar, or wind energy.

beavy oil includes Grade Nos. 4, 5, and 6, and residual fuel oils. Light oil includes Grade No. 2 heating oil, kerosene, and jet fuel.

c Biomass fuels are not included, except those consumed at electric utilities and those added to motor gasoline.

-No consumption, including cases where adjustments were made. See "Consumption Adjustments for Process Fuel and Intermediate Products."

Sources: Data sources, estimation procedures, and assumptions are described in the "Documentation" section of this report. 
Energy Expenditure Estimates by Sector, Nebraska $1970,1975,1980$, and 1985-1991

(Million Dollars)

\begin{tabular}{|c|c|c|c|c|c|c|c|c|c|c|}
\hline Sector and Energy Source & 1970 & 1975 & 1980 & 1985 & 1986 & 1987 & 1988 & 1989 & 1990 & 1991 \\
\hline 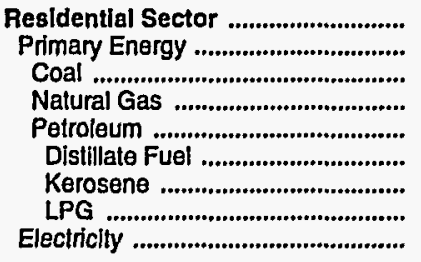 & $\begin{array}{r}167.4 \\
80.3 \\
.3 \\
49.6 \\
30.4 \\
1.4 \\
3.0 \\
26.1 \\
87.0\end{array}$ & $\begin{array}{r}249.4 \\
119.2 \\
.1 \\
68.9 \\
50.1 \\
2.6 \\
5.8 \\
41.7 \\
130.3\end{array}$ & $\begin{array}{r}433.0 \\
184.0 \\
.4 \\
133.5 \\
50.0 \\
14.4 \\
.4 \\
35.2 \\
249.1\end{array}$ & $\begin{array}{r}643.2 \\
277.7 \\
.3 \\
233.9 \\
43.5 \\
15.7 \\
2.2 \\
25.6 \\
365.5\end{array}$ & $\begin{array}{r}592.6 \\
220.0 \\
.1 \\
194.0 \\
26.0 \\
8.0 \\
.6 \\
17.3 \\
372.6\end{array}$ & $\begin{array}{r}580.5 \\
199.1 \\
.1 \\
169.5 \\
29.6 \\
5.3 \\
.4 \\
23.9 \\
381.4\end{array}$ & $\begin{array}{r}629.1 \\
224.1 \\
.7 \\
194.0 \\
29.4 \\
4.9 \\
.5 \\
.5 \\
23.9 \\
405.0\end{array}$ & $\begin{array}{r}-657.8 \\
243.8 \\
.1 \\
200.7 \\
42.9 \\
7.8 \\
.3 \\
34.8 \\
414.0\end{array}$ & $\begin{array}{r}648.4 \\
225.4 \\
.1 \\
.1 \\
190.9 \\
34.4 \\
6.6 \\
.2 \\
.2 \\
27.6 \\
423.0\end{array}$ & $\begin{array}{r}680.7 \\
245.7 \\
.3 \\
207.3 \\
38.1 \\
7.2 \\
7.2 \\
.2 \\
30.7 \\
435.0\end{array}$ \\
\hline 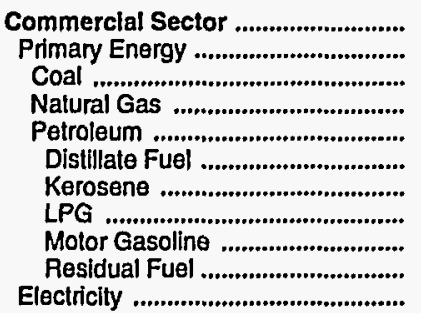 & $\begin{array}{r}89.9 \\
31.7 \\
.1 \\
24.7 \\
6.8 \\
1.2 \\
.3 \\
.3 \\
2.8 \\
1.7 \\
.8 \\
58.3\end{array}$ & $\begin{array}{r}143.1 \\
56.2 \\
.1 \\
42.9 \\
13.3 \\
2.5 \\
1.0 \\
5.1 \\
3.0 \\
1.7 \\
86.9\end{array}$ & $\begin{array}{r}298.5 \\
120.0 \\
.4 \\
99.1 \\
20.5 \\
6.8 \\
.6 \\
4.7 \\
7.9 \\
.5 \\
178.5\end{array}$ & $\begin{array}{r}538.2 \\
211.0 \\
.4 \\
166.0 \\
44.6 \\
31.7 \\
.5 \\
4.4 \\
8.0 \\
- \\
327.2\end{array}$ & $\begin{array}{r}487.3 \\
160.0 \\
.1 \\
142.8 \\
17.1 \\
6.8 \\
.2 \\
4.7 \\
5.4 \\
- \\
327.3\end{array}$ & $\begin{array}{r}479.5 \\
145.7 \\
.1 \\
126.6 \\
19.0 \\
7.3 \\
.1 \\
6.1 \\
5.5 \\
333.8\end{array}$ & $\begin{array}{r}516.1 \\
165.1 \\
.8 \\
147.7 \\
16.6 \\
5.3 \\
. \\
6.0 \\
5.1 \\
.2 \\
351.0\end{array}$ & $\begin{array}{r}532.1 \\
158.1 \\
.1 \\
140.8 \\
17.1 \\
5.3 \\
.1 \\
5.6 \\
5.6 \\
.6 \\
374.0\end{array}$ & $\begin{array}{r}541.9 \\
162.9 \\
.1 \\
140.8 \\
22.0 \\
7.3 \\
.8 \\
5.9 \\
7.7 \\
.3 \\
379.0\end{array}$ & $\begin{array}{r}561.8 \\
172.8 \\
.2 \\
155.9 \\
16.7 \\
4.7 \\
.1 \\
6.5 \\
5.0 \\
.3 \\
389.0\end{array}$ \\
\hline 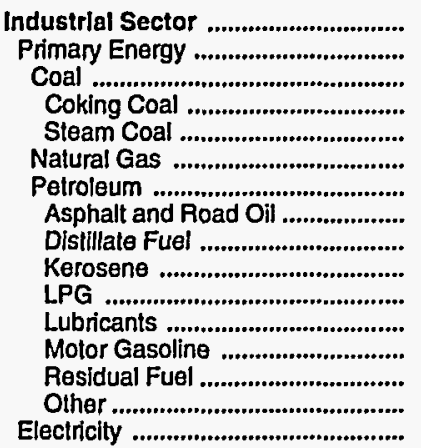 & $\begin{array}{r}92.3 \\
67.4 \\
.8 \\
-\overline{8} \\
17.0 \\
49.6 \\
5.3 \\
14.0 \\
.6 \\
3.4 \\
4.9 \\
21.0 \\
.3 \\
.1\end{array}$ & $\begin{array}{r}230.9 \\
177.0 \\
4.8 \\
-\overline{0} \\
4.8 \\
49.2 \\
122.9 \\
10.3 \\
42.3 \\
1.5 \\
16.5 \\
8.8 \\
41.1 \\
.8 \\
1.6 \\
54.0\end{array}$ & $\begin{array}{r}486.6 \\
363.7 \\
8.7 \\
8 . \overline{7} \\
101.1 \\
253.9 \\
18.6 \\
98.1 \\
.9 \\
51.0 \\
3.6 \\
77.7 \\
.3 \\
3.7 \\
123.0\end{array}$ & $\begin{array}{r}589.9 \\
441.4 \\
11.9 \\
11 . \\
11.9 \\
119.4 \\
310.0 \\
15.8 \\
177.4 \\
1.0 \\
33.9 \\
4.0 \\
70.7 \\
1.7 \\
5.6 \\
148.5\end{array}$ & $\begin{array}{r}446.1 \\
299.7 \\
10.6 \\
10 . \overline{1} \\
66.3 \\
622.7 \\
24.9 \\
240.4 \\
90.4 \\
3.2 \\
41.0 \\
3.5 \\
45.5 \\
3.5 \\
10.7\end{array}$ & $\begin{array}{r}479.4 \\
330.4 \\
9.4 \\
-\overline{9} \\
9.4 \\
83.2 \\
237.8 \\
31.7 \\
87.1 \\
2.1 \\
49.2 \\
3.2 \\
49.6 \\
3.4 \\
11.5 \\
149.0\end{array}$ & $\begin{array}{r}488.3 \\
330.3 \\
7.8 \\
7 . \overline{-} \\
7.8 \\
92.1 \\
230.5 \\
29.7 \\
83.5 \\
1.2 \\
57.6 \\
3.5 \\
40.4 \\
4.3 \\
10.2 \\
158.0\end{array}$ & $\begin{array}{r}534.2 \\
350.2 \\
7.9 \\
7 . \overline{9} \\
79.9 \\
253.1 \\
23.2 \\
106.1 \\
.3 \\
56.1 \\
3.3 \\
46.8 \\
3.4 \\
13.9 \\
184.0\end{array}$ & $\begin{array}{r}572.2 \\
378.2 \\
6.6 \\
- \\
6.6 \\
76.5 \\
295.1 \\
29.0 \\
139.3 \\
.5 \\
57.7 \\
3.4 \\
47.1 \\
3.3 \\
14.7 \\
194.0\end{array}$ & $\begin{array}{r}556.3 \\
361.3 \\
9.4 \\
- \\
9.4 \\
68.3 \\
283.5 \\
29.9 \\
136.1 \\
.3 \\
50.0 \\
3.5 \\
46.6 \\
2.2 \\
14.9 \\
195.0\end{array}$ \\
\hline 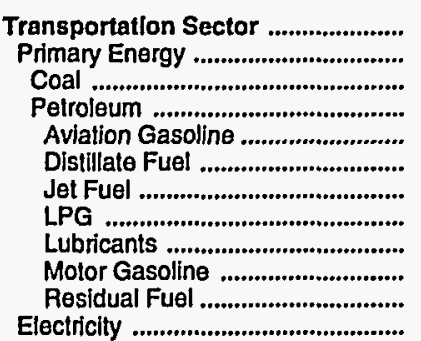 & $\begin{array}{r}317.0 \\
317.0 \\
317.0 \\
2.2 \\
24.4 \\
7.3 \\
.9 \\
9.8 \\
271.7 \\
.7 \\
-\end{array}$ & $\begin{array}{r}578.3 \\
578.3 \\
578.3 \\
2.5 \\
67.2 \\
19.3 \\
2.1 \\
13.6 \\
472.2 \\
1.5 \\
-\end{array}$ & $\begin{array}{r}1,233.0 \\
1,233.0 \\
-\overline{1} \\
1,233.0 \\
9.7 \\
210.2 \\
56.2 \\
3.3 \\
30.3 \\
923.3 \\
- \\
-\end{array}$ & $\begin{array}{r}1,176.5 \\
1,176.5 \\
-\overline{1,176.5} \\
4.9 \\
268.1 \\
45.9 \\
1.4 \\
33.8 \\
822.4 \\
-\end{array}$ & $\begin{array}{r}978.4 \\
978.4 \\
978.4 \\
978.4 \\
5.0 \\
281.8 \\
32.8 \\
1.1 \\
29.3 \\
628.5 \\
-\end{array}$ & $\begin{array}{r}1,040.1 \\
1,040.1 \\
\overline{1,0} \\
1,040.1 \\
3.4 \\
320.3 \\
32.9 \\
1.4 \\
27.0 \\
655.2 \\
.\end{array}$ & $\begin{array}{r}1,072.3 \\
1,072.3 \\
-\overline{1} \\
1,072.3 \\
3.6 \\
342.3 \\
34.2 \\
1.4 \\
29.9 \\
660.8 \\
- \\
-\end{array}$ & $\begin{array}{r}1,172.8 \\
1,172.8 \\
1,172.8 \\
3.9 \\
340.4 \\
37.1 \\
1.7 \\
27.9 \\
761.8 \\
-\end{array}$ & $\begin{array}{r}1,342.2 \\
1,342.2 \\
1,342.2 \\
3.9 \\
397.2 \\
50.0 \\
2.1 \\
29.0 \\
860.1 \\
- \\
-\end{array}$ & $\begin{array}{r}1,294.6 \\
1,294.6 \\
- \\
1,294.6 \\
3.7 \\
391.2 \\
37.7 \\
1.9 \\
29.8 \\
830.2 \\
= \\
-\end{array}$ \\
\hline 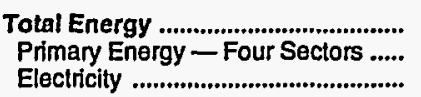 & $\begin{array}{l}666.6 \\
496.3 \\
170.3\end{array}$ & $\begin{array}{r}1,201.8 \\
930.6 \\
271.2\end{array}$ & $\begin{array}{r}2,451.2 \\
1,900.6 \\
550.6\end{array}$ & $\begin{array}{r}2,947.8 \\
2,106.6 \\
841.2\end{array}$ & $\begin{array}{r}2,504.4 \\
1,658.1 \\
846.3\end{array}$ & $\begin{array}{r}2,579.5 \\
1,715.3 \\
864.2\end{array}$ & $\begin{array}{r}2,705.8 \\
1,791.8 \\
914.0\end{array}$ & $\begin{array}{r}2,896.9 \\
1,924.9 \\
972.0\end{array}$ & $\begin{array}{r}3,104.7 \\
2,108.7 \\
996.0\end{array}$ & $\begin{array}{l}3,093.4 \\
2,074.3 \\
1,019.0\end{array}$ \\
\hline 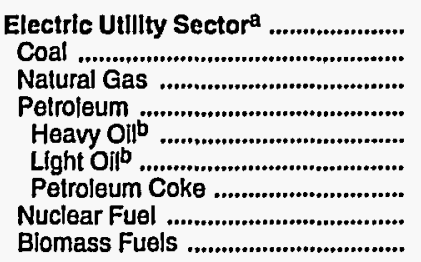 & $\begin{array}{r}22.3 \\
8.5 \\
12.8 \\
1.0 \\
.6 \\
.5 \\
- \\
- \\
-\end{array}$ & $\begin{array}{r}68.1 \\
23.4 \\
23.3 \\
10.5 \\
7.2 \\
3.3 \\
11 . \overline{0} \\
-\end{array}$ & $\begin{array}{r}164.7 \\
109.8 \\
20.5 \\
6.7 \\
3.6 \\
3.1 \\
-\overline{7} \\
27.7 \\
-\end{array}$ & $\begin{array}{r}158.7 \\
122.9 \\
4.4 \\
2.1 \\
2 . \overline{1} \\
29 . \overline{-} \\
-\end{array}$ & $\begin{array}{r}167.8 \\
107.7 \\
5.3 \\
1.9 \\
.8 \\
1.1 \\
-\overline{5} \\
52.8 \\
-\end{array}$ & $\begin{array}{r}169.4 \\
104.5 \\
4.4 \\
1.6 \\
.8 \\
.8 \\
-\overline{0} \\
59.0 \\
-\end{array}$ & $\begin{array}{r}166.5 \\
112.3 \\
5.2 \\
2.4 \\
1.1 \\
1.3 \\
46 . \overline{5} \\
-\end{array}$ & $\begin{array}{r}168.9 \\
104.7 \\
5.9 \\
2.1 \\
.8 \\
1.2 \\
-\overline{5} \\
56.3 \\
-\end{array}$ & $\begin{array}{r}161.1 \\
103.4 \\
7.2 \\
1.3 \\
1.3 \\
\overline{-} \\
49.3 \\
-\end{array}$ & $\begin{array}{r}169.5 \\
108.5 \\
6.8 \\
.8 \\
. \\
.7 \\
53.4 \\
-\end{array}$ \\
\hline Primary Energy - Five Sectors ${ }^{\circ} .$. & 518.6 & 998.7 & $2,065.3$ & $2,265.3$ & $1,825.8$ & $1,884.7$ & $1,958.3$ & $2,093.8$ & $2,269.8$ & $2,243.9$ \\
\hline
\end{tabular}

a There are no direct fuel costs for hydroelectric, geothermal, centralized solar, or wind energy.

b Heavy oil includes Grade Nos. 4,5, and 6, and residual tuel oils. Light oil includes Grade No. 2 heating oil, kerosene, and jet fuel.

c Blomass fuels are not included, except those consumed at electric utilities and those added to motor gasoline.

- No consumption, including cases where adjustments were made. See "Consumption Adjustments for Process Fuel and Intermediate Products."

- Value less than 0.05 million dollars.

Note: Totals may not equal sum of components due to independent rounding.

Sources: Data sources, estimation procedures, and assumptions are described in the "Documentation" section of this report. 
N Energy Price and Expenditure Estimates by Source, Nevada

E 1970,1975, 1980, and 1985-1991

\begin{tabular}{|c|c|c|c|c|c|c|c|c|c|c|}
\hline Energy Source & 1970 & 1975 & 1980 & 1985 & 1986 & 1987 & 1988 & 1989 & 1990 & 1991 \\
\hline & \multicolumn{10}{|c|}{ Prices in Dollars per Million Btu } \\
\hline Coal & 0.37 & 0.35 & 1.06 & 1.62 & 1.40 & 1.40 & 1.37 & 1.52 & 1.49 & 1.41 \\
\hline 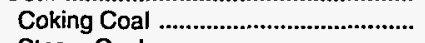 & - & - & - & - & - & - & - & - & - & - \\
\hline 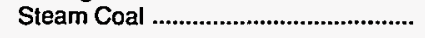 & .37 & .35 & 1.06 & 1.62 & 1.40 & 1.40 & 1.37 & 1.52 & 1.49 & 1.41 \\
\hline 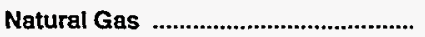 & .61 & 1.31 & 3.10 & 5.44 & 4.60 & 4.25 & 4.49 & 3.84 & 3.68 & 3.72 \\
\hline 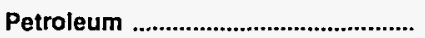 & 1.95 & 3.40 & 7.67 & 7.77 & 5.82 & 5.82 & 6.03 & 6.96 & 7.87 & 7.47 \\
\hline 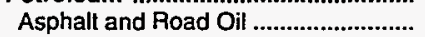 & .59 & 1.83 & 3.69 & 4.78 & 4.34 & 3.27 & 3.28 & 2.73 & 2.60 & 3.27 \\
\hline 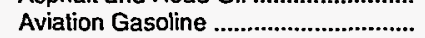 & 2.17 & 3.45 & 9.02 & 9.99 & 8.41 & 7.55 & 7.41 & 8.28 & 9.32 & 8.71 \\
\hline 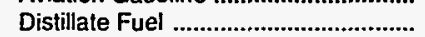 & 1.29 & 2.75 & 6.97 & 7.32 & 5.44 & 5.70 & 5.83 & 6.92 & 7.59 & 7.33 \\
\hline Jet Fuel & .76 & 2.12 & 6.59 & 6.22 & 4.25 & 4.27 & 4.08 & 4.79 & 6.26 & 5.00 \\
\hline 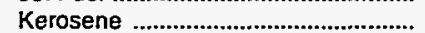 & .77 & 2.42 & - & 14.41 & 12.71 & 8.60 & 9.20 & 12.05 & 10.02 & 9.48 \\
\hline LPG & 2.91 & 4.03 & 7.07 & 11.46 & 10.85 & 10.13 & 10.07 & 12.60 & 11.36 & 12.14 \\
\hline 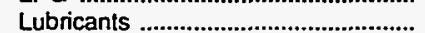 & 5.08 & 7.48 & 14.36 & 17.61 & 15.59 & 12.70 & 14.61 & 13.30 & 13.40 & 15.42 \\
\hline Motor Gasoline & 3.07 & 4.74 & 9.96 & 8.77 & 6.75 & 6.79 & 7.32 & 8.19 & 9.10 & 8.83 \\
\hline 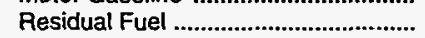 & .58 & 1.98 & 3.58 & 4.45 & 2.04 & 2.41 & 2.39 & 2.73 & 2.93 & 3.69 \\
\hline Other ${ }^{2}$ & .43 & - & 4.04 & 3.39 & - & - & - & - & - & - \\
\hline 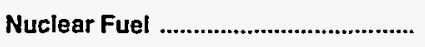 & - & - & - & - & - & - & - & - & - & - \\
\hline Blomass Fuels at Utllities ................. & - & - & - & - & - & - & - & - & - & - \\
\hline Primary Energy - Five Sectors ${ }^{b}$.... & 1.32 & 1.82 & 4.69 & 4.89 & 3.58 & 3.69 & 3.69 & 4.20 & 4.56 & 4.27 \\
\hline $\begin{array}{l}\text { Electric Utility Fuelc .......................... } \\
\text { Electricity Purchased by End Users }\end{array}$ & $\begin{array}{r}.36 \\
3.89\end{array}$ & $\begin{array}{r}.59 \\
6.86\end{array}$ & $\begin{array}{r}1.68 \\
13.18\end{array}$ & $\begin{array}{r}1.79 \\
16.75\end{array}$ & $\begin{array}{r}1.47 \\
17.16\end{array}$ & $\begin{array}{r}1.47 \\
15.45\end{array}$ & $\begin{array}{r}1.47 \\
15.93\end{array}$ & $\begin{array}{r}1.62 \\
15.46\end{array}$ & $\begin{array}{r}1.59 \\
15.77\end{array}$ & $\begin{array}{r}1.48 \\
16.47\end{array}$ \\
\hline \multirow[t]{2}{*}{ 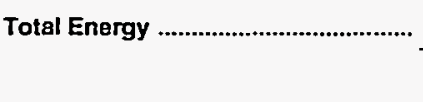 } & 1.96 & 3.52 & 8.18 & 9.03 & 7.81 & 7.37 & 7.73 & 8.24 & 8.99 & 8.79 \\
\hline & \multicolumn{10}{|c|}{ Expenditures in Millions of Dollars } \\
\hline 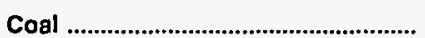 & 6.4 & 35.8 & 99.1 & 204.2 & 225.5 & 217.1 & 250.5 & 259.3 & 247.3 & 253.8 \\
\hline Coking Coal & - & - & - & - & - & - & - & - & - & - \\
\hline 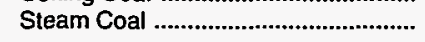 & 6.4 & 35.8 & 99.1 & 204.2 & 225.5 & 217.1 & 250.5 & 259.3 & 247.3 & 253.8 \\
\hline Natural Gas & 34.5 & 85.5 & 191.5 & 222.8 & 162.3 & 173.6 & 213.0 & 245.9 & 243.0 & 245.1 \\
\hline Petroleum ................................................ & 176.6 & 393.4 & $1,125.9$ & $1,055.0$ & 821.8 & 905.0 & $1,003.6$ & 1,212.7 & $1,349.6$ & $1,289.0$ \\
\hline 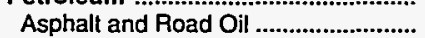 & 2.4 & 10.2 & 15.0 & 26.8 & 16.3 & 18.7 & 20.3 & 25.3 & 18.7 & 23.3 \\
\hline 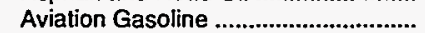 & 2.0 & 3.4 & 9.4 & 5.3 & 5.2 & 3.8 & 4.5 & 4.9 & 5.2 & 4.9 \\
\hline 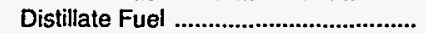 & 21.2 & 41.1 & 160.9 & 230.4 & 172.6 & 214.8 & 229.6 & 299.9 & 325.2 & 302.6 \\
\hline 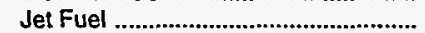 & 19.2 & 69.2 & 266.2 & 197.0 & 140.5 & 152.5 & 145.3 & 162.6 & 212.9 & 178.9 \\
\hline 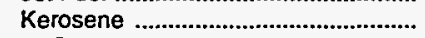 & .1 & .4 & - & 4.3 & 3.7 & 1.7 & 1.5 & 1.8 & 1.1 & 1.2 \\
\hline LPG & 9.2 & 7.4 & 22.9 & 40.6 & 33.5 & 32.1 & 36.8 & 72.2 & 58.9 & 46.6 \\
\hline 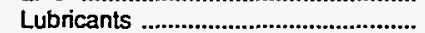 & 3.2 & 5.4 & 9.4 & 10.5 & 9.1 & 8.4 & 9.3 & 8.7 & 9.0 & 9.3 \\
\hline Motor Gasoline & 118.7 & 239.7 & 587.0 & 535.6 & 432.9 & 465.1 & 541.6 & 626.3 & 710.2 & 712.1 \\
\hline 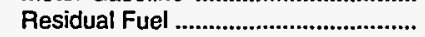 & .5 & 16.7 & 55.0 & 4.4 & 8.0 & 7.8 & 14.8 & 11.1 & 8.4 & 10.2 \\
\hline Othera & $\bullet$ & - & .1 & .1 & - & - & - & - & - & - \\
\hline 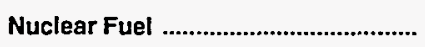 & - & - & - & - & - & - & - & - & - & - \\
\hline Btomass Fuels at Utilities ................. & - & - & - & - & - & - & - & - & - & - \\
\hline Primary Energy - Five Sectors ${ }^{b} \ldots$ & 217.5 & 514.7 & $1,416.5$ & $1,481.9$ & $1,209.6$ & $1,295.7$ & $1,467.1$ & $1,717.8$ & $1,839.9$ & $1,787.9$ \\
\hline $\begin{array}{l}\text { Electric Utility Fuelc .......................... } \\
\text { Electricity Purchased by End Users }\end{array}$ & $\begin{array}{r}-15.1 \\
75.7\end{array}$ & $\begin{array}{l}-79.8 \\
179.0\end{array}$ & $\begin{array}{r}-226.1 \\
468.2\end{array}$ & $\begin{array}{r}-238.1 \\
634.3\end{array}$ & $\begin{array}{r}-249.1 \\
655.8\end{array}$ & $\begin{array}{r}-239.1 \\
638.7\end{array}$ & $\begin{array}{r}-290.8 \\
717.3\end{array}$ & $\begin{array}{r}-315.8 \\
773.1\end{array}$ & $\begin{array}{r}-301.8 \\
879.9\end{array}$ & $\begin{array}{r}-296.7 \\
914.1\end{array}$ \\
\hline 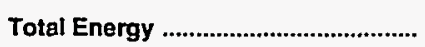 & 278.1 & 613.9 & $1,658.6$ & $1,878.1$ & $1,616.2$ & $1,695.4$ & $1,893.6$ & $2,175.1$ & $2,418.0$ & $2,405.3$ \\
\hline
\end{tabular}

a Includes petroleum coke used at electric utilities.

b Biomass fuels are not included, except those consumed at electric utilities and those added to motor gasoline.

c There are no direct fuel costs for hydroelectric, geothermal, centralized solar, or wind energy.

-No consumption, including cases where adjustments were made. See "Consumption Adjusiments for Process Fuel and Intermediate Products."

- Value less than 0.05 million dollars.

Note: Expenditure totals may not equal sum of components due to independent rounding.

Sources: Data sources, estimation procedures, and assumptions are described in the "Documentation" section of this report. 


\begin{tabular}{|c|c|c|c|c|c|c|c|c|c|c|}
\hline Sector and Energy Source & 1970 & 1975 & 1980 & 1985 & 1986 & 1987 & 1988 & 1989 & 1990 & 1991 \\
\hline 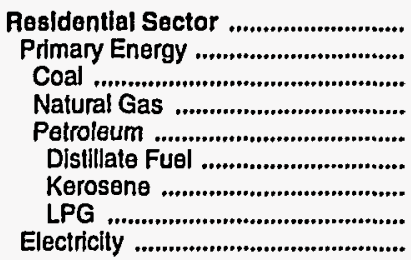 & $\begin{array}{r}2.70 \\
1.76 \\
1.31 \\
1.39 \\
2.51 \\
1.27 \\
3.52 \\
4.46\end{array}$ & $\begin{array}{r}4.30 \\
2.18 \\
1.55 \\
1.83 \\
3.72 \\
2.82 \\
- \\
4.90 \\
7.54\end{array}$ & $\begin{array}{r}8.74 \\
4.58 \\
5.13 \\
3.87 \\
8.31 \\
6.92 \\
- \\
9.28 \\
14.21\end{array}$ & $\begin{array}{r}12.59 \\
7.61 \\
4.54 \\
6.63 \\
10.68 \\
7.55 \\
15.04 \\
12.40 \\
18.83\end{array}$ & $\begin{array}{r}12.41 \\
6.63 \\
4.49 \\
5.62 \\
10.22 \\
7.26 \\
14.46 \\
11.84 \\
19.28\end{array}$ & $\begin{array}{r}11.09 \\
6.00 \\
4.11 \\
5.29 \\
8.59 \\
5.79 \\
11.54 \\
11.19 \\
17.05\end{array}$ & $\begin{array}{r}11.70 \\
6.53 \\
3.83 \\
5.89 \\
8.93 \\
6.02 \\
11.99 \\
10.97 \\
17.58\end{array}$ & $\begin{array}{r}11.32 \\
6.94 \\
4.14 \\
5.38 \\
12.70 \\
8.26 \\
16.46 \\
14.68 \\
16.78\end{array}$ & $\begin{array}{r}11.26 \\
6.61 \\
5.03 \\
5.49 \\
11.10 \\
6.76 \\
13.48 \\
13.10 \\
16.72\end{array}$ & $\begin{array}{r}11.32 \\
6.39 \\
4.07 \\
5.41 \\
11.21 \\
6.58 \\
13.11 \\
13.42 \\
17.28\end{array}$ \\
\hline 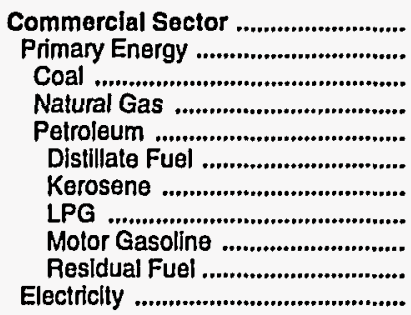 & $\begin{array}{r}2.15 \\
.77 \\
.52 \\
.70 \\
1.34 \\
1.12 \\
.77 \\
1.16 \\
3.07 \\
.62 \\
4.74\end{array}$ & $\begin{array}{r}3.88 \\
1.59 \\
.82 \\
1.45 \\
2.99 \\
2.62 \\
2.42 \\
2.45 \\
4.74 \\
2.00 \\
8.01\end{array}$ & $\begin{array}{r}7.73 \\
4.29 \\
1.36 \\
3.68 \\
6.78 \\
6.60 \\
- \\
4.99 \\
9.96 \\
3.53 \\
15.39\end{array}$ & $\begin{array}{r}11.26 \\
6.17 \\
1.58 \\
5.77 \\
8.02 \\
7.76 \\
9.06 \\
9.63 \\
8.77 \\
4.80 \\
18.24\end{array}$ & $\begin{array}{r}10.74 \\
4.89 \\
1.66 \\
4.63 \\
5.76 \\
5.32 \\
4.52 \\
9.06 \\
6.75 \\
2.30 \\
18.66\end{array}$ & $\begin{array}{r}9.36 \\
4.47 \\
1.53 \\
4.27 \\
5.03 \\
4.60 \\
4.65 \\
8.52 \\
6.79 \\
2.64 \\
16.59\end{array}$ & $\begin{array}{r}10.01 \\
4.84 \\
1.49 \\
4.64 \\
5.72 \\
5.09 \\
4.97 \\
8.58 \\
7.32 \\
2.32 \\
16.94\end{array}$ & $\begin{array}{r}10.10 \\
4.84 \\
1.62 \\
4.30 \\
7.48 \\
6.73 \\
6.59 \\
10.08 \\
8.19 \\
2.52 \\
16.85\end{array}$ & $\begin{array}{r}10.51 \\
4.77 \\
1.51 \\
4.25 \\
7.50 \\
6.79 \\
7.19 \\
9.03 \\
9.10 \\
2.85 \\
17.39\end{array}$ & $\begin{array}{r}10.44 \\
4.58 \\
1.51 \\
4.19 \\
7.26 \\
6.38 \\
6.46 \\
9.29 \\
8.83 \\
2.54 \\
17.88\end{array}$ \\
\hline 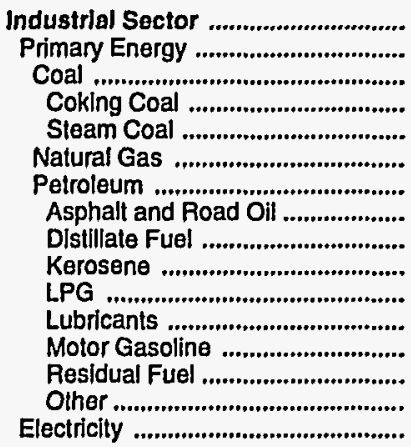 & $\begin{array}{r}1.02 \\
.75 \\
.52 \\
-52 \\
.52 \\
.52 \\
1.04 \\
.59 \\
.96 \\
.77 \\
1.16 \\
5.08 \\
3.07 \\
.49 \\
.43 \\
2.14\end{array}$ & $\begin{array}{r}2.18 \\
1.61 \\
.82 \\
. \overrightarrow{82} \\
1.06 \\
2.25 \\
1.83 \\
2.25 \\
2.42 \\
2.45 \\
7.48 \\
4.74 \\
1.83 \\
-\overrightarrow{4} \\
4.23\end{array}$ & $\begin{array}{r}7.20 \\
3.67 \\
1.36 \\
1 . \overline{6} \\
2.83 \\
5.10 \\
3.69 \\
5.56 \\
-\overline{0} \\
4.99 \\
14.36 \\
9.96 \\
3.75 \\
4.04 \\
11.63\end{array}$ & $\begin{array}{r}8.06 \\
5.72 \\
1.58 \\
-58 \\
1.58 \\
4.05 \\
6.97 \\
4.78 \\
7.97 \\
9.06 \\
9.63 \\
17.61 \\
8.77 \\
4.80 \\
3.39 \\
12.91\end{array}$ & $\begin{array}{r}7.56 \\
4.01 \\
1.66 \\
- \\
1.66 \\
3.76 \\
4.46 \\
4.34 \\
3.97 \\
4.52 \\
9.06 \\
15.59 \\
6.75 \\
2.30 \\
- \\
13.36\end{array}$ & $\begin{array}{r}6.75 \\
3.82 \\
1.53 \\
- \\
1.53 \\
3.72 \\
4.20 \\
3.27 \\
4.09 \\
4.65 \\
8.52 \\
12.70 \\
6.79 \\
2.64 \\
-\overline{12.46}\end{array}$ & $\begin{array}{r}6.81 \\
3.98 \\
1.49 \\
-\overline{1} \\
1.49 \\
3.84 \\
4.34 \\
3.28 \\
4.37 \\
4.97 \\
8.58 \\
14.61 \\
7.32 \\
2.32 \\
13.01\end{array}$ & $\begin{array}{r}7.28 \\
4.80 \\
1.62 \\
- \\
1.62 \\
4.83 \\
5.19 \\
2.73 \\
5.80 \\
6.59 \\
10.08 \\
13.30 \\
8.19 \\
2.52 \\
- \\
12.99\end{array}$ & $\begin{array}{r}7.98 \\
4.94 \\
1.51 \\
- \\
1.51 \\
3.98 \\
5.67 \\
2.60 \\
6.32 \\
7.19 \\
9.03 \\
13.40 \\
9.10 \\
2.85 \\
- \\
13.76\end{array}$ & $\begin{array}{r}8.04 \\
4.59 \\
1.51 \\
- \\
1.51 \\
4.06 \\
5.24 \\
3.27 \\
5.67 \\
6.46 \\
9.29 \\
15.42 \\
8.83 \\
2.54 \\
-\overline{145} \\
14.53\end{array}$ \\
\hline 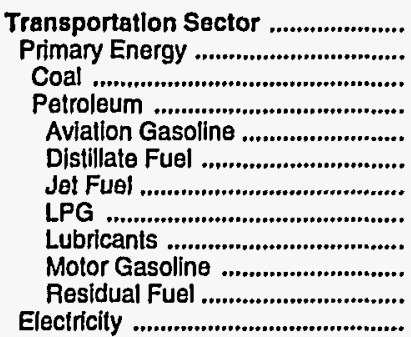 & $\begin{array}{r}2.08 \\
2.08 \\
.52 \\
2.08 \\
2.17 \\
1.50 \\
.76 \\
1.16 \\
5.08 \\
3.07 \\
.60 \\
-\end{array}$ & $\begin{array}{r}3.66 \\
3.66 \\
.82 \\
3.66 \\
3.45 \\
3.01 \\
2.12 \\
2.45 \\
7.48 \\
4.74 \\
2.36 \\
-\end{array}$ & $\begin{array}{r}8.44 \\
8.44 \\
- \\
8.44 \\
9.02 \\
7.36 \\
6.59 \\
4.99 \\
14.36 \\
9.96 \\
- \\
-\end{array}$ & $\begin{array}{r}7.79 \\
7.79 \\
- \\
7.79 \\
9.99 \\
6.97 \\
6.22 \\
9.63 \\
17.61 \\
8.77 \\
- \\
-\end{array}$ & $\begin{array}{r}5.96 \\
5.96 \\
- \\
5.96 \\
8.41 \\
6.01 \\
4.25 \\
9.06 \\
15.59 \\
6.75 \\
1.86 \\
-\end{array}$ & $\begin{array}{r}6.07 \\
6.07 \\
- \\
6.07 \\
7.55 \\
6.59 \\
4.27 \\
8.52 \\
12.70 \\
6.79 \\
- \\
-\end{array}$ & $\begin{array}{r}6.40 \\
6.40 \\
- \\
6.40 \\
7.41 \\
6.87 \\
4.08 \\
8.58 \\
14.61 \\
7.32 \\
- \\
-\end{array}$ & $\begin{array}{r}7.27 \\
7.27 \\
- \\
7.27 \\
8.28 \\
7.76 \\
4.79 \\
10.08 \\
13.30 \\
8.19 \\
- \\
-\end{array}$ & $\begin{array}{r}8.36 \\
8.36 \\
- \\
8.36 \\
9.32 \\
8.97 \\
6.26 \\
9.03 \\
13.40 \\
9.10 \\
- \\
-\end{array}$ & $\begin{array}{r}7.86 \\
7.86 \\
- \\
7.86 \\
8.71 \\
8.89 \\
5.00 \\
9.29 \\
15.42 \\
8.83 \\
- \\
-\end{array}$ \\
\hline $\begin{array}{l}\text { Total Energy .................................... } \\
\text { Primary Energy - Four Sectors ..... } \\
\text { Electricity ......................................... }\end{array}$ & $\begin{array}{l}1.96 \\
1.65 \\
3.89\end{array}$ & $\begin{array}{l}3.52 \\
2.93 \\
6.86\end{array}$ & $\begin{array}{r}8.18 \\
7.12 \\
13.18\end{array}$ & $\begin{array}{r}9.03 \\
7.31 \\
16.75\end{array}$ & $\begin{array}{r}7.81 \\
5.69 \\
17.16\end{array}$ & $\begin{array}{r}7.37 \\
5.60 \\
15.45\end{array}$ & $\begin{array}{r}7.73 \\
5.89 \\
15.93\end{array}$ & $\begin{array}{r}8.24 \\
6.55 \\
15.46\end{array}$ & $\begin{array}{r}8.99 \\
7.22 \\
15.77\end{array}$ & $\begin{array}{r}8.79 \\
6.84 \\
16.47\end{array}$ \\
\hline 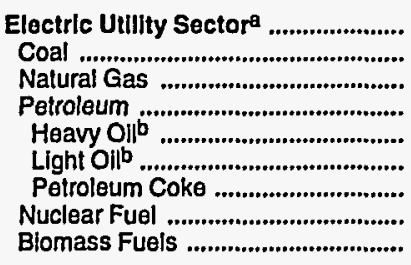 & $\begin{array}{l}.36 \\
.30 \\
.38 \\
.62 \\
.61 \\
.70 \\
- \\
- \\
-\end{array}$ & $\begin{array}{r}.59 \\
.34 \\
1.09 \\
2.00 \\
1.98 \\
2.47 \\
- \\
- \\
-\end{array}$ & $\begin{array}{r}1.68 \\
1.05 \\
2.59 \\
3.60 \\
3.58 \\
5.58 \\
- \\
- \\
-\end{array}$ & $\begin{array}{r}1.79 \\
1.62 \\
4.07 \\
4.91 \\
3.71 \\
6.12 \\
- \\
- \\
-\end{array}$ & $\begin{array}{r}1.47 \\
1.39 \\
3.04 \\
2.06 \\
1.97 \\
3.94 \\
- \\
- \\
-\end{array}$ & $\begin{array}{r}1.47 \\
1.40 \\
2.58 \\
2.51 \\
2.36 \\
4.41 \\
- \\
- \\
-\end{array}$ & $\begin{array}{r}1.47 \\
1.36 \\
2.72 \\
2.51 \\
2.40 \\
4.05 \\
- \\
- \\
-\end{array}$ & $\begin{array}{r}1.62 \\
1.52 \\
2.11 \\
2.97 \\
2.74 \\
5.19 \\
- \\
- \\
-\end{array}$ & $\begin{array}{r}1.59 \\
1.49 \\
1.96 \\
3.50 \\
2.93 \\
6.47 \\
- \\
- \\
-\end{array}$ & $\begin{array}{r}1.48 \\
1.41 \\
1.73 \\
4.05 \\
3.86 \\
5.20 \\
- \\
- \\
-\end{array}$ \\
\hline Prlmary Energy - Flve Sectors c .. & 1.32 & 1.82 & 4.69 & 4.89 & 3.58 & 3.69 & 3.69 & 4.20 & $\mathbf{4 . 5 6}$ & 4.27 \\
\hline
\end{tabular}

a There are no direct fuel costs for hydroelectric, geothermal, centralized solar, or wind energy.

b Heavy oil includes Grade Nos, 4, 5, and 6, and residual fuel oils. Light oil includes Grade No. 2 heating oil, kerosene, and jet fuel.

c Blomass fuels are not included, except those consumed at electric utilities and those added to motor gasoline.

- No consumption, including cases where adjustments were made. See "Consumption Adjustments for Process Fuel and Intermediate Products."

Sources: Data sources, estimation procedures, and assumptions are described in the "Documentation" section of this report. 


\section{N Energy Expenditure Estimates by Sector, Nevada \\ E $1970,1975,1980$, and 1985-1991 \\ V (Million Dollars)}

\section{A \\ A}

\begin{tabular}{|c|c|c|c|c|c|c|c|c|c|c|}
\hline Sector and Energy Source & 1970 & 1975 & 1980 & 1985 & 1986 & 1987 & 1988 & 1989 & 1990 & 1991 \\
\hline 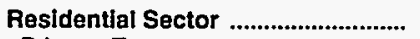 & 52.6 & 104.0 & 255.1 & 399.4 & 380.0 & 372.8 & 424.0 & 448.7 & 462.1 & 493.2 \\
\hline 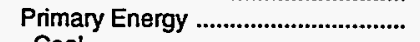 & 22.4 & 31.9 & 75.9 & 134.3 & 110.4 & 108.9 & 126.0 & 152.7 & 146.1 & 152.2 \\
\hline Coal & .7 & .1 & .2 & .1 & .1 & .1 & .1 & .1 & .2 & .1 \\
\hline 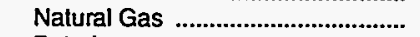 & 10.9 & 21.6 & 53.6 & 88.7 & 72.9 & 75.1 & 89.7 & 93.0 & 97.1 & 107.3 \\
\hline 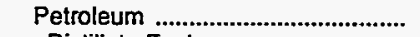 & 10.7 & 10.1 & 22.1 & 45.6 & 37.4 & 33.7 & 36.3 & 59.6 & 48.9 & 44.8 \\
\hline 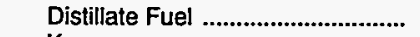 & 2.4 & 4.4 & 7.5 & 12.5 & 10.4 & 11.0 & 10.2 & 12.1 & 9.4 & 8.5 \\
\hline 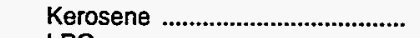 & - & $\overline{-}$ & - & 4.0 & 3.5 & 1.3 & 1.1 & 1.3 & .6 & .8 \\
\hline 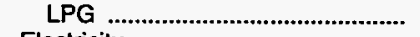 & 8.3 & 5.8 & 14.6 & 29.1 & 23.5 & 21.4 & 25.0 & 46.1 & 38.8 & 35.6 \\
\hline 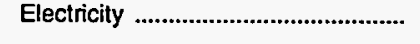 & 30.3 & 72.1 & 179.2 & 265.1 & 269.5 & 263.9 & 298.0 & 296.0 & 316.0 & 341.0 \\
\hline Commercial Sector ............................. & 43.7 & 106.6 & 151.1 & 310.4 & 297.9 & 296.0 & 322.1 & 338.3 & 358.8 & 377.7 \\
\hline 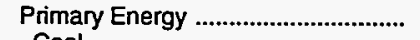 & 10.3 & 28.1 & 57.9 & 98.4 & 78.0 & 84.4 & 89.1 & 91.3 & 88.8 & 92.7 \\
\hline Coal & .5 & .1 & .1 & - & .1 & 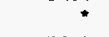 & $\because$ & 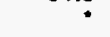 & .1 & 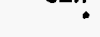 \\
\hline 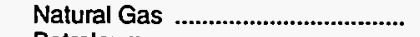 & 7.3 & 23.2 & 39.6 & 74.9 & 56.2 & 59.0 & 68.7 & 67.1 & 66.0 & 73.6 \\
\hline 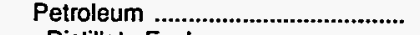 & 2.5 & 4.8 & 18.3 & 23.4 & 21.7 & 25.4 & 20.4 & 24.1 & 22.7 & 19.0 \\
\hline 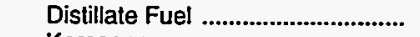 & 1.0 & 2.0 & 13.6 & 14.6 & 15.3 & 19.2 & 13.5 & 14.8 & 13.8 & 10.9 \\
\hline 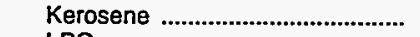 & $\dot{-}$ & .2 & - & .2 & .1 & .1 & .2 & .2 & .1 & .1 \\
\hline LPG & .5 & .5 & 1.4 & 4.0 & 3.2 & 2.9 & 3.4 & 5.6 & 4.7 & 4.3 \\
\hline 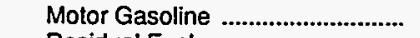 & .8 & 1.7 & 3.2 & 3.8 & 2.9 & 3.0 & 3.1 & 3.5 & 4.0 & 3.6 \\
\hline 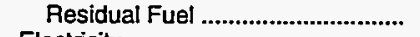 & .1 & .4 & .2 & .8 & .2 & .2 & .1 & 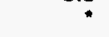 & 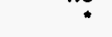 & $\bullet$ \\
\hline 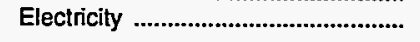 & 33.4 & 78.6 & 93.2 & 212.0 & 219.9 & 211.6 & 233.0 & 247.0 & 270.0 & 285.0 \\
\hline Industrial Sector .................................. & 29.7 & 66.4 & 273.4 & 300.9 & 247.9 & 261.0 & 311.3 & 425.6 & 494.6 & 459.4 \\
\hline 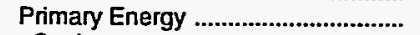 & 17.7 & 38.1 & 77.6 & 143.7 & 81.6 & 97.7 & 125.0 & 195.5 & 200.6 & 171.3 \\
\hline Coal & .9 & 1.5 & 4.6 & 4.1 & 4.1 & 4.0 & 4.2 & 6.1 & 5.9 & 6.9 \\
\hline 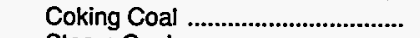 & - & - & - & - & - & - & - & - & - & - \\
\hline 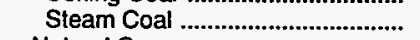 & .9 & 1.5 & 4.6 & 4.1 & 4.1 & 4.0 & 4.2 & 6.1 & 5.9 & 6.9 \\
\hline 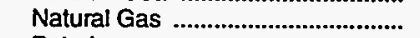 & 5.8 & 11.4 & 21.9 & 24.2 & 12.1 & 20.7 & 24.8 & 35.3 & 30.8 & 25.5 \\
\hline 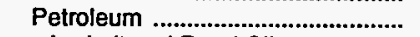 & 11.0 & 25.2 & 51.1 & 115.4 & 65.4 & 73.1 & 96.0 & 154.0 & 163.9 & 138.9 \\
\hline 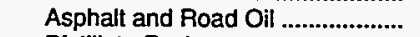 & 2.4 & 10.2 & 15.0 & 26.8 & 16.3 & 18.7 & 20.3 & 25.3 & 18.7 & 23.3 \\
\hline 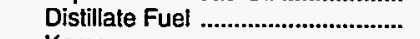 & 4.7 & 9.3 & 21.1 & 71.1 & 34.4 & 38.1 & 58.7 & 99.7 & 120.0 & 98.0 \\
\hline 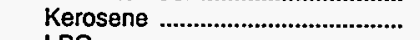 & $\bullet$ & .2 & - & $\bullet$ & .1 & .3 & .1 & .3 & .3 & .3 \\
\hline LPG & .4 & 1.0 & 6.9 & 6.5 & 6.0 & 7.3 & 7.7 & 19.8 & 14.6 & 6.0 \\
\hline 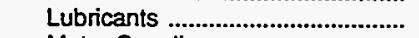 & .7 & 1.2 & 2.2 & 2.4 & 2.1 & 1.9 & 2.2 & 2.0 & 2.1 & 2.2 \\
\hline Motor Gasoline & 2.7 & 2.9 & 5.8 & 6.0 & 4.9 & 5.5 & 5.6 & 6.4 & 8.1 & 8.3 \\
\hline Residual Fuel .................................. & .1 & .5 & • & 2.5 & 1.6 & 1.3 & 1.5 & .7 & .1 & .9 \\
\hline 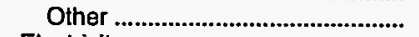 & • & - & .1 & .1 & - & - & - & - & - & - \\
\hline 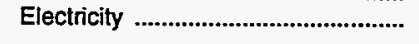 & 12.0 & 28.3 & 195.8 & 157.2 & 166.3 & 163.2 & 186.4 & 230.1 & 294.0 & 288.1 \\
\hline Transportation Sector ......................... & 152.1 & 336.9 & 978.9 & 867.5 & 690.5 & 765.7 & 836.1 & 962.6 & $1,102.6$ & $1,075.0$ \\
\hline 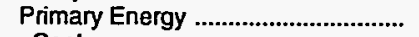 & 152.1 & 336.9 & 978.9 & 867.5 & 690.5 & 765.7 & 836.1 & 962.6 & $1,102.6$ & $1,075.0$ \\
\hline Coal & $\bullet$ & 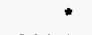 & - & - & - & - & - & - & - & - \\
\hline Petroleum & 152.1 & 336.9 & 978.9 & 867.5 & 690.5 & 765.7 & 836.1 & 962.6 & $1,102.6$ & $1,075.0$ \\
\hline Aviation Gasoline ........................... & 2.0 & 3.4 & 9.4 & 5.3 & 5.2 & 3.8 & 4.5 & 4.9 & 5.2 & 4.9 \\
\hline 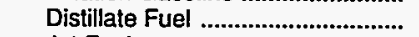 & 13.0 & 24.7 & 118.0 & 130.3 & 111.9 & 145.7 & 145.5 & 171.2 & 178.7 & 183.1 \\
\hline Jet Fuel & 19.2 & 69.2 & 266.2 & 197.0 & 140.5 & 152.5 & 145.3 & 162.6 & 212.9 & 178.9 \\
\hline LPG & $\cdot$ & .1 & .1 & 1.1 & .7 & .5 & .7 & .7 & .7 & .7 \\
\hline 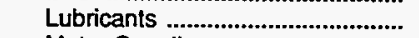 & 2.6 & 4.2 & 7.3 & 8.1 & 7.0 & 6.5 & 7.2 & 6.7 & 6.9 & 7.1 \\
\hline Motor Gasoline & 115.3 & 235.2 & 578.0 & 525.7 & 425.0 & 456.6 & 532.9 & 616.5 & 698.1 & 700.2 \\
\hline 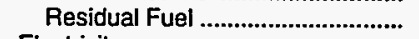 & 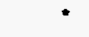 & .1 & - & - & $\bullet$ & - & - & - & - & - \\
\hline 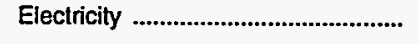 & - & - & - & - & - & - & - & - & - & - \\
\hline 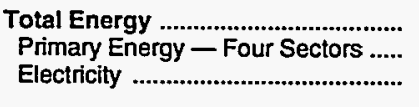 & $\begin{array}{r}278.1 \\
202.5 \\
75.7\end{array}$ & $\begin{array}{l}613.9 \\
434.9 \\
179.0\end{array}$ & $\begin{array}{r}1,658.6 \\
1,190.4 \\
468.2\end{array}$ & $\begin{array}{r}1,878.1 \\
1,243.9 \\
634.3\end{array}$ & $\begin{array}{r}1,616.2 \\
960.4 \\
655.8\end{array}$ & $\begin{array}{r}1,695.4 \\
1,056.6 \\
638.7\end{array}$ & $\begin{array}{r}1,893.6 \\
1,176.3 \\
717.3\end{array}$ & $\begin{array}{r}2,175.1 \\
1,402.0 \\
773.1\end{array}$ & $\begin{array}{r}2,418.0 \\
1,538.1 \\
879.9\end{array}$ & $\begin{array}{r}2,405.3 \\
1,491.2 \\
914.1\end{array}$ \\
\hline Electric Utility Sector a ...................... & 15.1 & 79.8 & 226.1 & 238.1 & 249.1 & 239.1 & 290.8 & 315.8 & 301.8 & 296.7 \\
\hline Coal & 4.3 & 34.1 & 94.2 & 199.9 & 221.2 & 213.0 & 246.2 & 253.0 & 241.1 & 246.8 \\
\hline 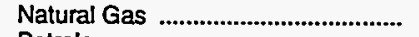 & 10.5 & 29.3 & 76.4 & 35.0 & 21.1 & 18.9 & 29.8 & 50.4 & 49.1 & 38.6 \\
\hline 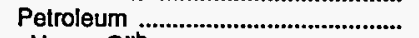 & .4 & 16.5 & 55.5 & 3.1 & 6.8 & 7.2 & 14.8 & 12.4 & 11.6 & 11.3 \\
\hline 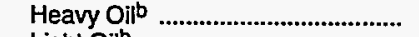 & .3 & 15.7 & 54.8 & 1.2 & 6.2 & 6.3 & 13.2 & 10.4 & 8.2 & 9.2 \\
\hline Light Oilb & .1 & .8 & .7 & 1.9 & .6 & .9 & 1.6 & 2.0 & 3.4 & 2.0 \\
\hline 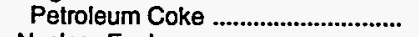 & - & - & - & - & - & - & - & - & - & - \\
\hline 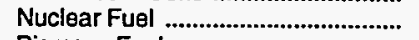 & - & - & - & - & - & - & - & - & - & - \\
\hline 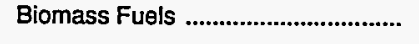 & - & - & - & - & - & - & - & - & - & - \\
\hline Primary Energy - Flve Sectorsc .. & 217.5 & 514.7 & $1,416.5$ & $1,481.9$ & $1,209.6$ & $1,295.7$ & $1,467.1$ & $1,717.8$ & $1,839.9$ & $1,787.9$ \\
\hline
\end{tabular}

There are no direct fuel costs for hydroelectric, geothermal, centralized solar, or wind energy.

beavy oil includes Grade Nos. 4, 5, and 6, and residual fuel oils. Light oil includes Grade No. 2 heating oil, kerosene, and jet fuel.

c Biomass fuels are not included, except those consumed at electric utilities and those added to motor gasoline.

- No consumption, including cases where adjustments were made. See "Consumption Adjustments for Process Fuel and Intermediate Products."

- Value less than 0.05 million dollars.

Note: Totals may not equal sum of components due to independent rounding.

Sources: Data sources, estimation procedures, and assumptions are described in the "Documentation" section of this report. 


\begin{tabular}{|c|c|c|c|c|c|c|c|c|c|c|}
\hline Energy Source & 1970 & 1975 & 1980 & 1985 & 1986 & 1987 & 1988 & 1989 & 1990 & 1991 \\
\hline & \multicolumn{10}{|c|}{ Prices in Dollars per Million Btu } \\
\hline 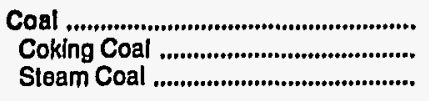 & $\begin{array}{r}0.37 \\
.37\end{array}$ & $\begin{array}{r}1.22 \\
1.22\end{array}$ & $\begin{array}{r}1.60 \\
1.60\end{array}$ & $\begin{array}{r}2.03 \\
2.03\end{array}$ & $\begin{array}{r}2.01 \\
2.01\end{array}$ & $\begin{array}{r}1.93 \\
1.93\end{array}$ & $\begin{array}{r}1.79 \\
1.79\end{array}$ & $\begin{array}{r}1.75 \\
1.75\end{array}$ & $\frac{1.82}{-82}$ & $\frac{1.81}{1.81}$ \\
\hline 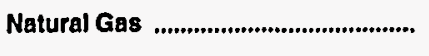 & 1.65 & 2.24 & 4.27 & 6.44 & 6.53 & 5.75 & 5.50 & 6.06 & 6.38 & 6.12 \\
\hline 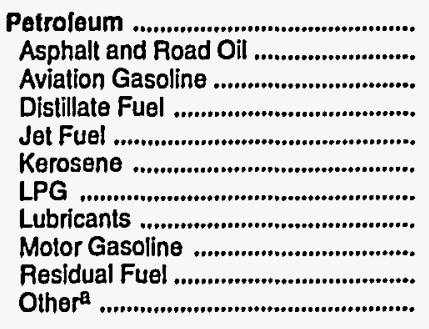 & $\begin{array}{r}1.60 \\
.70 \\
2.17 \\
1.38 \\
.75 \\
1.50 \\
2.06 \\
5.08 \\
2.92 \\
.42 \\
1.76\end{array}$ & $\begin{array}{l}3.24 \\
2.05 \\
3.45 \\
2.80 \\
2.10 \\
3.09 \\
3.53 \\
7.48 \\
4.54 \\
1.85 \\
4.01\end{array}$ & $\begin{array}{r}7.29 \\
4.00 \\
9.02 \\
6.97 \\
6.51 \\
8.05 \\
6.89 \\
14.36 \\
10.11 \\
3.82 \\
8.04\end{array}$ & $\begin{array}{r}7.78 \\
5.20 \\
9.99 \\
7.51 \\
6.53 \\
8.71 \\
11.42 \\
17.61 \\
9.26 \\
3.81 \\
10.67\end{array}$ & $\begin{array}{r}5.44 \\
4.86 \\
8.41 \\
5.52 \\
4.93 \\
6.34 \\
10.87 \\
15.59 \\
7.32 \\
2.02 \\
9.89\end{array}$ & $\begin{array}{r}5.92 \\
3.54 \\
7.55 \\
5.54 \\
4.18 \\
6.33 \\
10.68 \\
12.70 \\
7.60 \\
2.67 \\
10.07\end{array}$ & $\begin{array}{r}5.82 \\
3.34 \\
7.41 \\
5.66 \\
4.22 \\
6.48 \\
10.55 \\
14.61 \\
7.82 \\
1.94 \\
8.53\end{array}$ & $\begin{array}{r}6.43 \\
3.17 \\
8.28 \\
6.33 \\
4.83 \\
7.39 \\
10.32 \\
13.30 \\
8.60 \\
2.33 \\
12.52\end{array}$ & $\begin{array}{r}7.24 \\
3.34 \\
9.32 \\
7.30 \\
6.40 \\
8.56 \\
11.55 \\
13.40 \\
9.66 \\
2.43 \\
10.75\end{array}$ & $\begin{array}{r}7.30 \\
3.05 \\
8.71 \\
6.69 \\
5.36 \\
7.55 \\
12.82 \\
15.42 \\
9.55 \\
1.90 \\
11.29\end{array}$ \\
\hline Nuclear Fuel ......................................... & - & - & - & - & - & - & - & - & 1.03 & .84 \\
\hline Blomass Fuels at Utllttles ................. & - & - & - & - & - & - & - & - & - & - \\
\hline Primary Energy - Flve Sectors ${ }^{b}$.... & 1.41 & 2.88 & 6.16 & 6.41 & 5.05 & 5.30 & 5.17 & 5.72 & 5.37 & 4.74 \\
\hline $\begin{array}{l}\text { Electric Utility Fuelc ............................ } \\
\text { Electricity Purchased by End Users }\end{array}$ & $\begin{array}{r}.36 \\
6.74\end{array}$ & $\begin{array}{r}1.43 \\
12.68\end{array}$ & $\begin{array}{r}2.68 \\
19.55\end{array}$ & $\begin{array}{r}2.46 \\
23.28\end{array}$ & $\begin{array}{r}1.95 \\
22.14\end{array}$ & $\begin{array}{r}2.20 \\
23.25\end{array}$ & $\begin{array}{r}1.83 \\
24.24\end{array}$ & $\begin{array}{r}2.01 \\
24.49\end{array}$ & $\begin{array}{r}1.58 \\
26.67\end{array}$ & $\begin{array}{r}1.22 \\
26.76\end{array}$ \\
\hline \multirow[t]{2}{*}{ 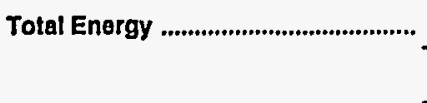 } & 2.20 & 4.38 & 9.59 & 10.68 & 8.89 & 9.08 & 9.63 & 10.17 & 11.28 & 11.08 \\
\hline & \multicolumn{10}{|c|}{ Expenditures in Millions of Dollars } \\
\hline 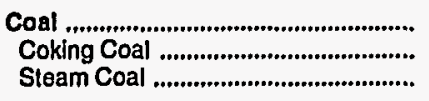 & $\begin{array}{r}10.1 \\
10 . \overline{1}\end{array}$ & $\begin{array}{r}31.9 \\
31.9\end{array}$ & $\begin{array}{r}46.9 \\
46 . \overline{9}\end{array}$ & $\begin{array}{r}80.5 \\
80 . \overline{5}\end{array}$ & $\begin{array}{r}50.4 \\
50 . \overline{4}\end{array}$ & $\begin{array}{r}60.9 \\
60 . \overline{9}\end{array}$ & $\begin{array}{r}58.7 \\
-\overline{7}\end{array}$ & $\begin{array}{r}55.3 \\
-\overrightarrow{3}\end{array}$ & $\begin{array}{r}57.2 \\
\overline{57.2}\end{array}$ & $\begin{array}{r}62.7 \\
-\overline{7}\end{array}$ \\
\hline Natural Gas ........................................... & 11.2 & 17.2 & 41.0 & 69.7 & 68.8 & 70.7 & 73.2 & 86.0 & 92.2 & 86.9 \\
\hline 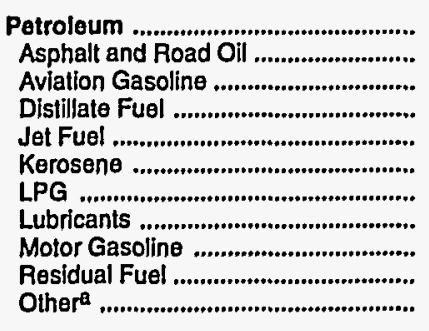 & $\begin{array}{r}225.2 \\
2.5 \\
.4 \\
61.9 \\
4.2 \\
6.6 \\
6.5 \\
2.2 \\
124.4 \\
14.7 \\
1.6\end{array}$ & $\begin{array}{r}441.9 \\
5.9 \\
.6 \\
116.9 \\
10.3 \\
8.1 \\
18.7 \\
3.2 \\
223.4 \\
53.2 \\
1.7\end{array}$ & $\begin{array}{r}971.1 \\
6.7 \\
1.8 \\
236.1 \\
27.3 \\
15.5 \\
31.3 \\
7.3 \\
498.1 \\
135.5 \\
11.6\end{array}$ & $\begin{array}{r}989.6 \\
29.5 \\
1.2 \\
229.4 \\
18.4 \\
44.5 \\
65.3 \\
8.1 \\
502.8 \\
82.4 \\
8.1\end{array}$ & $\begin{array}{r}834.0 \\
17.8 \\
1.6 \\
185.7 \\
16.5 \\
13.7 \\
66.5 \\
7.0 \\
427.7 \\
90.1 \\
7.3\end{array}$ & $\begin{array}{r}952.6 \\
18.3 \\
1.1 \\
243.5 \\
14.6 \\
16.7 \\
80.3 \\
6.5 \\
471.6 \\
92.4 \\
7.5\end{array}$ & $\begin{array}{r}948.5 \\
9.5 \\
1.4 \\
224.4 \\
16.6 \\
18.1 \\
80.3 \\
7.2 \\
506.7 \\
77.6 \\
6.7\end{array}$ & $\begin{array}{r}1,093.7 \\
15.6 \\
1.4 \\
278.6 \\
20.0 \\
22.5 \\
93.8 \\
6.7 \\
554.9 \\
90.5 \\
9.7\end{array}$ & $\begin{array}{r}1,111.5 \\
26.6 \\
1.0 \\
269.2 \\
22.7 \\
12.9 \\
88.9 \\
6.9 \\
594.2 \\
80.3 \\
8.8\end{array}$ & $\begin{array}{r}1,044.7 \\
13.3 \\
1.1 \\
247.6 \\
18.9 \\
13.8 \\
76.5 \\
7.1 \\
608.8 \\
48.0 \\
9.6\end{array}$ \\
\hline Nuclear Fuel ............................................ & - & - & - & - & - & - & - & - & 45.0 & 61.4 \\
\hline Blomass Fuels at Utilltles .................. & - & - & - & - & - & - & - & - & - & - \\
\hline Prlmary Energy - Five Sectors ${ }^{b}$.... & 246.4 & 491.0 & $1,059.1$ & $1,139.8$ & 953.2 & $1,084.2$ & $1,080.4$ & $1,234.9$ & $1,305.9$ & $1,255.8$ \\
\hline $\begin{array}{l}\text { Electric Utility Fuelc } \\
\text { Electricity Purchased by End Users }\end{array}$ & $\begin{array}{r}-15.6 \\
83.5\end{array}$ & $\begin{array}{r}-58.2 \\
207.7\end{array}$ & $\begin{array}{r}-150.9 \\
394.5\end{array}$ & $\begin{array}{r}-131.5 \\
588.4\end{array}$ & $\begin{array}{r}-104.3 \\
594.8\end{array}$ & $\begin{array}{r}-118.2 \\
664.3\end{array}$ & $\begin{array}{r}-115.5 \\
731.9\end{array}$ & $\begin{array}{r}-127.3 \\
759.1\end{array}$ & $\begin{array}{r}-156.9 \\
817.1\end{array}$ & $\begin{array}{r}-149.3 \\
800.0\end{array}$ \\
\hline Total Enargy ........................................ & 314.3 & 640.5 & $1,302.6$ & $1,596.7$ & $1,443.7$ & $1,630.4$ & $1,696.9$ & $1,866.7$ & $1,966.1$ & $1,906.4$ \\
\hline
\end{tabular}

ancludes petroleum coke used at electric utilities.

b Biomass fuels are not included, except those consumed at electric utilities and those added to motor gasoline.

c There are no direct fuel costs for hydroelectric, geothermal, centralized solar, or wind energy.

-No consumption, including cases where adjustments were made. See "Consumption Adjustments for Process Fuel and Intermediate Products."

Note: Expenditure totals may not equal sum of components due to independent rounding.

Sources: Data sources, estimation procedures, and assumptions are described in the "Documentation" section of this report. 


\section{N Energy Price Estimates by Sector, New Hampshire \\ E 1970, 1975, 1980, and 1985-1991}

W (Dollars per Million Btu)

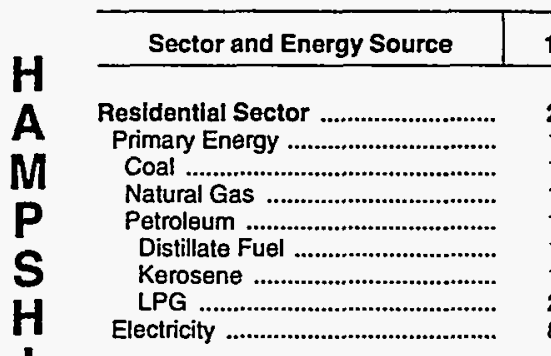

Commercial Sector ............... 3.12

Coal En

Natural Gas

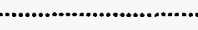

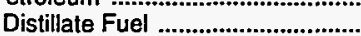

Kerosene

LPG

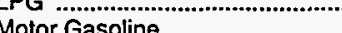

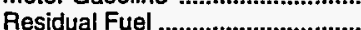

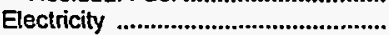

Industrial Sector

Primary Energy

Coal

Coking coal

Steam Coal

(n)..........................

Petroleum .

Asphalt and Road Oil .....................

Distillate Fuel ....................................

Kerosene

LPG

Lubricants

Residuasoline

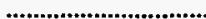

Other

Electricity ..........................................

Transportation Sector ..................... 2.60

Primary Energy ...................................

Coal .

Petroleum ........................................

Aviation Gasoline

Distillate Fuel

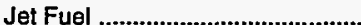

LPG

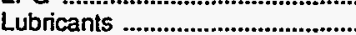

Motor Gasoline

Residual Fuel

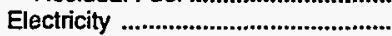

Total Energy ....................................

Primary Energy - Four Sectors .....

Electricity ...........................................

Electric UtIllty Sectora

Coal .

Natural Gas

Petroleum

Heavy Oib

Light Oib

Petroleum Coke

Nuclear Fuel

Biomass Fuels

1.23

.95
1.42

1.14

1.11

.74
1.37

2.92

2.92
.34

8.80

1.20
.67
.95

Primary Energy - Five Sectorsc ..

\section{1.7}

1.77
6.74

1975

\begin{tabular}{l|l|}
1980 & \\
\hline
\end{tabular}

\begin{tabular}{l|r}
1985 & 1986 \\
\hline
\end{tabular}

1987

1988

1989

$\begin{array}{rr}4.65 & 10.17 \\ 2.98 & 7.03 \\ 2.62 & 3.90 \\ 2.62 & 4.57 \\ 3.01 & 7.48 \\ 2.87 & 7.24 \\ 3.16 & 8.15 \\ 4.70 & 9.22 \\ 14.25 & 20.93\end{array}$

12.17

11.22

7.89
4.39

6.96

8.07

7.38

8.81

11.14

26.15

6.18

6.18
2.38

2.65

2.10

2.54

2.46

2.54

2.43

4.54

1.85

15.39

\subsection{6}

5.36

1.69

4.05

5.95

6.44
6.27

4.78

10.11

3.76

24.30

\section{$\begin{array}{ll}3.55 & 8.68\end{array}$}

3.55

2.06
2.65
-

2.65

1.44

2.08

2.05

2.29

2.54

2.43

7.48

4.54

1.85

$\begin{array}{rr}9.42 & 8.04 \\ & 15.82\end{array}$

$4.27 \quad 9.62$

$4.27 \quad 9.62$

2.65

4.27

3.45

2.90

2.09

2.43

7.48

4.54

1.90

$9 . \overline{62}$

9.02

7.38

6.51

4.78
14.36

10.11

3.18

4.38

4.38
3.34

3.34
12.68

9.59

9.59
7.85

19.55

13.16

6.69

2.40

7.31

6.71

6.86

11.75

9.26

4.20

25.55

6.40

4.93
6.88

6.88
6.30

6.30
5.47

6.53

10.35
25.02

\subsection{8}

4.94

2.13

4.33

4.29

3.91

11.70

7.32

2.23
24.03

$10.47 \quad 8.63$

5.87

2.40

$2 . \overline{0}$

5.41

6.09

5.20

5.99

6.86

11.75

17.61
9.26

4.20

10.7

10.67
19.32

4.01

2.13

$2 . \overline{13}$

5.04

3.98

4.86

3.41

3.91

11.70

15.59
7.32

23

98

9.89
18.22

$9.16 \quad 7.20$

9.16

7.20

$9 . \overline{6}$

9.99

7.20

8.41

7.11

4.93

6.53

11.75

17.61

11.70
15.59

7.32

1.32

-

1.97

$10.68 \quad 8.89$

8.11

23.28

6.26

22.14

$2.68 \quad 2.46$

$\begin{array}{ll}1.43 & 2.68 \\ 1.21 & 1.60\end{array}$

$2.46 \quad 1.95$

1.95

$11.16 \quad 11$

6.32
4.04

6.32

6.33

5.52

6.58

10.37

26.68

10.34

5.04

2.39

5.65

4.84

4.51

4.76
11.14

7.60

2.86

25.01

\subsection{9}

4.47

2.39

$2 . \overline{39}$

4.22

4.50

3.54

4.15

4.76

11.14

12.70
7.60

2.86

2.86

18.71

11.81
6.46

6.46
4.08

6.13

6.54

5.64

6.73

10.37

27.75

11.95

7.14

4.09
6.72

6.72
7.23

6.44

7.68

10.37
27.97

10.97 
(Million Dollars)

\begin{tabular}{|c|c|c|c|c|c|c|c|c|c|c|}
\hline Sector and Energy Source & 1970 & 1975 & 1980 & 1985 & 1986 & 1987 & 1988 & 1989 & 1990 & 1991 \\
\hline 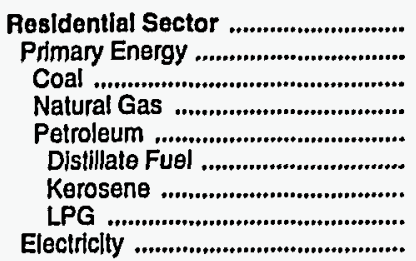 & $\begin{array}{r}113.1 \\
71.3 \\
.1 \\
7.3 \\
63.9 \\
53.0 \\
6.3 \\
4.6 \\
41.8\end{array}$ & $\begin{array}{r}229.3 \\
124.9 \\
.1 \\
9.9 \\
114.8 \\
95.5 \\
7.3 \\
12.1 \\
104.5\end{array}$ & \begin{tabular}{r|}
380.5 \\
203.6 \\
.2 \\
20.2 \\
183.2 \\
148.4 \\
14.9 \\
19.9 \\
177.0
\end{tabular} & $\begin{array}{r}504.9 \\
250.5 \\
.5 \\
33.6 \\
216.4 \\
139.4 \\
42.7 \\
34.3 \\
254.4\end{array}$ & $\begin{array}{r}454.7 \\
192.2 \\
.9 \\
36.1 \\
155.3 \\
103.3 \\
13.1 \\
38.9 \\
262.5\end{array}$ & $\begin{array}{r}522.2 \\
225.3 \\
.6 \\
36.4 \\
188.2 \\
126.7 \\
15.0 \\
46.5 \\
296.9\end{array}$ & $\begin{array}{r}555.1 \\
227.2 \\
.7 \\
37.2 \\
189.3 \\
121.3 \\
16.7 \\
51.3 \\
328.0\end{array}$ & $\begin{array}{r}625.3 \\
287.2 \\
.5 \\
43.1 \\
243.6 \\
161.6 \\
20.4 \\
61.6 \\
338.0\end{array}$ & $\begin{array}{r}621.3 \\
265.3 \\
.7 \\
43.7 \\
220.8 \\
146.6 \\
11.7 \\
62.5 \\
356.0\end{array}$ & $\begin{array}{r}596.9 \\
248.9 \\
1.4 \\
40.0 \\
207.5 \\
137.2 \\
12.0 \\
58.2 \\
348.0\end{array}$ \\
\hline 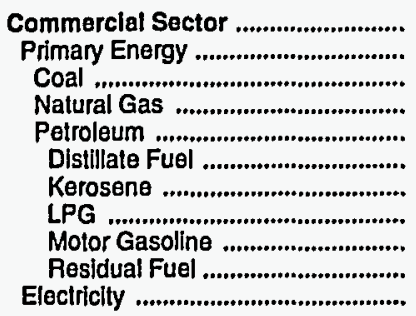 & $\begin{array}{r}29.8 \\
8.8 \\
.1 \\
3.2 \\
5.5 \\
4.1 \\
.1 \\
.4 \\
.7 \\
.2 \\
21.0\end{array}$ & $\begin{array}{r}63.8 \\
17.4 \\
.1 \\
5.5 \\
11.7 \\
8.5 \\
.2 \\
1.1 \\
1.2 \\
.7 \\
46.4\end{array}$ & $\begin{array}{r}165.4 \\
73.3 \\
17.0 \\
56.3 \\
39.2 \\
.3 \\
1.8 \\
6.2 \\
8.8 \\
92.0\end{array}$ & $\begin{array}{r}207.2 \\
69.3 \\
.2 \\
31.2 \\
37.9 \\
21.5 \\
1.6 \\
6.4 \\
6.1 \\
2.3 \\
137.9\end{array}$ & $\begin{array}{r}213.7 \\
72.8 \\
.3 \\
29.0 \\
43.6 \\
22.4 \\
.4 \\
7.8 \\
5.6 \\
7.3 \\
140.9\end{array}$ & $\begin{array}{r}254.0 \\
91.0 \\
.3 \\
26.7 \\
64.0 \\
44.0 \\
1.0 \\
8.8 \\
5.1 \\
5.1 \\
163.0\end{array}$ & $\begin{array}{r}262.3 \\
82.3 \\
.3 \\
28.5 \\
53.5 \\
30.2 \\
1.1 \\
9.5 \\
5.8 \\
6.8 \\
180.0\end{array}$ & $\begin{array}{r}284.4 \\
94.3 \\
.2 \\
33.5 \\
60.6 \\
34.6 \\
1.6 \\
10.7 \\
5.8 \\
7.9 \\
190.0\end{array}$ & $\begin{array}{r}306.1 \\
101.1 \\
.3 \\
34.1 \\
66.6 \\
39.3 \\
.9 \\
10.0 \\
3.7 \\
12.6 \\
205.0\end{array}$ & $\begin{array}{r}300.7 \\
91.7 \\
.6 \\
31.9 \\
59.1 \\
36.7 \\
.7 \\
9.4 \\
2.8 \\
9.6 \\
209.0\end{array}$ \\
\hline 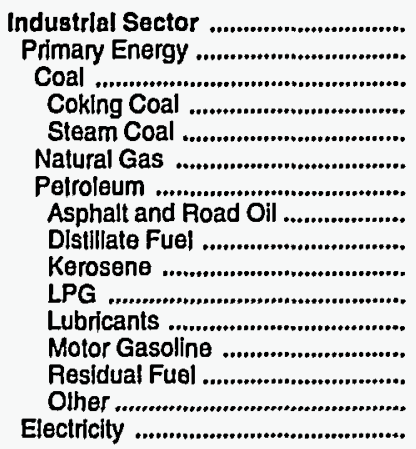 & $\begin{array}{r}39.6 \\
18.8 \\
.2 \\
- \\
.2 \\
.7 \\
18.0 \\
2.5 \\
2.0 \\
.2 \\
1.4 \\
.5 \\
.6 \\
9.1 \\
1.6 \\
20.7\end{array}$ & $\begin{array}{r}105.9 \\
49.0 \\
.4 \\
- \\
.4 \\
1.6 \\
47.0 \\
5.9 \\
5.7 \\
.6 \\
5.5 \\
1.0 \\
.7 \\
26.1 \\
1.7 \\
56.9\end{array}$ & $\begin{array}{r}200.4 \\
75.0 \\
.4 \\
.4 \\
.4 \\
3.9 \\
70.7 \\
6.7 \\
18.6 \\
.3 \\
8.3 \\
2.0 \\
1.4 \\
21.7 \\
11.6 \\
125.5\end{array}$ & $\begin{array}{r}310.4 \\
114.3 \\
2.4 \\
2 . \overline{4} \\
5.0 \\
107.0 \\
29.5 \\
13.4 \\
.2 \\
23.5 \\
2.3 \\
3.0 \\
27.0 \\
8.1 \\
196.1\end{array}$ & $\begin{array}{r}278.8 \\
87.4 \\
.2 \\
- \\
.2 \\
3.8 \\
83.4 \\
17.8 \\
6.8 \\
.2 \\
19.1 \\
2.0 \\
2.6 \\
27.7 \\
7.3 \\
191.4\end{array}$ & $\begin{array}{r}306.1 \\
101.7 \\
.2 \\
-5 \\
.2 \\
7.5 \\
94.0 \\
18.3 \\
12.9 \\
.7 \\
24.2 \\
1.8 \\
2.6 \\
25.9 \\
7.5 \\
204.4\end{array}$ & $\begin{array}{r}295.6 \\
71.6 \\
.1 \\
-\overline{1} \\
.1 .3 \\
64.2 \\
9.5 \\
11.3 \\
.3 \\
18.9 \\
2.0 \\
2.8 \\
12.7 \\
6.7 \\
224.0\end{array}$ & $\begin{array}{r}318.9 \\
87.9 \\
1.0 \\
1.0 \\
9.4 \\
77.6 \\
15.6 \\
14.7 \\
.4 \\
21.0 \\
1.9 \\
4.1 \\
10.1 \\
9.7 \\
231.0\end{array}$ & $\begin{array}{r}352.8 \\
96.8 \\
1.8 \\
-\overline{1} \\
1.8 \\
14.3 \\
80.7 \\
26.6 \\
14.4 \\
.3 \\
15.7 \\
1.9 \\
2.8 \\
10.2 \\
8.8 \\
256.0\end{array}$ & $\begin{array}{r}318.1 \\
75.1 \\
3.4 \\
- \\
3.4 \\
14.9 \\
56.8 \\
13.3 \\
13.3 \\
1.0 \\
8.5 \\
2.0 \\
2.5 \\
6.5 \\
9.6 \\
243.0\end{array}$ \\
\hline 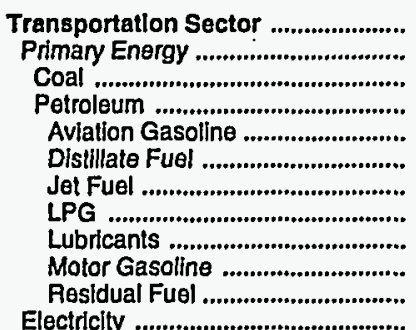 & $\begin{array}{r}131.9 \\
131.9 \\
131.9 \\
.4 \\
2.4 \\
4.2 \\
1.7 \\
123.1 \\
\end{array}$ & $\begin{array}{r}241.5 \\
241.5 \\
241.5 \\
.6 \\
7.1 \\
10.2 \\
2.2 \\
221.4 \\
.1 \\
-\end{array}$ & $\begin{array}{r}556.4 \\
556.4 \\
- \\
556.4 \\
1.8 \\
29.5 \\
27.0 \\
1.3 \\
5.2 \\
490.5 \\
1.0\end{array}$ & $\begin{array}{r}574.2 \\
574.2 \\
574 . \overline{2} \\
1.2 \\
54.1 \\
18.4 \\
1.0 \\
5.8 \\
493.6 \\
-\end{array}$ & $\begin{array}{r}496.6 \\
496.6 \\
-\overline{6} \\
496.6 \\
1.6 \\
52.6 \\
16.5 \\
.7 \\
5.1 \\
419.5 \\
.6\end{array}$ & $\begin{array}{r}548.1 \\
548.1 \\
- \\
548.1 \\
1.1 \\
59.3 \\
14.6 \\
.8 \\
4.7 \\
463.9 \\
3.8 \\
-\end{array}$ & $\begin{array}{r}583.9 \\
583.9 \\
- \\
583.9 \\
1.4 \\
60.3 \\
16.6 \\
.6 \\
5.2 \\
498.1 \\
1.7 \\
-\end{array}$ & $\begin{array}{r}638.1 \\
638.1 \\
- \\
638.1 \\
1.4 \\
66.2 \\
20.0 \\
.5 \\
4.8 \\
545.0 \\
.3 \\
-\end{array}$ & $\begin{array}{r}685.9 \\
685.9 \\
- \\
685.9 \\
1.0 \\
67.7 \\
22.7 \\
.6 \\
5.0 \\
587.7 \\
1.2 \\
-\end{array}$ & $\begin{array}{r}690.8 \\
690.8 \\
- \\
690.8 \\
1.1 \\
59.4 \\
18.9 \\
.4 \\
5.1 \\
603.5 \\
2.3 \\
-\end{array}$ \\
\hline $\begin{array}{l}\text { Total Energy .................................... } \\
\text { Primary Energy - Four Sectors ..... } \\
\text { Electriclty .......................................... }\end{array}$ & $\begin{array}{r}314.3 \\
230.9 \\
83.5\end{array}$ & $\begin{array}{l}640.5 \\
432.8 \\
207.7\end{array}$ & $\begin{array}{r}1,302.6 \\
908.2 \\
394.5\end{array}$ & $\begin{array}{r}1,596.7 \\
1,008.3 \\
588.4\end{array}$ & $\begin{array}{r}1,443.7 \\
848.9 \\
594.8\end{array}$ & $\begin{array}{r}1,630.4 \\
966.0 \\
664.3\end{array}$ & $\begin{array}{r}1,696.9 \\
964.9 \\
731.9\end{array}$ & $\begin{array}{r}1,866.7 \\
1,107.7 \\
759.1\end{array}$ & $\begin{array}{r}1,966.1 \\
1,149.0 \\
817.1\end{array}$ & $\begin{array}{r}1,906.4 \\
1,106.5 \\
800.0\end{array}$ \\
\hline 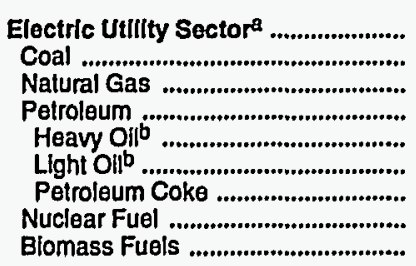 & $\begin{array}{r}15.6 \\
9.7 \\
-\overline{5.9} \\
5.5 \\
.4 \\
- \\
-\end{array}$ & $\begin{array}{r}58.2 \\
31.3 \\
.2 \\
26.7 \\
26.4 \\
.3 \\
- \\
- \\
-\end{array}$ & $\begin{array}{r}150.9 \\
46.3 \\
-\overline{6} \\
104.6 \\
104.0 \\
.7 \\
- \\
- \\
-\end{array}$ & $\begin{array}{r}131.5 \\
77.4 \\
54 . \overline{1} \\
53.0 \\
1.1 \\
- \\
-\end{array}$ & $\begin{array}{r}104.3 \\
49.1 \\
- \\
55.2 \\
54.5 \\
.7 \\
- \\
- \\
-\end{array}$ & $\begin{array}{r}118.2 \\
59.9 \\
58.3 \\
57.6 \\
.7 \\
- \\
- \\
-\end{array}$ & $\begin{array}{r}115.5 \\
57.7 \\
.1 \\
57.7 \\
56.3 \\
1.3 \\
- \\
- \\
-\end{array}$ & $\begin{array}{r}127.3 \\
53.5 \\
.1 \\
73.7 \\
72.2 \\
1.5 \\
- \\
- \\
-\end{array}$ & $\begin{array}{r}156.9 \\
54.3 \\
- \\
57.5 \\
56.3 \\
1.2 \\
- \\
45.0 \\
-\end{array}$ & $\begin{array}{r}149.3 \\
57.4 \\
- \\
30.5 \\
29.5 \\
1.0 \\
- \\
61.4 \\
-\end{array}$ \\
\hline Primary Energy - Flve Sectors ${ }^{c}$.. & 246.4 & 491.0 & $1,059.1$ & $1,139.8$ & 953.2 & $1,084.2$ & $1,080.4$ & $1,234.9$ & $1,305.9$ & $1,255.8$ \\
\hline
\end{tabular}

a There are no direct fuel costs for hydroelectric, geothermal, centralized solar, or wind energy.

b Heavy oll Includes Grade Nos. 4, 5, and 6, and residual fuel oils. Light oil includes Grade No. 2 heating oil, kerosene, and jet fuel.

c Blomass fuels are not included, except those consumed at electric utitities and those added to motor gasoline.

-No consumption, including cases where adjustments were made. See "Consumption Adjustments for Process Fuel and Intermediate Products."

- Value less than 0.05 million dollars.

Note: Totals may not equal sum of components due to independent rounding.

Sources: Data sources, estimation procedures, and assumptions are described in the "Documentation" section of this report. 
N Energy Price and Expenditure Estimates by Source, New Jersey

E $1970,1975,1980$, and 1985-1991

\begin{tabular}{|c|c|c|c|c|c|c|c|c|c|c|}
\hline Energy Source & 1970 & 1975 & 1980 & 1985 & 1986 & 1987 & 1988 & 1989 & 1990 & 1991 \\
\hline & \multicolumn{10}{|c|}{ Prices in Dollars per Million Btu } \\
\hline 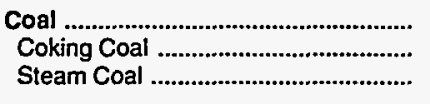 & $\begin{array}{r}0.45 \\
.58 \\
.44\end{array}$ & $\begin{array}{r}1.58 \\
1.58\end{array}$ & $\begin{array}{r}1.81 \\
1.81\end{array}$ & $\frac{1.92}{1.92}$ & $\frac{1.80}{1.80}$ & $\begin{array}{r}1.76 \\
- \\
1.76\end{array}$ & $\begin{array}{r}1.71 \\
1.71\end{array}$ & $\begin{array}{r}1.75 \\
1.75\end{array}$ & $\begin{array}{r}1.79 \\
1.79\end{array}$ & $\begin{array}{r}1.77 \\
1.77\end{array}$ \\
\hline 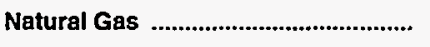 & 1.28 & 2.29 & 4.15 & 6.18 & 5.86 & 5.04 & 4.97 & 5.11 & 5.05 & 4.91 \\
\hline 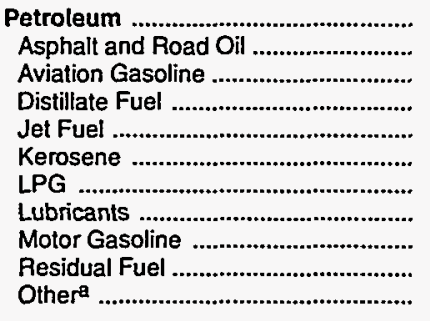 & $\begin{array}{r}1.42 \\
.68 \\
2.17 \\
1.29 \\
.72 \\
1.18 \\
1.63 \\
5.08 \\
2.99 \\
.45 \\
1.10\end{array}$ & $\begin{array}{l}3.27 \\
1.89 \\
3.45 \\
2.73 \\
2.03 \\
2.86 \\
3.59 \\
7.48 \\
4.79 \\
2.08 \\
2.70\end{array}$ & $\begin{array}{r}7.17 \\
3.65 \\
9.02 \\
6.75 \\
6.26 \\
6.35 \\
5.72 \\
14.36 \\
9.94 \\
4.53 \\
7.07\end{array}$ & $\begin{array}{r}7.48 \\
4.89 \\
9.99 \\
7.92 \\
5.76 \\
8.91 \\
11.86 \\
17.61 \\
8.95 \\
4.35 \\
7.86\end{array}$ & $\begin{array}{r}5.47 \\
4.16 \\
8.41 \\
6.23 \\
3.84 \\
6.74 \\
11.50 \\
15.59 \\
6.89 \\
2.34 \\
4.56\end{array}$ & $\begin{array}{r}5.75 \\
3.39 \\
7.55 \\
6.05 \\
4.01 \\
6.12 \\
11.03 \\
12.70 \\
7.25 \\
2.99 \\
5.31\end{array}$ & $\begin{array}{r}5.73 \\
3.07 \\
7.41 \\
6.00 \\
3.79 \\
5.79 \\
10.64 \\
14.61 \\
7.58 \\
2.40 \\
4.44\end{array}$ & $\begin{array}{r}6.28 \\
2.71 \\
8.28 \\
6.49 \\
4.34 \\
6.58 \\
9.41 \\
13.30 \\
8.48 \\
2.83 \\
5.15\end{array}$ & $\begin{array}{r}7.22 \\
2.86 \\
9.32 \\
7.85 \\
5.60 \\
8.15 \\
10.97 \\
13.40 \\
9.03 \\
3.25 \\
6.05\end{array}$ & $\begin{array}{r}6.85 \\
2.70 \\
8.71 \\
7.38 \\
4.79 \\
8.09 \\
10.14 \\
15.42 \\
8.91 \\
2.63 \\
5.50\end{array}$ \\
\hline Nuclear Fuel .......................................... & .20 & .18 & .34 & .71 & .73 & .81 & .68 & .66 & .61 & .61 \\
\hline Biomass Fuels at Utilities ................. & - & - & - & - & - & - & - & - & - & - \\
\hline Primary Energy - Five Sectors ${ }^{b}$.... & 1.31 & 2.99 & 6.04 & 6.20 & 4.99 & 4.82 & 4.76 & 5.13 & 5.61 & 5.34 \\
\hline $\begin{array}{l}\text { Electric Utility Fuelc } \\
\text { Electricity Purchased by End Users }\end{array}$ & $\begin{array}{r}.42 \\
6.24\end{array}$ & $\begin{array}{r}1.71 \\
13.61\end{array}$ & $\begin{array}{r}2.67 \\
21.26\end{array}$ & $\begin{array}{r}1.90 \\
28.18\end{array}$ & $\begin{array}{r}1.47 \\
27.56\end{array}$ & $\begin{array}{r}1.49 \\
25.16\end{array}$ & $\begin{array}{r}1.30 \\
24.97\end{array}$ & $\begin{array}{r}1.45 \\
25.95\end{array}$ & $\begin{array}{r}1.20 \\
26.63\end{array}$ & $\begin{array}{r}1.12 \\
27.76\end{array}$ \\
\hline \multirow[t]{2}{*}{ 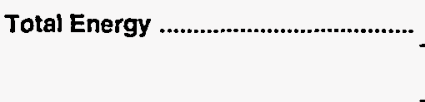 } & 1.95 & 4.26 & 8.34 & 9.62 & 8.13 & 7.92 & 8.03 & 8.53 & 9.25 & 9.18 \\
\hline & \multicolumn{10}{|c|}{ Expenditures in Millions of Dollars } \\
\hline 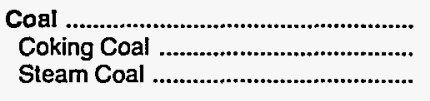 & $\begin{array}{r}55.6 \\
5.3 \\
50.4\end{array}$ & $\begin{array}{r}95.9 \\
-\overline{9}\end{array}$ & $\begin{array}{r}124.5 \\
-\overline{-} \\
124.5\end{array}$ & $\begin{array}{r}198.1 \\
198 . \overline{1}\end{array}$ & $\begin{array}{r}140.2 \\
-\overline{2} \\
140.2\end{array}$ & $\begin{array}{r}158.9 \\
- \\
158.9\end{array}$ & $\begin{array}{r}138.5 \\
-\overline{5} \\
138.5\end{array}$ & $\begin{array}{r}164.2 \\
-\overline{2}\end{array}$ & $\begin{array}{r}144.4 \\
- \\
144.4\end{array}$ & $\begin{array}{r}109.3 \\
109.3\end{array}$ \\
\hline 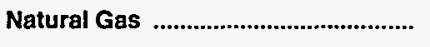 & 413.8 & 556.5 & $1,434.3$ & $2,371.8$ & $2,097.3$ & $2,150.0$ & $2,087.1$ & $2,360.1$ & $2,184.4$ & $2,295.8$ \\
\hline 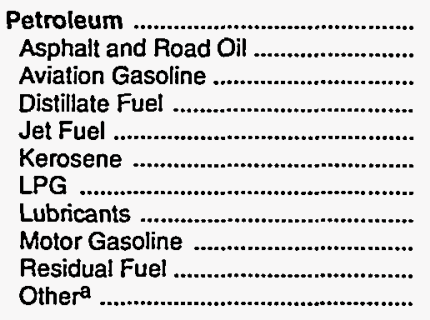 & $\begin{array}{r}2,001.4 \\
26.3 \\
1.7 \\
468.7 \\
26.9 \\
12.2 \\
40.9 \\
60.2 \\
1,040.8 \\
215.4 \\
108.3\end{array}$ & $\begin{array}{r}4,088.2 \\
62.9 \\
1.6 \\
947.8 \\
71.4 \\
19.6 \\
95.0 \\
79.0 \\
1,951.3 \\
575.0 \\
284.7\end{array}$ & $\begin{array}{r}8,945.0 \\
105.7 \\
3.8 \\
2,072.7 \\
308.7 \\
61.0 \\
134.3 \\
206.5 \\
3,797.7 \\
1,419.1 \\
835.5\end{array}$ & $\begin{array}{r}8,834.9 \\
153.6 \\
9.3 \\
1,861.2 \\
1,430.6 \\
70.9 \\
300.8 \\
230.4 \\
3,546.4 \\
644.1 \\
587.5\end{array}$ & $\begin{array}{r}6,934.9 \\
153.5 \\
6.8 \\
1,629.6 \\
852.8 \\
46.7 \\
265.5 \\
199.5 \\
2,921.4 \\
441.1 \\
417.9\end{array}$ & $\begin{array}{r}7,230.2 \\
119.3 \\
7.7 \\
1,542.6 \\
984.5 \\
45.7 \\
308.4 \\
183.8 \\
3,090.8 \\
457.5 \\
489.9\end{array}$ & $\begin{array}{r}7,103.4 \\
88.4 \\
5.7 \\
1,612.5 \\
876.3 \\
45.3 \\
279.5 \\
203.9 \\
3,230.9 \\
339.0 \\
422.0\end{array}$ & $\begin{array}{r}7,818.3 \\
72.4 \\
5.3 \\
1,700.8 \\
1,084.9 \\
57.4 \\
205.9 \\
190.3 \\
3,625.8 \\
392.2 \\
483.4\end{array}$ & $\begin{array}{r}8,185.8 \\
68.0 \\
5.6 \\
1,595.2 \\
1,470.6 \\
33.7 \\
162.6 \\
197.4 \\
3,694.1 \\
303.1 \\
655.6\end{array}$ & $\begin{array}{r}7,671.4 \\
56.1 \\
4.4 \\
1,427.9 \\
1,132.4 \\
28.2 \\
241.1 \\
203.1 \\
3,727.9 \\
278.4 \\
571.8\end{array}$ \\
\hline 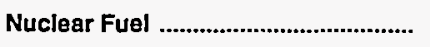 & 7.6 & 6.1 & 27.9 & 135.8 & 117.2 & 197.0 & 175.7 & 162.2 & 155.8 & 162.9 \\
\hline Biomass Fuels at Utilitles .................. & - & - & - & - & - & - & - & - & - & - \\
\hline Primary Energy - Five Sectors ${ }^{b} \ldots$ & $2,478.5$ & $4,746.7$ & $10,531.7$ & $11,540.6$ & $9,289.5$ & $9,736.2$ & $9,504.7$ & $10,504.9$ & $10,670.4$ & $10,239.5$ \\
\hline $\begin{array}{l}\text { Electric Utility Fuelc ......................... } \\
\text { Electricity Purchased by End Users }\end{array}$ & $\begin{array}{r}-182.1 \\
799.5\end{array}$ & $\begin{array}{r}-451.6 \\
1,966.1\end{array}$ & $\begin{array}{r}-881.5 \\
3,538.5\end{array}$ & $\begin{array}{r}-730.2 \\
5,148.3\end{array}$ & $\begin{array}{r}-479.1 \\
5,228.8\end{array}$ & $\begin{array}{r}-655.2 \\
5,017.5\end{array}$ & $\begin{array}{r}-574.2 \\
5,265.5\end{array}$ & $\begin{array}{r}-654.6 \\
5,583.2\end{array}$ & $\begin{array}{r}-478.0 \\
5,688.7\end{array}$ & $\begin{array}{r}-454.0 \\
6,088.4\end{array}$ \\
\hline 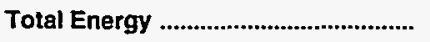 & $3,096.0$ & $6,261.3$ & $13,188.7$ & $15,958.8$ & $14,039.2$ & $14,098.5$ & $14,196.1$ & $15,433.5$ & $15,881.1$ & $15,873.9$ \\
\hline
\end{tabular}

Includes petroleum coke used at electric utilities.

b Biomass fuels are not included, except those consumed at electric utilities and those added to motor gasoline.

c There are no direct fuel costs for hydroelectric, geothermal, centralized solar, or wind energy.

-No consumption, including cases where adjustments were made. See "Consumption Adjustments for Process Fuel and Intermediate Products."

Note: Expenditure totals may not equal sum of components due to independent rounding.

Sources: Data sources, estimation procedures, and assumptions are described in the "Documentation" section of this report. 


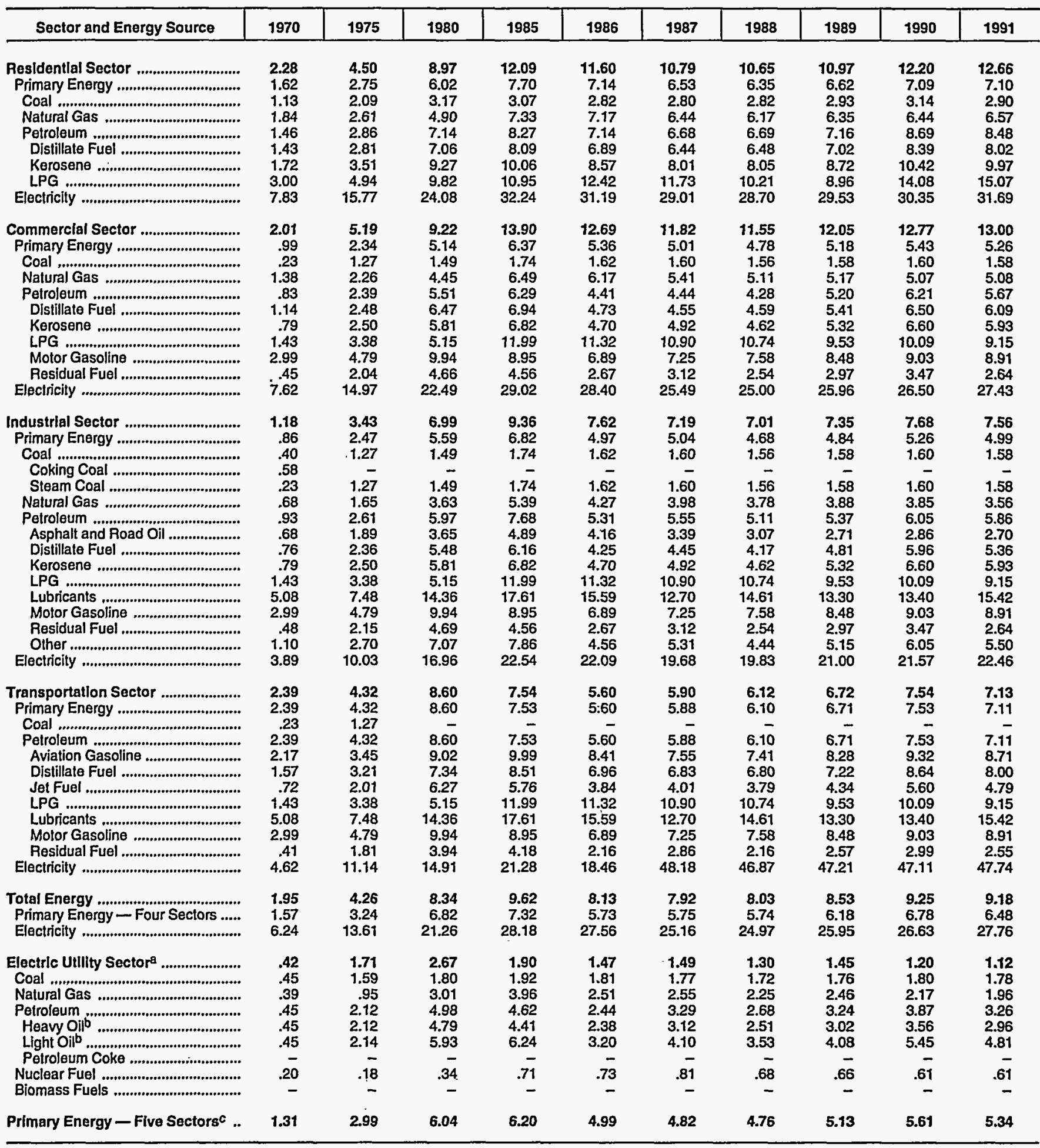

a There are no direct fuel costs for hydroelectric, geothermal, centralized solar, or wind energy

b Heavy oil includes Grade Nos. 4, 5, and 6, and residual fuel oils. Light oil includes Grade No. 2 heating oil, kerosene, and jet fuel.

c Blomass fuels are not included, except those consumed at electric utilities and those added to motor gasoline.

- No consumption, including cases where adjustments were made. See "Consumption Adjustments for Process Fuel and Intermediate Products."

Sources: Data sources, estimation procedures, and assumptions are described in the "Documentation" section of this report. 
N Energy Expenditure Estimates by Sector, New Jersey

E 1970, 1975, 1980, and 1985-1991

W (Million Dollars)

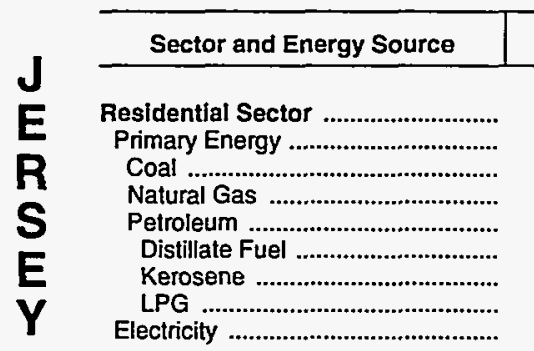

Commercial Sector

Primary Energy ..

Coal

Gas

Petroleum

Distillate Fuel

Kerosene

LPG

Motor Gasoline

Electricity

Industrial Sector

Primary Energy

Coal

Coking Coal

(n....................

Natural Gas

Petroleum .........................................

Asphalt and Road Oil ...................

Distillate Fuel .

Kerosene

LPG

Lubricants

.........................

Residual Fuel

Other .

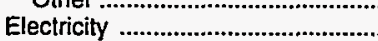

Transportation Sector

Primary Energy ................................... Coal

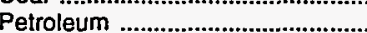

Aviation Gasoline ...........................

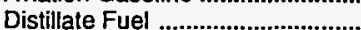

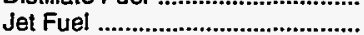

LPG

Lubricants

Motor Gasoline

Residual Fuel

Electricity

Total Energy .

Primary Energy - Four Sectors .....

Electricity

Electric Utillity Sectora

Coal

Natural Gas

Petroleum

Heavy Oilb

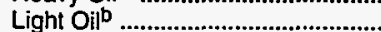

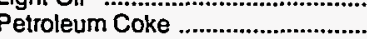

Nuclear Fuel .

Biomass Fuels

Primary Energy - Five Sectors ${ }^{c}$..

1970

88
55
26
29
32

$1,657.8$

2.4

264.7

291.6

74.6

7.5
9.5

324.1

$\mathbf{4 7 8 . 8}$
197.9

197.9
.3

79.3

118.2

74.0

1.3
8

.8
9.6

32.5

280.9

560.5

366.5

366.5
7.5

5.3

2.2

51.4

307.6

26.3

38.6

3.4

30.0

42.5

6.3

52.1

108.3
194.0

173.9

$1,173.9$
$1,173.4$

$1,173.4$

1.7

$$
\begin{aligned}
& 78.3 \\
& 26.9
\end{aligned}
$$

26.9
.6

17.7

$1,024.9$
23.3

096.0

$3,096.0$
$2,296.4$

$\begin{array}{r}799.5 \\ \hline\end{array}$

182.1

45.4

18.4

110.6

107.5

107.5
3.2

$7 . \overline{6}$

$2,478.5$

527.3

5010

8.0

17.7

780.0

$1,085.2$

378.0

.9
124.2

252.9

149.4

2.4

2.1

15.9

83.0
707.2

$1,277.1$

799.8
2.0

20

75.5

722.4

62.9

109.5
8.7

73.9
51.6

51.6
5.9

125.3

284.7

477.3

$2,241.2$

$2,239.5$

1.6
166.5

166.5
64.9

64.9
1.2
27.5

$1,929.5$

48.3
1.7

$6,261.3$

$4,295.2$

$4,295.2$
$1,966.1$

90.8

90.8
8.4

346.2

$6 . \overline{1}$

$4,746.7$

1975

$\begin{array}{rr}1,657.8 & 3,062.8 \\ 877.8 & 1,721.3\end{array}$

2.2
348.4

$2,239.5$

$1,721.3$

$2,075.2$

$\begin{array}{rr}691.2 & 4.4 \\ & 1,130.9\end{array}$

$1,027.7$

985.9

13.8

$1,341.5$

$2,259.8 \quad 2,984.5$

$\begin{array}{rr}964.5 & 914.0 \\ .8 & 1.9\end{array}$

$278.0 \quad 553.5$

685.8

345.2

1.3

2.6
15.5

321.1

$1,295.3$

$3,002.0$

358.6

227.9

3.0

31.0

89.7

$2,070.5$

$3,038.6$

$2,101.8$
1.2

$1,856.8$

15.0

$15 . \overline{0}$

433.0

$1,408.9$

153.6

89.3

16.2

252.8

161.2

21.7

126.5

587.5

$1,181.8$

$5,970.8$

$4,862.5$

$\begin{array}{rr}4,862 . \overline{5} & 5,964 . \overline{4}\end{array}$

3.8

438.1

284.6

667.6
$1,430.6$

4.8

$62.1 \quad 69.3$

$3,774.5 \quad 3,493.6$

7.5

$3,493.6$
289.3

6.4

$13,188.7$

$9,650.2$

$15,958.8$

$10,810.5$

$10,810.5$
$5,148.3$

$\begin{array}{lll}451.6 & 881.5 & 730.2\end{array}$

120.2

247.6

730.2
176.8

254.5

163.1

138.7

$\begin{array}{rr}96.7 & 24.4 \\ - & - \\ 27.9 & 135.8\end{array}$

$\begin{array}{rr}96.7 & 24.4 \\ -\overline{9} & \overline{-} \\ 27.9 & 135.8\end{array}$

389.1

$10,531.7 \quad 11,540.6$

$9,736.2$

$1,163.4$

771.1

1.3

$1,113.0$

46.4

$1,925.0$

47.6
$1,911.3$

$3,017.9$

2,872.7

$2,149.3$

$2,059.6$

417.9

$1,148.7$

489.9

$1,031.3$

$4,600.9$

4,909.3

1988

\begin{tabular}{l|l|l|}
\hline 1989 & 1990 & 1991 \\
\hline
\end{tabular}

\begin{tabular}{c|c|c|}
1986 & 1987 & 1988 \\
\hline $3,862.0$ & $3,751.0$ & $3,903.1$
\end{tabular}

$4,080.7$

$1,880.0$

1.1
$1,147.1$

731.9

659.9

21.5

$2,023.0$

$3,003.9$

826.5

.4
530.9

295.1

211.4

3.0

9.4

25.8

45.6
$2,177.4$

2411.0

$2,411.0$
$1,359.5$

$\begin{array}{r}10.2 \\ \hline\end{array}$

$10 \overline{2}$

290.6

290.6
$1,058.6$

88.4

77.5

20.8
216.3

216.3
142.6

20.9

70.2

422.0

$1,051.5$

$4,878.2$

$4,864.6$ 


\begin{tabular}{|c|c|c|c|c|c|c|c|c|c|c|}
\hline Energy Source & 1970 & 1975 & 1980 & 1985 & 1986 & 1987 & 1988 & 1989 & 1990 & 1991 \\
\hline & \multicolumn{10}{|c|}{ Prices in Dollars per Million Btu } \\
\hline 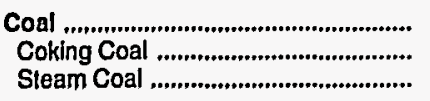 & $\begin{array}{r}0.14 \\
.14\end{array}$ & $\begin{array}{r}0.23 \\
.23\end{array}$ & $\begin{array}{r}0.56 \\
.56\end{array}$ & $\begin{array}{r}1.09 \\
1.09\end{array}$ & $\frac{1.14}{1.14}$ & $\frac{1.21}{1.21}$ & $\begin{array}{r}1.18 \\
1.18\end{array}$ & $\frac{1.24}{1.24}$ & $\frac{1.32}{1.32}$ & $\begin{array}{r}1.38 \\
1.38\end{array}$ \\
\hline 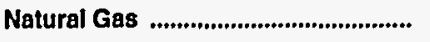 & .39 & .75 & 2.66 & 4.60 & 4.24 & 3.68 & 3.44 & 3.61 & 3.84 & 3.62 \\
\hline 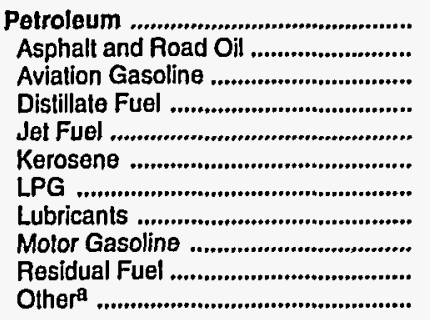 & $\begin{array}{r}1.92 \\
.58 \\
2.17 \\
1.07 \\
.76 \\
1.03 \\
1.35 \\
5.08 \\
2.94 \\
.34 \\
.50\end{array}$ & $\begin{array}{l}3.43 \\
1.84 \\
3.45 \\
2.42 \\
2.12 \\
2.26 \\
3.17 \\
7.48 \\
4.72 \\
1.66 \\
1.37\end{array}$ & $\begin{array}{r}7.80 \\
3.67 \\
9.02 \\
6.80 \\
6.59 \\
6.91 \\
5.84 \\
14.36 \\
9.58 \\
3.80 \\
2.65\end{array}$ & $\begin{array}{r}7.85 \\
4.79 \\
9.99 \\
6.53 \\
6.24 \\
7.31 \\
8.21 \\
17.61 \\
9.14 \\
3.98 \\
2.59\end{array}$ & $\begin{array}{r}6.04 \\
4.35 \\
8.41 \\
4.87 \\
4.39 \\
4.33 \\
6.88 \\
15.59 \\
7.02 \\
2.01 \\
2.84\end{array}$ & $\begin{array}{r}6.32 \\
3.19 \\
7.55 \\
5.50 \\
4.28 \\
4.93 \\
7.20 \\
12.70 \\
7.46 \\
2.56 \\
3.26\end{array}$ & $\begin{array}{r}6.27 \\
3.39 \\
7.41 \\
5.31 \\
4.17 \\
4.26 \\
6.35 \\
14.61 \\
7.44 \\
1.87 \\
2.85\end{array}$ & $\begin{array}{r}6.98 \\
2.87 \\
8.28 \\
6.34 \\
4.79 \\
5.13 \\
6.27 \\
13.30 \\
8.24 \\
2.16 \\
3.53\end{array}$ & $\begin{array}{r}8.01 \\
2.76 \\
9.32 \\
7.27 \\
6.01 \\
6.05 \\
7.99 \\
13.40 \\
9.23 \\
2.75 \\
4.30\end{array}$ & $\begin{array}{r}7.47 \\
3.45 \\
8.71 \\
6.89 \\
4.84 \\
5.58 \\
6.10 \\
15.42 \\
9.10 \\
2.27 \\
3.53\end{array}$ \\
\hline 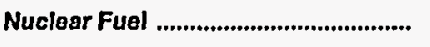 & - & - & - & - & - & - & - & - & - & - \\
\hline Blomass Fuels at Utilities .................. & - & - & - & - & - & - & - & - & - & - \\
\hline Prlmary Energy - Flve Sectors ${ }^{b}$.... & .85 & 1.63 & 3.71 & 3.99 & 3.43 & 3.47 & 3.37 & 3.67 & 4.17 & 4.25 \\
\hline $\begin{array}{l}\text { Electric Utility Fuelc } \\
\text { Electricity Purchased by End Users }\end{array}$ & 5.62 & $\begin{array}{r}.45 \\
7.99\end{array}$ & $\begin{array}{r}1.02 \\
15.52\end{array}$ & $\begin{array}{r}1.33 \\
21.20\end{array}$ & $\begin{array}{r}1.31 \\
22.14\end{array}$ & $\begin{array}{r}1.29 \\
21.83\end{array}$ & $\begin{array}{r}1.25 \\
21.76\end{array}$ & $\begin{array}{r}1.32 \\
21.55\end{array}$ & $\begin{array}{r}1.37 \\
20.99\end{array}$ & $\begin{array}{r}1.42 \\
21.09\end{array}$ \\
\hline \multirow[t]{2}{*}{ Total Energy ........................................... } & 1.46 & 2.88 & 7.08 & 9.26 & 8.01 & 8.00 & 7.79 & 8.37 & 9.16 & 8.70 \\
\hline & \multicolumn{10}{|c|}{ Expenditures In Millions of Dollars } \\
\hline 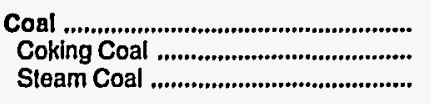 & $\begin{array}{r}14.3 \\
14.3\end{array}$ & $\begin{array}{r}30.0 \\
30.0\end{array}$ & $\begin{array}{r}114.2 \\
114 . \overline{2}\end{array}$ & $\begin{array}{r}293.7 \\
293 . \overline{7}\end{array}$ & $\begin{array}{r}274.3 \\
274.3\end{array}$ & $\begin{array}{r}315.1 \\
- \\
315.1\end{array}$ & $\begin{array}{r}312.9 \\
312.9\end{array}$ & $\begin{array}{r}346.0 \\
346 . \overline{0}\end{array}$ & $\begin{array}{r}363.4 \\
363.4\end{array}$ & $\begin{array}{r}322.0 \\
- \\
322.0\end{array}$ \\
\hline Natural Gas ......................................... & 80.7 & 134.8 & 394.1 & 350.8 & 303.8 & 280.8 & 320.8 & 355.4 & 348.8 & 352.9 \\
\hline 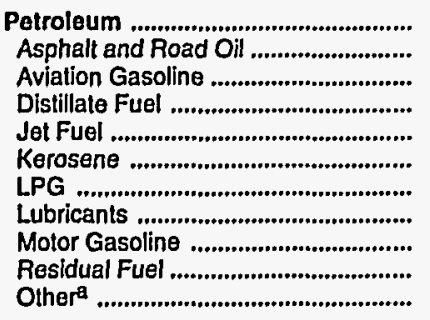 & $\begin{array}{r}292.3 \\
4.6 \\
1.2 \\
33.6 \\
12.9 \\
5.8 \\
22.0 \\
8.3 \\
202.9 \\
.4 \\
.5\end{array}$ & $\begin{array}{r}653.3 \\
19.9 \\
1.4 \\
94.7 \\
30.9 \\
8.4 \\
41.3 \\
14.4 \\
409.2 \\
31.0 \\
2.0\end{array}$ & $\begin{array}{r}1,507.8 \\
27.7 \\
7.6 \\
315.6 \\
96.0 \\
52.5 \\
98.6 \\
28.9 \\
850.8 \\
23.5 \\
6.6\end{array}$ & $\begin{array}{r}1,483.4 \\
47.7 \\
4.8 \\
323.9 \\
97.7 \\
7.9 \\
88.8 \\
32.2 \\
859.3 \\
19.0 \\
2.0\end{array}$ & $\begin{array}{r}1,147.0 \\
46.7 \\
4.4 \\
275.5 \\
66.8 \\
1.7 \\
43.9 \\
27.9 \\
674.8 \\
2.7 \\
2.6\end{array}$ & $\begin{array}{r}1,270.6 \\
43.7 \\
3.3 \\
341.2 \\
70.0 \\
1.7 \\
40.4 \\
25.7 \\
740.5 \\
1.2 \\
2.9\end{array}$ & $\begin{array}{r}1,253.8 \\
47.6 \\
2.1 \\
316.2 \\
64.1 \\
1.2 \\
34.7 \\
28.5 \\
755.8 \\
1.2 \\
2.6\end{array}$ & $\begin{array}{r}1,447.4 \\
31.7 \\
4.0 \\
331.4 \\
74.7 \\
2.0 \\
154.9 \\
26.6 \\
817.1 \\
1.8 \\
3.0\end{array}$ & $\begin{array}{r}1,699.4 \\
26.6 \\
4.0 \\
386.4 \\
96.2 \\
1.9 \\
251.7 \\
27.6 \\
898.7 \\
2.0 \\
4.3\end{array}$ & $\begin{array}{r}1,694.4 \\
34.9 \\
4.1 \\
378.4 \\
76.8 \\
2.0 \\
249.8 \\
28.4 \\
914.9 \\
1.1 \\
4.0\end{array}$ \\
\hline Nuclear Fuel ,.......................................... & - & - & - & - & - & - & - & - & - & - \\
\hline Biomass Fuels at Utilities ................. & - & - & - & - & - & - & - & - & - & - \\
\hline Primary Energy — Flve Sectors ${ }^{b} \ldots$ & 387.3 & 818.1 & $2,016.1$ & $2,127.9$ & $1,725.1$ & $1,866.5$ & $1,887.5$ & $2,148.8$ & $2,411.6$ & $2,369.2$ \\
\hline $\begin{array}{l}\text { Electric Utility Fuelc ........................... } \\
\text { Electricity Purchased by End Users }\end{array}$ & $\begin{array}{r}-32.0 \\
106.6\end{array}$ & $\begin{array}{r}-95.4 \\
179.5\end{array}$ & $\begin{array}{r}-268.0 \\
460.2\end{array}$ & $\begin{array}{r}-392.6 \\
836.0\end{array}$ & $\begin{array}{r}-342.2 \\
874.2\end{array}$ & $\begin{array}{r}-359.9 \\
893.3\end{array}$ & $\begin{array}{r}-359.3 \\
923.0\end{array}$ & $\begin{array}{r}-407.0 \\
958.2\end{array}$ & $\begin{array}{r}-414.2 \\
963.6\end{array}$ & $\begin{array}{r}-371.1 \\
987.1\end{array}$ \\
\hline Total Energy .......................................... & 461.8 & 902.3 & $2,208.2$ & $2,571.3$ & $2,257.1$ & $2,400.0$ & $2,451.2$ & $2,700.0$ & $2,961.0$ & $2,985.2$ \\
\hline
\end{tabular}

a Includes petroleum coke used at electric utilities.

b Biomass fuels are not included, except those consumed at electric utilities and those added to motor gasoline.

c There are no direct fuel costs for hydroelectric, geothermal, centralized solar, or wind energy.

- No consumption, including cases where adjustments were made. See "Consumption Adjustments for Process Fuel and Intermediate Products."

Note: Expenditure totals may not equal sum of components due to independent rounding.

Sources: Data sources, estimation procedures, and assumptions are described in the "Documentation" section of this report. 
N Energy Price Estimates by Sector, New Mexico

E 1970, 1975, 1980, and 1985-1991

W (Dollars per Million Btu)

\begin{tabular}{|c|c|c|c|c|c|c|c|c|c|c|}
\hline Sector and Energy Source & 1970 & 1975 & 1980 & 1985 & 1986 & 1987 & 1988 & 1989 & 1990 & 1991 \\
\hline 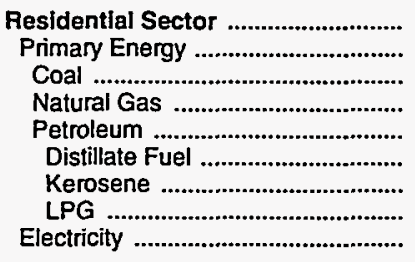 & $\begin{array}{r}1.78 \\
1.00 \\
.90 \\
.86 \\
1.60 \\
.98 \\
1.49 \\
1.61 \\
8.15\end{array}$ & $\begin{array}{r}3.06 \\
1.64 \\
-\overline{1.24} \\
4.12 \\
2.82 \\
3.05 \\
4.16 \\
10.47\end{array}$ & $\begin{array}{r}6.66 \\
3.78 \\
2.54 \\
3.17 \\
7.29 \\
6.79 \\
7.95 \\
7.19 \\
18.89\end{array}$ & $\begin{array}{r}11.10 \\
6.33 \\
2.83 \\
5.59 \\
8.59 \\
6.92 \\
8.62 \\
8.62 \\
25.48\end{array}$ & $\begin{array}{r}11.01 \\
5.50 \\
2.78 \\
5.28 \\
7.03 \\
5.09 \\
6.34 \\
7.16 \\
26.43\end{array}$ & $\begin{array}{r}10.34 \\
4.96 \\
2.40 \\
4.61 \\
7.66 \\
4.81 \\
5.99 \\
7.78 \\
26.39\end{array}$ & $\begin{array}{r}10.54 \\
5.03 \\
2.12 \\
4.87 \\
6.46 \\
4.74 \\
5.91 \\
6.50 \\
26.42\end{array}$ & $\begin{array}{r}11.23 \\
5.73 \\
2.43 \\
5.45 \\
7.44 \\
5.34 \\
6.65 \\
7.48 \\
26.41\end{array}$ & $\begin{array}{r}11.13 \\
6.04 \\
2.41 \\
5.37 \\
9.24 \\
6.47 \\
8.06 \\
9.28 \\
26.22\end{array}$ & $\begin{array}{r}11.24 \\
5.89 \\
2.36 \\
5.18 \\
10.39 \\
5.96 \\
7.42 \\
10.46 \\
26.63\end{array}$ \\
\hline 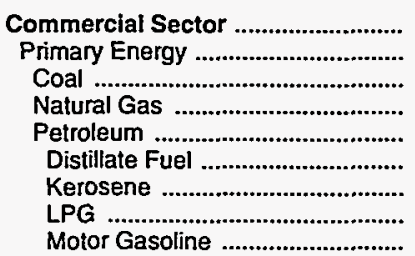 & $\begin{array}{r}1.37 \\
.50 \\
.56 \\
.44 \\
1.34 \\
.92 \\
1.01 \\
1.12 \\
2.94\end{array}$ & $\begin{array}{r}2.74 \\
.94 \\
- \\
.74 \\
3.03 \\
2.62 \\
2.22 \\
2.60 \\
4.72\end{array}$ & $\begin{array}{r}6.79 \\
3.50 \\
.88 \\
2.79 \\
6.85 \\
6.57 \\
6.80 \\
5.36 \\
9.58 \\
\end{array}$ & $\begin{array}{r}12.49 \\
5.58 \\
1.41 \\
5.34 \\
6.58 \\
5.62 \\
6.95 \\
7.27 \\
9.14\end{array}$ & $\begin{array}{r}11.59 \\
4.08 \\
1.38 \\
4.04 \\
4.43 \\
3.23 \\
3.44 \\
6.51 \\
7.02\end{array}$ & $\begin{array}{r}11.19 \\
3.83 \\
1.40 \\
3.67 \\
4.49 \\
3.78 \\
4.32 \\
6.06 \\
7.46\end{array}$ & $\begin{array}{l}9.66 \\
3.22 \\
1.33 \\
3.08 \\
4.25 \\
3.34 \\
3.78 \\
6.11 \\
7.44\end{array}$ & $\begin{array}{r}0.55 \\
3.71 \\
1.18 \\
3.54 \\
4.90 \\
3.89 \\
4.87 \\
6.00 \\
8.24\end{array}$ & $\begin{array}{r}11.49 \\
4.49 \\
1.30 \\
4.19 \\
5.88 \\
4.74 \\
5.89 \\
7.67 \\
9.23\end{array}$ & $\begin{array}{r}11.47 \\
4.16 \\
1.36 \\
3.98 \\
5.31 \\
4.41 \\
5.38 \\
5.51 \\
9.10\end{array}$ \\
\hline 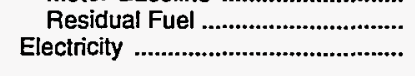 & 5.78 & $7 . \overline{90}$ & $15 . \overline{95}$ & $\begin{array}{r}4.00 \\
22.57\end{array}$ & 23.41 & 22.49 & $23 . \overline{10}$ & 22.68 & 22.22 & 22.56 \\
\hline 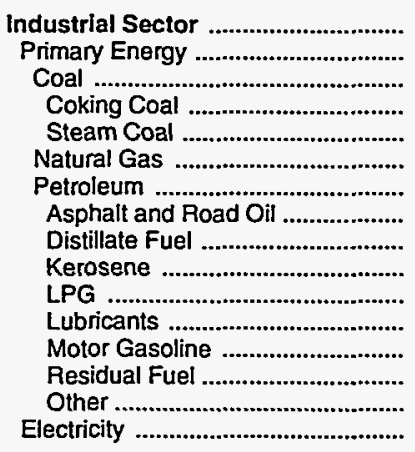 & $\begin{array}{r}.65 \\
.49 \\
.56 \\
- \\
.56 \\
.25 \\
1.01 \\
.58 \\
.95 \\
1.01 \\
1.12 \\
5.08 \\
2.94 \\
.41 \\
.50 \\
3.44\end{array}$ & $\begin{array}{r}1.52 \\
1.27 \\
- \\
- \\
- \\
.58 \\
2.13 \\
1.84 \\
2.05 \\
2.22 \\
2.60 \\
7.48 \\
4.72 \\
1.60 \\
1.37 \\
5.54\end{array}$ & $\begin{array}{r}4.95 \\
4.06 \\
.88 \\
- \\
.88 \\
2.46 \\
5.34 \\
3.67 \\
6.42 \\
6.80 \\
5.36 \\
14.36 \\
9.58 \\
3.82 \\
2.65 \\
12.11\end{array}$ & $\begin{array}{r}7.66 \\
5.45 \\
1.41 \\
-\overline{1} \\
1.41 \\
3.67 \\
5.87 \\
4.79 \\
6.11 \\
6.95 \\
7.27 \\
17.61 \\
9.14 \\
4.00 \\
2.59 \\
16.01\end{array}$ & $\begin{array}{r}6.57 \\
3.74 \\
1.38 \\
- \\
1.38 \\
3.04 \\
3.90 \\
4.35 \\
3.02 \\
3.44 \\
6.51 \\
15.59 \\
7.02 \\
2.09 \\
2.84 \\
16.64\end{array}$ & $\begin{array}{r}6.32 \\
3.81 \\
1.40 \\
- \\
1.40 \\
3.30 \\
3.94 \\
3.19 \\
3.80 \\
4.32 \\
6.06 \\
12.70 \\
7.46 \\
2.86 \\
3.26 \\
16.56\end{array}$ & $\begin{array}{r}6.06 \\
3.63 \\
1.33 \\
- \\
1.33 \\
3.16 \\
3.79 \\
3.39 \\
3.32 \\
3.78 \\
6.11 \\
14.61 \\
7.44 \\
1.81 \\
2.85 \\
15.56\end{array}$ & $\begin{array}{r}6.42 \\
4.44 \\
1.18 \\
- \\
1.18 \\
2.97 \\
4.89 \\
2.87 \\
4.28 \\
4.87 \\
6.00 \\
13.30 \\
8.24 \\
1.96 \\
3.53 \\
15.53\end{array}$ & $\begin{array}{r}7.28 \\
5.62 \\
1.30 \\
- \\
1.30 \\
3.49 \\
6.11 \\
2.76 \\
5.18 \\
5.89 \\
7.67 \\
13.40 \\
9.23 \\
2.62 \\
4.30 \\
14.61\end{array}$ & $\begin{array}{r}6.32 \\
4.84 \\
1.36 \\
- \\
1.36 \\
3.39 \\
5.16 \\
3.45 \\
4.73 \\
5.38 \\
5.51 \\
15.42 \\
9.10 \\
2.01 \\
3.53 \\
14.18\end{array}$ \\
\hline $\begin{array}{c}\text { Transportation Sector ....................... } \\
\text { Primary Energy ................................. }\end{array}$ & $\begin{array}{l}2.28 \\
2.28\end{array}$ & $\begin{array}{l}4.03 \\
4.03\end{array}$ & $\begin{array}{l}8.69 \\
8.69\end{array}$ & $\begin{array}{r}8.47 \\
8.47\end{array}$ & $\begin{array}{l}6.65 \\
6.65\end{array}$ & $\begin{array}{l}7.02 \\
7.02\end{array}$ & $\begin{array}{l}6.99 \\
6.99\end{array}$ & $\begin{array}{l}7.69 \\
7.69\end{array}$ & $\begin{array}{l}8.68 \\
8.68\end{array}$ & $\begin{array}{l}8.39 \\
8.39\end{array}$ \\
\hline 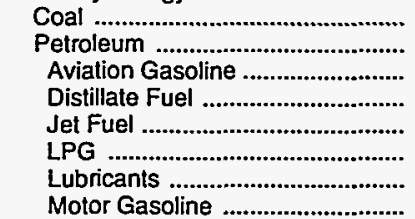 & $\begin{array}{r}.56 \\
2.28 \\
2.17 \\
1.15 \\
.76 \\
1.12 \\
5.08 \\
2.94\end{array}$ & $\begin{array}{l}4 . \overline{-} \\
3.45 \\
2.62 \\
2.12 \\
2.60 \\
7.48 \\
4.72\end{array}$ & $\begin{array}{r}-\overline{8} \\
8.69 \\
9.02 \\
6.97 \\
6.59 \\
5.36 \\
14.36 \\
9.58\end{array}$ & $\begin{array}{r}8 . \overline{7} \\
9.99 \\
6.98 \\
6.24 \\
7.27 \\
17.61 \\
9.14\end{array}$ & $\begin{array}{r}6.65 \\
8.41 \\
6.29 \\
4.39 \\
6.51 \\
15.59 \\
7.02\end{array}$ & $\begin{array}{r}7.02 \\
7.55 \\
6.89 \\
4.28 \\
6.06 \\
12.70 \\
7.46\end{array}$ & $\begin{array}{r}-\overline{6} \\
7.41 \\
6.67 \\
4.17 \\
6.11 \\
14.61 \\
7.44\end{array}$ & $\begin{array}{r}7.69 \\
8.28 \\
7.30 \\
4.79 \\
6.00 \\
13.30 \\
8.24\end{array}$ & $\begin{array}{r}8.68 \\
9.32 \\
8.26 \\
6.01 \\
7.67 \\
13.40 \\
9.23\end{array}$ & $\begin{array}{r}-\overline{8} \\
8.39 \\
7.81 \\
7.85 \\
4.84 \\
5.51 \\
15.42 \\
9.10\end{array}$ \\
\hline $\begin{array}{l}\text { Residual Fuel } \\
\text { Electricity }\end{array}$ & .38 & $\begin{array}{r}4.16 \\
- \\
-\end{array}$ & - & $\begin{array}{r}0.14 \\
- \\
-\end{array}$ & $\begin{array}{r}.06 \\
- \\
-\end{array}$ & $\begin{array}{r}1.40 \\
- \\
-\end{array}$ & $\begin{array}{r}1.44 \\
- \\
-\end{array}$ & $\begin{array}{r}0 .<4 \\
- \\
-\end{array}$ & $\begin{array}{r}5.20 \\
- \\
-\end{array}$ & $\begin{array}{r}5.10 \\
- \\
-\end{array}$ \\
\hline $\begin{array}{l}\text { Total Energy ................................ } \\
\text { Primary Energy - Four Sectors ...... } \\
\text { Electricity ............................................ }\end{array}$ & $\begin{array}{l}1.46 \\
1.20 \\
5.62\end{array}$ & $\begin{array}{l}2.88 \\
2.49 \\
7.99\end{array}$ & $\begin{array}{r}7.08 \\
6.20 \\
15.52\end{array}$ & $\begin{array}{r}9.26 \\
7.28 \\
21.20\end{array}$ & $\begin{array}{r}8.01 \\
5.71 \\
22.14\end{array}$ & $\begin{array}{r}8.00 \\
5.81 \\
21.83\end{array}$ & $\begin{array}{r}7.79 \\
5.61 \\
21.76\end{array}$ & $\begin{array}{r}8.37 \\
6.26 \\
21.55\end{array}$ & $\begin{array}{r}9.16 \\
7.20 \\
20.99\end{array}$ & $\begin{array}{r}8.70 \\
6.75 \\
21.09\end{array}$ \\
\hline 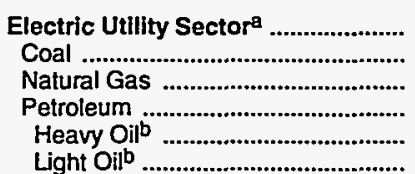 & $\begin{array}{l}.20 \\
.14 \\
.30 \\
.23 \\
.23 \\
.27\end{array}$ & $\begin{array}{r}.45 \\
.23 \\
.69 \\
1.70 \\
1.70 \\
1.89\end{array}$ & $\begin{array}{r}1.02 \\
.56 \\
2.47 \\
5.21 \\
3.70 \\
6.53\end{array}$ & $\begin{array}{l}1.33 \\
1.09 \\
3.48 \\
4.98 \\
3.71 \\
6.20\end{array}$ & $\begin{array}{l}1.31 \\
1.13 \\
3.31 \\
2.69 \\
1.65 \\
3.79\end{array}$ & $\begin{array}{l}1.29 \\
1.21 \\
2.36 \\
3.58 \\
2.14 \\
4.47\end{array}$ & $\begin{array}{l}1.25 \\
1.18 \\
2.14 \\
3.17 \\
1.97 \\
4.22\end{array}$ & $\begin{array}{l}1.32 \\
1.24 \\
2.13 \\
3.99 \\
2.74 \\
4.98\end{array}$ & $\begin{array}{l}1.37 \\
1.32 \\
1.91 \\
4.70 \\
3.09 \\
6.22\end{array}$ & $\begin{array}{l}1.42 \\
1.38 \\
1.70 \\
5.11 \\
3.86 \\
5.35\end{array}$ \\
\hline 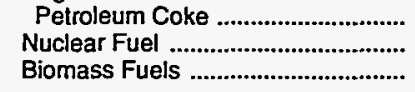 & - & $\begin{array}{l}- \\
- \\
-\end{array}$ & $\begin{array}{l}- \\
-\end{array}$ & $\begin{array}{l}- \\
-\end{array}$ & $\begin{array}{l}- \\
- \\
-\end{array}$ & $\begin{array}{l}- \\
-\end{array}$ & - & $\begin{array}{l}- \\
- \\
-\end{array}$ & $\begin{array}{l}- \\
- \\
-\end{array}$ & - \\
\hline Primary Energy - Flve Sectors ${ }^{c}$.. & .85 & 1.63 & 3.71 & 3.99 & 3.43 & 3.47 & 3.37 & 3.67 & 4.17 & 4.25 \\
\hline
\end{tabular}

a There are no direct fuel costs for hydroelectric, geothermal, centralized solar, or wind energy.

b Heavy oil includes Grade Nos. 4, 5, and 6, and residual fuel oils. Light oil includes Grade No. 2 heating oil, kerosene, and jet fuel.

c Biomass fuels are not included, except those consumed at electric utilities and those added to motor gasoline.

- No consumption, including cases where adjustments were made. See "Consumption Adjustments for Process Fuel and Intermediate Products."

Sources: Data sources, estimation procedures, and assumptions are described in the "Documentation" section of this report. 


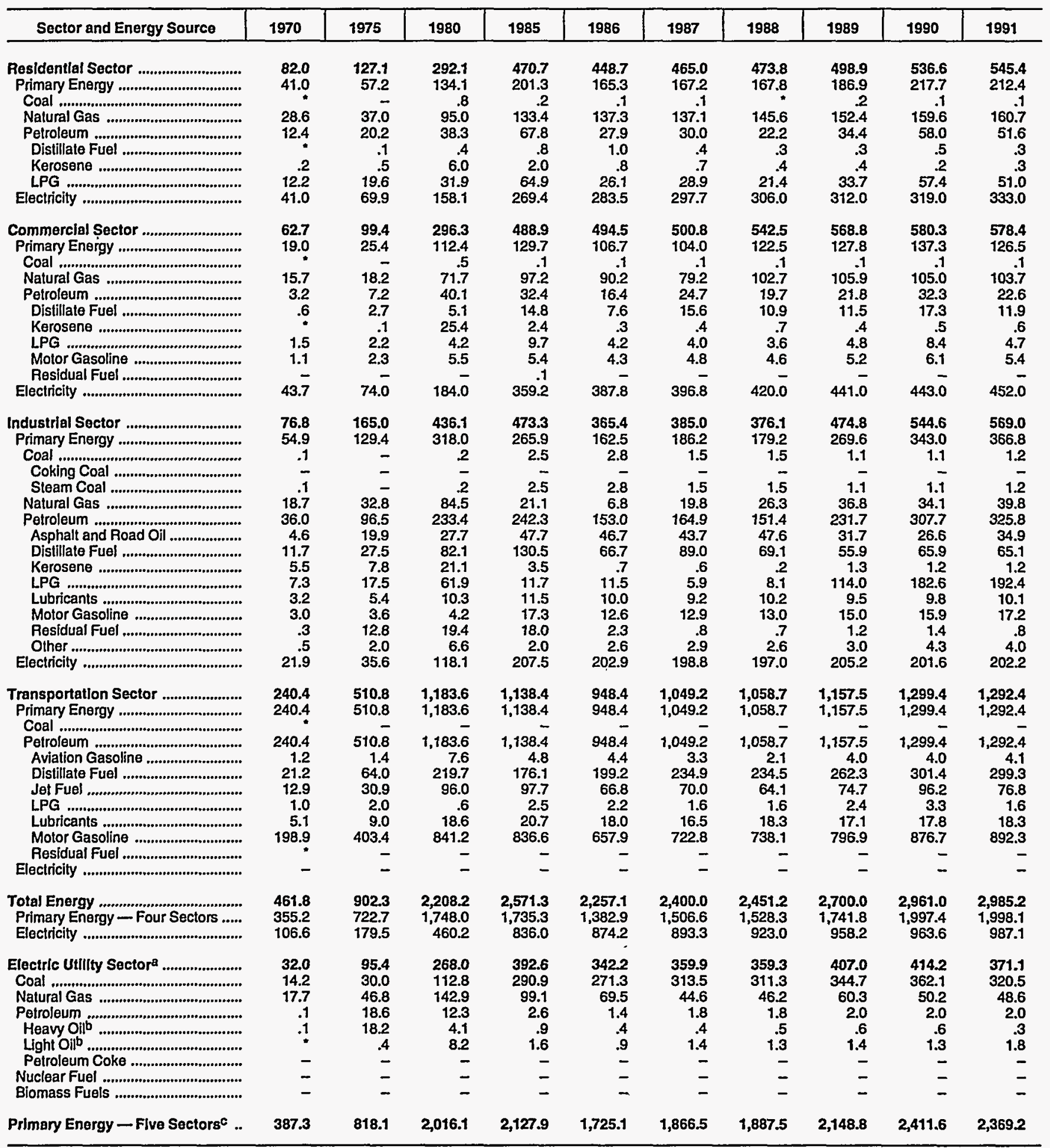

a There are no direct fuel costs for hydroelectric, geothermal, centralized solar, or wind energy.

b Heavy oil includes Grade Nos. 4, 5, and 6, and residual fuel oils. Light oil includes Grade No. 2 heating oil, kerosene, and jet fuel.

c Blomass fuels are not included, except those consumed at electric utilities and those added to motor gasoline.

-No consumption, including cases where adjustments were made. See "Consumption Adjustments for Process Fuel and Intermediate Products."

Value less than 0.05 million dollars.

Note: Totals may not equal sum of components due to independent rounding.

Sources: Data sources, estimation procedures, and assumptions are described in the "Documentation" section of this report. 


\section{N Energy Price and Expenditure Estimates by Source, New York \\ E 1970,1975, 1980, and 1985-1991}

\begin{tabular}{|c|c|c|c|c|c|c|c|c|c|c|}
\hline Energy Source & 1970 & 1975 & 1980 & 1985 & 1986 & 1987 & 1988 & 1989 & 1990 & 1991 \\
\hline & \multicolumn{10}{|c|}{ Prices In Dollars per Million Btu } \\
\hline 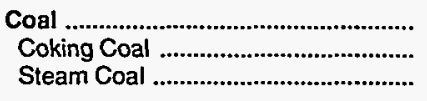 & $\begin{array}{r}0.51 \\
.58 \\
.49\end{array}$ & $\begin{array}{l}1.53 \\
2.14 \\
1.27\end{array}$ & $\begin{array}{l}1.78 \\
2.38 \\
1.56\end{array}$ & $\begin{array}{l}1.81 \\
1.88 \\
1.80\end{array}$ & $\begin{array}{l}1.72 \\
1.76 \\
1.71\end{array}$ & $\begin{array}{l}1.61 \\
1.59 \\
1.62\end{array}$ & $\begin{array}{l}1.62 \\
1.61 \\
1.62\end{array}$ & $\begin{array}{l}1.63 \\
1.67 \\
1.62\end{array}$ & $\begin{array}{l}1.66 \\
1.71 \\
1.66\end{array}$ & $\begin{array}{l}1.65 \\
1.72 \\
1.64\end{array}$ \\
\hline 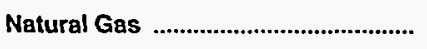 & 1.07 & 2.16 & 4.10 & 5.94 & 5.65 & 5.06 & 5.09 & 5.38 & 5.25 & 5.18 \\
\hline 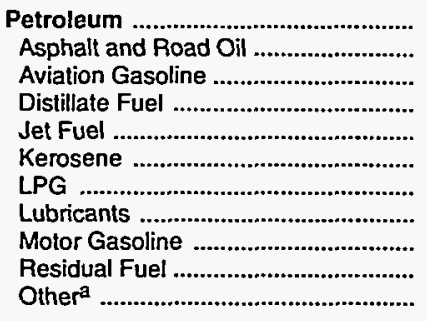 & $\begin{array}{r}1.36 \\
.70 \\
2.17 \\
1.24 \\
.72 \\
1.39 \\
2.24 \\
5.08 \\
2.92 \\
.43 \\
1.17\end{array}$ & $\begin{array}{l}2.96 \\
1.84 \\
3.45 \\
2.66 \\
2.02 \\
3.06 \\
3.99 \\
7.48 \\
4.80 \\
1.93 \\
2.67\end{array}$ & $\begin{array}{r}6.93 \\
3.67 \\
9.02 \\
6.78 \\
6.27 \\
7.78 \\
7.43 \\
14.36 \\
10.26 \\
4.10 \\
7.05\end{array}$ & $\begin{array}{r}7.39 \\
4.81 \\
9.99 \\
7.92 \\
6.51 \\
8.31 \\
11.40 \\
17.61 \\
8.79 \\
4.38 \\
6.57\end{array}$ & $\begin{array}{r}5.37 \\
4.34 \\
8.41 \\
6.25 \\
4.42 \\
6.76 \\
10.50 \\
15.59 \\
6.60 \\
2.61 \\
4.41\end{array}$ & $\begin{array}{r}5.69 \\
3.73 \\
7.55 \\
6.09 \\
4.55 \\
6.59 \\
10.13 \\
12.70 \\
6.99 \\
3.10 \\
5.77\end{array}$ & $\begin{array}{r}5.41 \\
3.18 \\
7.41 \\
6.11 \\
4.15 \\
6.71 \\
10.01 \\
14.61 \\
7.16 \\
2.52 \\
4.89\end{array}$ & $\begin{array}{r}6.01 \\
2.81 \\
8.28 \\
6.64 \\
4.70 \\
6.82 \\
11.02 \\
13.30 \\
7.82 \\
2.99 \\
5.66\end{array}$ & $\begin{array}{r}7.09 \\
2.96 \\
9.32 \\
8.13 \\
6.03 \\
8.82 \\
12.76 \\
13.40 \\
8.83 \\
3.64 \\
6.10\end{array}$ & $\begin{array}{r}7.11 \\
2.76 \\
8.71 \\
7.98 \\
5.18 \\
8.58 \\
13.53 \\
15.42 \\
9.44 \\
2.73 \\
4.52\end{array}$ \\
\hline Nuclear Fuel .............................................. & .20 & .31 & .56 & .67 & .61 & .63 & .57 & .65 & .65 & .55 \\
\hline Biomass Fuels at Utilities ................. & - & - & 1.74 & - & - & .95 & - & - & - & - \\
\hline Primary Energy - Five Sectors ${ }^{b}$.... & 1.17 & 2.60 & 5.45 & 5.87 & 4.76 & 4.75 & 4.56 & 4.97 & 5.50 & 5.36 \\
\hline $\begin{array}{l}\text { Electric Utility Fuelc ....................... } \\
\text { Electricity Purchased by End Users }\end{array}$ & $\begin{array}{r}.42 \\
6.72\end{array}$ & $\begin{array}{r}1.53 \\
14.04\end{array}$ & $\begin{array}{r}2.68 \\
19.64\end{array}$ & $\begin{array}{r}2.54 \\
26.97\end{array}$ & $\begin{array}{r}1.79 \\
26.07\end{array}$ & $\begin{array}{r}2.01 \\
25.56\end{array}$ & $\begin{array}{r}1.80 \\
25.09\end{array}$ & $\begin{array}{r}2.06 \\
26.08\end{array}$ & $\begin{array}{r}2.20 \\
27.51\end{array}$ & $\begin{array}{r}1.74 \\
28.68\end{array}$ \\
\hline \multirow[t]{2}{*}{ 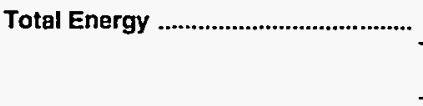 } & 1.81 & 4.11 & 8.08 & 10.38 & 9.07 & 9.01 & 8.99 & 9.72 & 10.64 & 10.84 \\
\hline & \multicolumn{10}{|c|}{ Expenditures in Millions of Dollars } \\
\hline 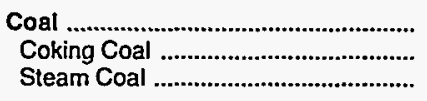 & $\begin{array}{r}308.4 \\
96.4 \\
212.0\end{array}$ & $\begin{array}{l}477.1 \\
197.8 \\
279.3\end{array}$ & $\begin{array}{l}558.6 \\
197.6 \\
361.0\end{array}$ & $\begin{array}{r}546.0 \\
58.5 \\
487.6\end{array}$ & $\begin{array}{r}435.5 \\
58.1 \\
377.4\end{array}$ & $\begin{array}{r}473.5 \\
61.6 \\
411.9\end{array}$ & $\begin{array}{r}539.2 \\
72.6 \\
466.6\end{array}$ & $\begin{array}{r}589.7 \\
74.1 \\
515.6\end{array}$ & $\begin{array}{r}574.9 \\
62.2 \\
512.7\end{array}$ & $\begin{array}{r}566.9 \\
56.6 \\
510.4\end{array}$ \\
\hline 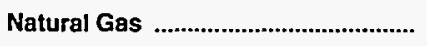 & 771.3 & $1,255.2$ & $3,087.1$ & $4,637.2$ & $4,213.2$ & $3,999.6$ & $4,090.8$ & $4,627.0$ & $4,614.1$ & $4,630.4$ \\
\hline 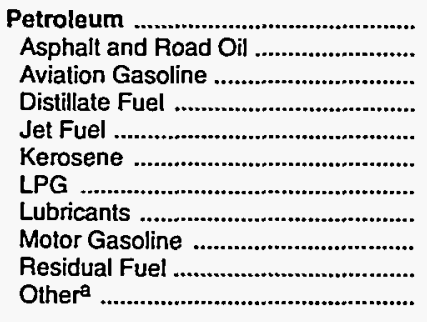 & $\begin{array}{r}3,611.9 \\
26.0 \\
2.7 \\
803.3 \\
155.5 \\
55.3 \\
37.2 \\
67.8 \\
2,005.9 \\
409.7 \\
48.5\end{array}$ & $\begin{array}{r}7,618.7 \\
69.9 \\
4.8 \\
1,626.9 \\
441.7 \\
90.3 \\
70.3 \\
88.4 \\
3,368.0 \\
1,740.1 \\
118.2\end{array}$ & $\begin{array}{r}14,901.7 \\
121.3 \\
14.6 \\
2,862.3 \\
1,275.3 \\
101.8 \\
139.0 \\
182.1 \\
6,865.7 \\
2,964.1 \\
375.5\end{array}$ & $\begin{array}{r}12,246.0 \\
230.1 \\
11.1 \\
2,861.6 \\
139.0 \\
250.5 \\
202.3 \\
203.2 \\
6,299.5 \\
1,827.8 \\
220.7\end{array}$ & $\begin{array}{r}9,549.9 \\
185.3 \\
10.9 \\
2,568.6 \\
91.8 \\
117.3 \\
186.4 \\
176.0 \\
4,740.2 \\
1,308.1 \\
165.4\end{array}$ & $\begin{array}{r}10,257.5 \\
162.0 \\
4.8 \\
2,590.2 \\
72.8 \\
155.4 \\
202.6 \\
162.1 \\
5,234.5 \\
1,495.6 \\
177.5\end{array}$ & $\begin{array}{r}10,110.5 \\
168.7 \\
3.9 \\
2,684.5 \\
113.6 \\
200.3 \\
190.2 \\
179.8 \\
5,018.8 \\
1,398.8 \\
151.9\end{array}$ & $\begin{array}{r}11,040.5 \\
92.7 \\
3.7 \\
2,963.2 \\
158.9 \\
185.5 \\
224.1 \\
167.8 \\
5,480.3 \\
1,590.5 \\
173.8\end{array}$ & $\begin{array}{r}12,382.0 \\
108.4 \\
3.6 \\
3,141.0 \\
183.5 \\
114.2 \\
257.0 \\
174.1 \\
6,419.2 \\
1,756.9 \\
223.9\end{array}$ & $\begin{array}{r}11,822.0 \\
117.0 \\
2.8 \\
2,861.6 \\
147.6 \\
128.8 \\
353.9 \\
179.2 \\
6,644.2 \\
1,164.1 \\
222.8\end{array}$ \\
\hline 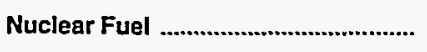 & 9.2 & 44.9 & 118.3 & 175.2 & 146.3 & 154.6 & 148.3 & 159.1 & 164.7 & 169.4 \\
\hline Biomass Fuels at Utilities .................. & - & - & 2 & - & - & $\cdot$ & - & - & - & - \\
\hline Primary Energy - Flve Sectors ${ }^{b}$.... & $4,700.7$ & $9,395.9$ & $18,666.0$ & $17,604.4$ & $14,344.9$ & $14,885.3$ & $14,888.8$ & $16,416.4$ & $17,735.7$ & $17,188.6$ \\
\hline $\begin{array}{l}\text { Electric Utility Fuelc .......................... } \\
\text { Electricity Purchased by End Users }\end{array}$ & $\begin{array}{r}-335.3 \\
2,006.4\end{array}$ & $\begin{array}{r}-1,326.8 \\
4,580.0\end{array}$ & $\begin{array}{r}-2,427.3 \\
7,044.4\end{array}$ & $\begin{array}{l}-2,320.5 \\
10,369.9\end{array}$ & $\begin{array}{l}-1,558.8 \\
10,220.2\end{array}$ & $\begin{array}{r}-1,917.7 \\
10,328.0\end{array}$ & $\begin{array}{l}-1,892.7 \\
10,706.6\end{array}$ & $\begin{array}{r}-2,303.0 \\
11,352.8\end{array}$ & $\begin{array}{r}-2,383.6 \\
12,087.5\end{array}$ & $\begin{array}{r}-1,848.0 \\
12,665.0\end{array}$ \\
\hline 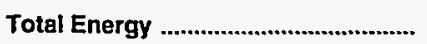 & $6,371.7$ & $12,649.1$ & $23,283.2$ & $25,653.8$ & $23,006.3$ & $23,295.6$ & $23,702.7$ & $25,466.1$ & $27,439.5$ & $28,005.6$ \\
\hline
\end{tabular}

a Includes petroleum coke used at electric utilities.

b Biomass fuels are not included, except those consumed at electric utilities and those added to motor gasoline.

c There are no direct fuel costs for hydroelectric, geothemal, centralized solar, or wind energy.

-No consumption, including cases where adjustments were made. See "Consumption Adjustments for Process Fuel and Intermediate Products."

"Value less than 0.05 million dollars.

Note: Expenditure totals may not equal sum of components due to independent rounding.

Sources: Data sources, estimation procedures, and assumptions are described in the "Documentation" section of this report. 
(Dollars per Million Btu)

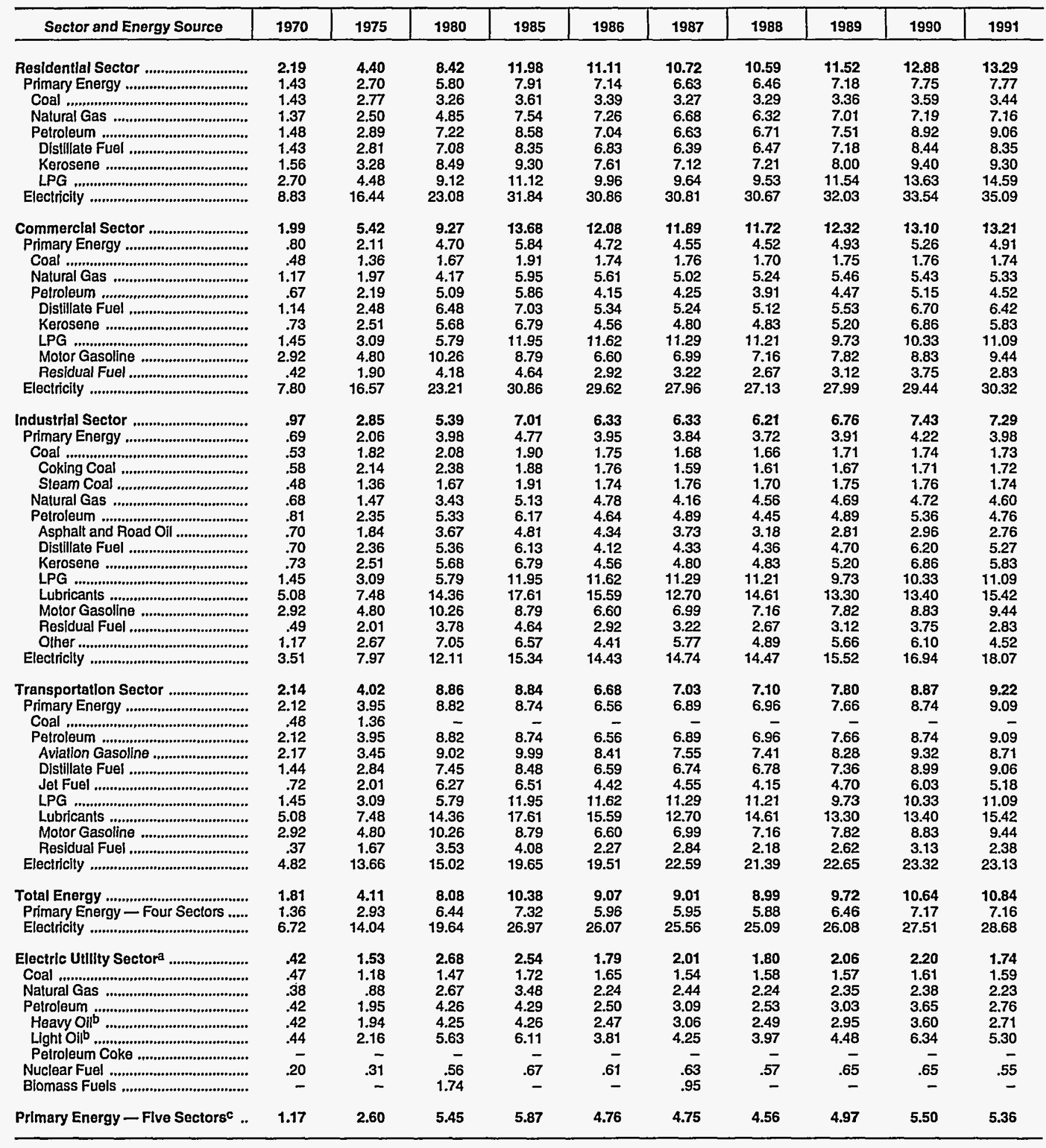

a There are no direct fuel costs for hydroelectric, geothermal, centralized solar, or wind energy.

b Heavy oil includes Grade Nos. 4, 5, and 6, and residual fuel oils. Light oil inciudes Grade No. 2 heating oil, kerosene, and jet fuel.

c Biomass fuels are not included, except those consumed at electric utilities and those added to motor gasoline.

-No consumption, including cases where adjustments were made. See "Consumption Adjustments for Process Fuel and Intermediale Products.

Sources: Data sources, estimation procedures, and assumptions are described in the "Documentation" section of this report. 
N Energy Expenditure Estimates by Sector, New York

E $1970,1975,1980$, and 1985-1991

W (Million Dollars)

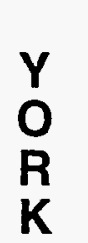

\begin{tabular}{|c|c|c|c|c|c|c|c|c|c|c|}
\hline Sector and Energy Source & 1970 & 1975 & 1980 & 1985 & 1986 & 1987 & 1988 & 1989 & 1990 & 1991 \\
\hline 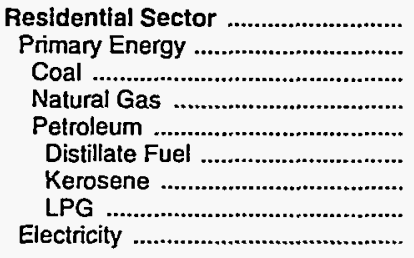 & $\begin{array}{r}1,844.5 \\
1,076.5 \\
12.8 \\
484.5 \\
579.2 \\
501.4 \\
49.4 \\
28.3 \\
768.0\end{array}$ & \begin{tabular}{r|}
$3,490.4$ \\
$1,879.9$ \\
14.2 \\
830.2 \\
$1,035.4$ \\
914.6 \\
69.6 \\
51.2 \\
$1,610.5$
\end{tabular} & $\begin{array}{r}5,798.8 \\
3,390.0 \\
13.7 \\
1,654.8 \\
1,721.5 \\
1,554.5 \\
82.9 \\
84.1 \\
2,408.8\end{array}$ & $\begin{array}{r}7,860.2 \\
4,301.5 \\
17.7 \\
2,478.1 \\
1,805.7 \\
1,506.7 \\
169.7 \\
129.3 \\
3,558.6\end{array}$ & $\begin{array}{r}7,654.9 \\
4,098.6 \\
17.1 \\
2,511.7 \\
1,569.8 \\
1,355.5 \\
95.3 \\
118.9 \\
3,556.3\end{array}$ & $\begin{array}{r}7,638.0 \\
3,928.2 \\
14.6 \\
2,300.8 \\
1,612.8 \\
1,347.9 \\
129.7 \\
135.2 \\
3,709.8\end{array}$ & $\begin{array}{r}7,926.5 \\
4,006.4 \\
11.6 \\
2,322.2 \\
1,672.6 \\
1,373.0 \\
170.2 \\
129.4 \\
3,920.1\end{array}$ & $\begin{array}{r}8,533.9 \\
4,393.8 \\
12.0 \\
2,633.2 \\
1,748.6 \\
1,455.7 \\
125.7 \\
167.1 \\
4,140.1\end{array}$ & $\begin{array}{r}8,526.0 \\
4,111.9 \\
11.5 \\
2,501.1 \\
1,599.3 \\
1,303.6 \\
94.1 \\
201.6 \\
4,414.0\end{array}$ & $\begin{array}{r}8,785.5 \\
4,095.5 \\
11.2 \\
2,490.9 \\
1,593.4 \\
1,216.4 \\
110.6 \\
266.3 \\
4,690.1\end{array}$ \\
\hline 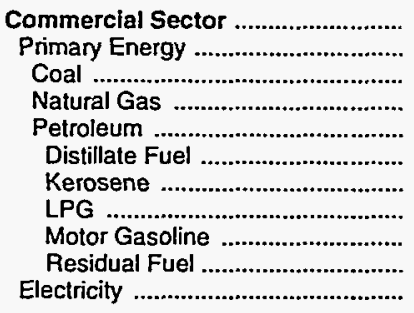 & $\begin{array}{r}1,325.0 \\
439.9 \\
3.2 \\
166.0 \\
270.8 \\
135.5 \\
2.6 \\
2.7 \\
16.1 \\
113.8 \\
885.0\end{array}$ & $\begin{array}{r}3,056.9 \\
919.0 \\
6.2 \\
256.7 \\
656.0 \\
273.8 \\
6.0 \\
6.2 \\
29.3 \\
340.7 \\
2,137.9\end{array}$ & $\begin{array}{r}5,194.8 \\
1,982.8 \\
7.0 \\
690.4 \\
1,285.4 \\
546.7 \\
5.4 \\
9.4 \\
55.7 \\
668.1 \\
3,212.0\end{array}$ & $\begin{array}{r}7,298.8 \\
2,138.1 \\
10.4 \\
1,010.8 \\
1,117.0 \\
484.4 \\
33.2 \\
24.5 \\
88.3 \\
486.6 \\
5,160.7\end{array}$ & $\begin{array}{r}7,079.0 \\
1,951.2 \\
11.1 \\
966.2 \\
973.9 \\
512.3 \\
5.9 \\
24.5 \\
64.3 \\
367.0 \\
5,127.8\end{array}$ & \begin{tabular}{r|}
$6,826.2$ \\
$1,795.3$ \\
8.8 \\
864.3 \\
922.1 \\
451.3 \\
8.6 \\
28.0 \\
50.2 \\
384.0 \\
$5,031.0$
\end{tabular} & $\begin{array}{r}6,995.6 \\
1,839.4 \\
7.3 \\
1,013.5 \\
818.6 \\
439.2 \\
5.7 \\
26.9 \\
41.6 \\
305.3 \\
5,156.2\end{array}$ & $\begin{array}{r}7,413.7 \\
2,016.6 \\
7.2 \\
1.103 .7 \\
905.7 \\
498.8 \\
15.3 \\
24.9 \\
55.4 \\
311.4 \\
5,397.1\end{array}$ & $\begin{array}{r}7,776.1 \\
2,111.3 \\
6.3 \\
1,090.0 \\
1,015.0 \\
506.1 \\
10.5 \\
27.0 \\
55.4 \\
416.2 \\
5,664.8\end{array}$ & $\begin{array}{r}7,825.3 \\
1,957.7 \\
6.4 \\
1,091.9 \\
859.3 \\
477.0 \\
7.0 \\
35.7 \\
35.5 \\
304.0 \\
5,867.6\end{array}$ \\
\hline 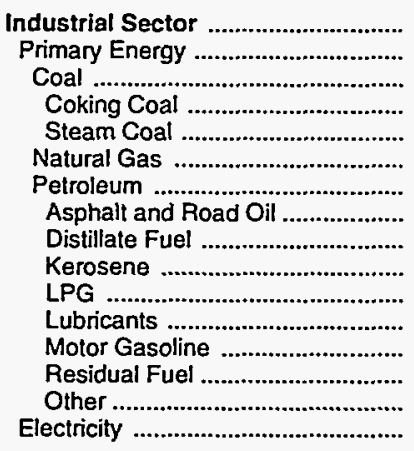 & $\begin{array}{r}903.2 \\
581.2 \\
164.5 \\
96.4 \\
68.1 \\
80.0 \\
336.7 \\
26.0 \\
68.8 \\
3.3 \\
5.6 \\
30.9 \\
50.3 \\
103.2 \\
48.5 \\
322.1\end{array}$ & $\begin{array}{r}1,961.1 \\
1,226.5 \\
283.3 \\
197.8 \\
85.5 \\
156.0 \\
787.2 \\
69.9 \\
216.9 \\
14.8 \\
11.4 \\
45.3 \\
34.1 \\
276.6 \\
118.2 \\
734.6\end{array}$ & $\begin{array}{r}3,374.0 \\
2,056.0 \\
304.2 \\
197.6 \\
106.6 \\
398.4 \\
1,353.4 \\
121.3 \\
289.8 \\
13.4 \\
43.8 \\
89.5 \\
82.7 \\
337.3 \\
375.5 \\
1,318.1\end{array}$ & $\begin{array}{r}3,238.1 \\
1,737.7 \\
180.4 \\
58.5 \\
121.9 \\
526.2 \\
1,031.1 \\
230.1 \\
172.1 \\
47.6 \\
42.2 \\
99.8 \\
56.5 \\
162.0 \\
220.7 \\
1,500.4\end{array}$ & $\begin{array}{r}2,673.8 \\
1,289.9 \\
143.1 \\
58.1 \\
85.0 \\
425.4 \\
721.5 \\
185.3 \\
75.5 \\
16.1 \\
38.5 \\
86.4 \\
43.4 \\
110.9 \\
165.4 \\
1,383.9\end{array}$ & $\begin{array}{r}2,662.8 \\
1,246.4 \\
141.3 \\
61.6 \\
79.6 \\
398.7 \\
706.4 \\
162.0 \\
97.2 \\
17.1 \\
35.8 \\
79.6 \\
47.1 \\
90.1 \\
177.5 \\
1,416.4\end{array}$ & $\begin{array}{r}2,702.4 \\
1,241.8 \\
150.7 \\
72.6 \\
78.1 \\
412.8 \\
678.2 \\
168.7 \\
93.9 \\
24.4 \\
28.9 \\
88.3 \\
53.1 \\
69.0 \\
151.9 \\
1,460.6\end{array}$ & $\begin{array}{r}2,896.6 \\
1,263.8 \\
160.9 \\
74.1 \\
86.8 \\
449.8 \\
653.0 \\
92.7 \\
104.8 \\
44.5 \\
26.7 \\
82.4 \\
57.0 \\
71.1 \\
173.8 \\
1,632.7\end{array}$ & $\begin{array}{r}3,154.0 \\
1,338.5 \\
143.0 \\
62.2 \\
80.8 \\
473.7 \\
721.8 \\
108.4 \\
123.0 \\
9.7 \\
22.7 \\
85.5 \\
52.8 \\
95.7 \\
223.9 \\
1,815.5\end{array}$ & $\begin{array}{r}3,296.4 \\
1,378.5 \\
142.4 \\
56.6 \\
85.9 \\
561.4 \\
674.6 \\
117.0 \\
93.4 \\
11.1 \\
45.5 \\
88.0 \\
54.4 \\
42.4 \\
222.8 \\
1,918.0\end{array}$ \\
\hline 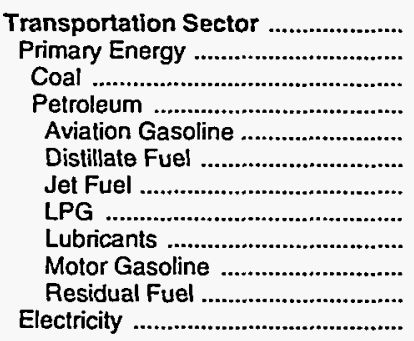 & $\begin{array}{r}2,299.1 \\
2,267.8 \\
.2 \\
2,267.6 \\
2.7 \\
89.5 \\
155.5 \\
.6 \\
36.9 \\
1,939.4 \\
43.0 \\
31.3\end{array}$ & $\begin{array}{r}4,140.7 \\
4,043.8 \\
4,043.7 \\
4.8 \\
173.7 \\
423.1 \\
1.4 \\
43.1 \\
3,304.6 \\
93.0 \\
96.9\end{array}$ & $\begin{array}{r}8,915.6 \\
8,809.9 \\
- \\
8,809.9 \\
14.6 \\
447.5 \\
1,274.5 \\
1.7 \\
92.7 \\
6,727.2 \\
251.7 \\
105.6\end{array}$ & $\begin{array}{r}7,256.8 \\
7,106.5 \\
- \\
7,106.5 \\
11.1 \\
669.3 \\
139.0 \\
6.3 \\
103.4 \\
6,154.6 \\
22.7 \\
150.3\end{array}$ & $\begin{array}{r}5,598.6 \\
5,446.3 \\
- \\
5,446.3 \\
10.9 \\
595.4 \\
91.8 \\
4.6 \\
89.5 \\
4,632.5 \\
21.8 \\
152.3\end{array}$ & $\begin{array}{r}6,168.7 \\
5,997.8 \\
- \\
5,997.8 \\
4.8 \\
658.1 \\
72.8 \\
3.6 \\
82.5 \\
5,137.1 \\
38.9 \\
170.9\end{array}$ & $\begin{array}{r}6,078.2 \\
5,908.5 \\
- \\
5,908.5 \\
3.9 \\
728.4 \\
113.6 \\
5.0 \\
91.5 \\
4,924.1 \\
42.0 \\
169.7\end{array}$ & $\begin{array}{r}6,622.0 \\
6,439.1 \\
- \\
6,439.1 \\
3.7 \\
809.0 \\
158.9 \\
5.5 \\
85.4 \\
5,367.9 \\
8.8 \\
182.8\end{array}$ & $\begin{array}{r}7,983.5 \\
7,790.3 \\
- \\
7,790.3 \\
3.6 \\
1,170.8 \\
183.5 \\
5.7 \\
88.6 \\
6,311.0 \\
27.1 \\
193.2\end{array}$ & $\begin{array}{r}8,098.4 \\
7,909.0 \\
- \\
7,909.0 \\
2.8 \\
1,047.4 \\
147.6 \\
6.3 \\
91.2 \\
6,554.3 \\
59.3 \\
189.4\end{array}$ \\
\hline 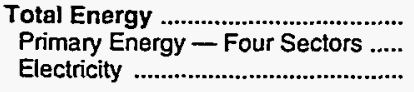 & $\begin{array}{l}6,371.7 \\
4,365.4 \\
2,006.4\end{array}$ & $\begin{array}{r}12,649.1 \\
8,069.1 \\
4,580.0\end{array}$ & $\begin{array}{r}23,283.2 \\
16,238.8 \\
7,044.4\end{array}$ & $\begin{array}{l}25,653.8 \\
15,283.9 \\
10,369.9\end{array}$ & $\begin{array}{l}23,006.3 \\
12,786.1 \\
10,220.2\end{array}$ & $\begin{array}{l}23,295.6 \\
12,967.6 \\
10,328.0\end{array}$ & $\begin{array}{l}23,702.7 \\
12,996.1 \\
10,706.6\end{array}$ & $\begin{array}{l}25,466.1 \\
14,113.4 \\
11,352.8\end{array}$ & $\begin{array}{l}27,439.5 \\
15,352.0 \\
12,087.5\end{array}$ & $\begin{array}{l}28,005.6 \\
15,340.6 \\
12,665.0\end{array}$ \\
\hline 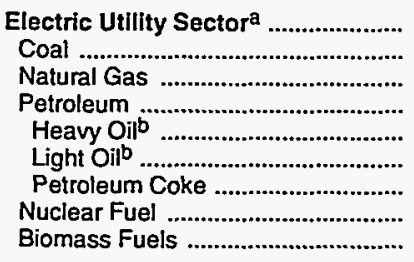 & $\begin{array}{r}335.3 \\
127.6 \\
40.9 \\
157.7 \\
149.6 \\
8.1 \\
-\overline{2} \\
9 .\end{array}$ & $\begin{array}{r}1,326.8 \\
173.3 \\
12.2 \\
1,096.4 \\
1,029.8 \\
66.6 \\
\overline{-} \\
44.9 \\
-\end{array}$ & $\begin{array}{r}2,427.3 \\
233.8 \\
343.4 \\
1,731.5 \\
1,706.9 \\
24.5 \\
-\overline{-} \\
118.3 \\
.2\end{array}$ & $\begin{array}{r}2,320.5 \\
337.5 \\
622.1 \\
1,185.7 \\
1,156.5 \\
29.2 \\
\overline{-} \\
175.2 \\
-\end{array}$ & $\begin{array}{r}1,558.8 \\
264.2 \\
309.9 \\
838.4 \\
808.5 \\
29.9 \\
- \\
146.3 \\
-\end{array}$ & $\begin{array}{r}1,917.7 \\
308.9 \\
435.8 \\
1,018.3 \\
982.7 \\
35.7 \\
-\overline{6} \\
154.6\end{array}$ & $\begin{array}{r}1,892.7 \\
369.5 \\
342.3 \\
1,032.6 \\
982.6 \\
50.0 \\
- \\
148.3 \\
-\end{array}$ & $\begin{array}{r}2,303.0 \\
409.5 \\
440.2 \\
1,294.1 \\
1,199.2 \\
94.9 \\
- \\
159.1 \\
-\end{array}$ & $\begin{array}{r}2,383.6 \\
414.1 \\
549.3 \\
1,255.5 \\
1,218.0 \\
37.5 \\
- \\
164.7 \\
-\end{array}$ & $\begin{array}{r}1,848.0 \\
406.8 \\
486.2 \\
785.7 \\
758.4 \\
27.3 \\
- \\
169.4 \\
-\end{array}$ \\
\hline Primary Energy - Five Sectors ${ }^{c}$.. & $4,700.7$ & $9,395.9$ & $18,666.0$ & $17,604.4$ & $14,344.9$ & $14,885.3$ & $14,888.8$ & $16,416.4$ & $17,735.7$ & $17,188.6$ \\
\hline
\end{tabular}

a There are no direct fuel costs for hydroelectric, geothermal, centralized solar, or wind energy.

b Heavy oil includes Grade Nos. 4, 5, and 6, and residual fuel oils. Light oil includes Grade No. 2 heating oil, kerosene, and jet fuel.

c Biomass fuels are not included, except those consumed at electric utilities and those added to motor gasoline.

- No consumption, including cases where adjustments were made. See "Consumption Adjustments for Process Fuel and Intermediate Products."

Value less than 0.05 million dollars.

Note: Totals may not equal sum of components due to independent rounding.

Sources: Data sources, estimation procedures, and assumptions are described in the "Documentation" section of this report. 


\begin{tabular}{|c|c|c|c|c|c|c|c|c|c|c|}
\hline Energy Source & 1970 & 1975 & 1980 & 1985 & 1986 & 1987 & 1988 & 1989 & 1990 & 1991 \\
\hline & \multicolumn{10}{|c|}{ Prices in Dollars per Million Btu } \\
\hline 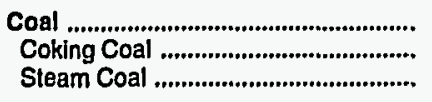 & $\begin{array}{r}0.43 \\
.43\end{array}$ & $\frac{1.12}{1.12}$ & $\frac{1.58}{1.58}$ & $\begin{array}{r}1.97 \\
-\overline{7}\end{array}$ & $\begin{array}{r}1.85 \\
1.85\end{array}$ & $\frac{1.79}{1.79}$ & $\begin{array}{r}1.77 \\
1.77\end{array}$ & $\begin{array}{r}1.77 \\
-\overline{7}\end{array}$ & $\begin{array}{r}1.79 \\
1.79\end{array}$ & $\begin{array}{r}1.77 \\
-\overline{7}\end{array}$ \\
\hline 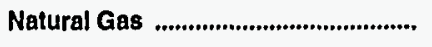 & .69 & 1.57 & 3.55 & 5.29 & 4.77 & 4.66 & 4.46 & 4.56 & 4.19 & 4.09 \\
\hline 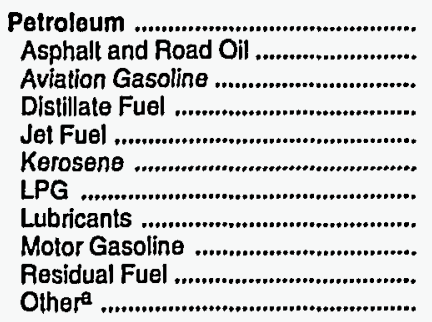 & $\begin{array}{r}1.94 \\
.67 \\
2.17 \\
1.13 \\
.73 \\
1.32 \\
1.89 \\
5.08 \\
2.82 \\
.46 \\
1.10\end{array}$ & $\begin{array}{l}3.68 \\
1.80 \\
3.45 \\
2.74 \\
2.03 \\
2.86 \\
3.24 \\
7.48 \\
4.55 \\
1.90 \\
2.54\end{array}$ & $\begin{array}{r}8.15 \\
3.57 \\
9.02 \\
6.80 \\
6.46 \\
7.67 \\
6.11 \\
14.36 \\
9.91 \\
3.72 \\
7.44\end{array}$ & $\begin{array}{r}8.21 \\
4.94 \\
9.99 \\
7.52 \\
5.77 \\
8.57 \\
9.72 \\
17.61 \\
9.03 \\
4.45 \\
7.23\end{array}$ & $\begin{array}{r}6.12 \\
4.22 \\
8.41 \\
5.75 \\
3.85 \\
6.60 \\
9.02 \\
15.59 \\
6.69 \\
2.72 \\
4.78\end{array}$ & $\begin{array}{r}6.46 \\
3.23 \\
7.55 \\
5.71 \\
3.96 \\
5.60 \\
8.71 \\
12.70 \\
7.33 \\
2.94 \\
5.90\end{array}$ & $\begin{array}{r}6.32 \\
3.18 \\
7.41 \\
5.60 \\
3.71 \\
5.74 \\
8.76 \\
14.61 \\
7.26 \\
2.34 \\
4.96\end{array}$ & $\begin{array}{r}7.11 \\
2.89 \\
8.28 \\
6.37 \\
4.30 \\
6.78 \\
8.82 \\
13.30 \\
8.10 \\
2.70 \\
5.76\end{array}$ & $\begin{array}{r}8.41 \\
2.95 \\
9.32 \\
8.05 \\
5.65 \\
8.38 \\
10.31 \\
13.40 \\
9.44 \\
3.11 \\
6.38\end{array}$ & $\begin{array}{r}8.12 \\
3.08 \\
8.71 \\
7.56 \\
4.79 \\
7.85 \\
11.09 \\
15.42 \\
9.23 \\
2.37 \\
5.10\end{array}$ \\
\hline Nuclear Fual ........................................... & - & .29 & .36 & .54 & .53 & .56 & .59 & .55 & .54 & .52 \\
\hline Blomass Fuels at Utllitles ................ & - & - & - & - & - & - & - & - & - & - \\
\hline Prlmary Energy - Flve Sectors ${ }^{b}$.... & 1.20 & 2.47 & 4.63 & 4.78 & 3.80 & 3.87 & 3.83 & 4.05 & 4.63 & 4.40 \\
\hline $\begin{array}{l}\text { Electric Utility Fuelc .......................... } \\
\text { Electricity Purchased by End Users }\end{array}$ & $\begin{array}{r}.41 \\
4.17\end{array}$ & $\begin{array}{l}1.05 \\
7.92\end{array}$ & $\begin{array}{r}1.48 \\
11.72\end{array}$ & $\begin{array}{r}1.56 \\
17.46\end{array}$ & $\begin{array}{r}1.46 \\
18.16\end{array}$ & $\begin{array}{r}1.29 \\
18.05\end{array}$ & $\begin{array}{r}1.30 \\
18.03\end{array}$ & $\begin{array}{r}1.31 \\
18.33\end{array}$ & $\begin{array}{r}1.33 \\
18.74\end{array}$ & $\begin{array}{r}1.27 \\
18.96\end{array}$ \\
\hline \multirow[t]{2}{*}{ Total Energy ..................................... } & 2.02 & 4.11 & 8.02 & 9.55 & 8.38 & 8.61 & 8.49 & 9.17 & 10.01 & 9.95 \\
\hline & \multicolumn{10}{|c|}{ Expenditures in Millions of Dollars } \\
\hline 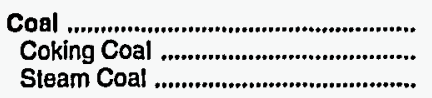 & 210.3 & $\begin{array}{r}533.2 \\
533 . \overline{2}\end{array}$ & 985.6 & $\begin{array}{r}1,084.5 \\
1,084 . \overline{5}\end{array}$ & $\begin{array}{r}1,077.4 \\
- \\
1,077.4\end{array}$ & $\begin{array}{r}894.0 \\
894.0\end{array}$ & $\begin{array}{r}912.8 \\
-\overline{8}\end{array}$ & $\begin{array}{r}982.3 \\
982.3\end{array}$ & $\begin{array}{r}945.7 \\
-\overline{7}\end{array}$ & $\begin{array}{r}925.6 \\
-\overline{6}\end{array}$ \\
\hline Natural Gas ......................................... & 102.8 & 178.1 & 529.3 & 705.5 & 644.6 & 677.9 & 662.9 & 718.8 & 656.5 & 675.7 \\
\hline 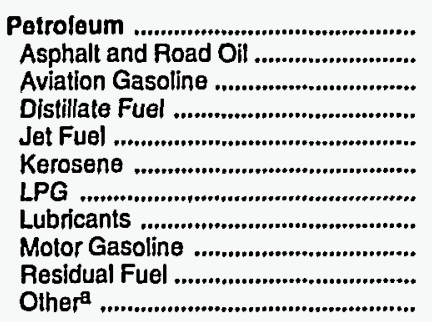 & $\begin{array}{r}1,201.9 \\
16.1 \\
1.7 \\
149.3 \\
18.7 \\
86.6 \\
39.1 \\
26.2 \\
835.7 \\
19.7 \\
8.8\end{array}$ & $\begin{array}{r}2,354.1 \\
36.4 \\
3.8 \\
339.0 \\
42.3 \\
94.7 \\
77.6 \\
42.8 \\
1,599.1 \\
92.9 \\
25.5\end{array}$ & $\begin{array}{r}5,423.4 \\
73.3 \\
9.8 \\
955.5 \\
185.3 \\
141.7 \\
179.1 \\
105.0 \\
3,448.9 \\
211.1 \\
113.6\end{array}$ & $\begin{array}{r}5,663.5 \\
113.2 \\
8.8 \\
1,087.3 \\
213.6 \\
231.9 \\
264.4 \\
117.2 \\
3,362.0 \\
174.3 \\
90.8\end{array}$ & $\begin{array}{r}4,527.8 \\
127.0 \\
9.6 \\
924.9 \\
152.9 \\
147.5 \\
239.4 \\
101.4 \\
2,601.7 \\
108.4 \\
115.0\end{array}$ & $\begin{array}{r}4,874.3 \\
86.3 \\
8.3 \\
943.6 \\
171.2 \\
116.3 \\
278.6 \\
93.5 \\
2,947.9 \\
100.7 \\
127.8\end{array}$ & $\begin{array}{r}5,012.1 \\
94.8 \\
8.8 \\
1,028.8 \\
172.1 \\
156.3 \\
245.8 \\
103.7 \\
3,015.8 \\
76.7 \\
109.4\end{array}$ & $\begin{array}{r}5,302.8 \\
72.3 \\
9.6 \\
1,003.9 \\
184.0 \\
129.6 \\
295.3 \\
96.7 \\
3,306.4 \\
80.3 \\
124.7\end{array}$ & $\begin{array}{r}6,025.9 \\
82.4 \\
10.0 \\
1,175.0 \\
174.2 \\
77.2 \\
322.1 \\
100.4 \\
3,823.8 \\
101.2 \\
159.5\end{array}$ & $\begin{array}{r}5,849.0 \\
78.2 \\
7.5 \\
1,054.3 \\
129.5 \\
86.2 \\
413.2 \\
103.3 \\
3,734.6 \\
91.1 \\
151.1\end{array}$ \\
\hline 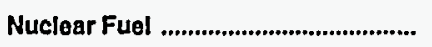 & - & 4.4 & 22.9 & 111.8 & 115.7 & 171.2 & 183.3 & 173.4 & 150.4 & 169.8 \\
\hline Blomass Fuels at Utilitles .................. & - & - & - & - & - & - & - & - & - & - \\
\hline Prlmary Energy - Five Sectors ${ }^{b}$.... & $1,515.0$ & $3,069.8$ & $6,961.2$ & $7,565.3$ & $6,365.6$ & $6,617.4$ & $6,771.2$ & $7,177.2$ & $7,778.6$ & $7,620.0$ \\
\hline $\begin{array}{l}\text { Electric Utility Fuelc ........................... } \\
\text { Electricity Purchased by End Users }\end{array}$ & $\begin{array}{r}-190.7 \\
576.2\end{array}$ & $\begin{array}{r}-473.6 \\
1,393.1\end{array}$ & $\begin{array}{r}-967.2 \\
2,553.8\end{array}$ & $\begin{array}{r}-1,097.2 \\
4,305.3\end{array}$ & $\begin{array}{r}-1,079.1 \\
4,797.8\end{array}$ & $\begin{array}{r}-960.0 \\
5,051.7\end{array}$ & $\begin{array}{r}-989.4 \\
5,228.3\end{array}$ & $\begin{array}{r}-1,058.0 \\
5,479.4\end{array}$ & $\begin{array}{r}-973.5 \\
5,715.3\end{array}$ & $\begin{array}{r}-992.1 \\
5,973.0\end{array}$ \\
\hline 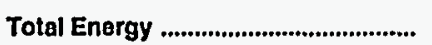 & $1,900.4$ & $3,989.4$ & $8,547.8$ & $10,773.4$ & $10,084.3$ & $10,709.1$ & $11,010.0$ & $11,598.7$ & $12,520.4$ & $12,600.9$ \\
\hline
\end{tabular}

Includes petroleum coke used at electric utilities.

b Biomass fuels are not included, except those consumed at electric utilities and those added to motor gasoline.

c There are no direct fuel costs for hydroelectric, geothermal, centralized solar, or wind energy.

- No consumption, including cases where adjustments were made. See "Consumption Adjustments for Process Fuel and Intermediate Products."

Note: Expenditure totals may not equal sum of components due to independent rounding.

Sources: Data sources, estimation procedures, and assumptions are described in the "Documentation" section of this report. 
N Energy Price Estimates by Sector, North Carolina $1970,1975,1980$, and 1985-1991

R (Dollars per Million Btu)

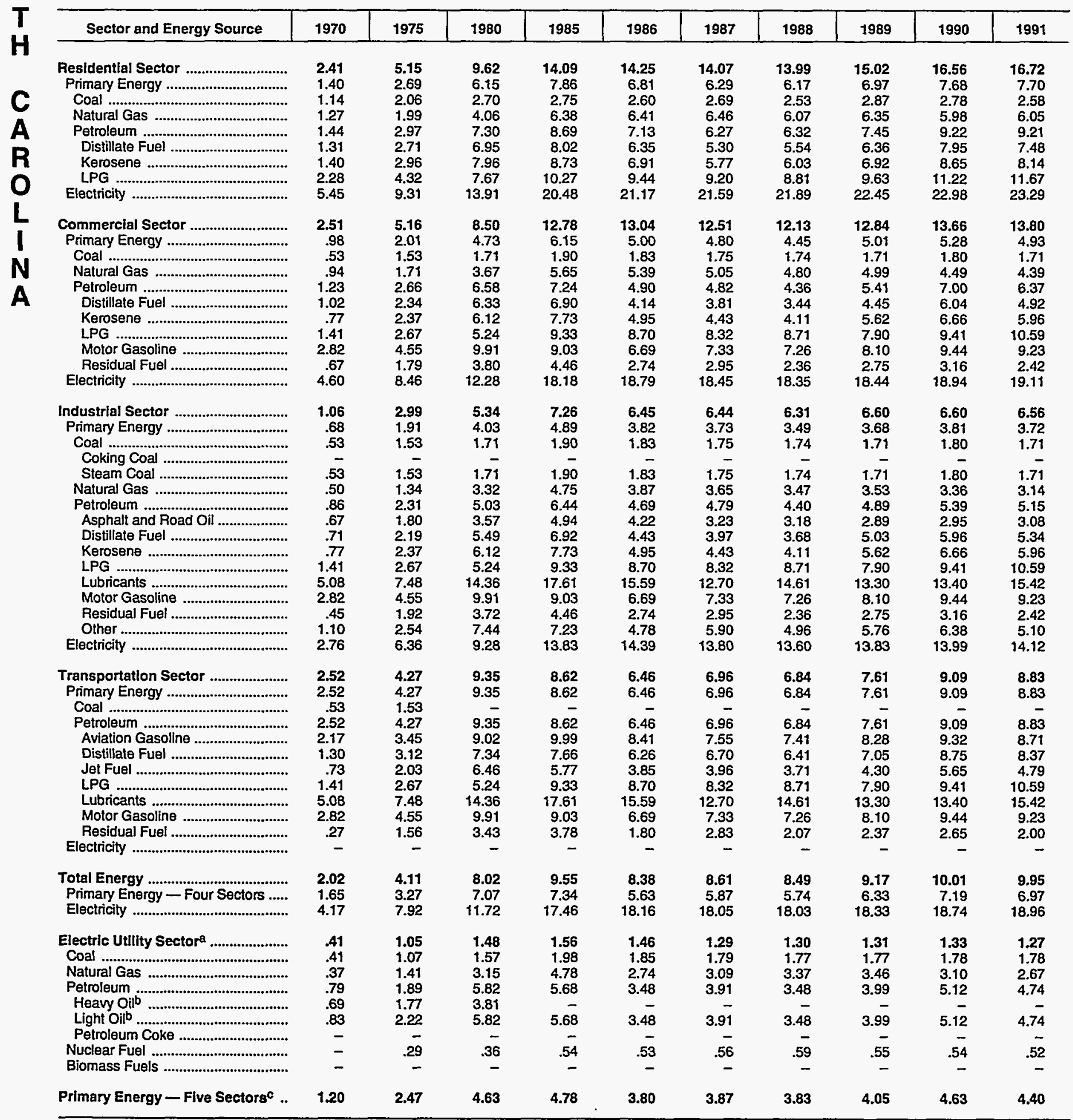

a There are no direct fuel costs for hydroelectric, geothermal, centralized solar, or wind energy.

beavy oil includes Grade Nos. 4, 5, and 6, and residual fuel olls. Light oll includes Grade No. 2 heating oil, kerosene, and jet fuel.

c Biomass fuels are not included, except those consumed at electric utilities and those added to motor gasoline.

-No consumption, including cases where adjustments were made. See "Consumption Adjustments for Process Fuel and Intermediate Products."

Sources: Data sources, estimation procedures, and assumptions are described in the "Documentation" section of this report. 


\begin{tabular}{|c|c|c|c|c|c|c|c|c|c|c|}
\hline Sector and Energy Source & 1970 & 1975 & 1980 & 1985 & 1986 & 1987 & 1988 & 1989 & 1990 & 1991 \\
\hline 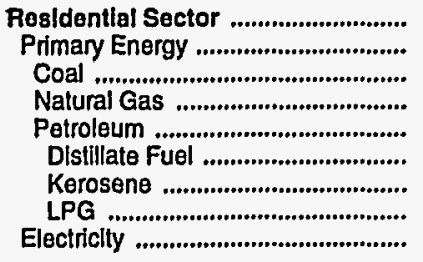 & $\begin{array}{r}483.8 \\
211.3 \\
4.1 \\
35.6 \\
171.6 \\
65.9 \\
79.8 \\
25.9 \\
272.5\end{array}$ & $\begin{array}{r}897.9 \\
294.6 \\
6.3 \\
55.6 \\
232.8 \\
114.6 \\
82.2 \\
36.0 \\
603.3\end{array}$ & $\begin{array}{r}1,789.6 \\
633.0 \\
3.9 \\
139.6 \\
489.4 \\
285.2 \\
124.0 \\
80.2 \\
1,156.6\end{array}$ & $\begin{array}{r}2,614.2 \\
737.7 \\
4.7 \\
189.1 \\
543.9 \\
228.0 \\
197.7 \\
118.2 \\
1,876.5\end{array}$ & $\begin{array}{r}2,768.3 \\
636.9 \\
3.5 \\
209.8 \\
423.5 \\
184.1 \\
130.1 \\
109.3 \\
2,131.4\end{array}$ & $\begin{array}{r}2,975.1 \\
653.9 \\
3.8 \\
239.1 \\
410.9 \\
175.5 \\
105.0 \\
130.5 \\
2,321.2\end{array}$ & $\begin{array}{r}3,090.4 \\
684.4 \\
4.5 \\
239.9 \\
440.0 \\
185.0 \\
139.4 \\
115.6 \\
2,406.0\end{array}$ & $\begin{array}{r}3,230.4 \\
719.4 \\
3.8 \\
253.2 \\
462.3 \\
173.1 \\
118.1 \\
171.1 \\
2,511.0\end{array}$ & $\begin{array}{r}3,226.4 \\
627.4 \\
3.8 \\
216.0 \\
407.6 \\
164.6 \\
69.0 \\
173.9 \\
2,599.0\end{array}$ & $\begin{array}{r}3,390.9 \\
657.9 \\
2.2 \\
237.0 \\
418.7 \\
139.6 \\
77.3 \\
201.9 \\
2,733.0\end{array}$ \\
\hline 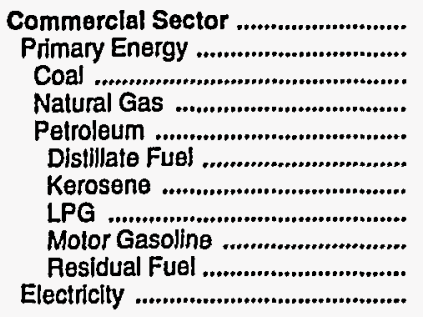 & $\begin{array}{r}196.5 \\
44.3 \\
3.6 \\
20.7 \\
20.0 \\
10.1 \\
1.0 \\
2.8 \\
5.3 \\
.8 \\
152.2\end{array}$ & $\begin{array}{r}420.8 \\
83.8 \\
8.6 \\
37.7 \\
37.4 \\
19.4 \\
1.6 \\
3.9 \\
9.9 \\
2.6 \\
337.0\end{array}$ & $\begin{array}{r}827.5 \\
230.1 \\
4.6 \\
97.1 \\
128.3 \\
61.7 \\
4.1 \\
9.7 \\
41.1 \\
11.7 \\
597.4\end{array}$ & $\begin{array}{r}1,516.2 \\
327.3 \\
6.0 \\
146.2 \\
175.1 \\
106.4 \\
10.7 \\
18.9 \\
30.0 \\
9.0 \\
1,188.9\end{array}$ & $\begin{array}{r}1,591.3 \\
254.2 \\
4.6 \\
141.7 \\
107.9 \\
58.4 \\
4.8 \\
17.8 \\
22.8 \\
4.1 \\
1,337.2\end{array}$ & $\begin{array}{r}1,671.4 \\
279.4 \\
4.6 \\
156.4 \\
118.4 \\
65.1 \\
3.5 \\
20.8 \\
27.8 \\
1.2 \\
1,392.0\end{array}$ & $\begin{array}{r}1,731.5 \\
284.5 \\
5.8 \\
160.4 \\
118.3 \\
61.9 \\
6.0 \\
20.1 \\
26.1 \\
4.2 \\
1,447.0\end{array}$ & $\begin{array}{r}1,823.5 \\
296.5 \\
4.2 \\
170.4 \\
121.9 \\
61.0 \\
5.6 \\
24.8 \\
26.6 \\
3.9 \\
1,526.9\end{array}$ & $\begin{array}{r}1,938.3 \\
289.3 \\
4.6 \\
144.8 \\
139.9 \\
68.1 \\
3.0 \\
25.7 \\
38.5 \\
4.5 \\
1,649.0\end{array}$ & $\begin{array}{r}1,987.7 \\
265.8 \\
2.7 \\
155.4 \\
107.6 \\
52.2 \\
3.2 \\
32.3 \\
18.2 \\
1.8 \\
1,722.0\end{array}$ \\
\hline 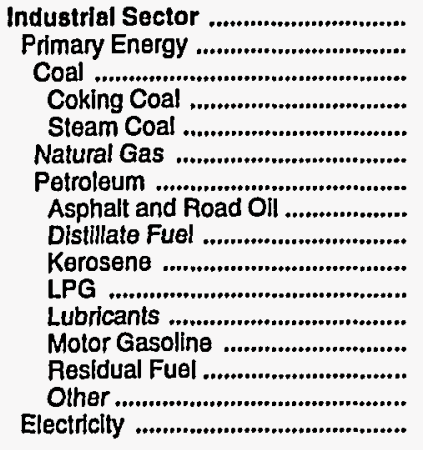 & $\begin{array}{r}319.4 \\
168.0 \\
28.7 \\
28 . \overline{7} \\
38.4 \\
100.8 \\
16.1 \\
18.6 \\
5.8 \\
10.1 \\
10.1 \\
14.9 \\
16.5 \\
8.8 \\
151.4\end{array}$ & $\begin{array}{r}878.6 \\
425.7 \\
53.2 \\
53.2 \\
54.2 \\
84.6 \\
287.9 \\
36.4 \\
54.6 \\
10.9 \\
36.6 \\
20.2 \\
18.7 \\
85.1 \\
25.5 \\
452.8\end{array}$ & $\begin{array}{r}1,838.7 \\
1,038.9 \\
57.3 \\
5-\overline{5} \\
287.3 \\
694.6 \\
73.3 \\
132.0 \\
13.7 \\
88.2 \\
49.7 \\
26.8 \\
197.3 \\
113.6 \\
799.8\end{array}$ & $\begin{array}{r}2,450.3 \\
1,210.4 \\
106.1 \\
- \\
106.1 \\
367.2 \\
737.1 \\
113.2 \\
130.5 \\
23.5 \\
121.1 \\
55.5 \\
39.5 \\
163.0 \\
90.8 \\
1,239.9\end{array}$ & $\begin{array}{r}2,394.5 \\
1,065.3 \\
116.2 \\
-\overline{1} \\
116.2 \\
289.8 \\
659.3 \\
127.0 \\
118.4 \\
12.5 \\
107.0 \\
48.0 \\
28.6 \\
102.7 \\
115.0 \\
1,329.2\end{array}$ & $\begin{array}{r}2,324.6 \\
986.1 \\
110.3 \\
-\overline{3} \\
110.3 \\
278.7 \\
597.1 \\
86.3 \\
87.6 \\
7.9 \\
123.5 \\
44.3 \\
31.6 \\
88.0 \\
127.8 \\
1,338.5\end{array}$ & $\begin{array}{r}2,288.0 \\
912.7 \\
109.4 \\
-\overline{1} \\
109.4 \\
258.9 \\
544.4 \\
94.8 \\
79.5 \\
10.9 \\
105.4 \\
49.1 \\
28.2 \\
67.1 \\
109.4 \\
1,375.3\end{array}$ & $\begin{array}{r}2,388.7 \\
947.2 \\
108.5 \\
- \\
108.5 \\
289.3 \\
549.4 \\
72.3 \\
103.8 \\
5.8 \\
95.0 \\
45.8 \\
35.6 \\
66.4 \\
124.7 \\
1,441.5\end{array}$ & $\begin{array}{r}2,528.7 \\
1,061.3 \\
133.2 \\
-\overline{3} \\
133.2 \\
287.9 \\
640.2 \\
82.4 \\
100.7 \\
5.3 \\
116.9 \\
47.5 \\
39.8 \\
88.1 \\
159.5 \\
1,467.3\end{array}$ & $\begin{array}{r}2,579.2 \\
1,061.2 \\
116.3 \\
116.3 \\
116.3 \\
275.0 \\
670.0 \\
78.2 \\
92.5 \\
5.7 \\
171.9 \\
48.9 \\
41.7 \\
79.9 \\
151.1 \\
1,518.0\end{array}$ \\
\hline Transportation Sector ...................... & $\begin{array}{l}900.7 \\
900.7\end{array}$ & $\begin{array}{l}1,792.1 \\
1,792.1\end{array}$ & $\begin{array}{l}4,092.0 \\
4,092.0\end{array}$ & $\begin{array}{l}4,192.7 \\
4,192.7\end{array}$ & $\begin{array}{l}3,330.0 \\
3,330.0\end{array}$ & $\begin{array}{l}3,738.0 \\
3,738.0\end{array}$ & $\begin{array}{l}3,900.1 \\
3,900.1\end{array}$ & $\begin{array}{l}4,156.2 \\
4,156.2\end{array}$ & $\begin{array}{l}4,827.1 \\
4,827.1\end{array}$ & $\begin{array}{l}4,643.0 \\
4,643.0\end{array}$ \\
\hline 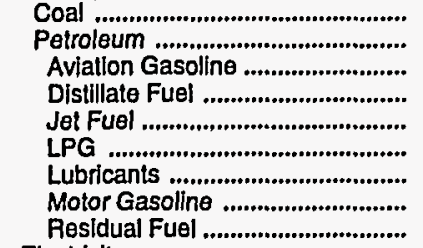 & $\begin{array}{r}900.7 \\
1.7 \\
47.8 \\
18.7 \\
.3 \\
16.1 \\
815.5 \\
.6\end{array}$ & \begin{tabular}{r|}
$1,792.1$ \\
3.8 \\
149.2 \\
42.3 \\
1.1 \\
22.6 \\
$1,570.5$ \\
2.6
\end{tabular} & $\begin{array}{r}4,092 . \overline{0} \\
9.8 \\
457.5 \\
185.3 \\
1.0 \\
55.3 \\
3,381.0 \\
2.1\end{array}$ & $\begin{array}{r}4,192 . \overline{7} \\
8.8 \\
607.6 \\
213.6 \\
6.2 \\
61.7 \\
3,292.5 \\
2.3\end{array}$ & $\begin{array}{r}3,330 . \overline{0} \\
9.6 \\
556.9 \\
152.9 \\
5.4 \\
53.4 \\
2,550.3 \\
1.5\end{array}$ & $\begin{array}{r}3,738.0 \\
8.3 \\
605.5 \\
171.2 \\
3.8 \\
49.2 \\
2,888.5 \\
11.6\end{array}$ & $\begin{array}{r}3,900 . \overline{1} \\
8.8 \\
693.0 \\
172.1 \\
4.7 \\
54.6 \\
2,961.5 \\
5.4\end{array}$ & $\begin{array}{r}4,156 . \overline{2} \\
9.6 \\
653.0 \\
184.0 \\
4.5 \\
50.9 \\
3,244.2 \\
10.0\end{array}$ & $\begin{array}{r}4,827 . \overline{1} \\
10.0 \\
830.4 \\
174.2 \\
5.5 \\
52.8 \\
3,745.5 \\
8.7\end{array}$ & $\begin{array}{r}4,643.0 \\
7.5 \\
760.4 \\
129.5 \\
7.1 \\
54.4 \\
3,674.8 \\
9.4\end{array}$ \\
\hline Electricity .............................................. & - & - & - & - & - & - & - & - & - & - \\
\hline $\begin{array}{l}\text { Total Energy ...................................... } \\
\text { Primary Energy - Four Sectors ...... } \\
\text { Electricity .......................................... }\end{array}$ & $\begin{array}{r}1,900.4 \\
1,324.3 \\
576.2\end{array}$ & $\begin{array}{l}3,989.4 \\
2,596.2 \\
1,393.1\end{array}$ & $\begin{array}{l}8,547.8 \\
5,994.0 \\
2,553.8\end{array}$ & $\begin{array}{r}10,773.4 \\
6,468.1 \\
4,305.3\end{array}$ & $\begin{array}{r}10,084.3 \\
5,286.5 \\
4,797.8\end{array}$ & $\begin{array}{r}10,709.1 \\
5,657.4 \\
5,051.7\end{array}$ & $\begin{array}{r}11,010.0 \\
5,781.7 \\
5,228.3\end{array}$ & $\begin{array}{r}11,598.7 \\
6,119.2 \\
5,479.4\end{array}$ & $\begin{array}{r}12,520.4 \\
6,805.1 \\
5,715.3\end{array}$ & $\begin{array}{r}12,600.9 \\
6,627.9 \\
5,973.0\end{array}$ \\
\hline 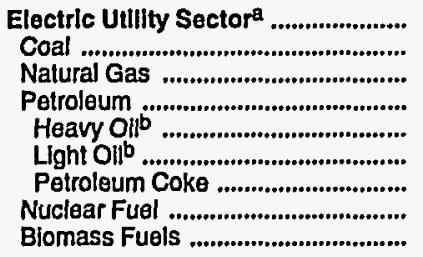 & $\begin{array}{r}190.7 \\
173.8 \\
8.0 \\
8.9 \\
1.9 \\
6.9 \\
- \\
-\end{array}$ & $\begin{array}{r}473.6 \\
465.1 \\
.1 \\
3.9 \\
2.6 \\
1.2 \\
-\overline{4} \\
-\end{array}$ & $\begin{array}{r}967.2 \\
919.7 \\
5.5 \\
19.0 \\
\vdots \\
19.0 \\
- \\
22.9 \\
-\end{array}$ & $\begin{array}{r}1,097.2 \\
967.8 \\
2.9 \\
14.7 \\
-\overline{7} \\
14.7 \\
-\overline{8} \\
111.8 \\
-\end{array}$ & $\begin{array}{r}1,079.1 \\
953.1 \\
3.2 \\
7.1 \\
-\overline{1} \\
7.1 \\
- \\
115.7 \\
-\end{array}$ & $\begin{array}{r}960.0 \\
775.2 \\
3.7 \\
9.9 \\
\overline{-} \\
9.9 \\
\overline{-} \\
171 . \overline{2} \\
-\end{array}$ & $\begin{array}{r}989.4 \\
793.1 \\
3.7 \\
9.3 \\
- \\
9.3 \\
- \\
183.3 \\
-\end{array}$ & $\begin{array}{r}1,058.0 \\
865.7 \\
6.0 \\
12.9 \\
-\overline{9} \\
12.9 \\
-\overline{4} \\
173.4 \\
-\end{array}$ & $\begin{array}{r}973.5 \\
804.0 \\
7.9 \\
11.1 \\
11 . \overline{1} \\
-\overline{4} \\
150.4 \\
-\end{array}$ & $\begin{array}{r}992.1 \\
804.4 \\
8.3 \\
9.6 \\
-\overline{6} \\
9 . \overline{8} \\
169 . \overline{-}\end{array}$ \\
\hline Primary Energy - Flve Sectorsc .. & $1,515.0$ & $3,069.8$ & $6,961.2$ & $7,565,3$ & $6,365.6$ & $6,617.4$ & $6,771.2$ & $7,177.2$ & $7,778.6$ & $7,620.0$ \\
\hline
\end{tabular}

a There are no direct fuel costs for hydroelectric, geothermal, centralized solar, or wind energy.

b Heavy oll includes Grade Nos. 4, 5, and 6, and residual fuel oils. Light oil includes Grade No. 2 heating oil, kerosene, and jet fuel.

c Blomass fuels are not Included, except those consumed at electric utilities and those added to motor gasoline.

-No consumption, including cases where adjustments were made. See "Consumption Adjustments for Process Fuel and Intermediate Products." Value less than 0.05 million dollars.

Note: Totals may not equal sum of components due to independent rounding.

Sources: Data sources, estimation procedures, and assumptions are described in the "Documentation" section of this report. 


\begin{tabular}{|c|c|c|c|c|c|c|c|c|c|c|}
\hline Energy Source & 1970 & 1975 & 1980 & 1985 & 1986 & 1987 & 1988 & 1989 & 1990 & 1991 \\
\hline & \multicolumn{10}{|c|}{ Prices in Dollars per Million Btu } \\
\hline 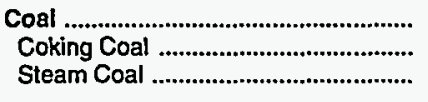 & $\begin{array}{r}0.34 \\
. \\
.34\end{array}$ & $\begin{array}{r}0.42 \\
.42\end{array}$ & $\begin{array}{r}0.67 \\
-67\end{array}$ & $\frac{1.44}{1.44}$ & $\begin{array}{r}1.43 \\
1 . \overline{4}\end{array}$ & $\begin{array}{r}1.35 \\
1.35\end{array}$ & $\frac{1.15}{1.15}$ & $\frac{1.21}{1.21}$ & $\begin{array}{r}1.18 \\
1.18\end{array}$ & $\begin{array}{r}1.16 \\
1.16\end{array}$ \\
\hline 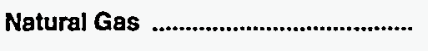 & .78 & 1.26 & 3.41 & 4.97 & 4.73 & 4.65 & 4.34 & 4.08 & 4.12 & 4.15 \\
\hline 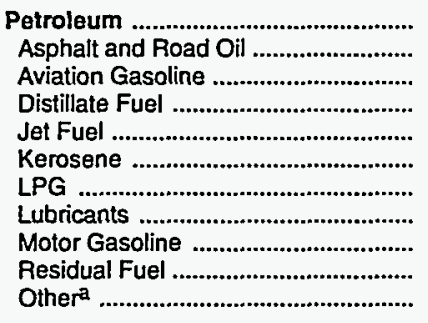 & $\begin{array}{r}1.83 \\
.79 \\
2.17 \\
1.07 \\
.75 \\
1.47 \\
1.87 \\
5.08 \\
2.83 \\
.91 \\
.51\end{array}$ & $\begin{array}{l}3.55 \\
2.08 \\
3.45 \\
2.66 \\
2.09 \\
2.84 \\
3.29 \\
7.48 \\
4.69 \\
1.80 \\
1.40\end{array}$ & $\begin{array}{r}7.70 \\
3.85 \\
9.02 \\
6.59 \\
6.47 \\
6.30 \\
6.12 \\
14.36 \\
9.97 \\
3.58 \\
2.48\end{array}$ & $\begin{array}{r}7.94 \\
5.17 \\
9.99 \\
6.96 \\
6.44 \\
8.03 \\
8.37 \\
17.61 \\
9.64 \\
3.49 \\
2.31\end{array}$ & $\begin{array}{r}5.84 \\
4.07 \\
8.41 \\
4.90 \\
4.90 \\
4.58 \\
7.48 \\
15.59 \\
6.92 \\
1.93 \\
1.53\end{array}$ & $\begin{array}{r}6.27 \\
3.74 \\
7.55 \\
5.73 \\
4.64 \\
5.59 \\
5.28 \\
12.70 \\
7.53 \\
1.61 \\
1.50\end{array}$ & $\begin{array}{r}6.33 \\
3.53 \\
7.41 \\
5.39 \\
4.37 \\
4.52 \\
5.08 \\
14.61 \\
8.05 \\
2.49 \\
1.45\end{array}$ & $\begin{array}{r}6.94 \\
3.13 \\
8.28 \\
6.12 \\
4.77 \\
5.91 \\
5.39 \\
13.30 \\
8.88 \\
2.58 \\
1.74\end{array}$ & $\begin{array}{r}8.06 \\
3.32 \\
9.32 \\
7.41 \\
6.11 \\
7.28 \\
7.01 \\
13.40 \\
9.87 \\
2.64 \\
2.02\end{array}$ & $\begin{array}{r}7.68 \\
3.36 \\
8.71 \\
6.72 \\
5.17 \\
6.58 \\
7.73 \\
15.42 \\
9.57 \\
2.35 \\
1.72\end{array}$ \\
\hline 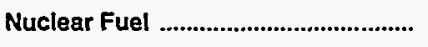 & - & - & - & - & - & - & - & - & - & - \\
\hline Biomass Fuels at Utilities ................... & - & - & - & - & - & - & - & - & - & - \\
\hline Primary Energy - Flve Sectors ${ }^{b}$.... & 1.26 & 2.23 & 3.66 & 3.30 & 2.71 & 2.69 & 2.41 & 2.61 & 2.73 & 2.69 \\
\hline $\begin{array}{l}\text { Electric Utility Fuelc } \\
\text { Electricity Purchased by End Users }\end{array}$ & $\begin{array}{r}.25 \\
7.04\end{array}$ & $\begin{array}{r}.27 \\
8.57\end{array}$ & $\begin{array}{r}.57 \\
11.96\end{array}$ & $\begin{array}{r}.89 \\
17.11\end{array}$ & $\begin{array}{r}.83 \\
17.30\end{array}$ & $\begin{array}{r}.80 \\
17.15\end{array}$ & $\begin{array}{r}.71 \\
16.93\end{array}$ & $\begin{array}{r}.70 \\
16.74\end{array}$ & $\begin{array}{r}.69 \\
16.86\end{array}$ & $\begin{array}{r}.71 \\
16.90\end{array}$ \\
\hline \multirow[t]{2}{*}{ Total Energy .......................................... } & 1.97 & 3.47 & 7.28 & 7.08 & 5.90 & 5.86 & 5.91 & 6.04 & 6.60 & 6.48 \\
\hline & \multicolumn{10}{|c|}{ Expenditures in Millions of Dollars } \\
\hline 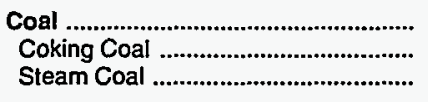 & $\begin{array}{r}19.5 \\
19.5\end{array}$ & $28 . \overline{-}$ & $\begin{array}{r}110.2 \\
110 . \overline{2}\end{array}$ & $\begin{array}{l}436.0 \\
436 . \overline{0}\end{array}$ & $\begin{array}{r}444.2 \\
444 . \overline{2}\end{array}$ & $\begin{array}{r}430.8 \\
\overline{-} \\
430.8\end{array}$ & $\begin{array}{r}424.0 \\
\overline{-} \\
424.0\end{array}$ & $\begin{array}{r}436.6 \\
\overline{-} \\
436.6\end{array}$ & $\begin{array}{r}443.9 \\
443.9\end{array}$ & $\begin{array}{r}441.5 \\
- \\
441.5\end{array}$ \\
\hline 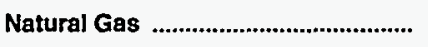 & 14.9 & 31.1 & 77.6 & 118.4 & 103.3 & 89.4 & 103.4 & 105.4 & 98.9 & 110.1 \\
\hline 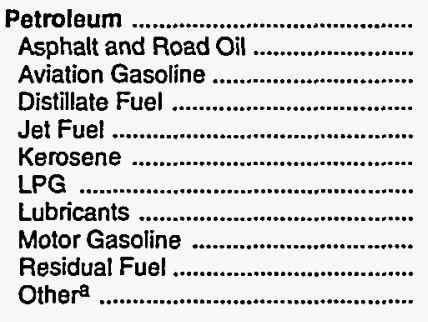 & $\begin{array}{r}201.2 \\
7.3 \\
1.0 \\
30.9 \\
8.3 \\
2.0 \\
12.1 \\
5.1 \\
130.2 \\
3.2 \\
.8\end{array}$ & $\begin{array}{r}393.6 \\
14.6 \\
1.5 \\
68.8 \\
20.9 \\
1.1 \\
19.3 \\
7.2 \\
247.6 \\
10.0 \\
2.6\end{array}$ & $\begin{array}{r}938.4 \\
19.2 \\
2.9 \\
312.6 \\
59.7 \\
.5 \\
29.0 \\
15.5 \\
480.1 \\
13.6 \\
5.3\end{array}$ & $\begin{array}{r}887.8 \\
35.9 \\
.2 \\
303.8 \\
58.3 \\
.7 \\
16.3 \\
17.2 \\
446.7 \\
6.2 \\
2.3\end{array}$ & $\begin{array}{r}657.0 \\
23.7 \\
1.6 \\
210.9 \\
43.5 \\
.4 \\
46.4 \\
14.9 \\
311.7 \\
3.1 \\
.8\end{array}$ & $\begin{array}{r}682.5 \\
22.0 \\
1.1 \\
227.5 \\
31.5 \\
.2 \\
33.8 \\
13.8 \\
348.8 \\
2.6 \\
1.3\end{array}$ & $\begin{array}{r}681.3 \\
22.4 \\
1.2 \\
212.4 \\
31.0 \\
.4 \\
29.5 \\
29.5 \\
15.3 \\
363.7 \\
4.0 \\
1.4\end{array}$ & $\begin{array}{r}750.8 \\
19.2 \\
1.3 \\
249.9 \\
34.5 \\
.4 \\
34.6 \\
14.2 \\
391.7 \\
3.5 \\
1.5\end{array}$ & $\begin{array}{r}826.6 \\
17.9 \\
1.3 \\
291.9 \\
39.0 \\
.2 \\
35.4 \\
14.8 \\
420.1 \\
4.0 \\
1.8\end{array}$ & $\begin{array}{r}830.6 \\
17.3 \\
1.2 \\
290.1 \\
31.5 \\
.4 \\
55.7 \\
15.2 \\
414.9 \\
2.8 \\
1.4\end{array}$ \\
\hline 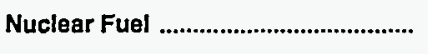 & - & - & - & - & - & - & - & - & - & - \\
\hline Blomass Fuels at Utillties ................. & - & - & - & - & - & - & - & - & - & - \\
\hline Primery Energy - Five Sectors ${ }^{b} \ldots$ & 235.6 & 453.5 & $1,126.3$ & $1,442.2$ & $1,204.5$ & $1,202.7$ & $1,208.7$ & $1,292.8$ & $1,369.3$ & $1,382.2$ \\
\hline 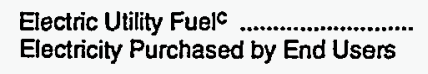 & $\begin{array}{r}-12.3 \\
67.3\end{array}$ & $\begin{array}{r}-15.8 \\
108.0\end{array}$ & $\begin{array}{l}-87.9 \\
210.2\end{array}$ & $\begin{array}{r}-203.4 \\
407.5\end{array}$ & $\begin{array}{r}-188.0 \\
401.2\end{array}$ & $\begin{array}{r}-183.8 \\
383.2\end{array}$ & $\begin{array}{r}-201.8 \\
407.3\end{array}$ & $\begin{array}{r}-189.4 \\
400.6\end{array}$ & $\begin{array}{r}-198.3 \\
400.8\end{array}$ & $\begin{array}{r}-209.5 \\
415.8\end{array}$ \\
\hline 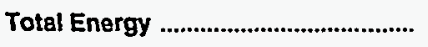 & 290.6 & 545.7 & $1,248.6$ & $1,646.2$ & $1,417.7$ & $1,402.1$ & $1,414.2$ & $1,503.9$ & $1,571.8$ & $1,588.5$ \\
\hline
\end{tabular}

Includes petroleum coke used at electric utilities.

b Biomass fuels are not included, except those consumed at electric utilities and those added to motor gasoline.

c There are no direct fuel costs for hydroelectric, geothermal, centralized solar, or wind energy.

-No consumption, including cases where adjustments were made. See "Consumption Adjustments for Process Fuel and Intermediate Products."

Note: Expenditure totals may not equal sum of components due to independent rounding.

Sources: Data sources, estimation procedures, and assumptions are described in the "Documentation" section of this report. 
Energy Price Estimates by Sector, North Dakota

$1970,1975,1980$, and 1985-1991

(Dollars per Million Btu)

\begin{tabular}{|c|c|c|c|c|c|c|c|c|c|c|}
\hline Sector and Energy Source & 1970 & 1975 & 1980 & 1985 & 1986 & 1987 & 1988 & 1989 & 1990 & 1991 \\
\hline 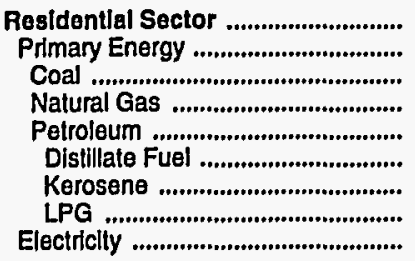 & $\begin{array}{r}2.54 \\
1.37 \\
1.56 \\
.99 \\
1.62 \\
1.28 \\
1.65 \\
2.07 \\
7.80\end{array}$ & $\begin{array}{l}3.95 \\
2.25 \\
3.09 \\
1.51 \\
3.02 \\
2.55 \\
2.69 \\
3.51 \\
9.18\end{array}$ & $\begin{array}{r}7.52 \\
5.12 \\
1.96 \\
3.66 \\
7.04 \\
6.92 \\
7.39 \\
7.48 \\
13.14\end{array}$ & $\begin{array}{r}10.18 \\
5.97 \\
1.74 \\
5.26 \\
7.57 \\
7.48 \\
8.09 \\
8.46 \\
18.02\end{array}$ & $\begin{array}{r}9.56 \\
4.98 \\
1.53 \\
5.08 \\
5.19 \\
4.84 \\
5.24 \\
6.13 \\
18.20\end{array}$ & $\begin{array}{r}10.00 \\
5.14 \\
1.45 \\
5.22 \\
5.28 \\
5.34 \\
5.77 \\
5.13 \\
18.44\end{array}$ & $\begin{array}{r}9.57 \\
4.74 \\
1.15 \\
4.88 \\
4.85 \\
4.66 \\
5.04 \\
5.24 \\
18.26\end{array}$ & $\begin{array}{r}9.57 \\
5.08 \\
1.13 \\
4.46 \\
6.20 \\
5.55 \\
6.00 \\
7.32 \\
18.01\end{array}$ & $\begin{array}{r}10.24 \\
5.55 \\
1.10 \\
4.55 \\
7.23 \\
6.87 \\
7.42 \\
7.98 \\
18.35\end{array}$ & $\begin{array}{r}9.82 \\
5.48 \\
3.81 \\
4.61 \\
6.66 \\
6.38 \\
6.89 \\
7.09 \\
18.18\end{array}$ \\
\hline 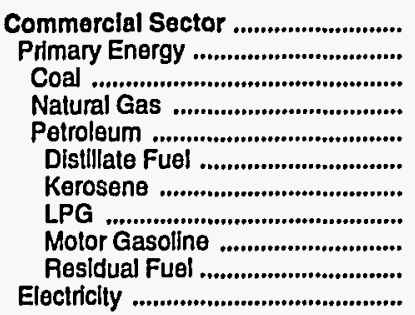 & $\begin{array}{r}1.74 \\
.89 \\
.74 \\
.67 \\
1.45 \\
1.06 \\
-\overline{1.30} \\
2.83 \\
.84 \\
6.62\end{array}$ & $\begin{array}{l}2.23 \\
1.43 \\
1.26 \\
1.11 \\
2.23 \\
2.34 \\
- \\
2.63 \\
4.69 \\
1.69 \\
7.84\end{array}$ & $\begin{array}{r}5.39 \\
4.06 \\
2.63 \\
3.26 \\
5.63 \\
6.45 \\
5.23 \\
9.97 \\
3.78 \\
12.16\end{array}$ & $\begin{array}{r}8.78 \\
5.03 \\
3.21 \\
4.81 \\
6.47 \\
6.42 \\
7.38 \\
8.32 \\
9.64 \\
3.49 \\
17.54\end{array}$ & $\begin{array}{r}8.67 \\
4.36 \\
3.11 \\
4.59 \\
4.26 \\
3.47 \\
3.98 \\
8.25 \\
6.92 \\
1.92 \\
17.53\end{array}$ & $\begin{array}{r}9.14 \\
4.43 \\
2.80 \\
4.53 \\
4.70 \\
4.19 \\
5.03 \\
5.37 \\
7.53 \\
1.61 \\
17.25\end{array}$ & $\begin{array}{r}8.55 \\
4.14 \\
2.66 \\
4.25 \\
4.46 \\
3.31 \\
3.96 \\
4.95 \\
8.05 \\
2.49 \\
17.41\end{array}$ & $\begin{array}{r}8.05 \\
3.96 \\
2.74 \\
3.99 \\
4.61 \\
4.17 \\
5.19 \\
3.60 \\
8.88 \\
2.58 \\
16.95\end{array}$ & $\begin{array}{r}8.93 \\
4.23 \\
2.82 \\
4.06 \\
6.16 \\
5.17 \\
6.48 \\
6.16 \\
9.87 \\
2.64 \\
17.07\end{array}$ & $\begin{array}{r}8.98 \\
4.31 \\
2.70 \\
4.15 \\
6.40 \\
4.53 \\
5.63 \\
8.34 \\
9.57 \\
2.35 \\
17.12\end{array}$ \\
\hline 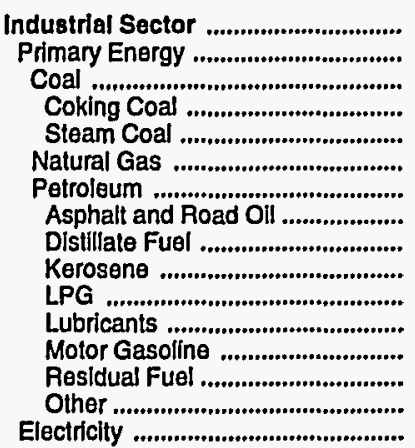 & $\begin{array}{r}1.52 \\
1.30 \\
.74 \\
\overline{.74} \\
.38 \\
.38 \\
1.45 \\
.79 \\
.79 \\
.85 \\
1.30 \\
5.08 \\
2.83 \\
.94 \\
.51 \\
5.95\end{array}$ & $\begin{array}{l}3.12 \\
2.73 \\
1.26 \\
- \\
1.26 \\
1.00 \\
3.16 \\
2.08 \\
2.72 \\
2.90 \\
2.63 \\
7.48 \\
4.69 \\
1.94 \\
1.40 \\
8.00\end{array}$ & $\begin{array}{r}5.87 \\
5.37 \\
2.63 \\
- \\
2.63 \\
2.58 \\
6.07 \\
3.85 \\
5.50 \\
5.76 \\
5.23 \\
14.36 \\
9.97 \\
3.19 \\
2.48 \\
9.94\end{array}$ & $\begin{array}{r}4.96 \\
4.31 \\
3.21 \\
-\overline{3} \\
3.21 \\
4.19 \\
6.72 \\
5.17 \\
6.70 \\
7.38 \\
8.32 \\
17.61 \\
9.64 \\
3.49 \\
2.31 \\
15.27\end{array}$ & $\begin{array}{r}4.17 \\
3.56 \\
3.11 \\
-\overline{3.11} \\
3.92 \\
4.60 \\
4.07 \\
3.61 \\
3.98 \\
8.25 \\
15.59 \\
6.92 \\
1.92 \\
1.53 \\
15.60\end{array}$ & $\begin{array}{r}3.89 \\
3.34 \\
2.80 \\
-\overline{2} \\
2.80 \\
3.09 \\
4.83 \\
3.74 \\
4.57 \\
5.03 \\
5.37 \\
12.70 \\
7.53 \\
1.61 \\
1.50 \\
15.05\end{array}$ & $\begin{array}{r}3.75 \\
3.12 \\
2.66 \\
- \\
2.66 \\
3.23 \\
4.33 \\
3.53 \\
3.60 \\
3.96 \\
4.95 \\
14.61 \\
8.05 \\
2.49 \\
1.45 \\
14.44\end{array}$ & $\begin{array}{r}3.85 \\
3.27 \\
2.74 \\
-\overline{1} \\
2.74 \\
3.38 \\
4.76 \\
3.13 \\
4.71 \\
5.19 \\
3.60 \\
13.30 \\
8.88 \\
2.58 \\
1.74 \\
14.56\end{array}$ & $\begin{array}{r}4.04 \\
3.56 \\
2.82 \\
- \\
2.82 \\
3.24 \\
5.77 \\
3.32 \\
5.89 \\
6.48 \\
6.16 \\
13.40 \\
9.87 \\
2.64 \\
2.02 \\
13.99\end{array}$ & $\begin{array}{r}3.98 \\
3.48 \\
2.70 \\
- \\
2.70 \\
3.05 \\
5.57 \\
3.36 \\
5.12 \\
5.63 \\
8.34 \\
15.42 \\
9.57 \\
2.35 \\
1.72 \\
14.30\end{array}$ \\
\hline 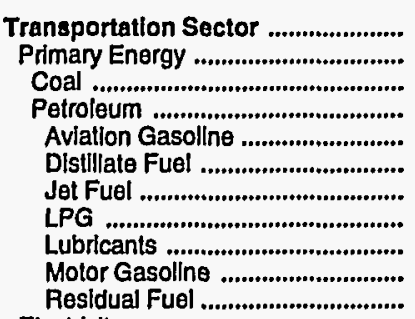 & $\begin{array}{r}2.19 \\
2.19 \\
.74 \\
2.19 \\
2.17 \\
1.33 \\
.75 \\
1.30 \\
5.08 \\
2.83 \\
.83\end{array}$ & $\begin{array}{l}3.95 \\
3.95 \\
1.26 \\
3.95 \\
3.45 \\
2.67 \\
2.09 \\
2.63 \\
7.48 \\
4.69 \\
-\end{array}$ & $\begin{array}{r}8.74 \\
8.74 \\
-\overrightarrow{84} \\
9.02 \\
7.23 \\
6.47 \\
5.23 \\
14.36 \\
9.97 \\
-\end{array}$ & $\begin{array}{r}8.65 \\
8.65 \\
-6 \\
8.65 \\
9.99 \\
7.12 \\
6.44 \\
8.32 \\
17.61 \\
9.64 \\
-\end{array}$ & $\begin{array}{r}6.64 \\
6.64 \\
-\overline{6} \\
6.64 \\
8.41 \\
6.46 \\
4.90 \\
8.25 \\
15.59 \\
6.92 \\
2.34\end{array}$ & $\begin{array}{r}7.16 \\
7.16 \\
- \\
7.16 \\
7.55 \\
6.97 \\
4.64 \\
5.37 \\
12.70 \\
7.53 \\
-\end{array}$ & $\begin{array}{r}7.49 \\
7.49 \\
7.49 \\
7.41 \\
7.14 \\
4.37 \\
4.95 \\
14.61 \\
8.05 \\
-\end{array}$ & $\begin{array}{r}8.17 \\
8.17 \\
8.17 \\
8.28 \\
7.73 \\
4.77 \\
3.60 \\
13.30 \\
8.88 \\
-\end{array}$ & $\begin{array}{r}9.28 \\
9.28 \\
-\overline{9} \\
9.28 \\
9.32 \\
8.96 \\
6.11 \\
6.16 \\
13.40 \\
9.87 \\
-\end{array}$ & $\begin{array}{r}8.92 \\
8.92 \\
-\overline{8} \\
8.92 \\
8.71 \\
8.50 \\
5.17 \\
8.34 \\
15.42 \\
9.57 \\
-\end{array}$ \\
\hline 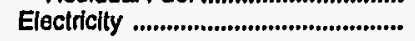 & - & - & - & - & - & - & - & - & - & - \\
\hline 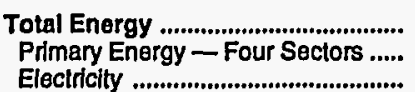 & $\begin{array}{l}1.97 \\
1.62 \\
7.04\end{array}$ & $\begin{array}{l}3.47 \\
3.03 \\
8.57\end{array}$ & $\begin{array}{r}7.28 \\
6.75 \\
11.96\end{array}$ & $\begin{array}{r}7.08 \\
5.93 \\
17.11\end{array}$ & $\begin{array}{r}5.90 \\
4.68 \\
17.30\end{array}$ & $\begin{array}{r}5.86 \\
4.70 \\
17.15\end{array}$ & $\begin{array}{r}5.91 \\
4.67 \\
16.93\end{array}$ & $\begin{array}{r}6.04 \\
4.90 \\
16.74\end{array}$ & $\begin{array}{r}6.60 \\
5.46 \\
16.86\end{array}$ & $\begin{array}{r}6.48 \\
5.32 \\
16.90\end{array}$ \\
\hline 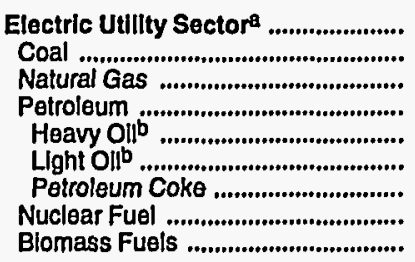 & $\begin{array}{r}.25 \\
.25 \\
.35 \\
.96 \\
.90 \\
1.23 \\
= \\
-\end{array}$ & $\begin{array}{r}.27 \\
.26 \\
.66 \\
1.94 \\
1.93 \\
2.12 \\
= \\
=\end{array}$ & $\begin{array}{r}.57 \\
.56 \\
2.47 \\
6.07 \\
\overline{67} \\
= \\
= \\
-\end{array}$ & $\begin{array}{r}.89 \\
.88 \\
4.74 \\
5.52 \\
-\overline{52} \\
5 . \\
= \\
-\end{array}$ & $\begin{array}{r}.83 \\
.82 \\
5.22 \\
3.71 \\
\overline{3} \\
3.71 \\
= \\
-\end{array}$ & $\begin{array}{r}.80 \\
.79 \\
4.43 \\
4.19 \\
\overline{-19} \\
= \\
=\end{array}$ & $\begin{array}{r}.71 \\
.70 \\
4.29 \\
3.81 \\
-\overline{3} \\
- \\
- \\
-\end{array}$ & $\begin{array}{r}.70 \\
.69 \\
4.39 \\
4.41 \\
4 . \overline{1} \\
= \\
=\end{array}$ & $\begin{array}{r}.69 \\
.69 \\
3.86 \\
5.60 \\
- \\
5.60 \\
= \\
=\end{array}$ & $\begin{array}{r}.71 \\
.71 \\
4.34 \\
4.49 \\
4 . \overline{-} \\
- \\
-\end{array}$ \\
\hline Prlmary Enorgy —Flve Sectors ${ }^{C}$.. & 1.26 & 2.23 & 3.66 & $\mathbf{3 . 3 0}$ & 2.71 & 2.69 & 2.41 & 2.61 & 2.73 & 2.69 \\
\hline
\end{tabular}

a There are no direct fuel costs for hydroelectric, geothermal, centralized solar, or wind energy.

6 Heavy oll includes Grade Nos. 4, 5, and 6, and residual fuel oils. Light oil includes Grade No. 2 heating oil, kerosene, and jet fuel.

c Blomass fuels are not included, except those consumed at electric utilities and those added to motor gasoline.

- No consumptlon, including cases where adjustments were made. See "Consumption Adjustments for Process Fuel and Intermediate Products."

Sources: Data sources, estimation procedures, and assumptions are described in the "Documentation" section of this report. 


\section{N Energy Expenditure Estimates by Sector, North Dakota \\ O $1970,1975,1980$, and $1985-1991$}

$\mathbf{R}$ (Million Dollars)

\begin{tabular}{|c|c|c|c|c|c|c|c|c|c|c|}
\hline Sector and Energy Source & 1970 & 1975 & 1980 & 1985 & 1986 & 1987 & 1988 & 1989 & 1990 & 1991 \\
\hline 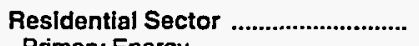 & 66.8 & 104.4 & 210.1 & 299.1 & 278.1 & 260.2 & 278.7 & 287.4 & 281.8 & 303.2 \\
\hline 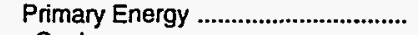 & 29.6 & 44.9 & 100.0 & 114.0 & 94.7 & 84.8 & 88.6 & 99.3 & 96.8 & 111.1 \\
\hline 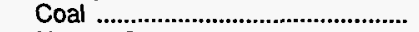 & 1.2 & 2.3 & 1.3 & 1.6 & 1.2 & .7 & .7 & .9 & .7 & 2.5 \\
\hline 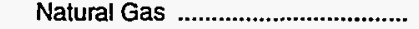 & 8.4 & 15.4 & 37.1 & 57.9 & 49.5 & 44.1 & 47.1 & 46.0 & 43.2 & 49.8 \\
\hline 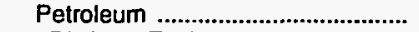 & 20.0 & 27.3 & 61.6 & 54.5 & 43.9 & 40.0 & 40.8 & 52.4 & 52.9 & 58.8 \\
\hline Distillate Fuel ........................................ & 8.2 & 11.5 & 47.3 & 48.8 & 29.8 & 27.8 & 26.2 & 29.5 & 33.8 & 33.5 \\
\hline 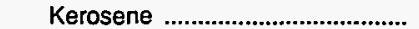 & 1.8 & .3 & .2 & .6 & .2 & .2 & .2 & .3 & .2 & .3 \\
\hline LPG & 10.0 & 15.4 & 14.0 & 5.2 & 13.9 & 12.0 & 14.4 & 22.6 & 18.9 & 25.0 \\
\hline 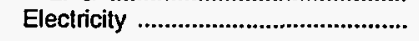 & 37.2 & 59.5 & 110.1 & 185.1 & 183.5 & 175.4 & 190.0 & 188.0 & 185.0 & 192.0 \\
\hline 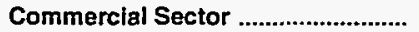 & 27.9 & 49.0 & 127.8 & 202.2 & 181.2 & 167.4 & 174.4 & 173.3 & 191.4 & 201.6 \\
\hline 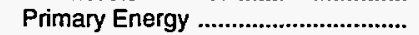 & 12.2 & 27.5 & 80.3 & 81.0 & 61.3 & 51.4 & 56.4 & 58.3 & 57.4 & 61.6 \\
\hline 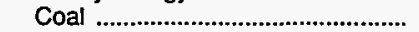 & 1.0 & 1.7 & 3.2 & 5.4 & 4.7 & 2.5 & 3.2 & 4.1 & 3.4 & 3.3 \\
\hline Natural Gas ........................................... & 5.8 & 13.7 & 37.8 & 51.7 & 43.5 & 37.6 & 44.0 & 44.5 & 42.9 & 46.6 \\
\hline 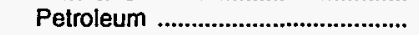 & 5.4 & 12.0 & 39.2 & 23.9 & 13.2 & 11.4 & 9.2 & 9.8 & 11.1 & 11.7 \\
\hline 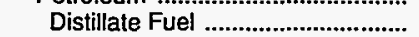 & 1.5 & 2.4 & 24.1 & 18.1 & 6.3 & 5.9 & 3.0 & 4.5 & 4.5 & 4.2 \\
\hline 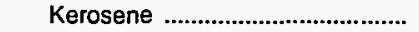 & - & - & - & & & & & & & \\
\hline LPG & 1.1 & 2.0 & 1.7 & .9 & 3.3 & 2.2 & 2.4 & 2.0 & 2.6 & 5.2 \\
\hline 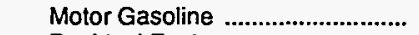 & 2.2 & 2.3 & 3.8 & 3.5 & 2.6 & 2.9 & 3.1 & 2.8 & 3.6 & 2.2 \\
\hline Residual Fuel ....................................... & .5 & 5.2 & 9.5 & 1.4 & .9 & .3 & .7 & .4 & .4 & .1 \\
\hline 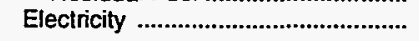 & 15.7 & 21.5 & 47.5 & 121.2 & 119.9 & 116.0 & 118.0 & 115.0 & 133.9 & 140.0 \\
\hline 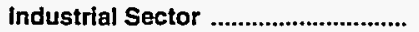 & 7.3 & 143.2 & 279.1 & 556.5 & 515.4 & 500.5 & 462.2 & 506.7 & 507.4 & 504.9 \\
\hline 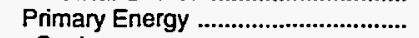 & 63.0 & 116.2 & 226.5 & 455.3 & 417.5 & 408.6 & 362.9 & 409.2 & 425.6 & 421.1 \\
\hline Coal & 5.4 & 9.4 & 20.2 & 228.0 & 251.5 & 245.1 & 219.4 & 244.0 & 243.2 & 228.0 \\
\hline 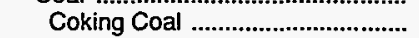 & - & - & - & - & - & - & - & - & - & - \\
\hline 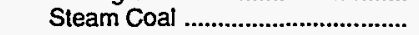 & 5.4 & 9.4 & 20.2 & 228.0 & 251.5 & 245.1 & 219.4 & 244.0 & 243.2 & 228.0 \\
\hline Natural Gas .................................. & .7 & 1.9 & 2.6 & 8.7 & 10.3 & 7.7 & 12.3 & 15.0 & 12.9 & 13.7 \\
\hline Petroleum & 56.9 & 105.0 & 203.7 & 218.6 & 155.7 & 155.8 & 131.3 & 150.1 & 169.5 & 179.4 \\
\hline 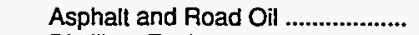 & 7.3 & 14.6 & 19.2 & 35.9 & 23.7 & 22.0 & 22.4 & 19.2 & 17.9 & 17.3 \\
\hline 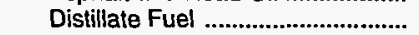 & 10.0 & 25.6 & 78.8 & 108.4 & 64.6 & 68.4 & 51.5 & 76.3 & 89.0 & 91.2 \\
\hline 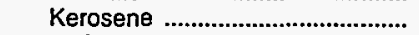 & .3 & .8 & .3 & .1 & .2 & $\because$ & .1 & 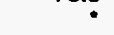 & & .1 \\
\hline LPG & 1.0 & 1.8 & 13.0 & 9.9 & 28.5 & 19.3 & 12.4 & 9.8 & 13.7 & 25.1 \\
\hline 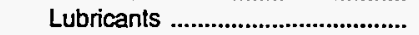 & .9 & 1.0 & 2.3 & 2.5 & 2.2 & 2.0 & 2.2 & 2.1 & 2.2 & 2.2 \\
\hline 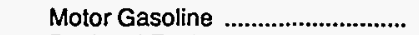 & 34.4 & 54.1 & 80.7 & 54.7 & 33.6 & 40.6 & 37.9 & 38.2 & 41.2 & 39.4 \\
\hline 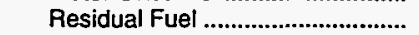 & 2.3 & 4.6 & 4.1 & 4.8 & 2.2 & 2.3 & 3.3 & 3.0 & 3.7 & 2.7 \\
\hline 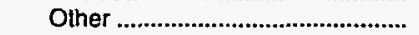 & .8 & 2.6 & 5.3 & 2.3 & .8 & 1.3 & 1.4 & 1.5 & 1.8 & 1.4 \\
\hline 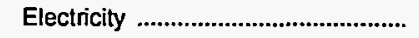 & 14.3 & 27.0 & 52.6 & 101.1 & 97.9 & 91.8 & 99.3 & 97.5 & 81.9 & 83.8 \\
\hline Transportation Sector .......................... & 118.6 & 249.1 & 631.5 & 588.4 & 443.0 & 474.1 & 499.0 & 536.5 & 591.3 & 578.8 \\
\hline 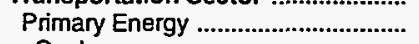 & 118.6 & 249.1 & 631.5 & 588.4 & 443.0 & 474.1 & 499.0 & 536.5 & 591.3 & 578.8 \\
\hline 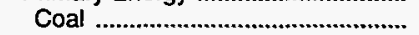 & & & & & & & & & & \\
\hline 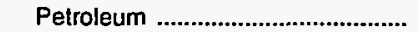 & 118.6 & 249.1 & 631.5 & 588.4 & 443.0 & 474.1 & 499.0 & 536.5 & 591.3 & 578.8 \\
\hline Aviation Gasoline .......................... & 1.0 & 1.5 & 2.9 & .2 & 1.6 & 1.1 & 1.2 & 1.3 & 1.3 & 1.2 \\
\hline 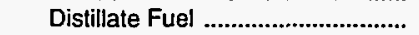 & 11.1 & 29.2 & 159.9 & 126.3 & 108.9 & 124.1 & 130.8 & 137.7 & 162.7 & 159.4 \\
\hline 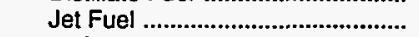 & 8.3 & 20.9 & 59.7 & 58.3 & 43.5 & 31.5 & 31.0 & 34.5 & 39.0 & 31.5 \\
\hline LPG & & & .2 & .3 & .7 & .3 & .3 & .2 & .3 & .5 \\
\hline 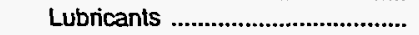 & 4.2 & 6.2 & 13.2 & 14.7 & 12.7 & 11.7 & 13.0 & 12.2 & 12.6 & 13.0 \\
\hline Motor Gasoline ...................................... & 93.6 & 191.2 & 395.6 & 388.6 & 275.5 & 305.4 & 322.7 & 350.6 & 375.3 & 373.3 \\
\hline 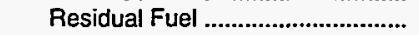 & .2 & - & - & - & * & - & - & - & - & - \\
\hline Electricity & - & - & - & - & - & - & - & - & - & - \\
\hline 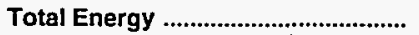 & 290.6 & $\mathbf{5 4 5 . 7}$ & $1,248.6$ & $1,646.2$ & $1,417.7$ & $1,402.1$ & $1,414.2$ & $1,503.9$ & $1,571.8$ & $1,588.5$ \\
\hline Primary Energy — Four Sectors ..... & 223.3 & 437.7 & $1,038.3$ & $1,238.7$ & $1,016.5$ & $1,018.9$ & $1,006.9$ & $1,103.3$ & $1,171.0$ & $1,172.7$ \\
\hline 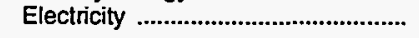 & 67.3 & 108.0 & 210.2 & 407.5 & 401.2 & 383.2 & 407.3 & 400.6 & 400.8 & 415.8 \\
\hline Electric Utility Sector ${ }^{\mathrm{a}}$ & 12.3 & 15.8 & 87.9 & 203.4 & 188.0 & 183.8 & 201.8 & 189.4 & 198.3 & 209.5 \\
\hline Coal & 12.0 & 15.4 & 85.5 & 201.1 & 186.8 & 182.5 & 200.8 & 187.6 & 196.5 & 207.7 \\
\hline 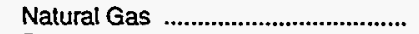 & .1 & .1 & * & * & & & & & & \\
\hline 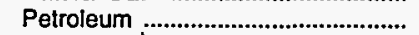 & .2 & .2 & 2.4 & 2.4 & 1.2 & 1.2 & 1.0 & 1.9 & 1.8 & 1.8 \\
\hline 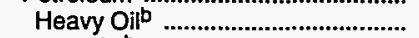 & .1 & .2 & - & - & - & - & - & - & - & - \\
\hline 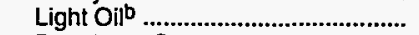 & $*$ & $*$ & 2.4 & 2.4 & 1.2 & 1.2 & 1.0 & 1.9 & 1.8 & 1.8 \\
\hline 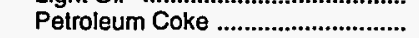 & - & - & - & - & - & - & - & - & - & - \\
\hline 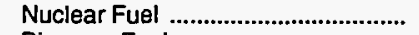 & - & - & - & - & - & - & - & - & - & - \\
\hline 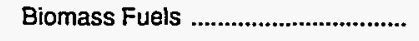 & - & - & - & - & - & - & - & - & - & - \\
\hline Primary Energy — Five Sectors ${ }^{c}$.. & 235.6 & 453.5 & $1,126.3$ & $1,442.2$ & $1,204.5$ & $1,202.7$ & $1,208.7$ & $1,292.8$ & $1,369.3$ & $1,382.2$ \\
\hline
\end{tabular}

a There are no direct fuel costs for hydroelectric, geothermal, centralized solar, or wind energy.

b Heavy oil includes Grade Nos. 4, 5, and 6, and residual fuel oils. Light oil includes Grade No. 2 heating oil, kerosene, and jet fuel.

c Biomass fuels are not included, except those consumed at electric utilities and those added to motor gasoline.

- No consumption, including cases where adjustments were made. See "Consumption Adjustments for Process Fuel and Intermediate Products."

- Value less than 0.05 million dollars.

Note: Totals may not equal sum of components due to independent rounding.

Sources: Data sources, estimation procedures, and assumptions are described in the "Documentation" section of this report. 


\begin{tabular}{|c|c|c|c|c|c|c|c|c|c|c|}
\hline Energy Source & 1970 & 1975 & 1980 & 1985 & 1986 & 1987 & 1988 & 1989 & 1990 & 1991 \\
\hline & \multicolumn{10}{|c|}{ Prices in Dollars per Million Btu } \\
\hline 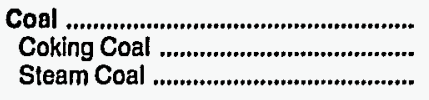 & $\begin{array}{r}0.35 \\
.42 \\
.33\end{array}$ & $\begin{array}{l}1.14 \\
1.57 \\
1.03\end{array}$ & $\begin{array}{l}1.56 \\
2.00 \\
1.47\end{array}$ & $\begin{array}{l}1.72 \\
2.05 \\
1.68\end{array}$ & $\begin{array}{l}1.66 \\
2.04 \\
1.62\end{array}$ & $\begin{array}{l}1.56 \\
1.72 \\
1.55\end{array}$ & $\begin{array}{l}1.53 \\
1.73 \\
1.51\end{array}$ & $\begin{array}{l}1.51 \\
1.75 \\
1.48\end{array}$ & $\begin{array}{l}1.54 \\
1.80 \\
1.51\end{array}$ & $\begin{array}{l}1.49 \\
1.72 \\
1.48\end{array}$ \\
\hline 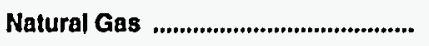 & .74 & 1.30 & 3.27 & 5.32 & 5.11 & 4.70 & 4.55 & 4.65 & 4.53 & 4.53 \\
\hline 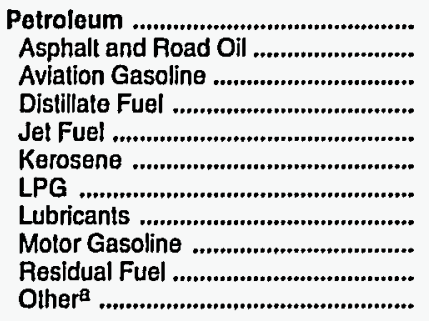 & $\begin{array}{r}2.17 \\
.74 \\
2.17 \\
1.13 \\
.74 \\
1.11 \\
1.73 \\
5.08 \\
2.93 \\
.61 \\
1.14\end{array}$ & $\begin{array}{l}3.79 \\
2.03 \\
3.45 \\
2.53 \\
2.09 \\
2.72 \\
3.73 \\
7.48 \\
4.73 \\
2.14 \\
2.36\end{array}$ & $\begin{array}{r}7.70 \\
3.66 \\
9.02 \\
6.44 \\
6.38 \\
6.87 \\
5.48 \\
14.36 \\
9.45 \\
3.34 \\
6.93\end{array}$ & $\begin{array}{r}8.63 \\
4.43 \\
9.99 \\
7.66 \\
6.04 \\
7.76 \\
9.51 \\
17.61 \\
9.15 \\
4.21 \\
7.57\end{array}$ & $\begin{array}{r}6.49 \\
3.70 \\
8.41 \\
5.81 \\
3.85 \\
6.07 \\
8.83 \\
15.59 \\
6.78 \\
2.60 \\
6.09\end{array}$ & $\begin{array}{r}6.77 \\
3.23 \\
7.55 \\
6.02 \\
4.04 \\
5.96 \\
8.37 \\
12.70 \\
7.36 \\
2.84 \\
6.34\end{array}$ & $\begin{array}{r}6.80 \\
3.23 \\
7.41 \\
5.89 \\
3.92 \\
5.80 \\
8.44 \\
14.61 \\
7.43 \\
2.50 \\
5.44\end{array}$ & $\begin{array}{r}7.32 \\
2.99 \\
8.28 \\
6.62 \\
4.49 \\
6.41 \\
9.31 \\
13.30 \\
8.18 \\
2.62 \\
5.83\end{array}$ & $\begin{array}{r}8.34 \\
3.13 \\
9.32 \\
7.79 \\
5.73 \\
7.72 \\
10.41 \\
13.40 \\
9.35 \\
2.60 \\
6.20\end{array}$ & $\begin{array}{r}8.17 \\
2.96 \\
8.71 \\
7.39 \\
4.95 \\
7.05 \\
10.04 \\
15.42 \\
9.23 \\
2.35 \\
5.65\end{array}$ \\
\hline Nuclear Fuel ......................................... & - & - & 28 & 1.09 & .72 & .73 & 1.33 & 1.32 & 124 & 1.14 \\
\hline Blomass Fuels at Utilities .................. & .65 & .92 & 1.74 & .79 & .32 & .95 & .87 & (d) & $(d)$ & $(d)$ \\
\hline Primary Energy — Five Sectors ${ }^{b} \ldots$ & .97 & 2.00 & 4.08 & 4.81 & 4.01 & 3.92 & 3.86 & 4.05 & 4.34 & 4.21 \\
\hline $\begin{array}{l}\text { Electric Utility Fue }\left.\right|^{c} \text {........................... } \\
\text { Electricity Purchased by End Users }\end{array}$ & $\begin{array}{r}.30 \\
4.68\end{array}$ & $\begin{array}{r}.98 \\
7.94\end{array}$ & $\begin{array}{r}1.50 \\
12.97\end{array}$ & $\begin{array}{r}1.70 \\
16.90\end{array}$ & $\begin{array}{r}1.63 \\
17.23\end{array}$ & $\begin{array}{r}1.51 \\
16.97\end{array}$ & $\begin{array}{r}1.52 \\
16.90\end{array}$ & $\begin{array}{r}1.48 \\
16.83\end{array}$ & $\begin{array}{r}1.50 \\
17.33\end{array}$ & $\begin{array}{r}1.45 \\
17.98\end{array}$ \\
\hline \multirow[t]{2}{*}{ 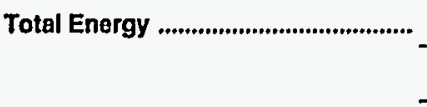 } & 1.50 & 3.05 & 6.23 & 8.25 & 7.34 & 7.34 & 7.23 & 7.63 & 8.23 & 8.38 \\
\hline & \multicolumn{10}{|c|}{ Expenditures In Millions of Dollers } \\
\hline 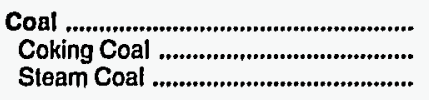 & $\begin{array}{l}556.1 \\
146.6 \\
409.5\end{array}$ & $\begin{array}{r}1,847.6 \\
519.3 \\
1,328.3\end{array}$ & $\begin{array}{r}2,390.1 \\
549.5 \\
1,840.7\end{array}$ & $\begin{array}{r}2,383.9 \\
287.8 \\
2,096.1\end{array}$ & $\begin{array}{r}2,373.3 \\
283.0 \\
2,090.3\end{array}$ & $\begin{array}{r}2,239.8 \\
246.8 \\
1,993.0\end{array}$ & $\begin{array}{r}2,260.2 \\
252.4 \\
2,007.8\end{array}$ & $\begin{array}{r}2,208.0 \\
247.6 \\
1,960.4\end{array}$ & $\begin{array}{r}2,194.3 \\
239.0 \\
1,955.3\end{array}$ & $\begin{array}{r}2,109.9 \\
170.7 \\
1,939.3\end{array}$ \\
\hline Natural Gas ......................................... & 769.2 & $1,243.3$ & $2,887.6$ & $3,944.8$ & $3,711.1$ & $3,382.8$ & $3,688.3$ & $3,826.1$ & $3,390.6$ & $3,509.1$ \\
\hline 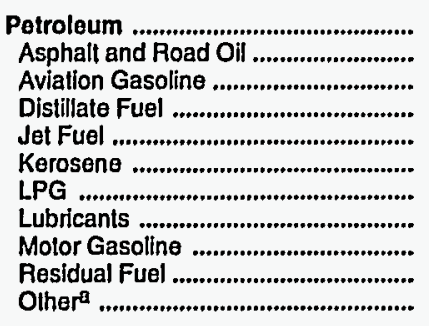 & $\begin{array}{r}2,223.4 \\
44.3 \\
7.8 \\
224.5 \\
24.4 \\
40.7 \\
56.5 \\
111.9 \\
1,637.3 \\
17.6 \\
58.5\end{array}$ & $\begin{array}{r}4,355.9 \\
118.0 \\
8.5 \\
621.6 \\
70.7 \\
55.5 \\
127.6 \\
163.8 \\
2,949.3 \\
117.1 \\
123.6\end{array}$ & $\begin{array}{r}9,908.7 \\
178.1 \\
21.5 \\
1,828.0 \\
259.2 \\
95.5 \\
883.2 \\
332.7 \\
5,623.4 \\
122.1 \\
565.1\end{array}$ & $\begin{array}{r}9,047.6 \\
186.3 \\
16.6 \\
1,605.0 \\
245.3 \\
75.2 \\
934.9 \\
371.3 \\
5,224.6 \\
33.6 \\
354.7\end{array}$ & $\begin{array}{r}6,767.6 \\
180.3 \\
15.9 \\
1,211.8 \\
215.8 \\
54.0 \\
462.7 \\
321.4 \\
3,986.7 \\
24.5 \\
294.5\end{array}$ & $\begin{array}{r}7,292.4 \\
193.3 \\
9.1 \\
1,175.1 \\
246.2 \\
49.3 \\
478.9 \\
296.1 \\
4,477.8 \\
27.0 \\
339.5\end{array}$ & $\begin{array}{r}7,231.7 \\
136.4 \\
12.4 \\
1,271.1 \\
203.8 \\
57.4 \\
335.2 \\
328.5 \\
4,569.4 \\
32.7 \\
284.9\end{array}$ & $\begin{array}{r}8,069.9 \\
210.5 \\
10.5 \\
1,473.4 \\
263.6 \\
48.5 \\
440.8 \\
306.6 \\
4,994.1 \\
29.1 \\
292.8\end{array}$ & $\begin{array}{r}8,750.8 \\
205.0 \\
11.2 \\
1,663.1 \\
343.5 \\
39.4 \\
407.9 \\
318.0 \\
5,394.3 \\
20.5 \\
347.9\end{array}$ & $\begin{array}{r}8,420.8 \\
176.5 \\
9.4 \\
1,534.9 \\
295.1 \\
38.8 \\
398.2 \\
327.3 \\
5,325.8 \\
13.3 \\
301.3\end{array}$ \\
\hline Nuclear Fuel ........................................ & - & - & 6.4 & 23.0 & 2 & 59.0 & 120.8 & 179.7 & 141.3 & 182.1 \\
\hline Blomass Fuels at Utillties ................. & * & $\bullet$ & $\bullet$ & 2.2 & .9 & 3.5 & 3.2 & (d) & (d) & (d) \\
\hline Primary Energy - Five Sectors $b$.... & $3,548.7$ & $7,446.8$ & $15,193.0$ & $15,401.3$ & $12,853.2$ & $12,977.5$ & $13,304.1$ & $14,283.8$ & $14,477.0$ & $14,221.9$ \\
\hline $\begin{array}{l}\text { Electric Utility Fuele ........................... } \\
\text { Electricity Purchased by End Users }\end{array}$ & $\begin{array}{r}-245.5 \\
1,344.2\end{array}$ & $\begin{array}{r}-1,046.9 \\
2,773.5\end{array}$ & $\begin{array}{r}-1,729.9 \\
4,904.7\end{array}$ & $\begin{array}{r}-1,919.7 \\
7,080.8\end{array}$ & $\begin{array}{r}-1,870.3 \\
7,209.8\end{array}$ & $\begin{array}{r}-1,844.2 \\
7,450.9\end{array}$ & $\begin{array}{r}-1,915.4 \\
7,649.7\end{array}$ & $\begin{array}{r}-1,980.6 \\
8,033.5\end{array}$ & $\begin{array}{r}-1,920.0 \\
8,321.6\end{array}$ & $\begin{array}{r}-1,960.3 \\
8,827.3\end{array}$ \\
\hline Total Energy ........................................... & $4,647.4$ & $9,173.4$ & $18,367.8$ & $20,562.4$ & $18,192.6$ & $18,584.2$ & $19,038.4$ & $20,336.6$ & $20,878.6$ & $21,088.9$ \\
\hline
\end{tabular}

a Includes petroleum coke used at electric utilities.

b Blomass fuels are not included, except those consumed at electric utilities and those added to motor gasoline.

c There are no direct fuel cosls for hydroelectric, geothermal, centralized solar, or wind energy.

d Utilities used biomass fuels at no charge or received a fee for accepting them.

-No consumption, including cases where adjustments were made. See "Consumption Adjustments for Process Fuel and Intermediate Products."

"Value less than 0.05 million dollars.

Nole; Expenditure totals may not equal sum of components due to independent rounding

Sources: Data sources, estimation procedures, and assumptions are described in the "Documentation" section of this report. 


\begin{tabular}{|c|c|c|c|c|c|c|c|c|c|c|}
\hline Sector and Energy Source & 1970 & 1975 & 1980 & 1985 & 1986 & 1987 & 1988 & 1989 & 1990 & 1991 \\
\hline 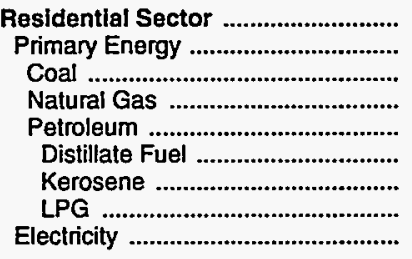 & $\begin{array}{r}1.69 \\
.98 \\
1.05 \\
.88 \\
1.54 \\
1.41 \\
1.42 \\
2.14 \\
6.99\end{array}$ & $\begin{array}{r}3.12 \\
1.74 \\
2.62 \\
1.47 \\
2.96 \\
2.51 \\
2.90 \\
4.53 \\
10.93\end{array}$ & $\begin{array}{r}6.37 \\
3.92 \\
3.07 \\
3.49 \\
6.94 \\
6.63 \\
8.07 \\
7.66 \\
16.29\end{array}$ & $\begin{array}{r}9.77 \\
6.02 \\
3.00 \\
5.79 \\
8.36 \\
7.55 \\
8.43 \\
10.09 \\
22.49\end{array}$ & $\begin{array}{r}9.56 \\
5.65 \\
2.86 \\
5.55 \\
6.99 \\
5.88 \\
6.57 \\
9.59 \\
22.45\end{array}$ & $\begin{array}{r}9.45 \\
5.27 \\
2.74 \\
5.12 \\
6.81 \\
5.66 \\
6.32 \\
8.88 \\
22.56\end{array}$ & $\begin{array}{r}9.25 \\
5.16 \\
2.65 \\
5.01 \\
6.57 \\
5.66 \\
6.32 \\
8.40 \\
22.24\end{array}$ & $\begin{array}{r}9.53 \\
5.41 \\
2.69 \\
5.11 \\
8.06 \\
6.18 \\
6.90 \\
11.42 \\
22.79\end{array}$ & $\begin{array}{r}10.21 \\
5.52 \\
2.80 \\
5.09 \\
9.16 \\
7.43 \\
8.30 \\
12.05 \\
23.58\end{array}$ & $\begin{array}{r}10.34 \\
5.41 \\
2.64 \\
5.06 \\
8.34 \\
6.89 \\
7.70 \\
10.71 \\
23.91\end{array}$ \\
\hline 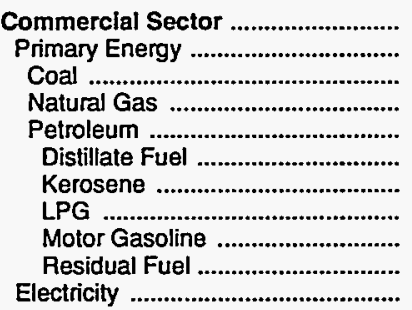 & $\begin{array}{r}1.87 \\
.76 \\
.40 \\
.75 \\
1.26 \\
1.20 \\
.84 \\
1.39 \\
2.93 \\
.69 \\
6.33\end{array}$ & $\begin{array}{r}3.54 \\
1.51 \\
1.31 \\
1.31 \\
2.74 \\
2.33 \\
2.48 \\
2.83 \\
4.73 \\
2.20 \\
10.10\end{array}$ & $\begin{array}{r}7.07 \\
3.76 \\
1.34 \\
3.26 \\
7.13 \\
6.28 \\
6.01 \\
5.34 \\
9.45 \\
3.58 \\
15.71\end{array}$ & $\begin{array}{r}10.76 \\
5.24 \\
1.49 \\
5.34 \\
6.98 \\
6.10 \\
6.93 \\
9.43 \\
9.15 \\
4.18 \\
20.91\end{array}$ & $\begin{array}{r}10.47 \\
4.81 \\
1.45 \\
5.11 \\
5.25 \\
3.72 \\
4.56 \\
8.60 \\
6.78 \\
2.60 \\
20.73\end{array}$ & $\begin{array}{r}10.25 \\
4.62 \\
1.39 \\
4.68 \\
5.82 \\
4.15 \\
4.98 \\
8.20 \\
7.36 \\
2.83 \\
20.29\end{array}$ & $\begin{array}{r}10.00 \\
4.60 \\
1.39 \\
4.57 \\
5.87 \\
3.73 \\
4.47 \\
8.46 \\
7.43 \\
2.51 \\
19.97\end{array}$ & $\begin{array}{r}10.63 \\
4.79 \\
1.39 \\
4.70 \\
6.49 \\
4.32 \\
5.47 \\
8.17 \\
8.18 \\
2.60 \\
20.66\end{array}$ & $\begin{array}{r}11.28 \\
4.61 \\
1.44 \\
4.50 \\
7.19 \\
5.49 \\
6.41 \\
9.36 \\
9.35 \\
2.54 \\
21.30\end{array}$ & $\begin{array}{r}11.53 \\
4.65 \\
1.41 \\
4.56 \\
6.74 \\
4.85 \\
5.57 \\
9.59 \\
9.23 \\
2.30 \\
21.56\end{array}$ \\
\hline 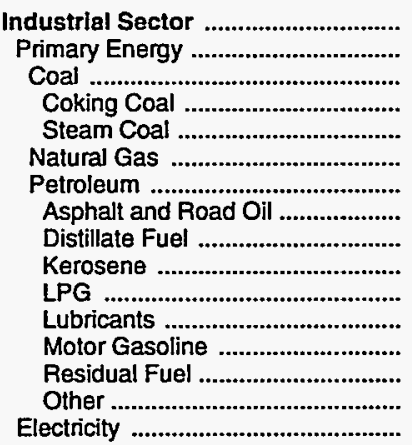 & $\begin{array}{r}.83 \\
.60 \\
.41 \\
.42 \\
.40 \\
.57 \\
1.21 \\
.74 \\
.77 \\
.84 \\
1.39 \\
5.08 \\
2.93 \\
.55 \\
1.14 \\
2.90\end{array}$ & $\begin{array}{l}2.16 \\
1.60 \\
1.47 \\
1.57 \\
1.31 \\
1.08 \\
2.59 \\
2.03 \\
2.31 \\
2.48 \\
2.83 \\
7.48 \\
4.73 \\
2.17 \\
2.36 \\
5.61\end{array}$ & $\begin{array}{r}4.43 \\
3.56 \\
1.79 \\
2.00 \\
1.34 \\
3.01 \\
5.73 \\
3.66 \\
5.45 \\
6.01 \\
5.34 \\
14.36 \\
9.45 \\
3.31 \\
6.93 \\
9.73\end{array}$ & $\begin{array}{r}6.19 \\
4.69 \\
1.78 \\
2.05 \\
1.49 \\
4.66 \\
7.94 \\
4.43 \\
6.25 \\
6.93 \\
9.43 \\
17.61 \\
9.15 \\
4.18 \\
7.57 \\
11.75\end{array}$ & $\begin{array}{r}5.67 \\
3.89 \\
1.75 \\
2.04 \\
1.45 \\
4.48 \\
6.11 \\
3.70 \\
4.11 \\
4.56 \\
8.60 \\
15.59 \\
6.78 \\
2.60 \\
6.09 \\
12.12\end{array}$ & $\begin{array}{r}5.43 \\
3.61 \\
1.56 \\
1.72 \\
1.39 \\
4.09 \\
5.85 \\
3.23 \\
4.49 \\
4.98 \\
8.20 \\
12.70 \\
7.36 \\
2.83 \\
6.34 \\
11.80\end{array}$ & $\begin{array}{r}5.22 \\
3.41 \\
1.56 \\
1.73 \\
1.39 \\
3.94 \\
5.66 \\
3.23 \\
4.04 \\
4.47 \\
8.46 \\
14.61 \\
7.43 \\
2.51 \\
5.44 \\
11.79\end{array}$ & $\begin{array}{r}5.41 \\
3.59 \\
1.59 \\
1.75 \\
1.39 \\
4.02 \\
5.49 \\
2.99 \\
4.94 \\
5.47 \\
8.17 \\
13.30 \\
8.18 \\
2.60 \\
5.83 \\
11.36\end{array}$ & $\begin{array}{r}5.66 \\
3.69 \\
1.63 \\
1.80 \\
1.44 \\
3.92 \\
5.94 \\
3.13 \\
5.78 \\
6.41 \\
9.36 \\
13.40 \\
9.35 \\
2.54 \\
6.20 \\
11.81\end{array}$ & $\begin{array}{r}5.83 \\
3.69 \\
1.55 \\
1.72 \\
1.41 \\
3.92 \\
5.82 \\
2.96 \\
5.03 \\
5.57 \\
9.59 \\
15.42 \\
9.23 \\
2.30 \\
5.65 \\
12.31\end{array}$ \\
\hline 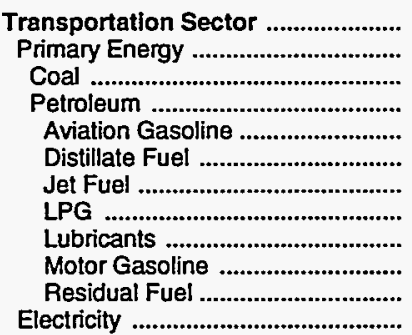 & $\begin{array}{r}2.66 \\
2.66 \\
.40 \\
2.66 \\
2.17 \\
1.26 \\
.74 \\
1.39 \\
5.08 \\
2.93 \\
.64 \\
4.05\end{array}$ & $\begin{array}{l}4.39 \\
4.38 \\
1.31 \\
4.39 \\
3.45 \\
2.76 \\
2.08 \\
2.83 \\
7.48 \\
4.73 \\
1.61 \\
7.63\end{array}$ & $\begin{array}{r}8.87 \\
8.87 \\
- \\
8.87 \\
9.02 \\
6.95 \\
6.38 \\
5.34 \\
14.36 \\
9.45 \\
3.02 \\
13.51\end{array}$ & $\begin{array}{r}8.92 \\
8.92 \\
- \\
8.92 \\
9.99 \\
8.28 \\
6.04 \\
9.43 \\
17.61 \\
9.15 \\
-\overline{10} \\
22.10\end{array}$ & $\begin{array}{r}6.61 \\
6.61 \\
- \\
6.61 \\
8.41 \\
6.47 \\
3.85 \\
8.60 \\
15.59 \\
6.78 \\
2.51 \\
23.15\end{array}$ & $\begin{array}{r}7.06 \\
7.06 \\
- \\
7.06 \\
7.55 \\
6.70 \\
4.04 \\
8.20 \\
12.70 \\
7.36 \\
2.84 \\
17.79\end{array}$ & $\begin{array}{r}7.13 \\
7.13 \\
- \\
7.13 \\
7.41 \\
6.57 \\
3.92 \\
8.46 \\
14.61 \\
7.43 \\
2.16 \\
17.01\end{array}$ & $\begin{array}{r}7.79 \\
7.79 \\
- \\
7.79 \\
8.28 \\
7.22 \\
4.49 \\
8.17 \\
13.30 \\
8.18 \\
2.32 \\
17.59\end{array}$ & $\begin{array}{r}8.95 \\
8.95 \\
- \\
8.95 \\
9.32 \\
8.44 \\
5.73 \\
9.36 \\
13.40 \\
9.35 \\
2.70 \\
17.97\end{array}$ & $\begin{array}{r}8.78 \\
8.78 \\
- \\
8.78 \\
8.71 \\
8.22 \\
4.95 \\
9.59 \\
15.42 \\
9.23 \\
2.26 \\
17.84\end{array}$ \\
\hline $\begin{array}{l}\text { Total Energy ............................... } \\
\text { Primary Energy - Four Sectors ...... } \\
\text { Electricity }\end{array}$ & $\begin{array}{l}1.50 \\
1.17 \\
4.68\end{array}$ & $\begin{array}{l}3.05 \\
2.41 \\
7.94\end{array}$ & $\begin{array}{r}6.23 \\
5.24 \\
12.97\end{array}$ & $\begin{array}{r}8.25 \\
6.51 \\
16.90\end{array}$ & $\begin{array}{r}7.34 \\
5.33 \\
17.23\end{array}$ & $\begin{array}{r}7.34 \\
5.32 \\
16.97\end{array}$ & $\begin{array}{r}7.23 \\
5.22 \\
16.90\end{array}$ & $\begin{array}{r}7.63 \\
5.63 \\
16.83\end{array}$ & $\begin{array}{r}8.23 \\
6.11 \\
17.33\end{array}$ & $\begin{array}{r}8.38 \\
6.05 \\
17.98\end{array}$ \\
\hline 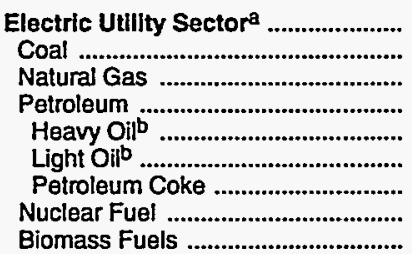 & $\begin{array}{r}.30 \\
.29 \\
.39 \\
.72 \\
.69 \\
.75 \\
- \\
- \\
.65\end{array}$ & $\begin{array}{r}.98 \\
.95 \\
1.19 \\
2.29 \\
2.18 \\
2.35 \\
- \\
- \\
.92\end{array}$ & $\begin{array}{r}1.50 \\
1.48 \\
2.90 \\
5.11 \\
3.58 \\
5.72 \\
-\frac{28}{1.74}\end{array}$ & $\begin{array}{r}1.70 \\
1.69 \\
5.09 \\
5.71 \\
4.43 \\
6.09 \\
- \\
1.09 \\
.79\end{array}$ & $\begin{array}{r}1.63 \\
1.63 \\
4.67 \\
3.56 \\
2.73 \\
3.71 \\
- \\
.72 \\
.32\end{array}$ & $\begin{array}{r}1.51 \\
1.56 \\
3.04 \\
3.93 \\
3.00 \\
4.10 \\
- \\
.73 \\
.95\end{array}$ & $\begin{array}{r}1.52 \\
1.52 \\
3.46 \\
3.19 \\
2.45 \\
3.77 \\
- \\
1.33 \\
.87\end{array}$ & $\begin{array}{r}1.48 \\
1.49 \\
3.13 \\
3.43 \\
2.66 \\
4.26 \\
- \\
1.32 \\
\text { (d) }\end{array}$ & $\begin{array}{r}1.50 \\
1.51 \\
2.55 \\
4.84 \\
3.12 \\
5.39 \\
- \\
1.24 \\
\text { (d) }\end{array}$ & $\begin{array}{r}1.45 \\
1.48 \\
2.18 \\
4.35 \\
2.58 \\
4.91 \\
- \\
1.14 \\
\left({ }^{d}\right)\end{array}$ \\
\hline Primary Energy - Five Sectorsc .. & .97 & 2.00 & 4.08 & 4.81 & 4.01 & 3.92 & 3.86 & 4.05 & 4.34 & 4.21 \\
\hline
\end{tabular}

There are no direct fuel costs for hydroelectric, geothermal, centralized solar, or wind energy.

b Heavy oil includes Grade Nos. 4, 5, and 6, and residual fuel oils. Light oil includes Grade No. 2 heating oil, kerosene, and jet fuel.

c Biomass fuels are not included, except those consumed at electric utilities and those added to motor gasoline.

d Utilities used biomass fuels at no charge or received a fee for accepting them.

- No consumption, including cases where adjustments were made. See "Consumption Adjustments for Process Fuel and Intermediate Products."

Sources: Data sources, estimation procedures, and assumptions are described in the "Documentation" section of this report. 
Energy Expenditure Estimates by Sector, Ohio $1970,1975,1980$, and 1985-1991 (Million Dollars)

\begin{tabular}{|c|c|c|c|c|c|c|c|c|c|c|}
\hline Sector and Energy Source & 1970 & 1975 & 1980 & 1985 & 1986 & 1987 & 1988 & 1989 & 1990 & 1991 \\
\hline 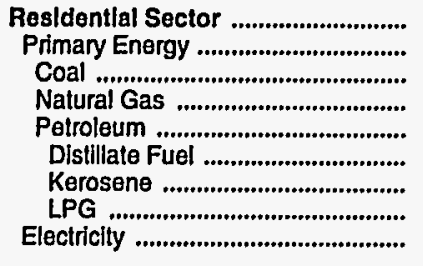 & $\begin{array}{r}1,090.9 \\
559.8 \\
13.8 \\
414.0 \\
132.0 \\
76.5 \\
24.1 \\
31.5 \\
531.1\end{array}$ & $\begin{array}{r}1,980.1 \\
940.4 \\
23.3 \\
643.4 \\
273.6 \\
157.8 \\
33.8 \\
82.0 \\
1,039.7\end{array}$ & $\begin{array}{r}3,675.4 \\
1,815.5 \\
14.0 \\
1,396.3 \\
405.3 \\
286.8 \\
46.5 \\
72.0 \\
1,859.9\end{array}$ & \begin{tabular}{r|}
$4,967.8$ \\
$2,363.4$ \\
21.7 \\
$1,978.7$ \\
363.0 \\
196.7 \\
45.0 \\
121.3 \\
$2,604.3$
\end{tabular} & $\begin{array}{r}4,943.5 \\
2,245.2 \\
25.3 \\
1,898.7 \\
321.2 \\
157.0 \\
44.0 \\
120.2 \\
2,698.4\end{array}$ & $\begin{array}{r}4,899.0 \\
2,072.9 \\
18.8 \\
1,746.7 \\
307.4 \\
137.1 \\
38.4 \\
131.9 \\
2,826.2\end{array}$ & \begin{tabular}{r|}
$5,101.8$ \\
$2,163.8$ \\
16.4 \\
$1,826.7$ \\
320.6 \\
153.4 \\
45.1 \\
122.2 \\
$2,938.0$
\end{tabular} & $\begin{array}{r}5,329.0 \\
2,312.0 \\
12.4 \\
1,910.7 \\
388.9 \\
164.6 \\
34.2 \\
190.1 \\
3,017.0\end{array}$ & $\begin{array}{r}5,085.2 \\
2,036.1 \\
15.5 \\
1,631.0 \\
389.6 \\
176.5 \\
29.4 \\
183.7 \\
3,049.0\end{array}$ & $\begin{array}{r}5,420.9 \\
2,080.9 \\
11.0 \\
1,698.7 \\
371.2 \\
169.4 \\
29.5 \\
172.3 \\
3,340.0\end{array}$ \\
\hline 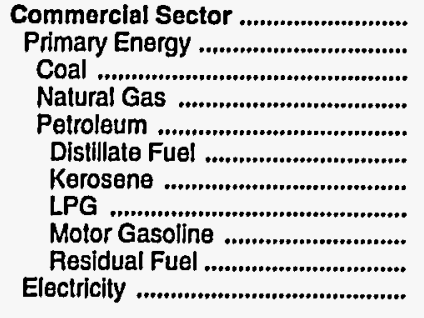 & $\begin{array}{r}545.7 \\
176.6 \\
9.5 \\
140.0 \\
27.1 \\
13.0 \\
.7 \\
3.6 \\
6.2 \\
3.6 \\
369.2\end{array}$ & $\begin{array}{r}1,023.2 \\
332.5 \\
21.5 \\
227.6 \\
83.5 \\
29.0 \\
1.5 \\
9.0 \\
23.7 \\
20.1 \\
690.7\end{array}$ & $\begin{array}{r}2,031.3 \\
781.1 \\
11.2 \\
551.1 \\
218.8 \\
94.8 \\
4.4 \\
8.9 \\
102.2 \\
8.5 \\
1,250.2\end{array}$ & $\begin{array}{r}3,041.6 \\
959.5 \\
19.7 \\
799.0 \\
140.9 \\
72.4 \\
17.3 \\
20.0 \\
29.0 \\
2.2 \\
2,082.1\end{array}$ & $\begin{array}{r}3,061.9 \\
906.4 \\
23.7 \\
743.7 \\
138.9 \\
46.1 \\
4.9 \\
19.0 \\
66.3 \\
2.6 \\
2,155.5\end{array}$ & $\begin{array}{r}3,092.7 \\
893.1 \\
17.6 \\
718.7 \\
156.8 \\
51.1 \\
5.3 \\
21.5 \\
77.9 \\
.9 \\
2,199.6\end{array}$ & $\begin{array}{r}3,237.2 \\
966.5 \\
15.9 \\
754.3 \\
196.4 \\
48.5 \\
6.7 \\
21.7 \\
118.2 \\
1.2 \\
2,270.7\end{array}$ & $\begin{array}{r}3,396.4 \\
965.8 \\
11.7 \\
791.4 \\
162.6 \\
42.4 \\
7.4 \\
24.0 \\
88.5 \\
.3 \\
2,430.6\end{array}$ & $\begin{array}{r}3,357.3 \\
823.7 \\
14.8 \\
671.9 \\
136.9 \\
52.8 \\
6.9 \\
25.2 \\
51.7 \\
.4 \\
2,533.6\end{array}$ & $\begin{array}{r}3,559.4 \\
850.7 \\
10.8 \\
715.9 \\
123.9 \\
45.6 \\
5.7 \\
27.2 \\
44.8 \\
.6 \\
2,708.7\end{array}$ \\
\hline 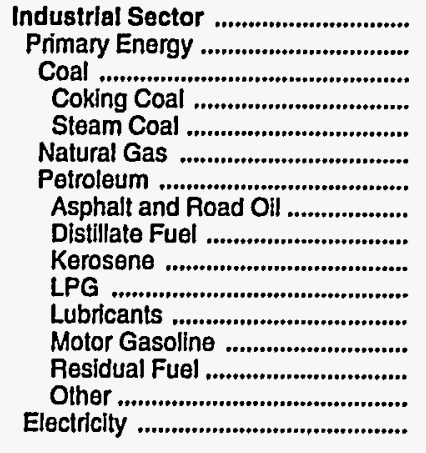 & $\begin{array}{r}1,252.9 \\
809.5 \\
301.9 \\
146.6 \\
155.3 \\
206.6 \\
301.1 \\
44.3 \\
50.5 \\
15.9 \\
20.7 \\
73.7 \\
29.7 \\
7.9 \\
58.5 \\
443.4\end{array}$ & $\begin{array}{r}2,870.3 \\
1,828.4 \\
815.2 \\
519.3 \\
296.0 \\
366.0 \\
647.2 \\
118.0 \\
149.7 \\
20.2 \\
34.7 \\
90.2 \\
37.7 \\
73.0 \\
123.6 \\
1,042.0\end{array}$ & $\begin{array}{r}5,786.1 \\
3,993.6 \\
723.6 \\
549.5 \\
174.1 \\
926.5 \\
2,343.4 \\
178.1 \\
396.8 \\
44.5 \\
797.9 \\
208.6 \\
57.3 \\
95.1 \\
565.1 \\
1,792.6\end{array}$ & $\begin{array}{r}5,917.6 \\
3,526.4 \\
473.5 \\
287.8 \\
185.7 \\
1,163.5 \\
1,889.4 \\
186.3 \\
243.0 \\
12.9 \\
780.7 \\
232.8 \\
51.6 \\
27.5 \\
354.7 \\
2,391.2\end{array}$ & $\begin{array}{r}5,076.7 \\
2,723.9 \\
470.0 \\
283.0 \\
187.0 \\
1,065.9 \\
1,188.0 \\
180.3 \\
139.2 \\
5.1 \\
310.6 \\
201.5 \\
36.3 \\
20.4 \\
294.5 \\
2,352.8\end{array}$ & $\begin{array}{r}5,017.1 \\
2,594.2 \\
438.4 \\
246.8 \\
191.6 \\
914.7 \\
1,241.1 \\
193.3 \\
138.3 \\
5.6 \\
315.0 \\
185.7 \\
39.8 \\
23.9 \\
339.5 \\
2,422.9\end{array}$ & $\begin{array}{r}5,000.4 \\
2,561.8 \\
457.6 \\
252.4 \\
205.2 \\
1,103.9 \\
1,000.3 \\
136.4 \\
121.7 \\
5.6 \\
180.5 \\
205.9 \\
40.1 \\
25.3 \\
284.9 \\
2,438.6\end{array}$ & $\begin{array}{r}5,244.2 \\
2,660.8 \\
407.2 \\
247.6 \\
159.6 \\
1,120.9 \\
1,132.6 \\
210.5 \\
150.9 \\
6.9 \\
215.4 \\
192.2 \\
43.7 \\
20.1 \\
292.8 \\
2,583.4\end{array}$ & $\begin{array}{r}5,406.6 \\
2,670.0 \\
405.4 \\
239.0 \\
166.4 \\
1,084.4 \\
1,180.1 \\
205.0 \\
173.1 \\
3.2 \\
186.8 \\
199.4 \\
47.5 \\
17.4 \\
347.9 \\
2,736.6\end{array}$ & $\begin{array}{r}5,285.4 \\
2,509.2 \\
336.4 \\
170.7 \\
165.7 \\
1,087.4 \\
1,085.4 \\
176.5 \\
153.7 \\
3.6 \\
188.6 \\
205.2 \\
46.7 \\
9.9 \\
301.3 \\
2,776.2\end{array}$ \\
\hline 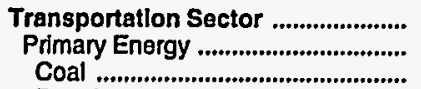 & $\begin{array}{r}1,757.9 \\
1,757.3 \\
.4\end{array}$ & $\begin{array}{r}3,299.8 \\
3,298.6 \\
.1\end{array}$ & $\begin{array}{r}6,874.9 \\
6,872.9\end{array}$ & $\begin{array}{r}6,635.5 \\
6,632.3 \\
-\end{array}$ & $\begin{array}{r}5,110.6 \\
5,107.4\end{array}$ & $\begin{array}{r}5,575.4 \\
5,573.1\end{array}$ & $\begin{array}{r}5,698.9 \\
5,696.6\end{array}$ & $\begin{array}{r}6,367.1 \\
6,364.6\end{array}$ & $\begin{array}{r}7,029.6 \\
7,027.2 \\
-\end{array}$ & $\begin{array}{r}6,823.1 \\
6,820.8 \\
-\end{array}$ \\
\hline 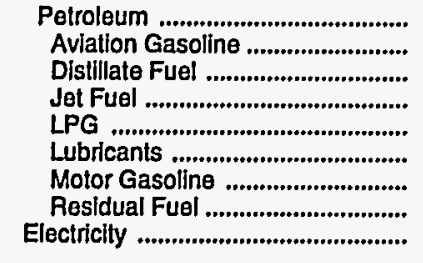 & \begin{tabular}{r|}
$1,756.8$ \\
7.8 \\
81.2 \\
24.4 \\
.7 \\
38.3 \\
$1,601.5$ \\
3.1 \\
.6
\end{tabular} & $\begin{array}{r}3,298.5 \\
8.5 \\
251.5 \\
69.2 \\
1.9 \\
73.6 \\
2,887.8 \\
6.0 \\
1.2\end{array}$ & $\begin{array}{r}6,872.9 \\
21.5 \\
994.9 \\
259.2 \\
4.4 \\
124.1 \\
5,463.9 \\
4.8 \\
2.0\end{array}$ & $\begin{array}{r}6,632.3 \\
16.6 \\
1,074.9 \\
245.3 \\
12.9 \\
138.5 \\
5,144.0 \\
-\overline{0}\end{array}$ & $\begin{array}{r}5,107.4 \\
15.9 \\
858.8 \\
215.8 \\
12.9 \\
119.9 \\
3,884.0 \\
.1 \\
3.2\end{array}$ & $\begin{array}{r}5,573.1 \\
9.1 \\
836.2 \\
246.2 \\
10.5 \\
110.5 \\
4,360.1 \\
.5 \\
2.3\end{array}$ & $\begin{array}{r}5,696.6 \\
12.4 \\
935.9 \\
203.8 \\
10.8 \\
122.5 \\
4,411.1 \\
.2 \\
2.3\end{array}$ & $\begin{array}{r}6,364.6 \\
10.5 \\
1,102.9 \\
263.6 \\
11.2 \\
114.4 \\
4,861.9 \\
.1 \\
2.4\end{array}$ & $\begin{array}{r}7,027.2 \\
11.2 \\
1,246.5 \\
343.5 \\
12.2 \\
118.6 \\
5,295.1 \\
.1 \\
2.4\end{array}$ & $\begin{array}{r}6,820.8 \\
9.4 \\
1,149.6 \\
295.1 \\
10.1 \\
122.1 \\
5,234.3 \\
.1 \\
2.3\end{array}$ \\
\hline $\begin{array}{l}\text { Total Energy ..................................... } \\
\text { Primary Energy - Four Sectors ...... } \\
\text { Electricity ......................................... }\end{array}$ & $\begin{array}{l}4,647.4 \\
3,303.2 \\
1,344.2\end{array}$ & $\begin{array}{l}9,173.4 \\
6,399.9 \\
2,773.5\end{array}$ & $\begin{array}{r}18,367.8 \\
13,463.1 \\
4,904.7\end{array}$ & $\begin{array}{r}20,562.4 \\
13,481.6 \\
7,080.8\end{array}$ & $\begin{array}{r}18,192.6 \\
10,982.8 \\
7,209.8\end{array}$ & $\begin{array}{r}18,584.2 \\
11,133.3 \\
7,450.9\end{array}$ & $\begin{array}{r}19,038.4 \\
11,388.7 \\
7,649.7\end{array}$ & $\begin{array}{r}20,336.6 \\
12,303.1 \\
8,033.5\end{array}$ & $\begin{array}{r}20,878.6 \\
12,557.0 \\
8,321.6\end{array}$ & $\begin{array}{r}21,088.9 \\
12,261.5 \\
8,827.3\end{array}$ \\
\hline 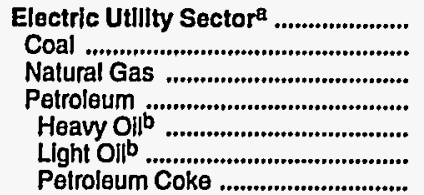 & $\begin{array}{r}245.5 \\
230.5 \\
8.6 \\
6.4 \\
3.0 \\
3.4 \\
-\end{array}$ & $\begin{array}{r}1,046.9 \\
987.4 \\
6.3 \\
53.2 \\
18.0 \\
35.2 \\
-\end{array}$ & $\begin{array}{r}1,729.9 \\
1,641.4 \\
13.7 \\
68.3 \\
13.6 \\
54.7 \\
-\end{array}$ & $\begin{array}{r}1,919.7 \\
1,869.0 \\
3.6 \\
22.0 \\
3.9 \\
18.0 \\
-\end{array}$ & $\begin{array}{r}1,870.3 \\
1,854.3 \\
2.9 \\
12.1 \\
1.4 \\
10.7 \\
-\end{array}$ & $\begin{array}{r}1,844.2 \\
1,764.9 \\
2.7 \\
14.1 \\
1.7 \\
12.4 \\
-\end{array}$ & $\begin{array}{r}1,915.4 \\
1,770.4 \\
3.4 \\
17.7 \\
6.0 \\
11.7 \\
-\end{array}$ & $\begin{array}{r}1,980.6 \\
1,776.7 \\
3.1 \\
21.1 \\
8.5 \\
12.6 \\
\end{array}$ & $\begin{array}{r}1,920.0 \\
1,758.7 \\
3.2 \\
16.9 \\
2.7 \\
14.2 \\
-\end{array}$ & $\begin{array}{r}1,960.3 \\
1,751.8 \\
7.1 \\
19.4 \\
2.7 \\
16.7 \\
-\end{array}$ \\
\hline 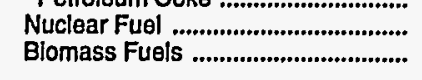 & $\overline{-}$ & $\overline{-}$ & $6 . \overline{4}$ & $\begin{array}{r}23 . \overline{0} \\
2.2\end{array}$ & $\overline{2}$ & $\begin{array}{r}59 . \overline{0} \\
3.5\end{array}$ & $\begin{array}{r}120 . \overline{8} \\
3.2\end{array}$ & $(\mathrm{~d}) \overline{7}$ & $\begin{array}{l}141 . \overline{3} \\
(\mathrm{~d})\end{array}$ & (d) \\
\hline Prlmary Energy - Flve Sectorsc .. & $3,548.7$ & $7,446.8$ & $15,193.0$ & $15,401.3$ & $12,853.2$ & $12,977.5$ & $13,304.1$ & $14,283.8$ & $14,477.0$ & $14,221.9$ \\
\hline
\end{tabular}

a There are no direct fuel costs for hydroelectric, geothermal, centralized solar, or wind energy.

b Heavy oil includes Grade Nos. 4, 5, and 6, and residual fuel oils. Light oil includes Grade No. 2 heating oil, kerosene, and jet fuel.

c Blomass fuels are not included, except those consumed at electric utilities and those added to motor gasoline.

Utilitles used blomass fuels at no charge or received a fee for accepting them.

-No consumption, including cases where adjustments were made. See "Consumption Adjustments for Process Fuel and Intermediate Products."

-Value less than 0.05 million dollars.

Note: Totals may not equal sum of components due to independent rounding.

Sources: Data sources, estimation procedures, and assumptions are described in the "Documentation" section of this report. 
- Energy Price and Expenditure Estimates by Source, Oklahoma 1970, 1975, 1980, and 1985-1991

\begin{tabular}{|c|c|c|c|c|c|c|c|c|c|c|}
\hline Energy Source & 1970 & 1975 & 1980 & 1985 & 1986 & 1987 & 1988 & 1989 & 1990 & 1991 \\
\hline & \multicolumn{10}{|c|}{ Prices in Dollars per Million Btu } \\
\hline Coal & 0.57 & 0.96 & 1.25 & 1.69 & 1.65 & 1.58 & 1.50 & 1.36 & 1.40 & 1.32 \\
\hline 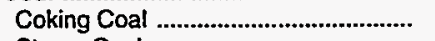 & - & - & - & - & - & - & - & - & - & - \\
\hline 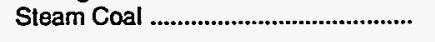 & .57 & .96 & 1.25 & 1.69 & 1.65 & 1.58 & 1.50 & 1.36 & 1.40 & 1.32 \\
\hline 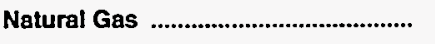 & .35 & .75 & 1.96 & 3.41 & 3.11 & 2.76 & 2.80 & 2.86 & 2.80 & 2.78 \\
\hline 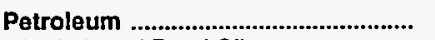 & 1.97 & 3.52 & 7.97 & 7.43 & 6.02 & 6.31 & 6.15 & 6.68 & 7.83 & 7.41 \\
\hline 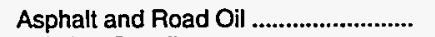 & .68 & 1.86 & 3.60 & 4.20 & 4.58 & 3.44 & 3.24 & 3.15 & 3.14 & 3.40 \\
\hline 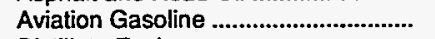 & 2.17 & 3.45 & 9.02 & 9.99 & 8.41 & 7.55 & 7.41 & 8.28 & 9.32 & 8.71 \\
\hline 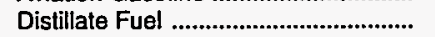 & .90 & 2.36 & 6.77 & 5.54 & 5.36 & 5.63 & 5.52 & 6.21 & 7.49 & 6.93 \\
\hline 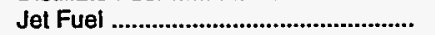 & .72 & 2.01 & 6.34 & 5.87 & 3.94 & 3.90 & 3.75 & 4.27 & 5.93 & 4.73 \\
\hline Kerosene & .66 & 2.41 & 6.52 & 4.07 & 4.81 & 3.91 & 4.76 & 5.98 & 7.46 & 6.75 \\
\hline LPG & 1.42 & 2.91 & 6.03 & 7.21 & 7.92 & 7.40 & 5.45 & 5.70 & 6.41 & 6.44 \\
\hline 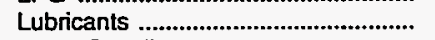 & 5.08 & 7.48 & 14.36 & 17.61 & 15.59 & 12.70 & 14.61 & 13.30 & 13.40 & 15.42 \\
\hline 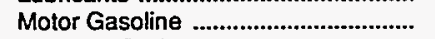 & 2.82 & 4.52 & 9.79 & 8.76 & 6.30 & 7.08 & 7.12 & 7.79 & 9.00 & 8.72 \\
\hline 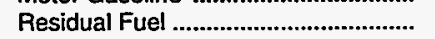 & .50 & 1.58 & 3.23 & 3.41 & 2.09 & 2.18 & 1.81 & 2.04 & 2.46 & 1.77 \\
\hline Othera & .58 & 1.45 & 2.90 & 3.36 & 3.04 & 2.89 & 2.44 & 2.87 & 3.32 & 2.93 \\
\hline 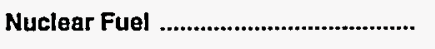 & - & - & - & - & - & - & - & - & - & - \\
\hline Blomass Fuels at Utilities .................. & - & - & - & - & - & - & - & - & - & - \\
\hline Primary Energy - Five Sectors ${ }^{b} \ldots$. & 1.03 & 1.90 & 4.04 & 4.56 & 3.87 & 3.73 & 3.65 & 3.84 & 4.20 & 4.02 \\
\hline $\begin{array}{l}\text { Electric Utility Fuelc .......................... } \\
\text { Electricity Purchased by End Users }\end{array}$ & $\begin{array}{r}.19 \\
5.76\end{array}$ & $\begin{array}{r}.61 \\
6.64\end{array}$ & $\begin{array}{r}1.63 \\
11.80\end{array}$ & $\begin{array}{r}2.30 \\
17.23\end{array}$ & $\begin{array}{r}2.17 \\
17.83\end{array}$ & $\begin{array}{r}2.08 \\
16.48\end{array}$ & $\begin{array}{r}2.04 \\
16.20\end{array}$ & $\begin{array}{r}2.02 \\
16.28\end{array}$ & $\begin{array}{r}2.05 \\
16.08\end{array}$ & $\begin{array}{r}1.92 \\
16.99\end{array}$ \\
\hline \multirow[t]{2}{*}{ 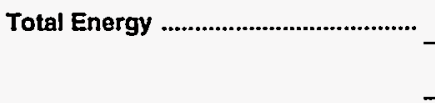 } & 1.83 & 3.06 & 6.43 & 7.58 & 6.96 & 6.59 & 6.48 & 6.68 & 7.37 & 7.25 \\
\hline & \multicolumn{10}{|c|}{ Expenditures in Milllons of Dollars } \\
\hline Coal & 0.1 & 0.5 & 132.5 & 400.2 & 360.2 & 379.9 & 404.1 & 366.5 & 388.1 & 384.4 \\
\hline 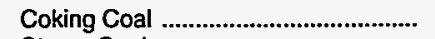 & - & - & - & - & - & - & - & $\overline{-}$ & - & - \\
\hline 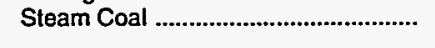 & .1 & .5 & 132.5 & 400.2 & 360.2 & 379.9 & 404.1 & 366.5 & 388.1 & 384.4 \\
\hline 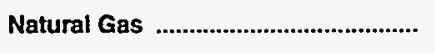 & 152.7 & 392.2 & $1,209.5$ & $1,633.3$ & $1,416.3$ & $1,305.6$ & $1,307.5$ & $1,363.3$ & $1,307.5$ & $1,262.6$ \\
\hline 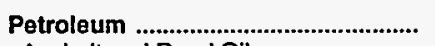 & 636.9 & $1,329.1$ & $3,202.7$ & $3,206.5$ & $2,317.5$ & $2,396.2$ & $2,402.3$ & $2,658.7$ & $3,085.7$ & $2,944.2$ \\
\hline 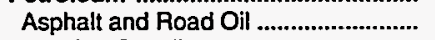 & 20.7 & 69.9 & 115.1 & 111.7 & 99.8 & 62.4 & 76.7 & 57.5 & 73.1 & 77.5 \\
\hline 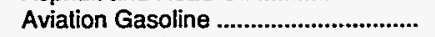 & 4.9 & 5.4 & 14.9 & 11.0 & 10.6 & 6.8 & 6.5 & 6.9 & 6.9 & 4.9 \\
\hline 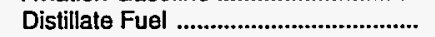 & 28.7 & 128.1 & 478.2 & 592.0 & 434.6 & 457.6 & 479.1 & 533.6 & 669.9 & 572.5 \\
\hline Jet Fuel & 17.2 & 43.2 & 170.5 & 190.6 & 129.8 & 161.8 & 150.7 & 220.5 & 259.8 & 257.1 \\
\hline 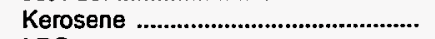 & 4.1 & 4.5 & 12.6 & 2.6 & 2.1 & 1.4 & 2.4 & 4.1 & 1.6 & 1.2 \\
\hline LPG & 50.3 & 99.1 & 196.2 & 204.8 & 168.5 & 145.9 & 96.2 & 116.9 & 80.6 & 116.1 \\
\hline 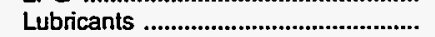 & 19.2 & 36.8 & 118.1 & 131.8 & 114.1 & 105.1 & 116.6 & 108.8 & 112.9 & 116.2 \\
\hline 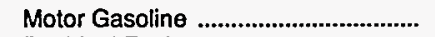 & 481.9 & 913.4 & $2,038.2$ & $1,940.7$ & $1,342.7$ & $1,436.3$ & $1,454.1$ & $1,590.1$ & $1,851.0$ & $1,778.0$ \\
\hline Residual Fuel ......................................... & 2.2 & 5.7 & 13.1 & 2.4 & 3.2 & 3.3 & 5.2 & 3.7 & 7.6 & 2.0 \\
\hline Other ${ }^{a}$ & 7.7 & 23.1 & 45.7 & 18.9 & 12.2 & 15.7 & 14.9 & 16.7 & 22.3 & 18.8 \\
\hline 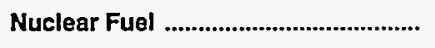 & - & - & - & - & - & - & - & - & - & - \\
\hline Blomass Fuels at Utilities .................. & - & - & - & - & - & - & - & - & - & - \\
\hline Primary Energy - Five Sectors ${ }^{b} \ldots .$. & 789.7 & $1,721.8$ & $4,544.7$ & $5,240.0$ & $4,094.0$ & $4,081.7$ & $4,113.8$ & $4,388.6$ & $4,781.3$ & $4,591.3$ \\
\hline $\begin{array}{l}\text { Electric Utility Fuelc } \\
\text { Electricity Purchased by End Users }\end{array}$ & $\begin{array}{r}-46.8 \\
311.7\end{array}$ & $\begin{array}{r}-190.0 \\
509.6\end{array}$ & $\begin{array}{r}-727.3 \\
1,211.3\end{array}$ & $\begin{array}{r}-988.5 \\
2,141.2\end{array}$ & $\begin{array}{r}-883.8 \\
2,158.5\end{array}$ & $\begin{array}{r}-882.1 \\
2,014.1\end{array}$ & $\begin{array}{r}-900.4 \\
2,047.7\end{array}$ & $\begin{array}{r}-891.0 \\
2,040.0\end{array}$ & $\begin{array}{r}-904.7 \\
2,316.2\end{array}$ & $\begin{array}{r}-862.2 \\
2,266.6\end{array}$ \\
\hline 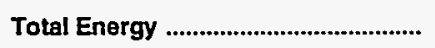 & $1,054.5$ & $2,041.4$ & $5,028.7$ & $6,392.8$ & $5,368.7$ & $5,213.7$ & $5,261.1$ & $5,537.6$ & $6,192.8$ & $5,995.6$ \\
\hline
\end{tabular}

a Includes petroleum coke used at electric utilities.

- Biomass fuels are not included, except those consumed at electric utilities and those added to motor gasoline.

c There are no direct fuel costs for hydroelectric, geothermal, centralized solar, or wind energy.

- No consumption, including cases where adjustments were made. See "Consumption Adjustments for Process Fuel and Intermediate Products." Note: Expenditure totals may not equal sum of components due to independent rounding.

Sources: Data sources, estimation procedures, and assumptions are described in the "Documentation" section of this report. 


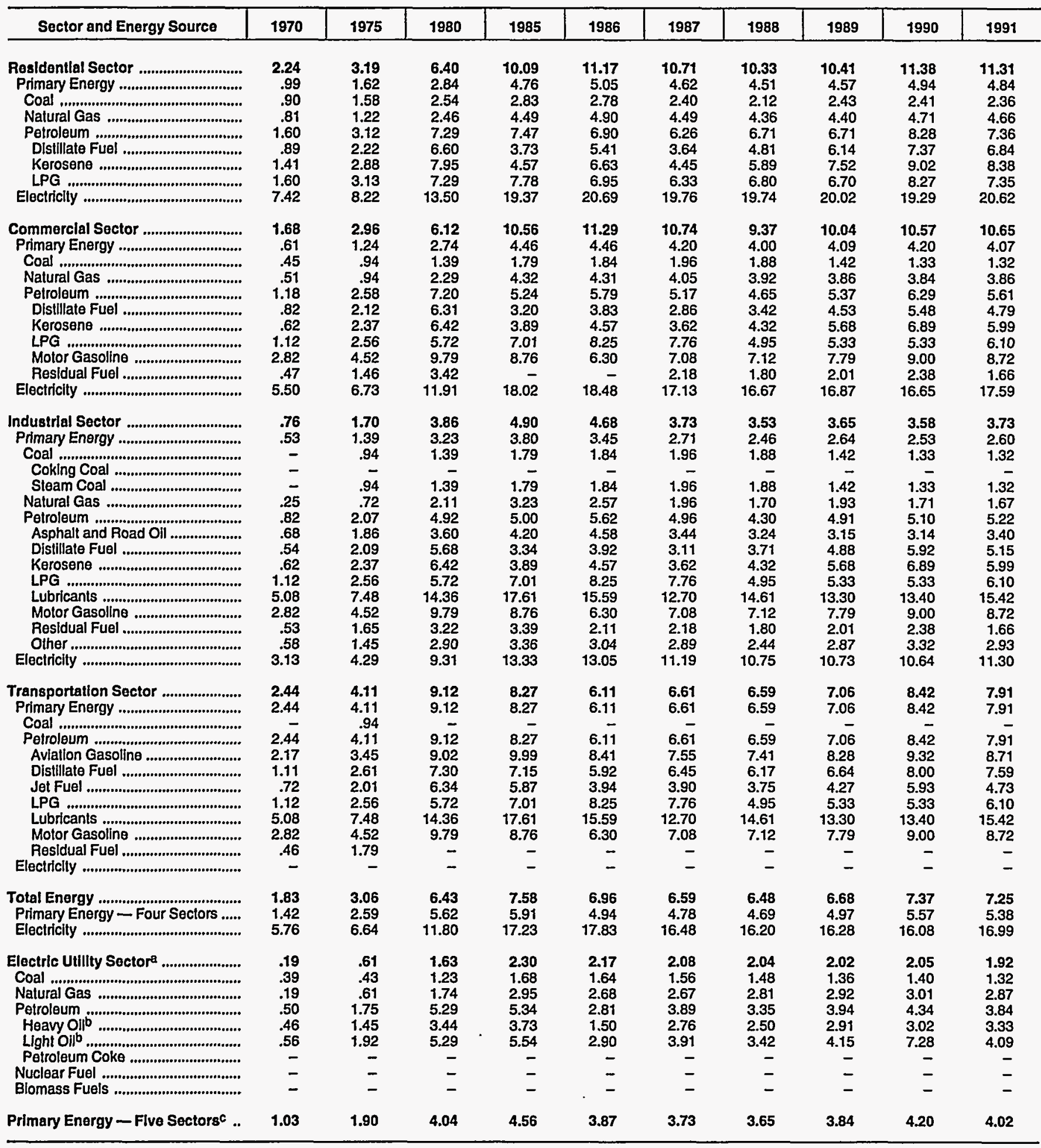

a There are no direct fuel costs for hydroelectric, geothermal, centralized solar, or wind energy.

b Heavy oll includes Grade Nos. 4, 5, and 6, and residual fuel oils. Light oil includes Grade No. 2 heating oil, kerosene, and jet fuel.

c Blomass fuels are not included, except those consumed at electric utilities and those added to motor gasoline.

-No consumptlon, Including cases where adjustments were made. See "Consumption Adjustments for Process Fuel and Intermediate Products."

Sources: Data sources, estimation procedures, and assumptions are described in the "Documentation" section of this report. 


\section{O Energy Expenditure Estimates by Sector, Oklahoma \\ K $1970,1975,1980$, and 1985-1991}

(Million Dollars)

\begin{tabular}{|c|c|c|c|c|c|c|c|c|c|c|}
\hline Sector and Energy Source & 1970 & 1975 & 1980 & 1985 & 1986 & 1987 & 1988 & 1989 & 1990 & 1891 \\
\hline $\begin{array}{l}\text { Residentlal Sector } \\
\text { Primary Energy }\end{array}$ & $\begin{array}{l}285.3 \\
100.7\end{array}$ & $\begin{array}{l}422.0 \\
163.3\end{array}$ & $\begin{array}{l}804.6 \\
237.8\end{array}$ & $\begin{array}{r}1,359.4 \\
407.8\end{array}$ & $\begin{array}{r}1,353.9 \\
372.4\end{array}$ & $\begin{array}{r}1,279.0 \\
329.5\end{array}$ & $\begin{array}{r}1,334.9 \\
359.9\end{array}$ & $\begin{array}{r}1,323.1 \\
361.1\end{array}$ & $\begin{array}{r}1,477.7 \\
353.7\end{array}$ & $\begin{array}{r}1,441.6 \\
363.6\end{array}$ \\
\hline 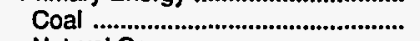 & & & .6 & .1 & .1 & & .1 & & & \\
\hline 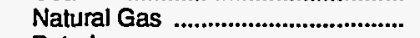 & 65.1 & 97.3 & 188.5 & 348.3 & 333.7 & 297.1 & 325.3 & 323.1 & 315.0 & 326.6 \\
\hline 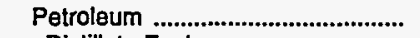 & 35.5 & 66.0 & 48.6 & 59.4 & 38.7 & 32.3 & 34.5 & 38.1 & 38.7 & 36.9 \\
\hline Distillate Fuel ......................................... & & .2 & .6 & 1.8 & .9 & .2 & .8 & • & • & \\
\hline 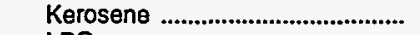 & .4 & .4 & .9 & .8 & .3 & .6 & .8 & .8 & .5 & .5 \\
\hline 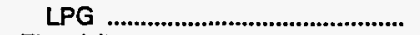 & 35.1 & 65.4 & 47.1 & 56.8 & $\mathbf{3 7 . 4}$ & 31.6 & 32.9 & 37.2 & 38.2 & 36.5 \\
\hline 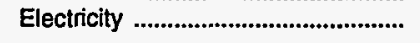 & 184.6 & 258.7 & 566.8 & 951.6 & 981.5 & 949.6 & 975.0 & 962.0 & $1,124.0$ & $1,078.0$ \\
\hline Commerclal Sector ................................ & 115.4 & 219.6 & 509.6 & 937.9 & 921.4 & 840.5 & 915.6 & 874.0 & 962.7 & 945.4 \\
\hline 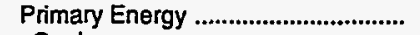 & 32.5 & 63.1 & 143.8 & 218.0 & 186.7 & 162.9 & 225.6 & 190.0 & 186.7 & 185.4 \\
\hline Coal & & & .6 & .1 & .1 & .1 & .2 & & & \\
\hline 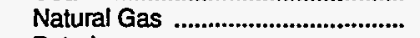 & 22.9 & 39.1 & 108.4 & 179.8 & 160.9 & 135.5 & 194.8 & 151.7 & 145.9 & 154.8 \\
\hline 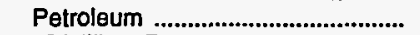 & 9.6 & 24.0 & 34.8 & 38.2 & 25.7 & 27.2 & 30.5 & 38.2 & 40.8 & 30.6 \\
\hline 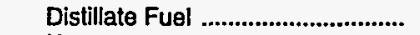 & .5 & 5.0 & 11.6 & 13.1 & 6.3 & 6.8 & 12.4 & 16.8 & 17.2 & 13.5 \\
\hline 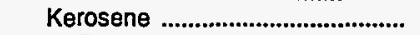 & .8 & 1.4 & .5 & .4 & .1 & .1 & 1.0 & 2.8 & .5 & .3 \\
\hline 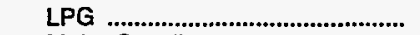 & 4.3 & 9.4 & 6.5 & 9.0 & 7.8 & 6.8 & 4.2 & 5.2 & 4.3 & 5.3 \\
\hline 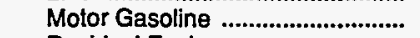 & 3.4 & 6.3 & 15.5 & 15.6 & 11.4 & 13.3 & 12.8 & 12.8 & 17.6 & 10.6 \\
\hline 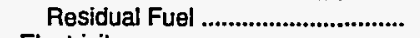 & .6 & 1.8 & .6 & - & - & .2 & .1 & .6 & 1.2 & .8 \\
\hline Electricity & 82.9 & 156.5 & 365.8 & 719.9 & 734.7 & 677.6 & 690.0 & 684.0 & 776.0 & 760.0 \\
\hline 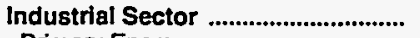 & 122.6 & 352.2 & $1,110.5$ & $1,495.1$ & $1,236.9$ & 1,074.1 & 976.5 & $1,072.3$ & $1,079.9$ & $1,088.7$ \\
\hline 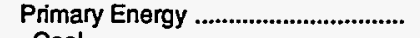 & 78.3 & 257.7 & 831.9 & $1,025.4$ & 794.6 & 687.2 & 593.8 & 678.3 & 663.8 & 660.1 \\
\hline 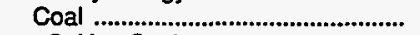 & - & .4 & 7.8 & 32.6 & 30.2 & 25.3 & 22.5 & 20.0 & 16.8 & 21.3 \\
\hline 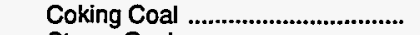 & - & - & - & & - & - & - & - & - & - \\
\hline 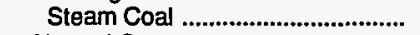 & - & .4 & 7.8 & 32.6 & 30.2 & 25.3 & 22.5 & 20.0 & 16.8 & 21.3 \\
\hline 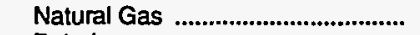 & 18.1 & 66.6 & 310.6 & 486.9 & 369.8 & 346.9 & 269.4 & 345.4 & 315.5 & 282.9 \\
\hline 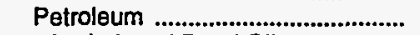 & 60.2 & 190.6 & 513.5 & 505.8 & 394.7 & 315.0 & 302.0 & 312.9 & 331.4 & 356.0 \\
\hline Asphalt and Road Oil .................... & 20.7 & 69.9 & 115.1 & 111.7 & 99.8 & 62.4 & 76.7 & 57.5 & 73.1 & 77.5 \\
\hline 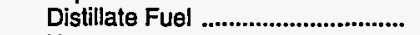 & 6.3 & 49.3 & 122.5 & 134.9 & 79.2 & 53.0 & 68.2 & 78.9 & 106.6 & 95.8 \\
\hline 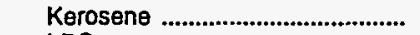 & 2.9 & 2.7 & 11.1 & 1.4 & 1.6 & .7 & .5 & .4 & .6 & .4 \\
\hline LPG & 8.7 & 19.7 & 137.7 & 135.5 & 120.1 & 104.8 & 57.3 & 72.8 & 36.2 & 71.9 \\
\hline 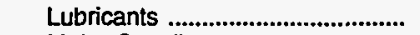 & 5.1 & 12.4 & 50.4 & 56.3 & 48.7 & 44.9 & 49.8 & 46.5 & 48.2 & 49.6 \\
\hline 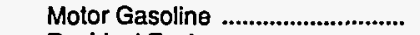 & 7.6 & 10.4 & 18.4 & 44.9 & 30.0 & 30.5 & 29.7 & 37.1 & 39.2 & 41.0 \\
\hline 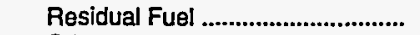 & 1.2 & 3.2 & 12.5 & 2.2 & 3.1 & 3.0 & 5.0 & 3.0 & 5.3 & 1.0 \\
\hline Other & 7.7 & 23.1 & 45.7 & 18.9 & 12.2 & 15.7 & 14.9 & 16.7 & 22.3 & 18.8 \\
\hline 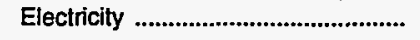 & 44.2 & 94.5 & 278.6 & 469.7 & 442.2 & 386.9 & 382.7 & 394.0 & 416.1 & 428.6 \\
\hline Transportation Sector ........................... & 531.2 & $1,047.7$ & $2,604.0$ & $2,600.4$ & $1,856.5$ & $2,020.1$ & $2,034.1$ & $2,268.1$ & $2,672.5$ & $2,519.9$ \\
\hline 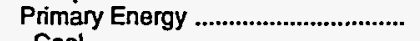 & 531.2 & $1,047.7$ & $2,604.0$ & $2,600.4$ & $1,856.5$ & $2,020.1$ & $2,034.1$ & $2,268.1$ & $2,672.5$ & $2,519.9$ \\
\hline Coal & & & & & & & & & & \\
\hline 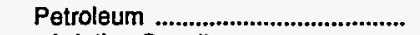 & 531.2 & $1,047.7$ & $2,604.0$ & $2,600.4$ & $1,856.5$ & $2,020.1$ & $2,034.1$ & $2,268.1$ & $2,672.5$ & $2,519.9$ \\
\hline Aviation Gasoline ........................... & 4.9 & 5.4 & 14.9 & 11.0 & 10.6 & 6.8 & 6.5 & 6.9 & 6.9 & 4.9 \\
\hline 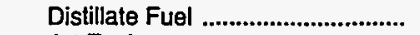 & 21.7 & 73.0 & 341.7 & 439.7 & 346.3 & 396.1 & 396.7 & 436.5 & 545.0 & 462.5 \\
\hline 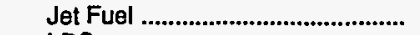 & 17.2 & 43.2 & 170.5 & 190.6 & 129.8 & 161.8 & 150.7 & 220.5 & 259.8 & 257.1 \\
\hline 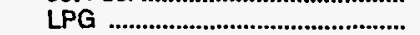 & 2.2 & 4.5 & 4.9 & 3.4 & 3.2 & 2.6 & 1.8 & 1.7 & 1.9 & 2.4 \\
\hline 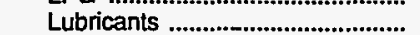 & 14.1 & 24.4 & 67.7 & 75.5 & 65.4 & 60.2 & 66.8 & 62.4 & 64.7 & 66.6 \\
\hline Motor Gasoline ................................ & 470.9 & 896.7 & $2,004.2$ & $1,880.2$ & $1,301.2$ & $1,392.6$ & $1,411.7$ & $1,540.2$ & $1,794.2$ & $1,726.5$ \\
\hline 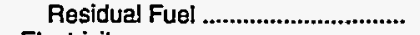 & .2 & .5 & - & - & - & - & - & - & - & - \\
\hline Electricity & - & - & - & - & - & - & - & - & - & - \\
\hline 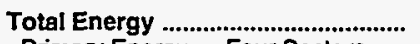 & $1,054.5$ & $2,041.4$ & $5,028.7$ & $6,392.8$ & $5,368.7$ & $5,213.7$ & $5,261.1$ & $5,537.6$ & $6,192.8$ & $5,995.6$ \\
\hline Primary Energy — Four Sectors ..... & 742.8 & $1,531.8$ & $3,817.4$ & $4,251.6$ & $3,210.2$ & $3,199.6$ & $3,213.4$ & $3,497.5$ & $3,876.6$ & $3,729.0$ \\
\hline Electricity & 311.7 & 509.6 & $1,211.3$ & $2,141.2$ & $2,158.5$ & $2,014.1$ & $2,047.7$ & $2,040.0$ & $2,316.2$ & $2,266.6$ \\
\hline 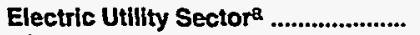 & 46.8 & 190.0 & 727.3 & 988.5 & 883.8 & 882.1 & 900.4 & 891.0 & 804.7 & 862.2 \\
\hline 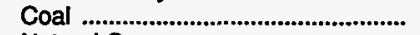 & & & 123.5 & 367.4 & 329.8 & 354.5 & 381.3 & 346.5 & 371.3 & 363.1 \\
\hline 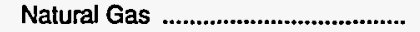 & 46.5 & 189.1 & 602.0 & 618.3 & 551.9 & 526.0 & 518.0 & 543.1 & 531.1 & 498.2 \\
\hline Petroleum & .4 & .9 & 1.8 & 2.7 & 2.0 & 1.6 & 1.2 & 1.5 & 2.3 & .9 \\
\hline 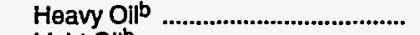 & .2 & .3 & $\cdot$ & .2 & .1 & • & .1 & .2 & 1.1 & .3 \\
\hline 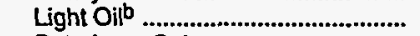 & .2 & .6 & 1.8 & 2.5 & 1.9 & 1.5 & 1.1 & 1.3 & 1.2 & .6 \\
\hline Petroleum Coke ....................................... & - & - & - & - & - & - & - & - & - & - \\
\hline 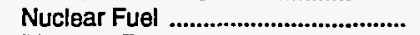 & - & - & - & - & - & - & - & - & - & - \\
\hline 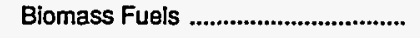 & - & - & - & - & - & - & - & - & - & - \\
\hline Primary Energy - Five Sector8 ${ }^{\circ}$.. & 789.7 & $1,721.8$ & $4,544.7$ & $5,240.0$ & $4,094.0$ & $4,081.7$ & $4,113.8$ & $4,388.6$ & $4,781.3$ & $4,591.3$ \\
\hline
\end{tabular}

a There are no direct fuel costs for hydroelectric, geothermal, centralized solar, or wind energy.

b Heavy oil includes Grade Nos. 4, 5, and 6, and residual fuel oils. Light oil includes Grade No. 2 heating oil, kerosene, and jet fuel.

c Biomass fuels are not included, except those consumed at electric utifities and those added to motor gasoline.

-No consumption, including cases where adjustments were made. See "Consumption Adjustments for Process Fuel and Intermediate Products."

Value less than 0.05 million dollars.

Note: Totals may not equal sum of components due to independent rounding.

Sources: Data sources, estimation procedures, and assumptions are described in the "Documentation" section of this report. 


\begin{tabular}{|c|c|c|c|c|c|c|c|c|c|c|}
\hline Energy Source & 1970 & 1975 & 1980 & 1985 & 1986 & 1987 & 1988 & 1989 & 1990 & 1991 \\
\hline & \multicolumn{10}{|c|}{ Prices in Dollars per Milllon Btu } \\
\hline 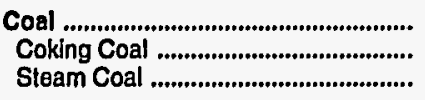 & $\begin{array}{r}0.57 \\
-57\end{array}$ & $\begin{array}{r}1.04 \\
1.04\end{array}$ & $\begin{array}{r}1.72 \\
1.72\end{array}$ & $\frac{2.15}{2.15}$ & $\begin{array}{r}2.47 \\
2.47\end{array}$ & $\begin{array}{r}2.43 \\
2.43\end{array}$ & $\begin{array}{r}2.43 \\
2.43\end{array}$ & $\begin{array}{r}1.76 \\
1.76\end{array}$ & $\begin{array}{r}1.22 \\
1.22\end{array}$ & $\begin{array}{r}1.17 \\
1.17\end{array}$ \\
\hline 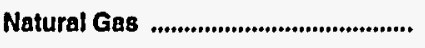 & .81 & 1.44 & 4.69 & $\mathbf{5 . 6 0}$ & 4.94 & 4.55 & 4.80 & 4.17 & 4.28 & 4.01 \\
\hline 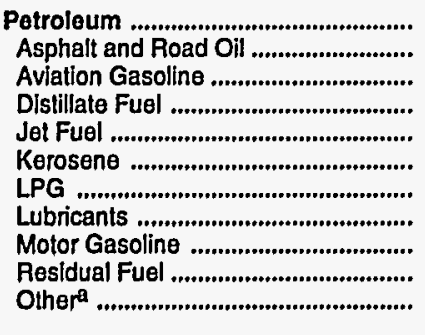 & $\begin{array}{r}1.88 \\
.58 \\
2.17 \\
1.21 \\
.73 \\
1.48 \\
2.21 \\
5.08 \\
2.83 \\
.51 \\
.78\end{array}$ & $\begin{array}{l}3.49 \\
1.78 \\
3.45 \\
2.62 \\
2.04 \\
2.84 \\
4.17 \\
7.48 \\
4.45 \\
2.06 \\
1.61\end{array}$ & $\begin{array}{r}7.86 \\
3.60 \\
9.02 \\
6.62 \\
6.21 \\
7.61 \\
7.09 \\
14.36 \\
9.75 \\
3.92 \\
4.04\end{array}$ & $\begin{array}{r}7.96 \\
4.42 \\
9.99 \\
7.98 \\
6.16 \\
9.85 \\
9.18 \\
17.61 \\
8.87 \\
4.70 \\
6.43\end{array}$ & $\begin{array}{r}5.86 \\
4.47 \\
8.41 \\
5.32 \\
4.24 \\
5.98 \\
9.58 \\
15.59 \\
6.92 \\
2.52 \\
2.80\end{array}$ & $\begin{array}{r}6.01 \\
4.27 \\
7.55 \\
5.75 \\
4.33 \\
6.31 \\
8.71 \\
12.70 \\
7.12 \\
2.62 \\
2.43\end{array}$ & $\begin{array}{r}6.12 \\
3.95 \\
7.41 \\
5.88 \\
4.04 \\
5.79 \\
8.54 \\
14.61 \\
7.48 \\
2.35 \\
2.19\end{array}$ & $\begin{array}{r}6.90 \\
3.19 \\
8.28 \\
6.77 \\
4.76 \\
7.48 \\
10.19 \\
13.30 \\
8.50 \\
2.55 \\
2.39\end{array}$ & $\begin{array}{r}7.77 \\
3.15 \\
9.32 \\
7.72 \\
5.93 \\
8.35 \\
10.37 \\
13.40 \\
9.45 \\
3.50 \\
2.50\end{array}$ & $\begin{array}{r}7.43 \\
3.28 \\
8.71 \\
7.78 \\
5.01 \\
8.21 \\
10.84 \\
15.42 \\
9.14 \\
2.56 \\
2.19\end{array}$ \\
\hline Nuclear Fuel ............................................ & - & .20 & .36 & .54 & .52 & .45 & .46 & .46 & .44 & .43 \\
\hline Blomass Fuols at Utilitles ................. & .65 & .92 & 1.74 & - & - & - & .87 & .33 & .85 & 1.63 \\
\hline Primary Energy - Flvo Sectors ${ }^{\mathrm{b}}$.... & 1.63 & 2.97 & 6.28 & 6.29 & 4.84 & 5.19 & 5.12 & $\mathbf{5 . 5 9}$ & 6.03 & 6.07 \\
\hline $\begin{array}{l}\text { Electric Utility Fuelc ............................. } \\
\text { Electricity Purchased by End Users }\end{array}$ & $\begin{array}{r}.48 \\
2.90\end{array}$ & $\begin{array}{l}2.04 \\
4.13\end{array}$ & $\begin{array}{r}.59 \\
7.59\end{array}$ & $\begin{array}{r}.67 \\
13.08\end{array}$ & $\begin{array}{r}.52 \\
12.95\end{array}$ & $\begin{array}{r}.45 \\
12.88\end{array}$ & $\begin{array}{r}.47 \\
12.61\end{array}$ & $\begin{array}{r}.99 \\
12.49\end{array}$ & $\begin{array}{r}.78 \\
12.25\end{array}$ & $\begin{array}{r}1.01 \\
12.45\end{array}$ \\
\hline \multirow[t]{2}{*}{ 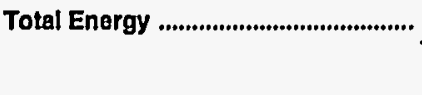 } & 1.87 & 3.22 & 7.34 & 8.77 & 7.36 & 7.41 & 7.42 & 7.85 & 8.32 & 8.07 \\
\hline & \multicolumn{10}{|c|}{ Expenditures In Mililions of Dollars } \\
\hline 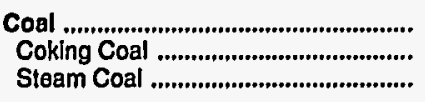 & $\begin{array}{r}1.7 \\
1 . \overline{7}\end{array}$ & $\begin{array}{r}2.8 \\
2 . \overline{8}\end{array}$ & $\begin{array}{r}20.8 \\
20.8\end{array}$ & $\begin{array}{r}21.6 \\
21.6\end{array}$ & $\begin{array}{r}7.2 \\
\overline{-} \\
7.2\end{array}$ & $\begin{array}{r}8.9 \\
-\overline{9}\end{array}$ & $\begin{array}{r}7.6 \\
-\overline{6}\end{array}$ & $\begin{array}{r}12.0 \\
12.0\end{array}$ & $\begin{array}{r}19.1 \\
-\overline{1} \\
19.1\end{array}$ & $\begin{array}{r}38.5 \\
- \\
38.5\end{array}$ \\
\hline Natural Gas ............................................ & 68.7 & 139.9 & 320.9 & 432.9 & 340.2 & 337.2 & 388.0 & 429.7 & 438.3 & 472.4 \\
\hline 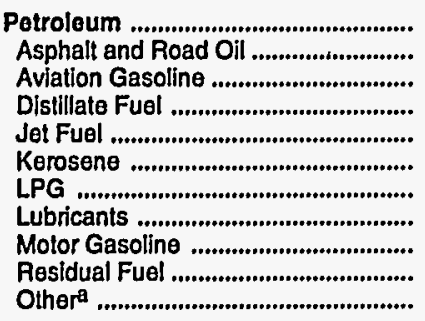 & \begin{tabular}{r|}
541.1 \\
8.3 \\
3.3 \\
89.2 \\
8.6 \\
1.8 \\
10.2 \\
23.7 \\
371.2 \\
18.5 \\
6.3
\end{tabular} & $\begin{array}{r}1,039.9 \\
38.0 \\
3.0 \\
199.4 \\
24.0 \\
3.6 \\
10.0 \\
30.8 \\
675.3 \\
45.4 \\
10.4\end{array}$ & $\begin{array}{r}2,584.6 \\
59.2 \\
11.8 \\
643.9 \\
86.5 \\
4.8 \\
31.7 \\
65.4 \\
1,562.9 \\
100.0 \\
18.1\end{array}$ & $\begin{array}{r}2,507.2 \\
83.3 \\
7.1 \\
715.0 \\
74.3 \\
3.8 \\
44.7 \\
73.0 \\
1,353.9 \\
142.9 \\
9.2\end{array}$ & $\begin{array}{r}1,909.8 \\
65.9 \\
8.2 \\
461.1 \\
62.5 \\
1.0 \\
52.9 \\
63.2 \\
1,088.0 \\
86.9 \\
20.0\end{array}$ & $\begin{array}{r}2,040.8 \\
60.6 \\
4.8 \\
543.0 \\
71.4 \\
.6 \\
47.5 \\
58.2 \\
1,144.3 \\
83.8 \\
26.6\end{array}$ & $\begin{array}{r}2,196.3 \\
63.5 \\
3.7 \\
563.9 \\
72.7 \\
.7 \\
49.3 \\
64.6 \\
1,263.3 \\
91.0 \\
23.8\end{array}$ & $\begin{array}{r}2,452.2 \\
59.4 \\
4.2 \\
640.6 \\
90.9 \\
2.1 \\
60.5 \\
60.3 \\
1,423.0 \\
86.4 \\
24.8\end{array}$ & $\begin{array}{r}2,760.5 \\
63.3 \\
5.7 \\
767.2 \\
111.3 \\
1.2 \\
52.1 \\
62.5 \\
1,566.3 \\
98.9 \\
32.1\end{array}$ & $\begin{array}{r}2,693.2 \\
57.9 \\
5.5 \\
731.8 \\
103.5 \\
1.0 \\
61.1 \\
64.4 \\
1,541.7 \\
101.7 \\
24.6\end{array}$ \\
\hline Nuclear Fuel ........................................... & - & - & 21.4 & 40.6 & 39.5 & 21.1 & 31.4 & 26.1 & 28.6 & 6.8 \\
\hline Blomass Fuels at Utllitles .................. & .3 & - & 2.9 & - & - & - & .9 & .1 & - & - \\
\hline Primary Energy - Five Sectors ${ }^{b}$.... & 611.8 & $1,182.6$ & $2,950.6$ & $3,002.3$ & $2,296.7$ & $2,408.1$ & $2,624.1$ & $2,920.1$ & $3,246.5$ & $3,210.8$ \\
\hline $\begin{array}{l}\text { Electric Utility Fuelc ........................... } \\
\text { Electricity Purchased by End Users }\end{array}$ & $\begin{array}{r}-.8 \\
248.3\end{array}$ & $\begin{array}{r}-.4 \\
458.4\end{array}$ & $\begin{array}{l}-41.1 \\
950.4\end{array}$ & $\begin{array}{r}-54.5 \\
1,573.1\end{array}$ & $\begin{array}{r}-39.6 \\
1,549.2\end{array}$ & $\begin{array}{r}-21.1 \\
1,657.4\end{array}$ & $\begin{array}{r}-32.3 \\
1,692.0\end{array}$ & $\begin{array}{r}-75.5 \\
1,774.0\end{array}$ & $\begin{array}{r}-67.9 \\
1,797.0\end{array}$ & $\begin{array}{r}-58.1 \\
1,855.0\end{array}$ \\
\hline Total Enorgy ......................................... & 859.3 & $1,640.6$ & $3,860.0$ & $4,520.9$ & $3,806.3$ & $4,044.3$ & $4,283.8$ & $4,618.6$ & $4,975.6$ & $5,007.7$ \\
\hline
\end{tabular}

a Includes petroleum coke used at electric utilities.

b Biomess fuels are not included, except those consumed at electric utilities and those added to motor gasoline.

c There are no direct fuel costs for hydroelectric, geothermal, centralized solar, or wind energy.

- No consumption, including cases where adjustments were made. See "Consumption Adjustments for Process Fuel and Intermediate Products."

Value less than 0.05 million dollars.

Note: Expenditure tolals may not equal sum of components due to independent rounding.

Sources: Data sources, estimation procedures, and assumptions are described in the "Documentation" section of this report. 


\section{- Energy Price Estimates by Sector, Oregon \\ R 1970, 1975, 1980, and 1985-1991 \\ E (Dollars per Million Btu)}

\begin{tabular}{|c|c|c|c|c|c|c|c|c|c|c|}
\hline Sector and Energy Source & 1970 & 1975 & 1980 & 1985 & 1986 & 1987 & 1988 & 1989 & 1990 & 1991 \\
\hline 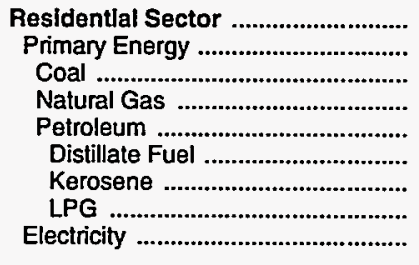 & $\begin{array}{r}2.47 \\
1.53 \\
.95 \\
1.45 \\
1.63 \\
1.41 \\
2.79 \\
2.66 \\
3.65\end{array}$ & $\begin{array}{l}3.78 \\
2.42 \\
1.14 \\
2.11 \\
3.03 \\
2.80 \\
3.82 \\
5.27 \\
5.27\end{array}$ & $\begin{array}{l}8.04 \\
6.20 \\
4.26 \\
5.36 \\
7.35 \\
7.02 \\
9.80 \\
9.00 \\
9.37\end{array}$ & $\begin{array}{r}10.78 \\
6.94 \\
3.67 \\
6.73 \\
7.24 \\
7.00 \\
9.85 \\
8.73 \\
13.72\end{array}$ & $\begin{array}{r}10.67 \\
6.18 \\
3.79 \\
6.47 \\
5.77 \\
5.08 \\
7.14 \\
10.89 \\
13.84\end{array}$ & $\begin{array}{r}10.93 \\
6.21 \\
3.76 \\
6.44 \\
5.84 \\
5.23 \\
7.36 \\
9.83 \\
14.07\end{array}$ & $\begin{array}{r}10.87 \\
6.23 \\
3.37 \\
6.64 \\
5.51 \\
5.11 \\
7.19 \\
9.16 \\
14.02\end{array}$ & $\begin{array}{r}10.82 \\
6.30 \\
3.66 \\
5.98 \\
6.89 \\
5.78 \\
8.14 \\
15.93 \\
13.97\end{array}$ & $\begin{array}{r}10.95 \\
6.69 \\
3.77 \\
6.13 \\
7.82 \\
6.99 \\
9.84 \\
13.92 \\
13.85\end{array}$ & $\begin{array}{r}11.01 \\
6.52 \\
5.21 \\
5.95 \\
8.01 \\
6.73 \\
9.47 \\
14.26 \\
14.11\end{array}$ \\
\hline 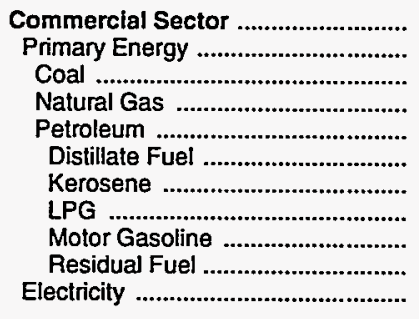 & $\begin{array}{r}2.29 \\
1.16 \\
.53 \\
1.22 \\
1.14 \\
1.22 \\
.93 \\
1.12 \\
2.83 \\
.79 \\
3.90\end{array}$ & $\begin{array}{l}3.67 \\
2.21 \\
1.04 \\
1.79 \\
2.68 \\
2.60 \\
2.58 \\
2.76 \\
4.45 \\
2.45 \\
5.20\end{array}$ & $\begin{array}{l}7.29 \\
5.66 \\
2.24 \\
4.88 \\
6.39 \\
6.71 \\
6.54 \\
5.40 \\
9.75 \\
4.90 \\
8.86\end{array}$ & $\begin{array}{r}10.91 \\
6.24 \\
2.48 \\
6.06 \\
6.58 \\
6.42 \\
9.86 \\
9.45 \\
8.87 \\
4.12 \\
14.96\end{array}$ & $\begin{array}{r}10.46 \\
5.08 \\
2.47 \\
5.47 \\
4.49 \\
4.59 \\
3.21 \\
9.06 \\
6.92 \\
2.10 \\
14.82\end{array}$ & $\begin{array}{r}10.44 \\
4.77 \\
2.42 \\
5.17 \\
4.23 \\
3.96 \\
4.72 \\
8.28 \\
7.12 \\
2.65 \\
15.00\end{array}$ & $\begin{array}{r}10.01 \\
4.86 \\
2.42 \\
5.24 \\
4.30 \\
4.16 \\
4.52 \\
8.38 \\
7.48 \\
2.55 \\
14.19\end{array}$ & $\begin{array}{r}10.07 \\
4.71 \\
2.37 \\
4.64 \\
4.91 \\
4.77 \\
5.25 \\
8.55 \\
8.50 \\
2.39 \\
14.17\end{array}$ & $\begin{array}{r}10.15 \\
5.17 \\
2.54 \\
4.74 \\
5.96 \\
5.91 \\
6.74 \\
9.03 \\
9.45 \\
3.03 \\
14.05\end{array}$ & $\begin{array}{r}10.23 \\
4.79 \\
2.65 \\
4.61 \\
5.29 \\
5.28 \\
6.05 \\
9.29 \\
9.14 \\
2.36 \\
14.29\end{array}$ \\
\hline 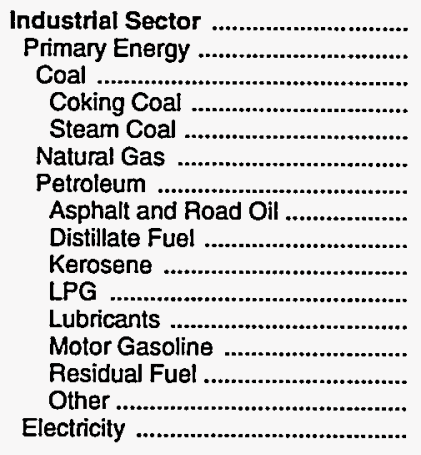 & $\begin{array}{r}.78 \\
.67 \\
.53 \\
- \\
.53 \\
.46 \\
.83 \\
.58 \\
.80 \\
.93 \\
1.12 \\
5.08 \\
2.83 \\
.33 \\
.78 \\
1.26\end{array}$ & $\begin{array}{r}1.73 \\
1.59 \\
1.04 \\
- \\
1.04 \\
.92 \\
2.16 \\
1.78 \\
2.29 \\
2.58 \\
2.76 \\
7.48 \\
4.45 \\
1.85 \\
1.61 \\
2.13\end{array}$ & $\begin{array}{r}4.56 \\
4.53 \\
2.24 \\
-\overline{2} \\
2.24 \\
4.21 \\
4.84 \\
3.60 \\
5.62 \\
6.54 \\
5.40 \\
14.36 \\
9.75 \\
3.39 \\
4.04 \\
4.65\end{array}$ & $\begin{array}{r}6.93 \\
5.58 \\
2.48 \\
-\overline{28} \\
2.48 \\
4.65 \\
6.42 \\
4.42 \\
8.72 \\
9.86 \\
9.45 \\
17.61 \\
8.87 \\
4.12 \\
6.43 \\
10.32\end{array}$ & $\begin{array}{r}5.61 \\
3.78 \\
2.47 \\
-\overline{2} \\
2.47 \\
3.73 \\
3.88 \\
4.47 \\
2.84 \\
3.21 \\
9.06 \\
15.59 \\
6.92 \\
2.10 \\
2.80 \\
10.08\end{array}$ & $\begin{array}{r}5.66 \\
3.77 \\
2.42 \\
- \\
2.42 \\
3.30 \\
4.15 \\
4.27 \\
4.18 \\
4.72 \\
8.28 \\
12.70 \\
7.12 \\
2.65 \\
2.43 \\
9.91\end{array}$ & $\begin{array}{r}5.68 \\
3.84 \\
2.42 \\
- \\
2.42 \\
3.64 \\
4.04 \\
3.95 \\
4.00 \\
4.52 \\
8.38 \\
14.61 \\
7.48 \\
2.55 \\
2.19 \\
9.82\end{array}$ & $\begin{array}{r}5.80 \\
3.87 \\
2.37 \\
- \\
2.37 \\
3.40 \\
4.29 \\
3.19 \\
4.64 \\
5.25 \\
8.55 \\
13.30 \\
8.50 \\
2.39 \\
2.39 \\
9.69\end{array}$ & $\begin{array}{r}5.71 \\
4.00 \\
2.54 \\
- \\
2.54 \\
3.39 \\
4.55 \\
3.15 \\
5.96 \\
6.74 \\
9.03 \\
13.40 \\
9.45 \\
3.03 \\
2.50 \\
9.27\end{array}$ & $\begin{array}{r}5.58 \\
3.84 \\
2.65 \\
- \\
2.65 \\
3.31 \\
4.48 \\
3.28 \\
5.35 \\
6.05 \\
9.29 \\
15.42 \\
9.14 \\
2.36 \\
2.19 \\
9.23\end{array}$ \\
\hline 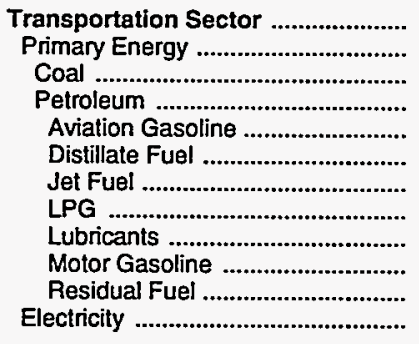 & $\begin{array}{r}2.41 \\
2.41 \\
.53 \\
2.41 \\
2.17 \\
1.34 \\
.73 \\
1.12 \\
5.08 \\
2.83 \\
.71 \\
-\end{array}$ & $\begin{array}{r}3.98 \\
3.98 \\
1.04 \\
3.98 \\
3.45 \\
2.69 \\
2.04 \\
2.76 \\
7.48 \\
4.45 \\
2.21 \\
-\end{array}$ & $\begin{array}{r}8.81 \\
8.81 \\
- \\
8.81 \\
9.02 \\
6.96 \\
6.21 \\
5.40 \\
14.36 \\
9.75 \\
4.14 \\
-\end{array}$ & $\begin{array}{r}8.40 \\
8.40 \\
- \\
8.40 \\
9.99 \\
8.27 \\
6.16 \\
9.45 \\
17.61 \\
8.87 \\
5.02 \\
-\end{array}$ & $\begin{array}{r}6.39 \\
6.39 \\
- \\
6.39 \\
8.41 \\
6.16 \\
4.24 \\
9.06 \\
15.59 \\
6.92 \\
2.86 \\
-\end{array}$ & $\begin{array}{r}6.54 \\
6.54 \\
- \\
6.54 \\
7.55 \\
6.63 \\
4.33 \\
8.28 \\
12.70 \\
7.12 \\
2.60 \\
17.04\end{array}$ & $\begin{array}{r}6.69 \\
6.69 \\
- \\
6.69 \\
7.41 \\
6.81 \\
4.04 \\
8.38 \\
14.61 \\
7.48 \\
2.26 \\
13.54\end{array}$ & $\begin{array}{r}7.50 \\
7.50 \\
- \\
7.50 \\
8.28 \\
7.77 \\
4.76 \\
8.55 \\
13.30 \\
8.50 \\
2.57 \\
14.24\end{array}$ & $\begin{array}{r}8.54 \\
8.54 \\
- \\
8.54 \\
9.32 \\
8.54 \\
5.93 \\
9.03 \\
13.40 \\
9.45 \\
3.59 \\
13.90\end{array}$ & $\begin{array}{r}7.99 \\
7.99 \\
- \\
7.99 \\
8.71 \\
8.63 \\
5.01 \\
9.29 \\
15.42 \\
9.14 \\
2.58 \\
15.56\end{array}$ \\
\hline $\begin{array}{l}\text { Total Energy ............................... } \\
\text { Primary Energy - Four Sectors ...... } \\
\text { Electricity ....................................... }\end{array}$ & $\begin{array}{l}1.87 \\
1.63 \\
2.90\end{array}$ & $\begin{array}{l}3.22 \\
2.97 \\
4.13\end{array}$ & $\begin{array}{l}7.34 \\
7.26 \\
7.59\end{array}$ & $\begin{array}{r}8.77 \\
7.46 \\
13.08\end{array}$ & $\begin{array}{r}7.36 \\
5.68 \\
12.95\end{array}$ & $\begin{array}{r}7.41 \\
5.72 \\
12.88\end{array}$ & $\begin{array}{r}7.42 \\
5.85 \\
12.61\end{array}$ & $\begin{array}{r}7.85 \\
6.38 \\
12.49\end{array}$ & $\begin{array}{r}8.32 \\
7.04 \\
12.25\end{array}$ & $\begin{array}{r}8.07 \\
6.69 \\
12.45\end{array}$ \\
\hline 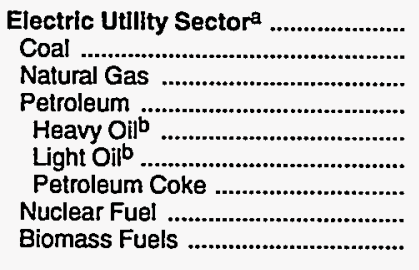 & $\begin{array}{r}.48 \\
- \\
.37 \\
.80 \\
.80 \\
.83 \\
- \\
- \\
.65\end{array}$ & $\begin{array}{r}2.04 \\
- \\
1.27 \\
2.31 \\
- \\
2.31 \\
- \\
.20 \\
.92\end{array}$ & $\begin{array}{r}.59 \\
1.41 \\
4.29 \\
6.53 \\
- \\
6.53 \\
- \\
.36 \\
1.74\end{array}$ & $\begin{array}{r}.67 \\
2.00 \\
-\overline{5} \\
5.67 \\
-\overline{5} \\
- \\
.54 \\
-\end{array}$ & $\begin{array}{r}.52 \\
- \\
2.8 \overline{1} \\
3.24 \\
- \\
3.24 \\
- \\
.52 \\
-\end{array}$ & $\begin{array}{r}.45 \\
- \\
\overline{-} \\
4.10 \\
\overline{0} \\
4.10 \\
\overline{45} \\
-\end{array}$ & $\begin{array}{r}.47 \\
- \\
- \\
4.03 \\
- \\
4.03 \\
- \\
.46 \\
.87\end{array}$ & $\begin{array}{r}.99 \\
1.56 \\
2.92 \\
4.53 \\
- \\
4.53 \\
- \\
.46 \\
.33\end{array}$ & $\begin{array}{r}.78 \\
1.08 \\
3.03 \\
3.47 \\
-\overline{37} \\
-\overline{44} \\
.85\end{array}$ & $\begin{array}{r}1.01 \\
1.08 \\
1.57 \\
4.75 \\
- \\
4.75 \\
- \\
.43 \\
1.63\end{array}$ \\
\hline Primary Energy - Five Sectors ${ }^{c}$.. & 1.63 & 2.97 & 6.28 & 6.29 & 4.84 & 5.19 & 5.12 & 5.59 & 6.03 & 6.07 \\
\hline
\end{tabular}

a There are no direct fuel costs for hydroelectric, geothermal, centralized solar, or wind energy.

b Heavy oil includes Grade Nos. 4, 5, and 6, and residual fuel oils. Light oil includes Grade No. 2 heating oil, kerosene, and jet fuel.

c Biomass fuels are not included, except those consumed at electric utilities and those added to motor gasoline.

-No consumption, including cases where adjustments were made. See "Consumption Adjustments for Process Fuel and intermediate Products."

Sources: Data sources, estimation procedures, and assumptions are described in the "Documentation" section of this report. 
Energy Expenditure Estimates by Sector, Oregon

$1970,1975,1980$, and 1985-1991

(Million Dollars)

\begin{tabular}{|c|c|c|c|c|c|c|c|c|c|c|}
\hline Sector and Energy Source & 1970 & 1975 & 1980 & 1985 & 1986 & 1987 & 1988 & 1989 & 1990 & 1991 \\
\hline 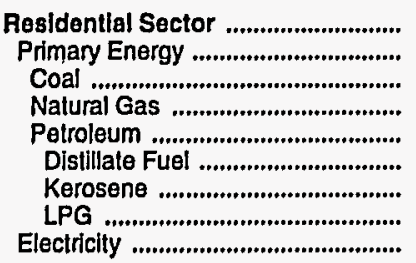 & $\begin{array}{r}188.1 \\
65.4 \\
.2 \\
29.8 \\
35.3 \\
25.6 \\
1.0 \\
8.7 \\
122.8\end{array}$ & $\begin{array}{r}327.8 \\
110.3 \\
.1 \\
63.1 \\
47.1 \\
39.0 \\
1.0 \\
7.1 \\
217.4\end{array}$ & $\begin{array}{r}640.2 \\
207.3 \\
.6 \\
103.1 \\
103.5 \\
82.5 \\
2.1 \\
19.0 \\
432.9\end{array}$ & $\begin{array}{r}944.3 \\
264.2 \\
14 \\
148.8 \\
115.4 \\
96.8 \\
2.3 \\
16.3 \\
680.0\end{array}$ & $\begin{array}{r}852.5 \\
204.6 \\
126.1 \\
78.6 \\
60.5 \\
.9 \\
17.2 \\
647.8\end{array}$ & $\begin{array}{r}851.2 \\
192.9 \\
.1 \\
124.1 \\
68.7 \\
53.2 \\
.4 \\
15.1 \\
658.3\end{array}$ & $\begin{array}{r}893.4 \\
207.3 \\
.1 \\
141.4 \\
65.8 \\
54.9 \\
.4 \\
10.6 \\
686.0\end{array}$ & $\begin{array}{r}944.9 \\
225.9 \\
.2 \\
139.3 \\
86.4 \\
63.6 \\
1.8 \\
21.0 \\
719.0\end{array}$ & $\begin{array}{r}966.2 \\
239.3 \\
.1 \\
146.6 \\
92.6 \\
72.7 \\
.7 \\
19.2 \\
727.0\end{array}$ & $\begin{array}{r}1,013.6 \\
245.6 \\
16 \\
161.4 \\
84.2 \\
58.3 \\
.7 \\
25.2 \\
768.0\end{array}$ \\
\hline 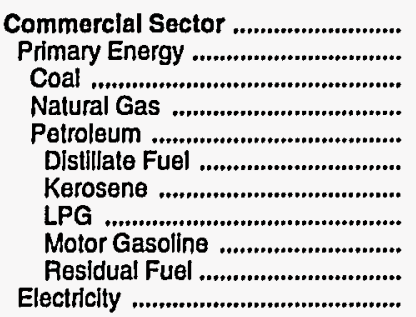 & $\begin{array}{r}126.1 \\
37.4 \\
.3 \\
14.5 \\
22.6 \\
11.5 \\
.2 \\
.6 \\
3.7 \\
6.6 \\
88.7\end{array}$ & $\begin{array}{r}225.8 \\
69.7 \\
.2 \\
29.6 \\
39.8 \\
18.8 \\
.5 \\
.7 \\
5.1 \\
14.8 \\
156.1\end{array}$ & $\begin{array}{r}509.3 \\
193.4 \\
.6 \\
77.5 \\
115.3 \\
70.0 \\
1.4 \\
2.0 \\
14.9 \\
27.0 \\
316.0\end{array}$ & $\begin{array}{r}718.7 \\
191.1 \\
.1 \\
118.9 \\
72.0 \\
51.8 \\
1.4 \\
3.1 \\
10.8 \\
4.9 \\
527.6\end{array}$ & $\begin{array}{r}668.8 \\
145.6 \\
94.2 \\
51.3 \\
35.8 \\
.1 \\
2.5 \\
8.5 \\
4.3 \\
523.2\end{array}$ & $\begin{array}{r}693.3 \\
141.4 \\
.1 \\
88.8 \\
52.5 \\
37.4 \\
.1 \\
2.2 \\
9.1 \\
3.7 \\
552.0\end{array}$ & $\begin{array}{r}700.9 \\
152.3 \\
.2 \\
98.7 \\
53.4 \\
36.8 \\
.2 \\
1.7 \\
9.3 \\
5.3 \\
548.6\end{array}$ & $\begin{array}{r}705.0 \\
143.3 \\
.2 \\
97.2 \\
45.9 \\
29.9 \\
.2 \\
2.0 \\
9.8 \\
4.0 \\
561.7\end{array}$ & $\begin{array}{r}746.3 \\
166.6 \\
.1 \\
99.2 \\
67.4 \\
46.0 \\
.3 \\
2.2 \\
13.4 \\
5.5 \\
579.7\end{array}$ & $\begin{array}{r}756.4 \\
151.9 \\
106.1 \\
45.8 \\
30.6 \\
.1 \\
2.9 \\
8.4 \\
3.8 \\
604.6\end{array}$ \\
\hline 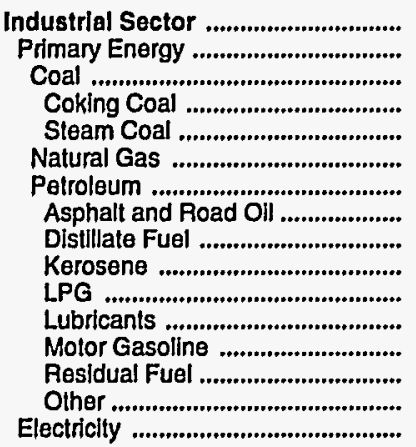 & $\begin{array}{r}119.1 \\
82.2 \\
1.2 \\
1 . \overline{2} \\
23.9 \\
57.1 \\
8.3 \\
14.8 \\
.6 \\
.8 \\
8.7 \\
10.7 \\
7.0 \\
6.3 \\
36.8\end{array}$ & $\begin{array}{r}268.3 \\
183.5 \\
2.5 \\
2 . \overline{2} \\
2.5 \\
47.2 \\
133.9 \\
38.0 \\
35.1 \\
2.1 \\
2.1 \\
8.6 \\
13.1 \\
24.5 \\
10.4 \\
84.8\end{array}$ & $\begin{array}{r}650.4 \\
448.7 \\
8.5 \\
\overline{8} \\
8.5 \\
138.8 \\
301.5 \\
59.2 \\
128.4 \\
1.4 \\
9.5 \\
19.3 \\
21.4 \\
44.2 \\
18.1 \\
201.6\end{array}$ & $\begin{array}{r}862.3 \\
496.9 \\
7.5 \\
- \\
7.5 \\
165.2 \\
324.2 \\
83.3 \\
128.5 \\
.1 \\
18.9 \\
21.5 \\
22.4 \\
40.3 \\
9.2 \\
365.4\end{array}$ & $\begin{array}{r}725.4 \\
347.3 \\
7.1 \\
- \\
7.1 \\
119.9 \\
220.2 \\
65.9 \\
41.0 \\
28.0 \\
18.6 \\
18.2 \\
28.5 \\
20.0 \\
378.2\end{array}$ & $\begin{array}{r}827.3 \\
380.6 \\
8.8 \\
-\overline{8} \\
8.8 \\
124.3 \\
247.6 \\
60.6 \\
74.1 \\
24.9 \\
17.2 \\
18.0 \\
26.2 \\
26.6 \\
446.7\end{array}$ & $\begin{array}{r}859.5 \\
402.5 \\
7.2 \\
\overline{-} \\
7.2 \\
147.9 \\
247.3 \\
63.5 \\
67.9 \\
30.9 \\
19.0 \\
16.4 \\
25.8 \\
23.8 \\
457.0\end{array}$ & $\begin{array}{r}889.5 \\
396.5 \\
3.5 \\
\overline{-} \\
3.5 \\
154.0 \\
238.9 \\
59.4 \\
78.4 \\
.1 \\
31.7 \\
17.8 \\
21.3 \\
5.5 \\
24.8 \\
493.0\end{array}$ & $\begin{array}{r}930.4 \\
440.4 \\
3.6 \\
- \\
3.6 \\
169.6 \\
267.1 \\
63.3 \\
98.7 \\
.2 \\
24.7 \\
18.4 \\
21.0 \\
8.6 \\
32.1 \\
490.0\end{array}$ & $\begin{array}{r}904.0 \\
422.0 \\
5.0 \\
-\overline{5} \\
5.0 \\
187.7 \\
229.3 \\
57.9 \\
71.4 \\
.1 \\
27.7 \\
19.0 \\
23.5 \\
5.2 \\
24.6 \\
482.0\end{array}$ \\
\hline 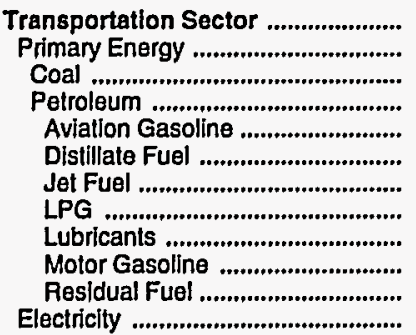 & $\begin{array}{r}426.0 \\
426.0 \\
426.0 \\
3.3 \\
37.4 \\
8.6 \\
.1 \\
15.0 \\
356.7 \\
4.8 \\
-\end{array}$ & $\begin{array}{r}818.7 \\
818.7 \\
818.7 \\
3.0 \\
106.2 \\
24.0 \\
.1 \\
22.3 \\
657.1 \\
6.1 \\
-\end{array}$ & $\begin{array}{r}2,060.1 \\
2,060.1 \\
- \\
2,060.1 \\
11.8 \\
358.9 \\
86.5 \\
1.3 \\
46.1 \\
1,526.7 \\
28.8 \\
-\end{array}$ & $\begin{array}{r}1,995.5 \\
1,995.5 \\
- \\
1,995.5 \\
7.1 \\
437.8 \\
74.3 \\
6.5 \\
51.5 \\
1,320.7 \\
97.6 \\
-\end{array}$ & $\begin{array}{r}1,559.6 \\
1,559.6 \\
-\overline{-} \\
1,559.6 \\
8.2 \\
323.8 \\
62.5 \\
5.1 \\
44.6 \\
1,061.3 \\
54.1 \\
-\end{array}$ & $\begin{array}{r}1,672.4 \\
1,672.0 \\
- \\
1,672.0 \\
4.8 \\
378.3 \\
71.4 \\
5.3 \\
41.1 \\
1,117.2 \\
53.9 \\
.4\end{array}$ & $\begin{array}{r}1,830.1 \\
1,829.7 \\
- \\
1,829.7 \\
3.7 \\
404.2 \\
72.7 \\
6.2 \\
45.5 \\
1,237.5 \\
59.9 \\
.4\end{array}$ & $\begin{array}{r}2,079.3 \\
2,078.9 \\
- \\
2,078.9 \\
4.2 \\
466.7 \\
90.9 \\
5.8 \\
42.5 \\
1,391.9 \\
76.9 \\
.4\end{array}$ & $\begin{array}{r}2,332.7 \\
2,332.3 \\
- \\
2,332.3 \\
5.7 \\
548.7 \\
111.3 \\
6.0 \\
44.1 \\
1,531.8 \\
84.8 \\
.4\end{array}$ & $\begin{array}{r}2,333.7 \\
2,333.3 \\
- \\
2,333.3 \\
5.5 \\
570.9 \\
103.5 \\
5.3 \\
45.4 \\
1,509.8 \\
92.8 \\
.4\end{array}$ \\
\hline $\begin{array}{l}\text { Total Energy .................................... } \\
\text { Primary Energy - Four Sectors ..... } \\
\text { Electricity .......................................... }\end{array}$ & $\begin{array}{l}859.3 \\
611.0 \\
248.3\end{array}$ & $\begin{array}{r}1,640.6 \\
1,182.2 \\
458.4\end{array}$ & $\begin{array}{r}3,860.0 \\
2,909.5 \\
950.4\end{array}$ & $\begin{array}{l}4,520.9 \\
2,947.8 \\
1,573.1\end{array}$ & $\begin{array}{l}3,806.3 \\
2,257.1 \\
1,549.2\end{array}$ & $\begin{array}{l}4,044.3 \\
2,387.0 \\
1,657.4\end{array}$ & $\begin{array}{l}4,283.8 \\
2,591.8 \\
1,692.0\end{array}$ & $\begin{array}{l}4,618.6 \\
2,844.6 \\
1,774.0\end{array}$ & $\begin{array}{l}4,975.6 \\
3,178.6 \\
1,797.0\end{array}$ & $\begin{array}{l}5,007.7 \\
3,152.7 \\
1,855.0\end{array}$ \\
\hline 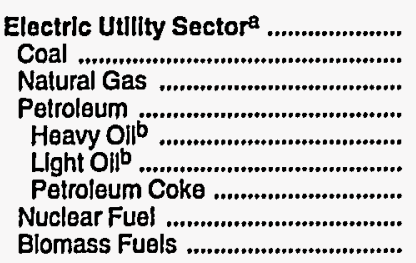 & $\begin{array}{l}.8 \\
.4 \\
.1 \\
.1 \\
- \\
.3\end{array}$ & $\begin{array}{l}.4 \\
\div \\
.4 \\
\overline{4} \\
\overline{-} \\
-\end{array}$ & $\begin{array}{r}41.1 \\
11.2 \\
1.4 \\
4.2 \\
\overline{-} \\
4 . \overline{2} \\
21 . \overline{4} \\
2.9\end{array}$ & $\begin{array}{r}54.5 \\
13.9 \\
\overline{1} \\
\overline{1} \\
. \overline{1} \\
40 . \overline{6} \\
-\end{array}$ & $\begin{array}{r}39.6 \\
- \\
.1 \\
-1 \\
\overline{-} \\
39.5 \\
-\end{array}$ & $\begin{array}{r}21.1 \\
- \\
\overline{-} \\
\overline{-} \\
21.1 \\
-\end{array}$ & $\begin{array}{r}32.3 \\
\overline{-} \\
\overline{-} \\
\overline{-} \\
31 . \overline{4} \\
.9\end{array}$ & $\begin{array}{r}75.5 \\
8.1 \\
39.2 \\
2.0 \\
-\overline{0} \\
2.0 \\
-\overline{1} \\
26.1 \\
.1\end{array}$ & $\begin{array}{r}67.9 \\
15.3 \\
22.9 \\
1.1 \\
-\overline{1} \\
1.1 \\
- \\
28.6\end{array}$ & $\begin{array}{r}58.1 \\
33.5 \\
17.2 \\
.6 \\
\overline{6} \\
. \\
6.8\end{array}$ \\
\hline Primary Energy - Flve Sectors ${ }^{c}$.. & 611.8 & $1,182.6$ & $2,950.6$ & $3,002.3$ & $2,296.7$ & $2,408.1$ & $2,624.1$ & $2,920.1$ & $3,246.5$ & $3,210.8$ \\
\hline
\end{tabular}

a There are no direct fuel costs for hydroelectric, geothermal, centralized solar, or wind energy.

b Heavy oll includes Grade Nos. 4, 5, and 6, and residual fuel oils. Light oil includes Grade No. 2 heating oil, kerosene, and jet fuel.

c Blomass fuels are not included, except those consumed at electric utilities and those added to motor gasoline.

-No consumplion, including cases where adjustments were made. See "Consumption Adjustments for Process Fuel and Intermediate Products."

Value less than 0.05 million dollars.

Note: Totals may not equal sum of components due to independent rounding.

Sources: Dala sources, estimation procedures, and assumptions are described in the "Documentation" section of this report. 


\begin{tabular}{|c|c|c|c|c|c|c|c|c|c|c|}
\hline Energy Source & 1970 & 1975 & 1980 & 1985 & 1986 & 1987 & 1988 & 1989 & 1990 & 1991 \\
\hline & \multicolumn{10}{|c|}{ Prices in Dollars per Million Btu } \\
\hline 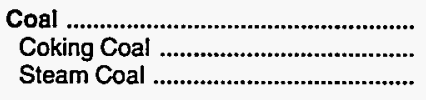 & $\begin{array}{r}0.39 \\
.44 \\
.35\end{array}$ & $\begin{array}{l}1.21 \\
1.52 \\
1.03\end{array}$ & $\begin{array}{l}1.59 \\
2.20 \\
1.35\end{array}$ & $\begin{array}{l}1.64 \\
1.88 \\
1.58\end{array}$ & $\begin{array}{l}1.55 \\
1.76 \\
1.52\end{array}$ & $\begin{array}{l}1.49 \\
1.59 \\
1.47\end{array}$ & $\begin{array}{l}1.50 \\
1.61 \\
1.47\end{array}$ & $\begin{array}{l}1.50 \\
1.67 \\
1.47\end{array}$ & $\begin{array}{l}1.57 \\
1.71 \\
1.54\end{array}$ & $\begin{array}{l}1.59 \\
1.75 \\
1.55\end{array}$ \\
\hline 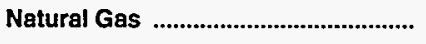 & .87 & 1.53 & 3.37 & 5.74 & 5.38 & 4.84 & 4.70 & 4.96 & 5.34 & 5.38 \\
\hline 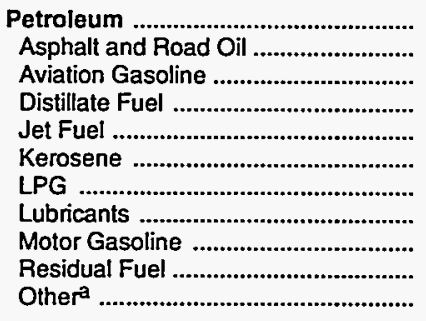 & $\begin{array}{r}1.71 \\
.78 \\
2.17 \\
1.17 \\
.72 \\
1.39 \\
1.82 \\
5.08 \\
2.92 \\
.47 \\
1.15\end{array}$ & $\begin{array}{l}3.45 \\
1.97 \\
3.45 \\
2.65 \\
2.01 \\
2.88 \\
3.39 \\
7.48 \\
4.72 \\
2.02 \\
2.35\end{array}$ & $\begin{array}{r}7.67 \\
3.72 \\
9.02 \\
6.70 \\
6.27 \\
7.75 \\
6.16 \\
14.36 \\
9.71 \\
4.30 \\
6.54\end{array}$ & $\begin{array}{r}8.06 \\
4.95 \\
9.99 \\
7.73 \\
5.84 \\
8.37 \\
9.75 \\
17.61 \\
9.01 \\
4.38 \\
7.15\end{array}$ & $\begin{array}{r}5.87 \\
4.01 \\
8.41 \\
5.87 \\
3.91 \\
6.41 \\
8.86 \\
15.59 \\
6.54 \\
2.45 \\
5.52\end{array}$ & $\begin{array}{r}6.07 \\
3.25 \\
7.55 \\
5.88 \\
4.02 \\
6.13 \\
8.49 \\
12.70 \\
7.03 \\
2.95 \\
5.54\end{array}$ & $\begin{array}{r}6.02 \\
3.04 \\
7.41 \\
5.84 \\
3.85 \\
6.22 \\
8.86 \\
14.61 \\
7.01 \\
2.34 \\
4.74\end{array}$ & $\begin{array}{r}6.59 \\
2.81 \\
8.28 \\
6.32 \\
4.44 \\
6.92 \\
9.29 \\
13.30 \\
7.79 \\
2.83 \\
5.44\end{array}$ & $\begin{array}{r}7.91 \\
2.83 \\
9.32 \\
7.79 \\
5.59 \\
8.32 \\
11.40 \\
13.40 \\
9.35 \\
3.20 \\
5.64\end{array}$ & $\begin{array}{r}7.96 \\
2.76 \\
8.71 \\
7.61 \\
4.81 \\
8.08 \\
12.19 \\
15.42 \\
9.44 \\
2.31 \\
5.80\end{array}$ \\
\hline Nuclear Fuel ......................................... & .21 & .25 & .42 & .92 & .78 & .78 & .84 & .88 & .83 & .77 \\
\hline Blomass Fuels at Utilities ................ & - & - & - & - & - & - & - & - & - & - \\
\hline Primary Energy - Five Sectors ${ }^{\mathrm{b}}$.... & .97 & 2.02 & 4.04 & 4.45 & 3.56 & 3.56 & 3.48 & 3.72 & 4.02 & 4.04 \\
\hline $\begin{array}{l}\text { Electric Utility Fuelc .......................... } \\
\text { Electricity Purchased by End Users }\end{array}$ & $\begin{array}{r}.34 \\
5.23\end{array}$ & $\begin{array}{r}.93 \\
10.37\end{array}$ & $\begin{array}{r}1.55 \\
15.17\end{array}$ & $\begin{array}{r}1.61 \\
21.24\end{array}$ & $\begin{array}{r}1.35 \\
21.86\end{array}$ & $\begin{array}{r}1.36 \\
21.48\end{array}$ & $\begin{array}{r}1.35 \\
20.92\end{array}$ & $\begin{array}{r}1.38 \\
21.68\end{array}$ & $\begin{array}{r}1.32 \\
22.46\end{array}$ & $\begin{array}{r}1.28 \\
23.51\end{array}$ \\
\hline \multirow[t]{2}{*}{ 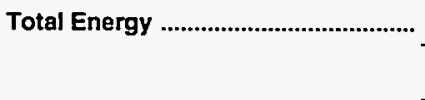 } & 1.47 & 3.26 & 6.41 & 8.48 & 7.66 & 7.45 & 7.31 & 7.77 & 8.58 & 8.94 \\
\hline & \multicolumn{10}{|c|}{ Expenditures in Millions of Dollars } \\
\hline 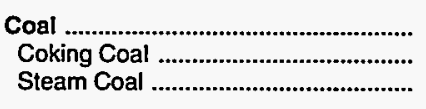 & $\begin{array}{l}656.6 \\
317.5 \\
339.1\end{array}$ & $\begin{array}{r}1,991.7 \\
913.7 \\
1,078.1\end{array}$ & $\begin{array}{l}2,594.6 \\
1,005.0 \\
1,589.6\end{array}$ & $\begin{array}{r}2,307.2 \\
492.9 \\
1,814.3\end{array}$ & $\begin{array}{r}2,042.7 \\
330.9 \\
1,711.8\end{array}$ & $\begin{array}{r}2,051.5 \\
357.1 \\
1,694.3\end{array}$ & $\begin{array}{r}2,193.0 \\
437.4 \\
1,755.6\end{array}$ & $\begin{array}{r}2,199.6 \\
442.2 \\
1,757.4\end{array}$ & $\begin{array}{r}2,244.7 \\
480.0 \\
1,764.7\end{array}$ & $\begin{array}{r}2,163.0 \\
412.9 \\
1,750.1\end{array}$ \\
\hline 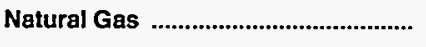 & 653.4 & 964.8 & $2,489.5$ & $3,444.8$ & $3,147.9$ & $2,944.4$ & $3,012.8$ & $3,293.4$ & $3,291.5$ & $3,285.9$ \\
\hline 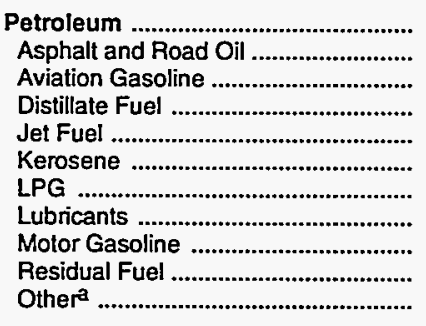 & $\begin{array}{r}2,447.5 \\
34.0 \\
7.3 \\
429.1 \\
36.9 \\
33.6 \\
32.7 \\
118.5 \\
1,559.6 \\
157.4 \\
38.6\end{array}$ & $\begin{array}{r}4,720.6 \\
74.2 \\
7.4 \\
1,039.9 \\
97.3 \\
55.5 \\
76.5 \\
152.0 \\
2,695.2 \\
441.3 \\
81.4\end{array}$ & $\begin{array}{r}10,484.8 \\
127.1 \\
15.3 \\
2,665.1 \\
360.1 \\
121.5 \\
163.8 \\
354.3 \\
5,507.0 \\
798.1 \\
372.4\end{array}$ & $\begin{array}{r}9,331.7 \\
161.3 \\
10.5 \\
2,418.9 \\
334.6 \\
168.9 \\
262.3 \\
395.4 \\
4,825.9 \\
483.8 \\
270.1\end{array}$ & $\begin{array}{r}7,082.5 \\
158.5 \\
10.7 \\
1,850.0 \\
219.3 \\
138.5 \\
269.5 \\
342.3 \\
3,576.6 \\
348.8 \\
168.3\end{array}$ & $\begin{array}{r}7,587.5 \\
141.9 \\
5.6 \\
1,976.3 \\
239.6 \\
101.3 \\
258.6 \\
315.4 \\
3,927.4 \\
427.8 \\
193.5\end{array}$ & $\begin{array}{r}7,603.6 \\
110.4 \\
7.1 \\
1,996.8 \\
255.1 \\
130.2 \\
192.4 \\
349.8 \\
4,084.0 \\
312.7 \\
165.2\end{array}$ & $\begin{array}{r}8,318.6 \\
125.1 \\
7.4 \\
2,258.4 \\
242.5 \\
120.4 \\
228.0 \\
326.5 \\
4,453.3 \\
372.1 \\
185.0\end{array}$ & $\begin{array}{r}9,444.3 \\
140.3 \\
6.8 \\
2,444.5 \\
380.7 \\
78.0 \\
255.3 \\
338.7 \\
5,246.8 \\
338.3 \\
214.8\end{array}$ & $\begin{array}{r}9,243.7 \\
113.6 \\
5.1 \\
2,346.4 \\
310.5 \\
81.7 \\
324.9 \\
348.6 \\
5,310.8 \\
214.3 \\
187.9\end{array}$ \\
\hline 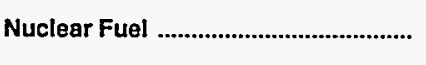 & 1.1 & 44.3 & 55.4 & 262.2 & 336.6 & 293.8 & 342.6 & 369.7 & 511.5 & 474.0 \\
\hline Blomass Fuels at Utilitles ................. & - & - & - & - & - & - & - & - & - & - \\
\hline Primary Energy - Five Sectors ${ }^{b} \ldots$ & $3,758.7$ & $7,721.4$ & $15,624.2$ & $15,345.9$ & $12,609.7$ & $12,877.2$ & $13,152.0$ & $14,181.3$ & $15,492.0$ & $15,166.6$ \\
\hline $\begin{array}{l}\text { Electric Utility Fuelc .......................... } \\
\text { Electricity Purchased by End Users }\end{array}$ & $\begin{array}{r}-296.5 \\
1,330.2\end{array}$ & $\begin{array}{r}-1,047.7 \\
3,060.5\end{array}$ & $\begin{array}{r}-1,997.2 \\
5,096.8\end{array}$ & $\begin{array}{r}-2,233.1 \\
7,203.2\end{array}$ & $\begin{array}{r}-2,058.5 \\
7,568.9\end{array}$ & $\begin{array}{r}-2,014.1 \\
7,789.5\end{array}$ & $\begin{array}{r}-2,092.5 \\
7,994.3\end{array}$ & $\begin{array}{r}-2,169.5 \\
8,360.8\end{array}$ & $\begin{array}{r}-2,215.1 \\
8,736.6\end{array}$ & $\begin{array}{r}-2,122.4 \\
9,235.0\end{array}$ \\
\hline 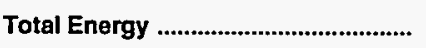 & $4,792.4$ & $9,734.3$ & $18,723.9$ & $20,315.9$ & $18,120.1$ & $18,652.6$ & $19,053.8$ & $20,372.6$ & $22,013.5$ & $22,279.2$ \\
\hline
\end{tabular}

Includes petroleum coke used at electric utilities.

b Biomass fuels are not included, except those consumed at electric utilities and those added to motor gasoline.

c There are no direct fuel costs for hydroelectric, geothermal, centralized solar, or wind energy.

-No consumption, including cases where adjustments were made. See "Consumption Adjustments for Process Fuel and Intermediate Products."

Note: Expenditure totals may not equal sum of components due to independent rounding.

Sources: Data sources, estimation procedures, and assumptions are described in the "Documentation" section of this report. 


\begin{tabular}{|c|c|c|c|c|c|c|c|c|c|c|}
\hline Sector and Energy Source & 1970 & 1975 & 1980 & 1985 & 1986 & 1987 & 1988 & 1989 & 1990 & 1991 \\
\hline 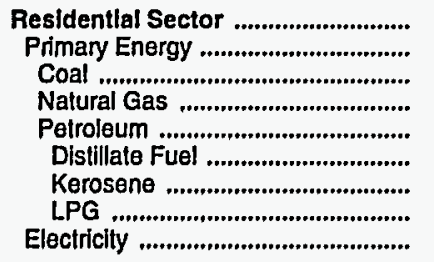 & $\begin{array}{l}1.98 \\
1.26 \\
1.03 \\
1.20 \\
1.41 \\
1.35 \\
1.57 \\
2.47 \\
7.15\end{array}$ & $\begin{array}{r}3.97 \\
2.32 \\
2.57 \\
1.89 \\
2.89 \\
2.81 \\
3.12 \\
4.42 \\
12.80\end{array}$ & $\begin{array}{r}7.17 \\
4.92 \\
2.70 \\
3.73 \\
7.10 \\
6.95 \\
8.05 \\
9.00 \\
17.42\end{array}$ & $\begin{array}{r}10.75 \\
6.95 \\
2.83 \\
6.50 \\
8.12 \\
7.82 \\
8.68 \\
11.63 \\
25.05\end{array}$ & $\begin{array}{r}10.48 \\
6.13 \\
2.76 \\
6.17 \\
6.50 \\
6.22 \\
6.90 \\
9.88 \\
25.96\end{array}$ & $\begin{array}{r}10.41 \\
5.80 \\
2.72 \\
5.82 \\
6.21 \\
5.88 \\
6.52 \\
10.30 \\
25.96\end{array}$ & $\begin{array}{r}10.19 \\
5.68 \\
2.69 \\
5.59 \\
6.23 \\
5.95 \\
6.60 \\
9.26 \\
25.44\end{array}$ & $\begin{array}{r}10.57 \\
6.10 \\
2.79 \\
5.92 \\
6.85 \\
6.50 \\
7.22 \\
11.08 \\
26.09\end{array}$ & $\begin{array}{r}11.94 \\
6.80 \\
2.96 \\
6.37 \\
8.30 \\
7.84 \\
8.70 \\
12.94 \\
27.02\end{array}$ & $\begin{array}{r}12.34 \\
6.88 \\
2.64 \\
6.53 \\
8.23 \\
7.62 \\
8.46 \\
13.85 \\
28.09\end{array}$ \\
\hline 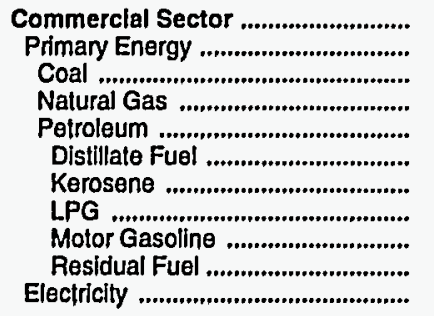 & $\begin{array}{r}1.90 \\
.90 \\
.32 \\
.93 \\
1.12 \\
1.09 \\
.74 \\
1.40 \\
2.92 \\
.47 \\
6.71\end{array}$ & $\begin{array}{r}4.48 \\
1.94 \\
1.25 \\
1.67 \\
2.56 \\
2.48 \\
2.52 \\
2.84 \\
4.72 \\
2.02 \\
11.88\end{array}$ & $\begin{array}{r}7.57 \\
3.95 \\
1.33 \\
3.49 \\
6.08 \\
6.39 \\
6.01 \\
5.36 \\
9.71 \\
4.43 \\
16.63\end{array}$ & $\begin{array}{r}11.38 \\
5.76 \\
1.61 \\
5.99 \\
6.56 \\
6.78 \\
7.14 \\
8.92 \\
9.01 \\
4.70 \\
23.28\end{array}$ & $\begin{array}{r}11.06 \\
4.97 \\
1.52 \\
5.61 \\
4.66 \\
4.72 \\
4.64 \\
8.54 \\
6.54 \\
2.71 \\
23.66\end{array}$ & $\begin{array}{r}10.68 \\
4.68 \\
1.46 \\
5.23 \\
4.66 \\
4.69 \\
5.02 \\
7.82 \\
7.03 \\
3.05 \\
22.76\end{array}$ & $\begin{array}{r}10.38 \\
4.46 \\
1.45 \\
4.91 \\
4.34 \\
4.31 \\
4.78 \\
8.59 \\
7.01 \\
2.54 \\
22.37\end{array}$ & $\begin{array}{r}10.79 \\
4.82 \\
1.44 \\
5.16 \\
5.15 \\
5.16 \\
5.50 \\
8.20 \\
7.79 \\
3.02 \\
23.26\end{array}$ & $\begin{array}{r}12.06 \\
5.54 \\
1.47 \\
5.79 \\
6.23 \\
6.11 \\
6.39 \\
10.33 \\
9.35 \\
3.46 \\
24.01\end{array}$ & $\begin{array}{r}12.45 \\
5.46 \\
1.43 \\
5.80 \\
6.13 \\
5.98 \\
6.04 \\
11.09 \\
9.44 \\
2.51 \\
24.65\end{array}$ \\
\hline 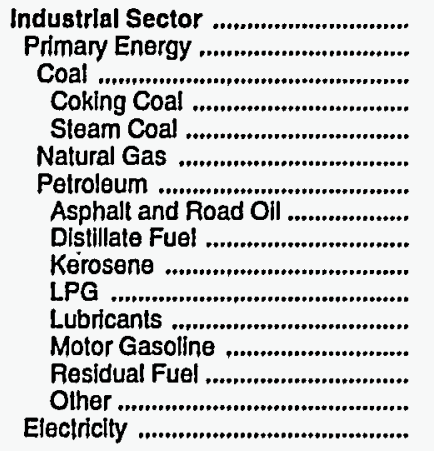 & $\begin{array}{r}.78 \\
.55 \\
.41 \\
.44 \\
.32 \\
.57 \\
.98 \\
.78 \\
.70 \\
.74 \\
1.40 \\
5.08 \\
2.92 \\
.50 \\
1.15 \\
3.55\end{array}$ & $\begin{array}{l}2.24 \\
1.62 \\
1.47 \\
1.52 \\
1.25 \\
1.07 \\
2.55 \\
1.97 \\
2.38 \\
2.52 \\
2.84 \\
7.48 \\
4.72 \\
2.07 \\
2.35 \\
7.99\end{array}$ & $\begin{array}{r}4.30 \\
3.13 \\
2.03 \\
2.20 \\
1.33 \\
3.00 \\
6.03 \\
3.72 \\
5.67 \\
6.01 \\
5.36 \\
14.36 \\
9.71 \\
4.07 \\
6.74 \\
12.87\end{array}$ & $\begin{array}{r}6.08 \\
3.96 \\
1.81 \\
1.88 \\
1.61 \\
4.77 \\
7.78 \\
4.95 \\
6.45 \\
7.14 \\
8.92 \\
17.61 \\
9.01 \\
4.70 \\
7.98 \\
17.07\end{array}$ & $\begin{array}{r}5.94 \\
3.54 \\
1.68 \\
1.76 \\
1.52 \\
4.21 \\
5.86 \\
4.01 \\
4.20 \\
4.64 \\
8.54 \\
15.59 \\
6.54 \\
2.71 \\
6.46 \\
17.31\end{array}$ & $\begin{array}{r}5.41 \\
3.16 \\
1.55 \\
1.59 \\
1.46 \\
3.52 \\
5.47 \\
3.25 \\
4.53 \\
5.02 \\
7.82 \\
12.70 \\
7.03 \\
3.05 \\
6.57 \\
16.91\end{array}$ & $\begin{array}{r}5.20 \\
2.98 \\
1.57 \\
1.61 \\
1.45 \\
3.50 \\
5.46 \\
3.04 \\
4.32 \\
4.78 \\
8.59 \\
14.61 \\
7.01 \\
2.54 \\
5.74 \\
16.18\end{array}$ & $\begin{array}{r}5.39 \\
3.14 \\
1.61 \\
1.67 \\
1.44 \\
3.76 \\
5.49 \\
2.81 \\
4.97 \\
5.50 \\
8.20 \\
13.30 \\
7.79 \\
3.02 \\
6.58 \\
16.86\end{array}$ & $\begin{array}{r}5.60 \\
3.31 \\
1.65 \\
1.71 \\
1.47 \\
4.02 \\
5.83 \\
2.83 \\
5.78 \\
6.39 \\
10.33 \\
13.40 \\
9.35 \\
3.46 \\
6.54 \\
17.51\end{array}$ & $\begin{array}{r}5.93 \\
3.35 \\
1.65 \\
1.75 \\
1.43 \\
3.88 \\
6.25 \\
2.76 \\
5.46 \\
6.04 \\
11.09 \\
15.42 \\
9.44 \\
2.51 \\
6.91 \\
18.43\end{array}$ \\
\hline 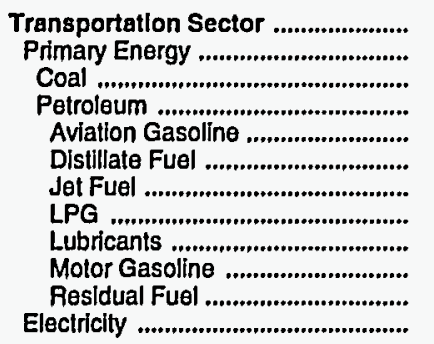 & \begin{tabular}{r|}
2.48 \\
2.48 \\
.32 \\
2.48 \\
2.17 \\
1.35 \\
.72 \\
1.40 \\
5.08 \\
2.92 \\
.42 \\
3.66
\end{tabular} & $\begin{array}{l}4.16 \\
4.15 \\
1.25 \\
4.15 \\
3.45 \\
2.64 \\
2.01 \\
2.84 \\
7.48 \\
4.72 \\
1.80 \\
8.41\end{array}$ & $\begin{array}{r}8.86 \\
8.85 \\
- \\
8.85 \\
9.02 \\
7.05 \\
6.27 \\
5.36 \\
14.36 \\
9.71 \\
3.76 \\
15.14\end{array}$ & $\begin{array}{r}8.67 \\
8.65 \\
- \\
8.65 \\
9.99 \\
8.35 \\
5.84 \\
8.92 \\
17.61 \\
9.01 \\
4.14 \\
21.08\end{array}$ & $\begin{array}{r}6.29 \\
6.27 \\
- \\
6.27 \\
8.41 \\
6.52 \\
3.91 \\
8.54 \\
15.59 \\
6.54 \\
2.23 \\
20.55\end{array}$ & $\begin{array}{r}6.64 \\
6.61 \\
- \\
6.61 \\
7.55 \\
6.73 \\
4.02 \\
7.82 \\
12.70 \\
7.03 \\
2.78 \\
29.63\end{array}$ & $\begin{array}{r}6.60 \\
6.57 \\
- \\
6.57 \\
7.41 \\
6.66 \\
3.85 \\
8.59 \\
14.61 \\
7.01 \\
2.08 \\
29.15\end{array}$ & $\begin{array}{r}7.34 \\
7.31 \\
- \\
7.31 \\
8.28 \\
7.05 \\
4.44 \\
8.20 \\
13.30 \\
7.79 \\
2.47 \\
29.79\end{array}$ & $\begin{array}{r}8.72 \\
8.69 \\
- \\
8.69 \\
9.32 \\
8.79 \\
5.59 \\
10.33 \\
13.40 \\
9.35 \\
2.82 \\
31.66\end{array}$ & $\begin{array}{r}8.68 \\
8.64 \\
- \\
8.64 \\
8.71 \\
8.56 \\
4.81 \\
11.09 \\
15.42 \\
9.44 \\
2.10 \\
32.74\end{array}$ \\
\hline $\begin{array}{l}\text { Total Energy ..................................... } \\
\text { Primary Energy - Four Sectors ...... } \\
\text { Electricity ........................................... }\end{array}$ & $\begin{array}{l}1.47 \\
1.15 \\
5.23\end{array}$ & $\begin{array}{r}.3 .26 \\
2.48 \\
. \quad 10.37\end{array}$ & $\begin{array}{r}6.41 \\
5.27 \\
-15.17\end{array}$ & $\begin{array}{r}8.48 \\
6.37 \\
21.24\end{array}$ & $\begin{array}{r}7.66 \\
5.23 \\
21.86\end{array}$ & $\begin{array}{r}7.45 \\
5.08 \\
21.48\end{array}$ & $\begin{array}{r}7.31 \\
4.97 \\
20.92\end{array}$ & $\begin{array}{r}7.77 \\
5.37 \\
21.68\end{array}$ & $\begin{array}{r}8.58 \\
6.10 \\
22.46\end{array}$ & $\begin{array}{r}8.94 \\
6.22 \\
23.51\end{array}$ \\
\hline 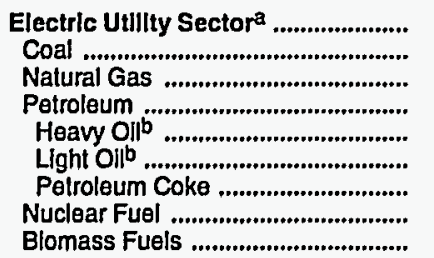 & $\begin{array}{l}.34 \\
.31 \\
.41 \\
.47 \\
.47 \\
.49 \\
- \\
.21 \\
-\end{array}$ & $\begin{array}{r}.93 \\
.95 \\
1.47 \\
2.12 \\
2.07 \\
2.27 \\
-\overline{.25} \\
-\end{array}$ & $\begin{array}{r}1.55 \\
1.33 \\
3.60 \\
4.60 \\
4.52 \\
5.85 \\
.72 \\
.42 \\
-\end{array}$ & $\begin{array}{r}1.61 \\
1.56 \\
5.08 \\
4.30 \\
4.32 \\
5.85 \\
1.27 \\
.92 \\
-\end{array}$ & $\begin{array}{r}1.35 \\
1.49 \\
3.61 \\
2.47 \\
2.42 \\
3.79 \\
1.09 \\
.78 \\
-\end{array}$ & $\begin{array}{r}1.36 \\
1.44 \\
3.35 \\
2.95 \\
2.99 \\
4.01 \\
1.05 \\
.78 \\
-\end{array}$ & $\begin{array}{r}1.35 \\
1.45 \\
3.33 \\
2.39 \\
2.37 \\
3.56 \\
1.01 \\
.84 \\
-\end{array}$ & $\begin{array}{r}1.38 \\
1.45 \\
3.48 \\
2.87 \\
2.87 \\
4.07 \\
.94 \\
.88 \\
-\end{array}$ & $\begin{array}{r}1.32 \\
1.52 \\
2.95 \\
3.32 \\
3.31 \\
5.48 \\
.90 \\
.83 \\
-\end{array}$ & $\begin{array}{r}1.28 \\
1.55 \\
2.95 \\
2.48 \\
2.40 \\
4.76 \\
.84 \\
.77 \\
-\end{array}$ \\
\hline PrImary Energy - Flve Sectors ${ }^{c}$.. & .97 & 2.02 & 4.04 & 4.45 & 3.56 & 3.56 & 3.48 & 3.72 & 4.02 & 4.04 \\
\hline
\end{tabular}

There are no direct fuel costs for hydroelectric, geothermal, centralized solar, or wind energy.

b Heavy oil includes Grade Nos. 4, 5, and 6, and residual fuel oils. Light oil includes Grade No. 2 heating oil, kerosene, and jet fuel.

c Biomass fuels are not included, except those consumed at electric utilities and those added to motor gasoline.

-No consumption, including cases where adjustments were made. See "Consumption Adjustments for Process Fuel and Intermediate Products."

Sources: Data sources, estimation procedures, and assumptions are described in the "Documentation" section of this report. 
P Energy Expenditure Estimates by Sector, Pennsylvania

E $1970,1975,1980$, and 1985-1991

N (Million Dollars)

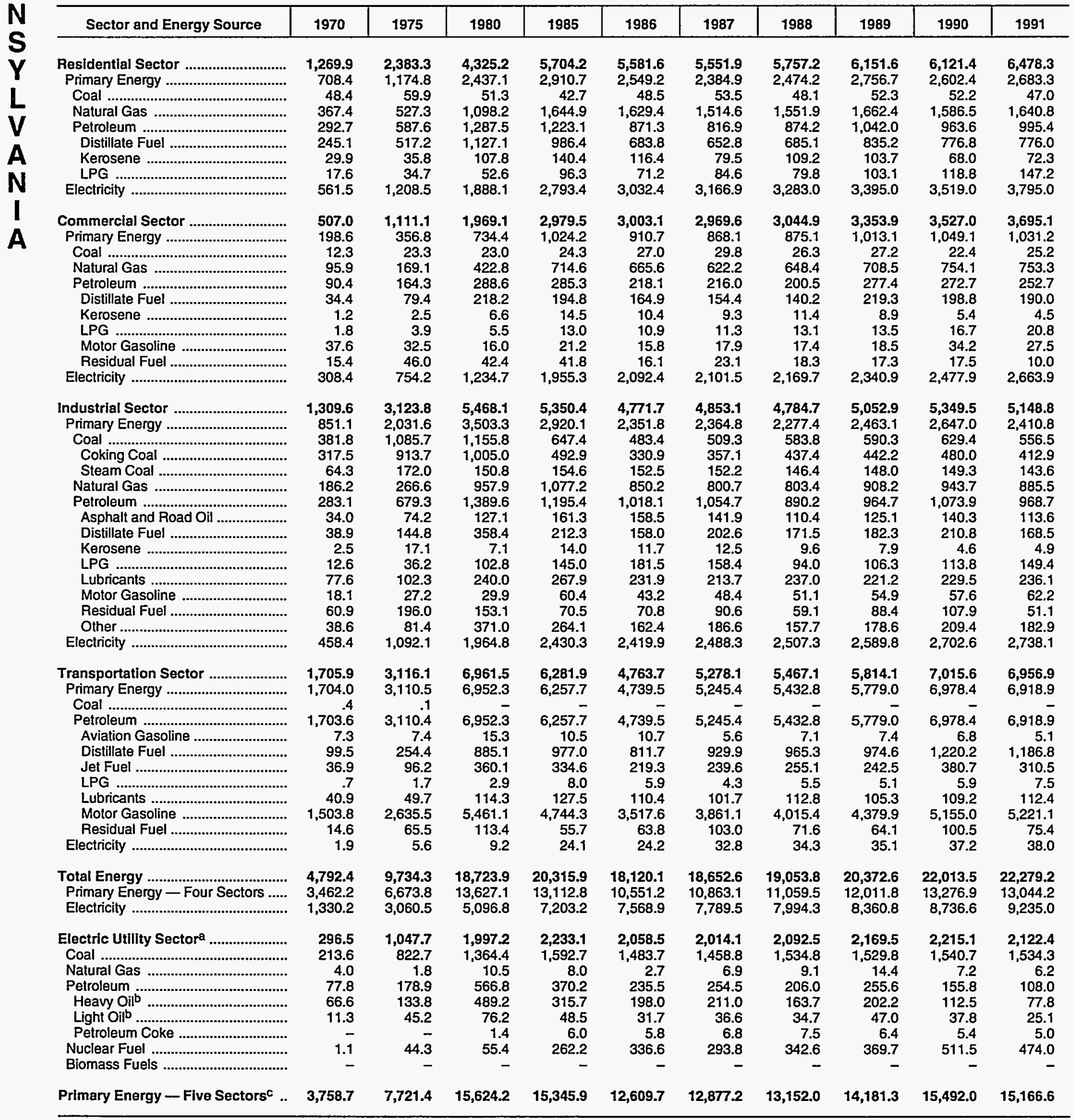

a There are no direct fuel costs for hydroelectric, geothermal, centralized solar, or wind energy.

b Heavy oil includes Grade Nos. 4, 5, and 6, and residual fuel oils. Light oil includes Grade No. 2 heating oil, kerosene, and jet fuel.

c Biomass fuels are not included, except those consumed at electric utilities and those added to motor gasoline.

- No consumption, including cases where adjustments were made. See "Consumption Adjustments for Process Fuel and Intermediate Products."

Note: Totals may not equal sum of components due to independent rounding.

Sources: Data sources, estimation procedures, and assumptions are described in the "Documentation" section of this report. 
Energy Price and Expenditure Estimates by Source, Rhode Island $1970,1975,1980$, and 1985-1991

\begin{tabular}{|c|c|c|c|c|c|c|c|c|c|c|}
\hline Energy Source & 1970 & 1975 & 1980 & 1985 & 1986 & 1987 & 1988 & 1989 & 1990 & 1991 \\
\hline & \multicolumn{10}{|c|}{ Prices in Dollars per Million Btu } \\
\hline 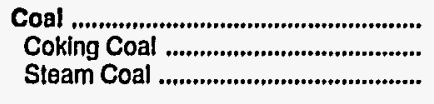 & $\begin{array}{r}0.94 \\
.94\end{array}$ & $\begin{array}{r}2.64 \\
2.64\end{array}$ & 2.37 & $\frac{3.03}{3.03}$ & $\begin{array}{r}2.30 \\
2.30\end{array}$ & $\begin{array}{r}3.09 \\
3 . \overline{0}\end{array}$ & $\begin{array}{r}2.54 \\
- \\
2.54\end{array}$ & $\begin{array}{r}2.70 \\
2.70\end{array}$ & $\begin{array}{r}3.56 \\
3.56\end{array}$ & $\begin{array}{r}3.51 \\
3.51\end{array}$ \\
\hline Natural Gas ........................................... & 1.38 & 2.74 & 5.09 & 6.66 & 6.77 & 5.49 & 5.94 & 6.21 & 5.83 & 5.94 \\
\hline 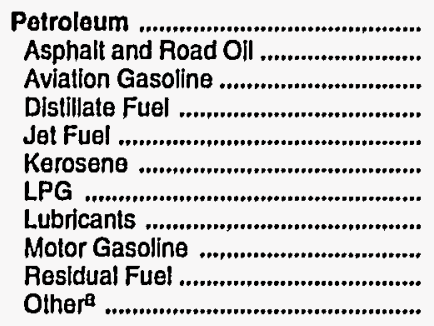 & $\begin{array}{r}1.41 \\
.66 \\
2.17 \\
1.35 \\
.75 \\
1.50 \\
1.77 \\
5.08 \\
2.90 \\
.43 \\
1.86\end{array}$ & $\begin{array}{l}3.17 \\
1.86 \\
3.45 \\
2.76 \\
2.09 \\
2.98 \\
3.50 \\
7.48 \\
4.50 \\
1.92 \\
4.18\end{array}$ & $\begin{array}{r}7.59 \\
3.58 \\
9.02 \\
7.06 \\
6.51 \\
7.44 \\
6.57 \\
14.36 \\
9.72 \\
4.03 \\
8.20\end{array}$ & $\begin{array}{r}7.64 \\
5.18 \\
9.99 \\
8.08 \\
6.10 \\
8.91 \\
11.70 \\
17.61 \\
9.13 \\
4.66 \\
7.37\end{array}$ & $\begin{array}{r}6.01 \\
4.86 \\
8.41 \\
6.37 \\
4.25 \\
6.90 \\
10.62 \\
15.59 \\
7.36 \\
2.67 \\
10.73\end{array}$ & $\begin{array}{r}6.40 \\
3.54 \\
7.55 \\
6.24 \\
4.18 \\
6.84 \\
11.19 \\
12.70 \\
7.96 \\
3.10 \\
10.73\end{array}$ & $\begin{array}{r}6.36 \\
3.34 \\
7.41 \\
6.37 \\
4.04 \\
6.76 \\
11.00 \\
14.61 \\
8.40 \\
2.47 \\
10.84\end{array}$ & $\begin{array}{r}7.15 \\
3.17 \\
8.28 \\
7.06 \\
4.75 \\
7.69 \\
9.28 \\
13.30 \\
9.11 \\
2.89 \\
10.00\end{array}$ & $\begin{array}{r}8.20 \\
3.34 \\
9.32 \\
8.53 \\
6.03 \\
8.90 \\
12.46 \\
13.40 \\
10.03 \\
3.40 \\
9.71\end{array}$ & $\begin{array}{r}8.50 \\
3.05 \\
8.71 \\
7.85 \\
5.23 \\
7.85 \\
13.91 \\
15.42 \\
10.09 \\
2.86 \\
9.71\end{array}$ \\
\hline Nuclear Fuel ........................................... & - & - & - & - & - & - & - & - & - & - \\
\hline Blomass Fuels at Utilities .................. & - & - & - & - & - & - & - & - & - & - \\
\hline Primary Energy — Five Sectors ${ }^{b}$.... & 1.40 & 3.11 & 7.06 & 7.43 & 6.13 & 6.18 & 6.17 & 6.91 & 7.56 & 7.53 \\
\hline $\begin{array}{l}\text { Electric Utility Fuelc ........................... } \\
\text { Electricity Purchased by End Users }\end{array}$ & $\begin{array}{r}.43 \\
6.85\end{array}$ & $\begin{array}{r}1.84 \\
13.78\end{array}$ & $\begin{array}{r}3.91 \\
20.67\end{array}$ & $\begin{array}{r}3.82 \\
24.73\end{array}$ & $\begin{array}{r}2.39 \\
23.40\end{array}$ & $\begin{array}{r}2.50 \\
23.93\end{array}$ & $\begin{array}{r}2.28 \\
23.28\end{array}$ & $\begin{array}{r}2.65 \\
24.24\end{array}$ & $\begin{array}{r}2.60 \\
26.85\end{array}$ & $\begin{array}{r}2.23 \\
29.75\end{array}$ \\
\hline \multirow[t]{2}{*}{ Total Energy ........................................ } & 1.91 & 4.16 & 9.06 & 9.67 & 8.50 & 8.62 & 8.54 & 9.43 & 10.61 & 10.52 \\
\hline & \multicolumn{10}{|c|}{ Expendltures in Millions of Dollars } \\
\hline 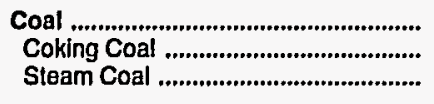 & $\begin{array}{r}0.2 \\
\overline{.2}\end{array}$ & $\begin{array}{r}0.4 \\
. \overline{4}\end{array}$ & $\begin{array}{r}0.4 \\
-4\end{array}$ & $\frac{0.6}{.6}$ & $\begin{array}{r}1.6 \\
1.6\end{array}$ & $\begin{array}{r}0.4 \\
\overline{4}\end{array}$ & $\begin{array}{r}11.1 \\
11 . \bar{y}\end{array}$ & $\begin{array}{r}1.8 \\
1 . \overline{8}\end{array}$ & $\begin{array}{r}0.5 \\
\overline{5}\end{array}$ & $\begin{array}{r}0.4 \\
\overline{.4}\end{array}$ \\
\hline Natural Gas ........................................... & 35.2 & 64.3 & 142.7 & 204.6 & 182.9 & 202.3 & 187.0 & 215.8 & 213.7 & 330.8 \\
\hline 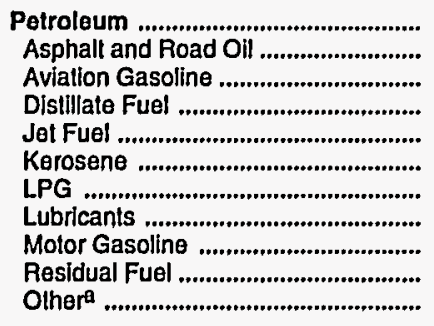 & $\begin{array}{r}234.1 \\
4.1 \\
1.6 \\
67.9 \\
.6 \\
3.7 \\
2.5 \\
3.9 \\
122.0 \\
25.7 \\
2.2\end{array}$ & $\begin{array}{r}434.3 \\
16.5 \\
5.0 \\
128.5 \\
3.2 \\
2.2 \\
6.5 \\
4.4 \\
211.9 \\
52.9 \\
3.4\end{array}$ & $\begin{array}{r}794.4 \\
24.7 \\
12.2 \\
207.0 \\
12.8 \\
3.5 \\
7.1 \\
11.5 \\
429.7 \\
63.9 \\
22.0\end{array}$ & $\begin{array}{r}856.3 \\
102.3 \\
1.5 \\
209.6 \\
17.1 \\
6.8 \\
21.1 \\
12.8 \\
415.5 \\
65.5 \\
4.1\end{array}$ & $\begin{array}{r}708.0 \\
47.7 \\
1.5 \\
196.7 \\
9.3 \\
6.6 \\
22.6 \\
11.1 \\
345.4 \\
63.2 \\
4.0\end{array}$ & $\begin{array}{r}748.4 \\
41.7 \\
1.6 \\
220.1 \\
12.5 \\
4.3 \\
27.4 \\
10.2 \\
381.1 \\
45.2 \\
4.4\end{array}$ & $\begin{array}{r}773.9 \\
38.6 \\
1.7 \\
220.3 \\
14.5 \\
4.4 \\
22.7 \\
11.3 \\
409.6 \\
47.2 \\
3.5\end{array}$ & $\begin{array}{r}786.6 \\
33.7 \\
1.9 \\
242.8 \\
19.4 \\
2.7 \\
17.1 \\
10.6 \\
424.3 \\
31.0 \\
3.1\end{array}$ & $\begin{array}{r}824.2 \\
36.3 \\
2.0 \\
230.4 \\
26.4 \\
2.7 \\
22.6 \\
11.0 \\
459.0 \\
30.8 \\
3.0\end{array}$ & $\begin{array}{r}780.5 \\
9.3 \\
1.3 \\
231.7 \\
19.1 \\
2.3 \\
23.4 \\
11.3 \\
459.8 \\
19.8 \\
2.4\end{array}$ \\
\hline Nuclear Fuol ......................................... & - & - & - & - & - & - & - & - & - & - \\
\hline Blomass Fuels at Utilities ................. & - & - & - & - & - & - & - & - & - & - \\
\hline Primary Energy - Five Sectors ${ }^{b}$.... & 269.6 & 498.9 & 937.5 & $1,061.5$ & 892.6 & 951.1 & 972.1 & $1,004.2$ & $1,038.3$ & $1,111.6$ \\
\hline $\begin{array}{l}\text { Electric Utility Fuelc } \\
\text { Electricity Purchased by End Users }\end{array}$ & $\begin{array}{r}-9.3 \\
90.7\end{array}$ & $\begin{array}{r}-18.1 \\
209.3\end{array}$ & $\begin{array}{r}-47.5 \\
361.9\end{array}$ & $\begin{array}{r}-27.4 \\
458.2\end{array}$ & $\begin{array}{r}-22.3 \\
451.6\end{array}$ & $\begin{array}{r}-26.8 \\
485.1\end{array}$ & $\begin{array}{r}-22.4 \\
494.0\end{array}$ & $\begin{array}{l}-17.7 \\
526.0\end{array}$ & $\begin{array}{r}-20.6 \\
588.1\end{array}$ & $\begin{array}{r}-5.7 \\
650.0\end{array}$ \\
\hline 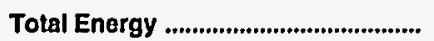 & 350.9 & 690.1 & $1,252.0$ & $1,492.3$ & $1,321.9$ & $1,409.4$ & $1,443.6$ & $1,512.5$ & $1,605.9$ & $1,755.9$ \\
\hline
\end{tabular}

Includes petroleum coke used at electric utilities.

b Blomass fuels are not included, except those consumed at electric utilities and those added to motor gasoline.

c There are no direct fuel costs for hydroelectric, geolhermal, centralized solar, or wind energy.

-No consumption, including cases where adjustments were made. See "Consumption Adjustments for Process Fuel and Intermediate Products."

Note: Expenditure totals may not equal sum of components due to independent rounding.

Sources: Data sources, estimation procedures, and assumptions are described in the "Documentation" section of this report.

Energy Information Administration 


\section{R Energy Price Estimates by Sector, Rhode Island \\ H 1970, 1975, 1980, and 1985-1991 \\ O (Dollars per Million Btu)}

\begin{tabular}{|c|c|c|c|c|c|c|c|c|c|c|}
\hline Sector and Energy Source & 1970 & 1975 & 1980 & 1985 & 1986 & 1987 & 1988 & 1989 & 1990 & 1991 \\
\hline 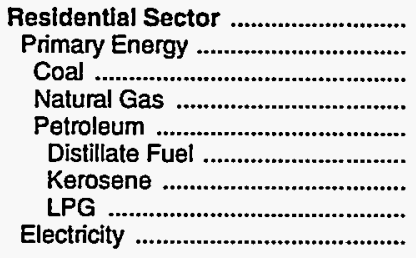 & $\begin{array}{r}2.19 \\
1.59 \\
.98 \\
1.79 \\
1.52 \\
1.49 \\
1.70 \\
2.55 \\
8.44\end{array}$ & $\begin{array}{r}4.33 \\
2.94 \\
2.62 \\
3.04 \\
2.90 \\
2.85 \\
3.16 \\
5.49 \\
15.43\end{array}$ & $\begin{array}{r}9.08 \\
6.60 \\
4.47 \\
5.58 \\
7.33 \\
7.29 \\
8.15 \\
8.57 \\
22.64\end{array}$ & $\begin{array}{r}10.90 \\
8.03 \\
4.39 \\
7.62 \\
8.33 \\
8.15 \\
8.93 \\
11.44 \\
26.77\end{array}$ & $\begin{array}{r}9.90 \\
6.86 \\
4.19 \\
7.25 \\
6.53 \\
6.33 \\
6.93 \\
9.82 \\
25.42\end{array}$ & $\begin{array}{r}9.81 \\
6.60 \\
3.85 \\
6.63 \\
6.59 \\
6.31 \\
6.91 \\
11.24 \\
25.93\end{array}$ & $\begin{array}{r}9.58 \\
6.52 \\
3.99 \\
6.43 \\
6.61 \\
6.39 \\
7.00 \\
11.13 \\
25.28\end{array}$ & $\begin{array}{r}10.43 \\
7.10 \\
3.96 \\
6.94 \\
7.25 \\
7.18 \\
7.86 \\
8.50 \\
26.34\end{array}$ & $\begin{array}{r}11.84 \\
7.82 \\
4.21 \\
7.03 \\
8.73 \\
8.38 \\
9.18 \\
13.81 \\
28.86\end{array}$ & $\begin{array}{r}12.38 \\
7.78 \\
4.07 \\
7.42 \\
8.17 \\
7.71 \\
8.44 \\
15.22 \\
32.17\end{array}$ \\
\hline 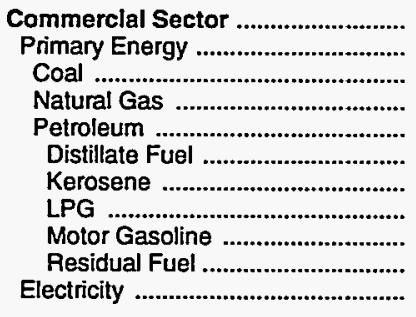 & $\begin{array}{r}2.08 \\
1.00 \\
.90 \\
1.44 \\
.85 \\
1.10 \\
.78 \\
1.20 \\
2.90 \\
.44 \\
7.02\end{array}$ & $\begin{array}{r}5.23 \\
2.39 \\
2.65 \\
2.71 \\
2.28 \\
2.44 \\
2.59 \\
2.66 \\
4.50 \\
1.81 \\
13.84\end{array}$ & $\begin{array}{r}10.70 \\
5.44 \\
1.67 \\
5.00 \\
6.05 \\
6.46 \\
- \\
5.29 \\
9.72 \\
3.96 \\
20.45\end{array}$ & $\begin{array}{r}12.55 \\
6.36 \\
2.38 \\
6.45 \\
6.28 \\
7.45 \\
8.34 \\
12.03 \\
9.13 \\
4.96 \\
24.56\end{array}$ & $\begin{array}{r}10.08 \\
4.87 \\
2.08 \\
6.21 \\
4.13 \\
5.69 \\
6.06 \\
11.25 \\
7.36 \\
2.85 \\
23.01\end{array}$ & $\begin{array}{r}10.72 \\
5.18 \\
2.36 \\
5.38 \\
4.97 \\
5.57 \\
6.01 \\
11.14 \\
7.96 \\
3.42 \\
23.26\end{array}$ & $\begin{array}{r}10.84 \\
5.04 \\
2.53 \\
5.65 \\
4.47 \\
5.45 \\
5.59 \\
10.88 \\
8.40 \\
2.83 \\
22.62\end{array}$ & $\begin{array}{r}11.88 \\
5.68 \\
2.60 \\
6.24 \\
5.05 \\
5.93 \\
5.95 \\
10.22 \\
9.11 \\
3.06 \\
23.52\end{array}$ & $\begin{array}{r}13.12 \\
5.79 \\
2.62 \\
6.04 \\
5.57 \\
7.25 \\
8.26 \\
10.80 \\
10.03 \\
3.35 \\
26.28\end{array}$ & $\begin{array}{r}13.62 \\
5.47 \\
2.65 \\
5.86 \\
5.09 \\
6.39 \\
6.57 \\
11.94 \\
10.09 \\
2.92 \\
28.97\end{array}$ \\
\hline 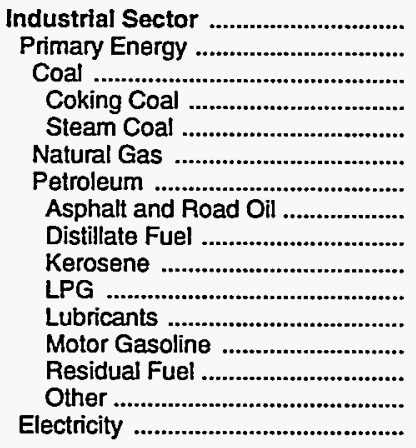 & $\begin{array}{r}1.06 \\
.66 \\
.90 \\
- \\
.90 \\
.85 \\
.62 \\
.66 \\
.71 \\
.78 \\
1.20 \\
5.08 \\
2.90 \\
.42 \\
1.86 \\
4.83\end{array}$ & $\begin{array}{r}3.20 \\
2.15 \\
2.65 \\
- \\
2.65 \\
2.10 \\
2.17 \\
1.86 \\
2.34 \\
2.59 \\
2.66 \\
7.48 \\
4.50 \\
2.05 \\
4.18 \\
11.36\end{array}$ & $\begin{array}{r}7.27 \\
4.91 \\
1.67 \\
- \\
1.67 \\
4.45 \\
5.07 \\
3.58 \\
5.65 \\
6.18 \\
5.29 \\
14.36 \\
9.72 \\
4.24 \\
8.20 \\
18.39\end{array}$ & $\begin{array}{r}7.48 \\
5.59 \\
2.38 \\
-\overline{2} \\
2.38 \\
5.70 \\
5.58 \\
5.18 \\
7.28 \\
8.34 \\
12.03 \\
17.61 \\
9.13 \\
4.96 \\
7.37 \\
21.93\end{array}$ & $\begin{array}{r}7.31 \\
4.83 \\
2.08 \\
- \\
2.08 \\
5.58 \\
4.78 \\
4.86 \\
5.29 \\
6.06 \\
11.25 \\
15.59 \\
7.36 \\
2.85 \\
10.73 \\
20.90\end{array}$ & $\begin{array}{r}7.14 \\
4.58 \\
2.36 \\
- \\
2.36 \\
5.23 \\
4.46 \\
3.54 \\
5.25 \\
6.01 \\
11.14 \\
12.70 \\
7.96 \\
3.42 \\
10.73 \\
21.89\end{array}$ & $\begin{array}{r}6.41 \\
3.96 \\
2.53 \\
- \\
2.53 \\
4.70 \\
4.10 \\
3.34 \\
4.88 \\
5.59 \\
10.88 \\
14.61 \\
8.40 \\
2.83 \\
10.84 \\
21.10\end{array}$ & $\begin{array}{r}7.25 \\
4.20 \\
2.60 \\
- \\
2.60 \\
5.03 \\
4.02 \\
3.17 \\
5.20 \\
5.95 \\
10.22 \\
13.30 \\
9.11 \\
3.06 \\
10.00 \\
21.98\end{array}$ & $\begin{array}{r}8.11 \\
4.54 \\
2.59 \\
- \\
2.59 \\
5.18 \\
4.36 \\
3.34 \\
7.21 \\
8.26 \\
10.80 \\
13.40 \\
10.03 \\
3.35 \\
9.71 \\
24.46\end{array}$ & $\begin{array}{r}7.69 \\
5.16 \\
- \\
- \\
- \\
5.25 \\
4.84 \\
3.05 \\
5.74 \\
6.57 \\
11.94 \\
15.42 \\
10.09 \\
2.92 \\
9.71 \\
27.09\end{array}$ \\
\hline 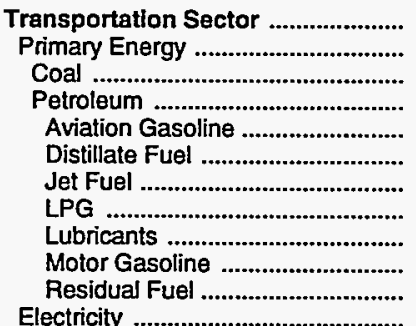 & $\begin{array}{r}2.17 \\
2.17 \\
- \\
2.17 \\
2.17 \\
1.36 \\
.75 \\
1.20 \\
5.08 \\
2.90 \\
.41 \\
-\end{array}$ & $\begin{array}{l}4.19 \\
4.19 \\
2.65 \\
4.19 \\
3.45 \\
2.90 \\
2.09 \\
2.66 \\
7.48 \\
4.50 \\
1.71 \\
-\end{array}$ & $\begin{array}{r}9.40 \\
9.40 \\
- \\
9.40 \\
9.02 \\
7.41 \\
6.51 \\
5.29 \\
14.36 \\
9.72 \\
3.34 \\
-\end{array}$ & $\begin{array}{r}9.03 \\
9.03 \\
- \\
9.03 \\
9.99 \\
8.89 \\
6.10 \\
12.03 \\
17.61 \\
9.13 \\
- \\
-\end{array}$ & $\begin{array}{r}7.28 \\
7.28 \\
- \\
7.28 \\
8.41 \\
7.26 \\
4.25 \\
11.25 \\
15.59 \\
7.36 \\
2.20 \\
-\end{array}$ & $\begin{array}{r}7.57 \\
7.57 \\
- \\
7.57 \\
7.55 \\
6.96 \\
4.18 \\
11.14 \\
12.70 \\
7.96 \\
2.55 \\
-\end{array}$ & $\begin{array}{r}7.87 \\
7.87 \\
-\overline{7} \\
7.87 \\
7.41 \\
7.36 \\
4.04 \\
10.88 \\
14.61 \\
8.40 \\
2.05 \\
-\end{array}$ & $\begin{array}{r}8.60 \\
8.60 \\
- \\
8.60 \\
8.28 \\
7.81 \\
4.75 \\
10.22 \\
13.30 \\
9.11 \\
2.50 \\
\end{array}$ & $\begin{array}{r}9.71 \\
9.71 \\
- \\
9.71 \\
9.32 \\
9.93 \\
6.03 \\
10.80 \\
13.40 \\
10.03 \\
3.42\end{array}$ & $\begin{array}{r}9.71 \\
9.71 \\
- \\
9.71 \\
8.71 \\
9.39 \\
5.23 \\
11.94 \\
15.42 \\
10.09 \\
2.16\end{array}$ \\
\hline $\begin{array}{l}\text { Total Energy ................................... } \\
\text { Primary Energy - Four Sectors ...... } \\
\text { Electricity .......................................... }\end{array}$ & $\begin{array}{l}1.91 \\
1.53 \\
6.85\end{array}$ & $\begin{array}{r}4.16 \\
3.19 \\
13.78\end{array}$ & $\begin{array}{r}9.06 \\
7.37 \\
20.67\end{array}$ & $\begin{array}{r}9.67 \\
7.62 \\
24.73\end{array}$ & $\begin{array}{r}8.50 \\
6.39 \\
23.40\end{array}$ & $\begin{array}{r}8.62 \\
6.45 \\
23.93\end{array}$ & $\begin{array}{r}8.54 \\
6.43 \\
23.28\end{array}$ & $\begin{array}{r}9.43 \\
7.11 \\
24.24\end{array}$ & $\begin{array}{r}10.61 \\
7.87 \\
26.85\end{array}$ & $\begin{array}{r}10.52 \\
7.63 \\
29.75\end{array}$ \\
\hline $\begin{array}{l}\text { Electric Utillty Sectora } \\
\text { Coal } \\
\text { Natural Gas } \\
\text { Petroleum } \\
\text { Heavy Oib } \\
\text { Light Oib }\end{array}$ & $\begin{array}{l}.43 \\
- \\
.39 \\
.44 \\
.44 \\
.48\end{array}$ & $\begin{array}{r}1.84 \\
1 . \overline{15} \\
1.84 \\
1.84 \\
2.00\end{array}$ & $\begin{array}{r}3.91 \\
-\overline{3} \\
4.00 \\
3.97 \\
6.03\end{array}$ & $\begin{array}{r}3.82 \\
-\overrightarrow{-} \\
3.37 \\
4.08 \\
4.03 \\
5.83\end{array}$ & $\begin{array}{r}2.39 \\
- \\
2.39 \\
2.37 \\
3.55\end{array}$ & $\begin{array}{r}2.50 \\
2.32 \\
2.70 \\
2.68 \\
3.38\end{array}$ & $\begin{array}{r}2.28 \\
-\overline{9} \\
2.28 \\
2.25 \\
3.71\end{array}$ & $\begin{array}{r}2.65 \\
- \\
2.41 \\
2.77 \\
2.70 \\
4.23\end{array}$ & $\begin{array}{r}2.60 \\
-\overline{2} \\
3.17 \\
3.68 \\
5.53\end{array}$ & $\begin{array}{l}2.23 \\
1 . \overline{8} \\
2.70 \\
2.41 \\
4.70\end{array}$ \\
\hline 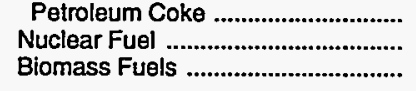 & $\begin{array}{l}- \\
-\end{array}$ & $\begin{array}{l}- \\
-\end{array}$ & $\begin{array}{l}- \\
-\end{array}$ & $\begin{array}{l}- \\
-\end{array}$ & $\begin{array}{l}- \\
- \\
-\end{array}$ & $\begin{array}{l}- \\
- \\
-\end{array}$ & $\begin{array}{l}- \\
- \\
-\end{array}$ & $\begin{array}{l}- \\
- \\
-\end{array}$ & $\begin{array}{l}- \\
- \\
-\end{array}$ & - \\
\hline Primary Energy - Flve Sectors ${ }^{c}$.. & 1.40 & 3.11 & 7.06 & 7.43 & 6.13 & 6.18 & 6.17 & 6.91 & 7.56 & 7.53 \\
\hline
\end{tabular}

There are no direct fuel costs for hydroelectric, geothermal, centralized solar, or wind energy.

b Heavy oil includes Grade Nos. 4, 5, and 6, and residual fuel oils. Light oil includes Grade No. 2 heating oil, kerosene, and jet fuel.

c Biomass fuels are not included, except those consumed at electric utilities and those added to motor gasoline.

-No consumption, including cases where adjustments were made. See "Consumption Adjustments for Process Fuel and Intermediate Products."

Sources: Data sources, estimation procedures, and assumptions are described in the "Documentation" section of this report. 
(Million Dollars)

\begin{tabular}{|c|c|c|c|c|c|c|c|c|c|c|}
\hline Sector and Energy Source & 1970 & 1975 & 1980 & 1985 & 1986 & 1987 & 1988 & 1989 & 1990 & 1991 \\
\hline 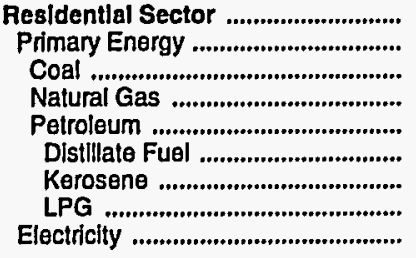 & $\begin{array}{r}117.5 \\
77.5 \\
.1 \\
21.9 \\
55.5 \\
50.7 \\
3.2 \\
1.5 \\
40.0\end{array}$ & $\begin{array}{r}223.2 \\
134.5 \\
.1 \\
40.2 \\
94.1 \\
89.6 \\
1.6 \\
3.0 \\
88.7\end{array}$ & $\begin{array}{r}367.9 \\
225.8 \\
.2 \\
79.5 \\
146.1 \\
140.0 \\
2.5 \\
3.6 \\
142.1\end{array}$ & $\begin{array}{r}478.8 \\
298.8 \\
.3 \\
118.0 \\
180.5 \\
162.4 \\
6.6 \\
11.5 \\
180.0\end{array}$ & $\begin{array}{r}425.5 \\
246.5 \\
.3 \\
120.2 \\
126.0 \\
110.5 \\
6.4 \\
9.2 \\
179.0\end{array}$ & $\begin{array}{r}441.5 \\
248.1 \\
.2 \\
114.0 \\
133.8 \\
117.4 \\
4.0 \\
12.5 \\
193.4\end{array}$ & $\begin{array}{r}465.5 \\
265.4 \\
.2 \\
116.7 \\
148.6 \\
134.1 \\
3.8 \\
10.7 \\
200.0\end{array}$ & $\begin{array}{r}487.5 \\
274.5 \\
.2 \\
130.4 \\
143.9 \\
132.9 \\
2.5 \\
8.5 \\
213.0\end{array}$ & $\begin{array}{r}502.8 \\
268.8 \\
.3 \\
128.0 \\
140.5 \\
124.6 \\
2.0 \\
13.9 \\
234.0\end{array}$ & $\begin{array}{r}530.5 \\
270.5 \\
.2 \\
132.5 \\
137.7 \\
120.7 \\
1.7 \\
15.4 \\
260.0\end{array}$ \\
\hline 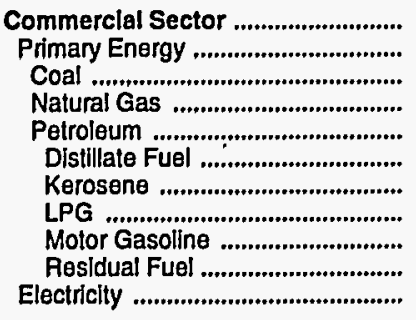 & $\begin{array}{r}51.1 \\
20.3 \\
.1 \\
7.5 \\
12.8 \\
9.4 \\
. \\
.1 \\
.6 \\
2.7 \\
30.8\end{array}$ & $\begin{array}{r}113.5 \\
39.1 \\
.1 \\
11.6 \\
27.3 \\
19.3 \\
\vdots \\
.3 \\
1.0 \\
6.9 \\
74.4\end{array}$ & $\begin{array}{r}197.1 \\
65.1 \\
34.5 \\
30.6 \\
23.2 \\
. \overline{4} \\
2.5 \\
4.5 \\
132.0\end{array}$ & $\begin{array}{r}271.9 \\
90.9 \\
.1 \\
50.6 \\
40.2 \\
19.2 \\
.2 \\
2.1 \\
1.5 \\
17.2 \\
181.0\end{array}$ & $\begin{array}{r}271.6 \\
93.6 \\
.1 \\
42.9 \\
50.6 \\
26.7 \\
.1 \\
1.8 \\
1.3 \\
20.5 \\
178.1\end{array}$ & $\begin{array}{r}285.9 \\
95.8 \\
.1 \\
52.0 \\
43.7 \\
28.9 \\
.2 \\
2.2 \\
1.5 \\
10.9 \\
190.2\end{array}$ & $\begin{array}{r}284.6 \\
88.7 \\
.1 \\
48.4 \\
40.1 \\
25.6 \\
.1 \\
1.8 \\
1.6 \\
11.0 \\
196.0\end{array}$ & $\begin{array}{r}306.8 \\
95.8 \\
.1 \\
56.2 \\
39.5 \\
26.9 \\
.2 \\
1.8 \\
1.8 \\
8.8 \\
211.0\end{array}$ & $\begin{array}{r}336.4 \\
95.4 \\
.1 \\
50.1 \\
45.2 \\
28.4 \\
.1 \\
1.9 \\
2.0 \\
12.7 \\
241.0\end{array}$ & $\begin{array}{r}357.7 \\
93.7 \\
.1 \\
49.8 \\
43.8 \\
28.9 \\
. \\
2.1 \\
1.9 \\
10.8 \\
264.0\end{array}$ \\
\hline 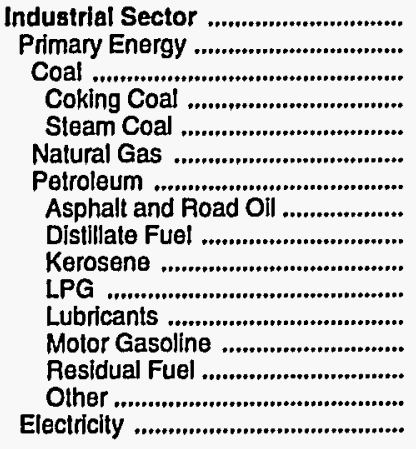 & $\begin{array}{r}45.0 \\
25.1 \\
: \\
5.0 \\
50.1 \\
4.1 \\
2.8 \\
.4 \\
.7 \\
1.5 \\
8.3 \\
8.3 \\
2.2 \\
19.9\end{array}$ & $\begin{array}{r}114.7 \\
68.5 \\
.1 \\
-1 \\
.1 \\
12.4 \\
56.0 \\
16.5 \\
6.0 \\
.6 \\
2.9 \\
1.8 \\
.1 \\
24.7 \\
3.4 \\
46.2\end{array}$ & $\begin{array}{r}198.3 \\
110.5 \\
.2 \\
.2 \\
.2 \\
23.1 \\
87.2 \\
24.7 \\
13.6 \\
1.1 \\
2.9 \\
5.4 \\
.1 \\
17.4 \\
22.0 \\
87.8\end{array}$ & $\begin{array}{r}285.7 \\
188.5 \\
.2 \\
\overline{.} \\
.2 \\
27.2 \\
161.0 \\
102.3 \\
10.5 \\
6.5 \\
6.5 \\
6.0 \\
1.3 \\
30.3 \\
4.1 \\
97.3\end{array}$ & $\begin{array}{r}214.4 \\
119.9 \\
1.2 \\
1.2 \\
19.8 \\
98.8 \\
47.7 \\
8.8 \\
.1 \\
10.9 \\
5.2 \\
1.2 \\
20.9 \\
4.0 \\
94.6\end{array}$ & $\begin{array}{r}224.4 \\
122.8 \\
.1 \\
- \\
.1 \\
23.6 \\
99.1 \\
41.7 \\
16.6 \\
.1 \\
12.3 \\
4.8 \\
1.2 \\
18.0 \\
4.4 \\
101.6\end{array}$ & $\begin{array}{r}208.1 \\
110.1 \\
10.9 \\
-\overline{10.9} \\
10.9 \\
21.5 \\
77.7 \\
38.6 \\
7.7 \\
.5 \\
9.3 \\
5.3 \\
1.5 \\
11.3 \\
3.5 \\
98.0\end{array}$ & $\begin{array}{r}196.0 \\
94.0 \\
1.6 \\
1 . \overline{6} \\
23.9 \\
68.6 \\
33.7 \\
9.4 \\
6.1 \\
6.1 \\
5.0 \\
1.7 \\
9.6 \\
3.1 \\
102.0\end{array}$ & $\begin{array}{r}208.8 \\
95.8 \\
- \\
= \\
23.3 \\
72.5 \\
36.3 \\
9.9 \\
.7 \\
6.1 \\
5.1 \\
1.8 \\
9.7 \\
3.0 \\
113.0\end{array}$ & $\begin{array}{r}310.0 \\
184.0 \\
\overline{-} \\
\overline{-} \\
145.1 \\
38.9 \\
9.3 \\
7.7 \\
6 \\
5.3 \\
5.3 \\
1.4 \\
7.0 \\
2.4 \\
126.0\end{array}$ \\
\hline 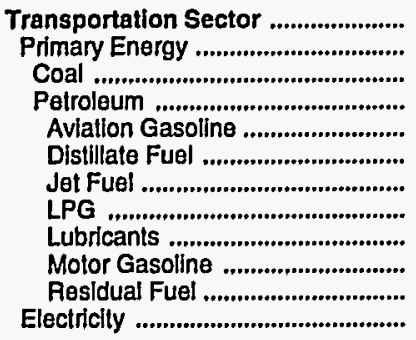 & $\begin{array}{r}137.4 \\
137.4 \\
137.4 \\
1.6 \\
4.8 \\
.6 \\
.1 \\
2.4 \\
121.4 \\
6.5 \\
-\end{array}$ & $\begin{array}{r}238.7 \\
238.7 \\
238.7 \\
5.0 \\
13.3 \\
3.2 \\
.3 \\
2.6 \\
210.8 \\
3.5 \\
-\end{array}$ & $\begin{array}{r}488.7 \\
488.7 \\
488.7 \\
12.2 \\
29.2 \\
12.8 \\
.2 \\
6.1 \\
427.1 \\
1.2 \\
-\end{array}$ & $\begin{array}{r}455.9 \\
455.9 \\
-\overline{9} \\
455.9 \\
1.5 \\
16.9 \\
17.1 \\
1.0 \\
6.8 \\
412.7 \\
-\end{array}$ & $\begin{array}{r}410.3 \\
410.3 \\
410.3 \\
40.5 \\
50.0 \\
9.3 \\
.7 \\
5.9 \\
342.8 \\
.1 \\
-\end{array}$ & $\begin{array}{r}457.6 \\
457.6 \\
457.6 \\
1.6 \\
56.7 \\
12.5 \\
.3 \\
5.4 \\
378.4 \\
2.7 \\
-\end{array}$ & $\begin{array}{r}485.5 \\
485.5 \\
485.5 \\
1.7 \\
52.0 \\
14.5 \\
.8 \\
6.0 \\
406.6 \\
3.8 \\
-\end{array}$ & $\begin{array}{r}522.2 \\
522.2 \\
522.2 \\
1.9 \\
72.7 \\
19.4 \\
.7 \\
5.6 \\
420.8 \\
1.1 \\
-\end{array}$ & $\begin{array}{r}557.8 \\
557.8 \\
- \\
557.8 \\
2.0 \\
66.9 \\
26.4 \\
.8 \\
5.8 \\
455.2 \\
.7 \\
-\end{array}$ & $\begin{array}{r}557.6 \\
557.6 \\
557.6 \\
1.3 \\
74.0 \\
19.1 \\
.6 \\
6.0 \\
456.5 \\
.1 \\
-\end{array}$ \\
\hline 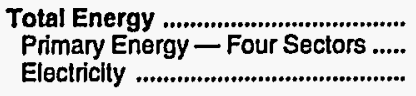 & $\begin{array}{r}350.9 \\
260.3 \\
90.7\end{array}$ & $\begin{array}{l}690.1 \\
480.8 \\
209.3\end{array}$ & $\begin{array}{r}1,252.0 \\
890.1 \\
361.9\end{array}$ & $\begin{array}{r}1,492.3 \\
1,034.1 \\
458.2\end{array}$ & $\begin{array}{r}1,321.9 \\
870.3 \\
451.6\end{array}$ & $\begin{array}{r}1,409.4 \\
924.2 \\
485.1\end{array}$ & $\begin{array}{r}1,443.6 \\
949.6 \\
494.0\end{array}$ & $\begin{array}{r}1,512.5 \\
986.5 \\
526.0\end{array}$ & $\begin{array}{r}1,605.9 \\
1,017.8 \\
588.1\end{array}$ & $\begin{array}{r}1,755.9 \\
1,105.9 \\
650.0\end{array}$ \\
\hline 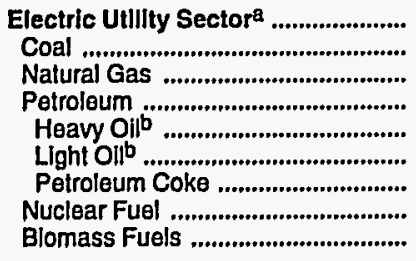 & $\begin{array}{r}9.3 \\
\overline{.9} \\
8.4 \\
8.2 \\
.2 \\
- \\
- \\
-\end{array}$ & $\begin{array}{r}18.1 \\
\square \\
18.1 \\
17.8 \\
.3 \\
= \\
=\end{array}$ & $\begin{array}{r}47.5 \\
- \\
5.7 \\
41.8 \\
40.8 \\
1.0 \\
= \\
=\end{array}$ & $\begin{array}{r}27.4 \\
8 . \overline{8} \\
18.6 \\
17.9 \\
.7 \\
- \\
- \\
-\end{array}$ & $\begin{array}{r}22.3 \\
- \\
22 . \overline{3} \\
21.7 \\
.6 \\
= \\
=\end{array}$ & $\begin{array}{r}26.8 \\
12 . \overline{8} \\
14.1 \\
13.6 \\
.5 \\
- \\
- \\
-\end{array}$ & $\begin{array}{r}22.4 \\
\overline{.4} \\
22.0 \\
21.1 \\
.9 \\
- \\
- \\
-\end{array}$ & $\begin{array}{r}17.7 \\
-\overline{5} \\
12.4 \\
11.5 \\
.9 \\
- \\
=\end{array}$ & $\begin{array}{r}20.6 \\
12 . \overline{3} \\
8.3 \\
7.7 \\
.6 \\
= \\
= \\
-\end{array}$ & $\begin{array}{r}5.7 \\
\overline{-} \\
3.3 \\
2.4 \\
1.9 \\
.5 \\
- \\
- \\
-\end{array}$ \\
\hline Primary Energy - Five Sectors ${ }^{c}$.. & 269.6 & 498.9 & 937.5 & $1,061.5$ & 892.6 & 951.1 & 972.1 & $1,004.2$ & $1,038.3$ & $1,111.6$ \\
\hline
\end{tabular}

There are no direct fuel costs for hydroelectric, geothermal, centralized solar, or wind energy.

Heavy oil includes Grade Nos. 4,5, and 6, and residual fuel oils. Light oil includes Grade No. 2 heating oil, kerosene, and jet fuel.

c Blomass fuels are not Included, except those consumed at electric utilities and those added to motor gasoline.

- No consumption, including cases where adjustments were made. See "Consumption Adjustments for Process Fuel and Intermediate Products."

-Value less than 0.05 million dollars.

Note: Totals may not equal sum of components due to independent rounding.

Sources: Dala sources, estimation procedures, and assumptions are described in the "Documentation" section of this report. 


\begin{tabular}{|c|c|c|c|c|c|c|c|c|c|c|}
\hline Energy Source & 1970 & 1975 & 1980 & 1985 & 1986 & 1987 & 1988 & 1989 & 1990 & 1991 \\
\hline & \multicolumn{10}{|c|}{ Prices in Dollars per Million Btu } \\
\hline 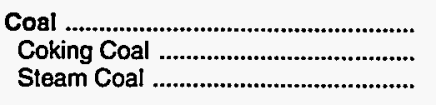 & $\begin{array}{r}0.47 \\
.47\end{array}$ & $\begin{array}{l}1.25 \\
1 . \overline{25}\end{array}$ & $\begin{array}{r}1.60 \\
1.60\end{array}$ & $\frac{1.88}{1 . \overline{8}}$ & $\frac{1.82}{1 . \overline{2}}$ & $\frac{1.74}{1 . \overline{74}}$ & $\frac{1.75}{1.75}$ & $\begin{array}{l}1.71 \\
1 . \overline{71}\end{array}$ & $\frac{1.73}{1.73}$ & $\begin{array}{r}1.64 \\
1.64\end{array}$ \\
\hline Natural Gas & .57 & 1.16 & 3.07 & 5.06 & 4.40 & 4.60 & 4.25 & 4.22 & 4.01 & 3.66 \\
\hline $\begin{array}{l}\text { Petroleum } \\
\text { Asphalt and Road Oil } \\
\text { Aviation Gasoline } \\
\text { Distillate Fuel } \\
\text { Jet Fuel } \\
\text { Kerosene } \\
\text { LPG }\end{array}$ & $\begin{array}{r}1.89 \\
.67 \\
2.17 \\
1.03 \\
.73 \\
1.43 \\
2.04 \\
5.08 \\
2.75 \\
.42 \\
1.58\end{array}$ & $\begin{array}{l}3.43 \\
1.78 \\
3.45 \\
2.68 \\
2.03 \\
3.02 \\
3.56 \\
7.48 \\
4.35 \\
1.40 \\
3.34\end{array}$ & $\begin{array}{r}8.07 \\
3.58 \\
9.02 \\
6.84 \\
6.46 \\
7.92 \\
5.77 \\
14.36 \\
10.18 \\
3.43 \\
6.70\end{array}$ & $\begin{array}{r}7.98 \\
4.99 \\
9.99 \\
7.06 \\
6.11 \\
8.01 \\
9.87 \\
17.61 \\
8.84 \\
4.36 \\
6.03\end{array}$ & $\begin{array}{r}5.98 \\
4.18 \\
8.41 \\
5.76 \\
4.23 \\
7.25 \\
9.78 \\
15.59 \\
6.40 \\
2.50 \\
3.74\end{array}$ & $\begin{array}{r}6.25 \\
3.20 \\
7.55 \\
5.82 \\
4.35 \\
5.82 \\
8.31 \\
12.70 \\
7.08 \\
2.93 \\
3.91\end{array}$ & $\begin{array}{r}6.12 \\
3.17 \\
7.41 \\
5.69 \\
4.19 \\
6.56 \\
10.16 \\
14.61 \\
7.08 \\
2.30 \\
3.35\end{array}$ & $\begin{array}{r}6.83 \\
2.90 \\
8.28 \\
6.15 \\
4.74 \\
6.51 \\
9.26 \\
13.30 \\
7.84 \\
2.70 \\
4.23\end{array}$ & $\begin{array}{r}7.83 \\
2.90 \\
9.32 \\
7.68 \\
6.07 \\
8.30 \\
10.35 \\
13.40 \\
8.80 \\
3.11 \\
4.72\end{array}$ & $\begin{array}{r}7.56 \\
3.06 \\
8.71 \\
7.21 \\
5.12 \\
7.75 \\
10.82 \\
15.42 \\
8.56 \\
2.42 \\
4.35\end{array}$ \\
\hline 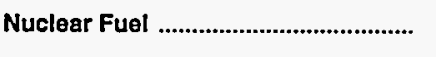 & .19 & .19 & .44 & .62 & .61 & .64 & .59 & .58 & .53 & .52 \\
\hline Biomass Fuels at Utilities ..................... & - & - & - & - & - & - & - & - & - & - \\
\hline Primary Energy - Five Sectors ${ }^{b} \ldots$ & 1.20 & 1.86 & 4.09 & 3.80 & 2.98 & 3.00 & 2.91 & 3.17 & 3.45 & 3.30 \\
\hline $\begin{array}{l}\text { Electric Utility Fuelc ........................ } \\
\text { Electricity Purchased by End Users }\end{array}$ & $\begin{array}{r}.42 \\
3.98\end{array}$ & $\begin{array}{r}.56 \\
7.72\end{array}$ & $\begin{array}{r}1.14 \\
11.11\end{array}$ & $\begin{array}{r}1.11 \\
15.99\end{array}$ & $\begin{array}{r}1.03 \\
16.25\end{array}$ & $\begin{array}{r}1.03 \\
16.41\end{array}$ & $\begin{array}{r}1.00 \\
16.40\end{array}$ & $\begin{array}{r}.99 \\
16.53\end{array}$ & $\begin{array}{r}.94 \\
16.39\end{array}$ & $\begin{array}{r}.90 \\
16.50\end{array}$ \\
\hline \multirow[t]{2}{*}{ Total Energy ........................................ } & 1.83 & 3.79 & 7.25 & 8.85 & 7.86 & 8.09 & 7.95 & 8.39 & 8.89 & 8.78 \\
\hline & \multicolumn{10}{|c|}{ Expenditures in Millions of Dollars } \\
\hline 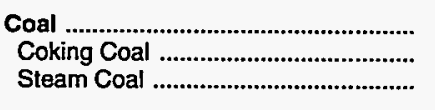 & $65 . \overline{3}$ & $\begin{array}{r}174.7 \\
174 . \overline{7}\end{array}$ & $\begin{array}{r}392.2 \\
392 . \overline{2}\end{array}$ & $\begin{array}{r}493.6 \\
493 . \overline{6}\end{array}$ & $\begin{array}{r}480.7 \\
\overline{-} \\
480 . \overline{7}\end{array}$ & $\begin{array}{r}513.0 \\
513.0\end{array}$ & $\begin{array}{r}526.9 \\
-\overline{9}\end{array}$ & $\begin{array}{r}514.1 \\
514 . \overline{1}\end{array}$ & $\begin{array}{r}499.0 \\
499.0\end{array}$ & $\begin{array}{r}478.2 \\
478 . \overline{2}\end{array}$ \\
\hline 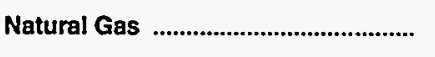 & $91.4^{\circ}$ & 143.3 & 441.2 & 495.3 & 435.6 & 488.0 & 479.3 & 495.2 & 525.9 & 492.8 \\
\hline 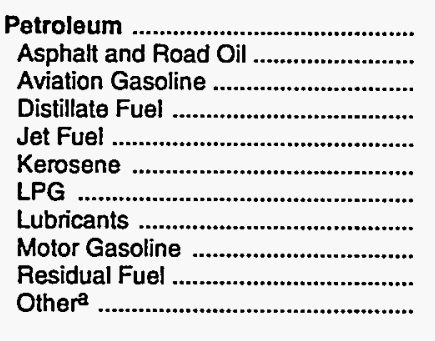 & $\begin{array}{r}569.7 \\
9.9 \\
2.5 \\
56.7 \\
12.4 \\
19.3 \\
22.6 \\
11.9 \\
415.8 \\
14.2 \\
4.4\end{array}$ & $\begin{array}{r}1,166.8 \\
28.9 \\
2.5 \\
130.7 \\
29.5 \\
17.5 \\
42.3 \\
20.9 \\
809.3 \\
67.5 \\
17.5\end{array}$ & $\begin{array}{r}2,937.1 \\
36.4 \\
6.8 \\
424.9 \\
107.1 \\
60.7 \\
67.4 \\
47.3 \\
1,899.0 \\
155.3 \\
132.2\end{array}$ & $\begin{array}{r}2,808.2 \\
45.3 \\
6.9 \\
482.8 \\
105.3 \\
67.4 \\
112.4 \\
52.8 \\
1,751.7 \\
80.0 \\
103.7\end{array}$ & $\begin{array}{r}2,161.0 \\
57.4 \\
6.6 \\
392.7 \\
72.7 \\
48.5 \\
102.6 \\
45.7 \\
1,321.6 \\
37.8 \\
75.4\end{array}$ & $\begin{array}{r}2,314.1 \\
51.6 \\
4.6 \\
401.8 \\
75.4 \\
44.9 \\
110.0 \\
42.1 \\
1.448 .6 \\
45.3 \\
89.9\end{array}$ & $\begin{array}{r}2,319.4 \\
69.3 \\
4.7 \\
417.9 \\
73.4 \\
55.2 \\
131.2 \\
46.7 \\
1,393.6 \\
47.3 \\
80.1\end{array}$ & $\begin{array}{r}2,698.6 \\
44.5 \\
5.0 \\
447.8 \\
80.3 \\
52.6 \\
125.5 \\
43.6 \\
1,754.5 \\
46.5 \\
98.2\end{array}$ & $\begin{array}{r}3,142.2 \\
38.1 \\
4.8 \\
650.4 \\
97.4 \\
31.0 \\
109.9 \\
45.2 \\
1,987.6 \\
47.9 \\
130.0\end{array}$ & $\begin{array}{r}3,057.0 \\
39.4 \\
7.9 \\
642.3 \\
77.1 \\
37.4 \\
141.0 \\
46.5 \\
1,912.0 \\
37.0 \\
116.4\end{array}$ \\
\hline Nuclear Fuel & * & 40.6 & 83.4 & 214.5 & 233.9 & 269.0 & 257.5 & 251.7 & 242.8 & 240.5 \\
\hline 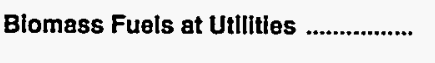 & - & - & - & - & - & - & - & - & - & - \\
\hline Primary Energy - Five Sectors ${ }^{b} \ldots$. & 726.5 & $1,525.4$ & $3,854.0$ & $4,011.6$ & $3,311.2$ & $3,584.2$ & $3,583.1$ & $3,959.6$ & $4,409.9$ & $4,268.4$ \\
\hline $\begin{array}{l}\text { Electric Utility Fuelc } \\
\text { Electricity Purchased by End Users }\end{array}$ & $\begin{array}{r}-65.0 \\
294.7\end{array}$ & $\begin{array}{r}-205.4 \\
782.8\end{array}$ & $\begin{array}{r}-467.6 \\
1,412.5\end{array}$ & $\begin{array}{r}-601.3 \\
2,523.7\end{array}$ & $\begin{array}{r}-598.7 \\
2,741.1\end{array}$ & $\begin{array}{r}-670.6 \\
2,909.0\end{array}$ & $\begin{array}{r}-677.1 \\
2,955.0\end{array}$ & $\begin{array}{r}-672.7 \\
3,038.0\end{array}$ & $\begin{array}{r}-656.9 \\
3,113.0\end{array}$ & $\begin{array}{r}-640.7 \\
3,212.0\end{array}$ \\
\hline 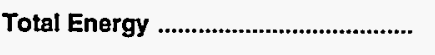 & 956.2 & $2,102.7$ & $4,798.9$ & $5,934.0$ & $5,453.6$ & $5,822.7$ & $5,861.0$ & $6,324.9$ & $6,865.9$ & $6,839.7$ \\
\hline
\end{tabular}

a Includes petroleum coke used at electric utilities.

biomass fuels are not included, except those consumed at electric utilities and those added to motor gasoline.

c There are no direct fuel costs for hydroelectric, geothermal, centralized solar, or wind energy.

-No consumption, including cases where adjustments were made. See "Consumption Adjustments for Process Fuel and Intermediate Products."

Value less than 0.05 million dollars.

Note: Expenditure totals may not equal sum of components due to independent rounding.

Sources: Data sources, estimation procedures, and assumptions are described in the "Documentation" section of this report. 
Energy Price Estimates by Sector, South Carolina

1970, 1975, 1980, and 1985-1991

(Dollars per Million Btu)

\begin{tabular}{|c|c|c|c|c|c|c|c|c|c|c|}
\hline Sector and Energy Source & 1970 & 1975 & 1980 & 1985 & 1986 & 1987 & 1988 & 1989 & 1990 & 1991 \\
\hline 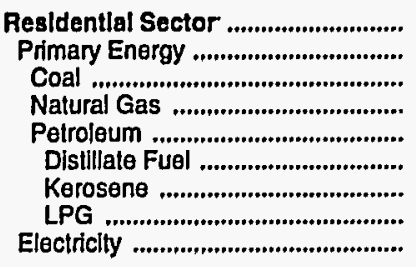 & $\begin{array}{l}2.82 \\
1.51 \\
1.20 \\
1.32 \\
1.64 \\
1.30 \\
1.58 \\
2.46 \\
5.64\end{array}$ & $\begin{array}{l}5.78 \\
2.71 \\
2.47 \\
2.08 \\
3.29 \\
2.69 \\
3.16 \\
4.28 \\
9.60\end{array}$ & $\begin{array}{r}9.73 \\
5.76 \\
3.19 \\
4.06 \\
7.50 \\
6.95 \\
8.27 \\
7.47 \\
13.69\end{array}$ & $\begin{array}{r}14.90 \\
7.44 \\
3.48 \\
6.44 \\
8.38 \\
7.19 \\
8.25 \\
9.72 \\
20.54\end{array}$ & $\begin{array}{r}15.20 \\
7.06 \\
3.31 \\
6.35 \\
8.14 \\
6.66 \\
7.64 \\
10.26 \\
20.89\end{array}$ & $\begin{array}{r}14.69 \\
6.32 \\
3.20 \\
6.41 \\
6.39 \\
5.29 \\
6.07 \\
7.93 \\
21.03\end{array}$ & $\begin{array}{r}15.03 \\
7.12 \\
3.26 \\
6.55 \\
7.90 \\
6.01 \\
6.90 \\
10.62 \\
20.89\end{array}$ & $\begin{array}{r}15.21 \\
7.09 \\
3.34 \\
6.56 \\
7.62 \\
5.90 \\
6.77 \\
10.02 \\
21.08\end{array}$ & $\begin{array}{r}16.32 \\
7.87 \\
3.34 \\
6.97 \\
9.01 \\
7.57 \\
8.69 \\
10.57 \\
20.95\end{array}$ & $\begin{array}{r}16.21 \\
7.76 \\
3.15 \\
6.80 \\
8.95 \\
7.06 \\
8.10 \\
11.00 \\
21.17\end{array}$ \\
\hline 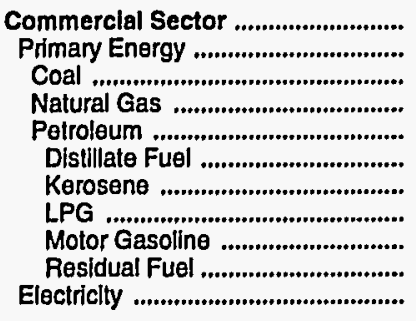 & $\begin{array}{r}2.35 \\
.93 \\
.50 \\
.86 \\
1.28 \\
1.01 \\
.63 \\
1.39 \\
2.75 \\
.46 \\
4.85\end{array}$ & $\begin{array}{l}4.84 \\
1.58 \\
1.53 \\
1.22 \\
2.58 \\
2.32 \\
2.29 \\
2.68 \\
4.35 \\
1.15 \\
8.55\end{array}$ & $\begin{array}{r}7.65 \\
3.57 \\
1.70 \\
3.11 \\
6.70 \\
6.33 \\
5.15 \\
4.24 \\
10.18 \\
3.41 \\
12.07\end{array}$ & $\begin{array}{r}12.85 \\
5.92 \\
1.77 \\
5.60 \\
7.07 \\
6.18 \\
6.93 \\
10.09 \\
8.84 \\
4.50 \\
18.01\end{array}$ & $\begin{array}{r}12.63 \\
4.97 \\
1.77 \\
5.47 \\
5.45 \\
4.35 \\
5.20 \\
9.12 \\
6.40 \\
2.67 \\
18.37\end{array}$ & $\begin{array}{r}12.70 \\
5.08 \\
1.70 \\
5.45 \\
5.10 \\
3.80 \\
4.43 \\
8.76 \\
7.08 \\
2.97 \\
18.33\end{array}$ & $\begin{array}{r}12.51 \\
5.03 \\
1.67 \\
5.44 \\
4.98 \\
3.74 \\
4.46 \\
9.59 \\
7.08 \\
2.40 \\
18.03\end{array}$ & $\begin{array}{r}13.23 \\
5.39 \\
1.69 \\
5.51 \\
5.29 \\
4.14 \\
5.22 \\
8.27 \\
7.84 \\
2.81 \\
18.11\end{array}$ & $\begin{array}{r}13.93 \\
6.11 \\
1.74 \\
5.74 \\
7.13 \\
5.75 \\
6.35 \\
10.05 \\
8.80 \\
3.25 \\
17.92\end{array}$ & $\begin{array}{r}14.02 \\
5.61 \\
1.71 \\
5.41 \\
6.52 \\
4.64 \\
5.62 \\
10.60 \\
8.56 \\
2.59 \\
18.15\end{array}$ \\
\hline 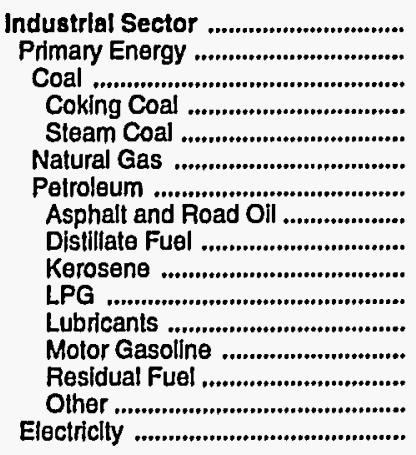 & $\begin{array}{r}.87 \\
.57 \\
.50 \\
-50 \\
.50 \\
.45 \\
.83 \\
.67 \\
.58 \\
.63 \\
1.39 \\
5.08 \\
2.75 \\
.40 \\
1.58 \\
2.41\end{array}$ & $\begin{array}{r}2.48 \\
1.56 \\
1.53 \\
- \\
1.53 \\
1.00 \\
2.27 \\
1.78 \\
2.12 \\
2.29 \\
2.68 \\
7.48 \\
4.35 \\
1.82 \\
3.34 \\
5.80\end{array}$ & $\begin{array}{r}4.40 \\
3.35 \\
1.70 \\
- \\
1.70 \\
2.89 \\
4.88 \\
3.58 \\
4.62 \\
5.15 \\
4.24 \\
14.36 \\
10.18 \\
3.53 \\
6.70 \\
8.56\end{array}$ & $\begin{array}{r}6.40 \\
4.17 \\
1.77 \\
- \\
1.77 \\
4.57 \\
6.24 \\
4.99 \\
6.21 \\
6.93 \\
10.09 \\
17.61 \\
8.84 \\
4.50 \\
6.03 \\
12.02\end{array}$ & $\begin{array}{r}5.85 \\
3.30 \\
1.77 \\
- \\
1.77 \\
3.62 \\
4.49 \\
4.18 \\
4.65 \\
5.20 \\
9.12 \\
15.59 \\
6.40 \\
2.67 \\
3.74 \\
12.00\end{array}$ & $\begin{array}{r}5.91 \\
3.31 \\
1.70 \\
- \\
1.70 \\
3.82 \\
4.30 \\
3.20 \\
3.96 \\
4.43 \\
8.76 \\
12.70 \\
7.08 \\
2.97 \\
3.91 \\
12.27\end{array}$ & $\begin{array}{r}5.63 \\
3.05 \\
1.67 \\
- \\
1.67 \\
3.34 \\
3.91 \\
3.17 \\
4.00 \\
4.46 \\
9.59 \\
14.61 \\
7.08 \\
2.40 \\
3.35 \\
12.42\end{array}$ & $\begin{array}{r}5.84 \\
3.24 \\
1.69 \\
- \\
1.69 \\
3.37 \\
4.42 \\
2.90 \\
4.67 \\
5.22 \\
8.27 \\
13.30 \\
7.84 \\
2.81 \\
4.23 \\
12.48\end{array}$ & $\begin{array}{r}5.86 \\
3.42 \\
1.74 \\
-\overline{1} \\
1.74 \\
3.26 \\
4.95 \\
2.90 \\
5.68 \\
6.35 \\
10.05 \\
13.40 \\
8.80 \\
3.25 \\
4.72 \\
12.24\end{array}$ & $\begin{array}{r}5.79 \\
3.22 \\
1.71 \\
-\overline{1} \\
1.71 \\
2.87 \\
4.82 \\
3.06 \\
5.03 \\
5.62 \\
10.60 \\
15.42 \\
8.56 \\
2.59 \\
4.35 \\
12.20\end{array}$ \\
\hline 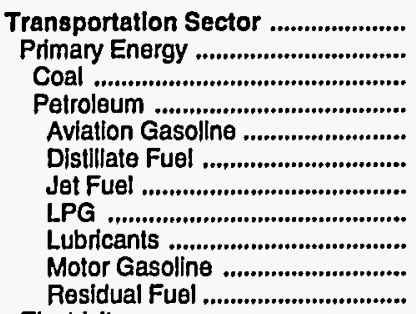 & $\begin{array}{r}2.34 \\
2.34 \\
.50 \\
2.34 \\
2.17 \\
1.32 \\
.73 \\
1.39 \\
5.08 \\
2.75 \\
.41\end{array}$ & $\begin{array}{l}4.04 \\
4.04 \\
1.53 \\
4.04 \\
3.45 \\
3.01 \\
2.03 \\
2.68 \\
7.48 \\
4.35 \\
1.52\end{array}$ & $\begin{array}{r}9.42 \\
9.42 \\
- \\
9.42 \\
9.02 \\
7.63 \\
6.46 \\
4.24 \\
14.36 \\
10.18 \\
2.90\end{array}$ & $\begin{array}{r}8.39 \\
8.39 \\
- \\
8.39 \\
9.99 \\
7.36 \\
6.11 \\
10.09 \\
17.61 \\
8.84 \\
3.82\end{array}$ & $\begin{array}{r}6.19 \\
6.19 \\
- \\
6.19 \\
8.41 \\
6.00 \\
4.23 \\
9.12 \\
15.59 \\
6.40 \\
2.01\end{array}$ & $\begin{array}{r}6.77 \\
6.77 \\
-\overline{6} \\
6.77 \\
7.55 \\
6.48 \\
4.35 \\
8.76 \\
12.70 \\
7.08 \\
2.80\end{array}$ & $\begin{array}{r}6.69 \\
6.69 \\
- \\
6.69 \\
7.41 \\
6.27 \\
4.19 \\
9.59 \\
14.61 \\
7.08 \\
1.98\end{array}$ & $\begin{array}{r}7.44 \\
7.44 \\
- \\
7.44 \\
8.28 \\
6.82 \\
4.74 \\
8.27 \\
13.30 \\
7.84 \\
2.38\end{array}$ & $\begin{array}{r}8.49 \\
8.49 \\
- \\
8.49 \\
9.32 \\
8.17 \\
6.07 \\
10.05 \\
13.40 \\
8.80 \\
2.58\end{array}$ & $\begin{array}{r}8.15 \\
8.15 \\
- \\
8.15 \\
8.71 \\
7.77 \\
5.12 \\
10.60 \\
15.42 \\
8.56 \\
2.06\end{array}$ \\
\hline Electricity ......................................... & - & - & - & - & - & - & - & - & - & - \\
\hline $\begin{array}{l}\text { Total Energy .................................... } \\
\text { Primary Energy - Four Sectors ...... } \\
\text { Electricity ....................................... }\end{array}$ & $\begin{array}{l}1.83 \\
1.47 \\
3.98\end{array}$ & $\begin{array}{l}3.79 \\
2.91 \\
7.72\end{array}$ & $\begin{array}{r}7.25 \\
6.34 \\
11.11\end{array}$ & $\begin{array}{r}8.85 \\
6.65 \\
15.99\end{array}$ & $\begin{array}{r}7.86 \\
5.16 \\
16.25\end{array}$ & $\begin{array}{r}8.09 \\
5.37 \\
16.41\end{array}$ & $\begin{array}{r}7.95 \\
5.22 \\
16.40\end{array}$ & $\begin{array}{r}8.39 \\
5.76 \\
16.53\end{array}$ & $\begin{array}{r}8.89 \\
6.44 \\
16.39\end{array}$ & $\begin{array}{r}8.78 \\
6.21 \\
16.50\end{array}$ \\
\hline 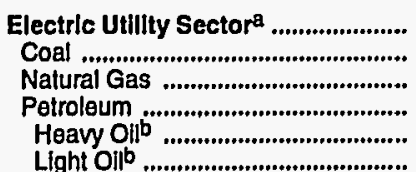 & $\begin{array}{l}.42 \\
.43 \\
.37 \\
.52 \\
.46 \\
.70\end{array}$ & $\begin{array}{r}.56 \\
1.14 \\
.71 \\
1.17 \\
1.14 \\
2.41\end{array}$ & $\begin{array}{l}1.14 \\
1.56 \\
2.41 \\
3.91 \\
3.44 \\
5.78\end{array}$ & $\begin{array}{l}1.11 \\
1.91 \\
4.54 \\
5.72 \\
3.94 \\
5.73\end{array}$ & $\begin{array}{l}1.03 \\
1.82 \\
2.03 \\
3.61 \\
2.16 \\
3.64\end{array}$ & $\begin{array}{l}1.03 \\
1.74 \\
3.35 \\
3.98 \\
2.93 \\
4.00\end{array}$ & $\begin{array}{l}1.00 \\
1.76 \\
1.81 \\
3.72 \\
2.22 \\
3.74\end{array}$ & $\begin{array}{l}.99 \\
1.71 \\
2.22 \\
4.07 \\
2.71 \\
4.26\end{array}$ & $\begin{array}{r}.94 \\
1.72 \\
1.72 \\
6.00 \\
3.02 \\
6.22\end{array}$ & $\begin{array}{r}.90 \\
1.63 \\
1.49 \\
4.54 \\
2.22 \\
4.75\end{array}$ \\
\hline 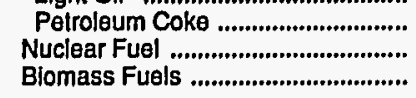 & .19 &.$\overline{19}$ &. & $\frac{-}{62}$ & $\begin{array}{r}61 \\
-\end{array}$ &. & $\begin{array}{r}-59 \\
-\end{array}$ & $\begin{array}{r}.58 \\
-\end{array}$ & .5 & .52 \\
\hline PrImary Energy - Flve Sectorsc .. & 1.20 & 1.86 & 4.09 & 3.80 & 2.98 & 3.00 & 2.91 & 3.17 & $\mathbf{3 . 4 5}$ & 3.30 \\
\hline
\end{tabular}

a There are no direct fuel costs for hydroelectric, geothermal, centralized solar, or wind energy.

beavy oll includes Grade Nos. 4, 5, and 6, and residual fuel oils. Light oil includes Grade No. 2 heating oil, kerosene, and jet fuel.

c Blomass fuels are not included, except those consumed at electric utilities and those added to motor gasoline.

- No consumption, including cases where adjustments were made. See "Consumption Adjustments for Process Fuel and Intermediate Products."

Sources: Data sources, estimation procedures, and assumptions are described in the "Documentation" section of this report. 
S Energy Expenditure Estimates by Sector, South Carolina

$1970,1975,1980$, and 1985-1991

(Million Dollars)

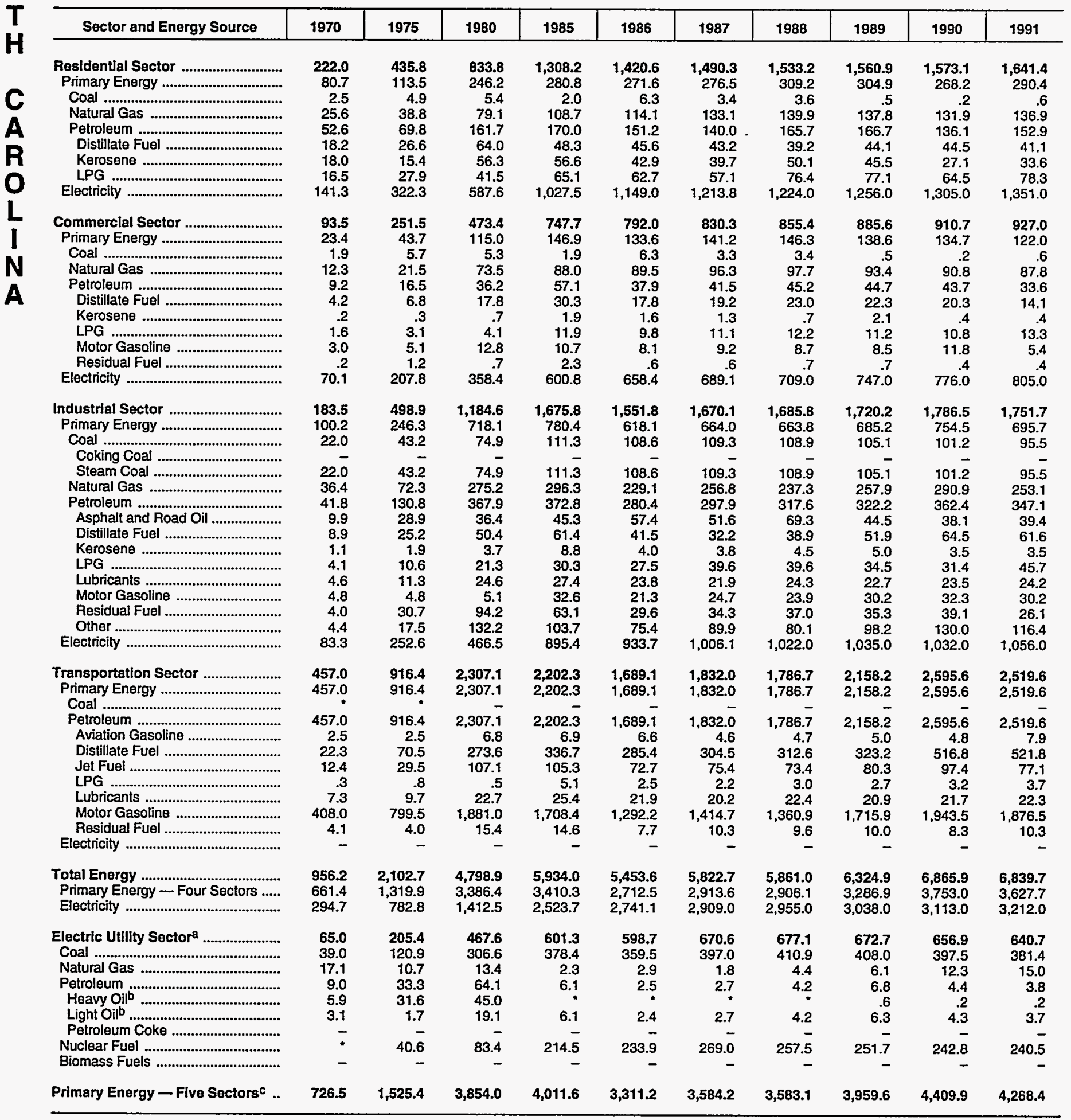

a There are no direct fuel costs for hydroelectric, geothermal, centralized solar, or wind energy.

b Heavy oil includes Grade Nos. 4, 5, and 6, and residual fuel oils. Light oil includes Grade No. 2 heating oil, kerosene, and jet fuel.

c Biomass fuels are not included, except those consumed at electric utilities and those added to motor gasoline.

-No consumption, including cases where adjustments were made. See "Consumption Adjustments for Process Fuel and Intermediate Products."

"Value less than 0.05 million dollars.

Note: Totals may not equal sum of components due to independent rounding.

Sources: Data sources, estimation procedures, and assumptions are described in the "Documentation" section of this report. 


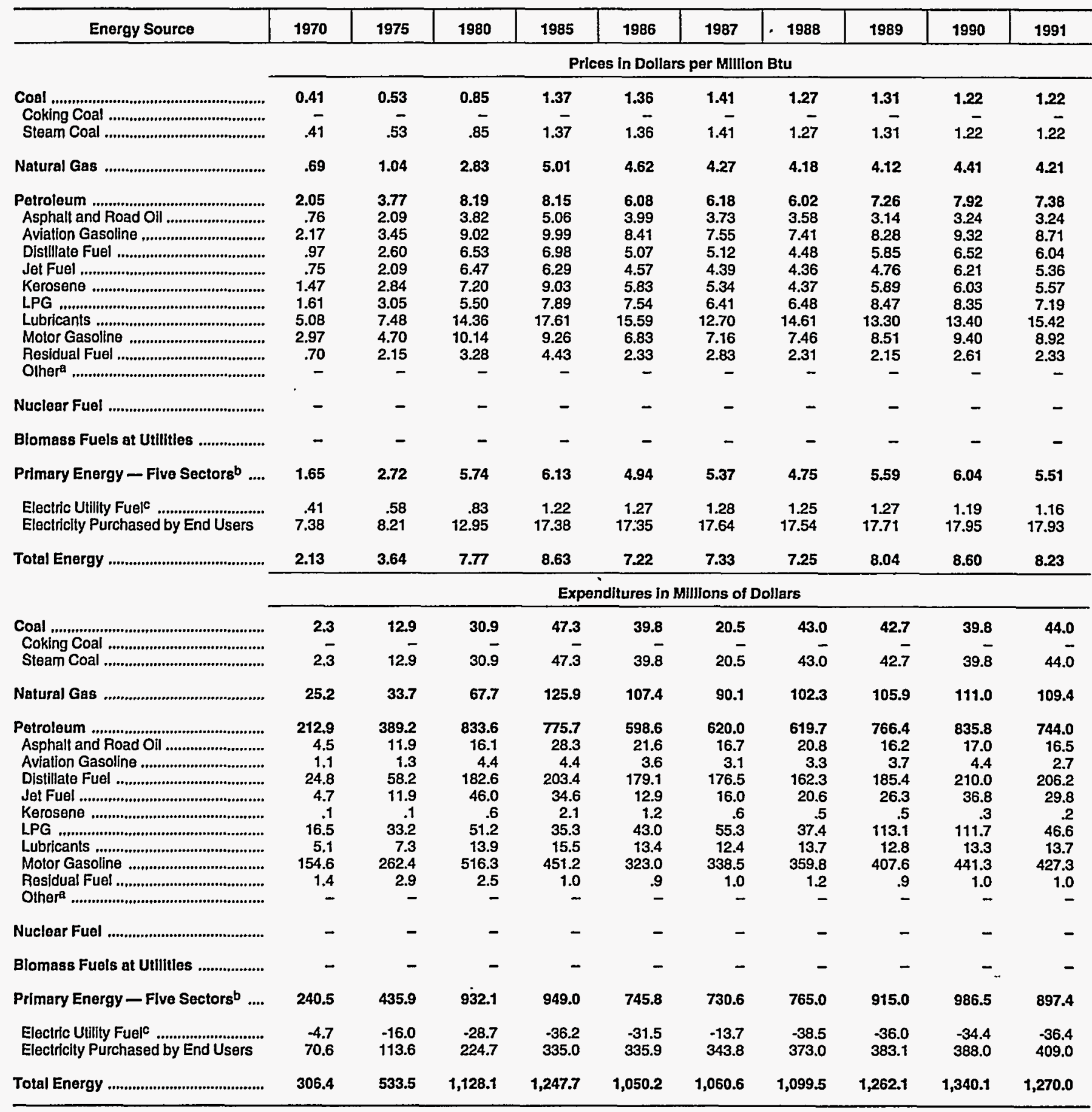

a Includes petroleum coke used at electric utilities.

blomass fuels are not included, except those consumed at electric utilities and those added to motor gasoline.

c There are no direct fuel costs for hydroelectric, geothemal, centralized solar, or wind energy.

-No consumplion, including cases where adjustments were made. See "Consumption Adjustments for Process Fuel and Intermediate Products."

Note: Expenditure totals may not equal sum of components due to independent rounding.

Sources: Data sources, estimation procedures, and assumptions are described in the "Documentation" section of this report. 


\section{S Energy Price Estimates by Sector, South Dakota}

\section{O 1970, 1975, 1980, and 1985-1991}

(Dollars per Million Btu)

\begin{tabular}{|c|c|c|c|c|c|c|c|c|c|c|}
\hline Sector and Energy Source & 1970 & 1975 & 1980 & 1985 & 1986 & 1987 & 1988 & 1989 & 1990 & 1991 \\
\hline $\begin{array}{l}\text { Residential Sector } \\
\text { Primary Energy } \\
\text { Coal } \\
\text { Natural Gas } \\
\text { Petroleum } \\
\text { Distillate Fuel } \\
\text { Kerosene } \\
\text { LPG }\end{array}$ & $\begin{array}{l}2.41 \\
1.30 \\
1.75 \\
1.04 \\
1.60 \\
1.28 \\
1.57 \\
1.78 \\
7.76\end{array}$ & $\begin{array}{l}3.82 \\
2.23 \\
3.61 \\
1.40 \\
3.14 \\
2.55 \\
2.91 \\
3.41 \\
8.97\end{array}$ & $\begin{array}{r}7.89 \\
4.84 \\
3.48 \\
3.14 \\
6.89 \\
6.92 \\
7.83 \\
6.84 \\
14.52\end{array}$ & $\begin{array}{r}10.69 \\
6.42 \\
2.65 \\
5.69 \\
7.66 \\
7.64 \\
9.27 \\
7.56 \\
19.13\end{array}$ & $\begin{array}{r}9.99 \\
5.55 \\
3.05 \\
5.24 \\
5.96 \\
5.45 \\
6.61 \\
6.92 \\
19.44\end{array}$ & $\begin{array}{r}9.73 \\
4.89 \\
2.97 \\
4.81 \\
4.97 \\
4.64 \\
5.63 \\
5.31 \\
19.93\end{array}$ & $\begin{array}{r}9.70 \\
4.72 \\
2.58 \\
4.81 \\
4.61 \\
3.95 \\
4.78 \\
5.64 \\
19.62\end{array}$ & $\begin{array}{r}10.54 \\
6.28 \\
2.65 \\
4.77 \\
7.95 \\
5.35 \\
6.49 \\
10.56 \\
19.95\end{array}$ & $\begin{array}{r}10.36 \\
5.79 \\
2.62 \\
5.06 \\
6.48 \\
5.52 \\
6.70 \\
7.20 \\
20.35\end{array}$ & $\begin{array}{r}10.35 \\
5.21 \\
2.97 \\
4.85 \\
5.70 \\
5.13 \\
6.22 \\
6.40 \\
20.25\end{array}$ \\
\hline $\begin{array}{l}\text { Commercial Sector } \\
\text { Primary Energy } \\
\text { Coal } \\
\text { Natural Gas } \\
\text { Petroleum } \\
\text { Distillate Fuel } \\
\text { Kerosene } \\
\text { LPG } \\
\text { Motor Gasoline } \\
\text { Residual Fuel } \\
\text { Electricity }\end{array}$ & $\begin{array}{r}1.94 \\
.76 \\
.49 \\
.63 \\
1.22 \\
1.06 \\
-\overline{1} \\
1.13 \\
2.97 \\
.66 \\
7.53\end{array}$ & $\begin{array}{r}2.71 \\
1.31 \\
1.04 \\
.99 \\
2.54 \\
2.34 \\
-\overline{2} \\
2.28 \\
4.70 \\
2.21 \\
8.82\end{array}$ & $\begin{array}{r}5.99 \\
3.69 \\
1.79 \\
2.72 \\
6.24 \\
6.45 \\
-\overline{4} \\
4.36 \\
10.14 \\
3.08 \\
13.11\end{array}$ & $\begin{array}{r}9.17 \\
5.09 \\
2.42 \\
4.56 \\
7.27 \\
6.56 \\
7.54 \\
8.32 \\
9.26 \\
4.44 \\
17.53\end{array}$ & $\begin{array}{r}8.53 \\
4.53 \\
1.96 \\
4.32 \\
5.43 \\
3.85 \\
4.35 \\
8.25 \\
6.83 \\
2.33 \\
17.68\end{array}$ & $\begin{array}{r}8.52 \\
4.46 \\
1.85 \\
4.17 \\
5.10 \\
3.65 \\
4.37 \\
7.76 \\
7.16 \\
2.84 \\
17.52\end{array}$ & $\begin{array}{r}8.66 \\
4.17 \\
1.76 \\
4.02 \\
4.56 \\
2.80 \\
3.35 \\
7.73 \\
7.46 \\
2.35 \\
17.66\end{array}$ & $\begin{array}{r}8.86 \\
4.39 \\
1.76 \\
3.92 \\
5.83 \\
4.02 \\
5.00 \\
7.13 \\
8.51 \\
2.15 \\
17.57\end{array}$ & $\begin{array}{r}9.42 \\
4.79 \\
1.79 \\
4.13 \\
6.81 \\
4.16 \\
5.22 \\
9.36 \\
9.40 \\
2.61 \\
18.13\end{array}$ & $\begin{array}{r}9.18 \\
4.26 \\
1.73 \\
3.97 \\
5.55 \\
3.64 \\
4.53 \\
8.34 \\
8.92 \\
2.33 \\
18.17\end{array}$ \\
\hline 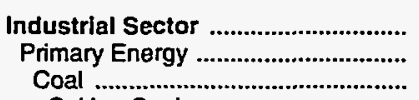 & $\begin{array}{r}1.42 \\
1.34 \\
.49\end{array}$ & $\begin{array}{l}2.95 \\
2.63 \\
1.04\end{array}$ & $\begin{array}{l}6.02 \\
5.53 \\
1.79\end{array}$ & $\begin{array}{l}6.51 \\
5.80 \\
2.42\end{array}$ & $\begin{array}{l}5.19 \\
4.19 \\
1.96\end{array}$ & $\begin{array}{l}5.42 \\
4.24 \\
i .85\end{array}$ & $\begin{array}{l}4.93 \\
3.60 \\
1.76\end{array}$ & $\begin{array}{l}5.79 \\
4.60 \\
1.76\end{array}$ & $\begin{array}{l}6.29 \\
5.13 \\
1.79\end{array}$ & $\begin{array}{l}5.53 \\
4.10 \\
1.73\end{array}$ \\
\hline Coking Coal & .49 & $1 . \overline{4}$ & $1 . \overline{79}$ & $2 . \overline{42}$ & $1 . \overline{96}$ & $\overline{1.85}$ & $\overline{1.76}$ & $\overline{1.76}$ & $1 . \overline{9}$ & $\overline{1.73}$ \\
\hline 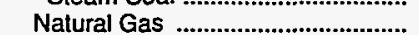 & .32 & .60 & 2.35 & 4.11 & 3.53 & 3.04 & 3.12 & 3.04 & 3.73 & 3.25 \\
\hline Petroleum & 1.56 & 3.15 & 6.44 & 6.89 & 4.63 & 4.78 & 3.94 & 5.35 & 5.94 & 4.79 \\
\hline Asphalt and Road Oil ....................... & .76 & 2.09 & 3.82 & 5.06 & 3.99 & 3.73 & 3.58 & 3.14 & 3.24 & 3.24 \\
\hline 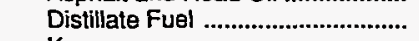 & .73 & 2.57 & 5.65 & 6.85 & 3.95 & 3.97 & 3.04 & 4.54 & 4.74 & 4.12 \\
\hline 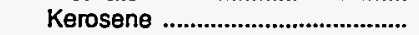 & .78 & 2.74 & 5.92 & 7.54 & 4.35 & 4.37 & 3.35 & 5.00 & 5.22 & 4.53 \\
\hline 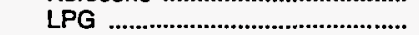 & 1.13 & 2.28 & 4.36 & 8.32 & 8.25 & 7.76 & 7.73 & 7.13 & 9.36 & 8.34 \\
\hline 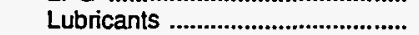 & 5.08 & 7.48 & 14.36 & 17.61 & 15.59 & 12.70 & 14.61 & 13.30 & 13.40 & 15.42 \\
\hline Motor Gasoline ................................... & 2.97 & 4.70 & 10.14 & 9.26 & 6.83 & 7.16 & 7.46 & 8.51 & 9.40 & 8.92 \\
\hline 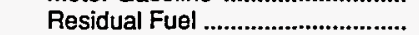 & .70 & 2.02 & 3.34 & 4.44 & 2.33 & 2.84 & 2.35 & 2.15 & 2.61 & 2.33 \\
\hline Other & - & - & - & - & - & - & - & - & - & - \\
\hline 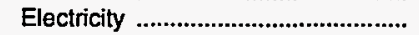 & 4.72 & 6.00 & 9.70 & 12.34 & 12.59 & 13.41 & 13.51 & 13.82 & 13.62 & 13.58 \\
\hline $\begin{array}{l}\text { Transportation Sector } \\
\text { Primary Energy }\end{array}$ & $\begin{array}{l}2.56 \\
2.56\end{array}$ & $\begin{array}{l}4.23 \\
4.23\end{array}$ & $\begin{array}{l}9.21 \\
9.21\end{array}$ & $\begin{array}{l}8.64 \\
8.64\end{array}$ & 6.77 & $\begin{array}{l}7.02 \\
7.02\end{array}$ & 7.17 & $\begin{array}{l}8.04 \\
8.04\end{array}$ & $\begin{array}{l}8.99 \\
8.99\end{array}$ & $\begin{array}{l}8.58 \\
8.58\end{array}$ \\
\hline & $\begin{array}{r}2.50 \\
.49\end{array}$ & $\begin{array}{l}4.23 \\
1.04\end{array}$ & $\begin{array}{l}9.21 \\
-\end{array}$ & 8.64 & $\begin{array}{r}6.77 \\
-\end{array}$ & $\begin{array}{r}7.02 \\
-\end{array}$ & $\begin{array}{r}7.17 \\
-\end{array}$ & $\begin{array}{r}8.04 \\
-\end{array}$ & $\begin{array}{r}8.99 \\
-\end{array}$ & $\begin{array}{l}8.58 \\
-\end{array}$ \\
\hline Petroleum & 2.56 & 4.23 & 9.21 & 8.64 & 6.77 & 7.02 & 7.17 & 8.04 & 8.99 & 8.58 \\
\hline Aviation Gasoline ............................... & 2.17 & 3.45 & 9.02 & 9.99 & 8.41 & 7.55 & 7.41 & 8.28 & 9.32 & 8.71 \\
\hline Distillate Fuel ................................... & 1.32 & 2.72 & 7.12 & 6.93 & 6.38 & 6.83 & 6.66 & 7.44 & 8.57 & 8.34 \\
\hline Jet Fuel ........................................... & .75 & 2.09 & 6.47 & 6.29 & 4.57 & 4.39 & 4.36 & 4.76 & 6.21 & 5.36 \\
\hline 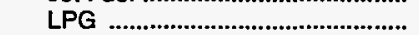 & 1.13 & 2.28 & 4.36 & 8.32 & 8.25 & 7.76 & 7.73 & 7.13 & 9.36 & 8.34 \\
\hline 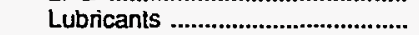 & 5.08 & 7.48 & 14.36 & 17.61 & 15.59 & 12.70 & 14.61 & 13.30 & 13.40 & 15.42 \\
\hline 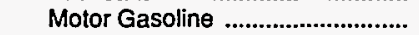 & 2.97 & 4.70 & 10.14 & 9.26 & 6.83 & 7.16 & 7.46 & 8.51 & 9.40 & 8.92 \\
\hline 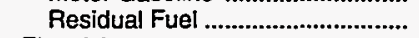 & .65 & 1.82 & - & - & - & - & - & 2.07 & 1.82 & - \\
\hline 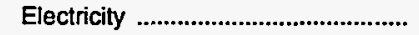 & - & - & - & - & - & - & - & - & - & - \\
\hline Total Energy & 2.13 & 3.64 & 7.77 & 8.63 & 7.22 & 7.33 & 7.25 & 8.04 & 8.60 & 8.23 \\
\hline Primary Energy - Four Sectors ...... & 1.75 & 3.16 & 7.07 & 7.29 & 5.67 & 5.72 & 5.58 & 6.49 & 7.09 & 6.54 \\
\hline 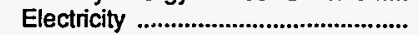 & 7.38 & 8.21 & 12.95 & 17.38 & 17.35 & 17.64 & 17.54 & 17.71 & 17.95 & 17.93 \\
\hline Electric Utllity Sector ${ }^{a}$.............................. & .41 & .58 & .83 & 1.22 & 1.27 & 1.28 & 1.25 & 1.27 & 1.19 & 1.16 \\
\hline 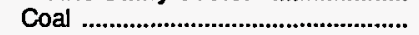 & .35 & .48 & .76 & 1.18 & 1.24 & 1.23 & 1.21 & 1.24 & 1.15 & 1.13 \\
\hline 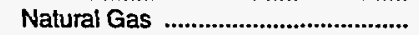 & .33 & .64 & 1.97 & 3.73 & 3.49 & 2.43 & 2.28 & 2.58 & 2.60 & 1.76 \\
\hline Petroleum & .74 & 2.22 & 6.03 & 5.75 & 3.64 & 4.23 & 3.65 & 4.50 & 5.65 & 4.88 \\
\hline Heavy Oid & .70 & 2.19 & 3.07 & 3.99 & 2.33 & 2.39 & 2.06 & - & - & - \\
\hline 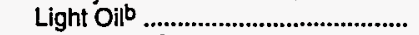 & .97 & 2.29 & 6.50 & 5.81 & 3.68 & 4.34 & 4.02 & 4.50 & 5.65 & 4.88 \\
\hline 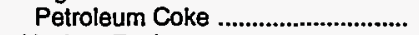 & - & - & - & - & - & - & - & - & - & - \\
\hline 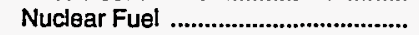 & - & - & - & - & - & - & - & - & - & - \\
\hline 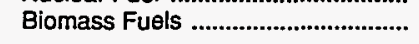 & - & - & - & - & - & - & - & - & - & - \\
\hline Primary Energy - Flve Sectors ${ }^{c}$.. & 1.65 & 2.72 & 5.74 & 6.13 & 4.94 & 5.37 & 4.75 & 5.59 & 6.04 & 5.51 \\
\hline
\end{tabular}

There are no direct fuel costs for hydroelectric, geothermal, centralized solar, or wind energy.

b Heavy oil includes Grade Nos. 4, 5, and 6, and residual fuel oils. Light oil includes Grade No. 2 heating oil, kerosene, and jet fuel.

c Biomass fuels are not included, except those consumed at electric utilities and those added to motor gasoline.

-No consumption, including cases where adjustments were made. See "Consumption Adjustments for Process Fuel and Intermediate Products."

Sources: Data sources, estimation procedures, and assumptions are described in the "Documentation" section of this report. 
(Million Dollars)

\begin{tabular}{|c|c|c|c|c|c|c|c|c|c|c|}
\hline Sector and Energy Source & 1970 & 1975 & 1980 & 1985 & 1986 & 1987 & 1988 & 1989 & 1990 & 1991 \\
\hline 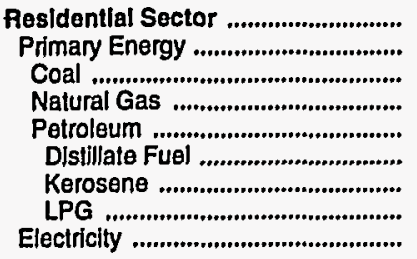 & $\begin{array}{r}76.0 \\
34.0 \\
.4 \\
14.3 \\
19.4 \\
5.7 \\
.1 \\
13.5 \\
42.0\end{array}$ & $\begin{array}{r}114.4 \\
51.1 \\
.5 \\
16.7 \\
33.9 \\
8.5 \\
25.3 \\
63.3\end{array}$ & $\begin{array}{r}223.8 \\
93.9 \\
.4 \\
33.1 \\
60.5 \\
30.7 \\
.4 \\
29.3 \\
129.9\end{array}$ & $\begin{array}{r}300.4 \\
119.7 \\
.3 \\
65.3 \\
54.1 \\
33.1 \\
1.8 \\
19.1 \\
180.7\end{array}$ & \begin{tabular}{r|}
293.9 \\
111.2 \\
.5 \\
55.6 \\
55.1 \\
33.0 \\
.9 \\
21.2 \\
182.7
\end{tabular} & $\begin{array}{r}276.4 \\
94.2 \\
.1 \\
45.2 \\
48.9 \\
23.1 \\
.5 \\
25.2 \\
182.3\end{array}$ & $\begin{array}{r}288.5 \\
93.5 \\
.1 \\
52.5 \\
41.0 \\
21.1 \\
.4 \\
19.5 \\
195.0\end{array}$ & $\begin{array}{r}337.7 \\
138.7 \\
.1 \\
55.0 \\
83.6 \\
28.1 \\
.3 \\
55.2 \\
199.0\end{array}$ & $\begin{array}{r}322.8 \\
123.8 \\
.1 \\
52.4 \\
71.3 \\
25.9 \\
.2 \\
45.2 \\
199.0\end{array}$ & $\begin{array}{r}314.2 \\
104.2 \\
.1 \\
55.4 \\
48.7 \\
24.0 \\
.2 \\
24.5 \\
210.0\end{array}$ \\
\hline 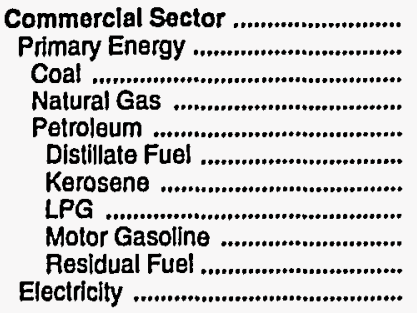 & $\begin{array}{r}35.7 \\
11.6 \\
.2 \\
7.2 \\
4.2 \\
1.9 \\
-\overline{1.5} \\
.8 \\
.1 \\
24.1\end{array}$ & $\begin{array}{r}49.4 \\
19.5 \\
.3 \\
11.4 \\
7.8 \\
3.1 \\
-\overline{3.0} \\
1.4 \\
.3 \\
29.9\end{array}$ & $\begin{array}{r}95.3 \\
44.4 \\
.4 \\
23.1 \\
20.9 \\
13.7 \\
-\overline{3} \\
3.3 \\
3.5 \\
.4 \\
51.0\end{array}$ & $\begin{array}{r}177.6 \\
66.2 \\
.5 \\
46.0 \\
19.7 \\
10.6 \\
. \\
3.7 \\
4.8 \\
111.5\end{array}$ & $\begin{array}{r}153.2 \\
56.5 \\
.6 \\
39.8 \\
16.1 \\
6.1 \\
4 \\
4.5 \\
5.4 \\
.1 \\
96.7\end{array}$ & $\begin{array}{r}152.4 \\
55.0 \\
.1 \\
34.6 \\
20.3 \\
8.8 \\
6 \\
6.5 \\
4.9 \\
.1 \\
97.4\end{array}$ & $\begin{array}{r}156.1 \\
50.1 \\
.1 \\
34.4 \\
15.6 \\
5.6 \\
4 \\
4.7 \\
4.9 \\
.3 \\
106.0\end{array}$ & $\begin{array}{r}160.6 \\
52.6 \\
.1 \\
35.2 \\
17.3 \\
5.2 \\
. \\
6.6 \\
5.3 \\
.3 \\
108.1\end{array}$ & $\begin{array}{r}167.6 \\
55.6 \\
.1 \\
35.9 \\
19.6 \\
5.0 \\
. \\
10.4 \\
3.8 \\
.4 \\
112.0\end{array}$ & $\begin{array}{r}170.1 \\
51.1 \\
.1 \\
38.3 \\
12.8 \\
4.1 \\
5.6 \\
2.5 \\
.5 \\
119.0\end{array}$ \\
\hline 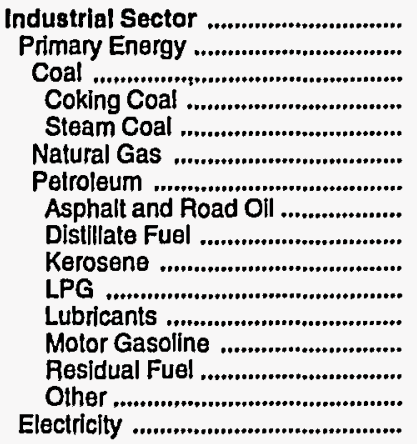 & $\begin{array}{r}57.5 \\
53.0 \\
- \\
2.2 \\
50.7 \\
4.5 \\
9.9 \\
1.3 \\
.4 \\
34.5 \\
.2 \\
-5\end{array}$ & $\begin{array}{r}107.6 \\
87.3 \\
1.2 \\
1 . \overline{1} \\
3.2 \\
3.5 \\
82.6 \\
11.9 \\
24.5 \\
4.5 \\
.9 \\
40.1 \\
.7 \\
\\
20.4\end{array}$ & \begin{tabular}{r|}
227.6 \\
183.9 \\
4.4 \\
$-\overline{4}$ \\
4.4 \\
11.0 \\
168.5 \\
16.1 \\
54.0 \\
.2 \\
17.5 \\
.3 \\
78.5 \\
2.0 \\
4 \\
4.8
\end{tabular} & $\begin{array}{r}210.4 \\
167.6 \\
11.7 \\
11 . \overline{7} \\
14.6 \\
141.3 \\
28.3 \\
66.6 \\
.2 \\
11.7 \\
.3 \\
33.8 \\
.4 \\
-\overline{4} \\
42.9\end{array}$ & $\begin{array}{r}195.9 \\
139.4 \\
8.2 \\
8 . \overline{8} \\
8.2 \\
11.9 \\
119.3 \\
21.6 \\
58.6 \\
.3 \\
16.6 \\
.3 \\
21.3 \\
.8 \\
- \\
56.5\end{array}$ & $\begin{array}{r}200.8 \\
136.7 \\
7.4 \\
-\overline{4} \\
7.4 \\
10.1 \\
119.2 \\
16.7 \\
55.4 \\
.1 \\
22.2 \\
.3 \\
23.7 \\
.8 \\
- \\
64.1\end{array}$ & $\begin{array}{r}196.2 \\
124.2 \\
6.1 \\
- \\
6.1 \\
14.8 \\
103.3 \\
20.8 \\
47.3 \\
.1 \\
12.6 \\
.3 \\
21.3 \\
.8 \\
-\overline{0} \\
72.0\end{array}$ & $\begin{array}{r}245.4 \\
169.4 \\
7.8 \\
-\overline{8} \\
7.8 \\
15.3 \\
146.2 \\
16.2 \\
54.1 \\
.2 \\
50.7 \\
.3 \\
24.2 \\
.6 \\
-\overline{6} \\
76.0\end{array}$ & $\begin{array}{r}259.7 \\
182.7 \\
6.9 \\
- \\
6.9 \\
22.0 \\
153.8 \\
17.0 \\
56.5 \\
.1 \\
55.4 \\
.3 \\
24.0 \\
.6 \\
- \\
77.0\end{array}$ & $\begin{array}{r}216.3 \\
136.3 \\
8.7 \\
\\
8.7 \\
15.4 \\
112.2 \\
16.5 \\
56.1 \\
.1 \\
16.0 \\
.3 \\
22.7 \\
.5 \\
-5 \\
80.0\end{array}$ \\
\hline 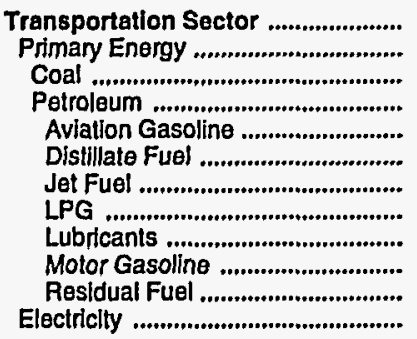 & $\begin{array}{r}137.2 \\
137.2 \\
137.2 \\
1.1 \\
7.1 \\
4.7 \\
.2 \\
4.7 \\
119.3 \\
-\end{array}$ & $\begin{array}{r}262.1 \\
262.1 \\
262.1 \\
1.3 \\
21.1 \\
11.9 \\
.5 \\
6.3 \\
220.8 \\
-\end{array}$ & $\begin{array}{r}581.3 \\
581.3 \\
581.3 \\
4.4 \\
82.0 \\
46.0 \\
1.1 \\
13.6 \\
434.3 \\
- \\
-\end{array}$ & $\begin{array}{r}559.3 \\
559.3 \\
559.3 \\
4.4 \\
91.8 \\
34.6 \\
.7 \\
15.2 \\
412.7 \\
- \\
-\end{array}$ & $\begin{array}{r}407.3 \\
407.3 \\
- \\
407.3 \\
3.6 \\
80.6 \\
12.9 \\
.8 \\
13.1 \\
296.3 \\
-\end{array}$ & $\begin{array}{r}431.0 \\
431.0 \\
-\overline{0} \\
431.0 \\
3.1 \\
88.7 \\
16.0 \\
1.3 \\
12.1 \\
310.0 \\
- \\
-\end{array}$ & $\begin{array}{r}458.6 \\
458.6 \\
- \\
458.6 \\
3.3 \\
87.2 \\
20.6 \\
.5 \\
13.4 \\
333.6 \\
- \\
-\end{array}$ & $\begin{array}{r}518.4 \\
518.4 \\
- \\
518.4 \\
3.7 \\
97.2 \\
26.3 \\
.5 \\
12.5 \\
378.2 \\
- \\
-\end{array}$ & $\begin{array}{r}590.0 \\
590.0 \\
- \\
590.0 \\
4.4 \\
121.5 \\
36.8 \\
.8 \\
13.0 \\
413.5 \\
- \\
-\end{array}$ & $\begin{array}{r}569.4 \\
569.4 \\
-\overline{569.4} \\
2.7 \\
121.0 \\
29.8 \\
.4 \\
13.4 \\
402.1 \\
-\end{array}$ \\
\hline $\begin{array}{l}\text { Total Energy ..................................... } \\
\text { Primary Energy - Four Sectors ..... } \\
\text { Electricity ........................................ }\end{array}$ & $\begin{array}{r}306.4 \\
235.8 \\
70.6\end{array}$ & $\begin{array}{l}533.5 \\
419.9 \\
113.6\end{array}$ & $\begin{array}{r}1,128.1 \\
903.5 \\
224.7\end{array}$ & $\begin{array}{r}1.247 .7 \\
912.7 \\
335.0\end{array}$ & $\begin{array}{r}1,050.2 \\
714.3 \\
335.9\end{array}$ & $\begin{array}{r}1,060.6 \\
716.9 \\
343.8\end{array}$ & $\begin{array}{r}1,099.5 \\
726.5 \\
373.0\end{array}$ & $\begin{array}{r}1,262.1 \\
879.0 \\
383.1\end{array}$ & $\begin{array}{r}1,340.1 \\
952.1 \\
388.0\end{array}$ & $\begin{array}{r}1,270.0 \\
861.0 \\
409.0\end{array}$ \\
\hline 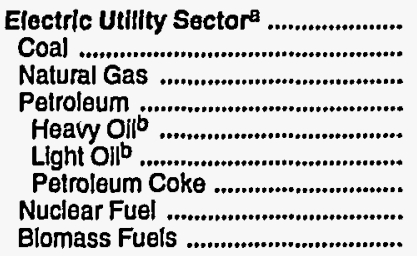 & $\begin{array}{l}4.7 \\
1.8 \\
1.5 \\
1.5 \\
1.2 \\
.3 \\
- \\
- \\
-\end{array}$ & $\begin{array}{r}16.0 \\
11.0 \\
2.1 \\
2.9 \\
2.0 \\
.9 \\
- \\
- \\
-\end{array}$ & $\begin{array}{r}28.7 \\
25.8 \\
.5 \\
2.4 \\
.2 \\
2.2 \\
- \\
- \\
-\end{array}$ & $\begin{array}{r}36.2 \\
34.8 \\
.1 \\
1.3 \\
1.3 \\
- \\
-\end{array}$ & $\begin{array}{r}31.5 \\
30.5 \\
.1 \\
.8 \\
.8 \\
- \\
- \\
-\end{array}$ & $\begin{array}{r}13.7 \\
12.9 \\
.2 \\
.6 \\
. \\
.5 \\
- \\
- \\
-\end{array}$ & $\begin{array}{r}38.5 \\
36.7 \\
.5 \\
1.3 \\
.1 \\
1.1 \\
- \\
- \\
-\end{array}$ & $\begin{array}{r}36.0 \\
34.7 \\
.3 \\
.9 \\
-9 \\
.9 \\
- \\
-\end{array}$ & $\begin{array}{r}34.4 \\
32.8 \\
.6 \\
1.1 \\
- \\
1.1 \\
- \\
- \\
-\end{array}$ & $\begin{array}{r}36.4 \\
35.1 \\
.3 \\
1.0 \\
1.0 \\
- \\
-\end{array}$ \\
\hline Primary Energy - Flve Sectorsc ... & 240.5 & 435.9 & 932.1 & 949.0 & 745.8 & 730.6 & 765.0 & 915.0 & 986.5 & 897.4 \\
\hline
\end{tabular}

a There are no direct fuel costs for hydroelectric, geothermal, centralized solar, or wind energy.

beavy oil includes Grade Nos. 4, 5, and 6, and residual fuel oils. Light oil includes Grade No. 2 heating oil, kerosene, and jet fuel.

c Biomass fuels are not included, except those consumed at electric utilities and those added to motor gasoline.

- No consumption, including cases where adjustments were made. See "Consumption Adjustments for Process Fuel and Intermediate Products."

Value less than 0.05 million dollars.

Note; Totals may not equal sum of components due to independent rounding.

Sources: Dala sources, estimation procedures, and assumptions are described in the "Documentation" section of this report. 


\section{T Energy Price and Expenditure Estimates by Source, Tennessee \\ E 1970, 1975, 1980, and 1985-1991}

\begin{tabular}{|c|c|c|c|c|c|c|c|c|c|c|}
\hline Energy Source & 1970 & 1975 & 1980 & 1985 & 1986 & 1987 & 1988 & 1989 & 1990 & 1991 \\
\hline & \multicolumn{10}{|c|}{ Prices in Dollars per Million Btu } \\
\hline 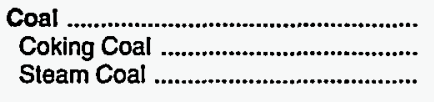 & $\begin{array}{r}0.26 \\
.38 \\
.25\end{array}$ & $\begin{array}{r}0.91 \\
1.60 \\
.91\end{array}$ & $\begin{array}{l}1.54 \\
1.81 \\
1.54\end{array}$ & $\begin{array}{l}1.55 \\
1.93 \\
1.55\end{array}$ & $\begin{array}{l}1.44 \\
1.75 \\
1.44\end{array}$ & $\begin{array}{l}1.38 \\
1.63 \\
1.38\end{array}$ & $\begin{array}{l}1.35 \\
1.70 \\
1.35\end{array}$ & $\begin{array}{l}1.36 \\
1.74 \\
1.35\end{array}$ & $\begin{array}{l}1.35 \\
1.83 \\
1.35\end{array}$ & $\begin{array}{l}1.28 \\
1.80 \\
1.28\end{array}$ \\
\hline 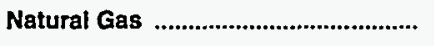 & .54 & .93 & 2.69 & 4.46 & 4.20 & 3.69 & 3.75 & 3.87 & 3.98 & 3.91 \\
\hline $\begin{array}{l}\text { Petroleum } \\
\text { Asphalt and Road Oil } \\
\text { Aviation Gasoline .................................. } \\
\text { Distillate Fuel } \\
\text { Jet Fuel . } \\
\text { Kerosene } \\
\text { LPG } \\
\text { Lubricants } \\
\text { Motor Gasoline } \\
\text { Residual Fuel } \\
\text { Other }\end{array}$ & $\begin{array}{r}2.06 \\
.67 \\
2.17 \\
1.11 \\
.73 \\
1.19 \\
1.92 \\
5.08 \\
2.84 \\
.36 \\
.81\end{array}$ & $\begin{array}{l}3.76 \\
1.79 \\
3.45 \\
2.68 \\
2.03 \\
2.93 \\
3.44 \\
7.48 \\
4.58 \\
1.78 \\
2.24\end{array}$ & $\begin{array}{r}8.37 \\
3.56 \\
9.02 \\
6.80 \\
6.39 \\
7.21 \\
6.42 \\
14.36 \\
9.89 \\
3.36 \\
6.24\end{array}$ & $\begin{array}{r}7.89 \\
4.76 \\
9.99 \\
6.44 \\
5.83 \\
6.99 \\
8.97 \\
17.61 \\
8.85 \\
4.80 \\
6.79\end{array}$ & $\begin{array}{r}6.38 \\
4.89 \\
8.41 \\
5.85 \\
3.81 \\
5.35 \\
8.65 \\
15.59 \\
7.01 \\
2.83 \\
4.06\end{array}$ & $\begin{array}{r}6.66 \\
3.24 \\
7.55 \\
6.23 \\
4.01 \\
5.48 \\
8.70 \\
12.70 \\
7.44 \\
2.88 \\
4.74\end{array}$ & $\begin{array}{r}6.58 \\
3.05 \\
7.41 \\
6.00 \\
3.82 \\
5.55 \\
8.91 \\
14.61 \\
7.43 \\
2.64 \\
3.91\end{array}$ & $\begin{array}{r}7.19 \\
2.86 \\
8.28 \\
6.63 \\
4.34 \\
6.21 \\
9.40 \\
13.30 \\
8.25 \\
2.68 \\
4.69\end{array}$ & $\begin{array}{r}8.22 \\
2.94 \\
9.32 \\
7.89 \\
5.58 \\
6.93 \\
10.88 \\
13.40 \\
9.40 \\
3.14 \\
5.64\end{array}$ & $\begin{array}{r}7.84 \\
3.30 \\
8.71 \\
7.34 \\
4.82 \\
6.38 \\
9.69 \\
15.42 \\
9.00 \\
2.34 \\
4.92\end{array}$ \\
\hline 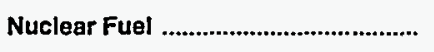 & - & - & .38 & .78 & - & - & .83 & .89 & .84 & .85 \\
\hline Blomass Fuels at Utilities .................. & - & - & - & - & - & - & - & - & - & - \\
\hline Primary Energy - Five Sectors ${ }^{b}$.... & 1.02 & 2.13 & 4.43 & 4.26 & 3.88 & 3.95 & 3.75 & 3.87 & 4.22 & 3.98 \\
\hline $\begin{array}{l}\text { Electric Utility Fuelc ........................... } \\
\text { Electricity Purchased by End Users }\end{array}$ & 2.85 & $\begin{array}{r}.89 \\
5.83\end{array}$ & $\begin{array}{r}1.57 \\
10.67\end{array}$ & $\begin{array}{r}1.41 \\
14.65\end{array}$ & $\begin{array}{r}1.43 \\
15.59\end{array}$ & $\begin{array}{r}1.38 \\
15.98\end{array}$ & $\begin{array}{r}1.30 \\
16.19\end{array}$ & $\begin{array}{r}1.23 \\
15.91\end{array}$ & $\begin{array}{r}1.24 \\
15.59\end{array}$ & $\begin{array}{r}1.15 \\
15.29\end{array}$ \\
\hline \multirow[t]{2}{*}{ 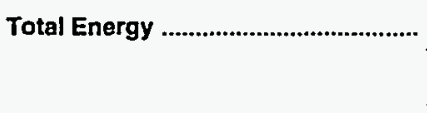 } & 1.71 & 3.54 & 7.30 & 8.16 & 7.52 & 7.67 & 7.72 & 8.00 & 8.55 & 8.30 \\
\hline & \multicolumn{10}{|c|}{ Expendltures in Millions of Dollars } \\
\hline 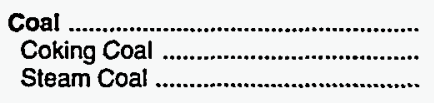 & $\begin{array}{r}103.1 \\
2.5 \\
100.7\end{array}$ & $\begin{array}{r}431.0 \\
8.9 \\
422.1\end{array}$ & $\begin{array}{r}888.2 \\
5.0 \\
883.2\end{array}$ & $\begin{array}{r}929.3 \\
8.0 \\
921.3\end{array}$ & $\begin{array}{r}873.1 \\
5.1 \\
867.9\end{array}$ & $\begin{array}{r}822.3 \\
3.3 \\
819.0\end{array}$ & $\begin{array}{r}822.4 \\
3.6 \\
818.8\end{array}$ & $\begin{array}{r}765.2 \\
3.6 \\
761.6\end{array}$ & $\begin{array}{r}812.8 \\
3.3 \\
809.6\end{array}$ & $\begin{array}{r}725.0 \\
.9 \\
724.2\end{array}$ \\
\hline 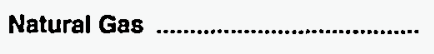 & 123.6 & 186.1 & 570.9 & 813.4 & 742.6 & 689.5 & 747.2 & 797.6 & 804.8 & 829.9 \\
\hline 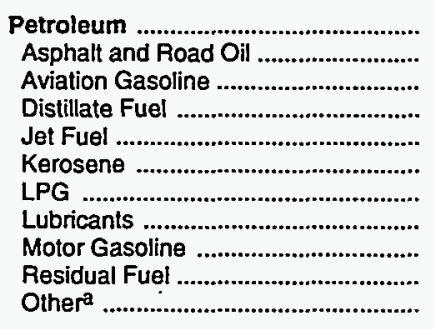 & $\begin{array}{r}831.6 \\
16.2 \\
1.3 \\
70.8 \\
13.6 \\
27.9 \\
23.0 \\
25.4 \\
625.1 \\
1.1 \\
27.2\end{array}$ & $\begin{array}{r}1,870.9 \\
44.8 \\
1.2 \\
272.8 \\
45.1 \\
38.0 \\
48.8 \\
60.3 \\
1,292.7 \\
4.3 \\
62.9\end{array}$ & $\begin{array}{r}4,365.1 \\
79.8 \\
13.2 \\
759.3 \\
149.8 \\
62.7 \\
65.3 \\
108.0 \\
2,853.4 \\
28.2 \\
245.4\end{array}$ & $\begin{array}{r}4,235.0 \\
139.3 \\
7.8 \\
835.9 \\
160.1 \\
43.9 \\
73.1 \\
120.6 \\
2,698.2 \\
9.6 \\
146.6\end{array}$ & $\begin{array}{r}3,608.1 \\
134.9 \\
8.5 \\
771.9 \\
127.8 \\
14.5 \\
83.7 \\
104.4 \\
2,221.8 \\
7.1 \\
133.4\end{array}$ & $\begin{array}{r}3,875.3 \\
98.3 \\
7.1 \\
818.9 \\
129.0 \\
20.9 \\
82.5 \\
96.2 \\
2,460.3 \\
4.4 \\
157.7\end{array}$ & $\begin{array}{r}3,702.6 \\
81.8 \\
6.8 \\
823.4 \\
91.3 \\
31.5 \\
100.4 \\
106.7 \\
2,316.9 \\
5.5 \\
138.4\end{array}$ & $\begin{array}{r}4,137.5 \\
108.1 \\
7.6 \\
901.5 \\
106.8 \\
28.4 \\
118.8 \\
99.6 \\
2,600.6 \\
5.7 \\
160.4\end{array}$ & $\begin{array}{r}4,657.8 \\
113.0 \\
8.2 \\
1,097.0 \\
131.7 \\
17.2 \\
113.2 \\
103.3 \\
2,846.3 \\
4.6 \\
223.3\end{array}$ & $\begin{array}{r}4,271.2 \\
117.3 \\
6.4 \\
966.9 \\
93.2 \\
12.4 \\
112.0 \\
106.3 \\
2,655.1 \\
4.0 \\
197.8\end{array}$ \\
\hline 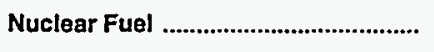 & - & - & 2.1 & 81.1 & - & - & 34.9 & 149.2 & 125.9 & 151.1 \\
\hline Blomass Fuels at Utilities .................. & - & - & - & - & - & - & - & - & - & - \\
\hline Primary Energy - Five Sectors ${ }^{b} \ldots$ & $1,058.3$ & $2,487.9$ & $5,826.4$ & $6,058.7$ & $5,223.7$ & $5,387.1$ & $5,307.1$ & $5,849.5$ & $6,401.4$ & $5,977.3$ \\
\hline $\begin{array}{l}\text { Electric Utility Fuelc } \\
\text { Electricity Purchased by End Use.............. }\end{array}$ & $\begin{array}{r}-80.9 \\
504.6\end{array}$ & $\begin{array}{r}-376.4 \\
1,357.0\end{array}$ & $\begin{array}{r}-804.8 \\
2,656.5\end{array}$ & $\begin{array}{r}-846.9 \\
3,409.7\end{array}$ & $\begin{array}{r}-716.0 \\
3,559.5\end{array}$ & $\begin{array}{r}-682.9 \\
3,754.3\end{array}$ & $\begin{array}{r}-718.6 \\
3,975.4\end{array}$ & $\begin{array}{r}-773.6 \\
4,030.7\end{array}$ & $\begin{array}{r}-803.6 \\
4,054.9\end{array}$ & $\begin{array}{r}-745.1 \\
4,041.4\end{array}$ \\
\hline 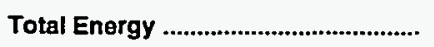 & $1,482.0$ & $3,468.5$ & $7,678.1$ & $8,621.5$ & $8,067.2$ & $8,458.4$ & $8,563.9$ & $9,106.7$ & $9,652.7$ & $9,273.6$ \\
\hline
\end{tabular}

Includes petroleum coke used at electric utilities.

biomass fuels are not included, except those consumed at electric utilities and those added to motor gasoline.

c There are no direct fuel costs for hydroelectric, geothermal, centraiized solar, or wind energy.

- No consumption, including cases where adjustments were made. See "Consumption Adjustments for Process Fuel and Intermediate Products."

Note: Expenditure totals may not equal sum of components due to independent rounding.

Sources: Data sources, estimation procedures, and assumptions are described in the "Documentation" section of this report. 
Energy Price Estimates by Sector, Tennessee $1970,1975,1980$, and 1985-1991

(Dollars per Million Btu)

\begin{tabular}{|c|c|c|c|c|c|c|c|c|c|c|}
\hline Sector and Energy Source & 1970 & 1975 & 1980 & 1985 & 1986 & 1987 & 1988 & 1989 & 1990 & 1991 \\
\hline 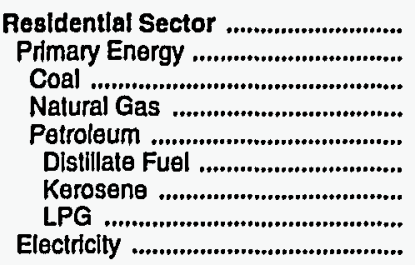 & $\begin{array}{r}2.15 \\
1.15 \\
.74 \\
.89 \\
1.82 \\
1.24 \\
1.62 \\
2.16 \\
3.34\end{array}$ & $\begin{array}{l}4.46 \\
1.93 \\
1.75 \\
1.25 \\
3.56 \\
2.49 \\
3.38 \\
3.84 \\
6.62\end{array}$ & $\begin{array}{r}7.79 \\
3.73 \\
1.97 \\
2.85 \\
7.95 \\
6.89 \\
9.09 \\
7.65 \\
10.43\end{array}$ & $\begin{array}{r}10.97 \\
5.44 \\
1.85 \\
4.96 \\
7.95 \\
6.59 \\
7.09 \\
9.23 \\
14.28\end{array}$ & $\begin{array}{r}11.63 \\
5.24 \\
1.67 \\
4.81 \\
7.76 \\
5.30 \\
5.71 \\
8.96 \\
15.26\end{array}$ & $\begin{array}{r}11.96 \\
4.99 \\
1.79 \\
4.53 \\
7.55 \\
5.25 \\
5.65 \\
9.09 \\
16.03\end{array}$ & $\begin{array}{r}12.11 \\
5.00 \\
1.52 \\
4.51 \\
7.67 \\
5.53 \\
5.95 \\
9.06 \\
16.74\end{array}$ & $\begin{array}{r}12.11 \\
5.33 \\
1.61 \\
4.68 \\
8.63 \\
5.90 \\
6.35 \\
10.25 \\
16.65\end{array}$ & $\begin{array}{r}12.53 \\
5.67 \\
1.77 \\
4.94 \\
10.19 \\
6.59 \\
7.09 \\
11.90 \\
16.68\end{array}$ & $\begin{array}{r}12.36 \\
5.63 \\
1.75 \\
5.02 \\
9.28 \\
6.12 \\
6.58 \\
10.57 \\
16.54\end{array}$ \\
\hline 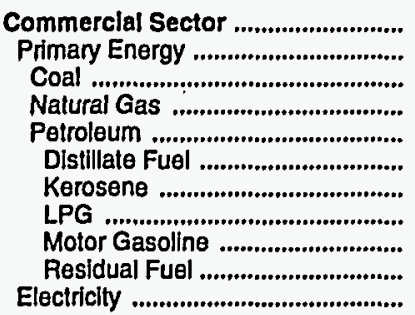 & $\begin{array}{r}1.87 \\
.75 \\
.35 \\
.70 \\
1.46 \\
1.06 \\
.78 \\
1.27 \\
2.84 \\
.42 \\
4.97\end{array}$ & $\begin{array}{l}3.48 \\
1.38 \\
1.17 \\
1.09 \\
2.88 \\
2.29 \\
2.32 \\
2.39 \\
4.58 \\
1.77 \\
8.27\end{array}$ & $\begin{array}{r}7.97 \\
3.57 \\
1.39 \\
2.95 \\
7.05 \\
6.49 \\
6.16 \\
4.96 \\
9.89 \\
3.44 \\
13.29\end{array}$ & $\begin{array}{r}8.95 \\
5.04 \\
1.60 \\
4.75 \\
6.04 \\
5.66 \\
6.78 \\
8.66 \\
8.85 \\
4.80 \\
17.05\end{array}$ & $\begin{array}{r}9.40 \\
4.49 \\
1.54 \\
4.53 \\
4.63 \\
3.80 \\
4.55 \\
8.30 \\
7.01 \\
2.83 \\
17.96\end{array}$ & $\begin{array}{r}9.31 \\
4.29 \\
1.43 \\
4.20 \\
5.09 \\
4.12 \\
5.16 \\
8.25 \\
7.44 \\
2.88 \\
17.67\end{array}$ & $\begin{array}{r}9.30 \\
4.30 \\
1.40 \\
4.25 \\
5.22 \\
3.93 \\
4.90 \\
8.71 \\
7.43 \\
2.62 \\
18.03\end{array}$ & $\begin{array}{r}9.94 \\
4.45 \\
1.40 \\
4.35 \\
6.17 \\
4.43 \\
5.75 \\
8.23 \\
8.25 \\
2.69 \\
18.03\end{array}$ & $\begin{array}{r}10.62 \\
4.76 \\
1.40 \\
4.64 \\
6.98 \\
4.96 \\
6.49 \\
9.36 \\
9.40 \\
3.16 \\
18.02\end{array}$ & $\begin{array}{r}10.51 \\
4.69 \\
1.42 \\
4.61 \\
6.45 \\
4.34 \\
5.64 \\
8.34 \\
9.00 \\
2.48 \\
17.99\end{array}$ \\
\hline 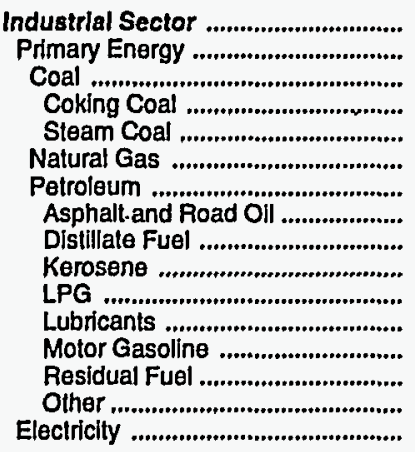 & $\begin{array}{r}.93 \\
.54 \\
.35 \\
.38 \\
.35 \\
.38 \\
.86 \\
.67 \\
.72 \\
.78 \\
1.27 \\
5.08 \\
2.84 \\
.36 \\
.81 \\
2.05\end{array}$ & $\begin{array}{r}2.56 \\
1.38 \\
1.22 \\
1.60 \\
1.17 \\
.73 \\
2.28 \\
1.79 \\
2.11 \\
2.32 \\
2.39 \\
7.48 \\
4.58 \\
1.89 \\
2.24 \\
4.87\end{array}$ & $\begin{array}{r}5.10 \\
3.36 \\
1.41 \\
1.81 \\
1.39 \\
2.54 \\
5.51 \\
3.56 \\
5.50 \\
6.16 \\
4.96 \\
14.36 \\
9.89 \\
3.36 \\
6.24 \\
9.71\end{array}$ & $\begin{array}{r}6.81 \\
3.87 \\
1.61 \\
1.93 \\
1.60 \\
4.11 \\
6.37 \\
4.76 \\
5.91 \\
6.78 \\
8.66 \\
17.61 \\
8.85 \\
4.80 \\
6.79 \\
14.22\end{array}$ & $\begin{array}{r}6.54 \\
3.33 \\
1.55 \\
1.75 \\
1.54 \\
3.77 \\
4.87 \\
4.89 \\
3.96 \\
4.55 \\
8.30 \\
15.59 \\
7.01 \\
2.83 \\
4.06 \\
15.12\end{array}$ & $\begin{array}{r}6.35 \\
3.04 \\
1.43 \\
1.63 \\
1.43 \\
3.05 \\
4.68 \\
3.24 \\
4.49 \\
5.16 \\
8.25 \\
12.70 \\
7.44 \\
2.88 \\
4.74 \\
15.39\end{array}$ & $\begin{array}{r}6.36 \\
2.95 \\
1.40 \\
1.70 \\
1.40 \\
3.16 \\
4.38 \\
3.05 \\
4.26 \\
4.90 \\
8.71 \\
14.61 \\
7.43 \\
2.62 \\
3.91 \\
15.15\end{array}$ & $\begin{array}{r}6.20 \\
3.06 \\
1.41 \\
1.74 \\
1.40 \\
3.27 \\
4.53 \\
2.86 \\
5.01 \\
5.75 \\
8.23 \\
13.30 \\
8.25 \\
2.69 \\
4.69 \\
14.51\end{array}$ & $\begin{array}{r}6.18 \\
3.32 \\
1.41 \\
1.83 \\
1.40 \\
3.29 \\
5.09 \\
2.94 \\
5.65 \\
6.49 \\
9.36 \\
13.40 \\
9.40 \\
3.16 \\
5.64 \\
13.74\end{array}$ & $\begin{array}{r}5.96 \\
3.18 \\
1.42 \\
1.80 \\
1.42 \\
3.12 \\
4.86 \\
3.30 \\
4.91 \\
5.64 \\
8.34 \\
15.42 \\
9.00 \\
2.48 \\
4.92 \\
13.21\end{array}$ \\
\hline 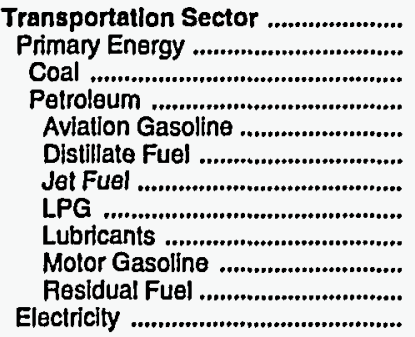 & $\begin{array}{r}2.49 \\
2.49 \\
.35 \\
2.49 \\
2.17 \\
1.28 \\
.73 \\
1.27 \\
5.08 \\
2.84 \\
.42 \\
4.97\end{array}$ & $\begin{array}{l}4.19 \\
4.19 \\
1.17 \\
4.19 \\
3.45 \\
3.02 \\
2.03 \\
2.39 \\
7.48 \\
4.58 \\
1.67 \\
8.27\end{array}$ & $\begin{array}{r}9.20 \\
9.20 \\
-\overline{9} \\
9.20 \\
9.02 \\
7.25 \\
6.39 \\
4.96 \\
14.36 \\
9.89 \\
3.45 \\
13.29\end{array}$ & $\begin{array}{r}8.29 \\
8.29 \\
- \\
8.29 \\
9.99 \\
6.73 \\
5.83 \\
8.66 \\
17.61 \\
8.85 \\
- \\
17.05\end{array}$ & $\begin{array}{r}6.73 \\
6.73 \\
- \\
6.73 \\
8.41 \\
6.47 \\
3.81 \\
8.30 \\
15.59 \\
7.01 \\
- \\
17.96\end{array}$ & $\begin{array}{r}7.10 \\
7.10 \\
- \\
7.10 \\
7.55 \\
6.75 \\
4.01 \\
8.25 \\
12.70 \\
7.44 \\
1.92 \\
19.96\end{array}$ & $\begin{array}{r}7.07 \\
7.07 \\
- \\
7.07 \\
7.41 \\
6.49 \\
3.82 \\
8.71 \\
14.61 \\
7.43 \\
3.01 \\
20.34\end{array}$ & $\begin{array}{r}7.76 \\
7.76 \\
\overrightarrow{7} \\
7.76 \\
8.28 \\
6.96 \\
4.34 \\
8.23 \\
13.30 \\
8.25 \\
2.37 \\
19.95\end{array}$ & $\begin{array}{r}8.96 \\
8.96 \\
- \\
8.96 \\
9.32 \\
8.36 \\
5.58 \\
9.36 \\
13.40 \\
9.40 \\
2.22 \\
20.12\end{array}$ & $\begin{array}{r}8.56 \\
8.56 \\
- \\
8.56 \\
8.71 \\
7.84 \\
4.82 \\
8.34 \\
15.42 \\
9.00 \\
1.71 \\
20.09\end{array}$ \\
\hline $\begin{array}{l}\text { Total Energy .................................... } \\
\text { Primary Energy - Four Sectors ..... } \\
\text { Electricity ........................................... }\end{array}$ & $\begin{array}{l}1.71 \\
1.42 \\
2.85\end{array}$ & $\begin{array}{l}3.54 \\
2.82 \\
5.83\end{array}$ & $\begin{array}{r}7.30 \\
6.25 \\
10.67\end{array}$ & $\begin{array}{r}8.16 \\
6.32 \\
14.65\end{array}$ & $\begin{array}{r}7.52 \\
5.34 \\
15.59\end{array}$ & $\begin{array}{r}7.67 \\
5.42 \\
15.98\end{array}$ & $\begin{array}{r}7.72 \\
5.31 \\
16.19\end{array}$ & $\begin{array}{r}8.00 \\
5.73 \\
15.91\end{array}$ & $\begin{array}{r}8.55 \\
6.44 \\
15.59\end{array}$ & $\begin{array}{r}8.30 \\
6.13 \\
15.29\end{array}$ \\
\hline 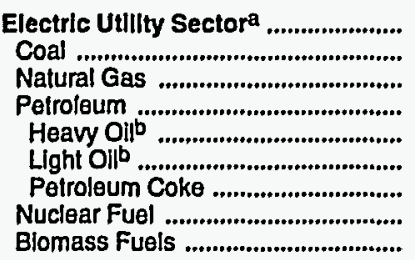 & $\begin{array}{l}.23 \\
.23 \\
.25 \\
- \\
- \\
- \\
- \\
-\end{array}$ & $\begin{array}{r}.89 \\
.87 \\
-\overline{19} \\
\overline{-} \\
2.19 \\
- \\
-\end{array}$ & $\begin{array}{r}1.57 \\
1.56 \\
2.33 \\
6.39 \\
- \\
6.39 \\
-\overline{3} \\
-\end{array}$ & $\begin{array}{r}1.41 \\
1.54 \\
- \\
5.85 \\
\overline{5} \\
5.85 \\
- \\
.78 \\
-\end{array}$ & $\begin{array}{r}1.43 \\
1.42 \\
- \\
3.37 \\
- \\
3.37 \\
- \\
- \\
-\end{array}$ & $\begin{array}{r}1.38 \\
1.37 \\
- \\
3.93 \\
- \\
3.93 \\
- \\
- \\
-\end{array}$ & $\begin{array}{r}1.30 \\
1.33 \\
2.39 \\
3.51 \\
- \\
3.51 \\
- \\
.83 \\
-\end{array}$ & $\begin{array}{r}1.23 \\
1.34 \\
2.74 \\
3.95 \\
-\overline{3.95} \\
-\overline{89} \\
-\end{array}$ & $\begin{array}{r}1.24 \\
1.34 \\
2.72 \\
5.61 \\
- \\
5.61 \\
- \\
.84 \\
-\end{array}$ & $\begin{array}{r}1.15 \\
1.25 \\
2.44 \\
4.98 \\
\overline{-} \\
4.98 \\
\overline{-} \\
.85 \\
-\end{array}$ \\
\hline Primary Energy - Five Sectorsc .. & 1.02 & 2.13 & 4.43 & 4.26 & 3.88 & 3.95 & 3.75 & 3.87 & 4.22 & 3.98 \\
\hline
\end{tabular}

a There are no direct fuel costs for hydroelectric, geothermal, centralized solar, or wind energy.

b Heavy oll includes Grade Nos. 4, 5, and 6, and residual fuel oils. Light oil includes Grade No. 2 heating oil, kerosene, and jet fuel.

c Biomass fuels are not included, except those consumed at electric utilities and those added to motor gasoline.

-No consumption, including cases where adjustments were made. See "Consumption Adjustments for Process Fuel and Intermediate Products."

Sources: Data sources, estimation procedures, and assumptions are described in the "Documentation" section of this report. 


\section{T Energy Expenditure Estimates by Sector, Tennessee \\ E $1970,1975,1980$, and 1985-1991 \\ $N$ (Million Dollars)}

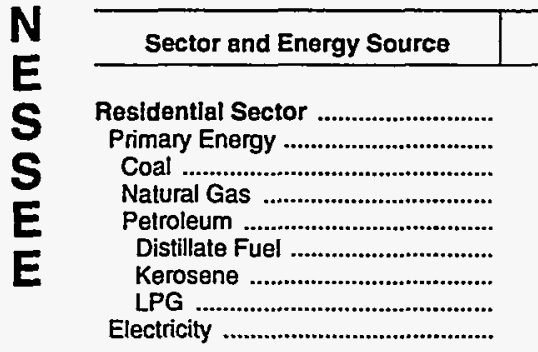

Commercial Sector ..

Primary Energy

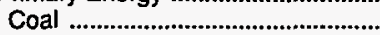

Naturai Gas

Petroleum

Distillate Fuel

Kerosene

LPG .

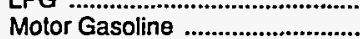

Residual Fuel

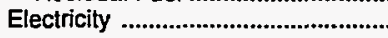

Industrial Sector ................................

Primary Energy

Coal

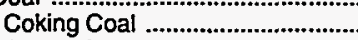

Steam Coal

Natural Gas

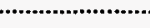

Asphalt and Road Oil .....................

Distillate Fuel .

Kerosene

LPG .

Lubricants

Motor Gasoline

Residual Fuel

Eleclricity ..........................................

Transportation Sector ......................

Primary Energy ...................................

Coal

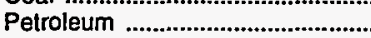

Aviation Gasoline

Distillate Fuel

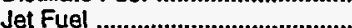

LPG .

Lubricants

Motor Gasoline .......................

Residual Fuet

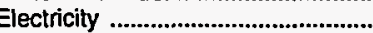

Total Energy .....................................

Primary Energy - Four Sectors ....

Electricity .............................................

Electrlc Utillty Sector ${ }^{a}$.

Coal

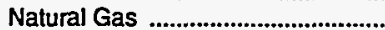

Petroleum

Heary Oilb

Petroleum Coke

Nuclear Fuel ...

Biomass Fuels

\begin{tabular}{|l|l|l|l|l|}
\hline 1970 & 1975 & 1980 & 1985 & 1986 \\
\hline
\end{tabular}

\begin{tabular}{|l|l|l|l|}
1975 & 1980 & 1985 & 1986 \\
\hline
\end{tabular}

$\begin{array}{lllll}288.7 & 650.3 & 1,149.1 & 1,529.1 & 1,610.8\end{array}$

84.5

129.7

216.5

284.5

$\begin{array}{llll}42.5 & 56.8 & 129.8 & 202.0\end{array}$

$38.7 \quad 68.1$

$1.2 \quad 3.4$

$18.6 \quad 25.3$

$18.9 \quad 39.5$

$204.2 \quad 520.6$

129.8
82.8

12.4

28.3

42.2

932.6

$\begin{array}{lll}153.4 & 289.4 & 853.7\end{array}$

$\begin{array}{lll}45.6 & 79.4 & 209.2\end{array}$

$\begin{array}{rrr}2.9 & 5.8 & 5.1 \\ 30.4 & 47.9 & 132.1\end{array}$

$\begin{array}{lll}12.2 & 25.7 & 72.0\end{array}$

$\begin{array}{ll}2.6 & 7.9\end{array}$

38.4

3.4
3.6

4.8
24.2

24.2

$\begin{array}{rr}2.0 & 4.3 \\ 5.9 & 10.1\end{array}$

$107.8 \quad 210.0$

644.5

202.0
79.8

9.9
29.7

$1,244.6$

263.2

1.1
199.9

62.2

5.1

46.5
, 347.6

924.1

855.1

350.9

4.3
213.2

133.4

101.7

6.4
6.7

15.6

2.9

$\begin{array}{llll}340.0 & 975.9 & 2,067.7 & 2,680.1\end{array}$

259.1

1.9
199.0

58.2

31.2

2.3

7.6
14.8

2.3

595.9

147.4

349.5

988.3

$1,088.3$

$2,569.9$

954.0

158.6

5.1
153.5

5.0
89.4

52.0

81.4

306.4

8.0

156.6

398.1

343.7

451.6

134.9

$\begin{array}{rr}44.8 & 79.8 \\ 57.6 & 136.3\end{array}$

139.3

119.5

30.8

3.9

21.0

54.9

29.9

1.9

2.3

$\begin{array}{rr}27.2 & 62.9 \\ 192.6 & 626.3\end{array}$

$1,079.4$

$1,591.8$

$699.9 \quad 1,553.0$

$699.9 \quad 1,553.0$

$3,607.6$

$3,488.2$

$3,607.6$

$3,488.2$

$699.8 \quad 1,553.0$

$1.3 \quad 1.2$

$3,607 . \overline{6}$

13.2

$3,488 . \overline{2}$

7.8
596.7

160.1

45.1

149.8

160.1
5.2

$\begin{array}{rr}.5 & 1.1 \\ 15.1 & 36 . \\ 615.7 & 1,279 .\end{array}$

58.9

65.7
652.7

84.5

47.5

21.3

$1,616.0$

$3,031.4$

$3,031.4$

$3,302.3$

$3,031.4$

8.5
646.5

127.8

6.1

56.9

7.1

129.0

3.6

52.4

-

$2,422.0$

\section{$1,482.0 \quad 3,468.5$}

\section{$7,678.1$}

$8,621.5$

$8,067.2$
$4,507.8$

$8,458.4$

$4,704.2$

$3,754.3$

8,563.9

$3,409.7$

$3,559.5$

$9,106.7$

$5,076.0$

$4,030.7$

$9,652.7$

$5,597.8$

$4,054.9$

$5,232$.

376.4

804.8

$846.9 \quad 716.0$

$\begin{array}{rr}682.9 & 718.6 \\ 677.8 & 675.7 \\ - & .6\end{array}$

$\begin{array}{lll}773.6 & 803.6 & 745.1\end{array}$

$616.1 \quad 668.5 \quad 585.5$

$\begin{array}{rrr}711.4 & 677.8 & 675.7 \\ 4 . \overline{5} & 5 . \overline{1} & 7.5\end{array}$

$\overline{-}$

$5 . \overline{1}$

$7 . \overline{5}$
$34 . \overline{9}$

8.2

1.6
7.6

.5
7.9

70

149.2

125.

$151 . \overline{1}$

Primary Energy - Flve Sectorsc ..

$1,058.3$

$2,487.9$

$5,826.4$

$6,058.7$

$5,223.7$

$5,387.1$

$5,307.1$

$5,849.5$

$6,401.4$

$5,977.3$

a There are no direct fuel costs for hydroelectric, geothermal, centralized solar, or wind energy.

b Heavy oil includes Grade Nos. 4, 5, and 6, and residual fuel oils. Light oil includes Grade No. 2 heating oil, kerosene, and jet fuel.

c Biomass fuels are not included, except those consumed at electric utilities and those added to motor gasoline.

- No consumption, including cases where adjustments were made. See "Consumption Adjustments for Process Fuel and Intermediate Products."

- Value less than 0.05 million dollars.

Note: Totals may not equal sum of components due to independent rounding.

Sources: Data sources, estimation procedures, and assumptions are described in the "Documentation" section of this report. 


\begin{tabular}{|c|c|c|c|c|c|c|c|c|c|c|}
\hline Energy Source & 1970 & 1975 & 1980 & 1985 & 1986 & 1987 & 1988 & 1989 & 1990 & 1991 \\
\hline & \multicolumn{10}{|c|}{ Prices in Dollars per Million Btu } \\
\hline 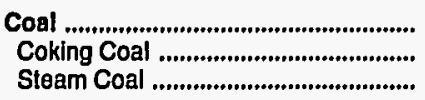 & $\begin{array}{r}0.39 \\
.38 \\
.86\end{array}$ & $\begin{array}{r}0.61 \\
1.60 \\
.46\end{array}$ & $\begin{array}{l}1.22 \\
1.81 \\
1.19\end{array}$ & $\begin{array}{l}1.60 \\
1.93 \\
1.59\end{array}$ & $\begin{array}{l}1.55 \\
1.75 \\
1.55\end{array}$ & $\begin{array}{r}1.54 \\
1.54\end{array}$ & $\begin{array}{r}1.47 \\
-\overline{7}\end{array}$ & $\begin{array}{r}1.44 \\
1.44\end{array}$ & $\frac{1.44}{1.44}$ & $\begin{array}{r}1.49 \\
1.49\end{array}$ \\
\hline Natural Gas ,..................................... & .29 & .89 & 2.17 & 3.38 & 2.62 & 2.38 & 2.44 & 2.51 & 2.47 & 2.30 \\
\hline 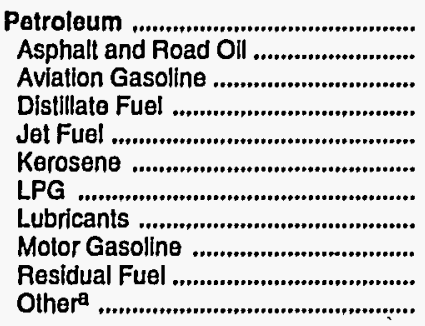 & $\begin{array}{r}1.55 \\
.74 \\
2.17 \\
.94 \\
.72 \\
.76 \\
1.07 \\
5.08 \\
2.66 \\
.42 \\
.79\end{array}$ & $\begin{array}{l}3.03 \\
1.77 \\
3.45 \\
2.35 \\
2.01 \\
2.24 \\
2.49 \\
7.48 \\
4.36 \\
1.61 \\
2.49\end{array}$ & $\begin{array}{r}6.63 \\
3.79 \\
9.02 \\
6.73 \\
6.34 \\
6.91 \\
5.29 \\
14.36 \\
9.26 \\
2.49 \\
6.59\end{array}$ & $\begin{array}{r}6.53 \\
4.20 \\
9.99 \\
6.43 \\
5.67 \\
7.57 \\
4.42 \\
17.61 \\
8.79 \\
4.00 \\
7.00\end{array}$ & $\begin{array}{r}4.84 \\
4.58 \\
8.41 \\
4.74 \\
3.56 \\
3.59 \\
4.26 \\
15.59 \\
6.47 \\
1.45 \\
4.00\end{array}$ & $\begin{array}{r}5.12 \\
3.35 \\
7.55 \\
5.44 \\
3.85 \\
4.39 \\
3.70 \\
12.70 \\
7.19 \\
2.59 \\
4.83\end{array}$ & $\begin{array}{r}4.88 \\
3.21 \\
7.41 \\
5.07 \\
3.57 \\
3.85 \\
3.57 \\
14.61 \\
7.32 \\
1.84 \\
4.02\end{array}$ & $\begin{array}{r}5.05 \\
2.84 \\
8.28 \\
6.04 \\
4.16 \\
5.22 \\
2.69 \\
13.30 \\
7.92 \\
2.43 \\
4.86\end{array}$ & $\begin{array}{r}6.23 \\
2.94 \\
9.32 \\
6.74 \\
5.41 \\
4.89 \\
4.13 \\
13.40 \\
9.16 \\
2.93 \\
5.95\end{array}$ & $\begin{array}{r}5.99 \\
3.33 \\
8.71 \\
6.58 \\
4.64 \\
4.56 \\
4.21 \\
15.42 \\
9.09 \\
2.97 \\
5.13\end{array}$ \\
\hline Nuclear Fuel ....................................... & - & - & - & - & - & - & .37 & .42 & .56 & .49 \\
\hline Blomass Fuels at Utilitles ................ & .65 & .92 & 1.74 & .79 & .32 & .95 & - & .35 & .35 & .35 \\
\hline Primary Energy - Flve Sectors ${ }^{b}$.... & .85 & 1.93 & 4.22 & 4.61 & 3.54 & 3.58 & 3.45 & $\mathbf{3 . 5 0}$ & 4.02 & 3.83 \\
\hline $\begin{array}{l}\text { Electric Utility Fuele ............................ } \\
\text { Electricity Purchased by End Users }\end{array}$ & $\begin{array}{r}.25 \\
4.52\end{array}$ & $\begin{array}{r}.73 \\
6.81\end{array}$ & $\begin{array}{r}1.65 \\
12.69\end{array}$ & $\begin{array}{r}2.44 \\
18.58\end{array}$ & $\begin{array}{r}1.92 \\
16.91\end{array}$ & $\begin{array}{r}1.80 \\
16.32\end{array}$ & $\begin{array}{r}1.74 \\
16.58\end{array}$ & $\begin{array}{r}1.73 \\
16.79\end{array}$ & $\begin{array}{r}1.67 \\
17.09\end{array}$ & $\begin{array}{r}1.61 \\
17.91\end{array}$ \\
\hline \multirow[t]{2}{*}{ Total Energy ........................................ } & 1.28 & 2.80 & 6.00 & 7.04 & 5.72 & $\mathbf{5 . 7 0}$ & 5.53 & $\mathbf{5 . 6 3}$ & 6.43 & 6.31 \\
\hline & \multicolumn{10}{|c|}{ Expenditures in Millions of Dollars } \\
\hline 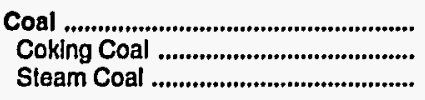 & $\begin{array}{r}11.9 \\
11.6 \\
.2\end{array}$ & $\begin{array}{r}120.2 \\
41.0 \\
79.2\end{array}$ & $\begin{array}{r}892.5 \\
47.9 \\
844.6\end{array}$ & $\begin{array}{r}1,832.9 \\
20.9 \\
1,812.0\end{array}$ & $\begin{array}{r}1,798.1 \\
3.6 \\
1,794.5\end{array}$ & $\begin{array}{r}1,850.9 \\
- \\
1,850.9\end{array}$ & $\begin{array}{r}1,852.4 \\
- \\
1,852.4\end{array}$ & $\begin{array}{r}1,910.4 \\
\frac{-}{1,910.4}\end{array}$ & $\begin{array}{r}1,918.8 \\
- \\
1,918.8\end{array}$ & $\begin{array}{r}1,981.4 \\
- \\
1,981.4\end{array}$ \\
\hline Natural Gas .......................................... & 804.9 & $2,361.3$ & $6,838.0$ & $9,815.8$ & $7,021.2$ & $6,513.9$ & $7,247.2$ & $7,783.9$ & $\mathbf{7 , 3 1 1 . 4}$ & $6,954.4$ \\
\hline 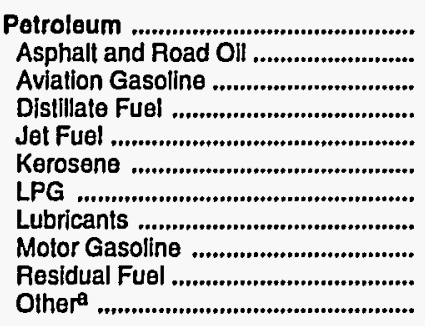 & $\begin{array}{r}3,376.3 \\
58.0 \\
22.0 \\
176.6 \\
97.4 \\
32.5 \\
611.9 \\
129.6 \\
1,976.0 \\
36.0 \\
236.4\end{array}$ & $\begin{array}{r}8,323.8 \\
95.7 \\
22.8 \\
735.9 \\
306.2 \\
91.3 \\
1,452.4 \\
196.2 \\
4,020.6 \\
383.7 \\
1,019.0\end{array}$ & $\begin{array}{r}23,998.1 \\
274.5 \\
57.5 \\
2,823.7 \\
1,098.5 \\
601.2 \\
3,670.4 \\
465.0 \\
8,805.7 \\
969.9 \\
5,231.6\end{array}$ & $\begin{array}{r}24,077.8 \\
329.2 \\
66.4 \\
3,522.2 \\
2,383.1 \\
33.3 \\
4,077.4 \\
519.0 \\
9,479.5 \\
710.5 \\
2,957.1\end{array}$ & $\begin{array}{r}17,866.1 \\
414.4 \\
65.4 \\
2,382.8 \\
1,614.0 \\
13.8 \\
3,859.8 \\
449.3 \\
7,119.0 \\
251.2 \\
1,696.4\end{array}$ & $\begin{array}{r}19,126.5 \\
279.8 \\
43.8 \\
2,796.5 \\
1,839.5 \\
14.6 \\
3,675.5 \\
413.9 \\
7,735.2 \\
351.5 \\
1,976.1\end{array}$ & $\begin{array}{r}19,122.5 \\
307.9 \\
37.9 \\
2,550.8 \\
1,911.9 \\
5.1 \\
3,807.1 \\
459.1 \\
8,038.9 \\
278.0 \\
1,725.8\end{array}$ & $\begin{array}{r}19,933.7 \\
182.7 \\
34.3 \\
3,090.2 \\
2,189.7 \\
20.8 \\
3,048.2 \\
428.5 \\
8,458.3 \\
437.5 \\
2,043.4\end{array}$ & $\begin{array}{r}24,420.1 \\
273.0 \\
39.4 \\
3,230.1 \\
2,931.6 \\
5.5 \\
4,327.4 \\
444.5 \\
9,831.0 \\
505.9 \\
2,831.6\end{array}$ & $\begin{array}{r}23,612.7 \\
206.8 \\
28.8 \\
3,244.5 \\
2,399.2 \\
2.4 \\
4,719.7 \\
457.5 \\
9,485.2 \\
524.9 \\
2,543.5\end{array}$ \\
\hline Nuclear Fuel ......................................... & - & - & - & - & - & - & 15.1 & 44.6 & 94.9 & 103.3 \\
\hline Blomass Fuels at Utilities ................ & .7 & .9 & 1.4 & 2.5 & 1.0 & 2.3 & - & .7 & 1.0 & 1.0 \\
\hline Primary Energy - Five Sectorsb .... & $4,193.8$ & $10,806.1$ & $31,730.0$ & $35,729.0$ & $26,686.5$ & $27,493.6$ & $28,237.2$ & $29,673.3$ & $33,746.1$ & $32,652.8$ \\
\hline $\begin{array}{l}\text { Electric Utility Fue|c } \\
\text { Electricity Purchased by End User................ }\end{array}$ & $\begin{array}{r}-267.6 \\
1,421.0\end{array}$ & $\begin{array}{r}-1,099.3 \\
2,895.0\end{array}$ & $\begin{array}{r}-3,576.1 \\
7,434.5\end{array}$ & $\begin{array}{r}-5,652.8 \\
13,119.7\end{array}$ & $\begin{array}{r}-4,302.1 \\
11,920.6\end{array}$ & $\begin{array}{r}-4,036.5 \\
11,645.3\end{array}$ & $\begin{array}{r}-4,073.9 \\
12,325.7\end{array}$ & $\begin{array}{r}-4,245.4 \\
12,782.0\end{array}$ & $\begin{array}{l}-4,165.2 \\
13,431.1\end{array}$ & $\begin{array}{r}-4,063.1 \\
14,270.8\end{array}$ \\
\hline Total Energy & $5,347.2$ & $12,601.8$ & $35,588.4$ & $43,195.9$ & $34,304.9$ & $35,102.4$ & $36,489.0$ & $38,209.9$ & $43,012.0$ & $42,860.5$ \\
\hline
\end{tabular}

a Includes petroleum coke used at electric utilities.

b Blomass fuels are not included, except those consumed at electric utilities and those added to motor gasoline.

c There are no direct fuel costs for hydroelectric, geothermal, centralized solar, or wind energy.

- No consumplion, including cases where adjustments were made. See "Consumption Adjustments for Process Fuel and Intermediate Products."

Note: Expenditure totals may not equal sum of components due to independent rounding.

Sources: Data sources, estimation procedures, and assumptions are described in the "Documentation" section of this report. 


\section{T Energy Price Estimates by Sector, Texas \\ E 1970, 1975, 1980, and 1985-1991 \\ X (Dollars per Million Btu)}

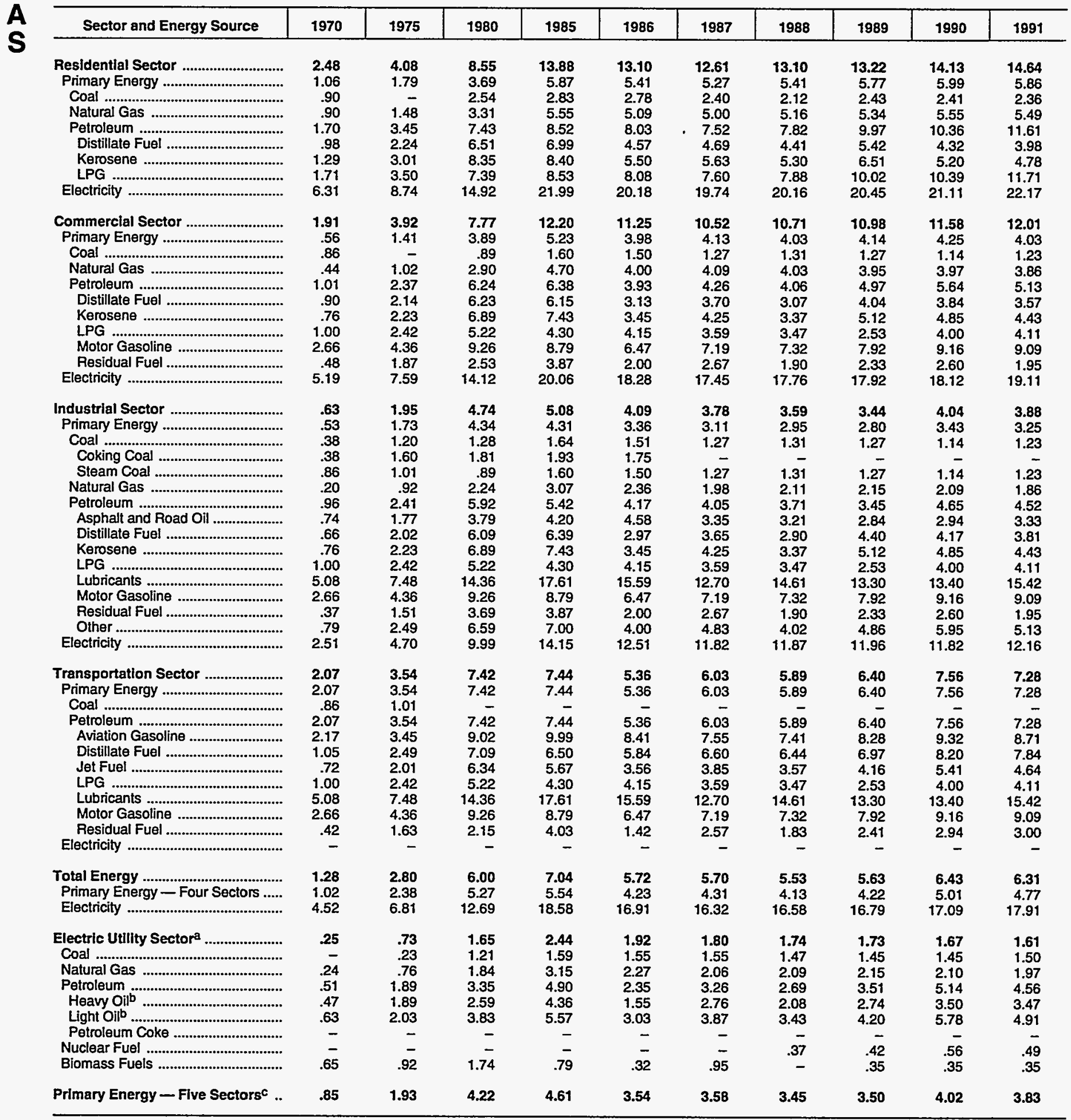

a There are no direct fuel costs for hydroelectric, geothermal, centralized solar, or wind energy.

b Heavy oil includes Grade Nos. 4, 5, and 6, and residual fuel oils. Light oil includes Grade No. 2 heating oil, kerosene, and jet fuel.

c Biomass fuels are not included, except those consumed at electric utilities and those added to motor gasoline.

-No consumption, including cases where adjustments were made. See "Consumption Adjustments for Process Fuel and Intermediate Products."

Sources: Data sources, estimation procedures, and assumptions are described in the "Documentation" section of this report. 


\begin{tabular}{|c|c|c|c|c|c|c|c|c|c|c|}
\hline Sector and Energy Source & 1970 & 1975 & 1980 & 1985 & 1986 & 1987 & 1988 & 1989 & 1990 & 1991 \\
\hline 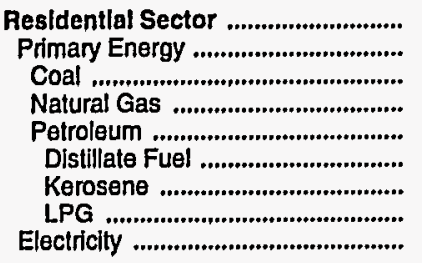 & $\begin{array}{r}1,015.6 \\
314.3 \\
. \\
213.8 \\
100.5 \\
.8 \\
.2 \\
99.5 \\
701.2\end{array}$ & $\begin{array}{r}1,726.1 \\
506.5 \\
- \\
353.8 \\
152.7 \\
3.5 \\
.7 \\
148.5 \\
1,219.6\end{array}$ & $\begin{array}{r}3,852.3 \\
942.0 \\
765.9 \\
176.1 \\
.3 \\
9.4 \\
166.4 \\
2,910.3\end{array}$ & $\begin{array}{r}6,839.0 \\
1,457.1 \\
.2 \\
1,226.8 \\
230.2 \\
1.6 \\
5.4 \\
223.3 \\
5,381.8\end{array}$ & $\begin{array}{r}6,214.2 \\
1,229.5 \\
.3 \\
1,032.8 \\
196.4 \\
.6 \\
1.4 \\
194.4 \\
4,984.7\end{array}$ & $\begin{array}{r}6,307.3 \\
1,298.3 \\
.6 \\
1,097.7 \\
200.1 \\
2.2 \\
1.9 \\
195.9 \\
5,009.0\end{array}$ & $\begin{array}{r}6,623.5 \\
1,309.5 \\
.8 \\
1,127.5 \\
181.2 \\
.8 \\
1.8 \\
178.6 \\
5,314.0\end{array}$ & $\begin{array}{r}7,076.7 \\
1,520.7 \\
.3 \\
1,277.0 \\
243.4 \\
.4 \\
1.8 \\
241.2 \\
5,556.0\end{array}$ & $\begin{array}{r}7,396.7 \\
1,449.7 \\
.2 \\
1,217.6 \\
231.9 \\
.1 \\
.8 \\
231.1 \\
5,947.0\end{array}$ & $\begin{array}{r}7,802.0 \\
1,441.0 \\
.2 \\
1,268.8 \\
172.0 \\
.1 \\
.9 \\
171.0 \\
6,361.0\end{array}$ \\
\hline 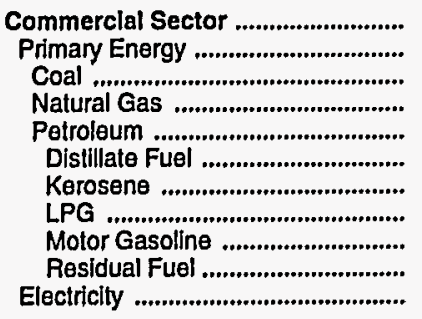 & $\begin{array}{r}511.7 \\
106.4 \\
66.3 \\
40.1 \\
4.4 \\
15.6 \\
10.3 \\
9.7 \\
.2 \\
405.2\end{array}$ & $\begin{array}{r}1,115.4 \\
238.3 \\
- \\
122.6 \\
115.7 \\
20.8 \\
53.1 \\
18.1 \\
15.7 \\
7.9 \\
877.2\end{array}$ & $\begin{array}{r}3,079.0 \\
956.5 \\
\\
504.3 \\
452.2 \\
103.1 \\
126.9 \\
20.7 \\
160.5 \\
40.9 \\
2.122 .5\end{array}$ & $\begin{array}{r}5,327.5 \\
1,211.5 \\
.2 \\
741.3 \\
470.0 \\
343.3 \\
10.6 \\
19.9 \\
90.2 \\
6.1 \\
4,116.0\end{array}$ & $\begin{array}{r}4,634.1 \\
807.2 \\
.3 \\
613.0 \\
193.8 \\
98.7 \\
3.5 \\
17.6 \\
70.9 \\
3.1 \\
3,827.0\end{array}$ & $\begin{array}{r}4,677.1 \\
957.4 \\
.6 \\
666.7 \\
290.1 \\
176.2 \\
2.0 \\
16.3 \\
86.5 \\
9.0 \\
3,719.8\end{array}$ & $\begin{array}{r}4,920.7 \\
950.8 \\
.9 \\
734.8 \\
215.1 \\
99.9 \\
.8 \\
13.9 \\
94.0 \\
6.5 \\
3,970.0\end{array}$ & $\begin{array}{r}5,089.5 \\
966.5 \\
.2 \\
750.8 \\
215.4 \\
91.6 \\
11.8 \\
10.8 \\
97.0 \\
4.4 \\
4,123.0\end{array}$ & $\begin{array}{r}5,291.4 \\
914.3 \\
.2 \\
713.5 \\
200.6 \\
73.3 \\
.7 \\
15.7 \\
109.8 \\
1.2 \\
4,377.0\end{array}$ & $\begin{array}{r}5,585.5 \\
881.5 \\
.2 \\
728.9 \\
152.4 \\
61.4 \\
.3 \\
10.6 \\
77.5 \\
2.7 \\
4,704.0\end{array}$ \\
\hline 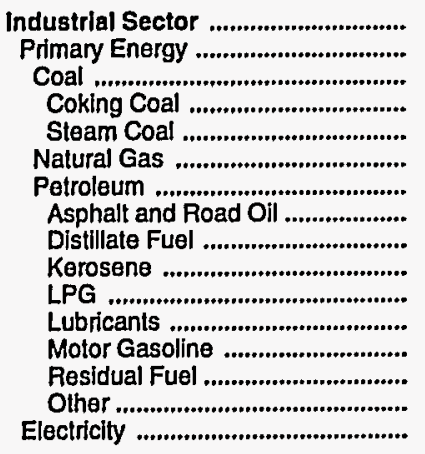 & $\begin{array}{r}1,514.4 \\
1,199.9 \\
11.8 \\
11.6 \\
.2 \\
258.3 \\
929.8 \\
58.0 \\
33.9 \\
16.7 \\
481.0 \\
79.6 \\
19.7 \\
4.5 \\
236.4 \\
314.5\end{array}$ & $\begin{array}{r}4,527.0 \\
3,728.8 \\
93.3 \\
41.0 \\
52.3 \\
834.9 \\
2,800.6 \\
95.7 \\
168.1 \\
37.5 \\
1,241.2 \\
117.3 \\
22.8 \\
99.0 \\
1,019.0 \\
798.2\end{array}$ & $\begin{array}{r}16,088.6 \\
13,687.0 \\
80.9 \\
47.9 \\
32.9 \\
2,840.6 \\
10,765.5 \\
274.5 \\
701.9 \\
464.9 \\
3,470.9 \\
298.7 \\
22.9 \\
300.1 \\
5,231.6 \\
2,401.7\end{array}$ & $\begin{array}{r}16,529.5 \\
12,907.7 \\
138.5 \\
20.9 \\
117.6 \\
3,940.8 \\
8,828.4 \\
329.2 \\
1,016.2 \\
17.4 \\
3,824.9 \\
333.4 \\
217.1 \\
133.2 \\
2,957.1 \\
3,621.9\end{array}$ & $\begin{array}{r}12,682.6 \\
9,573.8 \\
108.7 \\
3.6 \\
105.1 \\
2,775.0 \\
6,690.0 \\
414.4 \\
474.3 \\
8.9 \\
3,636.3 \\
288.6 \\
154.3 \\
16.7 \\
1,696.4 \\
3,108.9\end{array}$ & $\begin{array}{r}12,226.7 \\
9,310.2 \\
78.4 \\
- \\
78.4 \\
2,507.9 \\
6,723.8 \\
279.8 \\
558.1 \\
10.7 \\
3,457.3 \\
265.9 \\
159.1 \\
16.8 \\
1,976.1 \\
2,916.5\end{array}$ & $\begin{array}{r}12,815.1 \\
9,773.4 \\
68.3 \\
- \\
68.3 \\
3,131.1 \\
6,574.0 \\
307.9 \\
466.0 \\
2.6 \\
3,608.6 \\
295.0 \\
158.8 \\
9.3 \\
1,725.8 \\
3,041.7\end{array}$ & $\begin{array}{r}12,796.6 \\
9,693.6 \\
79.1 \\
- \\
79.1 \\
3,476.9 \\
6,137.7 \\
182.7 \\
644.2 \\
7.2 \\
2,792.1 \\
275.3 \\
187.7 \\
5.0 \\
2,043.4 \\
3,103.0\end{array}$ & $\begin{array}{r}14,683.8 \\
11,576.7 \\
69.9 \\
- \\
69.9 \\
3,188.7 \\
8,318.2 \\
273.0 \\
627.6 \\
4.1 \\
4,073.6 \\
285.6 \\
207.5 \\
15.2 \\
2,831.6 \\
3,107.1\end{array}$ & $\begin{array}{r}14,515.3 \\
11,309.5 \\
77.8 \\
-\overline{7} \\
77.8 \\
2,913.3 \\
8,318.4 \\
206.8 \\
512.4 \\
1.2 \\
4,533.0 \\
293.9 \\
220.4 \\
7.2 \\
2,543.5 \\
3,205.7\end{array}$ \\
\hline 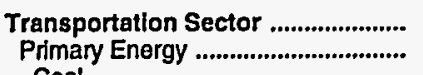 & $\begin{array}{l}2,305.6 \\
2,305.6\end{array}$ & $\begin{array}{l}5,233.3 \\
5,233.3\end{array}$ & $\begin{array}{l}12,568.4 \\
12,568.4\end{array}$ & $\begin{array}{l}14,499.9 \\
14,499.9\end{array}$ & $\begin{array}{l}10,773.9 \\
10,773.9\end{array}$ & $\begin{array}{l}11,891.3 \\
11,891.3\end{array}$ & $\begin{array}{l}12,129.6 \\
12,129.6\end{array}$ & $\begin{array}{l}13,247.1 \\
13,247.1\end{array}$ & $\begin{array}{l}15,640.2 \\
15,640.2\end{array}$ & $\begin{array}{l}14,957.6 \\
14,957.6\end{array}$ \\
\hline 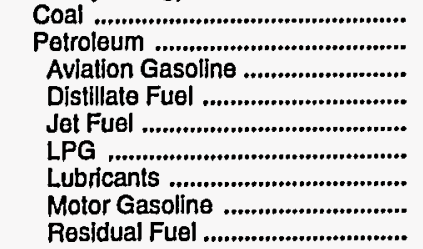 & $\begin{array}{r}2,305.5 \\
22.0 \\
137.5 \\
97.4 \\
21.1 \\
50.0 \\
1,946.6 \\
30.9\end{array}$ & $\begin{array}{r}5,233.3 \\
22.8 \\
542.6 \\
306.2 \\
44.6 \\
78.9 \\
3,982.0 \\
256.2\end{array}$ & $\begin{array}{r}12,568 . \overline{4} \\
57.5 \\
1,993.2 \\
1,098.5 \\
12.4 \\
166.3 \\
8,622.3 \\
618.1\end{array}$ & $\begin{array}{r}14,499.9 \\
66.4 \\
2,136.1 \\
2,383.1 \\
9.4 \\
185.6 \\
9,172.2 \\
547.0\end{array}$ & $\begin{array}{r}10,773 . \overline{9} \\
65.4 \\
1,800.9 \\
1,614.0 \\
11.6 \\
160.6 \\
6,893.7 \\
227.7\end{array}$ & $\begin{array}{r}11,891 . \overline{3} \\
43.8 \\
2,048.6 \\
1,839.5 \\
5.9 \\
148.0 \\
7,489.6 \\
315.8\end{array}$ & $\begin{array}{r}12,129.6 \\
37.9 \\
1,971.0 \\
1,911.9 \\
5.9 \\
164.2 \\
7,786.0 \\
252.7\end{array}$ & $\begin{array}{r}13,247 . \overline{1} \\
34.3 \\
2,297.4 \\
2,189.7 \\
4.2 \\
153.2 \\
8,173.6 \\
394.7\end{array}$ & $\begin{array}{r}15,640 . \overline{2} \\
39.4 \\
2,505.6 \\
2,931.6 \\
7.0 \\
158.9 \\
9,513.7 \\
484.0\end{array}$ & $\begin{array}{r}14,957.6 \\
28.8 \\
2,660.7 \\
2,399.2 \\
5.1 \\
163.6 \\
9,187.4 \\
512.8\end{array}$ \\
\hline Electriclty .......................................... & - & - & - & - & - & - & - & - & - & - \\
\hline $\begin{array}{l}\text { Total Energy ................................... } \\
\text { Primary Energy - Four Sectors ...... } \\
\text { Electricity ........................................ }\end{array}$ & $\begin{array}{l}5,347.2 \\
3,926.2 \\
1,421.0\end{array}$ & $\begin{array}{r}12,601.8 \\
9,706.8 \\
2,895.0\end{array}$ & $\begin{array}{r}35,588.4 \\
28,153.9 \\
7,434.5\end{array}$ & $\begin{array}{l}43,195.9 \\
30,076.2 \\
13,119.7\end{array}$ & $\begin{array}{l}34,304.9 \\
22,384.4 \\
11,920.6\end{array}$ & $\begin{array}{l}35,102.4 \\
23,457.1 \\
11,645.3\end{array}$ & $\begin{array}{l}36,489.0 \\
24,163.3 \\
12,325.7\end{array}$ & $\begin{array}{l}38,209.9 \\
25,427.9 \\
12,782.0\end{array}$ & $\begin{array}{l}43,012.0 \\
29,581.0 \\
13,431.1\end{array}$ & $\begin{array}{l}42,860.5 \\
28,589.7 \\
14,270.8\end{array}$ \\
\hline 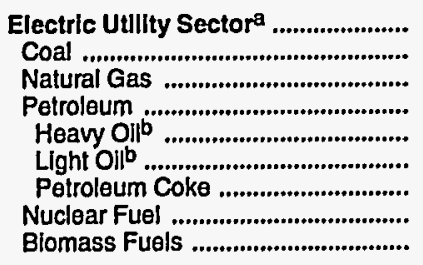 & $\begin{array}{r}267.6 \\
-\overline{5} \\
266.5 \\
.5 \\
.3 \\
.2 \\
- \\
\overline{.7}\end{array}$ & $\begin{array}{r}1,099.3 \\
26.9 \\
1,050.0 \\
21.5 \\
20.6 \\
.9 \\
- \\
-9\end{array}$ & $\begin{array}{r}3,576.1 \\
811.7 \\
2,727.1 \\
35.9 \\
10.7 \\
25.1 \\
- \\
- \\
1.4\end{array}$ & $\begin{array}{r}5,652.8 \\
1,694.0 \\
3,907.0 \\
49.3 \\
24.2 \\
25.1 \\
- \\
- \\
2.5\end{array}$ & $\begin{array}{r}4,302.1 \\
1,688.7 \\
2,600.4 \\
12.0 \\
3.6 \\
8.3 \\
- \\
- \\
1.0\end{array}$ & $\begin{array}{r}4,036.5 \\
1,771.4 \\
2,241.5 \\
21.3 \\
9.9 \\
11.4 \\
- \\
- \\
2.3\end{array}$ & $\begin{array}{r}4,073.9 \\
1,782.4 \\
2,253.8 \\
22.6 \\
9.6 \\
13.1 \\
-\overline{1} \\
15.1 \\
-\end{array}$ & $\begin{array}{r}4,245.4 \\
1,830.8 \\
2,279.2 \\
90.0 \\
33.4 \\
56.6 \\
-\overline{6} \\
44.6 \\
.7\end{array}$ & $\begin{array}{r}4,165.2 \\
1,848.5 \\
2,191.6 \\
29.2 \\
5.6 \\
23.6 \\
-\overline{9} \\
94.9 \\
1.0\end{array}$ & $\begin{array}{r}4,063.1 \\
1,903.1 \\
2,043.5 \\
12.2 \\
2.3 \\
10.0 \\
- \\
103.3 \\
1.0\end{array}$ \\
\hline Prlmary Energy - Flve Sectorsc .. & $4,193.8$ & $10,806.1$ & $31,730.0$ & $35,729.0$ & $26,686.5$ & $27,493.6$ & $28,237.2$ & $29,673.3$ & $33,746.1$ & $32,652.8$ \\
\hline
\end{tabular}

There are no direct fuel costs for hydroelectric, geothermal, centralized solar, or wind energy.

b Heavy oll includes Grade Nos. 4,5, and 6, and residual fuel oils. Light oil includes Grade No. 2 heating oil, kerosene, and jet fuel.

c Biomass fuels are not included, except those consumed at electric utilities and those added to motor gasoline.

- No consumplion, including cases where adjustments were made. See "Consumption Adjustments for Process Fuel and Intermediate Products."

- Value less than 0.05 million dollars.

Nole: Totals may not equal sum of components due to independent rounding.

Sources: Data sources, estimation procedures, and assumptions are described in the "Documentation" section of this report. 


\section{U Energy Price and Expenditure Estimates by Source, Utah}

T 1970, 1975, 1980, and 1985-1991

A

\begin{tabular}{|c|c|c|c|c|c|c|c|c|c|c|}
\hline Energy Source & 1970 & 1975 & 1980 & 1985 & 1986 & 1987 & 1988 & 1989 & 1990 & 1991 \\
\hline & \multicolumn{10}{|c|}{ Prices in Dollars per Million Btu } \\
\hline 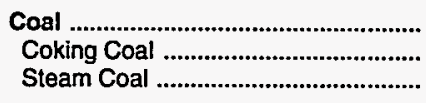 & $\begin{array}{r}0.38 \\
.43 \\
.28\end{array}$ & $\begin{array}{r}0.92 \\
1.38 \\
.55\end{array}$ & $\begin{array}{l}1.35 \\
1.97 \\
1.16\end{array}$ & $\begin{array}{l}1.48 \\
1.93 \\
1.39\end{array}$ & $\begin{array}{l}1.46 \\
1.75 \\
1.42\end{array}$ & $\begin{array}{r}1.25 \\
1.25\end{array}$ & $\begin{array}{l}1.34 \\
2.02 \\
1.26\end{array}$ & $\begin{array}{l}1.31 \\
1.92 \\
1.25\end{array}$ & $\begin{array}{l}1.24 \\
1.84 \\
1.18\end{array}$ & $\begin{array}{l}1.28 \\
1.99 \\
1.21\end{array}$ \\
\hline 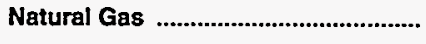 & .57 & 1.07 & 2.33 & 4.01 & 4.41 & 4.16 & 3.94 & 3.99 & 4.17 & 4.21 \\
\hline 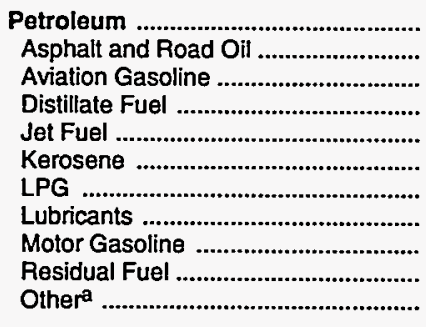 & $\begin{array}{r}1.67 \\
.57 \\
2.17 \\
1.09 \\
.76 \\
.76 \\
2.12 \\
5.08 \\
2.75 \\
.39 \\
.51\end{array}$ & $\begin{array}{l}3.28 \\
1.79 \\
3.45 \\
2.61 \\
2.12 \\
2.42 \\
4.37 \\
7.48 \\
4.52 \\
1.71 \\
1.41\end{array}$ & $\begin{array}{r}7.48 \\
3.65 \\
9.02 \\
6.54 \\
6.59 \\
5.82 \\
5.68 \\
14.36 \\
9.80 \\
3.70 \\
2.85\end{array}$ & $\begin{array}{r}7.91 \\
4.85 \\
9.99 \\
6.69 \\
6.25 \\
9.95 \\
8.69 \\
17.61 \\
9.09 \\
3.86 \\
3.68\end{array}$ & $\begin{array}{r}5.99 \\
4.38 \\
8.41 \\
5.43 \\
4.05 \\
4.46 \\
6.83 \\
15.59 \\
6.78 \\
2.36 \\
1.53\end{array}$ & $\begin{array}{r}6.28 \\
3.23 \\
7.55 \\
5.78 \\
4.19 \\
5.49 \\
6.70 \\
12.70 \\
7.44 \\
2.16 \\
1.50\end{array}$ & $\begin{array}{r}6.48 \\
3.32 \\
7.41 \\
5.85 \\
3.95 \\
5.30 \\
6.82 \\
14.61 \\
7.71 \\
1.98 \\
1.45\end{array}$ & $\begin{array}{r}6.95 \\
2.73 \\
8.28 \\
6.67 \\
4.60 \\
8.65 \\
8.49 \\
13.30 \\
8.24 \\
1.67 \\
1.74\end{array}$ & $\begin{array}{r}7.85 \\
2.70 \\
9.32 \\
8.00 \\
5.75 \\
10.07 \\
8.65 \\
13.40 \\
9.09 \\
2.67 \\
2.02\end{array}$ & $\begin{array}{r}7.36 \\
3.25 \\
8.71 \\
7.60 \\
5.13 \\
9.76 \\
9.22 \\
15.42 \\
8.81 \\
2.31 \\
1.72\end{array}$ \\
\hline 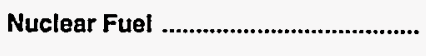 & - & - & - & - & - & - & - & - & - & - \\
\hline Blomass Fuels at Utilities ................ & - & - & - & - & - & - & - & - & - & - \\
\hline Primary Energy - Five Sectors ${ }^{b} \ldots$ & 1.02 & 2.04 & 4.05 & 4.28 & 3.79 & 3.39 & 3.27 & 3.34 & 3.53 & 3.60 \\
\hline $\begin{array}{l}\text { Electric Utility Fuelc ...................... } \\
\text { Electricity Purchased by End Users }\end{array}$ & $\begin{array}{r}.25 \\
5.31\end{array}$ & $\begin{array}{r}.51 \\
7.06\end{array}$ & $\begin{array}{r}1.20 \\
13.11\end{array}$ & $\begin{array}{r}1.39 \\
19.05\end{array}$ & $\begin{array}{r}1.42 \\
19.56\end{array}$ & $\begin{array}{r}1.27 \\
19.15\end{array}$ & $\begin{array}{r}1.27 \\
18.41\end{array}$ & $\begin{array}{r}1.25 \\
17.10\end{array}$ & $\begin{array}{r}1.18 \\
16.09\end{array}$ & $\begin{array}{r}1.21 \\
16.08\end{array}$ \\
\hline \multirow[t]{2}{*}{ 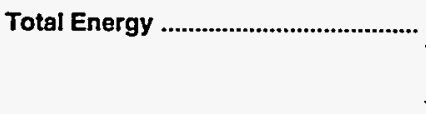 } & 1.31 & 2.59 & 5.77 & 7.24 & 6.93 & 7.32 & 6.76 & 6.78 & 7.12 & 6.95 \\
\hline & \multicolumn{10}{|c|}{ Expenditures in Millions of Dollars } \\
\hline 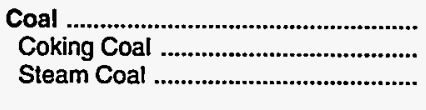 & $\begin{array}{r}30.1 \\
22.7 \\
7.4\end{array}$ & $\begin{array}{r}107.0 \\
71.7 \\
35.3\end{array}$ & $\begin{array}{r}227.1 \\
77.9 \\
149.2\end{array}$ & $\begin{array}{r}295.0 \\
64.8 \\
230.2\end{array}$ & $\begin{array}{r}275.0 \\
36.9 \\
238.1\end{array}$ & $\begin{array}{r}342.0 \\
342.0\end{array}$ & $\begin{array}{r}451.3 \\
63.8 \\
387.5\end{array}$ & $\begin{array}{r}452.0 \\
60.6 \\
391.4\end{array}$ & $\begin{array}{r}455.0 \\
60.8 \\
394.2\end{array}$ & $\begin{array}{r}441.1 \\
63.5 \\
377.6\end{array}$ \\
\hline 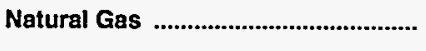 & 61.5 & 113.6 & 255.6 & 439.9 & 365.0 & 348.4 & 376.3 & 406.0 & 419.4 & 512.8 \\
\hline 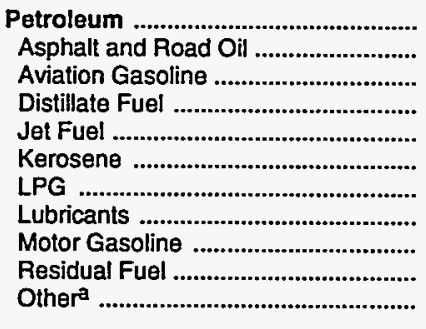 & $\begin{array}{r}252.9 \\
6.0 \\
1.9 \\
32.4 \\
7.6 \\
1.1 \\
6.7 \\
7.9 \\
177.5 \\
10.3 \\
1.5\end{array}$ & $\begin{array}{r}612.1 \\
14.5 \\
2.8 \\
137.5 \\
22.4 \\
2.0 \\
15.4 \\
10.5 \\
357.3 \\
43.5 \\
6.2\end{array}$ & $\begin{array}{r}1,399.3 \\
35.8 \\
6.3 \\
319.7 \\
96.4 \\
3.4 \\
23.8 \\
26.1 \\
799.6 \\
74.8 \\
13.4\end{array}$ & $\begin{array}{r}1,281.0 \\
50.8 \\
4.7 \\
231.5 \\
133.0 \\
1.8 \\
43.4 \\
29.1 \\
775.3 \\
1.7 \\
9.7\end{array}$ & $\begin{array}{r}1,062.2 \\
37.7 \\
4.7 \\
231.3 \\
98.4 \\
.6 \\
36.0 \\
25.2 \\
624.7 \\
.7 \\
3.0\end{array}$ & $\begin{array}{r}1,132.9 \\
30.6 \\
3.8 \\
227.9 \\
116.8 \\
.9 \\
37.5 \\
23.2 \\
687.1 \\
1.5 \\
3.5\end{array}$ & $\begin{array}{r}1,187.0 \\
23.5 \\
4.2 \\
249.6 \\
110.4 \\
.8 \\
33.2 \\
25.7 \\
735.7 \\
.6 \\
3.3\end{array}$ & $\begin{array}{r}1,229.5 \\
30.3 \\
4.4 \\
239.9 \\
131.7 \\
.5 \\
44.8 \\
24.0 \\
749.2 \\
.2 \\
4.4\end{array}$ & $\begin{array}{r}1,404.0 \\
24.7 \\
5.0 \\
342.1 \\
171.0 \\
.8 \\
33.9 \\
24.9 \\
794.2 \\
2.1 \\
5.4\end{array}$ & $\begin{array}{r}1,419.8 \\
61.9 \\
5.2 \\
344.7 \\
158.3 \\
.9 \\
23.3 \\
25.7 \\
795.4 \\
.3 \\
4.0\end{array}$ \\
\hline 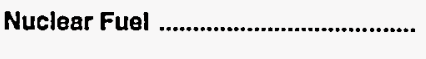 & - & - & - & - & - & - & - & - & - & - \\
\hline Blomass Fuels at Utillties .................. & - & - & - & - & - & - & - & - & - & - \\
\hline Primary Energy - Five Sectors ${ }^{b}$.... & 344.5 & 832.7 & $1,882.0$ & $2,015.9$ & $1,702.2$ & $1,823.2$ & $2,014.6$ & $2,087.6$ & $2,278.5$ & $2,373.7$ \\
\hline $\begin{array}{l}\text { Electric Utility Fuelc ........................ } \\
\text { Electricity Purchased by End Users }\end{array}$ & $\begin{array}{r}-6.4 \\
92.0\end{array}$ & $\begin{array}{r}-26.2 \\
186.9\end{array}$ & $\begin{array}{r}-141.2 \\
469.3\end{array}$ & $\begin{array}{r}-208.0 \\
830.7\end{array}$ & $\begin{array}{r}-222.5 \\
852.9\end{array}$ & $\begin{array}{r}-331.1 \\
861.6\end{array}$ & $\begin{array}{r}-367.6 \\
895.9\end{array}$ & $\begin{array}{r}-367.7 \\
853.9\end{array}$ & $\begin{array}{r}-370.7 \\
830.9\end{array}$ & $\begin{array}{r}-362.7 \\
857.3\end{array}$ \\
\hline Total Energy .......................................... & 430.2 & 993.4 & $2,210.1$ & $2,638.6$ & $2,332.6$ & $2,353.7$ & $2,542.8$ & $2,573.8$ & $2,738.6$ & $2,868.4$ \\
\hline
\end{tabular}

a Includes petroleum coke used at electric utilities.

b Biomass fuels are not included, except those consumed at electric utilities and those added to motor gasoline.

c There are no direct fuel costs for hydroelectric, geothermal, centralized solar, or wind energy.

-No consumption, including cases where adjustments were made. See "Consumption Adjustments for Process Fuel and Intermediate Products."

Note: Expenditure totals may not equal sum of components due to independent rounding.

Sources: Data sources, estimation procedures, and assumptions are described in the "Documentation" section of this report. 
Energy Price Estimates by Sector, Utah $1970,1975,1980$, and 1985-1991

(Dollars per Million Btu)

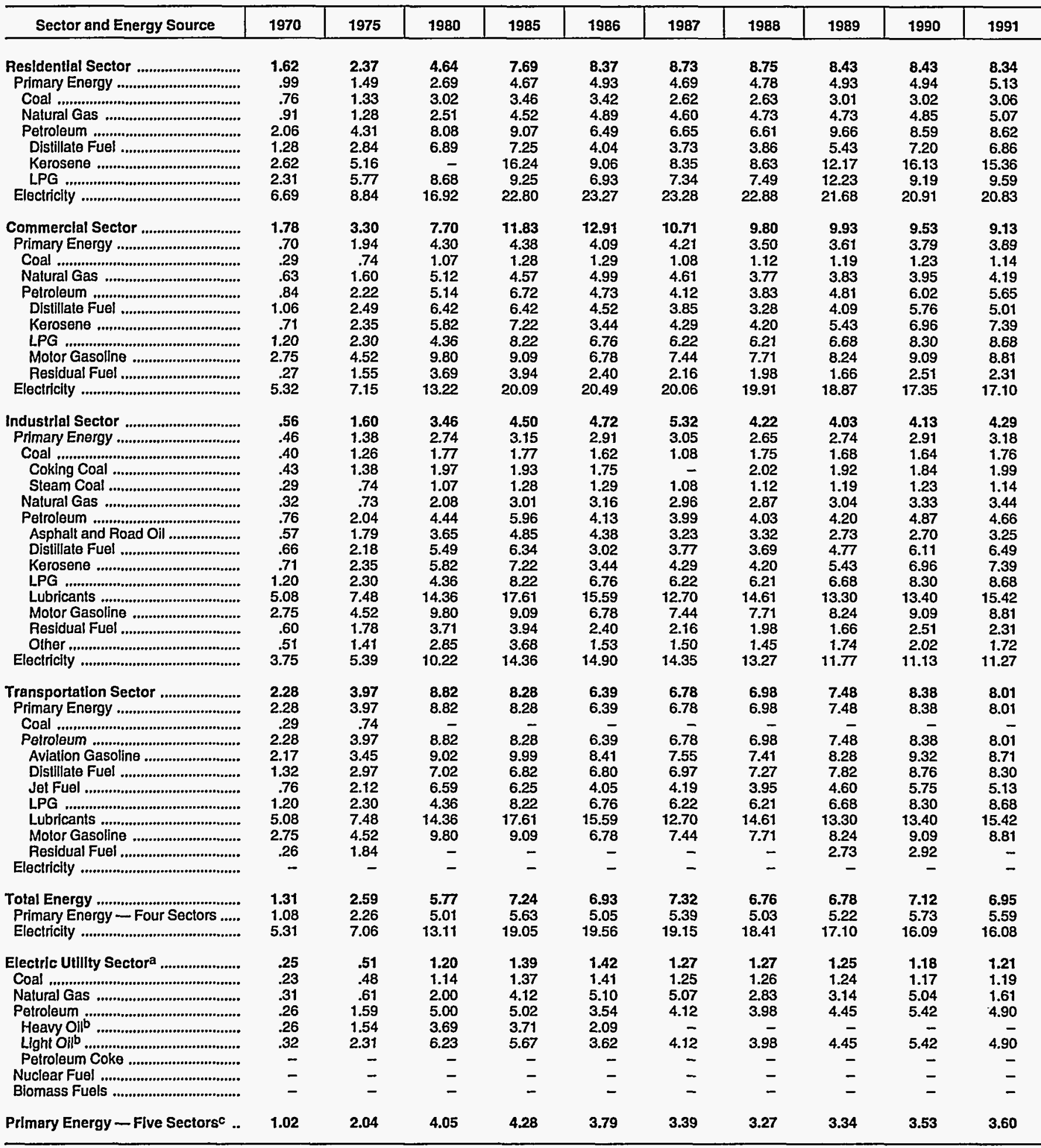

a There are no direct fuel costs for hydroelectric, geothermal, centralized solar, or wind energy.

b Heavy oll includes Grade Nos. 4, 5, and 6, and residual fuel oils. Light oil includes Grade No. 2 heating oil, kerosene, and jet fuel.

c Blomass fuels are not included, except those consumed at electric utilities and those added to motor gasoline.

-No consumption, including cases where adjustments were made. See "Consumption Adjustments for Process Fuel and Intermediate Products."

Sources: Data sources, estimation procedures, and assumptions are described in the "Documentation" section of this report. 
U Energy Expenditure Estimates by Sector, Utah

T 1970, 1975, 1980, and 1985-1991

A (Million Dollars)

H

\begin{tabular}{|c|c|c|c|c|c|c|c|c|c|c|}
\hline Sector and Energy Source & 1970 & 1975 & 1980 & 1985 & 1986 & 1987 & 1988 & 1989 & 1990 & 1991 \\
\hline 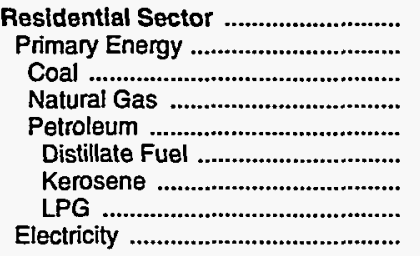 & $\begin{array}{r}84.4 \\
45.9 \\
.7 \\
37.9 \\
7.2 \\
1.1 \\
.1 \\
6.0 \\
38.5\end{array}$ & $\begin{array}{r}167.5 \\
92.3 \\
1.5 \\
72.8 \\
18.1 \\
5.9 \\
.1 \\
12.1 \\
75.2\end{array}$ & $\begin{array}{r}359.4 \\
179.4 \\
5.8 \\
158.0 \\
15.6 \\
4.5 \\
- \\
11.1 \\
179.9\end{array}$ & $\begin{array}{r}627.6 \\
317.5 \\
7.2 \\
285.3 \\
25.0 \\
3.1 \\
.9 \\
21.0 \\
310.1\end{array}$ & $\begin{array}{r}607.1 \\
290.4 \\
5.3 \\
267.4 \\
17.7 \\
1.8 \\
.2 \\
15.7 \\
316.7\end{array}$ & $\begin{array}{r}545.3 \\
229.1 \\
2.7 \\
206.4 \\
20.0 \\
2.2 \\
.4 \\
17.4 \\
316.1\end{array}$ & $\begin{array}{r}564.6 \\
240.6 \\
4.2 \\
215.9 \\
20.6 \\
3.0 \\
.3 \\
17.3 \\
324.0\end{array}$ & $\begin{array}{r}572.9 \\
264.9 \\
5.6 \\
232.2 \\
27.2 \\
5.8 \\
.4 \\
21.0 \\
308.0\end{array}$ & $\begin{array}{r}559.1 \\
256.1 \\
6.5 \\
229.3 \\
20.3 \\
5.7 \\
.4 \\
14.1 \\
303.0\end{array}$ & $\begin{array}{r}620.9 \\
303.9 \\
7.6 \\
275.1 \\
21.3 \\
6.4 \\
.4 \\
14.4 \\
317.0\end{array}$ \\
\hline 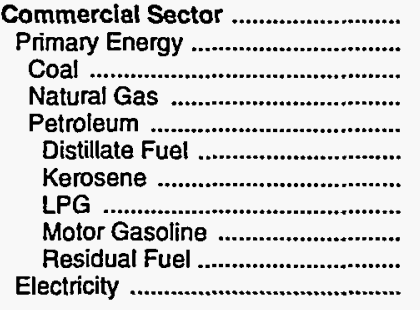 & $\begin{array}{r}49.0 \\
14.8 \\
.5 \\
6.0 \\
8.2 \\
3.2 \\
.2 \\
.6 \\
2.9 \\
1.4 \\
34.3\end{array}$ & $\begin{array}{r}106.9 \\
46.4 \\
1.5 \\
9.2 \\
35.8 \\
18.8 \\
.4 \\
.9 \\
5.0 \\
10.7 \\
60.5\end{array}$ & $\begin{array}{r}216.4 \\
74.7 \\
3.8 \\
1.8 \\
69.0 \\
38.4 \\
1.1 \\
1.0 \\
4.1 \\
24.4 \\
141.7\end{array}$ & $\begin{array}{r}391.3 \\
76.3 \\
4.9 \\
41.7 \\
29.6 \\
20.2 \\
.8 \\
3.3 \\
4.2 \\
1.1 \\
315.0\end{array}$ & $\begin{array}{r}383.6 \\
56.3 \\
3.7 \\
21.9 \\
30.6 \\
24.0 \\
.1 \\
2.7 \\
3.2 \\
.6 \\
327.3\end{array}$ & $\begin{array}{r}433.4 \\
100.6 \\
2.0 \\
73.8 \\
24.8 \\
16.5 \\
.4 \\
2.6 \\
3.6 \\
1.5 \\
332.9\end{array}$ & $\begin{array}{r}438.5 \\
96.6 \\
3.3 \\
73.1 \\
20.2 \\
13.3 \\
.1 \\
2.5 \\
3.6 \\
.6 \\
342.0\end{array}$ & $\begin{array}{r}422.9 \\
89.9 \\
4.1 \\
68.7 \\
17.1 \\
10.9 \\
.1 \\
2.0 \\
3.9 \\
.1 \\
333.0\end{array}$ & $\begin{array}{r}413.9 \\
94.9 \\
4.9 \\
69.8 \\
20.3 \\
12.1 \\
.2 \\
2.3 \\
4.6 \\
1.2 \\
319.0\end{array}$ & $\begin{array}{r}437.5 \\
112.5 \\
5.2 \\
86.8 \\
20.5 \\
13.7 \\
.4 \\
2.3 \\
3.8 \\
.3 \\
325.0\end{array}$ \\
\hline 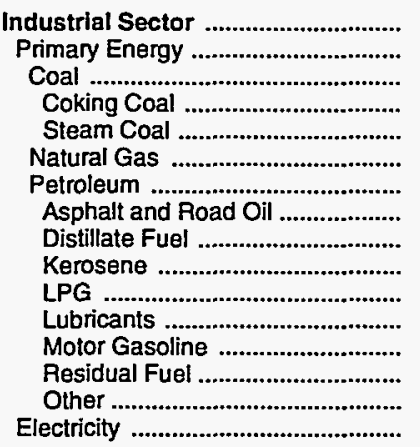 & $\begin{array}{r}89.2 \\
70.0 \\
26.4 \\
22.7 \\
3.6 \\
16.5 \\
27.1 \\
6.0 \\
6.0 \\
.8 \\
.1 \\
2.9 \\
3.8 \\
6.0 \\
1.5 \\
19.2\end{array}$ & $\begin{array}{r}268.0 \\
216.8 \\
81.2 \\
71.7 \\
9.5 \\
29.9 \\
105.7 \\
14.5 \\
40.9 \\
1.5 \\
2.3 \\
3.3 \\
6.3 \\
30.5 \\
6.2 \\
51.2\end{array}$ & $\begin{array}{r}524.1 \\
376.4 \\
89.9 \\
77.9 \\
12.0 \\
86.0 \\
200.6 \\
35.8 \\
70.9 \\
2.2 \\
11.5 \\
9.2 \\
8.5 \\
49.1 \\
13.4 \\
147.7\end{array}$ & $\begin{array}{r}534.8 \\
329.1 \\
78.3 \\
64.8 \\
13.5 \\
111.8 \\
139.0 \\
50.8 \\
40.8 \\
.1 \\
16.8 \\
10.3 \\
10.5 \\
9 \\
9.7 \\
205.7\end{array}$ & $\begin{array}{r}438.0 \\
229.1 \\
47.6 \\
36.9 \\
10.7 \\
74.4 \\
107.1 \\
37.7 \\
34.2 \\
.3 \\
15.6 \\
8.9 \\
7.5 \\
3.0 \\
208.9\end{array}$ & $\begin{array}{r}391.7 \\
179.1 \\
12.1 \\
- \\
12.1 \\
66.7 \\
100.3 \\
30.6 \\
33.7 \\
.1 \\
16.1 \\
8.2 \\
7.9 \\
3.5 \\
312.6\end{array}$ & $\begin{array}{r}493.6 \\
263.7 \\
79.1 \\
63.8 \\
15.3 \\
86.8 \\
97.8 \\
23.5 \\
41.2 \\
.3 \\
11.9 \\
9.1 \\
8.5 \\
3.3 \\
229.9\end{array}$ & $\begin{array}{r}509.8 \\
296.9 \\
79.1 \\
60.6 \\
18.4 \\
103.0 \\
114.9 \\
30.3 \\
42.8 \\
.1 \\
20.3 \\
8.5 \\
8.4 \\
4.4 \\
4.4 \\
212.9\end{array}$ & $\begin{array}{r}522.9 \\
314.0 \\
80.1 \\
60.8 \\
19.3 \\
115.8 \\
118.1 \\
24.7 \\
53.6 \\
.2 \\
16.0 \\
8.8 \\
9.4 \\
. \\
5.4 \\
208.9\end{array}$ & $\begin{array}{r}\mathbf{5 9 6 . 0} \\
380.7 \\
77.0 \\
63.5 \\
13.4 \\
141.9 \\
161.8 \\
61.9 \\
71.6 \\
.1 \\
5.3 \\
9.1 \\
9.7 \\
. \\
4.0 \\
215.3\end{array}$ \\
\hline 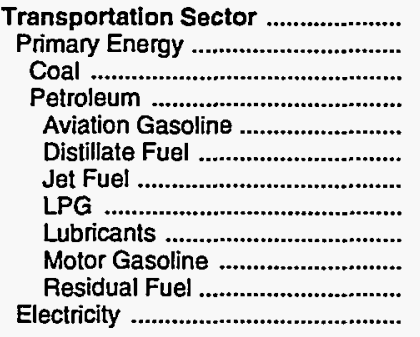 & $\begin{array}{r}207.5 \\
207.5 \\
207.5 \\
1.9 \\
22.1 \\
7.6 \\
5.0 \\
170.9 \\
- \\
-\end{array}$ & $\begin{array}{r}451.0 \\
451.0 \\
451.0 \\
2.8 \\
71.7 \\
22.4 \\
.1 \\
7.2 \\
346.0 \\
.8 \\
-\end{array}$ & $\begin{array}{r}1,110.3 \\
1,110.3 \\
- \\
1,110.3 \\
6.3 \\
203.5 \\
96.4 \\
.2 \\
16.9 \\
787.0 \\
- \\
-\end{array}$ & $\begin{array}{r}1,085.0 \\
1,085.0 \\
-\overline{0} \\
1,085.0 \\
4.7 \\
165.5 \\
133.0 \\
2.2 \\
18.8 \\
760.7 \\
-\end{array}$ & $\begin{array}{r}903.9 \\
903.9 \\
- \\
903.9 \\
4.7 \\
168.6 \\
98.4 \\
1.9 \\
16.3 \\
614.0 \\
- \\
-\end{array}$ & $\begin{array}{r}983.4 \\
983.4 \\
- \\
983.4 \\
3.8 \\
170.9 \\
116.8 \\
1.3 \\
15.0 \\
675.5 \\
- \\
-\end{array}$ & $\begin{array}{r}1,046.0 \\
1,046.0 \\
- \\
1,046.0 \\
4.2 \\
189.7 \\
110.4 \\
1.5 \\
16.6 \\
723.6 \\
- \\
-\end{array}$ & $\begin{array}{r}1,068.1 \\
1,068.1 \\
- \\
1,068.1 \\
4.4 \\
178.1 \\
131.7 \\
1.4 \\
15.5 \\
736.9 \\
-\end{array}$ & $\begin{array}{r}1,242.8 \\
1,242.8 \\
- \\
1,242.8 \\
5.0 \\
268.1 \\
171.0 \\
1.5 \\
16.1 \\
780.2 \\
.9 \\
-\end{array}$ & $\begin{array}{r}1,214.0 \\
1,214.0 \\
- \\
1,214.0 \\
5.2 \\
250.7 \\
158.3 \\
1.4 \\
16.6 \\
781.9 \\
- \\
-\end{array}$ \\
\hline $\begin{array}{l}\text { Total Energy .................................. } \\
\text { Primary Energy - Four Sectors ...... } \\
\text { Electricity ........................................... }\end{array}$ & $\begin{array}{r}430.2 \\
338.2 \\
92.0\end{array}$ & $\begin{array}{l}993.4 \\
806.5 \\
186.9\end{array}$ & $\begin{array}{r}2,210.1 \\
1,740.8 \\
469.3\end{array}$ & $\begin{array}{r}2,638.6 \\
1,807.9 \\
830.7\end{array}$ & $\begin{array}{r}2,332.6 \\
1,479.7 \\
852.9\end{array}$ & $\begin{array}{r}2,353.7 \\
1,492.2 \\
861.6\end{array}$ & $\begin{array}{r}2,542.8 \\
1,646.9 \\
895.9\end{array}$ & $\begin{array}{r}2,573.8 \\
1,719.9 \\
853.9\end{array}$ & $\begin{array}{r}2,738.6 \\
1,907.8 \\
830.9\end{array}$ & $\begin{array}{r}2,868.4 \\
2,011.1 \\
857.3\end{array}$ \\
\hline 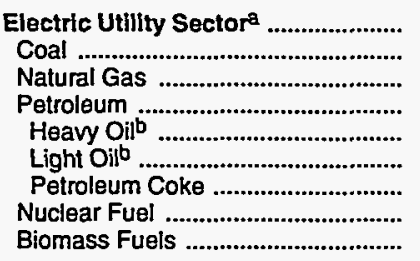 & $\begin{array}{l}6.4 \\
2.5 \\
1.0 \\
2.9 \\
2.8 \\
- \\
- \\
- \\
-\end{array}$ & $\begin{array}{r}26.2 \\
22.8 \\
1.8 \\
1.6 \\
1.5 \\
.1 \\
- \\
- \\
-\end{array}$ & $\begin{array}{r}141.2 \\
127.6 \\
9.8 \\
3.8 \\
1.4 \\
2.4 \\
- \\
- \\
-\end{array}$ & $\begin{array}{r}208.0 \\
204.6 \\
1.0 \\
2.4 \\
.6 \\
1.8 \\
- \\
- \\
-\end{array}$ & $\begin{array}{r}222.5 \\
218.4 \\
1.3 \\
2.8 \\
.1 \\
2.7 \\
- \\
- \\
-\end{array}$ & $\begin{array}{r}331.1 \\
325.1 \\
1.4 \\
4.5 \\
-\overline{5} \\
4.5 \\
- \\
- \\
-\end{array}$ & $\begin{array}{r}367.6 \\
364.7 \\
.6 \\
2.4 \\
- \\
2.4 \\
- \\
- \\
-\end{array}$ & $\begin{array}{r}367.7 \\
363.3 \\
2.2 \\
2.2 \\
-\overline{2} \\
2.2 \\
- \\
- \\
-\end{array}$ & $\begin{array}{r}370.7 \\
363.5 \\
4.6 \\
2.6 \\
-\overline{6} \\
2.6 \\
- \\
- \\
-\end{array}$ & $\begin{array}{r}362.7 \\
351.4 \\
8.9 \\
2.3 \\
-\overline{2} \\
2.3 \\
- \\
- \\
-\end{array}$ \\
\hline Primary Energy - Five Sectorsc .. & 344.5 & 832.7 & $1,882.0$ & $2,015.9$ & $1,702.2$ & $1,823.2$ & $2,014.6$ & $2,087.6$ & $2,278.5$ & $2,373.7$ \\
\hline
\end{tabular}

a There are no direct fuel costs for hydroelectric, geothermal, centralized solar, or wind energy.

beavy oil includes Grade Nos. 4, 5, and 6, and residual fuel oils. Light oil includes Grade No. 2 heating oil, kerosene, and jet fuel.

c Biomass fuels are not included, except those consumed at electric utilities and those added to motor gasoline.

-No consumption, including cases where adjustments were made. See "Consumption Adjustments for Process Fuel and Intermediate Products."

"Value less than 0.05 million dollars.

Note: Totals may not equal sum of components due to independent rounding

Sources: Data sources, estimation procedures, and assumptions are described in the "Documentation" section of this report. 
Energy Price and Expenditure Estimates by Source, Vermont $1970,1975,1980$, and 1985-1991

\begin{tabular}{|c|c|c|c|c|c|c|c|c|c|c|}
\hline Energy Source & 1970 & 1975 & 1980 & 1985 & 1986 & 1987 & 1988 & 1989 & 1990 & 1991 \\
\hline & \multicolumn{10}{|c|}{ Prlces In Dollars per Million Btu } \\
\hline 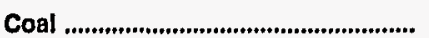 & 0.72 & 2.35 & 2.46 & 2.84 & 2.62 & 3.37 & 3.40 & 3.12 & 3.73 & 3.07 \\
\hline 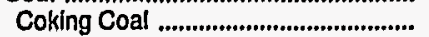 & - & - & - & - & - & - & - & - & - & - \\
\hline 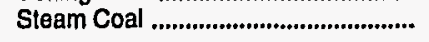 & .72 & 2.35 & 2.46 & 2.84 & 2.62 & 3.37 & 3.40 & 3.12 & 3.73 & 3.07 \\
\hline Natural Gas ........................................... & 1.41 & 1.87 & 5.62 & 5.59 & 5.00 & 4.92 & 4.51 & 4.56 & 4.65 & 4.51 \\
\hline 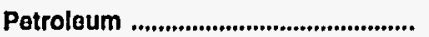 & 1.97 & 3.64 & 8.42 & 8.88 & 6.95 & 6.95 & 7.07 & 7.99 & 9.23 & 8.81 \\
\hline Asphalt and Road Oil ......................... & .68 & 1.91 & 3.66 & 5.20 & 4.86 & 3.55 & 3.39 & 3.17 & 3.34 & 3.05 \\
\hline Avlation Gasoline ................................ & 2.17 & 3.45 & 9.02 & 9.99 & 8.41 & 7.55 & 7.41 & 8.28 & 9.32 & 8.71 \\
\hline Distillate Fuel ....................................... & 1.37 & 2.77 & 7.01 & 8.13 & 6.36 & 6.22 & 6.23 & 6.74 & 8.15 & 7.66 \\
\hline 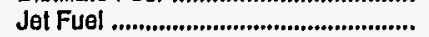 & .75 & 2.22 & 6.55 & 6.10 & 4.25 & 4.18 & 4.04 & 5.33 & 6.60 & 5.07 \\
\hline 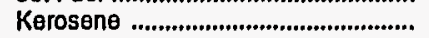 & 1.53 & 3.03 & 7.82 & 8.83 & 6.69 & 6.63 & 6.35 & 7.19 & 8.72 & 8.40 \\
\hline LPG & 2.15 & 4.12 & 7.56 & 11.71 & 10.39 & 10.38 & 9.37 & 12.19 & 13.14 & 14.29 \\
\hline 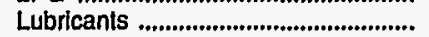 & 5.08 & 7.48 & 14.36 & 17.61 & 15.59 & 12.70 & 14.61 & 13.30 & 13.40 & 15.42 \\
\hline 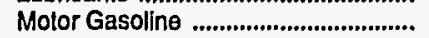 & 3.09 & 4.69 & 10.12 & 9.53 & 7.47 & 7.58 & 7.87 & 8.84 & 9.66 & 9.48 \\
\hline Residual Fuel .................................... & .66 & 1.92 & 4.05 & 4.54 & 2.92 & 3.15 & 2.54 & 2.83 & 3.32 & 2.51 \\
\hline Othera & 4.14 & 4.95 & 12.01 & 13.38 & 14.34 & 13.55 & 11.82 & 17.61 & 14.39 & 15.96 \\
\hline Nuclear Fuel ......................................... & - & .31 & .58 & .64 & .70 & .69 & .71 & .62 & .57 & .56 \\
\hline Blomass Fuels at Utllilies ................ & - & - & 1.74 & .79 & .32 & .95 & .87 & 2.92 & 2.82 & 2.60 \\
\hline Primary Energy - Flve Sectors ${ }^{b} \ldots$ & 1.92 & 2.41 & 5.67 & 5.93 & 5.31 & 4.75 & 4.68 & 5.40 & 6.00 & 5.64 \\
\hline $\begin{array}{l}\text { Electric Utility Fuelc ........................... } \\
\text { Electricity Purchased by End Users }\end{array}$ & 6.05 & $\begin{array}{r}.36 \\
10.33\end{array}$ & $\begin{array}{r}.69 \\
14.33\end{array}$ & $\begin{array}{r}.72 \\
20.81\end{array}$ & $\begin{array}{r}.73 \\
22.51\end{array}$ & $\begin{array}{r}.73 \\
24.16\end{array}$ & $\begin{array}{r}.74 \\
23.69\end{array}$ & $\begin{array}{r}.76 \\
23.46\end{array}$ & $\begin{array}{r}.66 \\
24.24\end{array}$ & $\begin{array}{r}.65 \\
25.30\end{array}$ \\
\hline \multirow[t]{2}{*}{ Total Energy ........................................... } & 2.40 & 4.40 & 9.29 & 10.53 & 9.16 & 9.46 & 9.54 & 10.27 & 11.60 & 11.29 \\
\hline & \multicolumn{10}{|c|}{ Expenditures In Millions of Dollars } \\
\hline 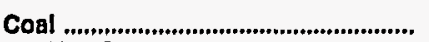 & 1.5 & 1.7 & 1.3 & 5.6 & 1.7 & 1.1 & 1.0 & 0.7 & 0.8 & 0.9 \\
\hline 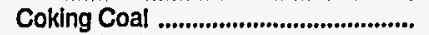 & - & - & - & - & - & - & - & - & - & - \\
\hline Steam Coal & 1.5 & 1.7 & 1.3 & 5.6 & 1.7 & 1.1 & 1.0 & .7 & .8 & .9 \\
\hline Natural Gas .......................................... & 3.8 & 7.5 & 22.2 & 27.7 & 24.7 & 25.1 & 24.8 & 27.6 & 31.0 & 31.5 \\
\hline Petroleum .............................................. & 145.6 & 250.8 & 518.8 & 586.5 & 466.9 & 506.4 & 534.8 & 602.2 & 632.9 & 657.9 \\
\hline Asphalt and Road Oil ......................... & 1.2 & .4 & 1.0 & 11.4 & 13.5 & 11.6 & 8.9 & 9.5 & .6 & 10.7 \\
\hline Avlation Gasoline ................................. & .2 & .2 & 1.1 & 1.1 & 1.2 & .8 & .6 & .7 & .7 & .7 \\
\hline 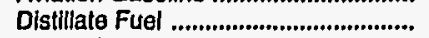 & 45.7 & 75.0 & 167.3 & 198.5 & 147.1 & 158.4 & 169.5 & 181.7 & 192.0 & 190.1 \\
\hline Jet Fuel & .5 & 2.2 & 5.6 & 6.7 & 3.1 & 4.2 & 3.2 & 6.6 & 6.6 & 3.9 \\
\hline 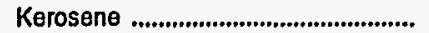 & 4.4 & 5.4 & 12.5 & 28.9 & 14.4 & 11.9 & 16.4 & 14.8 & 11.0 & 13.1 \\
\hline LPG & 4.4 & 12.8 & 18.5 & 33.4 & 32.8 & 41.8 & 39.6 & 67.5 & 66.8 & 84.4 \\
\hline Lubricants ............................................... & 2.0 & 2.5 & 5.9 & 6.5 & 5.7 & 5.2 & 5.8 & 5.4 & 5.6 & 5.8 \\
\hline Motor Gasoline ................................. & 82.5 & 140.2 & 288.9 & 290.9 & 234.0 & 259.4 & 281.3 & 304.2 & 337.8 & 337.2 \\
\hline Residual Fuel ........................................ & 3.7 & 9.6 & 12.0 & 3.5 & 8.7 & 6.7 & 3.8 & 3.4 & 5.0 & 4.2 \\
\hline Other & 1.0 & 2.5 & 5.9 & 5.6 & 6.4 & 6.5 & 5.8 & 8.4 & 6.8 & 7.9 \\
\hline Nuclear Fual ....................................... & - & 12.0 & 18.7 & 20.8 & 15.5 & 26.3 & 31.3 & 24.1 & 22.1 & 24.9 \\
\hline Blomass Fuels at Utilltles ................. & - & - & .9 & 2.3 & .3 & 1.5 & .9 & 5.5 & 2.7 & 2.9 \\
\hline Primary Energy $\longrightarrow$ Five Sectors ${ }^{b} \ldots$ & 150.9 & 272.0 & 561.9 & 642.9 & 509.1 & 560.5 & 592.8 & 660.2 & 689.6 & 718.1 \\
\hline $\begin{array}{l}\text { Electric Utility Fuelc ........................... } \\
\text { Electriclly Purchased by End Users }\end{array}$ & $\begin{array}{l}-2.2 \\
53.9\end{array}$ & $\begin{array}{r}-14.5 \\
105.6\end{array}$ & $\begin{array}{r}-23.4 \\
193.1\end{array}$ & $\begin{array}{r}-26.1 \\
285.1\end{array}$ & $\begin{array}{r}-17.3 \\
286.2\end{array}$ & $\begin{array}{l}-29.2 \\
337.8\end{array}$ & $\begin{array}{r}-33.9 \\
357.0\end{array}$ & $\begin{array}{r}-31.0 \\
366.0\end{array}$ & $\begin{array}{l}-26.8 \\
390.0\end{array}$ & $\begin{array}{r}-30.1 \\
406.1\end{array}$ \\
\hline Total Energy ......................................... & 202.6 & 363.0 & 731.7 & 901.9 & 778.1 & 869.0 & 915.9 & 995.2 & $1,052.8$ & $1,094.1$ \\
\hline
\end{tabular}

a Includes petroleum coke used at electric utitities.

b Blomass fuels are not included, except those consumed at electric utilities and those added to motor gasoline.

c There are no direct fuel costs for hydroelectric, geothermal, centralized solar, or wind energy.

-No consumption, including cases where adjustments were made. See "Consumption Adjustments for Process Fuel and Intermediate Products."

Note: Expenditure totals may not equal sum of components due to independent rounding.

Sources: Data sources, estimation procedures, and assumptions are described in the "Documentation" section of this report. 
V Energy Price Estimates by Sector, Vermont

E $1970,1975,1980$, and 1985-1991

R (Dollars per Million Btu)

$\mathbf{M}$
$\mathbf{O}$
$\mathbf{N}$
$\mathbf{T}$

\begin{tabular}{|c|c|c|c|c|c|c|c|c|c|c|}
\hline Sector and Energy Source & 1970 & 1975 & 1980 & 1985 & 1986 & 1987 & 1988 & 1989 & 1990 & 1991 \\
\hline 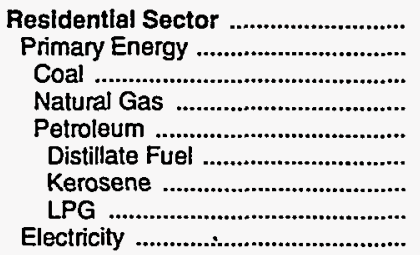 & $\begin{array}{l}2.24 \\
1.58 \\
1.37 \\
1.97 \\
1.57 \\
1.51 \\
1.63 \\
2.55 \\
6.68\end{array}$ & $\begin{array}{r}4.52 \\
3.04 \\
2.62 \\
2.62 \\
3.07 \\
2.87 \\
3.16 \\
4.72 \\
11.47\end{array}$ & $\begin{array}{r}9.65 \\
7.43 \\
4.42 \\
6.30 \\
7.56 \\
7.32 \\
8.15 \\
9.28 \\
15.76\end{array}$ & $\begin{array}{r}11.08 \\
8.41 \\
4.91 \\
6.33 \\
8.67 \\
8.08 \\
8.98 \\
11.79 \\
21.20\end{array}$ & $\begin{array}{r}10.15 \\
7.01 \\
4.71 \\
6.36 \\
7.11 \\
6.49 \\
7.22 \\
10.02 \\
23.15\end{array}$ & $\begin{array}{r}11.51 \\
6.91 \\
4.37 \\
6.31 \\
7.00 \\
6.23 \\
6.92 \\
10.04 \\
27.72\end{array}$ & $\begin{array}{r}11.21 \\
6.70 \\
4.51 \\
5.71 \\
6.83 \\
6.19 \\
6.88 \\
8.95 \\
26.66\end{array}$ & $\begin{array}{r}11.91 \\
7.97 \\
4.48 \\
5.70 \\
8.24 \\
6.79 \\
7.54 \\
12.77 \\
26.24\end{array}$ & $\begin{array}{r}13.58 \\
9.05 \\
4.73 \\
5.87 \\
9.49 \\
8.02 \\
8.92 \\
13.76 \\
27.22\end{array}$ & $\begin{array}{r}13.62 \\
9.22 \\
4.59 \\
6.31 \\
9.61 \\
7.71 \\
8.57 \\
15.17 \\
27.94\end{array}$ \\
\hline 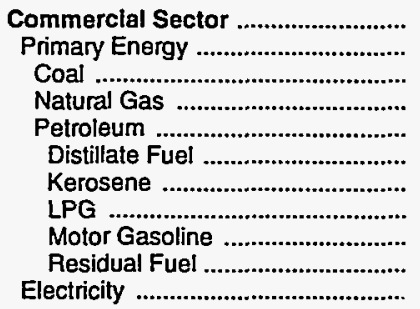 & $\begin{array}{r}2.18 \\
1.06 \\
.87 \\
1.43 \\
1.04 \\
1.11 \\
.92 \\
1.37 \\
3.09 \\
.79 \\
6.78\end{array}$ & $\begin{array}{r}4.51 \\
2.33 \\
2.60 \\
2.10 \\
2.35 \\
2.46 \\
2.65 \\
2.92 \\
4.69 \\
1.91 \\
11.34\end{array}$ & $\begin{array}{r}9.00 \\
5.90 \\
1.65 \\
6.22 \\
5.93 \\
6.48 \\
6.39 \\
5.59 \\
10.12 \\
4.09 \\
15.56\end{array}$ & $\begin{array}{r}12.72 \\
6.80 \\
2.38 \\
5.76 \\
7.93 \\
7.56 \\
7.67 \\
11.46 \\
9.53 \\
4.54 \\
24.02\end{array}$ & $\begin{array}{r}12.30 \\
5.54 \\
2.12 \\
5.19 \\
5.73 \\
5.77 \\
4.99 \\
11.25 \\
7.47 \\
2.92 \\
25.65\end{array}$ & $\begin{array}{r}13.16 \\
5.47 \\
2.36 \\
4.94 \\
5.71 \\
5.29 \\
5.52 \\
11.14 \\
7.58 \\
3.15 \\
24.53\end{array}$ & $\begin{array}{r}13.17 \\
5.55 \\
2.53 \\
4.72 \\
5.88 \\
5.42 \\
5.25 \\
10.88 \\
7.87 \\
2.54 \\
24.44\end{array}$ & $\begin{array}{r}13.10 \\
5.89 \\
2.60 \\
4.81 \\
6.28 \\
5.91 \\
6.03 \\
10.22 \\
8.84 \\
2.84 \\
24.23\end{array}$ & $\begin{array}{r}14.40 \\
6.54 \\
2.62 \\
5.12 \\
7.16 \\
7.09 \\
7.41 \\
10.80 \\
9.66 \\
3.33 \\
25.16\end{array}$ & $\begin{array}{r}14.27 \\
6.31 \\
2.57 \\
5.30 \\
6.69 \\
6.47 \\
6.79 \\
11.94 \\
9.48 \\
2.51 \\
26.42\end{array}$ \\
\hline 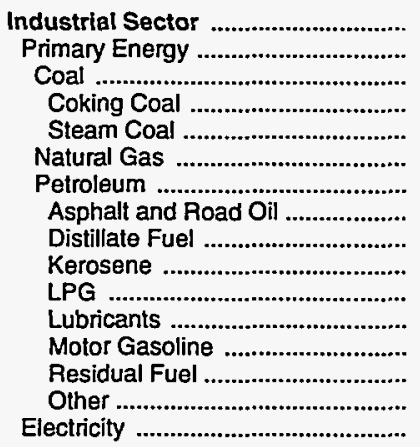 & $\begin{array}{r}1.71 \\
.96 \\
.87 \\
- \\
.87 \\
.85 \\
.97 \\
.68 \\
.84 \\
.92 \\
1.37 \\
5.08 \\
3.09 \\
.53 \\
4.14 \\
4.52\end{array}$ & $\begin{array}{r}3.74 \\
2.41 \\
2.60 \\
- \\
2.60 \\
1.44 \\
2.63 \\
1.91 \\
2.38 \\
2.65 \\
2.92 \\
7.48 \\
4.69 \\
1.93 \\
4.95 \\
7.61\end{array}$ & $\begin{array}{r}7.69 \\
5.73 \\
1.65 \\
- \\
1.65 \\
4.94 \\
5.96 \\
3.66 \\
5.84 \\
6.39 \\
5.59 \\
14.36 \\
10.12 \\
4.01 \\
12.01 \\
11.37\end{array}$ & $\begin{array}{r}10.87 \\
6.50 \\
2.38 \\
-\overline{2} \\
2.38 \\
4.91 \\
7.01 \\
5.20 \\
6.70 \\
7.67 \\
11.46 \\
17.61 \\
9.53 \\
4.54 \\
13.38 \\
18.40\end{array}$ & $\begin{array}{r}9.84 \\
5.05 \\
2.12 \\
- \\
2.12 \\
3.57 \\
5.33 \\
4.86 \\
4.36 \\
4.99 \\
11.25 \\
15.59 \\
7.47 \\
2.92 \\
14.34 \\
20.05\end{array}$ & $\begin{array}{r}9.08 \\
5.02 \\
2.36 \\
-\overline{2} \\
2.36 \\
3.52 \\
5.29 \\
3.55 \\
4.82 \\
5.52 \\
11.14 \\
12.70 \\
7.58 \\
3.15 \\
13.55 \\
19.79\end{array}$ & $\begin{array}{r}9.00 \\
4.62 \\
2.53 \\
- \\
2.53 \\
3.00 \\
4.94 \\
3.39 \\
4.58 \\
5.25 \\
10.88 \\
14.61 \\
7.87 \\
2.54 \\
11.82 \\
19.39\end{array}$ & $\begin{array}{r}9.53 \\
5.18 \\
2.60 \\
- \\
2.60 \\
3.05 \\
5.69 \\
3.17 \\
5.26 \\
6.03 \\
10.22 \\
13.30 \\
8.84 \\
2.84 \\
17.61 \\
19.21\end{array}$ & $\begin{array}{r}11.58 \\
6.31 \\
2.62 \\
- \\
2.62 \\
3.56 \\
7.34 \\
3.34 \\
6.47 \\
7.41 \\
10.80 \\
13.40 \\
9.66 \\
3.33 \\
14.39 \\
19.31\end{array}$ & $\begin{array}{r}10.06 \\
5.36 \\
2.57 \\
- \\
2.57 \\
3.03 \\
5.87 \\
3.05 \\
5.93 \\
6.79 \\
11.94 \\
15.42 \\
9.48 \\
2.51 \\
15.96 \\
20.66\end{array}$ \\
\hline 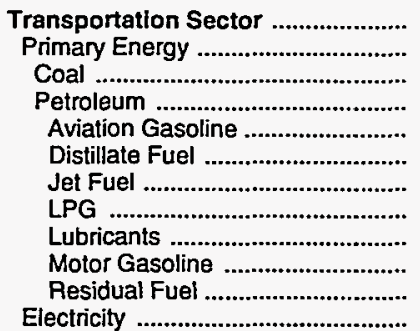 & $\begin{array}{r}2.94 \\
2.94 \\
.87 \\
2.94 \\
2.17 \\
1.43 \\
.75 \\
1.37 \\
5.08 \\
3.09 \\
.76 \\
-\end{array}$ & $\begin{array}{r}4.49 \\
4.49 \\
2.60 \\
4.49 \\
3.45 \\
2.90 \\
2.09 \\
2.92 \\
7.48 \\
4.69 \\
1.84 \\
-\end{array}$ & $\begin{array}{r}9.72 \\
9.72 \\
- \\
9.72 \\
9.02 \\
7.41 \\
6.51 \\
5.59 \\
14.36 \\
10.12 \\
- \\
-\end{array}$ & $\begin{array}{r}9.46 \\
9.46 \\
- \\
9.46 \\
9.99 \\
9.30 \\
6.10 \\
11.46 \\
17.61 \\
9.53 \\
- \\
-\end{array}$ & $\begin{array}{r}7.48 \\
7.48 \\
- \\
7.48 \\
8.41 \\
7.51 \\
4.25 \\
11.25 \\
15.59 \\
7.47 \\
- \\
-\end{array}$ & $\begin{array}{r}7.50 \\
7.50 \\
- \\
7.50 \\
7.55 \\
7.35 \\
4.18 \\
11.14 \\
12.70 \\
7.58 \\
2.64 \\
-\end{array}$ & $\begin{array}{r}7.78 \\
7.78 \\
- \\
7.78 \\
7.41 \\
7.51 \\
4.04 \\
10.88 \\
14.61 \\
7.87 \\
- \\
-\end{array}$ & $\begin{array}{r}8.61 \\
8.61 \\
- \\
8.61 \\
8.28 \\
7.88 \\
5.33 \\
10.22 \\
13.30 \\
8.84 \\
2.60 \\
-\end{array}$ & $\begin{array}{r}9.61 \\
9.61 \\
- \\
9.61 \\
9.32 \\
9.66 \\
6.60 \\
10.80 \\
13.40 \\
9.66 \\
2.76 \\
-\end{array}$ & $\begin{array}{r}9.39 \\
9.39 \\
- \\
9.39 \\
8.71 \\
9.14 \\
5.07 \\
11.94 \\
15.42 \\
9.48 \\
2.16 \\
\end{array}$ \\
\hline 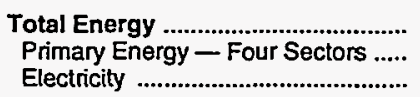 & $\begin{array}{l}2.40 \\
1.97 \\
6.05\end{array}$ & $\begin{array}{r}4.40 \\
3.56 \\
10.33\end{array}$ & $\begin{array}{r}9.29 \\
8.25 \\
14.33\end{array}$ & $\begin{array}{r}10.53 \\
8.57 \\
20.81\end{array}$ & $\begin{array}{r}9.16 \\
6.81 \\
22.51\end{array}$ & $\begin{array}{r}9.46 \\
6.82 \\
24.16\end{array}$ & $\begin{array}{r}9.54 \\
6.90 \\
23.69\end{array}$ & $\begin{array}{r}10.27 \\
7.73 \\
23.46\end{array}$ & $\begin{array}{r}11.60 \\
8.88 \\
24.24\end{array}$ & $\begin{array}{r}11.29 \\
8.51 \\
25.30\end{array}$ \\
\hline 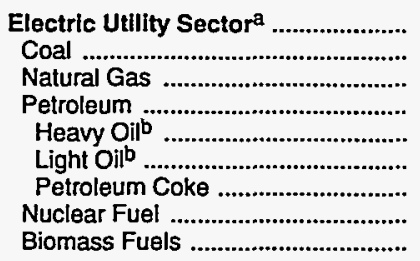 & $\begin{array}{l}.72 \\
.49 \\
- \\
.91 \\
.83 \\
.92 \\
- \\
- \\
-\end{array}$ & $\begin{array}{r}.36 \\
2.05 \\
1.17 \\
2.41 \\
1.95 \\
2.42 \\
- \\
.31 \\
-\end{array}$ & $\begin{array}{r}.69 \\
1.73 \\
4.50 \\
6.28 \\
-\overline{8} \\
6.28 \\
\overline{-} \\
.58 \\
1.74\end{array}$ & $\begin{array}{r}.72 \\
2.03 \\
4.84 \\
5.83 \\
-\overline{5} \\
5.83 \\
- \\
.64 \\
.79\end{array}$ & $\begin{array}{r}.73 \\
1.88 \\
2.26 \\
3.55 \\
- \\
3.55 \\
- \\
.70 \\
.32\end{array}$ & $\begin{array}{r}.73 \\
- \\
\overline{-} \\
3.38 \\
\overline{8} \\
3.38 \\
\overline{69} \\
.95\end{array}$ & $\begin{array}{r}.74 \\
- \\
- \\
3.71 \\
\overline{-} \\
3.71 \\
\overline{-} \\
.71 \\
.87\end{array}$ & $\begin{array}{r}.76 \\
- \\
2.38 \\
4.23 \\
- \\
4.23 \\
- \\
.62 \\
2.92\end{array}$ & $\begin{array}{r}.66 \\
- \\
2.42 \\
5.53 \\
- \\
5.53 \\
- \\
.57 \\
2.82\end{array}$ & $\begin{array}{r}.65 \\
- \\
1.74 \\
4.70 \\
- \\
4.70 \\
- \\
.56 \\
2.60\end{array}$ \\
\hline Primary Energy - Five Sectorsc ... & 1.92 & 2.41 & 5.67 & 5.93 & 5.31 & 4.75 & 4.68 & 5.40 & 6.00 & 5.64 \\
\hline
\end{tabular}

a There are no direct fuel costs for hydroelectric, geothermal, centralized solar, or wind energy.

b Heavy oil includes Grade Nos. 4, 5, and 6, and residual fuel oils. Light oil includes Grade No. 2 healing oil, kerosene, and jet fuel.

c Biomass fuels are not included, except those consumed at electric utilities and those added to motor gasoline.

-No consumption, including cases where adjustments were made. See "Consumption Adjustments for Process Fuel and Intermediate Products."

Sources: Data sources, estimation procedures, and assumptions are described in the "Documentation" section of this report. 
Energy Expenditure Estimates by Sector, Vermont

$1970,1975,1980$, and 1985-1991

(Million Dollars)

\begin{tabular}{|c|c|c|c|c|c|c|c|c|c|c|}
\hline Sector and Energy Source & 1970 & 1975 & 1980 & 1985 & 1986 & 1987 & 1988 & 1989 & 1990 & 1991 \\
\hline 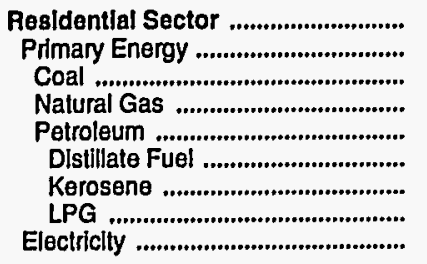 & $\begin{array}{r}71.8 \\
44.1 \\
.6 \\
2.1 \\
41.4 \\
34.0 \\
4.0 \\
3.4 \\
27.7\end{array}$ & $\begin{array}{r}125.1 \\
69.3 \\
.5 \\
3.0 \\
65.8 \\
51.9 \\
4.2 \\
9.7 \\
55.8\end{array}$ & $\begin{array}{r}219.8 \\
124.1 \\
.7 \\
8.1 \\
115.3 \\
92.5 \\
10.6 \\
12.1 \\
95.8\end{array}$ & $\begin{array}{r}278.9 \\
167.7 \\
2.3 \\
9.1 \\
156.3 \\
104.5 \\
26.2 \\
25.5 \\
111.2\end{array}$ & $\begin{array}{r}206.0 \\
114.7 \\
.7 \\
10.0 \\
104.0 \\
69.9 \\
11.9 \\
22.2 \\
91.3\end{array}$ & $\begin{array}{r}250.3 \\
117.0 \\
.7 \\
10.4 \\
106.0 \\
68.1 \\
9.8 \\
28.1 \\
133.3\end{array}$ & $\begin{array}{r}265.8 \\
122.8 \\
.6 \\
10.6 \\
111.7 \\
70.0 \\
12.0 \\
29.6 \\
143.0\end{array}$ & $\begin{array}{r}313.2 \\
164.2 \\
.3 \\
11.9 \\
152.0 \\
85.5 \\
11.9 \\
54.6 \\
149.0\end{array}$ & $\begin{array}{r}336.2 \\
168.2 \\
.5 \\
12.4 \\
155.3 \\
90.2 \\
9.8 \\
55.3 \\
168.0\end{array}$ & $\begin{array}{r}352.8 \\
182.7 \\
.3 \\
13.7 \\
168.6 \\
91.5 \\
12.0 \\
65.1 \\
170.0\end{array}$ \\
\hline 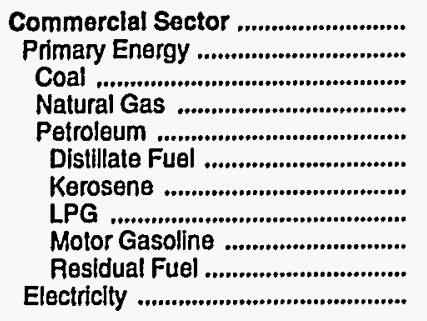 & $\begin{array}{r}23.2 \\
9.1 \\
.2 \\
.8 \\
8.1 \\
5.1 \\
.1 \\
.3 \\
.4 \\
2.1 \\
14.1\end{array}$ & $\begin{array}{r}45.0 \\
17.6 \\
.4 \\
1.6 \\
15.6 \\
9.1 \\
.2 \\
1.1 \\
.7 \\
4.5 \\
27.4\end{array}$ & $\begin{array}{r}88.3 \\
39.3 \\
.2 \\
5.1 \\
34.1 \\
23.4 \\
1.6 \\
1.3 \\
1.7 \\
6.1 \\
49.0\end{array}$ & $\begin{array}{r}121.1 \\
42.5 \\
1.6 \\
9.0 \\
31.9 \\
23.3 \\
1.6 \\
4.4 \\
2.0 \\
.7 \\
78.6\end{array}$ & $\begin{array}{r}124.2 \\
37.1 \\
.2 \\
8.6 \\
28.2 \\
18.1 \\
1.7 \\
4.4 \\
1.6 \\
2.5 \\
87.1\end{array}$ & $\begin{array}{r}158.4 \\
39.3 \\
.2 \\
8.9 \\
30.1 \\
20.1 \\
1.0 \\
5.5 \\
1.6 \\
1.8 \\
119.2\end{array}$ & $\begin{array}{r}166.9 \\
41.9 \\
.2 \\
9.1 \\
32.6 \\
21.8 \\
1.9 \\
6.4 \\
1.6 \\
1.0 \\
125.0\end{array}$ & $\begin{array}{r}174.7 \\
47.7 \\
.1 \\
9.9 \\
37.7 \\
24.9 \\
2.0 \\
7.7 \\
1.7 \\
1.5 \\
127.1\end{array}$ & $\begin{array}{r}177.6 \\
46.5 \\
.2 \\
10.3 \\
36.0 \\
23.2 \\
.5 \\
7.7 \\
2.1 \\
2.5 \\
131.0\end{array}$ & $\begin{array}{r}188.4 \\
50.4 \\
.1 \\
10.8 \\
39.4 \\
26.4 \\
.6 \\
9.0 \\
1.4 \\
2.1 \\
138.0\end{array}$ \\
\hline 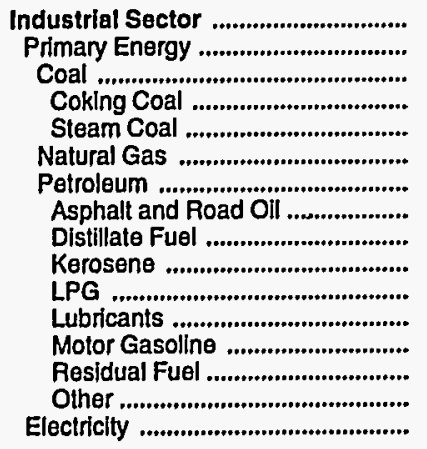 & $\begin{array}{r}21.7 \\
9.5 \\
.1 \\
\overline{.1} \\
.9 \\
8.5 \\
1.2 \\
2.3 \\
.2 \\
.6 \\
.5 \\
1.1 \\
1.5 \\
1.0 \\
12.1\end{array}$ & $\begin{array}{r}43.0 \\
20.7 \\
.1 \\
-1 \\
.1 \\
2.2 \\
18.3 \\
.4 \\
5.1 \\
1.0 \\
1.9 \\
.5 \\
1.9 \\
5.1 \\
2.5 \\
22.3\end{array}$ & $\begin{array}{r}94.0 \\
45.6 \\
.1 \\
. \overline{1} \\
7.9 \\
37.6 \\
1.0 \\
17.1 \\
.3 \\
5.0 \\
1.3 \\
1.0 \\
5.9 \\
5.9 \\
48.4\end{array}$ & $\begin{array}{r}153.4 \\
58.0 \\
.3 \\
. \overline{3} \\
.3 \\
9.1 \\
48.6 \\
11.4 \\
17.5 \\
1.1 \\
2.9 \\
1.5 \\
5.8 \\
2.8 \\
5.6 \\
95.3\end{array}$ & $\begin{array}{r}165.6 \\
57.8 \\
.2 \\
\overline{.} \\
.2 \\
6.1 \\
51.5 \\
13.5 \\
12.8 \\
.8 \\
5.8 \\
1.3 \\
4.7 \\
6.2 \\
6.4 \\
107.8\end{array}$ & $\begin{array}{r}142.3 \\
57.0 \\
.1 \\
-1 \\
.1 \\
5.8 \\
51.0 \\
11.6 \\
13.3 \\
1.0 \\
7.8 \\
1.2 \\
4.8 \\
4.8 \\
65 \\
85.3\end{array}$ & $\begin{array}{r}139.3 \\
50.3 \\
.2 \\
\overline{.2} \\
5.2 \\
45.0 \\
8.9 \\
15.4 \\
2.5 \\
3.1 \\
1.3 \\
5.1 \\
2.8 \\
5.8 \\
89.0\end{array}$ & $\begin{array}{r}144.1 \\
54.1 \\
.3 \\
\overline{.3} \\
5.7 \\
48.0 \\
9.5 \\
15.4 \\
.9 \\
4.8 \\
1.2 \\
5.9 \\
1.8 \\
8.4 \\
90.0\end{array}$ & $\begin{array}{r}134.5 \\
43.5 \\
.1 \\
\overline{.1} \\
6.6 \\
36.9 \\
.6 \\
17.6 \\
.7 \\
3.3 \\
1.3 \\
4.1 \\
2.4 \\
6.8 \\
91.0\end{array}$ & $\begin{array}{r}155.5 \\
57.5 \\
.5 \\
\overline{.5} \\
5.1 \\
51.9 \\
10.7 \\
15.4 \\
.4 \\
9.7 \\
1.3 \\
4.4 \\
2.1 \\
7.9 \\
98.0\end{array}$ \\
\hline 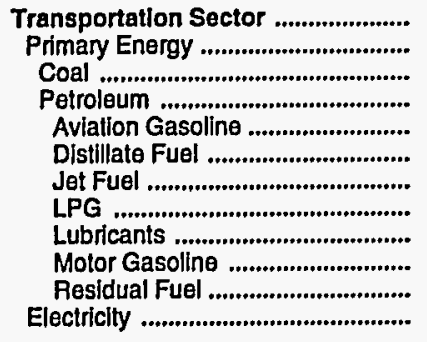 & $\begin{array}{r}86.0 \\
86.0 \\
86.0 \\
.2 \\
2.9 \\
.5 \\
1.5 \\
81.0 \\
-\end{array}$ & $\begin{array}{r}149.9 \\
149.9 \\
* \\
149.9 \\
.2 \\
8.5 \\
1.5 \\
2.1 \\
137.6 \\
-\end{array}$ & $\begin{array}{r}329.5 \\
329.5 \\
-\overline{3} \\
329.5 \\
1.1 \\
32.7 \\
4.9 \\
4 \\
4.5 \\
286.2 \\
-\end{array}$ & $\begin{array}{r}348.5 \\
348.5 \\
- \\
348.5 \\
1.1 \\
52.0 \\
6.7 \\
.6 \\
5.1 \\
283.1 \\
-\end{array}$ & $\begin{array}{r}282.2 \\
282.2 \\
282 . \overline{-} \\
1.2 \\
45.4 \\
3.1 \\
.4 \\
4.4 \\
227.7 \\
- \\
-\end{array}$ & $\begin{array}{r}318.0 \\
318.0 \\
318.0 \\
.8 \\
55.5 \\
4.2 \\
.4 \\
4.0 \\
253.0 \\
. \\
-\end{array}$ & $\begin{array}{r}343.9 \\
343.9 \\
343.9 \\
.6 \\
60.6 \\
3.2 \\
.4 \\
4.5 \\
274.6 \\
- \\
-\end{array}$ & $\begin{array}{r}363.3 \\
363.3 \\
- \\
363.3 \\
.7 \\
54.7 \\
6.6 \\
.4 \\
4.2 \\
296.6 \\
.1 \\
-\end{array}$ & $\begin{array}{r}404.5 \\
404.5 \\
- \\
404.5 \\
.7 \\
60.7 \\
6.6 \\
.4 \\
4.3 \\
331.7 \\
.1 \\
-\end{array}$ & $\begin{array}{r}397.4 \\
397.4 \\
- \\
397.4 \\
.7 \\
56.4 \\
3.9 \\
.5 \\
4.5 \\
331.5 \\
-\end{array}$ \\
\hline 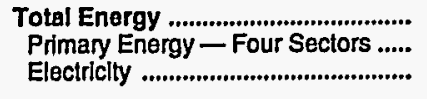 & $\begin{array}{r}202.6 \\
148.7 \\
53.9\end{array}$ & $\begin{array}{l}363.0 \\
257.5 \\
105.6\end{array}$ & $\begin{array}{l}731.7 \\
538.5 \\
193.1\end{array}$ & $\begin{array}{l}901.9 \\
616.7 \\
285.1\end{array}$ & $\begin{array}{l}778.1 \\
491.8 \\
286.2\end{array}$ & $\begin{array}{l}869.0 \\
531.2 \\
337.8\end{array}$ & $\begin{array}{l}915.9 \\
558.9 \\
357.0\end{array}$ & $\begin{array}{l}995.2 \\
629.2 \\
366.0\end{array}$ & $\begin{array}{r}1,052.8 \\
662.8 \\
390.0\end{array}$ & $\begin{array}{r}1,094.1 \\
688.0 \\
406.1\end{array}$ \\
\hline 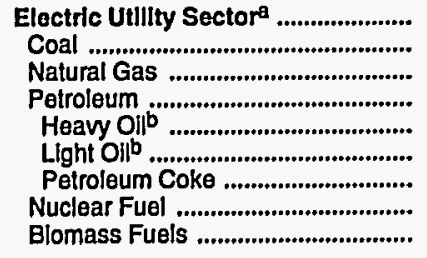 & $\begin{array}{r}2.2 \\
.7 \\
\overline{-} \\
1.6 \\
.1 \\
1.4 \\
- \\
- \\
-\end{array}$ & $\begin{array}{r}14.5 \\
.7 \\
.7 \\
1.2 \\
1.2 \\
\overline{-} \\
12.0 \\
-\end{array}$ & $\begin{array}{r}23.4 \\
.4 \\
1.1 \\
2.3 \\
-\overline{3} \\
2.3 \\
-\bar{y} \\
18.7 \\
.9\end{array}$ & $\begin{array}{r}26.1 \\
1.4 \\
.5 \\
1.1 \\
-\overline{-} \\
1.1 \\
-\overline{8} \\
20.8 \\
2.3\end{array}$ & $\begin{array}{r}17.3 \\
.6 \\
.9 \\
\overline{9} \\
.9 \\
\overline{1} \\
15.5 \\
.3\end{array}$ & $\begin{array}{r}29.2 \\
- \\
\overline{-} \\
1.4 \\
\overline{4} \\
1.4 \\
-\overline{4} \\
26 . \overline{3} \\
1.5\end{array}$ & $\begin{array}{r}33.9 \\
\overline{-} \\
1 . \overline{6} \\
\overline{-} \\
1.6 \\
\overline{\bar{c}} \\
31 . \overline{3} \\
.9\end{array}$ & $\begin{array}{r}31.0 \\
\overline{1} \\
1 . \overline{2} \\
\overline{-} \\
1.2 \\
24 . \overline{1} \\
5.5\end{array}$ & $\begin{array}{r}26.8 \\
\overline{\bar{y}} \\
1.7 \\
.2 \\
\overline{2} \\
\overline{2} \\
22 . \overline{1} \\
2 . \overline{7}\end{array}$ & $\begin{array}{r}30.1 \\
\overline{-} \\
1.9 \\
.4 \\
\overline{4} \\
.4 \\
\overline{4} \\
24 . \overline{9} \\
2.9\end{array}$ \\
\hline Primary Energy — Flve Sectors $c$.. & 150.9 & 272.0 & 561.9 & 642.9 & 509.1 & 560.5 & 592.8 & 660.2 & 689.6 & 718.1 \\
\hline
\end{tabular}

a There are no direct fuel costs for hydroelectric, geothermal, centralized solar, or wind energy.

beavy oil includes Grade Nos. 4,5, and 6, and residual fuel oils. Light oil includes Grade No. 2 heating oil, kerosene, and jet fuel.

c Biomass fuels are not included, except those consumed at electric utilities and those added to motor gasoline.

- No consumption, Including cases where adjustments were made. See "Consumption Adjustments for Process Fuel and Intermediate Products."

- Value less than 0.05 million dollars.

Note: Totals may not equal sum of components due to independent rounding.

Sources: Data sources, estimation procedures, and assumptions are described in the "Documentation" section of this report.

Energy Information Administration 
V Energy Price and Expenditure Estimates by Source, Virginia $1970,1975,1980$, and 1985-1991

\begin{tabular}{|c|c|c|c|c|c|c|c|c|c|c|}
\hline Energy Source & 1970 & 1975 & 1980 & 1985 & 1986 & 1987 & 1988 & 1989 & 1990 & 1991 \\
\hline & \multicolumn{10}{|c|}{ Prices in Dollars per Million Btu } \\
\hline 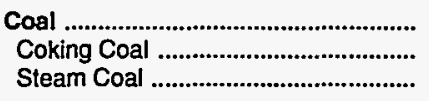 & $\begin{array}{r}0.41 \\
.40 \\
.41\end{array}$ & $\begin{array}{r}1.30 \\
1.30\end{array}$ & $\begin{array}{l}1.72 \\
1.86 \\
1.71\end{array}$ & $\begin{array}{l}1.80 \\
1.93 \\
1.78\end{array}$ & $\begin{array}{l}1.70 \\
1.75 \\
1.69\end{array}$ & $\begin{array}{l}1.60 \\
1.72 \\
1.59\end{array}$ & $\begin{array}{l}1.57 \\
1.73 \\
1.56\end{array}$ & $\begin{array}{l}1.57 \\
1.75 \\
1.56\end{array}$ & $\begin{array}{l}1.60 \\
1.80 \\
1.59\end{array}$ & $\begin{array}{l}1.57 \\
1.72 \\
1.56\end{array}$ \\
\hline 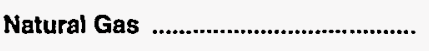 & .96 & 1.71 & 3.62 & 5.68 & 5.06 & 4.53 & 4.40 & 4.95 & 4.67 & 4.76 \\
\hline 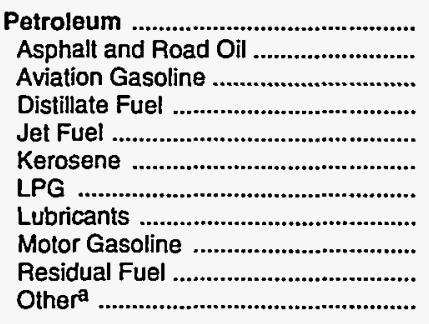 & $\begin{array}{r}1.49 \\
.68 \\
2.17 \\
1.14 \\
.73 \\
1.36 \\
1.95 \\
5.08 \\
2.85 \\
.31 \\
.75\end{array}$ & $\begin{array}{l}3.16 \\
1.82 \\
3.45 \\
2.60 \\
2.03 \\
2.93 \\
3.63 \\
7.48 \\
4.77 \\
1.80 \\
2.66\end{array}$ & $\begin{array}{r}7.47 \\
3.60 \\
9.02 \\
6.84 \\
6.46 \\
7.59 \\
6.33 \\
14.36 \\
9.97 \\
3.75 \\
7.25\end{array}$ & $\begin{array}{r}8.12 \\
4.93 \\
9.99 \\
7.77 \\
5.79 \\
8.15 \\
9.87 \\
17.61 \\
9.33 \\
4.26 \\
8.23\end{array}$ & $\begin{array}{r}5.88 \\
4.21 \\
8.41 \\
5.91 \\
3.83 \\
6.52 \\
9.24 \\
15.59 \\
6.97 \\
2.15 \\
5.62\end{array}$ & $\begin{array}{r}6.34 \\
3.29 \\
7.55 \\
6.09 \\
4.05 \\
6.02 \\
9.19 \\
12.70 \\
7.60 \\
2.99 \\
6.36\end{array}$ & $\begin{array}{r}6.37 \\
3.25 \\
7.41 \\
6.00 \\
3.77 \\
6.05 \\
9.06 \\
14.61 \\
7.85 \\
2.21 \\
5.42\end{array}$ & $\begin{array}{r}6.84 \\
2.96 \\
8.28 \\
6.54 \\
4.34 \\
6.60 \\
8.42 \\
13.30 \\
8.55 \\
2.67 \\
5.85\end{array}$ & $\begin{array}{r}7.96 \\
2.99 \\
9.32 \\
7.85 \\
5.53 \\
8.33 \\
11.25 \\
13.40 \\
9.46 \\
3.24 \\
6.56\end{array}$ & $\begin{array}{r}7.53 \\
3.10 \\
8.71 \\
7.42 \\
4.78 \\
7.58 \\
11.70 \\
15.42 \\
9.02 \\
2.03 \\
5.94\end{array}$ \\
\hline Nuclear Fuel ............................................ & - & .28 & .74 & .55 & .53 & .56 & .53 & .39 & .47 & .53 \\
\hline Blomass Fuels at Utillties .................. & - & - & - & - & - & - & - & - & - & - \\
\hline Primary Energy - Five Sectors ${ }^{b}$.... & 1.17 & 2.52 & 5.31 & 5.14 & 4.03 & 4.27 & 4.21 & 4.65 & 4.90 & 4.63 \\
\hline $\begin{array}{l}\text { Electric Utility Fuelc .......................... } \\
\text { Electricity Purchased by End Users }\end{array}$ & $\begin{array}{r}.35 \\
4.91\end{array}$ & $\begin{array}{l}1.24 \\
9.63\end{array}$ & $\begin{array}{r}2.00 \\
15.77\end{array}$ & $\begin{array}{r}1.18 \\
17.06\end{array}$ & $\begin{array}{r}1.14 \\
16.97\end{array}$ & $\begin{array}{r}1.23 \\
17.03\end{array}$ & $\begin{array}{r}1.11 \\
16.64\end{array}$ & $\begin{array}{r}1.28 \\
17.26\end{array}$ & $\begin{array}{r}1.06 \\
17.70\end{array}$ & $\begin{array}{r}1.05 \\
17.89\end{array}$ \\
\hline \multirow[t]{2}{*}{ 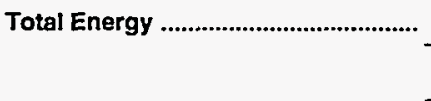 } & 1.80 & 3.97 & 8.02 & 8.79 & 7.52 & 7.68 & 7.65 & 8.29 & 8.85 & 8.73 \\
\hline & \multicolumn{10}{|c|}{ Expenditures in Millions of Dollars } \\
\hline 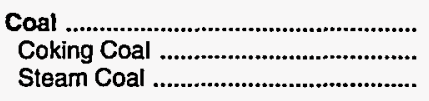 & $\begin{array}{r}113.6 \\
.3 \\
113.3\end{array}$ & $\begin{array}{r}220.7 \\
220 . \overline{7}\end{array}$ & $\begin{array}{r}398.1 \\
33.0 \\
365.1\end{array}$ & $\begin{array}{r}531.3 \\
45.7 \\
485.5\end{array}$ & $\begin{array}{r}512.1 \\
42.1 \\
470.0\end{array}$ & $\begin{array}{r}538.9 \\
43.4 \\
495.5\end{array}$ & $\begin{array}{r}537.5 \\
42.8 \\
494.7\end{array}$ & $\begin{array}{r}569.1 \\
44.0 \\
525.1\end{array}$ & $\begin{array}{r}532.6 \\
42.7 \\
489.9\end{array}$ & $\begin{array}{r}557.8 \\
44.9 \\
512.9\end{array}$ \\
\hline Natural Gas ......................................... & 126.6 & 205.0 & 548.0 & 783.7 & 706.0 & 707.2 & 701.7 & 851.4 & 830.8 & 818.0 \\
\hline 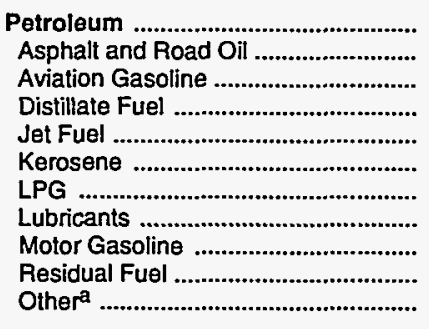 & $\begin{array}{r}1,105.6 \\
10.2 \\
3.9 \\
163.6 \\
44.9 \\
38.9 \\
17.8 \\
22.2 \\
727.8 \\
65.0 \\
11.3\end{array}$ & $\begin{array}{r}2,590.2 \\
28.1 \\
4.4 \\
344.3 \\
131.9 \\
37.7 \\
41.2 \\
33.3 \\
1.484 .6 \\
462.4 \\
22.4\end{array}$ & $\begin{array}{r}5,723.8 \\
62.6 \\
9.9 \\
980.1 \\
444.2 \\
73.9 \\
70.2 \\
82.9 \\
3,092.9 \\
575.1 \\
332.0\end{array}$ & $\begin{array}{r}5,516.5 \\
132.0 \\
6.6 \\
1,139.9 \\
357.1 \\
186.2 \\
138.4 \\
92.5 \\
3,086.1 \\
221.1 \\
156.4\end{array}$ & $\begin{array}{r}4,316.4 \\
124.2 \\
6.6 \\
976.9 \\
283.6 \\
103.8 \\
113.0 \\
80.1 \\
2,387.4 \\
158.2 \\
82.6\end{array}$ & $\begin{array}{r}4,837.2 \\
96.2 \\
2.8 \\
1,039.6 \\
327.8 \\
85.4 \\
138.0 \\
73.8 \\
2,782.4 \\
196.1 \\
95.2\end{array}$ & $\begin{array}{r}5,024.2 \\
77.6 \\
2.8 \\
1,138.9 \\
331.6 \\
104.5 \\
137.2 \\
81.8 \\
2,936.2 \\
133.6 \\
80.0\end{array}$ & $\begin{array}{r}5,346.4 \\
82.4 \\
3.1 \\
1,106.3 \\
383.1 \\
100.8 \\
135.0 \\
76.4 \\
3,183.1 \\
191.8 \\
84.4\end{array}$ & $\begin{array}{r}5,903.1 \\
93.3 \\
3.3 \\
1,276.8 \\
489.8 \\
64.9 \\
162.1 \\
79.2 \\
3,475.0 \\
152.2 \\
106.5\end{array}$ & $\begin{array}{r}\mathbf{5 , 5 0 1 . 0} \\
76.9 \\
5.2 \\
1,158.0 \\
377.6 \\
67.1 \\
193.8 \\
81.6 \\
3,340.1 \\
108.8 \\
91.9\end{array}$ \\
\hline Nuclear Fuel ......................................... & - & 27.7 & 92.8 & 131.5 & 120.4 & 108.9 & 119.0 & 60.1 & 119.6 & 135.2 \\
\hline Biomass Fuels at Utilities ................. & - & - & - & - & - & - & - & - & - & - \\
\hline Primary Energy - Five Sectors ${ }^{\mathrm{b}} \ldots$ & $1,345.8$ & $3,043.6$ & $6,762.6$ & $6,962.9$ & $5,655.0$ & $6,192.2$ & $6,382.4$ & $6,827.1$ & $7,386.1$ & $7,012.0$ \\
\hline 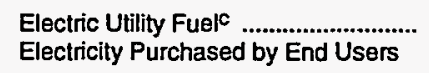 & $\begin{array}{r}-101.4 \\
494.4\end{array}$ & $\begin{array}{r}-455.1 \\
1,280.5\end{array}$ & $\begin{array}{r}-726.4 \\
2,581.5\end{array}$ & $\begin{array}{r}-514.6 \\
3,343.0\end{array}$ & $\begin{array}{r}-510.6 \\
3,643.1\end{array}$ & $\begin{array}{r}-538.8 \\
3,868.1\end{array}$ & $\begin{array}{r}-522.4 \\
3,964.8\end{array}$ & $\begin{array}{r}-568.3 \\
4,307.7\end{array}$ & $\begin{array}{r}-508.9 \\
4,374.4\end{array}$ & $\begin{array}{r}-532.0 \\
4,558.0\end{array}$ \\
\hline Total Energy & $1,738.8$ & $3,869.0$ & $8,617.8$ & $9,791.3$ & $8,787.5$ & $9,521.5$ & $9,824.9$ & $10,566.5$ & $11,251.5$ & $11,037.9$ \\
\hline
\end{tabular}

\footnotetext{
a Includes petroleum coke used at electric utilities.

b Biomass fuels are not included, except those consumed at electric utilities and those added to motor gasoline.

c There are no direct fuel costs for hydroelectric, geothermal, centralized solar, or wind energy.

-No consumption, including cases where adjustments were made. See "Consumption Adjustments for Process Fuel and Intermediate Products."

Note: Expenditure totals may not equal sum of components due to independent rounding.

Sources: Data sources, estimation procedures, and assumptions are described in the "Documentation" section of this report.
} 
Energy Price Estimates by Sector, Virginia $1970,1975,1980$, and 1985-1991

(Dollars per Million Btu)

\begin{tabular}{|c|c|c|c|c|c|c|c|c|c|c|}
\hline Sector and Energy Source & 1970 & 1975 & 1980 & 1985 & 1986 & 1987 & 1988 & 1989 & 1990 & 1991 \\
\hline 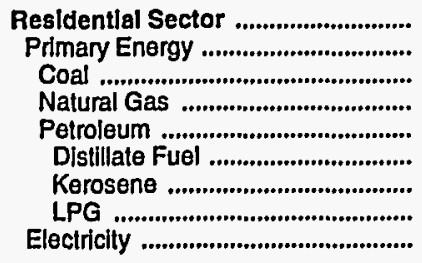 & $\begin{array}{l}2.45 \\
1.45 \\
1.34 \\
1.45 \\
1.45 \\
1.37 \\
1.44 \\
2.27 \\
6.11\end{array}$ & $\begin{array}{r}5.19 \\
2.60 \\
2.73 \\
2.20 \\
2.88 \\
2.69 \\
2.99 \\
4.43 \\
11.05\end{array}$ & $\begin{array}{r}10.23 \\
5.75 \\
3.85 \\
4.20 \\
7.32 \\
7.10 \\
7.96 \\
8.07 \\
17.80\end{array}$ & $\begin{array}{r}12.45 \\
7.51 \\
3.92 \\
6.76 \\
8.34 \\
7.89 \\
8.30 \\
10.48 \\
19.49\end{array}$ & $\begin{array}{r}12.11 \\
6.50 \\
3.59 \\
6.24 \\
6.84 \\
6.43 \\
6.76 \\
9.86 \\
19.48\end{array}$ & $\begin{array}{r}11.95 \\
6.00 \\
3.53 \\
5.63 \\
6.49 \\
5.93 \\
6.24 \\
10.07 \\
19.62\end{array}$ & $\begin{array}{r}11.76 \\
5.98 \\
3.49 \\
5.58 \\
6.48 \\
6.01 \\
6.32 \\
9.81 \\
19.29\end{array}$ & $\begin{array}{r}12.79 \\
6.69 \\
3.59 \\
6.33 \\
7.18 \\
6.49 \\
6.83 \\
11.03 \\
20.33\end{array}$ & $\begin{array}{r}14.29 \\
7.60 \\
3.48 \\
6.48 \\
9.15 \\
8.25 \\
8.68 \\
13.03 \\
21.24\end{array}$ & $\begin{array}{r}14.50 \\
7.45 \\
3.35 \\
6.53 \\
8.79 \\
7.54 \\
7.93 \\
13.55 \\
21.51\end{array}$ \\
\hline 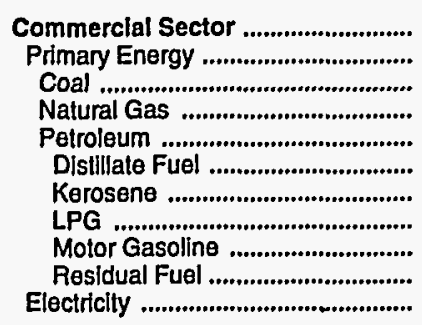 & $\begin{array}{r}2.53 \\
.94 \\
.42 \\
.94 \\
1.18 \\
1.08 \\
.65 \\
1.48 \\
2.85 \\
.32 \\
4.84\end{array}$ & $\begin{array}{l}5.50 \\
1.93 \\
1.47 \\
1.69 \\
2.60 \\
2.37 \\
2.36 \\
2.79 \\
4.77 \\
1.85 \\
9.49\end{array}$ & $\begin{array}{r}10.06 \\
4.30 \\
1.64 \\
3.71 \\
6.32 \\
6.46 \\
5.94 \\
4.60 \\
9.97 \\
3.91 \\
15.79\end{array}$ & $\begin{array}{r}12.09 \\
5.81 \\
1.69 \\
5.76 \\
6.72 \\
6.54 \\
6.81 \\
9.35 \\
9.33 \\
4.29 \\
17.35\end{array}$ & $\begin{array}{r}11.46 \\
4.62 \\
1.61 \\
5.07 \\
4.45 \\
4.62 \\
4.71 \\
8.72 \\
6.97 \\
2.40 \\
17.21\end{array}$ & $\begin{array}{r}11.21 \\
4.28 \\
1.55 \\
4.46 \\
4.54 \\
4.31 \\
4.54 \\
8.45 \\
7.60 \\
3.05 \\
17.12\end{array}$ & $\begin{array}{r}11.05 \\
4.19 \\
1.53 \\
4.28 \\
4.58 \\
4.12 \\
4.34 \\
8.49 \\
7.85 \\
2.32 \\
16.55\end{array}$ & $\begin{array}{r}11.74 \\
4.74 \\
1.56 \\
4.74 \\
5.22 \\
4.72 \\
5.40 \\
6.02 \\
8.55 \\
2.72 \\
16.89\end{array}$ & $\begin{array}{r}12.21 \\
5.10 \\
1.64 \\
4.73 \\
6.56 \\
6.11 \\
6.45 \\
9.21 \\
9.46 \\
3.31 \\
17.14\end{array}$ & $\begin{array}{r}12.32 \\
4.91 \\
1.61 \\
4.66 \\
6.02 \\
5.38 \\
5.64 \\
9.80 \\
9.02 \\
2.32 \\
17.16\end{array}$ \\
\hline 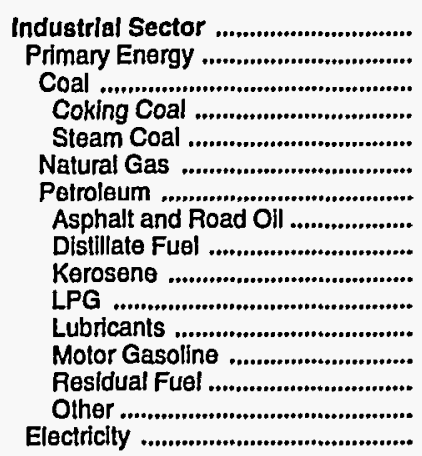 & $\begin{array}{r}.81 \\
.57 \\
.42 \\
.40 \\
.42 \\
.49 \\
.79 \\
.68 \\
.60 \\
.65 \\
1.48 \\
5.08 \\
2.85 \\
.34 \\
.96 \\
3.08\end{array}$ & $\begin{array}{l}2.50 \\
1.74 \\
1.47 \\
1.47 \\
1.08 \\
2.18 \\
1.82 \\
2.19 \\
2.36 \\
2.79 \\
7.48 \\
4.77 \\
1.81 \\
2.66 \\
7.37\end{array}$ & $\begin{array}{r}4.83 \\
3.77 \\
1.69 \\
1.86 \\
1.64 \\
2.99 \\
5.58 \\
3.60 \\
5.33 \\
5.94 \\
4.60 \\
14.36 \\
9.97 \\
3.58 \\
7.25 \\
12.19\end{array}$ & $\begin{array}{r}5.38 \\
4.10 \\
1.74 \\
1.93 \\
1.69 \\
4.60 \\
6.44 \\
4.93 \\
6.10 \\
6.81 \\
9.35 \\
17.61 \\
9.33 \\
4.29 \\
8.23 \\
12.47\end{array}$ & $\begin{array}{r}4.65 \\
3.22 \\
1.64 \\
1.75 \\
1.61 \\
3.81 \\
4.70 \\
4.21 \\
4.22 \\
4.71 \\
8.72 \\
15.59 \\
6.97 \\
2.40 \\
5.62 \\
12.07\end{array}$ & $\begin{array}{r}4.52 \\
3.10 \\
1.59 \\
1.72 \\
1.55 \\
3.50 \\
4.73 \\
3.29 \\
4.06 \\
4.54 \\
8.45 \\
12.70 \\
7.60 \\
3.05 \\
6.36 \\
12.12\end{array}$ & $\begin{array}{r}4.44 \\
2.93 \\
1.57 \\
1.73 \\
1.53 \\
3.22 \\
4.53 \\
3.25 \\
3.89 \\
4.34 \\
8.49 \\
14.61 \\
7.85 \\
2.32 \\
5.42 \\
11.95\end{array}$ & $\begin{array}{r}4.70 \\
3.11 \\
1.60 \\
1.75 \\
1.56 \\
3.76 \\
4.56 \\
2.96 \\
4.84 \\
5.40 \\
6.02 \\
13.30 \\
8.55 \\
2.72 \\
5.85 \\
12.33\end{array}$ & $\begin{array}{r}4.77 \\
3.28 \\
1.67 \\
1.80 \\
1.64 \\
3.52 \\
5.11 \\
2.99 \\
5.78 \\
6.45 \\
9.21 \\
13.40 \\
9.46 \\
3.31 \\
6.56 \\
12.51\end{array}$ & $\begin{array}{r}4.61 \\
3.10 \\
1.63 \\
1.72 \\
1.61 \\
3.66 \\
5.09 \\
3.10 \\
5.04 \\
5.64 \\
9.80 \\
15.42 \\
9.02 \\
2.32 \\
5.94 \\
12.40\end{array}$ \\
\hline 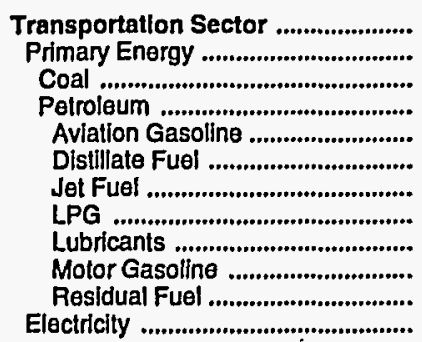 & $\begin{array}{r}1.95 \\
1.95 \\
.42 \\
1.95 \\
2.17 \\
1.25 \\
.73 \\
1.48 \\
5.08 \\
2.85 \\
.30 \\
-\end{array}$ & $\begin{array}{l}3.91 \\
3.91 \\
1.47 \\
3.91 \\
3.45 \\
2.72 \\
2.03 \\
2.79 \\
7.48 \\
4.77 \\
1.61\end{array}$ & $\begin{array}{r}8.73 \\
8.72 \\
8.72 \\
9.02 \\
7.27 \\
6.46 \\
4.60 \\
14.36 \\
9.97 \\
3.32 \\
14.65\end{array}$ & $\begin{array}{r}8.55 \\
8.55 \\
-\overline{8} \\
8.55 \\
9.99 \\
8.34 \\
5.79 \\
9.35 \\
17.61 \\
9.33 \\
4.18 \\
17.33\end{array}$ & $\begin{array}{r}6.29 \\
6.29 \\
6.29 \\
8.41 \\
6.34 \\
3.83 \\
8.72 \\
15.59 \\
6.97 \\
1.91 \\
18.20\end{array}$ & $\begin{array}{r}6.85 \\
6.85 \\
- \\
6.85 \\
7.55 \\
6.93 \\
4.05 \\
8.45 \\
12.70 \\
7.60 \\
2.81 \\
16.57\end{array}$ & $\begin{array}{r}6.90 \\
6.89 \\
-\overline{6} \\
7.89 \\
6.74 \\
3.77 \\
8.49 \\
14.61 \\
7.85 \\
1.99 \\
16.10\end{array}$ & $\begin{array}{r}7.53 \\
7.53 \\
-\overline{1} \\
7.53 \\
8.28 \\
7.26 \\
4.34 \\
6.02 \\
13.30 \\
8.55 \\
2.34 \\
15.09\end{array}$ & $\begin{array}{r}8.46 \\
8.46 \\
8.46 \\
9.32 \\
8.40 \\
5.53 \\
9.21 \\
13.40 \\
9.46 \\
3.03 \\
15.55\end{array}$ & $\begin{array}{r}8.01 \\
8.01 \\
8 . \overline{1} \\
8.01 \\
8.71 \\
8.10 \\
4.78 \\
9.80 \\
15.42 \\
9.02 \\
1.82 \\
15.68\end{array}$ \\
\hline 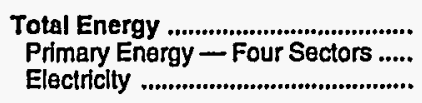 & $\begin{array}{l}1.80 \\
1.44 \\
4.91\end{array}$ & $\begin{array}{l}3.97 \\
3.08 \\
9.63\end{array}$ & $\begin{array}{r}8.02 \\
6.62 \\
15.77\end{array}$ & $\begin{array}{r}8.79 \\
7.02 \\
17.06\end{array}$ & $\begin{array}{r}7.52 \\
5.39 \\
16.97\end{array}$ & $\begin{array}{r}7.68 \\
5.58 \\
17.03\end{array}$ & $\begin{array}{r}7.65 \\
5.61 \\
16.64\end{array}$ & $\begin{array}{r}8.29 \\
6.10 \\
17.26\end{array}$ & $\begin{array}{r}8.85 \\
6.72 \\
17.70\end{array}$ & $\begin{array}{r}8.73 \\
6.42 \\
17.89\end{array}$ \\
\hline 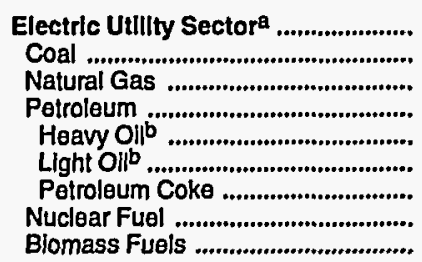 & $\begin{array}{r}.35 \\
.38 \\
.29 \\
.32 \\
.31 \\
.35 \\
.35 \\
-\end{array}$ & $\begin{array}{r}1.24 \\
1.14 \\
.99 \\
1.85 \\
1.84 \\
2.18 \\
-.28 \\
-\end{array}$ & $\begin{array}{r}2.00 \\
1.71 \\
2.89 \\
4.03 \\
3.94 \\
5.86 \\
-74 \\
-\end{array}$ & $\begin{array}{r}1.18 \\
1.80 \\
3.44 \\
4.60 \\
4.37 \\
5.57 \\
-55 \\
.5\end{array}$ & $\begin{array}{r}1.14 \\
1.71 \\
1.93 \\
2.16 \\
2.09 \\
3.39 \\
-53 \\
-\end{array}$ & $\begin{array}{r}1.23 \\
1.58 \\
2.42 \\
3.11 \\
3.06 \\
3.73 \\
- \\
.56 \\
-\end{array}$ & $\begin{array}{r}1.11 \\
1.55 \\
2.05 \\
2.38 \\
2.30 \\
3.38 \\
- \\
.53 \\
-\end{array}$ & $\begin{array}{r}1.28 \\
1.55 \\
2.48 \\
2.88 \\
2.78 \\
4.01 \\
.39 \\
-\end{array}$ & $\begin{array}{r}1.06 \\
1.54 \\
2.58 \\
4.14 \\
3.60 \\
5.83 \\
.47 \\
-\end{array}$ & $\begin{array}{r}1.05 \\
1.52 \\
1.82 \\
2.36 \\
2.13 \\
4.64 \\
- \\
.53 \\
-\end{array}$ \\
\hline Primary Energy - Flve Sectors $c$.. & 1.17 & 2.52 & 5.31 & 5.14 & 4.03 & 4.27 & 4.21 & 4.65 & 4.90 & 4.63 \\
\hline
\end{tabular}

a There are no direct fuel costs for hydroelectric, geothermal, centralized solar, or wind energy.

b Heavy oil includes Grade Nos. 4, 5, and 6, and residual fuel oils. Light oil includes Grade No. 2 heating oil, kerosene, and jet fuel.

c Blomass fuels are not included, except those consumed at electric utilities and those added to motor gasoline.

-No consumption, including cases where adjustments were made. See "Consumption Adjustments for Process Fuel and Intermediate Products."

Sources: Data sources, estimation procedures, and assumptions are described in the "Documentation" section of this report. 


\section{Energy Expenditure Estimates by Sector, Virginia 1970, 1975, 1980, and 1985-1991}

R (Million Dollars)

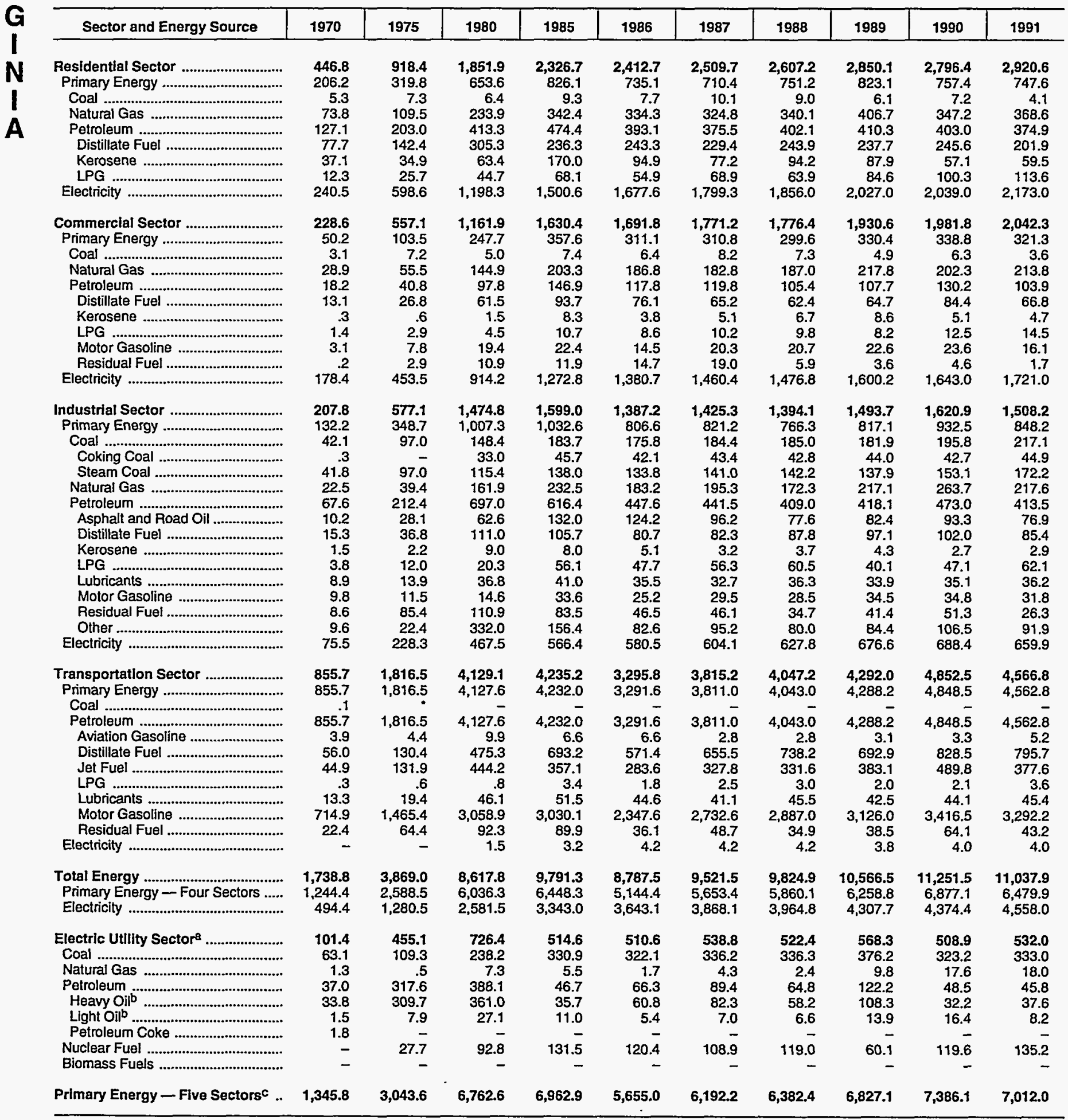

a There are no direct fuel costs for hydroelectric, geothermal, centralized solar, or wind energy.

b Heavy oil includes Grade Nos. 4, 5, and 6, and residual fuel oils. Light oil includes Grade No. 2 heating oil, kerosene, and jet fuel.

c Biomass fuels are not included, except those consumed at electric utilities and those added to motor gasoline.

-No consumption, including cases where adjustments were made. See "Consumption Adjustments for Process Fuel and Intermediate Products."

-Value less than 0.05 million dollars.

Note: Totals may not equal sum of components due to independent rounding.

Sources: Data sources, estimation procedures, and assumptions are described in the "Documentation" section of this report. 


\begin{tabular}{|c|c|c|c|c|c|c|c|c|c|c|}
\hline Energy Source & 1970 & 1975 & 1980 & 1985 & 1986 & 1987 & 1988 & 1989 & 1990 & 1991 \\
\hline & \multicolumn{10}{|c|}{ Prices in Dollars per Million Btu } \\
\hline 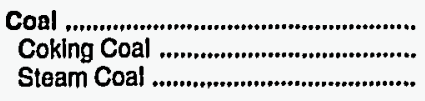 & $\begin{array}{r}0.54 \\
.54\end{array}$ & $\begin{array}{r}0.61 \\
-61\end{array}$ & $\frac{1.15}{1.15}$ & $\begin{array}{r}1.74 \\
-\overline{7}\end{array}$ & $\frac{1.78}{1.78}$ & $\frac{1.73}{1.73}$ & $\begin{array}{r}1.62 \\
1.62\end{array}$ & $\begin{array}{r}1.62 \\
-\overline{-} \\
1.62\end{array}$ & $\begin{array}{r}1.65 \\
1.65\end{array}$ & $\begin{array}{r}1.65 \\
1.65\end{array}$ \\
\hline 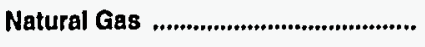 & .71 & 1.60 & 4.48 & 5.23 & 4.53 & 3.81 & 3.96 & 4.02 & 3.60 & 3.55 \\
\hline 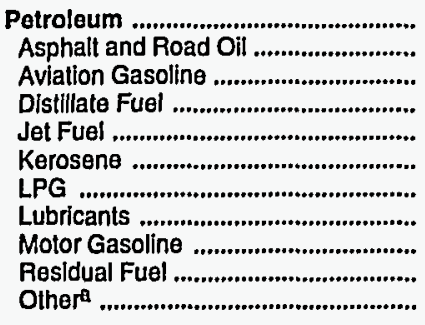 & $\begin{array}{r}1.71 \\
.66 \\
2.17 \\
1.18 \\
.73 \\
1.62 \\
2.50 \\
5.08 \\
2.92 \\
.32 \\
.60\end{array}$ & $\begin{array}{l}3.24 \\
1.77 \\
3.45 \\
2.55 \\
2.04 \\
3.07 \\
4.46 \\
7.48 \\
4.62 \\
1.93 \\
1.50\end{array}$ & $\begin{array}{r}7.11 \\
3.70 \\
9.02 \\
6.68 \\
6.21 \\
8.53 \\
6.78 \\
14.36 \\
9.92 \\
3.24 \\
2.97\end{array}$ & $\begin{array}{r}7.64 \\
4.17 \\
9.99 \\
7.80 \\
6.03 \\
7.22 \\
9.11 \\
17.61 \\
9.31 \\
4.53 \\
3.43\end{array}$ & $\begin{array}{r}5.67 \\
3.94 \\
8.41 \\
5.95 \\
4.01 \\
4.49 \\
9.21 \\
15.59 \\
7.29 \\
2.55 \\
3.05\end{array}$ & $\begin{array}{r}5.75 \\
3.21 \\
7.55 \\
6.22 \\
4.02 \\
4.76 \\
8.93 \\
12.70 \\
7.36 \\
2.59 \\
2.15\end{array}$ & $\begin{array}{r}5.57 \\
3.41 \\
7.41 \\
6.31 \\
3.79 \\
4.89 \\
8.98 \\
14.61 \\
7.40 \\
2.17 \\
1.95\end{array}$ & $\begin{array}{r}6.14 \\
3.29 \\
8.28 \\
7.06 \\
4.50 \\
6.04 \\
9.30 \\
13.30 \\
8.03 \\
2.19 \\
2.17\end{array}$ & $\begin{array}{r}7.06 \\
3.18 \\
9.32 \\
7.98 \\
5.68 \\
8.97 \\
10.05 \\
13.40 \\
9.45 \\
2.70 \\
2.28\end{array}$ & $\begin{array}{r}7.16 \\
3.40 \\
8.71 \\
7.78 \\
4.76 \\
8.58 \\
10.66 \\
15.42 \\
9.14 \\
5.18 \\
2.09\end{array}$ \\
\hline Nuclear Fuel .......................................... & .18 & .24 & .43 & .71 & .70 & .70 & .49 & .48 & .47 & .45 \\
\hline Blomass Fuels at Utilities ..................... & .65 & - & - & .79 & .32 & .95 & .87 & .79 & .61 & .76 \\
\hline Primary Energy - Five Sectors ${ }^{b}$.... & 1.42 & 2.51 & 5.83 & 5.92 & 4.72 & 4.72 & 4.57 & 4.98 & 5.64 & 5.73 \\
\hline $\begin{array}{l}\text { Electric Utility Fuelc .............................. } \\
\text { Electricity Purchased by End Users }\end{array}$ & $\begin{array}{r}.18 \\
2.02\end{array}$ & 2.76 & $\begin{array}{r}.91 \\
4.16\end{array}$ & $\begin{array}{l}1.17 \\
9.18\end{array}$ & $\begin{array}{l}1.06 \\
9.41\end{array}$ & $\begin{array}{l}1.28 \\
9.80\end{array}$ & $\begin{array}{r}1.13 \\
10.13\end{array}$ & $\begin{array}{r}1.31 \\
10.34\end{array}$ & $\begin{array}{r}1.09 \\
10.03\end{array}$ & $\begin{array}{l}1.16 \\
9.96\end{array}$ \\
\hline \multirow[t]{2}{*}{ Total Energy .......................................... } & 1.59 & 2.82 & 5.98 & 7.67 & 6.45 & 6.48 & 6.52 & 6.92 & 7.35 & 7.36 \\
\hline & \multicolumn{10}{|c|}{ Expenditures In Millions of Dollars } \\
\hline 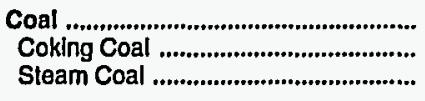 & $\begin{array}{r}3.2 \\
3 . \overline{2}\end{array}$ & $\begin{array}{r}46.9 \\
46 . \overline{9}\end{array}$ & $\begin{array}{r}104.2 \\
104 . \overline{2}\end{array}$ & $\begin{array}{r}163.4 \\
163.4\end{array}$ & $\begin{array}{r}112.7 \\
112 . \overline{7}\end{array}$ & $\begin{array}{r}165.3 \\
-\overline{3}\end{array}$ & $\begin{array}{r}160.9 \\
160.9\end{array}$ & $\begin{array}{r}157.5 \\
- \\
157.5\end{array}$ & $\begin{array}{r}141.3 \\
141.3\end{array}$ & $\begin{array}{r}147.0 \\
147.0\end{array}$ \\
\hline Natural Gas ......................................... & 97.2 & 242.3 & 530.5 & 686.4 & 510.2 & 476.0 & 551.3 & 627.7 & 554.3 & 590.0 \\
\hline 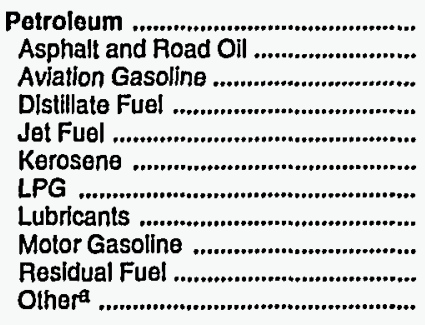 & $\begin{array}{r}812.2 \\
10.2 \\
3.8 \\
123.0 \\
43.3 \\
2.2 \\
15.3 \\
20.5 \\
553.3 \\
17.9 \\
22.7\end{array}$ & $\begin{array}{r}1,631.0 \\
34.1 \\
4.8 \\
248.4 \\
160.7 \\
6.0 \\
11.5 \\
28.2 \\
994.2 \\
82.8 \\
60.3\end{array}$ & $\begin{array}{r}3,938.8 \\
50.3 \\
16.2 \\
715.7 \\
419.5 \\
5.8 \\
33.4 \\
61.2 \\
2,222.4 \\
327.7 \\
86.5\end{array}$ & $\begin{array}{r}4,205.7 \\
56.4 \\
10.2 \\
923.8 \\
522.2 \\
49.6 \\
69.3 \\
68.3 \\
2,151.5 \\
314.2 \\
40.2\end{array}$ & $\begin{array}{r}3,463.7 \\
62.9 \\
9.7 \\
801.8 \\
385.1 \\
19.1 \\
67.5 \\
59.1 \\
1,798.4 \\
236.4 \\
23.7\end{array}$ & $\begin{array}{r}3,648.8 \\
48.3 \\
10.5 \\
766.2 \\
421.2 \\
23.2 \\
91.4 \\
54.5 \\
1,977.9 \\
213.1 \\
42.5\end{array}$ & $\begin{array}{r}3,652.0 \\
43.5 \\
8.0 \\
772.8 \\
440.3 \\
26.2 \\
74.8 \\
60.4 \\
1,974.4 \\
210.7 \\
40.9\end{array}$ & $\begin{array}{r}4,161.4 \\
57.1 \\
7.9 \\
864.4 \\
522.5 \\
24.4 \\
98.8 \\
56.4 \\
2,268.1 \\
208.5 \\
53.3\end{array}$ & $\begin{array}{r}4,923.2 \\
52.4 \\
14.7 \\
1,012.1 \\
716.0 \\
3.8 \\
77.2 \\
58.5 \\
2,639.3 \\
269.6 \\
79.6\end{array}$ & $\begin{array}{r}4,914.4 \\
67.0 \\
11.8 \\
902.9 \\
579.7 \\
3.4 \\
83.1 \\
60.2 \\
2,604.0 \\
552.2 \\
50.1\end{array}$ \\
\hline Nuclear Fuel ......................................... & 5.2 & 8.7 & 9.6 & 61.4 & 63.7 & 41.8 & 31.6 & 31.7 & 29.1 & 20.4 \\
\hline Blomass Fuols at Utillties ................. & • & - & - & 2.3 & .6 & 3.4 & 3.4 & 3.0 & 2.1 & 2.2 \\
\hline Prlmary Energy - Flve Sectors ${ }^{b} \ldots$ & 917.9 & $1,928.9$ & $4,583.0$ & $5,119.2$ & $4,151.0$ & $4,335.4$ & $4,399.1$ & $4,981.4$ & $5,650.0$ & $5,674.0$ \\
\hline $\begin{array}{l}\text { Electric Utility Fuelc ........................... } \\
\text { Electricity Purchased by End Users }\end{array}$ & $\begin{array}{r}-5.2 \\
316.8\end{array}$ & $\begin{array}{r}-46.5 \\
523.9\end{array}$ & $\begin{array}{l}-95.7 \\
953.4\end{array}$ & $\begin{array}{r}-203.6 \\
2,331.7\end{array}$ & $\begin{array}{r}-155.9 \\
2,312.1\end{array}$ & $\begin{array}{r}-194.0 \\
2,427.3\end{array}$ & $\begin{array}{r}-182.6 \\
2,800.5\end{array}$ & $\begin{array}{r}-221.5 \\
2,983.8\end{array}$ & $\begin{array}{r}-157.2 \\
3,033.7\end{array}$ & $\begin{array}{r}-152.5 \\
3,069.4\end{array}$ \\
\hline Total Energy & $1,229,4$ & $2,406.2$ & $5,440.7$ & $7,247.3$ & $6,307.1$ & $6,568.6$ & $7,017.0$ & 7,743.7 & $8,526.5$ & $8,590.8$ \\
\hline
\end{tabular}

a Includes petroleum coke used at electric utilities.

b Biomass fuels are not included, except those consumed at electric utilities and those added to motor gasoline.

c There are no direct fuel costs for hydroelectric, geothermal, centralized solar, or wind energy.

-No consumption, including cases where adjustments were made. See "Consumption Adjustments for Process Fuel and Intermediate Products."

-Value less than 0.05 million dollars.

Note: Expenditure totals may not equal sum of components due to independent rounding.

Sources: Data sources, estimation procedures, and assumptions are described in the "Documentation" section of this report. 
W Energy Price Estimates by Sector, Washington

A $1970,1975,1980$, and 1985-1991

S (Dollars per Million Btu)

\begin{tabular}{|c|c|c|c|c|c|c|c|c|c|c|}
\hline Sector and Energy Source & 1970 & 1975 & 1980 & 1985 & 1986 & 1987 & 1988 & 1989 & 1990 & 1991 \\
\hline 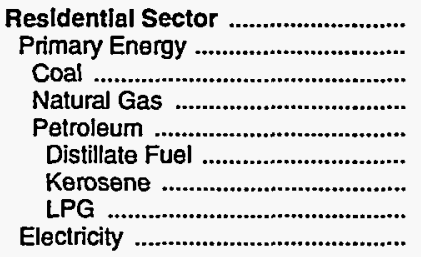 & $\begin{array}{r}2.12 \\
1.47 \\
.95 \\
1.33 \\
1.57 \\
1.40 \\
2.47 \\
3.05 \\
3.12\end{array}$ & $\begin{array}{l}3.23 \\
2.54 \\
1.14 \\
2.18 \\
2.97 \\
2.80 \\
3.61 \\
5.73 \\
3.94\end{array}$ & $\begin{array}{l}5.73 \\
5.99 \\
4.26 \\
5.05 \\
7.40 \\
7.27 \\
9.80 \\
8.12 \\
5.56\end{array}$ & $\begin{array}{r}9.53 \\
6.82 \\
3.67 \\
6.35 \\
7.88 \\
7.76 \\
9.92 \\
8.46 \\
11.14\end{array}$ & $\begin{array}{r}9.45 \\
5.93 \\
3.79 \\
5.76 \\
6.28 \\
5.95 \\
7.61 \\
9.77 \\
11.45\end{array}$ & $\begin{array}{r}9.83 \\
5.78 \\
3.76 \\
5.25 \\
6.62 \\
6.10 \\
7.80 \\
10.26 \\
12.20\end{array}$ & $\begin{array}{r}9.90 \\
5.72 \\
3.37 \\
5.36 \\
6.47 \\
6.03 \\
7.70 \\
10.31 \\
12.45\end{array}$ & $\begin{array}{r}10.19 \\
6.02 \\
3.66 \\
5.32 \\
7.61 \\
6.71 \\
8.58 \\
13.86 \\
12.69\end{array}$ & $\begin{array}{r}10.22 \\
6.02 \\
3.77 \\
4.87 \\
8.45 \\
7.90 \\
10.10 \\
12.32 \\
12.88\end{array}$ & $\begin{array}{r}9.99 \\
5.67 \\
5.21 \\
4.54 \\
8.70 \\
7.80 \\
9.97 \\
12.62 \\
12.79\end{array}$ \\
\hline 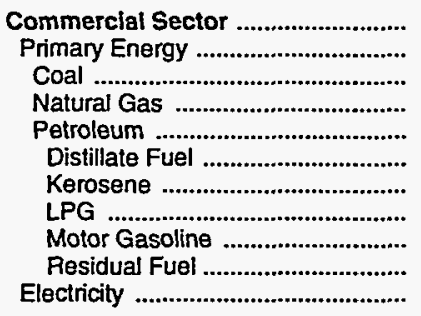 & $\begin{array}{r}1.90 \\
1.12 \\
.52 \\
1.05 \\
1.21 \\
1.21 \\
.84 \\
1.19 \\
2.92 \\
.33 \\
3.21\end{array}$ & $\begin{array}{r}2.94 \\
2.06 \\
.90 \\
1.75 \\
2.87 \\
2.60 \\
2.31 \\
2.67 \\
4.62 \\
2.45 \\
4.10\end{array}$ & $\begin{array}{l}5.35 \\
5.03 \\
2.28 \\
4.59 \\
6.76 \\
6.90 \\
7.04 \\
5.60 \\
9.92 \\
3.61 \\
5.67\end{array}$ & $\begin{array}{r}7.94 \\
5.62 \\
2.30 \\
5.24 \\
6.38 \\
6.53 \\
7.02 \\
9.34 \\
9.31 \\
4.05 \\
10.57\end{array}$ & $\begin{array}{r}8.22 \\
4.81 \\
2.22 \\
4.91 \\
4.82 \\
4.63 \\
4.27 \\
9.06 \\
7.29 \\
2.07 \\
10.95\end{array}$ & $\begin{array}{r}8.04 \\
4.51 \\
2.36 \\
4.36 \\
4.84 \\
4.55 \\
4.61 \\
8.52 \\
7.36 \\
2.58 \\
10.92\end{array}$ & $\begin{array}{r}7.94 \\
4.49 \\
2.34 \\
4.47 \\
4.66 \\
4.53 \\
4.71 \\
8.58 \\
7.40 \\
2.01 \\
11.01\end{array}$ & $\begin{array}{r}8.49 \\
4.80 \\
2.38 \\
4.54 \\
5.65 \\
5.31 \\
5.83 \\
8.09 \\
8.03 \\
2.33 \\
11.47\end{array}$ & $\begin{array}{r}8.63 \\
4.65 \\
2.45 \\
4.02 \\
6.52 \\
6.17 \\
6.80 \\
9.03 \\
9.45 \\
2.84 \\
11.63\end{array}$ & $\begin{array}{r}8.57 \\
4.29 \\
2.73 \\
3.94 \\
5.72 \\
5.38 \\
5.93 \\
9.29 \\
9.14 \\
2.30 \\
11.77\end{array}$ \\
\hline 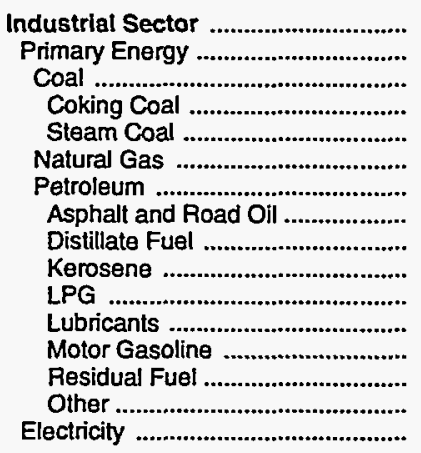 & $\begin{array}{r}.67 \\
.55 \\
.52 \\
- \\
.52 \\
.38 \\
.67 \\
.66 \\
.73 \\
.84 \\
1.19 \\
5.08 \\
2.92 \\
.33 \\
.60 \\
.97\end{array}$ & $\begin{array}{r}1.52 \\
1.58 \\
.90 \\
-\overline{.90} \\
1.29 \\
1.85 \\
1.77 \\
2.05 \\
2.31 \\
2.67 \\
7.48 \\
4.62 \\
1.78 \\
1.50 \\
1.37\end{array}$ & $\begin{array}{r}3.40 \\
4.08 \\
2.28 \\
- \\
2.28 \\
4.09 \\
4.19 \\
3.70 \\
6.06 \\
7.04 \\
5.60 \\
14.36 \\
9.92 \\
3.36 \\
2.97 \\
2.26\end{array}$ & $\begin{array}{r}5.40 \\
4.88 \\
2.30 \\
-\overline{2} \\
2.30 \\
4.58 \\
5.22 \\
4.17 \\
6.20 \\
7.02 \\
9.34 \\
17.61 \\
9.31 \\
4.05 \\
3.43 \\
6.23\end{array}$ & $\begin{array}{r}4.58 \\
3.52 \\
2.22 \\
-\overline{2} \\
2.22 \\
3.49 \\
3.65 \\
3.94 \\
3.78 \\
4.27 \\
9.06 \\
15.59 \\
7.29 \\
2.07 \\
3.05 \\
6.29\end{array}$ & $\begin{array}{r}4.50 \\
3.27 \\
2.36 \\
- \\
2.36 \\
2.78 \\
3.62 \\
3.21 \\
4.08 \\
4.61 \\
8.52 \\
12.70 \\
7.36 \\
2.58 \\
2.15 \\
6.71\end{array}$ & $\begin{array}{r}4.96 \\
3.09 \\
2.34 \\
-\overline{2} \\
2.34 \\
2.89 \\
3.27 \\
3.41 \\
4.16 \\
4.71 \\
8.58 \\
14.61 \\
7.40 \\
2.01 \\
1.95 \\
7.68\end{array}$ & $\begin{array}{r}5.35 \\
3.50 \\
2.38 \\
-\overline{2} \\
2.38 \\
2.84 \\
4.10 \\
3.29 \\
5.16 \\
5.83 \\
8.09 \\
13.30 \\
8.03 \\
2.33 \\
2.17 \\
7.77\end{array}$ & $\begin{array}{r}5.00 \\
3.49 \\
2.45 \\
-\overline{2} \\
2.45 \\
2.64 \\
4.19 \\
3.18 \\
6.01 \\
6.80 \\
9.03 \\
13.40 \\
9.45 \\
2.84 \\
2.28 \\
7.00\end{array}$ & $\begin{array}{r}4.92 \\
3.43 \\
2.73 \\
- \\
2.73 \\
2.71 \\
4.15 \\
3.40 \\
5.24 \\
5.93 \\
9.29 \\
15.42 \\
9.14 \\
2.30 \\
2.09 \\
6.72\end{array}$ \\
\hline 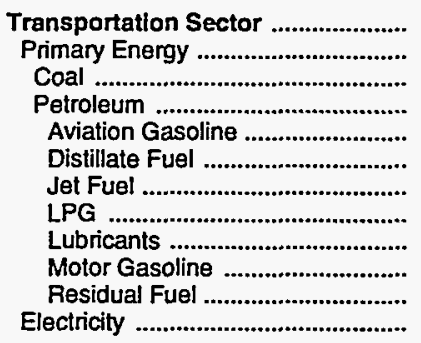 & $\begin{array}{r}2.23 \\
2.23 \\
.52 \\
2.23 \\
2.17 \\
1.32 \\
.73 \\
1.19 \\
5.08 \\
2.92 \\
.30 \\
2.16\end{array}$ & $\begin{array}{r}3.73 \\
3.73 \\
.90 \\
3.73 \\
3.45 \\
2.65 \\
2.04 \\
2.67 \\
7.48 \\
4.62 \\
2.14 \\
3.20\end{array}$ & $\begin{array}{r}7.86 \\
7.86 \\
- \\
7.86 \\
9.02 \\
6.72 \\
6.21 \\
5.60 \\
14.36 \\
9.92 \\
3.15 \\
4.26\end{array}$ & $\begin{array}{r}8.24 \\
8.24 \\
- \\
8.24 \\
9.99 \\
8.77 \\
6.03 \\
9.34 \\
17.61 \\
9.31 \\
5.02 \\
8.28\end{array}$ & $\begin{array}{r}6.07 \\
6.07 \\
- \\
6.07 \\
8.41 \\
6.71 \\
4.01 \\
9.06 \\
15.59 \\
7.29 \\
2.86 \\
8.39\end{array}$ & $\begin{array}{r}6.18 \\
6.18 \\
- \\
6.18 \\
7.55 \\
7.22 \\
4.02 \\
8.52 \\
12.70 \\
7.36 \\
2.60 \\
7.63\end{array}$ & $\begin{array}{r}6.00 \\
6.00 \\
- \\
6.00 \\
7.41 \\
7.24 \\
3.79 \\
8.58 \\
14.61 \\
7.40 \\
2.26 \\
8.49\end{array}$ & $\begin{array}{r}6.42 \\
6.42 \\
- \\
6.42 \\
8.28 \\
7.92 \\
4.50 \\
8.09 \\
13.30 \\
8.03 \\
2.18 \\
9.19\end{array}$ & $\begin{array}{r}7.50 \\
7.50 \\
- \\
7.50 \\
9.32 \\
9.04 \\
5.68 \\
9.03 \\
13.40 \\
9.45 \\
2.69 \\
9.15\end{array}$ & $\begin{array}{r}7.56 \\
7.56 \\
- \\
7.56 \\
8.71 \\
8.94 \\
4.76 \\
9.29 \\
15.42 \\
9.14 \\
5.36 \\
9.38\end{array}$ \\
\hline $\begin{array}{l}\text { Total Energy .................................... } \\
\text { Primary Energy - Four Sectors ...... } \\
\text { Electricity ......................................... }\end{array}$ & $\begin{array}{l}1.59 \\
1.48 \\
2.02\end{array}$ & $\begin{array}{l}2.82 \\
2.83 \\
2.77\end{array}$ & $\begin{array}{l}5.98 \\
6.59 \\
4.16\end{array}$ & $\begin{array}{l}7.67 \\
7.11 \\
9.18\end{array}$ & $\begin{array}{l}6.45 \\
5.46 \\
9.41\end{array}$ & $\begin{array}{l}6.48 \\
5.41 \\
9.80\end{array}$ & $\begin{array}{r}6.52 \\
5.27 \\
10.13\end{array}$ & $\begin{array}{r}6.92 \\
5.73 \\
10.34\end{array}$ & $\begin{array}{r}7.35 \\
6.41 \\
10.03\end{array}$ & $\begin{array}{l}7.36 \\
6.43 \\
9.96\end{array}$ \\
\hline 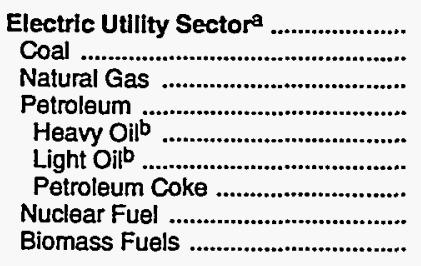 & $\begin{array}{l}.18 \\
- \\
- \\
.33 \\
.32 \\
.37 \\
- \\
.18 \\
.65\end{array}$ & $\begin{array}{r}.46 \\
.56 \\
-\overline{2} \\
2.49 \\
2.50 \\
2.41 \\
- \\
.24 \\
-\end{array}$ & $\begin{array}{r}.91 \\
.96 \\
3.43 \\
3.93 \\
3.58 \\
6.40 \\
-\overline{43} \\
-\end{array}$ & $\begin{array}{r}1.17 \\
1.65 \\
4.54 \\
5.72 \\
-\overline{7} \\
\overline{7} \\
.71 \\
.79\end{array}$ & $\begin{array}{r}1.06 \\
1.69 \\
2.81 \\
2.79 \\
3.16 \\
2.76 \\
-\overline{70} \\
.32\end{array}$ & $\begin{array}{r}1.28 \\
1.67 \\
2.50 \\
4.03 \\
2.82 \\
4.10 \\
- \\
.70 \\
.95\end{array}$ & $\begin{array}{r}1.13 \\
1.55 \\
3.05 \\
3.98 \\
2.66 \\
4.03 \\
-\overline{49} \\
.87\end{array}$ & $\begin{array}{r}1.31 \\
1.56 \\
5.02 \\
2.95 \\
2.54 \\
4.52 \\
-\overline{48} \\
.79\end{array}$ & $\begin{array}{r}1.09 \\
1.58 \\
3.03 \\
5.09 \\
3.05 \\
5.14 \\
- \\
.47 \\
.61\end{array}$ & $\begin{array}{r}1.16 \\
1.55 \\
3.83 \\
5.74 \\
6.19 \\
5.72 \\
- \\
.45 \\
.76\end{array}$ \\
\hline Primary Energy - Five Sectors ${ }^{c}$.. & 1.42 & 2.51 & 5.83 & 5.92 & 4.72 & 4.72 & 4.57 & 4.98 & 5.64 & 5.73 \\
\hline
\end{tabular}

a There are no direct fuel costs for hydroelectric, geothermal, centralized solar, or wind energy.

b Heavy oil includes Grade Nos. 4, 5, and 6, and residual fuel oils. Light oil includes Grade No. 2 heating oil, kerosene, and jet fuel.

c Biomass fuels are not included, except those consumed at electric utilities and those added to motor gasoline.

-No consumption, including cases where adjustments were made. See "Consumption Adjustments for Process Fuel and Intermediate Products."

Sources: Data sources, estimation procedures, and assumptions are described in the "Documentation" section of this report. 
Energy Expenditure Estimates by Sector, Washington $1970,1975,1980$, and 1985-1991

(Million Dollars)

\begin{tabular}{|c|c|c|c|c|c|c|c|c|c|c|}
\hline Sector and Energy Source & 1970 & 1975 & 1980 & 1985 & 1986 & 1987 & 1988 & 1989 & 1990 & 1991 \\
\hline 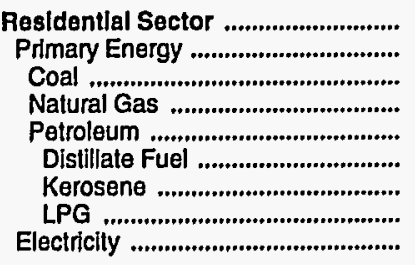 & $\begin{array}{r}280.8 \\
117.3 \\
.3 \\
44.8 \\
72.2 \\
57.4 \\
1.6 \\
13.2 \\
163.5\end{array}$ & $\begin{array}{r}427.3 \\
169.4 \\
.2 \\
78.1 \\
91.1 \\
78.3 \\
4.2 \\
8.6 \\
258.0\end{array}$ & $\begin{array}{r}794.5 \\
330.6 \\
5.5 \\
158.0 \\
167.2 \\
144.9 \\
3.6 \\
18.7 \\
463.8\end{array}$ & $\begin{array}{r}1,447.8 \\
386.0 \\
6.5 \\
217.8 \\
161.7 \\
140.0 \\
4.8 \\
16.8 \\
1,061.8\end{array}$ & $\begin{array}{r}1,340.9 \\
305.5 \\
2.6 \\
179.1 \\
123.8 \\
106.4 \\
2.2 \\
15.2 \\
1,035.3\end{array}$ & $\begin{array}{r}1,370.4 \\
297.6 \\
1.5 \\
161.5 \\
134.5 \\
107.7 \\
1.8 \\
25.0 \\
1,072.8\end{array}$ & $\begin{array}{r}1,480.2 \\
324.2 \\
3.0 \\
192.4 \\
128.8 \\
106.2 \\
2.6 \\
20.0 \\
1,156.0\end{array}$ & $\begin{array}{r}1,595.1 \\
354.1 \\
2.5 \\
210.6 \\
140.9 \\
107.3 \\
2.6 \\
31.0 \\
1,241.0\end{array}$ & $\begin{array}{r}1,640.6 \\
374.6 \\
1.9 \\
202.5 \\
170.1 \\
138.0 \\
2.8 \\
29.3 \\
1,266.0\end{array}$ & $\begin{array}{r}1,679.6 \\
375.6 \\
3.3 \\
216.3 \\
156.1 \\
112.8 \\
2.6 \\
40.7 \\
1,304.0\end{array}$ \\
\hline 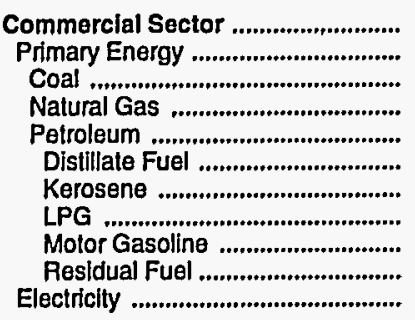 & $\begin{array}{r}116.7 \\
43.0 \\
.3 \\
20.4 \\
22.3 \\
15.7 \\
.1 \\
.9 \\
4.7 \\
1.0 \\
73.6\end{array}$ & $\begin{array}{r}242.3 \\
97.0 \\
.2 \\
58.2 \\
38.6 \\
23.0 \\
.3 \\
.7 \\
9.1 \\
5.5 \\
145.3\end{array}$ & $\begin{array}{r}502.6 \\
234.8 \\
5.4 \\
148.7 \\
80.7 \\
43.1 \\
.7 \\
2.3 \\
24.9 \\
9.7 \\
267.8\end{array}$ & $\begin{array}{r}1,095.0 \\
411.2 \\
7.6 \\
193.3 \\
210.4 \\
162.4 \\
8.2 \\
3.3 \\
17.4 \\
19.0 \\
683.8\end{array}$ & $\begin{array}{r}950.1 \\
247.2 \\
2.8 \\
161.7 \\
82.7 \\
65.2 \\
1.3 \\
2.5 \\
11.8 \\
1.8 \\
702.9\end{array}$ & $\begin{array}{r}981.0 \\
246.9 \\
1.8 \\
145.6 \\
99.5 \\
61.7 \\
21.1 \\
3.7 \\
12.1 \\
.9 \\
734.1\end{array}$ & $\begin{array}{r}1,059.4 \\
281.7 \\
3.9 \\
168.3 \\
109.5 \\
69.8 \\
23.2 \\
2.9 \\
10.8 \\
2.8 \\
777.7\end{array}$ & $\begin{array}{r}1,080.4 \\
272.8 \\
3.1 \\
180.2 \\
89.5 \\
52.8 \\
21.5 \\
3.2 \\
11.0 \\
1.0 \\
807.6\end{array}$ & $\begin{array}{r}1,110.5 \\
256.9 \\
2.3 \\
160.3 \\
94.3 \\
75.2 \\
.6 \\
.6 \\
3.8 \\
13.9 \\
1.0 \\
853.6\end{array}$ & $\begin{array}{r}1,122.3 \\
239.7 \\
3.2 \\
169.7 \\
66.8 \\
50.4 \\
.6 \\
5.3 \\
9.1 \\
1.5 \\
882.6\end{array}$ \\
\hline 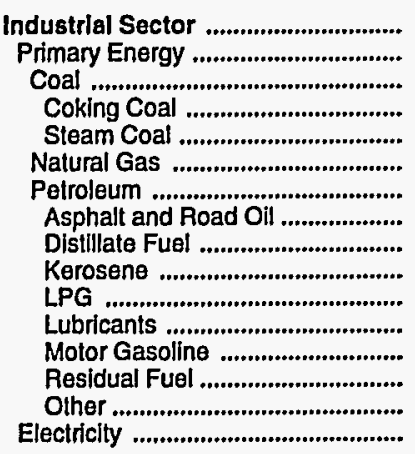 & $\begin{array}{r}198.1 \\
118.4 \\
2.7 \\
2 . \overline{7} \\
32.0 \\
83.7 \\
10.2 \\
19.6 \\
.5 \\
1.1 \\
8.2 \\
8.4 \\
13.1 \\
22.7 \\
79.7\end{array}$ & $\begin{array}{r}446.2 \\
325.6 \\
9.8 \\
-\overline{-} \\
9.8 \\
106.0 \\
209.8 \\
34.1 \\
44.8 \\
1.5 \\
1.8 \\
8.7 \\
10.6 \\
47.9 \\
60.3 \\
120.6\end{array}$ & $\begin{array}{r}903.4 \\
681.7 \\
16.2 \\
-\overline{-} \\
16.2 \\
220.5 \\
445.0 \\
50.3 \\
150.7 \\
1.5 \\
10.5 \\
17.6 \\
14.5 \\
113.3 \\
86.5 \\
221.7\end{array}$ & $\begin{array}{r}1,316.9 \\
731.1 \\
10.3 \\
-\overline{10.3} \\
274.9 \\
445.8 \\
56.4 \\
99.4 \\
36.6 \\
38.0 \\
19.6 \\
33.8 \\
121.8 \\
40.2 \\
585.8\end{array}$ & $\begin{array}{r}1,096.5 \\
523.0 \\
16.4 \\
-\overline{1} \\
16.4 \\
169.0 \\
337.6 \\
62.9 \\
75.3 \\
15.7 \\
40.4 \\
17.0 \\
28.4 \\
74.1 \\
23.7 \\
573.5\end{array}$ & $\begin{array}{r}1,158.6 \\
538.6 \\
13.9 \\
13 . \overline{9} \\
13.9 \\
168.6 \\
356.1 \\
48.3 \\
86.6 \\
.4 \\
55.1 \\
15.6 \\
28.4 \\
79.2 \\
42.5 \\
620.0\end{array}$ & $\begin{array}{r}1,374.1 \\
507.6 \\
12.3 \\
-\overline{1} \\
12.3 \\
185.1 \\
310.2 \\
43.5 \\
68.6 \\
.4 \\
43.2 \\
17.3 \\
26.3 \\
70.0 \\
40.9 \\
866.5\end{array}$ & $\begin{array}{r}1,483.4 \\
548.6 \\
11.8 \\
\overline{-} \\
11.8 \\
193.8 \\
343.1 \\
57.1 \\
110.1 \\
.2 \\
57.2 \\
16.2 \\
29.4 \\
19.5 \\
53.3 \\
934.8\end{array}$ & $\begin{array}{r}1,513.4 \\
599.7 \\
12.7 \\
-\overline{12.7} \\
190.8 \\
396.1 \\
52.4 \\
155.2 \\
.4 \\
34.4 \\
16.8 \\
32.5 \\
24.7 \\
79.6 \\
913.7\end{array}$ & $\begin{array}{r}1,433.2 \\
550.8 \\
11.7 \\
-\overline{1} \\
11.7 \\
203.4 \\
335.7 \\
67.0 \\
121.0 \\
.2 \\
29.0 \\
17.3 \\
38.1 \\
13.1 \\
50.1 \\
882.3\end{array}$ \\
\hline 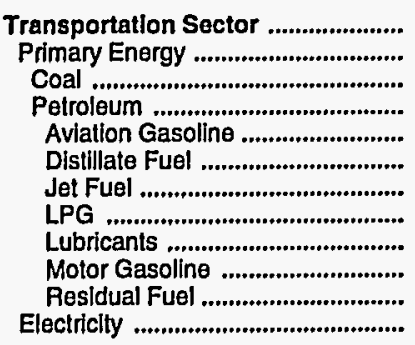 & $\begin{array}{r}633.9 \\
633.9 \\
633.9 \\
3.8 \\
30.3 \\
43.3 \\
.2 \\
12.3 \\
540.2 \\
3.8 \\
\end{array}$ & $\begin{array}{r}1,290.3 \\
4.8 \\
102.2 \\
160.7 \\
.4 \\
19.4 \\
974.5 \\
28.3 \\
\end{array}$ & $\begin{array}{r}3,240.3 \\
3,240.3 \\
- \\
3,240.3 \\
16.2 \\
375.8 \\
419.5 \\
1.9 \\
43.6 \\
2,183.1 \\
200.3\end{array}$ & $\begin{array}{r}3,387.6 \\
3,387.3 \\
3,387.3 \\
10.2 \\
521.4 \\
522.2 \\
11.1 \\
48.7 \\
2,100.2 \\
173.4 \\
.4\end{array}$ & $\begin{array}{r}2,919.7 \\
2,919.3 \\
-\overline{2} \\
2,919.3 \\
9.7 \\
554.4 \\
385.1 \\
9.4 \\
42.2 \\
1,758.2 \\
160.5 \\
.3\end{array}$ & $\begin{array}{r}3,058.6 \\
3,058.3 \\
- \\
3,058.3 \\
10.5 \\
509.7 \\
421.2 \\
7.6 \\
38.8 \\
1,937.4 \\
133.0 \\
.3\end{array}$ & $\begin{array}{r}3,103.3 \\
3,103.0 \\
3,103.0 \\
8.0 \\
527.9 \\
440.3 \\
8.7 \\
43.1 \\
1,937.2 \\
137.9 \\
.3\end{array}$ & $\begin{array}{r}3,584.8 \\
3,584.4 \\
3,584 . \overline{4} \\
7.9 \\
593.1 \\
522.5 \\
7.4 \\
40.2 \\
2,227.8 \\
185.5 \\
.4\end{array}$ & $\begin{array}{r}4,262.1 \\
4,261.7 \\
4,261 . \overline{7} \\
14.7 \\
642.8 \\
716.0 \\
9.6 \\
41.7 \\
2,592.9 \\
243.9 \\
.4\end{array}$ & $\begin{array}{r}4,355.7 \\
4,355.3 \\
- \\
4,355.3 \\
11.8 \\
618.2 \\
579.7 \\
8.2 \\
42.9 \\
2,556.9 \\
537.6 \\
.4\end{array}$ \\
\hline 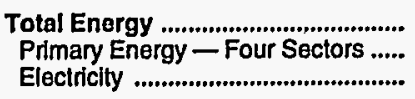 & $\begin{array}{r}1,229.4 \\
912.6 \\
316.8\end{array}$ & $\begin{array}{r}2,406.2 \\
1,882.3 \\
523.9\end{array}$ & $\begin{array}{r}5,440.7 \\
4,487.4 \\
953.4\end{array}$ & $\begin{array}{l}7,247.3 \\
4,915.6 \\
2,331.7\end{array}$ & $\begin{array}{l}6,307.1 \\
3,995.0 \\
2,312.1\end{array}$ & $\begin{array}{l}6,568.6 \\
4,141.3 \\
2,427.3\end{array}$ & $\begin{array}{l}7,017.0 \\
4,216.6 \\
2,800.5\end{array}$ & $\begin{array}{l}7,743.7 \\
4,759.9 \\
2,983.8\end{array}$ & $\begin{array}{l}8,526.5 \\
5,492.8 \\
3,033.7\end{array}$ & $\begin{array}{l}8,590.8 \\
5,521.5 \\
3,069.4\end{array}$ \\
\hline 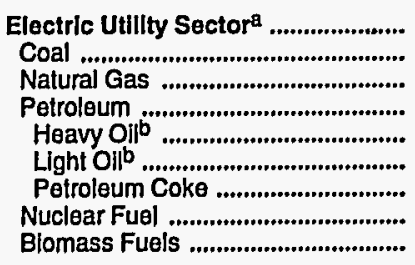 & $\begin{array}{r}5.2 \\
\vdots \\
5 . \\
5\end{array}$ & $\begin{array}{r}46.5 \\
36.7 \\
1 . \overline{2} \\
1.1 \\
.1 \\
\overline{-} \\
8 . \overline{-}\end{array}$ & $\begin{array}{r}95.7 \\
77.1 \\
3.3 \\
5.7 \\
4.5 \\
1.1 \\
-\overline{6} \\
9.6\end{array}$ & $\begin{array}{r}203.6 \\
139.0 \\
.4 \\
.6 \\
\overline{.6} \\
. \overrightarrow{-} \\
61.4 \\
2.3\end{array}$ & $\begin{array}{r}155.9 \\
90.9 \\
.4 \\
.3 \\
. \\
.3 \\
63.7 \\
.6\end{array}$ & $\begin{array}{r}194.0 \\
148.1 \\
.2 \\
.4 \\
.4 \\
. \overline{4} \\
41.8 \\
3.4\end{array}$ & $\begin{array}{r}182.6 \\
141.6 \\
5.5 \\
.4 \\
.4 \\
31 . \overline{6} \\
3.4\end{array}$ & $\begin{array}{r}221.5 \\
140.0 \\
43.2 \\
3.5 \\
2.4 \\
1.1 \\
31 . \overline{7} \\
3.0\end{array}$ & $\begin{array}{r}157.2 \\
124.4 \\
.6 \\
.9 \\
.9 \\
. \overline{2} \\
29.1 \\
2.1\end{array}$ & $\begin{array}{r}152.5 \\
128.9 \\
.6 \\
.5 \\
.5 \\
.5 \\
20.4 \\
2.2\end{array}$ \\
\hline Primary Energy - Five Sectorsc .. & 917.9 & 1,928.9 & $4,583.0$ & $5,119.2$ & $4,151.0$ & $4,335.4$ & $4,399.1$ & $4,981.4$ & $5,650.0$ & $5,674.0$ \\
\hline
\end{tabular}

There are no direct fuel costs for hydroelectric, geothermal, centralized solar, or wind energy.

b Heavy oil includes Grade Nos. 4,5, and 6, and residual fuel oils. Light oil includes Grade No. 2 heating oil, kerosene, and jet fuel.

c Biomass fuels are not included, except those consumed at electric utilities and those added to motor gasoline.

- No consumption, Including cases where adjustments were made. See "Consumption Adjustments for Process Fuel and Intermediate Products."

- Value less than 0.05 million dollars.

Note: Totals may not equal sum of components due to independent rounding.

Sources: Data sources, estimation procedures, and assumptions are described in the "Documentation" section of this report. 
W Energy Price and Expenditure Estimates by Source, West Virginia

E 1970, 1975, 1980, and 1985-1991

\begin{tabular}{|c|c|c|c|c|c|c|c|c|c|c|}
\hline Energy Source & 1970 & 1975 & 1980 & 1985 & 1986 & 1987 & 1988 & 1989 & 1990 & 1991 \\
\hline & \multicolumn{10}{|c|}{ Prices in Dollars per Million Btu } \\
\hline 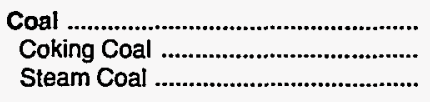 & $\begin{array}{r}0.31 \\
.40 \\
.28\end{array}$ & $\begin{array}{r}1.02 \\
1.51 \\
.94\end{array}$ & $\begin{array}{l}1.46 \\
1.86 \\
1.41\end{array}$ & $\begin{array}{l}1.61 \\
1.93 \\
1.59\end{array}$ & $\begin{array}{l}1.50 \\
1.75 \\
1.49\end{array}$ & $\begin{array}{l}1.43 \\
1.72 \\
1.41\end{array}$ & $\begin{array}{l}1.44 \\
1.73 \\
1.42\end{array}$ & $\begin{array}{l}1.43 \\
1.75 \\
1.41\end{array}$ & $\begin{array}{l}1.47 \\
1.80 \\
1.45\end{array}$ & $\begin{array}{l}1.51 \\
1.72 \\
1.50\end{array}$ \\
\hline Natural Gas & .62 & 1.16 & 3.18 & 5.28 & 4.99 & 4.48 & 4.08 & 4.08 & 4.40 & 4.59 \\
\hline 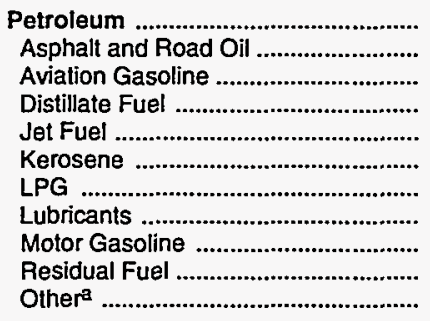 & $\begin{array}{r}1.77 \\
.68 \\
2.17 \\
1.40 \\
.73 \\
1.49 \\
1.67 \\
5.08 \\
2.86 \\
.58 \\
.87\end{array}$ & $\begin{array}{l}3.62 \\
1.81 \\
3.45 \\
3.36 \\
2.05 \\
2.83 \\
3.25 \\
7.48 \\
4.61 \\
1.89 \\
2.87\end{array}$ & $\begin{array}{r}8.06 \\
3.58 \\
9.02 \\
7.24 \\
6.46 \\
8.19 \\
6.16 \\
14.36 \\
9.96 \\
3.33 \\
7.19\end{array}$ & $\begin{array}{r}8.38 \\
4.91 \\
9.99 \\
8.01 \\
6.87 \\
8.10 \\
9.02 \\
17.61 \\
9.19 \\
4.01 \\
7.37\end{array}$ & $\begin{array}{r}5.85 \\
4.19 \\
8.41 \\
5.87 \\
5.14 \\
6.41 \\
8.02 \\
15.59 \\
7.00 \\
2.57 \\
3.95\end{array}$ & $\begin{array}{r}6.29 \\
3.23 \\
7.55 \\
5.97 \\
4.72 \\
6.18 \\
8.36 \\
12.70 \\
7.34 \\
2.81 \\
4.81\end{array}$ & $\begin{array}{r}6.11 \\
3.35 \\
7.41 \\
5.88 \\
4.45 \\
6.17 \\
10.13 \\
14.61 \\
7.52 \\
2.38 \\
3.98\end{array}$ & $\begin{array}{r}6.84 \\
3.13 \\
8.28 \\
6.62 \\
4.99 \\
7.00 \\
7.84 \\
13.30 \\
8.52 \\
2.65 \\
4.82\end{array}$ & $\begin{array}{r}8.03 \\
3.07 \\
9.32 \\
8.04 \\
6.41 \\
8.59 \\
10.79 \\
13.40 \\
9.96 \\
2.68 \\
5.92\end{array}$ & $\begin{array}{r}7.67 \\
3.11 \\
8.71 \\
7.16 \\
5.58 \\
7.62 \\
11.12 \\
15.42 \\
9.92 \\
1.92 \\
5.23\end{array}$ \\
\hline Nuclear Fuel & - & - & - & - & - & - & - & - & - & - \\
\hline Blomass Fuels at Utilities .................. & .65 & - & - & - & - & - & - & - & - & - \\
\hline Primary Energy - Five Sectors ${ }^{b}$.... & .68 & 1.58 & 3.16 & 3.22 & 2.62 & 2.64 & 2.59 & 2.74 & 3.13 & 3.17 \\
\hline $\begin{array}{l}\text { Electric Utility Fuelc } \\
\text { Electricity Purchased by End Users }\end{array}$ & $\begin{array}{r}.26 \\
3.96\end{array}$ & $\begin{array}{r}.88 \\
8.30\end{array}$ & $\begin{array}{r}1.43 \\
10.58\end{array}$ & $\begin{array}{r}1.62 \\
14.19\end{array}$ & $\begin{array}{r}1.50 \\
14.81\end{array}$ & $\begin{array}{r}1.43 \\
14.77\end{array}$ & $\begin{array}{r}1.45 \\
14.10\end{array}$ & $\begin{array}{r}1.43 \\
14.04\end{array}$ & $\begin{array}{r}1.48 \\
13.90\end{array}$ & $\begin{array}{r}1.53 \\
14.22\end{array}$ \\
\hline \multirow[t]{2}{*}{ 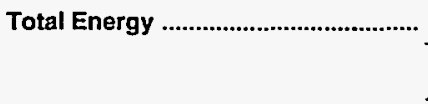 } & 1.13 & 2.79 & 5.78 & 7.34 & 5.99 & 6.09 & 5.78 & 6.08 & 6.71 & 6.84 \\
\hline & \multicolumn{10}{|c|}{ Expenditures in Millions of Dollars } \\
\hline 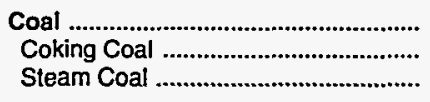 & $\begin{array}{r}187.2 \\
55.3 \\
131.9\end{array}$ & $\begin{array}{l}833.9 \\
178.3 \\
655.6\end{array}$ & $\begin{array}{r}1,253.8 \\
190.2 \\
1,063.6\end{array}$ & $\begin{array}{r}1,399.0 \\
72.4 \\
1,326.6\end{array}$ & $\begin{array}{r}1,315.2 \\
89.0 \\
1,226.2\end{array}$ & $\begin{array}{r}1,241.9 \\
81.0 \\
1,160.8\end{array}$ & $\begin{array}{r}1,319.5 \\
94.3 \\
1,225.2\end{array}$ & $\begin{array}{r}1,326.2 \\
91.5 \\
1,234.7\end{array}$ & $\begin{array}{r}1,286.3 \\
93.1 \\
1,193.2\end{array}$ & $\begin{array}{r}1,208.8 \\
86.8 \\
1,122.0\end{array}$ \\
\hline 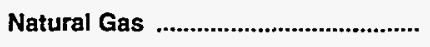 & 108.3 & 171.0 & 415.1 & 510.6 & 472.4 & 444.9 & 434.9 & 475.4 & 471.2 & 454.5 \\
\hline 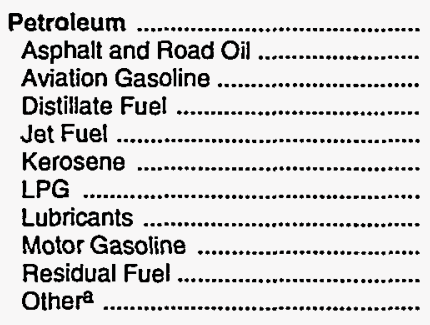 & $\begin{array}{r}383.5 \\
3.9 \\
.9 \\
31.9 \\
1.2 \\
2.7 \\
7.8 \\
21.1 \\
237.6 \\
7.5 \\
69.0\end{array}$ & $\begin{array}{r}927.6 \\
11.3 \\
1.0 \\
114.2 \\
2.8 \\
5.2 \\
18.1 \\
31.1 \\
467.7 \\
26.2 \\
250.0\end{array}$ & $\begin{array}{r}2,380.6 \\
17.0 \\
3.0 \\
441.1 \\
12.9 \\
23.0 \\
77.7 \\
58.4 \\
1,014.2 \\
24.8 \\
708.4\end{array}$ & $\begin{array}{r}1,950.0 \\
14.0 \\
1.9 \\
451.7 \\
9.0 \\
32.0 \\
36.9 \\
65.2 \\
894.0 \\
22.2 \\
423.1\end{array}$ & $\begin{array}{r}1,376.7 \\
15.7 \\
2.1 \\
261.1 \\
6.3 \\
21.3 \\
33.2 \\
56.4 \\
686.3 \\
15.5 \\
278.8\end{array}$ & $\begin{array}{r}1,518.0 \\
11.5 \\
1.3 \\
312.3 \\
5.6 \\
18.2 \\
36.5 \\
52.0 \\
743.9 \\
8.1 \\
328.6\end{array}$ & $\begin{array}{r}1,537.2 \\
19.5 \\
1.4 \\
310.6 \\
6.2 \\
20.4 \\
44.0 \\
57.6 \\
781.3 \\
8.3 \\
287.8\end{array}$ & $\begin{array}{r}1,765.1 \\
16.9 \\
1.6 \\
388.4 \\
10.6 \\
20.2 \\
42.4 \\
53.8 \\
871.5 \\
14.9 \\
344.8\end{array}$ & $\begin{array}{r}2,143.5 \\
14.8 \\
1.7 \\
456.3 \\
9.8 \\
14.4 \\
59.9 \\
55.8 \\
1,021.8 \\
18.7 \\
490.3\end{array}$ & $\begin{array}{r}2,011.8 \\
10.9 \\
1.5 \\
400.2 \\
6.7 \\
13.0 \\
71.4 \\
57.4 \\
1,007.4 \\
9.5 \\
433.7\end{array}$ \\
\hline 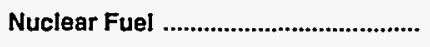 & - & - & - & - & - & - & - & - & - & - \\
\hline Biomass Fuels at Utilities ................. & - & - & - & - & - & - & - & - & - & - \\
\hline Primary Energy - Five Sectors ${ }^{b}$.... & 679.1 & $1,932.5$ & $4,049.5$ & $3,859.5$ & $3,164.4$ & $3,204.7$ & $3,291.6$ & $3,566.8$ & $3,901.0$ & $3,675.1$ \\
\hline $\begin{array}{l}\text { Electric Utility Fuelc ............................ } \\
\text { Electricity Purchased by End Users }\end{array}$ & $\begin{array}{r}-89.9 \\
204.3\end{array}$ & $\begin{array}{r}-531.0 \\
477.3\end{array}$ & $\begin{array}{r}-997.7 \\
748.8\end{array}$ & $\begin{array}{r}-1,261.8 \\
1,000.4\end{array}$ & $\begin{array}{r}-1,156.2 \\
1,029.6\end{array}$ & $\begin{array}{r}-1,093.2 \\
1,053.0\end{array}$ & $\begin{array}{r}-1,148.1 \\
1,068.2\end{array}$ & $\begin{array}{r}-1,155.4 \\
1,084.9\end{array}$ & $\begin{array}{r}-1,108.0 \\
1,088.8\end{array}$ & $\begin{array}{r}-1,056.7 \\
1,133.0\end{array}$ \\
\hline 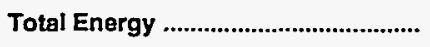 & 793.5 & $1,878.7$ & $3,800.5$ & $3,598.1$ & $3,037.8$ & $3,164.6$ & $3,211.7$ & $3,496.3$ & $3,881.9$ & $3,751.4$ \\
\hline
\end{tabular}

Inciudes petroleum coke used at electric utilities.

biomass fuels are not included, except those consumed at electric utilities and those added to motor gasoline.

c There are no direct fuel costs for hydroelectric, geothermal, centralized solar, or wind energy.

-No consumption, including cases where adjustments were made. See "Consumption Adjustments for Process Fuel and Intermediate Products."

- Value less than 0.05 million dollars.

Note: Expenditure totals may not equal sum of components due to independent rounding.

Sources: Data sources, estimation procedures, and assumptions are described in the "Documentation" section of this report. 
Energy Price Estimates by Sector, West Virginia $1970,1975,1980$, and 1985-1991

(Dollars per Million Btu)

\begin{tabular}{|c|c|c|c|c|c|c|c|c|c|c|}
\hline Sector and Energy Source & 1970 & 1975 & 1980 & 1985 & 1986 & 1987 & 1988 & 1989 & 1990 & 1991 \\
\hline 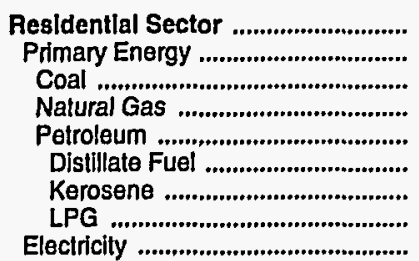 & $\begin{array}{r}1.76 \\
.91 \\
.66 \\
.87 \\
1.75 \\
1.37 \\
1.64 \\
2.49 \\
6.41\end{array}$ & $\begin{array}{r}3.51 \\
1.57 \\
1.22 \\
1.40 \\
3.27 \\
2.69 \\
3.17 \\
4.97 \\
10.47\end{array}$ & $\begin{array}{r}6.39 \\
4.11 \\
1.59 \\
3.48 \\
7.36 \\
6.65 \\
8.48 \\
8.94 \\
12.64\end{array}$ & $\begin{array}{r}9.94 \\
6.20 \\
1.66 \\
5.99 \\
8.27 \\
7.42 \\
8.82 \\
9.61 \\
17.38\end{array}$ & $\begin{array}{r}9.91 \\
5.85 \\
1.37 \\
5.90 \\
6.26 \\
5.65 \\
6.72 \\
7.26 \\
17.84\end{array}$ & $\begin{array}{r}9.84 \\
5.53 \\
1.23 \\
5.57 \\
6.15 \\
5.78 \\
6.88 \\
6.08 \\
17.87\end{array}$ & $\begin{array}{r}9.50 \\
5.29 \\
1.24 \\
5.11 \\
6.99 \\
5.67 \\
6.74 \\
11.03 \\
17.39\end{array}$ & $\begin{array}{r}9.59 \\
5.45 \\
1.29 \\
5.34 \\
6.80 \\
6.34 \\
7.54 \\
6.99 \\
17.31\end{array}$ & $\begin{array}{r}10.45 \\
6.30 \\
1.43 \\
6.03 \\
9.09 \\
7.57 \\
9.01 \\
12.50 \\
17.29\end{array}$ & $\begin{array}{r}10.74 \\
6.35 \\
1.31 \\
6.06 \\
8.88 \\
7.14 \\
8.49 \\
13.00 \\
17.32\end{array}$ \\
\hline 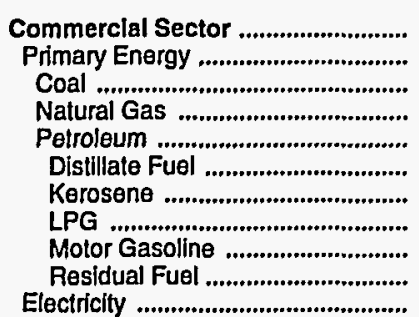 & $\begin{array}{r}1.83 \\
.69 \\
.35 \\
.69 \\
1.57 \\
1.08 \\
.77 \\
1.45 \\
2.86 \\
.86 \\
5.81\end{array}$ & $\begin{array}{r}3.36 \\
1.29 \\
1.33 \\
1.18 \\
2.77 \\
2.37 \\
2.46 \\
2.76 \\
4.61 \\
1.82 \\
10.00\end{array}$ & $\begin{array}{r}6.28 \\
3.44 \\
1.44 \\
3.24 \\
7.04 \\
6.24 \\
6.85 \\
5.80 \\
9.96 \\
4.02 \\
12.59\end{array}$ & $\begin{array}{r}9.86 \\
5.85 \\
1.43 \\
5.64 \\
7.41 \\
6.62 \\
7.18 \\
8.87 \\
9.19 \\
4.01 \\
16.64\end{array}$ & $\begin{array}{r}9.61 \\
5.11 \\
1.33 \\
5.47 \\
5.20 \\
4.42 \\
5.35 \\
8.22 \\
7.00 \\
2.57 \\
16.95\end{array}$ & $\begin{array}{r}9.48 \\
4.88 \\
1.25 \\
5.10 \\
5.81 \\
4.75 \\
4.84 \\
9.03 \\
7.34 \\
2.81 \\
16.61\end{array}$ & $\begin{array}{r}8.63 \\
4.68 \\
1.23 \\
4.81 \\
5.24 \\
4.56 \\
4.93 \\
9.78 \\
7.52 \\
2.38 \\
16.04\end{array}$ & $\begin{array}{r}8.65 \\
4.84 \\
1.23 \\
4.88 \\
6.15 \\
5.34 \\
5.87 \\
8.15 \\
8.52 \\
2.65 \\
16.06\end{array}$ & $\begin{array}{r}9.16 \\
5.42 \\
1.28 \\
5.44 \\
7.60 \\
6.55 \\
7.54 \\
10.15 \\
9.96 \\
2.68 \\
15.85\end{array}$ & $\begin{array}{r}9.60 \\
5.66 \\
1.29 \\
5.69 \\
6.78 \\
5.64 \\
5.95 \\
10.59 \\
9.92 \\
1.92 \\
16.00\end{array}$ \\
\hline 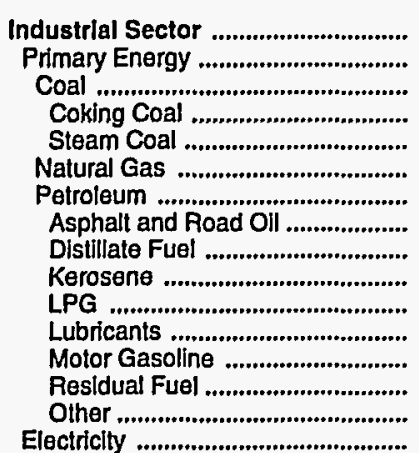 & $\begin{array}{r}.66 \\
.53 \\
.38 \\
.40 \\
.35 \\
.45 \\
.96 \\
.68 \\
.71 \\
.77 \\
1.45 \\
5.08 \\
2.86 \\
.48 \\
.87 \\
2.63\end{array}$ & $\begin{array}{l}2.11 \\
1.76 \\
1.43 \\
1.51 \\
1.33 \\
.98 \\
2.80 \\
1.81 \\
2.27 \\
2.46 \\
2.76 \\
7.48 \\
4.61 \\
1.92 \\
2.87 \\
6.56\end{array}$ & $\begin{array}{r}4.32 \\
3.90 \\
1.70 \\
1.86 \\
1.44 \\
2.91 \\
6.75 \\
3.58 \\
6.15 \\
6.85 \\
5.80 \\
14.36 \\
9.96 \\
3.33 \\
7.19 \\
8.58\end{array}$ & $\begin{array}{r}5.22 \\
4.38 \\
1.64 \\
1.93 \\
1.43 \\
4.39 \\
7.30 \\
4.91 \\
6.43 \\
7.18 \\
8.87 \\
17.61 \\
9.19 \\
4.01 \\
7.37 \\
10.77\end{array}$ & $\begin{array}{r}3.95 \\
3.05 \\
1.53 \\
1.75 \\
1.33 \\
3.85 \\
4.41 \\
4.19 \\
4.78 \\
5.35 \\
8.22 \\
15.59 \\
7.00 \\
2.57 \\
3.95 \\
11.26\end{array}$ & $\begin{array}{r}4.05 \\
3.16 \\
1.46 \\
1.72 \\
1.25 \\
3.24 \\
5.00 \\
3.23 \\
4.33 \\
4.84 \\
9.03 \\
12.70 \\
7.34 \\
2.81 \\
4.81 \\
11.26\end{array}$ & $\begin{array}{r}3.66 \\
2.80 \\
1.46 \\
1.73 \\
1.23 \\
2.66 \\
4.43 \\
3.35 \\
4.41 \\
4.93 \\
9.78 \\
14.61 \\
7.52 \\
2.38 \\
3.98 \\
10.57\end{array}$ & $\begin{array}{r}3.87 \\
3.05 \\
1.46 \\
1.75 \\
1.23 \\
2.71 \\
5.06 \\
3.13 \\
5.25 \\
5.87 \\
8.15 \\
13.30 \\
8.52 \\
2.65 \\
4.82 \\
10.52\end{array}$ & $\begin{array}{r}4.31 \\
3.56 \\
1.50 \\
1.80 \\
1.28 \\
2.75 \\
6.08 \\
3.07 \\
6.75 \\
7.54 \\
10.15 \\
13.40 \\
9.96 \\
2.68 \\
5.92 \\
10.44\end{array}$ & $\begin{array}{r}4.27 \\
3.44 \\
1.49 \\
1.72 \\
1.29 \\
2.75 \\
5.52 \\
3.11 \\
5.33 \\
5.95 \\
10.59 \\
15.42 \\
9.92 \\
1.92 \\
5.23 \\
10.74\end{array}$ \\
\hline 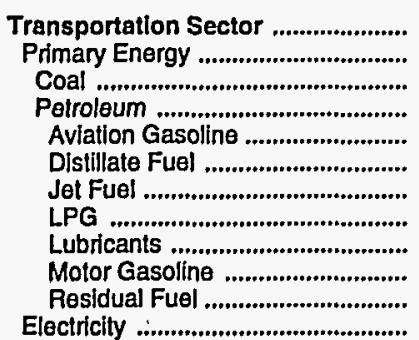 & $\begin{array}{r}2.67 \\
2.67 \\
.35 \\
2.68 \\
2.17 \\
1.72 \\
.73 \\
1.45 \\
5.08 \\
2.86 \\
.85 \\
-\end{array}$ & $\begin{array}{l}4.50 \\
4.50 \\
1.33 \\
4.50 \\
3.45 \\
3.97 \\
2.03 \\
2.76 \\
7.48 \\
4.61 \\
=\end{array}$ & $\begin{array}{r}9.61 \\
9.61 \\
- \\
9.61 \\
9.02 \\
8.36 \\
6.46 \\
5.80 \\
14.36 \\
9.96 \\
- \\
-\end{array}$ & $\begin{array}{r}9.14 \\
9.14 \\
- \\
9.14 \\
9.99 \\
8.76 \\
6.87 \\
8.87 \\
17.61 \\
9.19 \\
4.29 \\
-\end{array}$ & $\begin{array}{r}7.05 \\
7.05 \\
- \\
7.05 \\
8.41 \\
6.85 \\
5.14 \\
8.22 \\
15.59 \\
7.00 \\
- \\
-\end{array}$ & $\begin{array}{r}7.31 \\
7.31 \\
- \\
7.31 \\
7.55 \\
7.04 \\
4.72 \\
9.03 \\
12.70 \\
7.34 \\
- \\
-\end{array}$ & $\begin{array}{r}7.42 \\
7.42 \\
7 . \overline{7} \\
7.41 \\
6.86 \\
4.45 \\
9.78 \\
14.61 \\
7.52 \\
- \\
-\end{array}$ & $\begin{array}{r}8.26 \\
8.26 \\
- \\
8.26 \\
8.28 \\
7.50 \\
4.99 \\
8.15 \\
13.30 \\
8.52 \\
- \\
-\end{array}$ & $\begin{array}{r}9.71 \\
9.71 \\
- \\
9.71 \\
9.32 \\
8.95 \\
6.41 \\
10.15 \\
13.40 \\
9.96 \\
- \\
-\end{array}$ & $\begin{array}{r}9.52 \\
9.52 \\
- \\
9.52 \\
8.71 \\
8.23 \\
5.58 \\
10.59 \\
15.42 \\
9.92 \\
- \\
-\end{array}$ \\
\hline 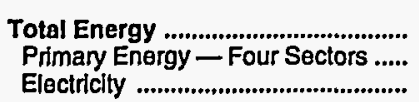 & $\begin{array}{r}1.13 \\
.90 \\
3.96\end{array}$ & $\begin{array}{l}2.79 \\
2.28 \\
8.30\end{array}$ & $\begin{array}{r}5.78 \\
5.20 \\
10.58\end{array}$ & $\begin{array}{r}7.34 \\
6.19 \\
14.19\end{array}$ & $\begin{array}{r}5.99 \\
4.58 \\
14.81\end{array}$ & $\begin{array}{r}6.09 \\
4.71 \\
14.77\end{array}$ & $\begin{array}{r}5.78 \\
4.47 \\
14.10\end{array}$ & $\begin{array}{r}6.08 \\
4.85 \\
14.04\end{array}$ & $\begin{array}{r}6.71 \\
5.59 \\
13.90\end{array}$ & $\begin{array}{r}6.84 \\
5.58 \\
14.22\end{array}$ \\
\hline 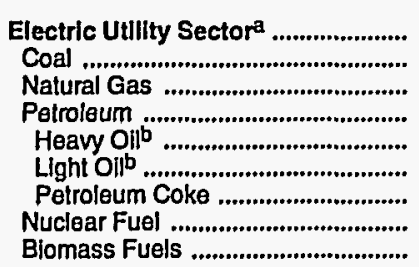 & $\begin{array}{r}.26 \\
.25 \\
.32 \\
.94 \\
.94 \\
.94 \\
- \\
-65\end{array}$ & $\begin{array}{r}.88 \\
.87 \\
.60 \\
1.84 \\
1.83 \\
2.44 \\
- \\
- \\
-\end{array}$ & $\begin{array}{r}1.43 \\
1.41 \\
2.98 \\
6.30 \\
-\overline{6} \\
6.30 \\
- \\
-\end{array}$ & $\begin{array}{r}1.62 \\
1.60 \\
4.78 \\
6.00 \\
-\overline{0} \\
- \\
- \\
-\end{array}$ & $\begin{array}{r}1.50 \\
1.50 \\
4.03 \\
3.71 \\
\overline{-} \\
3.71 \\
- \\
- \\
-\end{array}$ & $\begin{array}{r}1.43 \\
1.42 \\
3.94 \\
4.36 \\
- \\
4.36 \\
- \\
- \\
-\end{array}$ & $\begin{array}{r}1.45 \\
1.44 \\
3.89 \\
3.96 \\
\overline{-} \\
3.96 \\
- \\
= \\
-\end{array}$ & $\begin{array}{r}1.43 \\
1.43 \\
4.59 \\
4.50 \\
- \\
4.50 \\
- \\
- \\
-\end{array}$ & $\begin{array}{r}1.48 \\
1.47 \\
5.13 \\
5.72 \\
5.72 \\
- \\
- \\
-\end{array}$ & $\begin{array}{r}1.53 \\
1.52 \\
3.63 \\
5.37 \\
\overline{5} \\
- \\
- \\
-\end{array}$ \\
\hline Primary Energy - Five Sectors' ${ }^{c}$. & .68 & 1.58 & 3.16 & 3.22 & 2.62 & 2.64 & 2.59 & 2.74 & 3.13 & 3.17 \\
\hline
\end{tabular}

a There are no direct fuel costs for hydroelectric, geothermal, centralized solar, or wind energy.

b Heavy oil Includes Grade Nos. 4, 5, and 6, and residual fuel oils. Light oil includes Grade No. 2 heating oil, kerosene, and jet fuel.

c Blomass fuels are not included, except those consumed at electric utilities and those added to motor gasoline.

-No consumplion, including cases where adjustments were made. See "Consumption Adjustments for Process Fuel and Intermediate Products."

Sources: Data sources, estimation procedures, and assumptions are described in the "Documentation" section of this report. 
W Energy Expenditure Estimates by Sector, West Virginia

E 1970, 1975, 1980, and 1985-1991

S (Million Dollars)

\begin{tabular}{|c|c|c|c|c|c|c|c|c|c|c|}
\hline Sector and Energy Source & 1970 & 1975 & 1980 & 1985 & 1986 & 1987 & 1988 & 1989 & 1990 & 1891 \\
\hline 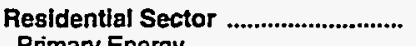 & 135.3 & 273.1 & 538.5 & 681.3 & 698.0 & 697.3 & 704.1 & 715.0 & 714.8 & 742.1 \\
\hline 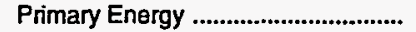 & 59.7 & 95.2 & 253.6 & 283.2 & 272.9 & 255.3 & 256.2 & 264.0 & 267.8 & 263.1 \\
\hline Coal & 1.1 & 2.4 & 2.1 & 1.2 & 1.4 & 1.5 & 1.2 & 1.7 & 2.2 & 1.1 \\
\hline 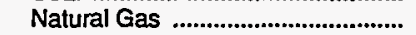 & 51.7 & 74.5 & 173.6 & 234.7 & 229.8 & 213.1 & 207.3 & 213.5 & 210.6 & 211.6 \\
\hline Petroleum .......................................... & 7.0 & 18.3 & $\pi 7.9$ & 47.3 & 41.7 & 40.7 & 47.6 & 48.8 & 54.9 & 50.3 \\
\hline 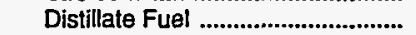 & 2.0 & 9.1 & 45.3 & 20.0 & 18.4 & 21.4 & 18.9 & 23.8 & 25.3 & 22.3 \\
\hline 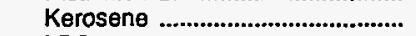 & 2.5 & 3.1 & 19.6 & 19.5 & 17.3 & 13.4 & 15.2 & 14.8 & 10.7 & 9.5 \\
\hline LPG & 2.5 & 6.1 & 13.0 & 7.8 & 6.0 & 6.0 & 13.5 & 10.2 & 18.9 & 18.5 \\
\hline 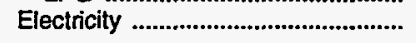 & 75.6 & 177.9 & 284.9 & 398.1 & 425.1 & 442.0 & 448.0 & 451.0 & 447.0 & 479.0 \\
\hline 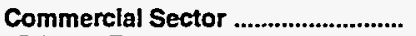 & 62.5 & 137.8 & 252.4 & 403.6 & 398.0 & 392.7 & 416.0 & 435.6 & 443.3 & 456.6 \\
\hline 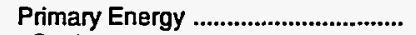 & 18.1 & 40.3 & 95.2 & 150.2 & 130.9 & 123.1 & 147.0 & 160.7 & 168.3 & 166.6 \\
\hline Coal & 1.0 & 4.9 & 3.5 & 1.8 & 2.4 & 2.9 & 2.2 & 3.0 & 3.7 & 2.0 \\
\hline Natural Gas .................................... & 15.3 & 30.2 & 73.4 & 103.7 & 94.2 & 92.0 & 116.1 & 122.3 & 124.7 & 128.5 \\
\hline 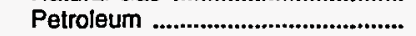 & 1.8 & 5.2 & 18.3 & 44.7 & 34.3 & 28.2 & 28.6 & 35.3 & 39.9 & 36.1 \\
\hline 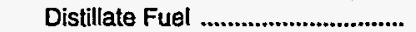 & .6 & 2.9 & 9.5 & 23.3 & 19.3 & 12.5 & 9.5 & 15.4 & 16.9 & 17.0 \\
\hline 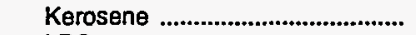 & .1 & .1 & 1.4 & 5.2 & 1.7 & 1.6 & 2.2 & 2.5 & 2.0 & 2.2 \\
\hline 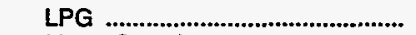 & .3 & .6 & 1.5 & 1.3 & 1.2 & 1.6 & 2.1 & 2.1 & 2.7 & 2.7 \\
\hline Motor Gasoline .................................. & .8 & 1.4 & 5.7 & 14.8 & 12.0 & 12.4 & 12.2 & 13.8 & 17.2 & 13.7 \\
\hline Residual Fuel ..................................... & & .1 & .1 & .1 & .2 & .1 & 2.6 & 1.5 & 1.1 & .6 \\
\hline 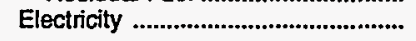 & 44.4 & 97.5 & 157.1 & 253.4 & 267.0 & 269.6 & 269.0 & 275.0 & 275.0 & 290.0 \\
\hline 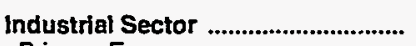 & 327.8 & 905.7 & $1,731.5$ & $1,283.3$ & $1,082.2$ & $1,120.3$ & $1,094.1$ & $1,206.3$ & $1,401.6$ & $\mathbf{1 , 2 7 1 . 2}$ \\
\hline 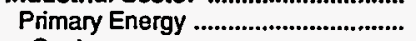 & 243.5 & 703.8 & $1,424.8$ & 934.4 & 744.7 & 778.8 & 743.0 & 847.4 & $1,034.8$ & 907.2 \\
\hline Coal & 97.9 & 304.0 & 275.7 & 147.6 & 164.7 & 155.0 & 176.4 & 177.3 & 185.3 & 160.1 \\
\hline 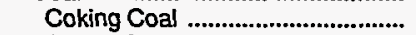 & 55.3 & 178.3 & 190.2 & 72.4 & 89.0 & 81.0 & 94.3 & 91.5 & 93.1 & 86.8 \\
\hline Steam Coal ................................... & 42.6 & 125.7 & 85.6 & 75.2 & 75.7 & 73.9 & 82.1 & 85.7 & 92.2 & 73.4 \\
\hline 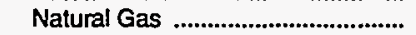 & 41.2 & 66.1 & 167.9 & 171.6 & 147.3 & 138.8 & 111.2 & 139.0 & 135.2 & 113.8 \\
\hline Petroleum …………………….... & 104.5 & 333.7 & 981.2 & 615.1 & 432.8 & 485.0 & 455.3 & 531.1 & 714.3 & 633.2 \\
\hline 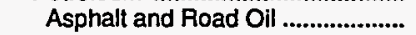 & 3.9 & 11.3 & 17.0 & 14.0 & 15.7 & 11.5 & 19.5 & 16.9 & 14.8 & 10.9 \\
\hline 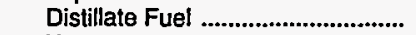 & 4.5 & 19.1 & 125.3 & 69.7 & 51.4 & 63.6 & 66.2 & 78.8 & 104.3 & 79.2 \\
\hline 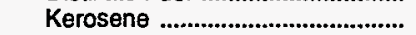 & .2 & 2.0 & 2.0 & 7.2 & 2.3 & 3.2 & 2.9 & 2.8 & 1.7 & 1.3 \\
\hline LPG & 5.0 & 11.2 & 62.9 & 27.2 & 25.4 & 28.4 & 27.6 & 29.5 & 37.6 & 49.6 \\
\hline 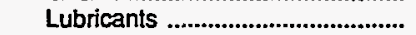 & 15.4 & 20.3 & 36.6 & 40.9 & 35.4 & 32.6 & 36.1 & 33.7 & 35.0 & 36.0 \\
\hline 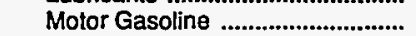 & 1.7 & 1.9 & 4.3 & 11.1 & 8.4 & 9.1 & 9.3 & 11.1 & 13.0 & 13.5 \\
\hline 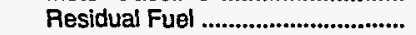 & 4.8 & 17.9 & 24.7 & 22.1 & 15.4 & 8.1 & 5.7 & 13.4 & 17.6 & 8.9 \\
\hline Other & 69.0 & 250.0 & 708.4 & 423.1 & 278.8 & 328.6 & 287.8 & 344.8 & 490.3 & 433.7 \\
\hline 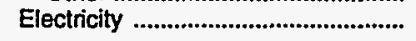 & 84.3 & 201.9 & 306.7 & 348.9 & 337.5 & 341.4 & 351.2 & 358.9 & 366.8 & 364.0 \\
\hline Transportation Sector ........................... & 267.9 & 562.1 & $1,278.1$ & $1,229.9$ & 859.7 & 954.3 & 997.4 & $1,139.4$ & $1,322.2$ & $1,281.5$ \\
\hline 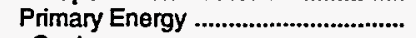 & 267.9 & 562.1 & $1,278.1$ & $1,229.9$ & 859.7 & 954.3 & 997.4 & $1,139.4$ & $1,322.2$ & $1,281.5$ \\
\hline Coal & .1 & & & & & & - & - & & \\
\hline 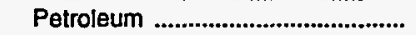 & 267.7 & 562.1 & $1,278.1$ & $1,229.9$ & 859.7 & 954.3 & 997.4 & $1,139.4$ & $1,322.2$ & $1,281.5$ \\
\hline Aviation Gasoline ........................... & & 1.0 & & 1.9 & 2.1 & 1.3 & 1.4 & 1.6 & 1.7 & 1.5 \\
\hline 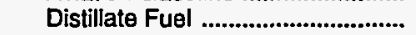 & 24.8 & 83.0 & 236.1 & 325.9 & 163.8 & 205.2 & 207.7 & 259.9 & 297.6 & 271.0 \\
\hline 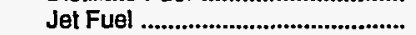 & 1.2 & 2.7 & 12.8 & 9.0 & 6.3 & 5.6 & 6.2 & 10.6 & 9.8 & 6.7 \\
\hline LPG & .1 & .1 & .3 & .7 & .6 & .5 & .8 & .6 & .7 & .7 \\
\hline 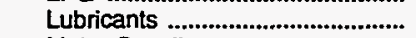 & 5.7 & 10.9 & 21.8 & 24.3 & 21.0 & 19.4 & 21.5 & 20.1 & 20.8 & 21.4 \\
\hline 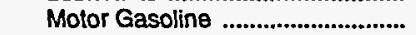 & 235.1 & 464.3 & $1,004.2$ & 868.1 & 665.9 & 722.3 & 759.8 & 846.6 & 991.6 & 980.2 \\
\hline 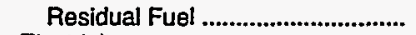 & . & - & - & & - & - & - & - & - & - \\
\hline Electricity & - & - & - & - & - & - & - & - & - & - \\
\hline 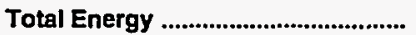 & 793.5 & $1,878.7$ & $3,800.5$ & $3,598.1$ & $3,037.8$ & $3,164.6$ & $3,211.7$ & $3,496.3$ & $3,881.9$ & $3,751.4$ \\
\hline Primary Energy - Four Sectors ..... & 589.2 & $1,401.5$ & $3,051.8$ & $2,597.7$ & $2,008.2$ & $2,111.5$ & $2,143.5$ & $2,411.4$ & $2,793.0$ & $2,618.4$ \\
\hline 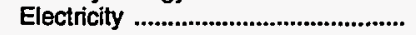 & 204.3 & 477.3 & 748.8 & $1,000.4$ & $1,029.6$ & $1,053.0$ & $1,068.2$ & $1,084.9$ & $1,088.8$ & $1,133.0$ \\
\hline 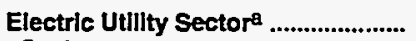 & 89.9 & 531.0 & 997.7 & $1,261.8$ & $1,156.2$ & $1,093.2$ & 1,148.1 & $1,155.4$ & $1,108.0$ & $1,056.7$ \\
\hline 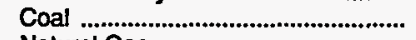 & 87.1 & 522.5 & 972.5 & $1,248.3$ & $1,146.7$ & $1,082.5$ & $1,139.6$ & $1,144.3$ & $1,095.1$ & $1,045.6$ \\
\hline Natural Gas ................................................ & .2 & .1 & .2 & .6 & 1.2 & .9 & .3 & .6 & .7 & .5 \\
\hline Petroleum & 2.6 & 8.3 & 25.1 & 12.9 & 8.2 & 9.7 & 8.2 & 10.6 & 12.3 & 10.6 \\
\hline Heavy Oib & 2.5 & 8.2 & - & - & - & - & - & - & - & - \\
\hline 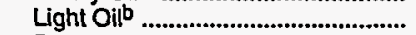 & & .2 & 25.1 & 12.9 & 8.2 & 9.7 & 8.2 & 10.6 & 12.3 & 10.6 \\
\hline 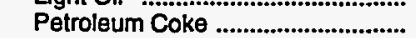 & - & - & - & - & - & - & - & - & - & - \\
\hline 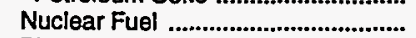 & - & - & - & - & - & - & - & - & - & - \\
\hline 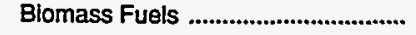 & - & - & - & - & - & - & - & - & - & - \\
\hline Primary Energy — Five Sectors ${ }^{c}$.. & 679.1 & $1,932.5$ & $4,049.5$ & $3,859.5$ & $3,164.4$ & $\mathbf{3 , 2 0 4 . 7}$ & $3,291.6$ & $3,566.8$ & $3,901.0$ & $3,675.1$ \\
\hline
\end{tabular}

\footnotetext{
a There are no direct fuel costs for hydroelectric, geothermal, centralized solar, or wind energy.

beavy oll includes Grade Nos. 4, 5, and 6, and residual fuel oils. Light oil includes Grade No. 2 heating oil, kerosene, and jet fuel.

c Biomass fuels are not included, except those consumed at electric utilities and those added to motor gasoline.

-No consumption, including cases where adjustments were made. See "Consumption Adjustments for Process Fuel and Intermediate Products." -Value less than 0.05 million dollars.

Note: Totals may not equal sum of components due to independent rounding.

Sources: Data sources, estimation procedures, and assumptions are described in the "Documentation" section of this report.
} 


\begin{tabular}{|c|c|c|c|c|c|c|c|c|c|c|}
\hline Energy Source & 1970 & 1975 & 1980 & 1985 & 1986 & 1987 & 1988 & 1989 & 1990 & 1991 \\
\hline & \multicolumn{10}{|c|}{ Prices In Dollars per Million Btu } \\
\hline 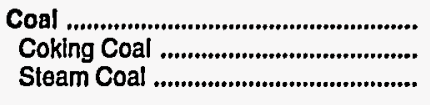 & $\begin{array}{r}0.51 \\
.53 \\
.51\end{array}$ & $\begin{array}{l}1.05 \\
1.80 \\
1.03\end{array}$ & $\begin{array}{l}1.44 \\
2.27 \\
1.43\end{array}$ & $\begin{array}{l}1.76 \\
2.08 \\
1.76\end{array}$ & $\frac{1.68}{1.68}$ & $\begin{array}{r}1.53 \\
1.53\end{array}$ & $\begin{array}{r}1.53 \\
-\overline{5}\end{array}$ & $\begin{array}{r}1.50 \\
1.50\end{array}$ & $\begin{array}{r}1.41 \\
1.41\end{array}$ & $\begin{array}{r}1.41 \\
1.41\end{array}$ \\
\hline Natural Gas ........................................... & .79 & 1.30 & 3.43 & 5.37 & 5.14 & 5.03 & 4.88 & 4.61 & 4.56 & 4.40 \\
\hline 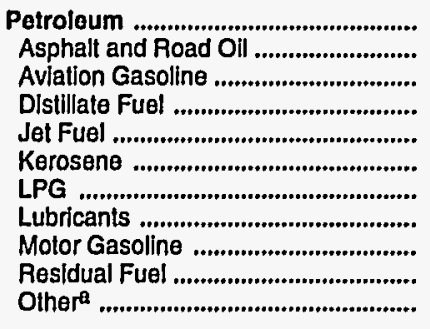 & $\begin{array}{r}1.89 \\
.76 \\
2.17 \\
1.07 \\
.74 \\
1.17 \\
1.88 \\
5.08 \\
2.65 \\
.57 \\
1.65\end{array}$ & $\begin{array}{l}3.65 \\
2.05 \\
3.45 \\
2.47 \\
2.08 \\
2.71 \\
3.60 \\
7.48 \\
4.54 \\
1.79 \\
3.21\end{array}$ & $\begin{array}{r}8.13 \\
3.85 \\
9.02 \\
6.59 \\
6.38 \\
7.05 \\
6.51 \\
14.36 \\
9.43 \\
3.48 \\
8.73\end{array}$ & $\begin{array}{r}8.68 \\
4.87 \\
9.99 \\
7.66 \\
6.19 \\
8.20 \\
8.55 \\
17.61 \\
9.33 \\
4.59 \\
8.73\end{array}$ & $\begin{array}{r}6.63 \\
4.09 \\
8.41 \\
5.78 \\
4.45 \\
6.01 \\
8.14 \\
15.59 \\
7.00 \\
2.99 \\
7.37\end{array}$ & $\begin{array}{r}6.92 \\
3.37 \\
7.55 \\
6.15 \\
4.28 \\
6.29 \\
7.34 \\
12.70 \\
7.49 \\
2.55 \\
7.90\end{array}$ & $\begin{array}{r}6.82 \\
3.34 \\
7.41 \\
5.96 \\
4.11 \\
5.90 \\
7.04 \\
14.61 \\
7.55 \\
2.45 \\
6.86\end{array}$ & $\begin{array}{r}7.56 \\
2.94 \\
8.28 \\
6.66 \\
4.63 \\
6.52 \\
10.18 \\
13.30 \\
8.29 \\
2.40 \\
7.73\end{array}$ & $\begin{array}{r}8.51 \\
3.13 \\
9.32 \\
7.81 \\
5.99 \\
7.68 \\
9.78 \\
13.40 \\
9.38 \\
2.41 \\
7.79\end{array}$ & $\begin{array}{r}8.22 \\
3.10 \\
8.71 \\
7.21 \\
5.26 \\
7.11 \\
8.42 \\
15.42 \\
9.18 \\
2.35 \\
7.38\end{array}$ \\
\hline Nuclear Fuel .......................................... & .15 & .32 & .47 & .58 & .51 & .48 & .43 & .45 & .48 & .45 \\
\hline Blomass Fuels at Utllitles ................. & .65 & - & 1.74 & .79 & .32 & .95 & .87 & .75 & .68 & .73 \\
\hline Primary Energy - Flve Sectors ${ }^{b}$.... & 1.16 & 2.12 & 4.42 & 5.01 & 4.15 & 4.14 & 4.14 & 4.38 & 4.63 & 4.49 \\
\hline $\begin{array}{l}\text { Electric Utility Fuelc ........................... } \\
\text { Electricity Purchased by End Users }\end{array}$ & $\begin{array}{r}.39 \\
6.02\end{array}$ & $\begin{array}{r}.71 \\
8.88\end{array}$ & $\begin{array}{r}1.25 \\
13.34\end{array}$ & $\begin{array}{r}1.42 \\
16.87\end{array}$ & $\begin{array}{r}1.33 \\
16.97\end{array}$ & $\begin{array}{r}1.23 \\
16.41\end{array}$ & $\begin{array}{r}1.21 \\
16.14\end{array}$ & $\begin{array}{r}1.21 \\
15.99\end{array}$ & $\begin{array}{r}1.15 \\
15.77\end{array}$ & $\begin{array}{r}1.15 \\
16.01\end{array}$ \\
\hline \multirow[t]{2}{*}{ 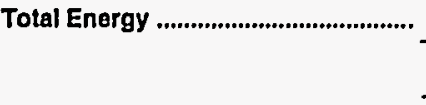 } & 1.75 & 3.28 & 6.85 & 8.61 & 7.71 & 7.68 & 7.51 & 7.73 & 8.22 & 8.05 \\
\hline & \multicolumn{10}{|c|}{ Expenditures in Millions of Dollars } \\
\hline 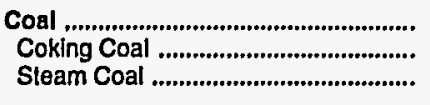 & $\begin{array}{r}196.2 \\
5.0 \\
191.2\end{array}$ & $\begin{array}{r}285.6 \\
12.0 \\
273.6\end{array}$ & $\begin{array}{r}472.2 \\
12.3 \\
459.9\end{array}$ & $\begin{array}{r}636.0 \\
.1 \\
635.9\end{array}$ & $\begin{array}{r}623.8 \\
-\overline{8} \\
623.8\end{array}$ & $\begin{array}{r}590.9 \\
590.9\end{array}$ & $\begin{array}{r}601.6 \\
601.6\end{array}$ & $\begin{array}{r}589.5 \\
-\overline{5}\end{array}$ & $\begin{array}{r}560.2 \\
-\overline{2} \\
560.2\end{array}$ & $\begin{array}{r}573.5 \\
573.5\end{array}$ \\
\hline 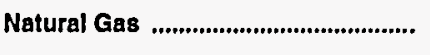 & 267.1 & 474.2 & $1,184.8$ & $1,634.5$ & $1,408.5$ & $1,374.5$ & $1,515.0$ & $1,491.3$ & $1,371.6$ & $1,420.9$ \\
\hline 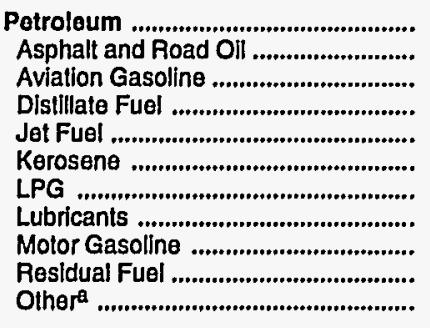 & $\begin{array}{r}952.8 \\
23.6 \\
3.6 \\
161.6 \\
6.7 \\
19.9 \\
54.3 \\
30.6 \\
633.6 \\
8.8 \\
10.0\end{array}$ & $\begin{array}{r}1,896.3 \\
41.1 \\
3.0 \\
382.3 \\
26.0 \\
15.0 \\
112.5 \\
41.9 \\
1,230.6 \\
19.3 \\
24.5\end{array}$ & $\begin{array}{r}3,832.2 \\
77.1 \\
5.6 \\
863.2 \\
86.1 \\
8.9 \\
143.2 \\
88.8 \\
2,457.8 \\
27.6 \\
73.8\end{array}$ & $\begin{array}{r}3,776.2 \\
54.6 \\
5.1 \\
1,007.8 \\
57.8 \\
10.9 \\
164.3 \\
99.1 \\
2,280.9 \\
9.3 \\
86.4\end{array}$ & $\begin{array}{r}2,905.3 \\
55.8 \\
4.6 \\
738.9 \\
39.1 \\
3.2 \\
157.5 \\
85.8 \\
1,743.8 \\
14.5 \\
62.3\end{array}$ & $\begin{array}{r}3,029.5 \\
53.5 \\
3.2 \\
756.9 \\
34.8 \\
4.1 \\
150.1 \\
79.0 \\
1,864.8 \\
13.8 \\
69.3\end{array}$ & $\begin{array}{r}3,231.3 \\
75.8 \\
3.5 \\
838.7 \\
30.9 \\
2.3 \\
153.9 \\
87.6 \\
1,967.6 \\
12.8 \\
58.2\end{array}$ & $\begin{array}{r}3,612.4 \\
74.3 \\
5.4 \\
942.2 \\
34.9 \\
2.3 \\
256.6 \\
81.8 \\
2,139.3 \\
12.2 \\
63.4\end{array}$ & $\begin{array}{r}3,971.6 \\
76.4 \\
5.7 \\
1,048.6 \\
47.9 \\
2.1 \\
233.7 \\
84.8 \\
2,390.6 \\
13.2 \\
68.6\end{array}$ & $\begin{array}{r}3,899.8 \\
68.5 \\
4.6 \\
966.2 \\
37.6 \\
2.0 \\
256.3 \\
87.3 \\
2,405.6 \\
8.6 \\
63.0\end{array}$ \\
\hline Nuclear Fuel ............................................. & .3 & 36.6 & 50.3 & 69.2 & 62.2 & 59.1 & 52.7 & 52.1 & 57.9 & 53.4 \\
\hline Blomass Fuels at UtIlitles ................. & .1 & - & 1.1 & .7 & .4 & 1.5 & 1.5 & 1.4 & 1.2 & 1.2 \\
\hline Primary Energy - Flve Sectors ${ }^{b}$.... & $1,416.4$ & $2,692.7$ & $5,540.7$ & $6,116.6$ & $5,000.2$ & $5,055.6$ & $5,402.1$ & $5,746.8$ & $5,962.5$ & $5,948.7$ \\
\hline $\begin{array}{l}\text { Electric Utility Fuelc } \\
\text { Electricity Purchased by End Users }\end{array}$ & $\begin{array}{r}-109.2 \\
501.0\end{array}$ & $\begin{array}{r}-245.2 \\
932.2\end{array}$ & $\begin{array}{r}-494.9 \\
1,669.5\end{array}$ & $\begin{array}{r}-612.9 \\
2,601.0\end{array}$ & $\begin{array}{r}-595.6 \\
2,674.4\end{array}$ & $\begin{array}{r}-571.4 \\
2,491.2\end{array}$ & $\begin{array}{r}-574.9 \\
2,609.1\end{array}$ & $\begin{array}{r}-565.7 \\
2,613.8\end{array}$ & $\begin{array}{r}-544.4 \\
2,620.3\end{array}$ & $\begin{array}{r}-557.3 \\
2,760.5\end{array}$ \\
\hline Total Energy ........................................... & $1,808.2$ & $3,379.7$ & $6,715.3$ & $8,104.7$ & $7,078.9$ & $6,975.3$ & $7,436.3$ & $7,794.9$ & $8,038.4$ & $8,151.9$ \\
\hline
\end{tabular}

a Includes petroleum coke used at electric utilities.

b Blomass fuels are not included, except those consumed at electric utilities and those added to motor gasoline.

c There are no direct fuel costs for hydroelectric, geothermal, centralized solar, or wind energy.

- No consumptlon, including cases where adjustments were made. See "Consumption Adjustments for Process Fuel and Intermediate Products."

Note: Expenditure totals may not equal sum of components due to independent rounding.

Sources: Data sources, estimation procedures, and assumptions are described in the "Documentation" section of this report. 


\section{W Energy Price Estimates by Sector, Wisconsin \\ I 1970, 1975, 1980, and 1985-1991 \\ S (Dollars per Million Btu)}

\begin{tabular}{|c|c|c|c|c|c|c|c|c|c|c|}
\hline Sector and Energy Source & 1970 & 1975 & 1980 & 1985 & 1986 & 1987 & 1988 & 1989 & 1990 & 1991 \\
\hline 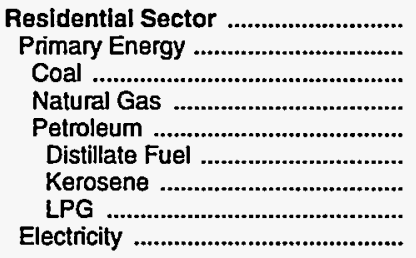 & $\begin{array}{l}2.06 \\
1.33 \\
1.63 \\
1.22 \\
1.42 \\
1.21 \\
1.47 \\
2.07 \\
6.75\end{array}$ & $\begin{array}{r}3.48 \\
2.24 \\
3.10 \\
1.71 \\
2.94 \\
2.57 \\
2.97 \\
4.14 \\
10.04\end{array}$ & $\begin{array}{r}6.85 \\
4.78 \\
3.92 \\
3.81 \\
6.82 \\
6.60 \\
8.11 \\
7.69 \\
15.04\end{array}$ & $\begin{array}{r}10.03 \\
6.80 \\
4.26 \\
6.41 \\
7.75 \\
7.44 \\
8.45 \\
8.72 \\
19.73\end{array}$ & $\begin{array}{r}9.78 \\
6.17 \\
3.86 \\
6.14 \\
6.26 \\
5.72 \\
6.50 \\
8.05 \\
20.07\end{array}$ & $\begin{array}{r}9.55 \\
6.02 \\
3.72 \\
5.94 \\
6.23 \\
5.69 \\
6.45 \\
7.69 \\
19.69\end{array}$ & $\begin{array}{r}9.20 \\
5.89 \\
3.83 \\
5.84 \\
6.02 \\
5.59 \\
6.35 \\
7.29 \\
19.52\end{array}$ & $\begin{array}{r}9.49 \\
6.31 \\
3.84 \\
5.61 \\
8.18 \\
6.14 \\
6.97 \\
12.12 \\
19.56\end{array}$ & $\begin{array}{r}9.80 \\
6.37 \\
3.37 \\
5.71 \\
8.17 \\
7.13 \\
8.09 \\
10.03 \\
19.44\end{array}$ & $\begin{array}{r}9.59 \\
6.14 \\
3.43 \\
5.57 \\
7.60 \\
6.78 \\
7.69 \\
8.91 \\
19.73\end{array}$ \\
\hline 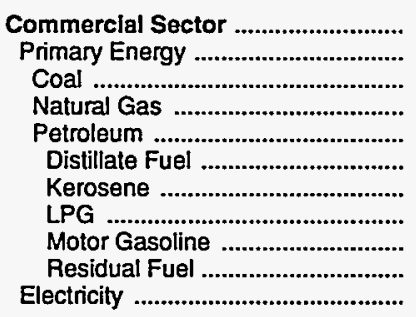 & $\begin{array}{r}2.05 \\
.84 \\
.66 \\
.82 \\
1.08 \\
1.04 \\
.83 \\
1.35 \\
2.65 \\
.59 \\
7.28\end{array}$ & $\begin{array}{r}3.55 \\
1.50 \\
1.51 \\
1.29 \\
2.43 \\
2.39 \\
2.41 \\
2.63 \\
4.54 \\
1.66 \\
10.13\end{array}$ & $\begin{array}{r}6.92 \\
3.80 \\
1.47 \\
3.43 \\
6.21 \\
6.30 \\
5.72 \\
5.33 \\
9.43 \\
4.31 \\
15.25\end{array}$ & $\begin{array}{r}9.51 \\
5.50 \\
2.11 \\
5.14 \\
6.73 \\
6.43 \\
6.98 \\
8.32 \\
9.33 \\
4.50 \\
18.90\end{array}$ & $\begin{array}{r}10.06 \\
4.93 \\
2.02 \\
4.96 \\
4.92 \\
4.29 \\
4.57 \\
8.25 \\
7.00 \\
2.90 \\
18.78\end{array}$ & $\begin{array}{r}9.47 \\
4.76 \\
1.90 \\
4.74 \\
5.13 \\
4.63 \\
5.16 \\
6.78 \\
7.49 \\
2.55 \\
17.93\end{array}$ & $\begin{array}{r}9.09 \\
4.64 \\
1.95 \\
4.64 \\
4.83 \\
4.40 \\
4.37 \\
6.69 \\
7.55 \\
2.46 \\
17.63\end{array}$ & $\begin{array}{r}8.87 \\
4.60 \\
1.87 \\
4.42 \\
5.35 \\
5.15 \\
5.75 \\
6.59 \\
8.29 \\
2.40 \\
17.33\end{array}$ & $\begin{array}{r}9.29 \\
5.04 \\
1.80 \\
4.72 \\
6.36 \\
5.65 \\
7.06 \\
9.36 \\
9.38 \\
2.41 \\
17.03\end{array}$ & $\begin{array}{r}9.09 \\
4.77 \\
1.78 \\
4.59 \\
5.57 \\
4.88 \\
6.17 \\
7.61 \\
9.18 \\
2.35 \\
17.17\end{array}$ \\
\hline 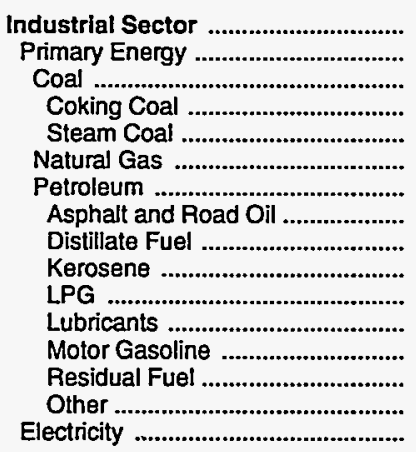 & $\begin{array}{r}1.00 \\
.76 \\
.65 \\
.53 \\
.66 \\
.54 \\
1.14 \\
.76 \\
.76 \\
.83 \\
1.35 \\
5.08 \\
2.65 \\
.57 \\
2.05 \\
4.23\end{array}$ & $\begin{array}{l}2.18 \\
1.65 \\
1.55 \\
1.80 \\
1.51 \\
1.03 \\
2.70 \\
2.05 \\
2.23 \\
2.41 \\
2.63 \\
7.48 \\
4.54 \\
2.06 \\
3.29 \\
6.63\end{array}$ & $\begin{array}{r}4.56 \\
3.61 \\
1.55 \\
2.27 \\
1.47 \\
3.12 \\
5.95 \\
3.85 \\
5.18 \\
5.72 \\
5.33 \\
14.36 \\
9.43 \\
3.31 \\
8.78 \\
10.10\end{array}$ & $\begin{array}{r}6.35 \\
4.69 \\
2.11 \\
2.08 \\
2.11 \\
4.44 \\
7.57 \\
4.87 \\
6.30 \\
6.98 \\
8.32 \\
17.61 \\
9.33 \\
4.50 \\
8.84 \\
12.64\end{array}$ & $\begin{array}{r}5.97 \\
4.10 \\
2.02 \\
-\overline{2} \\
2.02 \\
4.18 \\
5.73 \\
4.09 \\
4.13 \\
4.57 \\
8.25 \\
15.59 \\
7.00 \\
2.90 \\
7.37 \\
12.71\end{array}$ & $\begin{array}{r}5.85 \\
4.13 \\
1.90 \\
-\overline{0} \\
1.90 \\
4.32 \\
5.46 \\
3.37 \\
4.66 \\
5.16 \\
6.78 \\
12.70 \\
7.49 \\
2.55 \\
7.90 \\
12.31\end{array}$ & $\begin{array}{r}5.60 \\
3.91 \\
1.95 \\
- \\
1.95 \\
4.05 \\
4.97 \\
3.34 \\
3.94 \\
4.37 \\
6.69 \\
14.61 \\
7.55 \\
2.46 \\
6.86 \\
12.01\end{array}$ & $\begin{array}{r}5.49 \\
3.79 \\
1.87 \\
- \\
1.87 \\
3.71 \\
5.21 \\
2.94 \\
5.19 \\
5.75 \\
6.59 \\
13.30 \\
8.29 \\
2.40 \\
7.73 \\
11.91\end{array}$ & $\begin{array}{r}5.51 \\
3.82 \\
1.80 \\
-\overline{1} \\
1.80 \\
3.37 \\
5.92 \\
3.13 \\
6.37 \\
7.06 \\
9.36 \\
13.40 \\
9.38 \\
2.41 \\
7.79 \\
11.69\end{array}$ & $\begin{array}{r}5.41 \\
3.67 \\
1.78 \\
- \\
1.78 \\
3.14 \\
5.77 \\
3.10 \\
5.56 \\
6.17 \\
7.61 \\
15.42 \\
9.18 \\
2.35 \\
7.38 \\
11.81\end{array}$ \\
\hline 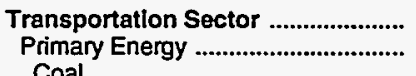 & $\begin{array}{l}2.49 \\
2.49\end{array}$ & $\begin{array}{l}4.24 \\
4.24\end{array}$ & $\begin{array}{l}8.99 \\
8.99\end{array}$ & $\begin{array}{l}9.18 \\
9.18\end{array}$ & $\begin{array}{l}6.95 \\
6.95\end{array}$ & $\begin{array}{l}7.40 \\
7.40\end{array}$ & $\begin{array}{l}7.42 \\
7.42\end{array}$ & $\begin{array}{l}8.08 \\
8.08\end{array}$ & $\begin{array}{l}9.21 \\
9.21\end{array}$ & $\begin{array}{l}8.99 \\
8.99\end{array}$ \\
\hline 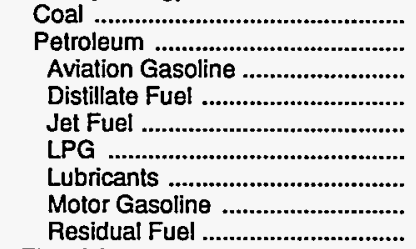 & $\begin{array}{r}.66 \\
2.49 \\
2.17 \\
1.33 \\
.74 \\
1.35 \\
5.08 \\
2.65 \\
.55\end{array}$ & $\begin{array}{l}1.51 \\
4.24 \\
3.45 \\
2.62 \\
2.08 \\
2.63 \\
7.48 \\
4.54 \\
1.44\end{array}$ & $\begin{array}{r}-\overline{8.99} \\
9.02 \\
7.28 \\
6.38 \\
5.33 \\
14.36 \\
9.43 \\
3.80\end{array}$ & $\begin{array}{r}-\overline{9.18} \\
9.99 \\
8.69 \\
6.19 \\
8.32 \\
17.61 \\
9.33 \\
4.71\end{array}$ & $\begin{array}{r}6.95 \\
8.41 \\
6.69 \\
4.45 \\
8.25 \\
15.59 \\
7.00 \\
3.60\end{array}$ & $\begin{array}{r}7.40 \\
7.55 \\
7.15 \\
4.28 \\
6.78 \\
12.70 \\
7.49 \\
-\end{array}$ & $\begin{array}{r}7.42 \\
7.41 \\
7.00 \\
4.11 \\
6.69 \\
14.61 \\
7.55 \\
2.37\end{array}$ & $\begin{array}{r}8.08 \\
8.28 \\
7.51 \\
4.63 \\
6.59 \\
13.30 \\
8.29 \\
2.66\end{array}$ & $\begin{array}{r}9 . \overline{-} \\
9.32 \\
8.79 \\
5.99 \\
9.36 \\
13.40 \\
9.38 \\
2.80\end{array}$ & $\begin{array}{r}8 . \overline{99} \\
8.71 \\
8.40 \\
5.26 \\
7.61 \\
15.42 \\
9.18 \\
2.29\end{array}$ \\
\hline 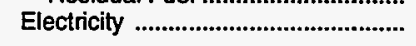 & - & - & - & - & - & - & - & - & - & - \\
\hline $\begin{array}{l}\text { Total Energy .................................. } \\
\text { Primary Energy - Four Sectors ...... } \\
\text { Electricity .......................................... }\end{array}$ & $\begin{array}{l}1.75 \\
1.38 \\
6.02\end{array}$ & $\begin{array}{l}3.28 \\
2.65 \\
8.88\end{array}$ & $\begin{array}{r}6.85 \\
5.90 \\
13.34\end{array}$ & $\begin{array}{r}8.61 \\
6.99 \\
16.87\end{array}$ & $\begin{array}{r}7.71 \\
5.80 \\
16.97\end{array}$ & $\begin{array}{r}7.68 \\
5.93 \\
16.41\end{array}$ & $\begin{array}{r}7.51 \\
5.83 \\
16.14\end{array}$ & $\begin{array}{r}7.73 \\
6.13 \\
15.99\end{array}$ & $\begin{array}{r}8.22 \\
6.67 \\
15.77\end{array}$ & $\begin{array}{r}8.05 \\
6.42 \\
16.01\end{array}$ \\
\hline 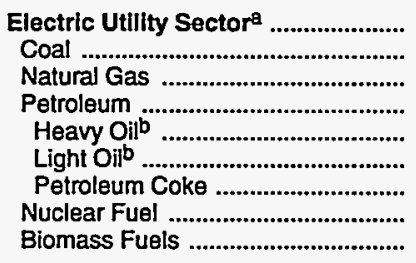 & $\begin{array}{l}.39 \\
.39 \\
.42 \\
.54 \\
.56 \\
.67 \\
.36 \\
.15 \\
.65\end{array}$ & $\begin{array}{r}.71 \\
.86 \\
.82 \\
1.93 \\
1.65 \\
2.30 \\
.72 \\
.32 \\
-\end{array}$ & $\begin{array}{r}1.25 \\
1.42 \\
2.94 \\
5.35 \\
4.28 \\
5.58 \\
1.17 \\
.47 \\
1.74\end{array}$ & $\begin{array}{r}1.42 \\
1.71 \\
4.11 \\
5.12 \\
-\overline{5} \\
5.48 \\
1.38 \\
.58 \\
.79\end{array}$ & $\begin{array}{r}1.33 \\
1.62 \\
3.73 \\
3.60 \\
- \\
3.60 \\
- \\
.51 \\
.32\end{array}$ & $\begin{array}{r}1.23 \\
1.47 \\
3.92 \\
3.97 \\
- \\
3.97 \\
-\overline{48} \\
.95\end{array}$ & $\begin{array}{r}1.21 \\
1.46 \\
3.25 \\
3.65 \\
- \\
3.65 \\
-\overline{43} \\
.87\end{array}$ & $\begin{array}{r}1.21 \\
1.45 \\
3.10 \\
4.10 \\
-\overline{0} \\
4.10 \\
- \\
.45 \\
.75\end{array}$ & $\begin{array}{r}1.15 \\
1.36 \\
2.93 \\
5.26 \\
-\overline{6} \\
5.26 \\
-\overline{48} \\
.68\end{array}$ & $\begin{array}{r}1.15 \\
1.36 \\
2.70 \\
4.46 \\
-\overline{6} \\
4.46 \\
\overline{45} \\
.73\end{array}$ \\
\hline Primary Energy - Five Sectorsc ... & 1.16 & 2.12 & 4.42 & 5.01 & 4.15 & 4.14 & 4.14 & 4.38 & 4.63 & 4.49 \\
\hline
\end{tabular}

a There are no direct fuel costs for hydroelectric, geothemal, centralized solar, or wind energy.

b Heavy oil includes Grade Nos. 4, 5, and 6, and residual fuel oils. Light oil includes Grade No. 2 heating oil, kerosene, and jet fuel.

c Biomass fuels are not included, except those consumed at electric utilities and those added to motor gasoline.

-No consumption, including cases where adjustments were made. See "Consumption Adjustments for Process Fuel and Intermediate Products."

Sources: Data sources, estimation procedures, and assumptions are described in the "Documentation" section of this report. 
Energy Expenditure Estimates by Sector, Wisconsin $1970,1975,1980$, and 1985-1991

(Million Dollars)

\begin{tabular}{|c|c|c|c|c|c|c|c|c|c|c|}
\hline Sector and Energy Source & 1970 & 1975 & 1980 & 1985 & 1986 & 1987 & 1988 & 1989 & 1990 & 1991 \\
\hline 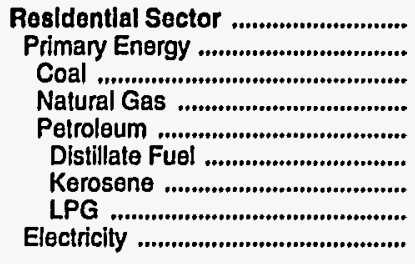 & $\begin{array}{r}512.5 \\
286.3 \\
15.5 \\
131.2 \\
139.5 \\
82.3 \\
13.4 \\
43.9 \\
226.2\end{array}$ & $\begin{array}{r}882.0 \\
478.4 \\
11.9 \\
209.5 \\
257.0 \\
164.8 \\
8.9 \\
83.2 \\
403.6\end{array}$ & $\begin{array}{r}1,575.9 \\
878.3 \\
1.7 \\
473.2 \\
403.4 \\
313.4 \\
5.7 \\
84.3 \\
697.6\end{array}$ & $\begin{array}{r}2,233.8 \\
1,136.1 \\
1.0 \\
751.6 \\
383.5 \\
278.4 \\
9.3 \\
95.7 \\
1,097.7\end{array}$ & $\begin{array}{r}2,128.4 \\
994.8 \\
1.1 \\
687.3 \\
306.5 \\
214.2 \\
2.6 \\
89.6 \\
1,133.5\end{array}$ & $\begin{aligned} 1,948.2 \\
911.5 \\
2.9 \\
617.9 \\
290.7 \\
191.5 \\
3.7 \\
95.5 \\
1,036.7\end{aligned}$ & $\begin{array}{r}2,115.4 \\
1,024.4 \\
2.6 \\
714.7 \\
307.2 \\
212.4 \\
1.9 \\
92.9 \\
1,091.0\end{array}$ & $\begin{array}{r}2,194.8 \\
1,109.9 \\
.6 \\
716.3 \\
392.9 \\
193.0 \\
1.6 \\
198.4 \\
1,085.0\end{array}$ & $\begin{array}{r}2,087.9 \\
1,000.9 \\
.2 \\
654.6 \\
346.0 \\
192.5 \\
1.3 \\
152.2 \\
1,087.0\end{array}$ & $\begin{array}{r}2,236.9 \\
1,068.8 \\
.3 \\
696.1 \\
372.4 \\
202.4 \\
1.3 \\
168.7 \\
1,168.0\end{array}$ \\
\hline 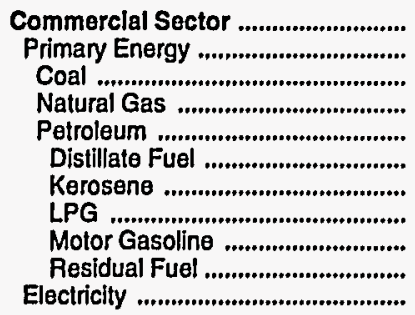 & $\begin{array}{r}229.6 \\
76.1 \\
11.7 \\
45.5 \\
18.8 \\
11.5 \\
.6 \\
5.0 \\
.8 \\
.9 \\
153.5\end{array}$ & $\begin{array}{r}425.5 \\
137.1 \\
10.7 \\
88.6 \\
37.8 \\
24.9 \\
.6 \\
9.3 \\
1.2 \\
1.8 \\
288.4\end{array}$ & $\begin{array}{r}868.0 \\
346.5 \\
1.2 \\
266.9 \\
78.5 \\
61.8 \\
1.8 \\
10.3 \\
3.8 \\
.8 \\
521.5\end{array}$ & $\begin{array}{r}1,311.2 \\
531.6 \\
.9 \\
378.3 \\
152.5 \\
118.8 \\
.7 \\
16.1 \\
13.9 \\
3.0 \\
779.6\end{array}$ & $\begin{array}{r}1,142.3 \\
352.5 \\
1.1 \\
277.1 \\
74.3 \\
43.1 \\
.1 \\
16.2 \\
10.3 \\
4.6 \\
789.8\end{array}$ & $\begin{array}{r}1,100.0 \\
355.2 \\
2.8 \\
276.0 \\
76.4 \\
48.4 \\
.1 \\
14.9 \\
11.2 \\
1.9 \\
744.8\end{array}$ & $\begin{array}{r}1,170.3 \\
392.3 \\
2.4 \\
313.3 \\
76.6 \\
46.2 \\
.2 \\
15.0 \\
11.4 \\
3.8 \\
778.0\end{array}$ & $\begin{array}{r}1,184.1 \\
408.1 \\
.5 \\
311.2 \\
96.3 \\
60.5 \\
.2 \\
.2 \\
19.0 \\
12.1 \\
4.5 \\
776.0\end{array}$ & $\begin{array}{r}1,199.0 \\
420.0 \\
.1 \\
315.2 \\
104.7 \\
60.3 \\
.3 \\
25.1 \\
15.7 \\
3.3 \\
779.0\end{array}$ & $\begin{array}{r}1,246.8 \\
426.8 \\
.3 \\
330.5 \\
96.0 \\
55.7 \\
.3 \\
25.4 \\
11.9 \\
2.6 \\
820.0\end{array}$ \\
\hline 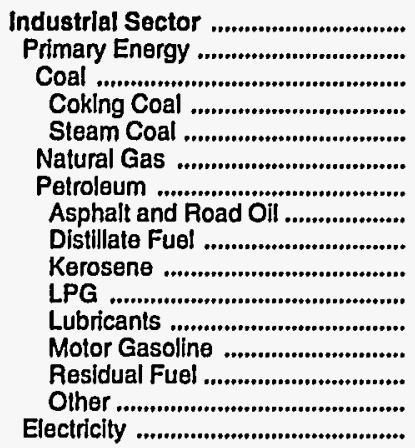 & $\begin{array}{r}407.6 \\
286.3 \\
78.0 \\
5.0 \\
73.0 \\
77.3 \\
131.0 \\
23.6 \\
35.1 \\
5.9 \\
5.0 \\
13.6 \\
34.4 \\
3.9 \\
9.5 \\
121.3\end{array}$ & $\begin{array}{r}744.3 \\
50.1 \\
84.6 \\
12.0 \\
72.6 \\
159.5 \\
260.0 \\
41.1 \\
92.9 \\
5.5 \\
19.1 \\
19.3 \\
48.4 \\
9.3 \\
24.4 \\
240.2\end{array}$ & $\begin{array}{r}1,390.1 \\
939.7 \\
84.6 \\
12.3 \\
72.3 \\
404.2 \\
450.9 \\
77.1 \\
108.3 \\
1.3 \\
47.0 \\
43.2 \\
80.9 \\
19.4 \\
73.7 \\
450.4\end{array}$ & $\begin{array}{r}1,735.0 \\
1,011.4 \\
104.7 \\
.1 \\
104.6 \\
499.2 \\
407.5 \\
54.6 \\
112.5 \\
.8 \\
47.0 \\
48.3 \\
55.7 \\
2.2 \\
86.2 \\
723.7\end{array}$ & $\begin{array}{r}1,626.4 \\
875.4 \\
100.6 \\
-\overline{1} \\
100.6 \\
437.5 \\
337.3 \\
55.8 \\
82.5 \\
.5 \\
47.5 \\
41.8 \\
39.2 \\
7.7 \\
62.3 \\
751.0\end{array}$ & $\begin{array}{r}1,602.0 \\
892.3 \\
86.9 \\
-\overline{8} \\
86.9 \\
472.1 \\
333.4 \\
53.5 \\
83.8 \\
.3 \\
36.7 \\
38.5 \\
39.3 \\
12.0 \\
69.3 \\
709.7\end{array}$ & $\begin{array}{r}\mathbf{1 , 6 5 0 . 7} \\
910.5 \\
89.7 \\
89 . \overline{1} \\
89.7 \\
478.2 \\
342.7 \\
75.8 \\
79.6 \\
.2 \\
42.7 \\
42.7 \\
34.5 \\
8.9 \\
58.2 \\
740.1\end{array}$ & $\begin{array}{r}1,658.1 \\
905.2 \\
86.7 \\
-\overline{-} \\
86.7 \\
457.4 \\
361.2 \\
74.3 \\
101.5 \\
.5 \\
36.3 \\
39.8 \\
37.8 \\
7.6 \\
63.4 \\
752.9\end{array}$ & $\begin{array}{r}1,654.8 \\
900.5 \\
85.0 \\
-\overline{-} \\
85.0 \\
394.8 \\
420.7 \\
76.4 \\
133.4 \\
.4 \\
52.4 \\
41.3 \\
38.2 \\
9.8 \\
68.6 \\
754.3\end{array}$ & $\begin{array}{r}1,660.3 \\
887.8 \\
81.3 \\
81 . \overline{3} \\
886.9 \\
419.7 \\
68.5 \\
132.8 \\
.3 \\
58.3 \\
42.5 \\
48.1 \\
6.0 \\
63.0 \\
772.5\end{array}$ \\
\hline 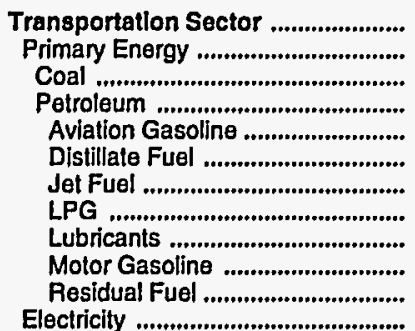 & $\begin{array}{r}658.5 \\
658.5 \\
.1 \\
658.4 \\
3.6 \\
32.3 \\
6.7 \\
.4 \\
17.0 \\
598.4 \\
-\end{array}$ & $\begin{array}{r}1,328.0 \\
1,328.0 \\
1,328.0 \\
3.0 \\
92.4 \\
25.5 \\
.9 \\
22.6 \\
1,181.0 \\
2.6 \\
\end{array}$ & $\begin{array}{r}2,881.3 \\
2,881.3 \\
2,881 . \overline{3} \\
5.6 \\
363.6 \\
86.1 \\
1.6 \\
45.5 \\
2,373.2 \\
5.6 \\
\end{array}$ & $\begin{array}{r}2,824.7 \\
2,824.7 \\
2,824 . \overline{7} \\
5.1 \\
490.1 \\
57.8 \\
5.5 \\
50.8 \\
2,211.3 \\
4.1\end{array}$ & $\begin{aligned} 2,181.8 \\
2,181.8 \\
2,181.8 \\
4.6 \\
393.6 \\
39.1 \\
4.2 \\
44.0 \\
1,694.2 \\
2.2 \\
\end{aligned}$ & $\begin{array}{r}2,325.1 \\
2,325.1 \\
- \\
2,325.1 \\
3.2 \\
429.2 \\
34.8 \\
3.1 \\
40.5 \\
1,814.3 \\
-\end{array}$ & $\begin{array}{r}2,499.9 \\
2,499.9 \\
2,- \\
2,499.9 \\
3.5 \\
495.5 \\
30.9 \\
3.3 \\
44.9 \\
1,921.7 \\
.1\end{array}$ & $\begin{array}{r}2,757.9 \\
2,757.9 \\
- \\
2,757.9 \\
5.4 \\
583.3 \\
34.9 \\
2.9 \\
41.9 \\
2,089.4 \\
.1\end{array}$ & $\begin{array}{r}3,096.8 \\
3,096.8 \\
3,096.8 \\
5.7 \\
658.9 \\
47.9 \\
4.0 \\
43.5 \\
2,336.7 \\
-\end{array}$ & $\begin{array}{r}3,007.8 \\
3,007.8 \\
-\overline{3}, 007.8 \\
4.6 \\
571.4 \\
37.6 \\
3.8 \\
44.8 \\
2,345.7 \\
-\end{array}$ \\
\hline 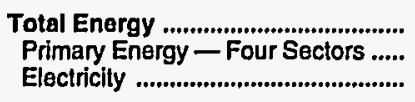 & $\begin{array}{r}1,808.2 \\
1,307.2 \\
501.0\end{array}$ & $\begin{array}{r}3,379.7 \\
2,447.5 \\
932.2\end{array}$ & $\begin{array}{l}6,715.3 \\
5,045.8 \\
1,669.5\end{array}$ & $\begin{array}{l}8,104.7 \\
5,503.7 \\
2,601.0\end{array}$ & $\begin{array}{l}7,078.9 \\
4,404.5 \\
2,674.4\end{array}$ & $\begin{array}{l}6,975.3 \\
4.484 .1 \\
2,491.2\end{array}$ & $\begin{array}{l}7,436.3 \\
4,827.2 \\
2,609.1\end{array}$ & $\begin{array}{l}7,794.9 \\
5,181.1 \\
2,613.8\end{array}$ & $\begin{array}{l}8,038.4 \\
5,418.1 \\
2,620.3\end{array}$ & $\begin{array}{l}8,151.9 \\
5,391.4 \\
2,760.5\end{array}$ \\
\hline 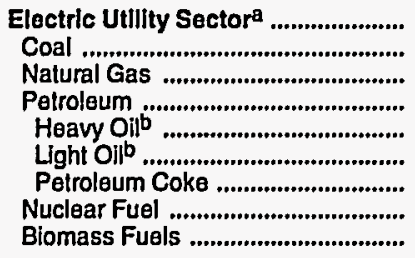 & $\begin{array}{r}109.2 \\
90.8 \\
13.1 \\
5.0 \\
4.0 \\
.5 \\
.5 \\
.3 \\
.1\end{array}$ & $\begin{array}{r}245.2 \\
178.3 \\
16.7 \\
13.6 \\
5.7 \\
7.7 \\
.2 \\
36.6 \\
-\end{array}$ & $\begin{array}{r}494.9 \\
384.7 \\
40.6 \\
18.1 \\
1.8 \\
16.2 \\
.1 \\
50.3 \\
1.1\end{array}$ & $\begin{array}{r}612.9 \\
529.4 \\
5.4 \\
8.2 \\
.0 \\
8.0 \\
.2 \\
69.2 \\
.7\end{array}$ & $\begin{array}{r}595.6 \\
520.9 \\
6.7 \\
5.4 \\
-\overline{-} \\
5.4 \\
62 . \overline{2} \\
.4\end{array}$ & $\begin{array}{r}571.4 \\
498.4 \\
8.5 \\
4.0 \\
-\overline{0} \\
4.0 \\
59.1 \\
1.5\end{array}$ & $\begin{array}{r}574.9 \\
506.9 \\
8.9 \\
4.9 \\
4.9 \\
52 . \overline{7} \\
1.5\end{array}$ & $\begin{array}{r}565.7 \\
501.7 \\
6.4 \\
4.0 \\
-\overline{-} \\
4.0 \\
52.1 \\
1.4\end{array}$ & $\begin{array}{r}544.4 \\
474.9 \\
7.0 \\
3.5 \\
3.5 \\
57.9 \\
1.2\end{array}$ & $\begin{array}{r}557.3 \\
491.6 \\
7.4 \\
3.8 \\
-\overline{-} \\
3.8 \\
53.4 \\
1.2\end{array}$ \\
\hline Primary Energy — Flve Sectorsc .. & $1,416.4$ & $2,692.7$ & $5,540.7$ & $6,116.6$ & $5,000.2$ & $5,055.6$ & $5,402.1$ & $5,746.8$ & $5,962.5$ & $5,948.7$ \\
\hline
\end{tabular}

There are no direct fuel costs for hydroelectric, geothermal, centralized solar, or wind energy.

b Heavy oil includes Grade Nos. 4, 5, and 6, and residual fuel oils. Light oil includes Grade No. 2 heating oil, kerosene, and jet fuel.

c Blomass fuels are not included, except those consumed at electric utilities and those added to motor gasoline.

- No consumption, including cases where adjustments were made. See "Consumption Adjustments for Process Fuel and Intermediate Products."

Value less than 0.05 million dollars.

Note: Totals may not equal sum of components due to independent rounding.

Sources: Data sources, estimation procedures, and assumptions are described in the "Documentation" section of this report. 


\section{W Energy Price and Expenditure Estimates by Source, Wyoming}

Y $1970,1975,1980$, and 1985-1991

\begin{tabular}{|c|c|c|c|c|c|c|c|c|c|c|}
\hline Energy Source & 1970 & 1975 & 1980 & 1985 & 1986 & 1987 & 1988 & 1989 & 1990 & 1991 \\
\hline & \multicolumn{10}{|c|}{ Prices in Dollars per Million Btu } \\
\hline Coal & 0.16 & 0.31 & 0.70 & 1.00 & 0.98 & 0.91 & 0.88 & 0.89 & 0.86 & 0.86 \\
\hline 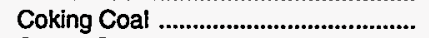 & - & - & - & - & - & - & - & - & - & - \\
\hline 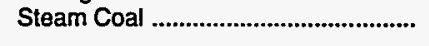 & .16 & .31 & .70 & 1.00 & .98 & .91 & .88 & .89 & .86 & .86 \\
\hline 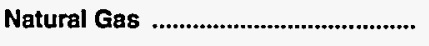 & .38 & .71 & 2.45 & 4.28 & 4.01 & 3.93 & 3.75 & 3.82 & 3.57 & 3.48 \\
\hline Petroleum & 1.73 & 3.28 & 7.16 & 7.67 & 6.08 & 5.82 & 5.98 & 6.84 & 7.58 & 7.42 \\
\hline 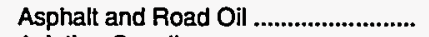 & .58 & 1.80 & 3.66 & 4.82 & 4.36 & 3.21 & 3.40 & 2.88 & 2.71 & 3.43 \\
\hline 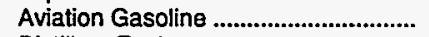 & 2.17 & 3.45 & 9.02 & 9.99 & 8.41 & 7.55 & 7.41 & 8.28 & 9.32 & 8.71 \\
\hline 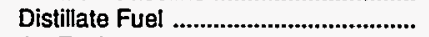 & 1.11 & 2.51 & 6.44 & 7.32 & 5.81 & 5.53 & 5.80 & 6.66 & 7.56 & 7.10 \\
\hline Jet Fuel & .76 & 2.12 & 6.59 & 6.53 & 4.56 & 4.61 & 4.62 & 5.06 & 6.45 & 6.05 \\
\hline 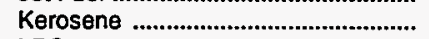 & .96 & 2.47 & 5.76 & 10.22 & 5.27 & 4.37 & 4.79 & 5.68 & 6.66 & 6.86 \\
\hline LPG & 1.64 & 3.46 & 5.77 & 8.13 & 7.22 & 6.33 & 6.21 & 7.47 & 8.01 & 9.53 \\
\hline 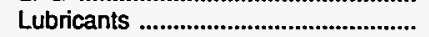 & 5.08 & 7.48 & 14.36 & 17.61 & 15.59 & 12.70 & 14.61 & 13.30 & 13.40 & 15.42 \\
\hline Motor Gasoline & 2.93 & 4.77 & 10.28 & 8.87 & 6.72 & 6.88 & 6.84 & 7.79 & 8.66 & 8.40 \\
\hline 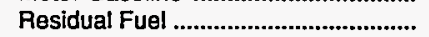 & .55 & 1.71 & 3.56 & 3.14 & 2.06 & 1.83 & 1.65 & 1.88 & 2.46 & 2.26 \\
\hline Othera & .51 & 1.39 & 2.53 & 2.37 & 1.53 & 1.50 & 1.45 & 1.74 & 2.02 & 1.72 \\
\hline Nuclear Fuel ......................................... & - & - & - & - & - & - & - & - & - & - \\
\hline Blomass Fuels at Utillties .................. & - & - & - & - & - & - & - & - & - & - \\
\hline Primary Energy - Five Sectors ${ }^{b}$.... & .84 & 1.51 & 3.00 & 2.54 & 2.30 & 2.05 & 2.03 & 2.28 & 2.26 & 2.22 \\
\hline $\begin{array}{l}\text { Electric Utility Fuelc ......................... } \\
\text { Electricity Purchased by End Users }\end{array}$ & $\begin{array}{r}.14 \\
4.53\end{array}$ & $\begin{array}{r}.26 \\
4.63\end{array}$ & $\begin{array}{r}.59 \\
7.45\end{array}$ & $\begin{array}{r}.93 \\
12.54\end{array}$ & $\begin{array}{r}.93 \\
13.13\end{array}$ & $\begin{array}{r}.88 \\
12.91\end{array}$ & $\begin{array}{r}.85 \\
12.81\end{array}$ & $\begin{array}{r}.85 \\
12.66\end{array}$ & $\begin{array}{r}.84 \\
12.37\end{array}$ & $\begin{array}{r}.84 \\
12.53\end{array}$ \\
\hline \multirow[t]{2}{*}{ 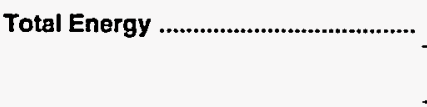 } & 1.31 & 2.53 & 5.79 & 6.87 & 6.12 & 5.97 & 6.07 & 6.45 & 6.41 & 6.23 \\
\hline & \multicolumn{10}{|c|}{ Expenditures In Milllons of Dollars } \\
\hline 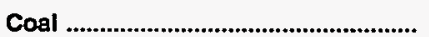 & 10.2 & 39.8 & 187.2 & 406.6 & 328.3 & 389.3 & 390.5 & 374.6 & 395.9 & 387.8 \\
\hline 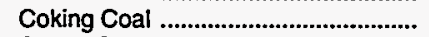 & - & - & - & - & - & - & - & - & - & - \\
\hline Steam Coal & 10.2 & 39.8 & 187.2 & 406.6 & 328.3 & 389.3 & 390.5 & 374.6 & 395.9 & 387.8 \\
\hline 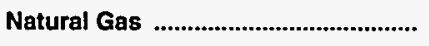 & 28.4 & 36.4 & 91.6 & 176.5 & 148.5 & 125.2 & 131.2 & 135.2 & 162.9 & 179.9 \\
\hline 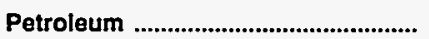 & 152.0 & 359.5 & $1,097.6$ & 825.5 & 613.3 & 664.1 & 682.8 & 799.0 & 828.8 & 77.4 \\
\hline 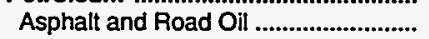 & 4.2 & 7.2 & 28.2 & 53.6 & 46.5 & 31.3 & 23.6 & 17.6 & 17.1 & 23.1 \\
\hline 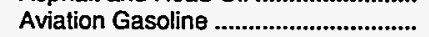 & 2.8 & 3.8 & 4.9 & 2.6 & 2.1 & 1.9 & 2.0 & 1.6 & 1.7 & 1.2 \\
\hline 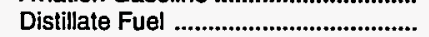 & 32.7 & 111.2 & 496.4 & 326.9 & 233.4 & 282.4 & 318.0 & 379.4 & 422.8 & 364.6 \\
\hline Jet Fuel & .5 & 1.5 & 6.0 & 5.6 & 3.7 & 5.2 & 5.0 & 4.5 & 5.1 & 4.6 \\
\hline 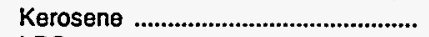 & 1.8 & 2.4 & 2.0 & 1.2 & .3 & .3 & .3 & .2 & .2 & .4 \\
\hline LPG & 10.8 & 21.6 & 42.7 & 51.1 & 52.8 & 59.9 & 43.6 & 64.8 & 37.0 & 44.1 \\
\hline 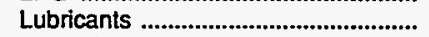 & 3.5 & 7.0 & 18.1 & 20.2 & 17.5 & 16.1 & 17.9 & 16.7 & 17.3 & 17.8 \\
\hline 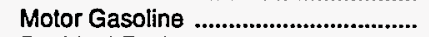 & 90.8 & 184.4 & 458.9 & 357.2 & 252.3 & 262.6 & 267.0 & 309.1 & 321.4 & 317.9 \\
\hline 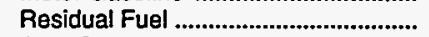 & 2.7 & 13.6 & 24.0 & 1.4 & 1.4 & .3 & 1.2 & $\because$ & 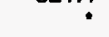 & 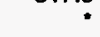 \\
\hline Other ${ }^{\mathrm{a}}$ & 2.1 & 6.9 & 16.4 & 5.8 & 3.3 & 4.0 & 4.1 & 5.0 & 6.1 & 3.6 \\
\hline Nuclear Fuel ......................................... & - & - & - & - & - & - & - & - & - & - \\
\hline Blomass Fuels at Uttlities .................. & - & - & - & - & - & - & - & - & - & - \\
\hline Primary Energy - Five Sectors ${ }^{b}$.... & 190.6 & 435.7 & $1,376.3$ & $1,408.6$ & $1,090.1$ & $1,178.5$ & $1,204.5$ & $1,308.8$ & $1,387.6$ & $1,345.1$ \\
\hline $\begin{array}{l}\text { Electric Utility Fuelc .......................... } \\
\text { Electricity Purchased by End Users }\end{array}$ & $\begin{array}{r}-8.9 \\
46.9\end{array}$ & $\begin{array}{r}-30.3 \\
70.0\end{array}$ & $\begin{array}{r}-140.7 \\
176.1\end{array}$ & $\begin{array}{r}-346.3 \\
427.3\end{array}$ & $\begin{array}{r}-283.5 \\
433.6\end{array}$ & $\begin{array}{r}-345.3 \\
449.3\end{array}$ & $\begin{array}{r}-350.0 \\
465.3\end{array}$ & $\begin{array}{r}-328.6 \\
466.9\end{array}$ & $\begin{array}{r}-349.8 \\
481.6\end{array}$ & $\begin{array}{r}-340.2 \\
487.6\end{array}$ \\
\hline 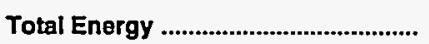 & 228.5 & 475.4 & $1,411.7$ & $1,489.7$ & $1,240.2$ & $1,282.6$ & $1,319.8$ & $1,447.2$ & $1,519.4$ & $1,492.5$ \\
\hline
\end{tabular}

a Includes petroleum coke used at electric utilities.

Biomass fuels are not included, except those consumed at electric utilities and those added to motor gasoline.

c There are no direct fuel costs for hydroelectric, geothermal, centralized solar, or wind energy.

-No consumption, including cases where adjustments were made. See "Consumption Adjustments for Process Fuel and Intermediate Products."

Note: Expenditure totals may not equal sum of components due to independent rounding.

Sources: Data sources, estimation procedures, and assumptions are described in the "Documentation" section of this report. 


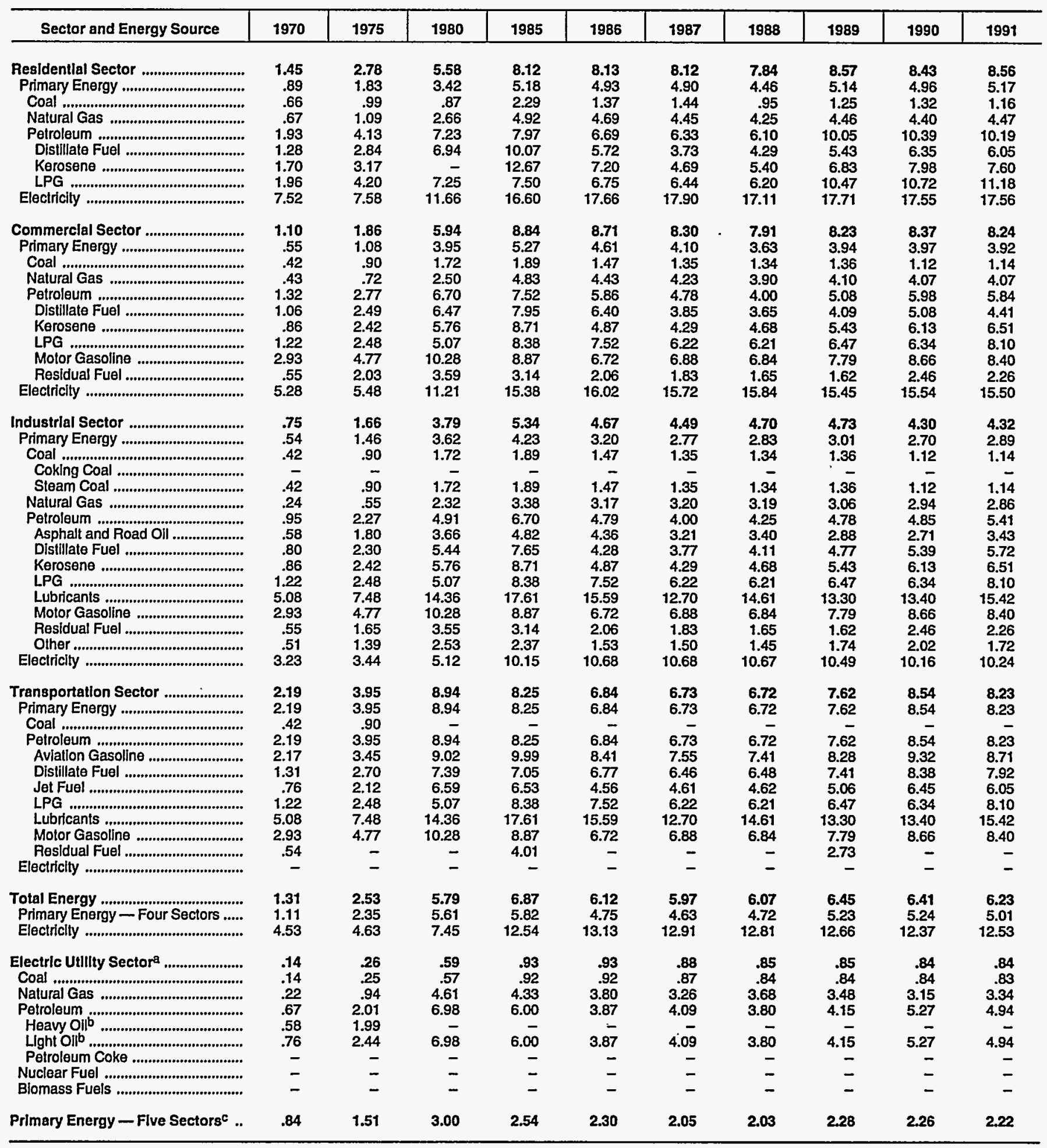

There are no direct fuel costs for hydroelectric, geothermal, centralized solar, or wind energy.

b Heavy oll includes Grade Nos. 4, 5, and 6, and residual fuel oils. Light oil includes Grade No. 2 heating oil, kerosene, and jet fuel.

c Blomass fuels are not included, except those consumed at electric utilities and those added to motor gasoline.

-No consumption, including cases where adjustments were made. See "Consumption Adjustments for Process Fuel and Intermediate Products."

Sources: Data sources, estimation procedures, and assumptions are described in the "Documentation" section of this report. 


\section{W Energy Expenditure Estimates by Sector, Wyoming}

Y 1970,1975, 1980, and 1985-1991

O (Million Dollars)

\begin{tabular}{|c|c|c|c|c|c|c|c|c|c|c|}
\hline Sector and Energy Source & 1970 & 1975 & 1980 & 1985 & 1986 & 1987 & 1988 & 1989 & 1990 & 1991 \\
\hline 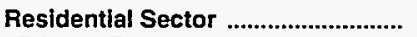 & 35.7 & 51.3 & 102.2 & 195.4 & 184.8 & 182.6 & 176.8 & 184.4 & 179.7 & 194.4 \\
\hline 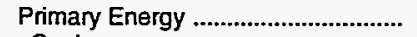 & 20.2 & 28.3 & 46.1 & 92.6 & 83.7 & 82.8 & 73.8 & 80.4 & 76.7 & 85.4 \\
\hline Coal & .1 & .3 & .6 & 1.5 & .8 & .9 & .8 & 1.0 & 1.2 & 1.3 \\
\hline 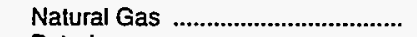 & 12.3 & 12.3 & 27.5 & 74.2 & 62.8 & 49.9 & 52.2 & 55.5 & 55.6 & 56.8 \\
\hline Pelroleum .......................................... & 7.9 & 15.6 & 18.1 & 17.0 & 20.1 & 32.0 & 20.8 & 23.9 & 19.9 & 27.2 \\
\hline 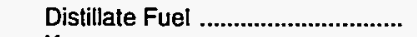 & .1 & .4 & .9 & 3.0 & .9 & .7 & .8 & 1.1 & .9 & 3.1 \\
\hline 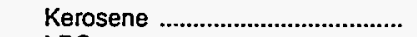 & .4 & .2 & - & .6 & .1 & .1 & $\because$ & $\because$ & .1 & .1 \\
\hline LPG & 7.4 & 15.0 & 17.1 & 13.4 & 19.1 & 31.2 & 20.0 & 22.8 & 18.9 & 24.1 \\
\hline 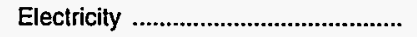 & 15.5 & 23.0 & 56.1 & 102.8 & 101.1 & 99.8 & 103.0 & 104.0 & 103.0 & 109.0 \\
\hline 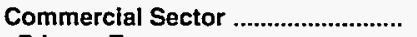 & 21.2 & 27.9 & 84.0 & 198.3 & 186.0 & 170.6 & 171.0 & 167.3 & 174.4 & 183.8 \\
\hline 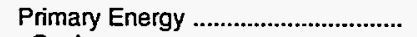 & 9.4 & 13.4 & 40.4 & 76.5 & 63.2 & 53.8 & 51.0 & 50.2 & 51.4 & 54.8 \\
\hline 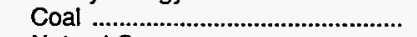 & .1 & .5 & 2.1 & 2.3 & 1.7 & 1.6 & 2.2 & 2.1 & 1.9 & 2.4 \\
\hline 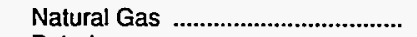 & 6.1 & 6.9 & 13.2 & 46.4 & 37.4 & 37.7 & 35.8 & 37.0 & 37.7 & 39.2 \\
\hline 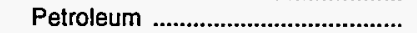 & 3.2 & 5.9 & 25.1 & 27.8 & 24.0 & 14.5 & 13.0 & 11.1 & 11.8 & 13.2 \\
\hline 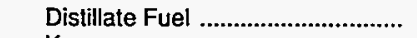 & .2 & .9 & 16.1 & 20.4 & 14.6 & 6.1 & 5.7 & 5.9 & 6.4 & 6.2 \\
\hline Kerosene & .7 & .6 & .8 & .3 & 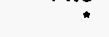 & .1 & .1 & .1 & 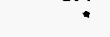 & .1 \\
\hline LPG & .8 & 1.6 & 2.1 & 2.6 & 3.8 & 5.3 & 3.5 & 2.5 & 2.0 & 3.1 \\
\hline Motor Gasoline ............................. & 1.3 & 1.8 & 5.5 & 3.1 & 4.3 & 2.6 & 2.4 & 2.6 & 3.4 & 3.8 \\
\hline 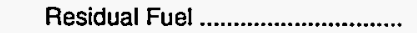 & .2 & 1.1 & .6 & 1.4 & 1.4 & .3 & 1.2 & 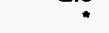 & $\because$ & 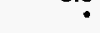 \\
\hline 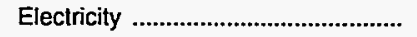 & 11.8 & 14.5 & 43.5 & 121.8 & 122.9 & 116.8 & 120.0 & 117.0 & 123.0 & 129.0 \\
\hline 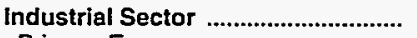 & 59.2 & 154.8 & 490.1 & 566.2 & 465.2 & 451.2 & 447.3 & 483.7 & 505.0 & 539.0 \\
\hline Primary Energy .................................... & 39.7 & 122.4 & 413.6 & 363.5 & 255.5 & 218.5 & 205.0 & 237.8 & 249.4 & 289.4 \\
\hline Coal & 1.7 & 10.6 & 49.6 & 62.2 & 45.6 & 44.6 & 41.0 & 46.1 & 46.3 & 47.7 \\
\hline 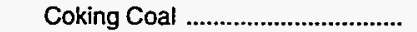 & - & - & - & - & - & - & - & - & - & - \\
\hline 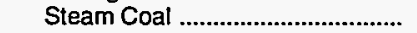 & 1.7 & 10.6 & 49.6 & 62.2 & 45.6 & 44.6 & 41.0 & 46.1 & 46.3 & 47.7 \\
\hline 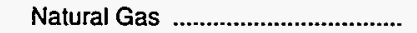 & 9.5 & 16.7 & 50.0 & 55.3 & 47.8 & 37.3 & 42.5 & 42.4 & 69.3 & 83.6 \\
\hline 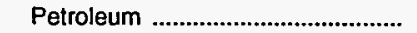 & 28.5 & 95.0 & 314.0 & 246.1 & 162.2 & 136.7 & 121.5 & 149.3 & 133.8 & 158.1 \\
\hline Asphalt and Road Oil ................... & 4.2 & 7.2 & 28.2 & 53.6 & 46.5 & 31.3 & 23.6 & 17.6 & 17.1 & 23.1 \\
\hline 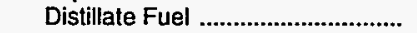 & 8.9 & 47.3 & 198.0 & 122.6 & 61.2 & 58.3 & 53.2 & 63.6 & 71.3 & 88.6 \\
\hline 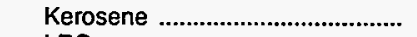 & .8 & 1.6 & 1.3 & .3 & .2 & .2 & .1 & .1 & .1 & .1 \\
\hline LPG & 2.1 & 4.0 & 22.0 & 33.6 & 28.5 & 22.3 & 18.9 & 38.6 & 15.5 & 15.5 \\
\hline 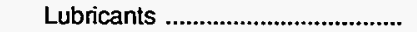 & .9 & 2.1 & 5.0 & 5.6 & 4.8 & 4.4 & 4.9 & 4.6 & 4.8 & 4.9 \\
\hline 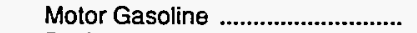 & 8.5 & 14.8 & 19.7 & 24.7 & 17.7 & 16.3 & 16.6 & 19.7 & 18.9 & 22.1 \\
\hline 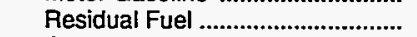 & .9 & 11.1 & 23.4 & 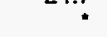 & 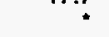 & 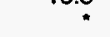 & 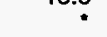 & 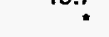 & 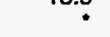 & 26. \\
\hline Other & 2.1 & 6.9 & 16.4 & 5.8 & 3.3 & 4.0 & 4.1 & 5.0 & 6.1 & 3.6 \\
\hline Electricity & 19.6 & 32.5 & 76.5 & 202.7 & 209.6 & 232.7 & 242.3 & 245.9 & 255.6 & 249.6 \\
\hline Transportation Sector ......................... & 112.4 & 241.4 & 735.4 & 529.6 & 404.2 & 478.1 & 524.7 & 611.8 & 660.3 & 575.3 \\
\hline 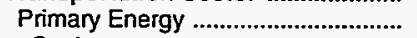 & 112.4 & 241.4 & 735.4 & 529.6 & 404.2 & 478.1 & 524.7 & 611.8 & 660.3 & 575.3 \\
\hline Coal & & 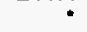 & - & - & - & - & - & - & - & - \\
\hline 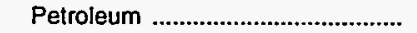 & 112.3 & 241.4 & 735.4 & 529.6 & 404.2 & 478.1 & 524.7 & 611.8 & 660.3 & 575.3 \\
\hline 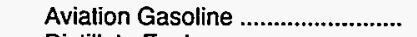 & 2.8 & 3.8 & 4.9 & 2.6 & 2.1 & 1.9 & 2.0 & 1.6 & 1.7 & 1.2 \\
\hline 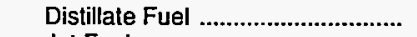 & 23.4 & 62.4 & 276.4 & 176.1 & 154.0 & 214.5 & 255.6 & 305.9 & 341.2 & 263.2 \\
\hline 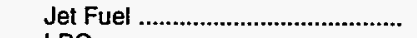 & .5 & 1.5 & 6.0 & 5.6 & 3.7 & 5.2 & 5.0 & 4.5 & 5.1 & 4.6 \\
\hline LPG & .4 & 1.1 & 1.4 & 1.4 & 1.4 & 1.1 & 1.2 & .8 & .6 & 1.4 \\
\hline 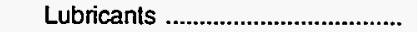 & 2.6 & 4.9 & 13.1 & 14.6 & 12.7 & 11.7 & 12.9 & 12.1 & 12.5 & 12.9 \\
\hline Motor Gasoline ............................. & 81.0 & 167.8 & 433.7 & 329.4 & 230.3 & 243.7 & 248.0 & 286.8 & 299.1 & 292.0 \\
\hline Residual Fuel & 1.6 & - & - & 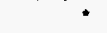 & - & - & - & 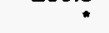 & - & - \\
\hline 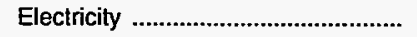 & - & - & - & - & - & - & - & - & - & - \\
\hline 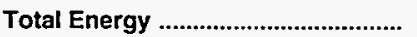 & 228.5 & 475.4 & $1,411.7$ & $1,489.7$ & $1,240.2$ & $1,282.6$ & $1,319.8$ & $1,447.2$ & $1,519.4$ & $1,492.5$ \\
\hline Primary Energy - Four Sectors ..... & 181.7 & 405.4 & $1,235.6$ & $1,062.3$ & 806.6 & 833.3 & 854.5 & 980.2 & $1,037.8$ & $1,004.9$ \\
\hline 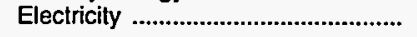 & 46.9 & 70.0 & 176.1 & 427.3 & 433.6 & 449.3 & 465.3 & 466.9 & 481.6 & 487.6 \\
\hline Electric Utility Sector ${ }^{a}$ & 8.9 & 30.3 & 140.7 & 346.3 & 283.5 & 345.3 & 350.0 & 328.6 & 349.8 & 340.2 \\
\hline Coal & 8.3 & 28.4 & 134.9 & 340.7 & 280.2 & 342.2 & 346.6 & 325.4 & 346.6 & 336.4 \\
\hline 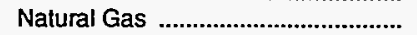 & .5 & .4 & .9 & .6 & .5 & .3 & .7 & .3 & .2 & .3 \\
\hline 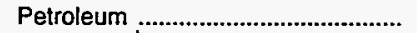 & .1 & 1.5 & 5.0 & 5.0 & 2.8 & 2.8 & 2.7 & 2.9 & 3.0 & 3.5 \\
\hline 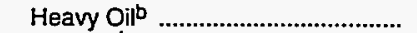 & * & 1.4 & - & - & - & - & - & - & - & - \\
\hline 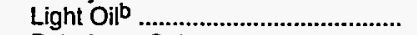 & .1 & .1 & 5.0 & 5.0 & 2.8 & 2.8 & 2.7 & 2.9 & 3.0 & 3.5 \\
\hline 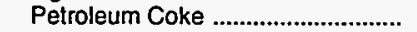 & - & - & - & - & - & - & - & - & - & - \\
\hline 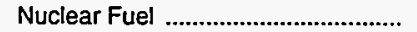 & - & - & - & - & - & - & - & - & - & - \\
\hline 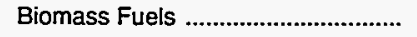 & - & - & - & - & - & - & - & - & - & - \\
\hline Primary Energy - Five Sectors ${ }^{c}$.. & 190.6 & 435.7 & $1,376.3$ & $1,408.6$ & $1,090.1$ & $1,178.5$ & $1,204.5$ & $1,308.8$ & $1,387.6$ & $1,345.1$ \\
\hline
\end{tabular}

a There are no direct fuel costs for hydroelectric, geothermal, centralized solar, or wind energy.

beavy oil includes Grade Nos. 4, 5, and 6, and residual fuel oils. Light oil includes Grade No. 2 heating oil, kerosene, and jet fuel.

c Biomass fuels are not included, except those consumed at electric utilities and those added to motor gasoline.

-No consumption, including cases where adjustments were made. See "Consumption Adjustments for Process Fuel and Intermediate Products."

"Value less than 0.05 million dollars.

Note: Totals may not equal sum of components due to independent rounding.

Sources: Data sources, estimation procedures, and assumptions are described in the "Documentation" section of this report. 
Appendix A

\section{Documentation}




\section{Documentation}

\section{Section 1. Overview}

This appendix documents data sources and procedures used to develop the estimates in the State Energy Price and Expenditure Data System (SEPEDS) that are presented in the "Statistical Tables" of this report. Information is provided for each of the four major energy sources: coal, natural gas, petroleum, and electricity. The last section describes adjustments for consumption of process fuel and intermediate products. Many of the price estimates used in SEPEDS were developed by the Battelle Pacific Northwest Laboratories under contract to EIA.

\section{Price Estimation Methodologies}

Price data in the State Energy Price and Expenditure Report are expressed in dollars per million Btu. If the source data are in physical units, they are divided by the appropriate conversion factors to create the Btu prices. Estimated prices are used only when specific State-level prices are not available for a given energy source and sector. In some cases, prices for energy consumed in one sector in a State are assigned to another sector in the same State. Specific examples are: commercial and transportation steam coal use industrial steam coal prices; commercial kerosene uses industrial kerosene prices; commercial and transportation liquefied petroleum gases (LPG) use industrial LPG prices; transportation lubricants uses industrial lubricants prices; and commercial and industrial motor gasoline use transportation motor gasoline prices.

In addition, there are a few cases where State-level prices could not be identified for any economic sector for a given energy source for some or all years. In these instances, a national-level price is used for all States for a given year. The procedures for estimating these national-level prices are presented in the body of the documentation under each energy source as appropriate. The cases where a nationallevel price is assigned to all States in all years are: transportation use of aviation gasoline; industrial and transportation use of lubricants; and the components of other petroleum products used in the industrial sector.

Finally, within a given energy source and sector where price data are usually available, there are some cases of missing prices. Two general approaches are used to assign or estimate prices in cases where consumption occurs but no price is directly available from the data sources. The first approach is to assign an adjacent State price or the simple average of adjacent States' prices. When this approach is not feasible, the consumption-weighted price from the Census division or region or the Petroleum Administration for Defense district or subdistrict in which the State is located is assigned. Nuclear fuel price estimation procedures are unique and are described in the nuclear fuel documentation.

Three State groupings used in the report-U.S. Census regions and divisions, Federal regions, and Petroleum Administration for Defense districts-are shown in Figures $A 1, A 2$, and $A 3$, respectively, on the following pages. Throughout the documentation, the term "State" includes the District of Columbia. States are often designated by the two-letter postal code abbreviations shown on the maps.

\section{Expenditures}

Energy consumption estimates used to calculate expenditures in SEPEDS are from the Energy Information Administration (EIA) State Energy Data Report 1991, Consumption Estimates, DOE/EIA0214(91), published in May 1993. The data system from which the consumption report is developed is called the State Energy Data System (SEDS). Full documentation of SEDS data sources and the methods used to estimate energy consumption are described in the consumption report.

Note: Throughout this report, the term "State" includes the District of Columbia. 
To calculate energy expenditures, SEDS consumption is adjusted to remove quantities of process fuel and intermediate products used in the industrial and transportation sectors that are not purchased directly by end users. Energy expenditures, in million dollars, are calculated by multiplying SEPEDS prices for each fuel in dollars per million Btu times the SEDS adjusted consumption for the fuel in billion Btu.

\section{End-Use Sector, State, and National Consumption-Weighted Average Prices}

Aggregated prices shown in this report are the consumption-weighted averages of the various energy sources included in the aggregation. The SEPEDS calculation is performed by summing the expenditures for the energy sources and dividing by the sum of the corresponding consumption values. For example, the average price for residential petroleum is the sum of residential expenditures for distillate fuel oil, kerosene, and liquefied petroleum gas divided by the sum of residential consumption of those fuels.

Primary Energy and Electricity. Within each enduse sector, the sum of all fuels used directly as energy, i.e., coal, natural gas, and petroleum products, is called primary energy. Electricity is considered a secondary energy source because it is generated from the primary energy sources consumed by electric utilities. Within each sector, the primary energy price is calculated by summing the expenditures for coal (the sum of coking coal and steam coal), natural gas, and petroleum (the sum of all petroleum products used by the sector) and dividing by the sum of all the consumption for those fuels. The average price of all energy consumed by each sector is the sum of the expenditures for primary en- ergy and electricity divided by the sum of the consumption of the primary energy sources and electricity.

State and National Totals. The price for "Primary Energy - Four Sectors" is the sum of the residential, commercial, industrial, and transportation sectors' expenditures for primary energy fuels divided by the sum of the end-use sectors' consumption of the primary energy sources. The total electricity price is the sum of electricity expenditures for the four sectors divided by the four sectors' electricity consumption. The "Total Energy" average price, then, is the sum of the four sectors' primary energy and electricity expenditures divided by the corresponding consumption values.

Electric Utility Sector. Electric utilities comprise a fifth energy consuming sector, although they are not considered an end-use sector. The average price paid by electric utilities for primary energy sources is the sum of their expenditures for coal, natural gas, petroleum products, nuclear fuel, and biomass fuels divided by the quantities consumed.

Primary Energy - Five Sectors. The average price for the "Primary Energy - Five Sectors" shown in the SEPER tables is the sum of the four end-use sectors' and electric utility sector expenditures for coal, natural gas, petroleum, and other fuels divided by the sum of five sectors' consumption of those fuels. The distinction between this average price and the "Total Energy" average price is that this price includes all fossil and other fuels consumed, including those used to generate electricity. The "Total Energy" price is the average price of fuels consumed directly as energy by the four end-use sectors and the much higher price of the four sectors' electricity consumption. 
Figure A1. U.S. Census Regions and Divisions

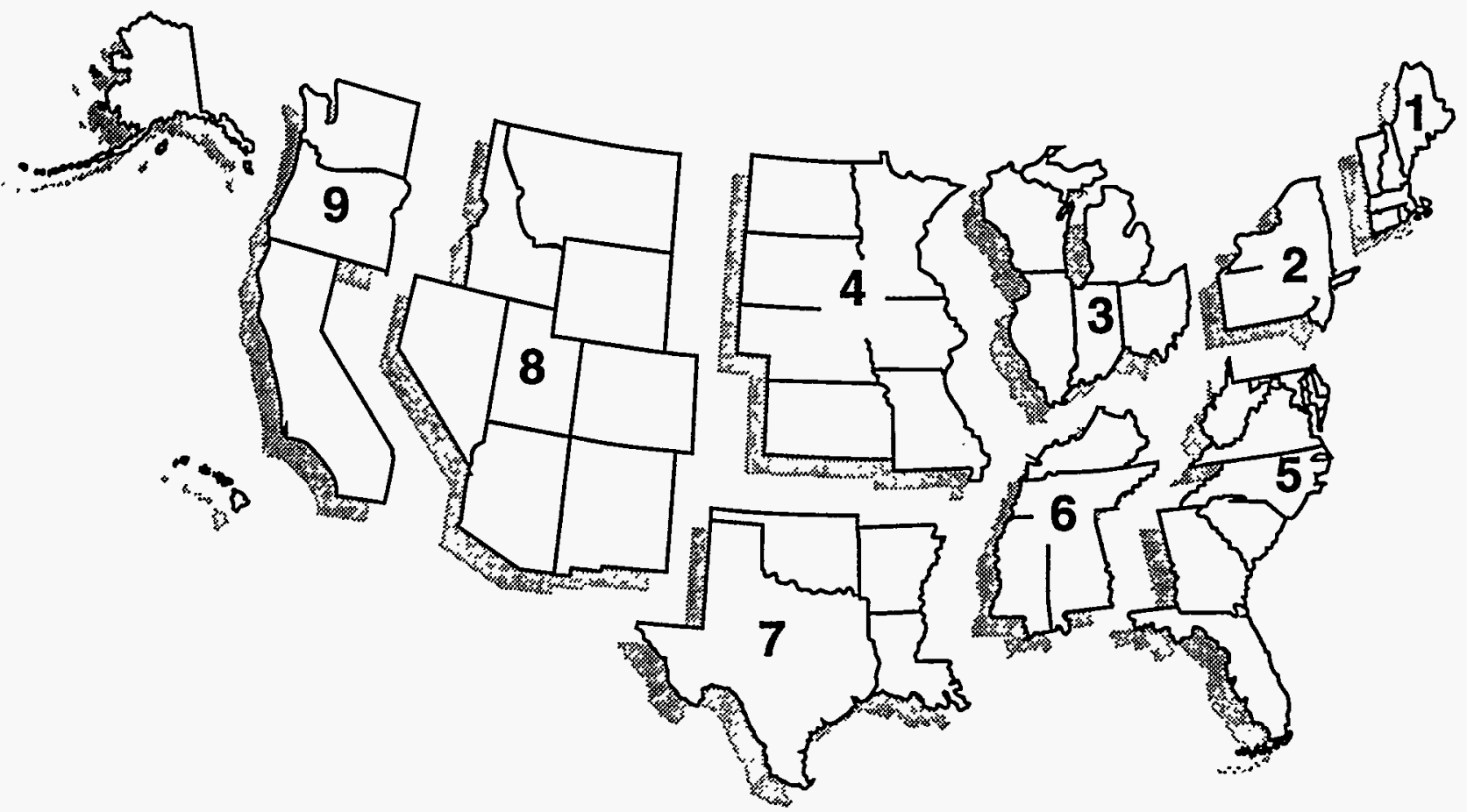

$\begin{array}{llll}\begin{array}{l}\text { Region 1 } \\ \text { Northeast }\end{array} & \begin{array}{l}\text { Region 2 } \\ \text { Midwest }\end{array} & \begin{array}{l}\text { Region 3 } \\ \text { South }\end{array} & \begin{array}{l}\text { Region 4 } \\ \text { West }\end{array} \\ \begin{array}{l}\text { Division 1 } \\ \text { (New England) }\end{array} & \begin{array}{l}\text { Division 3 } \\ \text { (East North Central) }\end{array} & \begin{array}{l}\text { Division 5 } \\ \text { (South Atlantic) }\end{array} & \begin{array}{l}\text { Division 8 } \\ \text { (Mountain) }\end{array} \\ \text { Connecticut (CT) } & \text { Illinois (IL) } & \text { Delaware (DE) } & \text { Arizona (AZ) } \\ \text { Maine (ME) } & \text { Indiana (IN) } & \text { District of Columbia (DC) } & \text { Colorado (CO) } \\ \text { Massachusetts (MA) } & \text { Michigan (MI) } & \text { Florida (FL) } & \text { Idaho (ID) } \\ \text { New Hampshire (NH) } & \text { Ohio (OH) } & \text { Georgia (GA) } & \text { Montana (MT) } \\ \text { Rhode Island (RI) } & \text { Wisconsin (WI) } & \text { Maryland (MD) } & \text { Nevada (NV) } \\ \text { Vermont (VT) } & \text { Division 4 } & \text { North Carolina (NC) } & \text { New Mexico (NM) } \\ & \text { (West North Central) } & \text { South Carolina (SC) } & \text { Utah (UT) } \\ \text { Division 2 } & \text { Virginia (VA) } & \text { Wyoming (WY) } \\ \text { (Middle Atlantic) } & \text { Iowa (IA) } & \text { West Virginia (WV) } & \text { Division 9 } \\ \text { New Jersey (NJ) } & \text { Kansas (KS) } & & \text { Pacific) } \\ \text { New York (NY) } & \text { Minnesota (MN) } & \text { Division 6 } & \text { Alaska (AK) } \\ \text { Pennsylvania (PA) } & \text { Missouri (MO) } & \text { (East South Central) } & \text { California (CA) } \\ & \text { Nebraska (NE) } & \text { Alabama (AL) } & \text { Hawaii (HI) } \\ & \text { North Dakota (ND) } & \text { Kentucky (KY) } & \text { Oregon (OR) } \\ & \text { South Dakota (SD) } & \text { Mississippi (MS) } & \text { Washington (WA) } \\ & & \text { Tennessee (TN) } & \\ & & & \text { Division 7 } \\ & & \text { (West South Central) } & \\ & & \text { Arkansas (AR) } & \\ & & \text { Louisiana (LA) } & \\ & & \text { Oklahoma (OK) } & \\ & & \text { Texas (TX) } & \end{array}$




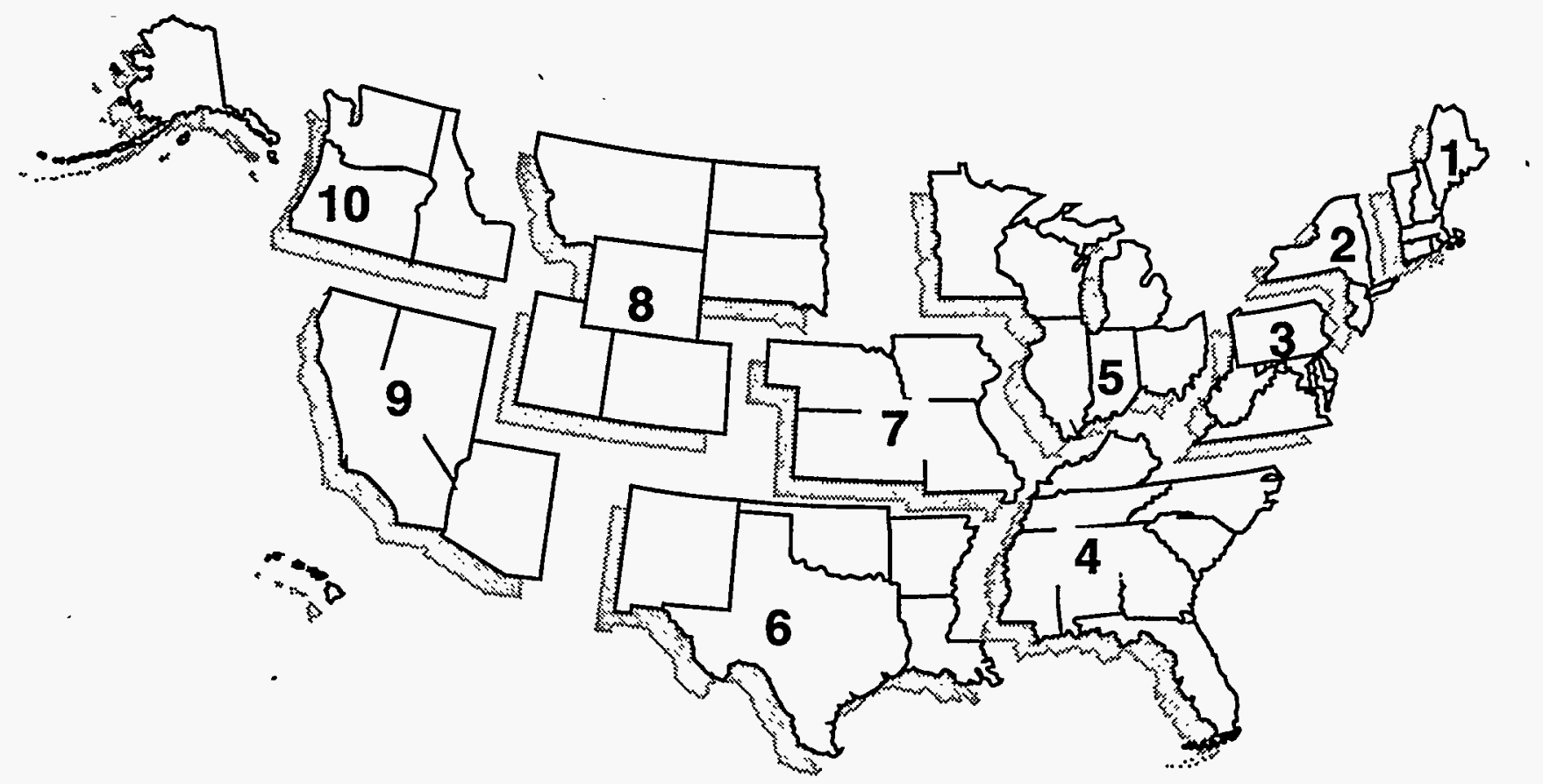

Region 1

New England

Connecticut (CT)

Maine (ME)

Massachusetts (MA)

New Hampshire (NH)

Rhode Island (RI)

Vermont (VT)

Region 2

New York/New Jersey

New Jersey (NJ)

New York (NY)

\section{Region 3}

Mid Atlantic

Delaware (DE)

District of Columbia (DC)

Maryland (MD)

Pennsylvania (PA)

Virginia (VA)

West Virginia (WV)
Region 4

South Atlantic

Alabama (AL)

Florida (FL)

Georgia (GA)

Kentucky (KY)

Mississippi (MS)

North Carolina (NC)

South Carolina (SC)

Tennessee (TN)

Region 5

Midwest

Illinois (IL)

Indiana (IN)

Michigan (MI)

Minnesota (MN)

Ohio (OH)

Wisconsin (WI)
Region 6

Southwest

Arkansas (AR)

Louisiana (LA)

New Mexico (NM)

Oklahoma (OK)

Texas (TX)

\section{Region 7}

Central

Iowa (IA)

Kansas (KS)

Missouri (MS)

Nebraska (NE)
Region 8

North Central

Colorado (CO)

Montana (MT)

North Dakota (ND)

South Dakota (SD)

Utah (UT)

Wyoming (WY)

Region 9

West

Arizona

California (CA)

Hawaii (HI)

Nevada (NV)

\section{Region 10}

Northwest

Alaska (AK)

Idaho (ID)

Oregon (OR)

Washington (WA) 


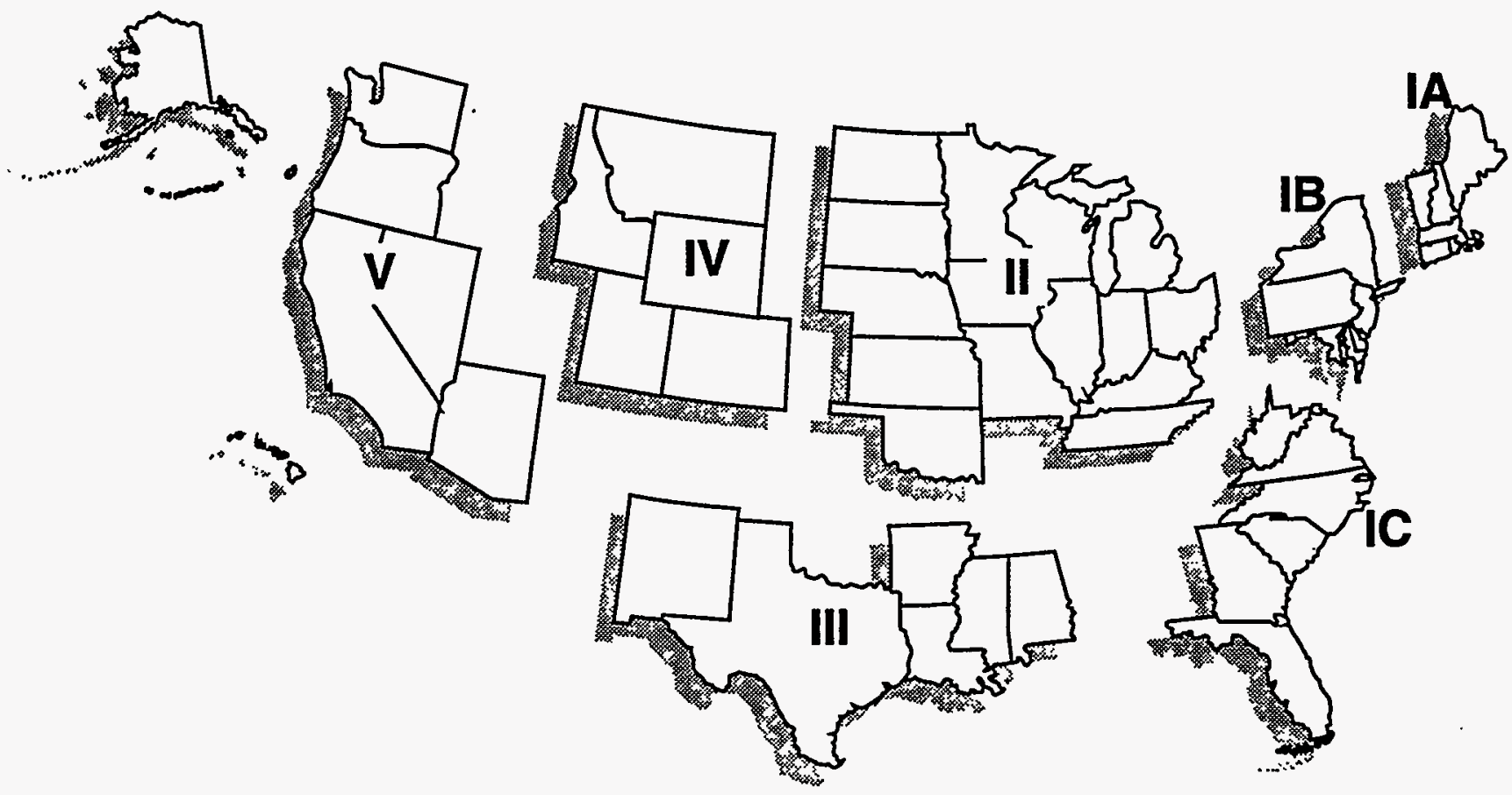

District IA

Connecticut (CT)

Maine (ME)

Massachusetts (MA)

New Hampshire (NH)

Rhode Island (RI)

Vermont (VT)

\section{District IB}

Delaware (DE)

District of Columbia (DC)

Maryland (MD)

New Jersey (NJ)

New York (NY)

Pennsyivania (PA)
District II

Illinois (IL)

Indiana (IN)

Iowa (IA)

Kansas (KS)

Kentucky (KY)

Michigan (MI)

Minnesota (MN)

Missouri (MO)

Nebraska (NE)

North Dakota (ND)

Ohio (OH)

Oklahoma (OK)

South Dakota (SD)

Tennessee (TN)

Wisconsin (WI)
District III

Alabama (AL)

Arkansas (AR)

Louisiana (LA)

Mississippi (MS)

New Mexico (NM)

Texas (TX)

\section{District IV}

Colorado (CO)

Idaho (ID)

Montana (MT)

Utah (UT)

Wyoming (WY)
District V

Alaska (AK)

Arizona (AZ)

California (CA)

Hawaii (HI)

Nevada (NV)

Oregon (OR)

Washington (WA)

\section{District IC}

Florida (FL)

Georgia (GA)

North Carolina (NC)

South Carolina (SC)

Virginia (VA)

West Virginia (WV) 
(SAHS) uraf

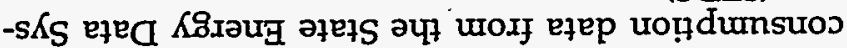

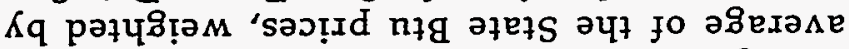

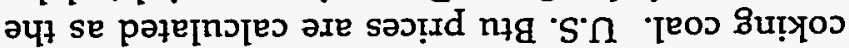

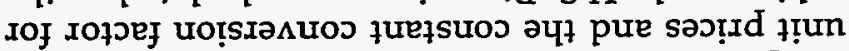

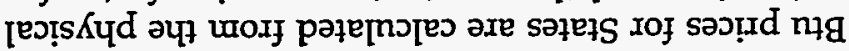

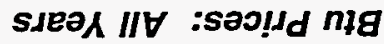

\begin{tabular}{|c|c|c|}
\hline HO & $066 \mathrm{l}-\angle 86 \mathrm{k}$ & \\
\hline$\lambda$ 서 & $986 L-\varepsilon 86 \downarrow$ & \\
\hline$M M{ }^{\prime} \forall \wedge$ & Z86L-8L6L & MM \\
\hline IM IN & L86L ‘ $9861-0861$ & \\
\hline IM NW 'IW & $6 \angle 61$ & \\
\hline$I M$ 'NW & $8 \angle 6 L-0<6 L$ & $I M$ \\
\hline HO & L66เ-L86L & \\
\hline 서 & 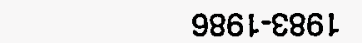 & \\
\hline$\Lambda M^{\prime} \forall \Lambda$ & 286L-8L6L & \\
\hline$M M$ & $\angle L 6 L ' 9 \angle 6 L '$ ' $\angle 6 L$ '0L6L & $\forall \wedge$ \\
\hline $\mathrm{NI}$ & L66L-886L & \\
\hline$x \perp$ & $986 \mathrm{~L}-\varepsilon 86 \mathrm{~L}$ & \\
\hline In 'O " $\forall 0$ & ट86L-0<6L & in \\
\hline 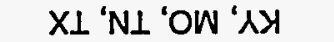 & $\angle 86 L-0 \angle 6 L$ & $X \perp$ \\
\hline $7 \forall$ & L66L-886L & \\
\hline 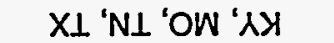 & $\angle 86 L-0 \angle 6 L$ & $\mathrm{~N} \perp$ \\
\hline$\forall d$ & เ66เ-886ト & \\
\hline AN 'OW & $286 L-2 \angle 6 L$ & \\
\hline AN 'rN 'OW & $1 \angle 61 t^{\circ} 0 \angle 61$ & AN \\
\hline AN 'rN ‘ aW & LL6L'0 $0 \angle 6+$ & $r N$ \\
\hline ד & 8861 & \\
\hline$X \perp{ }^{\prime} N \perp$ 'OW ' & $\angle 86 L-0 \angle 6 L$ & OW \\
\hline IM NW 'IN & $6 \angle 61$ & \\
\hline IM NW & $8 \angle 6 L-0 \angle 6 L$ & NW \\
\hline $\mathrm{HO}$ & L66เ-886เ & \\
\hline$I M^{\prime} I W$ & L86L's86L-086L & \\
\hline IM'NW 'IW & $6 \angle 6 \mathrm{t}$ & IN \\
\hline$\forall d$ & L66L-E86L & \\
\hline AN 'rN ‘OW & $1 \angle 6+t^{\circ} 0 \angle 6 \downarrow$ & OW \\
\hline $\mathrm{HO}$ & 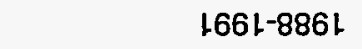 & \\
\hline$X \perp ' N \perp$ 'OW 'AY & $\angle 86 L-0 \angle 6 L$ & ᄉᄉ्र \\
\hline & L66L-986L & 기 \\
\hline $1 n^{\prime} \mathrm{O}, \forall \supset$ & $286 \perp-0<6 \downarrow$ & ०० \\
\hline In 'O ' $\forall 0$ & ट86เ-0<61 & $\forall O$ \\
\hline pau6!ISS $\forall$ SaJ!גd ә|EIS & sjead & әีE1S \\
\hline 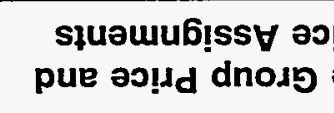 & $\begin{array}{l}\text { d әjełs luose!p } \\
\text { eis jeos bu!yos }\end{array}$ & \\
\hline
\end{tabular}

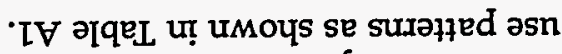

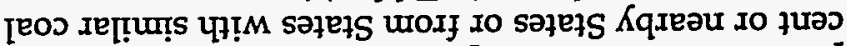

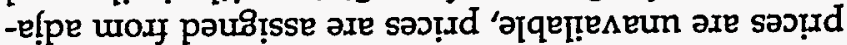

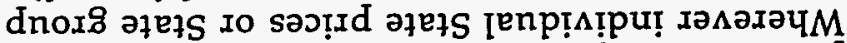

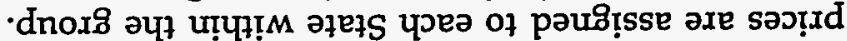

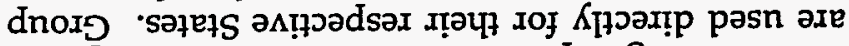
sajụd әңе

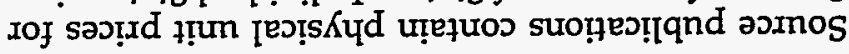

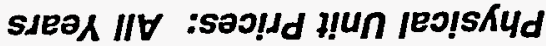

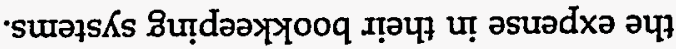

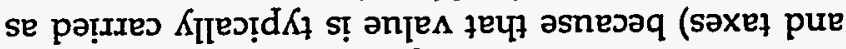

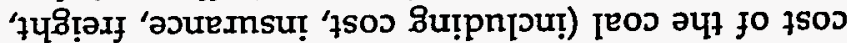

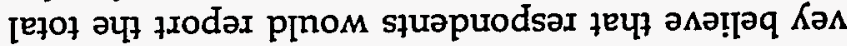

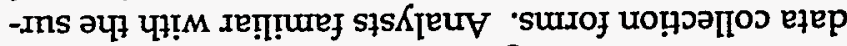

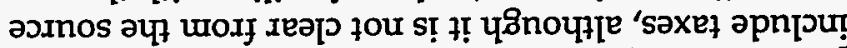

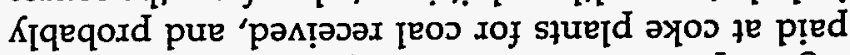

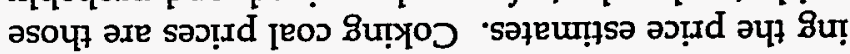

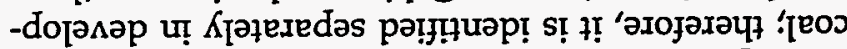

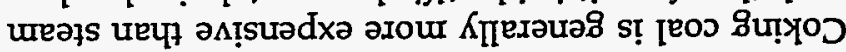

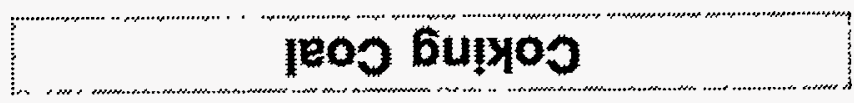

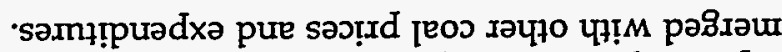

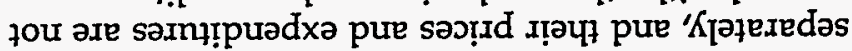

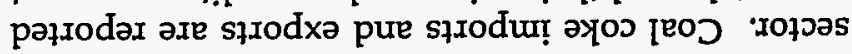

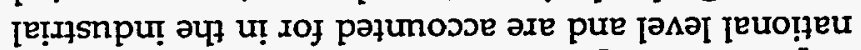

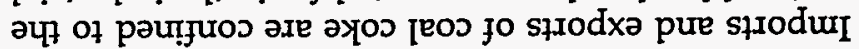

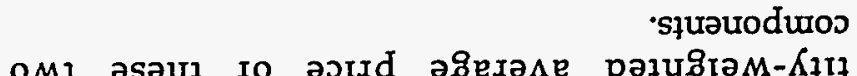

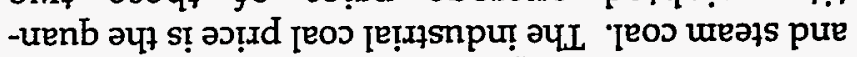

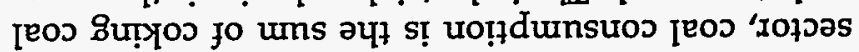
[ет̣,

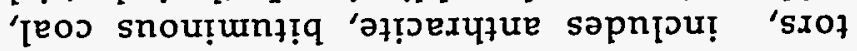

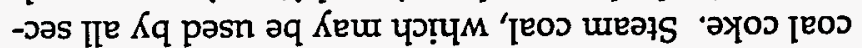

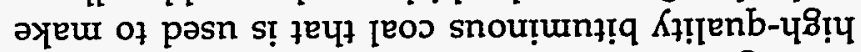

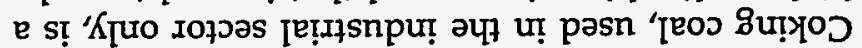

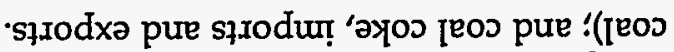

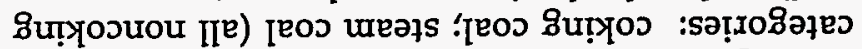

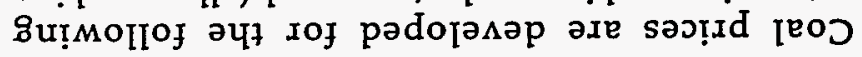




\section{Data Sources}

Prices

1981 forward: Energy Information Administration, Quarterly Coal Report, October-December issue, Table A3.

E
E
C
T
I
C
U
I
I
I
$T$
$Y$

1977-1980: Energy Information Administration, Coke and Coal Chemicals, Table 19 (1977), Table 15 (1978), and Table 7 (1979, 1980).

1970-1976: Bureau of Mines, U.S. Department of the Interior, Minerals Yearbook, "Coke and Coal Chemicals" chapter, Table 22.

\section{Consumption}

1970 forward: Energy Information Administration, State Energy Data System, coking coal consumption.

\section{Conversion Factor: All Years}

26.80 million Btu per short ton.

\section{Steam Coal}

Steam coal is used in all sectors. Price data are generally available in the electric utility, residential, and industrial sectors. However, no price data are directly available in the transportation and commercial sectors. The industrial sector steam coal prices are assigned as price estimates in these two sectors. Data sources and computational procedures for estimating coal prices are discussed by sector. Estimates of the amount of steam coal consumed by sector are from SEDS and are adjusted for process fuel consumption in the industrial sector. (See the "Consumption Adjustments for Process Fuel and Intermediate Products" section on page 251.)

\section{Electric Utility Sector}

\section{Btu Prices: 1973 Forward}

State Btu prices, including all taxes, are from Cost and Quality of Fuels for Electric Utility Plants (CEQ) for 1973 forward and are converted from cents to dollars per million Btu. Where individual State prices are unavailable, quantity-weighted Census division prices are assigned as shown in Table A2.

\section{Btu Prices: 1970 Through 1972}

Btu prices for States are from the Statistical Yearbook and are converted from cents to dollars. DE, DC, and MD are each assigned the combined price for the three States. The steam coal electric utility sector AK price for 1971 is estimated as discussed below.

\section{Alaska Prices: All Years}

Since CEQ data do not cover AK, Btu prices for AK are from other sources or estimated for 1971 and for 1973 forward. For the years 1970, 1972, 1974, 1976, 1977, and 1979 through 1991, prices are directly from the Statistical Yearbook. Prices for 1971, 1973, 1975, and 1978 are estimated from the Statistical Yearbook prices for the United States and the average ratio of AK-to-U.S. prices for the years when AK prices are available. The 1971 and 1973 estimated prices are based on the average ratio for 1970 and 1972, while the 1975 and 1978 estimated prices are based on the average ratio for 1974, 1976, 1977, and 1979.

\section{U.S. Prices: All Years}

U.S. Btu prices are calculated as the average of the State Btu prices, weighted by consumption data from SEDS.

\section{Data Sources}

Prices

1973 forward: Energy Information Administration, Cost and Quality of Fuels for Electric Utility Plants, Table 3 (1973-1979), Table 51 (1980-1982), Table 50 (1983, 1984), Table 40 (1985-1989), Table 7 (1990 forward).

Table A2. Electric Utility Sector Price Assignments, 1973 Forward

\begin{tabular}{cll}
\hline State & \multicolumn{1}{c}{ Years } & $\begin{array}{c}\text { State/Census Division } \\
\text { Prices Assigned }\end{array}$ \\
\hline CT & $1975-1979$ & New England \\
DC & 1976 & MD, VA \\
OK & 1973,1974 & West South Central \\
& 1975 & CO, KS, MO, NM, TX \\
OR & 1983,1989 & Pacific Contiguous \\
RI & 1974 & MA \\
VT & $1980,1983-1986$ & New England \\
\hline
\end{tabular}


1970 forward: Edison Electric Institute, Statistical Yearbook of the Electric Utility Industry, table titled,

"Analysis of Fuel for Electric Generation: Total Electric Utility Industry" (1970-1988), Table 29 (1989 forward).

\section{Consumption}

1970 forward: Energy Information Administration, State Energy Data System, electric utility sector coal consumption.

\section{Conversion Factors: All Years}

Btu prices are directly from the data sources; no explicit conversion factors are used.

\section{Residential Sector}

Residential sector steam coal prices are the average delivered prices for coal purchased by residential customers and include taxes.

\section{Prices: 1979 Forward}

Residential steam coal.Btu prices for 1979 forward. are not available. The residential steam coal prices calculated for 1974 through 1978 from Gas Househeating Survey (GHS) and the average Btu spot prices from $C \mathcal{E} Q$ for 1974 through 1978 are used to estimate a regression equation. Electric utility coal spot prices from CEQ for 1979 forward are converted from cents per million Btu to dollars per million Btu and used in the regression equation to estimte residential steam coal prices for 1979 forward. AK residential coal prices are estimated using a different methodology described on page 186.

Several States are missing C\&Q spot prices, both in the 1974 through 1978 data and in the 1979 forward data used to calculate prices. For these missing data, $C E Q$ prices are assigned from other States for use in the regression as shown in Table A3.

Price estimates for 1974 through 1978 for some States are not available because there was no consumption. To calculate prices for 1979 forward, these States are assigned the final prices from selected States as shown in Table A4. Because the CEQ spot price for ND in 1991, when used in the regression, results in a negative number, the $\mathrm{MN}$ final price is assigned to ND.
Table A3. Residential Sector Coal Spot Price Assignments from C\&Q, 1979 Forward

\begin{tabular}{|c|c|c|}
\hline State & Years & $\begin{array}{l}\text { State Prices } \\
\text { Assigned }\end{array}$ \\
\hline $\mathrm{CO}$ & 1979,1981 & KS \\
\hline \multirow[t]{3}{*}{ CT } & 1975 & NY \\
\hline & $1976-1979$ & NH \\
\hline & $1980-1987$ & MA \\
\hline DC & $1976-1991$ & MD \\
\hline \multirow[t]{4}{*}{ ID } & $1974,1979-1982$ & NV \\
\hline & $1975-1977$ & $\mathrm{SD}$ \\
\hline & $\cdot 1978$ & ND \\
\hline & 1983-1991 & $\mathrm{CO}$ \\
\hline \multirow[t]{2}{*}{ MA } & 1975 & $V T$ \\
\hline & $1976-1979$ & NH \\
\hline \multirow[t]{3}{*}{ ME } & $1974,1975,1981,1983$ & VT \\
\hline & $1976-1980,1982$ & NH \\
\hline & $1984-1991$ & MA \\
\hline \multirow[t]{4}{*}{ MT } & $1974,1975,1978$ & ND \\
\hline & 1976,1977 & SD \\
\hline & $1979-1982$ & NV \\
\hline & $1986,1990,1991$ & $\mathrm{CO}$ \\
\hline \multirow[t]{2}{*}{ ND } & 1976,1977 & SD \\
\hline & $1979-1988,1990$ & MN \\
\hline \multirow[t]{2}{*}{$\mathrm{NH}$} & $1974,1975,1981,1983$ & VT \\
\hline & 1984,1985 & MA \\
\hline \multirow{5}{*}{$\begin{array}{l}\text { NV } \\
\text { RI }\end{array}$} & $1975-1978,1983-1989,1991$ & $\mathrm{CO}$ \\
\hline & 1974 & CT \\
\hline & 1975 & $\mathrm{VT}$ \\
\hline & $1976-1979$ & NH \\
\hline & $1980-1991$ & MA \\
\hline \multirow[t]{2}{*}{ SD } & 1978,1984 & ND \\
\hline & $1979-1983,1986,1987,1989,1991$ & MN \\
\hline \multirow[t]{2}{*}{ UT } & $1975-1978,1980,1983$ & $\mathrm{CO}$ \\
\hline & 1979 & NV \\
\hline \multirow[t]{2}{*}{$\mathrm{VT}$} & 1976,1980 & NH \\
\hline & 1984-1991 & MA \\
\hline \multirow[t]{3}{*}{ WA } & 1970 & OR \\
\hline & $1974-1978,1983-1985$ & $\mathrm{CO}$ \\
\hline & $1979-1982$ & NV \\
\hline WY & $1974-1976,1978,1982,1983,1985$ & $\mathrm{CO}$ \\
\hline
\end{tabular}

In addition, several States are assigned the simple average of the final prices of adjacent States as shown in Table A4.

\section{Prices: 1971 Through 1978}

For 1971 through 1978, Btu steam coal prices are calculated using data from GHS. The price for a State is equal to the simple average of the city/utility price observations for that State. For 1971 and 1972, GHS reports physical unit prices rather than Btu prices, (as published for 1973 through 1978) and therefore, 
Table A4. Residential Sector Spot Coal Final Price Assignments, 1979 Forward

\begin{tabular}{|c|c|c|}
\hline State & Years & $\begin{array}{l}\text { State and Averaged } \\
\text { Final Prices Assigned }\end{array}$ \\
\hline \multirow[t]{3}{*}{ AR } & $\begin{array}{l}1980,1982,1984,1985, \\
1987-1991\end{array}$ & AL \\
\hline & 1981 & MO, OK, TN, TX \\
\hline & 1983 & MO, MS, OK, TN \\
\hline$A Z$ & $\begin{array}{l}1982,1984,1985 \\
1987,1988,1990,1991\end{array}$ & $\begin{array}{l}\text { CA, NM, NV, UT } \\
\text { UT }\end{array}$ \\
\hline \multirow[t]{2}{*}{$\mathrm{CA}$} & $1979-1985$ & NV \\
\hline & $1987-1991$ & WA \\
\hline FL & $1980-1991$ & GA \\
\hline LA & $\begin{array}{l}1980,1982,1984,1986, \\
1988,1991\end{array}$ & $A L$ \\
\hline \multirow[t]{2}{*}{ MS } & $\begin{array}{l}1979,1980,1983,1984 \\
1986-1991\end{array}$ & $A L$ \\
\hline & 1985 & $A L, A R, T N$ \\
\hline MT & $1986-1989$ & WY \\
\hline NM & 1979-1991 & $\mathrm{CO}$ \\
\hline OK & $1979-1991$ & $\mathrm{CO}$ \\
\hline OR & $\begin{array}{l}1979,1980,1982-1991 \\
1981\end{array}$ & $\begin{array}{l}\text { WA } \\
C A, I D, N V, W A\end{array}$ \\
\hline $\mathrm{TX}$ & $1980-1982,1985-1991$ & $\mathrm{CO}$ \\
\hline
\end{tabular}

the State-level conversion factors for this sector from SEDS are used to convert to Btu prices for those years. AK residential coal prices are estimated using a different methodology described on page 186.

A simple average of price observations in $\mathrm{CT}, \mathrm{MA}$, $\mathrm{ME}, \mathrm{NH}, \mathrm{RI}$, and VT is assigned to each of these States. To impute other missing prices in the 1971 through 1978 period, States are assigned simple averages of adjacent State prices or directly assigned the single price of an adjacent or nearby State as listed in Table A5.

\section{Prices: 1970}

Since State-level coal price data for 1970 are not available from either GHS or CEQ, the 1970 residential sector coal prices are calculated using the 1971 through 1978 data from the Statistical Yearbook for the 39 States with some reported coal use from 1971 through 1983 and regression analysis.

For estimating the 1970 prices, States missing Statistical Yearbook data are assigned prices as follows: ID/1970 through 1978 from MT; MA/1976 through 1978 from CT; ME/1970 through 1978 from NH; RI/1973, 1975 through 1978 from CT; and WA/1970 through 1972 from OR. DC, DE, and MD
Table A5. Residential Sector Spot Coal Price Assignments, 1971-1978

\begin{tabular}{|c|c|c|}
\hline State & Years & $\begin{array}{l}\text { State Assigned or } \\
\text { Averaged Prices }\end{array}$ \\
\hline $\mathrm{AL}$ & 1971 & TN \\
\hline AR & 1977,1978 & $\mathrm{AL}$ \\
\hline CA & $\begin{array}{l}1971,1972,1974 \\
1978\end{array}$ & NV \\
\hline DC & 1971-1978 & MD \\
\hline $\mathrm{DE}$ & $\begin{array}{l}1971,1972,1974, \\
1976,1977\end{array}$ & MD \\
\hline \multirow[t]{2}{*}{ GA } & 1971 & NC, TN \\
\hline & 1972 & AL, NC, TN \\
\hline ID & 1977 & MT, UT, WY \\
\hline KS & 1971,1972 & $\mathrm{CO}, \mathrm{MO}$ \\
\hline \multirow[t]{2}{*}{ MN } & 1971 & $|A, N D, W|$ \\
\hline & 1972 & $|A, W|$ \\
\hline MS & 1978 & $A L$ \\
\hline \multirow[t]{2}{*}{ MT } & 1971 & ID, ND, WY \\
\hline & 1972,1973 & ID, WY \\
\hline \multirow[t]{3}{*}{ ND } & 1972 & $|A, W|$ \\
\hline & 1973 & MN, SD \\
\hline & 1974 & $M N, M T, S D$ \\
\hline \multirow[t]{2}{*}{ NE } & 1971,1972 & $\mathrm{CO}, \mathrm{IA}, \mathrm{MO}, \mathrm{WY}$ \\
\hline & 1975 & $\mathrm{CO}, \mathrm{IA}, \mathrm{KS}, \mathrm{MO}, \mathrm{SD}, \mathrm{WY}$ \\
\hline NJ & $\begin{array}{l}1971,1972,1974, \\
1977,1978\end{array}$ & $D E, N Y, P A$ \\
\hline NM & 1971 & co \\
\hline \multirow[t]{2}{*}{ NV } & 1971, 1972, 1975 & ID, UT \\
\hline & 1973 & ID, OR, UT \\
\hline OK & $1971-1978$ & $\mathrm{CO}$ \\
\hline OR & $1971-1978$ & WA \\
\hline SC & 1971,1972 & NC \\
\hline \multirow[t]{2}{*}{ SD } & 1971 & IA, ND, WY \\
\hline & 1972 & IA, WY \\
\hline TX & $1971-1974,1977$ & co \\
\hline UT & 1974,1978 & CO, ID, NV, WY \\
\hline \multirow[t]{2}{*}{ WA } & 1971, 1972, 1974 & ID \\
\hline & 1977 & MT, UT, WY \\
\hline WV & 1971,1972 & $\mathrm{KY}, \mathrm{MD}, \mathrm{OH}, \mathrm{PA}, \mathrm{VA}$ \\
\hline
\end{tabular}

are all assigned the combined Statistical Yearbook price for those States. Wherever individual State prices are unavailable, prices are assigned from an adjacent or nearby State as follows: CA from NV; NM from CO; OK from CO; OR from WA; and TX from $\mathrm{CO}$. AK residential coal prices are estimated using a different methodology described below.

\section{Alaska Prices: All Years}

AK's Btu prices for 1981 through 1986 and 1990 forward are estimated from the WA State prices during that period. The average ratio of AK-to-WA prices 
during 1970 through 1977 is applied to the WA price each year to estimate the AK price for the year. There is no price for AK in 1978 through 1980 and 1987 through 1989 because the State had no reported consumption during those years.

AK physical unit prices for 1970 through 1977 are estimated using the ratio of AK-to-U.S. utility sector prices.

\section{U.S. Prices: All Years}

U.S. Btu prices are calculated as the average of the State Btu prices, weighted by consumption data from SEDS.

\section{Data Sources}

\section{Prices}

1974 forward: Energy Information Administration, Cost and Quality of Fuels for Electric Plants, average spot coal prices, Table 2 (1974-1979); Table 44 (19801982); Table 49 (1983, 1984); Table 39 (1985-1989); and Table 8 (1990 forward).

1971-1978: American Gas Association, Gas Househeating Survey, table titled, "Competitive Fuel Prices."

1970-1978: Edison Electric Institute, Statistical Yearbook of the Electric Utility Industry, Table 435.

\section{Consumption}

1970 forward: Energy Information Administration, State Energy Data System, residential sector coal consumption.

\section{Conversion Factors: 1971, 1972}

Energy Information Administration, State Energy Data Report 1991, Consumption Estimates, Table D8.

\section{Commercial Sector}

Commercial sector prices are assigned from industrial steam coal prices. States without physical unit industrial steam coal prices were assigned the prices from adjacent States as follows: CT/1980 from MA; DC/1980 through 1991 from MD; RI/1982, 1983, 1990 , and 1991 from MA; OK/1990 from KS. The Btu price for RI/1982 is calculated using the RI/1981 conversion factor and for RI/1990 using the U.S. average anthracite factor. U.S. Btu prices are calculated as the average of the State Btu prices, weighted by consumption data from SEDS.

\section{Industrial Sector}

For 1980 forward, prices are based on the average price of coal receipts at manufacturing plants. For 1970 through 1979, prices are based on the average cost of coal sold to manufacturing firms.

\section{Physical Unit Prices: 1980 Forward}

For 1984 forward, State prices are from the Quarterly Coal Report (QCR). The annual price is estimated as the average of the quarterly prices weighted by the quarterly receipts. For 1980 through 1983, prices are directly from EIA-3 data. Published prices in average cost per ton are used directly when available, while missing prices are estimated using simple averages or price assignments from adjacent States. Although it is not clear from the source data collection forms, the prices probably include taxes. Analysts familiar with the survey believe that respondents would report the total cost of the coal (including cost, insurance, freight, and taxes) because that value is typically carried as the expense in their bookkeeping systems.

Missing State data are estimated using simple averages of the published data for adjacent States. In a few cases, only a single adjacent State or Census division price is available for the estimation. The adjacent State and Census division price assignments used for estimations are shown in Table A6.

\section{Physical Unit Prices: 1971, 1974 Through 1979}

For 1971 and 1974 through 1979, available cost and quantity of bituminous coal, lignite, and anthracite from Annual Survey of Manufactures (ASM) or Census of Manufactures (CM) are used to calculate prices as average cost per unit of sales for covered States. (States with undisclosed data are not considered covered). Although it is not clear from the data sources, the prices probably include taxes as explained for prices in 1980 forward.

For States with industrial steam coal use and for which $A S M$ or CM data are not available in 1971 and 1974 through 1979, adjacent State simple averages of available $A S M / C M$ data are used to impute prices. The assigned prices from adjacent States are shown in Table A7. 
C Table A6. Industrial Sector Steam Coal Price
Estimates for 1980 Forward

\begin{tabular}{|c|c|c|}
\hline State & Years & $\begin{array}{l}\text { Prices Used } \\
\text { in the Estimation }\end{array}$ \\
\hline$A Z$ & $\begin{array}{l}1980 \\
1981,1984,1985\end{array}$ & $\begin{array}{l}\text { CA, UT } \\
\text { CA, CO, UT }\end{array}$ \\
\hline CO & 1980 & KS, UT \\
\hline CT & $1981-1991$ & New England \\
\hline DC & 1980,1981 & MD \\
\hline DE & $1980-1991$ & MD \\
\hline FL & 1980 & $\mathrm{AL}, \mathrm{GA}$ \\
\hline HI & $1982-1991$ & $\mathrm{CA}$ \\
\hline LA & $1980-1985,1989-1991$ & $A R, T X$ \\
\hline \multirow[t]{2}{*}{ MA } & $1980-1983$ & NY \\
\hline & $1984-1991$ & New England \\
\hline ME & $1980-1983$ & NY \\
\hline MS & $1980-1991$ & $\begin{array}{l}\text { New England } \\
\text { Al AR TN }\end{array}$ \\
\hline \multirow[t]{2}{*}{ MT } & 1983 & ID, WY \\
\hline & 1984-1991 & ID \\
\hline \multirow[t]{2}{*}{ ND } & 1980-1982 & MN, MT \\
\hline & $\begin{array}{l}1983-1991 \\
1980\end{array}$ & $\begin{array}{l}\text { MN } \\
\text { IA. KS, MO }\end{array}$ \\
\hline NE & $1982,1983,1986-1991$ & CO, IA, KS, MO, WY \\
\hline NH & $\begin{array}{l}1980-1983 \\
1984-1991\end{array}$ & NY \\
\hline NJ & $1980-1991$ & NY, PA \\
\hline \multirow[t]{4}{*}{ NM } & 1980 & TX, UT \\
\hline & $1981,1984-1988$ & $\mathrm{CO}, \mathrm{OK}, \mathrm{TX}$ \\
\hline & 1982,1983 & $\mathrm{AZ}, \mathrm{CO}, \mathrm{OK}, \mathrm{TX}$ \\
\hline & $1989-1991$ & $\mathrm{AZ}, \mathrm{CO}, \mathrm{TX}$ \\
\hline \multirow[t]{2}{*}{ NV } & $1980,1981,1984-1991$ & CA, ID, UT \\
\hline & 1983 & $\mathrm{AZ}, \mathrm{CA}, \mathrm{ID}, \mathrm{UT}$ \\
\hline \multirow{2}{*}{$\begin{array}{l}\text { OK } \\
\text { OR }\end{array}$} & $1980,1989-1991$ & $A R, K S, M O, T X$ \\
\hline & $\begin{array}{l}1980,1981,1983-1991 \\
1982\end{array}$ & $\begin{array}{l}\text { CA, ID, WA } \\
C A, I D, N V, W A\end{array}$ \\
\hline \multirow[t]{2}{*}{ RI } & 1980,1981 & NY \\
\hline & 1984-1989 & New England \\
\hline \multirow[t]{5}{*}{ SD } & 1980 & IA, MN, MT \\
\hline & 1981 & IA, MN, MT, NE \\
\hline & 1982 & IA, MN, MT, WY \\
\hline & $1983,1986-1991$ & IA, MN, WY \\
\hline & 1984,1985 & IA, MN, NE \\
\hline \multirow[t]{2}{*}{ VT } & $1980-1983$ & NY \\
\hline & 1984-1991 & New England \\
\hline WV & 1980 & $\mathrm{KY}, \mathrm{MD}, \mathrm{OH}, \mathrm{PA}, \mathrm{VA}$ \\
\hline \multirow[t]{2}{*}{ WY } & 1980 & ID, MT, UT \\
\hline & $\begin{array}{l}1981 \\
1984,1985\end{array}$ & $\begin{array}{l}\text { CO, ID, MT, NE, UT } \\
\text { CO, ID, NE, UT }\end{array}$ \\
\hline
\end{tabular}

Table A7. Industrial Sector Steam Coal Price Estimates for 1971 and 1974-1979

\begin{tabular}{|c|c|c|}
\hline State & Years & $\begin{array}{l}\text { State Prices Used } \\
\text { in the Estimation }\end{array}$ \\
\hline AR & $\begin{array}{l}1971,1972,1974,1975 \\
1979\end{array}$ & $\begin{array}{l}\text { MO, TN } \\
\text { MO, TN, TX }\end{array}$ \\
\hline$A Z$ & $\begin{array}{l}1971 \\
1974-1978\end{array}$ & $\begin{array}{l}\text { CA, NV, UT } \\
\text { CA, UT }\end{array}$ \\
\hline CO & $\begin{array}{l}1974-1978 \\
1979\end{array}$ & $\begin{array}{l}\text { KS, NE, UT } \\
\text { UT }\end{array}$ \\
\hline CT & $\begin{array}{l}1974-1978 \\
1979\end{array}$ & $\begin{array}{l}\text { MA, NY } \\
\text { NY }\end{array}$ \\
\hline DC & $1971,1974-1979$ & $M D, V A$ \\
\hline $\begin{array}{l}\text { DE } \\
F L\end{array}$ & $\begin{array}{l}1971,1974-1979 \\
1979\end{array}$ & $\begin{array}{l}\text { MD, NJ, PA } \\
\text { AL, GA }\end{array}$ \\
\hline ID & $\begin{array}{l}1974 \\
1975-1978 \\
1979\end{array}$ & $\begin{array}{l}\text { OR, UT } \\
\text { UT } \\
\text { UT, WA }\end{array}$ \\
\hline KS & 1979 & MO \\
\hline LA & $\begin{array}{l}1978 \\
1979\end{array}$ & $\begin{array}{l}\text { AR } \\
\text { TX }\end{array}$ \\
\hline MA & 1979 & NY \\
\hline ME & $\begin{array}{l}1975-1978 \\
1979\end{array}$ & $\begin{array}{l}\text { MA } \\
\text { NY }\end{array}$ \\
\hline MS & $\begin{array}{l}\text { 1971, 1974, 1975, } 1979 \\
1976-1978\end{array}$ & $\begin{array}{l}\text { AL, TN } \\
A L, A R, T N\end{array}$ \\
\hline MT & $\begin{array}{l}1974-1978 \\
1979\end{array}$ & $\begin{array}{l}\text { MN, NE, UT } \\
\text { MN, UT }\end{array}$ \\
\hline ND & $1974-1979$ & MN \\
\hline NE & 1979 & IA, MO \\
\hline $\mathrm{NH}$ & $1971,1974-1979$ & MA \\
\hline NM & $\begin{array}{l}1971 \\
1974,1976-1978 \\
1979\end{array}$ & $\begin{array}{l}\text { CO, OK, TX, UT } \\
\text { KS, UT } \\
\text { UT }\end{array}$ \\
\hline NV & $\begin{array}{l}1974 \\
1975-1979\end{array}$ & $\begin{array}{l}\text { CA, OR, UT } \\
\text { CA, UT }\end{array}$ \\
\hline OK & $\begin{array}{l}1974,1975 \\
1976-1978 \\
1979\end{array}$ & $\begin{array}{l}\text { KS, MO } \\
\text { AR, KS, MO } \\
\text { MO, TX }\end{array}$ \\
\hline OR & $\begin{array}{l}1975-1978 \\
1979\end{array}$ & $\begin{array}{l}C A \\
C A, W A\end{array}$ \\
\hline RI & $\begin{array}{l}1971,1974-1976,1978 \\
1979\end{array}$ & $\begin{array}{l}\text { MA } \\
\text { NY }\end{array}$ \\
\hline SD & $\begin{array}{l}1971,1974 \\
1975-1978 \\
1979\end{array}$ & $\begin{array}{l}\text { IA } \\
\text { IA, MN, NE } \\
\text { IA, MN }\end{array}$ \\
\hline$T X$ & $\begin{array}{l}1974,1975 \\
1976-1978\end{array}$ & $\begin{array}{l}\text { KS } \\
\text { AR, KS }\end{array}$ \\
\hline VT & $\begin{array}{l}1971,1974-1978 \\
1979\end{array}$ & $\begin{array}{l}\text { MA } \\
\text { NY }\end{array}$ \\
\hline WA & $\begin{array}{l}1974 \\
1975-1978\end{array}$ & $\begin{array}{l}\mathrm{CA}, \mathrm{OR} \\
\mathrm{CA}\end{array}$ \\
\hline WY & $\begin{array}{l}1974-1978 \\
1979\end{array}$ & $\begin{array}{l}\text { NE, UT } \\
\text { UT }\end{array}$ \\
\hline
\end{tabular}


Table A8. Industrial Sector Price Assignments Used in the Regression Equation for 1971, and 1974-1979

\begin{tabular}{lll}
\hline State & Years & $\begin{array}{c}\text { State } \\
\text { Prices Assigned }\end{array}$ \\
\hline AR & $1973-1977$ & MO \\
CA & $1970-1977$ & NV \\
CT & $1975-1977$ & NY \\
DC & 1976,1977 & MD \\
ID & $1970-1977$ & MT \\
MA & 1976,1977 & NH \\
ME & $1970-1977$ & NH \\
OK & $1973-1975$ & KS \\
OR & $1973-1977$ & WA \\
TX & 1970 & NM \\
WA & $1970-1972$ & OR \\
& & \\
\hline
\end{tabular}

\section{Physical Unit Prices: 1970, 1972, 1973}

Steam coal industrial sector prices for 1970,1972, and 1973 (years for which no $A S M / C M$ prices are available) are estimated using regression techniques. Values for the independent variable are steam coal electric utility sector physical unit prices and values for the dependent variable are the steam coal industrial physical unit prices (from $A S M$ or estimated as described above) for 1971, and 1974 through 1977. A few States are assigned electric utility prices for the dependent variable in the regression as shown in Table A8.

Wherever individual State prices remain unavailable after the estimation using the above regression techniques, prices are assigned from adjacent or nearby States as shown in Table A9.

\section{Physical Unit Prices: Alaska, All Years}

Steam coal industrial sector prices are estimated for Alaska based on the assumption that the ratio of the

Table A9. Industrial Sector Final Price Assignments for 1970, 1972 and 1973

\begin{tabular}{lll}
\hline State & \multicolumn{1}{c}{ Years } & \multicolumn{1}{c}{ State } \\
Prices Assigned
\end{tabular}

Alaska price to the U.S. price in the industrial sector is the same as the ratio of the AK price to the U.S. price in the electric utility sector for each year.

\section{Btu Prices: All Years}

Btu prices for States are calculated from the physical unit prices and the conversion factors, which vary by State and by year. U.S. Btu prices are calculated as the average of the State Btu prices, weighted by consumption data from SEDS, adjusted for process fuel and coking coal consumption.

\section{Data Sources}

Prices

1984 forward: Energy Information Administration, Quarterly Coal Report, Tables 16 and 17 (1984 1st quarter through 1985 2nd quarter and 1986, 1987, and 1988 1st quarters); Tables 18 and 19 (1985 3rd and 4th quarters, 1986, 1987, and 1988 2nd quarters through 4th quarters, and 1989 forward 1st quarter through 4th quarter).

1980-1983: Energy Information Administration, "Quarterly Coal Consumption Report: Manufacturing Plants," (Form EIA-3). Only published data are used from Table 25 (1980), Table 11 (1981, 1982), and Table 2 (1983).

1971, 1974-1979: Bureau of the Census, U.S. Department of Commerce, Annual Survey of Manufactures and Census of Manufactures, Table 4 (1971) and Table 3 (1974-1979).

1970, 1972, 1973: Steam coal electric utility physical unit prices.

\section{Consumption}

1970 forward: Energy Information Administration, State Energy Data System, industrial sector coal consumption.

\section{Conversion Factors: All Years}

Energy Information Administration, State Energy Data Report 1991, Consumption Estimates, Tables D10 and D11.

The conversion factor for $\mathrm{CT} / 1990$ is assigned from $\mathrm{CT} / 1989$, the $\mathrm{NH} / 1976$ conversion factor is assigned from $\mathrm{NH} / 1975$, the $\mathrm{RI} / 1977$ conversion factor is 
C assigned from RI/1976, and the VT/1986 conversion factor is assigned from VT/1985.

\section{Transportation Sector}

C Transportation sector steam coal prices are assigned O from industrial sector steam coal prices. U.S. Btu K prices are calculated as the average of the State Btu E prices, weighted by consumption data from SEDS.

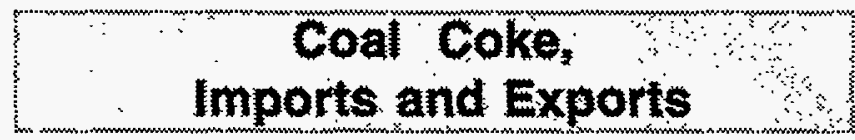

Imports and exports of coal coke are components of total U.S. energy consumption and are accounted for in the industrial sector. Prices and values of imports and exports are developed only for the United States; no attempt is made to estimate State-level prices or expenditures. Prices are f.a.s. (free alongside ship) values and do not include taxes. The quantities of U.S. coal coke imports and exports are from SEDS.

\section{Physical Unit Prices: All Years}

For 1980 forward, the Coke Plant Report, the Quarterly Coal Report, and Bureau of the Census computer tapes provide physical unit coal coke import and export prices in dollars per short ton. For 1970 through 1979, Coke and Coal Chemicals, International Coal, and the Minerals Yearbook provide coal coke import and export physical unit quantities and values in short tons and dollars, respectively. Values are equivalent to expenditures.

\section{Btu Prices: All Years}

For 1980 forward, Btu prices are computed by dividing the physical unit prices by the conversion factor. For 1970 through 1979, physical unit prices are com- puted by dividing the import and export values by their respective quantities, and Btu prices are computed by dividing the physical unit prices by the conversion factor.

\section{Data Sources}

Prices

1989 forward: Bureau of the Census, U.S. Department of Commerce, computer tapes for "Monthly Report IM 145" and "Monthly Report EM 522."

1981-1988: Energy Information Administration, Quarterly Coal Report, October-December issues, Tables A11 and A13 (1981-1985) and Tables A10 and A12 (1986-1988).

1980: Energy Information Administration, Coke Plant Report, Tables 7 and 8.

1978-1979: Energy Information Administration, Coke and Coal Chemicals 1979, Tables 5 and 6.

1977: National Coal Association, International Coal 1980, tables titled, "U.S. Imports of Solid Fuels and Customs Value" and "U.S. Exports of Coke and Value."

1976: Energy Information Administration, Coke and Coal Chemicals, Tables 19 and 20.

1970-1975: Bureau of Mines, U.S. Department of the Interior, Minerals Yearbook, "Coke and Coal Chemicals" chapter, Tables 19 and 20.

\section{Consumption}

1970 forward: Energy Information Administration, State Energy Data System, U.S. imports and exports of coal coke.

\section{Conversion Factor: All Years}

24.8 million Btu per short ton. 


\section{Section 3. Natural Gas}

Natural gas prices are developed for the residential, commercial, industrial, and electric utility sectors. The transportation sector is not covered here because natural gas used for transportation is consumed solely in pipeline operations and is discussed in Section 6, "Consumption Adjustments for Process Fuel and Intermediate Products," on page 251. Reported natural gas prices are retail prices for sales of natural gas to ultimate users.

In general, taxes are included in the prices. However, taxes collected by a utility from an end user and turned over to a Government authority frequently are not included in the revenues reported in the Natural Gas Annual or Natural Gas Production and Consumption and, therefore, are not included in the prices. Taxes paid by the utility (rather than the end user) are considered operating costs and are passed on to the end user as part of the rate. Therefore, Federal, State, business, and property taxes are typically included in the prices, while sales and other point-ofpurchase taxes typically are not.

Estimates of the amount of natural gas consumed by the residential, commercial, industrial, and electric utility sectors are from the State Energy Data System (SEDS). Estimates for the industrial sector are adjusted to remove estimated refinery consumption and lease and plant use of natural gas in each State. (See the discussion in Section 6, "Consumption Adjustments for Process Fuel and Intermediate Products," on page 251.)

\section{Residential, Commercial, and Industrial Sectors}

\section{Prices: 1987 Forward}

All natural gas physical unit prices by State for the residential, commercial, and industrial sectors are from the Natural Gas Annual (NGA).

\section{Prices: 1970 Through 1986}

All natural gas physical unit prices for the residential, commercial, and industrial sectors are calculated from value and quantity of sales data from the Natural Gas Annual or its predecessor report, Natural Gas Production and Consumption. State prices are calculated directly from the data sources as average revenue per unit of sales by natural gas utilities. Prices for each of the three sectors are calculated by dividing the value of natural gas, reported in thousands of dollars, by the quantity of natural gas sold, as reported in million cubic feet.

For 1970 though 1979, both the value and quantity of sales data from the NGA are reported as composites for $M D$ and $D C$ and for $M E, N H$, and VT. In each case, the combined prices are assigned to each of the States in the composite.

\section{Btu Prices: All Years}

State Btu prices for all years are calculated using the physical unit price series and the State-level nonelectric utility conversion factors. U.S. Btu prices are calculated as the average of the State Btu prices, weighted by consumption data from SEDS, adjusted for process fuel consumption in the industrial sector.

\section{Data Sources}

Prices

1980 forward: Energy Information Administration, Natural Gas Annual, Volume 1, Table 11 (1980), Table 14 (1981-1985), Table 15 (1986), Table 19 (1987), and Table 22 (1988 forward).

1970-1979: Bureau of Mines, U.S. Department of the Interior, Natural Gas Production and Consumption, Table $6(1970,1979)$ and Table 7 (1971-1978). 
Consumption

1970 forward: Energy Information Administration, State Energy Data System, residential, commercial, and industrial natural gas consumption.

\section{Conversion Factors: All Years}

G Energy Information Administration, State Energy A Data Report 1991, Consumption Estimates, Tables D4 $\mathbf{S}$ and D5.

\section{Electric Utility Sector}

\section{Prices: $1973,1974,1983$ Forward}

Prices for States are reported by Cost and Quality of Fuels for Electric Utility Plants (CEQ) for gas consumed at steam-electric plants only. Btu prices are from $C \mathcal{E} Q$, converted from cents to dollars per million Btu.

Where individual State prices are unavailable from CEQ, they are developed from the NGA. Prior to 1987, physical unit prices are calculated by dividing the value of natural gas, reported in thousands of dollars, by the quantity of natural gas sold, reported in million cubic feet. For 1987 forward, physical unit prices are directly from the NGA. Table A10 lists the States and years for which NGA data are used. Btu prices are calculated from the physical unit prices using State-level electric utility conversion factors.

Prices are not available from either CEQ or NGA for CT/1973; ID/1974, 1987; NH/1983; and OR/1983, $1984,1986,1989,1990$. In these cases, quantityweighted Census division prices from CEQ are assigned. In addition, prices for VT/1986 and WA/1986, 1987, 1990 use quantity-weighted Census division prices from $C \mathcal{E} Q$ for a more consistent price level than available from the NGA.

\section{Prices: 1980 Through 1982}

State Btu and physical unit prices for 1980 through 1982 are from CEQ for all reporting plants. Physical unit prices are directly from the data source, while Btu prices are converted from cents to dollars per million Btu.
Table A10. Natural Gas Electric Utility Sector Prices from NGA, 1973 Forward

\begin{tabular}{ll}
\hline State & \multicolumn{1}{c}{ Years } \\
\hline AK & $1973-1990$ \\
CT & $1974-1976$ \\
ID & $1983-1986$ \\
MD & $1973,1974,1983-1985$ \\
NC & $1983-1990$ \\
ND & $1973,1974,1976-1986$ \\
NH & $1973,1974,1977,1987-1989$ \\
PA & 1973 \\
RI & 1976,1980 \\
SC & 1977 \\
SD & $1983-1990$ \\
TN & $1976,1980,1981,1983,1988-1991$ \\
UT & 1988,1989 \\
VT & $1983-1985,1989,1990$ \\
WA & $1978,1983-1985,1988,1989$ \\
WY & 1973,1975 \\
\end{tabular}

Note: NGA includes predecessor publications.

\section{Prices: 1975 Through 1979}

State prices are reported separately by $C \mathcal{E} Q$ for gas consumed at steam-electric plants and gas consumed at combustion turbine and internal combustion units. Weighted-average Btu prices are calculated using the two $C E Q$ prices and the respective gas deliveries for steam-electric and combustion use. The $\mathrm{NH} / 1977$ price is not available from $C \mathcal{E} Q$. A combined price is computed from value and quantity of sales data from the NGA for ME, NH, and VT and assigned to $\mathrm{NH} / 1977$.

\section{Prices: 1970 Through 1972}

State prices for 1970 through 1972 are from Natural Gas Production and Consumption and are calculated similarly to prices for the residential, commercial, and industrial sectors. Prices, as average revenue per unit of sales, are computed from value and quantity of sales data from the source reports. A combined price is reported for NH and VT for 1971 and 1972, and each of these States is assigned the combined price. State Btu prices are calculated from the physical unit prices using the State-level electric utility conversion factors.

\section{U.S. Prices: All Years}

U.S. Btu prices are calculated as the average of the State Btu prices, weighted by consumption data from SEDS. 
1976-1979: Energy Information Administration,

Prices

Energy Data Reports, Natural Gas Production and Consumption, Table 7 (1976-1978) and Table 6 (1979).

1973 forward: Energy Information Administration, Cost and Quality of Fuels for Electric Utility Plants, tables numbered as follows:

1970-1975: Bureau of Mines, U.S. Department of the Interior, Natural Gas Production and Consumption, Table 6 (1970) and Table 7 (1971-1975).

\section{Consumption}

\begin{tabular}{llc} 
Years & Price Data & Volume Data \\
\hline 1973, 1974 & Table 10 & Table 9 \\
1975-1979 & Table 10, 16 & Table 9, 15 \\
$1980-1982$ & Table 48 & - \\
1983,1984 & Table 53 & - \\
$1985-1987$ & Table 43 & - \\
1988,1989 & Table 44 & - \\
1990,1991 & Table 19 & - \\
\hline
\end{tabular}

1980 forward: Energy Information Administration, Natural Gas Annual, Volume 1, Table 11 (1980), Table 14 (1981-1985), Table 15 (1986), Table 19 (1987), and Table 22 (1988 forward).

1970 forward: Energy Information Administration, State Energy Data System, electric utility natural gas consumption.

\section{Conversion Factors}

Btu prices that are calculated directly from Cost and Quality of Fuels for Electric Utility Plants (CEQ) require no conversion factors. When Natural Gas Annual (NGA) data are used to develop prices that are missing from $C \mathcal{E} Q$, conversion factors are used from the following source.

1970 forward: Energy Information Administration, State Energy Data Report 1991, Consumption Estimates, Tables D2 and D3. 


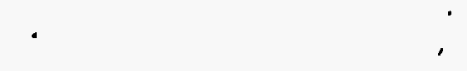

1 


\section{Section 4. Petroleum}

\section{Asphalt and Road OII}

Asphalt and road oil prices are developed for the industrial sector only because SEDS assumes that all asphalt and road oil consumption occurs in the industrial sector. Asphalt and road oil are primarily used for paving (79 percent of consumption in 1970 and 86 percent in 1991) with the remaining products used for roofing and sealing. Taxes are not included in the prices because most street and highway paving is done under contract to State, county, and other public authorities who are typically exempted from paying taxes.

\section{Physical Unit Prices: All Years}

Asphalt prices in physical units are developed from monthly reports in the Engineering News Record, a construction industry weekly magazine published by McGraw-Hill, Inc. The source data consist of monthly reports from correspondents in 20 U.S. cities with price quotes for tank cars, drums, or both, for the three major types of asphalt products: asphalt cement (AC-20), asphalt emulsion (rapid set and slow set), and asphalt cutback.

For 1986 forward, the tank car price is used. However, for 1986 and 1987, the drum price is used if a tank car price is not available. For 1970 through 1985, when both tank car and drum prices are available, a simple average of the two prices is used. When only one price is available, that price is used.

Asphalt prices are developed by calculating a simple average annual price from the monthly prices for each city for the three products. City prices are assigned to States. CA, OH (1970 through 1985), and $P A$ have prices from two cities; in these cases, simple averages of the two city prices are used. No States have prices from more than two cities. States with no prices are assigned a Census division simple average price. State average asphalt prices are calculated as the quantity-weighted average prices of the three products for each State. If there is no Census division price, the simple average of the prices for the other Census divisions within that Census region is used. Quantity data for 1970 through 1980 are from the Bureau of Mines and EIA reports on sales of asphalt. Quantity data for 1981 forward are from the Report on Sales of Asphalt in the U.S. published by the Asphalt Institute. Non-paving asphalts are assumed to have the price of paving asphalt cement.

For 1970 through 1982, asphalt and road oil are estimated as separate data series. Asphalt prices are estimated as discussed above. Road oil prices are assumed to equal asphalt emulsion prices because specific prices are not available from any source.

\section{Btu Prices: All Years}

Asphalt prices in dollars per ton are converted to dollars per gallon by dividing by 235 gallons per ton for asphalt cement, 241 gallons per ton for emulsion, or 248.6 gallons per ton for cutback. These prices are then multiplied by 42 gallons per barrel and divided by 6.636 million Btu per barrel to get dollars per million Btu. Road oil unit prices of dollars per ton are converted to dollars per million Btu using the constant conversion factors of 5.5 barrels per ton and 6.636 million Btu per barrel. The average price of all asphalt and road oil is computed as the consumption weighted average of the individual product prices.

U.S. Btu prices are calculated as the average of the State Btu prices, weighted by consumption data from SEDS.

\section{Data Sources}

Prices

1970 forward: McGraw-Hill, Inc., Engineering News Record.

Quantities for Calculating Weighted Average Prices

1981 forward: Asphalt Institute, Asphalt Usage, United States and Canada. 
A 1978-1980: Energy Information Administration, V Energy Data Reports, Sales of Asphalt, Table 2.

1977: Energy Information Administration, Energy Data Reports, Asphalt Sales, Annual, Table 2.

1971-1976: Bureau of Mines, U.S. Department of the Interior, Mineral Industry Survey, Asphalt Sales, Annual, Table 2.

1970: Bureau of Mines, U.S. Department of the Interior, Mineral Industry Survey, Asphalt Shipments, Annual, Table 2.

\section{Consumption}

1970 forward: Energy Information Administration, State Energy Data System, industrial sector, asphalt and road oil consumption.

\section{Conversion Factors: All Years}

235 gallons per ton of asphalt cement; 241 gallons per ton of emulsion; 248.6 gallons per ton of cutback; 42 gallons per barrel; 5.5 barrels per ton of road oil; 6.636 million Btu per barrel.

\section{Aviation Gasoline}

Aviation gasoline prices are developed for the transportation sector. Estimates of the amount of aviation gasoline consumed by the transportation sector are from SEDS. Aviation gasoline prices are national averages, excluding taxes, developed from several sources, depending on the years. In all cases, physical unit prices are developed and then converted to Btu prices. Federal and State excise taxes, as well as State and local sales taxes, are not included.

\section{Physical Unit Prices: 1976 Forward}

Aviation gasoline prices for 1978 forward are assumed to be the national average refiners sales prices to end users published in the EIA's Annual Energy Review. The 1976 and 1977 prices are assumed to be the national average retail prices published in the ELA's Monthly Energy Review.

\section{Physical Unit Prices: 1970 Through 1975}

For 1970 through 1975, aviation gasoline prices are not available. Prices are derived by dividing the na- tional motor gasoline prices for those years by the 1976 national motor gasoline price and applying that percent changes to the 1976 the national aviation gasoline price.

\section{Btu Prices: All Years}

Aviation gasoline Btu prices are calculated by converting the physical unit prices from cents per gallon to dollars per barrel ( 42 gallons per barrel) and, then, to dollars per million Btu (5.048 million Btu per barrel).

\section{Data Sources}

Prices

1978 forward: Energy Information Administration, Annual Energy Review 1992, Table 5.21, row titled "Sales Prices to End Users: Aviation Gasoline."

1976, 1977: Energy Information Administration, Monthly Energy Review, April 1984, page 106, column titled "Aviation Gasoline, Retail."

1970-1975: Energy Information Administration, Annual Energy Review 1989, Table 70, column titled "Motor Gasoline, Leaded Regular, Nominal."

\section{Consumption}

1970 forward: Energy Information Administration, State Energy Data System, transportation sector, aviation gasoline consumption.

\section{Conversion Factor: All Years}

5.048 million Btu per barrel.

\section{Distillate Fuel}

Distillate fuel prices are developed for all sectors. Distillate fuel in the transportation sector is assumed to be diesel fuel. Estimates of the amount of distillate fuel consumed in each sector are from SEDS. Estimated consumption for the industrial sector is adjusted to remove the estimated refinery consumption of distillate fuel in each State. (See the discussion in Section 6, "Consumption Adjustments for Process Fuel and Intermediate Products," on page 251.) 


\section{Residential Sector}

Residential distillate prices are developed from a variety of data sources, depending on the years involved. In all cases, physical unit prices for States are developed first, then Btu prices are calculated using the physical unit prices and the conversion factor. The prices contained in this series are the retail prices paid by consumers for residential heating oil, including taxes.

\section{Physical Unit Prices: 1991}

Physical unit distillate prices in cents per gallon (excluding taxes) are available for 24 States from the $P M A$. State-level prices for the remaining 27 States are estimated using physical unit prices derived for 1990 in SEPEDS (described below) and the 1991 PMA Petroleum Administration for Defense (PAD) district prices. The estimation procedures are described below and include the addition of State general sales taxes.

1. State prices in cents per gallon are available from the PMA for the following 24 States: $\mathrm{AK}, \mathrm{CT}$, $\mathrm{DC}, \mathrm{DE}, \mathrm{ID}, \mathrm{IL}, \mathrm{IN}, \mathrm{MA}, \mathrm{MD}, \mathrm{ME}, \mathrm{MI}, \mathrm{MN}, \mathrm{NH}$, NJ, NY, OH, OR, PA, RI, VA, VT, WA, WI, and WV. Prices for these States are converted from cents to dollars per gallon, and State general sales taxes from the Bureau of the Census, State Government Tax Collections (SGTC) are added.

2. For the remaining 27 States that do not have prices in the PMA, prices are estimated using the 1990 SEPEDS physical unit and PMA PAD district prices for 1990 and 1991 . The following steps are used to estimate the prices:

a. For 1990, the Subdistrict IC price is withheld in the $P M A$ and the average of the VA and WV prices is used as the Subdistrict IC price.

b. The 1990 State prices derived from AGA and $P M A$, as described below, are adjusted by the percentage change in the 1990 and 1991 prices for each State's PMA PAD district or subdistrict.

c. The State general sales taxes from SGTC are added.

\section{Physical Unit Prices: 1983 Through 1990}

For 1983 through 1990, physical unit distillate prices in cents per gallon (excluding taxes) are generally available for 24 States from the Petroleum Marketing Annual (PMA). State-level prices for the States without $P M A$ prices are estimated using price data from the American Gas Association (AGA), SEDS consumption data, and PMA Petroleum Administration for Defense (PAD) district prices. The estimation procedures are described below and include the addition of State general sales taxes.

1. State prices in cents per gallon are generally available from the $P M A$ for the following 24 States: AK, CT, DC, DE, ID, IL, IN, MA, MD, ME, MI, MN, NH, NJ, NY, OH, OR, PA, RI, VA, VT, WA, WI, and WV. Prices for these States are converted from cents to dollars per gallon, and State general sales taxes from the Bureau of the Census, State Government Tax Collections (SGTC) are added.

2. For the States that do not have prices in the $P M A$, prices are estimated using AGA fuel oil prices, SEDS consumption data, and PMA PAD district prices for Districts II, III, IV, and V and Subdistrict IC (all the States in PAD Subdistricts IA and IB have published prices). The following steps are used to estimate the prices:

a. Distillate prices from the PMA for PAD Districts II, III, IV, and V and Subdistrict IC are converted from cents per gallon to dollars per gallon.

b. The AGA lists fuel oil prices by company for the principal city served in dollars per million Btu, including State sales taxes. A simple average of the city-level prices is used to derive a State-level price for each of the States without PMA prices. These AGA State averages are converted from dollars per million Btu to dollars per gallon using the AGA conversion factor of 7.194 gallons per million Btu. State general sales taxes from SGTC are subtracted to give State averages comparable to the PMA prices.

c. The AGA State prices derived in Step b. are combined into PAD district averages using SEDS consumption to weight each State's values. This procedure gives AGA consumption-weighted average prices for PAD Districts II, III, IV, and V and Subdistrict IC that are comparable to the volume-weighted PAD district prices published in the PMA. The AGA PAD district averages are calculated using only the available States; if a State does 
not appear in the survey, it is not included in the PAD calculation.

d. Adjustment factors, ratios of the PMA PAD district price divided by the AGA derived PAD district price, are calculated for PAD Districts II, III, IV, and V, and Subdistrict IC.

e. Prices for the States not published in the $P \dot{M} A$ are calculated by multiplying the AGA State prices derived in Step b. by the appropriate PAD district adjustment factor from Step d. and adding State general sales taxes from the SGTC.

f. States that do not have prices in either the $P M A$ or the AGA are assigned a PMA PAD district price, and State general sales taxes from the SGTC are added. The States with assigned PAD prices are as shown in Table A11.

\section{Physical Unit Prices: 1978 Through 1982}

Procedures for the 1978 through 1982 period are similar to those for 1983 forward except for changes in data sources. Annual physical unit prices are either directly from the Monthly Energy Review (MER) or are calculated from monthly regional price data, also from the MER. These data were collected on Form EIA-9A (formerly EIA Form 9 and FEA Form. P112-M1) and include taxes. Price data from Platt's Oil Price Handbook and Oilmanac (Platt's) and SEDS consumption data for 1978-1982 are used to compute State prices when only regional data are available. These calculations are described step-by-step below.

1. Annual State physical unit prices are generally available from the MER for the same 24 States covered by the PMA in 1983 forward. These 24 States compose all of Federal Regions 1, 2, 3, 5, and 10 (see Figure A2 on page 180). Prices for these States exclude taxes and are converted to dollars per gallon.

2. Of the States without $M E R$ prices, the 22 in Federal Regions 4, 7, 8, and 9 have annual prices estimated from the monthly Federal regional prices published in the $M E R$. No regional prices are available for Federal Region 6 for the 19781982 period, and some monthly prices are missing in regions 7, 8, and 9 in 1980, 1981, and 1982.

a. Missing monthly prices for Federal regions are estimated with assigned prices as follows:
Table A11. Distillate Residential Sector

Price Assignments, 1983-1990

\begin{tabular}{lll}
\hline State & \multicolumn{1}{c}{ Years } & Prices Assigned \\
\hline AR & 1988 & PAD District III \\
CA & 1984 & PAD District V \\
HI & $1983-1990$ & PAD District V \\
IL & 1986 & PAD District II \\
KS & 1986,1989 & PAD District II \\
LA & 1986 & PAD District III \\
MS & $1983,1985,1986$ & PAD District III \\
NM & $1984-1990$ & PAD District III \\
OK & $1986,1989,1990$ & PAD District II \\
SD & 1986 & PAD District II \\
UT & 1985 & PAD District IV \\
& & P \\
\hline
\end{tabular}

the Region 9 November 1980 price is assigned for December 1980; an average of the Region 7 July and October 1982 prices is assigned to August and September 1982; an average of Region 8 June and September 1982 prices is assigned to July and August 1982; and an average of Region 3 August and October 1982 prices is assigned for the missing September 1982 price. Imputation of missing Region 6 prices for 1978 through 1982 and missing Region 9 prices for 1981 and 1982 is discussed later.

b. The simple average of monthly State-level normal heating degree-day data is averaged for all the States within each of the 10 Federal regions and used to estimate average Federal region heating dégree-days. $\mathrm{AK}, \mathrm{DC}$, and $\mathrm{HI}$ are assigned the monthly heating degree-days from $M N, M D$, and $F L$, respectively.

c. Weighted average annual physical unit distillate prices for the residential sector are calculated for Federal Regions 4, 7, 8, and 9 (except for Region 9 in 1981 and 1982) using the regional normal heating degree-days and the monthly regional prices from the MER.

d. In 1981, only March and May prices are available for Federal Region 9. To estimate the average annual price for this region, the relationship between the U.S. annual heating oil price (from the MER) and the U.S. March and May prices is expressed as a ratio and used with the Region 9 March and May prices to estimate the 1981 annual Region 9 price. 


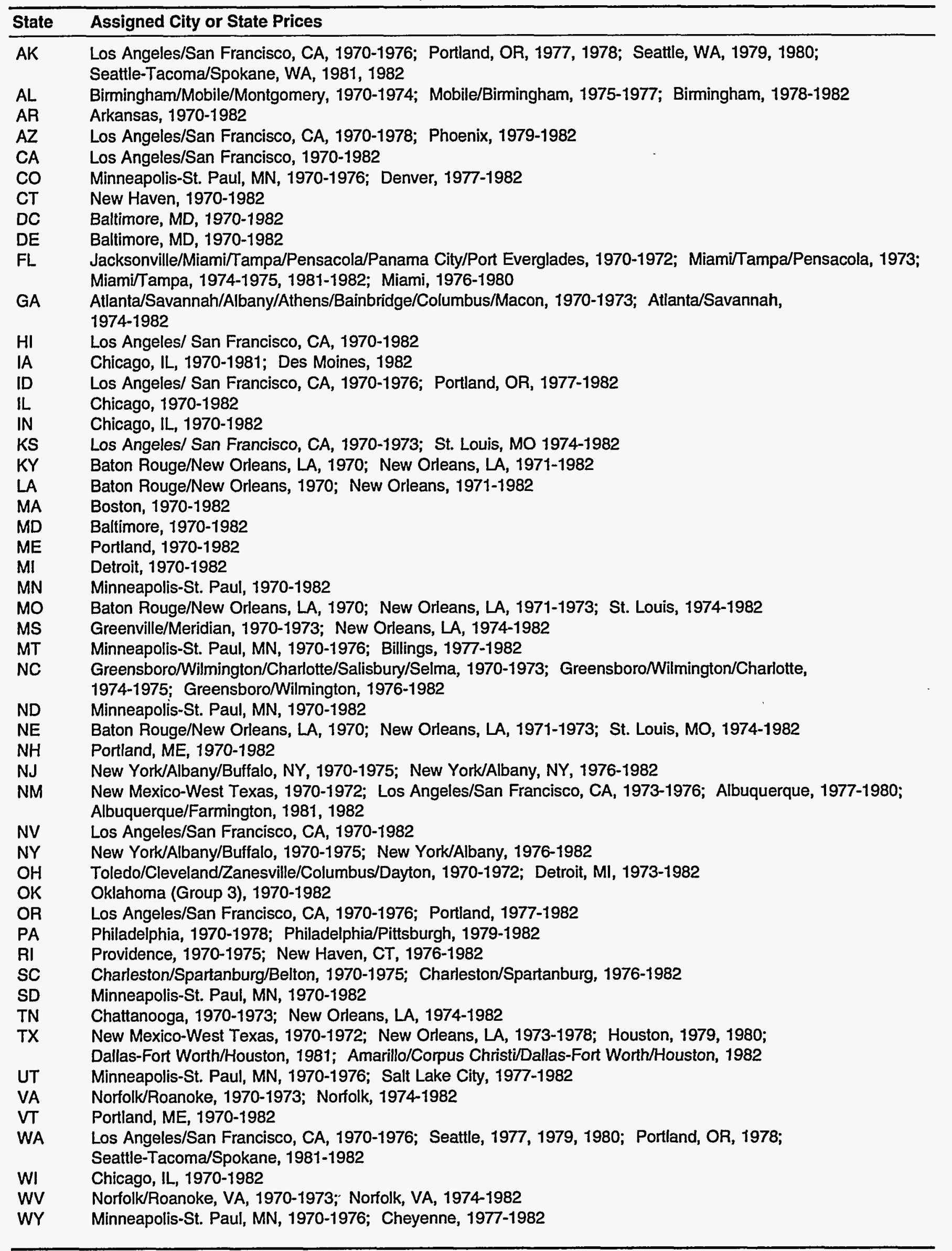


e. City-level prices from Platt's are assigned to States as shown in Table A12. The assigned State-level Platt's prices for States are consumption-weighted into Federal regions using residential sector consumption data from SEDS.

f. Adjustment factors, ratios of the regional MER distillate prices to the regional Platt'sbased distillate prices, are calculated for Federal Regions 4, 7, 8, and 9 (except for 1982).

g. Since there are no monthly regional distillate prices from the MER for Federal Region 6 for 1978 through 1982 and Federal Region 9 for .1982, the adjustment factors for these regions are based on the adjustment factors for previous time periods. The Region 6 adjustment factor for each of the years in the 1978 through 1982 period is equal to 1.1313 , which is the average of the adjustment factor for the West South Central Census Division for 1976 and 1977. The Region 9 adjustment factor for 1982 is equal to 1.1995 , which is the average adjustment factor for Region 9 from 1978 through 1981.

$h$. The residential sector distillate State prices for the 27 States in Federal Regions 4, 6, 7, 8, and 9 are calculated by multiplying the regional adjustment factors for each year and the Statelevel assigned Platt's prices.

\section{Physical Unit Prices: 1975 Through 1977}

For the years 1975 through 1977, no State-level data are available, and regional data from Form EIA-9A are available only at the Census division level, except for Federal region prices for November and December of 1977. Using a methodology similar to that described above for the allocation of regional data to States, adjustment factors are calculated at the regional level and applied to Platt's price data assigned to States. The resulting prices implicitly include average regional taxes but do not reflect individual State differences.

1. Monthly regional price data for 1975 and 1976 are reported in the MER only for Census divisions. In 1977, however, monthly price data are reported for Census divisions for January through October and for Federal regions for November and December. The Federal region prices for November and December are assigned to their respective States and reaggregated into
Census divisions in order to create a consistent set of monthly Census division prices for 1977. Annual residential sector distillate consumption data from SEDS are used to do the reaggregation.

2. The Census division monthly price data from the MER for 1975, 1976, and the first 10 months of 1977 are used with the estimated Census division price data for November and December 1977 to estimate State-level prices.

a. Missing monthly prices in the East South Central Division for June and November 1975, and the Mountain Division for March and July 1975 are estimated using an average of the prices for the month preceding and the month following the missing month. Missing November and December West South Central Division prices in 1977 are estimated with the assignment of the October price to both months. No monthly price data are available for the West South Central Division in 1975; Step "f." below discusses how the calculations are handled for this division.

b. The monthly State-level normal heating degree-day data are averaged for the States within each Census division to estimate regional monthly heating degree-days. AK, DC, and $\mathrm{HI}$ are assigned the monthly heating degree-days from $\mathrm{MN}, \mathrm{MD}$, and $\mathrm{FL}$, respectively.

c. Weighted average annual distillate prices for Census divisions are calculated using the monthly Census division price data from the $M E R$ and the normal heating degree-days estimated for Census divisions.

d. City-level No. 2 fuel oil refinery and terminal prices from Platt's for 1975 through 1977 are assigned to States as shown in Table A12. The assigned Platt's prices for States are consumption-weighted into Census divisions using residential sector consumption data from SEDS.

e. Adjustment factors, ratios of the MER distillate Census division prices to the Platt's distillate Census division prices, are calculated.

f. Since there are no 1975 MER price data for the West South Central Division from which to calculate an adjustment factor, the 1975 adjustment factor for this region is assumed to be equal to the simple average of the West 
South Central adjustment factors for 1976 and 1977 (i.e., 1.1313).

g. The residential sector distillate State prices for all States are calculated by multiplying the regional adjustment factors for each year by the State-level assigned Platt's prices.

\section{Physical Unit Prices: 1970 Through 1974}

There are no regional or State-level distillate price data directly available for the 1970 through 1974 period. To estimate State prices, regional average prices are first derived from the relationship between U.S. prices and Federal region prices for 1975 through 1980. State prices are then estimated from the regional prices using a methodology similar to that described for 1978 through 1982. The resulting prices implicitly include average regional taxes but do not reflect individual State differences.

1. The first step in the estimation of residential distillate prices for the 1970 through 1974 time period, is to develop an equation that uses U.S. prices to estimate prices for Federal regions. Regression techniques are used for this purpose. U.S. prices for 1975 through 1980 from the Annual Energy Review (AER) are used as the independent variable for developing the equation; annual Federal region prices are used as the dependent variable. Federal region prices for 1978 through 1980 are calculated above, but $M E R$ prices for 1975 through 1977 are for Census divisions. To convert these annual Census division prices into Federal region prices, the estimated State prices for 1975 through 1977 are aggregated into Federal regions using SEDS consumption data.

2. Regression techniques are applied to the pooled Federal region price data (dependent variable) and the U.S. prices from the AER (independent variable) for 1975 through 1980 . U.S. prices for 1970 through 1974 are input to estimate annual Federal region prices for 1970 through 1974.

3. City-level prices from Platt's for 1970 through 1974 are assigned to States as shown in Table A12. The assigned State-level Platt's prices are consumption-weighted into Federal regions using residential sector distillate consumption data from SEDS.

4. Adjustment factors, ratios of the regional MER distillate Federal region prices to the Platt's- based distillate Federal region prices, are calculated.

5. The residential sector distillate prices for all States are calculated by multiplying the regional adjustment factors for each year by the Statelevel assigned Platt's prices.

\section{Btu Prices: All Years}

Btu prices for States are calculated by converting the physical unit prices from dollars per gallon to dollars per barrel (42 gallons per barrel) and then to dollars per million Btu (5.825 million Btu per barrel). U.S. Btu prices are calculated as the average of the State Btu prices, weighted by consumption data from SEDS.

\section{Data Sources}

Prices

1986 forward: Energy Information Administration, Petroleum Marketing Annual, Table 37 (1986-1988) and Table 39 (1989 forward), column titled, "To Residential Consumers."

1983-1990: American Gas Association, Residential Gas Market Survey (1989, 1990), Gas Househeating Survey (1983-1988), Appendix 2, "Competitive Fuel Prices," column titled, "Fuel Oil."

1983 forward: Bureau of the Census, U.S. Department of Commerce, State Government Tax Collections, table titled, "State Government Excises on General Sales, Motor Fuel, and Cigarettes, Beginning and End of Fiscal Year."

1983-1985: Energy Information Administration, Petroleum Marketing Annual 1985, Volume 1, Table 26.

1970-1982: McGraw-Hill, Inc., Platt's Oil Price Handbook and Oilmanac, refinery and terminal prices for No. 2 fuel oil, average of highs and lows.

1975-1982: National Oceanic and Atmospheric Administration, U.S. Department of Commerce, State, Regional, and National Monthly and Seasonal Heating Degree-Days Weighted by Population (1980 Census), Historical Climatology Series 5-1, table titled, "1951-80 State Pop. Wgt'd Heating Degree-Days."

1975-1982: Energy Information Administration, Monthly Energy Review, table titled, "Residential Heating Oil Prices by Region," February 1978, page 
67 (1975, 1976); April 1980, page 83 (1977, 1978); July 1982, page 87 (1979-1982).

1970-1982: Energy Information Administration, Annual Energy Review 1988. Table 67, "Motor Gasoline and Residential Heating Oil Prices, 1949-1988."

\section{Consumption}

1970 forward: Energy Information Administration, State Energy Data System, residential sector distillate consumption.

\section{Conversion Factor: All years}

5.825 million Btu per barrel

\section{Commercial Sector}

Commercial sector distillate prices are estimated by two general methods, one for prices 1983 forward, and the other for the 1970 through 1982 prices. For 1983 forward, retail prices paid by commercial/institutional establishments (excluding taxes) are from Petroleum Marketing Annual (PMA) supplemented with prices derived from the American Gas Association (AGA) Residential Gas Market Survey. State general sales taxes from the Bureau of the Census are added. For 1970 through 1982, commercial distillate prices are based on refinery and terminal (wholesale) prices from Platt's and markups from Energy Prices: 1960-73 that include taxes. For both time periods, physical unit prices are calculated from the data sources, and Btu prices are computed using the physical unit prices and the conversion factor.

\section{Physical Unit Prices: 1991}

Physical unit distillate prices in cents per gallon (excluding taxes) are available for 24 States from the $P M A$. State-level prices for the remaining 27 States are estimated using physical unit prices derived for 1990 in SEPEDS (described below) and the 1991 PMA Petroleum Administration for Defense (PAD) district prices. The estimation procedures are described below.

1. State prices in cents per gallon are available from the PMA for the following 24 States: $A K, C T$, $\mathrm{DC}, \mathrm{DE}, \mathrm{ID}, \mathrm{IL}, \mathrm{IN}, \mathrm{MA}, \mathrm{MD}, \mathrm{ME}, \mathrm{MI}, \mathrm{MN}, \mathrm{NH}$, NJ, NY, OH, OR, PA, RI, VA, VT, WA, WI, and WV. Prices for these States are converted from cents to dollars per gallon, and State general sales taxes from the Bureau of the Census, State Government Tax Collections (SGTC) are added.

2. For the remaining 27 States that do not have prices in the $P M A$, prices are estimated using the 1990 SEPEDS physical unit and PMA PAD district prices for 1990 and 1991. The following steps are used to estimate the prices:

a. For 1990, the Subdistrict IC price is withheld in the PMA and the average of the VA and WV prices is used as the Subdistrict IC price. For 1991, the PAD District III price is with held in the PMA. The ratio of the PAD District II commercial consumers price to the PAD District II industrial price is applied to the PAD District III industrial price to estimate the withheld commercial price.

b. The 1990 State prices derived from AGA and $P M A$, as described below, are adjusted by the percentage change in the 1990 and 1991 prices for each State's PMA PAD district or subdistrict.

c. The State general sales taxes from SGTC are added.

\section{Physical Unit Prices: 1983 Through 1990}

For 1983 through 1990, physical unit distillate prices in cents per gallon (excluding taxes) are available for approximately 24 States from the PMA. State-level prices for the remaining 27 States are estimated using price data from the American Gas Association (AGA), SEDS consumption data, and PMA Petroleum Administration for Defense (PAD) district prices. The estimation procedures are described below.

1. State prices in cents per gallon are generally available from the $P M A$ for the following 24 States: AK, CT, DC, DE, ID, IL, IN, MA, MD, ME, MI, MN, NH, NJ, NY, OH, OR, PA, RI, VA, VT, WA, WI, and WV. Prices for these States are converted from cents to dollars per gallon, and State general sales taxes from the Bureau of the Census, State Government Tax Collections (SGTC) are added.

2. For the remaining 27 States that do not have prices in the $P M A$, prices are estimated using AGA fuel oil prices, SEDS consumption data, and PMA PAD district prices for Districts II, III, IV, and V and Subdistrict IC (all the States in PAD Subdistricts IA and IB have published 
prices). For 1990, the Subdistrict IC price is . withheld in the PMA and the average of the VA. and WV prices is used as the Subdistrict IC price. The following steps are used to estimate the prices:

a. Distillate prices from the PMA for PAD Districts II, III, IV, and V and Subdistrict IC are converted from cents per gallon to dollars per gallon.

b. The AGA lists fuel oil prices by company for the principal city served in dollars per million Btu, including State sales taxes. A simple average of the city-level prices is used to derive a State-level price for each of the 27 States without PMA prices. These AGA State averages are converted from dollars per million Btu to dollars per gallon using the AGA conversion factor of 7.194 gallons per million Btu. State general sales taxes from SGTC are subtracted to give State averages comparable to the PMA prices.

c. The AGA State prices derived in Step b. are combined into PAD district averages using SEDS consumption to weight each State's values. This procedure gives AGA consumption-weighted average prices for PAD Districts II, III, IV, and V and Subdistrict IC that are comparable to the volume-weighted PAD district prices published in the PMA. The AGA PAD district averages are calculated using only the available States; if a State does not appear in the survey, it is not included in the PAD district calculation.

d. Adjustment factors, ratios of the PMA PAD district price divided by the AGA derived PAD district price, are calculated for PAD Districts II, III, IV, and V, and Subdistrict IC.

e. Prices for the 27 States not published in the PMA are calculated by multiplying the AGA State prices derived in Step $b$. by the appropriate PAD district adjustment factor from Step $d$. and adding State general sales taxes from the SGTC.

f. States that do not have prices in either the PMA or the AGA are assigned a PMA PAD district price, and State general sales taxes from the SGTC are added. The States with assigned prices are as shown in Table A13.
Table A13. Distillate Commercial Sector

Price Assignments, 1983-1990

\begin{tabular}{llll}
\hline State & \multicolumn{1}{c}{ Years } & Prices Assigned & S \\
\hline AR & 1988 & PAD District III & T \\
CA & 1984 & PAD District V & I \\
HI & $1983-1990$ & PAD District V & L \\
KS & 1986,1989 & PAD District II & L \\
LA & 1986 & PAD District III & A \\
MO & 1986 & PAD District II & T \\
MS & $1983,1985,1986$ & PAD District III & E \\
NM & $1984-1990$ & PAD District III & \\
OK & $1986,1989,1990$ & PAD District II & \\
SD & 1986 & PAD District II & F \\
UT & 1985 & PAD District IV & U \\
& & & E \\
\hline & & & L
\end{tabular}

\section{Physical Unit Prices: 1970 Through 1982}

Commercial sector distillate physical unit prices for 1970 through 1982 are calculated using Platt's prices assigned to States and commercial sector markups estimated from Energy Prices: 1960-73. The resulting estimates implicitly include State-specific taxes.

1. The first step is to compute the markups. Energy Prices contains single price estimates for small commercial users and two price estimates for large commercial users for 10 cities: Boston, MA; Albany, NY; New York, NY; Charlotte, NC; Washington, DC; Chicago, IL; Detroit MI; Minneapolis/St. Paul, MN; St. Louis, MO; and Seattle, WA. First, a simple average of the two large commercial prices is calculated for each city except for Albany and New York. In this case, all four large commercial prices are averaged together, since cities are assigned to their respective States.

2. For the nine States covered by the Energy Prices data (noted in Step 1), the markup of the reported prices from Energy Prices over the assigned Platt's prices (Table A12 on page 199) and the markup of the residential prices calculated above for 1970 through 1972 over the Platt's prices are calculated.

3. At this point, residential and commercial sector retail markups have been computed for nine States for each of the years 1970 through 1972. The next step is to calculate the average retail markup for the 3-year period for each sector. A simple average of the markup ratios is calculated. 


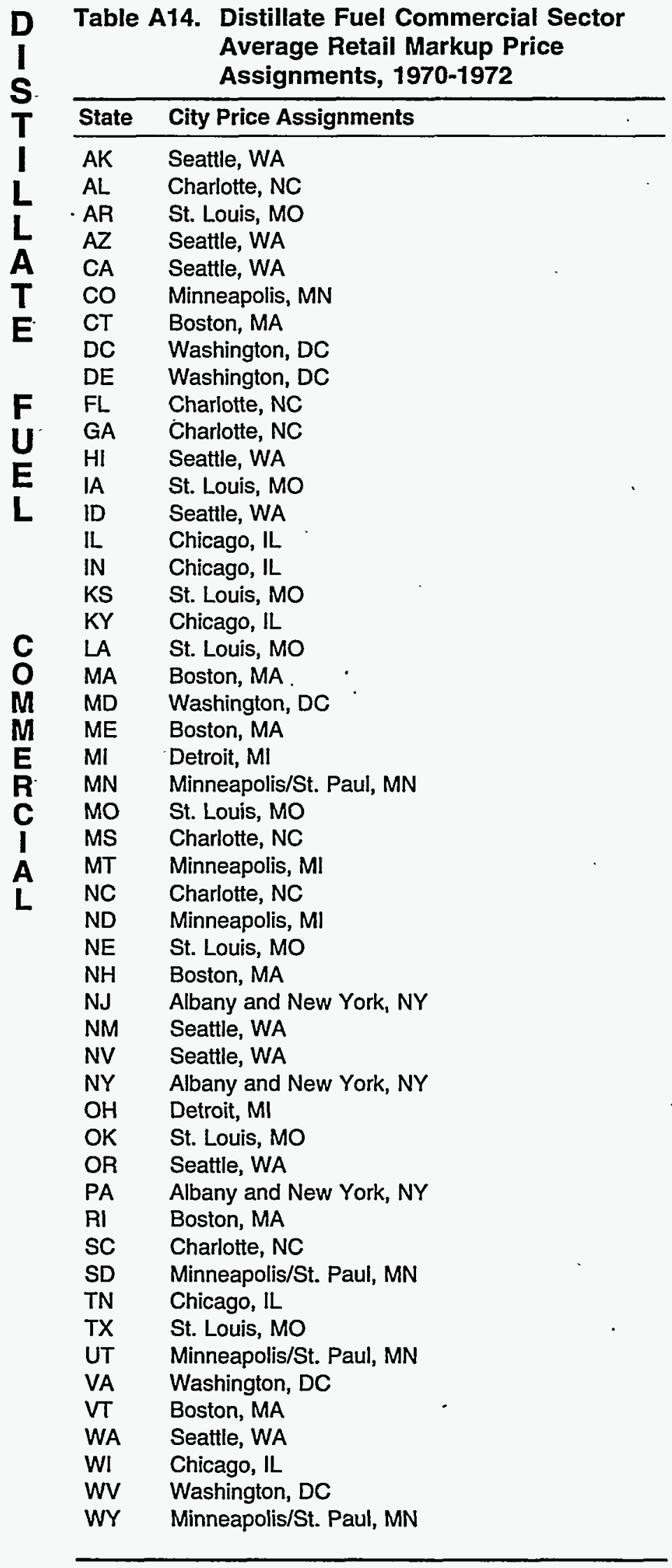

4. The average commercial and residential sector retail markups for the nine available States are assigned as shown in Table A14.

5. To translate the average commercial and residential markups for 1970 through 1972 into the estimated commercial sector retail markups to be used for 1970 through 1982, the relationship between these two markups is used with the residential markups calculated for all States for each year. The calculation of the residential markups is the same procedure used in Step 2 above.

6. The commercial sector adjustment factors for each State for each of the years 1970 through 1982 are multiplied by the corresponding Platt's prices for 1970 through 1982 to calculate the final commercial sector physical unit prices.

\section{Btu Prices: All Years}

Btu prices for States are calculated by converting the physical unit prices from cents to dollars per gallon, then to dollars per barrel (42 gallons per barrel), and finally, to dollars per million Btu (5.825 million Btu per barrel). U.S. prices are calculated as the average of the State Btu prices, weighted by consumption data from SEDS.

\section{Data Sources}

Prices

1986 forward: Energy Information Administration, Petroleum Marketing Annual, Table 37 (1986-1988) and Table 39 (1989 forward), column titled, "To Commercial/Institutional Consumers."

1983-1990: American Gas Association, Residential Gas Market Survey $(1989,1990)$, Gas Househeating Survey (1983-1988), Appendix 2, "Competitive Fuel Prices," column titled, "Fuel Oil."

1983 forward: Bureau of the Census, U.S. Department of Commerce, State Government Tax Collections, table titled, "State Government Excises on General Sales, Motor Fuel, and Cigarettes: Beginning and End of Fiscal Year."

1983-1985: Energy Information Administration, Petroleum Marketing Annual 1985, Volume 1, Table 26. 
1970-1982: McGraw-Hill, Inc., Platt's Oil Price Handbook and Oilmanac, refinery and terminal prices for No. 2 fuel oil, average of highs and lows.

1970-1982: Foster Associates, Inc., 1974, Energy Prices 1960-73, Tables 4-c and 5-b.

\section{Consumption}

1970 forward: Energy Information Administration, State Energy Data System, commercial sector distillate consumption.

\section{Conversion Factor: All Years}

5.825 million Btu per barrel

\section{Electric Utility Sector}

The electric utility price for distillate fuel is the average delivered cost of No. 2 fuel oil receipts at electric utilities (See Light Oil (Utilities) on page 216). For 1973 forward, these prices are from Cost and Quality of Fuels for Electric Utility Plants; for 1970 through 1972, prices from the Statistical Yearbook of the Electric Utility Industry are used with regression analysis. Btu prices are developed directly from the data sources and include all applicable taxes.

\section{Prices: 1973 Forward}

Btu prices for the years 1973 forward are based directly on the Btu prices reported in Cost and Quality of Fuels (CEQ). For 1973, 1974, and 1980 forward, Btu prices are directly from the data source. For 1975 through 1979, weighted average Btu prices are calculated from prices and consumption data reported separately for steam-electric plants and for combustion turbine and internal combustion units. Because 1973 was the first year $C E Q$ was published, there are many States with missing data for that year. The estimation of the missing 1973 State prices follows the same procedure as prices for 1970 through 1972 and is discussed under that section.

Wherever individual State prices are unavailable, quantity-weighted Census division prices from $C \mathcal{E} Q$ are assigned as shown in Table A15.

For 1973, 1974, and 1983 forward, Btu prices in cents per million Btu are from $C E Q$ and converted to dollars per million Btu. For 1975 through 1979, Btu prices in cents per million Btu are from $C E Q$ with their corresponding delivered quantities, weighted
Table A15. Distillate Electric Utility Census Division Price Assignments from $C \& Q, 1973$ Forward

\begin{tabular}{ll}
\hline State & \multicolumn{1}{c}{ Years } \\
\hline CA & $1983-1985,1987,1988,1990,1991$ \\
CT & 1973 \\
DC & 1973 \\
DE & 1973 \\
ID & $1973,1974,1976,1980-1991$ \\
MD & 1973 \\
ME & 1973,1974 \\
MT & $1973-1975,1977,1983$ \\
NH & 1973,1974 \\
NJ & 1973,1974 \\
OR & 1987,1988 \\
RI & $1976-1991$ \\
SD & 1973,1974 \\
TN & 1973 \\
VT & $1973,1974,1978,1983-1991$ \\
WA & $1973-1977$ \\
WV & 1973 \\
WY & 1973 \\
\end{tabular}

into overall prices, and converted to dollars per million Btu. For 1980 through 1982, Btu prices are reported in $C E Q$ for all reporting plants and are converted to dollars per million Btu. U.S. Btu prices for all years are calculated as the average of the State Btu prices, weighted by consumption data from SEDS.

\section{Prices: 1970 Through 1972}

Btu prices for 1970 through 1972 (and AK and $\mathrm{HI}$ for 1973) are estimated using data from Statistical Yearbook of the Electric Utility Industry. U.S. prices are then computed using the State-level prices and the electric utility distillate consumption data from SEDS.

1. Regression techniques are used to arrive at the equation for estimating electric utility sector distillate prices for the 1970 through 1972 (or 1973) period. AL is treated as the reference State. The regression equation uses Statistical Yearbook State-level prices for 1974 through 1980 as the independent variable and the State-level prices calculated above for 1974 through 1980 as the dependent variable. Substituting Btu prices for 1970 through 1972 (or 1973) from the Statistical Yearbook into the regression equation yields the estimated electric utility sector State-level distillate prices. 
2. Wherever individual State prices are unavailable, quantity-weighted Census division prices are assigned as follows: ID/1970 through 1972; TN/1970; and WA/1970, 1971.

3. U.S. Btu prices are calculated as the average of the State Btu prices, weighted by consumption data from SEDS.

\section{Alaska and Hawaii Prices}

Since CEQ does not cover AK/1973 forward or HII/1973 through 1982, prices for these States are estimated. The ratio of the reported CEQ U.S. price and the Statistical Yearbook U.S. price is multiplied by the Statistical Yearbook State price for each year to estimate the price. AK prices for 1971, 1975, and 1978, for which Statistical Yearbook data are missing, are estimated from the average ratio of AK prices to U.S. prices in adjacent years (e.g., 1975 is calculated as the average of the. 1974 and 1976 prices). Prices for 1970 through 1973 are calculated as described in the "Prices: 1970-1972" section. Prices for HI/1983 forward are from C\&Q.

\section{Data Sources}

Prices

1973 forward: Energy Information Administration, Cost and Quality of Fuels for Electric Utility Plants; Table 6 (1973, 1974); Tables 5, 6, 12, 13 (1975-1979); Table 45 (1980-1982); Table 51 (1983, 1984); Table 41 (1985-1989); and Table 14 (1990 forward).

1970 forward: Edison Electric Institute, Statistical Yearbook of the Electric Utility Industry, table titled, "Analysis of Fuel for Electric Generation-Total Electric Utility Industry," (1970-1988); and table titled, "Fossil Fuels Used for Electric Generation Total Electric Utility Industry," (1990 forward).

\section{Consumption}

1970 forward: Energy Information Administration, State Energy Data System, electric utility sector distillate consumption.

\section{Conversion Factors}

Btu prices are calculated directly from data sources; no explicit conversion factors are needed for any years for the electric utility sector.

\section{Industrial Sector}

The industrial sector distillate prices are developed using several different data sources and estimation methods, depending on the years involved. For 1983 forward, sales prices of No. 2 fuel oil to industrial consumers (excluding taxes) are from the Petroleum Marketing Annual (PMA) supplemented-with prices derived from the American Gas Association (AGA) Residential Gas Market Survey. State general sales taxes from the Bureau of the Census are added. For 1970 through 1982, prices are the average cost of distillate to manufacturing firms and implicitly include taxes that reflect individual State differences.

\section{Physical Unit Prices: 1991}

Physical unit distillate prices in cents per gallon (excluding taxes) are available for 21 States from the $P M A$. State-level prices for the remaining 30 States are estimated using physical unit prices derived for 1990 in SEPEDS (described below) and the 1991 PMA Petroleum Administration for Defense (PAD) district prices. The estimation procedures are described below.

1. State prices in cents per gallon are available from the PMA for the following 21 States: $A K, C T$, $\mathrm{DE}$, IL, IN, MA, MD, ME, MI, MN, NH, NJ, NY, $\mathrm{OH}, \mathrm{PA}, \mathrm{RI}, \mathrm{VA}$; VT, WA, WI, and WV. Prices for these States are converted from cents to dollars per gallon, and State general sales taxes from the Bureau of the Census, State Government Tax Collections (SGTC) are added.

2. For the remaining 30 States that do not have prices in the $P M A$, prices are estimated using the 1990 SEPEDS physical unit and PMA PAD district prices for 1990 and 1991. The following steps are used to estimate the prices:

a. The 1990 State prices derived from $A G A$ and $P M A$, as described below, are adjusted by the percentage change in the 1990 and 1991 prices for each State's PMA PAD district or subdistrict.

b. The State general sales taxes from SGTC are added.

\section{Physical Unit Prices: 1983 Through 1990}

For 1983 through 1990, physical unit distillate prices in cents per gallon (excluding taxes) are generally available for 24 States from the PMA. State-level 
prices for the remaining 27 States are estimated using price data from the American Gas Association (AGA), SEDS consumption data, and PMA Petroleum Administration for Defense (PAD) district prices. The estimation procedures are described below.

1. State prices in cents per gallon are generally available from the $P M A$ for the following 24 States: AK, CT, DC, DE, ID, IL, IN, MA, MD, ME, MI, MN, NH, NJ, NY, OH, OR, PA, RI, VA, VT, WA, WI, and WV. Prices for these States are converted from cents to dollars per gallon, and State general sales taxes from the Bureau of the Census, State Government Tax Collections (SGTC) are added.

2. For the remaining 27 States that do not have prices in the $P M A$, prices are estimated using AGA fuel oil prices, SEDS consumption data, and PMA PAD district prices for Districts II, III, IV, and V and Subdistrict IC (all the States in PAD Subdistricts IA and IB have published prices). The following steps are used to estimate the prices:

a. Distillate prices from the PMA for PAD Districts II, III, IV, and V and Subdistrict IC are converted from cents per gallon to dollars per gallon.

b. The AGA lists fuel oil prices by company for the principal city served in dollars per million $\mathrm{Btu}$, including State sales taxes. A simple average of the city-level prices is used to derive a State-level price for each of the 27 States without PMA prices. These AGA State averages are converted from dollars per million Btu to dollars per gallon using the AGA conversion factor of 7.194 gallons per million Btu. State general sales taxes from SGTC are subtracted to give State averages comparable to the PMA prices.

c. The AGA State prices derived in Step b. are combined into PAD district averages using SEDS consumption to weight each State's values. This procedure gives AGA consumption-weighted average prices for PAD Districts II, III, IV, and V and Subdistrict IC that are comparable to the volume-weighted PAD district prices published in the PMA. The AGA PAD district averages are calculated using only the available States; if a State does not appear in the survey, it is not included in the PAD calculation.
Table A16. Distillate Industrial Sector PADD

Price Assignments, 1983-1990

\begin{tabular}{lll}
\hline State & \multicolumn{1}{c}{ Years } & Prices Assigned \\
\hline AK & 1988 & PAD District V \\
AR & 1988 & PAD District III \\
CA & 1984 & PAD District V \\
HI & $1983-1990$ & PAD District V \\
KS & 1986,1989 & PAD District II \\
LA & 1986 & PAD District III \\
MS & $1983,1985,1986$ & PAD District III \\
NM & $1984-1990$ & PAD District III \\
OK & $1986,1989,1990$ & PAD District II \\
SD & 1986 & PAD District II \\
UT & 1985 & PAD District IV \\
WY & 1991 & PAD District IV \\
\end{tabular}

d. Adjustment factors, ratios of the PMA PAD district price divided by the AGA derived PAD district price, are calculated for PAD Districts II, III, IV, and V, and Subdistrict IC.

e. Prices for the 27 States not published in the PMA are calculated by multiplying the AGA State prices derived in Step $b$. by the appropriate PAD district adjustment factor from Step d. and adding State general sales taxes from the SGTC.

f. States that do not have prices in either the $P M A$ or the AGA are assigned a PMA PAD district price, and State general sales taxes from the SGTC are added. The States with assigned prices are shown in Table A16.

\section{Physical Unit Prices: 1982}

In 1984, the Bureau of the Census announced that State-level fuel cost and quantity information would no longer be published in either the Annual Survey of Manufactures (ASM) or Census of Manufactures (CM). In addition, the $P M A$, the source for 1983 forward industrial sector distillate price data, did not contain 1982 prices. Because of this lack of price data, the 1982 industrial sector distillate prices are estimated, based on the relationship of industrial sector prices to electric utility sector prices for 1978 through 1981 . The 1983 prices are not used in the estimation because they exclude taxes, while the 1978 through 1981 prices include taxes.

1. In order to calculate the average ratios of industrial-to-electric utility distillate prices, electric 


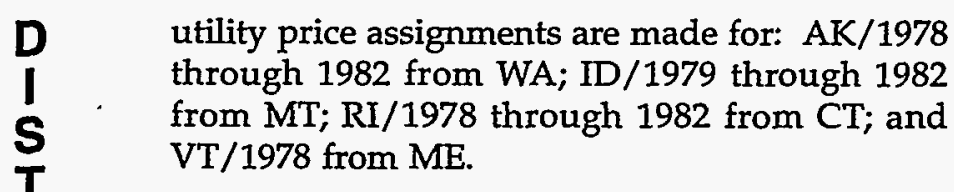

2. The average 1978 through 1981 ratios of industrial-to-electric utility sector distillate prices are calculated for each State.

3. Prices for 1982 are estimated by multiplying the average ratios by the electric utility data for 1982.

\section{Physical Unit Prices: 1971, 1974 Through 1981}

For the years 1971 and 1974 through 1981, industrial sector distillate prices are calculated directly from cost and quantity data from the Annual Survey of Manufactures (ASM) or Census of Manufactures (CM) for all States where data are available. Taxes are included in the prices. There are no missing prices for 1971. Six States are missing some ASM cost and quantity data for the 1974 through 1981 period. Cost and quantity data for these States are estimated as the simple average of the cost and quantity data for their adjacent States: The States, the years for which data are estimated, and the adjacent States used to make the estimation are shown in Table A17.

\section{Physical Unit Prices: 1970, 1972, 1973}

Since $A S M$ and $C M$ data are not available for these years, the prices must be estimated. Physical unit prices are based on the ratio of $1971 \mathrm{CM}$ prices to the 1971 assigned Platt's prices (Table A12 on page 199). The resulting ratios for each State are used with the Platt's assigned prices for 1970, 1972, and 1973 to impute prices.

1. The first step is to calculate State-level ratios between prices calculated from the 1971 CM cost and quantity data and the 1971 assigned Platt's prices. There are no missing States in either of these two sets of prices.

2. State-level physical unit prices for 1970,1972 , and 1973 are estimated by multiplying the 1971 ratio by the assigned State-level Platt's prices for each respective year.

\section{Btu Prices: All Years}

Btu prices for States are calculated by converting the physical unit prices from cents to dollars per gallon,
Table A17. Distillate Industrial Sector

Price Assignments, 1974-1981

\begin{tabular}{lcl}
\hline State & \multicolumn{1}{c}{ Years } & \multicolumn{1}{c}{ State Prices Used } \\
\hline HI & $1979-1981$ & CA \\
ND & $1979-1981$ & MN, MT, SD \\
NM & $1974-1979$ & AZ, CO, TX \\
NV & $1974-1981$ & AZ, CA, ID, OR, UT \\
OK & $1974-1978$ & AR, CO, KS, MO, TX \\
WY & $1974-1981$ & CO, ID, MT, NE, SD, UT \\
\hline
\end{tabular}

then to dollars per barrel (42 gallons per barrel) and, finally to dollars per million Btu (5.825 million Btu per barrel). U.S. Btu prices are calculated as the average of the State Btu prices, weighted by consumption data from SEDS, adjusted for process fuel consumption.

\section{Data Sources}

Prices

1986 forward: Energy Information Administration, Petroleum Marketing Annual, Table 37 (1986-1988) and Table 39 (1989 forward), column titled, "To Industrial Consumers."

1983-1990: American Gas Association, Residential Gas Market Survey (1989, 1990), Gas Househeating Survey (1983-1988), Appendix 2, "Competitive Fuel Prices," column titled, "Fuel Oil."

1983 forward: Bureau of the Census, U.S. Department of Commerce, State Government Tax Collections, table titled, "State Government Excises on General Sales, Motor Fuel, and Cigarettes: Beginning and End of Fiscal Year."

1983-1985: Energy Information Administration, Petroleum Marketing Annual 1985, Volume 1, Table 26.

1970-1982: McGraw-Hill, Inc., Platt's Oil Price Handbook and Oilmanac, refinery and terminal prices for No. 2 fuel oil, average of highs and lows.

1971, 1977, and 1981: Bureau of the Census, U.S. Department of Commerce, Census of Manufactures, Table 4 (1971) and Table $3(1977,1981)$.

1974-1976 and 1978-1980: Bureau of the Census, U.S. Department of Commerce, Annual Survey of Manufactures, Table 3. 


\section{Consumption}

1970 forward: Energy Information Administration, State Energy Data System, industrial sector distillate consumption.

\section{Conversion Factor: All Years}

5.825 million Btu per barrel

\section{Transportation Sector}

Consumption of distillate fuel in the transportation sector includes distillate fuel used for vessel bunkering and for military and railroad use, plus on-highway diesel fuel use. Since on-highway diesel fuel use accounts for the largest portion of this sector-increasing from 55 percent in 1970 to 77 percent in 1991-prices and expenditures are calculated using diesel prices. State physical unit prices for 1986 forward are from the Petroleum Marketing Annual (PMA). Physical unit prices for earlier years are calculated using PMA prices and consumption data from Highway Statistics to weight monthly or quarterly prices from Agricultural Prices into annual prices. For all years, physical unit prices are calculated from the data sources, while Btu prices are calculated from the physical unit prices and the conversion factor.

\section{Physical Unit Prices: 1986 Forward}

Diesel fuel physical unit prices for 1986 forward are based on the annual State-level price data available from the PMA for approximately 24 States and monthly tax rate information from Highway Statistics. Generally, the PMA provides physical unit prices, excluding taxes, for AK, CT, DE, DC, ID, IL, IN, ME, MD, MA, MI, MN, NH, NJ, NY, OH, OR, PA, RI, VT, VA, WA, WI, and WV. State and Federal excise taxes on diesel fuel are added to PMA prices to derive final physical unit prices, which are converted to dollars per gallon. In cases where the tax rate is not constant through the year, the months' rates are averaged, weighted by the number of months each rate was in effect. State and local sales and other general taxes are not included.

For the remaining States for which no State-level physical unit prices are published, the PMA PAD district prices for diesel fuel and motor gasoline and State motor gasoline prices are used. The State diesel fuel price is estimated as the ratio of the PAD district diesel fuel price to the PAD district motor gasoline price times the State motor gasoline price. The use of the ratio assumes that the relationship between the motor gasoline State and PAD district prices is similar to that of the diesel fuel State and PAD district prices. Motor gasoline prices to end users at all refiners company outlets are used. When a State has no price available in that data series, the motor gasoline price to end users by all types of sellers through company outlets is used. States with no published diesel fuel or motor gasoline prices are assigned the motor gasoline price of an adjacent State. For 1991, $\mathrm{DC}$ is assigned the MD price. State and Federal excise taxes are added as described above.

\section{Physical Unit Prices: 1983 Through 1985}

Diesel fuel physical unit prices for 1983 through 1985 are based on the annual State-level price data available from the PMA and monthly State and Federal tax rate information from Highway Statistics for 24 States. The prices for the remaining 27 States are calculated using Agricultural Prices as outlined in the 1977 through 1982 methodology.

For the 24 States of AK, CT, DE, DC, ID, IL, IN, MA, MD, ME, MI, MN, NH, NJ, NY, OH, OR, PA, RI, VA, $\mathrm{VT}, \mathrm{WA}, \mathrm{WI}$, and $\mathrm{WV}$, the PMA provides physical unit prices, excluding taxes. In 1983 through 1985, the DC price is missing, and the MD price is assigned. In 1983, RI has no price and the PAD District IA average is assigned. A simple average of monthly State and Federal excise taxes is calculated as a combined average tax and added to the PMA price for a final physical unit price. State and local sales and other general taxes are not included.

\section{Physical Unit Prices: 1977 Through 1982}

Monthly prices from Agricultural Prices and monthly special fuels consumption data from Highway Statistics are collected for the States. MD prices are assigned to DC. Prices include State and local per gallon taxes. Federal taxes and State and local sales and other general taxes are not included.

The volume-weighted annual diesel physical unit prices for States and the United States are calculated using the monthly Agricultural Prices price data, weighted by the monthly Highway Statistics consumption data. AK/1977 through 1982 prices are estimated based on the assumption that the ratio of AK-to-U.S. diesel fuel price is the same as the ratio of the AK-to-U.S. motor gasoline price each year. 


\section{Data Sources}

Quarterly prices from Agricultural Prices and monthly special fuels consumption data from Highway Statistics are collected for the States. Prices include State and local per gallon taxes. Federal taxes and State and local sales taxes and other general taxes are not included.

1. Prices for 1970 through 1972 are reported in cents per gallon and must be converted to dollars per gallon. Prices for 1973 through 1976 are already reported in dollars per gallon.

2. For 1971 through 1973 , State-level prices are not available for CT, MA, ME, NH, RI, and VT. Each is assigned the New England price for the 3 years.

3. The third quarter $\mathrm{DE}$ price is assigned to the missing fourth quarter DE price in 1972.

4. The combined MD/DE prices reported in 1973 are assigned to each of the States.

5. For 1970 through $1976, \mathrm{MD}$ (or MD/DE) prices are assigned to $D C$.

The monthly special fuels consumption for 1970 through 1976 are converted into quarterly consumption by summing the months for each quarter.

The consumption-weighted annual diesel physical unit prices for the States are calculated using the quarterly weights and quarterly prices. For 1970 through 1972, the quarterly prices from Agriculture Prices are converted from cents per gallon to dollars per gallon. For 1973 forward, the prices are already in dollars per gallon in the source. AK/1970 through 1976 prices are estimated based on the assumption that the ratio of AK-to-U.S. diesel fuel price is the same as the ratio of AK-to-U.S. motor gasoline price each year.

\section{Btu Prices: All Years}

Btu prices for States are calculated by converting the physical unit prices from cents per gallon to dollars per barrel (42 gallons per barrel) and, then, to dollars per million Btu (5.825 million Btu per barrel). U.S. Btu prices are calculated as the average of the State Btu prices, weighted by consumption from SEDS.

\section{Prices}

1986 forward: Energy Information Administration, Petroleum Marketing Annual, Table 36 (1986-1988) and Table 38 (1989 forward), column titled, "Sales to End Users, Through Company-Operated Retail Outlets," for diesel fuel prices; Table 29 (1986-1988) and Table 30 (1989 forward), column titled "All Refiners, Sales to End Users, Through Company Outlets," for motor gasoline prices; and Table 28 (1986-1988) and Table 29 (1989 forward), column titled "Motor Gasoline Average, Through Company Outlets," for additional motor gasoline prices.

1983-1985: Energy Information Administration, Petroleum Marketing Annual 1985, Volume 1, Table 25, column titled, "Sales to End Users, Sales Through Company-Operated Retail Outlets."

1970-1985: Crop Reporting Board, U.S. Department of Agriculture, Agriculture Prices, tables generally titled, "Motor Supplies: Average Price Paid by Farmers for Motor Fuel" for 1970-1979, and "Diesel Fuel: Average Price Paid by States" for 1980-1985.

1970 forward: Federal Highway Administration, U.S. Department of Transportation, Highway Statistics, Table MF-121T for State tax rates, Table FE101 for Federal tax rates, and Table MF-25 for special fuels consumption data. Table MF-25 is not included in the 1976 volume but is publicly available directly from the Federal Highway Administration.

\section{Consumption}

1970 forward: Energy Information Administration, State Energy Data System, transportation sector distillate consumption.

\section{Conversion Factor: All Years}

5.825 million Btu per barrel

\section{Heavy OII (Utilities)}

For all years, the price of heavy oil consumed at electric utilities is the average cost of No. 6 fuel oil (residual fuel oil) as reported in Cost and Quality of Fuels for Electric Utility Plants. (See Residual Fuel Electric Utility Sector on page 231.) 
Jet fuel prices are estimated for all years for the transportation sector and for 1972 through 1982 for the electric utility sector. After 1982, electric utility use of jet fuel is reported as light oil. (See Light Oil (Utilities) on page 216).

\section{Transportation Sector}

Consumption estimates from SEDS are developed for two types of jet fuel: kerosene type (used primarily by commercial aircraft and accounting for 88 percent of all jet fuel in 1991) and naphtha type (used primarily by military aircraft and accounting for the remaining 12 percent in 1991). Prices are developed for kerosene-type jet fuel and are used. as the price for both types of jet fuel. Taxes are not included in the prices.

\section{Physical Unit Prices: 1985 Forward}

Transportation sector jet fuel prices for 1985 forward are based on data from EIA's Petroleum Marketing Annual (PMA). Annual prices to end users are available for all States in most cases. Prices are converted to dollars per gallon. States without prices are assigned adjacent State or PAD subdistrict prices as shown in Table A18.

\section{Physical Unit Prices: 1983, 1984}

Prices for 1983 and 1984 are based on data from ELA's Petroleum Marketing Monthly (PMM). Monthly jet fuel prices in cents per gallon (excluding taxes) are available from the PMM for the PAD districts and subdistricts for January 1983 through August 1984. The monthly prices for each of the PAD districts or subdistricts (IA, IB, IC, II, III, IV, and V) are assigned to each State within a district whenever the State monthly price is unavailable. State-level data are available from September 1984 through December 1984. Annual prices are the simple averages of the monthly prices and are assigned directly to their respective States. These prices are converted to dollars per gallon. Missing 1984 prices are assigned values as shown in Table A19.

\section{Physical Unit Prices: 1976 Through 1982}

State-level jet fuel prices for 1976 through 1982 are calculated from the Producer Prices and Price Indexes

\begin{tabular}{lll}
\hline State & \multicolumn{1}{c}{ Years } & $\begin{array}{c}\text { PAD District or State } \\
\text { Prices Assigned }\end{array}$ \\
\hline DC & $1985-1988,1990,1991$ & MD \\
DE & 1987 & PAD Subdistrict IB \\
ME & $1985,1990,1991$ & PAD Subdistrict IA \\
NH & 1987 & PAD Subdistrict IA \\
RI & $1985-1988$ & PAD Subdistrict IA \\
VT & $1985-1988,1991$ & PAD Subdistrict IA \\
\hline
\end{tabular}

(PPI) monthly indices for Census divisions and the jet fuel base prices by State for July 1975. The monthly price for each Census division is equal to the PPI monthly index times the jet fuel base price for July 1975 for that Census division. Census division monthly prices are assigned to each State within the Census division, and annual jet fuel prices are computed as simple averages of the monthly State prices.

\section{Physical Unit Prices: 1970 Through 1975}

Jet fuel physical unit State-level prices for the 1970 through 1975 period are based on U.S. annual wholesale prices from the PPI and the relationship of these prices to wholesale kerosene prices reported in Platt's. The U.S. prices are converted to Census division prices which are then assigned directly to States.

Table A19. Jet Fuel Transportation Sector Price Assignments, 1983 and 1984

\begin{tabular}{lll}
\hline State & \multicolumn{1}{c}{ Months } & \multicolumn{1}{c}{$\begin{array}{c}\text { PAD District or State } \\
\text { Prices Assigned }\end{array}$} \\
\hline CT & September & PAD Subdistrict IA \\
DC & September-December & MD \\
ID & September & PAD District IV \\
KS & November, December & PAD District II \\
ME & September-December & PAD Subdistrict IA \\
MS & October & PAD District III \\
ND & December & PAD District II \\
NE & October & PAD District II \\
NH & September, November, & PAD Subdistrict IA \\
& December & \\
NM & October-December & PAD District III \\
OR & November & PAD District V \\
RI & September-December & PAD Subdistrict IA \\
VT & September-December & PAD Subdistrict IA \\
WV & September & PAD Subdistrict IC \\
& & \\
\hline
\end{tabular}


J Preliminary U.S. jet fuel prices from the PPI for 1973 through 1980 are calculated using the annual jet fuel price indices, the jet fuel U.S. base price for July 1975 (0.276 dollars per gallon), and the U.S. index for July 1975 (235.8). The index for 1973 is assumed to be F equal to a simple average of the 11 available monthly $\mathbf{U}$ indices.

The calculated preliminary U.S. jet fuel prices from the PPI are used as the dependent variable in a regression equation for 1973 through 1980 where the wholesale kerosene prices from Platt's are the independent variable. The regression equation is used to estimate U.S. annual jet fuel prices for 1970 through 1972.

Jet fuel prices for Census divisions are estimated using the preliminary U.S. prices derived above for 1970 through 1975 (calculated directly from the PPI data for 1973 through 1975 and estimated for 1970 through 1972). These prices are used as inputs to a regression equation which establishes a linear relationship between preliminary U.S. prices and Census division prices for the years 1970 through 1975. Census division prices are assigned to each State within the Census division.

\section{Btu Prices: All Years}

Btu prices for States are calculated from the physical unit prices and the Btu conversion factor. U.S. Btu prices are calculated as the average of the State Btu prices, weighted by consumption data from SEDS.

\section{Data Sources}

Prices

1985 forward: Energy Information Administration, Petroleum Marketing Annual, Table 21, column titled, "Kerosene-Type Jet Fuel" (1985); and Table 33 (19861988) and Table 35 (1989 forward), column titled, "Kerosene-Type Jet Fuel, Sales to End Users."

1983, 1984: Energy Information Administration, Petroleum Marketing Monthly, Table 14 (January 1984), Table 19 (February-September 1984), and Table 25 (October 1984-January 1985).

1973-1982: Bureau of Labor Statistics, U.S. Department of Labor, Producer Prices and Price Indexes, Supplement, table titled, "Producer price indexes for refined petroleum products by region."
1970-1975: McGraw Hill, Inc., Platt's Oil Price Handbook and Oilmanac, 57th Edition, page 480.

\section{Consumption}

1970 forward: Energy Information Administration, State Energy Data System, transportation sector jet fuel consumption.

\section{Conversion Factor: All Years}

5.670 million Btu per barrel

\section{Electric Utility Sector}

Jet fuel electric utility consumption estimates are from SEDS for 1972 through 1982 only. For 1970 and 1971, no parallel series is available, and for the years after 1982, the series is a part of light oil. All applicable taxes are included in the prices.

\section{Btu Prices: 1975 Through 1982}

For the States that consumed kerosene-type jet fuel at electric utilities during these years, the Btu prices are directly from Cost and Quality of Fuels for Electric Utility Plants (CEQ).

\section{Btu Prices: 1972 Through 1974}

Because $C \& Q$ prices are not available for 1972 through 1974, prices are estimated from CEQ prices for 1975 and 1976 and Agricultural Prices data for 1972 through 1976.

1. Simple annual averages of Agricultural Prices quarterly values are calculated for 1972 through 1976. New England Census Division prices are assigned to CT, MA, ME, NH, RI, and VT.

2. The average annual prices based on Agricultural Prices values for 1975 and 1976 are used as the independent variables in a regression where the dependent variables are State-level prices based on CEQ prices for 1975 and 1976.

3. State-level price estimates for 1972 through 1974 are derived from the results of the regression analysis and the Agricultural Prices values for 1972 through 1974. 


\section{U.S. Btu Prices: All Years}

U.S. Btu prices are calculated as the average of the State Btu prices, weighted by consumption data from SEDS.

\section{Data Sources}

\section{Prices}

1975-1982: Energy Information Administration, Cost and Quality of Fuels for Electric Utility Plants, Tables 6 and 13 (1975), Table 13 (1976-1979), and Table 47 (1980-1982).

1972-1976: Crop Reporting Board, U.S. Department of Agriculture, Agriculture Prices, table titled, "Household Supplies: Average Prices Paid by Farmers for Lawn Mowers and Petroleum Products."

\section{Consumption}

1972-1982: Energy Information Administration, State Energy Data System, electric utility sector kerosenetype jet fuel consumption.

\section{Conversion Factors: All Years}

Because Btu prices are available directly from the data sources, no conversion factors are used.

\section{Kerosene}

Kerosene prices are developed for the residential and industrial sectors, and the industrial sector prices are assigned to the commercial sector. Estimates of the amount of kerosene consumed by the residential, commercial, and industrial sectors are from State Energy Data System (SEDS).

\section{Residential Sector}

\section{Physical Unit Prices: 1985 Forward}

In July 1985, the Bureau of Labor Statistics ceased publication of the Producer Prices and Price Indexes (PPI) data series that is used to estimate State Energy Price and Expenditure Data System residential kerosene prices for earlier years. An alternative approach is therefore applied that maintains continuity with the previously published prices. This approach is used to estimate physical unit prices for all States with residential kerosene consumption.

The approach used to estimate residential kerosene prices for 1985 forward is based on the fact that average U.S. kerosene and distillate fuel prices from 1980 through 1984 are highly correlated. Residential kerosene prices for 1985 forward are estimated by. applying the percentage changes in residential distillate fuel prices for each year to the residential kerosene prices for the previous year in each State. Taxes are included in the distillate fuel oil prices and are, therefore, reflected in the kerosene price estimates.

\section{Physical Unit Prices: 1977 Through 1984}

Because Agricultural Prices kerosene price data are not available after 1976, monthly Census division PPI prices and price indices are used as the basis for the residential kerosene series from 1977 through 1984. To maintain consistency in the series, the PPI prices are multiplied by an adjustment factor that accounts for the relationship between PPI and Agricultural Prices data for quarters in which the two series overlap. In the description of computational procedures below, the adjustment factor is derived first, the PPI prices for 1977 through 1984 are estimated, and the final kerosene physical unit and Btu prices for States are calculated. The final residential sector kerosene prices approximate the average prices paid by farmers. Taxes are included in the source data from Agricultural Prices and are, therefore, reflected in the final price estimates.

The first step is to compute the adjustment factor relating PPI and Agricultural Prices data.

1. Monthly PPI prices for the 18 months covered from July 1975 through December 1976 are calculated from the July 1975 base prices and monthly indices for Census divisions.

2. The calculated Census division monthly prices are assigned to each State within the respective Census division.

3. Volume-weighted quarterly PPI-based prices for States are calculated using the monthly volume weights developed from Retail Sales and Inventories sales data for "other distillate fuel oil."

4. The adjustment factor relating $P P I$ and Agricultural Prices data is calculated as the simple average of the ratios of the quarterly kerosene 
price by State from Agricultural Prices to the calculated quarterly PPI -based kerosene prices by State.

The next step is the calculation of monthly State-level prices from PPI kerosene Census division data for 1977 through 1984.

1. Monthly Census division PPI prices are calculated using the July 1975 base prices and the monthly price indices for 1977 through 1984. The missing monthly indices for February, June, July, and October 1980 for the East South Central Division are assumed to be equal to the index for the preceding month.

2. Census division monthly prices are assigned to each State within the respective Census division.

The next step is the calculation of annual physical unit State prices.

1. Annual PPI-based physical unit prices for States are computed from the monthly PPI prices and the monthly consumption weights.

2. Final residential kerosene prices for States are estimated as the product of the annual PPI-based State price and the adjustment factor calculated above.

\section{Physical Unit Prices: 1970 Through 1976}

Physical unit prices for States are calculated from quarterly price data in Agricultural Prices and consumption weights derived from Retail Sales and Inventories of Fuel Oil. Taxes are included in the source data.

The quarterly physical unit price data from Agricultural Prices for 1970 through 1976 are published in several different forms. The first step in the calculation of prices for these years is to organize the published Agricultural Prices data into a consistent form.

1. For 1971 through 1973 , no quarterly prices are available for CT, MA, ME, NH, RI, and VT. Each of these States is assigned the quarterly prices reported for the New England Census Division.

2. For 1973 , combined $M D / D E$ quarterly prices are reported instead of separate State prices. For this year, the combined prices are assigned to both States.
3. No prices are reported for $A K, D C / 1970$ through 1976. Quarterly weighted Census division prices are assigned to $\mathrm{AK}$, and $\mathrm{MD}$ prices are assigned to $\mathrm{DC}$ for all 7 years.

In order to weight the quarterly prices from Agricultural Prices into annual State prices, monthly quantity weights are calculated from Retail Sales and Inventories of Fuel Oil. This assumes that the "other distillate oil" consumption data by PAD districts is kerosene.

1. Monthly weights are computed using simple averaging of all available "other distillate oil" sales data for each month for each PAD district. Since data are available from November 1978 to March 1981, some months have averages based on three data points while others are based on one or two data points. For example, the average weight for March is the simple average of the 1979, 1980, and 1981 March volumes published in Retail Sales and Inventories of Fuel Oil.

2. Each month's share of average annual sales is calculated by PAD district from the average monthly sales figures. These shares, which become the monthly weights, are then assigned to each State within its respective district.

Final State annual kerosene physical unit prices are calculated as the weighted average of the Agricultural Prices quarterly prices. The monthly weights (shares) are converted to quarterly weights by summing the shares for months within a particular quarter. These same weights are used with the Statelevel price data for each year from 1970 to 1976.

\section{Alaska Btu Prices: 1970 Through 1979, 1983, 1984}

Kerosene residential prices for AK are estimated based on the assumption that the ratio of AK-to-U.S. kerosene residential prices is the same as the ratio of AK-to-U.S. distillate fuel residential prices.

\section{Btu Prices: All Years}

Btu prices for States are calculated from the physical unit prices and the conversion factor. U.S. Btu prices are calculated as the average of the State Btu prices, weighted by consumption data from SEDS. 
Prices

1985 forward: Residential sector distillate fuel price estimates (in physical units) from the State Energy Price and Expenditure Data System.

1975-1984: Bureau of Labor Statistics, U.S. Department of Labor, Producer Prices and Price Indexes, Supplement, table titled, "Producer price indexes for refined petroleum products by region."

1978-1981: Energy Information Administration, $R e-$ tail Sales and Inventories of Fuel Oil, Table 2.

1970-1976: Crop Reporting Board, U.S. Department of Agriculture, Agricultural Prices, table titled, "Household Supplies: Average Price Paid by Farmers for Lawn Mowers and Petroleum Products."

\section{Consumption}

1970 forward: Energy Information Administration, State Energy Data System, residential sector kerosene consumption.

\section{Conversion Factor: All Years}

5.670 million Btu per barrel

\section{Commercial Sector}

State prices from the industrial sector are assigned to the commercial sector. Although there is no industrial consumption of kerosene in DC in 1989 and 1990 an industrial price is calculated to be assigned to the commercial sector consumption.

\section{Industrial Sector}

The industrial sector kerosene prices are based on wholesale price and price index data and on the industrial sector distillate prices. The procedures vary slightly for 1985 forward, 1975 through 1984, and 1970 through 1974. In all years, physical unit prices are calculated first, then Btu prices are computed using the physical unit prices and the conversion factor. Prices approximate an average kerosene price for the manufacturing sector. Taxes are included in the distillate fuel oil prices and are, therefore, reflected in the kerosene price estimates.
The discontinuation of the published PPI data from the Bureau of Labor Statistics affected the estimation of industrial sector kerosene prices. To estimate prices for 1985 forward, the percentage change in industrial sector distillate fuel prices for each year is applied to the industrial sector kerosene prices for the previous year on a State-by-State basis.

\section{Physical Unit Prices: 1975 Through 1984}

Physical unit industrial kerosene prices for 1975 through 1984 are estimated from PPI base prices and indices for kerosene and No. 2 distillate oil, and from the industrial sector distillate prices in physical units. The ratio of $P P I$ kerosene prices to $P P I$ distillate prices is used as an adjustment factor to estimate kerosene prices.

Annual wholesale prices are calculated from PPI annual indices for kerosene and No. 2 distillate fuel oil and their respective July 1975 base prices for Census divisions: Annual average distillate price indices for 1976 are estimated as the simple average of monthly indices. Census division prices for both kerosene and fuel oil No. 2 are assigned to each State within the respective Census divisions. The industrial sector physical unit kerosene prices for States are computed using the distillate industrial physical unit prices and the ratio of PPI kerosene prices to PPI fuel oil No. 2 prices.

\section{Physical Unit Prices: 1970 Through 1974}

Physical unit State-level for 1970 through 1974 are estimated from the distillate industrial prices and the average ratio of kerosene to distillate prices from $P P I$ for 1975 through 1978. The average annual wholesale price ratio between kerosene and fuel oil No. 2 (distillate) is calculated from PPI-based data for the years 1975 through 1978. State-level kerosene industrial physical unit prices are calculated as the product of the ratios and the industrial sector distillate prices for 1970 through 1974.

\section{Btu Prices: All Years}

Btu prices for States are computed by converting the physical unit prices in dollars per gallon to dollars per barrel (42 gallons per barrel) and, then, to dollars per million Btu (5.670 million Btu per barrel). U.S. Btu prices are calculated as the average of the State 
L Btu prices, weighted by consumption data from SEDS.

\section{Data Sources}

Prices

1970 forward: Industrial sector distillate fuel price estimates (in physical units) from the State Energy Price and Expenditure Data System.

1975-1985: Bureau of Labor Statistics, U.S. Department of Labor, Producer Prices and Price Indexes, Supplement, table titled "Producer price indexes for refined petroleum products by region."

\section{Consumption}

1970 forward: Energy Information Administration, State Energy Data System, industrial sector kerosene consumption.

\section{Conversion Factor: All Years}

5.670 million Btu per barrel

\section{Light Oil (Utilities)}

Beginning with 1980, the price of light oil consumed at electric utilities is the average delivered cost of No. 2 fuel oil as reported in Cost and Quality of Fuels for Electric Utility Plants. For earlier years, the price is the consumption-weighted average of the kerosenetype jet fuel price and No. 2 fuel oil. (See also Distillate Fuel Electric Utility Sector on page 205 and Jet Fuel Electric Utility Sector on page 212).

\section{Liquefied Petroleum Gases}

Liquefied petroleum gases (LPG) prices are developed for the residential and the industrial sectors. State prices for the commercial and transportation sectors are assigned from the industrial sector. Estimates of the amount of LPG consumed by sector are from the State Energy Data System (SEDS), and are adjusted for process fuel and intermediate product consumption in the industrial sector. (See the discussion under "Consumption Adjustments for Process Fuel-and Intermediate Products" on page 251.)

\section{Residential Sector}

Residential sector prices are the average delivered prices of LPG to residential consumers in areas where natural gas is available as a competing fuel. Taxes are included in the prices. For 1973 forward, Btu prices are reported in the data source. For 1970 through 1972, physical unit prices are calculated first and Btu prices are calculated from the physical unit prices and the conversion factors. Prices for $\mathrm{AK}$ and $\mathrm{HI}$ in all years are estimated by a different methodology described in a separate section on page 218.

\section{Btu Prices: 1991}

Propane prices from the American Gas Association (AGA) are not available for 1991. Propane prices from the EIA Petroleum Marketing Annual (PMA) are used to calculate the percentage change in propane prices between 1990 and 1991 for each PAD district or subdistrict. These percentages are applied to the 1990 State residential LPG prices from the State Energy Price and Expenditure Data System (SEPEDS) to estimate 1991 prices for the contiguous 48 States and the District of Columbia. Prices for LPG in AK and $\mathrm{HI}$ are developed using the methodology described on page 218.

In 1990, prices for PAD Subdistricts IA and IB and PAD District $V$ are unavailable, and in 1991, prices for PAD Subdistrict IA and PAD District V are unavailable. To estimate the missing PAD district prices, a ratio of the end-users price to the resale price for propane published in the PMA is calculated and applied to the known resale price for the PAD districts and subdistricts that do not have an endusers price. For 1990, the PAD District I end-users-to-resale ratio is multiplied by the PAD Subdistricts IA and IB resale prices to estimate an end-users price for those Subdistricts. For 1991, the PAD Subdistrict IB end-users-to-resale ratio is multiplied by the PAD Subdistrict IA resale prices to estimate an end-users price. For both years, the U.S. end-users-to-resale price ratio is applied to the PAD District $\mathrm{V}$ resale price to estimate a PAD District $\mathrm{V}$ end-users price.

\section{Btu Prices: 1973 Through 1990}

Propane prices by city are reported by the American Gas Association (AGA) directly in dollars per million Btu including taxes. The simple average of available city prices is used as the State annual average. Outlier prices are omitted from consideration for Arkansas Louisiana Gas for AR/1989; City of Long 
Beach for CA/1989, 1990; Public Service Electric \& Gas for NJ/1989; Central Hudson Gas and Electric for NY/1979 through 1981; Orange \& Rockland Utilities for NY/1989, 1990; Pike County Light \& Power for PA/1989, 1990; and Northwestern Public Service for $\mathrm{SD} / 1989$.

To estimate missing prices (other than $\mathrm{AK}$ and $\mathrm{HI}$, which are described in a separate section that follows), simple averages of adjacent States' prices are used as shown in Table A20. Estimated data for one State are not used to estimate prices for another State.

\section{Physical Unit Prices: 1971, 1972}

Physical unit residential LPG prices are based on the city-level propane prices reported by AGA in cents per gallon. Prices for missing States are estimated. The AGA prices are the average delivered prices for propane purchased by residential consumers as of December 31.

1. City-level propane prices from AGA are assigned to their respective States. The AL/1971 price for the Phoenix City Utilities System is omitted as an outlier.

2. Physical unit prices for a State are calculated directly from the available city/utility price observations reported by AGA. Final physical unit prices are equal to the simple average of the price observations for each State.

3. MD prices are assigned for missing DC prices. AK and $\mathrm{HI}$ prices are discussed in a separate section that follows.

\section{Physical Unit Prices: 1970}

Since AGA did not publish LPG prices prior to 1971, the residential sector LPG prices for 1970 are estimated. To maintain continuity with the AGA prices for 1971 forward, prices for 1970 are estimated using simple regression analysis. The relationship between AGA data for 1971 and 1972 and corresponding Agricultural Prices data is the basis for the estimation.

1. Before regression analysis can be applied, Agricultural Prices data for 1970 through 1972 are prepared for 49 States (no AK or HI prices are available). These prices include taxes. Development of $\mathrm{AK}$ and $\mathrm{HI}$ prices are described in a separate section on page 218.
Table A20. LPG Residential Sector

Price Assignments, 1973 Forward

\begin{tabular}{lll}
\hline State & \multicolumn{1}{c}{ Years } & $\begin{array}{l}\text { State Prices Used } \\
\text { in the Estimation }\end{array}$ \\
\hline AR & 1977 & MO, MS, OK, TN, TX \\
CT & 1990 & MA, NY, RI \\
DC & $1973-1983,1990$ & MD \\
DE & 1976,1984 & MD, NJ, PA \\
ID & 1977 & MT, NV, OR, UT, WA, WY \\
LA & 1977 & MS, TX \\
ME & $1973-1977,1985$, & MA, NH, VT \\
& 1986 & \\
MO & 1986 & IA, IL, KS \\
ND & 1973 & MN, MT, SD \\
NM & 1987,1988 & AZ, CO, UT \\
NV & 1973,1975 & AZ, CA, ID, OR, UT, WY \\
OR & 1976 & CA, ID, NV, WA \\
SD & 1986 & MN, MT, ND \\
UT & $1974,1978,1985$ & AZ, CO, ID, NV, WY \\
VT & 1979 & MA, NH, NY \\
& & \\
\hline
\end{tabular}

a. State-level prices for small purchases, representing residential end users, for 1970 through 1972 are published by Agricultural Prices in cents per pound. When price per pound data are not available, price per gallon data, representing larger volume purchases, are used. These prices per gallon are multiplied by 0.543 , the average ratio of price per pound to price per gallon for the United States for 1970 through 1972, to create uniform input data in price per pound.

b. For 1971 and 1972, the price reported for the New England Region is assigned to CT, MA, $\mathrm{ME}, \mathrm{NH}, \mathrm{RI}$, and VT.

c. Data in cents per pound are converted to dollars per gallon by multiplying by the propane conversion factor of 4.2 pounds per gallon, from the Petroleum Products Handbook and dividing by 100 .

Table A21. LPG Residential Agricultural Prices Assigned to Estimate 1970 Prices

\begin{tabular}{lll}
\hline State & Years & State Prices Used \\
\hline DC & $1970-1972$ & MD \\
NV & 1970,1971 & AZ, CA, ID, UT \\
OR & $1971-1972$ & CA, ID \\
UT & 1972 & AZ, CO, ID, NV, WY \\
WA & $1970-1972$ & CA, ID \\
\hline
\end{tabular}

$$
\text { L }
$$


d. Missing prices use adjacent States' average prices as shown in Table A21.

2. The physical unit AGA prices and Agricultural Prices data for 1971 through 1972 (excluding AK and $\mathrm{HI}$ ) are used with simple regression analysis to estimate final physical unit LPG residential prices.

\section{Alaska and Hawaii Prices: All Years}

Prices cannot be estimated for AK and HI using adjacent State price assignments. Missing prices for these two States are estimated by computing ratios of the AK or HI prices to the U.S. prices from the AGA for years when AK or HI data are available and applying these ratios to the U.S. prices in years where prices need to be estimated.

1. AGA prices for $A K$ are available in 1972 and 1980. The 1972 AK-to-US ratio is used to estimate prices for 1970, 1971, and 1973 through 1979. The 1980 AK-to-US price ratio is used to estimate prices for 1981 through 1991.

2. AGA prices for $H I$ are available in 1971,1977 through 1979, and 1989. The 1971 HI-to-US AGA is used to estimate prices for 1970 and 1972 through 1974. The average ratio of the HI-to-US prices for 1977 through 1979 is used to estimate prices for 1975, 1976, and 1980 through 1984. The 1989 HI-to-US ratio is used to estimate prices for 1985 through 1988, 1990, and 1991.

\section{Btu Prices: All Years}

For 1973 forward, Btu prices for States are directly from the data sources. For 1970 through 1972, Btu prices for States are calculated by converting the physical unit prices by using the factors cited in Table A25 on page 221. U.S. Btu prices are calculated as the average of the State Btu prices, weighted by consumption data from SEDS.

\section{Data Sources}

Prices

1991: Energy Information Administration, State Energy Price and Expenditure Data System, 1990 residential sector LPG prices.
1991: Energy Information Administration, Petroleum Marketing Annual, Table 35 (1990 and 1991), columns titled, "Propane (Consumer Grade)."

1971 through 1990: American Gas Association (AGA), Gas Househeating Survey (1971-1988), Residential Gas Market Survey (1989 and 1990) Appendix 2, "Competitive Fuel Prices."

1970-1972: Crop Reporting Board, U.S. Department of Agriculture, Agricultural Prices, table titled, "Average Price Paid by Farmers for Lawn Mowers and Petroleum Products, Specified Dates, by State," column titled, "L.P. Gas."

\section{Consumption}

1970 forward: Energy Information Administration, State Energy Data System, residential sector LPG consumption.

\section{Conversion Factors}

1970-1972: Energy Information Administration, State Energy Data Report 1991, Consumption Estimates, Table D1, as shown in Table A25 on page 221.

1970-1972: 4.2 pounds per gallon from Guthrie, Virgil, ed., 1960. Petroleum Products Handbook. John Wiley and Sons, Inc., New York, New York: pages 35.

Conversion factors are not necessary for other years because Btu prices are available directly from the data sources.

\section{Commercial Sector}

State prices from the industrial sector are assigned to the commercial sector.

\section{Industrial Sector}

The industrial sector LPG prices are estimated from average LPG prices to manufacturing firms, average LPG prices to farmers, and refiner and gas plant operator sales to end users. Prices for 1985 forward are from the Petroleum Marketing Annual (PMA). Prices for 1978 through 1981 are from the Annual Survey of Manufactures (ASM) or the Census of Manufactures (CM), and prices for 1970 through 1977 and 1982 through 1984 are derived from Agricultural Prices and scaled to the $A S M / C M$ prices using the ratio of 
ASM/CM to Agricultural Prices LPG prices for the years 1978 through 1981, when both price series were available. Taxes are included in the 1970 through 1984 estimates but are not included in the prices for 1985 forward.

\section{Physical Unit Prices: 1985 Forward}

Industrial sector LPG physical unit State prices for 1985 forward are estimated using physical unit annual prices in the Petroleum Marketing Annual (PMA). Where prices are not available, the PAD district price is assigned as shown in Table A22.

When a PAD district price is not available, a consumption-weighted price is calculated using available prices for States within the district and the SEDS industrial sector consumption for the States. PAD district price calculations are made for PAD District $V$ for 1985 using $A K, C A, O R$, and WA consumption-weighted prices, for PAD Subdistrict IA for 1986 using CT and NH consumption-weighted prices, and for PAD Subdistrict IA for 1987 through 1988 using CT and MA consumption-weighted prices.

When a PAD district price is not available and there are no State data within the PAD district to develop a consumption-weighted average, a different methodology is used. In 1990, prices for PAD Subdistricts IA and IB and PAD District V are unavailable while no State data are available to create consumptionweighted estimates. In 1991, prices for PAD Subdistrict IA and PAD District V are unavailable. To estimate the missing PAD district prices, a ratio of the end-users price to the resale price for propane published in the PMA is calculated and applied to the known resale price for the PAD districts and subdistricts that do not have an end-users price. For 1990, the PAD District I end-users-to-resale ratio is multiplied by the PAD Subdistricts IA and IB resale prices to estimate an end-users price for those Subdistricts. For 1991, the PAD Subdistrict IB end-users-to-resale ratio is multiplied by the PAD Subdistrict IA resale prices to estimate an end-users price. For both years, the U.S. end-users-to-resale price ratio is applied to the PAD District V resale price to estimate a PAD District V end-users price.

\section{Physical Unit Prices: 1982 Through 1984, 1970 Through 1977}

Industrial sector LPG physical unit prices for 1982 through 1984 and 1970 through 1977 are estimated based on the relationship between State-level LPG
Table A22. LPG Industrial Sector PAD District Price Assignments, 1985 Forward

\begin{tabular}{|c|c|}
\hline State & Years \\
\hline AK & $1986-1988,1990,1991$ \\
\hline$A L$ & $1985-1988$ \\
\hline$A Z$ & $1985-1991$ \\
\hline $\mathrm{CA}$ & 1990,1991 \\
\hline $\mathrm{CO}$ & 1991 \\
\hline CT & 1990,1991 \\
\hline DC & $1985-1991$ \\
\hline $\mathrm{DE}$ & $1986-1991$ \\
\hline $\mathrm{FL}$ & 1990,1991 \\
\hline GA & $1985,1990,1991$ \\
\hline $\mathrm{HI}$ & $1985-1991$ \\
\hline IA & 1986, 1991. \\
\hline ID & $1986,1990,1991$ \\
\hline IN & 1990 \\
\hline KS & $1986-1989$ \\
\hline MA & 1986, 1990, 1991 \\
\hline MD & $1988,1990,1991$ \\
\hline ME & $1986-1991$ \\
\hline $\mathrm{MI}$ & $1985-1988,1990$ \\
\hline MN & $1985,1986,1988-1991$ \\
\hline MS & 1990,1991 \\
\hline MT & 1990,1991 \\
\hline NC & 1991 \\
\hline ND & $1985,1986,1991$ \\
\hline NE & $1986-1991$ \\
\hline $\mathrm{NH}$ & $1987-1991$ \\
\hline NV & $1985-1988,1990,1991$ \\
\hline NY & 1990,1991 \\
\hline $\mathrm{OH}$ & 1990 \\
\hline OK & 1986,1987 \\
\hline OR & $1986,1990,1991$ \\
\hline PA & 1990,1991 \\
\hline $\mathrm{Rl}^{\circ}$ & $1986-1991$ \\
\hline SD & $1985-1991$ \\
\hline TN & 1990,1991 \\
\hline UT & $1986-1988,1990,1991$ \\
\hline$V T$ & $1986-1991$ \\
\hline WA & $1986-1991$ \\
\hline WI & $1985,1986,1990$ \\
\hline WV & $1989-1991$ \\
\hline WY & 1987,1988 \\
\hline
\end{tabular}

prices from Agricultural Prices and the prices calculated from Annual Survey of Manufactures (ASM) or Census of Manufactures (CM) for 1978 through 1981.

1. Before the adjustment factor relating Agricultural Prices and $A S M / C M$ data is computed, monthly Agricultural Prices data are converted into annual prices and missing data are estimated.

a. Annual LPG prices are calculated as simple averages of the monthly prices from Agricul- 


\begin{tabular}{|c|c|c|}
\hline & Price As & $\begin{array}{l}\text { Sector } \\
\text { ents, 1970-1976 }\end{array}$ \\
\hline State & Years & $\begin{array}{l}\text { State Prices Used } \\
\text { in the Estimation }\end{array}$ \\
\hline CT & 1974 & NY \\
\hline MA & 1974 & NY \\
\hline ME & 1974 & NY \\
\hline NH & 1974 & NY \\
\hline NV & $1970-1971$ & $A Z, C A, I D, U T$ \\
\hline & $1973-1974$ & $A Z, C A, I D$ \\
\hline OR & $1970-1974$ & CA, ID \\
\hline RI & 1974 & NY \\
\hline & $1975-1976$ & $\mathrm{CT}, \mathrm{MA}, \mathrm{NY}$ \\
\hline UT & 1972 & $A Z, C O, I D, N V, W Y$ \\
\hline & $1973-1974$ & $A Z, C O, I D, W Y$ \\
\hline VT & 1974 & NY \\
\hline WA & $1970-1974$ & CA, ID \\
\hline
\end{tabular}

tural Prices for the years 1977 through 1984. The only States missing data are WV/1977 through 1981 and AK, DC, HI/1977 through 1984. WV is assigned the simple average of the $\mathrm{KY}, \mathrm{MD}, \mathrm{OH}, \mathrm{PA}$, and VA prices. $\mathrm{AK}, \mathrm{DC}$, and $\mathrm{HI}$ prices are discussed below.

b. The average ratio of $A S M / C M$-based final prices for 1978 through 1981 and the 1978 through 1981 Agricultural Prices annual prices is calculated for 48 States (excluding $A K, D C$, and $\mathrm{HI}$ ) as the simple average of the ratio over the 4 years. This average ratio is used as an adjustment factor.

2. Final industrial sector LPG prices for 1982 through 1984 and 1970 through 1977 are estimated using the State-level adjustment factors and annual average LPG prices from Agricultural Prices for these years.

a. Annual average LPG prices are calculated for 1982 through 1984 and 1970 through 1977 as the simple average of the monthly prices.

b. Agricultural Prices published annual average prices in dollars per gallon for all States in 1975 and 1976. For DE/1970 through 1974, MD/1970 through 1974, VA/1970 through 1974 , and WV/1970 through 1972 , only prices for small volume purchases in cents per pound were published. These are converted to cents per gallon by multiplying by 1.96 , the average ratio of cents per gallon to cents per pound for the United States for 1970 through 1974. c. For 1970 through 1972, Agricultural Prices are converted from cents per gallon to dollars per gallon.

d. For 1971 through 1973, the New England price per gallon reported by Agricultural Prices is assigned to CT, MA, ME, NH, RI, and VT.

e. MD prices are assigned to $D C / 1970$ through 1972, 1974 through 1977, and 1982 through 1984. The combined MD/DE/1973 price is assigned to $\mathrm{MD}, \mathrm{DE}$, and $\mathrm{DC}$.

f. Excluding $A K$ and HI, States missing Agricultural Prices LPG prices are assigned the simple average price of adjacent States. The States with missing data and the adjacent State assignments are shown in Table A23.

g. Industrial sector LPG physical unit prices for 1970 through 1977 and 1982 through 1984 for all States (except AK, DC, and HI) are calculated using the estimated annual Agricultural Prices data for the respective year and the State-level average ratios as adjustment factors.

3. AK/1970 through 1977, 1982 through 1984 and HI/1970 through 1977, 1982 through 1984 prices are estimated by using the relationship between $A S M / C M$ based prices for these States and the U.S. price reported by Agricultural Prices (1979 through 1981 for AK and 1978 through 1981 for $\mathrm{HI})$. The average ratio for the available years for the two States is calculated and used with the Agricultural Prices U.S. prices for the years to be estimated.

\section{Physical Unit Prices: 1978 Through 1981}

For 1978 through 1981, the industrial sector LPG prices are either calculated directly from cost and quantity data from the $A S M$ or the $C M$ or are estimated using the relationship of $A S M / C M$ data to LPG price data from Agricultural Prices.

1. For 1978 through 1981, industrial sector physical unit prices for LPG are calculated as the average cost per unit from cost and quantity data published in $A S M / C M$. Since sales are reported in pounds, the prices are converted to dollars per gallon. The conversion factor of 4.5 pounds per gallon is from $A S M / C M$.

2. The AK price for 1978 is the consumptionweighted average Census division price. In 
Table A24. LPG Industrial Sector

Price Assignments, 1978-1981

\begin{tabular}{lll}
\hline State & \multicolumn{1}{c}{ Years } & \multicolumn{1}{c}{ State Prices Used } \\
\hline AR & 1978 & LA, MO, MS, OK, TX \\
DC & $1978-1981$ & MD \\
LA & 1980 & AR, MS, TX \\
NM & $1979-1981$ & AZ, CO, OK, TX \\
WY & $1978-1981$ & CO, ID, MT, ND, NE, SD, UT
\end{tabular}

addition, four States have prices estimated as the simple average of the prices of adjacent States, and $D C$ is assigned the MD price as shown in Table A24.

\section{Btu Prices: All Years}

Btu prices for States and the United States are calculated from the physical unit prices and the conversion factors shown in Table A25. U.S. Btu prices are calculated as the average of the State Btu prices, weighted by consumption data from SEDS, adjusted for process fuel and intermediate product consumption.

\section{Data Sources}

Prices

1985 forward: Energy Information Administration, Petroleum Marketing Annual, Table 21 (1985), Table 33 (1986-1988), and Table 35 (1989 forward), columns titled "Propane (Consumer Grade)."

1970-1984: Crop Reporting Board, U.S. Department of Agriculture, Agricultural Prices, tables titled, "Average Price Paid by Farmers for Lawn Mowers and Petroleum Products, Specified Dates, by State," column titled, "L.P. Gas," (1970-1976); "Household Supplies: Average Price by Farmers" (1977-1979); "L.P. Gas: Average Price Paid by States" (1980); and "L.P. Gas: Average Price Paid by Months by States" (1981-1984).

1981: Bureau of the Census, U.S. Department of Commerce, 1982 Census of Manufactures, Fuels and Electric Energy Consumed, Part 2, States and Standard Metropolitan Statistical Areas by Major Industry Groups, Table 3, State-level quantity and cost of liquefied petroleum gases.
Table A25. LPG Btu Conversion Factors 1970 Forward

\begin{tabular}{cccc}
\hline Year & $\begin{array}{c}\text { Conversion } \\
\text { Factor }\end{array}$ & Year & $\begin{array}{c}\text { Conversion } \\
\text { Factor }\end{array}$ \\
\hline 1970 & 3.779 & 1981 & 3.643 \\
1971 & 3.772 & 1982 & 3.615 \\
1972 & 3.760 & 1983 & 3.614 \\
1973 & 3.746 & 1984 & 3.599 \\
1974 & 3.730 & 1985 & 3.603 \\
1975 & 3.715 & 1986 & 3.640 \\
1976 & 3.711 & 1987 & 3.659 \\
1977 & 3.677 & 1988 & 3.652 \\
1978 & 3.669 & 1989 & 3.683 \\
1979 & 3.680 & 1990 & 3.625 \\
1980 & 3.674 & 1991 & 3.614 \\
& & & \\
\hline
\end{tabular}

1978-1980: Bureau of the Census, U.S. Department of Commerce, Annual Survey of Manufactures, Fuels and Electric Energy Consumed, States by Industry Group and Standard Metropolitan Statistical Areas by Major Industry Group, Table 3, State-level quantity and cost of liquefied petroleum gases.

\section{Consumption}

1970 forward: Energy Information Administration, State Energy Data System, industrial sector LPG consumption.

\section{Conversion Factors}

1970 forward: Energy Information Administration, State Energy Data Report 1991, Consumption Estimates, Table D1, as shown in Table A25.

1978-1981: 4.5 pounds per gallon from Annual Survey of Manufactures, Appendix C.

\section{Transportation Sector}

State prices from the industrial sector are assigned to the transportation sector.

\section{Lubricants}

Lubricant prices are developed for the industrial sector and assigned to the transportation sector. State-level prices are not available for either sector; 
national-level prices are assigned to all States and do not include end-user taxes paid at the time of sale. Estimates of lubricant consumption by the industrial and transportation sectors are from the State Energy Data System (SEDS).

\section{Physical Unit Prices: All Years}

Prices of lubricants are estimated from three product categories:

1. Lubricating oils made in refineries (SIC 29117.21) and not made in refineries (SIC 29920.21).

2. Lubricating greases made in refineries (SIC 29117.31) and not made in refineries (SIC 29920.31).

3. Lubricating oils and greases, not specifically known (n.s.k.), made in refineries (SIC 29117.00) and not made in refineries (SIC 29920.00 for establishments with 10 employees or more and SIC 29920.02 for establishments with fewer than 10 employees).

For years where Census of Manufactures (CM) data are available (1967, 1972, 1977, and 1982), total shipments are calculated by adding the shipments for the three product categories. Shipments for the third product category are estimated for 29920.00 and 29920.02 by dividing their value of shipments sum by the weighted average cost of the product categories 29920.21 and 29920.31 .

Total shipments in each year for which $C M$ data are available is divided by the estimated SEDS total lubricants consumption (in physical units) for that year to establish a shipments-to-consumption ratio. Ratios for the years not covered by the $C M$ (i.e., 1968 through 1971, 1972 through 1976, and 1978 through 1981) are estimated by linear interpolation, and the 1982 value is used for 1983 forward. Total shipments for the years not covered by the $C M$ are estimated by multiplying SEDS consumption by the appropriate shipment-to-consumption ratio.

Estimated shipment prices are calculated by dividing the value of shipments shown in the CM or the $A n$ nual Survey of Manufactures by the estimated shipments for each product category. The shipment prices are used to represent wholesale prices.

End-user prices in dollars per barrel are estimated by multiplying the shipment prices by trade ratio factors that represent the wholesale-to-retail markup. The trade ratio factors are developed from Bureau of
Economic Analysis (BEA) data on computer tapes giving 1972 and 1977 data. For 1972, the sum of data called "purchasers value" for the three product categories is divided by the sum of the "producers value" for the three categories to derive a trade ratio. A similar calculation is made for 1977, but the terms "purchase value" and "basic value" are used in the source data.

The 1972 ratio is used for 1970 through 1972 and the 1977 ratio is used for 1977 forward. The values for 1973 through 1976 are estimated by linear interpolation using the 1972 and 1977 values. The trade ratios for 1982 and later years are not used because the range of petroleum products included in the ratios was expanded by BEA and the ratios no longer represent the specific mark-up for lubricants.

\section{Btu Prices: All Years}

Btu prices are obtained by dividing the prices in dollars per barrel by the conversion factor, 6.065 million Btu per barrel.

\section{Data Sources}

Prices

1970, 1971, 1973-1976, 1978-1981, and 1983 forward: Bureau of the Census, U.S. Department of Commerce, Annual Survey of Manufactures; Lubricating Oils and Greases (Industries 29117 and 29920).

1972, 1977, and 1982: Bureau of the Census, U.S. Department of Commerce, Census of Manufactures, Petroleum Refining; Lubricating Oils and Greases (Industries 29117 and 29920).

1972 and 1977: Bureau of Economic Analysis, U.S. Department of Commerce, Input-Output Table Work Tapes for SIC Codes 29117 and 29920).

\section{Consumption}

1970 forward: Energy Information Administration, State Energy Data System, lubricants consumption.

\section{Conversion Factor: All Years}

6.065 million Btu per barrel 
State-level prices that are not available from the $P M A$ are assigned as listed in Table A26.

Motor gasoline prices are developed for the transportation sector, and the transportation sector prices are assigned to the commercial and industrial sectors. Motor gasoline consumed in privately-owned vehicles is accounted for in the transportation sector. Estimates of motor gasoline consumed by the transportation, commercial, and industrial sectors used in calculating expenditures are from SEDS. Prices in this series are retail prices (usually service station prices) including taxes.

\section{Physical Unit Prices: 1983 Forward}

Motor gasoline physical unit prices for 1983 forward are based on annual State-level prices from the Petroleum Marketing Annual (PMA) except for prices for certain States and years, noted below, that are derived from the Consumer Prices: Energy (CPI). State and Federal motor gasoline taxes are added to the prices from the $P M A$; all taxes are included in the $C P I$ data. In cases where the tax rate is not constant through the year, the months' rates are averaged, weighted by the number of months each rate was in effect. Due to the lack of uniformity in application, State and local general Sales taxes are not included.

The average sales price (excluding taxes) of finished motor gasoline to end users through company outlets is used, under the assumption that this price most closely approximates retail motor gasoline prices. Finished motor gasoline includes leaded and unleaded motor gasoline and gasohol.

Table A26. Motor Gasoline Price Assignments, 1983 Forward

\begin{tabular}{lll}
\hline State & \multicolumn{1}{c}{ Years } & \multicolumn{1}{c}{ Source } \\
\hline AK & $1983-1986$ & CPI \\
CT & $1989-1991$ & PMA, PAD Subdistrict IA \\
DC & $1983-1991$ & $C P I$ \\
DE & 1991 & $P M A$, PAD Subdistrict IB \\
HI & $1983-1986$ & $C P I$ \\
HI & $1987-1990$ & See text \\
MD & $1985-1991$ & CPI (Baltimore) \\
ME & $1985-1988,1990$, & PMA, PAD Subdistrict IA \\
& 1991 & \\
MT & 1991 & PMA, PAD Subdistrict IV \\
SD & 1987,1991 & PMA, PAD District II \\
VT & $1989-1991$ & PMA, PAD Subdistrict IA \\
WY & 1985 & $P M A$, PAD District IV \\
& & \\
\hline
\end{tabular}

Monthly State tax information and Federal tax information are from Highway Statistics. The monthly State taxes are averaged to create an average annual tax for each State which is combined with the Federal tax to adjust the PMA price.

Monthly CPI city prices weighted by monthly consumption from Highway Statistics for AK/1983 through 1986, HI/1983 through 1986, DC/1983 forward, and MD/1985 forward are used to calculate annual prices for those States. CPI prices already include taxes.

Collection of motor gasoline price data for $\mathrm{HI}$ by the Bureau of Labor Statistics stopped in December 1986. The HI/1987 through 1990 prices are derived as follows: the average ratio of the $\mathrm{HI} C P I$ price (with $\mathrm{HI}$ State and Federal taxes subtracted) to the PAD District V price (with HI State and Federal taxes added) for the years 1983 through 1986 is multiplied by the PAD District V price for the current year and then taxes are added to estimate the HI/1987 through 1990 prices.

\section{Physical Unit Prices: 1982}

Monthly physical unit motor gasoline prices for 1982 are from the Platt's Oil Price Handbook and Oilmanac (Platt's) table, "AAA 'Fuel Gauge' Report," the CPI, or both. Table A27 summarizes price data availability by source. The Platt's prices are reported for both leaded and unleaded motor gasoline and for both full service and self service for all States except AK and HI. All available Platt's prices for 1982 are used in the calculation of motor gasoline prices. The continuity of these prices with prices published by Platt's in previous years suggests that taxes are included.

The available CPI monthly physical unit motor gasoline prices for 1982 are for all types of motor gasoline and cover 25 States as shown in Table A28. The CPI prices are assigned to any State that has a county included in the Standard Metropolitan Statistical Area (SMSA) definitions used by the Bureau of Labor Statistics. These "all types" prices cover leaded regular, unleaded regular, and leaded premium, including taxes. All the available CPI prices for 1982 are also used in the calculation of motor gasoline prices. Complete monthly data exist for the 25 States covered by the CPI. The CPI Detailed Report of April 1986 explicitly states that Federal, State, and local taxes are included. 
Table A27. Summary of Motor Gasoline Price Data by Year, 1970-1982

\begin{tabular}{|c|c|c|c|c|}
\hline Years & Source & Grades Covered & $\begin{array}{c}\text { Composite } \\
\text { Price }\end{array}$ & $\begin{array}{l}\text { Missing States } \\
\text { All Sources }\end{array}$ \\
\hline 1982 & $\begin{array}{l}\text { Platt's } \\
\text { CPI }\end{array}$ & $\begin{array}{l}\text { leaded } \\
\text { unleaded } \\
\text { leaded regular } \\
\text { leaded premium } \\
\text { unleaded regular }\end{array}$ & $\begin{array}{l}\text { no } \\
\text { no } \\
\text { yes } \\
\text { yes } \\
\text { yes }\end{array}$ & none \\
\hline $1979-1981$ & $\begin{array}{l}\text { Platt's } \\
\text { CPI }\end{array}$ & $\begin{array}{l}\text { leaded regular } \\
\text { leaded premium } \\
\text { unleaded regular } \\
\text { unleaded premium } \\
\text { leaded regular } \\
\text { leaded premium } \\
\text { unleaded regular }\end{array}$ & $\begin{array}{l}\text { no } \\
\text { no } \\
\text { no } \\
\text { no } \\
\text { yes } \\
\text { yes } \\
\text { yes }\end{array}$ & $\begin{array}{l}\text { AR, DE, ME, MS, MT, ND, NH, } \\
\text { OK, RI, SC, SD, VT, WV, WY }\end{array}$ \\
\hline 1978 & $\begin{array}{l}\text { Platt's } \\
C P I\end{array}$ & $\begin{array}{l}\text { leaded regular } \\
\text { leaded regular } \\
\text { leaded premium } \\
\text { unleaded regular }\end{array}$ & $\begin{array}{l}\text { no } \\
\text { yes } \\
\text { yes } \\
\text { yes }\end{array}$ & none \\
\hline 1976,1977 & $\begin{array}{l}\text { Platt's } \\
\text { CPI }\end{array}$ & $\begin{array}{l}\text { leaded regular } \\
\text { leaded regular } \\
\text { leaded premium } \\
\text { unleaded regular }\end{array}$ & $\begin{array}{l}\text { no } \\
\text { no } \\
\text { no } \\
\text { no }\end{array}$ & AK \\
\hline 1974,1975 & $\begin{array}{l}\text { Platt's } \\
\text { CPI }\end{array}$ & $\begin{array}{l}\text { leaded regular } \\
\text { leaded regular } \\
\text { leaded premium }\end{array}$ & $\begin{array}{l}\text { no } \\
\text { no } \\
\text { no }\end{array}$ & AK \\
\hline $1970-1973$ & Platt's & leaded regular & no & $\mathrm{AK}, \mathrm{HI}$ \\
\hline
\end{tabular}

To combine the product-specific Platt's prices with the "all types" prices published in the CPI, the Platt's prices are weighted into "all types" prices using annual U.S. share data for leaded and unleaded motor gasoline (no breakdowns for regular and premium are possible with current data limitations). The share data come from the Monthly Energy Review (MER).

Motor gasoline price data reported by Platt's for 1982 cover the following months: February, April, June, August, November, and December. The missing 6 months are assigned prices as follows: January is assigned the February price, and the other missing months are assigned the average price of the preceding and succeeding months. A missing February price for $\mathrm{MO}$ is assumed to be equal to the April price, and a missing price for $O R$ is assumed to be equal to the average of the April and August prices.

For States with data from Platt's only, prices by product type (leaded and unleaded) are first calculated as the simple average of full-service and self-service prices for that product for each month and State. The resulting prices are then weighted into monthly composite prices using U.S. leaded and unleaded shares of motor gasoline product supplied from the $M E R$. The following 26 States have data only from Platt's: AL, AR, AZ, CT, DE, IA, ID, LA, ME, MS, MT, NC, ND, NE, NH, NM, NV, OK, RI, SC, SD, TN, UT, VT, WV, and WY.

Platt's reports two prices for each motor gasoline product for each year: one full-service price and one self-service price. These two prices are combined using a simple average into a single product price for each State for each month.

The unleaded U.S. share of total motor gasoline consumption is reported in the $M E R$ as 52.1 percent in 1982. Assuming that the remaining motor gasoline consumption is leaded, the leaded portion of total consumption is 47.9 percent. These shares are used for all States and months to calculate the composite prices from the leaded and unleaded prices. 
Table A28. Motor Gasoline Price Assignments from Consumer Prices: Energy, 1978-1982

\begin{tabular}{ll}
\hline State & City Price Assignments \\
\hline AK & Anchorage \\
CA & Los Angeles-Long Beach-Anaheim, San Diego, \\
& San Francisco, Oakland \\
CO & Denver-Boulder \\
DC & Washington \\
FL & Miami \\
GA & Atlanta \\
HI & Honolulu \\
IL & Chicago-Northwestern Indiana, St. Louis \\
IN & Chicago-Northwestern Indiana, Cincinnati \\
KS & Kansas City \\
KY & Cincinnati \\
MA & Boston \\
MD & Baltimore, Washington \\
MI & Detroit \\
MN & Minneapolis-St. Paul \\
MO & St. Louis, Kansas City \\
NJ & New York-Northeastern NJ, Philadelphia \\
NY & New York-Northeastern NJ, Buffalo \\
OH & Cincinnati, Cleveland \\
OR & Portland \\
PA & Philadelphia, Northeastern PA, Pittsburgh \\
TX & Dallas-Ft. Worth, Houston \\
VA & Washington \\
WA & Seattle-Everett, Portland \\
WI & Milwaukee, Minneapolis-St. Paul \\
& \\
\hline
\end{tabular}

Note: All types of motor gasoline are included.

For AK and $\mathrm{HI}$, the only States with data only from the CPI, the "all types" monthly prices reported are used directly as monthly composite prices.

For States with price data from both Platt's and the $C P I$, the Platt's data are first combined into product type prices and weighted with the MER shares. The resulting combined prices for all motor gasoline types are averaged together with the combined $C P I$ city prices assigned to the respective month and State. The following 23 States have monthly composite prices computed in this way: $C A, C O, D C, F L$, GA, IL, IN, KS, KY, MA, MD, MI, MN, MO, NJ, NY, $\mathrm{OH}, \mathrm{OR}, \mathrm{PA}, \mathrm{TX}, \mathrm{VA}, \mathrm{WA}$, and WI.

1. Leaded and unleaded gasoline prices are calculated as simple averages of full-service and self-service prices from Platt's, and are then weighted into a composite price using $M E R$ shares of leaded and unleaded motor gasoline consumption.
Table A29. Motor Gasoline Price Assignments from Platt's, 1979-1981

\begin{tabular}{ll}
\hline State & City Price Assignments \\
\hline AL & Birmingham \\
AZ & Phoenix, Tucson \\
CA & Bakersfield, Fresno, Los Angeles, Sacramento, \\
& San Diego, San Francisco, Stockton \\
CO & Denver \\
CT & New Haven \\
DC & Washington \\
FL & Miami, Tampa-St. Petersburg \\
GA & Atlanta \\
IA & Des Moines \\
ID & Boise \\
IL & Chicago \\
IN & Indianapolis \\
KY & Louisville \\
LA & New Orleans \\
MA & Boston \\
MD & Baltimore \\
MI & Detroit \\
MN & Minneapolis \\
MO & Kansas City, St. Louis \\
NC & Charlotte \\
NE & Omaha \\
NJ & Newark \\
NM & Albuquerque \\
NV & Las Vegas, Reno \\
NY & Long Island, Rochester \\
OH & Cincinnati \\
OR & Portland \\
PA & Philadelphia, Pittsburgh. \\
TN & Memphis \\
TX & El Paso, Houston \\
UT & Salt Lake City \\
VA & Norfolk \\
WA & Seattle, Spokane \\
WI & Milwaukee \\
& \\
\hline &
\end{tabular}

2. Monthly "all types" motor gasoline prices covering leaded regular, leaded premium, and unleaded regular are directly from the $C P I$. If there is more than one CPI price observation for a month and State, the CPI prices are simple averages.

3. Using a simple average, the composite Platt's prices are combined with the "all types" CPI prices for that State. The resulting prices are the monthly composite prices for 1982 .

Annual physical unit prices for all States are calculated from the monthly motor gasoline prices 
$M$ calculated above and weighted by the monthly motor gasoline consumption volumes for States from Highway Statistics.

\section{Physical Unit Prices: 1979 Through 1981}

For 1979 through 1981, Platt's monthly motor gasoline prices are from a table titled "Platt's/Lundberg Summary." Prices are available for cities by product-type, by grade, and by type of service (full service, self service). Four products and grades of motor gasoline are covered: leaded regular, unleaded regular, leaded premium, and unleaded premium. These data cover 37 States and taxes are included. The CPI reports "all types" prices, including taxes, for the cities listed in Table A28. Platt's city price assignments to States for 1979 through 1981 are shown in Table A29.

The computation of monthly composite prices for 1979 through 1981 varies, depending on the available data sources for each State. Monthly composite prices are estimated for the 14 States which do not have reported price data from either data source. If both Platt's and the CPI report prices for a city, the CPI price is used.

1. For States with city price observations only from Platt's, prices for leaded and unleaded motor gasoline are combined using simple averaging, regardless of the type of service, and converted to dollars per gallon. The leaded and unleaded prices are then weighted together into a monthly composite price. The following 12 States have prices only from Platt's for 1979 through 1981: $A L, A Z, C T, I A, I D, L A, N C, N E, N M, N V, T N$, and UT.

a. The Platt's prices for 1981 end in September of that year; monthly prices by grade and service type for October, November, and December are assumed to be equal to the corresponding September prices.

b. Leaded and unleaded prices are calculated for each State by simple averaging of all prices available for each product (leaded, unleaded), regardless of service type or grade of motor gasoline (regular, premium). All city prices for each State are averaged together.

c. Leaded and unleaded shares of total motor gasoline consumption for the United States are calculated from the $M E R$ for each year 1979 through 1981 . The monthly product type prices are weighted into composite prices using the national leaded and unleaded shares as weights.

2. For States with city price observations only from the CPI, the monthly "all types" prices are used directly for States with only one price observation per month. For States with multiple observations, monthly prices are combined by simple averaging. States with $C P I$ data only are: $\mathrm{AK}, \mathrm{CO}, \mathrm{DC}, \mathrm{GA}, \mathrm{HI}, \mathrm{IL}, \mathrm{KS}, \mathrm{MA}, \mathrm{MD}, \mathrm{MI}, \mathrm{MN}$, $\mathrm{MO}, \mathrm{NJ}, \mathrm{OH}, \mathrm{OR}, \mathrm{PA}$, and WI.

3. For the eight States with price observations from both Platt's and the CPI (CA, FL, IN, KY, NY, TX, VA, and WA), monthly composite prices for 1979 through 1981 are calculated using three steps:

a. The Platt's prices are combined into single "all types" prices as described above, using leaded and unleaded grades of motor gasoline shares as weights.

b. The CPI prices are combined by State.

c. Using simple averaging, the composite Platt's price for each State is combined with the "all types" CPI price for that State. The resulting prices are the monthly composite prices for 1979 through 1981.

4. Fourteen States are not covered by price data from either Platt's or the CPI in 1979 through 1981. These States are AR, DE, ME, MS, MT, ND, NH, OK, RI, SC, SD, VT, WV, and WY. Monthly composite prices for these States are estimated using the monthly State-level composite prices for 1982 and Census region monthly prices from the CPI for 1979 through 1982.

a. The ratio between the 1982 State prices and the 1982 CPI Census region prices corresponding to each State is calculated for use as an adjustment factor in 1979, 1980, and 1981.

b. The monthly price for each of the 14 missing States is assumed to be the product of the 1982 Census region adjustment factor for that State times the monthly motor gasoline price for that Census region from the CPI.

Annual physical unit prices for all States are calculated from the monthly motor gasoline prices calculated above and weighted by the monthly motor gasoline consumption volumes for States from Highway Statistics. 
Table A30. Motor Gasoline Price Assignments from Platt's, 1970-1978

\begin{tabular}{ll}
\hline State & City Price Assignments \\
\hline AL & Birmingham \\
AR & Little Rock \\
AZ & Phoenix \\
CA & Los Angeles, San Francisco \\
CO & Denver \\
CT & Hartford \\
DC & Washington \\
DE & Wilmington \\
FL & Miami \\
GA & Atlanta \\
IA & Des Moines \\
ID & Boise \\
IL & Chicago \\
IN & Indianapolis \\
KS & Wichita \\
KY & Louisville \\
LA & New Orleans \\
MA & Boston \\
MD & Baltimore \\
ME & Portland \\
MI & Detroit \\
MN & Twin Cities \\
MO & St. Louis \\
MS & Jackson \\
MT & Great Falls \\
NC & Charlotte \\
ND & Fargo \\
NE & Omaha \\
NH & Manchester \\
NJ & Newark \\
NM & Albuquerque \\
NV & Reno \\
NY & Buffalo, New York \\
OH & Cincinnati, Cleveland \\
OK & Tulsa \\
OR & Portland \\
PA & Philadelphia \\
RI & Providence \\
SC & Charleston \\
SD & Huron \\
TN & Memphis \\
TX & Dallas, El Paso, Houston \\
UT & Salt Lake City \\
VA & Norfolk \\
VT & Burlington \\
WA & Seattle, Spokane \\
WI & Milwaukee \\
WV & Charleston \\
WY & Cheyenne \\
\hline & \\
\hline & \\
\hline
\end{tabular}

Physical Unit Prices: 1978

The Platt's monthly leaded regular motor gasoline prices cover all States except AK and HI. The Platt's city assignments to States are shown in Table A30. In 1978, the CPI motor gasoline coverage was expanded from 21 States to 25 States (28 SMSAs) and an "all types" price was published that covers leaded regular, leaded premium, and unleaded regular. The CPI SMSA assignments to States for 1978 through 1982 are shown in Table A28 on page 225. Both the CPI and the Platt's prices include taxes.

Since both sources report a single price for each city or SMSA, product weights are not needed to compute monthly composite prices. Instead, city price observations are assigned to States as shown in Table A28 and Table A30. Price observations are combined using simple averaging by State and month. If both Platt's and the CPI cover a city/SMSA, the CPI price is used. Platt's prices are converted to dollars per gallon; the CPI prices are already expressed in dollars. All States are covered by the data sources, so no imputation is required for 1978. The following 26 States have prices only from Platt's: $A L, A R, A Z, C T$, $\mathrm{DE}, \mathrm{IA}, \mathrm{ID}, \mathrm{LA}, \mathrm{ME}, \mathrm{MS}, \mathrm{MT}, \mathrm{NC}, \mathrm{ND}, \mathrm{NE}, \mathrm{NH}, \mathrm{NM}$, NV, OK, RI, SC, SD, TN, UT, VT, WV, and WY. The following 19 States are covered only by the $C P I$ : $A K$, CA, CO, DC, FL, GA, HI, IL, MA, MD, MI, MN, MO, NJ, NY, OH, OR, PA, and WI. Six States have price data from both sources: IN, KS, KY, TX, VA, and WA.

Annual physical unit prices for all States are calculated from the monthly motor gasoline prices calculated above and weighted by the monthly motor gasoline consumption volumes for States from Highway Statistics.

\section{Physical Unit Prices: 1976, 1977}

The calculation of monthly composite State prices for 1976 and 1977 depends upon the source of data. Different procedures are used for States with only Platt's data, States with only CPI data, and States with both Platt's and CPI data. If both data sources cover a city, only the CPI price is used for that city. City price assignments to States are given in Table A30 for Platt's and in Table A31 for the CPI. Prices from both sources include taxes. AK is the only State for which prices need to be estimated.

For States with data from Platt's only, the monthly prices reported in Platt's are either used directly or combined by simple averaging if there is more than one price observation for a State in a given month. 


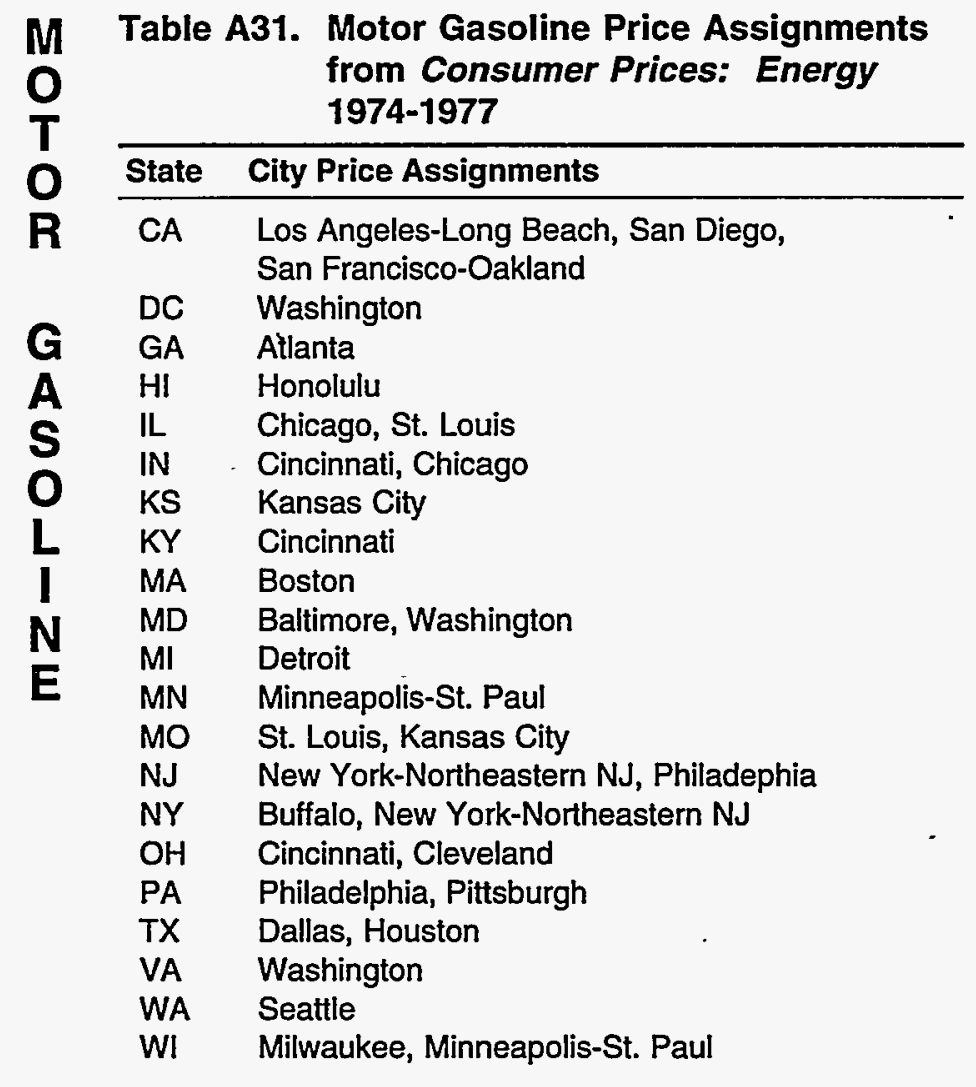

Note: Prices are available separately for leaded regular, leaded premium, and unleaded regular $(1976,1977)$; "all types" prices are not available.

The reported prices in cents per gallon are converted to dollars per gallon. Prices for the following 29 States are calculated using this procedure and cover only leaded regular motor gasoline: $A L, A R, A Z$, CO, CT, DE, FL, IA, ID, LA, ME, MS, MT, NC, ND, $\mathrm{NE}, \mathrm{NH}, \mathrm{NM}, \mathrm{NV}, \mathrm{OK}, \mathrm{OR}, \mathrm{RI}, \mathrm{SC}, \mathrm{SD}, \mathrm{TN}, \mathrm{UT}, \mathrm{VT}$, $W V$, and WY.

If State-level motor gasoline prices for 1976 and 1977 are available only from the CPI, monthly composite prices are calculated as weighted averages of leaded and unleaded prices. Prices for 15 States are calculated using data only from the CPI: CA, DC, GA, HI, IL, MA, MD, MI, MN, MO, NJ, NY, OH, PA, and WI.

1. The weights used in this process are national-level shares of leaded and unleaded motor gasoline product supplied. For 1977, the leaded and unleaded share of 0.725 and 0.275 , respectively, are from the $M E R$. For 1976, MER data for 1977 through 1984 are used to estimate the unleaded share using simple regression. The unleaded percentages for 1977 through 1984 are converted to shares and used to estimate leaded and unleaded shares of motor gasoline. The re- sulting 1976 leaded share is 0.744 and the unleaded share is 0.256 .

2. The next step is to calculate monthly composite leaded and unleaded prices for each State. If more than one CPI price observation is available for a particular grade of motor gasoline (leaded or unleaded) for a State in a given month, the CPI observations are combined by grade using simple averaging. Regular and premium prices are averaged for an estimate of State-level leaded prices.

3. Final monthly composite prices for 1976 and 1977 are calculated using the leaded and unleaded composite prices calculated above and the $M E R$-based leaded and unleaded shares as volume weights.

For States with price data from both Platt's and the $C P I$, all price observations are averaged together by product type. If both sources report prices for a city, the CPI price is used. Once composite leaded and unleaded prices have been calculated separately for each State, the leaded and unleaded consumption shares are used to weight the product-type prices into the final monthly composite motor gasoline prices. Six States are calculated with data from both Platt's and the CPI: IN, KS, KY, TX, VA, and WA.

1. Monthly leaded composite prices are calculated by combining Platt's prices with the CPI prices for leaded regular and premium motor gasoline by month, since the Platt's prices cover only regular leaded fuel. If both data sources cover a city, the CPI prices are used.

2. Since the CPI is the only source of unleaded gasoline price data for 1976 through 1977, monthly unleaded composite prices are calculated from CPI data only.

3. Final monthly composite prices for the six States with price data from both Platt's and the CPI are calculated using annual U.S. leaded and unleaded shares and leaded and unleaded monthly composite prices.

Prices for 1976 and 1977 for AK, the only State not covered by price data from either data source, are estimated based on the average relationship between the State and the national average price for years in which data are available. The national average price used for these estimations is a simple average of the prices of the 49 States for which data are available in all years (i.e., excluding $\mathrm{AK}$ and $\mathrm{HI}$ for all years). 
Annual prices for AK are estimated based on the average AK-to-U.S. price relationship for 1978 and 1979.

Annual physical unit prices (excluding AK) are calculated from the monthly motor gasoline prices calculated above and weighted by the monthly motor gasoline consumption volumes for States from Highway Statistics.

\section{Physical Unit Prices: 1974, 1975}

The Platt's price data for 1974 through 1975 cover only leaded regular motor gasoline. Beginning in 1974, motor gasoline price data are also available from the CPI for selected SMSAs. An SMSA price is assigned to each State with counties included in the definition of that SMSA; for the years 1974 through 1977, prices for 23 SMSAs cover 21 States. The State assignments of SMSA prices for 1974 through 1977 are given in Table A31 on page 228. For 1974 and 1975, CPI prices are reported separately for leaded regular and leaded premium motor gasoline. According to the CPI Detailed Report, April 1986, these prices include taxes; the Platt's prices also include taxes. AK is the only State not covered by either of these two data sources; prices for AK are imputed for 1974 and 1975.

The Platt's regular leaded prices and the CPI regular and premium leaded motor gasoline prices, including taxes, are assigned to their respective States as shown in Table A30 and Table A31. If both sources cover a city, the $C P I$ price is used. The following 29 States are covered only by Platt's: $A L, A R, A Z, C O$, CT, DE, FL, IA, ID, LA, ME, MS, MT, NC, ND, NE, NH, NM, NV, OK, OR, RI, SC, SD, TN, UT, VT, WV, and $W Y$. The following 15 States are covered only by CPI: CA, DC, GA, HI, IL, MA, MD, MI, MN, MO, NJ, NY, OH, PA, and WI. The following six States have both Platt's and CPI data for a particular city: IN, KS, KY, TX, VA, and WA.

All price observations assigned to a State, regardless of grade or data source, are added together and divided by the number of observations. As part of this calculation, Platt's prices are converted from cents per gallon to dollars per gallon.

Neither Platt's nor the CPI reports price data for AK. The methodology of the estimation of annual AK prices is the same as used in 1976 and 1977.

Annual physical unit prices for the remaining 50 States (excluding $A K$ ) are calculated from the monthly motor gasoline prices calculated above and weighted by the monthly motor gasoline consumption volumes for States from Highway Statistics.

\section{Physical Unit Prices: 1970 Through 1973}

Monthly motor gasoline physical unit prices for 1970 through 1973 are available only from Platt's, where city prices covering 49 States are reported in a table titled, "Service Station Prices: Gasoline (Including Taxes)." These prices, as shown in Table A27 are for leaded regular gasoline only and include taxes.

Monthly average city prices from Platt's are assigned to the State in which the city is located. Platt's city price assignments to States are given in Table A30.

Monthly composite prices for 1970 through 1973 are equal to the reported monthly Platt's prices or, if more than one city is available for a given State in a certain month, a simple average of the assigned city prices. The reported prices are converted from cents to dollars per gallon.

Platt's does not report data for either AK or HI for 1970 through 1973. The methodology of the estimation of AK and HI prices is the same as used for 1976 and 1977.

Annual physical unit prices (excluding $\mathrm{AK}$ and $\mathrm{HI}$ ) are calculated from the monthly motor gasoline prices weighted by the monthly motor gasoline consumption volumes for States from Highway Statistics.

\section{Btu Prices: All Years}

Btu prices for States are computed by converting the physical unit prices in dollars per gallon to dollars per barrel (42 gallons per barrel) and, then, to dollars per million Btu (5.253 million Btu per barrel). U.S. Btu prices are calculated as the average of the State Btu prices, weighted by consumption data from SEDS.

\section{Data Sources}

Prices

1986 forward: Energy Information Administration, Petroleum Marketing Annual, Table 29 (1986-1988) and Table 30 (1989 forward), column titled, "All Refiners, Sales to End Users, Through Company Outlets."

1983-1985: Energy Information Administration, $\mathrm{Pe}$ troleum Marketing Annual, 1985, Volume 1, Table 16, 
column titled, "All Refiners and Gas Plant Operators, Sales to End-users, Through Company Outlets."

1974 forward: Bureau of Labor Statistics, U.S. Department of Labor, Consumer Prices: Energy, computer printouts of monthly gasoline prices.

1970-1982: McGraw-Hill, Inc., Platt's Oil Price Handbook and Oilmanac; table titled "AAA 'Fuel-gauge' Report" (1982); table titled, "Platt's/Lundberg Summary," (1979-1981); and table titled, "Service Station Prices: Gasoline (Including Taxes)," (1970-1978).

1974-1982: Bureau of Labor Statistics, CPI Detailed Report, April 1986, Technical Notes, page 110.

1982: Energy Information Administration, Form EIA-25 "Prime Supplier Monthly Report" computer tape, unpublished data.

1976-1984: Energy Information Administration, Monthly Energy Review, January 1985, table titled, "Petroleum: Finished Motor Gasoline.Supply and Disposition."

Taxes

1970 forward: Federal Highway Administration, U.S. Department of Transportation, Highway Statistics, Tables MF-26 (1973 forward); MF-121T for State taxes (1983 forward); FE-101 for Federal taxes (1983 forward); and MF-23 and MF-25 (1970-1972).

\section{Consumption}

1970 forward: Energy Information Administration, State Energy Data System, transportation sector, motor gasoline consumption.

\section{Conversion Factor: All Years}

5.253 million Btu per barrel

Table A32. Petroleum Coke Electric Utility Price Assignments, 1972 Forward

\begin{tabular}{llc}
\hline State & Years & $\begin{array}{l}\text { State Prices } \\
\text { Assigned }\end{array}$ \\
\hline DE & $1981-1991$ & PA \\
KS & 1975 & MO \\
MO & 1983,1985 & MN \\
NY & 1974 & PA \\
WI & 1985 & MN \\
\hline
\end{tabular}

\section{Petroleum Coke (utilities)}

Petroleum coke is consumed by the electric utility and industrial sectors. The portion of petroleum coke consumed by the industrial sector ( 97 percent in 1991 ) is included in the category "other petroleum products," which is discussed in a subsequent section. The remaining petroleum coke is used for electricity generation in 12 States over the period 1970 forward. A maximum of seven States reported consumption in any single year. Estimates of the annual consumption of petroleum coke by electric utilities are from SEDS. Estimates of the deliveries of petroleum coke, used in the price calculation described below, are from the Cost and Quality of Fuels for Electric Utility Plants (CEQ). The $C \mathcal{E} Q$ prices are the delivered costs of the fuels and include all taxes, transportation, and other charges paid by the utilities.

\section{Btu Prices: 1972 Forward}

The principal data source for petroleum coke used by electric utilities is an EIA public use computer tape covering the period 1972 forward. From 1972 through 1982, steam plants with a maximum capacity of 25 megawatts were included. For 1983 and subsequent years, the reporting threshold was raised to 50 megawatts capacity. In addition to the computer tape, the data are also published, but not for the full period. From 1978 through 1982, CEQ was published monthly and annually; data for calculating petroleum coke prices are in only the monthly reports. For 1983 and subsequent years, $C E Q$ was published annually and includes petroleum coke prices for individual States and for the Nation.

The computer tape, Cost and Quality of Fuels for Electric Utility Plants (Form 423) Historic Files, shows quantity in short tons, estimated Btu per pound, and price in dollars per million Btu. The data are presented by plant, by State, and by month. The Btu price is calculated as the annual sum of the unit prices, weighted by the total Btu in each reported delivery, divided by the annual sum of the Btu delivered to all electric utility plants within the State. The States and years listed below have prices assigned as shown in Table A32.

The high DE prices prior to 1981 are actual reported prices. 
For the years 1970 and 1971, prices are estimated using the gross domestic product implicit price deflator. The deflator for 1970 or 1971 is divided by the 1972 deflator and the quotient is multiplied by the 1972 price for each State to develop the price estimates for 1970 and 1971. The deflators are 35.1 in 1970, 37.1 in 1971, and 38.8 in 1972.

Although SEDS has a consumption estimate for NJ in 1971 , there are no NJ price data for any year in the Form 423 Historic Files. Form 423 data for PA in 1972 are used to estimate a PA price for 1971, which is assigned to NJ. The Form 423 PA prices for 1972 and 1971 do not appear in SEPEDS since SEDS has no petroleum coke consumption in PA for those years.

\section{U.S. Btu Prices: All Years}

U.S. Btu prices are calculated as the average of the State Btu prices, weighted by consumption data from SEDS.

\section{Data Sources}

Prices

1972 forward: Energy Information Administration, Public Use Tape, "Cost and Quality of Fuels for Electric Utility Plants (Form 423) Historic Files," as published in the following reports:

- 1983 forward: Energy Information Administration, Cost and Quality of Fuels for Electric Utility Plants, Table 20 (1983, 1984), Table 12 (19851989), and Table 40 (1990 forward).

- 1978-1982: Energy Information Administration, Cost and Quality of Fuels for Electric Utility Plants, table titled, "Wood Chips, Refuse, and Petroleum Coke Used as Fuel by Steam-Electric Units."

1970-1972: Energy Information Administration, Annual Energy Review 1992, Appendix C. Gross Domestic Product and Implicit Price Deflator.

\section{Consumption}

1970 forward: Energy Information Administration, State Energy Data System, electric utility petroleum coke consumption.
No conversion factors are required; Btu prices are calculated directly from data sources.

Residual fuel prices are developed for the electric utility, industrial, commercial, and transportation sectors. Estimates of the amount of residual fuel consumed by sector are from SEDS and are adjusted for process fuel consumption in the industrial sector. (See the "Consumption Adjustments for Process Fuel and Intermediate Products" section on page 251.)

\section{Electric Utility Sector}

The electric utility price for residual fuel (heavy oil) is the average delivered cost of No. 6 fuel oil receipts at electric utilities. For 1973 forward, Btu prices are developed directly from the data sources. For 1970 through 1972, prices are estimated using simple regression analysis. All taxes, transportation, and other charges paid by utilities are included in the prices for all years.

\section{Btu Prices: 1973 Forward}

Electric utility sector residual fuel prices for 1973 forward are from Cost and Quality of Fuels for Electric Utility Plants (CEQ). For 1973 through 1979, Btu prices are calculated as the weighted average of contract and spot prices for No. 6 fuel oil. Table A33 lists the States and years for which consumption is indicated by SEDS but no price is shown in CEQ. For these States, the Census division price, as shown in $C E Q$, is assigned as the State price.

For 1983 forward, $C \& Q$ reports prices for steam electric plants of 50 megawatt capacity or greater. For 1980 through 1982, CEQ prices cover all reporting plants of 25 megawatt capacity or greater.

AK/1973 forward and HI/1973 through 1982 prices are developed using the ratio of the C\&Q U.S. price and the Statistical Yearbook U.S. price multiplied by the Statistical Yearbook State price. AK/1973, 1975, 1978 prices are based on the average ratio of U.S. and AK prices for 1974, 1976 and 1977, and 1979 and 1980. The U.S. prices for 1973, 1975, and 1978, are multiplied by the average ratio for the years that have prices. 


\section{R Btu Prices: 1970 Through 1972}

State-level Btu prices for 1970 through 1972 are estimated using regression techniques and price data from the Statistical Yearbook. The regression equations use Statistical Yearbook State-level prices for 1973 through 1980 as the independent variable and the State-level prices calculated above (including the estimations for $\mathrm{AK}$ and $\mathrm{HI}$ ) as the dependent variable. Pacific regional price averages are assigned for the missing WA/1970, 1971 prices. The average of 1970 and 1972 AK Statistical Yearbook prices is substituted for the missing 1971 AK price, and a similar calculation is made for the 1973, 1975, and 1978 prices.

\section{U.S. Btu Prices: All Years}

U.S. Btu prices are calculated as the average of the State Btu prices, weighted by consumption data from SEDS.

\section{Data Sources}

Prices

1973 forward: Energy Information Administration, Cost and Quality of Fuels for Electric Utility Plants, Table 6 (1973-1979), Table 45 (1980-1982), Table 51 (1983, 1984), Table 41 (1985-1989), and Table 14 (1990 forward).

1970 forward: Edison Electric Institute, Statistical Yearbook of the Electric Utility Industry, Table 43 (19701979), Table 26 (1980-1983), Table 28 (1984-1986), and Table 29 (1987 forward).

\section{Consumption}

1970 forward: Energy Information Administration, State Energy Data System, electric utility sector heavy oil consumption.

\section{Conversion Factors: All Years}

Because Btu prices are available directly from the data sources, no conversion factors are used.

\section{Industrial Sector}

The industrial sector residual fuel prices for 1984 forward are developed from refiner/reseller prices of residual fuel as published in the Petroleum Marketing Annual. Residual fuel prices for 1970 through 1983
Table A33. Residual Fuel Electric Utility Census Division Price Assignments, 1970 Forward

\begin{tabular}{ll}
\hline State & \multicolumn{1}{c}{ Years of Assigned Prices } \\
\hline AL & $1975-1979$ \\
AR & 1987 \\
AZ & $1984,1985,1991$ \\
CO & $1982,1987,1989-1991$ \\
DC & $1982-1991$ \\
GA & 1991 \\
IA & $1970-1985$ \\
IN & $1970-1979$ \\
KS & $1980,1981,1985-1987,1989-1991$ \\
KY & $1970-1979$ \\
MN & $1984,1985,1987-1990$ \\
MT & $1970-1979$ \\
NC & $1976,1977,1979,1980,1982,1984$ \\
ND & $1970-1979$ \\
NE & $1981-1983,1990,1991$ \\
NM & $1979-1982,1989-1991$ \\
NV & 1983,1985 \\
OK & $1977,1978,1980,1982-1987,1989,1991$ \\
OR & $1970,1973,1974$ \\
SC & $1983,1985-1991$ \\
SD & $1981-1988$ \\
TN & 1979 \\
UT & $1982.1983,1986$ \\
VT & $1970-1979$ \\
WA & $1970,1971,1975-1978,1981-1983,1986-1988$ \\
WV & $1970-1977,1979$ \\
WY & $1970-1979$ \\
& \\
\hline
\end{tabular}

are calculated or estimated using average costs of residual fuel to manufacturing firms published in two Bureau of the Census reports and Platt's Oil Price Handbook and Oilmanac. Price data in these sources are available for the years 1971 and 1974 through 1981; prices for 1970,1972, 1973, 1982, and 1983 are estimated. Prices for all years include taxes.

\section{Physical Unit Prices: 1984 Forward}

Residual fuel industrial sector physical unit prices are calculated using refiner/reseller prices to end users from the Petroleum Marketing Annual (PMA). The States that do not have $P M A$ prices are assigned their PAD district or subdistrict price as shown in Table A34, with the exception of AK. AK industrial residual fuel price for 1984 forward is based on the WA industrial residual fuel price and the ratio of the AK-to-WA industrial distillate fuel prices for each year. Tax data from Bureau of the Census publications are added to develop final sector prices. 
Table A34. Residual Fuel Industrial Sector Price Assignments, 1984 Forward

\begin{tabular}{|c|c|c|}
\hline State & Years & $\begin{array}{l}\text { PAD District Prices } \\
\text { Assigned }\end{array}$ \\
\hline AR & 1985 & PAD District III \\
\hline$A Z$ & 1984-1991 & PAD District V \\
\hline co & 1986, 1988, 1991 & PAD District IV \\
\hline ID & $1985,1986,1989-1991$ & PAD District IV \\
\hline MS & 1988,1991 & PAD District III \\
\hline ND & $1988-1991$ & PAD District II \\
\hline NM & 1984-1986, 1990, 1991 & PAD District III \\
\hline NV & 1986, 1988, 1991 & PAD District V \\
\hline OR & 1989 & PAD District V \\
\hline SD & 1990,1991 & PAD District II \\
\hline UT & $1989-1991$ & PAD District IV \\
\hline WV & 1984 & PAD Subdistrict IC \\
\hline WY & 1989-1991 & PAD District IV \\
\hline
\end{tabular}

Physical Unit Prices: 1982, 1983

After 1981, the U.S. Department of Commerce, Annual Survey of Manufacturers and the Census of Manufacturers (ASM/CM) ceased publication of fuelspecific State-level residual fuel data from which prices can be calculated. Prices for 1982 and 1983 are estimated from the average relationship between the ASM/CM-based prices generated for 1978 through 1981 and the assigned Platt's No. 6 fuel oil prices for 1978 through 1981 (Table A36). These average ratios are calculated at the State-level for all States except AK, which shows no industrial sector residual fuel use reported in SEDS for 1982 and 1983. Physical unit residual fuel industrial prices for 1982 and 1983 are calculated using the assigned Platt's prices for 1982 and 1983 (Table A36) and the State-level average ratios. The resulting estimates implicitly include taxes that reflect individual State differences.

\section{Physical Unit Prices: 1971, 1974 Through 1981}

For the years 1971 and 1974 through 1981, industrial sector residual prices are calculated directly from cost and quantity data reported by the $A S M / C M$. For all States with available cost and quantity data, prices are equal to the average cost of residual fuel to manufacturers. Taxes are included in the published cost data. Missing data for these years are assigned from the average prices of adjacent States as shown in Table A35.
Table A35. Residual Fuel Industrial Sector Price Assignments, 1971, 1974-1981

\begin{tabular}{cll}
\hline State & \multicolumn{1}{c}{ Years } & State Prices Used \\
\hline AK & 1980,1981 & HI, WA \\
DC & $1979-1981$ & MD, VA \\
MT & $1974-1979$ & ID, ND, SD \\
ND & 1980 & MN, MT, SD \\
NM & $1971,1974-1981$ & AZ, CO, TX \\
NV & $1974-1978$ & AZ, CA, ID, OR, UT \\
OK & $1974-1978,1980$ & AR, CO, KS, MO, TX \\
SD & 1981 & IA, MN, MT, ND, NE \\
WY & $1971,1974-1981$ & CO, NE, UT \\
\hline
\end{tabular}

Physical Unit Prices: 1970, 1972, 1973

Since $A S M / C M$ data are not available for 1970,1972 , or 1973, prices for these years must be estimated. Physical unit prices are based on the ratio of the 1971 CM prices to the 1971 assigned No. 6 fuel oil prices from Platt's Oil Price Handbook and Oilmanac (Table A36). The estimated $1971 C M$ prices for NM and WY are used in the calculations. The resulting ratios for each State are used with the Platt's assigned prices for 1970, 1972, and 1973 to estimate prices. The final estimates implicitly include State-specific taxes.

\section{Btu Prices: All Years}

Btu prices for States are calculated from the physical unit prices and the conversion factor. U.S. Btu prices are calculated as the average of the State Btu prices, weighted by consumption data from SEDS, adjusted for process fuel consumption.

\section{Data Sources}

Prices

1984 forward: Energy Information Administration, Petroleum Marketing Annual, Table A4 (1984-1990), Table A3 (1991).

1984 forward: Industrial sector distillate fuel price estimates from the SEPER data system (AK and WA only).

1970-1983: McGraw-Hill, Inc., Platt's Oil Price Handbook and Oilmanac, refinery and terminal prices for No. 6 fuel oil, average of highs and lows.

1971, 1977, 1981: Bureau of the Census, U.S. Department of Commerce, Census of Manufactures, Fuels and 


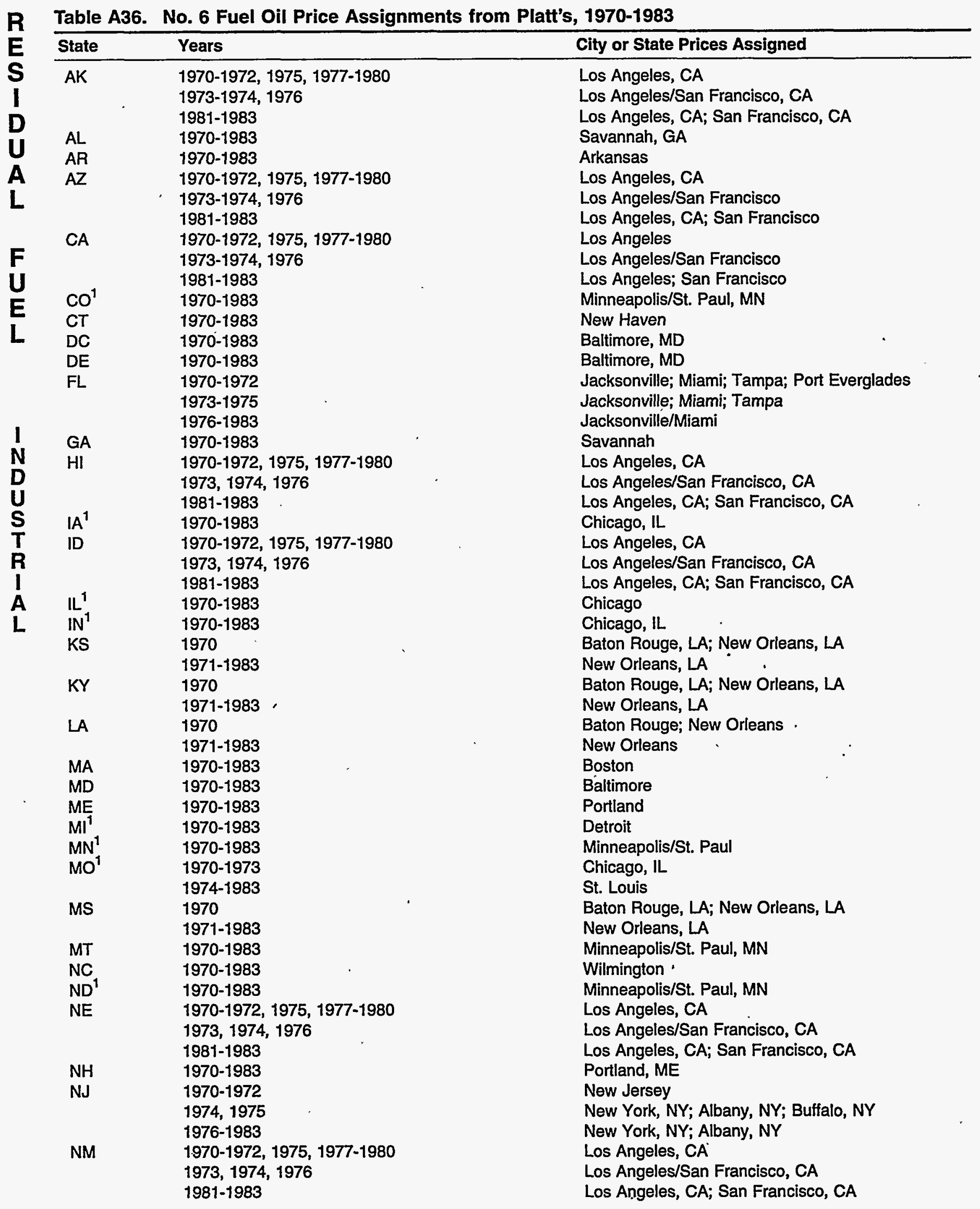

See footnotes at end of table. 
Table A36. No. 6 Fuel Oil Price Assignments from Platt's, 1970-1983 (Continued)

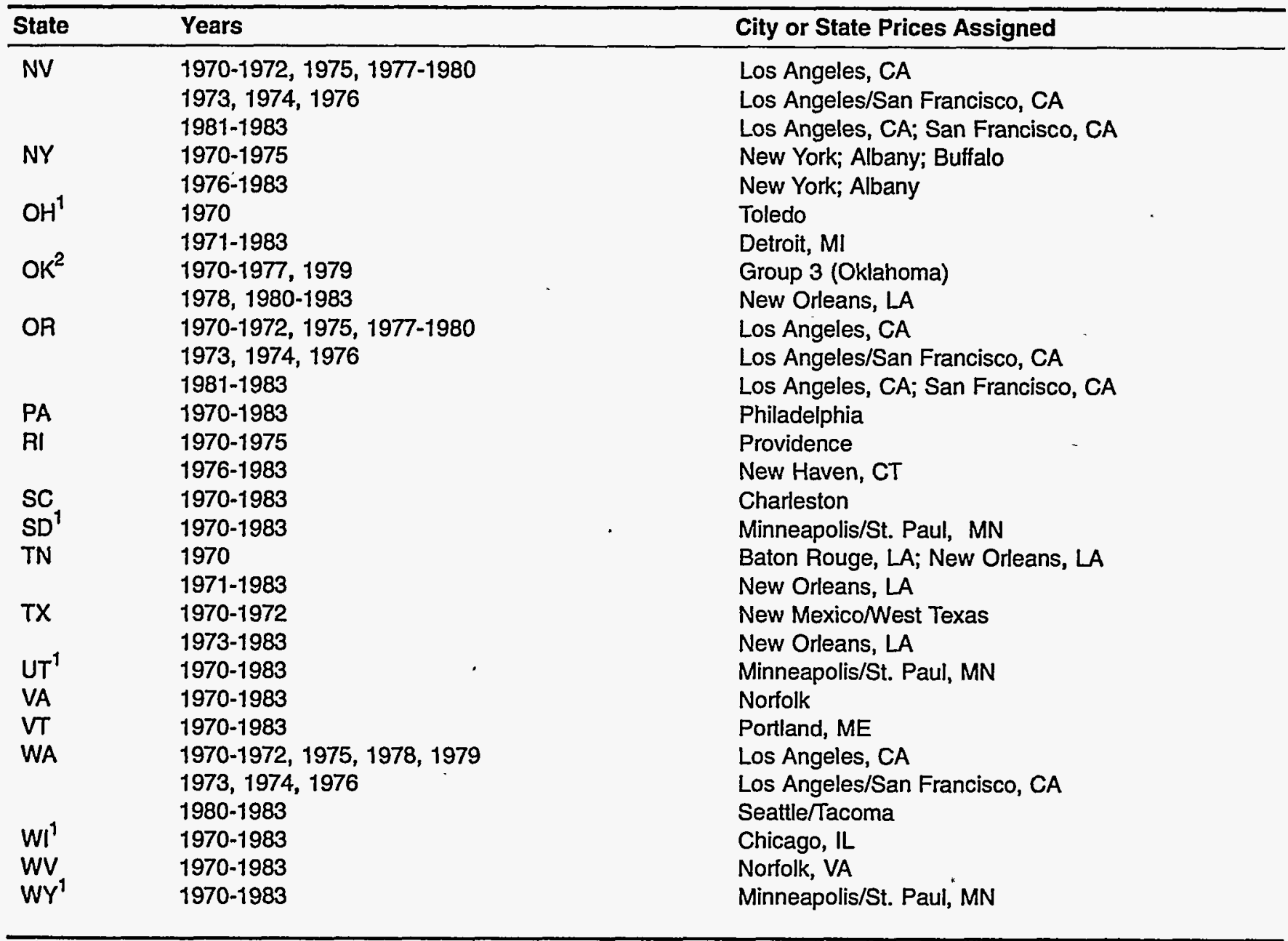

Raw data from Platt's must be converted from cents per gallon to dollars per barrel.

${ }^{2}$ As shown in Platt's.

Electric Energy Consumed, Part 2, Table 3. (Dates shown on the report covers are, respectively, 1972, 1977, and 1982.)

1974-1976 and 1978-1980: Bureau of the Census, U.S. Department of Commerce, Annual Survey of Manufactures, Fuels and Electric Energy Consumed, States by Industry Group, Table 3.

\section{Taxes}

1987 forward: Bureau of the Census, U.S. Department of Commerce, State Government Tax Collections, Table 8, column titled, "Percentage rate, September 1."

1984-1986: Bureau of the Census, U.S. Department of Commerce, Statistical Abstract of the United States, table titled, "State Government Tax Collections and
Excise Taxes," column titled, "Excise Taxes, General sales and gross receipts."

\section{Consumption}

1970 forward: Energy Information Administration, State Energy Data System, industrial sector residual consumption.

\section{Conversion Factor: All Years}

6.287 million Btu per barrel

\section{Commercial Sector}

For 1984 forward, State-level commercial sector residual fuel prices are developed from refiner/reseller 


\begin{tabular}{|c|c|c|}
\hline State & Years & $\begin{array}{l}\text { PAD District Prices } \\
\text { Assigned }\end{array}$ \\
\hline $\begin{array}{l}A Z \\
C O\end{array}$ & $\begin{array}{l}1984,1985,1988,1991 \\
1986\end{array}$ & $\begin{array}{l}\text { PAD District V } \\
\text { PAD District IV }\end{array}$ \\
\hline ID & $1985,1986,1989-1991$ & PAD District IV \\
\hline MS & 1988,1991 & PAD District II \\
\hline ND & $1988,1989-1991$ & PAD District II \\
\hline NM & 1984,1985 & PAD District III \\
\hline NV & $1986,1988,1991$ & PAD District V \\
\hline OR & 1989 & PAD District V \\
\hline$S D$ & 1990,1991 & PAD District II \\
\hline UT & $1989-1991$ & PAD District IV \\
\hline WV & 1984 & PAD Subdistrict IC \\
\hline WY & 1989-1991 & PAD District IV \\
\hline
\end{tabular}

C prices of residual fuel to end users published in the O PMA. For 1970 through 1983, commercial sector re-

\section{Physical Unit Prices: 1984 Forward}

Commercial sector residual fuel physical unit prices are calculated using refiner/reseller prices to end users from the PMA. The States that do not have $P M A$ prices are assigned their PAD district or subdistrict price (Table A37), with the exception of AK. AK commercial residual fuel price for 1984 forward is based on the WA commercial residual fuel price and the ratio of the AK-to-WA commercial distillate fuel prices for each year. Tax data from Bureau of the Census publications are added to develop final sector prices.

\section{Physical Unit Prices: 1976 Through 1983}

The commercial sector residual fuel physical unit prices for 1976 through 1983 are estimated from the electric utility sector residual prices for corresponding years and the tax-adjusted U.S. average retail residual fuel prices. The resulting price estimates implicitly include taxes that reflect individual State differences.

1. The first step in the estimation of the commercial residual fuel physical unit State prices is to convert the State-level tax rates reported in the Bureau of the Census publications into the vol-
Table A38. Residual Fuel Commercial Sector Price Assignments, 1970-1983

\begin{tabular}{|c|c|c|}
\hline State & Years & $\begin{array}{l}\text { State Prices Used } \\
\text { in the Estimation }\end{array}$ \\
\hline AL & $\begin{array}{l}1970-1974,1980 \\
1982,1983\end{array}$ & $\mathrm{FL}, \mathrm{GA}, \mathrm{MS}$ \\
\hline ID & $\begin{array}{l}1980,1981,1983 \\
1982\end{array}$ & $\begin{array}{l}\mathrm{CA}, \mathrm{CO} \\
\mathrm{CA}\end{array}$ \\
\hline IN & $1980-1983$ & IL, MI, OH \\
\hline $\mathrm{KY}$ & $1980-1983$ & IL, MO, OH, VA \\
\hline MT & $\begin{array}{l}1980,1983 \\
1982\end{array}$ & $\begin{array}{l}\mathrm{CO}, \mathrm{MN} \\
\mathrm{MN}\end{array}$ \\
\hline NC & 1981,1983 & GA, VA \\
\hline ND & $\begin{array}{l}1980,1983 \\
1981,1982\end{array}$ & $\begin{array}{l}M N, S D \\
M N\end{array}$ \\
\hline OR & $1975-1983$ & CA \\
\hline TN & $1970-1978,1980-1983$ & $A R, G A, M O, M S, V A$ \\
\hline VT & $1980-1983$ & $\mathrm{ME}, \mathrm{NH}, \mathrm{NY}$ \\
\hline WI & 1982,1983 & IL, MI, MN \\
\hline WV & $1980-1983$ & $\mathrm{MD}, \mathrm{OH}, \mathrm{PA}, \mathrm{VA}$ \\
\hline \multirow[t]{3}{*}{ WY } & 1980 & $\mathrm{CO}, \mathrm{NE}, \mathrm{SD}, \mathrm{UT}$ \\
\hline & 1981,1983 & $\mathrm{CO}$ \\
\hline & 1982 & MN \\
\hline
\end{tabular}

ume-weighted average U.S. sales tax rate using commercial residual consumption data from SEDS.

2. A preliminary U.S. residual fuel oil price, including taxes, is computed using the average U.S. tax rate estimated above and the annual average U.S. residual fuel price to end users (average retail price excluding taxes) from the Monthly Energy Review (MER).

3. Commercial sector physical unit residual fuel prices for States are computed using the electric - utility sector residual fuel prices. To do this calculation, the ratio of the State-level and U.S. prices in the commercial sector is assumed to be the same as the ratio of State and U.S. prices in the electric utility sector. Some States are missing electric utility prices for 1976 through 1983; these are estimated using adjacent States' average prices (Table A38).

\section{Physical Unit Prices: 1970 Through 1975}

Since no national or State-level retail residual prices are available from published data sources, commercial sector residual prices for 1970' through 1975 are estimated. The estimation method is based on the assumption that the average ratio of State-to-U.S. 
prices is the same in the commercial and electric utility sectors. The average ratio for 1976 through 1979 of the MER U.S. tax-adjusted prices to the electric utility sector U.S. prices is calculated and used as an adjustment factor with State-level electric utility sector prices for 1970-1975. The resulting price estimates implicitly include taxes that reflect individual State differences.

1. The average ratio of the MER tax-adjusted U.S. prices and the electric utility sector U.S. prices is calculated for 1976 through 1979.

2. State-level commercial sector residual fuel prices are calculated using the electric utility sector physical unit price series for 1970 through 1975 and the average ratio computed above. Price assignments for States missing electric utility sector data are shown in Table A38.

\section{Btu Prices: All Years}

Btu prices for States are calculated from the physical unit prices and the conversion factor. U.S. Btu prices are calculated as the average of the State Btu prices, weighted by consumption data from SEDS.

\section{Data Sources}

\section{Prices}

1984 forward: Energy Information Administration, Petroleum Marketing Annual, Table A4 (1984-1990), Table A3 (1991).

1984 forward: Commercial sector distillate fuel price estimates from the SEPER data system (AK and WA only).

1978-1983: Energy Information Administration, Monthly Energy Review, December 1988, table titled, "Refiner Sales Prices of Residual Fuel Oil," column titled, "Average Sales to End Users."

1976, 1977: Energy Information Administration, Monthly Energy Review, December 1983, table titled, "Average No. 6 Residual Fuel Oil Prices," column titled, "Average, Retail."

1970-1983: Electric utility sector residual fuel price estimates (in physical units) from the SEPER data system.
Taxes

1987 forward: Bureau of the Census, U.S. Department of Commerce, State Government Tax Collections, Table 8 , column titled, "Percentage rate, September 1."

1976-1986: Bureau of the Census, U.S. Department of Commerce, Statistical Abstract of the United States, table titled, "State Government Tax Collections and Excise Taxes," column titled, "Excise Taxes, General sales and gross receipts."

\section{Consumption}

1970 forward: Energy Information Administration, State Energy Data System, commercial sector residual fuel consumption.

\section{Conversion Factor: All Years}

6.287 million Btu per barrel

\section{Transportation Sector}

Residual fuel is consumed in the transportation sector for vessel bunkering, military use, and railroads. In 1970, vessels consumed 74 percent of the transportation use of residual fuel, and the military and railroads accounted for 24 percent and 2 percent, respectively. By 1991, vessel use had grown to 98 percent, military use had dropped to 2 percent, and the railroads' share was insignificant. Prices are developed for vessel bunkering, and electric utility prices are assigned to the military and railroad uses. Tax adjustments are made as described below. The transportation sector average price for each State and year is the consumption-weighted average of the prices of the three uses.

\section{Physical Unit Prices: All Years}

Vessel Bunkering. Physical unit prices are calculated from actual or estimated U.S. bunker $C$ prices and corresponding residual fuel electric utility sector State and U.S. prices. The ratio of U.S. bunker $C$ price to U.S. residual fuel electric utility sector price is multiplied by the State residual fuel electric utility sector price to obtain the State bunker $C$ price. Taxes are calculated as described for the commercial sector (1976 through 1983) and added, so that final State vessel burkering price estimates implicitly include State-specific taxes. Other 


\begin{tabular}{|c|c|c|}
\hline State & Years & $\begin{array}{l}\text { State Prices Used } \\
\text { in the Estimation }\end{array}$ \\
\hline AL & $1970-1974,1980-1986$ & FL, GA, MS \\
\hline CO & 1986 & $\mathrm{KS}, \mathrm{NM}, \mathrm{UT}$ \\
\hline CT & 1978 & $\mathrm{NH}, \mathrm{VT}$ \\
\hline \multirow[t]{2}{*}{$\mathrm{DC}$} & 1975 & $M D$ \\
\hline & 1978 & PA \\
\hline GA & 1978 & KY, MS \\
\hline ID & 1970,1979 & $\mathrm{CA}, \mathrm{CO}$ \\
\hline IL & 1975 & IA, IN, WI \\
\hline IN & $1980-1986$ & IL, MI, OH \\
\hline KS & 1975 & $\mathrm{MO}, \mathrm{NE}$ \\
\hline KY & $1980-1984$ & IL, MO, OH, VA \\
\hline MD & 1978 & $\mathrm{DE}, \mathrm{PA}$ \\
\hline ME & 1975 & $\mathrm{VT}$ \\
\hline$M N$ & 1986 & IL, MI \\
\hline MT & 1983-1985 & $\mathrm{CO}, \mathrm{MN}, \mathrm{SD}$ \\
\hline \multirow[t]{3}{*}{ NC } & 1975 & GA \\
\hline & 1978 & KY \\
\hline & $1981,1983,1985,1986$ & GA, VA \\
\hline \multirow[t]{2}{*}{ ND } & $1982-1984$ & MN, SD \\
\hline & 1986 & SD \\
\hline $\mathrm{NH}$ & 1975 & $\mathrm{VT}$ \\
\hline NM & 1983,1984 & $\mathrm{CO}$ \\
\hline NV & 1975,1978 & $\mathrm{CA}$ \\
\hline $\mathrm{OH}$ & 1975 & IN, MI \\
\hline OK & 1975 & MO, TX \\
\hline \multirow[t]{2}{*}{ OR } & 1972 & CA, WA \\
\hline & $1975-1986$ & $\mathrm{CA}$ \\
\hline \multirow[t]{2}{*}{ SC } & 1975,1984 & GA \\
\hline & 1978 & AL, FL \\
\hline SD & 1975,1978 & MN, ND \\
\hline \multirow[t]{3}{*}{$T N$} & $\begin{array}{l}1970,1971,1973,1974, \\
1976,1977,1980-1982\end{array}$ & AR, GA, MO, MS, VA \\
\hline & 1975 & $\mathrm{AR}, \mathrm{GA}, \mathrm{MO}, \mathrm{MS}$ \\
\hline & 1978 & AR, MO, MS \\
\hline \multirow[t]{2}{*}{ UT } & 1984 & $\mathrm{AZ}, \mathrm{CO}, \mathrm{NV}$ \\
\hline & 1975 & CO \\
\hline \multirow[t]{2}{*}{ VA } & 1975 & GA \\
\hline & 1978 & KY \\
\hline WA & 1984,1985 & $\mathrm{CA}$ \\
\hline \multirow[t]{2}{*}{ WI } & $1978,1982-1985$ & IL, MI, MN \\
\hline & 1986 & IL, MI \\
\hline WV & 1985 & $\mathrm{MD}, \mathrm{OH}, \mathrm{PA}, \mathrm{VA}$ \\
\hline WY & $1981,1982,1985$ & $\mathrm{CO}, \mathrm{MN}, \mathrm{SD}$ \\
\hline
\end{tabular}

procedures are described separately by groups of years.

1. For 1982 forward, national average prices for residual fuel with sulfur content greater than 1 percent are from the Annual Energy Review and used as proxies for bunker $C$ prices.
2. For 1975 through 1981 , national average bunker C prices are available from the Monthly Petroleum Product Price Report (MPPPR). Annual average U.S. prices for 1975 and 1976 are calculated ás the simple average of the monthly prices for each respective year because annual average prices are not shown in the MPPPR.

3. For 1970 through 1974 , no U.S. bunker C prices are available. To estimate State-level prices for these years, the average ratio of published bunker $C$ prices and electric utility sector prices for 1975 through 1979 is calculated and multiplied by the State-level electric utility prices for 1970 through 1974.

Missing State prices are assigned adjacent States' average prices as shown in Table A39.

Military and Railroad Use. For all years, electric utility sector residual fuel prices are assigned to military and railroad uses. The electric utility prices. include taxes. Since the military does not pay State taxes, their prices are adjusted to remove taxes. Estimation of the tax adjustment is described under the commercial sector.

In some cases States have no reported consumption of residual fuel oil in the electric utility sector and, therefore, no final price in that sector. Since the electric utilities sector prices are used as a base in the calculation of prices in the other sectors, intermediate prices are calculated for OR in 1971 and for all States that need prices in 1987 forward.

Average Prices. Transportation sector prices are the average of bunker fuel, military, and railroad prices, weighted by each category's share of total transportation consumption from SEDS.

\section{Btu Prices: All Years}

Btu prices for States are calculated from the physical unit prices and the residual fuel conversion factor. U.S. Btu prices are calculated as the average of the State Btu prices, weighted by consumption data from SEDS.

\section{Data Sources}

\section{Prices}

1982 forward: Energy Information Administration, Annual Energy Review 1992, Table 5.21, row titled, 
"Sales Prices to End Users, Residual Fuel Oil, Greater Than 1 Percent Sulfur Content."

1976-1981: Energy Information Administration, Monthly Petroleum Product Price Report, Table 3.

1975: National Energy Information Center, Federal Energy Administration, Monthly Petroleum Product Price Report, Table 3.

1970-1986: Electric utility sector residual fuel price estimates (in physical units) from SEPEDS.

\section{Taxes}

1987 forward: Bureau of the Census, U.S. Department of Commerce, State Government Tax Collections, Table 8, column titled, "Percentage rate, September 1."

1976-1986: Bureau of the Census, U.S. Department of Commerce, Statistical Abstract of the United States, table titled, "State Government Tax Collections and Excise Taxes," column titled, "Excise Taxes, General sales and gross receipts."

\section{Consumption}

1970 forward: Energy Information Administration, State Energy Data System, transportation sector residual fuel consumption including the subcategories for vessel bunkering, military, and railroad uses.

\section{Conversion Factor: All Years}

6.287 million Btu per barrel.

\section{Other Petroleum}

Sixteen separate products are included in the category called other petroleum:

1. Aviation gasoline blending components

2. Crude oil

3. Miscellaneous products $\left({ }^{*}\right)$

4. Motor gasoline blending components

5. Natural gasoline, including isopentane (19701983)

6. Pentanes plus (1984 forward)

7. Petrochemical feedstocks, naphtha $\left({ }^{*}\right)$

8. Petrochemical feedstocks, other oils $\left(^{*}\right)$

9. Petrochemical feedstocks, still gas (1970-1985) (*)

10. Petroleum coke (*)
11. Plant condensate (1970-1983)

12. Special naphthas $\left(^{*}\right)$

13. Still gas

14. Unfinished oils

15. Unfractionated stream (1970-1983)

16. Waxes $\left(^{*}\right)$

\section{Physical Unit Prices: All Years}

Of the 16 products, national-level prices are developed for the 7 noted with asterisks $\left({ }^{*}\right)$ above and described in the following paragraphs. All of these products are assigned to the industrial sector. Only national-level prices are developed because Statelevel price information is not available, and taxes are not included in any of the estimates. Expenditures for the other nine products are completely removed as process fuel or intermediate products. (See the "Consumption Adjustments for Process Fuel and Intermediate Products" section on page 251.)

Starting in 1984, three products-natural gasoline, plant condensate, and unfractionated stream-are dropped, and pentanes plus is added in the Energy Information Administration (EIA) reporting system. Natural gasoline (including isopentane) and plant condensate are reported together as the new product, pentanes plus. Unfractionated stream is dropped because its components are reported separately as liquefied petroleum gases.

\section{Miscellaneous Products}

The products in this subcategory vary from the inexpensive (absorption oils similar to kerosene) to the very expensive (hydraulic fluids). The price estimates are based on the evidence presented in the Minerals Yearbooks of the 1970's indicating that the greater part of the miscellaneous product line consists of finished petrochemicals, especially the aromatic hydrocarbons: benzene, toluene, and the xylenes.

Price estimates for 1972, 1977, 1982, and 1987 are from Census of Manufactures (CM) data on quantity and value of aromatics (product codes 2911054 and 2911056) and "other finished petroleum products" (product code 2911098) shipped by industry 2911. The ratio of miscellaneous-products-to-crude-oil price for these 4 years varies widely. Specifically, the ratio equals 1.91 for 1972, 2.15 for 1977, 1.56 for 1982, and 1.99 for 1987. Thus, to estimate miscellaneous products prices, the following ratios are used: 
1970 - 1974: 1.91 times the crude oil price 1975 - 1979: 2.15 times the crude oil price 1980 - 1984: 1.56 times the crude oil price. 1985 forward: 1.99 times the crude oil price.

\section{Price Data Sources}

1970 Forward: Energy Information Administration, Annual Energy Review 1992, Table 5.20, "Crude Oil Refiner Acquisition Cost, 1968-1992," column titled, "Composite, Nominal."

1972, 1977, 1982, 1987: Bureau of the Census, U.S. Department of Commerce, Census of Manufactures, data for Standard Industrial Code 2911 on "Quantity and Value of Shipments by All Producers" as shown in Table 6a from MC77-I-29A, Product Codes 2911054, 2911056, and 2911098 (1972 and 1977); and Table 6a from MC87-I-29A, Product Codes 2911D55 and 2911D57 (1982 and 1987).

\section{Petrochemical Feedstocks, Naphtha}

Naphthas for petrochemical feedstock use are those oils with boiling points less than $401^{\circ} \mathrm{F}$. Consumer prices for 1978 through 1980 are derived from the special Annual Survey of Manufactures (ASM) series on "Hydrocarbon, Coal, and Coke Materials Consumed," using data for industries in Standard Industrial Codes (SIC) 2869 (industrial organic chemicals) and 2821 (plastics materials, synthetic resins, and nonvulcanizable elastomers). A price estimate for 1982 is obtained from the CM, based on data for SIC 2869 only. Since the ratio of petrochemical-naphtha-to-crude-oil price is reasonably constant in 1978, 1979,1980 , and 1982, the simple average of the four ratios, 1.23 , is used to estimate prices for petrochemical feedstocks, naphthas, for all other years.

\section{Price Data Sources}

1970-1977, 1981, 1983 forward: Energy Information Administration, Annual Energy Review 1992, Table 5.20, "Crude Oil Refiner Acquisition Cost, 19681992," column titled, "Composite, Nominal."

1982: Bureau of the Census, U.S. Department of Commerce, 1982 Census of Manufactures, M82-I-28F$3(\mathrm{P})$, page 6, industry 2869.

1980: Bureau of the Census, U.S. Department of Commerce, 1980 Annual Survey of Manufactures, M80(AS)-4.3, page 9, industry 2821.
1978, 1979: Bureau of the Census, U.S. Department of Commerce, 1979 Annual Survey of Manufactures, M79(AS)-4.3, page 8, industries 2821 and 2869.

\section{Petrochemical Feedstocks, Other Oils}

Petrochemical feedstocks referred to as "other oils" or "gas oils" are those oils with boiling points equal to or greater than $401^{\circ} \mathrm{F}$. Consumer prices for 3 years are obtained from the data on gas oils presented in the special $A S M$ series on hydrocarbons consumed, using data for industries in SIC 2865 (cyclic crudes and intermediates). The other-oils-tocrude-oil price ratio is quite stable, and the average ratio for the 3-year period, 1.607, is used to estimate prices for petrochemical feedstocks, other oils, for all other years.

\section{Price Data Sources}

1970-1977, 1981 forward: Energy Information Administration, Annual Energy Review 1992, Table 5.20, "Crude Oil Refiner Acquisition Cost, 19681992," column titled, "Composite, Nominal."

1979, 1980: Bureau of the Census, U.S. Department of Commerce, 1980 Annual Survey of Manufactures, M80(AS)-4.3, page 9, industry 2865.

1978: Bureau of the Census, U.S. Department of Commerce, 1979 Annual Survey of Manufactures, M79(AS)-4.3, page 8, industry 2865 .

\section{Petrochemical Feedstocks, Still Gas (1970 Through 1985)}

The source data for still gas is a mixture of consumer prices and producer prices for industries in SIC 2869 and SIC 2911 (petroleum refining). The still-gas-tocrude-oil price ratio is somewhat variable because still gas is a highly variable gaseous mixture. Value and quantity are available for 1972, 1977 through 1980, and 1982. In imputing prices for years when data from the CM or ASM are not available, the average still-gas-to-crude-oil price ratio, 0.759 , is used. After 1985, EIA data series no longer report feedstock and refinery use of still gas separately and all SEDS industrial consumption is removed from SEPER. (See the "Consumption Adjustments for Process Fuel and Intermediate Products" section on page 251.)

\section{Price Data Sources}

1970, 1971, 1981, 1983-1985: Energy Information Administration, Annual Energy Review 1992, Table 5.20, 
"Crude Oil Refiner Acquisition Cost, 1968-1992," column titled, "Composite, Nominal."

1982: Bureau of the Census, U.S. Department of Commerce, 1987 Census of Manufactures, MC87-I-29A, Table 6a, industry 2911.

1979, 1980: Bureau of the Census, U.S. Department of Commerce, 1980 Annual Survey of Manufactures, M80(AS)-4.3, page 9, industry 2869.

1978: Bureau of the Census, U.S. Department of Commerce, 1979 Annual Survey of Manufactures, M79(AS)-4.3, page 28, industry 2869.

1972, 1977: Bureau of the Census, U.S. Department of Commerce, 1977 Census of Manufactures, MC77-129A, page 29A-20, industry 2911.

\section{Petroleum Coke}

Petroleum coke is consumed by the industrial and electric utility sectors. The portion of petroleum coke consumed by the electric utility sector (about 3 percent in 1991) is described in the Petroleum Coke (Utilities) section on page 230 . The remaining petroleum coke is marketed to industrial consumers in two forms, calcined and uncalcined. On a unit basis, the calcined coke is about three times as expensive as the uncalcined. Unit prices are calculated by dividing the value exported by the quantity exported, weighted by type of petroleum coke. The physical unit conversion factor for petroleum coke is 5 barrels per short ton.

\section{Price Data Sources}

1989 forward: Bureau of the Census, U.S. Department of Commerce, December issues of EM-545, Foreign and Domestic Exports, for Petroleum Coke, Not Calcined (Commodity no. 2713110000) and Petroleum Coke, Calcined, (Commodity no. 2713120000).

1988: Bureau of the Census, U.S. Department of Commerce, December issue of EM-522, U.S. Exports, Schedule B, Community by Country, Petroleum Coke, Except Calcined, 5213150, and Petroleum Coke, Calcined, 5175120.

1987: Bureau of the Census, U.S. Department of Commerce, December issue of EM-622, U.S. Exports, Schedule B, Commodity by Country, Petroleum Coke, Except Calcined, 5213150, and Petroleum Coke, Calcined, 5175120.
1986: Bureau of the Census, U.S. Department of Commerce, December issue of EM-546, U.S. Exports, Schedule B, Commodity by Country, Petroleum Coke, Except Calcined, 5213150, and Petroleum Coke, Calcined, 5175120.

1978-1985: Bureau of the Census, U.S. Department of Commerce, FT-446, U.S. Exports, Schedule B, Commodity by Country, Petroleum Coke, Except Calcined, 5213150, and Petroleum Coke, Calcined, 5175120.

1970-1977: Bureau of the Census, U.S. Department of Commerce, December issues of FT-410, U.S. Exports, Schedule B, Commodity by Country, Petroleum Coke, Except Calcined, 3329420, and Petroleum Coke, Calcined, 3329410.

\section{Special Naphthas}

Prices for special naphthas are the simple average of the "varnish makers and painters naphtha" average price and the "solvent naphtha" average price calculated from the city prices published in the Chemical Marketing Reporter. For 1984 forward, the prices are averaged from the first issue of each month; for 1974, 1979, and 1980, when petroleum prices were increasing rapidly, prices are averaged from 10 randomly-selected issues; and for all other years, prices are averaged from at least five randomly-selected issues.

\section{Price Data Source .}

1970 forward: Schnell Publishing Co., Inc., Chemical Marketing Reporter, selected monthly issues.

\section{Waxes}

The product-supplied information covers fully refined crystalline wax, other refined crystalline wax, and microcrystalline wax. A composite price index for these three waxes is listed in the Bureau of Labor Statistics publication Producer Prices and Producer Price Indexes since April 1974. Producer prices for 1967 for the three waxes are available from data in the 1967 Census of Manufactures. A weighted-average price for 1967 of $\$ 15.75$ per barrel is obtained by summing the values of shipments of the three waxes and dividing the sum by the total quantity shipped. Price estimates for 1974 through 1985 are derived by multiplying the published price indices by the estimated 1967 base price. For 1985, a simple average of monthly price indices for January through June is applied because the index for petroleum wax was discontinued after June 1985. Similarly, the index for 
1974 is estimated by averaging the October, November, and December values available for that year. Price estimates for 1970 through 1973, and 1986 forward are obtained by dividing the value of exports by the quantity exported. The physical unit conversion factors for wax are 280 pounds per barrel and 2.2046 pounds per kilogram.

\section{Price Data Sources}

1989 forward: Bureau of the Census, U.S. Department of Commerce, December issues of Report No. EM-545, titled Foreign and Domestic Exports for Paraffin Wax Less Than 0.75 Percent Oil (Commodity no. 2712200000) and Other Mineral Waxes NESOI (Commodity no. 2712900000).

1987, 1988: Bureau of the Census, U.S. Department of Commerce, December issues of Report No. EM546 (1987) and EM-522 (1988), titled U.S. Exports, Schedule B, Commodity by Country for Paraffin Wax and Other Petroleum Waxes Unblended incl Microcrystalline Wax (Commodity no. 4925200).

1986: Bureau of the Census, U.S. Department of Commerce, December issue of EM-546, U.S. Exports, Schedule B, Commodity by. Country for Paraffin Wax, Crystalline, Fully Refined (Commodity no. 4925210), Paraffin Wax, Crystalline, Except Fully Refined (Commodity no. 4925220), and Petroleum Waxes, NSPF incl Microcrystalline Wax (Commodity no. 4925240).

1974-1985: Bureau of Labor Statistics, U.S. Department of Labor, Producer Prices and Producer Price -Indexes, Annual Supplement, Commodity Code 0577.

1970-1973: Bureau of the Census, U.S. Department of Commerce, December issues of FT-410, U.S. Exports, Schedule B, Commodity by Country for Paraffin Wax, Crystalline, Fully Refined (Commodity no. 3326220), Paraffin Wax, Crystalline, Except Fully Refined (Commodity no. 3326230), and Microcrystalline Wax (Commodity no. 3326210).

(1967: Bureau of the Census, U.S. Department of Commerce, Census of Manufactures, 1967, page 29 A- . 15 , quantity and value of shipments of waxes.)
Table A40. Other Petroleum Products Conversion Factors

\begin{tabular}{lc}
\hline Petroleum Product & Heat Rate \\
\hline Miscellaneous Products & 5.796 \\
Petrochemical Feedstocks & \\
$\quad$ Naphtha & 5.248 \\
Other Oils & 5.825 \\
Still Gas & 6.000 \\
Petroleum Coke & 6.024 \\
Special Naphthas & 5.248 \\
Waxes & 5.537 \\
\hline
\end{tabular}

\section{Btu Prices: All Years}

Btu prices for the seven petroleum products are calculated by converting physical unit prices from dollars per barrel to dollars per million Btu using the conversion factors shown on this page. U.S. prices are developed for each product and assigned to the industrial sector in States and years where there is consumption. The State-level "other petroleum" price is the average of the seven petroleum products, weighted by SEDS consumption data. The variable State prices reflect the different mix of products consumed. U.S. Btu prices are calculated as the average of the State Btu prices, weighted by SEDS consumption data. All State and U.S. SEDS consumption data are adjusted to remove process fuel and intermediate product consumption.

Table A41 shows national-level estimated prices and expenditures by the other petroleum product components for the years 1970,1975, 1980, and 1985 forward.

\section{Conversion Factors: All Years}

Factors used to convert price data from physical unit prices to Btu prices are shown in Table A40. 
Table A41. Other Petroleum Price and Expenditure Estimates for the Industrial Sector, United States, 1970, 1975, 1980, 1985-1991

\begin{tabular}{|c|c|c|c|c|c|c|c|c|c|c|}
\hline Petroleum Product & 1970 & 1975 & 1980 & 1985 & 1986 & 1987 & 1988 & 1989 & 1990 & 1991 \\
\hline & \multicolumn{10}{|c|}{ Prices in Dollars per Million Btu } \\
\hline $\begin{array}{l}\text { Miscellaneous Products ....................... } \\
\text { Pelrochemical Feedstocks }\end{array}$ & 1.12 & 3.85 & 7.57 & 9.17 & 4.99 & 6.14 & 5.03 & 6.16 & 7.62 & 6.54 \\
\hline 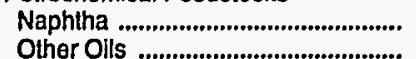 & $\begin{array}{l}.80 \\
.94\end{array}$ & $\begin{array}{l}2.43 \\
2.86\end{array}$ & $\begin{array}{l}6.68 \\
7.64\end{array}$ & $\begin{array}{l}6.27 \\
7.38\end{array}$ & $\begin{array}{l}3.41 \\
4.01\end{array}$ & $\begin{array}{l}4.20 \\
4.94\end{array}$ & $\begin{array}{l}3.44 \\
4.05\end{array}$ & $\begin{array}{l}4.21 \\
4.96\end{array}$ & $\begin{array}{l}5.21 \\
6.13\end{array}$ & $\begin{array}{l}4.47 \\
5.26\end{array}$ \\
\hline Stll Gas & .43 & 1.31 & 4.04 & 3.39 & 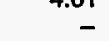 & - & 4.00 & - & 0.10 & $\begin{array}{l}5.20 \\
-\end{array}$ \\
\hline Petroleum Coke .................................. & .53 & 1.42 & 2.19 & 1.86 & 1.53 & 1.50 & 1.45 & 1.74 & 2.02 & 1.72 \\
\hline Special Naphthas ............................. & 1.96 & 3.12 & 10.48 & 10.87 & 10.73 & 10.73 & 10.84 & 10.00 & 9.71 & 9.71 \\
\hline 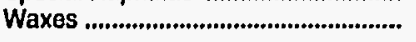 & 4.14 & 4.95 & 12.01 & 13.38 & 14.70 & 13.85 & 11.89 & 18.19 & 14.74 & 16.33 \\
\hline \multirow[t]{2}{*}{ Average Price ................................. } & .96 & 2.43 & 6.58 & 7.16 & 4.61 & 5.22 & 4.38 & 5.16 & 5.87 & 5.25 \\
\hline & \multicolumn{10}{|c|}{ Expenditures in Millions of Dollars } \\
\hline $\begin{array}{l}\text { Miscellaneous Products ...................... } \\
\text { Pelrochemical Feedstocks }\end{array}$ & 96.3 & 729.4 & $1,798.6$ & $1,308.2$ & 682.4 & 843.0 & 837.9 & 944.2 & $1,050.3$ & 997.6 \\
\hline 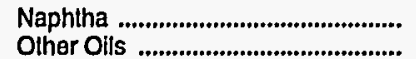 & $\begin{array}{l}239.3 \\
171.1\end{array}$ & $\begin{array}{l}683.3 \\
793.1\end{array}$ & $\begin{array}{l}3,173.1 \\
6,564.0\end{array}$ & $\begin{array}{l}1,478.5 \\
3,728.8\end{array}$ & $\begin{array}{l}1,164.1 \\
2,449.3\end{array}$ & $\begin{array}{l}1,459.4 \\
2,742.1\end{array}$ & $\begin{array}{l}1,222.6 \\
2,360.0\end{array}$ & $\begin{array}{l}1,636.6 \\
2,704.1\end{array}$ & $\begin{array}{l}1,811.3 \\
4,621.6\end{array}$ & $\begin{array}{l}1,335.5 \\
4,350.1\end{array}$ \\
\hline 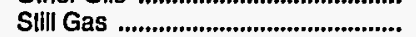 & 32.4 & 123.9 & 371.0 & 256.2 & - & - & 2,0 & $2,80+1$ & $4,0<1.0$ & $4,00$. \\
\hline 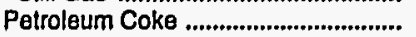 & 203.5 & 654.6 & 947.2 & 241.2 & 189.9 & 282.9 & 283.1 & 324.3 & 477.7 & 368.2 \\
\hline Special Naphthas ............................ & 323.0 & 450.3 & $2,022.4$ & $1,732.8$ & $1,393.8$ & $1,553.9$ & $1,236.5$ & $1,072.7$ & $1,040.4$ & 854.8 \\
\hline 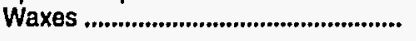 & 105.6 & 166.4 & 395.3 & 419.9 & 449.7 & 453.2 & 403.8 & 608.5 & 491.0 & 573.7 \\
\hline Total $\ldots \ldots \ldots \ldots \ldots \ldots \ldots$ & $1,171.2$ & $3,600.9$ & $15,271.5$ & $9,165.7$ & $6,329.3$ & $7,334.5$ & $6,343.8$ & $7,290.5$ & $9,492.2$ & $8,479.9$ \\
\hline
\end{tabular}

- Consumption data for this series are not available after 1985. See text.

Note: Expenditure totals may not equal sum of components due to independent rounding.

Source: State Energy Price and Expenditure Data System 1991. 



\section{Section 5. Electricity}

Electricity prices are retail prices for sales to ultimate users. Prices are developed for the residential, commercial, industrial, and transportation sectors. In general, taxes are included in the prices. However, taxes collected by a utility from an end user and turned over to a government authority are generally not included in the revenues reported in the Electric Power Annual or the Statistical Yearbook and are not included in the prices. Taxes paid by the utility (rather than the end user) are considered operating costs and are passed on to the end user as part of the rate. Therefore, Federal, State, business, and property taxes are typically included in the prices, while sales and other point-of-purchase taxes usually are not.

Consumption is based on sales by electric utilities to ultimate users. Electricity consumption data by State for the residential, commercial, industrial, and transportation sectors are obtained from the State Energy Data System (SEDS). Consumption of electricity in the industrial sector is adjusted for estimated refinery use in each State. (See the discussion in Section 6 "Consumption Adjustments for Process Fuel and Intermediate Products," on page 251.)

\section{Conversion Factor: All Years}

\section{3,412 Btu per kilowatthour}

\section{Physical Unit Prices: 1987 Forward}

For 1987 forward, physical unit prices for States are calculated for all four sectors as the average revenue per unit of sales for all electric utilities reported in a State. The calculation of physical unit prices uses the revenues and sales data from the Electric Power Annual. Data for the residential and industrial sectors are drawn from their respective columns. Data for the transportation sector are from the column titled "Other" that includes sales to railroads and railways. SEDS consumption is used to determine the portion of the data in the column titled "Other" that is not sold to the transportation sector. These data are added to the column titled "Commercial" to arrive at the revenues and sales data for the commercial sector.

For 1987 forward, prices are based on preliminary revenues and sales data in the given year, and are replaced with revised data in the following year. There are no missing prices in any of the four sectors.

\section{Physical Unit Prices: 1970 Through 1986}

For 1970 through 1986, preliminary physical unit prices for States are calculated for all four sectors as the average revenue per unit of sales for all electric utilities reported in a State. The calculation of physical prices uses the revenues and sales data from the Statistical Yearbook for each year in the series. Data for the residential sector and industrial sector are drawn from their respective columns. The commercial sector is the sum of the columns titled "Commercial," "Street and Highway Lighting," "Other Public Authorities," and "Interdepartmental." The transportation sector is the column titled "Railroads and Railways."

For 1980 through 1986, prices are based on preliminary revenues and sales data in the given year, and are replaced with revised data in the following year. The only exception to this rule is the revenues data for AR/1981; preliminary data are used in this case because of an apparent error in the revised data.

For 1970 through 1981, MD prices are assigned to DC. There are no other missing prices for the residential, commercial, and industrial sectors.

In the transportation sector, numerous price assignments are made due to the lack of sector-specific price data. Generally, electricity usage in the transportation sector is small; the sector's electricity use ranged from 0.1 percent to 0.2 percent of total U.S. electricity consumption in 1970 through 1986. From 1970 through 1986, only 15 States used measurable amounts of electricity in the transportation sector 
(CA, DC, FL, GA, IL, LA, MA, MD, NJ, NY, OH, PA, TN, VA, and WA). A few individual State prices are unavailable and are assigned the commercial sector prices: LA/1970 through 1986, and TN/1970 through 1986. (Prices are available for LA/1970, 1972, 1973, but those prices are replaced by commercial sector prices to maintain a consistent series for the State.) In addition, MA/1985, 1986 transportation prices are estimated by multiplying the MA/1985, 1986 commercial prices by the average of the ratios of the commercial-to-transportation sector prices for 1980 through 1984. Similarly, the VA/1977 transportation price is estimated by multiplying the VA/1977 commercial price by the average of the ratios of the commercial-to-transportation sectors prices for 1978 through 1982.

In order to reconcile national-level electricity prices based on the Statistical Yearbook with the EIA national-level electricity prices published in the Annual Energy Review (AER), yearly adjustment factors are calculated for the residential, commercial, and industrial sectors, as follows: a preliminary U.S. price for each sector is calculated as the average of the State prices, weighted by SEDS consumption. These preliminary U.S. prices are divided by the national-level electricity prices published in the $A E R$, and the quotient is used as an adjustment factor. The preliminary State prices are multiplied by the adjustment factor to produce the final physical unit State prices in those sectors. Since no transportation sector prices are published in the $A E R$, no adjustments are made to that sector and the final physical unit prices are derived solely from the Statistical Yearbook sales and revenue data. The annual adjustment factors for the residential, commercial, and industrial sectors are shown in Table A42.

\section{Btu Prices: All Years}

Btu prices for States are calculated by dividing the physical unit prices by the conversion factor. U.S. Btu prices are calculated as the average of the State Btu prices, weighted by consumption data from SEDS, adjusted for process fuel consumption in the industrial sector.

\section{Data Sources}

\section{Prices}

1987 forward: Energy Information Administration, Electric Power Annual 1988, Tables 19 and 21 (1987 data); Electric Power Annual, Tables 27 and 29 (19881990) and Tables 26 and 28 (1991).
Table A42. Annual Electricity Price Adjustment Factors

\begin{tabular}{cccc}
\hline Year & Residential & Commercial & Industrial \\
\hline 1970 & 1.05121 & 1.05712 & 1.06832 \\
1971 & 1.05632 & 1.05926 & 1.05504 \\
1972 & 1.05271 & 1.05514 & 1.05765 \\
1973 & 1.06626 & 1.06188 & 1.05991 \\
1974 & 1.09572 & 1.08098 & 1.08732 \\
1975 & 1.09257 & 1.08098 & 1.08732 \\
1976 & 1.07753 & 1.07755 & 1.06891 \\
1977 & 1.06746 & 1.07675 & 1.06820 \\
1978 & 1.06654 & 1.08273 & 1.06861 \\
1979 & 1.06986 & 1.08349 & 1.06441 \\
1980 & 1.04457 & 1.06109 & 1.06781 \\
1981 & 1.05821 & 1.06943 & 1.06523 \\
1982 & 1.06654 & 1.06351 & 1.05597 \\
1983 & 1.05421 & 1.05301 & 1.05537 \\
1984 & 0.99693 & 1.01924 & 0.99015 \\
1985 & 1.00010 & 1.02008 & 0.98355 \\
1986 & 0.99854 & 1.01518 & 0.98618 \\
& & & \\
\hline
\end{tabular}

Source: ElA calculations based on data from the Annual Energy Review and the Statistical Yearbook of the Electric Utility Industry.

1970-1986: Edison Electric Institute, Statistical Yearbook of the Electric Utility Industry, tables titled, "Revenues: Total Electric Utility Industry" and "Energy Sales: Total Electric Utility Industry."

1970-1986: Energy Information Administration, Annual Energy Review 1989, Table 95, "Retail Prices of Electricity Sold by Electric Utilities, 1960-1989."

\section{Consumption}

1970 forward: Energy Information Administration, State Energy Data System, electricity consumption by end-use sector.

\section{Nuclear Fuel for Generation of Electricity}

Nuclear fuel prices are developed for the electric utility sector. State-level data on the amount of electricity generated from nuclear power plants are from SEDS. Utilities operating nuclear plants report fuel costs per kilowatthour to the U.S. Department of Energy annually. These data are published on a plant-by-plant basis and include all taxes, transportation, and handling costs paid by the utilities. The 
State where the nuclear power plant is located is assigned the reported nuclear fuel cost. For States with two or more nuclear power plants, the average fuel cost, weighted by generation, is attributed to the State.

\section{Physical Unit Prices: All Years}

State-level nuclear fuel prices are estimated in two steps: (1) the total cost of fuels consumed at all nuclear power plants in a State is divided by their total generation of electricity, and (2) the cost per output created in step 1 is divided by the U.S.-level thermal conversion factor to create the price in dollars per million Btu. Occasionally, the fuel costs at nuclear power plants include small amounts of non-nuclear fuels, which are necessary to continue essential plant operations during refueling or maintenance of the reactor.

A price is estimated by another method for States where generation and fuel cost data are not available for years in which the State Energy Data Report (SEDR) shows nuclear electric power generation.

The ratio of the change in the national nuclear fuel price is used to interpolate or to project nuclear fuel prices for missing years within a State. The ratio of national prices used in the interpolation or projection of missing prices is the ratio before missing prices are assigned. The States and years for which nuclear prices are estimated are shown in Table A43.

CO/1985 and TN/1986, 1987 generation levels are negative, indicating that the nuclear power plants

Table A43. Nuclear Electricity Fuel Price Estimates, 1970 Forward

\begin{tabular}{ll}
\hline \multicolumn{1}{c}{ State } & \multicolumn{1}{c}{ Years } \\
\hline Alabama & $1973,1974,1976$ \\
Arizona & 1985 \\
Arkansas & 1980 \\
Colorado & $1977,1978,1982-1984,1986-1989$ \\
Georgia & 1974 \\
Maine & 1972 \\
Mississippi & 1984 \\
Missouri & 1984,1985 \\
North Carolina & 1982 \\
Ohio & 1986 \\
Oregon & 1975 \\
South Carolina & 1970,1985 \\
Tennessee & 1980 \\
Washington & $1970-1987$ \\
Wisconsin & 1970
\end{tabular}

used more energy than they supplied. In these cases the fuel prices and expenditures are set to zero. In 1986 , there is generation in CO but price data are not available. The CO/1986 price is estimated using the ratio of the 1984-to-1986 U.S. average prices and applying that ratio to the CO/1984 price.

For MO/1985, a large credit resulting from litigation is assigned to fuel costs, creating an artificially low price. The MO/1986 uranium price, which is in the range of other nuclear fuel plants, is used to estimate the 1985 price by applying the ratio of the 1986-to1985 national prices.

The 1985 Historical Plant Costs and Annual Production Expenses for Selected Electric Plants has a footnote for the Duke Power Catawba plant in SC stating that the reported production expenses represent only 12.5 percent of the actual production expenses. The production expenses used in the calculation for the Catawba plant are adjusted accordingly.

Prices for GA/1978, NC/1982, and $\mathrm{OH} / 1986$ are assigned because the prices calculated using reported data are significantly different from prices for other years for these States.

There are no prices available for WA/1970-1987; national prices are assigned for those years.

\section{Btu Prices: All Years}

Unit prices for nuclear fuel in dollars per kilowatthour of energy output are converted to dollars per million Btu of equivalent energy input using the annual thermal conversion factors shown in Table A44.

\section{Data Sources}

Prices

1988 forward: Energy Information Administration, Electric Plant Cost and Power Production Expenses, Table 16 (1988-1990) and Table 14 (1991).

1982-1987: Energy Information Administration, Historical Plant Costs and Annual Production Expenses for Selected Electric Plants, Table 18 (1982-1984), and Table 20 (1985-1987).

1979-1981: Energy Information Administration, Thermal Electric Plant Construction Cost and Annual Production Expenses, pages 267-279 (1979), Table 11 (1980 and 1981). 


\begin{tabular}{|c|c|c|c|}
\hline & \multicolumn{3}{|c|}{$\begin{array}{l}\text { Nuclear Steam-Electric Thermal } \\
\text { Conversion Factors } \\
\text { (Thousand Btu per Kilowatthour) }\end{array}$} \\
\hline Year & $\begin{array}{c}\text { Conversion } \\
\text { Factor }\end{array}$ & Year & $\begin{array}{c}\text { Conversion } \\
\text { Factor }\end{array}$ \\
\hline 1970 & 10.977 & 1981 & 11.030 \\
\hline 1971 & 10.837 & 1982 & 11.073 \\
\hline 1972 & 10.792 & 1983 & 10.905 \\
\hline 1973 & 10.903 & 1984 & 10.843 \\
\hline 1974 & 11.161 & 1985 & 10.813 \\
\hline 1975 & 11.013 & 1986 & 10.799 \\
\hline 1976 & 11.047 & 1987 & 10.776 \\
\hline 1977 & 10.769 & 1988 & 10.743 \\
\hline 1978 & 10.941 & 1989 & 10.724 \\
\hline 1979 & 10.879 & 1990 & 10.680 \\
\hline 1980 & 10.908 & 1991 & 10.740 \\
\hline
\end{tabular}

1975-1978: Energy Information Administration, Steam Electric Plant Construction Cost and Annual Production Expenses, "Section II-Nuclear Plants."

1970-1974: Federal Power Commission, Steam Electric Plant Construction Costs and Annual Production Expenses, data sheets for Nuclear Plants (1970-1973), and "Section II-Nuclear Plants" (1974).

\section{Consumption}

1970 forward: Energy Information Administration, State Energy Data System, electricity generated by nuclear power.

\section{Conversion Factors}

1970 forward: Energy Information Administration, State Energy Data Report 1991, Consumption Estimates, Table D1, as shown in Table A44.

Table A45. Biomass Fuels Used at Electric Utilities at No Cost or For a Fee, 1989 Forward

\begin{tabular}{ll}
\hline State & Years \\
\hline California & $1989-1991$ \\
Connecticut & $1989-1991$ \\
Hawaii & 1989,1990 \\
Montana & $1989-1991$ \\
Ohio & $1989-1991$ \\
\hline
\end{tabular}

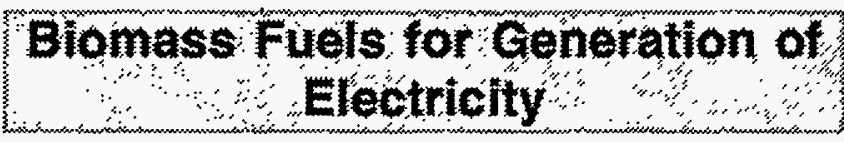

Biomass fuels prices are developed for the electric utility sector; data for biomass fuels consumed by other sectors are not available, except for ethanol added to motor gasoline. State-level data on the amount of electricity generated at electric utilities from biomass fuels are from SEDS (currently called "wood and waste" in SEDS) and are collected on Form EIA-759 (formerly, FPC-4). All electric utilities are required to report on EIA-759 but no price data are collected. State and national biomass fuels prices are developed for the electric utility sector from data reported on other EIA and Federal Energy Regulatory Commission (FERC) forms and from telephone surveys. Taxes are included in the prices for all years.

\section{Prices: All Years}

1989 Forward. State-level prices for biomass fuels used at electric utilities, in dollars per million Btu, are calculated from data obtained from FERC Form 1 and Form EIA-412 and by follow up telephone surveys of the electric utilities that are not required to submit those forms. For States with more than one utility using biomass fuels, a consumption-weighted average price is calculated. There are anomalies that are unique to biomass fuels used at utilities. In some cases, there is no charge for the fuel, and in some cases of municipal and industrial waste, the electric utilities charge a "tipping fee" for accepting truckloads of waste. Instead of paying for the fuel, the electric utility is paid to take the fuel. For States where all utilities pay nothing for the fuel or charge a fee for receiving it, listed in Table A45, a price of zero is assigned and no expenditure is included in calculating the average electric utility sector price for the State. The corresponding consumption is included in calculating the average price because biomass fuels are customarily "paid for" at the time of delivery, just like coal, natural gas, or petroleum, which are burned to produce electricity. The State and U.S. average electric utility fuel prices are, therefore, slightly lower because some of the fuel did not cost anything.

1983 Through 1988. The national price in dollars per million Btu is calculated from data obtained from FERC Form 1 and Form EIA-412 and by follow up telephone surveys of the electric utilities that report use of biomass fuels for generating electricity. The national price is the weighted-average price of those 
electric utilities able to provide price and consumption data and is assigned to all States.

Prices are erratic for biomass fuels used at utilities. In addition to the anomalies of no charge for the fuel and the "tipping fee" mentioned above, handling refuse-derived fuel is more labor intensive than handling conventional fossil fuels. The labor expenses are included in the plant's operating costs, not the fuel costs. Biomass fuels prices are also erratic because the demand is relatively small and the pricing mechanism, even for a single facility, may change from year to year. A price or quantity change by a single major user affects the national price more significantly than for any other fuel.

1978 Through 1982. National prices are derived from data collected on FPC Form 423 and published monthly by EIA in Cost and Quality of Fuels for Electric Utility Plants (CEQ) in a table titled "Wood Chips, Refuse, and Petroleum Coke Used as Fuel by SteamElectric Plants." For these years, fossil-fueled plants with a combined capacity of 25 megawatts or greater were required to report on FPC Form 423. Annual prices of biomass fuels sold to electric utilities are developed as quantity-weighted monthly prices for those plants where wood chips and refuse were used as fuel. Beginning in 1983, the reporting threshold was raised to 50 megawatts, and very few plants reported use of biomass fuels on the Form 423 in 1983 and subsequent years.

A detailed review of data in CEQ showed that some entries were in error by factors of 10,100 , or 1,000 . Accordingly, the following corrections were made. For 1982, the February, March, and April quantities for the Florida Power Corporation were divided by 1,000 to make them $0.08,0.04$, and 0.06 thousand short tons, respectively. The March, April, and May costs for Northern State Power were multiplied by 100 to make them $\$ 0.70$ per million Btu. For the 5 months from November 1979 through March 1980, the reported quantities of wood delivered to Burlington Electric Co. were divided by 10 , to place them in the range of 7.98 to 9.39 thousand short tons. For the 8 months from June 1978 through January 1979, seed corn delivered to the Logansport Indiana Electric Department were included in the biomass fuel. For
Table A46. Price Deflators Used for Biomass Fuels Prices, 1970-1977

\begin{tabular}{lccc}
\hline Years & Deflator & Years & Deflator \\
\hline 1970 & 35.1 & 1975 & 49.2 \\
1971 & 37.1 & 1976 & 52.3 \\
1972 & 38.8 & 1977 & 55.9 \\
1973 & 41.3 & 1978 & 60.3 \\
1974 & 44.9 & & \\
\hline
\end{tabular}

February 1978, the reported quantity of wood delivered to the United Power Associates was divided by 1,000 to make it 0.09 thousand short tons.

1970 Through 1977. The annual prices for wood chips are derived by deflating the 1978 price using the gross domestic product implicit price deflator based on 1987 dollars. The deflators are shown in Table A46.

\section{Data Sources}

Prices

1983 forward: Energy Information Administration, data reported on FERC Form 1, "Annual Report of Major Electric Utilities, Licensees and Others" and Form EIA-412, "Annual Report of Public Electric Utilities" and follow-up telephone surveys of the electric utilities that report use of biomass fuels for generating electricity.

1978-1982: Energy Information Administration, Cost and Quality of Fuels for Electric Utility Plants, table titled, "Wood Chips, Refuse, and Petroleum Coke Used as Fuel by Steam-Electric Plants."

1970-1978: Energy Information Administration, Annual Energy Review 1991, Appendix C, Gross Domestic Product and Implicit Price Deflator.

\section{Consumption}

1970 forward: Energy Information Administration, State Energy Data System, electricity generated from consumption of wood and waste. 



\section{Section 6. Consumption Adjustments for Process Fuel and Intermediate Products}

\begin{abstract}
Almost all aspects of energy production, processing, and distribution consume energy as an inherent part of those activities. Familiar examples include drilling for oil and gas, refining crude oil into finished petroleum products, transporting natural gas and petroleum by pipeline, and generating and delivering electricity to end users. Energy that is consumed to make energy more useable to the end user is called "process fuel." Energy products that are subsequently incorporated into another energy product for end-use consumption are called "intermediate products." Motor gasoline blending components are a familiar example of intermediate products which are consumed as part of the finished motor gasoline sold at service stations and other outlets.
\end{abstract}

The end user does not consume either process fuel or intermediate products directly, but he does pay for them, because the cost to the processor or distributor is passed on to the end user. In estimating energy expenditures, as is done in this report, process fuel and intermediate product consumption must be subtracted from the end-use sector in which they are included, either the industrial or transportation sector. If their use is not removed, the expenditures would be counted twice, first by the "processor" (producer, processor, or transporter) and again by the end user.

Process fuel consumption adjustments are incorporated into the data tables in this report, SEPER, for:

1. Fuel (petroleum, natural gas, coal) and electricity consumed at refineries

2. Crude oil lease, plant, and pipeline fuel

3. Natural gas lease and plant fuel

4. Natural gas pipeline fuel

5. Electrical system energy losses (i.e., energy consumed in the generation, transmission, and distribution of electricity)

Intermediate product consumption adjustments are incorporated into SEPER for:
1. Aviation gasoline blending components

2. Motor gasoline blending components

3. Natural gasoline (1970 through 1983)

4. Pentanes plus (1984 forward)

5. Plant condensate (1970 through 1983)

6. Unfinished oils

7. Unfractionated stream (1970 through 1983)

Starting in 1984, natural gasoline (including isopentane) and plant condensate are reported together as the new product, pentanes plus, and the components of unfractionated stream are reported separately under liquefied petroleum gases.

Table A48 shows estimates of the quantities of energy consumed by State as process fuel and intermediate products in 1990 and 1991. Estimates for 1970 through 1991 are available on the State Energy Price and Expenditure Data System (SEPEDS) personal computer diskettes.

\section{Procedures}

Refinery Fuel. Refinery fuel consumption of distillate fuel, residual fuel, liquefied petroleum gases, other petroleum (crude oil, petroleum coke, and still gas), natural gas, coal, and electricity is individually estimated for each source and subtracted from its State Energy Data System (SEDS) industrial sector total. Because crude oil consumption is not an individual fuel in SEDS for 1970 through 1982, the small amounts of crude oil that were used at refineries during those years were allocated to residual and distillate fuels consumed at refineries. The allocation from crude oil refinery use to residual and distillate fuels refinery use was made according to each fuel's share of the total crude oil used directly (including losses) as residual and distillate fuels (Petroleum Supply Annual Volume 1, Table 2). Over this period, residual fuel ranged from a low share of 75 percent in 1971 to 'a high share of 92 percent in 1976. Refinery consumption of still gas, excluding still gas consumed as petrochemical feedstocks, is subtracted from the SEDS industrial sector total for 1970 through 1985. Beginning in 1986, EIA data series no 
longer report refinery fuel and feedstock use separately, and all SEDS industrial still gas consumption is removed from SEPER.

Refinery fuel consumption data are available in the data sources by Petroleum Administration for Defense District (PADD) (1981 forward) and by State or group of States (1970 through 1980). Where Statelevel consumption data are not available, the State-level estimates are derived by allocating the district's or group's total consumption to the individual States within the district or group to the States that had operating refineries in a given year.

Table A47. Reallocations of Excess Refinery Fuel Consumption

\begin{tabular}{|c|c|c|c|c|}
\hline Year & Fuel & $\begin{array}{c}\text { Thousand } \\
\text { Barrels }\end{array}$ & $\begin{array}{c}\text { Excess } \\
\text { In: }\end{array}$ & $\begin{array}{c}\text { Re- } \\
\text { allocated }\end{array}$ \\
\hline 1971 & $\begin{array}{l}\text { Residual } \\
\text { Fuel }\end{array}$ & 294 & Kansas & Oklahoma \\
\hline 1973 & $\begin{array}{l}\text { Residual } \\
\text { Fuel }\end{array}$ & 45 & $\begin{array}{l}\text { Group 4: } \\
\text { Kentucky, } \\
\text { Tennessee }\end{array}$ & Illinois \\
\hline 1979 & LPG & 173 & Montana & Wyoming \\
\hline 1983 & $\begin{array}{l}\text { Petroeum } \\
\text { Coke }\end{array}$ & 3,091 & PADD I & PADD II \\
\hline 1984 & $\begin{array}{l}\text { Crude Oil } \\
\text { Petroleum } \\
\text { Coke }\end{array}$ & $\begin{array}{r}80 \\
992\end{array}$ & $\begin{array}{l}\text { PADD II } \\
\text { PADD I }\end{array}$ & $\begin{array}{l}\text { PADD III } \\
\text { PADD II }\end{array}$ \\
\hline \multirow[t]{3}{*}{1985} & $\begin{array}{l}\text { Petroleum } \\
\text { Coke }\end{array}$ & 80 & PADD II & PADD III \\
\hline & Crude Oil & 719 & PADD I & PADD ॥ \\
\hline & $\begin{array}{l}\text { Residual } \\
\text { Fuel }\end{array}$ & 212 & PADD IV & PADD V \\
\hline \multirow[t]{2}{*}{1986} & $\begin{array}{l}\text { Petroleum } \\
\text { Coke }\end{array}$ & 1,297 & PADD I & PADD II \\
\hline & $\begin{array}{l}\text { Residual. } \\
\text { Fuel }\end{array}$ & 403 & PADD IV & PADD V \\
\hline 1987 & $\begin{array}{l}\text { Residual } \\
\text { Fuel }\end{array}$ & 497 & PADD IV & PADD V \\
\hline 1988 & $\begin{array}{l}\text { Crude Oil } \\
\text { Residual } \\
\text { Fuel }\end{array}$ & $\begin{array}{r}8 \\
305\end{array}$ & $\begin{array}{l}\text { PADD III } \\
\text { PADD IV }\end{array}$ & $\begin{array}{l}\text { PADD V } \\
\text { PADD V }\end{array}$ \\
\hline 1989 & $\begin{array}{l}\text { Crude Oil } \\
\text { Crude Oil } \\
\text { Residual } \\
\text { Fuel }\end{array}$ & $\begin{array}{r}9 \\
15 \\
381\end{array}$ & $\begin{array}{l}\text { PADD I } \\
\text { PADD III } \\
\text { PADD IV }\end{array}$ & $\begin{array}{l}\text { PADD V } \\
\text { PADD V } \\
\text { PADD V }\end{array}$ \\
\hline 1990 & $\begin{array}{l}\text { Residual } \\
\text { Fuel }\end{array}$ & 332 & PADD IV & PADD V \\
\hline \multirow[t]{2}{*}{1991} & $\begin{array}{l}\text { Petroleum } \\
\text { Coke }\end{array}$ & 595 & PADD I & PADD II \\
\hline & $\begin{array}{l}\text { Residual } \\
\text { Fuel }\end{array}$ & 374 & PADD IV & PADD V \\
\hline
\end{tabular}

Source: ElA calculations based on data from the State Energy Data Report and the Petroleum Supply Annual.
Individual fuels are allocated to the refining States according to each State's share of the refining States' total industrial sector fuel consumption during the year. In some instances, estimated refinery fuel consumption exceeds the SEDS estimate for total industrial consumption of a fuel within a State. When this occurs, the excess refinery fuel consumption is reallocated as shown in Table A47.

Intermediate Products. Aviation gasoline blending components, motor gasoline blending components, natural gasoline (1970 through 1983), pentanes plus 1984 forward), plant condensate (1970 through 1983), unfinished oils, and unfractionated stream (1970 through 1983) are used at refineries and blending plants to make end-use petroleum products, particularly motor gasoline. Accordingly, consumption of these products is completely removed from SEPER.

Crude Oil Lease, Plant, and Pipeline Fuel. Industrial crude oil remaining after refinery fuel consumption is removed is assumed to be used as lease, plant, and pipeline fuel. Since these are process fuel uses, the remaining crude oil is removed from SEDS industrial sector consumption.

Natural Gas Lease and Plant Fuel. Natural gas consumed as lease and plant fuel is process fuel and is subtracted from SEDS industrial sector natural gas totals by State and year.

Natural Gas Pipeline Fuel. Transportation sector natural gas consumption shown in SEDS is used to power pipelines. As such, it is process fuel and is completely removed from the SEPER expenditure calculations.

Electrical System Energy Losses. The amount of energy lost during generation, transmission, and distribution of electricity (including plant use and unaccounted for electrical energy) is process fuel and is subtracted from sectoral energy consumption estimates used in SEPER. The energy losses are "paid for" when residential, commercial, industrial, and transportation sector consumers buy the electricity produced at electric utilities.

\section{Data Sources}

Capacity of Petroleum Refineries. 1982 forward: Energy Information Administration, Petroleum Supply Annual Volume 1, table titled, "Number and Capacity of Operable Petroleum Refineries," column heading, "Crude Capacity, Barrels per Calendar Day, Operating" (1982-1985), "Atmospheric Crude Oil 
Distillation Capacity, Barrels per Calendar Day, Operating" (1986 forward).

1979-1981: Energy Information Administration, Energy Data Reports, Petroleum Refineries in the United States and U.S. Territories, table titled, "Number and Capacity of Petroleum Refineries," column heading, "Crude Capacity, Barrels per Calendar Day, Operating."

1978: Energy Information Administration, Energy Data Reports, Petroleum Refineries in the United States and Puerto Rico, table titled, "Number and Capacity of Petroleum Refineries," column heading, "Crude Capacity, Barrels per Calendar Day, Operating."

1970-1977: Bureau of Mines, U.S. Department of the Interior, Mineral Industry Surveys, Petroleum Refineries in the United States and Puerto Rico, table titled, "Number and Capacity of Petroleum Refineries," column heading, "Crude Capacity, Barrels per Calendar Day, Operating."

Fuel Consumed at Refineries. 1981 forward: Energy Information Administration, Petroleum Supply Annual Volume 1, table titled, "Fuels Consumed at Refineries by PAD District." Data for 1991 are from a separately published ERRATA dated November 10, 1992, GPO Stock No. 061-003-00758-9.

1976-1980: Energy Information Administration, Energy Data Reports, Crude Petroleum, Petroleum Products, and Natural Gas Liquids, table titled, "Fuels
Consumed for All Purposes at Refineries in the United States, by States."

1970-1975: Bureau of Mines, U.S. Department of the Interior, Mineral Industry Surveys, Crude Petroleum, Petroleum Products, and Natural Gas Liquids, tables titled, "Fuels Consumed for All Purposes at Refineries in the United States, by States."

1970 forward: Energy Information Administration, State Energy Data System, industrial sector consumption estimates for aviation gasoline blending components, crude oil, motor gasoline blending components, natural gasoline (1970-1983), pentanes plus (1984 forward), petroleum coke, plant condensate (1970-1983), still gas (excluding still gas consumed as petrochemical feedstocks, 1970-1985), unfinished oil, and unfractionated stream (1970-1983).

Natural Gas Lease, Plant, and Pipeline Fuel Use. 1980 forward: Energy Information Administration, Natural Gas Annual, Table 10 (1980) and Table 13 (1981, 1982); Natural Gas Annual Volume 1, Table 13 (1983-1987) and Table 16 (1988); Natural Gas Annual, Table 16 (1989 forward).

1977-1979: Energy Information Administration, Energy Data Reports, "Natural Gas Production and Consumption," Table $6(1977,1978)$ and Table 5 (1979).

1970-1976: Bureau of Mines, U.S. Department of the Interior, Mineral Industry Surveys, "Natural Gas Production and Consumption," Table 5 (1970) and Table 6 (1971-1976). 


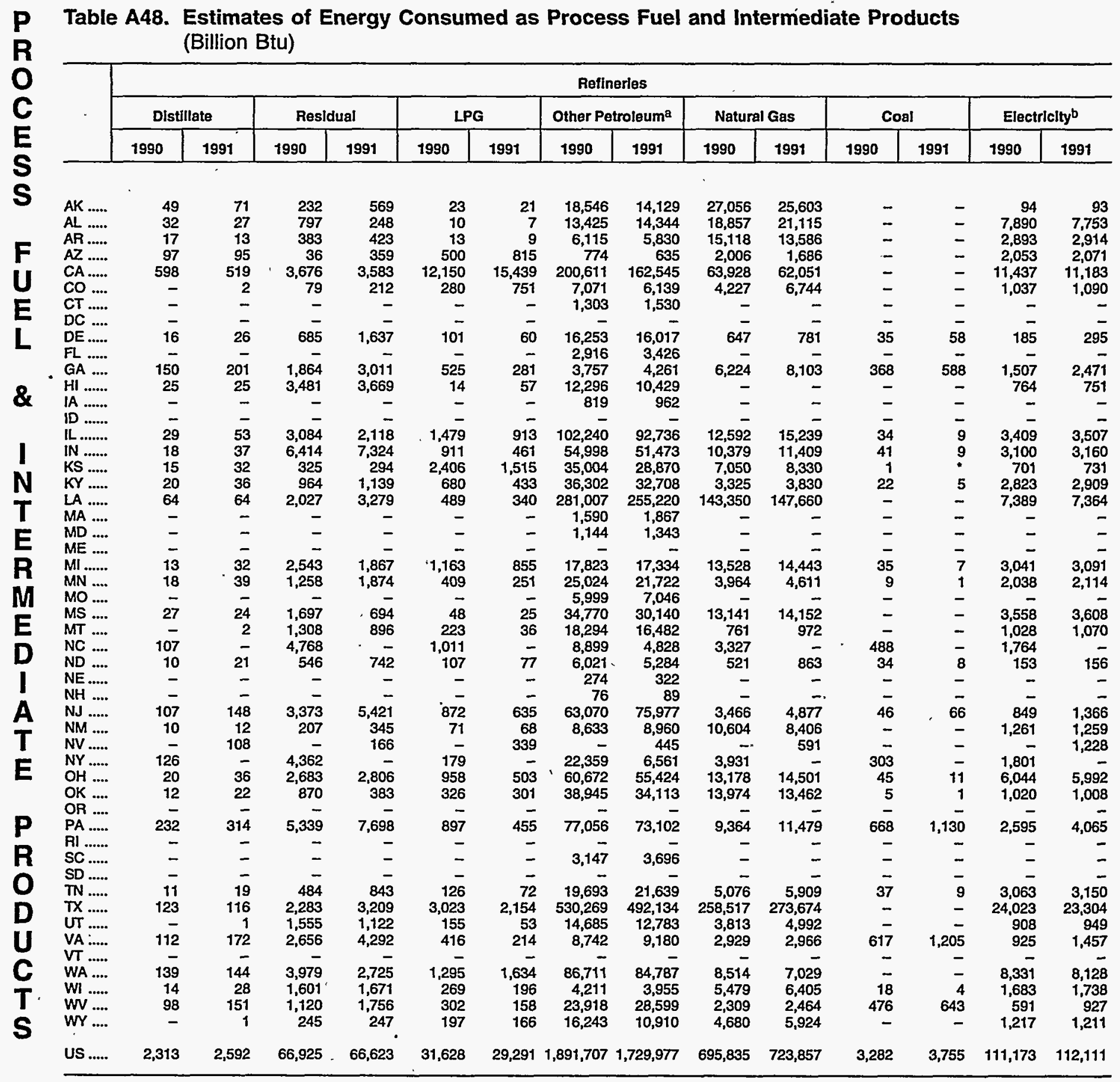

See footnotes at end of table. 
Table A48. Estimates of Energy Consumed as Process Fuel and Intermediate Praducts (Continued) (Billion Btu)

\begin{tabular}{|c|c|c|c|c|c|c|c|c|c|c|c|c|}
\hline & \multicolumn{2}{|c|}{$\begin{array}{c}\text { Total } \\
\text { Reflnerles }\end{array}$} & \multicolumn{2}{|c|}{$\begin{array}{l}\text { Crude Oll Lease, } \\
\text { Plant, and PIpeline Fuel }\end{array}$} & \multicolumn{2}{|c|}{$\begin{array}{c}\text { Natural Gas } \\
\text { Lease and Plant Fuel }\end{array}$} & \multicolumn{2}{|c|}{$\begin{array}{l}\text { Natural Gas } \\
\text { Plpeline Fuel }\end{array}$} & \multicolumn{2}{|c|}{$\begin{array}{c}\text { Electrical System } \\
\text { Energy Losses }\end{array}$} & \multicolumn{2}{|c|}{ Total } \\
\hline & 1990 & 1991 & 1990. & 1991 & 1990 & 1991 & 1990 & 1991 & 1990 & 1991 & 1990 & 1991 \\
\hline 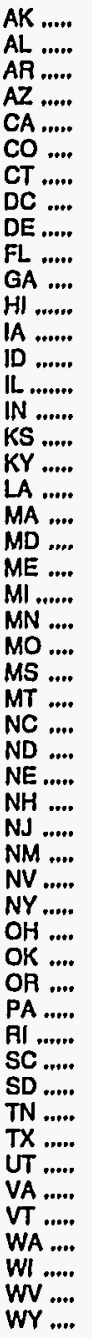 & $\begin{array}{r}46,001 \\
41,011 \\
24,539 \\
5,467 \\
292,400 \\
12,694 \\
1,303 \\
- \\
17,922 \\
2,916 \\
14,394 \\
16,581 \\
819 \\
122,868 \\
75,861 \\
45,503 \\
44,136 \\
434,326 \\
1,590 \\
1,144 \\
- \\
38,146 \\
32,720 \\
5,999 \\
53,241 \\
21,614 \\
20,365 \\
7,391 \\
274 \\
76 \\
71,781 \\
20,786 \\
- \\
33,062 \\
83,600 \\
55,153 \\
96,150 \\
108,969 \\
13,274 \\
28,812 \\
22,583\end{array}$ & $\begin{array}{r}40,486 \\
43,494 \\
22,774 \\
5,660 \\
255,319 \\
14,938 \\
1,530 \\
- \\
18,874 \\
3,426 \\
18,917 \\
14,930 \\
962 \\
114,575 \\
73,874 \\
39,772 \\
41,059 \\
413,926 \\
1,867 \\
1,343 \\
- \\
37,629 \\
30,612 \\
7,046 \\
48,643 \\
19,457 \\
4,828 \\
7,151 \\
322 \\
89 \\
88,491 \\
19,051 \\
2,878 \\
6,561 \\
79,273 \\
49,290 \\
98,240 \\
3,696 \\
104,448 \\
13,998 \\
34,699 \\
18,459\end{array}$ & 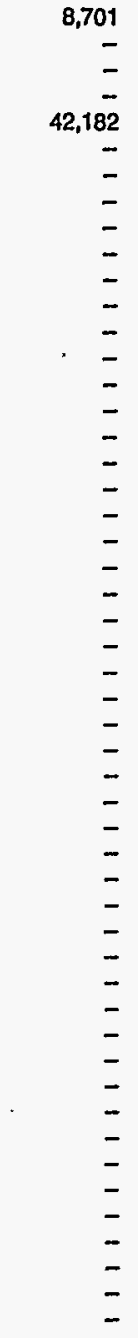 & 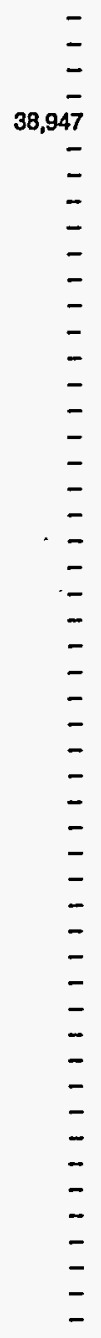 & $\begin{array}{r}183,832 \\
10,167 \\
7,155 \\
73 \\
23,432 \\
17,437 \\
- \\
- \\
- \\
1,865 \\
- \\
- \\
- \\
- \\
134 \\
13 \\
40,904 \\
3,275 \\
268,954 \\
- \\
1 \\
- \\
9,504 \\
- \\
- \\
7,489 \\
2,321 \\
- \\
7,161 \\
26 \\
-\end{array}$ & $\begin{array}{r}223,660 \\
8,861 \\
1,429 \\
46 \\
107,035 \\
24,594 \\
- \\
- \\
- \\
1,592 \\
- \\
- \\
- \\
- \\
145 \\
5 \\
43,782 \\
2,546 \\
204,913 \\
- \\
- \\
- \\
9,966 \\
- \\
- \\
4,724 \\
1,727 \\
- \\
12,116 \\
31 \\
- \\
- \\
3,310 \\
37,251\end{array}$ & $\begin{array}{r}1,620 \\
15,060 \\
8,708 \\
26,074 \\
20,770 \\
9,163 \\
502 \\
255 \\
4 \\
3,032 \\
7,540 \\
- \\
9,214 \\
5,223 \\
12,378 \\
8,583 \\
40,590 \\
25,610 \\
58,042 \\
1,277 \\
2,466 \\
5 \\
18,737 \\
12,065 \\
5,360 \\
38,941 \\
2,105 \\
6,482 \\
1,828 \\
3,480 \\
22 \\
2,690 \\
80,382 \\
816 \\
4,870 \\
10,381 \\
26,551 \\
9,221 \\
35,668 \\
121 \\
2,939 \\
112 \\
20,282 \\
110,494 \\
953 \\
7,235 \\
5 \\
5,205 \\
4,418 \\
9,316 \\
5,560\end{array}$ & $\begin{array}{r}2,602 \\
16,928 \\
8,478 \\
24,112 \\
19,003 \\
8,594 \\
539 \\
267 \\
4 \\
3,813 \\
7,633 \\
-7 \\
6,759 \\
4,740 \\
11,282 \\
4,683 \\
33,278 \\
20,923 \\
56,196 \\
1,077 \\
2,582 \\
3 \\
20,347 \\
13,493 \\
2,585 \\
35,696 \\
2,386 \\
6,406 \\
2,104 \\
2,333 \\
33 \\
2,990 \\
74,831 \\
353 \\
5,146 \\
9,445 \\
25,412 \\
9,090 \\
35,324 \\
165 \\
2,884 \\
344 \\
16,276 \\
85,219 \\
9,252 \\
6,914 \\
3 \\
8,912 \\
8,270\end{array}$ & $\begin{array}{r}38,625 \\
446,667 \\
203,972 \\
309,101 \\
1,573,420 \\
229,533 \\
202,645 \\
73,407 \\
61,745 \\
1,069,864 \\
599,575 \\
57,756 \\
219,417 \\
134,192 \\
831,658 \\
551,440 \\
202,358 \\
455,397 \\
475,738 \\
338,708 \\
369,209 \\
85,933 \\
613,937 \\
351,567 \\
401,937 \\
239,465 \\
97,827 \\
670,269 \\
52,279 \\
133,184 \\
66,931 \\
468,514 \\
103,016 \\
121,882 \\
963,944 \\
1,061,893 \\
316,813 \\
320,340 \\
855,315 \\
47,845 \\
414,811 \\
47,211 \\
575,013 \\
1,769,617 \\
114,799 \\
541,855 \\
35,153 \\
678,629 \\
366,707 \\
172,422 \\
87,721\end{array}$ & $\begin{array}{r}35,420 \\
454,034 \\
210,900 \\
310,329 \\
1,547,256 \\
233,268 \\
201,491 \\
75,603 \\
63,298 \\
1,085,162 \\
604,656 \\
50,156 \\
228,261 \\
133,818 \\
866,643 \\
571,247 \\
208,764 \\
476,032 \\
479,817 \\
332,192 \\
378,992 \\
84,409 \\
626,751 \\
361,542 \\
419,083 \\
244,857 \\
99,417 \\
684,576 \\
53,801 \\
137,968 \\
64,973 \\
479,656 \\
104,440 \\
123,286 \\
959,652 \\
1,080,135 \\
292,205 \\
323,694 \\
862,701 \\
47,482 \\
423,200 \\
49,574 \\
581,305 \\
1,782,338 \\
117,962 \\
556,990 \\
34,886 \\
687,521 \\
378,431 \\
175,192 \\
87,183\end{array}$ & $\begin{array}{r}278,779 \\
512,905 \\
244,374 \\
340,715 \\
1,952,204 \\
268,827 \\
204,450 \\
73,662 \\
79,672 \\
1,077,677 \\
621,510 \\
74,337 \\
229,450 \\
139,415 \\
967,037 \\
635,897 \\
329,355 \\
528,417 \\
1,237,061 \\
341,575 \\
372,820 \\
85,938 \\
680,323 \\
396,353 \\
413,296 \\
339,136 \\
123,866 \\
697,116 \\
68,659 \\
136,965 \\
67,029 \\
542,986 \\
273,788 \\
122,697 \\
1,002,740 \\
1,161,208 \\
512,317 \\
329,657 \\
993,388 \\
47,966 \\
420,897 \\
47,483 \\
623,862 \\
3,109,504 \\
158,336 \\
565,998 \\
35,158 \\
792,803 \\
384,399 \\
220,866 \\
161,381\end{array}$ & $\begin{array}{r}302,169 \\
523,316 \\
243,581 \\
340,147 \\
1,967,560 \\
281,394 \\
203,560 \\
75,869 \\
82,176 \\
1,093,992 \\
631,206 \\
65,087 \\
235,982 \\
138,558 \\
992,645 \\
649,809 \\
325,596 \\
540,560 \\
1,154,853 \\
335,137 \\
382,917 \\
84,412 \\
694,692 \\
405,646 \\
428,715 \\
333,920 \\
122,987 \\
695,810 \\
75,172 \\
140,654 \\
65,096 \\
571,137 \\
244,985 \\
126,573 \\
972,462 \\
1,170,395 \\
456,433 \\
332,846 \\
999,891 \\
47,648 \\
429,781 \\
50,308 \\
629,267 \\
2,994,877 \\
153,533 \\
583,730 \\
34,889 \\
797,220 \\
396,860 \\
227,113 \\
151,164\end{array}$ \\
\hline US ...... & $2,802,863$ & $2,668,207$ & 50,883 & 38,947 & $1,268,897$ & $1,183,556$ & 682,354 & 621,070 & $20,221,257$ & $20,472,547$ & $25,026,255$ & $24,984,327$ \\
\hline
\end{tabular}

a In this table, other petroleum consists of: crude oil, petroleum coke, and still gas consumed as process fuel; aviation gasoline blending components, motor gasoline blending components, pentanes plus, and unfinished oils used as intermediate products.

b Electricity is converted at the rate of 3,412 Btu per kilowatthour.

c Equals the sum of energy sources on the first page of this table.

- No consumption.

- Value less than 0.5 billion Btu.

Sources: Energy Information Administration. See "Consumption Adjustments for Process Fuel and Intermediate Products" in text. 
Appendix B

Summary of Changes 
,

, 


\section{Summary of Changes Since the State Energy Price and Expenditure Report 1990}

In this appendix, revisions in the State Energy Price and Expenditure Data System (SEPEDS) that appear in the tables of this State Energy Price and Expenditure Report (SEPER) are summarized by energy source. Revisions to prices and expenditures are noted, as well as revisions to consumption data that are used in the calculation of weighted-average prices. This appendix covers data revisions in all years contained in SEPEDS, 1970 through 1991, although only selected years are published in SEPER. Users of the SEPEDS personal computer (PC) diskettes, which contain data for all 22 years, may notice data changes that do not appear in the report due to the level of rounding in the published tables.

Price and expenditure revisions occur for several reasons: data sources for prices change; price estimation methodologies are revised or price assignment and estimation procedures are updated; data entries are corrected; or consumption estimates are revised. The first three kinds of changes affect State-level and U.S. prices directly. The fourth, a revised estimated consumption, affects only State prices that are estimated by consumption-weighted averaging of adjacent States and, similarly, affects U.S. average prices. Consumption estimates used in SEPER are from the State Energy Data System (SEDS), published as the State Energy Data Report 1991, Consumption Estimates (SEDR), which contains full documentation of the consumption estimation procedures. Consumption revisions are described in SEDR, Appendix E, "Summary of Changes." Since energy expenditure estimates depend on both the price and consumption estimates (including the consumption adjustments for process fuel and intermediate products), revision of either or both may cause revisions to the expenditures series.

\section{Coal}

Residential, Commercial, and Industrial Sectors in 1986 through 1990. Although State-level coal prices, with a few exceptions noted below, remained unchanged in all years, SEDS consumption revisions in the residential, commercial, and industrial sectors, as described in SEDR, Appendix E, caused small changes in consumption-weighted average industrial sector prices and U.S. average coal prices. In addition, most States' coal expenditures in the three sectors changed in 1986 through 1990 . The revisions are generally less than 1 percent and too small to be seen in the rounded data in SEPER tables, although they may be noticed by the users of SEPEDS PC diskettes.

SEDS residential and commercial coal consumption revisions included small quantities of coal in Louisiana in 1986 and Mississippi and Oklahoma in 1990, where there was previously estimated to be no consumption. The other significant commercial sector price change occurred for Alaska in 1990 where the price was revised from $\$ 2.45$ per million Btu to $\$ 2.76$ per million Btu. Large revisions in residential coal prices were caused by implementing different adjacent State price assignments for Montana in 1986 and for Arizona, Montana, and Nevada in 1990. The Montana prices decreased by 48 percent in 1986 and 75 percent in 1990 . The residential coal prices for $\mathrm{Ar}-$ izona and Nevada in 1990 increased by 69 percent and 22 percent, respectively.

ELA revised coal consumption at coke plants for 1986 forward, and SEDS made additional small revisions to estimated coal consumption by other industrial users. There are no revisions to industrial coal prices in SEPEDS with the exception of the Missouri coking coal price in 1988, where the previously estimated small quantity of coal consumption was changed to zero, and Rhode Island in 1990, where the previous consumption estimate of zero was revised to be a small quantity.

\section{Natural Gas}

Residential, Commercial, and Industrial Sectors in 1990. The 1990 industrial sector price for natural gas in Pennsylvania increased by $\$ 0.18$ million Btu due to a revision in the source data from the Natural Gas 
Annual. Numerous expenditures and several prices for 1990 were revised due to small consumption and conversion factor revisions in SEDS, as documented in SEDR, Appendix E. Most of these price and expenditure revisions are not visible in the published tables due to the level of rounding but may be noticed in the data on the SEPEDS PC diskettes. In the published SEPER, the 1990 Ohio residential natural gas price increased by $\$ 0.01$ per million Btu. Kentucky 1990 natural gas prices in both the residential and industrial sectors increased by $\$ 0.01$ per million Btu, and the commercial sector price increased by $\$ 0.02$ per million Btu.

\section{Petroleum}

\section{Lubricants}

Prices and Expenditures for 1970 through 1990. Small changes, generally less than 1 percent, occurred in lubricants prices, and therefore expenditures, for all States and end-use sectors in 1970 through $1990^{\circ}$ as a result of standardizing the price estimation calculations to use greater precision. Additional changes occurred in all lubricants price estimates for 1978 forward as a result of using the U.S. Department of Commerce, Bureau of Economic Analysis (BEA), 1977 trade-ratio factor to mark up producer prices to provide estimates for consumer prices. The previous edition of SEPER used the BEA 1982 trade-ratio factor in estimating the consumer prices for 1978 forward. The trade-ratio factor for 1982 (and later years) was replaced in SEPEDS because the range of petroleum products included in the ratio was expanded by $\mathrm{BEA}$ and no longer represents an appropriate markup for lubricants.

Larger revisions to lubricants price estimates, and resulting expenditures, occurred for all States in 1987, 1989 , and 1990 due to the incorporation of previously unavailable Bureau of the Census data on lubricant producer prices. Prices for all States and the U.S. average price decreased by 6 percent in 1987, 26 percent in 1989, and 39 percent in 1990.

\section{Residual Fuel}

Prices, 1987 through 1990. State prices for residual fuel in each of the consuming sectors are not revised; however, revisions to SEDS consumption in 1987 through 1990 as described in the SEDR, Appendix E, caused the State and U.S. consumption-weighted average prices for all residual fuel to be revised slightly, generally by less than 1 percent and often by amounts too small to be seen in the publication due to the level of rounding. The U.S. consumptionweighted average prices for the commercial and industrial sectors also were revised by 1 percent or less in 1987 through 1990.

Commercial, Industrial, and Transportation Sector Expenditures, 1987 through 1990 . The SEDS consumption revisions caused proportional State-level expenditure revisions in 1987 through 1990. Commercial sector expenditures also increased for all States, except Hawaii, by less than 1 percent. Hawaii commercial sector energy expenditures decreased by 96 percent in 1987, 46 percent in 1988, 54 percent in 1989, and 67 percent in 1990. The State-level commercial expenditure revisions caused the U.S. average to decrease by 3 percent in 1987 and 1988 and by 4 percent in 1989 and 1990. Industrial sector expenditures for residual fuel increased for all States and for the United States as a whole by 1 percent or less in 1987 through 1990, with the exception of Colorado, Montana, Utah, and Wyoming. In those States, because of consumption adjustments in SEPEDS for the refinery use of residual fuel, the expenditures were so small that, although the percentage changes were much larger, the values remained as asterisks in the SEPER tables. Transportation sector residual fuel expenditures for all States and the U.S. average increased by less than 1 percent in 1987 through 1990.

\section{Electricity}

\section{Electricity Consumed by End-Use Sectors}

Prices and Expenditures in 1990 . Electricity prices for the transportation and commercial sectors in 1990 were revised due to routine replacement of preliminary consumption values used in the price estimations for the District of Columbia and the 16 States with transportation electricity use as documented in SEDR, Appendix E. Electricity expenditures also revised in 1990 in proportion to the consumption changes. Most of the price and expenditure revisions are not visible in the published tables due to the level of rounding but may be noticed in the data on the SEPEDS PC diskettes. In the published SEPER, only two price changes can be seen-the Pennsylvania commercial sector price increased by $\$ 0.01$ per million Btu, and since the transportation consumption in Indiana was revised from zero to 36 billion Btu, the price changed from zero to $\$ 23.88$ per million Btu. 


\section{Biomass Fuels for Generation of Electricity}

Prices in 1989 and 1990. State-level prices for biomass fuels (previously called "wood and waste") burned at electric utilities were developed to replace the national average prices previously assigned to each State with biomass fuel consumption for 1989 and 1990. Five States, California, Colorado, Connecticut, Hawaii, and Montana had utilities that burned biomass fuels acquired at no cost or that charged a fee to receive the fuel. A price of zero was assigned to those States. Price revisions were quite significant in the other eight States with biomass fuels consumption. The revisions from the national average price of $\$ 0.55$ per million Btu previously used for all States in 1990 ranged from $\$ 0.35$ per million Btu for nitrogen consumed in Texas to $\$ 2.82$ per million Btu for the wood burned in Vermont. Similar revisions to prices occurred in 1989.

Expenditures in 1984 through 1990. Expenditures in 1989 and 1990 for biomass fuels burned at electric utilities were affected by the price changes described above. In addition, revisions to the Btu conversion factors in SEDS for 1984 through 1990 as described in $S E D R$, Appendix E, caused consumption and, therefore, SEPEDS expenditure changes for those years. Since a national average conversion factor was used for all States, each State's expenditures increased by the same percentage each year. The percentage increases were small-by 1 percent in 1984, 1985, and 1988 and by 2 percent in 1986 and 1987.

\section{Process Fuel and Intermediate Products}

Changes in consumption of process fuel and intermediate products in this edition of SEPER were caused, primarily, by industrial sector consumption revisions that affected the State allocations of various energy sources consumed by refineries. Due to the level of rounding in the published tables, some revisions are only noticeable on the SEPEDS PC diskettes. Diskette users may also detect changes of less than 0.01 percent that occurred due to refinements in calculation procedures.

\section{Refinery Fuel}

Other Petroleum. A change in the allocation factor for refinery use of crude oil in Petroleum Administration for Defense (PAD) District $V$ affected the overall consumption of other petroleum at refineries in Alaska and California in 1989 and 1990. The consumption changes are too small to be seen in SEPER
Table A48, but may be noticed in the data on the SEPEDS PC diskettes.

Natural Gas. Changes in 1990 industrial sector natural gas consumption described in Appendix E of $S E D R$ also impact refinery fuel consumption estimates used in SEPER. As indicated in the discussion in Section 6, "Consumption Adjustments for Process Fuel and Intermediate Products" on page 251, a change in total industrial sector natural gas consumption in any State can effect the allocation of refinery consumption of natural gas in every State in that State's PAD district. Thus, the SEPER State-level allocations changed in 30 of the 34 States that used natural gas as refinery fuel in 1990 . Only the four refining States in PAD District IV (Colorado, Montana, Utah, and Wyoming) did not change. All of the changes are less than 3.5 percent except for Michigan's increase of 13.3 percent.

Coal. Revisions to industrial sector coal consumption as described in SEDR, Appendix E, for 1986 through 1990 are reflected in the allocation of refinery coal usage to 19 States located in PAD Districts I, II, and III during each of those years. All of the changes are less than 1 percent and will be visible only to users of SEPEDS PC diskettes.

\section{Natural Gas Lease and Plant Fuel}

Changes in 1990 industrial sector natural gas consumption described in SEDR, Appendix $\mathrm{E}$, include the use of natural gas as lease and plant fuel. Consumption for five States and the United States total are affected, all less than 1 percent, except for Michigan, which increased by 13.2 percent.

\section{Crude Oil Lease, Plant; and Pipeline Fuel}

Recalculation of SEDS crude oil consumption estimates in greater precision caused estimates of crude oil used as lease and plant fuel in Alaska and California to change during 1989 and 1990 . The changes are less than 0.1 percent in both States for both years.

\section{Electrical System Energy Losses}

Changes in the fossil-fueled steam-electric power plant conversion factors for 1984 through 1989 described in SEDR, Appendix $\mathrm{E}$, are reflected in the electrical system energy losses shown as process fuel in SEPER. As noted in SEDR, changes in all States and all years are by less than 1 percent. 
Appendix C

\section{Additional \\ Measures of Energy Consumption, Expenditures, and Prices}




.

-

. 


\section{Additional Measures of Energy Consumption, Expenditures, and Prices}

The annual editions of the Energy Information Administration (EIA) State Energy Price and Expenditure Report (SEPER) provide detailed estimates of energy prices and expenditures, with expenditures given in nominal dollars. Energy expenditures are derived as the product of energy prices and energy consumption with the latter adjusted to exclude energy used or lost by energy industries. The fully adjusted measure of energy consumption is designated as net energy consumption and represents the energy sold to end users of energy in the domestic economy. The energy price and net energy consumption data underlying the current dollar energy expenditure estimates can also be used to derive additional energy-related measures: energy expenditures in constant 1987 dollars and a fixed-weight energy price index. These measures complement, but are not intended to replace, existing EIA data series.

In this appendix, the methodology used to derive the additional energy-related measures is presented and the measures are used to examine changes in energy consumption, expenditures, and prices throughout the period of 1970 through 1991. For purposes of analysis, information is presented for three shorter periods, as well as for the 22-year period. The first period, 1970 through 1973, was chosen to allow analysis of energy use and its relation to Gross Domestic Product (GDP) over a base period, before the sharp price increases that began in the last months of 1973. The second period, 1973 through 1981, includes the years of rapid price increases, which led to efforts to conserve energy and increase energy efficiency. The third period, 1981 through 1991, was selected to see whether the decline in real energy prices after 1981 resulted in a moderation of efforts to increase energy efficiency, relative to the earlier periods.

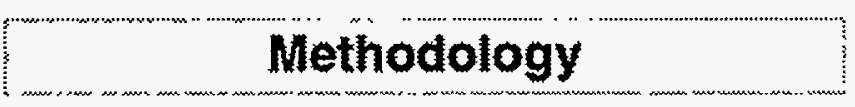

Net Energy Consumption

The net energy consumption data ${ }^{1}$ used in SEPER differ from the total energy consumption data currently published by EIA. The total (gross) consumption data include all energy sources before deduction of intermediate energy used or lost by energy industries in producing, processing, converting, generating, and distributing energy to end users. The net consumption data exclude certain categories of energy use, as outlined below. The major category of energy used or lost by energy industries is the losses incurred in the generation, transmission, and distribution of utility electricity. Additional important losses include a power plant's own use of electricity, unaccounted-for electric system energy losses, fuels and purchased electricity consumed by petroleum refineries, natural gas lease and plant fuel, natural gas used as pipeline fuel, and crude oil consumed as lease, plant, and pipeline fuel.

In addition to the exclusion of energy used or lost, two other adjustments are made to the total energy consumption data: industrial hydroelectricity (used as a power source by the company generating the power, and not selling it) is excluded, and several intermediate petroleum products, which are used in the production of other petroleum products or reflect petroleum supply-consumption balance entries, are also excluded. The fully adjusted measure thus derived is designated "net energy consumption" (Table C1). Table C7 in this appendix shows the reconciliation of total (gross) and net energy consumption data.

\footnotetext{
${ }^{1}$ The detailed estimates of net energy, classified by energy form and major end-use-sector, at both State and national levels, are included in the State Energy Price and Expenditure Data System. The net energy estimates are based on adjustments to total energy consumption data in EIA's State Energy Data Report 1991, Consumption Estimates, DOE/EIA-0214(91) (Washington, DC, May 1993).
} 


\section{A Constant-Dollar Energy Expenditures}

D

Table C1. Energy Consumption, 1970-1991

\begin{tabular}{|c|c|c|c|}
\hline Year & $\begin{array}{c}\text { Total } \\
\text { (trillion Btu) }\end{array}$ & $\begin{array}{c}\text { Net } \\
\text { (trillion Btu) }\end{array}$ & $\begin{array}{l}\text { Net/Total } \\
\text { (percent) }\end{array}$ \\
\hline $\begin{array}{c}1970 \ldots . . . . \\
1971 \ldots . . . \\
1972 \ldots . . . \\
1973 \ldots . . . \\
1974 \ldots . . .\end{array}$ & $\begin{array}{l}66,334 \\
67,789 \\
71,275 \\
74,351 \\
72,528\end{array}$ & $\begin{array}{l}50,138 \\
50,968 \\
53,273 \\
55,344 \\
53,409\end{array}$ & $\begin{array}{l}75.6 \\
75.2 \\
74.7 \\
74.4 \\
73.6\end{array}$ \\
\hline $\begin{array}{l}1975 \ldots . . . . \\
1976 . . . . . \\
1977 . . . . \\
1978 \ldots . . \\
1979 . . . . .\end{array}$ & $\begin{array}{l}70,569 \\
74,392 \\
76,317 \\
78,158 \\
78,920\end{array}$ & $\begin{array}{l}51,553 \\
54,276 \\
55,287 \\
56,419 \\
56,935\end{array}$ & $\begin{array}{l}73.1 \\
\cdot 73.0 \\
72.4 \\
72.2 \\
72.1\end{array}$ \\
\hline $\begin{array}{l}1980 \ldots . . . \\
1981 \ldots . . . \\
1982 \ldots . . . \\
1983 . . . . . \\
1984 \ldots . . .\end{array}$ & $\begin{array}{l}75,985 \\
74,022 \\
70,806 \\
70,486 \\
74,085\end{array}$ & $\begin{array}{l}54,140 \\
52,703 \\
49,827 \\
49,093 \\
51,847\end{array}$ & $\begin{array}{l}71.3 \\
71.2 \\
70.4 \\
69.6 \\
70.0\end{array}$ \\
\hline $\begin{array}{l}1985 \ldots . . . . . \\
1986 \ldots . . . . \\
1987 \ldots . . . . \\
1988 \ldots . . . \\
1989 . . . .\end{array}$ & $\begin{array}{l}74,054 \\
74,290 \\
76,840 \\
80,269 \\
81,317\end{array}$ & $\begin{array}{l}51,694 \\
51,820 \\
53,361 \\
55,802 \\
56,506\end{array}$ & $\begin{array}{l}69.8 \\
69.8 \\
69.4 \\
69.5 \\
69.5\end{array}$ \\
\hline $\begin{array}{l}1990 \ldots . . . . \\
1991 \ldots . . .\end{array}$ & $\begin{array}{l}81,143 \\
81,119\end{array}$ & $\begin{array}{l}56,084 \\
56,102\end{array}$ & $\begin{array}{l}69.1 \\
69.2\end{array}$ \\
\hline
\end{tabular}

Note: Calculations are based on unrounded data. Sources: Total Consumption-Table C7, column "Total (Gross)." Net Consumption-Table C7, column titled "Net". Net/Total Consumption-Net Btu divided by Total Btu.
Current- and constant-dollar energy expenditure estimates also can be used along with measures of U.S. gross domestic purchases of goods and services (published by the Bureau of Economic Analysis, U.S. Department of Commerce, as part of its system on national income and product accounts), to determine how much of total domestic purchases is accounted for by energy expenditures. The percentages are given in Table C5.

\section{Energy Price Measures}

The development of the constant-dollar energy expenditure series makes it possible to derive nominal

Table C2. Energy Expenditures and Expenditures/GDP Index, 1970-1991 $(1987=100)$

\begin{tabular}{|c|c|c|}
\hline Year & $\begin{array}{l}\text { Constant-Dollar } \\
\text { Expenditures } \\
\text { (million } 1987 \text { dollars) }\end{array}$ & $\begin{array}{l}\text { Constant-Dollar } \\
\text { Expenditures/GDP } \\
\text { Index }(1987=100)\end{array}$ \\
\hline $\begin{array}{l}1970 \ldots . . . \\
1971 \ldots . \\
1972 \ldots . \\
1973 . . . \\
1974 \ldots\end{array}$ & $\begin{array}{l}307,518 \\
318,458 \\
337,686 \\
353,506 \\
344,036\end{array}$ & $\begin{array}{l}123.4 \\
124.3 \\
125.4 \\
124.8 \\
122.2\end{array}$ \\
\hline $\begin{array}{l}1975 \ldots . . . \\
1976 \ldots . \\
1977 \ldots . . \\
1978 \ldots . \\
1979 . . .\end{array}$ & $\begin{array}{l}341,306 \\
360,377 \\
370,267 \\
380,539 \\
382,919\end{array}$ & $\begin{array}{l}122.2 \\
123.0 \\
120.9 \\
118.5 \\
116.3\end{array}$ \\
\hline $\begin{array}{l}1980 \ldots . . . \\
1981 \ldots \\
1982 \ldots . . . \\
1983 . . . \\
1984 \ldots\end{array}$ & $\begin{array}{l}369,392 \\
365,418 \\
354,387 \\
356,218 \\
373,085\end{array}$ & $\begin{array}{l}112.8 \\
109.7 \\
108.7 \\
105.2 \\
103.8\end{array}$ \\
\hline $\begin{array}{c}1985 . . . \\
1986 . . . \\
1987 \ldots \\
1988 \ldots \\
1989 . . . .\end{array}$ & $\begin{array}{l}376,089 \\
381,249 \\
393,525 \\
410,592 \\
416,610\end{array}$ & $\begin{array}{r}101.4 \\
99.9 \\
100.0 \\
100.4 \\
99.3\end{array}$ \\
\hline $\begin{array}{l}1990 \ldots . . . \\
1991 \ldots . .\end{array}$ & $\begin{array}{l}415,843 \\
418,631\end{array}$ & $\begin{array}{r}98.4 \\
100.2\end{array}$ \\
\hline
\end{tabular}

Note: Calculations are based on unrounded data.

Sources: Expenditures-ElA, State Energy Price and Expenditure Data System 1991.

Expenditures/GDP Index-Expenditures/GDP, converted to index, $1987=100.0$. (GDP data appear in Table $\mathbf{C} 6$, column titled "GDP (Billion 1987 Dollars)."

\footnotetext{
${ }^{2}$ Although this appendix does not provide estimates at the State level, the national estimates of constant-dollar energy expenditures are based on detailed energy consumption and price data for energy products, classified by end-use-sector, for each State.
} 
Table C3. Energy Price Indices, 1970-1991, $(1987=100)$

\begin{tabular}{|c|c|c|}
\hline \multirow[b]{2}{*}{ Year } & \multicolumn{2}{|c|}{ Energy Price Indices } \\
\hline & Nominal & Real \\
\hline $1970 \ldots$ & 27.8 & 76.7 \\
\hline $1971 \ldots$ & 29.0 & 76.0 \\
\hline $1972 \ldots$ & 29.7 & 74.7 \\
\hline $1973 \ldots$ & 32.1 & 76.1 \\
\hline $1974 \ldots . .$. & 44.4 & 95.7 \\
\hline $1975 \ldots$ & 50.2 & 98.9 \\
\hline $1976 \ldots$ & 53.5 & 99.8 \\
\hline $1977 \ldots$ & 59.0 & 102.7 \\
\hline $1978 \ldots$ & 62.4 & 101.9 \\
\hline $1979 \ldots .$. & 76.9 & 115.5 \\
\hline $1980 \ldots$ & 100.7 & 136.8 \\
\hline $1981 \ldots$ & 116.3 & 145.0 \\
\hline $1982 \ldots$ & 119.8 & 141.3 \\
\hline $1983 \ldots$ & 116.6 & 132.8 \\
\hline $1984 \ldots$. & 116.0 & 127.7 \\
\hline $1985 \ldots$ & 115.8 & 123.3 \\
\hline $1986 \ldots$ & 100.1 & 103.6 \\
\hline $1987 \ldots$ & 100.0 & 100.0 \\
\hline $1988 \ldots$ & 99.3 & 95.5 \\
\hline $1989 \ldots . .$. & 104.3 & 96.1 \\
\hline $1990 \ldots$ & 113.4 & 99.8 \\
\hline $1991 \ldots$ & 112.1 & 95.1 \\
\hline
\end{tabular}

Note: Calculations are based on unrounded data.

Sources: Nominal Fixed-Weight Energy Price Index-Based on net energy consumption (1987 fixed-quantity weights) and end-use price data from EIA, State Energy Price and Expenditure Data System 1991.

Real Fixed-Weight Price Index-Nominal energy fixed-weight price index/Gross Domestic Purchases benchmark-years-weighted price index. (Gross Domestic Purchases price data appear in appendix Table C6.)
Implicit price deflators, however, present a problem as indicators of changes in price because they also reflect changes in product mix (except for direct comparisons between any given year and the base year, with the product mix of the given year held. constant). Therefore, although they are convenient, their usefulness is limited. In order to compare price data for any year with price data for any other year, it is necessary to develop fixed-weight energy price indices. In concept, a fixed-weight price index excludes the effect of changes in product mix.

The methodology used to develop the fixed-weight energy price indices presented in this appendix is comparable to that used to develop the GDP fixedweight price index. The nominal fixed-weight energy price index is a measure of the average price of net energy consumption for each given year relative to the average price of net energy consumption in 1987, the weight-base year. The composition of net energy consumption is held constant at 1987 weights for each year in the series. The 1987 weights consist of detailed energy source and end-use sector categories (cells) for each State and the relative distribution of net energy consumption among the various States.

The nominal fixed-weight energy price index is derived by (a) multiplying 1987 net energy consumption in Btu for each of the cells by the related end-use price for each year, (b) aggregating the hypothetical expenditures derived by this procedure to the individual State and national levels for each year, and (c) dividing the hypothetical expenditures for each year by the actual expenditures in 1987. The real fixedweight energy price index is then obtained by dividing the nominal fixed-weight energy price index by the gross domestic product purchases benchmark-years-weighted price index ${ }^{3}$ (Table C3).

In order to avoid some of the problems involved in interpreting the movement in the energy implicit deflator, because the measure reflects both price and product mix changes, only the fixed-weight energy price series is analyzed in this appendix.

\footnotetext{
${ }^{3}$ The gross domestic purchases benchmark-years-weighted price index is used to convert the energy expenditure price index from nominal to real terms, instead of using the GDP fixed-weight price index because the latter is not available for years prior to 1982 and the scope of energy expenditures and prices is more consistent with that of gross domestic purchases, i.e., both include expenditures or purchases within the domestic economy, including imports but excluding exports.
} 


\section{Energy Consumption, Expenditures; and Prices}

\section{The Decline in the Net/Total Ratio}

Over time, the amount of energy used or lost by energy industries has accounted for an increasing proportion of total energy. In 1970, energy used or lost by energy industries amounted to about 24.4 percent of total energy consumed. By 1991, it had risen to 30.8 percent (Table C1). Of course, as the proportion of energy used or lost rose, the ratio of net energy consumption-energy actually delivered to end users-to total energy consumption registered a concomitant decline. The rate of decline in the ratio averaged about 0.4 percent per year from 1970 through 1991.

\section{The Shift to Higher-Priced Products}

When the effect on constant-dollar energy expenditures of the decline in the proportion of energy actually delivered to end users is taken into account by basing energy expenditure and price series on net energy consumption, it becomes possible to examine changes in the product mix. The difference between the rate of increase in constant-dollar energy expenditures and that of net energy consumption is an implicit measure of the rate of change in the mix of products supplied to end users (Table C4).

Table C4. Rates of Change in Energy Expenditures and Consumption, 1970-1991 (Percent)

\begin{tabular}{c|c|c|c}
\hline Period & $\begin{array}{c}\text { Constant- } \\
\text { Dollar } \\
\text { Expenditures }\end{array}$ & $\begin{array}{c}\text { Net Energy } \\
\text { Consumption }\end{array}$ & $\begin{array}{c}\text { Product } \\
\text { Mix } \\
\text { Effect }\end{array}$ \\
\hline $1970-73$ & 4.8 & 3.3 & 1.4 \\
$1973-81$ & .4 & -.6 & 1.0 \\
$1981-91$ & 1.4 & .6 & .7 \\
$1970-91$ & 1.5 & .5 & .9 \\
\hline
\end{tabular}

a Constant-dollar expenditure rate minus net energy consumption rate. Product-mix effect based on unrounded data. Note: Rates of change are average annual rates. c2.
When the rate of change in constant-dollar energy expenditures exceeds the rate of change in net energy consumption, the shift in product mix is towards higher-priced products. And, in fact, throughout the period of 1970 through 1991, constant-dollar energy expenditures rose more rapidly-or fell more slowly-than net energy consumption. For the period as a whole, the product-mix effect averaged 0.9 percent per year. But the product-mix effect was not uniform over the period; it declined from 1.4 percent per year in 1970 through 1973 to 1.0 percent per year in 1973 through 1981 and 0.7 percent per year in 1981 through 1991.

An examination of changes in the shares of electricity and motor gasoline over the 22-year period illustrates the product-mix effect. On a dollar-pernet-Btu basis, electricity and motor gasoline are relatively higher-priced sources of energy. In 1987, the base year used in calculating constant-dollar energy expenditures, the prices per million net Btu of electricity and motor gasoline averaged $\$ 18.74$ and $\$ 7.22$, respectively. By comparison, the average price for all other energy sources was $\$ 4.44$ per million net Btu. In 1970, electricity accounted for 9.3 percent of net energy consumption and motor gasoline accounted for 22.1 percent. By 1991, the shares of those higher-priced energy products had grown to 16.6 percent, and 24.6 percent, respectively.

\section{The Decline in Energy Intensity}

A second analytical use of the constant-dollar energy expenditure series is an examination of changes in the energy intensity of the economy. The change in the ratio of constant-dollar energy expenditures to constant-dollar GDP is one measure of energy intensity.

By definition, if the energy intensity of the economy were constant, the rate of change in constant-dollar energy expenditures would be identical to the rate of change in GDP. During 1970 through 1991, however, the rates of change differed. Furthermore, the change in the energy expenditure/GDP ratio was not uniform over the period. In 1970 through 1973, the energy expenditure/GDP ratio actually increased 0.4 percent per year (Figure C1).

In 1973 through 1981, the decline in energy intensity, as measured by the energy expenditure/GDP ratio, averaged 1.6 percent. The decline is largely attributable to dramatic increases in real energy prices. Such increases, by promoting energy conservation and energy efficiency, would tend to reduce energy 
Figure C1. Rates of Change in ConstantDollar Energy Expenditures/GDP Index, 1970-1991

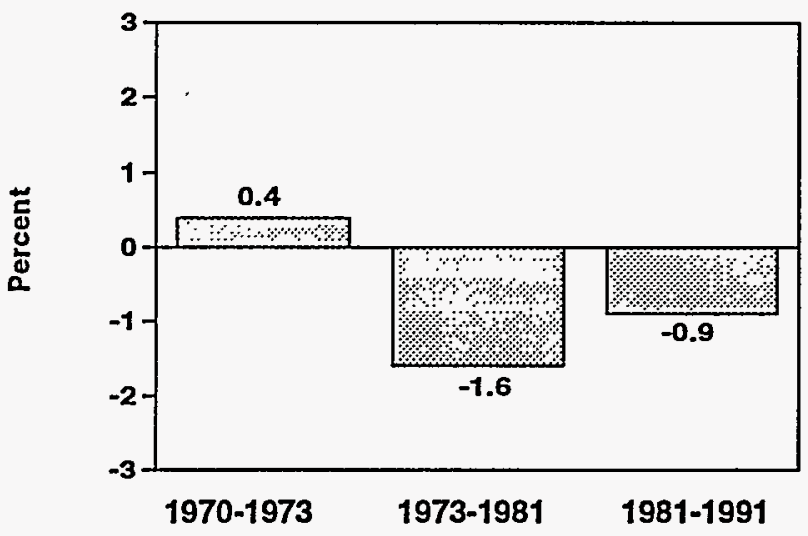

Note: Rates of change are average annual rates of change. Source: Based on data in Table C2.

consumption, and hence constant-dollar expenditures, relative to growth in the economy.

In 1981 through 1991, energy intensity continued to decline, averaging 0.9 percent per year, despite a substantial decline in the real price of energy. The price decline might have been expected to lead to an increase in energy expenditures relative to the growth in the economy. This apparent anomaly- the continuation of the decline in energy intensity although at a slower rate, despite the price decline-can be explained in large part by the continuing replacement of machinery, vehicles, appliances, structures, and other energy-consuming units with more efficient units. As the proportion of more efficient units rose, average energy efficiency increased. For example, U.S. passenger car efficiency increased faster in 1981 through 1991 than in 1973 through 1981. From 1973 to 1981 , the average number of miles traveled per gallon of fuel consumed rose from 13.30 to 15.94, an average annual increase of 2.3 percent. From 1981 to 1991 , the number rose from 15.94 to 21.68 , an average annual increase of 3.1 percent. $^{4}$

\section{Changes in Energy Prices}

For the period of 1970 through 1991 as a whole, the fixed-weight energy price index, in nominal terms, registered a sizeable increase of 6.9 percent per year. That average, however, masked significant variations between periods. In 1970 through 1973, prices in-
Table C5. Energy Expenditures as a Share of Gross Domestic Purchases, Current and Constant 1987 Dollars, 1970-1991

\begin{tabular}{|c|c|c|}
\hline \multirow[b]{2}{*}{ Year } & \multicolumn{2}{|c|}{$\begin{array}{l}\text { Energy Expenditures as a Share of } \\
\text { Gross Domestlc Purchases } \\
\text { (Percent) }\end{array}$} \\
\hline & $\begin{array}{c}\text { Based on } \\
\text { Current Dollars }\end{array}$ & $\begin{array}{c}\text { Based on } \\
\text { Constant Dollars }\end{array}$ \\
\hline $\begin{array}{l}1970 \ldots . . . \\
1971 \ldots . \\
1972 \ldots . \\
1973 \ldots . \\
1974 \ldots .\end{array}$ & $\begin{array}{r}8.2 \\
8.2 \\
8.0 \\
8.3 \\
10.5\end{array}$ & $\begin{array}{l}10.6 \\
10.6 \\
10.7 \\
10.7 \\
10.6\end{array}$ \\
\hline $\begin{array}{l}1975 \ldots . . \\
1976 \ldots . \\
1977 \ldots \\
1978 \ldots \\
1979 \ldots \\
. . . .\end{array}$ & $\begin{array}{l}10.9 \\
10.9 \\
11.0 \\
10.6 \\
11.8\end{array}$ & $\begin{array}{l}10.7 \\
10.6 \\
10.4 \\
10.2 \\
10.1\end{array}$ \\
\hline $\begin{array}{l}1980 \ldots . \\
1981 \ldots . \\
1982 \ldots . \\
1983 \ldots . \\
1984 \ldots .\end{array}$ & $\begin{array}{l}13.7 \\
14.0 \\
13.4 \\
12.0 \\
11.2\end{array}$ & $\begin{array}{l}9.9 \\
9.6 \\
9.4 \\
9.0 \\
8.7\end{array}$ \\
\hline $\begin{array}{l}1985 \ldots . \\
1986 \ldots \\
1987 \ldots . \\
1988 \ldots . \\
1989 \ldots \\
. . . .\end{array}$ & $\begin{array}{r}10.5 \\
8.7 \\
8.4 \\
8.1 \\
8.1\end{array}$ & $\begin{array}{l}8.5 \\
8.4 \\
8.4 \\
8.5 \\
8.5\end{array}$ \\
\hline $\begin{array}{l}1990 \ldots . . . \\
1991 \ldots .\end{array}$ & $\begin{array}{l}8.4 \\
8.2\end{array}$ & $\begin{array}{l}8.4 \\
8.6\end{array}$ \\
\hline
\end{tabular}

Sources: Energy Expenditures-Current Dollars, Table C6, Constant Dollars, Table C2.

Gross Domestic Purchases-Current and Constant Dollars, Table C6.

creased at the average annual rate of 5.0 percent, in nominal terms (Figure C2).

In 1973 through 1981, the rate jumped to 17.5 percent as world events-notably the Arab oil embargo of 1973 to 1974 and the Iranian crisis in 1979-boosted the price of oil. In 1981 through 1991, energy prices declined an average of 0.4 percent per year, in nominal terms.

However, removing the effects of general inflation modify these rates of change considerably for all three subperiods. The 5.0-percent-per-year increase

\footnotetext{
${ }^{4}$ Miles per gallon data are from EIA, Monthly Energy Review, May 1993, DOE/ELA-0035(93/05) (Washington, DC), Table 1.10.
} 
Figure C2. Rates of Change In Fixed-Weight Energy Price Indices, 1979-1990

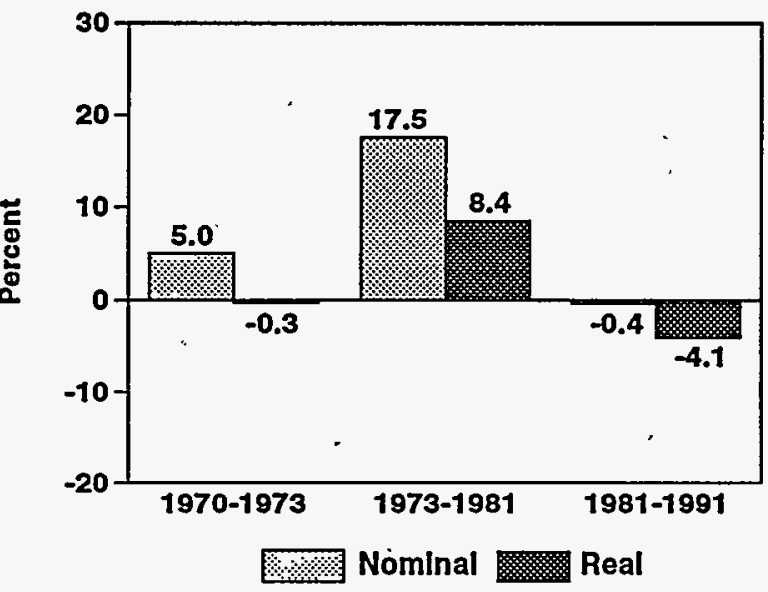

Note: Rates of change are average annual rates of change. Source: Based on data in Table C3.

in nominal terms in 1970 through 1973 is an actual decline of 0.3 percent per year in real terms. The increase in 1973 through 1981 is reduced from 17.5 percent per year, in nominal terms, to less than half that rate, 8.4 percent, in real terms.

In the period after 1981, the rate of decline in real terms is much larger than the nominal rate of decline, due to continued increases in the gross domestic purchase benchmark-weighted price index. The decline in nominal energy prices of 0.4 percent per year in 1981 through 1991 is a 4.1-percent-peryear reduction in real terms. Considering only the nominal rates of change in energy prices conceals the magnitude of the post-1981 decline. By 1991, the average price of energy, in real terms, was about 25 percent above the level of real energy prices in 1973, prior to the period of dramatic increase in energy prices. This represents a sharp reduction from the level reached in 1981 when real energy prices were 91 percent above the 1973 level.

\section{Energy Expenditures Percentage of Gross Domestic Purchases}

The development of energy expenditure data in both current and constant 1987 dollars, makes it possible to determine how much of U.S. gross domestic purchases of goods and services is accounted for by energy expenditures.
Measures of energy expenditures and gross domestic purchases are comparable in that both include products purchased in the domestic economy including imports and excluding exports. The percentages of energy purchases in gross domestic purchases, based on current and constant 1987 dollars are given in Table $\mathrm{C5}$. The percentages based on current-dollar measures show an almost continuous increase from 8.2 percent in 1970 to a peak of 14.0 percent in 1981, followed by an equally rapid decline to 8.1 percent in 1988. Since then the ratio has started to increase, with 1991 at 8.2 percent.

The energy expenditure-gross domestic purchases percentage based on constant 1987 dollars shows an entirely different pattern, remaining in the range 10.6 percent to 10.7 percent for the years 1970 through 1976 and then declining rapidly to a range of 8.4 percent to 8.6 percent for the years 1985 through 1991 .

Both the current- and constant-dollar percentages show that energy expenditures represent a significant proportion of total domestic purchases, although the percentages for the most recent years indicate a substantial reduction from their peaks in earlier years. In order to provide some perspective on energy expenditure's share of gross domestic purchases (i.e., how does energy's share compare with that of another major sector in the Nation's economy?), data in the national income and product accounts on expenditures in 1991 for new automobiles and trucks have been used to derive percentages of gross domestic purchases comparable to that for energy expenditures.

The expenditures for new automobiles and trucks, in current dollars, amounted to $\$ 214.1$ billion, or 3.8 percent of gross domestic purchases, as compared to $\$ 467.0$ billion or 8.2 percent for energy purchases. In constant dollars, expenditures for new automobiles and trucks accounted for 4.0 percent of gross domestic purchases as compared to 8.6 percent for energy expenditures. In both comparisons, the percentage for energy expenditures were more than double that for new automobiles and trucks. ${ }^{5}$

\section{Summary of Major Findings}

This appendix presents additional measures of energy consumption, expenditures, and prices, as well as examples of the kinds of analysis that these

\footnotetext{
${ }^{5}$ The basic data on 1991 expenditures for new automobiles and trucks are included in the April 1993 issue of the U.S. Bureau of Economic Analysis, Suroey of Current Business, Tables 8.3, 8.4, 8.5, and 8.6, page 29.
} 
series make possible. The major analytical findings of this appendix are as follows:

- The proportion of total energy consumption actually delivered to end users declined throughout 1970 to 1991 , from 75.6 percent to 69.2 percent.

- During the 22-year period, there was a shift towards higher-priced energy products in the mix of products delivered to end users.

- The energy intensity of the economy declined in response to the sharp increase in real energy prices in 1973 through 1981 and the decline continued in 1981 through 1991, despite a decrease in real prices after 1981. The apparent anomaly is explained in large part by the effect of replacement of energy-consuming stock with more energy efficient units.

- Real energy prices increased dramatically between 1973 and 1981, at a rate of 8.4 percent per year, with the level of real energy prices increasing by over 90 percent over the period. A major part of the increase was offset, however, by the decline in energy prices in the following period, at a rate of 4.1 percent per year, so that the level of real energy prices in 1991 was reduced to 25 percent above the 1973 level.

- In order to provide some perspective on the relative importance of energy expenditures in the Nation's economy, estimates of the percentage shares of energy expenditures in gross domestic purchases in both current and constant dollars, have been developed. The percentages based on current dollar measures show an almost continuous increase from 8.2 percent in 1970 to a peak of 14.0 percent in 1981 , followed by an equally rapid decline, with 1991 at 8.2 percent. The energy expenditures/gross domestic purchase percentage, based on constant 1987 dollars, shows an entirely different pattern, remaining in the range of 10.6 percent to 10.7 percent for the years 1970 through 1976, and then declining rapidly to a range of 8.4 percent to 8.6 percent for the years 1985 through 1991. The percentages for energy expenditures/gross domestic purchases in 1991, in both current and constant dollars, are more than double similar percentages for domestic expenditures for new automobiles and trucks. 


\begin{tabular}{|c|c|c|c|c|c|}
\hline \multirow[b]{2}{*}{ Year } & \multirow[b]{2}{*}{$\begin{array}{l}\text { Enargy Expenditures } \\
\text { (Million Current Dollars) }\end{array}$} & \multirow[b]{2}{*}{$\begin{array}{c}\text { GDP } \\
\text { (Billion } 1987 \text { Dollars) }\end{array}$} & \multicolumn{3}{|c|}{ Gross Domestic Purchase Measures } \\
\hline & & & (Billion Current Dollars) & (Billion 1987 Dollars) & $\begin{array}{c}\text { (Price Index, 1987=100, } \\
\text { Benchmark Weights) }\end{array}$ \\
\hline $\begin{array}{l}1970 \ldots . . . \\
1971 \ldots . . \\
1972 \ldots . . \\
1973 \ldots . \\
1974 \ldots .\end{array}$ & $\begin{array}{r}82,579 \\
89,781 \\
97,801 \\
111,638 \\
153,078\end{array}$ & $\begin{array}{l}2,873.9 \\
2,955.9 \\
3,107.1 \\
3,268.6 \\
3,248.1\end{array}$ & $\begin{array}{l}1,009.5 \\
1,100.2 \\
1,215.0 \\
1,349.0 \\
1,461.8\end{array}$ & $\begin{array}{l}2,909.1 \\
3,001.8 \\
3,163.6 \\
3,302.7 \\
3,252.2\end{array}$ & $\begin{array}{l}36.2 \\
38.1 \\
39.8 \\
42.2 \\
46.4\end{array}$ \\
\hline $\begin{array}{l}1975 \ldots . . \\
1976 \ldots . . \\
1977 \ldots . \\
1978 \ldots . \\
1979 \ldots .\end{array}$ & $\begin{array}{l}171,782 \\
193,660 \\
220,220 \\
238,877 \\
296,993\end{array}$ & $\begin{array}{l}3,221.7 \\
3,380.8 \\
3,533.3 \\
3,703.5 \\
3,796.8\end{array}$ & $\begin{array}{l}1,572.3 \\
1,770.7 \\
1,997.8 \\
2,258.8 \\
2,512.5\end{array}$ & $\begin{array}{l}3,198.6 \\
3,387.1 \\
3,561.1 \\
3,733.3 \\
3,807.4\end{array}$ & $\begin{array}{l}50.7 \\
53.6 \\
57.4 \\
61.2 \\
66.6\end{array}$ \\
\hline $\begin{array}{l}1980 \ldots . . . \\
1981 \ldots . \\
1982 \ldots \\
1983 . . . \\
1984 \ldots .\end{array}$ & $\begin{array}{l}373,900 \\
426,446 \\
424,809 \\
415,750 \\
433,462\end{array}$ & $\begin{array}{l}3,776.3 \\
3,843.1 \\
3,760.3 \\
3,906.6 \\
4,148.5\end{array}$ & $\begin{array}{l}2,722.8 \\
3,045.3 \\
3,170.2 \\
3,456.5 \\
3,879.9\end{array}$ & $\begin{array}{l}3,745.7 \\
3,821.2 \\
3,767.7 \\
3,962.8 \\
4,270.5\end{array}$ & $\begin{array}{l}73.6 \\
80.2 \\
84.8 \\
87.8 \\
90.8\end{array}$ \\
\hline $\begin{array}{l}1985 \ldots . . \\
1986 \ldots . \\
1987 \ldots . \\
1988 \ldots . \\
1989 \ldots . .\end{array}$ & $\begin{array}{l}435,444 \\
381,250 \\
393,525 \\
407,597 \\
434,338\end{array}$ & $\begin{array}{l}4,279.8 \\
4,404.5 \\
4,539.9 \\
4,718.6 \\
4,838.0\end{array}$ & $\begin{array}{l}4,154.3 \\
4,401.2 \\
4,683.0 \\
5,008.4 \\
5,330.5\end{array}$ & $\begin{array}{l}4,425.1 \\
4,559.6 \\
4,683.0 \\
4,822.6 \\
4,911.7\end{array}$ & $\begin{array}{r}93.9 \\
96.6 \\
100.0 \\
103.9 \\
108.6\end{array}$ \\
\hline $\begin{array}{l}1990 \ldots . . . \\
1991 \ldots . .\end{array}$ & $\begin{array}{l}469,468 \\
467,132\end{array}$ & $\begin{array}{l}4,877.5 \\
4,821.0\end{array}$ & $\begin{array}{l}5,591.1 \\
5,699.3\end{array}$ & $\begin{array}{l}4,929.3 \\
4,842.8\end{array}$ & $\begin{array}{l}113.6 \\
117.9\end{array}$ \\
\hline
\end{tabular}

Sources: Current-Dollar Energy Expenditures-Energy Information Administration (EIA), State Energy Price and Expenditure Data System 1991.

Gross Domestlc Product U.S. Department of Commerce (DOC), Bureau of Economic Analysis (BEA), Survey of Current Business, December 1992, Table 2. "Gross Domestic Product in Constant Dollars," column titled "GDP," p. 30.

Gross Domestlc Purchase Measures (Billion Current Dollars)-U.S. Department of Commerce, Bureau of Economic Analysls, Survay of Current Business,December, 1992, Table 1,"Gross Domestic Product," column titled "Gross Domestic Purchases," p. 27.

Gross Domestic Purches Measures (1987 Dollars)-U.S. Department of Commerce, Bureau of Commerce, Bureau of Economic Analysis, Survey of Current Business, December, 1992, Table 2, "Gross Domestic Product in Constant Dollars," column titled "Gross Domestic Purchases," p. 30.

Gross Domestic Purchase Measures, Price Index, 1987=100, Benchmark Weights, 1970-1988-U.S. Department of Commerce, Bureau of Economic Anayisis, Survey of Current Business, April, 1992, Table 3, "Fixed-Weighted and Alternative Price Indexes for Gross Domestic Product," column titled "Gross Domestic Purchases," p.48.1989-1991-Sunvey of Current Business, April 1993, Table 7.2, p. 35, line 23. 
Table C7. Total (Gross) — Net Energy Balance, 1970-1991 (Trillion Btu)

\begin{tabular}{|c|c|c|c|c|c|c|c|c|c|}
\hline Year & $\begin{array}{c}\text { Total } \\
\text { (Gross) }\end{array}$ & Net & $\begin{array}{c}\text { Electric } \\
\text { Utillty } \\
\text { Losses }\end{array}$ & $\begin{array}{c}\text { Refinery } \\
\text { Use }\end{array}$ & $\begin{array}{c}\text { Crude } \\
\text { Oll }\end{array}$ & $\begin{array}{c}\text { Natural } \\
\text { Gas } \\
\text { Lease } \\
\text { and Plant }\end{array}$ & $\begin{array}{c}\text { Natural } \\
\text { Gas } \\
\text { Pipelline }\end{array}$ & $\begin{array}{c}\text { Industrial } \\
\text { Hydro- } \\
\text { electric }\end{array}$ & $\begin{array}{l}\text { Total } \\
\text { Loss }\end{array}$ \\
\hline 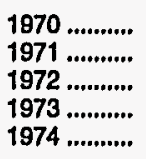 & $\begin{array}{l}66,334 \\
67,789 \\
71,275 \\
74,351 \\
72,528\end{array}$ & $\begin{array}{l}50,138 \\
50,968 \\
53,273 \\
55,344 \\
53,409\end{array}$ & $\begin{array}{l}11,517 \\
12,127 \\
13,110 \\
13,999 \\
14,198\end{array}$ & $\begin{array}{l}2,464 \\
2,442 \\
2,576 \\
2,690 \\
2,683\end{array}$ & $\begin{array}{l}- \\
- \\
- \\
-\end{array}$ & $\begin{array}{l}1,442 \\
1,456 \\
1,497 \\
1,539 \\
1,520\end{array}$ & $\begin{array}{l}740 \\
761 \\
786 \\
745 \\
684\end{array}$ & $\begin{array}{l}34 \\
34 \\
34 \\
35 \\
33\end{array}$ & $\begin{array}{l}16,197 \\
16,820 \\
18,003 \\
19,007 \\
19,119\end{array}$ \\
\hline 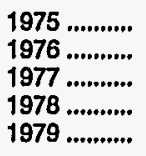 & $\begin{array}{l}70,569 \\
74,392 \\
76,317 \\
78,158 \\
78,920\end{array}$ & $\begin{array}{l}51,553 \\
54,276 \\
55,287 \\
56,419 \\
56,935\end{array}$ & $\begin{array}{l}14,384 \\
15,255 \\
16,060 \\
16,850 \\
17,063\end{array}$ & $\begin{array}{l}2,571 \\
2,590 \\
2,687 \\
2,623 \\
2,742\end{array}$ & $\begin{array}{l}- \\
- \\
- \\
-\end{array}$ & $\begin{array}{l}1,434 \\
1,679 \\
1,706 \\
1,694 \\
1,534\end{array}$ & $\begin{array}{l}595 \\
559 \\
544 \\
541 \\
613\end{array}$ & $\begin{array}{l}32 \\
33 \\
33 \\
32 \\
34\end{array}$ & $\begin{array}{l}19,017 \\
20,116 \\
21,030 \\
21,740 \\
21,985\end{array}$ \\
\hline 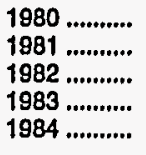 & $\begin{array}{l}75,985 \\
74,022 \\
70,806 \\
70,486 \\
74,085\end{array}$ & $\begin{array}{l}54,140 \\
52,703 \\
49,827 \\
49,093 \\
51,847\end{array}$ & $\begin{array}{l}17,387 \\
17,464 \\
17,100 \\
17,583 \\
18,157\end{array}$ & $\begin{array}{l}2,718 \\
2,204 \\
2,089 \\
2,122 \\
2,255\end{array}$ & $\begin{array}{l}- \\
- \\
\overline{140} \\
134\end{array}$ & $\begin{array}{r}1,058 \\
959 \\
1,144 \\
1,010 \\
1,113\end{array}$ & $\begin{array}{l}650 \\
660 \\
614 \\
505 \\
545\end{array}$ & $\begin{array}{l}33 \\
33 \\
33 \\
33 \\
33\end{array}$ & $\begin{array}{l}21,845 \\
21,320 \\
20,979 \\
21,394 \\
22,237\end{array}$ \\
\hline $\begin{array}{l}1985 . . . . . . . . . \\
1986 \ldots . . . . . . . . \\
1987 \ldots . . . . . . . \\
1988 . . . . . . . . . \\
1989 . . . . . . . .\end{array}$ & $\begin{array}{l}74,054 \\
74,290 \\
76,840 \\
80,269 \\
81,317\end{array}$ & $\begin{array}{l}51,694 \\
51,820 \\
53,361 \\
55,802 \\
56,506\end{array}$ & $\begin{array}{l}18,631 \\
18,593 \\
19,156 \\
19,887 \\
20,256\end{array}$ & $\begin{array}{l}2,046 \\
2,286 \\
2,485 \\
2,696 \\
2,711\end{array}$ & $\begin{array}{r}127 \\
103 \\
72 \\
85 \\
59\end{array}$ & $\begin{array}{r}1,001 \\
954 \\
1,194 \\
1,134 \\
1,103\end{array}$ & $\begin{array}{l}521 \\
501 \\
538 \\
633 \\
650\end{array}$ & $\begin{array}{l}33 \\
33 \\
33 \\
33 \\
33\end{array}$ & $\begin{array}{l}22,359 \\
22,470 \\
23,479 \\
24,468 \\
24,811\end{array}$ \\
\hline $\begin{array}{l}1990 \ldots . . . . . . . . \\
1991 . . . . . . . .\end{array}$ & $\begin{array}{l}81,143 \\
81,119\end{array}$ & $\begin{array}{l}56,084 \\
56,102\end{array}$ & $\begin{array}{l}20,221 \\
20,473\end{array}$ & $\begin{array}{l}2,803 \\
2,668\end{array}$ & $\begin{array}{l}51 \\
39\end{array}$ & $\begin{array}{l}1,269 \\
1,184\end{array}$ & $\begin{array}{l}682 \\
621\end{array}$ & $\begin{array}{l}33 \\
33\end{array}$ & $\begin{array}{l}25,059 \\
25,017\end{array}$ \\
\hline
\end{tabular}

Nole: Totals may not equal sum of components due to independent rounding.

Sources: Total (Gross)-EIA, State Energy Data Report 1991, Consumption Estimates, DOE/EIA-0214(91) (Washington, DC, May, 1993), p. 31, column titled, "Total" (trillion Btu).

Not-EIA, State Energy Price and Expenditure Data System 1991.

Electric UtIllty Losses-EIA, State Energy Data Report 1991, Consumption Estimates, Tables 13-16, pp. 32-35, sum of four end-use sectors' 'Electrical System Energy Losses" (Trillion Btu).

Refinery Uso-EIA, State Energy Price and Expenditure Data System 1991.

Crude Oll Lease, Plant, and PIpeline Use-EIA, State Energy Price and Expenditure Data System 1991.

Natural Gas Lease and Plant-EIA, State Energy Price and Expenditure Data System 1991.

Natural Gas PIpeline-EIA, State Energy Data Report 1991, Consumption Estimates, p. 35, column titled, "Natural Gas" (Trillion Btu)

Industrial Hydroelectric-EIA, State Energy Data Report 1991, Consumption Estimates, p. 34, column titled, "Industrial Hydropower" (Trillion Btu).

Total Loss-Sum of Electric Utility Losses; Refinery Use; Crude Oil as Lease, Plant, and Pipeline Fuel; Natural Gas Lease and Plant; Natural Gas Pipeline; and Industrial Hydroelectric. 
* , 


\section{Glossary}





\section{Glossary}

Asphalt: A dark-brown-to-black cement-like material containing bitumens as the predominant constituents obtained by petroleum processing. The definition includes crude asphalt as well as the following finished products: cements, fluxes, the asphalt content of emulsions (exclusive of water), and petroleum distillates blended with asphalt to make cutback asphalts.

ASTM: The American Society for Testing and Materials.

Aviation Gasoline Blending Components: Naphthas that are used for blending or compounding into finished aviation gasoline (e.g., straight-run gasoline, alkylate, and reformate). Excludes oxygenates (alcohols and ethers), butane, and pentanes plus.

Aviation Gasoline: All special grades of gasoline for use in aviation reciprocating engines, as given in ASTM Specification D910 and Military Specification MIL-G-5572. Aviation gasoline includes blending components.

Barrel (petroleum): A unit of volume equal to 42 U.S. gallons.

Biomass Fuels: Wood, waste, and alcohol fuels are the primary examples of biomass fuels. The term "biomass fuels" comprises biomass energy sources and biofuels. Biomass energy sources are essentially unprocessed; they are burned, as received, to produce thermal energy. Examples are wood, garbage, and corn. Biofuels result from the processing of biomass energy sources. In general, biofuels have a greater energy density and are more easily transported and used. Examples are wood chips, pellets, briquettes, and methanol (made from wood); refusederived fuel (made from garbage), and ethanol (made from corn).

British Thermal Unit (Btu): The quantity of heat needed to raise the temperature of 1 pound of water by $1^{\circ} \mathrm{F}$ at or near $39.2^{\circ} \mathrm{F}$. See Heat Content of a Quantity of Fuel, Gross and Heat Content of a Quantity of Fuel, Net.
Butane: A normally gaseous straight-chain or branched-chain hydrocarbon $\left(\mathrm{C}_{4} \mathrm{H}_{10}\right)$. It is extracted from natural gas or refinery gas streams. It includes isobutane and normal butane and is designated in ASTM Specification D1835 and Gas Processors Association Specifications for commercial butane.

- Isobutane: A normally gaseous branched-chain hydrocarbon. It is a colorless paraffinic gas that boils at a temperature of $10.9^{\circ} \mathrm{F}$. It is extracted from natural gas or refinery gas streams.

- Normal Butane: A normally gaseous straightchain hydrocarbon. It is a colorless paraffinic gas that boils at a temperature of $31.1^{\circ} \mathrm{F}$. It is extracted from natural gas or refinery gas streams.

Butylene: An olefinic hydrocarbon $\left(\mathrm{C}_{4} \mathrm{H}_{8}\right)$ recovered from refinery processes.

Coal: Includes all ranks of coal-anthracite, bituminous coal, subbituminous coal, and ligniteconforming to ASTM Specification D388.

- Coking Coal: Coal that meets the requirements for making coal coke. It must be low in ash and sulfur and form a coke that is capable of supporting the charge of iron ore and limestone in a blast furnace. Coking coal is usually a blend of two or more bituminous coals.

- Steam Coal: In this report, steam coal represents all noncoking coal.

Coal Coke: A hard, porous product made from baking bituminous coal in ovens at temperatures as high as $2,000^{\circ} \mathrm{F}$. It is used both as a fuel and as a reducing agent in smelting iron ore in a blast furnace.

Coke Plants: Plants where coal is carbonized in slot or beehive ovens for the manufacture of coke.

\section{Coking Coal: See Coal.}

Commercial Sector: The commercial sector, as defined economically, consists of business establishments that are not engaged in transportation or in manufacturing or other types of industrial 
activity (agriculture, mining, or construction). Commercial establishments include hotels, motels, restaurants, wholesale businesses, retail stores, laundries, and other service enterprises; religious and nonprofit organizations; health, social, and educational institutions; and Federal, State, and local governments. Street lights, pumps, bridges, and public services are also included if the establishment operating them is considered commercial.

Constant Dollars: Amounts expressed in constant dollars have been adjusted to remove the effect of changes in the purchasing power of the dollar. Prices expressed in constant dollars usually reflect buying power relative to a base year.

Conversion Factor: A number that translates units of one system into corresponding values of another system. Conversion factors can be used to translate physical units of measure for various fuels into Btu equivalents.

Crude Oil Used Directly: Crude oil consumed as fuel by crude oil pipelines and on crude oil leases.

Cubic Foot (natural gas): A unit of volume equal to 1 cubic foot at a pressure base of 14.73 pounds standard per square inch absolute and a temperature base of $60^{\circ} \mathrm{F}$.

Current Dollars: Amounts expressed in current dollars have not been adjusted to remove the effect of changes in the purchasing power of the dollar.

Degree-Day Normals: Simple arithmetic averages of monthly or annual degree-days over a long period of time (usually the 30-year period 1951 through 1980). The averages may be simple degree-day normals or population-weighted degree-day normals.

Degree-Days, Cooling (CDD): The number of degrees per day that the daily average temperature is above $65^{\circ} \mathrm{F}$. The daily average temperature is the mean of the maximum and minimum temperatures for a 24-hour period.

Degree-Days, Heating (HDD): The number of degrees per day that the daily average temperature is below $65^{\circ} \mathrm{F}$. The daily average temperature is the mean of the maximum and minimum temperatures for a 24-hour period.

Degree-Days, Population-Weighted: Heating or cooling degree-days weighted by the population of the area in which the degree-days are recorded. To compute State population-weighted degree-days, each State is divided into from one to nine climatically homogeneous divisions, which are assigned weights based on the ratio of the population of the division to the total population of the State. Degreeday readings for each division are multiplied by the corresponding population weight for each division and those products are then summed to arrive at the State population-weighted degree-day figure. To compute national population-weighted degree-days, the Nation is divided into nine Census regions comprised of from three to eight States, which are assigned weights based on the ratio of the population of the region to the total population of the Nation. Degree-day readings for each region are multiplied by the corresponding population weight for each region and those products are then summed to arrive at the national population-weighted degreeday figure.

Diesel Fuel: Fuel used for internal combustion in diesel engines; usually that fraction of crude oil that distills after kerosene. (See Distillate Fuel Oil.)

Distillate Fuel Oil: A general classification for one of the petroleum fractions produced in conventional distillation operations. Included are products known as No. 1, No. 2, and No. 4 fuel oils and No. 1, No. 2, and No. 4 diesel fuels. It is used primarily for space heating, on- and off-highway diesel engine fuel (including railroad engine fuel and fuel for agricultural machinery), and electric power generation.

Electrical System Energy Losses: The amount of energy lost during generation, transmission, and distribution of electricity, including plant and unaccounted-for uses.

Electricity Generation: The process of producing electric energy or transforming other forms of energy into electric energy. Also the amount of electric energy produced or expressed in watthours (Wh).

Electricity Sales: The amount of kilowatthours sold in a given period of time; usually grouped by classes of service, such as residential, commercial, industrial, and other. "Other" sales include sales for public street and highway lighting and other sales to public authorities, sales to railroads and railways, and interdepartmental sales.

Electric Power Plant: A station containing prime movers, electric generators, and auxiliary equipment for converting mechanical, chemical, and/or fission energy into electric energy. 
Electric Utility: A corporation, person, agency, authority, or other legal entity or instrumentality that owns and/or operates facilities for the generation, transmission, distribution, or sale of electric energy, primarily for use by the public, and that files forms listed in the Code of Federal Regulations, Title 18, Part 141. Facilities that qualify as cogenerators or small power producers under the Public Utility Regulatory Policies Act are not considered utilities.

Electric Utility Sector: The electric utility sector consists of privately and publicly owned establishments that generate, transmit, distribute, or sell electricity primarily for use by the public and that meet the definition of an electric utility. Nonutility power producers are not included in the electric utility sector.

End-Use Sectors: The residential, commercial, industrial, and transportation sectors of the economy.

Energy: The capacity for doing work as measured by the capability of doing work (potential energy) or the conversion of this capability to motion (kinetic energy). Energy has several forms, some of which are easily convertible and can be changed to another form useful for work. Most of the world's convertible energy comes from fossil fuels that are burned to produce heat that is then used as a transfer medium to mechanical or other means in order to accomplish tasks. Electrical energy is usually measured in kilowatthours, while heat energy is usually measured in British thermal units.

Ethane: A normally gaseous straight-chain hydrocarbon $\left(\mathrm{C}_{2} \mathrm{H}_{6}\right)$. It is a colorless, paraffinic gas that boils at a temperature of $-127.48^{\circ} \mathrm{F}$. It is extracted from natural gas and refinery gas streams.

Ethylene: An olefinic hydrocarbon $\left(\mathrm{C}_{2} \mathrm{H}_{4}\right)$ recovered from refinery processes or petrochemical processes.

Expenditures: In this report, "expenditures" refers to the money directly spent by consumers to purchase energy. Expenditures equal the amount of energy used by the consumer times the price per unit paid by the consumer. In calculating the amount of energy used, process fuel and intermediate products are not included.

Exports: Shipments of goods from the 50 States and the District of Columbia to foreign countries and to Puerto Rico, the Virgin Islands, and other U.S. possessions and territories.
Federal Energy Regulatory Commission (FERC): The Federal agency with jurisdiction over interstate electricity sales, wholesale electric rates, hydroelectric licensing, natural gas pricing, oil pipeline rates, and gas pipeline certification. FERC is an independent regulatory agency within the Department of Energy and is the successor to the Federal Power Commission.

Federal Power Commission (FPC): The predecessor agency of the Federal Energy Regulatory Commission. The Federal Power Commission was created by an Act of Congress under the Federal Water Power Act on June 10,1920. It was charged originally with regulating the electric power and natural gas industries. It was abolished on September 30, 1977, when the Department of Energy was created. Its functions were divided between the Department of Energy and the Federal Energy Regulatory Commission, an independent regulatory agency.

Fossil Fuel: Any naturally occurring organic fuel, such as petroleum, coal, and natural gas.

Gasohol: A blend of finished motor gasoline (leaded or unleaded) and alcohol (generally ethanol but sometimes methanol) limited to 10 percent by volume of alcohol. Gasohol is included in finished leaded and unleaded motor gasoline.

Geothermal Energy (as used at electric utilities): Hot water or steam extracted from geothermal reservoirs in the Earth's crust and supplied to steam turbines at electric utilities that drive generators to produce electricity.

Gross Domestic Product (GDP): The total value of goods and services produced by labor and property located in the United States. As long as the labor and property are located in the United States, the supplier (that is, the workers and, for property, the owners) may be either U.S. residents or residents of foreign countries.

Gross Domestic Product (GDP) Implicit Price Deflator: The implicit price deflator, published by the U.S. Department of Commerce, Bureau of Economic Analysis, is used to convert nominal figures to real figures.

Gross Domestic Purchases: The total purchases by U.S. residents for all goods and services wherever produced, including imports but excluding exports. It includes all personal consumption expenditures, gross private domestic investment, and government purchases (Federal, State, and local). 
Gross Domestic Purchases Price Index: This index measures prices paid by U.S. residents. It is derived from the prices of the components included in gross domestic purchases.

Heat Content of a Quantity of Fuel, Gross: The total amount of heat released when a fuel is burned. Coal, crude oil, and natural gas all include chemical compounds of carbon and hydrogen. When those fuels are burned, the carbon and hydrogen combine with oxygen in the air to produce carbon dioxide and water. Some of the energy released in burning goes into transforming the water into steam and is usually lost. The amount of heat spent in transforming the water into steam is counted as part of gross heat content but is not counted as part of net heat content. Also referred to as the higher heating value. Btu conversion factors typically used in EIA represent gross heat content.

Heat Content of a Quantity of Fuel, Net: The amount of useable heat energy released when a fuel is burned under conditions similar to those in which it is normally used. Also referred to as the lower heating value. Btu conversion factors typically used in EIA represent gross heat content.

Heavy Oil: The fuel oils remaining after the lighter oils have been distilled off during the refining process. Except for start-up and flame stabilization, virtually all petroleum used in steam-electric power plants is heavy oil.

Hydroelectric Power: The production of electricity from the kinetic energy of falling water.

Imports: Receipts of goods into the 50 States and the District of Columbia from foreign countries and from Puerto Rico, the Virgin Islands, and other U.S. possessions and territories.

Industrial Sector: The industrial sector comprises manufacturing industries, which make up the largest part of the sector, along with mining, construction, agriculture, fisheries, and forestry. Establishments in the sector range from steel mills, to small farms, to companies assembling electronic components.

Jet Fuel: The term includes kerosene-type jet fuel and naphtha-type jet fuel. Kerosene-type jet fuel is a kerosene-quality product used primarily for commercial turbojet and turboprop aircraft engines. Naphtha-type jet fuel is a fuel in the heavy naphthas range used primarily for military turbojet and turboprop aircraft engines.
Kerosene: A petroleum distillate that has a maximum distillation temperature of $401^{\circ} \mathrm{F}$ at the 10-percent recovery point, a final boiling point of $572^{\circ} \mathrm{F}$, and a minimum flash point of $100^{\circ} \mathrm{F}$. Included are the two grades designated in ASTM D3699 (No. 1-K and No. 2-K) and all grades of kerosene called range or stove oil. Kerosene is used in space heaters, cook stoves, and water heaters; it is suitable for use as an illuminant when burned in wick lamps.

Lease and Plant Fuel: Natural gas used in well, field, and lease operations (such as gas used in drilling operations, heaters, dehydrators, and field compressors), and as fuel in natural gas processing plants.

Light Oil: Lighter fuel oils distilled off during the refining process. Virtually all petroleum used in internal combustion and gas-turbine engines is light oil.

Liquefied Petroleum Gases (LPG): Ethane, ethyléne, propane, propylene, normal butane, butylene, and isobutane produced at refineries or natural gas processing plants, including plants that fractionate new natural gas plant liquids.

Lubricants: Substances used to reduce friction between bearing surfaces or as process materials either incorporated into other materials used as processing aids in the manufacturing of other products or as carriers of other materials. Petroleum lubricants may be produced either from distillates or residues. Other substances may be added to impart or improve certain required properties. Excluded are byproducts of lubricating oil refining, such as aromatic extracts derived from solvent extraction or tars derived from deasphalting. Included are all grades of lubricating oils from spindle oil to cylinder oil and those used in greases. Lubricant categories are paraffinic and naphthenic.

Miscellaneous Petroleum Products: All finished petroleum products not classified elsewhere-for example, petrolatum, lube refining byproducts (aromatic extracts and tars), absorption oils, ram-jet fuel, petroleum rocket fuels, synthetic natural gas feedstocks, and specialty oils.

Motor Gasoline: A complex mixture of relatively volatile hydrocarbons, with or without small quantities of additives, obtained by blending appropriate refinery streams to form a fuel suitable for use in spark-ignition engines. Motor gasoline includes 
both leaded and unleaded grades of finished motor gasoline, blending components, and gasohol.

Motor Gasoline Blending Components: Naphthas that will be used for blending or compounding into finished motor gasoline (e.g., straight-run gasoline, alkylate, reformate, benzene, toluene, and xylene). Excluded are oxygenates (alcohols and ethers), butane, and pentanes plus.

Natural Gas: A mixture of hydrocarbons (principally methane) and small quantities of various nonhydrocarbons existing in the gaseous phase or in solution with crude oil in underground reservoirs.

Natural Gasoline: A mixture of hydrocarbons, mostly pentanes and heavier, extracted from natural gas that meets specifications for natural gasoline set by the Gas Processors Association. Includes isopentane.

Nominal Price: The price paid for a product or service at the time of the transaction. The nominal price, which is expressed in current dollars, is not adjusted to remoye the effect of changes in the purchasing power of the dollar.

Nuclear Electric Power: Electricity generated by an electric power plant whose turbines are driven by steam generated in a reactor by heat from the fissioning of nuclear fuel.

Nuclear Electric Power Plant: A single-unit or multiunit facility in which heat produced in one or more reactors by the fissioning of nuclear fuel is used to drive one or more steam turbines.

Nuclear Fuel: Fissionable materials that have been enriched to a composition that, when placed in a nuclear reactor, will support a self-sustaining fission chain reaction, producing heat in a controlled manner for process use.

Pentanes Plus: A mixture of hydrocarbons, mostly pentanes and heavier, extracted from natural gas. Includes isopentane, natural gasoline, and plant condensate.

Petrochemical Feedstocks: Chemical feedstocks derived from petroleum principally for the manufacture of chemicals, synthetic rubber, and a variety of plastics. The categories reported are naphthas less than $401^{\circ} \mathrm{F}$ endpoint and other oils equal to or greater than $401^{\circ} \mathrm{F}$ endpoint.
Petroleum: A generic term applied to oil and oil products in all forms, such as crude oil, lease condensate, unfinished oils, petroleum products, natural gas plant liquids, and nonhydrocarbon compounds blended into finished petroleum products.

Petroleum Coke: A residue that is the final product of the condensation process in cracking. The product is either marketable petroleum coke or catalyst petroleum coke.

Petroleum Coke, Catalyst: The carbonaceous residue that is deposited on and deactivates the catalyst used in many catalytic operations (e.g., catalytic cracking). Carbon is deposited on the catalyst, thus deactivating the catalyst. The catalyst is reactivated by burning off the carbon, which is used as a fuel in the refining process. That carbon or coke is not recoverable in a concentrated form.

Petroleum Coke, Marketable: Those grades of coke produced in delayed or fluid cokers that may be recovered as relatively pure carbon. Marketable petroleum coke may be sold as is or further purified by calcining.

Petroleum Products: Products obtained from the processing of crude oil (including lease condensate), natural gas, and other hydrocarbon compounds. Petroleum products include unfinished oils, liquefied petroleum gases, pentanes plus, aviation gasoline, motor gasoline, naphtha-type jet fuel, kerosene-type jet fuel, kerosene, distillate fuel oil, residual fuel oil, petrochemical feedstocks, special naphthas, lubricants, waxes, petroleum coke, asphalt, road oil, still gas, and miscellaneous products.

Photovoltaic and Solar Thermal Energy (as used at electric utilities): Energy radiated by the sun as electromagnetic waves (electromagnetic radiation) that is converted at electric utilities into electricity by means of solar (photovoltaic) cells or concentrating (focusing) collectors.

Plant Condensate: One of the natural gas liquids, mostly pentanes and heavier hydrocarbons, recovered and separated as liquids at gas inlet separators or scrubbers in processing plants.

Primary Energy: All energy consumed by end users, excluding electricity but including the energy consumed at electric utilities to generate electricity. (In estimating energy expenditures, there are no fuel-associated expenditures for hydroelectric power, geothermal energy, solar energy, or wind energy, and 
the quantifiable expenditures for process fuel and intermediate products are excluded.)

Process Fuel: All energy consumed in the acquisition, processing, and transportation of energy. Quantifiable process fuel includes three categories: natural gas lease and plant operations, natural gas pipeline operations, and oil refinery operations.

Propane: A normally gaseous straight-chain hydrocarbon $\left(\mathrm{C}_{3} \mathrm{H}_{8}\right)$. It is a colorless paraffinic gas that boils at a temperature of $-43.67^{\circ} \mathrm{F}$. It is extracted from natural gas or refinery gas streams. It includes all products designated in ASTM Specification D1835 and Gas Processors Association Specifications for commercial propane and HD-5 propane.

Propylene: An olefinic hydrocarbon $\left(\mathrm{C}_{3} \mathrm{H}_{6}\right)$ recovered from refinery or petrochemical processes.

Real Price: A price that has been adjusted to remove the effect of changes in the purchasing power of the dollar. Real prices, which are expressed in constant dollars, usually reflect buying power relative to a base year.

Refinery (petroleum): An installation that manufactures finished petroleum products from crude oil, unfinished oils, natural gas liquids, other hydrocarbons, and alcohol.

Residential Sector: The residential sector is considered to consist of all private residences, whether occupied or vacant, owned or rented, including single-family homes, multifamily housing units, and mobile homes. Secondary homes, such as summer homes, are also included. Institutional housing, such as school dormitories, hospitals, and military barracks, generally are not included in the residential sector; they are included in the commercial sector.

Residual Fuel Oil: The heavier oils that remain after the distillate fuel oils and lighter hydrocarbons are distilled away in refinery operations and that conform to ASTM Specifications D396 and 975. Included are No. 5, a residual fuel oil of medium viscosity; Navy Special, for use in steam-powered vessels in government service and in shore power plants; and No. 6, which includes Bunker C fuel oil and is used for commercial and industrial heating, electricity generation, and to power ships. Imports of residual fuel oil include imported crude oil burned as fuel.

Road Oil: Any heavy petroleum oil, including residual asphaltic oil, used as a dust palliative and surface treatment on roads and highways. It is generally produced in six grades, from 0 , the most liquid, to 5 , the most viscous.

Short Ton (coal): A unit of weight equal to 2,000 pounds.

\section{SIC: See Standard Industrial Classification.}

Special Naphthas: All finished products within the naphtha boiling range that are used as paint thinners, cleaners, or solvents. Those products are refined to a specified flash point. Special naphthas include all commercial hexane and cleaning solvents conforming to ASTM Specifications D1836 and D484, respectively. Naphthas to be blended or marketed as motor gasoline or aviation gasoline, or that are to be used-as petrochemical and synthetic natural gas (SNG) feedstocks, are excluded.

Standard Industrial Classification (SIC): A set of codes developed by the Office of Management and Budget which categorizes industries into groups with similar economic activities.

\section{Steam Coal: See Coal.}

Still Gas (refinery gas): Any form or mixture of gas produced in refineries by distillation, cracking, reforming, and other processes. The principal constituents are methane, ethane, ethylene, normal butane, butylene, propane, and propylene. It is used primarily as refinery fuel and petrochemical feedstock.

Total Energy: All energy consumed by end users including electricity but excluding the energy consumed at electric utilities to generate electricity. (In estimating energy expenditures, there are no fuelassociated expenditures for hydroelectric power, geothermal energy, solar power, or wind energy, and the quantifiable expenditures for process fuel and intermediate products are excluded.)

Transportation Sector: The transportation sector consists of private and public vehicles that move people and commodities. Included are automobiles, trucks, buses, motorcycles, railroads and railways (including streetcars), aircraft, ships, barges, and natural gas pipelines.

Unfinished Oils: All oils requiring further refinery processing, except those requiring only mechanical blending. Included are naphthas and lighter oils, kerosene and light gas oils, heavy gas oils, and residuum. 
Unfractionated Streams: Mixtures of unsegregated natural gas liquid components, excluding those in plant condensate. This product is extracted from natural gas.

United States: The 50 States and the District of Columbia.

Waxes: Solid or semisolid materials derived from petroleum distillates or residues. Waxes are lightcolored, more or less translucent crystalline masses, slightly greasy to the touch, consisting of a mixture of solid hydrocarbons in which the paraffin series predominates. Included are all marketable waxes, whether crude scale or fully refined. Waxes are used primarily as industrial coating for surface protection.

Wind Energy (as used at electric utilities): The kinetic energy of wind converted at electric utilities into mechanical energy by wind turbines (i.e., blades rotating from a hub) that drive generators to produce electricity for distribution. 


\section{Historical Integrated Energy Data Reports from the Energy Information Administration}

The Monthly Energy Review* (DOE/EIA-0035) presents current monthly data on production, consumption, stocks, imports, exports, and prices of the principal energy commodities in the United States. Also included are data on international production of crude oil, consumption of petroleum products, petroleum stocks, and production of electricity from nuclear-powered facilities.

The Historical Monthly Energy Review* (DOE/EIA-0035(73-88)) presents monthly data from January 1973 through December 1988 for most of the series that are published for current months only in the Monthly Energy Review.

The Annual Energy Review* (DOE/EIA-0384) presents long-term historical annual energy data. Most series begin in 1949. U.S. energy consumption, production, trade, and prices are included. Major sections of the report are energy overview, consumption indicators, financial indicators, energy resources, petroleum, natural gas, coal, electricity, nuclear energy, renewable energy, and international energy.

The State Energy Data Report* (DOE/EIA-0214) presents estimates of annual energy consumption at the State and national levels by major sector (i.e., residential, commercial, industrial, transportation, and electric utilities) and by principal energy type for 1960 forward. The report includes documentation of the consumption estimates for each source of energy, the sources of all data, and a summary of changes made to historical data in the report since its previous release.

The State Energy Price and Expenditure Report* (DOE/EIA-0376) presents annual energy price and expenditure estimates at the State and national levels for selected years. The base year is 1970 . The estimates are presented by energy source (e.g., petroleum, natural gas, coal, and electricity) and by major sector (i.e., residential, commercial, industrial, transportation, and electric utilities). The report includes documentation of the price estimates for each type of energy, the sources of all data, and a summary of any changes made to historical data in the report since its previous release.

The International Energy Annual (DOE/EIA-0219) presents annual data for production, consumption, imports, and exports of primary energy commodities in more than 190 countries, dependencies, and areas of special sovereignty. Also included are prices of crude oil and petroleum products in selected countries. The data presented are derived largely from national publications, international organizations, and other authoritative sources. The data are converted to units of measurement and thermal values familiar to the American public.

The International Petroleum Statistics Report (DOE/EIA-0520) presents current monthly international petroleum data on production, consumption, imports, and stocks. Included are oil consumption and stocks for specific countries in the Organization for Economic Cooperation and Development (OECD). Also provided are the oil supply/consumption balances for the world in quarterly intervals and oil imports by OECD countries.

*Data for this report are also available on computer diskettes.

For further information, contact the:

National Energy Information Center, EI-231

Energy Information Administration

Forrestal Building, Room 1F-048

Washington, DC 20585

202-586-8800

TTY: For people who are deaf or hard of hearing: 202-586-1181

9 a.m. to 5 p.m., eastern time, M-F *U.S. G.P.0.:1993-343-169:80180 